\title{
National Health and Nutrition Examination Survey (NHANES)
}

Laboratory Procedures Manual

J uly 2009 
National Health and Nutrition

Examination Survey

\section{LABORATORY PROCEDURES MANUAL}

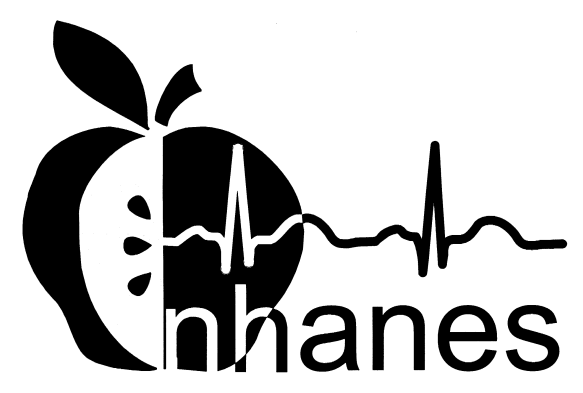

July 2009 


\section{TABLE OF CONTENTS}

\begin{tabular}{|c|c|c|c|c|}
\hline \multicolumn{4}{|c|}{ Chapter } & $\underline{\text { Page }}$ \\
\hline \multirow[t]{17}{*}{1} & \multicolumn{4}{|c|}{ OVERVIEW OF THE NATIONAL HEALTH AND NUTRITION } \\
\hline & \multicolumn{3}{|c|}{ 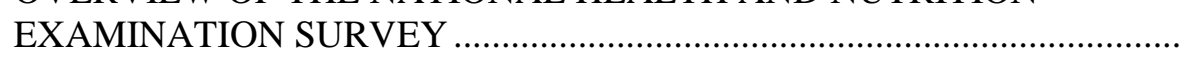 } & $1-1$ \\
\hline & 1.1 & \multicolumn{2}{|c|}{ History } & $1-1$ \\
\hline & 1.2 & \multicolumn{2}{|c|}{ 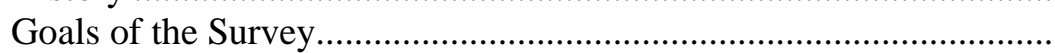 } & $1-4$ \\
\hline & 1.3 & \multicolumn{2}{|c|}{ 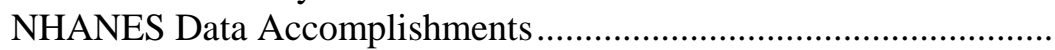 } & $1-4$ \\
\hline & 1.4 & \multicolumn{2}{|c|}{ 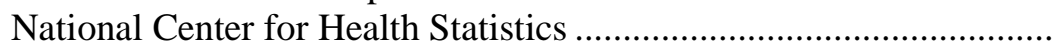 } & $1-6$ \\
\hline & 1.5 & \multicolumn{2}{|c|}{ 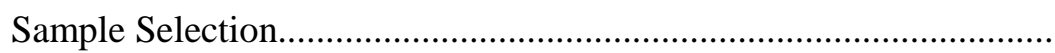 } & $1-6$ \\
\hline & 1.6 & \multicolumn{2}{|c|}{ 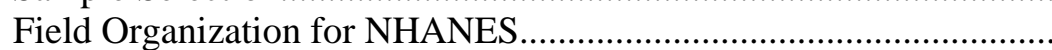 } & $1-7$ \\
\hline & 1.7 & \multicolumn{2}{|c|}{ Exams and Interviews in the Mobile Examination Center (MEC) ..... } & $1-10$ \\
\hline & & 1.7.1 & 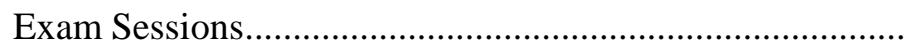 & $1-11$ \\
\hline & & 1.7.2 & 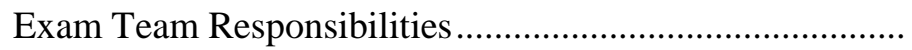 & $1-12$ \\
\hline & & 1.7 .3 & 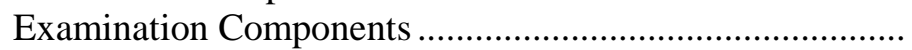 & $1-13$ \\
\hline & & 1.7 .4 & 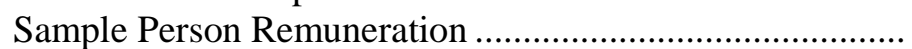 & $1-20$ \\
\hline & & 1.7 .5 & 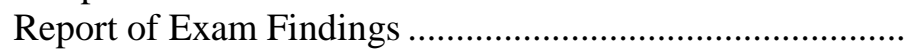 & $1-20$ \\
\hline & & 1.7.6 & 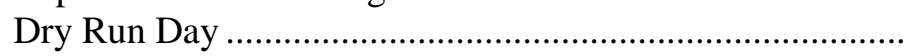 & $1-21$ \\
\hline & 1.8 & Integ & 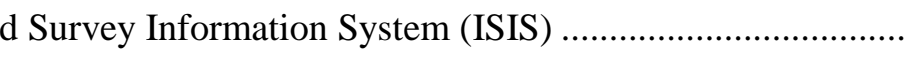 & $1-21$ \\
\hline & 1.9 & Confi & 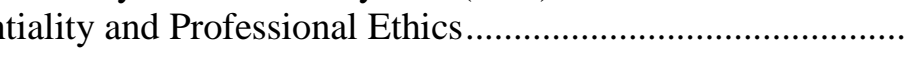 & $1-22$ \\
\hline \multirow[t]{10}{*}{2} & \multicolumn{3}{|c|}{ OVERVIEW AND LABORATORY TEAM RESPONSIBILITIES ................ } & $2-1$ \\
\hline & 2.1 & \multicolumn{2}{|c|}{ 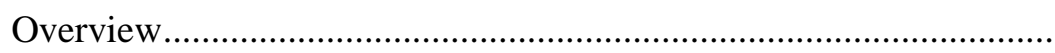 } & $2-1$ \\
\hline & 2.2 & \multicolumn{2}{|c|}{ The Laboratory Team } & $2-2$ \\
\hline & 2.3 & \multicolumn{2}{|c|}{ Tasks } & $2-2$ \\
\hline & 2.4 & \multicolumn{2}{|c|}{ 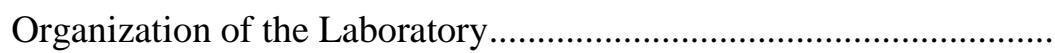 } & $2-3$ \\
\hline & 2.5 & \multicolumn{2}{|c|}{ 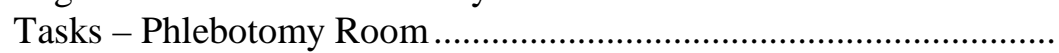 } & $2-5$ \\
\hline & 2.6 & \multicolumn{2}{|c|}{ 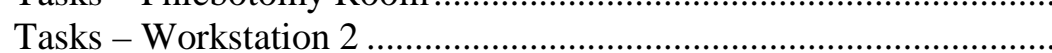 } & $2-5$ \\
\hline & 2.7 & \multicolumn{2}{|c|}{ 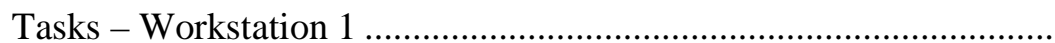 } & $2-7$ \\
\hline & 2.8 & \multicolumn{2}{|c|}{ Tasks - Workstation 3} & $2-8$ \\
\hline & 2.9 & \multicolumn{2}{|c|}{ 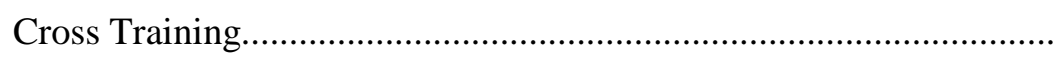 } & $2-9$ \\
\hline \multirow[t]{8}{*}{3} & LAF & RATO & 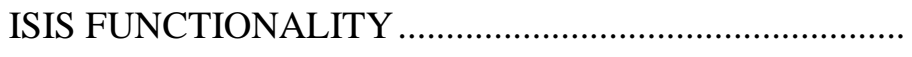 & $3-1$ \\
\hline & 3.1 & \multicolumn{2}{|c|}{ 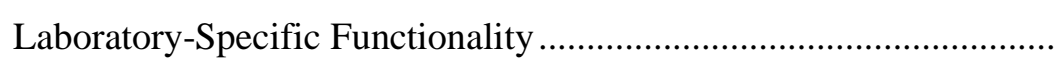 } & $3-1$ \\
\hline & 3.2 & \multicolumn{2}{|c|}{ Laboratory Heads-up Display } & $3-1$ \\
\hline & 3.3 & \multicolumn{2}{|c|}{ Laboratory Task Bar } & $3-3$ \\
\hline & 3.4 & \multicolumn{2}{|c|}{ 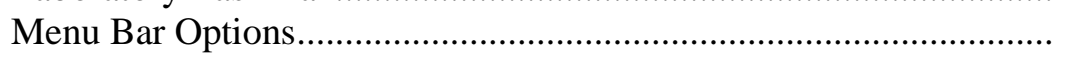 } & $3-4$ \\
\hline & & 3.4 .1 & 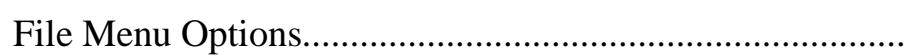 & $3-4$ \\
\hline & & 3.4 .2 & 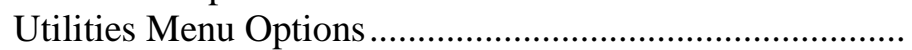 & $3-6$ \\
\hline & & 3.4 .3 & 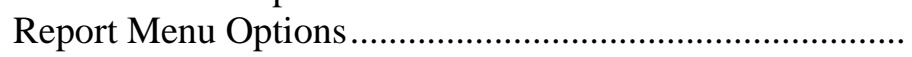 & $3-8$ \\
\hline
\end{tabular}




\section{TABLE OF CONTENTS (CONTINUED)}

3.4.4 Modules......................................................................... 3-9

3.4.5 Shipping ….............................................................. $\quad 3-10$

3.5 Informed Consent Exclusions............................................................ 3-11

3.6 Set Session Utility...................................................................... 3-14

3.7 Container Map ............................................................................. 3-16

3.8 Subsample Preview......................................................................... 3-19

3.9 Informed Consent Status................................................................... 3-21

3.10 Access a Processing Module............................................................... 3-23

3.11 Warning and Error Messages........................................................... 3-24

3.11.1 Not Filled Require a Comment ............................................ 3-25

3.11.2 Labels Must be Printed in Advance ................................... 3-25

3.12 Print an Ad Hoc Label .................................................................... 3-27

3.13 Coulter, Urine Scale, and Osmolality Icons..................................... 3-28

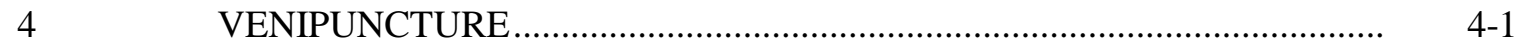

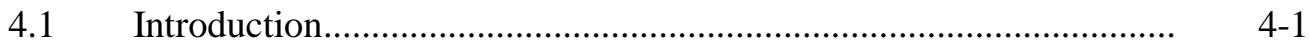

4.2 Equipment and Supplies .................................................................. 4.

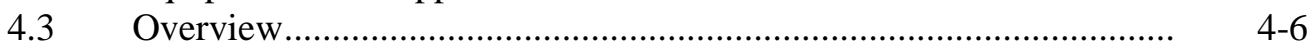

$4.4 \quad$ Gaining Cooperation........................................................

4.4.1 Refusal Conversion ........................................................... $\quad 4-12$

4.5 Performing the Venipuncture on SPs who do not Speak English...... 4-12

4.6 Open the Exam and Log the SP into the Exam.................................. 4-13

4.7 The Phlebotomy Interview.............................................................

4.7.1 Pregnancy Shared Exclusion Question .............................. 4 4-18

4.7.2 Safety Exclusion Questions.............................................. $\quad 4-21$

4.8 Administering the Fasting Questionnaire ........................................ 4-31

$4.9 \quad$ Venipuncture Procedures ............................................................... 4-38

4.9.1 Preparation of the Puncture Site........................................... 4-39

4.9.2 Venipuncture Technique for the BD Saf-T EZ $\AA$ Blood
Collection Set (19 Gauge)...................................................

4.9.3 Venipuncture Technique for the BD Safety-Lok ${ }^{\mathrm{TM}}$ Blood
Collection Set (21 and 23 Gauge) ........................................

Venipuncture Technique for the Eclipse Multisample
Needle ......................................................................... 


\section{TABLE OF CONTENTS (CONTINUED)}

4.9.5 Concluding the Venipuncture............................................ 4-46

$4.10 \quad$ Pediatric Venipuncture ................................................................. 4-48

4.10.1 Age-Specific Techniques in Phlebotomy Competency....... $\quad$ 4-50

4.11 Hand Venipuncture ........................................................................... 4-64

4.12 Recording the Results of the Venipuncture Procedure ....................... 4-65

4.13 Venipuncture Status ....................................................................... 4-69

4.14 Repeat Blood Draw....................................................................... 4-73

4.15 Phlebotomy Quality Control ......................................................... 4-75

4.15.1 Daily QC ........................................................................ 4-76

4.15.2 Supply Use Control Log................................................ 4 4-78

4.16 Red Cross Procedures for Handling Fainting (Syncope) .................... $\quad$ 4-81

4.17 How to Deal With System Failure ....................................................... 4-83

URINE SPECIMEN COLLECTION AND PROCESSING ..........................

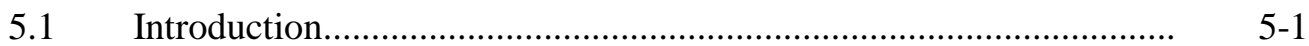

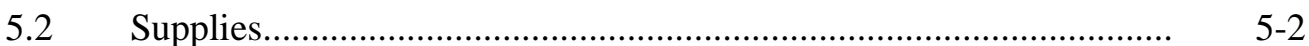

5.3 Urine Flow Rate Background .......................................................

5.4 Using the Scale for Urine Collection.................................................. 5-4

5.5 Document Urine Collection ..........................................................

5.6 Manually Documenting Urine Collection............................................

5.6.1 Manually Document Urine Collection With Barcode

Scanning .......................................................................... 5-23

5.6.2 Manually Document Urine Collection Without
Barcode Scanning........................................................... $\quad 5-24$

5.7 Urine Specimen Assays ............................................................. $5-27$

$5.8 \quad$ Urine Specimen Protocols .......................................................... $5-27$

5.9 Labeling Urine Processing Vessels.................................................. 5-29

5.10 Urine Specimen Processing ......................................................... 5 5

5.11 Record the Results of Urine Specimen Processing ............................ $\quad$ 5-30

5.12 Second and Third Urine Samples ................................................... $\quad 5-40$

5.13 Specimen Storage ........................................................................ $\quad 5-40$

5.14 How to Deal With System Failure ..................................................... 5-41 


\section{TABLE OF CONTENTS (continued)}

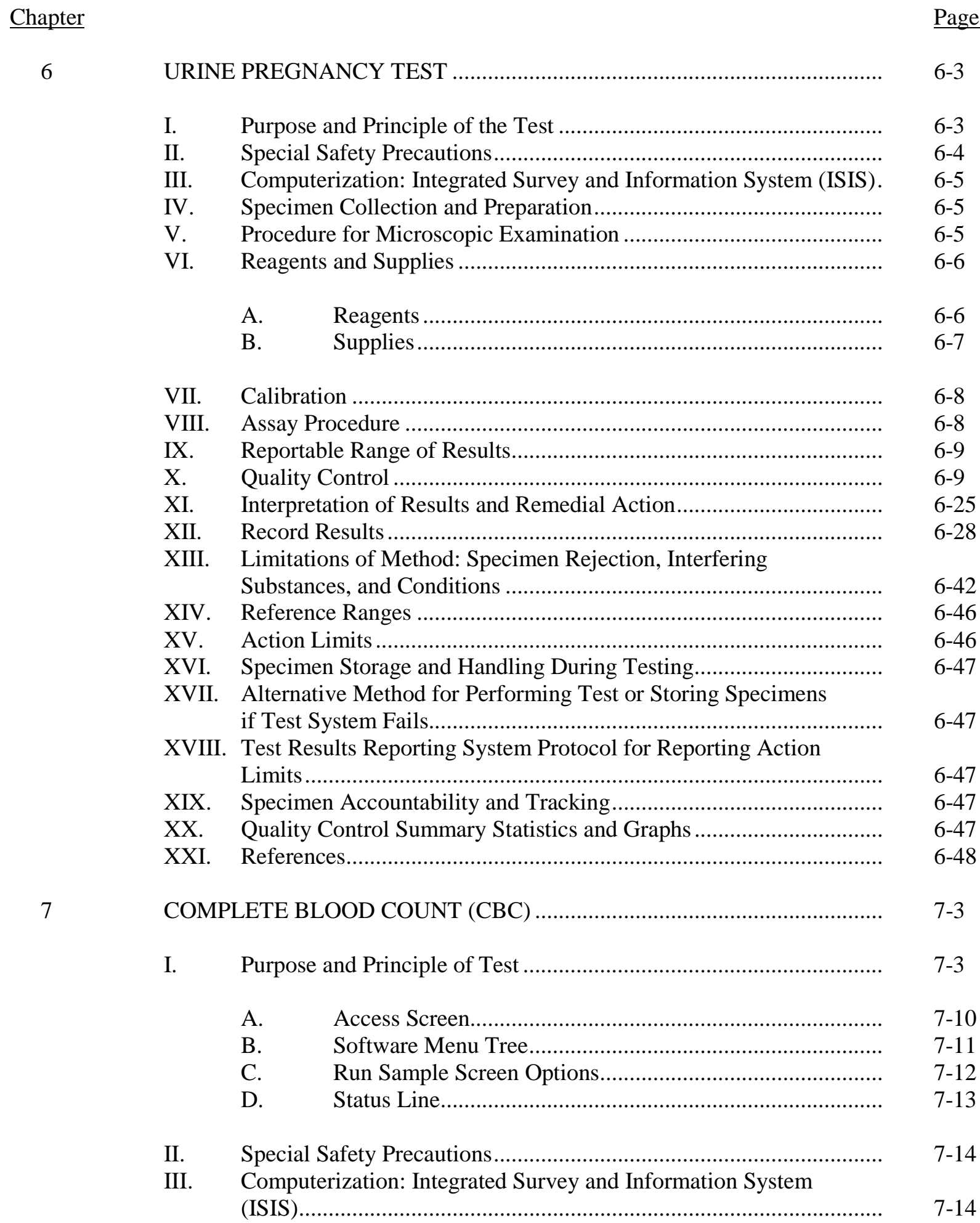




\section{TABLE OF CONTENTS (continued)}

IV. Specimen Collection and Preparation

A. Specimen Collection

V. Procedure for Microscopic Examination

VI. Reagents and Supplies

A. Reagents, Controls, and Calibrators................................... $\quad$ 7-15

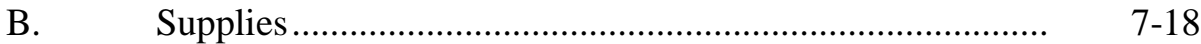

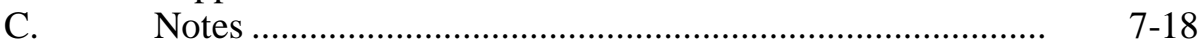

VII. Calibration .................................................................................

A. Perform Calibration:..........................................................

B. Verify the Calibration of Your Instrument:......................... 7-19

C. $\quad$ Pre-calibration, Reproducibility, and Carryover Check...... 7-21

D. $\quad$ CBC Calibration with S-CAL ${ }^{\circledR}$ Calibrator ….......................

VIII. Assay Procedure ............................................................................ $\quad$ 7-30

A. Run Start Up at the Beginning of the First Session Each

B. Run Controls ................................................................... $\quad$ $\quad$ 7-32

C. Run Sample Persons......................................................... $\quad$ 7-32

D. Daily Shut Down.............................................................. 7-38

E. Clean Cycle .....................................................................

F. $\quad$ Prolonged Shut Down ................................................ $\quad$ 7-39

G. Autopurge Cycle ............................................................. $\quad$ 7-40

H. Beginning and End of Stand Operations ........................... $\quad$ 7-41

IX. Coulter ${ }^{\circledR}$ Reportable Range of Results............................................ $\quad$ 7-54

X. Quality Control .............................................................................

A. Latron ${ }^{\circledR}$ Primer and Latex Controls.................................... 7-55

B. $\quad 5 C \circledR$ Cell Controls Tri Pack contains Normal, Abnormal I, Abnormal II ................................................. 7-58

C. Control Statistics and Graphs......................................... $\quad$ 7-62

D. $\quad$ Record 5C Cell and Latron Lot Numbers in ISIS ............... 7-65

E. Interlaboratory Quality Assurance Program (IQAP)........... 7-76

F. $\quad$ Proficiency Testing ........................................................ $\quad$ 7-78

G. Linearity for WBC, RBC, Hgb, and Plt Parameters............ $\quad$ 7-79 


\section{TABLE OF CONTENTS (continued)}

XI. Interpretation of Results and Remedial Action

A. Sample Person Hemoglobin and Hematocrit Review and Remedial Actions.

B. Sample Person Parameter Value Review and Remedial Actions.

C. HMX Parameter Codes ...

$7-116$

D. HMX Suspect Messages

$7-118$

E. Physician Review .....

XII. Limitations of Method: Specimen Rejection, Interfering Substances, and Conditions

A. Specimen Rejection

$7-123$

B. Interfering Substances and Conditions

XIII. Reference Ranges

$7-126$

XIV. Action Limits

$7-127$

XV. Specimen Storage and Handling During Testing.

$7-128$

A. Specimen Storage

XVI. Alternative Method for Performing Test or Storing Specimens if Test System Fails.

XVII. Test Results Reporting System: Protocol for Reporting Action Limits

XVIII. Quality Control Summary Statistics and Graphs

XIX. References.

8.1

Introduction.

8-1

8.2

8.3

Equipment and Supplies ......

8-1

Protocols

8-2

8.4

Blood Processing Age-Specific Protocols ........

8-7

8.5

Labeling Vessels

8-13

8.6

Equipment.

8.6.1 Benchtop Centrifuges

8.6.2 Laminar Flow Biological Safety Cabinet Operating Sequence 


\section{TABLE OF CONTENTS (continued)}

8.7 Blood Processing Procedures

8.7.1 Eppendorf Research Pro Pipettes ........................................ 8-22

8.7.2 Process the 3-mL. 4-mL, 7-mL, and 10-mL EDTA

8.7.3 Process the 2-mL Gray Tube for Glucose and OGTT......... 8-54

8.7.4 Process the 3-mL, 7-mL, 10-mL, and 15-mL Red Top Tubes for Pooled Sera.

8.7.5 Process the 10-mL Red Top Tubes for

8.7.6 Reagent Processing and Preparation ................................ 8 8-57

8.8 Record the Results of Specimen Processing ....................................... 8-57

8.9 Washed Packed Cell Processing ................................................................ 8-73

8.10 Blood Specimen Storage.................................................................. 8-81

8.10.1 Blood Specimen Storage Protocol......................................... 8-81

8.11 How to Deal With System Failure ..................................................... 8-83

PRINTING LABELS AND SPECIMEN SHIPMENT .................................. 9-1

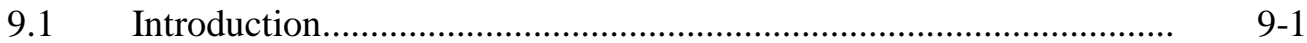

9.2 Equipment and Supplies ................................................................... 9-5

9.3 Print Vessel Labels and Label Blood Processing Racks...................... 9-5

9.3.1 Print Vessel Labels Using the Print Labels Module............ $\quad 9-7$

9.3.2 Print Vessel Labels Using the Heads-up Display................ 9 9-18

9.3.3 Rescheduled Appointments Report.................................... 9 9-20

9.4 Regeneration of Data .................................................................. $\quad 9-22$

9.5 Create New Container Labels .......................................................... 9-. 9-29

9.6 Trash or Reprint Container Labels.................................................. 9-43

9.6.1 Reprint Container Labels for Existing Containers .............. 9-46

9.7 Shipping Overview ...................................................................... $\quad 9-50$

9.8 Create Air Bills using FedEx Ship Manager...................................... 9-51

9.8.1 Create Air Bills for Domestic Shipments........................... 9-52

9.9 Close Containers ........................................................................... 9-77

9.10 Assign Containers to Shippers ........................................................... 9-81 


\section{TABLE OF CONTENTS (continued)}

9.11 Create Shipping Manifests

9.12 Informed Consent Exclusions

9.13 Packing Procedure for the Shippers

9.13.1 Pack Boxed Specimens ..................................................... 9-107

9.13.2 Pack Medium Shipping Containers for

Frozen Shipment

9.13.3 Pack Small Shipping Containers for Frozen Shipment

9.13.4 Pack Medium Shipping Container for Refrigerated Shipment

9.13.5 Label Shippers.

9.14 Container Protocol and Weekly Shipping List

Introduction

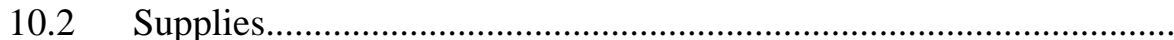

$10-2$

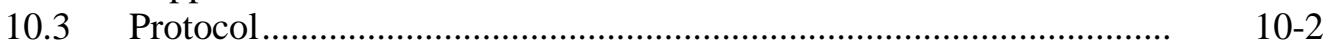

$10.4 \quad$ Labeling Vessels ........................................................................... 10

10.5 HPV Protocol.................................................................... 10

10.6 Record Processing Results....................................................... 10-3

10.7 Specimen Storage ....................................................................... 10.

10.8 Shipping Procedures ........................................................ $10-12$

10.8.1 Pack and Ship Vessel 78 at Room Temperature ................. 10-13

10.8.2 Label Shippers........................................................... $10-13$

10.9 How to Deal With System Failure ................................................ 10-14

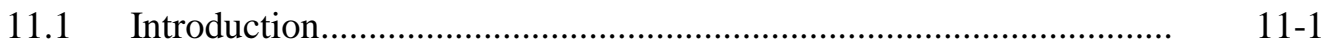

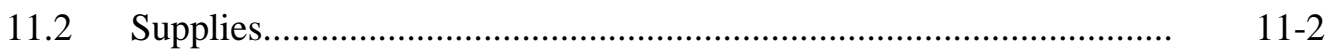

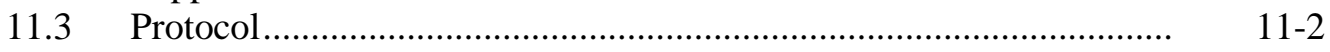

11.4 Labeling Vessels ....................................................................... $11-3$

11.5 HPV Rinse Protocol.................................................................. 11-3

11.6 Record Processing Results .............................................................. 11-4

11.7 Specimen Storage ......................................................................... 11-9

11.8 Shipping Procedures .................................................................. 11-9

11.9 How to Deal With System Failure ................................................. 11-10 
TABLE OF CONTENTS (continued)

Chapter

OSMOLALITY TESTING.....

Introduction.

$12-3$

I. Purpose and Principle of Test

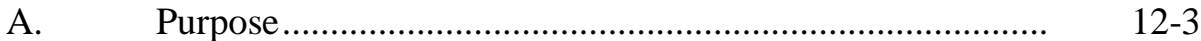

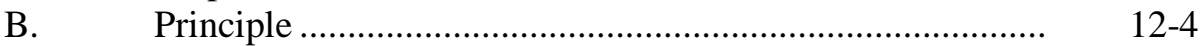

II. Special Safety Precautions............................................................... 12-6

III. Computerization: Integrated Survey and Information System (ISIS). 12-7

IV. Specimen Collection and Preparation ................................................ 12-7

A. Specimen Collection ..................................................... 12-7

B. Specimen Preparation...................................................... 12-7

V. Procedure for Microscopic Examination .......................................... 12-8

VI. Equipment, Reagents, and Supplies................................................ 12-8

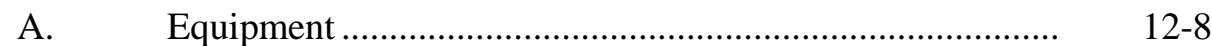

B. Controls and Calibrators ................................................... 12-9

C. Supplies ............................................................... 12-10

VII. Calibration ................................................................................. 12-11

A. Precalibration ................................................................. 12-11

B. $\quad$ Osmette Total Calibration with Standards .......................... 12-11

C. Osmette Troubleshooting Calibration with Standards When QC is Not Within the Acceptable Range .................. 12-12

VIII. Assay Procedure …....................................................................... 12-13

A. Osmette II Instrument Placement........................................ 12-14

B. Instrument Buttons and Functions...................................... 12-14

C. Proper Pipetting Technique ................................................. 12-15

D. Running SP Samples...................................................... 12-16

E. Daily Shut-down Procedures............................................ 12-18

F. $\quad$ Verifying the Pipette Calibration ......................................... 12-18

G. Cleaning, Opening, and Oiling the Osmette II Probe.......... 12-20

IX. Reportable Range of Results........................................................... 12-22 


\section{TABLE OF CONTENTS (continued)}

X. Quality Control

A. Standards and CON-TROL Principle

$12-22$

B. Standards and CON-TROL Storage and Handling

$12-22$

C. Quantimetrix Controls Principle

$12-23$

D. Quantimetrix Controls Storage and Handling.

$12-24$

E. Running Quality Control Samples

F. Record Precision Systems Standards and CON-TROLs in ISIS

G. Record Quantimetrix Human Urine Controls in ISIS

$12-32$

H. Proficiency Testing ......................................................... 12-54

I. Linearity for Osmolality.................................................... 12-54

XI. Interpretation of Results and Remedial Action................................. 12-54

A. Sample Person Parameter Value Review and Remedial

Actions.

$12-54$

B. Osmette II Instrument Messages

$12-68$

XII. Limitations of Method: Specimen Rejection, Interfering Substances and Conditions

A. Specimen Rejection......................................................... 12-69

B. Interfering Substances and Conditions................................ 12-69

XIII. Reference Ranges .......................................................................... 12-69

XIV. Action Limits ............................................................................... 12-69

XV. Specimen Storage and Handling during Testing ............................ $\quad 12-70$

A. Specimen Storage ........................................................ 12-70

XVI. Alternative Method for Performing Test or Storing Specimens if Test System Fails.

XVII. Test Results Reporting System: Protocol for Reporting Action Limits.....

XVIII. Quality Control Summary Statistics and Graphs ............................... 12-70

XIX. References................................................................................ 12-70

13.1 OGTT Application - Section Two (Blood Draw) ............................ 13-1

13.2 Laboratory Processing 


\section{TABLE OF CONTENTS (continued)}

\section{Chapter}

13.2.1 Laboratory Overview ......................................................... 13-8

13.2.2 Process the 2-mL Gray Top Tube for OGTT ....................... 13-8

13.2.3 Record the Results of Specimen Processing ....................... 13-9

13.2.4 Vessel 98 Storage Protocol .................................................. 13-20

13.2.5 Vessel 98 Shipping Protocol ................................................ 13-20

13.2.6 Performing the OGTT on SPs Who Do Not Speak English 13-22

14 QUALITY CONTROL AND QUALITY ASSURANCE - POLICIES

AND PROCEDURES ......................................................................... $14-1$

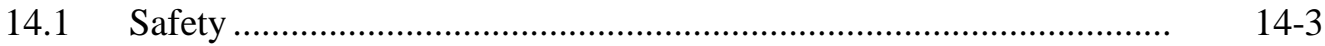

14.1.1 Introduction ................................................................ $14-3$

14.2 Specimen Submission and Handling .............................................. 14-3

14.2.1 Introduction ...................................................................

14.2.2 Identification of Specimens............................................... 14-4

14.2.3 Questions on Specimen Submission and Handling............. 14-4

14.3 Test Requisition ....................................................................... 14-4

14.3.1 Introduction .............................................................. 14-4

14.3.2 Laboratory Tests............................................................. 14-5

14.3.3 Human Subjects Review ................................................... 14-5

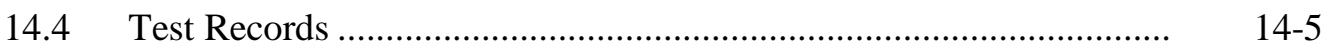

14.4.1 Introduction ....................................................................

14.4.2 Components of Test Records ........................................... 14-6

14.5 Test Report...................................................................... 14-6

14.5.1 Introduction.................................................................. 14-6

14.5.2 Review, Approval, and Release of Test Results ................. 14-6

14.5.3 List of Methods and Method Details.................................. 14-7

14.5.4 Format of the Test Report ................................................... 14-8

14.5.5 Format for Laboratory Report Cover Letter........................ 1 14-8

14.5.6 Format for Listing of Laboratory Results .......................... $\quad 14-8$

14.6 Quality Control ......................................................................... 14-9

14.6.1 Introduction ................................................................. $14-9$ 


\section{TABLE OF CONTENTS (continued)}

\section{Chapter}

14.6.2 Method Specific QC Procedures ......................................... 14-10

14.6.3 QC Definitions ................................................................. $14-10$

14.6.4 Overview of the Relationship between Internal QC, Proficiency Testing, and External QC................................ $14-12$

14.6.5 Internal ("Bench”) QC ....................................................... 14-13

14.6.6 Coulter Interlaboratory Quality Assurance

14.6.7 Proficiency Testing ........................................................ 14-15

14.6.8 Calibration Verification ................................................. 14-17

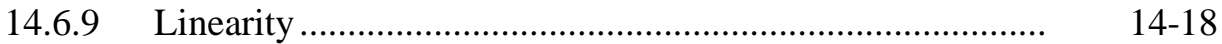

14.6.10 Quality Control Records ................................................ 14-19

14.7 Test Methods, Equipment, Reagents, Supplies, and Facilities ........... $\quad$ 14-20

14.7.1 Introduction ................................................................... 14-20

14.7.2 Labeling of Reagents, Solutions, Supplies.......................... 14-20

14.7.3 Facilities ......................................................................... 14-20

14.8 Analytical Procedure Manual .............................................................. 14-21

14.8.1 Introduction .................................................................... 14-21

14.8.2 Contents of the Analytical Procedure Documentation ........ 14-21

14.8.3 Approval and Record Maintenance..................................... 14-22

14.8.4 Method Performance Specifications .................................. 14-22

14.9 Equipment Maintenance and Function Checks................................ 14-23

14.9.1 Introduction ................................................................. 14-23

14.9.2 Eppendorf Pipette Calibration............................................ 14-23

14.9.3 Temperature Monitoring ..................................................... 14-24

14.10 Calibration and Calibration Verification .......................................... 14-26

14.10.1 Introduction..................................................................... 14-26

$\begin{array}{ll}\text { 14.10.2 Comparison of Methods Performed on Multiple } & \begin{array}{l}\text { Com } \\ \text { Instruments or at Multiple Sites ............................................ 14-27 }\end{array}\end{array}$

14.11 Remedial Actions............................................................................. 14-27

14.11.1 Introduction................................................................. $14-27$ 


\section{TABLE OF CONTENTS (continued)}

14.12 Integrated Survey Information System (ISIS)

14.12.1 Introduction

14.12.2 Integrated Survey and Information System Data

Down Times

14.12.3 Maintenance of LAN Hardware.......................................... 14-28

14.12.4 Backup Schedule of Integrated Survey and Information System Data

14.13 Communications and Complaints

14.13.1 Introduction

14.14 Quality Promotion

14.14.1 Introduction

14.15 Quality Assurance Program.

14.15.1 Introduction

14-30

14.15.2 Quality Audit: Survey participant Test Management...........

$14-31$

14.15.3

Quality Audit: Quality Control

$14-32$

14.15.4

Quality Audit: Personnel.

$14-32$

14.15.5 Quality Assurance Review with Staff

14-33

14.16 Personnel Training and Evaluation

14-33

15.1 Start of Stand Activities

15.3.1 General Inventory Procedures............................................. 15-8

15.3.2 End of Stand Inventory Procedures................................... 15-9

15.3.3 Mid-Stand Inventory Procedures ......................................... 15-10

15.3.4 Start of Stand Inventory Procedures...................................... 15-11

15.3.5 Consumables vs. Nonconsumables .................................... 15-12

15.3.6 Shipping Excess Inventory Back to the Warehouse............ 15-12

15.3.7 Tracking of Expired and Broken Inventory ......................... 15-13 


\section{TABLE OF CONTENTS (CONTINUED)}

Chapter

15.4 Dry Run

$15-13$

15.4.1 Phlebotomy Protocol and Procedures .................................. 15-14

15.4.2 Printing Labels, Urine Processing, and Pregnancy Testing 15-17

15.4.3 Urine Processing ............................................................... $15-30$

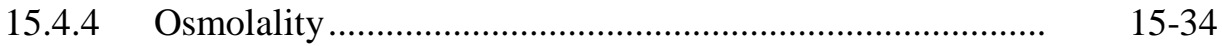

15.4.5 Hematology ............................................................... 15-35

15.4.6 Blood Processing and Storage ............................................. 15-35

15.4.7 Shipping Dry Run Specimens .......................................... 15-38

15.5 Centrifuges - Equipment Maintenance............................................ 15-39

15.6 Refrigerators and Freezers - Equipment Maintenance ........................ 15-40

15.7 Use of the Temperature Recording Instrument.................................. 15-41

15.8 Use of the Class II Type A Biological Safety Cabinet ........................ 15-43

15.9 Ambient Air Temperature............................................................... 15-57

15.10 Precision Instruments Osmette II....................................................... 15-57

15.11 Mettler Toledo Scale.................................................................... 15-61

15.12 Laboratory Quality Control Module .................................................. 15-64

15.13 Unusual Field Occurrence (UFO) ...................................................... 15-75

15.14 Hard Copy Supply Use Control Log................................................... 15-77

15.15 Eppendorf Pipette Care, Sterilization, Maintenance, and Calibration $\quad$ 15-79

15.16 Power Loss and Laboratory Operations............................................. 15-81

15.16.1 Scenario 1 - Power Loss during Exam Session with No Backup Generator.......................................................... 15-81

15.16.2 Scenario 2 - Power Loss when the MEC is Not Conducting Exams ............................................................. 15-84

15.16.3 Scenario 3 - Power Loss with a Backup Generator................. 15-85

List of Appendixes

$\underline{\text { Appendix }}$

A International Air Transport Association (IATA) Dangerous

Goods Regulations - January 2009.............................................................. A A

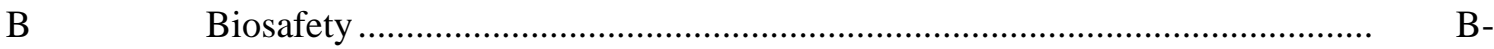

C Laboratory Protocol …......................................................................... C-1

D $\quad$ Laboratory and Phlebotomy Inventory …................................................. D-1 


\title{
TABLE OF CONTENTS (continued)
}

\author{
List of Tables
}

$\underline{\text { Table }}$

$\underline{\text { Page }}$

$5-1$

NHANES urine processing protocol - primary

NHANES urine processing protocol - VIP guest.

5-3 Storage and shipping protocol for urine.

\section{List of Figures}

$\underline{\text { Figure }}$

Laboratory layout.

$2-4$

$2-2$

Phlebotomy room layout

$2-4$

$4-1$

Floor plan for phlebotomy room.

4-4

4-2

VM 5000 series phlebotomy chair

Blood collection cart

12-2 Example of a urine collection bar-code label...

12-3 Example of Quantimetrix bar-code labels

\section{List of Exhibits}

$\underline{\text { Exhibit }}$ 


\title{
TABLE OF CONTENTS (continued)
}

\author{
List of Exhibits (continued)
}

\begin{tabular}{|c|c|}
\hline xhibit & \\
\hline $4-3$ & 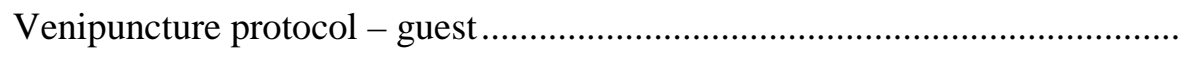 \\
\hline $4-4$ & 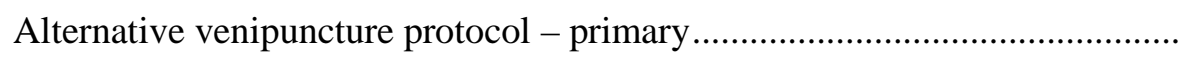 \\
\hline $4-5$ & Equipment and supplies - phlebotomy \\
\hline $4-6$ & 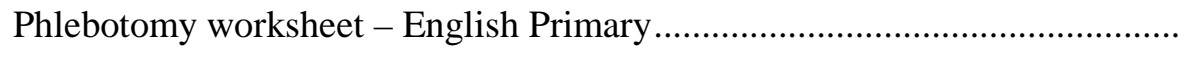 \\
\hline $4-7$ & 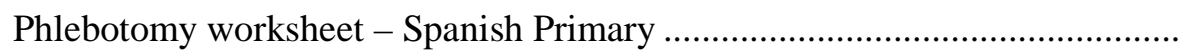 \\
\hline $5-1$ & 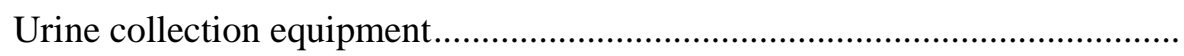 \\
\hline $5-2$ & 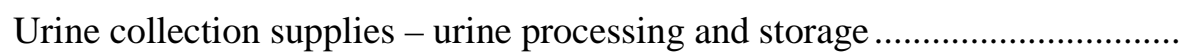 \\
\hline $5-3$ & 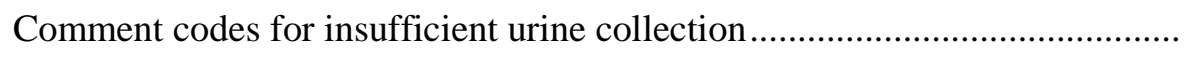 \\
\hline $5-4$ & 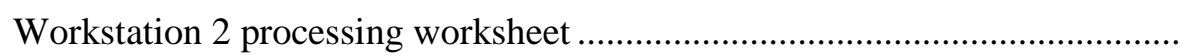 \\
\hline $8-1$ & 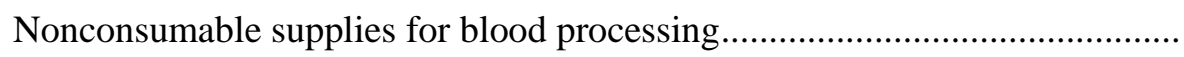 \\
\hline $8-2$ & 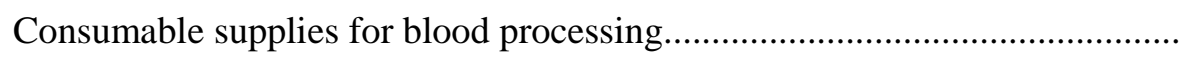 \\
\hline $8-3$ & 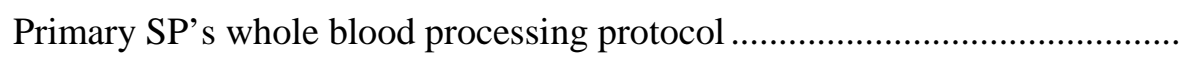 \\
\hline $8-4$ & 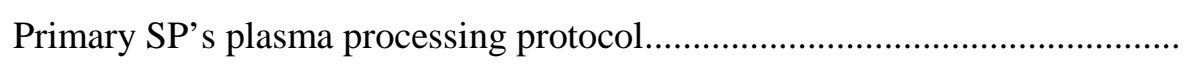 \\
\hline $8-5$ & Primary SP's packed cells processing protocol \\
\hline $8-6$ & Primary SP's serum processing protocol \\
\hline $8-7$ & 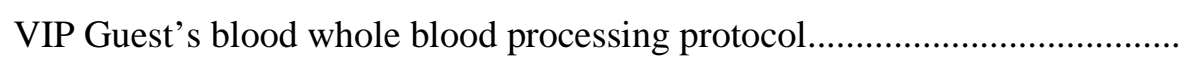 \\
\hline $8-8$ & VIP Guest's plasma processing protocol \\
\hline $8-9$ & 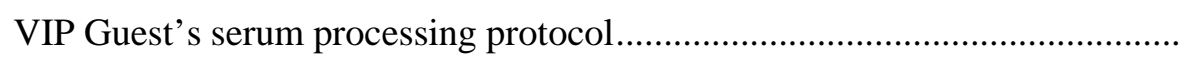 \\
\hline $8-10$ & Guest serum processing protocol \\
\hline $8-11$ & 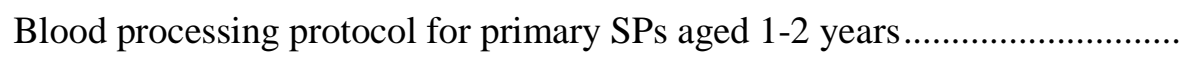 \\
\hline $8-12$ & 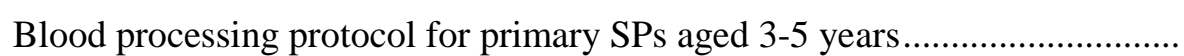 \\
\hline
\end{tabular}




\title{
TABLE OF CONTENTS (continued)
}

\author{
List of Exhibits (continued)
}

$\underline{\text { Exhibit }}$

$\underline{\text { Page }}$

8-13 Blood processing protocol for primary SPs aged 6-11 years............................ 8-8

8-14 Blood processing protocol for primary SPs aged 12-19 years.......................... 8-9

8-15 Blood processing protocol for primary SPs aged 20+ years .............................. 8-10

8-16 Blood processing protocol for VIP guest aged 1-2 years................................... 8-11

8-17 Blood processing protocol for VIP guest aged 3-5 years.................................. 8-11

8-18 Blood processing protocol for VIP guest aged 6-11 years................................ 8-12

8-19 Blood processing protocol for VIP guest aged 12+ years................................ 8-12

8-20 Blood processing protocol for guest aged 12+ years ...................................... 8-12

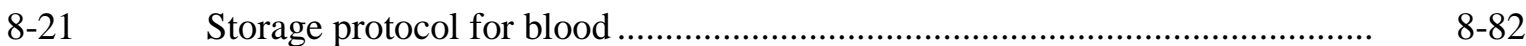

8-22 Workstation 1 processing worksheet ......................................................... $\quad$ 8-84

9-1 Blood and urine shipping protocol................................................................ 9-2

9-2 Supplies for labels/shipping .............................................................. $\quad 9-5$

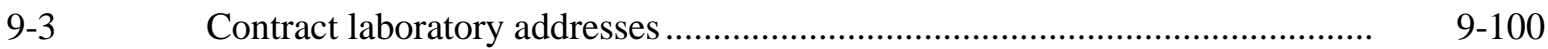

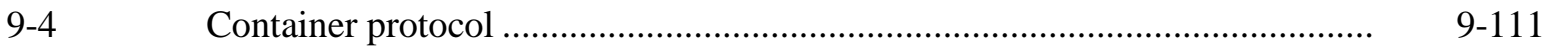

9-5 FedEx shipping list by lab name ...........................................................

9-6 FedEx shipping list by lab number …...................................................... $\quad$ 9-115

10-1 Equipment and supplies - HPV processing and storage $\quad$ E............................... 10-2

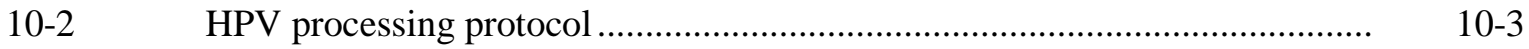

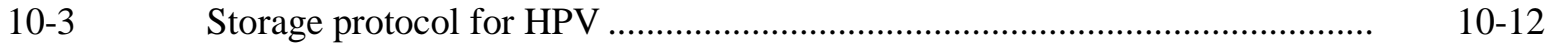

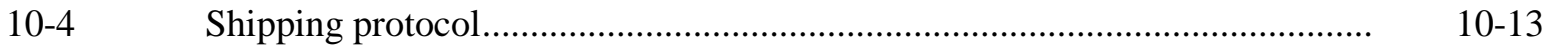




\title{
TABLE OF CONTENTS (continued)
}

\author{
List of Exhibits (continued)
}

\begin{tabular}{|c|c|}
\hline xhibit & \\
\hline $10-5$ & HPV Processing worksheet............................ \\
\hline $11-1$ & 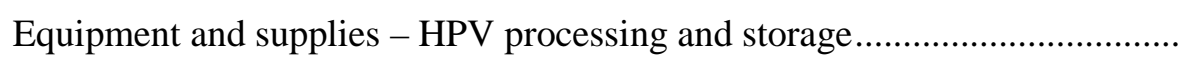 \\
\hline $11-2$ & 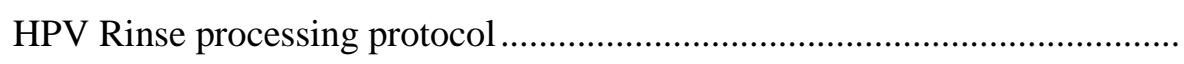 \\
\hline $11-3$ & Storage protocol for HPV \\
\hline $11-4$ & Shipping protocol. \\
\hline $11-5$ & 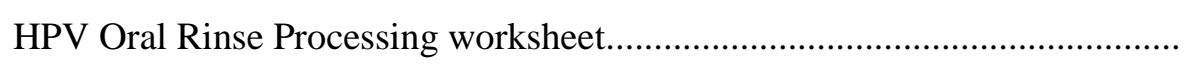 \\
\hline $12-1$ & 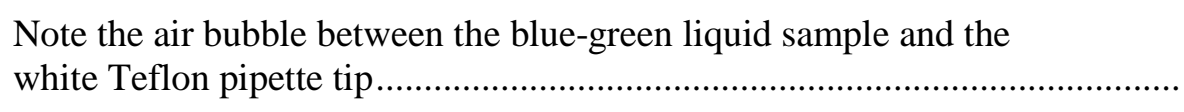 \\
\hline $13-1$ & Primary SP's OGTT processing protocol \\
\hline $13-2$ & 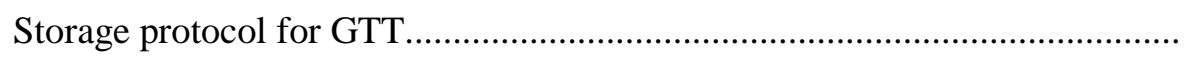 \\
\hline $13-3$ & 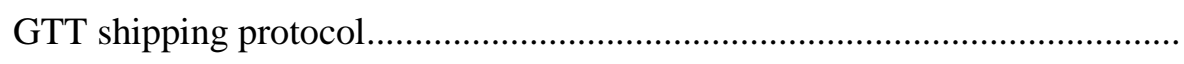 \\
\hline $13-4$ & 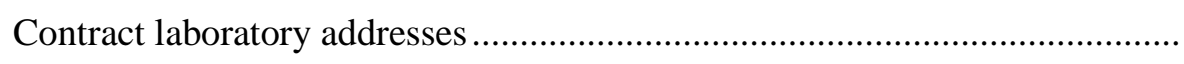 \\
\hline $15-1$ & 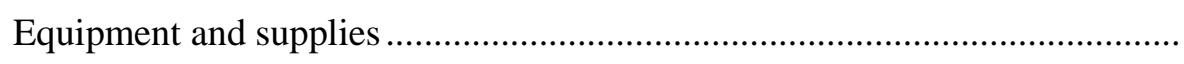 \\
\hline $15-2$ & 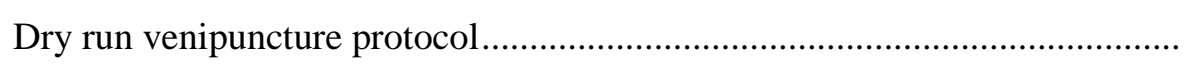 \\
\hline $15-3$ & Dry run urine processing protocol \\
\hline $15-4$ & 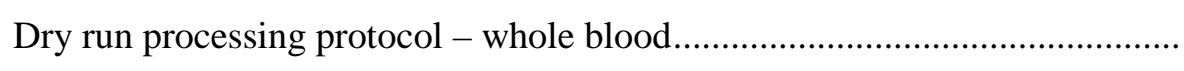 \\
\hline $15-5$ & 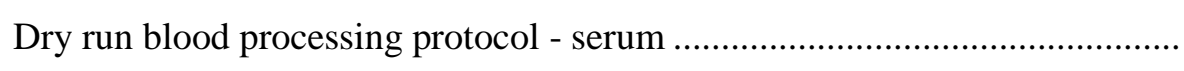 \\
\hline $15-6$ & 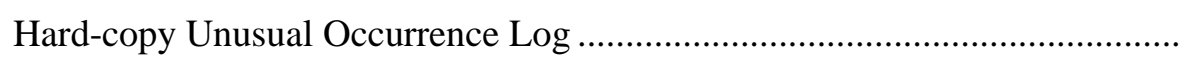 \\
\hline $15-7$ & Hard-copy Supply Use Control Log \\
\hline
\end{tabular}




\section{OVERVIEW OF THE NATIONAL HEALTH AND NUTRITION EXAMINATION SURVEY}

This chapter provides a general history and description of the National Health and Nutrition Examination Survey (NHANES), and an overview of the tasks that staff perform during the survey.

\subsection{History}

The National Health Survey Act, passed in 1956, provided the legislation authorizing for a survey to provide current statistical data on the amount, distribution, and effects of illness and disability in the United States. NHANES was created to fulfill the purpose of this act. The NHANES 99 for 2004 was the eighth in a series of national examination studies conducted in the United States since 1960, but effective in 2005, NHANES has become a continuous field survey with year-round data collection.

To fulfill the purposes of this act, it was recognized that data collection would involve at least three sources: (1) direct interviews with Americans of all ages; (2) clinical tests, measurements, and physical examinations on sample persons; and (3) facilities where persons received medical care such as hospitals, clinics, and doctors' offices.

To comply with the 1956 act, the National Center for Health Statistics (NCHS), a branch of the U.S. Public Health Service in the U.S. Department of Health and Human Services, has conducted eight separate examination surveys to collect interview and physical examination data.

The first three of these national health examination surveys were conducted in the 1960s:

1. 1960-62—National Health Examination Survey I (NHES I);

2. 1963-65—National Health Examination Survey II (NHES II); and

3. 1966-70—National Health Examination Survey III (NHES III).

NHES I focused on selected chronic disease of adults aged 18-79. NHES II and NHES III focused on the growth and development of children. The NHES II sample included children aged 6-11, 
while NHES III focused on youths aged 12-17. All three surveys had an approximate sample size of 7,500 individuals.

Beginning in 1970 a new emphasis was introduced. The study of nutrition and its relationship to health status gained importance as researchers began to discover links between dietary habits and disease. In response to this concern, under a directive from the Secretary of the Department of Health, Education and Welfare, the National Nutrition Surveillance System was instituted by NCHS. The purpose of this system was to measure the nutritional status of the U.S. population and changes over time. A special task force recommended that a continuing surveillance system include clinical observation and professional assessment as well as the recording of dietary intake patterns. Thus, the National Nutrition Surveillance System was combined with the National Health Examination Survey to form the National Health and Nutrition Examination Survey (NHANES). Five surveys of this type have been conducted since 1970:

1. 1971-75—National Health and Nutrition Examination Survey I (NHANES );

2. 1976-80—National Health and Nutrition Examination Survey II (NHANES II);

3. 1982-84-Hispanic Health and Nutrition Examination Survey (HHANES);

4. 1988-94—National Health and Nutrition Examination Survey (NHANES III); and

5. 1999-05—National Health and Nutrition Examination Survey (NHANES 99).

NHANES I, the first cycle of the NHANES studies, was conducted between 1971 and 1975. This survey was based on a national sample of about 28,000 persons between the ages of 1-74. Extensive data on health and nutrition were collected by interview, physical examination, and a battery of clinical measurements and tests from all members of the sample.

NHANES II began in 1976 with the goal of interviewing and examining 28,000 persons between the ages of 6 months and 74 years. This survey was completed in 1980. To establish a baseline for assessing changes over time, data collection for NHANES II was made comparable to NHANES I. This means that in both surveys many of the same measurements were taken in the same way, on the same age segment of the U.S. population.

While the NHANES I and NHANES II studies provided extensive information about the health and nutritional status of the general U.S. population, comparable data were not available for many 
of the ethnic groups within the United States. HHANES, conducted from 1982 to 1984, aimed at producing estimates of health and nutritional status for the three largest Hispanic subgroups in the United States-Mexican Americans, Cuban Americans, and Puerto Ricans-that were comparable to the estimates available for the general population. HHANES was similar in design to the previous HANES studies, interviewing and examining about 16,000 people in various regions across the country with large Hispanic populations.

NHANES III, conducted between 1988 and 1994, included about 40,000 people selected from households in 81 counties across the United States. As previously mentioned, the health status and characteristics of minority groups can be very different from that of nonminority groups. Thus, black Americans and Mexican Americans were selected in large proportions in NHANES III. Each of these groups comprised separately 30 percent of the sample. It was the first survey to include infants as young as 2 months of age and to include adults with no upper age limit. For the first time a home examination was developed for those persons who were unable or unwilling to come into the examination center but would agree to an abbreviated examination in their homes. To obtain reliable estimates, infants and young children (1-5 years) and older persons (60+ years) were sampled at a higher rate. NHANES III also placed an additional emphasis on the effects of the environment upon health. Data were gathered to measure the levels of pesticide exposure, the presence of certain trace elements in the blood, and the amounts of carbon monoxide present in the blood.

NHANES 99, conducted between 1999 and 2005, was the first of the series of surveys designed with the goal of becoming a continuous, annual survey. Each single year, and any combination of consecutive years of data collection, comprises a nationally representative sample of the U.S. population. This new design allows annual statistical estimates for broad groups and specific raceethnicity groups as well as flexibility in the content of the questionnaires and exam components. New technologic innovations in computer-assisted interviewing and data processing result in rapid and accurate data collection, data processing, and publication of results.

The number of people examined in a 12-month period is about the same as in previous NHANES-about 5,000 a year from 15 different locations across the Nation. The data from the NHANES are used by government agencies, state and community organizations, private researchers, consumer groups, companies, and health care providers. 
In addition to NHANES I, NHANES II, Hispanic HANES, and NHANES III, several other HANES projects have been underway since 1982. These projects have been a part of the HANES Epidemiological Follow-up Survey, a multiphase project that has been conducting follow-up interviews with the NHANES I survey population in order to provide a longitudinal picture of the health of the U.S. population.

\subsection{Goals of the Survey}

NHANES 2009, the current version of the survey, was designed to continue the collection of information about the health and diet of people in the United States. These data are used to fulfill specific goals. The overall goals of NHANES 2009 are to:

- Estimate the number and percent of persons in the U.S. population and designated subgroups with selected diseases and risk factors;

- Monitor trends in the prevalence, awareness, treatment, and control of selected diseases;

- Monitor trends in risk behaviors and environmental exposures;

- Analyze risk factors for selected diseases;

- Study the relationship between diet, nutrition, and health;

- Explore emerging public health issues and new technologies; and

- Establish a national probability sample of genetic material for future genetic testing.

\section{NHANES Data Accomplishments}

NHANES data have been used to influence policy and improve the health of the U.S. population in many ways since the survey series was established. For the past 40 years, the U.S. Public Health Service has been interviewing and examining tens of thousands of Americans. Currently, teams of doctors, nutritionists, and health technologists are conducting the National Health and Nutrition Examination Survey (NHANES) in communities across the United States. Information from the survey will be updated annually. 
Some of the contributions of the NHANES data include:

- Pediatric growth charts. Developed by the National Health and Nutrition Examination Survey, the NHANES growth charts are found on the walls of pediatricians' offices and clinics not only across the United States, but also around the world. With new information on younger babies, the current charts have been expanded and improved.

- Vitamins and minerals. The earlier NHANES showed that low iron levels were a serious problem for many people, including women of childbearing age, preschool children, and the elderly. As a result, the government decided to fortify grain and cereal with iron to correct this deficiency. The surveys also showed the need for folate to eliminate another deficiency and prevent birth defects. Today, we have a wealth of information at our fingertips and, indeed, on our food labels to help us make better choices about our diets. Much of that information comes directly from the National Health and Nutrition Examination Survey.

- Cholesterol levels. The first survey in the 1960s led public health officials to sound the alarm about the link between high cholesterol levels and the risk of heart disease. When NHANES started testing, one-third of adults had high cholesterol. Today fewer than 1 in 5 adults have high cholesterol. Changes in diet and lifestyle, all built on information from the national survey, have sharply reduced our risk of dying from a heart attack. These strategies have also helped doctors find ways to better treat heart attack patients and speed their recovery.

- Lead-free gasoline. It was NHANES that gave the first clear-cut evidence that Americans had too much lead in their blood. This led Congress, the Environmental Protection Agency, and others to phase out the use of lead as an additive in gasoline, and the results have been remarkable. By the 1990s, NHANES found that only 4 percent of Americans had elevated lead in their blood, but it remains a problem for certain groups, especially poor children living in old houses, in cities where lead paint was once common. The survey helps public health agencies pinpoint where lead still remains a problem.

As the NHANES survey continues to collect and update health information, there are other ways in which the information will be of use, including:

- Osteoporosis. This condition, in which bones get weaker as people grow old, is blamed for many of the fractures among the elderly. The National Health and Nutrition Examination Survey measures bone density of participants.

- Environmental smoke. Recent NHANES data found that nearly 9 out of 10 nonsmoking Americans were exposed to smoke either at home or on the job.

- Obesity. Despite the public health gains in recent years, more Americans are overweight than ever before. Today, more than half of the adults in the U.S. are overweight, and the number of overweight children and teens has doubled in the past 
decade. This has led public health experts to look for ways to improve both diet and fitness.

- Changes in Food/Diet. Today consumers can find a wide range of low fat and light foods in their grocery stores, from dinner entrees to snacks. As the food we eat changes, NHANES helps monitor whether these new foods and dietary changes actually are in the best interest of our health.

- Immunizations. The National Health and Nutrition Examination Survey has turned up important information about the extent of hepatitis B infections, and led to the recommendation that all infants and children be vaccinated against it. Though babies and children are the primary targets for immunizations, the survey also has alerted doctors to the importance of tetanus shots for older people.

\subsection{National Center for Health Statistics}

The Division of Health and Nutrition Examination Surveys (DHANES) is one of the survey divisions at the CDC's National Center for Health Statistics (NCHS).

DHANES is responsible for planning, operations, informatics, analysis, and reporting activities related to the family of health and nutrition examination surveys. These surveys range from the capstone cross-sectional National Health and Nutrition Examination Survey, to longitudinal studies, and more recent efforts with community-based studies. DHANES is comprised of more than 60 Federal employees who have training and expertise in diverse areas including public health, informatics, survey methodology, and statistics. The Federal staff are augmented by contractor staff working on- and offsite.

The Division consists of four branches and the Office of the Division Director. The DHANES Office of the Director coordinates the major activities in the Division. Each of the four branches consists of a multidisciplinary team with specific duties and responsibilities.

\subsection{Sample Selection}

A sample is defined as a representative part of a larger group. Since it is impossible to interview and examine everyone in the United States for NHANES, a representative sample is taken of the U.S. population. By studying a representative sample of the population, it is assumed that the findings would not have been too different had every person in the U.S. been studied. Because generalizations 
about the population will be made, it is extremely important that the sample be selected in a way that accurately represents the whole population. Statisticians calculate the size of the sample needed and take into consideration the geographic distribution and demographic characteristics of the population, such as age, gender, race, and income.

An introductory letter is sent to each household in the sample. A few weeks after the letter goes out, interviewers visit each listed household and use carefully designed screening procedures to determine whether any residents are eligible for the survey. If eligible residents are present, the interviewer then proceeds to introduce the study, presents the Sample Person (SP) with a survey brochure, and obtains a signed consent for the household interview. The brochure contains detailed information on the survey, the household interview, and the Mobile Examination Center (MEC) examination.

A signed consent form must be obtained from each eligible individual before the household interview can be conducted. A refusal to sign the consent form is considered a refusal to participate in the survey. After the interview is completed, the interviewer then explains the MEC exam, obtains another signed consent form for the MEC exam, and contacts the field office to schedule a MEC appointment for the SP. All SPs aged 12 years and older must sign the Examination Consent forms to participate in the MEC examination. Parental consent is also required for SPs under 18 years of age. SPs aged 7-11 years old are asked to sign the Examination Assent form. An additional consent form is required for consent to future general research and genetics testing for both adults (ages 18+) and parents of children under 18 years. This consent form gives permission to store a small sample of blood and urine for future specimen testing and to collect a sample for genetics testing. A refusal to sign the MEC consent or assent form is considered a refusal to participate in the examination phase of the survey. Examinations will not be performed on sample persons who do not sign a consent form.

\subsection{Field Organization for NHANES}

There are two levels of field organization for this study - the home office staff and the field staff.

- Home Office Staff from Westat - Project staff from Westat are responsible for overseeing the field teams and field work. 
- Field Office (FO) Staff - For this survey, an office will be opened at every survey location (stand). Each field office will have a Study Manager (SM), Office Manager (OM), a Field Manager (FM), and one Assistant Office Manager (AOM).

- $\quad$ The Study Manager (SM) is responsible for the overall management of operations at a stand.

- $\quad$ The Office Manager (OM) is responsible for the stand office operations and is the main conduit for the flow of work and information between the MEC and the household interviewing staff. She or he will supervise one or more local office clerks hired to assist with office activities. The OM reports to the SM.

- $\quad$ The Field Manager (FM) has primary responsibility for the supervision of the household interviewers. The FM also assists the SM and supervises the activities of the Assistant Office Managers. She or he will deal with administrative issues, ISIS problems, and preparations for the next stand.

- $\quad$ The Assistant Office Managers (AOMs) are primarily responsible for data entry into the Integrated Survey Information System (ISIS), editing data collection materials, and verification of interviewer work. The AOMs report to the FM and also work closely with the OM.

- $\quad$ Facilities Specialist (FES) - One Facilities and Equipment Specialist will travel with each field office team and caravan to oversee the maintenance and operation of the trailers.

- Household Interviewers - These staff members are primarily responsible for identifying and enrolling the survey participants, conducting the household interviews, and appointing the study participants for the MEC exam. Specifically, household interviewers will locate occupied residential dwelling units, administer the Screener to select eligible sample persons, obtain signed consents to the household interview, conduct the interviews, set up examination appointments, obtain consents for the MEC exam, conduct field reminders for MEC appointments, and assist in rescheduling broken, cancelled, and no-show appointments.

Several times a week, household interviewers visit the field office and report to the field manager. During the course of the study, interviewers also interact on a daily basis with other field office staff and home office staff.

- MEC Staff - These health professionals conduct the health exams. The survey includes two exam teams. There are 16 individuals on each traveling team: 1 MEC manager, 1 MEC coordinator, 1 licensed physician, 3 medical technologists, 4 health technologists, 2 registered dental hygienists, 2 MEC interviewers, 2 dietary interviewers, and 1 phlebotomist. In addition, local assistants are recruited, trained, and employed at each stand to assist the exam staff. 
The following section describes the steps that are always completed before the opening of a stand and provides an overview of the tasks that interviewers are expected to perform.

Steps completed prior to interviewing include:

1. Statisticians scientifically select certain segments in the sampling area. A segment is an area with definite boundaries, such as a city block or group of blocks containing a cluster of households.

2. Segments are listed by NHANES staff. Listing is the systematic recording on special forms of the address of every dwelling unit (DU) located within the segment. Commercial buildings and other structures not intended as living quarters are not listed.

3. A sample of dwelling units is selected from the listing forms. This sample is the group of addresses that interviewers visit in order to conduct interviews.

4. Immediately before data collection begins, an advance letter is sent to each dwelling unit with a mailing address. This letter briefly describes the study and informs the household that an interviewer will contact them in the near future.

Steps followed by the interviewers at a stand include:

1. Obtain assignment of sampled dwelling units to contact from the field manager.

2. Locate dwelling units using addresses on the Household Folders and listing/mapping materials in the Segment Folder.

3. Contact an adult who lives in the selected household (occupied residential dwelling unit) and administer the Screener using a laptop computer.

- The Screener is an interview that lists all the individuals who live in the household, divides the household into families, and collects all the demographic characteristics necessary to immediately determine if there are persons in the household eligible for further interviewing.

- $\quad$ All instructions necessary to determine eligibility and to select sample persons (SPs) are programmed in the CAPI Screener.

4. If all persons in a household are ineligible, no further work is done with the case. If eligible household members are identified, interviewers continue to conduct all the necessary tasks associated with the case.

5. In eligible households, obtain a signed interview consent form prior to completing the medical history and/or the family questionnaire. 
6. Administer the appropriate medical history CAPI interview to eligible respondents. The questions asked depend on the age of the SP.

7. Administer the Family questionnaire to one adult family member from each eligible family in the household.

8. Schedule a MEC appointment for each SP, coordinating the MEC schedule and the SP schedule.

9. Obtain signed consent form(s) for each SP for the examination, confirm the examination appointment(s) with the field office, and give each SP an appointment slip.

10. If there is more than one eligible family in a household, repeat this process with each additional family.

11. Record the result of each contact or attempted contact with the household on the Call Record located in the Household Folder.

Interviewers also support the survey by conducting field reminders prior to MEC appointments and reschedule broken, cancelled, or no-show MEC appointments. When an interview has been completed, interviewers edit their work, carefully reviewing all forms for completeness and legibility. Interviewers report in person to the field manager at the stand office for regularly scheduled conferences, usually every other day. During these conferences, interviewers discuss completed cases, discuss problems with incomplete cases, receive new case assignments, and report time, expenses, and production.

To ensure the accuracy and completeness of the survey, all interviewer work is edited by the field office staff, and then validated by recontacting respondents. After this review, supervisors provide interviewers with feedback concerning the quality of the work.

\subsection{Exams and Interviews in the Mobile Examination Center (MEC)}

Examinations and interviews are conducted in a mobile examination center (MEC), which is composed of four specially equipped trailers. Each trailer is approximately 48 feet long and 8 feet wide. The trailers are set up side-by-side and connected by enclosed passageways. During the main survey, detachable truck tractors drive the trailers from one geographic location to another. 
Exhibit 1-1 shows a floor plan for the MEC. The interior of the MEC is designed specifically for this survey. For example, the trailers are divided into specialized rooms to assure the privacy of each study participant during exams and interviews. Many customized features have been incorporated including an audiometry room that uses a soundproof booth, a wheelchair lift, and a wheelchair-accessible bathroom available to assist participants with mobility problems. Exhibit 1-2 shows the locations of the various exams within the MEC.

\subsubsection{Exam Sessions}

The MEC operates 5 days a week and includes weekday, evening, and weekend sessions. Two 4-hour sessions are scheduled each day with approximately 10-12 SPs per session. During a stand, workweeks rotate to offer a variety of MEC appointments on weekday mornings, afternoons, and evenings, and every weekend.

Exhibit 1-1. Floor plan of the MEC

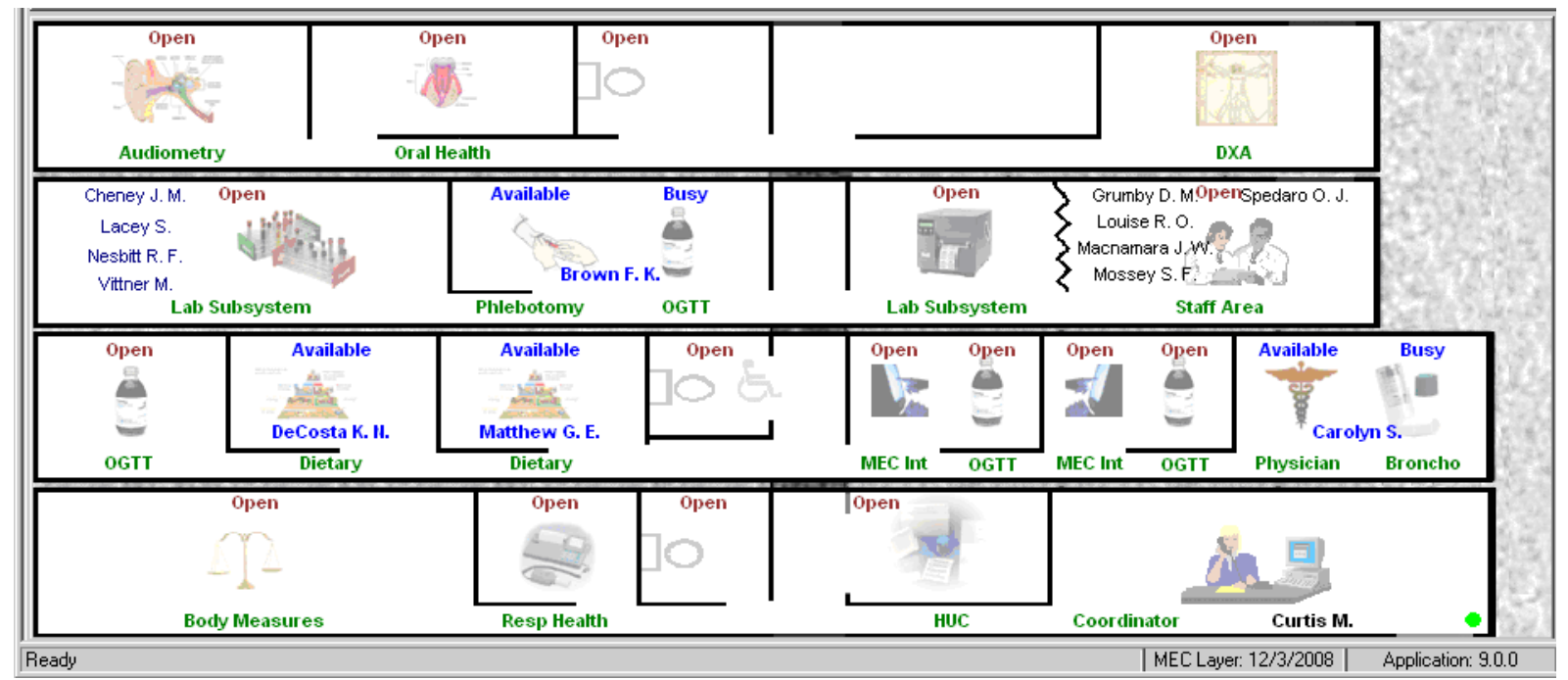


Exhibit 1-2. MEC exams and rooms

\begin{tabular}{|c|c|c|}
\hline Trailer & Room & Room Use \\
\hline \multirow[t]{5}{*}{ Trailer 1} & Coordinator & Welcoming and waiting area for SPs \\
\hline & Home Urine Collection & Home Urine Collection, Proxy exam \\
\hline & Respiratory Health & Exhaled Nitric Oxide \\
\hline & & Spirometry \\
\hline & Anthropometry & Body measurements \\
\hline \multirow[t]{6}{*}{ Trailer 2} & Physician & Physician examination \\
\hline & MEC Interview 1 & $\begin{array}{l}\text { Health interview, OGTT Trutol administration, } \\
\text { Proxy Exam }\end{array}$ \\
\hline & MEC Interview 2 & $\begin{array}{l}\text { Health interview, OGTT Trutol administration, } \\
\text { Proxy Exam }\end{array}$ \\
\hline & Dietary Interview 1 & Dietary interview \\
\hline & Dietary Interview 2 & Dietary interview \\
\hline & $\begin{array}{l}\text { Oral Glucose Tolerance Test } \\
\text { (OGTT) }\end{array}$ & Oral glucose tolerance Trutol administration \\
\hline \multirow[t]{5}{*}{ Trailer 3} & Phlebotomy/OGTT & Drawing of blood samples \\
\hline & & Oral glucose tolerance Trutol administration \\
\hline & Laboratory & Processing of biological samples \\
\hline & Label/shipping area & Lab area for labeling and shipping specimens \\
\hline & Staff area & Staff area that houses main computer system \\
\hline \multirow[t]{3}{*}{ Trailer 4} & Bone Density (DXA) & Bone Density scans \\
\hline & Oral Health & Dental examination \\
\hline & Audiometry & Hearing tests \\
\hline
\end{tabular}

\subsubsection{Exam Team Responsibilities}

There are 15 individuals on each exam team. In addition, a local assistant will be hired to assist the staff in managing examinee flow. The duties of the exam team members are summarized below:

- One MEC manager supervises the exam staff, oversees exam flow, and supports exam operations.

- One coordinator directs the flow of SPs through the MEC examination process. The coordinator manages all SP appointments, verifies that all components are completed for each SP, and exits SPs from the MEC.

- One physician conducts the medical examination and records results, reviews the results of the complete blood count and pregnancy test, and serves as the safety officer for the MEC. 
- One registered dental hygienist conducts the oral health exam and obtains the HPV specimen.

- Two health (MEC) interviewers administer questionnaires for physical and mental health information. The interviewers are also trained to administer the proxy exam, the OGTT exam, and the home urine collection exam.

- Two dietary interviewers administer the dietary questionnaire. The interviewers record a 24-hour dietary recall of the types and amounts of foods consumed by the SP in the last 24 hours. They are also trained to administer the OGTT and home urine collection exam.

- Four health technologists (some with radiologic technology and/or other health training) take and record body measurements, conduct respiratory health exams and bone density (DXA) scans, administer hearing tests, record for the oral health exam, conduct the home urine collection exam, and administer the Trutol solution for the glucose tolerance test.

- Three medical technologists conduct clinical laboratory tests on biological and environmental specimens, record the results of the tests, and prepare and ship specimens to various laboratories.

- One phlebotomist conducts the phlebotomy interview, administers the fasting questionnaire, and draws blood for laboratory tests and special studies like the volatile organic compound special study. The phlebotomist is also responsible for administering the Trutol and drawing a second blood sample for the GTT test. They are trained as a backup examiner for other MEC components like body measures and the home urine collection.

Each staff member is part of a team of professional persons with specific assignments that must be completed in order to accomplish the overall objective of the survey. Each individual must be aware of and respect the job demands placed upon other staff members, maintain an attitude of tolerance and consideration for fellow members of the team, and willingly perform extra tasks that may be assigned to support other staff members in the performance of their duties. MEC staff members may be requested to perform tasks not directly related to their specific professional skills in order to implement the overall data collection plan.

\subsubsection{Examination Components}

The full examination for an adult takes approximately $3^{1 / 2}$ hours, but the actual length depends on the SP's age. Some exams are done only on certain age groups so the exam profiles vary, even among adult SPs. The exam components are described briefly below and summarized in Exhibit 1-3. 
Exhibit 1-3. Examination components

\begin{tabular}{lc}
\hline \multicolumn{1}{c}{ Examination } & Age group \\
\hline Audiometry & $12-19$, and 70+ \\
\hline Bone Density (DXA) & All ages \\
\hline Body Measures & All ages \\
\hline Dietary Interview & $12+$ \\
\hline Glucose Tolerance & $1+$ \\
\hline Laboratory & $6+$ \\
\hline Urine Sample & $8+$ \\
\hline MEC (Health) Interview & $6+$ \\
\hline Home Urine Collection & $3+$ \\
\hline Oral Health & $1+$ \\
\hline Phlebotomy & All ages \\
\hline Physician & $8+$ \\
\hline Blood Pressure & $6-79$ \\
\hline Respiratory Health & $12+$ \\
$\quad$ Spirometry & Limited subsample \\
\hline Volatile Organic Compounds & \\
\hline
\end{tabular}

\section{- Audiometry}

The goals of the hearing exam are to obtain normative data on the hearing status of the adolescent and elderly U.S. population and to evaluate certain covariates that may be related to hearing loss, such as noise exposure. The hearing component tests youth aged 12-19 years, and adults aged 70 and over, by performing pure tone air conduction audiometry and tympanometry. Because air conduction thresholds alone cannot detect middle ear disease, tympanometry is conducted to identify potentially medically-correctible conditions which may be contributing to hearing impairment.

\section{- Body Composition}

The DXA component will use dual energy x-ray absorptiometry to evaluate skeletal health on SP's aged 8 years and older. Two DXA scans, AP spine and femur, will be used to assess overall skeletal changes that often occur with age by measuring bone mineral content (BMC) and bone mineral density (BMD). DXA measurements can 
also be used to provide information on early gender and ethnic changes in the rate of bone accretion and to determine the age when skeletal accretion ceases and when peak bone mass occurs.

\section{- Body Measures or Anthropometry}

All SPs will have body measurements taken. The exam will include height, weight, and other body measurements such as skinfold and arm girth, chest expansion and spine flexion. These measurements will be used to assess growth, obesity, and body fat distribution, and will provide information that can be used as a reference for later studies. Chest expansion and spinal flexion measures will provide data on the proportion of the population affected by spine disorders. Measurements of height and weight will allow for a revision of the child growth charts now in widespread use. Measuring body fat is important because it is associated with hypertension, adult diabetes, cardiovascular disease, gallstones, arthritis, and some forms of cancer. Furthermore, obesity and overweight can have an affect on the mental, physical, and social well-being of individuals.

\section{- Dietary Interview}

Dietary information has been collected in NHANES since the 1970s. Researchers and policymakers rely on NHANES data for detailed information about the foods and beverages that are consumed by the U.S. population. In addition to providing important national reference data on food and nutrient intakes that are obtained on all survey participants, the data help us to learn about food patterns of ethnic subgroups, the adequacy of diets consumed by young children and older persons, and the contribution of food to total nutrient intakes. Total nutrient intakes from food and dietary supplements can be computed by combining NHANES Dietary Recall data with the dietary interview supplement and antacid recall information. Many Federal agencies use NHANES data to evaluate Federal regulations in the areas of food fortification and human risk assessment analyses that are used to measure human exposure to contaminants that are found in food.

The goal of the dietary component is to estimate total intake of foods, food energy and nutrients, nonnutrient food components, and plain drinking water by the U.S. population; and assess dietary behaviors and the relationship of diet to health. Quantitative dietary intake data are obtained for all subjects by means of a 24-hour dietary recall interview using a computer-assisted dietary data entry system.

Two dietary interviews will be administered to all SPs. The primary dietary interview is administered in person in the MEC (the MEC In-person interview). At the end of the MEC dietary interview, the interviewers will schedule the SPs for a Phone Followup (PFU) 3-10 days later. The PFU is a follow-up dietary interview conducted by telephone by dietary interviewers from the home office. In addition, a second dietary phone interview is offered to all SPs aged 1 year and older who complete the MEC dietary interview. Proxy interviews will be conducted for children aged 1-11 years. Each household is also eligible to participate in an interview about food and food shopping. The adult who purchases the food for the household is asked to complete 
the interview. This interview, called the Flexible Consumer Behavior Survey, is also conducted by the home office dietary interviewers.

\section{- Home Urine Collection}

Microalbuminuria, or protein excretion in the urine, is an important indicator of early kidney disease, and is a prognostic indicator for diabetes, cardiovascular disease, and mortality. Microalbuminuria is measured by the urine albumin/creatinine ratio on the specimen provided on the day of the MEC exam. A second urine specimen is collected within 10 days of the MEC exam to compare the level of protein excretion with the first urine sample obtained in the MEC. During the home urine collection exam, the SP is given the necessary instructions and materials to successfully obtain and mail a urine specimen directly to a laboratory.

\section{- Oral Glucose Tolerance Test (OGTT or GTT)}

The purpose of this test is to reassess the prevalence of diabetes and impaired glucose tolerance (IGT) in the U.S. population. Persons with impaired glucose tolerance (IGT) - 15.6 percent of the U.S. population - are at high risk for developing diabetes. Also, IGT is an important risk factor for a number of other adverse health conditions and mortality. IGT is defined on the basis of an abnormal oral glucose tolerance test (OGTT). Persons without diabetes but with an OGTT 2-hr value of 140-199 mg/dl are considered to have IGT. Recent national and international randomized controlled trials have shown that diabetes can be delayed or prevented among persons with IGT.

The GTT will allow estimation of the prevalence of IGT and, thus, prediabetes in the U.S. population, surveillance of trends in the prevalence and awareness of these conditions, study of the risk factors for IGT and prediabetes, and examination of IGT as a risk factor for health conditions and mortality. Timely data on IGT and prediabetes are particularly important as the Nation initiates efforts to prevent diabetes among persons with prediabetes. These data on IGT and prediabetes are critical to targeting, designing, and evaluating prevention efforts.

A fasting glucose blood test is performed on all participants 12 years and older who are examined in the morning session after a 9-hour fast. After the venipuncture, participants are asked to drink 75 milligrams of Trutol ${ }^{\circledR}$ and to have a second venipuncture 2 hours (plus or minus 15 minutes) after consuming the Trutol. The blood glucose level from the second venipuncture will be used to determine if an SP has IGT.

\section{- Laboratory}

The laboratory component includes the collection and processing of various biological and environmental specimens including blood for subjects 1 year and older, and urine for subjects 6 years and older. On-site pregnancy testing excludes pregnant women from other examination components such as DXA. Complete Blood Counts (CBCs) are also performed in the MEC laboratory. All other specimen testing is performed by Federal, private, and university-based laboratories under contract to NCHS. 
Urine is collected from SPs aged 6 and older to assess kidney function, measure levels of mercury, heavy metals, iodine and arsenic, and assess environmental exposures to pesticides such as organophosphates, phthalates, polyaeromatic hydrocarbons, phytoestrogens, and perchlorates.

\section{- MEC (Health) Interview}

SPs aged 8 and older will have a health interview in the MEC. Generally, the questions asked in the MEC are considered to be more sensitive than the questions asked in the household. The MEC environment is believed to be a more appropriate setting for the administration of these questions. All eligible SPs will be asked questions in a face-to-face interview. In addition, persons who are 12-69 years old will be asked a series of more personal questions in complete privacy. The SP will listen to questions through a set of earphones and will enter responses by touching a computer screen.

Depending upon the age of the SP, the interview may consist of questions about tobacco, drug, and alcohol use, reproductive health (birth control practices, pregnancy and reproductive history, sexual activity), health behaviors (physical activity, weight history), kidney conditions and bowel health, arthritis, current health status and mental health, and exposure to certain chemicals. Children 8-11 years will only be asked questions about weight history.

\section{- Oral Health}

SPs aged 3 and older will receive an oral health exam conducted by registered dental hygienists. All examinees will be checked for tooth loss, cavities, restorations, and sealants. SPs 30 years and older will also be asked some questions about denture use and will be assessed for periodontal conditions; SPs aged 20-59 years will also be tested for human papilloma virus.

Oral and dental diseases affect many in the United States. Dental caries and tooth loss remain significant problems affecting the Nation's oral health. Although average dental caries rates for school-aged children have declined, nearly a half of all children still have caries. Additionally, more than 90 percent of adults in the United States have experienced caries. Dental sealants, an effective caries prevention measure, have been underutilized in the United States, with less than one-quarter of children aged 517 having them.

Over the past four decades, oral and dental health characteristics collected in national surveys supported by the Federal Government have been critical for monitoring health status, risk factors for disease, and access to preventive and treatment services. The 2005-06 NHANES oral health component will meet a critical need to continue monitoring trends in dental caries and tooth retention. More specifically, this NHANES will produce oral health data to monitor five oral health objectives in the Healthy People 2010 health promotion: dental caries experience, untreated dental decay, no permanent tooth loss, complete tooth loss, and dental sealants. 


\section{- Phlebotomy}

SPs aged 1 and older will have blood drawn. The amount drawn will depend on the person's age. It is important to draw blood from study participants for a number of reasons:

- $\quad$ Knowledge can be gained about how healthy a person is by measuring for various substances in his or her blood.

- $\quad$ Blood tests can provide early warnings of potential health problems, perhaps before physical signs appear. For example, a blood test for lead might indicate exposure to unsafe lead levels before an individual shows any physical signs of lead poisoning. Also, diabetes mellitus will be assessed by measures of plasma glucose, insulin, and glycohemoglobin in examinees ages 12 years and older. Diabetes is a large, growing, and costly public health problem in the United States and disproportionately affects racial and ethnic minorities. About 17 million Americans have diabetes and more than 1 million new cases of diabetes are diagnosed each year. Alarmingly, type 2 diabetes (formerly considered an adult disease) is now being diagnosed in children and adolescents and there has been a large increase in diagnosed diabetes among adults $<40$ years of age.

- $\quad$ Blood tests will also indicate the presence of STDs such as Chlamydia and gonorrhea, hepatitis, herpes, and HIV infection.

- $\quad$ Blood tests help in monitoring nutritional status, one of the key goals of NHANES. What researchers discover from this data can lead to health policy recommendations - the need for more vitamin fortification, for example.

- $\quad$ The blood testing also provides information about the levels of cholesterol and other blood lipids, another important study goal.

\section{- Physician}

All SPs see the physician. The physician measures blood pressure and pulse of all participants over 8 years of age, explains to female SPs how the HPV examination will be completed and explains the meaning of the STD/HIV test results to eligible SPs. The physician explains how SPs receive the results of STD/HIV tests so that the results remain totally confidential to the SP. Beginning in 2007, the physician will screen participants who qualify for the bronchodilator with spirometry exam, obtain consent for, and administer the bronchodilator. The physician must be present on the MEC before any exams can be conducted, and the physician is in charge of any medical emergency that occurs on the MEC.

\section{- Respiratory Health}

Beginning in 2007, respiratory health (RH) was incorporated in the NHANES study. $\mathrm{RH}$ will be collected by means of an assessment of lung function testing, or spirometry, and a measurement of exhaled nitric oxide (ENO). The objective of collecting this data is to assess the prevalence of asthma and adult chronic obstructive 
pulmonary disease in the U.S. population. All SPs aged 6-79 years will be eligible to participate in the component. SP's will be excluded from the RH exam if they answer positively to any pre-exam safety exclusion questions.

RH has three subcomponents: (1) spirometry, (2) ENO measurements, and (3) repeat spirometry measurements after the administration of a bronchodilator. For the third subcomponent, a subset of SPs whose baseline spirometry results show lung function values below a certain threshold, and will be provided with an opportunity to repeat spirometry after inhaling a bronchodilator medication. The MEC physician will first evaluate the participant to determine if the participant meets the requirements for safe bronchodilator administration. The physician will then obtain written informed consent, and then instruct the SP in the administration of the bronchodilator, and then administer the medication. Results from repeat spirometry testing following the bronchodilator could potentially provide a more detailed picture of each individual's breathing problem, i.e., an indication of whether the person is likely to have asthma or chronic obstructive pulmonary disease.

ENO provides a noninvasive marker measure of airway inflammation, a factor in asthma and possibly other lung diseases. Evaluation of airway inflammation, a precursor of asthma symptoms, is important in the investigation of underlying disease. Currently, there are no U.S. population-based estimates of ENO baseline levels for normal subjects, or for those persons with asthma and chronic obstructive pulmonary disease.

\section{- Volatile Organic Compounds/Perchlorate (VOC) Special Study}

The purpose of the VOC/Perchlorate study is to determine the prevalence of exposures to chemicals called Volatile Organic Compounds (VOCs) and Perchlorates. Volatile organic compounds are emitted as gases from certain solids or liquids. VOCs include a variety of chemicals, some of which may have short- and long-term adverse health effects. Concentrations of many VOCs are consistently higher indoors (up to ten times higher) than outdoors. VOCs are emitted by a wide array of products numbering in the thousands. Examples include paints and lacquers, paint strippers, cleaning supplies, pesticides, building materials and furnishings, office equipment such as copiers and printers, correction fluids and carbonless copy paper, graphics and craft materials including glues and adhesives, permanent markers, and photographic solutions.

Perchlorate is an oxidizer used in rocket fuel, explosives, and road flares, and it also occurs naturally in the atmosphere. Since the 1950s, unused amounts of the chemical have been dumped into unlined pits throughout the United States, where it has seeped into water supplies. The chemical is highly mobile and soluble in water, aiding its dispersion through the environment.

VOCs and Perchlorates are tested on a half-sample of participants over age 12; tap water from participants' homes is collected by the household interviewers and in the MEC, blood is collected from the participant during phlebotomy. 


\subsubsection{Sample Person Remuneration}

All examinees receive remuneration for the MEC visit as well as payment for transportation expenses. The MEC visit remuneration is age-related and may include an extra fasting incentive if the SP is eligible. SPs who complete the dietary phone interview and the Consumer Behavior Questionnaire also receive an incentive.

\subsubsection{Report of Exam Findings}

Examinees receive the results of many of the tests and exams conducted in the MEC, though some results are used only for research and are not reported.

One report, a Preliminary Report of Findings, is produced on the day of the SP's examination and includes results that are immediately available and require no further evaluation or interpretation. Before the examinee's departure from the MEC, the coordinator prints a report that can include height, weight, complete blood count, blood pressure and heart rate, and results from the oral health and audiometry examinations. The MEC physician reviews the blood pressure and complete blood count test results for abnormalities and discusses any problems with the SP (or his or her parent.) Approximately 12-16 weeks after the exam, NCHS mails the remainder of the examination results to the SP after appropriate clinical or quality reviews are completed. This Final Report of Findings will include all the results from the Preliminary Report of Findings and may include laboratory tests like liver and kidney function tests and lipids, bone density scan results, retinal image scan reports, and spirometry results. Seriously abnormal results are reported to the SP via telephone by NCHS before the remaining findings are mailed.

Certain tests, such as those for sexually transmitted diseases (Chlamydia, herpes simplex, and human papillomavirus) and human immunodeficiency virus (HIV) are released only to the sample person using a specially devised procedure requiring a unique password.

To further assist sample persons, an in-house NCHS survey response team is available to answer calls from NHANES participants regarding the results from the Report of Finding System. The response team effort works both as a triage mechanism and a surveillance system. A receipt and control 
record is kept on all sample person inquiries. Also available at no cost to sample persons is an 800 tollfree telephone number that can be accessed during regular scheduled business hours. The response team members include a physician, a nurse with a doctorate degree, and other staff who are trained to answer specific questions.

Tests and procedures conducted in the MEC are not considered diagnostic exams and are not a substitute for an evaluation by a medical professional. No clinical treatments or health interventions of any type are performed in the MEC. If a health problem is discovered during the course of the MEC exam, the physician offers to contact the examinee's personal healthcare provider or recommend a local physician or clinic for follow-up care. If a sample person is found to have a serious condition requiring immediate attention, the local rescue squad may be summoned or the SP will be advised to seek immediate medical treatment.

\subsubsection{Dry Run Day}

At the beginning of the examination period, one-half day is devoted to calibrating instruments, practicing MEC procedures, and collecting biological specimens that serve as blind quality control samples. A dry run day is scheduled immediately before the first exam day of every stand to make sure that all equipment is operational, supplies are adequate, and the facility is working properly. All procedures in the dry run are completed as though the actual exam session was being conducted. The only difference is that the examinees are volunteers who are not part of the sample for the survey. Volunteers may include local residents, local officials, or field employees or guests of NCHS.

\subsection{Integrated Survey Information System (ISIS)}

The Integrated Survey Information System (ISIS) is a computer-based infrastructure designed to support all survey operations including sample management, data collection, data editing, quality control, analysis, and delivery of NHANES data. With a collection of customized subsystems, the ISIS links the field office, mobile examination center, Westat home office, and NCHS during field operations. Each component in NHANES such as Dietary Interview has a computer application for direct data entry. Data collected in the Dietary Interview room of the mobile examination center is directly entered in the ISIS system computers. In addition, data from biomedical equipment such as the Dual 
Energy X-Ray Absorptiometry (DXA) scanner for body composition is directly downloaded to the ISIS system where it is displayed on the computer screen and stored in the system database.

\section{Confidentiality and Professional Ethics}

All information regarding this study must be kept strictly confidential except as required by law. This includes location of survey sites. Since this study is being conducted under a contract with the National Center for Health Statistics, the privacy of all information collected is protected by two public laws: Section 308(d) of the Public Health Service Act (42 U.S.C.242m) and the Privacy Act of 1974 (5 U.S.C. 552a).

Each person working on the study must be continuously aware of the responsibility to safeguard the rights of all the individuals participating in the study. Each participant should be treated courteously, not as a sample number. Never divulge names or any other information about study participants except to the research team. Refrain from any discussions about study participants, in or out of the MEC, which might be overheard by people not on the survey staff. All of the members of the research team are under the same legal, moral, and ethical obligations to protect the privacy of the SPs participating in the survey. No participant names will be included in any reports prepared about the survey and neither NCHS nor the contractor is allowed to release information that would identify study participants without the consent of the participants.

Cooperation from the public is essential to the success of survey research. A great deal of effort is expended in obtaining cooperation from many national, regional, state, and local officials and the general public. It is the responsibility of every field employee to build on the integrity of the survey to encourage continued access to study participants during current and future surveys. Professional conduct, both on and off the job, is extremely important.

Each staff member has a responsibility for promoting good public relations. The Public Health Service and the contractor will be judged by the actions of the staff both on and off duty; consequently staff must be discreet in speech and action. Personal appearance and behavior must be governed by these same considerations. Please be aware of the audience at all times and avoid statements or actions that could shed an unfavorable light on the survey. 
Staff will be asked to sign a pledge of confidentiality before the survey begins. This pledge states that they are prohibited by law from disclosing any information while working on the survey to anyone except authorized staff of NCHS and the contractor, and that they agree to abide by the contractor's Assurance of Confidentiality. 


\section{OVERVIEW AND LABORATORY TEAM RESPONSIBILITIES}

\section{$2.1 \quad$ Overview}

The laboratory component of NHANES includes the collection, processing, storage, and shipping of blood, urine, and other biological and environmental specimens. Collectively, these specimens provide data about the health status of the U.S. population.

The blood collection (venipuncture) procedure consists of (1) administering a questionnaire to screen for conditions that exclude the participant from the blood draw; (2) determining fasting status; and (3) performing a blood draw. The phlebotomist also collects one additional blood tube from SPs selected for the VOC (volatile organic compounds) special study and two EDTA tubes for genetic testing on consenting SPs aged 20+. They may administer a dextrose solution (Trutol) to and will collect an additional 2-mL gray top tube from SPs selected for the glucose tolerance (GT) component.

The purpose of urine collection and processing is to collect sufficient urine from participants aged 6 years and older to be able to (1) perform a pregnancy test on selected females aged 8 to 17 years and all females aged 18 to 59 years to exclude pregnant participants from other components that could put the participant at risk; (2) perform a test for osmolality; (3) allocate urine into vessels for storage and transport to multiple government and contact laboratories for analysis; and (4) calculate a urine flow rate, and (5) allocate urine into a vessel for future studies.

The purpose of blood processing is to (1) perform a complete blood count; (2) allocate blood (whole blood, plasma, washed packed cells, and serum) into vessels for transport to multiple government and contract laboratories for analysis; (3) allocate plasma and serum into vessels for future studies and process tubes for genetic testing; and (4) process serum for environmental testing.

The purpose of collecting and processing one vaginal swab is to be able to store and transport the samples to a laboratory for HPV (human papiloma virus) analysis.

The purpose of performing a complete blood count on blood specimens is to provide a study of blood cells and coagulation. 
The purpose of pregnancy testing is to exclude pregnant women aged 8-59 years from participating in the dual-energy x-ray absorptiometry (DXA) section of the body composition component of the MEC exam.

The purpose of the osmolality test is to measure the concentration of urine which can help both EPA and NCEH determine if data should be ignored because it's too dilute or too concentrated.

The purpose of conducting the special volatile organic compounds (VOCs) study is to collect blood to determine the prevalence of exposures to certain chemicals. A tap water sample is collected at the household level. This sample is tested for VOCs and perchlorate. The household water sample test results are linked back to the appropriate SPs that fall into the VOC/Perchlorate water subsample.

The purpose of collecting and processing an oral rinse for HPV is to be able to store and transport the samples to a laboratory for HPV (human papiloma virus) analysis. This sample is used to determine the prevalence and type of distribution of infection and to investigate the demographic and behavioral factors associated with infection.

\section{$2.2 \quad$ The Laboratory Team}

Each laboratory team includes three ASCP (American Society of Clinical Pathologists) certified medical technologists or medical laboratory technologists who have experience in all aspects of laboratory practice and a certified phlebotomist who has experience in venipuncture.

The chief medical technologist is the most senior member of the team. The chief medical technologist is responsible for overseeing all the activities of the medical technologists and phlebotomist in the MEC, and also quality control, equipment calibration, and maintenance. On a routine basis, the chief medical technologist performs the same duties as the other medical technologists.

\section{$2.3 \quad$ Tasks}

The phlebotomist's primary tasks include conducting the venipuncture, administering the Trutol solution, conducting a second venipuncture 2 hours after the Trutol solution has been consumed, 
assisting the medical technologists as needed, managing the phlebotomy and GTT component inventories, performing MEC equipment QC activities and recording the results, printing laboratory labels, labeling blood processing racks, and assisting with the shipping process at the end of each stand. In addition, the phlebotomist acts as a backup examiner and recorder for the body measures exam and oral health recorder for the oral health exam.

The medical technologist's tasks include printing labels and labeling vessels; performing complete blood counts (CBCs); conducting pregnancy tests; performing the osmolality test; processing blood, genetic tubes, urine, vaginal swabs, oral rinse, and tap water samples; performing MEC equipment QC activities and recording the results; and shipping specimens to contact laboratories. On a routine basis the medical technologists assist the phlebotomist as needed and manage the component inventory.

\subsection{Organization of the Laboratory}

The laboratory consists of two areas - the phlebotomy room and the laboratory (label/ship and environmental sample processing area, biological sample processing and hematology/pregnancy and osmolality testing) area. The phlebotomy room and laboratory area are located in trailer 4. Figures 2-1 and 2-2 illustrate the laboratory layout and phlebotomy room layout.

The phlebotomist performs the following activities in the phlebotomy room: collects blood, administers the Trutol solution, and collects a second blood sample from SPs who consumed the Trutol.

The medical technologist processes, stores, and ships biological and environmental samples, analyzes blood for a complete blood count, and performs pregnancy testing and they print labels, label blood processing racks, and prepare shipping documents. 


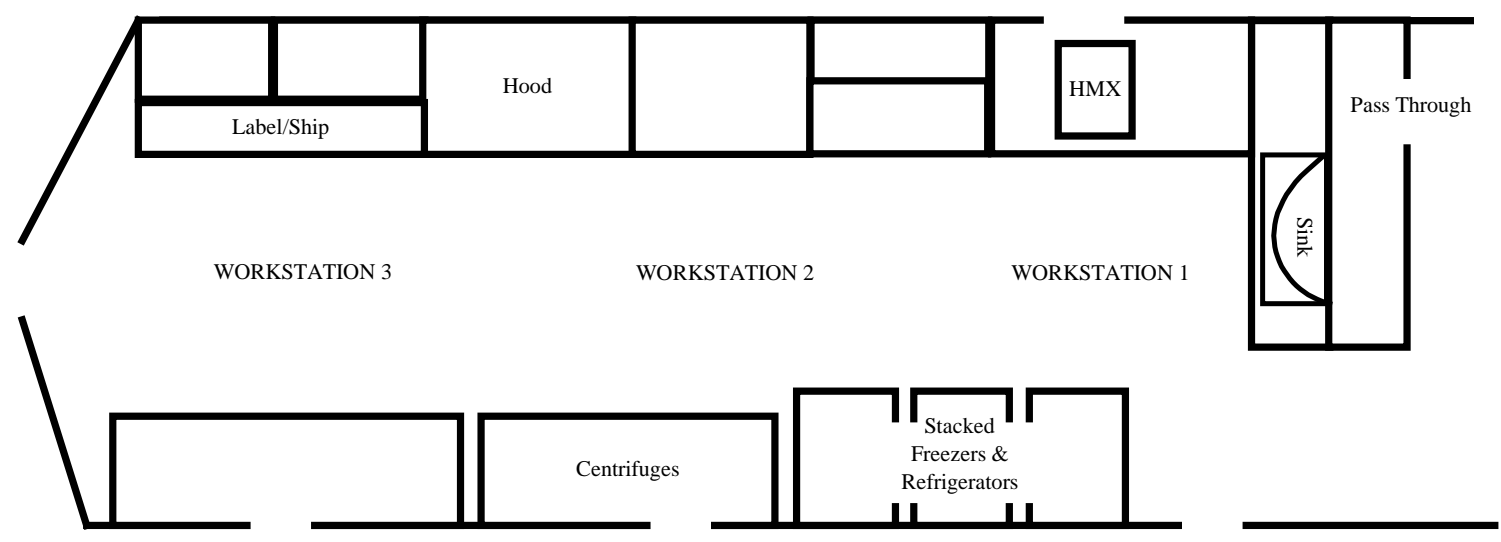

Figure 2-1. Laboratory layout

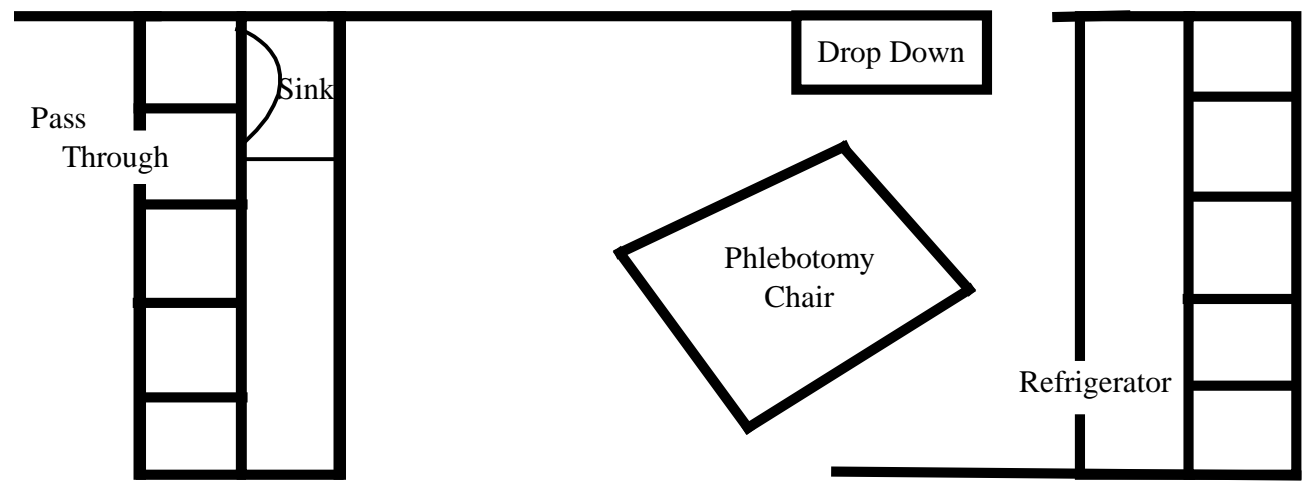

Figure 2-2. Phlebotomy room layout

\section{Laboratory Area Floor Plan}

The laboratory area contains three workstations designated workstation 1, 2, and 3. Water specimen processing, printing labels, labeling vials for each SP’s blood-processing rack, and shipping activities occur at workstation 3. Blood processing and storage occurs at workstation 2. Urine collection, urine processing and storage, pregnancy testing, osmolality testing, running CBCs, vaginal swab specimen processing, oral rinse specimen processing, and routine quality control activities occur at workstation 1. 
Tasks - Phlebotomy Room

- Set up supplies and check expiration dates of all items with lot numbers, such as the Vacutainer ${ }^{\circledR}$ tubes.

- Enter lot numbers and expiration dates into the automated Supply Use Control Log.

- Complete the phlebotomy examination, including conducting the venipuncture interview, administering the fasting questionnaire, and performing the venipuncture.

- Complete the OGTT examination including administering the Trutol solution to eligible SPs and performing a second blood draw on all GTT SPs who consumed the Trutol.

- Label and transfer all blood tubes to the laboratory area.

- Disinfect all the counters and blood draw chair at the beginning of each day.

- $\quad$ Take and record the QC readings.

- Maintain the appearance of the venipuncture area and the equipment.

- Monitor the inventory and restock supplies.

- Conduct the start and end of stand inventory counts.

- Clean the phlebotomy room, including the equipment, at the start and end of each stand.

- Assist in printing laboratory labels and label and assemble blood-processing racks.

- Assist the lab staff when they pack the shippers.

- Serve as the backup examiner and recorder in body measures and recorder in oral health.

\section{Tasks - Workstation 2}

- Stock blood processing and storage supplies.

- Receive blood specimens.

- Select the correct prelabeled rack and age appropriate template.

- Set the red top blood tubes aside to clot. 
- Refrigerate vessels 119 and 120 (Genetics).

- $\quad$ Refrigerate VOC blood (vessel 54) tube.

- $\quad$ Pipette whole blood into separate 2-mL cryovials from the 3-mL or 4-mL lavender tube for vessels 1 (Lead/Cad/HG) vessels 3 (RBC Folate), vessel 106 (RBC Folate), vessel 4 (Glycohem), vessel 5 (Mercury), and vessel 125 (HLA-B27).

- Make the dilution for RBC Folate vessels.

- Transfer the 3-mL or 4-mL lavender tube to the workstation 1 technologists for hematology.

- Centrifuge the second 3-mL or 4-mL lavender tube.

- $\quad$ Pipette the plasma from the second lavender tube into 2-mL cryovials for vessels 109 (PUFA/TFA) and 8 (Xtra Plasma).

- Centrifuge the 2-mL gray tube.

- $\quad$ Pipette the plasma from the 2-mL gray tube into a 2-mL cryovial for vessel 9 (Glucose).

- Centrifuge the 7-mL lavender tube.

- $\quad$ Pipette the plasma from the 7-mL lavender tube into a 5-mL cryovial for vessel 91 (SRM Pesticide).

- $\quad$ Centrifuge the red top tubes.

- Pool the serum from the 3-mL, 7-mL, 15-mL and single 10-mL red top tubes for each SP and mix well.

- Aliquot serum into separate 2-mL cryovials for the serum vessels according to the age-specific protocols.

- Pour serum from one of the dedicated $10-\mathrm{mL}$ red tubes into a 5-mL cryovial for vessel 29/90/92 and the serum from the second dedicated $10-\mathrm{mL}$ red tube into a 5-mL cryovial for vessel 89/93.

- $\quad$ Enter all blood processing results.

- Wash and lyse the packed cells from the EDTA tube(s) and transfer into a 2-mL cryovial for vessel 88 (Acrylamide) and a 2-mL cryovial for vessel 126 (PAH HB).

- Enter the blood processing results.

- Centrifuge the second 2-mL gray tube. 
- $\quad$ Pipette the plasma from the second 2-mL gray tube into a 2-mL cryovial for vessel 98 (OGTT).

- Enter the blood processing results.

- Refrigerate or freeze the processed vessels.

- Check contents of blood storage boxes (containers) using the container map module at the end of each session.

- Disinfect all the counters and the laminar flow hood at the beginning and end of each session.

- Maintain appearance of the blood processing area and the equipment.

- Monitor the inventory and restock supplies.

- Conduct the start and end of stand inventory counts.

- Clean the laboratory at the start and end of each stand.

- Assist in printing laboratory labels and label and assemble blood-processing racks.

\section{Tasks - Workstation 1}

- Perform start of stand, start of session, end of session, weekly, and end of stand quality control checks on laboratory equipment and record results.

- $\quad$ Prepare the ascorbic acid.

- Enter the ascorbic dates in the Supply Use Control Log.

- Stock supplies for urine processing, hematology, and pregnancy and osmolality testing.

- Verify and accept the urine volume and record the urine collection results.

- Run the pregnancy test controls.

- Run the osmolality test controls.

- Perform startup procedures and run quality control materials on the Coulter® HMX.

- Run specimens on the Coulter® HMX and enter results.

- Conduct the pregnancy test and enter results. 
- Conduct the osmolality test and enter results.

- Label urine vessels and aliquot into 2 or 5-mL cyrovials, 14-mL Falcon snap cap, or $10-\mathrm{mL}$ VWR screwcap tubes according to the processing protocol.

- Enter the urine processing results.

- Store the urine vessels.

- $\quad$ Process vaginal swab (vessel 78).

- Process oral health rinse (vessel 129).

- Check contents of urine, vaginal swab, , vaginal swab, and oral rinse boxes and bags using the container maps module at the end of each session.

- Disinfect all counters at the beginning and end of each session.

- Maintain the appearance of the urine processing/hematology area and the equipment.

- Monitor the inventory and restock supplies.

- Assist the phlebotomist with venipuncture.

- Conduct the start and end of stand inventory counts.

- Clean the laboratory at the start and end of each stand.

- Assist in printing laboratory labels and label and assemble blood-processing racks.

\section{$2.8 \quad$ Tasks - Workstation 3}

- Complete blood processing tasks for the first 45 minutes of each session.

- $\quad$ Process tap water (vessel 105) using the Water Collection module.

- Check contents of water container using the container maps module at the end of each processing session.

- $\quad$ Print blood, urine, pregnancy, and oral rinse testing labels.

- $\quad$ Prelabel the vials for each SP’s blood-processing rack.

- Generate container IDs at the start of stand.

- Assign a container ID label to one container for each vessel. 
- When shipping, close all open containers, create air bills, and assign containers to shippers.

- Print shipping manifests.

- Prepare blood (whole blood, serum, washed packed cells, and plasma), urine, vaginal swab, water, and oral rinse samples for shipping.

- Pack, weigh, label, and ship shippers.

- Open all new containers after shipping.

- Maintain appearance of the label/ship area and the equipment.

- Monitor inventory and restock supplies.

- Assist phlebotomist with venipuncture.

- Complete the Trutol section in the GTT examination as assigned.

- Conduct start and end of stand inventory counts.

- Clean the laboratory at the start and end of each stand.

\subsection{Cross Training}

Cross training the medical technologists and the phlebotomist increases individual's skills and provides scheduling flexibility and a safety net during staff absences. The primary phlebotomist helps the lab team setup and teardown. Medical technologists trained in phlebotomy (backup phlebotomists) work in phlebotomy on a routine basis to ensure competency and proficiency. The basic underlying assumption is that the blood draw must never be compromised. The primary phlebotomists must be available to step in and complete the blood draw even when backups are working in phlebotomy.

- When the backup phlebotomist is assigned to workstation 3 (label/ship):

- $\quad$ The primary phlebotomist works at workstation 3, except on Monday when the lab team ships. The backup phlebotomist always works their scheduled workstation 1 assignment on shipping Mondays.

- $\quad$ The backup phlebotomist works in phlebotomy when the primary phlebotomist works at workstation 3.

- The primary phlebotomist always performs the pediatric blood draws while the backup phlebotomist observes and holds the child. 
- If the backup phlebotomist encounters a difficult blood, then the primary phlebotomist comes into the room and they work together so that the blood draw is successful. This is intended as a learning experience; the backup phlebotomist can continue to learn from the primary phlebotomist.

- There are no rules for how many days the backup phlebotomist works in phlebotomy during each stand. The objective is at least 2-3 days per stand with emphasis on sessions with 8-10 adult SPs.

- On setup day the phlebotomist completes his or her room setup and assigned tasks before helping lab. On setup day the phlebotomist should, at a minimum, make the racks for dry run day. On teardown day he or she helps pack the shippers and assists the lab staff in other ways as directed by the chief medical technologist. 


\section{LABORATORY ISIS FUNCTIONALITY}

\subsection{Laboratory-Specific Functionality}

While MEC applications are custom built for each specific component, they share a common design. This commonality provides all component applications the same "look and feel” which minimizes reorienting technologists who rotate from component to component. A complete ISIS overview and these features and characteristics are described in the ISIS User Guide. The laboratory application has additional functionality that has been developed to accommodate and facilitate processing specimens and reporting results. This chapter describes the laboratory-specific functionality.

\section{Laboratory Heads-up Display}

Use the laboratory heads-up display to view the SPs scheduled into the session, the modules for which they are eligible, and their current process status. 
Use the heads-up display to access modules and print additional labels.

\begin{tabular}{|c|c|c|c|c|c|c|c|c|c|c|c|c|c|c|}
\hline File View & Untilities Repo & orts & Modules & Shippin & & Window Help & & & & & & & & \\
\hline iv & 圈 & 8 & 8 & D? & & & & & & & & & & \\
\hline & ppointments & for Se & ssion: & 430420 & & & & & & & bess & s Status & & \\
\hline SPID & Sample ID s & status & type & gender & age & name & UC & $U$ & $P$ & $O Z$ & $\mathrm{CB}$ & $\mathrm{B} \mathrm{gtt} P C$ & $\mathrm{CHPV}$ & OR \\
\hline 123442 & 123442 & $\mathrm{SC}$ & $\mathrm{P}$ & $\mathrm{F}$ & 36 & NELSON_3,JENNY & 0 & 0 & 0 & 0 & 0 & 00 & 0 & 0 \\
\hline 152141 & 152141 & $\mathrm{SC}$ & $P$ & $\mathrm{~F}$ & 19 & DRAKE_1, WENDY & 0 & 0 & 0 & 0 & 0 & 000 & 0 & 0 \\
\hline 161002 & 161002 & $\mathrm{sc}$ & $\mathrm{P}$ & M & 21 & MARTIN_1,THOMAS & 0 & 0 & $\cdot$ & 0 & 0 & 00 & $\cdot$ & 0 \\
\hline 164673 & 164673 & $\mathrm{SC}$ & $P$ & M & 34 & BROWN, ADAM & 0 & 0 & $\cdot$ & 0 & 0 & 00. & - & 0 \\
\hline 167942 & 167942 & $\mathrm{SC}$ & $\mathrm{P}$ & $F$ & 31 & HARRIS_2, MARIA & 0 & 0 & 0 & 0 & 0 & 000 & 0 & 0 \\
\hline 171098 & 171098 & $\mathrm{SC}$ & $P$ & $\mathrm{~F}$ & 73 & WALLACE_2, MILDRED & 0 & 0 & $\cdot$ & 0 & 0 & 000 & . & . \\
\hline 224633 & 224633 & $\mathrm{sc}$ & $P$ & M & 46 & SMITH_2, TYLER & 0 & 0 & . & 0 & 0 & 000 & . & 0 \\
\hline 238763 & 238763 & $\mathrm{SC}$ & $\mathrm{P}$ & $\mathrm{F}$ & 73 & WALLACE_3, MILDRED & 0 & 0 & $\cdot$ & 0 & 0 & 000 & . & . \\
\hline 240491 & 240491 & $\mathrm{SC}$ & $\mathrm{P}$ & $\mathrm{F}$ & 39 & WALLACE_3, JILL & 0 & 0 & 0 & 0 & 0 & 000 & 0 & 0 \\
\hline 244445 & 244445 & $\mathrm{SC}$ & $\mathrm{P}$ & $\mathrm{F}$ & 36 & NELSON_2,JENNY & 0 & 0 & 0 & 0 & 0 & 000 & 0 & 0 \\
\hline 248212 & 248212 & $\mathrm{sc}$ & $\mathrm{P}$ & M & 28 & TAYLOR_2, BEN & 0 & 0 & $\cdot$ & 0 & 0 & 00 & . & 0 \\
\hline 251401 & 251401 & $\mathrm{SC}$ & $P$ & M & 36 & NELSON_3,HOWARD & 0 & 0 & $\cdot$ & 0 & 0 & 000 & . & 0 \\
\hline
\end{tabular}

The heads-up display lists all SPs with appointments for the current session. It includes the SP ID, Sample ID, Status (SC=scheduled, LI=Logged-in, $\mathrm{CI}=$ checked-in, $\mathrm{BE}=$ being examined, $\mathrm{EX}=$ exited), Appointment Type ( $\mathrm{P}=$ primary, $\mathrm{G}=$ guest, VIP=VIP guest, $\mathrm{D}=$ dry run), Gender ( $\mathrm{M}=$ male, $\mathrm{F}=$ female), Age, and Name (last, first.)

The heads-up display provides the Process Status for the following modules: UC (urine collection), U (urine processing), P (pregnancy testing), OZ (Osmolality), CB (complete blood count), B (blood processing), gtt (glucose tolerance test or vessel 98 processing), PC (washed packed cell processing), HPV (HPV swab processing), and OR (oral rinse processing). The SP is ineligible for a module when the process status is - The SP is eligible for a module but no results have been recorded when the process status is $\mathrm{O}$. The SP is eligible for a module and some results have been recorded when the process status is $\odot$. The SP is eligible for a module, all results have been recorded, and the module is complete when the process status is $\mathbf{}$. 
The SP is eligible for a module $(\mathrm{O})$ but the process status for blood processing $(\mathrm{B})$, the complete blood count (CB), and packed cell processing (PC), will not enable until at least one blood tube has been recorded as collected in the phlebotomy component. Only SPs who have checked in are eligible for HPV processing. The process status is $\bigcirc$ for urine processing (U), pregnancy testing (P), and osmolality testing (OZ), until the SP's urine is marked as collected in the UC module. Once these samples are collected, the process status changes from $\bigcirc$ to $\bigcirc$. The gtt and OR modules will enable as soon as one SP has the OGTT blood tube drawn in phlebotomy and one SP has the oral rinse collected in the oral health examination. The process status regularly updates and changes after each result is saved.

The active SP is contained in a rectangular box. Use the heads-up display to select the correct SP. Select a different SP if the active SP is not the SP of choice.

\subsection{Laboratory Task Bar}

Monitor the activity in the laboratory task bar which is located on the bottom of the screen.

\begin{tabular}{|c|c|c|c|}
\hline Ready & |HUP: idle & |MEC Layer: 12/11/2008| Application: 9.0 .10 & Connected to Coordinator $104: 24$ FM \\
\hline 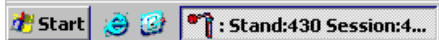 & & & बल० द्य $4: 24 \mathrm{P}$ \\
\hline
\end{tabular}

The laboratory task bar displays the following information:

- The status of the refresh routine such as when the labels are printing or the data are updating;

- The status of the heads-up display (HUP) such as when it is "idle;"

- The current version of the MEC layer;

- The current version of the application;

- The status of the connection to the coordinator; and

- The current time. 


\section{4}

\section{Menu Bar Options}

Each MEC application has tailored menu options to support the specific examination. The menu options below list all available options. The options marked with an asterisk appear on all MEC examination programs. Options are grayed (inverted) or not present when they are not available for a specific subsystem.

\subsubsection{File Menu Options}

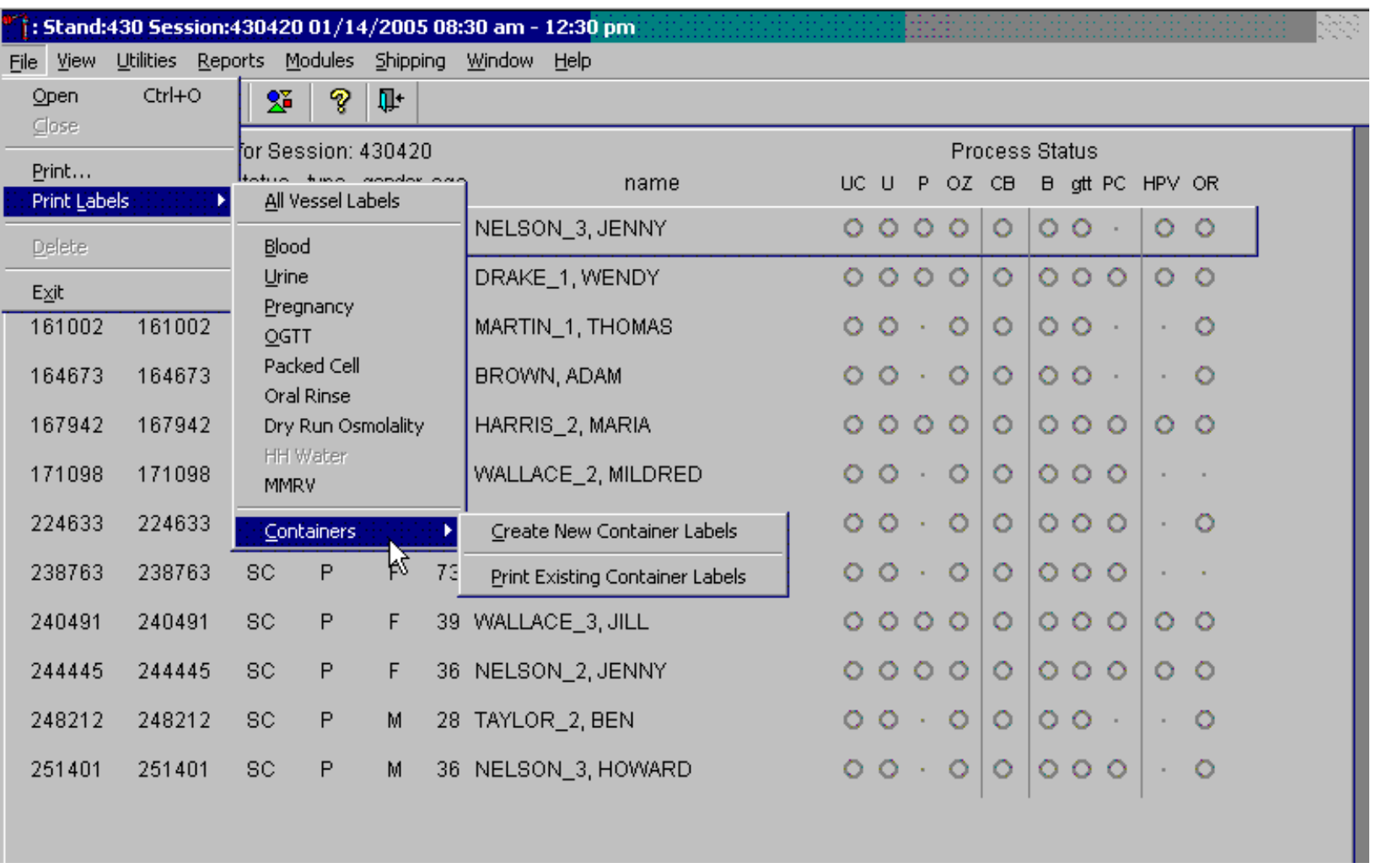


Asterisks (*) denote menu options common to all MEC examination components.

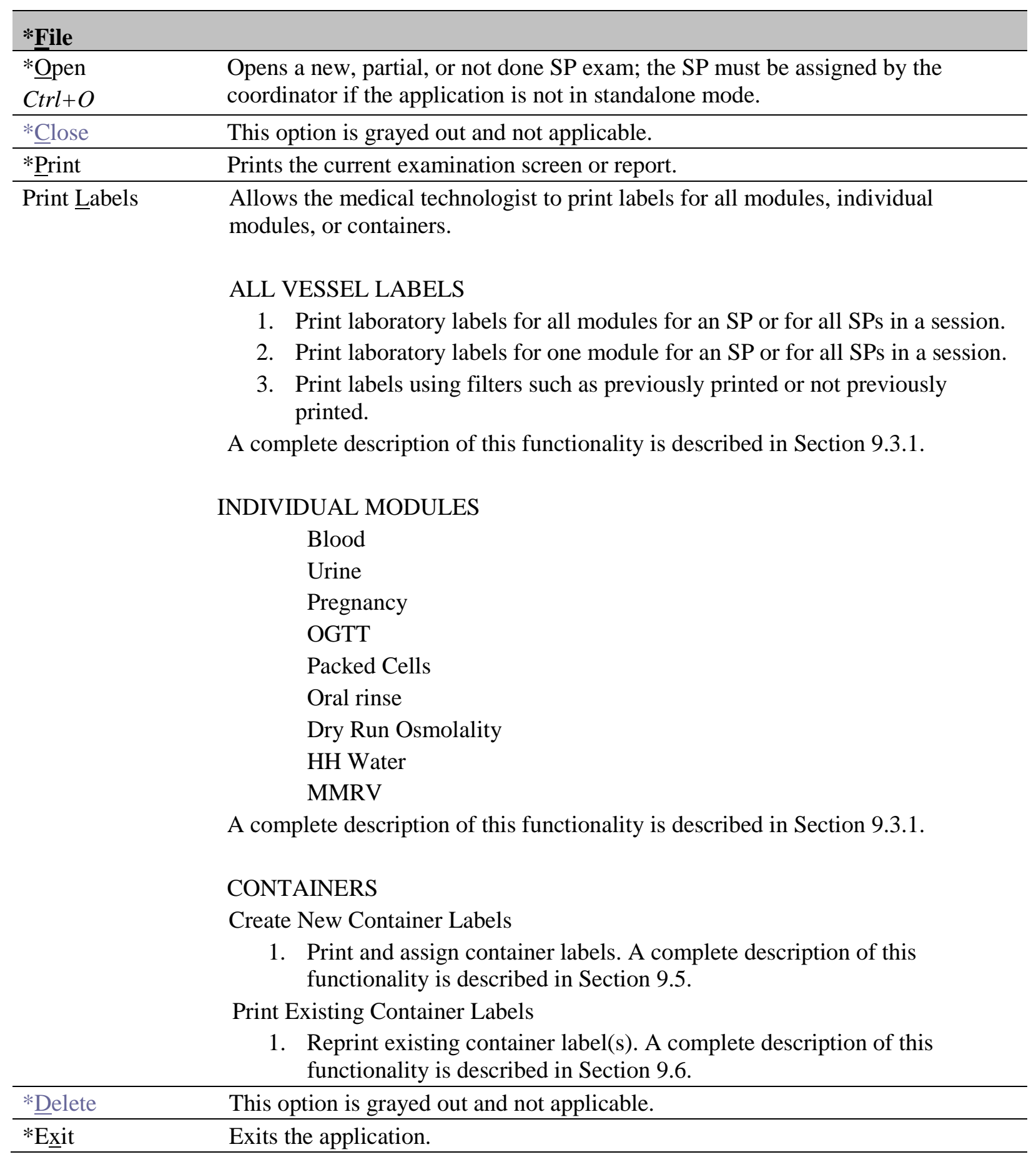




\subsubsection{Utilities Menu Options}

\begin{tabular}{|c|c|c|c|c|c|c|c|c|c|c|c|c|c|}
\hline \multicolumn{7}{|c|}{ [: Stand:430 Session:430420 01/14/2005 08:30 am - 12:30 pm } & & & & & & & \\
\hline 为 v & & & & & & & & & & & & & \\
\hline & \multirow{4}{*}{\multicolumn{4}{|c|}{$\begin{array}{l}\text { Exam Pause } \\
\text { Observations } \\
\text { IC Exclusions } \\
\text { Send Message } \\
\text { UFO Sighting } \\
\text { Set Session }\end{array}$}} & \multicolumn{9}{|c|}{ Process Status } \\
\hline SPID & & & & & age & name & UC U & P & $\mathrm{OZ}$ & $\mathrm{CB}$ & $\mathrm{B}$ gtt $\mathrm{PC}$ & HPV & OR \\
\hline 123442 & & & & & & NELSON_3,JENNY & 00 & 0 & 0 & 0 & 00 & 0 & 0 \\
\hline 152141 & & & & & & DRAKE_1, WENDY & 00 & 0 & 0 & 0 & 000 & 0 & 0 \\
\hline 161002 & \multirow{2}{*}{\multicolumn{2}{|c|}{ Englishi }} & \multirow{2}{*}{\multicolumn{2}{|c|}{$\begin{array}{l}\text { Ctril+E } \\
\text { Ctril+5 }\end{array}$}} & & MARTIN_1, THOMAS & 00 & $\cdot$ & 0 & 0 & 00. & . & 0 \\
\hline 164673 & & & & & 34 & BROWN, ADAM & 00 & $\cdot$ & 0 & 0 & 00 & & 0 \\
\hline 167942 & \multicolumn{4}{|c|}{$\begin{array}{l}\text { Ioolbars... } \\
\text { System Configuration }\end{array}$} & & HARRIS_2, MARIA & 00 & 0 & 0 & 0 & 000 & 0 & 0 \\
\hline 171098 & \multirow{2}{*}{\multicolumn{4}{|c|}{$\begin{array}{l}\text { Pregnancy QC } \\
\text { Coulter QC Lot Info }\end{array}$}} & & WALLACE_2, MILDRED & 00 & $\cdot$ & 0 & 0 & 000 & . & . \\
\hline 224633 & \multicolumn{3}{|c|}{ Osmolality QC } & & & SMITH_2,TYLER & 00 & . & 0 & 0 & 000 & & 0 \\
\hline 238763 & \multicolumn{4}{|c|}{ Regen SP Data } & & WALLACE_3, MILDRED & 00 & . & 0 & 0 & 000 & & . \\
\hline 240491 & 240491 & $\mathrm{SC}$ & $P$ & $\mathrm{~F}$ & 39 & WALLACE_3,JILL & 00 & 0 & 0 & 0 & 000 & & 0 \\
\hline 244445 & 244445 & $\mathrm{sc}$ & $P$ & $\mathrm{~F}$ & 36 & NELSON_2, JENNY & 00 & 0 & 0 & 0 & 000 & & 0 \\
\hline 248212 & 248212 & $\mathrm{sc}$ & $P$ & M & 28 & TAYLOR_2, BEN & 00 & . & 0 & 0 & 00 & & 0 \\
\hline 251401 & 251401 & $\mathrm{sc}$ & $P$ & M & 36 & NELSON_3,HOWARD & 00 & . & 0 & 0 & 000 & & 0 \\
\hline
\end{tabular}

\section{* Utilities}

Quality Control

Ctrl+Q
Opens the quality control dialog box to initiate quality control procedures. A complete description of this functionality is described for phlebotomy in Section 4.15 and for lab in Section 14.6.

\begin{tabular}{l}
${ }^{*}$ Exam $\underline{P}$ ause \\
\hline *Observation
\end{tabular}
This option is grayed out and not applicable. Submits an observation to the physician for possible referral.

IC Exclusions

This option is grayed out in the Laboratory application and is not applicable.

Posts an exclusion record for those SPs who do not want HIV, STD, PSA, or HPV tests performed, genetic tubes processed, or samples stored for future research; this utility is available only for coordinator, physician, and phlebotomy. A complete description of this functionality is described in Section 3.5.

*Send Message $\quad$ Opens a dialog box to send messages to the coordinator.

Ctrl+M

*UFO Sighting

Opens the Unusual Field Occurrence utility to document unusual equipment, SP, protocol, trailer, or supply issues.

Set Session

in the laboratory application. A complete description of this functionality is described in Section 3.6. 


\begin{tabular}{ll}
\hline *Utilities & \\
\hline $\begin{array}{l}\text { English } \\
\text { Ctrl+E }\end{array}$ & $\begin{array}{l}\text { This option is grayed out in the Laboratory application and is not } \\
\text { applicable. } \\
\text { Toggle option to set exam language to English. }\end{array}$ \\
\hline$\underline{\text { Spanish }}$ Ctrl+S & $\begin{array}{l}\text { This option is grayed out in the Laboratory application and is not } \\
\text { applicable. } \\
\text { Toggle option to set exam language to Spanish. }\end{array}$ \\
\hline *Toolbars & Configures placement of toolbars. \\
\hline System Configuration & Not applicable. \\
\hline Pregnancy QC & $\begin{array}{l}\text { Allows the laboratory medical technologist to access the Pregnancy QC } \\
\text { module. A complete description of this functionality is described in } \\
\text { Section 6.X. }\end{array}$ \\
\hline Coulter QC Lot info & $\begin{array}{l}\text { Allows the laboratory medical technologist to upload Coulter 5C Cell } \\
\text { control data for the Abnormal I, Normal, and Abnormal 2 control material } \\
\text { and enter control information for the Latron control material. }\end{array}$ \\
\hline Osmolality QC & $\begin{array}{l}\text { Allows laboratory medical technologist to add new Quantimetrix QC lot } \\
\text { information for level 1 and level 2. }\end{array}$ \\
\hline Regen SP Data & $\begin{array}{l}\text { Allows the laboratory medical technologists to regenerate or recalculate a } \\
\text { new profile for an SP. A complete description of this functionality is } \\
\text { described in Section 9.4. }\end{array}$ \\
\hline
\end{tabular}




\subsubsection{Report Menu Options}

\begin{tabular}{|c|c|c|c|c|c|c|c|c|c|c|c|c|c|c|}
\hline \multicolumn{12}{|c|}{ i: Stand:430 Session:430420 01/14/2005 08:30 am - 12:30 pm } & & & \\
\hline 为许 & 娄 $=$ & \multirow{2}{*}{\multicolumn{4}{|c|}{$\begin{array}{l}\text { Room Log } \\
\text { Session Preview }\end{array}$}} & \multirow[b]{3}{*}{ name } & & & & & & & & \\
\hline \multicolumn{2}{|c|}{$\begin{array}{r}\text { Appointm } \\
\text { SPID } \quad \text { Sampl }\end{array}$} & & & & & & \multicolumn{8}{|c|}{ Process Status } \\
\hline SP ID & Sampl & Contain & Map & & & & \multirow{2}{*}{$\begin{array}{c}\text { UC } \\
0\end{array}$} & & $\mathrm{P}$ & $\mathrm{OZ}$ & $\mathrm{CB}$ & $\mathrm{B}$ gtt PC & $\mathrm{HPV}$ & OR \\
\hline 123442 & 1234 & Subsam & e Prev & & & ELSON_3,JENNY & & 0 & 0 & 0 & 0 & 00 & 0 & 0 \\
\hline 152141 & 1521 & Reschec & led Ap & pointme & & RAKE_1, WENDY & 0 & 0 & 0 & 0 & 0 & 000 & 0 & 0 \\
\hline 161002 & $16100 z$ & Informe & Conse & it Stati & & TWARTIN_1, THOMAS & 0 & 0 & $\cdot 1$ & 0 & 0 & 00. & & 0 \\
\hline 164673 & 164673 & $\mathrm{sc}$ & $\mathrm{P}$ & $M$ & 34 & BROWN, ADAM & 0 & 0 & $\cdot 1$ & 0 & 0 & 00. & & 0 \\
\hline 167942 & 167942 & $\mathrm{sc}$ & $\mathrm{P}$ & $\mathrm{F}$ & 31 & HARRIS_2, MARIA & 0 & 0 & 0 & 0 & 0 & 000 & 0 & 0 \\
\hline 171098 & 171098 & $\mathrm{sc}$ & $\mathrm{P}$ & $\mathrm{F}$ & 73 & WALLACE_2, MILDRED & 0 & 0 & $\cdot 1$ & 0 & 0 & 000 & . & . \\
\hline 224633 & 224633 & $\mathrm{SC}$ & $P$ & M & 46 & SMITH_2,TYLER & 0 & 0 & $\cdot$ & 0 & 0 & 000 & & 0 \\
\hline 238763 & 238763 & $\mathrm{sc}$ & $\mathrm{P}$ & $\mathrm{F}$ & 73 & WALLACE_3, MILDRED & 0 & 0 & $\cdot 1$ & 0 & 0 & 000 & 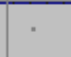 & · \\
\hline 240491 & 240491 & $\mathrm{sc}$ & $\mathrm{P}$ & $\mathrm{F}$ & 39 & WALLACE_3,JILL & 0 & 0 & 0 & 0 & 0 & 000 & 0 & 0 \\
\hline 244445 & 244445 & $\mathrm{SC}$ & $P$ & $\mathrm{~F}$ & 36 & NELSON_2, JENNY & 0 & 0 & 0 & 0 & 0 & 000 & 0 & 0 \\
\hline 248212 & 248212 & $\mathrm{sc}$ & $\mathrm{P}$ & $M$ & 28 & TAYLOR_2, BEN & 0 & 0 & $\cdot$ & 0 & 0 & 00. & & 0 \\
\hline 251401 & 251401 & $\mathrm{sc}$ & $P$ & $M$ & 36 & NELSON_3,HOWARD & 0 & 0 & . & 0 & 0 & 000 & & 0 \\
\hline
\end{tabular}

\begin{tabular}{ll}
\hline *Reports & This option is grayed out and not applicable. \\
\hline Show Results & $\begin{array}{l}\text { Lists the SPs who have completed the component, including appt/exam and } \\
\text { component status. }\end{array}$ \\
\hline *Room Log & Lists all SPs in current session. \\
\hline *Session Preview* & $\begin{array}{l}\text { Displays a list of laboratory containers and a graphic description of the } \\
\text { contents. A complete description of this functionality is described in } \\
\text { Section 3.7. }\end{array}$ \\
\hline Container Map & $\begin{array}{l}\text { List the SPs who are eligible for the various subsamples. A complete } \\
\text { description of this functionality is described in Section 3.8. }\end{array}$ \\
\hline Subsample Preview & $\begin{array}{l}\text { Lists the SPs who have rescheduled from a previous session into the current } \\
\text { session. A complete description of this functionality is described in Section } \\
\text { 9.3.3. }\end{array}$ \\
\hline Rescheduled & $\begin{array}{l}\text { Lists the various modules that require consent and displays the result of the } \\
\text { consent - excluded or not excluded. A complete description of this } \\
\text { functionality is described in Section 3.9. }\end{array}$ \\
\hline Informed Consent Status
\end{tabular}




\subsubsection{Modules}

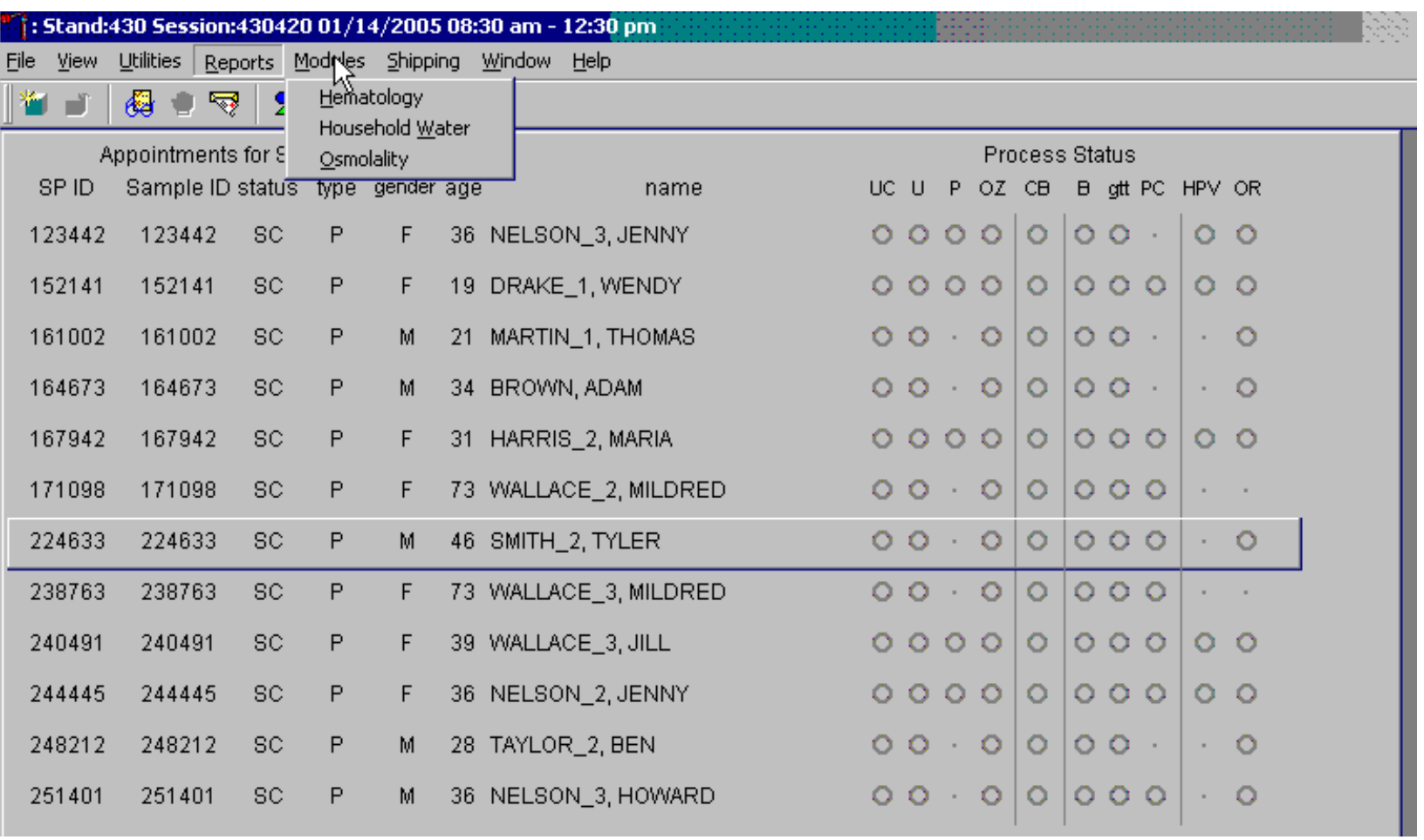

\section{Modules}

Hematology

Opens the laboratory hematology module. A complete description of this functionality is described in Section 7.XI.

Household Water

Opens the household water collection module. A complete description of this functionality is described in Chapter 4 of the Volatile Organic Compounds (VOC) and Perchlorate Study Procedures Manual.

Osmolality Open the osmolality module. A complete description of this functionality is described in Chapter 12. 


\subsubsection{Shipping}

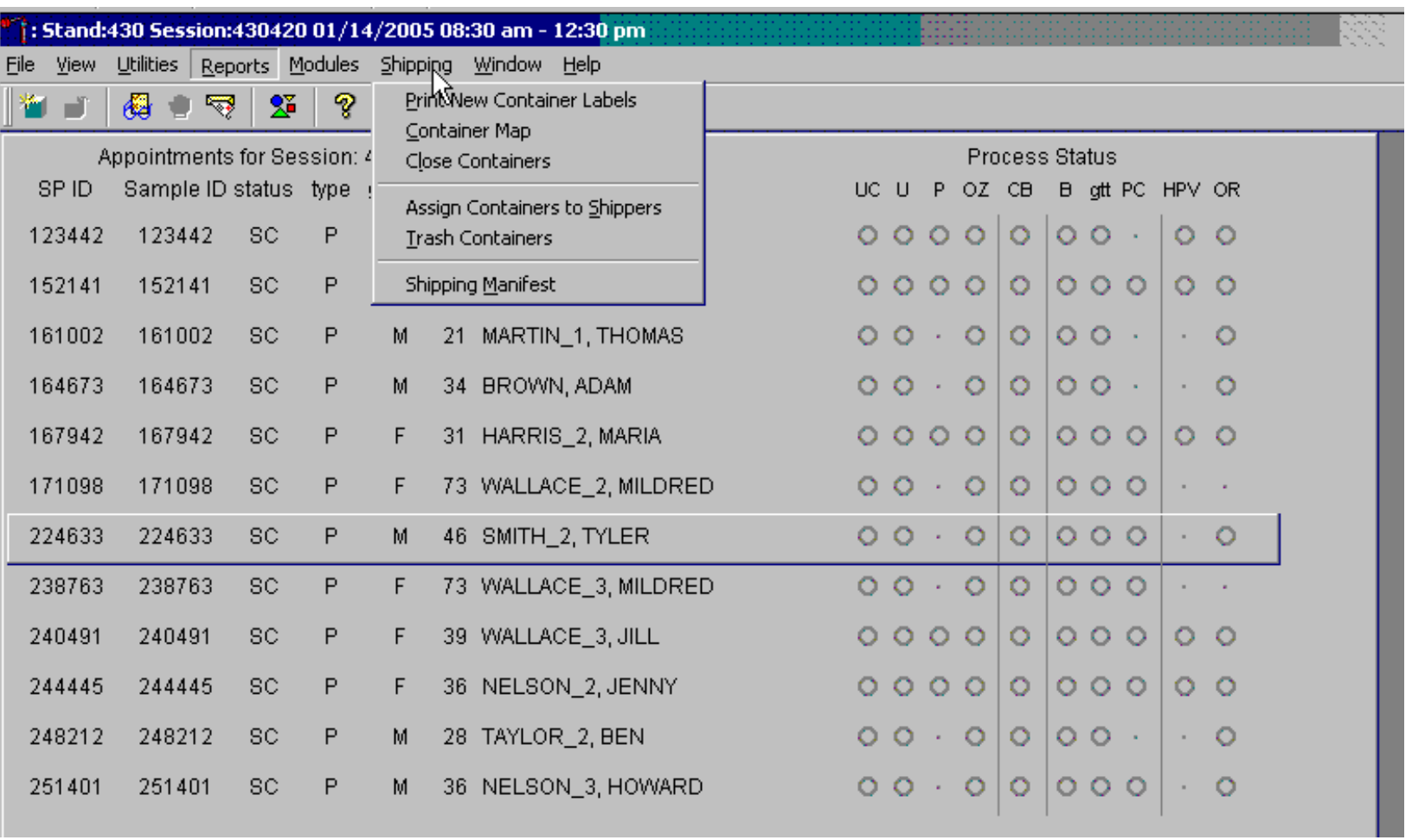

\section{Shipping}

Print New Container

Allows the medical technologist to print labels for containers such as a

Labels 5-inch box with $9 \times 9$ grid. A complete description of this functionality is described in Section 9.5.

Container Map

Allows the medical technologist to view a list of all containers with a graphic representation of the samples assigned to that container. A complete description of this functionality is described in Section 3.7.

\begin{tabular}{ll}
\hline Close Containers & $\begin{array}{l}\text { Allows the medical technologist to "close" active containers that have } \\
\text { been previously opened. A complete description of this functionality is } \\
\text { described in Section 9.9. }\end{array}$ \\
\hline $\begin{array}{l}\text { Assign Containers to } \\
\text { Shippers }\end{array}$ & $\begin{array}{l}\text { Allows the medical technologist to assign or associate a closed container } \\
\text { with a FedEx airbill number. A complete description of this functionality } \\
\text { is described in Section 9.10. }\end{array}$
\end{tabular}




\begin{tabular}{ll}
\hline Trash Containers & $\begin{array}{l}\text { Allows the medical technologist to delete container label numbers in the } \\
\text { database for labels that are damaged. A complete description of this } \\
\text { functionality is described in Section 9.6. }\end{array}$ \\
\hline Shipping Manifest & $\begin{array}{l}\text { Allows the medical technologist to print hard copy manifests and } \\
\text { electronically send email files to the contract laboratories. A complete } \\
\text { description of this functionality is described in Section 9.11. }\end{array}$ \\
\hline
\end{tabular}

\subsection{Informed Consent Exclusions}

If at any time during the phlebotomy or physician exam, the SP indicates that he or she does not want his or her biological specimens tested for HIV (AIDS), STD (sexually transmitted diseases include chlamydia), PSA, HPV, genetics, or future studies, then the staff enter this information using the IC Exclusions module. 
Access the IC Exclusions module.

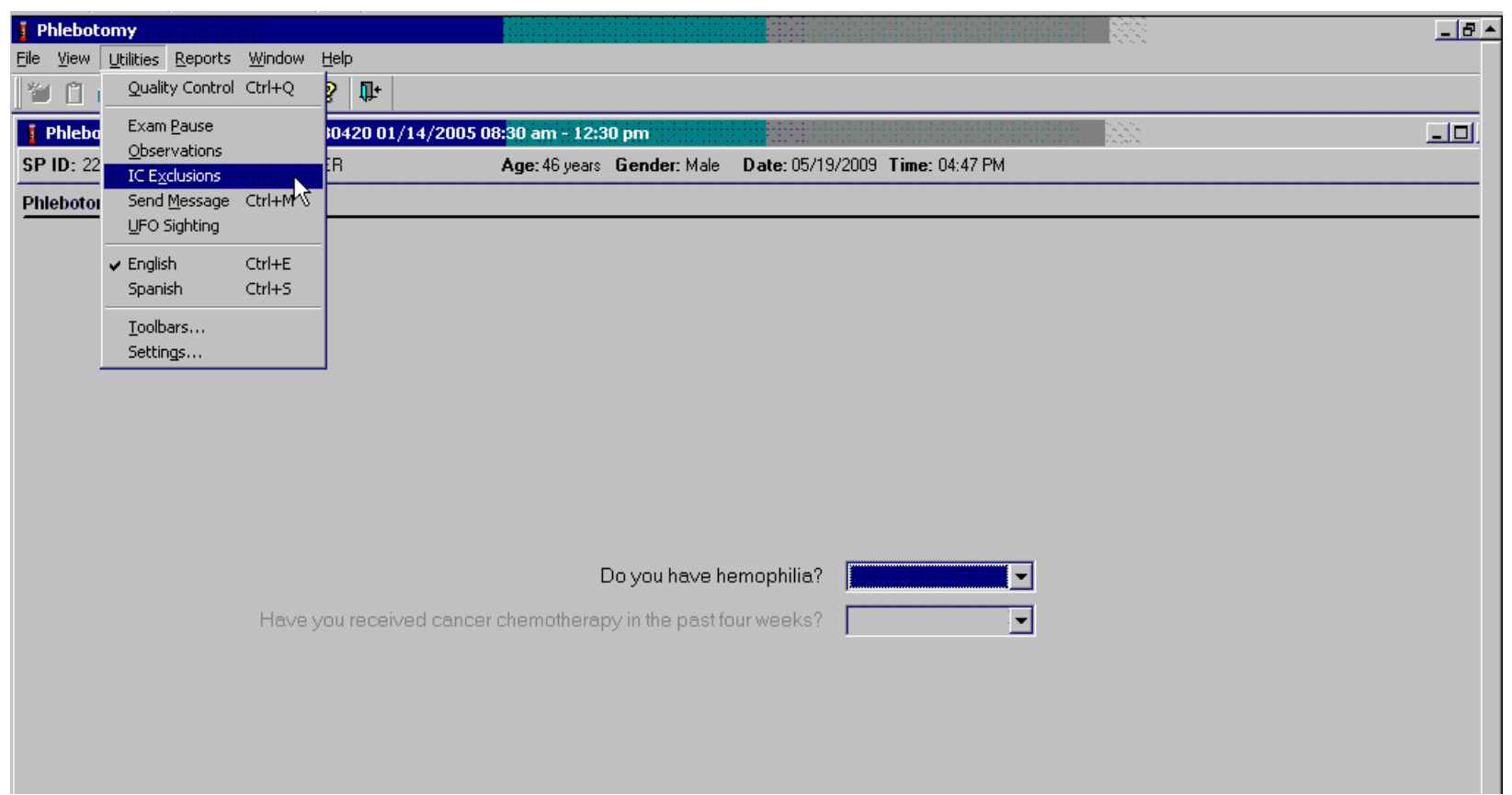

To access the IC (informed consent) Exclusions module, use the mouse to direct the mouse arrow to $\{$ Utilities $\}$ in the menu bar, left click, drag the arrow to $\{$ IC Exclusions\}, and left click, or type [Alt] [U/i], [X/X]. 
The IC Exclusions window displays all the specific exclusion item tests or groups of tests for which the SP is eligible and the status for each exclusion item.

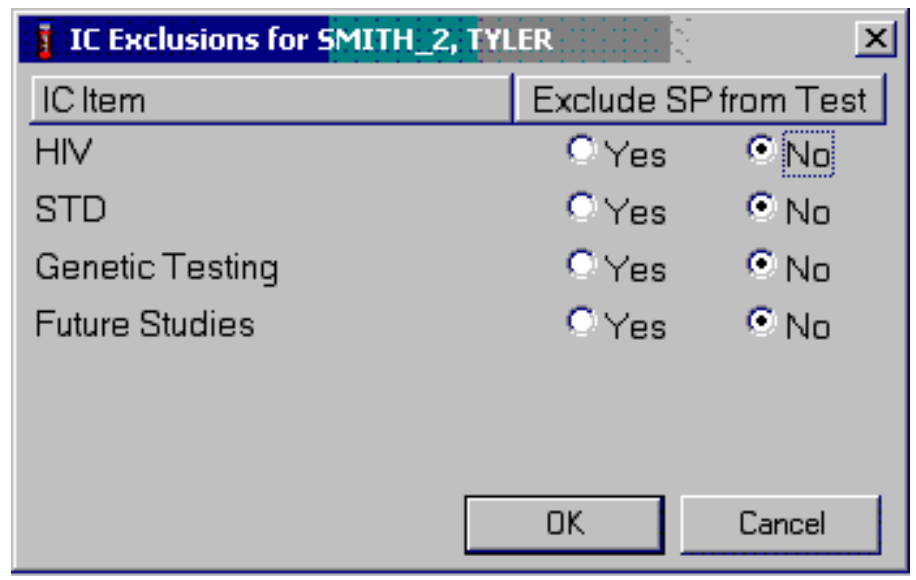

Record each item as directed by the SP. To mark each item, use the mouse to direct the mouse arrow to the "Yes" or "No" radio button and left click. To record these actions and save them to the database, use the mouse to direct the mouse arrow to the OK button and left click, or select [Enter]. To cancel these actions without saving the data to the database, use the mouse to direct the mouse arrow to the Cancel button and left click. 


\subsection{Set Session Utility}

Sign onto the computer terminal. Open the laboratory application. If the laboratory is conducting a MEC session, the heads-up display will default to the current session. Use the Set Session utility to access past or future sessions.

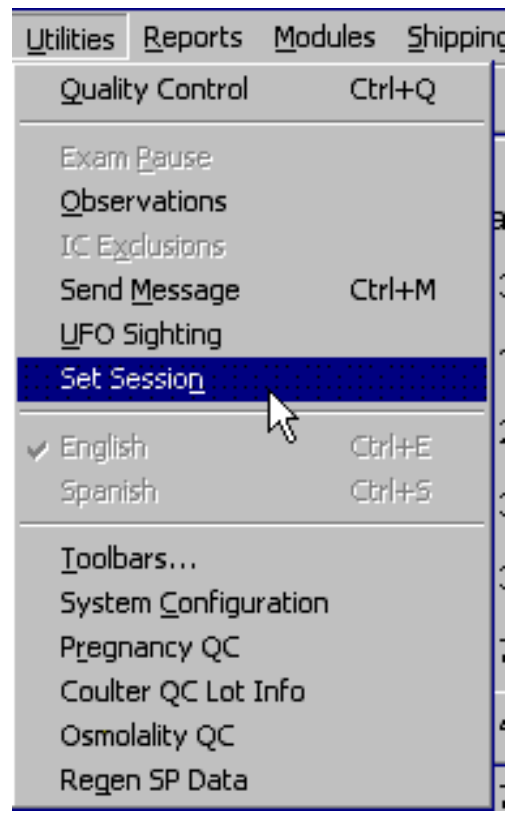

To access the Set Session utility, use the mouse to direct the mouse arrow to Utilities and left click. Drag the arrow to Set Session and left click. 
The Session PickUp list displays. Select the correct session from the pick list.

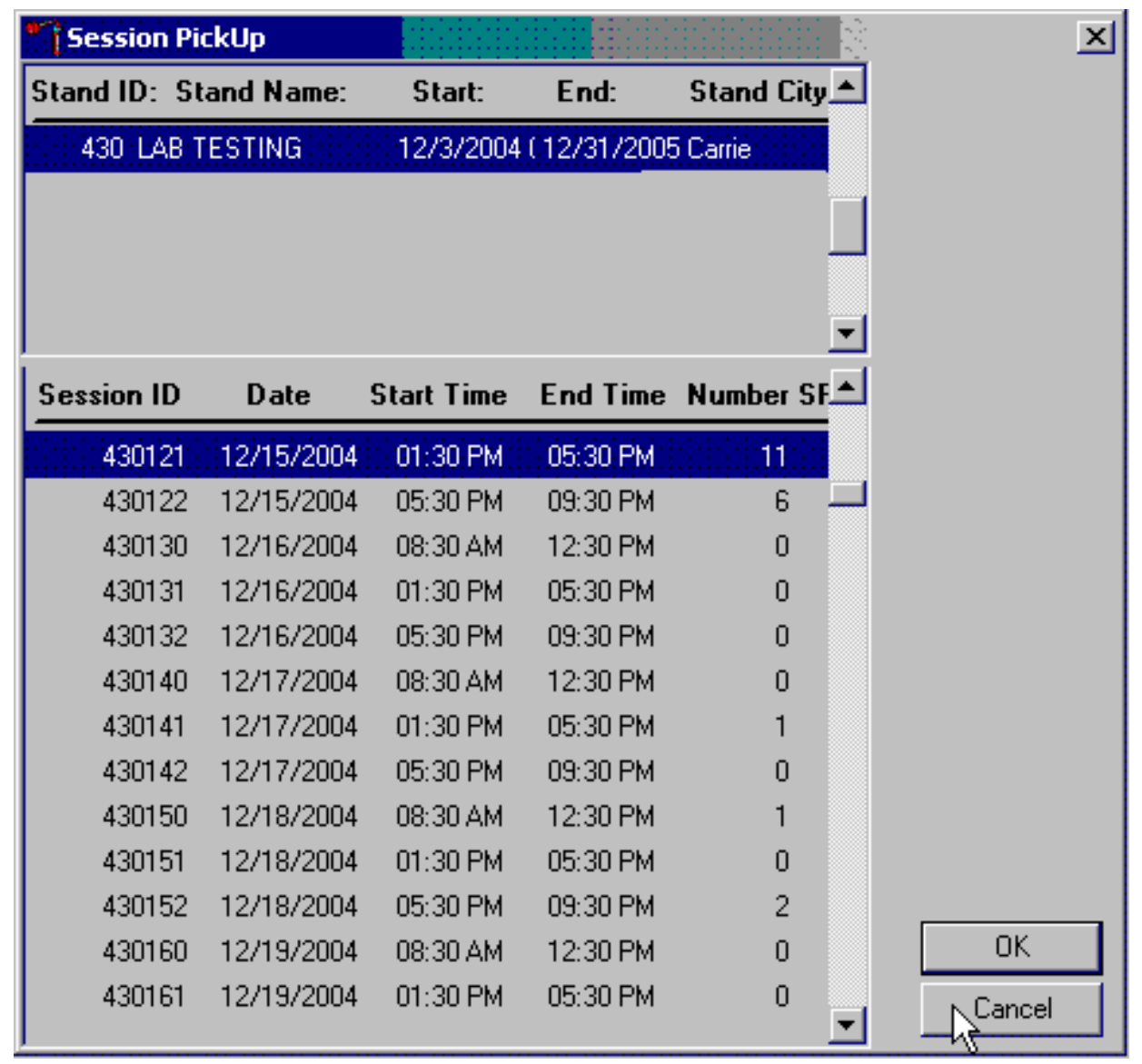

The Session PickUp window displays. The current stand is displayed in the top section of the window. Use the lower vertical scroll bar to view all sessions. Use the mouse to select the desired session. To proceed, hit the OK button or press [Enter]. To cancel and return to the previous session, click on the Cancel button. 
After selecting a session from the Session PickUp list and selecting OK, the laboratory heads-up screen displays.

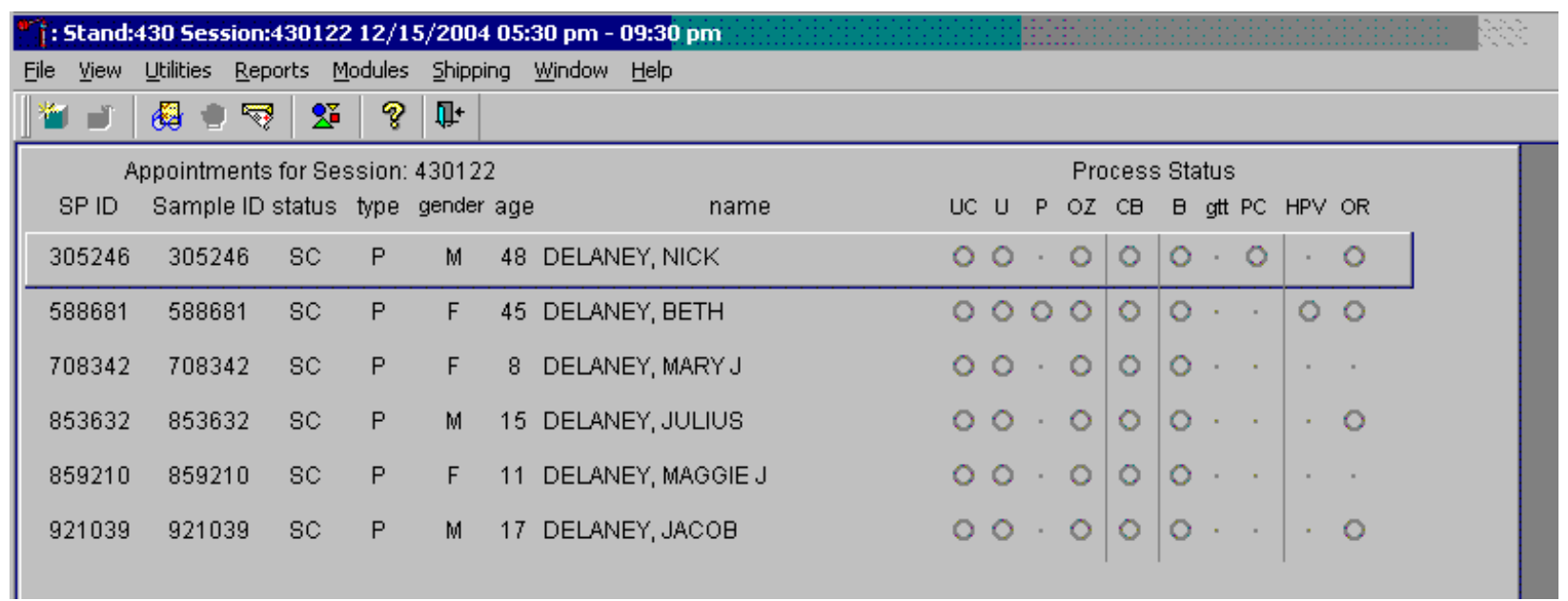

3.7

\section{Container Map}

Check the slot assignment of all containers at the end of each session.

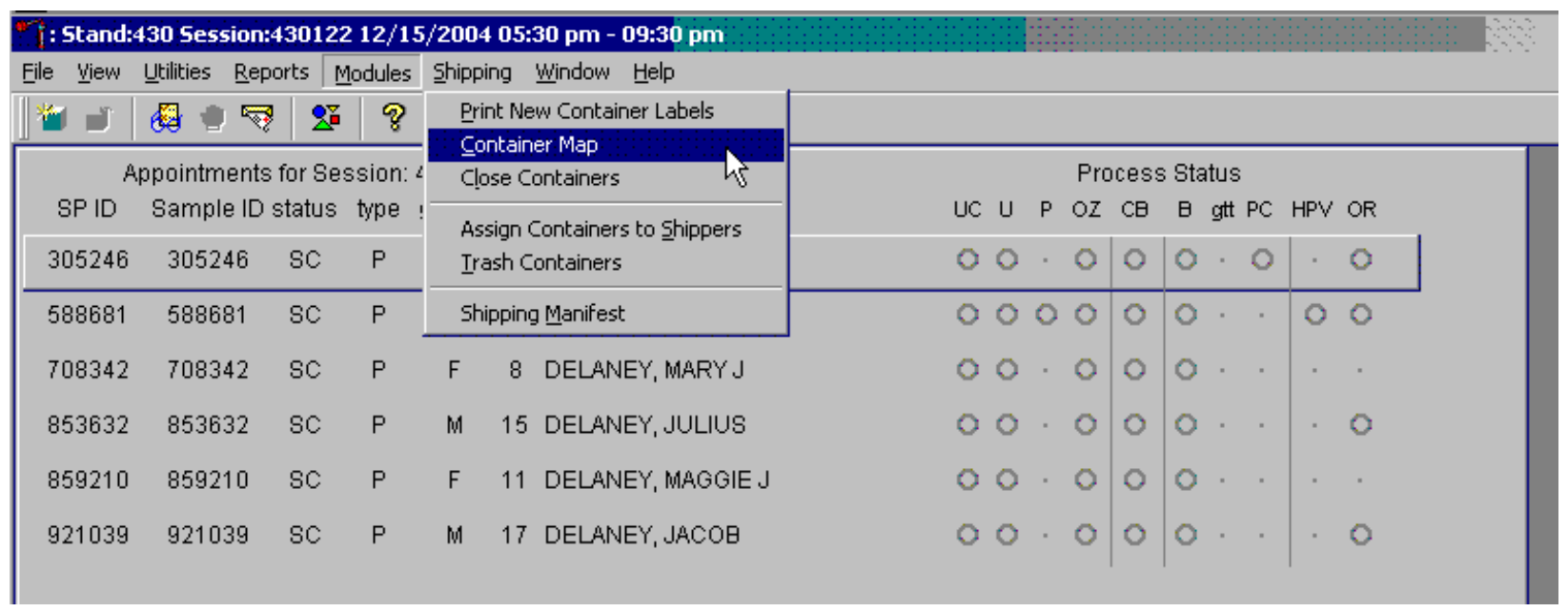

Access the Container Map report under the Reports or Shipping menu, or type [Alt] [R/r] [C/c] or [Alt] [S/s], [C/c]. 
Verify the contents of each container against the container map.

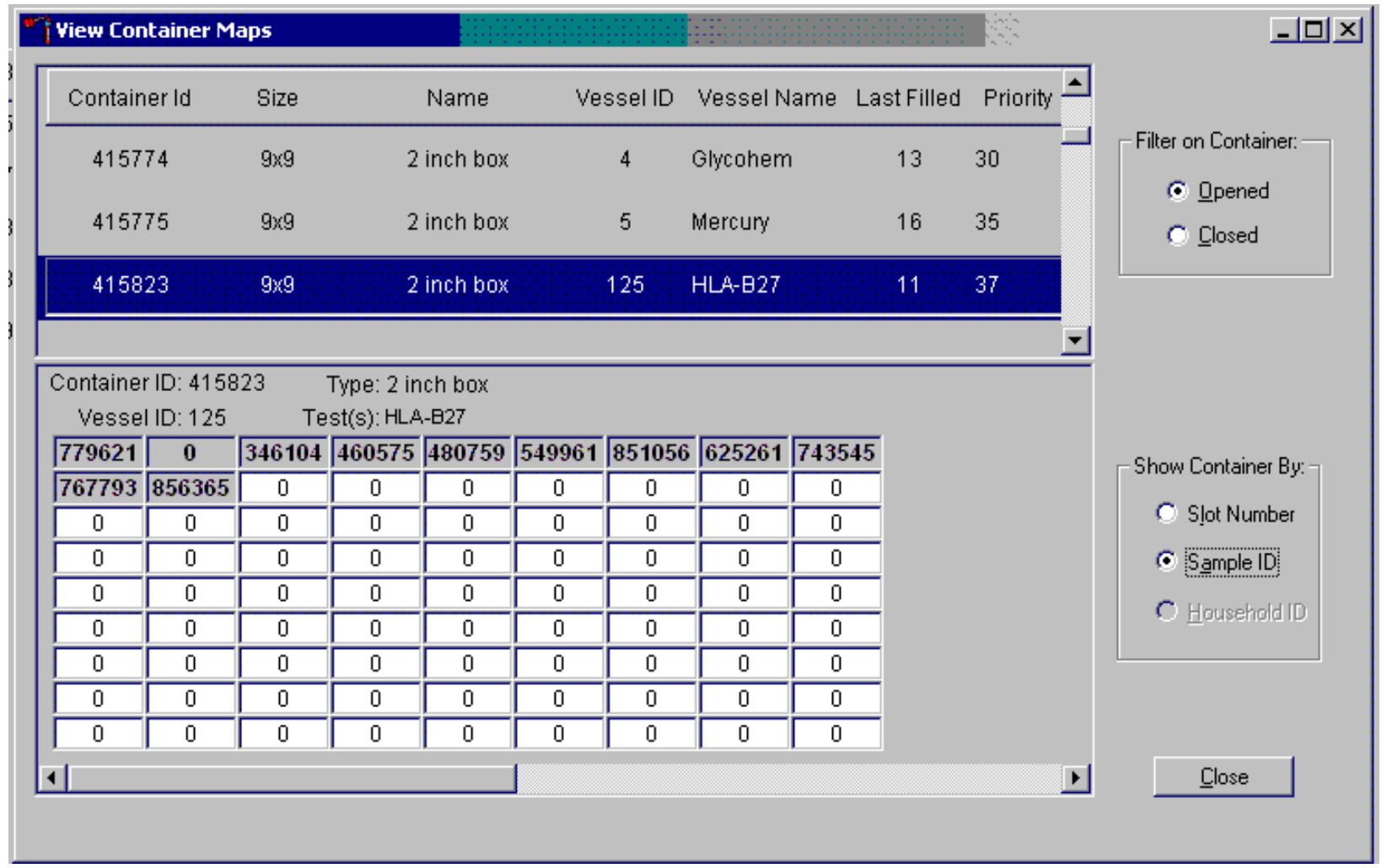

- If "Opened" under "Filter on Container" is selected, then all open containers will display.

- If "Sample ID” under "Show Container By:” is selected, then sample IDs will display in the assigned slot in the graphical representation of the container.

- Container IDs may be selected by mouse or by using the up and down keys.

- Verify the container ID on the box against the container ID listed on the screen and verify each vessel sample ID against its location in the map.

- Mark the specimens that have been checked by placing a black dot in the center of the vial cap. When subsequently checking the Container Map report for this container, begin checking the specimens that are not marked.

Continue checking each Container Map report for the remaining vessels. 
For the Household Water sample container, the Container Map report will display the household ID. Under "Show Container By:" the "Sample ID” is disabled and "Household ID” is the default selection.

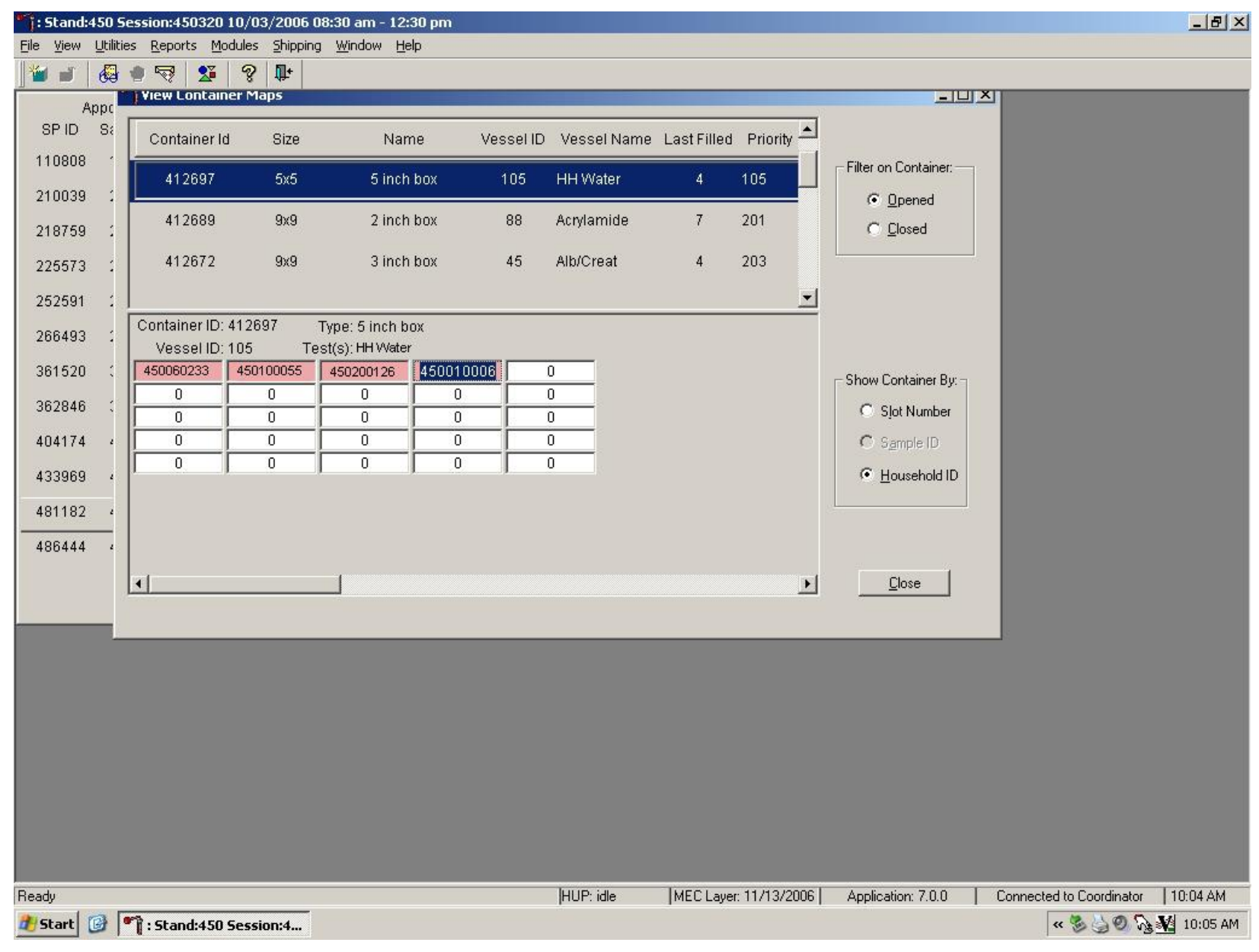

To exit the Container Map report, click on the Close button. 


\subsection{Subsample Preview}

Access the Subsample Preview to view all SPs in the current session and the selected subsamples for which they are eligible.

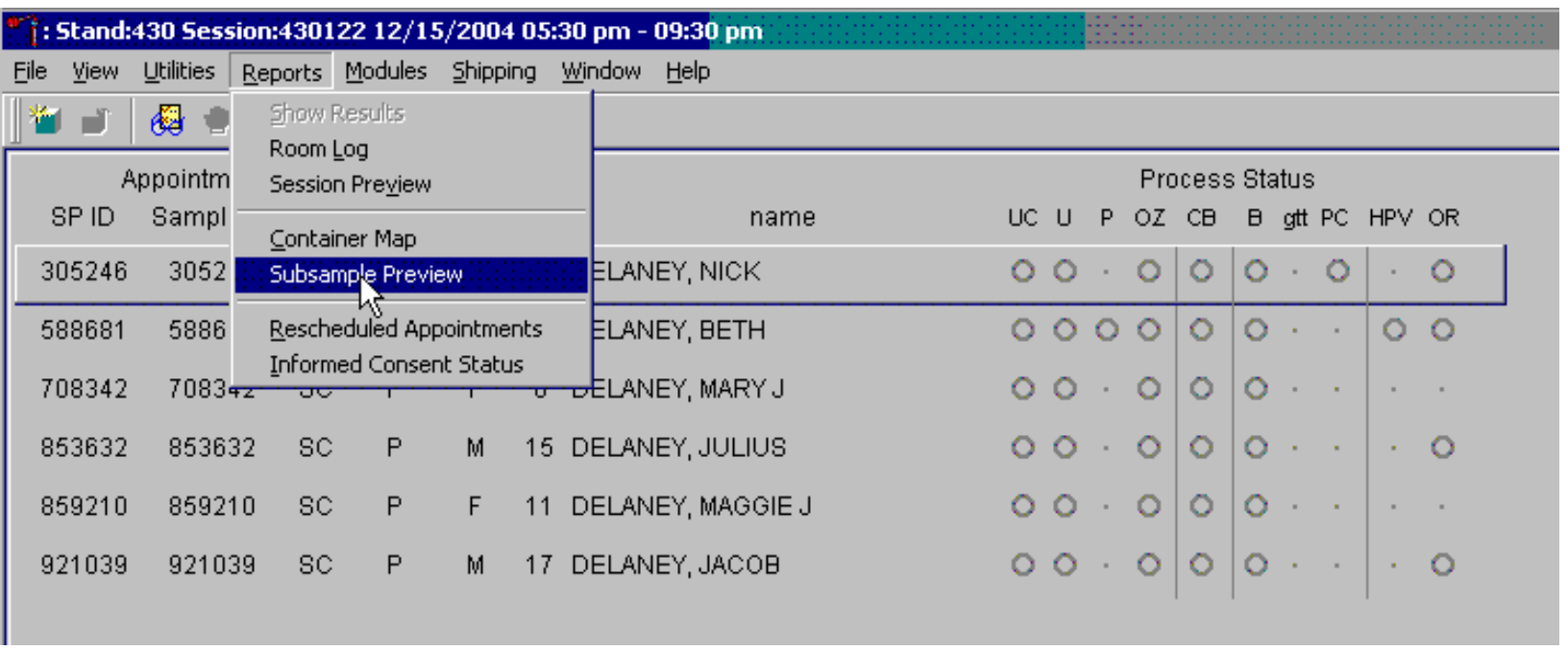

Access the Subsample Preview report under Reports in the menu bar. 
Review the Subsample Preview report.

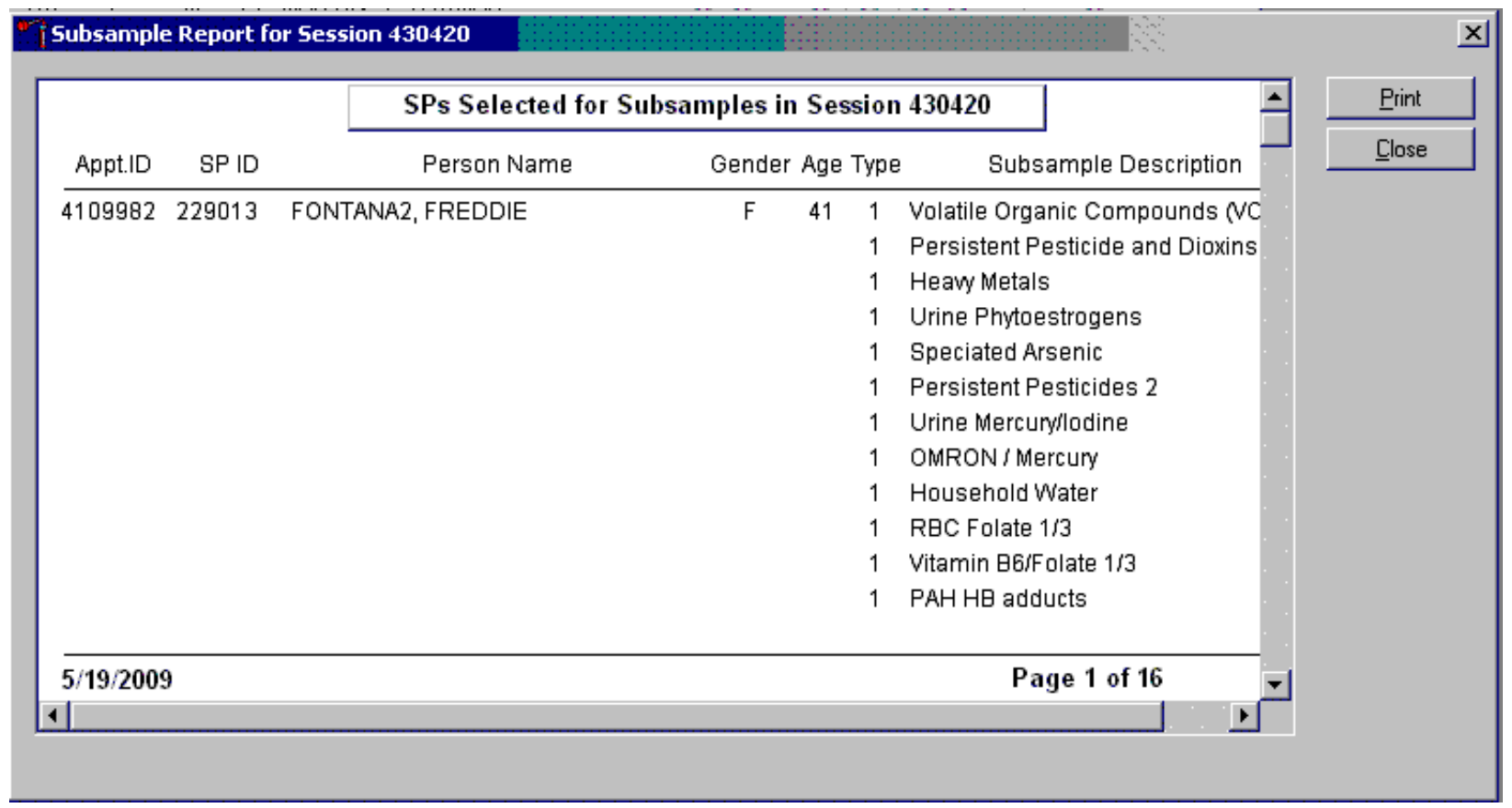

The Subsample Preview report includes all SPs assigned to the current session and the subsamples for which they are eligible. This report contains the appointment ID, SP ID, person name, gender, age, appointment type, and subsample description. Only SPs selected for inclusion in specific subsamples are eligible to have the related vessel filled. To view the entire report, use the scroll bar. To print a copy of the report, use the mouse to direct the mouse arrow to the Print button and left click. To exit and close the report, click on the Close button. 
Access the Informed Consent Status report to view SPs and the status of the informed consent result for various tests and/or groups of tests.

\begin{tabular}{|c|c|c|c|c|c|c|c|c|c|c|c|c|c|c|c|c|}
\hline File View & ㄴtilities & Reports & Modules & Shipp & ng & window Help & & & & & & & & & & \\
\hline 政 & 圈 & \multirow{2}{*}{\multicolumn{4}{|c|}{$\begin{array}{l}\text { Shows Results } \\
\text { Room Log } \\
\text { Session Preview }\end{array}$}} & \multirow[b]{3}{*}{ name } & \multirow{2}{*}{ UC } & & & & & & & & & \\
\hline \multirow{2}{*}{\multicolumn{2}{|c|}{ SPID $\begin{array}{r}\text { Appointm } \\
\text { Sampl }\end{array}$}} & & & & & & \multicolumn{10}{|c|}{ Process Status } \\
\hline & & \multirow{2}{*}{\multicolumn{4}{|c|}{$\begin{array}{l}\text { Container Map } \\
\text { Subsample Preview }\end{array}$}} & & & $\mathrm{U}$ & \multicolumn{2}{|c|}{$\mathrm{P} \quad \mathrm{OZ}$} & $\mathrm{ZCB}$ & $\mathrm{B}$ & \multicolumn{2}{|c|}{ gtt $P C$} & \multicolumn{2}{|c|}{ HPV O } \\
\hline 305246 & 3052 & & & & & ELANEY, NICK & 0 & 0 & - & 0 & 0 & 0 & - & 0 & $\cdot$ & 0 \\
\hline 588681 & 5886 & \multicolumn{4}{|c|}{ Rescheduled Appointments } & ELANEY, BETH & 0 & 0 & 0 & 0 & 0 & 0 & - & & 0 & \\
\hline 708342 & 70834 & \multicolumn{4}{|c|}{$\frac{\text { Informed Consent Status }}{\sqrt{L}}$} & fDLANEY, MARY J & 0 & & - & 0 & 0 & 0 & - & & & - \\
\hline 853632 & 85363 & $\mathrm{SC}$ & $\mathrm{P}$ & M & 15 & DELANEY, JULIUS & 0 & 0 & * & 0 & 0 & 0 & - & * & & 0 \\
\hline 859210 & 85921 & $\mathrm{SC}$ & $P$ & $\mathrm{~F}$ & 11 & DELANEY, MAGGIE J & 0 & 0 & - & 0 & 0 & 0 & - & - & & - \\
\hline 921039 & 92103 & $\mathrm{SC}$ & $P$ & M & 17 & DELANEY, JACOB & 0 & & . & 0 & 0 & 0 & . & $\cdot$ & & 0 \\
\hline
\end{tabular}

Access the Informed Consent Status report under the Reports menu in the menu bar. 
Review the SPs scheduled into the current session and the status of their informed consent by using the Informed Consent Status report.

\begin{tabular}{|c|c|c|c|c|c|c|c|c|c|}
\hline \multicolumn{3}{|c|}{ Consent Report for Session 430420} & \multirow[b]{3}{*}{ Std } & \multirow{3}{*}{$\begin{array}{c}\text { Genetic } \\
\text { Research }\end{array}$} & \multirow{3}{*}{$\begin{array}{c}\text { Future } \\
\text { Research }\end{array}$} & \multirow[b]{3}{*}{ HPV } & \multirow[b]{3}{*}{ PSA } & \multirow{3}{*}{$\Delta$} & \multirow{3}{*}{$\underline{\text { Print }}$} \\
\hline & \multirow[b]{2}{*}{ Sp Id } & \multirow[b]{2}{*}{ Hiv } & & & & & & & \\
\hline Appt Id & & & & & & & & & \\
\hline 4302791 & 123442 & $N$ & N & $Y$ & Y & N & - & & \\
\hline 4302792 & 251401 & N & N & Y & $Y$ & - & - & & \\
\hline 4302793 & 244445 & N & N & $Y$ & $\mathrm{Y}$ & $N$ & - & & \\
\hline 4302796 & 238763 & - & - & Y & $Y$ & - & - & & \\
\hline 4302798 & 240491 & N & N & $Y$ & Y & N & - & & \\
\hline 4302799 & 171098 & - & - & $Y$ & $Y$ & - & - & & \\
\hline 4302821 & 161002 & $N$ & N & Y & $Y$ & - & - & & \\
\hline 4302827 & 152141 & $N$ & $N$ & - & $Y$ & $N$ & - & & \\
\hline 4302840 & 164673 & $N$ & $N$ & $Y$ & $Y$ & - & - & & \\
\hline 4302890 & 248212 & $N$ & $N$ & $Y$ & $Y$ & - & - & $\square$ & \\
\hline 4302891 & 224633 & $N$ & $N$ & $N$ & $N$ & - & $N$ & & \\
\hline 4302927 & 167942 & $N$ & $N$ & $Y$ & $Y$ & $N$ & - & & \\
\hline & & & & & & & & \pm & \\
\hline
\end{tabular}

The consent report lists all SPs scheduled in the current session, their appointment and SP IDs, and the exclusion status for the following tests or groups of tests:

- HIV (vessels 22 - serum);

- $\quad$ STD (vessels 24 - HSV, 46 - Chlam);

- Genetic testing (vessels 119 and 120);

- $\quad$ Future research (vessels 8 - plasma, 32-39 - serum, 52 - urine);

- HPV Swab (vessel 78 - HPV swab); and

- $\quad$ PSA (vessel 75).

The status is "Y" or "Yes" if the SP is excluded and " $N$ " if the SP is not excluded. 
Select the correct SP and access the module of interest. Use the scroll bar to view all SPs scheduled into the session. The active SP is contained in a rectangular box. Use the heads-up display to select the correct SP. Select a different SP if the active SP is not the correct SP.

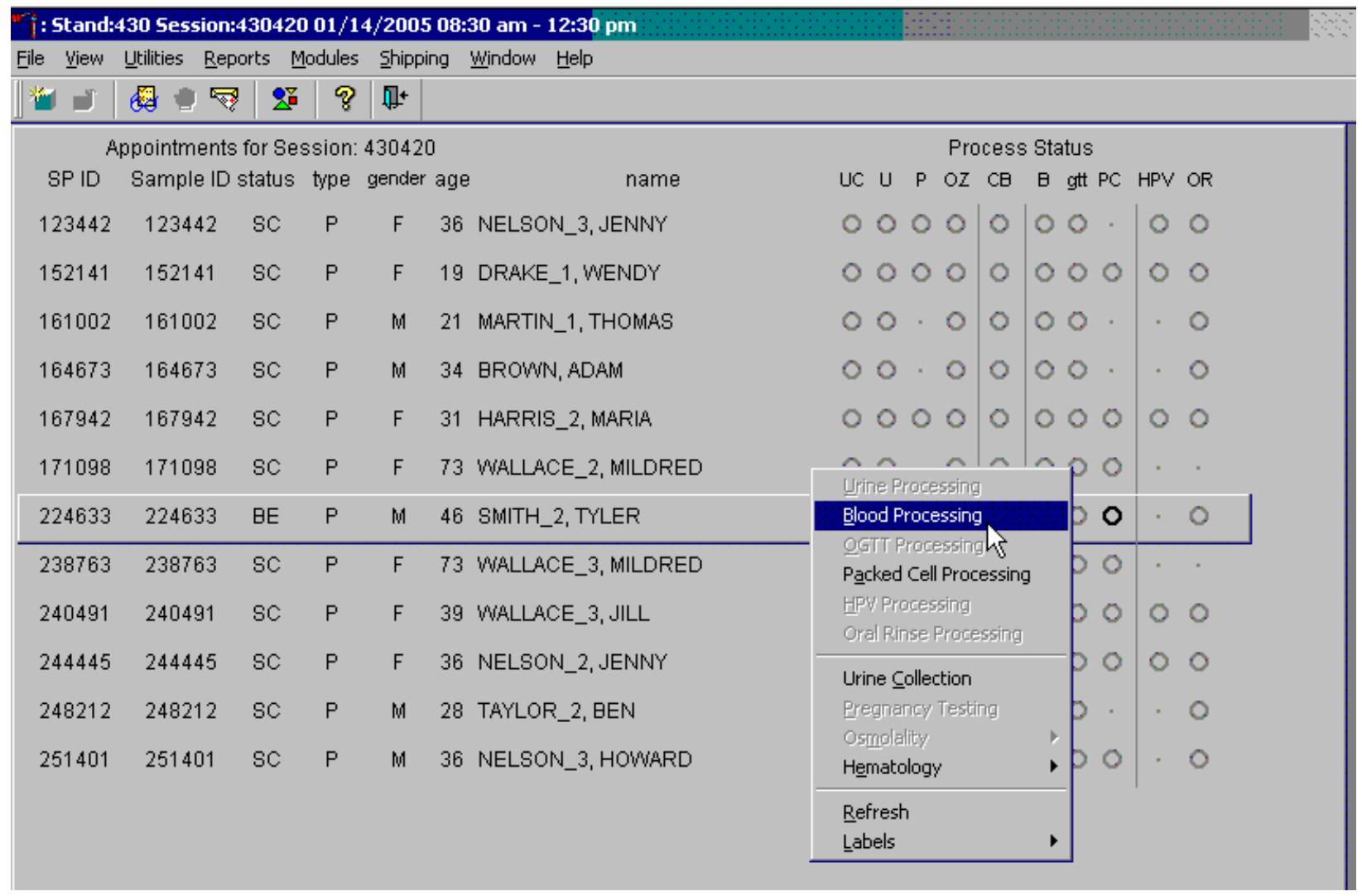

To select an SP, use the up and down keys to move up and down the list until the correct SP is highlighted or click on the SP. To access a specific processing module, click on the module on the heads-up screen: UP\{Urine Processing\}, BP\{Blood Processing\}, gtt\{OGTT Processing), (PC) Packed Cell Processing, HPV\{HPV Processing\}, OR \{Oral Rinse Processing\}, UC\{Urine Collection\}, $\mathrm{P}\{$ Pregnancy Testing $\}$, OZ \{Osmolality\}, or CB $\{$ Hematology $\}$ and left click. Alternatively, right click on an SP and select the correct module. 


\subsection{Warning and Error Messages}

Throughout the course of an examination, warning and error messages may appear when an attempt is made to perform an action the program cannot execute or requires confirmation to continue the action. The message normally appears with statements explaining the error condition. Complying with the error message statement will normally remedy the error. When required to confirm an action, such as deleting records, be sure the action is necessary because in most cases the action is irreversible.

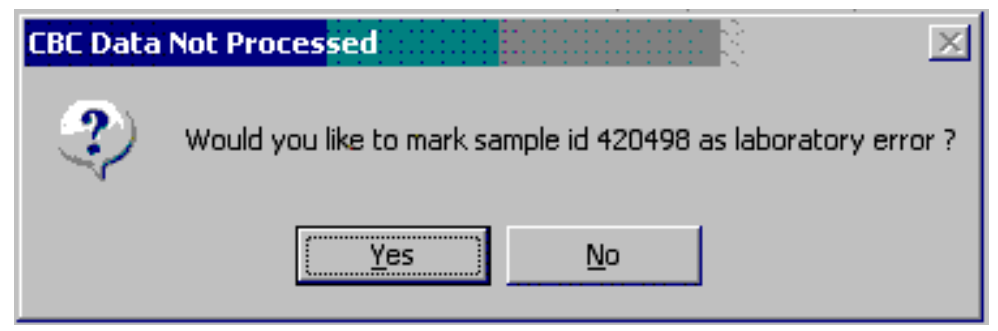

For example, if the Sample Person ID is incorrect in the SP Logon window, a warning message box displays indicating the problem and remedial instructions. To remove the warning message box, click on the OK button.

Warning messages require action.

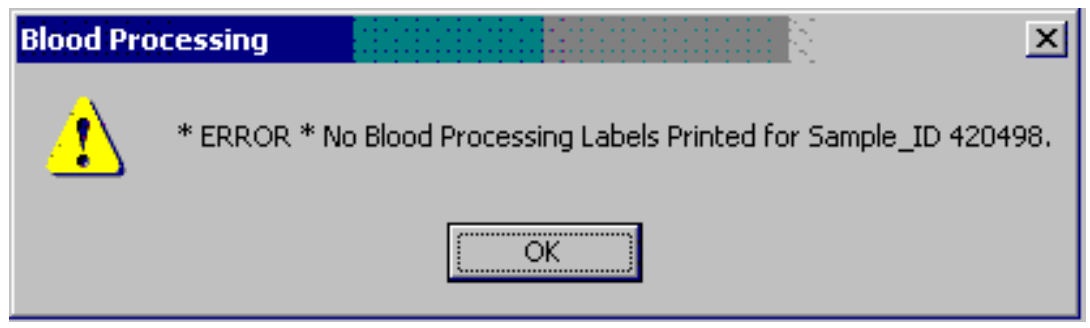

Remove the warning message and acknowledge the message by selecting the OK button.. 


\subsubsection{Not Filled Require a Comment}

Data cannot be saved until all vessels marked as Filled-’No” are assigned a comment.

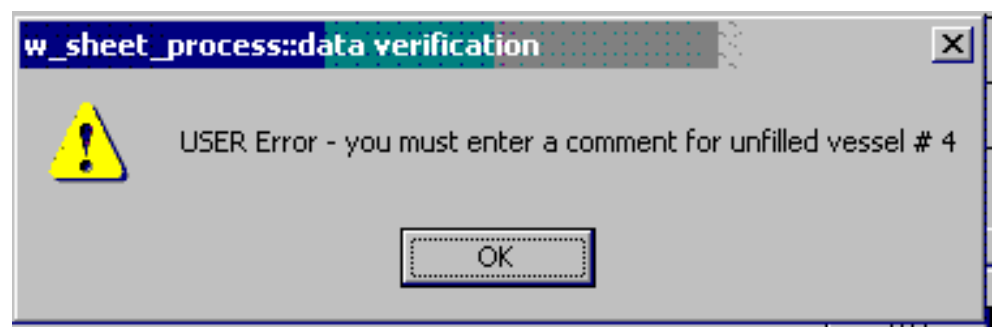

If the Save or the OK button is selected and all vessels are not marked as either Filled-"Yes” or Filled-”No,” a warning message box displays requesting a comment for each unfilled vessel. To remove the warning message box, click on the OK button.

\subsubsection{Labels Must be Printed in Advance}

If labels have not been printed in advance, a module-specific processing message box displays.

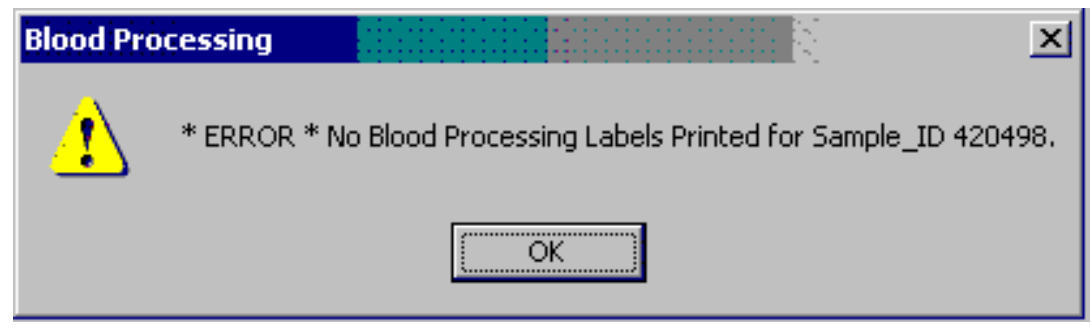

Specimen processing cannot occur unless labels have been printed in advance. To remove the warning message box, click on the OK button. Reprint the labels for all SPs or an individual SP in a session. 


\section{Printing Additional Labels}

Print additional labels for a particular SP using the heads-up display.

To print additional labels for a particular SP, right click on the SP and select $\{$ Labels $\}$, then drag the arrow to $\{$ All $\}$, $\{$ Blood $\}$, $\{$ Urine $\}$, $\{$ Pregnancy $\}$, $\{$ OGTT $\}$, $\{$ Packed Cell $\}$, or $\{$ Oral Rinse $\}$ and right click. Labels print for the SP in the Label/Ship area.

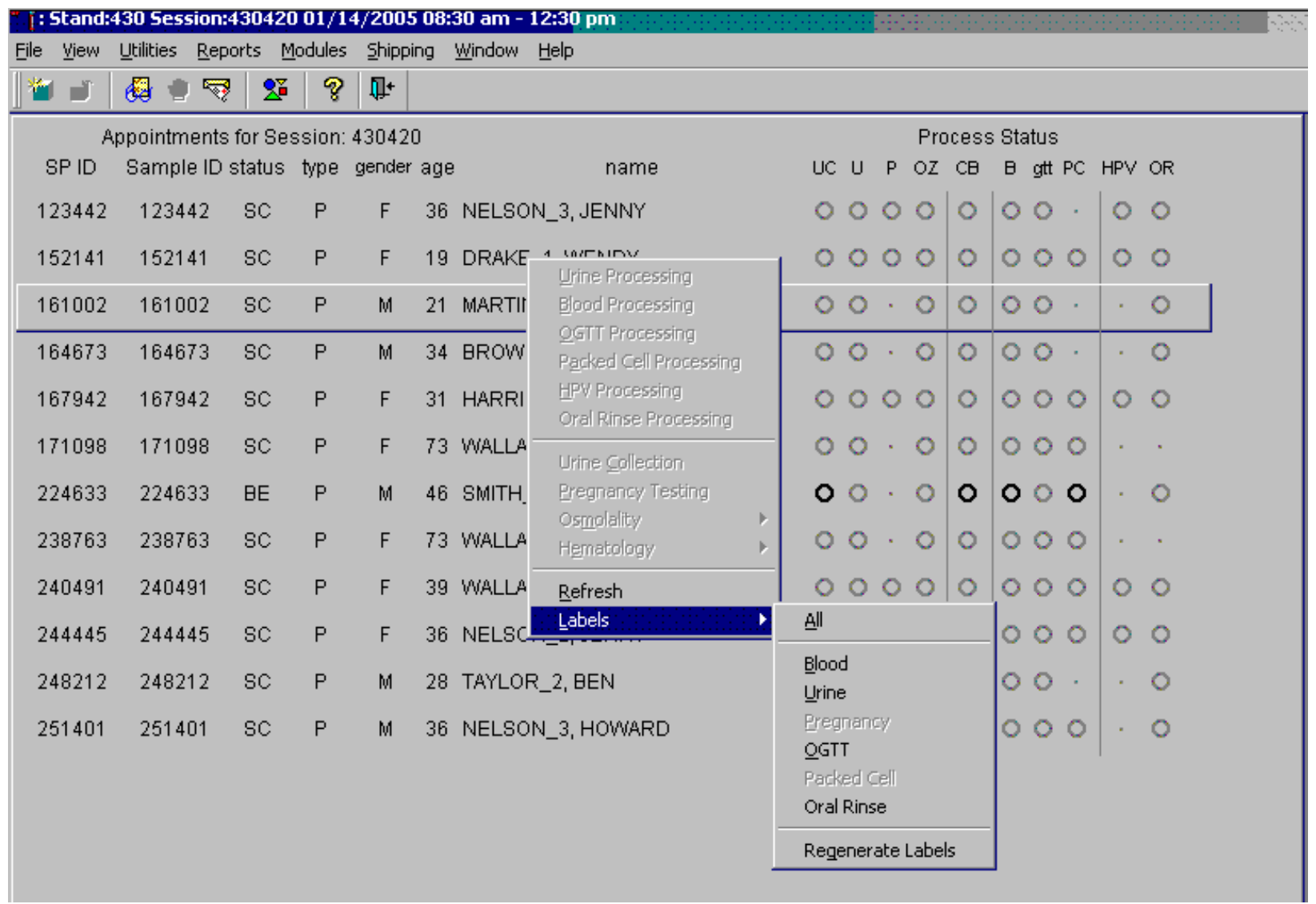




\subsection{2}

\section{Print an Ad Hoc Label}

Print an ad hoc label for a single vessel.

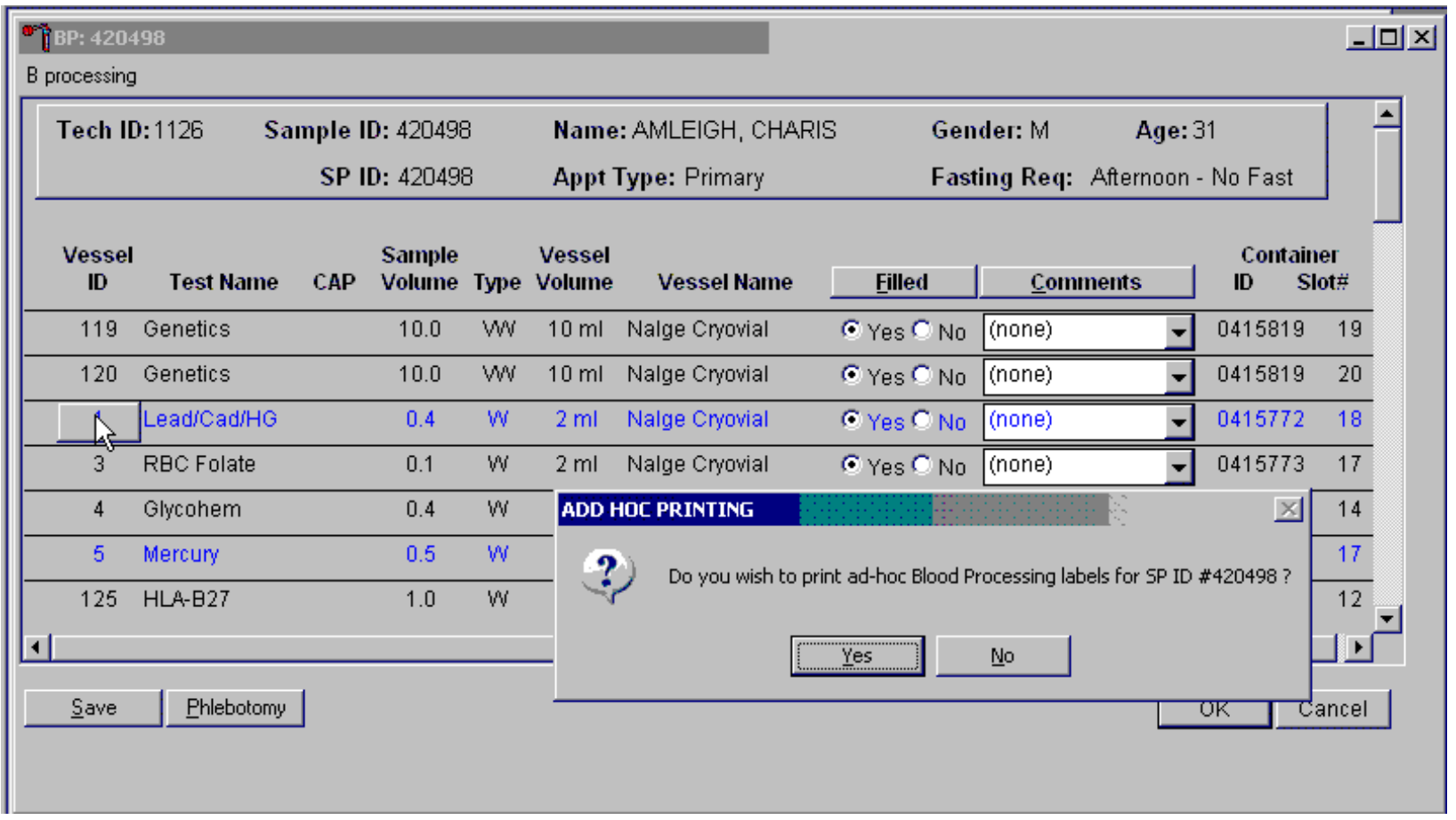

To print one ad hoc label for any one vessel in any of the processing modules, right click on the Vessel ID button. An ADD HOC PRINTING message text box displays. Respond to the message text box request. To print the label and remove the message text box, click on Yes or select [Enter]. To remove the message text box without printing a label, use the mouse to direct the mouse arrow to the No button and left click or type [N/n]. 


\section{Coulter}

The Hematology module does not need to be open before running SP samples on the HMX.

This is the NHANES Coulter Monitor. It must be open and running at the start of each session. This icon stores all HMX runs in the ISIS database. Open the icon by double-clicking on the Coulter icon on the desktop. The Coulter icon looks like this:

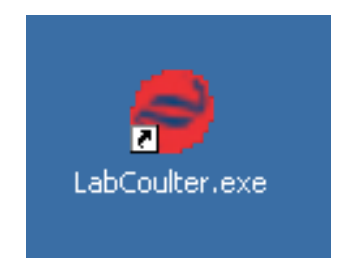

Note the red icon in the lower left hand corner of the system tray.

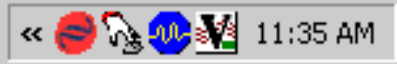

\section{Urine Scale}

Ensure that computer workstation 1 (Urine/Sink) is turned on and a technician is logged into the Laboratory application. Open the Urine Scale application by double clicking on the Urine Scale icon on the desktop. The Urine Scale icon looks like this:

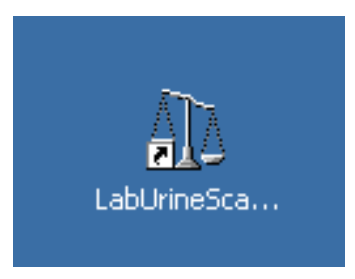

The Urine Scale application icon will appear in the system tray, similar to the Coulter application. This application captures data from the scale and logs it into the database. The Urine Scale 
application must be opened and in the system tray in order to capture any urine collection readings from the scale.

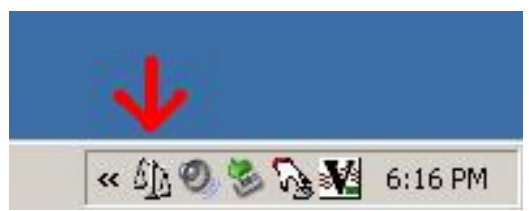

\section{Osmolality}

It is critical that the Osmo application must be running on the ISIS computer before running any SP samples. This application captures results from the Osmette II. Any samples run without the Osmo application running will be lost and will need to be rerun. To open the Osmo application, double click on the icon of a pipette on the desktop:

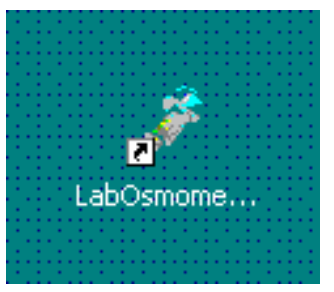

The application icon will display in the system tray when the application is running. It is very faint, but will turn green when data is transmitted from the Osmette to the ISIS computer.

\section{M.}

Double check to make sure this icon is visible before running SP samples. 


\section{VENIPUNCTURE}

\subsection{Introduction}

The blood collection procedure consists of (1) administering a questionnaire to screen for conditions that exclude the participant from the blood draw; (2) determining fasting status; and (3) performing a blood draw. The phlebotomist also collects two additional tubes for genetic testing, one additional blood tube from SPs selected for the VOC (volatile organic compounds) special study and administers a dextrose solution (Trutol) to and collects an additional 2-mL gray top tube from SPs selected for the Glucose Tolerance (GT) component.

The phlebotomist conducts an interview to screen SPs for conditions that exclude them from the blood draw. The phlebotomist also administers a fasting questionnaire to determine fasting compliance. The venipuncture protocol is dependent on the SP's age, appointment type, and session time. Exhibits 4-1 through 4-3 illustrate the various venipuncture protocols. These protocols indicate the types and numbers of tubes in priority order for each age group and person type. A chart located on the wall in the phlebotomy room also illustrates this information. It is extremely important to perform the venipuncture protocol as described for each SP. Use comments to describe differences between the actual tubes drawn and the established protocol. After the results of the blood draw are entered, labels automatically print for the blood tubes. The phlebotomist immediately labels collected tubes, places them through the pass-through window to the laboratory, and escorts the SP to the coordinator area or his or her next component. The phlebotomist may offer juice and crackers to SPs less than 12 years of age and SPs not selected for the GT component. 
Exhibit 4-1. Venipuncture protocol - primary

\begin{tabular}{|c|c|c|c|c|c|}
\hline & & & e in $\mathrm{Y}$ & & \\
\hline & $1-2$ & $3-5$ & $6-11$ & $12-19$ & $20+$ \\
\hline 3-mL Lavender & 1 & 2 & 1 & 2 & 1 \\
\hline 4-mL Lavender & & & 1 & 1 & 2 \\
\hline $\begin{array}{l}\text { 2-mL Gray } \\
\text { (Morning sessions only) }\end{array}$ & & & & 1 & 1 \\
\hline 3-mL Red & 2 & & & & \\
\hline 7-mL Red & & 2 & & & \\
\hline 15-mL Red & & & & 3 & 3 \\
\hline 10-mL Red & & & 2 & 1 & 1 \\
\hline 3-mL Red & & & 1 & & \\
\hline 10-mL Red ${ }^{\circ}$ & & & & 2 & 2 \\
\hline 10-mL Red ${ }^{1}$ & & & & 1 & 1 \\
\hline 7-mL EDTA ${ }^{1}$ & & & & 1 & 1 \\
\hline 10-mL EDTA $(20+)^{2}$ & & & & & 2 \\
\hline 10-mL Gray $(12+)^{3}$ & & & & 1 & 1 \\
\hline $\begin{array}{l}\text { 2-mL Gray }{ }^{4} \\
\text { (GTT morning sessions) }\end{array}$ & & & & 1 & 1 \\
\hline
\end{tabular}

${ }^{\circ}$ SPs selected for inclusion in the Persistent Pesticides or BFR subsamples

${ }^{1}$ SPs selected for inclusion in the Perfluoronated and Srm Pesticide subsamples

${ }^{2}$ Genetics tubes; must meet IC Exclusion criteria

${ }^{3}$ SPs selected for inclusion in the VOC subsample

${ }^{4}$ Glucose tolerance test

Exhibit 4-2. Venipuncture protocol - VIP guest

\begin{tabular}{lcccc}
\hline & \multicolumn{4}{c}{ Age in Years } \\
\cline { 2 - 5 } & $1-2$ & $3-5$ & $6-11$ & $12+$ \\
\hline 3-mL Lavender & 1 & 1 & 1 & 1 \\
\hline 4-mL Lavender & & & 1 \\
\hline 2-mL Gray & & & \\
(Morning sessions only) & & 1 & & \\
\hline 3-mL Red & 1 & & & 1 \\
\hline 7-mL Red & & & & 1 \\
\hline 15-mL Red & & & \\
\hline 10-mL Red
\end{tabular}


Exhibit 4-3. Venipuncture protocol - guest

\begin{tabular}{llc|}
\hline & \multicolumn{2}{c}{ Age in Years } \\
\cline { 2 - 3 } & $1-11$ & $12+$ \\
\hline 3-mL Lavender & 1 & 1 \\
\hline 3-mL Red & & 1 \\
\hline
\end{tabular}

If the veins of an SP appear too fragile to accommodate the size of the large red top tubes, substitute smaller red top tubes as an alternative protocol. Exhibit 4-4, Alternative venipuncture protocol - primary, illustrates the alternative size and number of tubes in boldface with the original tube protocol in parentheses. When the alternative protocol is substituted for the original protocol, a comment must be recorded. This protocol constitutes a deviation from the established standard; it should only be used in rare circumstances.

Exhibit 4-4. Alternative venipuncture protocol - primary

\begin{tabular}{lccc}
\hline & \multicolumn{3}{c}{ Age in Years } \\
\cline { 2 - 4 } & $3-5$ & $6-11$ & $12+$ \\
\hline 3-mL Red & $\mathbf{5}$ & $(1)$ & \\
\hline 7-mL Red & $(2)$ & $\mathbf{3}$ & $(3)$ \\
\hline 15-mL Red & & & $\mathbf{5}(1)$ \\
\hline 10-mL Red & & $(2)$ &
\end{tabular}

NOTE: Alternative venipuncture protocol tubes are shown in boldface; regular protocol tubes are shown in parentheses. 


\subsection{Equipment and Supplies}

The phlebotomy room contains a phlebotomy chair, drop-down bench, sink, data terminal, Dymo direct thermal label printer, refrigerator, phlebotomy cart, and cabinets. Figure 4-1 illustrates the floor plan for the phlebotomy room.

Figure 4-1. Floor plan for phlebotomy room

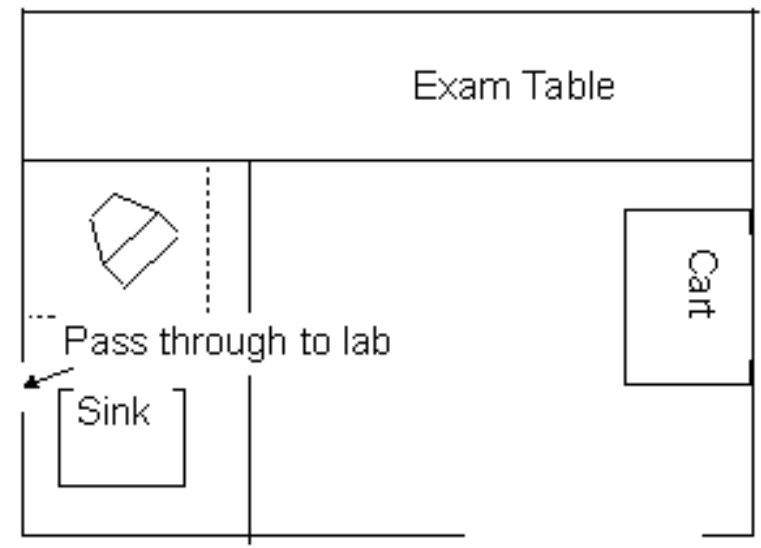

At the start and end of each stand, take a complete inventory using procedures described in Chapter 15, Section 15.3. 
The equipment and supplies used in venipuncture are listed in Exhibit 4-5.

Exhibit 4-5. Equipment and supplies - phlebotomy

\begin{tabular}{|c|c|}
\hline BD Hemogard Vacutainer ${ }^{\circledR}$ 3-mL EDTA & Stretch disposable tourniquet \\
\hline BD Hemogard Vacutainer ${ }^{\circledR}$ 4-mL EDTA & Latex tourniquet \\
\hline BD Hemogard Vacutainer® 2-mL Gray & Alcohol wipe \\
\hline BD Hemogard Vacutainer ${ }^{\circledR}$ 3-mL Red & $2 \times 2$ gauze square \\
\hline BD Hemogard Vacutainer ${ }^{\circledR}$ 7-mL Red & Co-Flex NL cohesive bandage \\
\hline Kendall Sherwood 15-mL Red & Adhesive bandage - Coverlet \\
\hline BD Vacutainer ${ }^{\circledR}$ 10-mL Red & Adhesive bandage - children \\
\hline BD Vacutainer® 10-mL Gray (VOC) & Transpore tape \\
\hline BD Hemogard Vacutainer® ${ }^{\circledR}$ 7-mL EDTA & Disposable baby wipes \\
\hline BD Hemogard Vacutainer ${ }^{\circledR}$ 10-mL EDTA & Baby oil \\
\hline BD Saf-T EZ® set 19 gauge & Surgical paper tape \\
\hline BD Luer adapter for use with the 19 gauge Saf- T EZ $®$ set & Cartoon stickers \\
\hline BD Vacutainer Safety-Lok ${ }^{\mathrm{TM}} 21$ gauge with adapter & Ammonia inhalant packet \\
\hline BD Vacutainer Safety-Lok ${ }^{\mathrm{TM}} 23$ gauge with adapter & Pillow and disposable pillow cover \\
\hline BD Vacutainer ${ }^{\circledR}$ multiple Eclipse 21 gauge needle & Squeeze ball \\
\hline BD Vacutainer ${ }^{\circledR}$ needle holder - single use & Juice and crackers \\
\hline Air sickness bag & List of reportable tests (handout) \\
\hline Heel warmer & Labels for Dymo printer \\
\hline \multirow[t]{2}{*}{ Liquid Band-Aid } & Sharps container 2 gallon \\
\hline & Biohazard bags 14 x 19 \\
\hline \multicolumn{2}{|l|}{ Non-Consumables } \\
\hline Step stool & Tube racks \\
\hline Phlebotomy cart & Floor mat \\
\hline Solar wall thermometer & CPR mask \\
\hline \multicolumn{2}{|l|}{ Obtain From Lab } \\
\hline Hand cream & Purell hand soap \\
\hline \multirow[t]{2}{*}{$\begin{array}{l}\text { Nonsterile, powder-free, nonlatex gloves -- small, medium, } \\
\text { large }\end{array}$} & Kleenex \\
\hline & Bleach bottle \\
\hline
\end{tabular}


For each SP, the phlebotomist routinely requires the following materials:

- Alcohol wipes

- 2"x 2" gauze squares

- Vacutainer ${ }^{\circledR}$ tubes of the appropriate size and type

- Disposable tourniquet

- $\quad$ Needle assembly

- Bandage

\subsection{Overview}

The coordinator tracks each SP through the MEC using the coordinator system. This system tracks the SP throughout the exam, including arrival, location during the session, and exit. The coordinator uses this system to direct the SP to the appropriate workstations in the MEC and to determine if all the appropriate examinations are complete. The MEC coordinator monitors exam component status using responses from examination stations.

It is critical to assign SPs aged $12+$ to phlebotomy as soon as possible at the start of a session, especially a morning session. It is also important to assess the fasting status of SPs aged 12+ assigned to a morning session because they are not eligible for the GT examination if they have not met their 9-hour fast requirement. The coordinator gives each SP a hard copy verification form as part of the check-in process. For SPs aged 12+ who are examined during a morning session, it includes the question, “Did you eat or drink anything, other than plain water, after 11:30 last night?” The SP records "Yes” or "No" in a check box and the coordinator enters this information into the coordinator application. The coordinator application then highlights (hot pink) the names of SPs who responded "Yes" since they have not met the 9-hour fast requirement. The coordinator uses the highlighted names to aid in prioritizing SPs for the phlebotomy component; SPs who are not highlighted are assigned to phlebotomy before SPs whose names are highlighted.

Each SP receives a bar-coded ID bracelet upon arrival at the MEC. The bracelet remains on the SP throughout the session. This bracelet contains the SP's ID number in bar code and eye-readable format. The phlebotomist "wands" the bracelet bar code with a bar code scanner (wand) to log the SP into 
the phlebotomy component. If necessary, the ID number can be entered manually by reading it from the bracelet and typing the numbers using the keyboard.

The phlebotomist is responsible for completing the venipuncture section of the phlebotomy exam.

\section{- Phlebotomy seating options}

For the blood draw, the seating options are dependent on the weight of the SP.

Figure 4-2. VM5000 series phlebotomy chair

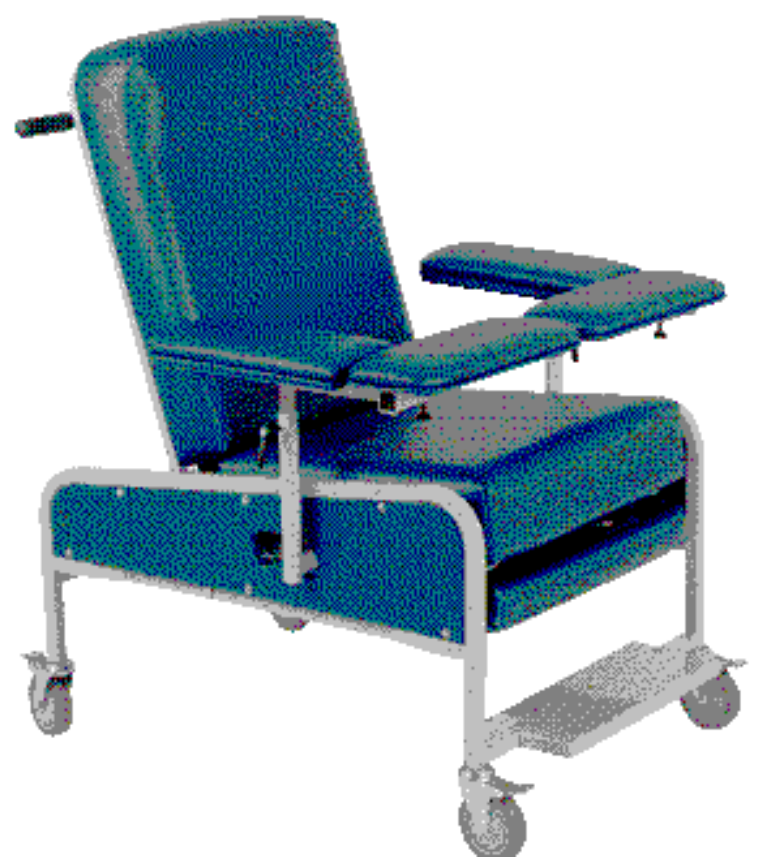

The VM5000-LXL phlebotomy chair features an extra wide 25” upholstered seat. There are three positions: upright, recline, and full recline. The VM5000 series is equipped with fully adjustable padded armrests that recline as the patient reclines. The chair includes a hand operated control to recline the chair with a touch of a button. The VM5000-LXL/XT provides a 25" seat to floor height and it includes casters.

There are two seating options for the blood draw and the choice is dependent on the weight of the SP. The chair weight restriction is $350 \mathrm{lbs}$. If the phlebotomist estimates that the weight of the SP is greater than 350 pounds then ask the SP to sit on the drop-down bench. If the phlebotomist estimates that 
the weight of the SP is less than 350 pounds and the SP will fit comfortably in the phlebotomy chair then ask the SP to sit in the chair. Roll wheelchairs completely into the room. If the SP feels faint, then recline the chair either before or during the blood draw as needed.

Lock the casters at all times except when repositioning the chair for those SPs over 350 lbs or wheelchair bound SPs. Position the four casters on the four floor marks. Screw in the arm rests. Raise and lower the front arm rest as needed. Raise and lower the right and left arm rests by using the black handle to loosen the screw. Push in the gray button to reposition the angle of the handle.

To recline the chair push the bottom button on the controller. The foot support will raise and the armrests will recline with the chair.

For SPs weighing more than $350 \mathrm{lbs}$, position the phlebotomy chair in front of the exterior door. For wheelchair bound SPs, position the chair in the right back corner of the room.

\section{- Blood collection cart}

Set up the appropriate blood collection cart in advance, making sure there are sufficient supplies for all SPs in the session. 
Figure 4-3. Blood collection cart

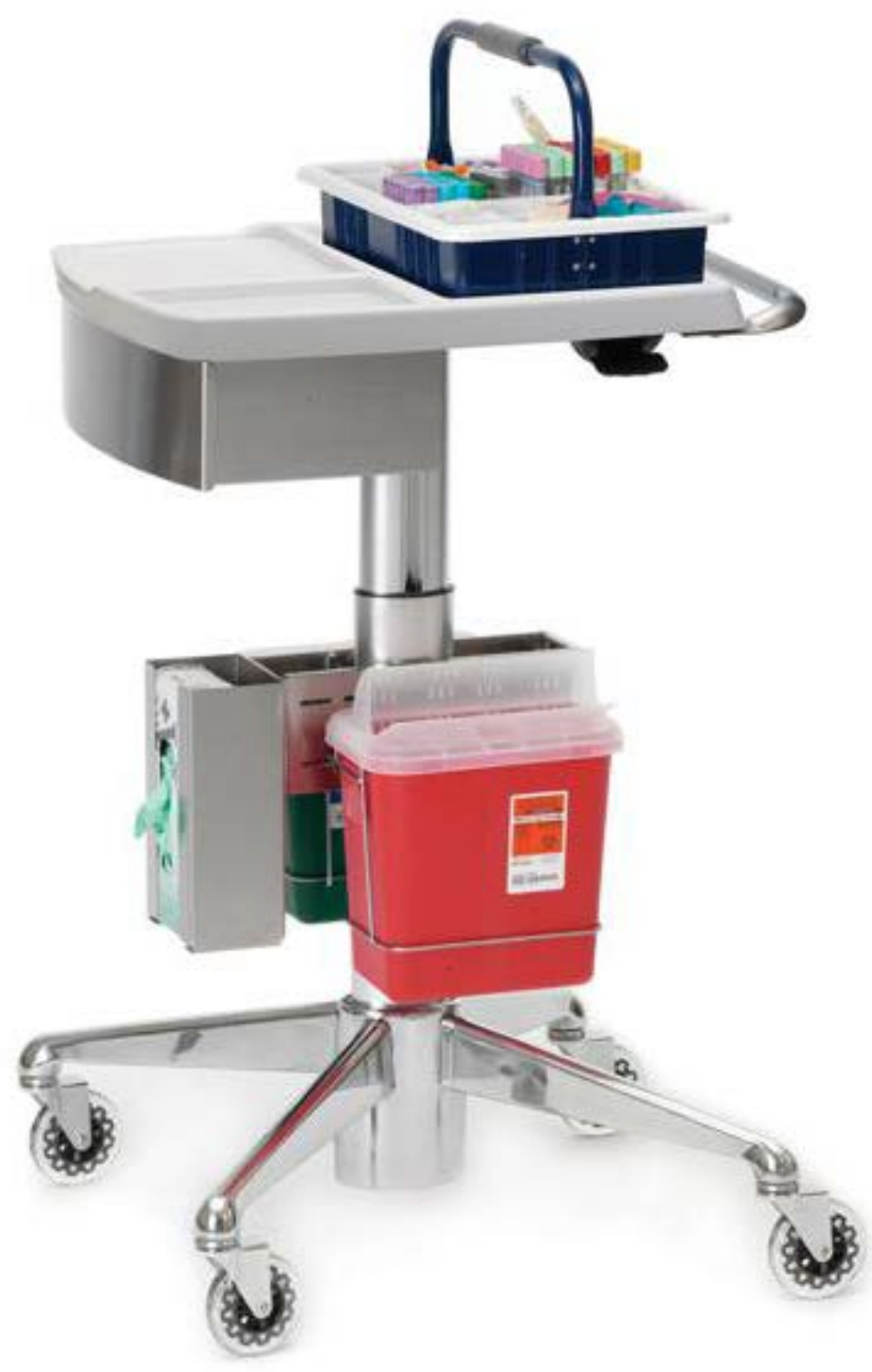

Adjust the height by lifting up on the black lever. Line an empty biohazard waste container with a biohazard bag and use it as a trash receptacle. Discard all sharps in the 8 quart biohazard container. Lock all needles in the drawer at the end of each day.

Access the phlebotomy application and open the phlebotomy exam. Log the SP into phlebotomy by scanning the bar code on the SP ID bracelet or manually typing the SP ID when the SP arrives in phlebotomy. 
Conduct the phlebotomy interview and administer the fasting questionnaire. The phlebotomy protocol screen displays. Draw the appropriate tubes, and enter the number of tubes collected next to the expected values. Labels generate automatically. Enter the appropriate comment(s) for any tube that was not collected. Label the blood tubes. Verify that the phlebotomy completion status is correct. The phlebotomy section status is "Complete" if all blood tubes are collected, "Partial" if some, but not all blood tubes are collected, and "Not Done" if no blood tubes are collected.

\subsection{Gaining Cooperation}

The coordinator will introduce the SP to the examination and briefly explain the examination process. The coordinator can answer any general questions the SP has about venipuncture. However, the phlebotomist must be prepared to answer all the questions the SP poses about the venipuncture procedure. In addition, the phlebotomist must convince the SP of the importance of cooperation in the venipuncture component of the examination.

Prepare to answer questions about the rationale for the venipuncture, the discomfort involved, the amount of blood being drawn, and the possibility of contracting an infectious disease from the process. To address SPs' concerns effectively, know the following information about the procedures used for the study:

\section{- Rationale}

Although the SP has provided much useful information in the household and individual interviews, the successful completion of the venipuncture component of NHANES is critical to the success of the study. Using the various specimens, researchers and laboratories are able to perform more than 250 different biochemical tests (SPs 12+), which provide detailed information about the SP's health and nutritional status. NHANES data produce descriptive statistics that measure and monitor the health of the U.S. population. Much of this information would not be available in any other way. The laboratories that conduct the analysis are considered the "gold standard" for their particular analyte(s).

\section{- Discomfort}

Venipuncture causes only minimal discomfort. A certified, experienced, phlebotomist performs venipunctures. A variety of blood collection needles are available so that the most appropriate size can be selected for each SP. 


\section{- Amount}

Phlebotomists draw the following amount of blood:

1-2 years primary SP, 9-mL (0.3 ounces), 0.6 tablespoons

3-5 years primary SP, 20-mL (0.7 ounces), 1.3 tablespoons

6-11 years primary SP, 30-mL (1.0 ounces), 2.0 tablespoons

12-19 years primary SP, 99-mL (3.4 ounces), 6.7 tablespoons

20+ years primary SP, 120-mL (4.1 ounces), 8.1 tablespoons

1-2 years VIP guest, 6-mL (0.2 ounces), 0.4 tablespoons

3-5 years VIP guest, 6-mL (0.2 ounces), 0.4 tablespoons

6-11 years VIP guest, 10 -mL ( 0.3 ounces), 0.7 tablespoons

12+ years VIP guest, $15-\mathrm{mL}$ (0.5 ounces), 1.0 tablespoons

1+ years guest and surplus, 3-mL (0.1 ounces), 0.2 tablespoons

12+ years guest and surplus, 6-mL (0.2 ounces), 0.4 tablespoons

An average adult male has 12 pints of blood and an average female has 9 pints. The Red Cross routinely draws $450-\mathrm{mL}$ (or one pint) during a routine donation. Their requirements limit donation to every 8 weeks. The Red Cross does allow autologous donations where an individual can donate his or her own blood before surgery. This consists of one unit per week for up to 6 consecutive weeks. The maximum volume drawn during a MEC exam amounts to slightly more than 25 percent $(128-\mathrm{mL})$ of the amount drawn from regular donors by the Red Cross. The body manufactures blood daily and replaces this volume of blood within 24 hours.

\section{- Infection control}

The supplies used for venipuncture are completely sterile and are used only once. There is absolutely no possibility of the SPs being infected by any blood-borne disease, such as hepatitis or AIDS, because of participation in the venipuncture component of the NHANES exam.

Gaining the cooperation of an SP is easier if the atmosphere in the phlebotomy room is pleasant and makes the SP feel comfortable. Below is a list of suggestions for creating a pleasant atmosphere in the phlebotomy room.

- Maintain a clean and uncluttered work area. This is especially important because of today's concern with blood-borne infectious diseases, such as hepatitis and AIDS. 
- Be aware of body image; a positive body image inspires confidence. Maintain a tidy appearance, erect posture, and a smile.

- Speak face to face with the subject and maintain eye contact. Staring at other areas in the room may cause the SP some uneasiness since it implies that he or she is not important. It also implies disinterest.

- Avoid nervous behaviors, such as squirming and tapping that can be distracting. The SP may begin to feel nervous, hurried, and anxious because of such behaviors.

\subsubsection{Refusal Conversion}

The coordinator should notify the phlebotomist of all venipuncture refusals so that the phlebotomist can attempt a refusal conversion.

The phlebotomist should discuss the condition of the SP's refusal with the coordinator before he or she attempts a conversion. If the coordinator indicates that the SP is an adamant refusal, the phlebotomist should not attempt to approach the SP or conduct the phlebotomy interview. If, however, the coordinator and the phlebotomist decide that the SP is a good candidate for a refusal conversion attempt, the phlebotomist may conduct the phlebotomy interview as part of the conversion attempt. If appropriate, the phlebotomist should enlist the help of the coordinator, the health technologist, and/or the MEC physician when attempting to convert refusals.

\subsection{Performing the Venipuncture on SPs who do not Speak English}

When the phlebotomist must administer the venipuncture procedure to an SP who does not speak English and the phlebotomist does not speak the language of the SP, a translator who does speak the language of the SP assists the phlebotomist.

The translator stays with the phlebotomist and the SP for the entire procedure. It is very important that the phlebotomist be able to communicate with the SP if the SP becomes ill during the venipuncture. 
Open and log onto the Phlebotomy application.

The message center window identifies the SP assigned to phlebotomy.

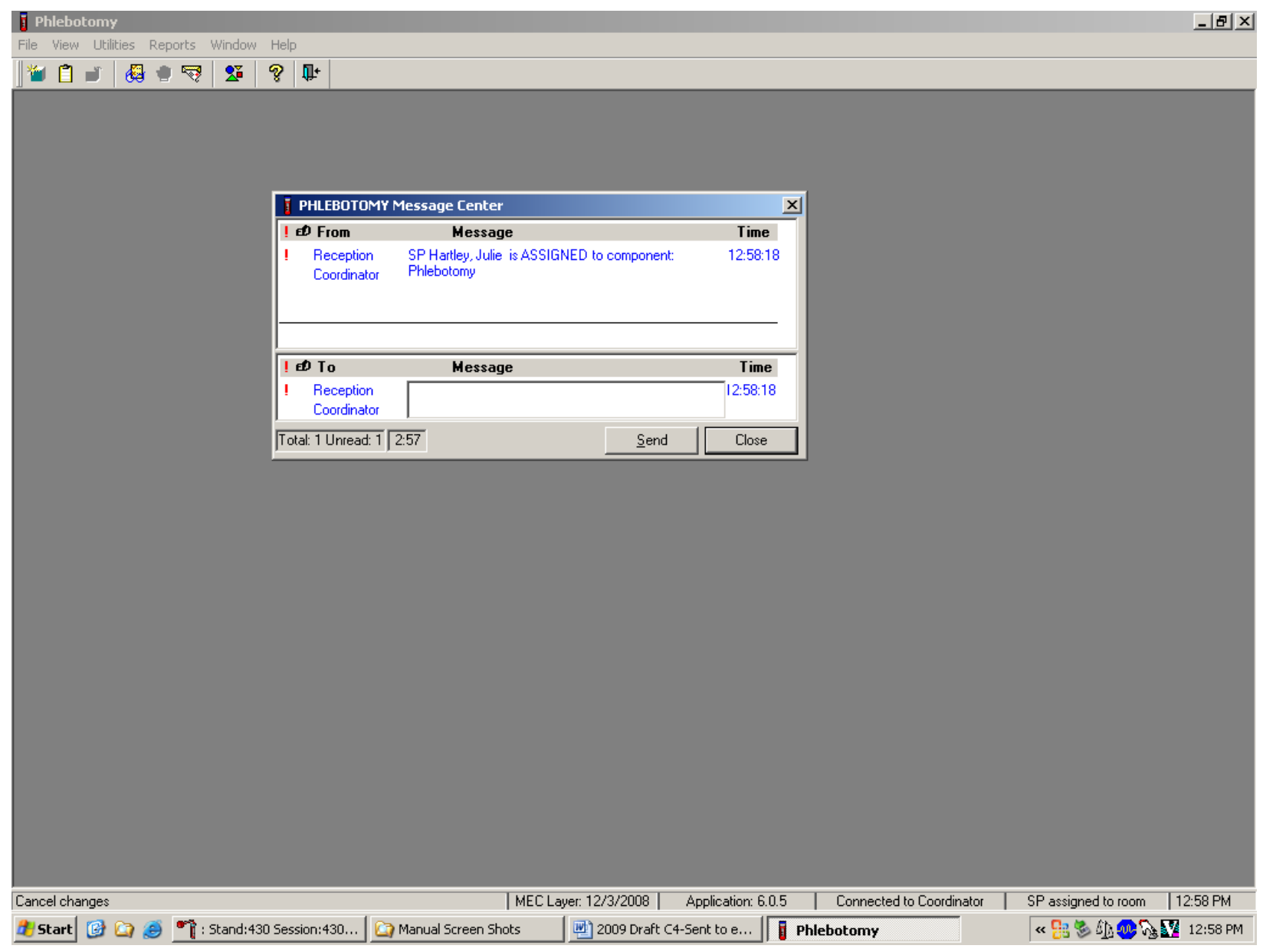

A Message Center message text box displays containing a message from the coordinator indicating the name of the SP who is assigned to the phlebotomy component. Enter an optional text message and, to send the message to the coordinator, use the mouse to direct the mouse arrow to the "Send" button and left click. To exit without sending a message to the coordinator, use the mouse to direct the mouse arrow to the "Close" button and left click, or select [Enter]. 
Open the Phlebotomy exam.

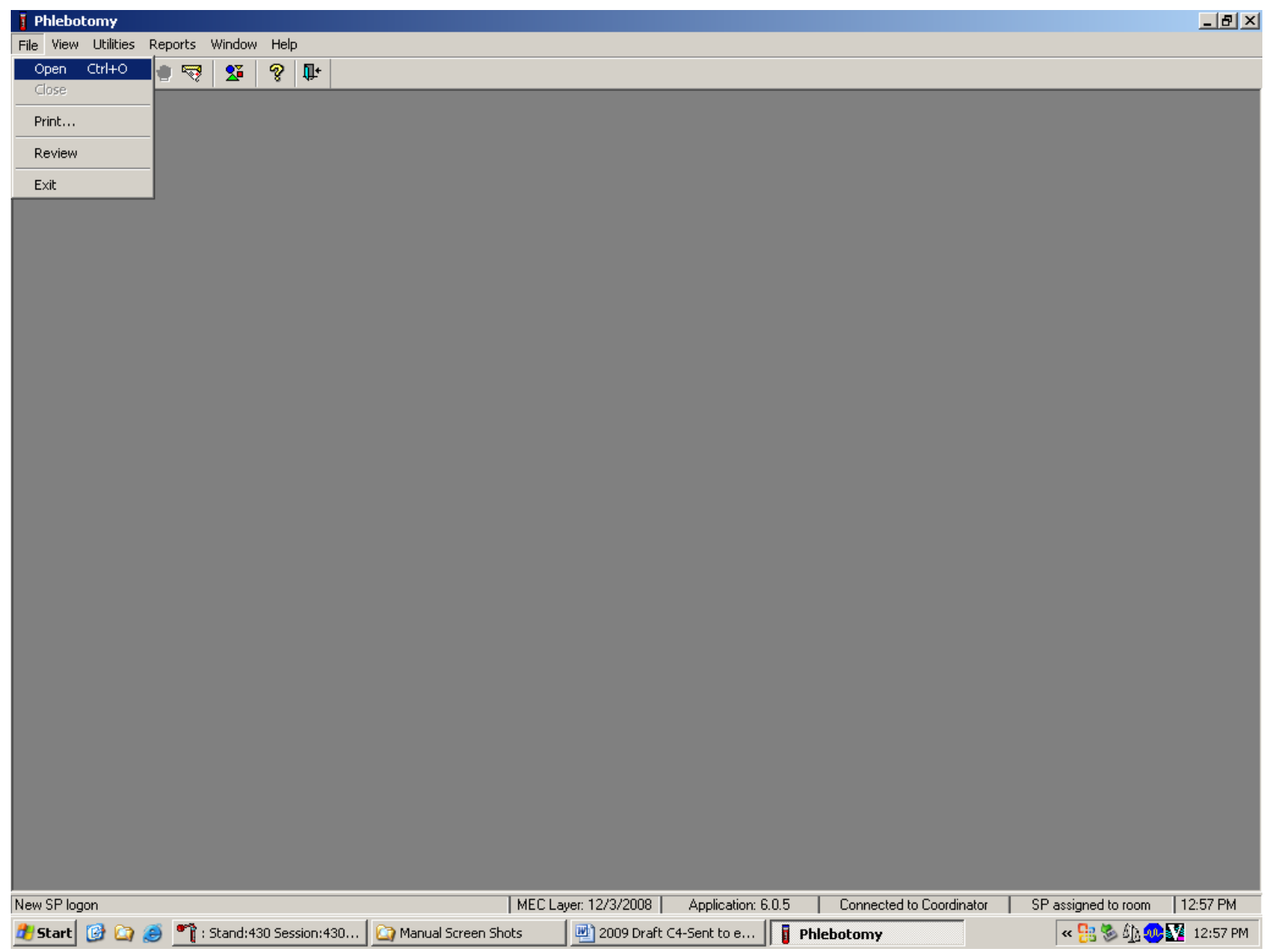

To open an exam, use the mouse to direct the mouse arrow to \{File $\}$ in the menu bar, left click, drag the arrow to \{Open\} and left click, or type [Alt] [F/f], [O/o], or [Ctrl] [O/o]. 
The SP Logon window displays.

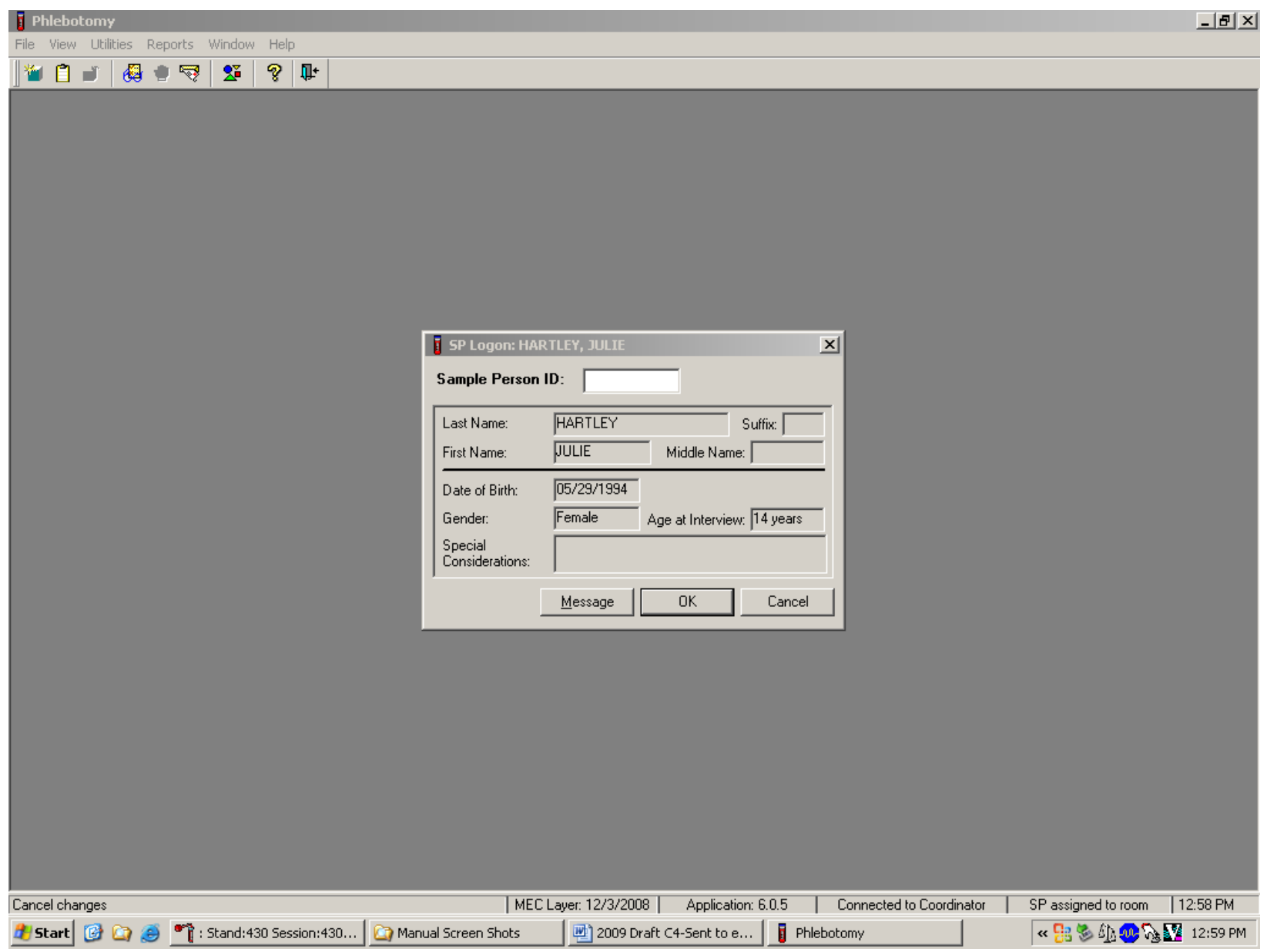

The SP Logon window displays for the SP assigned to the component. 
Log the SP into the component.

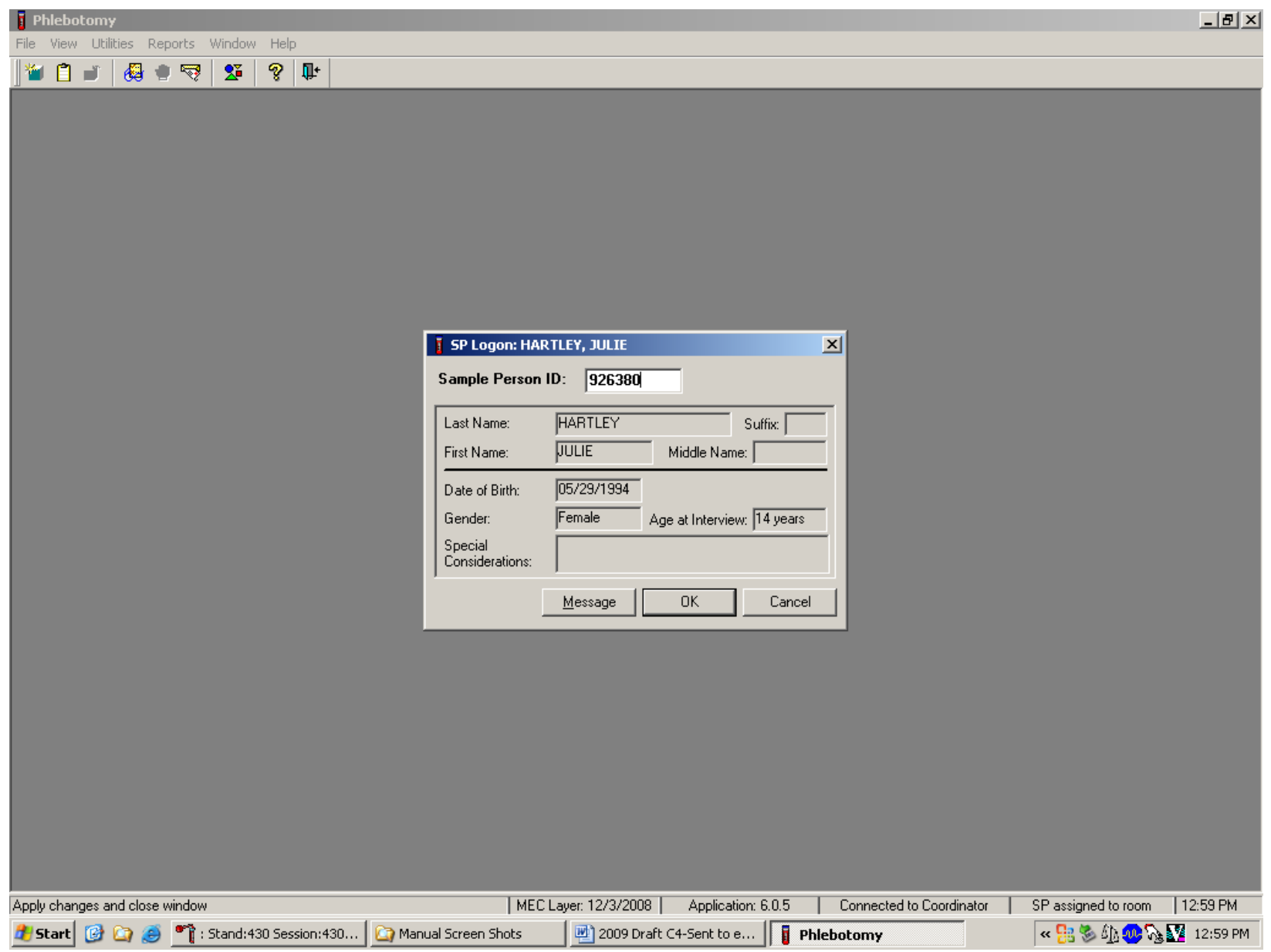

To log the SP into the component, either read the SP ID from the SP's bracelet and manually type this number into the Sample Person ID text box or use the bar code wand to scan the bracelet bar code. To continue, select [Enter] or use the mouse to direct the mouse arrow to the "OK" button and left click. To cancel the Logon process and to remove the window, use the mouse to direct the mouse arrow to the "Cancel” button and left click.

Verify all information that appears on the SP Logon window. If there is an error in any of this information, inform the coordinator immediately. The coordinator will verify and correct the information as necessary. 
The Phlebotomy Interview screen includes a shared exclusion pregnancy question for females aged 16-59 and two safety exclusion questions. There are only two reasons to exclude an SP from venipuncture-hemophilia, and having received chemotherapy within the past 4 weeks. Administer the interview (and fasting questionnaire) directly to SPs over the age of 16 or administer the interview questions (and fasting questionnaire) to the SP's parent or guardian for SPs under age 16.

Administer the phlebotomy interview immediately before performing the venipuncture. Read the text exactly and record the responses.

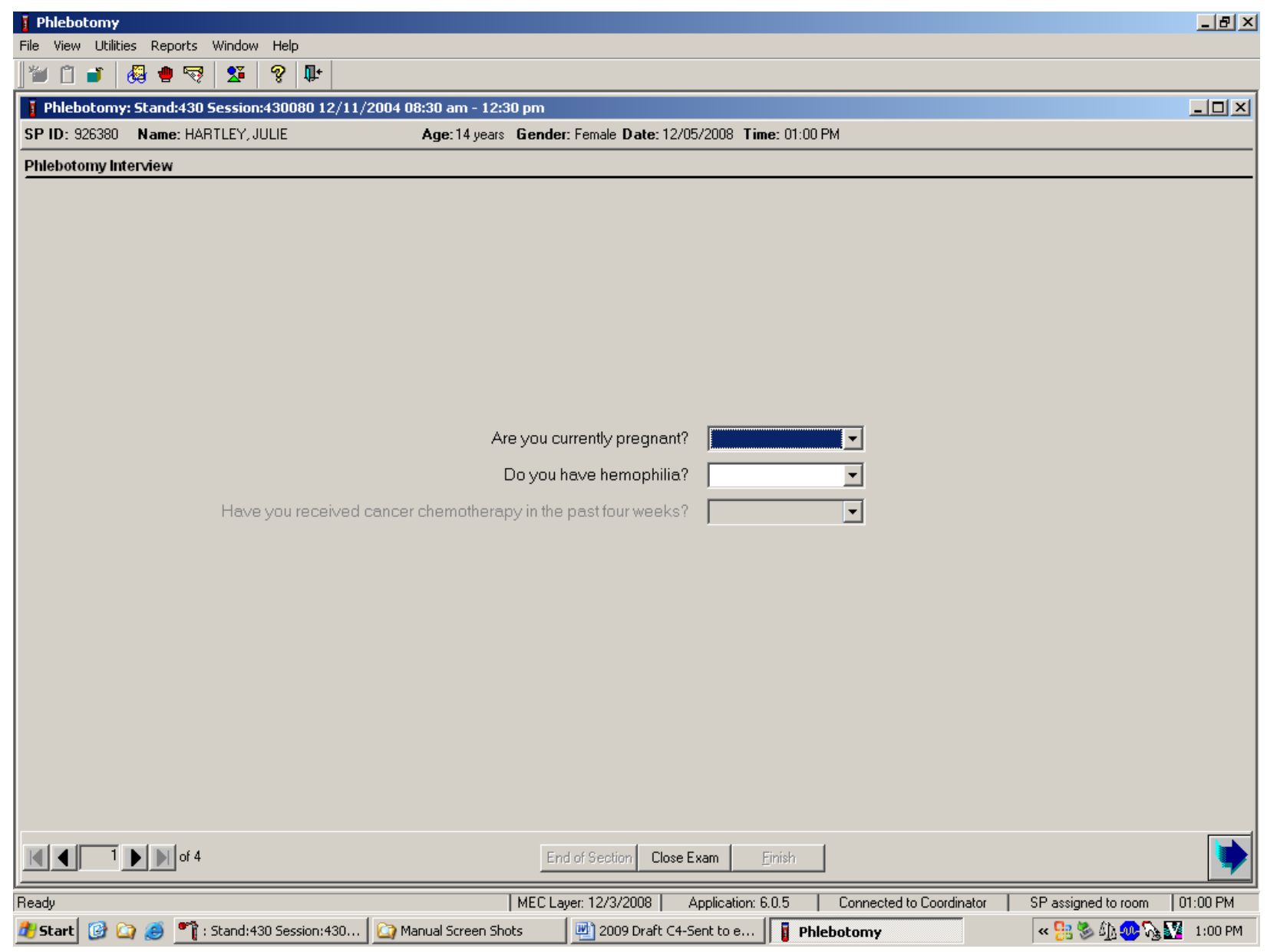




\subsubsection{Pregnancy Shared Exclusion Question}

For female SPs aged 12-59, the shared exclusion pregnancy questions are enabled and are asked during the MEC examination in the first examination where shared exclusions are asked. Each question is asked only once, even when the question is relevant for more than one component. Some examinations, like phlebotomy, do not require all the shared exclusions to be asked. In this situation, there may be some questions answered in one examination and then disabled in the remaining examinations. Shared exclusion questions are component specific. Answers provided during previous components appear on the screen for the next examiner to see, but the text box for data entry is disabled. Only shared exclusion questions relevant to the age and component that have not been answered are enabled. Example: If the SP goes to the physician component before phlebotomy, the questions about "pregnancy" and "how many weeks" would be asked by the physician. The "pregnancy" question would be disabled in the phlebotomy component.

- If the SP is a male, a female less than 12 years, or a female older than 60 years, the shared exclusion pregnancy questions will not be displayed.

- If the SP is a female aged 12-59, the first shared exclusion pregnancy question is displayed and enabled. 
Record the response to the shared exclusion question.

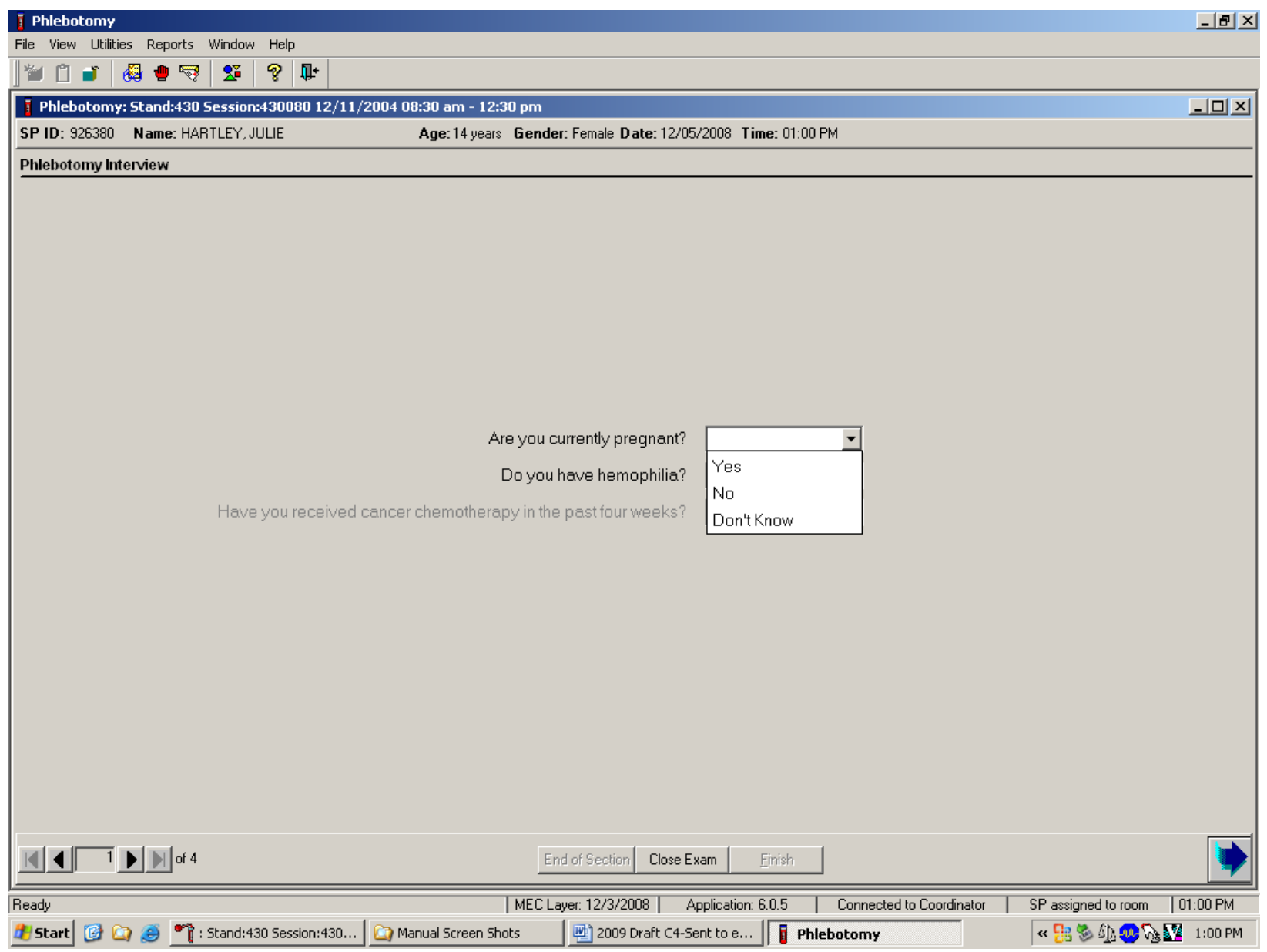

Record the response by typing [Y/y] for "Yes,” [N/n] for “No,” or [D/d] for “Don’t know.” Alternatively, use the mouse to direct the mouse arrow to the drop-down arrow on the drop-down list, left click to display the responses, and drag the mouse arrow to "Yes," "No," or “Don’t Know” and left click. Select [TAB] to move to the next text box. 
If the response to the question "Are you currently pregnant?" is "Yes," the SP is excluded from GTT due to pregnancy status. At the coordinator application, the component status for GT defaults to "Not Done” with the comment "Blocked: Not Done [SP pregnant]."

If the response to the question “Are you currently pregnant?" is "No" or "Don’t Know," the SP is not excluded from the GTT. Ask the next two phlebotomy safety exclusion questions.

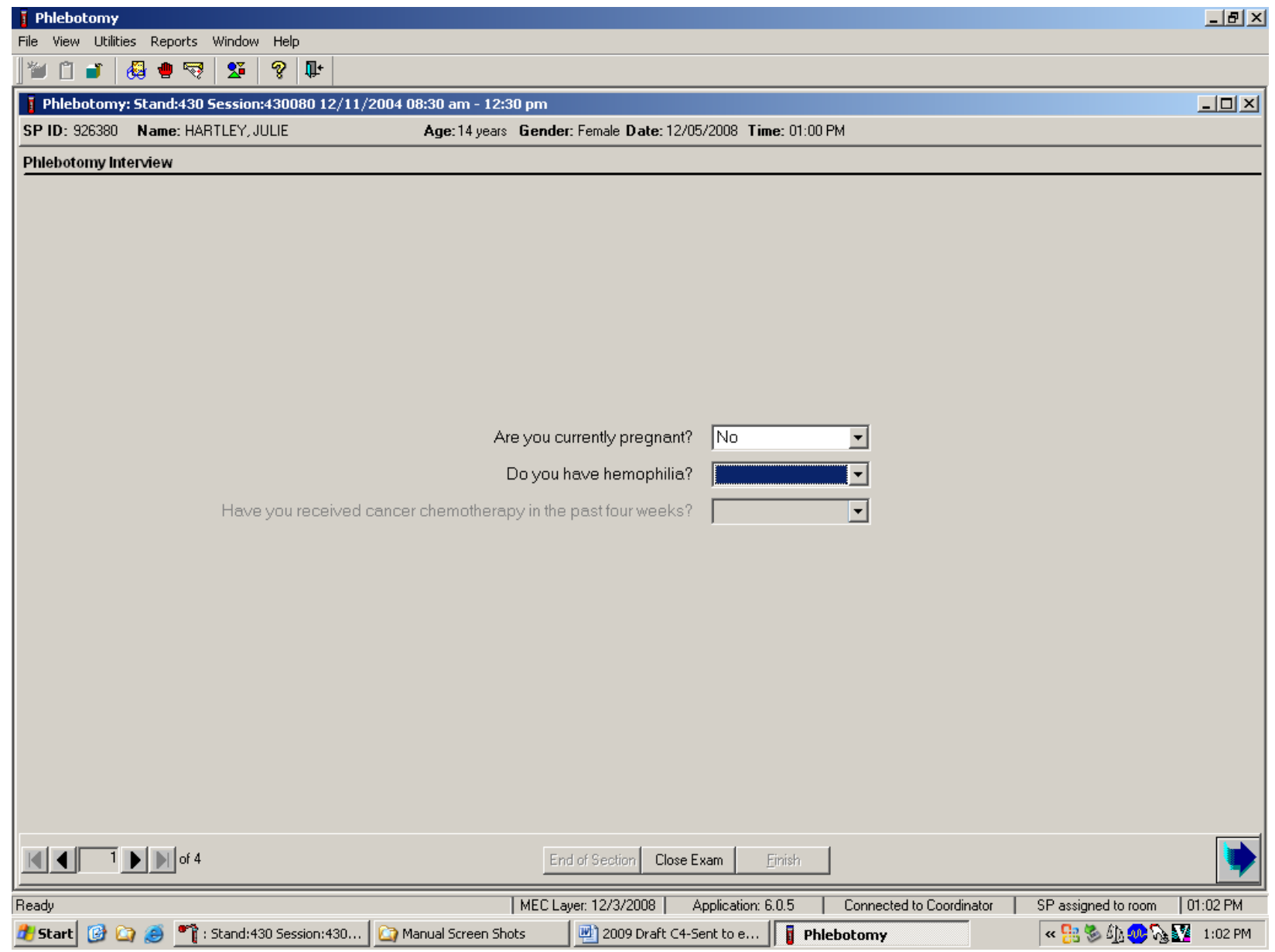

If the answer to the shared exclusion pregnancy question is "No" or "Don't know," then select [TAB] to proceed to the first safety exclusion question, "Do you have hemophilia?” 


\subsubsection{Safety Exclusion Questions}

Continue to conduct the phlebotomy interview by asking the question displayed on the screen, "Do you have hemophilia?"

\section{Q1: Do you have hemophilia?}

\begin{tabular}{l|l}
$\begin{array}{l}\text { Q1: This question asks whether the SP } \\
\text { has hemophilia, which is an exclusion } \\
\text { criterion for this procedure. }\end{array}$ & $\begin{array}{l}\text { Explain that we cannot perform phlebotomy on participants } \\
\text { who have hemophilia. Hemophilia is a rare disease where an } \\
\text { individual's blood does not clot. If an SP's relative has } \\
\text { hemophilia but the SP does not, the SP is not excluded. If the } \\
\text { SP is excluded, the Blood Draw Exclusion box displays. Read } \\
\text { the text in the box to the SP and escort the SP back to the } \\
\text { coordinator station. }\end{array}$ \\
\end{tabular}


Record the exact response.

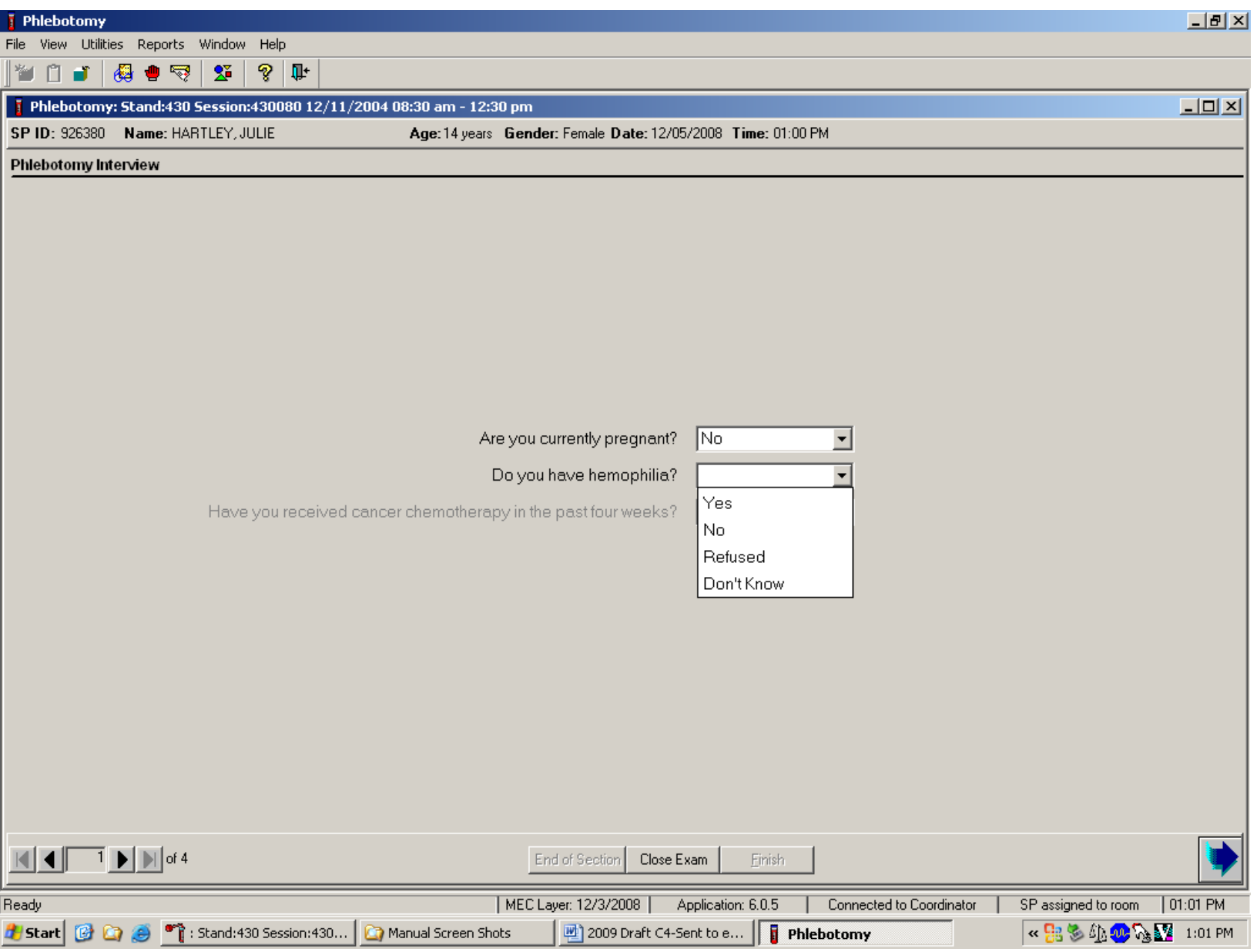

Record the response by typing [Y/y] for "Yes," [N/n] for "No," [R/r] if he or she refuses, or [D/d] for "Don't know." Alternatively, use the mouse to direct the mouse arrow to the drop-down arrow on the drop-down list, left click to display the responses, and drag the mouse arrow to "Yes," "No," "Refused,” or “Don't Know” and left click. If the response is "Yes,” "Refused,” or "Don’t Know,” use the mouse to direct the mouse arrow to the bright blue right arrow in the bottom right corner of the screen and left click or select [Enter]. 
If the SP is excluded from the phlebotomy exam due to hemophilia, the Blood Draw Exclusion informational message text box displays.

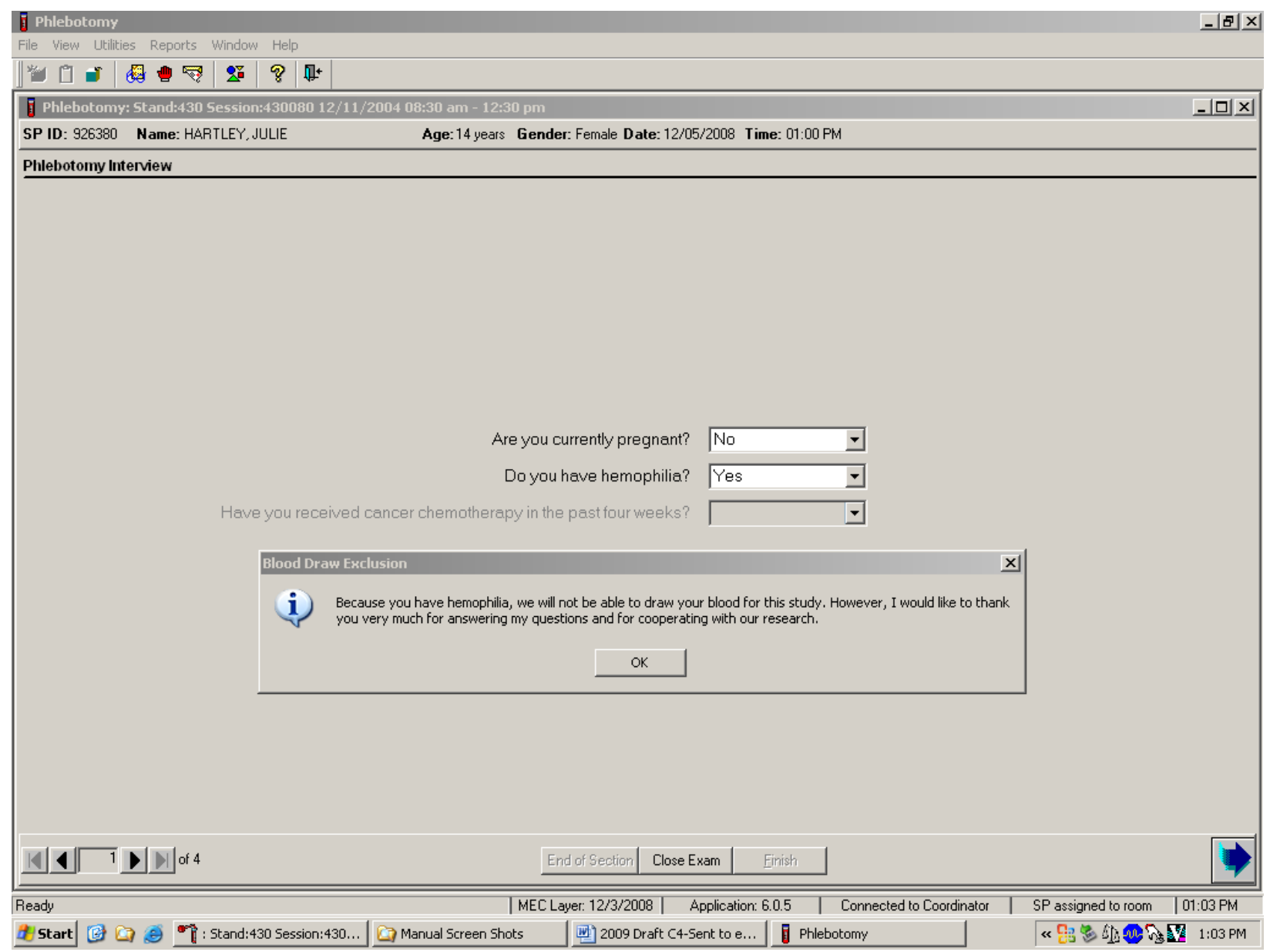

Read the script to the SP. To remove the Blood Draw Exclusion message text box, use the mouse to direct the mouse arrow to the "OK” button and left click, or select [Enter]. 
If the SP is excluded from the phlebotomy component due to hemophilia, an informational Exclusion message text box displays. In some cases more than one Exclusion message box will display, one after the other.

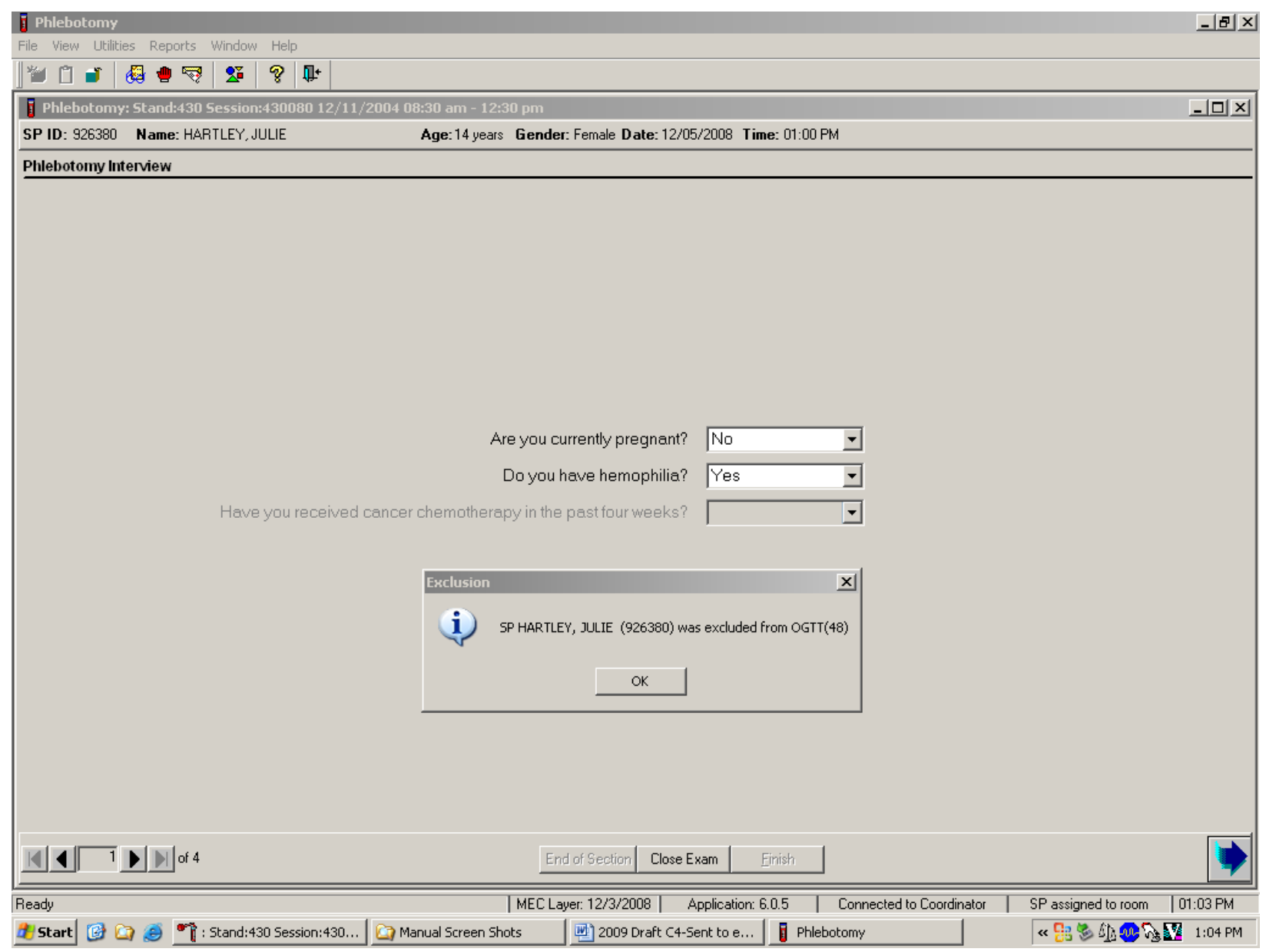

To remove the Exclusion message text box, use the mouse to direct the mouse arrow to the “OK” button and left click, or select [Enter]. 
Verify the status.

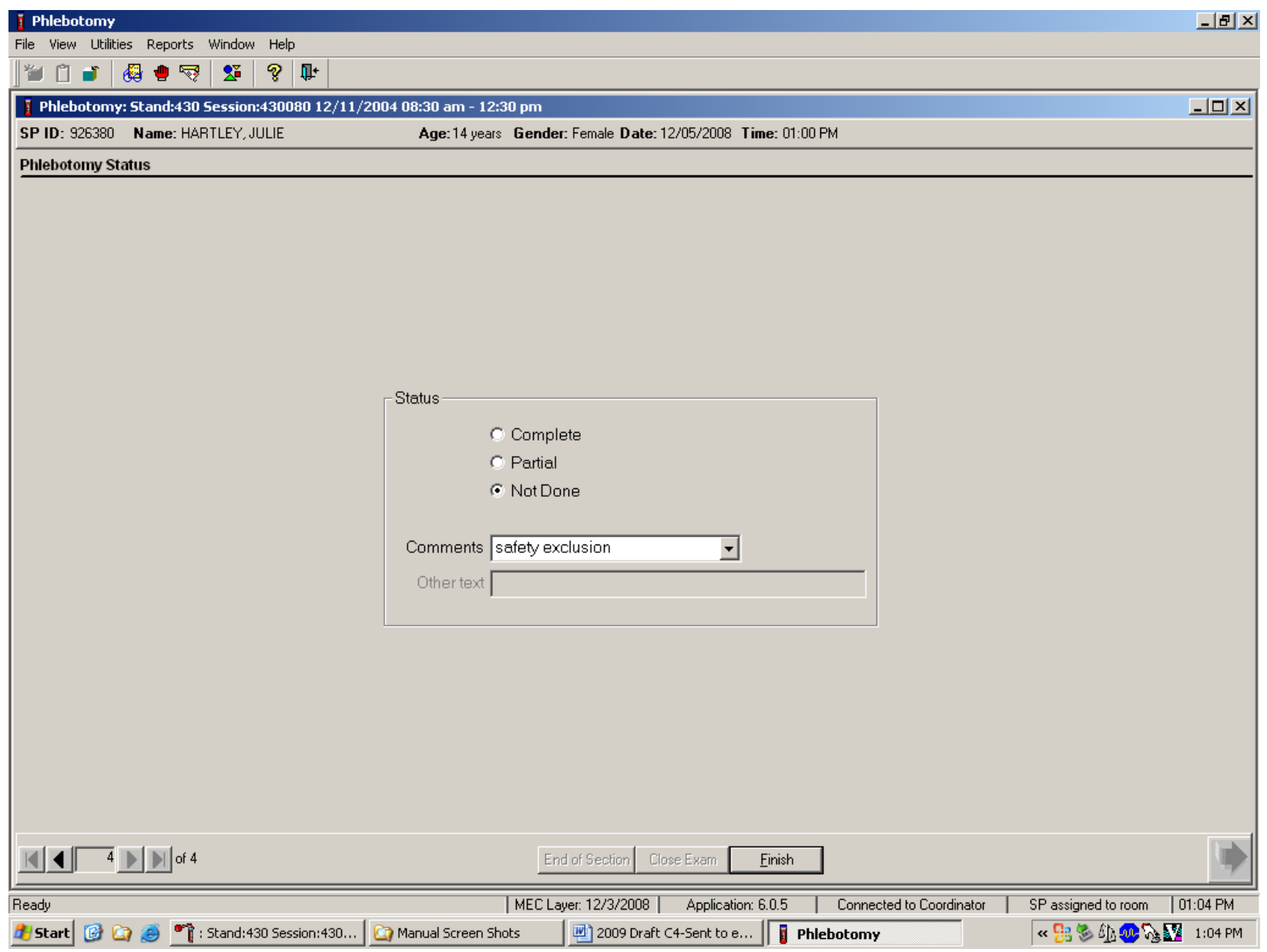

SPs excluded due to hemophilia are automatically coded by the application as "safety exclusion.” Escort the SP back to the coordinator or to the next component as directed by the Message Center. 
If the SP has not been excluded due to hemophilia, continue the phlebotomy interview by asking the second question displayed on the screen, "Have you received cancer chemotherapy in the past four weeks?”

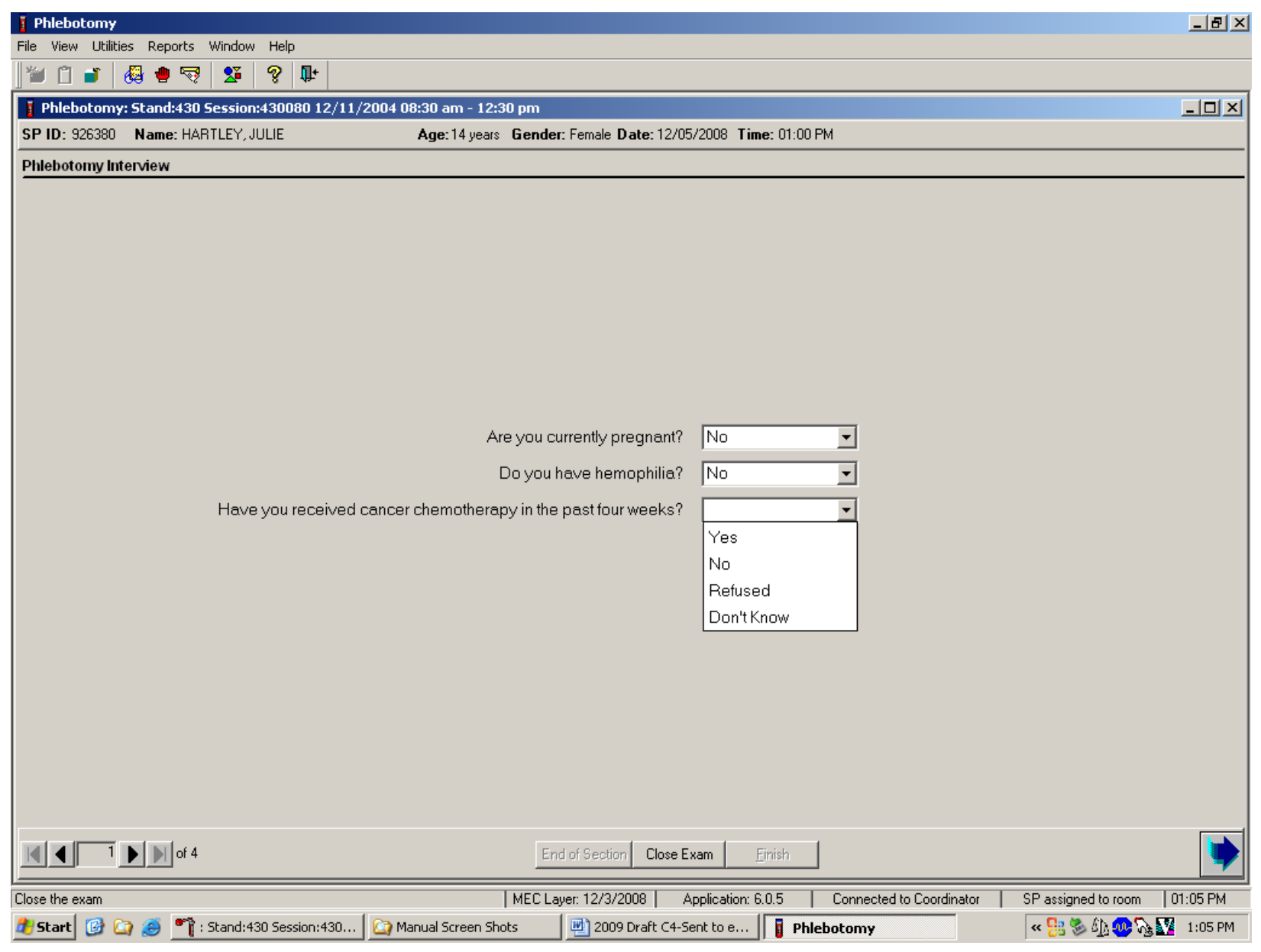

\section{Q2: Have you received cancer chemotherapy in the past four weeks?}

Q2: This question asks whether the SP has received chemotherapy in the past 4 weeks. This situation excludes the SP from this procedure.
Explain that we cannot perform phlebotomy on participants who have received cancer chemotherapy within the past 4 weeks. If the SP is excluded, the Blood Draw Exclusion box is displayed. Read the text in the box to the SP and escort him or her back to the coordinator station. 
Record the response by typing [Y/y] for "Yes," [N/n] for "No," [R/r] if he or she refuses, or [D/d] for "Don't know." Alternatively, use the mouse to direct the mouse arrow to the drop-down arrow on the drop-down list, left click to display the responses, and drag the mouse arrow to "Yes," "No," "Refused,” or "Don't Know” and left click. If the response is "Yes,” "Refused,” or "Don’t Know,” use the mouse to direct the mouse arrow to the bright blue right arrow in the bottom right corner of the screen and left click.

If the SP is excluded from the phlebotomy exam due to cancer chemotherapy, the Blood Draw Exclusion informational message text box displays.

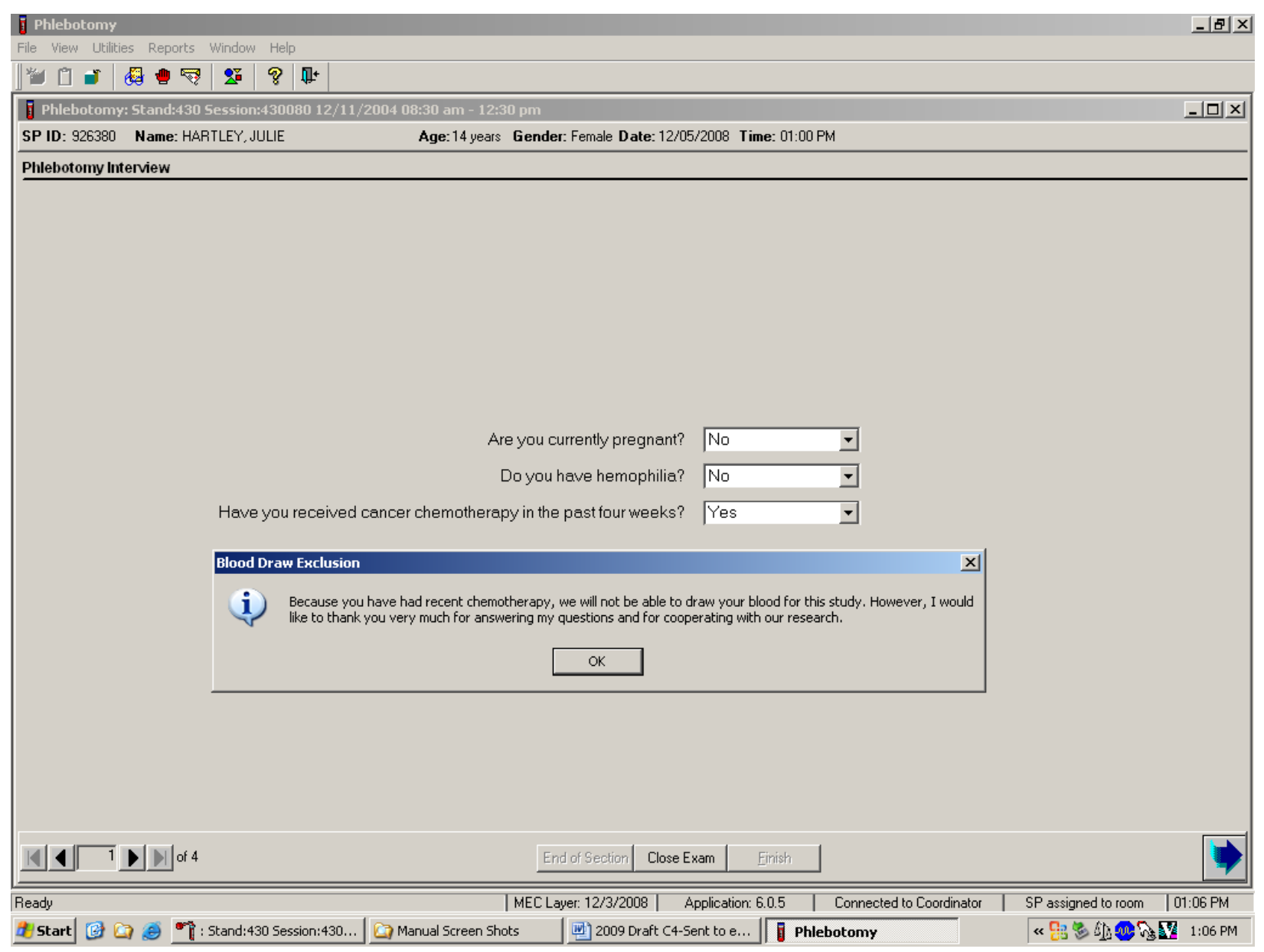

Read the script to the SP. To remove the Blood Draw Exclusion message box, use the mouse to direct the mouse arrow to the "OK" button and left click. 
If the SP is excluded from the phlebotomy component due to chemotherapy, an informational Exclusion message text box displays. In some cases more than one Exclusion message box will display, one after the other.

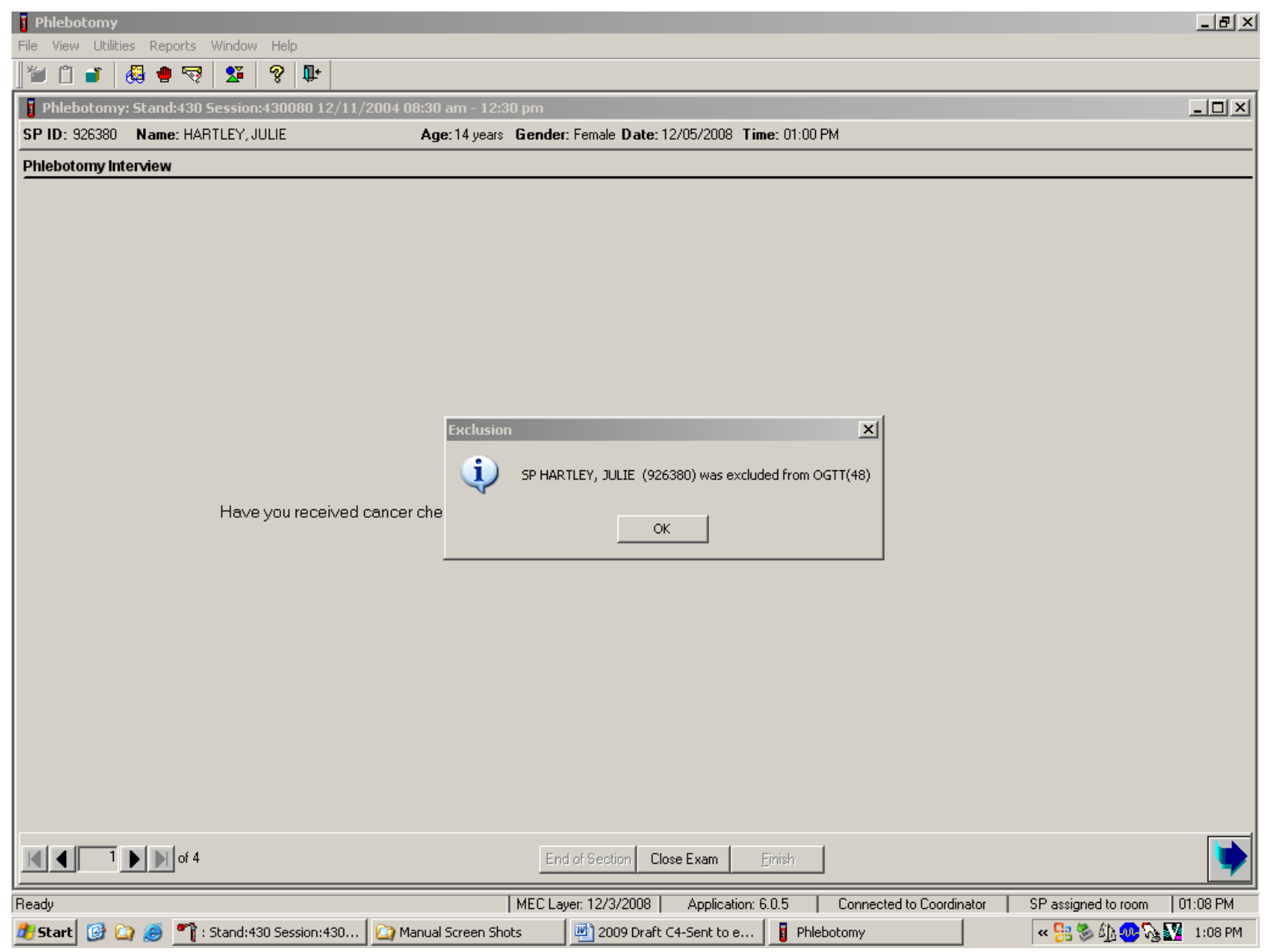

To remove the Exclusion message text box, use the mouse to direct the mouse arrow to the “OK” button and left click, or select [Enter]. To progress to the component status screen, use the mouse to direct the mouse arrow to the bright blue arrow in the bottom right corner and left click, or select [Enter]. 
Review the Status.

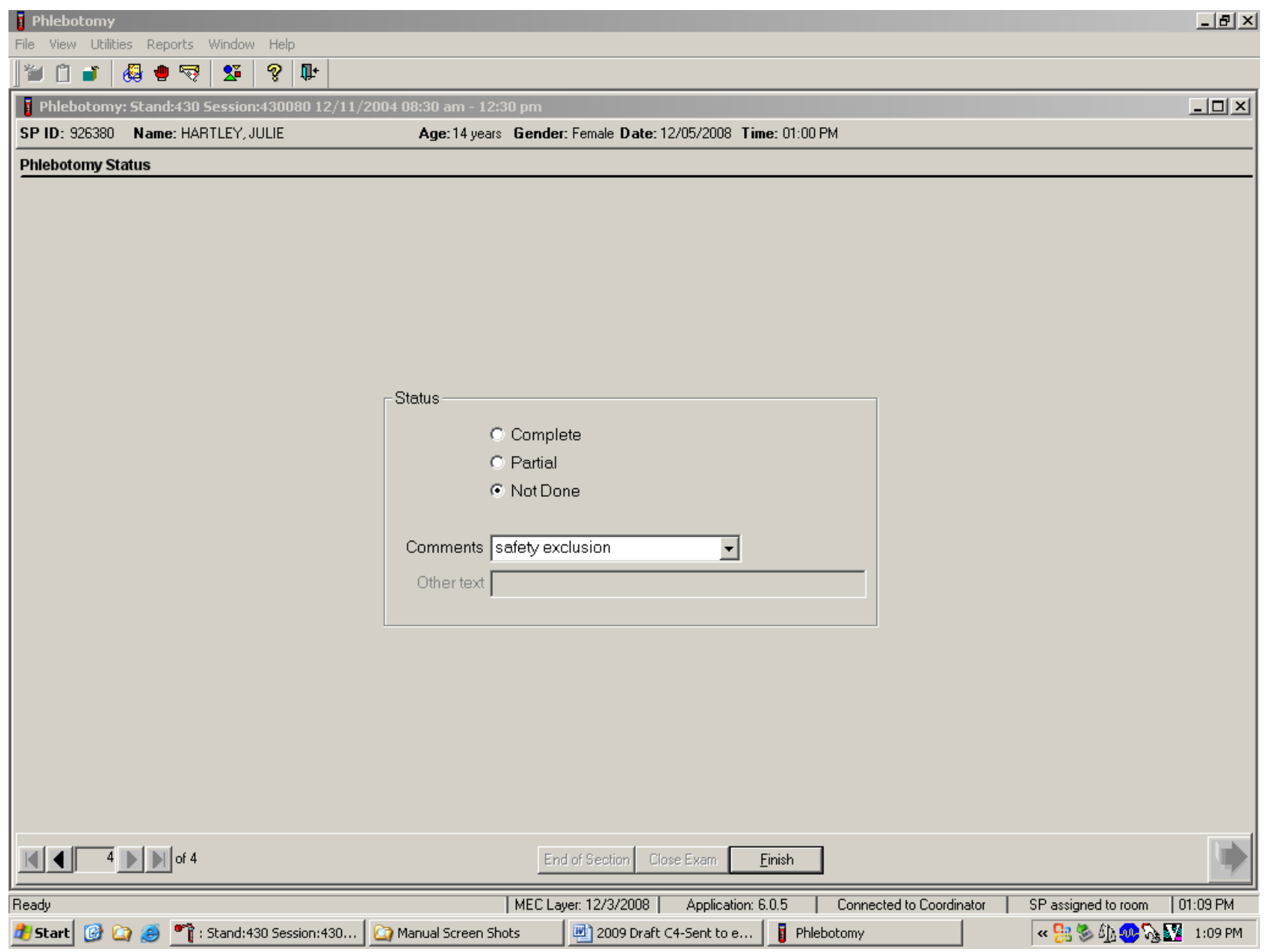

SPs excluded due to chemotherapy are automatically coded by the application as "safety exclusion.” Escort the SP back to the coordinator or to the next component as directed by the Message Center. 
If the response to both exclusion questions is "No," continue to the next screen.

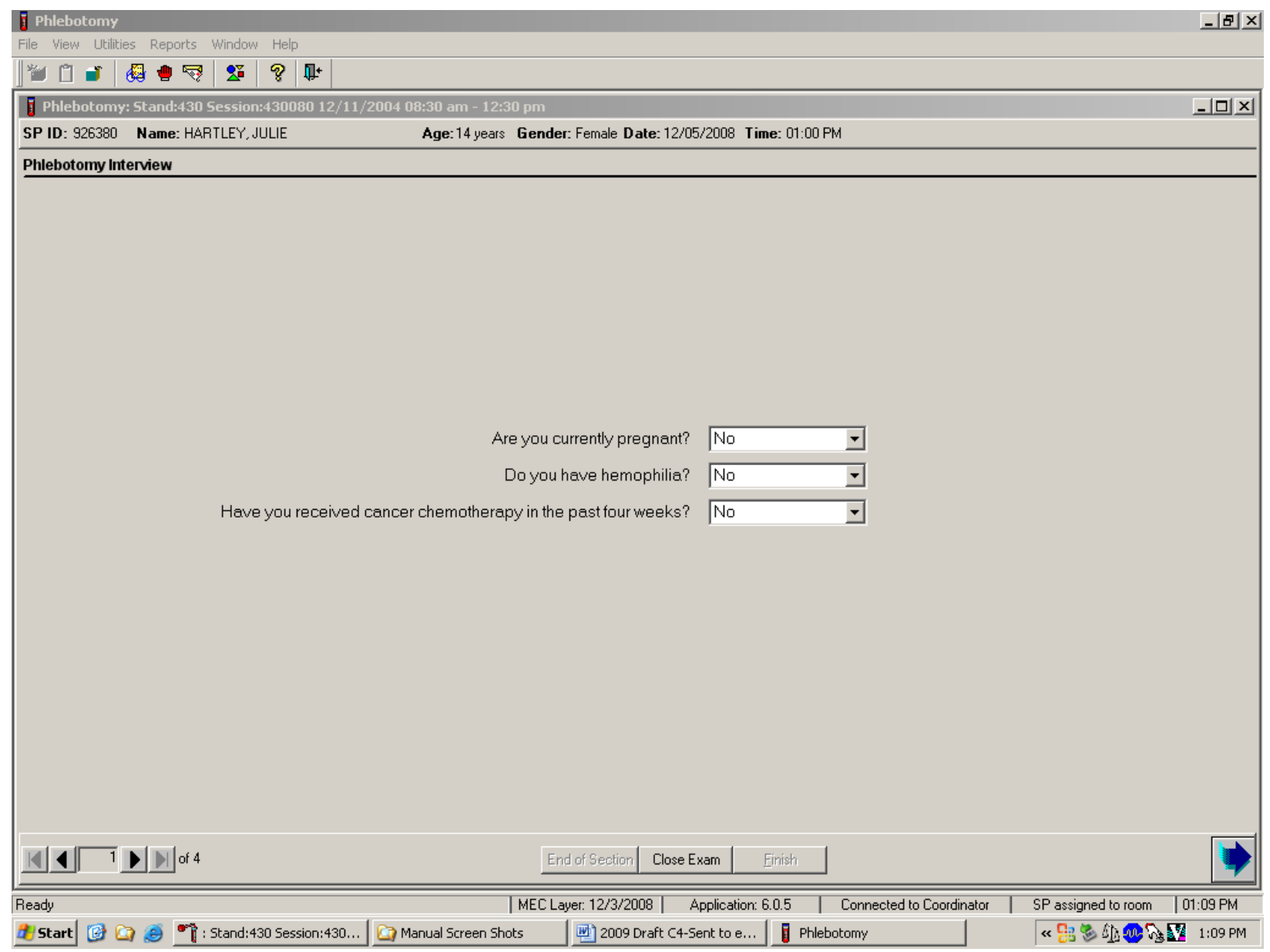

To progress to the fasting questionnaire screen, use the mouse to direct the mouse arrow to the bright blue arrow in the bottom right corner and left click, or select [Enter]. 


\subsection{Administering the Fasting Questionnaire}

Fasting status is critical to many of the laboratory analytes, and fasting data are required for correct interpretation of laboratory results. Fasting is especially critical because only SPs who have met the fast are eligible for the GT component. No primary SPs aged 1-11 or SPs assigned to afternoon or evening sessions are asked to fast. All other primary SPs aged 12+ appointed to a morning session are asked to fast for 9 hours. This includes diabetics taking insulin, although these SPs are always given the highest priority so the venipuncture can be completed as soon as possible after the SP checks into the MEC.

The phlebotomist administers the fasting questionnaire to all SPs, and the application calculates and displays the fasting time in number of hours and minutes. The phlebotomist then performs the venipuncture.

The overall objective is to perform phlebotomy on all eligible SPs. The coordinator must base the decision to assign an SP to phlebotomy on the greater goal of completing as many components as possible within the time constraints of the session, with phlebotomy as the highest priority component. Sometimes an SP insists on leaving the MEC before he or she has been assigned to phlebotomy. It is always preferable to get blood from this SP before he or she leaves the MEC. The coordinator and MEC manager are able to provide the best overall assessment of the status of a particular SP.

Guest, dry run, and VIP guests do not have a fasting requirement, even during morning sessions.

SPs may opt to return to the MEC to complete exams that were missed or to complete exams that were begun but not finished. These SPs are designated as "Partial” SPs. When the SP returns to the phlebotomy component, ask and record the fasting questions a second time so that the data are accurate. Delete any data in the text boxes (previous responses from the initial visit) and enter the new responses. 
Read the text exactly and record the time.

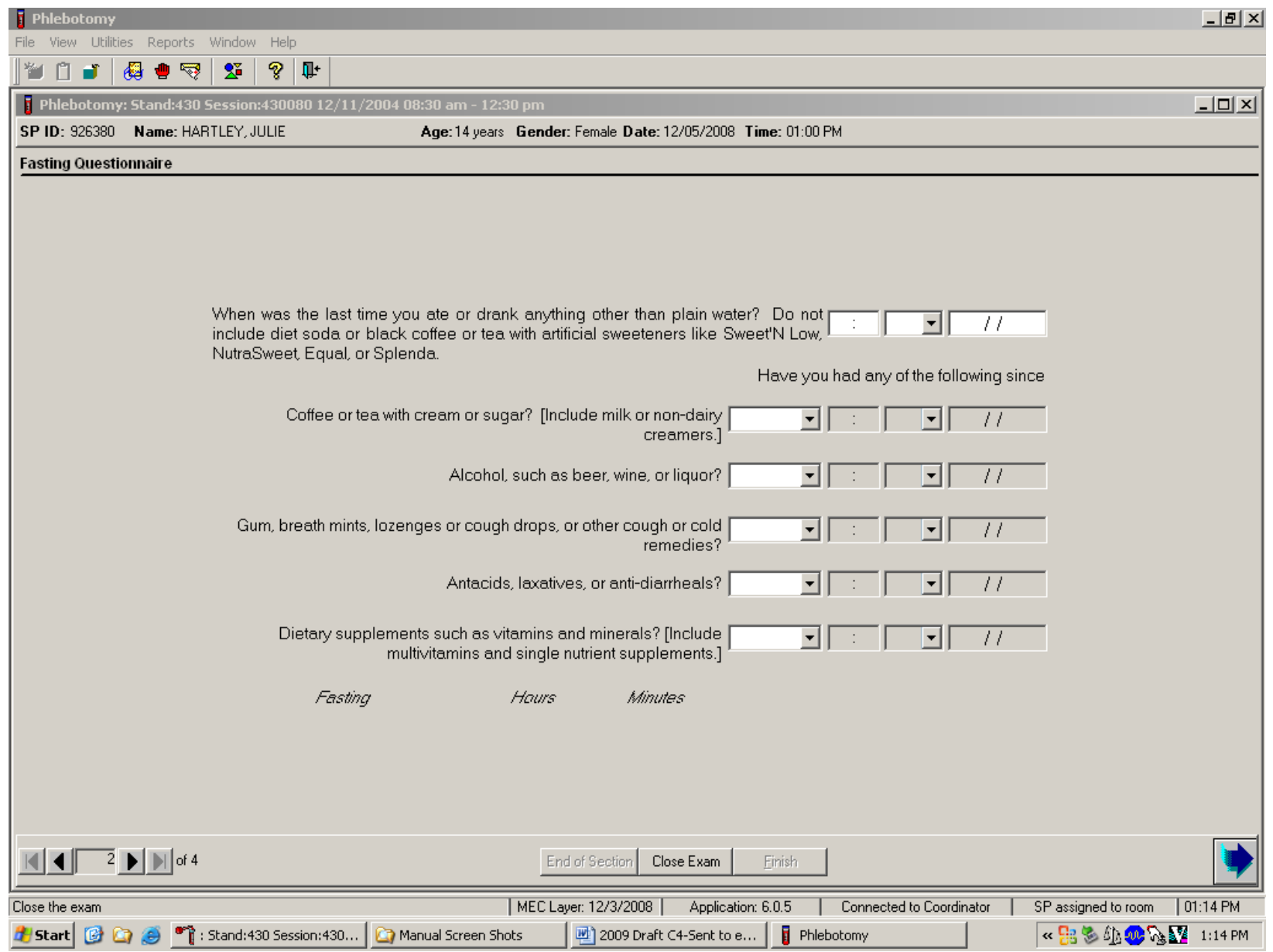

Q1: When was the last time you ate or drank anything other than plain water? Do not include diet soda or black coffee or tea with artificial sweeteners like Sweet'N Low, NutraSweet, Equal, or Splenda.

Q1: This question elicits the last time the SP ate or drank anything and determines fasting time.

SPs are allowed to consume diet soda, black coffee, or tea with artificial sweeteners like Sweet'N Low, NutraSweet, Equal, or Splenda since these have no effect on study analytes. Do not include flavored waters. 
Enter the response.

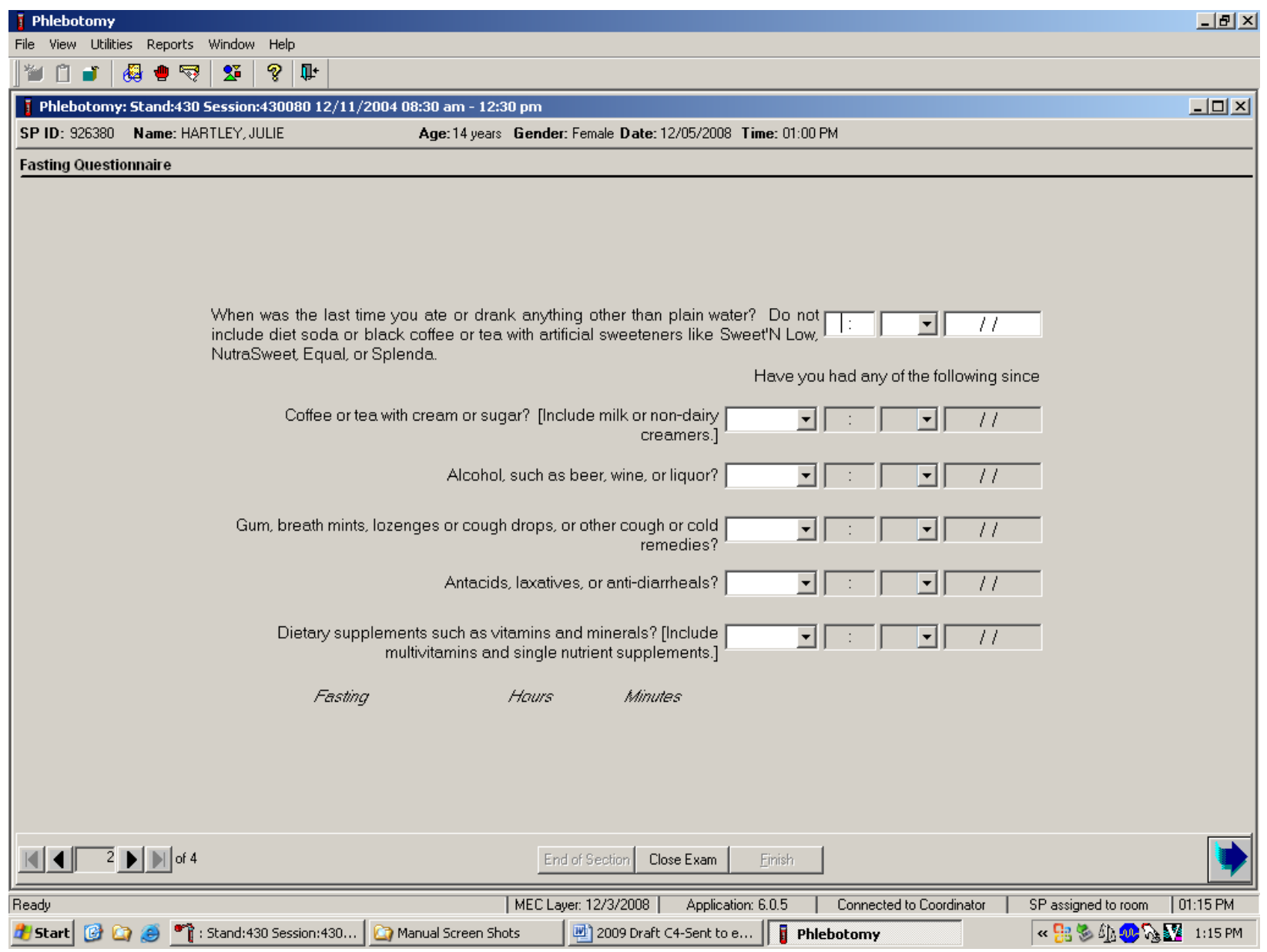

Type in the reported time using the numeric keys and select [Tab] to move to the AM/PM space. Type in [A/a] for times between midnight and 11:59 a.m. or [P/p] for times between 12:00 noon and 11:59 p.m. and select [Tab]. Alternatively, to select AM or PM, use the mouse to direct the mouse arrow to the drop-down arrow on the right side of the text box, left click, drag the mouse arrow to "AM," or "PM," and left click. 
Enter the date.

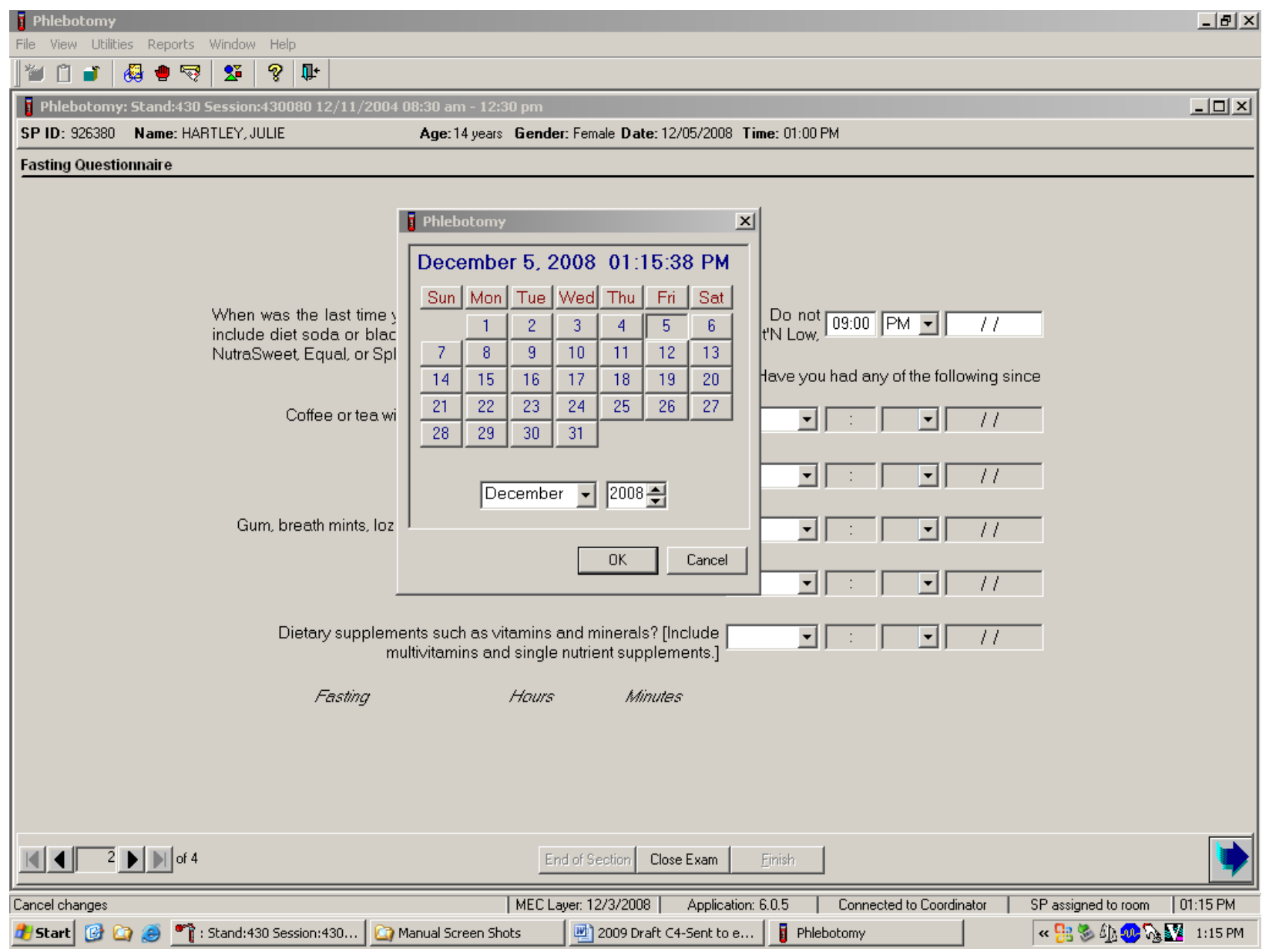

Type in the date using the keyboard's numeric keys and the mm/dd/yyyy format and select [Tab], or use the calendar to enter the date. To access the calendar, select [F2]. To select the correct month, use the mouse to direct the mouse arrow to the drop-down list, drag the arrow to the correct month (use the scroll bar if necessary), and left click. To select the correct day, use the mouse to direct the mouse arrow to the correct day on the displayed month and left click. To correct the year, use the mouse to direct the mouse arrow to the up-down controls on the spin box and toggle the number up and down. To transfer this date into the date space, use the mouse to direct the mouse arrow to the "OK" button and left click, or select [Enter]. To exit the calendar function, use the mouse to direct the mouse arrow to the "Cancel” button and left click. 
Continue administering the questionnaire. Verify the initial time response by asking the remaining questions.

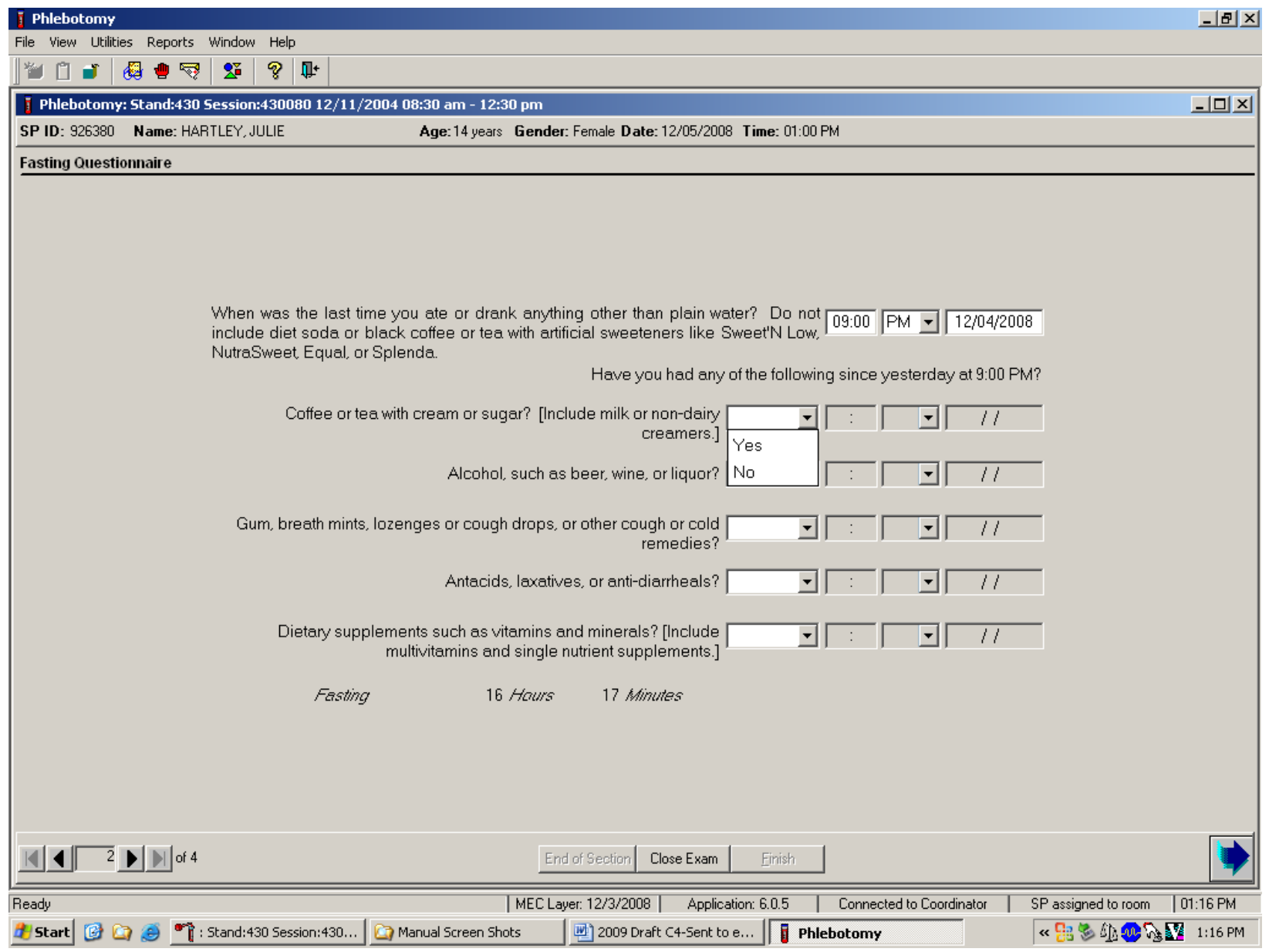




\section{Q2: Have you had any of the following since (time from question 1 inserted here)?}

\begin{tabular}{|c|c|}
\hline $\begin{array}{l}\text { Coffee or tea with cream or sugar? } \\
\text { [Include milk or non-dairy } \\
\text { creamers.] }\end{array}$ & $\begin{array}{l}\text { Black coffee is acceptable but coffee with any additives other than } \\
\text { artificial sweeteners is unacceptable. Include all milk products } \\
\text { such as half-and-half, light cream, non-fat milk, and all other non- } \\
\text { dairy creamers like Cremora or Coffee-Mate. }\end{array}$ \\
\hline $\begin{array}{l}\text { Alcohol, such as beer, wine, or } \\
\text { liquor? }\end{array}$ & $\begin{array}{l}\text { Alcohol includes all types of spirits including light beer and wine. } \\
\text { If the answer is "Yes," clarify the response. Ask the SP to describe } \\
\text { the activity, item consumed, and the correct time. }\end{array}$ \\
\hline $\begin{array}{l}\text { Gum, breath mints, lozenges, or } \\
\text { cough drops, or other cough or } \\
\text { cold remedies? }\end{array}$ & $\begin{array}{l}\text { Include sugar-free gum and mints in this category. Give the SP the } \\
\text { laminated card that lists common over-the-counter cough drops } \\
\text { and cough and cold remedies to refresh his or her memory if he or } \\
\text { she appears unsure about these items. If the answer is "Yes," } \\
\text { clarify the response. Ask the SP to describe the activity, item } \\
\text { consumed, and the correct time. }\end{array}$ \\
\hline $\begin{array}{l}\text { Antacids, laxatives, or anti- } \\
\text { diarrheals? }\end{array}$ & $\begin{array}{l}\text { Antacids neutralize stomach acids. Laxatives stimulate evacuation } \\
\text { of the bowels. Anti-diarrheals relieve diarrhea and cramping. } \\
\text { Include all over-the-counter antacids, laxatives, and anti- } \\
\text { diarrheals. Give the SP the laminated card that lists all over-the- } \\
\text { counter antacids, laxatives, and anti-diarrheals to refresh his or her } \\
\text { memory if he or she appears unsure about these items. If the } \\
\text { answer is "Yes," clarify the response. Ask the SP to describe the } \\
\text { activity, item consumed, and the correct time. }\end{array}$ \\
\hline $\begin{array}{l}\text { Dietary supplements such as } \\
\text { vitamins and minerals? [Include } \\
\text { multivitamins and single nutrient } \\
\text { supplements.] }\end{array}$ & $\begin{array}{l}\text { Vitamins refer to various relatively complex organic substances } \\
\text { occurring naturally in plant and animal tissue. They are essential in } \\
\text { small amounts for the control of metabolic processes. Many are } \\
\text { available over the counter as multivitamin-multimineral or single- } \\
\text { nutrient supplements like Vitamin C. Include all of these when } \\
\text { clarifying the response to this question. Give the SP the laminated } \\
\text { card that lists all over-the-counter vitamins, antioxidants, } \\
\text { multivitamins, multivitamins with minerals, and therapeutics to } \\
\text { refresh his or her memory if he or she appears unsure about these } \\
\text { items. If the answer is "Yes," clarify the response. Ask the SP to } \\
\text { describe the activity, item consumed, and the correct time. }\end{array}$ \\
\hline
\end{tabular}


To enter a "Yes" or "No" response, type [Y/y] for "Yes" and [N/n] for "No.” Use the up and down arrow keys to toggle between the two choices. Alternatively, use the mouse to direct the mouse arrow to the drop-down arrow on the response text box, select "Yes" or "No" and left click. If "No" is entered, the next response box is highlighted. If "Yes" is entered, the time and date text boxes are highlighted. Type in the reported time using the numeric keys and select [Tab] to move to the AM/PM space. Type in [A/a] for times between midnight and 11:59 a.m. or [P/p] for times between 12:00 noon and 11:59 p.m. and select [Tab]. Alternatively, to select AM or PM, use the mouse to direct the mouse arrow to the drop-down arrow on the right side of the text box, left click, drag the mouse arrow to "AM," or "PM," and left click.

Type in the date using the keyboard's numeric keys and the mm/dd/yyyy format and select [Tab], or use the calendar to enter the date. To access the calendar, select [F2]. To select the correct month, use the mouse to direct the mouse arrow to the drop-down list, drag the arrow to the correct month (use the scroll bar if necessary), and left click. To select the correct day, use the mouse to direct the mouse arrow to the correct day on the displayed month and left click. To correct the year, use the mouse to direct the mouse arrow to the up-down controls on the spin box and toggle the number up and down. To transfer this date into the date space, use the mouse to direct the mouse arrow to the "OK" button and left click, or select [Enter]. To exit the calendar function, use the mouse to direct the mouse arrow to the "Cancel” button and left click. 
Review the data in the Fasting Questionnaire.

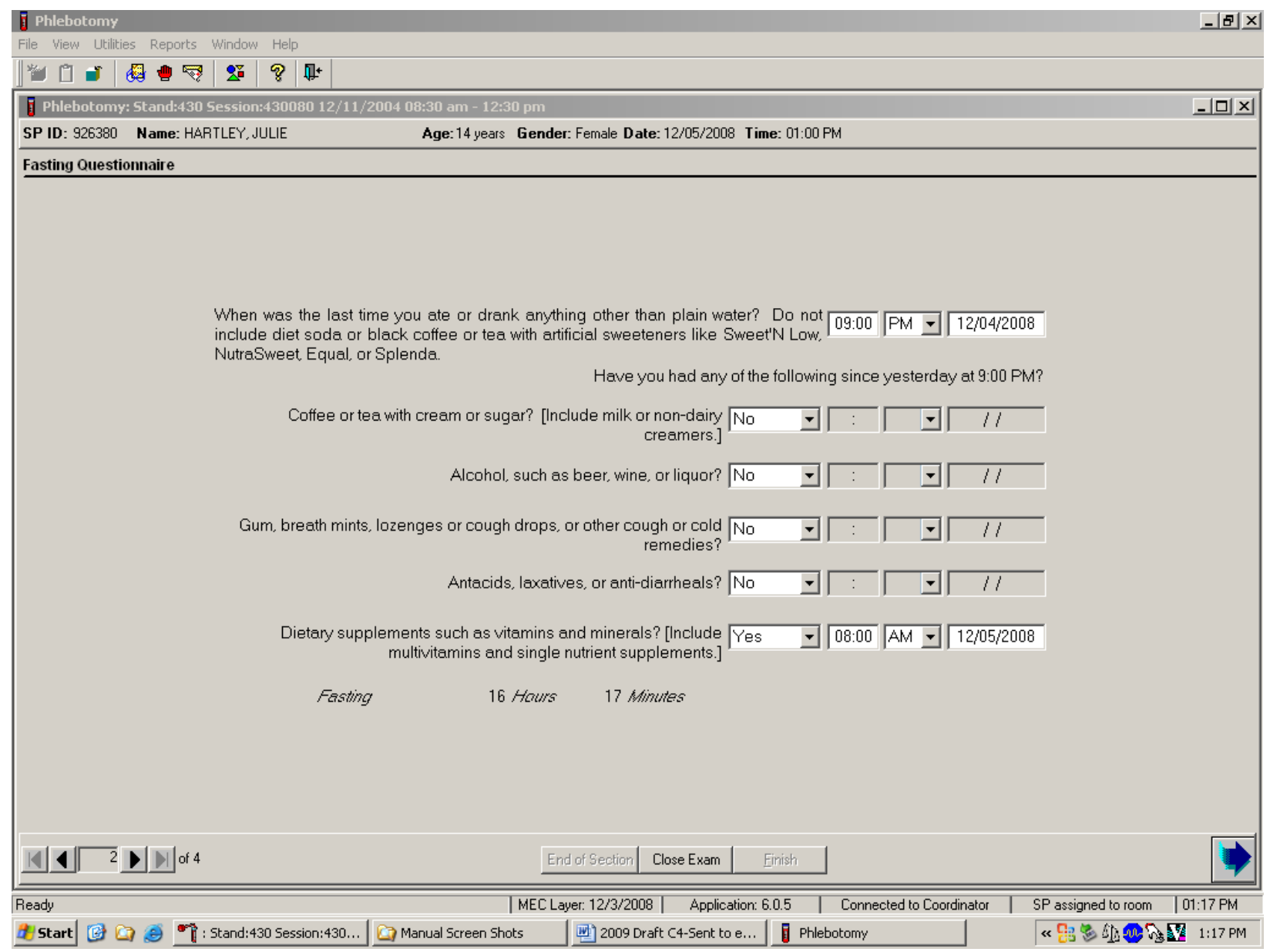

To progress to the next screen, use the mouse to direct the mouse arrow to the bright blue arrow in the bottom right corner and left click, or select [Enter].

Venipuncture should generally be performed using the median cubital, cephalic, or basilic veins in the left arm unless this arm is unsuitable. If the veins in the left arm are unsuitable, look for suitable veins in the right arm. If the veins in the antecubital space on both arms are not suitable, then look for veins in the forearm or dorsal side of the hand on the left arm/hand and then the right arm/hand. 
The Vacutainer ${ }^{\circledR}$ system of blood collection consists of glass or plastic tubes with colorcoded stoppers containing a premeasured vacuum that provides a controlled draw. Some tubes may contain additives to prohibit coagulation or inhibit glycolysis of blood cells. Draw the tubes in the order designated by the venipuncture protocol.

The exact quantity of blood drawn into each tube varies slightly with altitude, ambient temperature, and venous pressure. Fill tubes with additives completely to ensure proper ratio of blood to additive. Thoroughly mix the lavender and 2-mL and 10-mL gray top tubes immediately after drawing the tubes and place them on the specimen rocker until the tubes are labeled and passed through to the window to the laboratory.

Use Vacutainer ${ }^{\circledR}$ tubes at room temperature. Protect tubes from extreme temperatures and store in a cool place. It is important to note the expiration date printed on Vacutainer ${ }^{\circledR}$ tubes. Do not use expired tubes unless they are the only tubes available and they still contain a vacuum. Indicate if using expired tubes by using the "Other, specify” comment option on the venipuncture status screen.

Document the use of each Vacutainer® type in the automated Supply Use Control Log. When opening a new box, record the date, the lot number of the box, date in use, the expiration date, and tech ID number.

Allow two venipuncture attempts with the SP's verbal consent or with the parent's consent if an SP is a child or an infant.

If an SP faints or becomes ill, causing the termination of procedure without collecting all of the blood, repeat the procedure with the SP's consent after the SP has recovered.

\subsubsection{Preparation of the Puncture Site}

It is extremely important that the anticipated puncture site is thoroughly cleaned and all necessary equipment, including needles and tubes, are kept sterile and free from contamination. 
Follow the steps outlined below to prepare the puncture site.

- Place venipuncture equipment where it is readily available but not in danger of being upset. Keep extra equipment within easy reach.

- Thoroughly wash hands.

- Put on gloves.

- Place appropriate blood collection tubes in a test tube rack in the order dictated by the venipuncture protocol. If an SP exhibits nervousness, keep the tubes covered.

- Instruct the SP to sit on the exam table. Never attempt a venipuncture on a standing subject. Having the subject sit helps guard against any injury that might result if the subject faints. Place the SP in a supine position if it is impossible for the SP to sit upright during the procedure. Instruct the SP to extend the arm palm up and straight at the elbow. If the SP is a child or infant, have a medical technologist hold the SP in the proper position.

- Position the SP's arm on the armrest or a pillow so that the veins are readily accessible and you are able to work in a comfortable position. Be sure that the arm is in a downward position with the elbow lower than the heart to prevent backflow. Dispose of the pillow weekly for hygienic purposes. Change the pillowcase daily.

- Inspect the arm. Use the left arm unless unsuitable. The veins of choice are the cephalic, basilic, and median cubital veins located in the antecubital fossa area.

- If the veins in the antecubital space are not suitable, look for suitable veins on the forearm or on the dorsal side of the hand.

- Apply the tourniquet several inches above the selected site.

- Select a vein that is palpable and well fixed to surrounding tissue. Palpate even when the vein is visible. Use the following techniques if the veins do not distend quickly.

1. Massage the arm from wrist to elbow to force blood into the veins.

2. Tap the area sharply with the index and second finger two or three times to cause the veins to dilate.

3. Allow the arm to hang at the SP's side without a tourniquet to allow the veins to fill to their capacity.

4. Examine the SP's other arm; sometimes the veins in one arm are larger than in the other.

5. Check carefully for scar tissue or tendons near the vein. 
Apply the tourniquet for no more than 1 minute while searching for a vein and then release the tourniquet for 2 to 3 minutes. Avoid prolonged obstruction of blood flow by the tourniquet because it is uncomfortable for the SP and may alter certain results (e.g., cholesterol).

- Reapply the tourniquet when ready to perform the venipuncture.

- Cleanse the area with an alcohol wipe. Do not touch the side of the alcohol wipe that is in contact with the puncture site. Cleanse the area using a circular motion beginning with a narrow radius and moving outward so as not to cross over the area already cleansed.

- Repeat with a second alcohol wipe. Dry the cleansed area using a 2x2 gauze pad. The area should be completely dry before performing the venipuncture to reduce the burning sensation caused by alcohol penetrating the skin.

- Determine the correct needle size. Use a 19g, 21g, or $23 g$ butterfly or a $21 \mathrm{~g}$ multisample needle depending on the condition of the SP's veins. The $19 \mathrm{~g}$ butterfly should be suitable for most SPs. If the SP's veins appear fragile or small, use a $21 \mathrm{~g}$ or $23 g$ butterfly. If the SP is obese, use a $21 \mathrm{~g}$ multisample needle.

\subsubsection{Venipuncture Technique for the BD Saf-T EZ ${ }^{\circledR}$ Blood Collection Set (19 Gauge)}

1. Prepare the blood collection set. Obtain one blood collection set and one luer adapter. Open the blood collection package and inspect the unit. Open the blue adapter cover by twisting the two ends at the seal. Push the male luer adapter firmly and securely into the female end of the needle assembly. Attach a yellow tube holder onto the adapter.

2. Ask the SP to make a fist. Do not have the SP pump his or her fist since this action may alter certain results.

3. Fix the vein about one inch below the proposed point of entry by pulling the skin taut with the thumb of your less dominant hand.

4. Approach the vein in the same direction that the vein runs, holding the needle with bevel up and at a 15-degree angle to the SP's arm. 
5. Remove the needle cover. Grasp the wings and push the needle firmly and deliberately into the vein. Do not grasp the translucent needle shield to insert. If the needle is in the vein, a small amount of blood will appear in the butterfly tubing. Quickly push the first Vacutainer ${ }^{\circledR}$ tube down on the needle. If the needle is in the vein, blood will flow freely into the butterfly tubing. If no blood enters the tube and no bruise is forming, probe the vein until blood begins flowing into the tube. If no blood enters the tube and a bruise is forming, remove the needle. Place gauze squares over the puncture site and apply firm pressure to the puncture site for 3 minutes. Switch to the other arm using a new needle. Wait 10 minutes before beginning the procedure again if using the same arm for a second try.

6. Hold the tube with the tube stopper uppermost and with the tube lower than the needle to prevent backflow through the tube. It is very important to prevent possible backflow because of the possibility of adverse reactions to the SP.

7. As the Vacutainer ${ }^{\circledR}$ tube is filling, transfer the tube holder to the left (less dominant) hand, leaving the dominant hand free to pick up and change the tubes. If left-handed, do the reverse.

8. Fill all tubes completely. Make sure the tube contents do not touch the stopper or the end of the needle during the procedure.

9. Immediately invert the lavender and gray tubes to ensure proper mixing of blood and anticoagulant or additive. Place the lavender and gray tubes on the rocker. Do not invert or agitate the red top tubes.

10. Insert the next tube and push it down gently onto the adapter.

11. Because prolonged application causes vasoconstriction, remove the tourniquet after 2 minutes to ensure valid test results. If necessary (that is, if the blood flows more slowly), reapply the tourniquet after 2 minutes. If this is the last tube to fill, loosen the tourniquet when the tube begins filling and remove it as the last tube fills.

12. Fill tubes in the proper order, according to the protocol.

13. When the last tube has filled, remove the needle in a smooth quick motion. Avoid heavy pressure as the needle is being withdrawn because it may cause the point of the needle to cut the vein. To activate the shielding mechanism, either grab the tubing and hold the needle guard at the end closest to the tubing and pull the tubing until the needle guard locks in place or hold the needle guard at the end closest to the tubing, grab the wings and slide the wings back until the needle guard locks in place.

14. Discard the entire blood collection set, including the needle and adapter, in the Sharps needle disposal unit. Do not recap the needle. Discard tourniquet in trash. 


\subsubsection{Venipuncture Technique for the BD Safety-Lok ${ }^{\mathrm{TM}}$ Blood Collection Set (21 and 23 Gauge)}

The BD Vacutainer ${ }^{\mathrm{TM}}$ Push Button Blood Collection Set provides immediate protection against needle stick injury when properly activated within the vein, and in accordance with package insert directions.

1. Prepare the blood collection set. Open the package and inspect the unit. Check to ensure that the female luer adapter is firmly and securely attached on the male luer.

2. Ask the SP to make a fist. Do not have the SP pump his or her fist since this action may alter certain results.

3. Fix the vein about one inch below the proposed point of entry by pulling the skin taut with the thumb of your less dominant hand.

4. Approach the vein in the same direction that the vein runs, holding the needle with bevel up and at a 15-degree angle to the SP's arm.

5. Remove the needle sheath. With thumb and index finger, grasp wings together and access vein using standard needle insertion technique. If the needle is in the vein, a small amount of blood will appear in the butterfly tubing. Quickly push the first Vacutainer ${ }^{\circledR}$ tube down on the needle. If the needle is in the vein, blood will flow freely into the butterfly tubing. If no blood enters the tube and no bruise is forming, probe the vein until blood begins flowing into the tube. If no blood enters the tube and a bruise is forming, remove the needle. Place gauze squares over the puncture site and apply firm pressure to the puncture site for 3 minutes. Switch to the other arm using a new needle. Wait 10 minutes before beginning the procedure again if using the same arm for a second try.

6. Hold the tube with the tube stopper uppermost and with the tube lower than the needle to prevent backflow through the tube. It is very important to prevent possible backflow because of the possibility of adverse reactions to the SP.

7. As the Vacutainer ${ }^{\circledR}$ tube is filling, transfer the tube holder to the left (less dominant) hand, leaving the dominant hand free to pick up and change the tubes. If left-handed, do the reverse.

8. Fill all tubes completely. Make sure the tube contents do not touch the stopper or the end of the needle during the procedure.

9. Immediately invert the lavender and gray tubes to ensure proper mixing of blood and anticoagulant or additive. Place the lavender and gray tubes on the rocker.

10. Insert the next tube and push it down gently onto the adapter. 
11. Because prolonged application causes vasoconstriction, remove the tourniquet after 2 minutes to ensure valid test results. If necessary (that is, if the blood flows more slowly), reapply the tourniquet after 2 minutes. If this is the last tube to fill, loosen the tourniquet when the tube begins filling and remove it as the last tube fills.

12. Fill tubes in the proper order, according to the protocol.

13. When the last tube has filled, remove the Safety-Lok set by:

- $\quad$ Grasping the translucent yellow safety shield grip area with thumb and index finger while at the same time grasping the tubing, or

- $\quad$ Grasp either one wing or both wings and withdraw.

14. Activate the safety shield:

- $\quad$ One-Hand Technique. Hold tubing in hand and advance translucent yellow safety shield with thumb and index finger until a click is heard indicating that the needle is completely retracted and safety shield is locked in place over needle tip or...

- $\quad$ Modified One-Hand Technique. Apply pressure to site using your fingers. Withdraw blood collection set by grasping the translucent yellow safety shield grip area with the thumb and index finger. With opposite hand, grasp tubing between thumb and index finger while pushing the yellow safety shield forward until a click is heard indicating that the needle is completely retracted and the safety shield is locked in place or...

- $\quad$ Two-Hand Technique. Grasp either wing with one hand (A) and grip area of the yellow safety shield base with the other hand (B). Slide the wings back into the rear slot of the safety shield, until a click is heard indicating that the needle is completely retracted and locked into place over needle tip.

\section{Do not grasp safety shield by body; this will interfere with safety shield activation.}

15. Discard the entire blood collection set, including the needle and adapter, in the Sharps needle disposal unit. Do not recap the needle. Discard tourniquet in trash.

\subsubsection{Venipuncture Technique for the Eclipse Multisample Needle}

1. Prepare the needle. Holding both colored shields, twist and remove the white shield. Screw the needle onto the holder. Rotate safety shield back. Twist and pull needle shield straight off.

2. Place the first tube into the holder, securing it slightly, but not penetrating the stopper. 
3. Ask the SP to make a fist. Do not have the SP pump his or her fist since this may alter certain results.

4. Fix the vein about one inch below the proposed point of entry by pulling the skin taut with the thumb of your less dominant hand.

5. Approach the veins in the same direction that the vein runs, holding the needle with bevel up and at a 15-degree angle to the SP's arm.

6. Push the needle firmly and deliberately into the vein. Quickly push the first Vacutainer ${ }^{\circledR}$ tube down on the needle. If the needle is in the vein, blood will flow freely into the Vacutainer ${ }^{\circledR}$ tube. If no blood enters the tube but no bruise is forming, probe the vein until blood begins flowing into the tube. If no blood enters the tube and a bruise is forming, remove the needle. Place gauze squares over the puncture site and apply firm pressure to the puncture site for 3 minutes. Switch to the other arm using a new needle. Wait 10 minutes before beginning the procedure again, if using the same arm for a second try.

7. Hold the tube with the tube stopper uppermost and with the tube lower than the needle to prevent backflow through the tube. It is very important to prevent possible backflow because of the possibility of adverse reactions to the SP.

8. As the Vacutainer ${ }^{\circledR}$ tube is filling, transfer the tube holder to the left (less dominant) hand, leaving the right (dominant) hand free to pick up and change the tubes. If lefthanded, do the reverse.

9. Fill all tubes completely. Make sure the tube contents do not touch the stopper or the end of the needle during the procedure.

10. Immediately invert the lavender and gray tubes to ensure proper mixing of blood and anticoagulant or additive. Place the lavender and gray tubes on the rocker.

11. Insert the next tube and push it down gently onto the adapter.

12. Because prolonged application causes vasoconstriction, remove the tourniquet after 2 minutes to ensure valid test results. If necessary (that is, if the blood flows more slowly), reapply the tourniquet after 2 minutes. If this is the last tube to fill, loosen the tourniquet when the tube begins filling and remove it as the last tube fills.

13. Fill tubes in the proper order, according to the protocol.

14. When the last tube has filled, remove the needle in a quick, smooth motion. Avoid heavy pressure as the needle is being withdrawn because it may cause the point of the needle to cut the vein. Firmly push forward on the safety shield, lock into place, and inspect. After withdrawing the needle, immediately press clean gauze squares over the venipuncture site.

15. Discard the entire blood collection set, including the needle and adapter, in the Sharps needle disposal unit. Do not recap the needle. Discard tourniquet in trash. 
1. Have the SP place two fingers on the gauze to hold it in place, then ask the SP to raise the arm straight up, elevating the arm above the level of the heart, without bending the elbow. The SP should remain in this position for 2 to 3 minutes to help prevent hematomas.

2. Corresponding computer-generated bar code labels print automatically for the tubes drawn. Label the 10-mL gray top tube with the label containing the Vessel ID - 54. Label all other tubes with bar-coded labels with the bar code vertical on the tube. The time prints on the label.

3. Place the rack containing the blood collection tubes in the pass-through window located over the sink. Alternatively, deliver the rack to the laboratory, and distribute as described below:

- $\quad$ Place the lavender and gray tubes on the rocker located next to the centrifuge.

- $\quad$ Place the red top tubes upright in the test tube rack located next to the centrifuges in the laboratory.

4. Place all used needles in a Sharps container and dispose of all visibly contaminated waste in a biohazard bag.

5. Check the venipuncture site for clotting.

Venipuncture should generally be performed using the median cubital, cephalic, or basilic veins in the left arm unless this arm is unsuitable. If the veins in the left arm are unsuitable, look for suitable veins in the right arm. If the veins in the antecubital space on both arms are not suitable, then look for veins in the forearm or dorsal side of the hand on the left arm/hand and then the right arm/hand.

Use judgment when applying any bandage to elderly SPs. The skin of the elderly can be very thin and fragile and the Liquid Band-Aid ${ }^{\circledR}$ may irritate the skin when it is removed.

\section{If the venipuncture is performed on a vein in the antecubital space of the right arm:}

Apply Liquid Band-Aid to the puncture site. (If the SP is eligible for the GTT, remove the Liquid Band-Aid before the GTT venipuncture. After the GTT venipuncture is complete, reapply Liquid Band-Aid.) 


\section{If the venipuncture site is in the left arm:}

For all SPs aged 1-11 and SPs not eligible for GTT - Apply an adhesive bandage over the gauze pad. Instruct the SP to remove it in no less than 45 minutes if the bleeding has stopped. Also, suggest that the SP sit quietly for a few minutes. If bleeding continues, keep direct pressure on the site for 5 minutes or more.

For all SPs aged 12+ who are eligible for the GTT- Apply Liquid Band-Aid ${ }^{\circledR}$ to the puncture site in place of the regular adhesive bandage or CoFlex dressing. Liquid Band-Aid stops minor bleeding, reduces minor pain, and promotes fast healing on contact. It forms a clear, flexible, breathable seal. Apply once. Make sure puncture site has stopped bleeding.

To apply Liquid Band-Aid:

- $\quad$ Dry any excess blood.

- $\quad$ Open the activator package; hold the activator at the end of the stick. Do not touch the foam pad.

- $\quad$ Invert the liquid bandage bottle four times. Remove the cap. Squeeze four (4) drops of Liquid Band-Aid onto foam pad end of one activator.

- $\quad$ Allow the liquid to drop onto activator without touching the tip of the bottle to the foam end of the activator.

NOTE: Activator must be used to apply Liquid Band-Aid or product will not work.

- Immediately apply Liquid Band-Aid with the activator, covering the entire puncture site. Discard activator after use.

- $\quad$ Close bottle tightly after use.

- $\quad$ Do not touch the area for at least 1 minute until the seal forms.

- $\quad$ Apply once - do not repeat.

As the puncture site heals, liquid Band-Aid will naturally slough off.

To remove:

- $\quad$ Pour enough baby oil onto the Liquid Band-Aid seal to cover the area.

- $\quad$ Wait at least 1 minute.

- $\quad$ Wipe the area with a towelette. 
6. Report any adverse reaction to the venipuncture to the physician immediately and document the event by using the automated Unusual Field Occurrence utility.

7. If the SP has been fasting and is eligible for the GTT, then do not offer him or her a snack and juice. If the SP has not been fasting or is not eligible for the GTT, then offer him or her juice and crackers.

8. Escort the SP back to the coordinator.

9. If blood has spilled on the table or the arm board, wash the area with a 10 percent solution of bleach and water, then prepare the workstation for the next SP. Routinely disinfect the table or arm board with 10 percent bleach solution.

10. Wash hands and change gloves before approaching the next SP.

\subsection{0}

\section{Pediatric Venipuncture}

Pediatric venipuncture requires special techniques. Because you will be dealing with children of different age ranges and levels of understanding, it is important to be able to recognize at what stage a child is in early in the venipuncture process.

Infants and toddlers, aged 1 to 2 years, experience the world through their senses and do not have much of a language base. Therefore, verbal explanations are virtually meaningless. Expect crying and resistance from the start, even at the first touch. Restrain children of this age by enlisting the assistance of a medical technologist. The best techniques to use with these children are to maintain a reassuring tone, to reinforce that the child is a good boy or girl, and to draw the blood quickly. Be sure to reassure the child after the procedure and to use cartoon adhesive bandages and stickers when finished.

Preschoolers, aged 2 to 6 years, think concretely and in absolute terms; things are good or bad, right or wrong, painful or not painful. For these children, use simple, concrete terms when describing the procedure and its consequences. Always be honest. Try to avoid the word "take" which implies remove, "test" that implies pass or fail, and "fix" which implies broken. With children of this age, it is still important to emphasize that the child is a good boy or girl and to use colorful adhesive bandages and stickers. Try using distractions, such as holding something, counting things in the room, and breathing deeply. These techniques may work with the older children in this age group. The phlebotomist may have a medical technologist assist with most of these children. 
School-aged children, 7 to 12 years, have a better grasp of language and view the world more realistically. They understand past and future, that is, "this will be over soon," and relative terms, such as "this will hurt a little or a lot." Detailed explanations of the procedure are extremely effective. Again, always be honest. Include demonstrations, with a doll for example, whenever possible. Having the child help during the procedure distracts the child as well as letting him or her have some control. Be sure to reinforce how big a help he or she was after the draw. Give the child realistic choices, such as which arm to choose. You may need to consider having a medical technologist assist with some children even at this stage. Adhesive bandages continue to be important.

Although adolescents aged 12 to 18 years are beginning to think abstractly, they often regress when placed in stressful situations. It is best to assume that they will act younger than their chronological age. However, it is still very important to address the adolescent as an adult and not as a child. Provide detailed explanations and inform him or her that the best technique is being used. It is very important that the adolescent maintain control of the situation; therefore, give him or her some choices, as well as allowing him or her to help if offered. Be very clear about rules, such as remaining motionless, but do not restrain the adolescent.

Pediatric venipunctures are most successful when children are immobile, the veins are maximally distended, and all supplies are handy. Many children prefer a cloth or gauze under the tourniquet to prevent pinching of skin.

The usual site for pediatric venipunctures is the antecubital fossa. Most venipunctures in children are successful using a 23-gauge butterfly needle. Follow the general guidelines in Section 4.9 for performing venipunctures. 


\subsubsection{Age-Specific Techniques in Phlebotomy Competency}

Advance for Medical Laboratory Professionals

Vol. $14 \cdot$ Issue $6 \cdot$ Page 11

The Learning Scope:

Understanding and incorporating age-specific competence in evaluating the phlebotomist's performance

By Donna R. Kirven, BA, BPPVE, PBT(ASCP), CLA

\section{Learning Objectives}

At the conclusion of this article the participant will be able to:

State the importance of communicating and incorporating techniques in the performance of phlebotomy procedures based on the physical age and developmental stage of the patient.

Identify at least three major developmental considerations in the performance of phlebotomy procedures for each age-specific group.

Formulate an age-specific competency that can be used as part of the phlebotomy evaluation process.

All health care professions that involve direct patient contact require certain specialized techniques and competencies that are based on characteristics of the patient receiving service. The invasive procedures of phlebotomy are no exception.

Traditionally for some phlebotomists, the majority of their patients could be categorized in one or two age groups, while the experience of others includes all age groups. Age-specific concerns are age, growth, development and safety considerations that must be assessed and incorporated into phlebotomy practices for successful outcomes with all patient age groups.

Simply defined, age-specific competence is the knowledge, skills, ability and behaviors essential for providing care to specific populations of patients and their families. These considerations require expanded knowledge and an increased experience level of physical, psychological, and interpersonal assessment skills. The newly certified (or trained) phlebotomist should begin using that training on older children, and as confidence and technical skills are attained, progress to performing venipunctures on younger children and, subsequently, infants. ${ }^{1}$ Performing a venipuncture on pediatric patients can present a greater technical and emotional challenge to the phlebotomist, and without proper age-specific techniques, can result in an unnecessarily unpleasant experience for the child, the parents, and the phlebotomist.

To make quality health care services available to communities served by acute care facilities, age-specific phlebotomy techniques are now becoming a standard part of the phlebotomy training curriculum and annual competencies. 


\section{Factors in Age-Specific Techniques}

Age-specific techniques in phlebotomy involve much more than simply using a smaller tourniquet and collecting a minimum volume of blood. Considerations for motor skills, communication skills development, social needs, and physical and learning disabilities also are a part of the age-specific assessment. The rationale for age-specific techniques is based on the fact that different age groups of patients have special physical and psychosocial needs. Knowledge of the basic nutritional and social needs such as feeding, sucking, and physical affection in the infant patient can exemplify professionalism and confidence to anxious parents. Recognition of the increased potential for diminished hearing, vision and mobilization can dispel feelings of embarrassment and frustration in the elderly patient.

Safety is also an age-specific criteria issue. While the phlebotomist should exercise safe practices with all patients, it is important to be aware of the special safety precautions that should be used with pediatric, elderly, and disabled patients that are specifically attributable to age. Communication skills on the part of the patient as well as the phlebotomist are also important factors. When performing phlebotomy procedures on an infant, informing the parent or guardian of the details of the procedure in addition to reassurance can alleviate anxiety and build his or her trust before attempting the procedure. Becoming a highly qualified, experienced phlebotomist skilled in age-specific techniques requires good interpersonal skills such as integrity, effective communication and confidence, as well as demonstrated technical skill.

By incorporating awareness growth and development theories into phlebotomy training, the phlebotomist can provide service and perform procedures in a manner that meets the practical (quality specimen collection) and personal (trust, reassurance, respect) needs of patients and their family members. Table 1 shows six stages of development and the corresponding age divisions.

\section{The Neonate/Infant}

Venipunctures should not be performed on children under the age of 6 months unless the test ordered requires the volume and type of specimen that can only be obtained by a needle stick. Nonetheless, heel sticks, the most frequently used method performed for mandatory newborn screens that test for phenylketonuria (PKU), galactosemia, hypothyroidism, primary congenital hypothyroidism and several other hemoglobinopathies, ${ }^{2}$ are performed on a routine basis and require highly specialized skills and agespecific techniques.

The phlebotomist must select the appropriate method of obtaining a blood sample after considering two factors: the patient's age, and sample requirements for the test ordered. The novice phlebotomist must study anatomy and physiology, become familiar with special types of equipment specifically designed for the pediatric patient, observe various techniques as performed by experienced phlebotomists or nurses, and practice the techniques themselves to develop the necessary skills. They must make every attempt to collect the minimum amount of blood required for testing because of the small blood volume of infants. Removal of more than 10 percent of an infant's blood volume at one time can lead to cardiac arrest, ${ }^{1}$ while removal of large amounts of blood over an extended period of time can lead to anemia, possibly requiring the infant to undergo a blood transfusion. Improperly performing a heel incision, or using a safety lancet that is not of appropriate pediatric size on small children, can lead to puncture of the calcaneus bone, infection, and osteomyelitis.

Specific guidelines for performing a heel stick include:

Refrain from puncturing the anteromedial aspect or the posterior curve of the heel to avoid puncturing the underlying calcaneus. 
Hold the infant's foot securely but gently so as not to bruise his/her delicate tissue or restrict blood flow.

Avoid milking or squeezing the foot, which can dilute the blood with interstitial (tissue) fluid or cause hemolysis of the specimen.

Refrain from covering the puncture site with adhesive bandages that can macerate the infant's fragile skin underneath.

The emotional challenge of performing phlebotomy procedures on neonates requires that the phlebotomist be prepared to handle not only the infant's reaction to the procedure, but also the parent or guardian who may be present. It is important that the experienced phlebotomist build trust with the parent by communicating in a warm, compassionate manner while displaying a calm and confident approach. These types of interpersonal skills, in addition to requesting the parent's assistance in comforting the infant, can help calm parental anxiety and foster trust in the phlebotomist, allowing her to perform the procedure in a less frenzied environment. Consider the following:

\section{Case Study}

Jordan Smith is a 9-month old boy who has been brought to the outpatient laboratory by his parents for some lab work ordered by Jordan's pediatrician. Aside from the newborn screening performed shortly after his birth, Jordan has never had a blood test. His pediatrician has ordered electrolytes and a complete blood count.

Once Jordan's parents have completed the necessary laboratory registration, Monica, a phlebotomist with 15 years experience, having reviewed the requisition and assembled her phlebotomy supplies beforehand, calls Jordan and his parents into the outpatient drawing room. After introducing herself and completing the patient identification procedure with Jordan's mother, Monica greets Jordan and notices that Jordan's father appears to be frozen with fear, hasn't spoken a word and has large beads of perspiration covering his forehead. Jordan's mother, on the other hand, appears significantly less nervous and is engaged in physically and verbally comforting him. Her years of experience have taught Monica that she should address these observations before beginning the procedure. The dialogue proceeds as follows:

Monica: Mr. and Mrs. Smith, has Jordan ever had a blood test performed?

Mother: No.

Father: (Shakes his head designating "No")

Monica: I'd like to take a minute or two to explain the procedure to you, but first I'd like to reassure you that I will do my best to perform this procedure with as little discomfort as possible for Jordan and for you.

Mother: Thank you.

Father: (Mumbles “Thank you”)

Monica: Mr. Smith, you appear to be a little nervous, and that's quite understandable. Our phlebotomy staff here have an average of 10 years experience in performing these procedures on patients who are Jordan's age and younger. I understand your hesitation and respect your concern. If you feel that observing the procedure may be a bit too upsetting for you, you're welcome to have a seat in our waiting area while your wife remains here with us during the procedure, but if you prefer to stay that's fine too. 
Father: Well, it's just that he's so small—and do you really have to stick him with a needle to get blood, can't you just prick his foot or something like they did when he was born?

Monica: Children who are Jordan's age have usually begun to stand or walk, and in preparation for this, the skin of the foot has grown thicker and more resilient. To incise or cut the heel at his age and size, in the same method as that for newborns, would not allow sufficient blood flow required to complete the tests that his physician has ordered. Our standard practice for a patient Jordan's age is to obtain a blood specimen from a vein in the arm, which is believed to be a more efficient and less traumatic method of performing a blood test.

Father: How can you find a vein in a little arm like this?

Monica: That's a question that I'm often asked by parents. My training for collecting blood specimens from children required that I learn to feel the veins in little arms just as proficiently as I did in older arms, and after proper training and practice, I did. Sometimes, it's easier to find the veins in someone Jordan's size than it is to find them on an adult.

Father: So you've been doing this a long time?

Monica: I personally have more than 10 years experience drawing infants and children.

Father: Well, that's good to know. I feel a little bit better now so I think I'll stay in here.

Monica: That's great. Mrs. Smith do you have any questions?

Mother: No. I just want this done and over with.

Monica: Great! If I can get you to help me by holding Jordan just this way, it will really help us complete this procedure quickly and ensure that we have collected a quality specimen. Dad, you can help too by giving Jordan lots of encouragement and praise during the procedure.

\section{The Pre-School Child}

Although the pre-school aged patient has begun to verbally communicate in small statements, it is important that the phlebotomist explain the intended procedure in terminology appropriate for the child. As this patient age group is heavily engaged in security-seeking behavior, the presence of the phlebotomist - a stranger clad in a white lab coat — can often exacerbate their fear and increase their anxiety level even before they see a needle. Approach them slowly and gain their trust before handling equipment or touching their arms to look for a vein. ${ }^{2}$

One of the goals of age-specific techniques for this group is to attempt to keep the child calm and reduce crying which, if prolonged, can actually raise the white blood cell count and the blood $\mathrm{pH}$ level. ${ }^{3}$ Several methods can be used to accomplish this, some of which may not work for every child; however, the appropriate choices can be more easily made with the phlebotomist's increasing experience level.

Some of these methods are:

Prepare materials and gather equipment before you encounter the child. Doing this in the child's presence will allow more time for the child's anxiety to build.

Approach the child in a friendly, cheerful, and empathetic manner. Speak before you touch the child, even if the child cannot or will not respond. Introduce yourself and address the child by name. 
Speak in a soothing, non-threatening tone of voice. Even if the child does not completely understand what you are saying, a calming tone communicates a non-threatening intention.

Explain the procedure in terms that are appropriate for the child's age. This, coupled with a soothing tone of voice, can help to lessen the child's fear.

Do not say, "It will not hurt." Explain that some pain will be involved in the procedure, and that it is OK to say "ouch" or cry a bit, but that it is important to keep the arm as still as possible.

During the procedure, keep the child informed of how much longer it will be- "Just one more tube"while using appropriate praise for the child, even if he/she did not cooperate as much as possible. Phrases such as "You are doing such a good job of helping me. Thank you, (patient's name)" can provide reinforcing recognition that may encourage the child to approach his/her next phlebotomy experience with less anxiety.

\section{The School-Aged Patient}

Performing phlebotomy procedures on the 8-12 year old patient can be more of a physical challenge than an emotional one. This age group has mastered the meaning and use of the word "No," and is prepared to defend their fear with it. Proper immobilization of the pediatric patient is necessary to ensure the safety of the child as well as the phlebotomist. Instructing the parent/guardian on the correct "hugging" hold method for a child seated on the parent/guardian's lap, or the appropriate restraint for the prone child can allow the phlebotomist to collect the specimen in a manner that will also ensure accurate test results.

The school-aged patient has moved from security-seeking behavior to independence-seeking behavior and as such can exhibit unceasing curiosity. It is important that all phlebotomy equipment be kept out of reach of the child; however, be sure to respond to the child's inquiries as appropriate. Asking questions is an integral part of the learning process, and responding initiates effective communication skills. These young patients have begun to establish their identities, and the phlebotomist must communicate with them as individuals, in age-appropriate terminology.

Also, most of these children have begun the initial stages of puberty, so the phlebotomist must allow for the privacy of the child in preparation for the phlebotomy procedure. In an outpatient setting, if a urine specimen is ordered, give the child instructions for collection, in age-appropriate terminology, and allow him/her to attempt specimen collection assisted by the parent/guardian or alone.

\section{The Adolescent Patient}

In dealing with the adolescent patient, it is essential for the health care provider to remember the importance of demonstrating an understanding of the significance of peer relationships to the adolescent. Embarrassment can be this age group's mascot. The phlebotomist can implement this understanding by recognizing that some reassuring comments or approaches, if not age-appropriate, can actually instill more anxiety for the adolescent patient. Providing age-appropriate praise and positive feedback for this group of patients can lessen their anxiety.

Adolescents in the higher age range are usually engaged in thoughts, goals, and behavior that they believe will eliminate the perception of a child, and establish that of an adult. These behaviors can, however, manifest themselves in a sensitivity-heightened and non-interactive manner that can test the communication skills of the phlebotomist, as well as the parent/guardian. It is important to continue to initiate communication with adolescent patients and welcome their limited inquiries. 
The older adolescent male, for some unknown cause, possibly hormonal, can demonstrate a greater potential for fainting during a venipuncture procedure. One of the age-specific safety techniques that can be used with this patient age group is to more closely assess the patient before, during, and after the procedure. Listen for a previous history of syncope or light-headedness when first encountering the adolescent patient.

During the procedure, periodically check for pre-syncope signs such as profuse perspiration, change in skin pallor, and/or the onset of clammy hands. Another indication of pre-syncope can be the adolescent patient who is suddenly silent in the middle of a conversation or extremely talkative after having been previously silent.

It is important that the phlebotomist be familiar with the department's policy/procedure for dealing with patients who experience syncope, light-headedness, or dizziness.

\section{The Adult Patient}

Some of the typical conditions that commonly affect the adult patient are stress and depression, which are usually related to pressures of the workplace, marriage, childbearing, parenting, and social expectations. Additionally, these roles can induce a stronger identification with their parents or primary caregivers, known as generativity, as well as a closer reflection with their own mortality.

For the average adult, the focus of learning patterns and thinking lies mainly on problem solving. Accordingly, the phlebotomist must use effective communication skills by clearly and honestly informing the adult patient of the procedure while continually maintaining a professional, confident manner. Adult patients are more than willing to cooperate if they are aware of the benefits of their cooperation.

An adult patient can possess the same fear as that of a pediatric patient; however, the adult patient is usually more willing to allow the procedure as long as it is performed in a professional and concise manner. Conditions that may begin to affect the middle-aged adult patient are the onset of cardiovascular disease and menopause, stroke, and cancers. On the other hand, a significant percentage of adult patients who are directed toward maintaining good mental and physical health, optimal energy, and social activity exists. These patients are usually less stressed about phlebotomy procedures and view them as an integral part of the preventive health care process.

One of the most effective tools of good communication is eye contact. Eye contact promotes a sense of trust and honesty between the patient and phlebotomist; however, if a patient does not return the eye contact when being spoken to, he/she may prefer less direct eye contact due to cultural or modesty reasons. Experienced phlebotomists will take cues from the patient to monitor and satisfy the patient's comfort level needs.

When interacting with adult patients, the phlebotomist should refrain from engaging in negative body language such as crossed arms, wrinkled forehead, or frequently glancing at a clock or watch. These behaviors can detract from the professional image when communicating with adult patients.

\section{The Elderly Patient}

With the expanding life expectancy tables, many phlebotomists' patients are likely to be elderly. ${ }^{3}$ As with infants and younger patients, this can present both physical and psychological challenges for the health care professional. Likewise, working with the older adult can be extremely rewarding for the phlebotomist who continues to perfect her technical expertise and take the time to treat the patient with respect and compassion. 
Elderly patients may feel that they are in less control of their medical situation than other patients, may feel apprehensive about having the procedure performed, and embarrassed about feeling this way about it. As with all patients, elderly patients should be treated with consideration for their special needs and with tender, loving care. However, elderly patients are sometimes under the impression that they are a burden and become anxious about the procedure because they don't wish to be a bother. Phlebotomists can help to ease this anxiety by being friendly and cheerful, and taking the time to not only listen to their patients and talk with them, but also to encourage verbalization of their concerns and fears. This can help establish the patient's trust in the phlebotomist and dispel feelings of being a bother.

As people age, they usually undergo a number of physical changes that can have a significant impact on their safety and the effectiveness of normal collection procedures. The elderly patient experiences skin changes, such as the loss of elasticity and moisture, as well as the thinning of skin layers. Loss of supportive connective tissue can lead to "loosened" skin, and loss of muscle tissue and tone may allow for greater movement of veins. ${ }^{3}$ The phlebotomist must treat the skin of the elderly patient in a gentle manner. Sometimes the tourniquet that is applied in a tight or rough fashion can easily tear this delicate skin.

In this age group, patients may experience a decrease in peripheral circulation due to narrowed vessels that are the result of atherosclerosis ${ }^{4}$ and, combined with loss of vessel elasticity, become more fragile. Gently massaging the intended site rather than slapping or tapping the arm or hand can effectively increase circulation to the site. As skin layers thin, arteries can lay closer to the skin surface in elderly patients, so the phlebotomist must take care to palpate for the "best" vein, and ensure that it does not have a pulse.

Hearing loss is another disorder that is commonly found in the elderly patient. The phlebotomist should approach a patient who communicates that he/she has a hearing impairment by speaking in a slowed, deliberate and distinct voice to confirm that the patient understands. Some conditions, including Parkinson's disease and stroke, can lead to impaired or unclear speech. This can be a source of frustration for the elderly patient. The phlebotomist must allow the patient to complete his/her statements without being made to feel "rushed," and remember that speech difficulty does not imply difficulty hearing or understanding.

Arthritis also affects a large percentage of the elderly population and may restrict the patient from fully straightening or extending the fingers or arms. The phlebotomist should never force a patient's limb, but should ask the patient whether this action is painful or uncomfortable.

Tremor is also common in advanced aging ${ }^{3}$ and may present some difficulty for the patient to hold his/her arm steady during the venipuncture procedure.

A large number of adults over the age of 50 begin to experience loss of visual acuity and, as such, it is vitally important that the phlebotomist ensure that the entrance and floor areas are free of any debris that may not be visible to the elderly patient. Clear plastic needle covers and small pieces of tape can be falling hazards. Inpatient beds that are raised should be lowered before leaving the patient's room.

Poor nutrition and chronic degenerative disease may lead to emaciation, which can make the elderly patient more susceptible to infection due to loss of immune function, ${ }^{3}$ so these potential falling hazards should be eliminated to avoid cuts, lacerations, and broken limbs. 
Forgetfulness, confusion, and dementia are more commonly found in the elderly population. ${ }^{3}$ Reliability on the inpatient's armband to confirm identification is a must. Some confused patients may not fully understand your intentions and may lash out in defense. If the patient does not demonstrate a clear understanding of the procedure, the phlebotomist should check with the nurse and seek assistance if appropriate.

\section{Summary}

Age-specific care techniques are a requirement for all health care professions involving direct patient contact, such as phlebotomy. It is vitally important that these techniques be introduced and incorporated into all phlebotomy training programs, competencies, and continuing education. Learning these techniques requires not only a fundamental knowledge of human growth and development, but also the ability to combine technical expertise with age-specific interpersonal skills.

Incorporating the physical, psychological, and social needs of patients into the formal phlebotomy training curriculum can provide a vital foundation for all those who aspire to successfully perform phlebotomy procedures. Probationary and annual competencies should specifically address age-specific topics such as tone of voice, eye contact, and active listening skills. The implementation of age-specific techniques into the phlebotomy training curriculum and competency format will yield more effective phlebotomy practices and, subsequently, quality patient care. 


\section{REFERENCES}

1. Garza, D., Becan-McBride., K. Phlebotomy Handbook: Blood Collection Essentials, pediatric procedures, $6^{\text {th }}$ Edition. Upper Saddle River, NJ, Prentice Hall, 2002.

2. McCall, R., Tankersley, C. Pediatric venipuncture In: Phlebotomy Essentials, $2^{\text {nd }}$ Edition. Philadelphia, Lippencott-Raven, 1998.

3. Sommer, S., Warekois, R. Blood collection in special populations In: Phlebotomy: Worktext and Procedures Manual. Philadelphia, W.B. Saunders, 2002.

4. Klosinski, D. Collecting specimens from the elderly patient. Laboratory Medicine. 1997;28(8): 515-522.

Donna Kirven is phlebotomy education and training coordinator at John Muir Medical Center in Walnut Creek, CA.

http://www.advanceformlp.com/common/editorialsearch/viewer.aspx?FN=03mar10_mtp11.html\& $\mathrm{AD}=3 / 10 / 2003 \& \mathrm{FP}=\mathrm{mt}$ 
Vol. •Issue • Page LS answers

Learning Scope Questions

1. Which of the following is considered a personal need of the patient?
a) reassurance
b) respect
c) trust
d) all of the above

2. Which of the following age groups is heavily engaged in security-seeking behavior?
a) elderly
b) preschool
c) adolescent
d) school-aged

3. Immobilizing the pediatric patient can allow the phlebotomist to:
a) collect a specimen in a manner that will ensure accurate results.
b) build trust.
c) demonstrate confidence.
d) facilitate development and growth.

4. Which of the following age groups includes a population that demonstrates a greater potential for fainting during a venipuncture procedure?
a) elderly
b) preschool
c) adolescent
d) school-aged 
5. To monitor and satisfy the adult patient's comfort level needs, the experienced phlebotomist will take his/her cues from the:
a) physician.
b) nurse.
c) family.
d) patient.

6. To ease anxiety with the elderly patient, the phlebotomist can:
a) be friendly and cheerful.
b) listen and talk with the patient.
c) encourage verbalization of concerns and fears.
d) do all of the above.

7. One of the age-specific goals for the school-aged group is to:
a) facilitate bonding.
b) satisfy practical needs.
c) encourage commitment to task and contribution.
d) assess limiting physical conditions.

8. Infant cardiac arrest can result from removal of:
a) 10 percent of infant's blood volume over an extended period of time.
b) small amounts of blood over an extended period of time.
c) 10 percent of infant's blood volume at one time.
d) 2 percent of infant's blood volume at one time.

9. When performing venipuncture on the preschool patient, the phlebotomist should:
a) say nothing.
b) say, "it will not hurt."
c) not say, "it will not hurt."
d) say none of the above. 
10. The experienced phlebotomist must demonstrate an understanding of the importance of peer relationships when performing phlebotomy procedures with patients in the:
a) elderly age group.
b) adolescent age group.
c) adult age group.
d) preschool age group.

11. Some of the typical conditions that commonly affect the adult age group are:
a) diminished hearing.
b) loss of skin elasticity.
c) stress and depression.
d) embarrassment.

12. One of the conditions of advanced aging that can make it difficult for the elderly patient to hold his/her arm steady during the venipuncture procedure is:
a) skin layer thinning.
b) loss of visual acuity.
c) tremor.
d) poor nutrition.

13. Which of the following is considered one of the basic characteristics of the pre-school aged patient?
a) feeding
b) privacy
c) imagination
d) generativity 
14. Which of the following should be specifically addressed in probationary and annual competencies?
a) eye contact
b) tone of voice
c) active listening skills
d) all of the above

15. Age, growth and development, and safety considerations must be assessed and incorporated into phlebotomy practices for:
a) increased facility profit.
b) addressing practical patient needs only.
c) successful outcomes with all patient age groups.
d) enhanced interdepartmental communications.

16. In the case study, which of the following is a method that the phlebotomist used to build trust?
a) taking the time to explain the procedure
b) asking the parents if the patient had a blood test performed before
c) explaining the venipuncture procedure
d) all of the above

17. The phlebotomist in the case study demonstrated professional and timely assessment skills by:
a) explaining the procedure to the parents before beginning the procedure.
b) communicating her years of experience before beginning the procedure.
c) addressing the father's anxiety before beginning the procedure.
d) assembling the phlebotomy equipment before beginning the procedure. 
18. By thoroughly answering the parent's questions and communicating her length of experience, the phlebotomist in the case study demonstrated:
a) compassion.
b) proper immobilization technique.
c) satisfaction of practical needs.
d) confidence.

19. By explaining why the child was too old to have the specimen collected by skin puncture, the phlebotomist in the case study illustrated:
a) age appropriate terminology.
b) non-threatening tone of voice.
c) knowledge of growth and development.
d) respect for patient privacy.

20. In the case study, which of the following demonstrates that the phlebotomist addressed the personal needs of the patient and his parents?
a) explaining the procedure with compassion
b) reassuring the parents
c) addressing the father's anxiety
d) all of the above 


\subsection{Hand Venipuncture}

It is acceptable to use dorsal hand veins as the venipuncture site. This site should be used only if the antecubital veins are unsuitable, such as when the antecubital area is edematous, burned or scarred, there are casts on both arms, or the veins are thrombosed.

- Select a vein that is palpable, lightly brushing a finger across the back of the hand as it is palpated. This helps select the best vein and determines its direction. Palpate even when the vein is visible.

- Use a $21 \mathrm{~g}$ or $23 \mathrm{~g}$ butterfly blood collection set depending on the condition of the SP's veins.

- Follow the venipuncture technique described in Section 4.9.3 to draw the blood. 


\subsection{Recording the Results of the Venipuncture Procedure}

The Venipuncture screen displays the tube protocol for the SP.

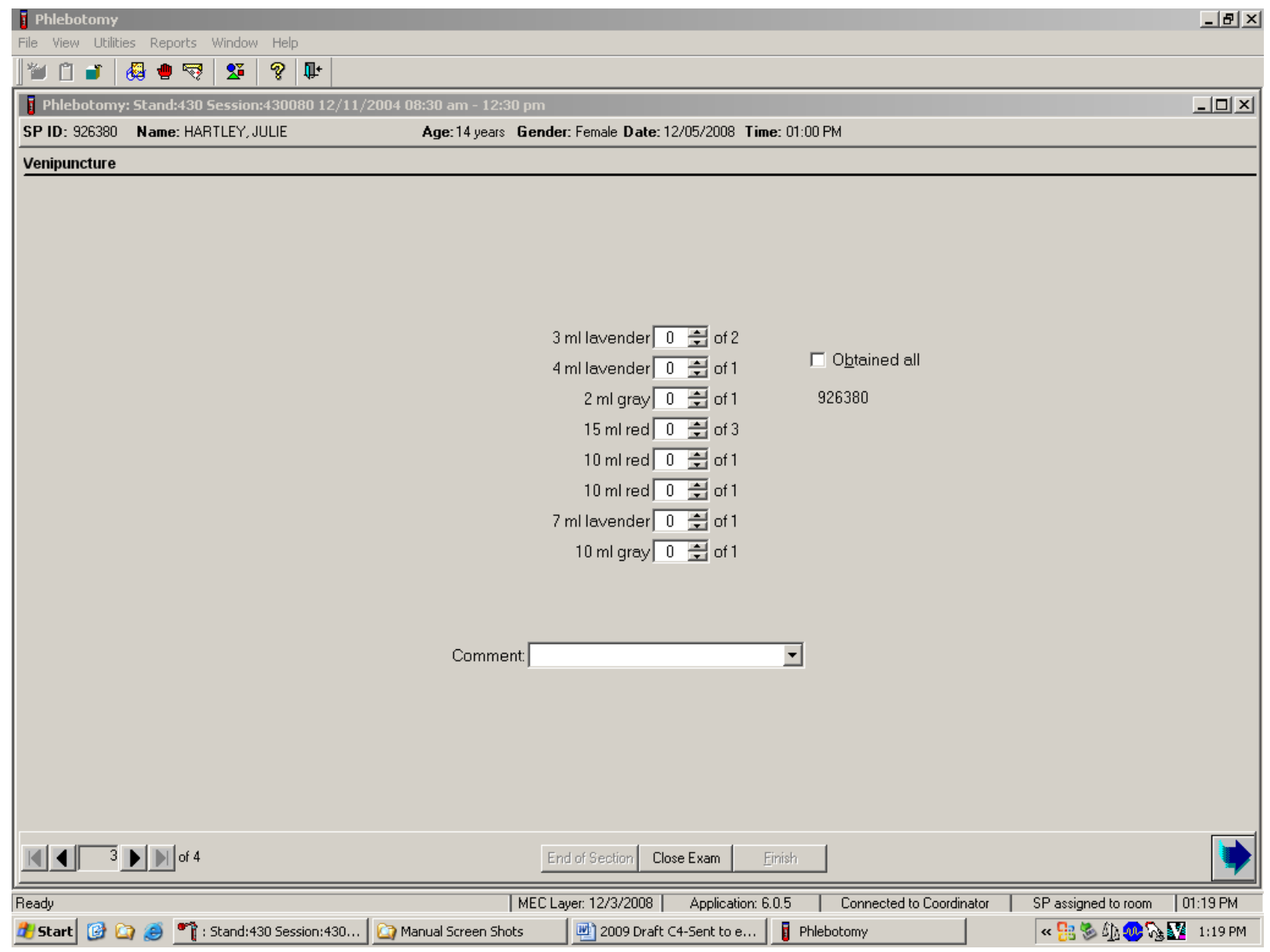

Each SP's profile is dependent on his or her age, appointment type, selection for inclusion into randomly-selected subsamples, and the session time. 
Immediately after completing the venipuncture, enter the results of the blood draw, the reasons for a tube not being drawn according to the protocol, and any comments about the venipuncture.

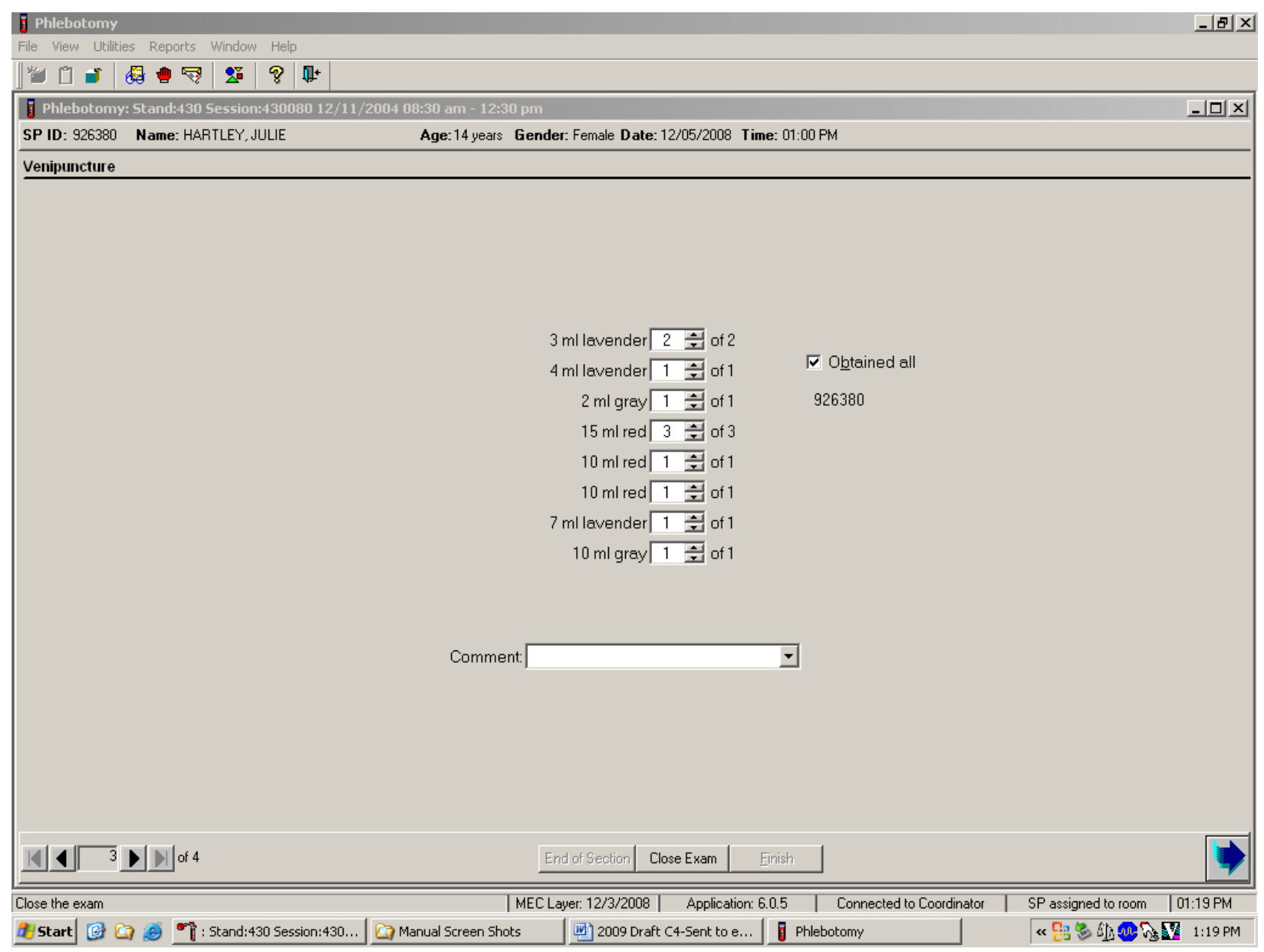

To record all tubes as filled or obtained, use the mouse to direct the mouse arrow to the “Obtained all” check box and left click, or type [Alt] [B/b]. This records a check mark in the box and marks all tubes as obtained. To mark individual tubes as filled or obtained, use the mouse to direct the mouse arrow to the up-down controls on the spin box and toggle the number of each tube up or down or type the correct number using the numeric keys. 
Document circumstances where the alternative protocol was used or the SP was in the supine position.

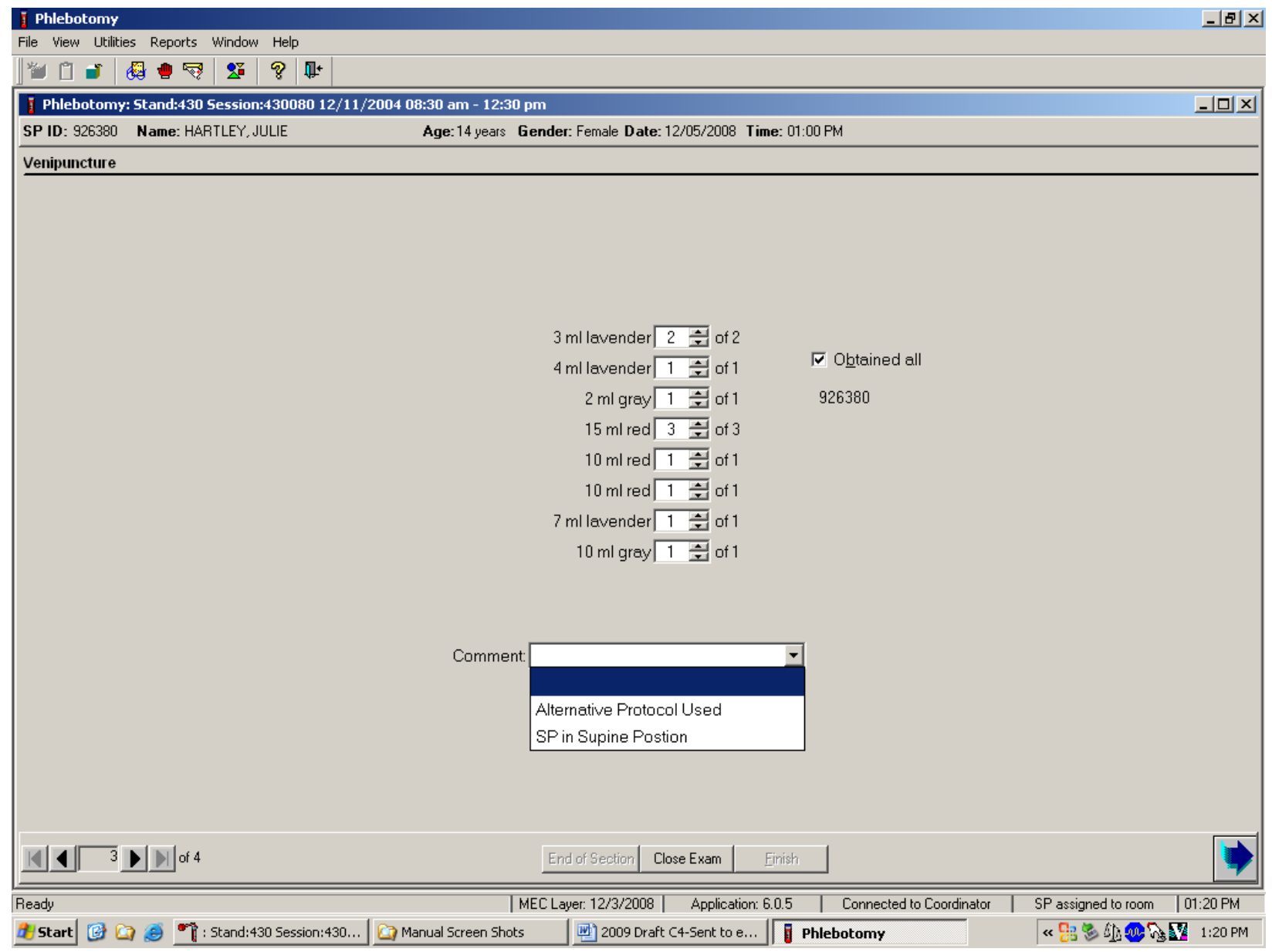

To record either "Alternative Protocol Used" or "SP in Supine Position” comments in the Comment text box, use the mouse to direct the mouse arrow to the arrow on the drop-down list, left click, drag the mouse arrow to the desired choice, and left click. Alternatively, type [A/a] for "Alternative Protocol Used" or [S/s] for "SP in Supine Position" and [Enter], or use the up and down arrows to toggle between the two choices and [Tab.]

To progress to the next screen, use the mouse to direct the mouse arrow to the bright blue arrow in the bottom right corner and left click, or select [Enter] when this blue arrow is highlighted. 
To reprint the Dymo labels, move forward one screen and back using the navigation arrows. A Print Labels informational text box displays. It is also possible to reprint labels in the review mode.

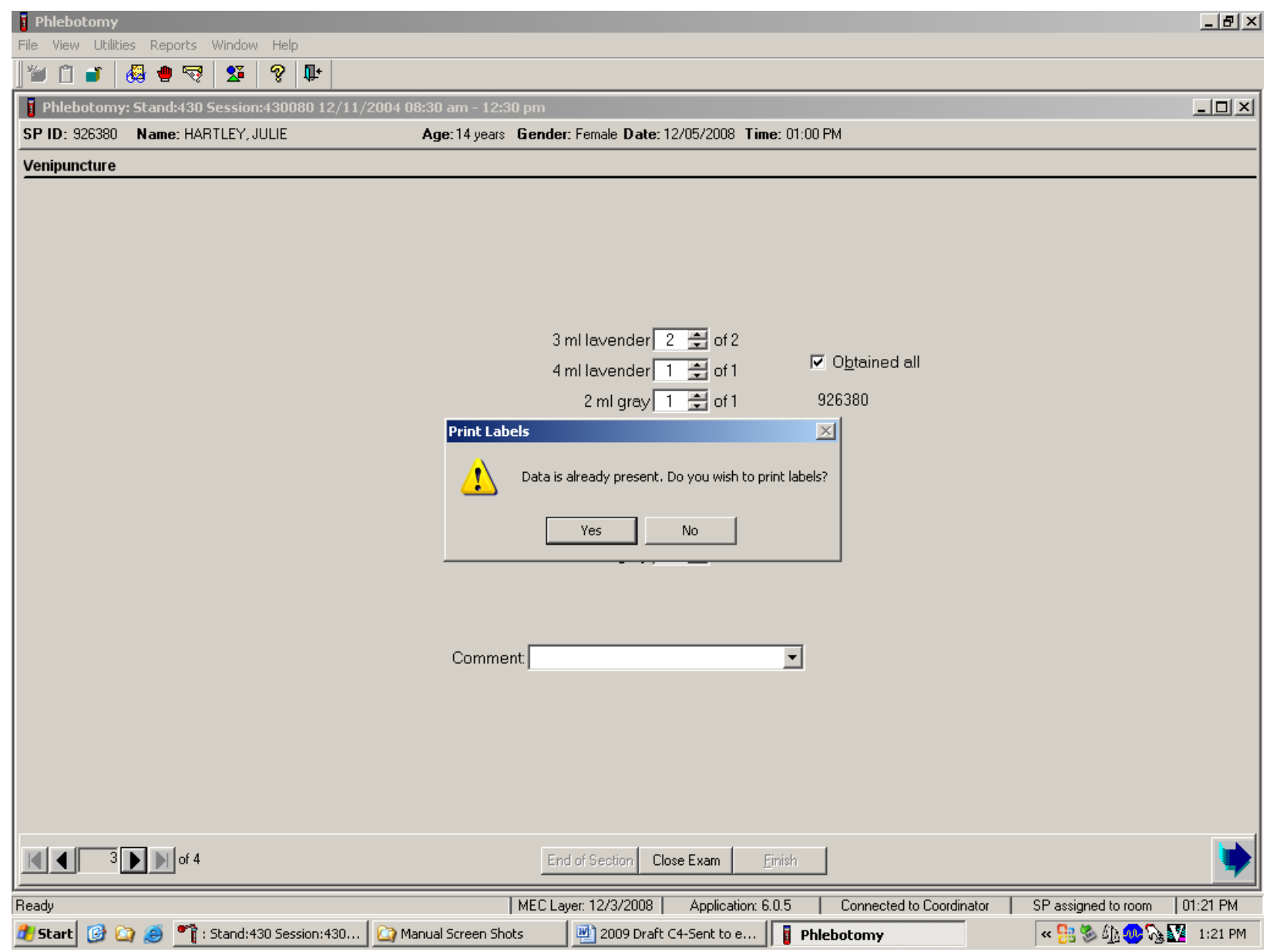

To reprint the labels, use the mouse to direct the mouse arrow to the arrow on the Yes button, and left click. To remove the Print Labels informational text box without reprinting the labels, use the mouse to direct the mouse arrow to the No button and left click. 


\subsection{Venipuncture Status}

Verify the venipuncture status.

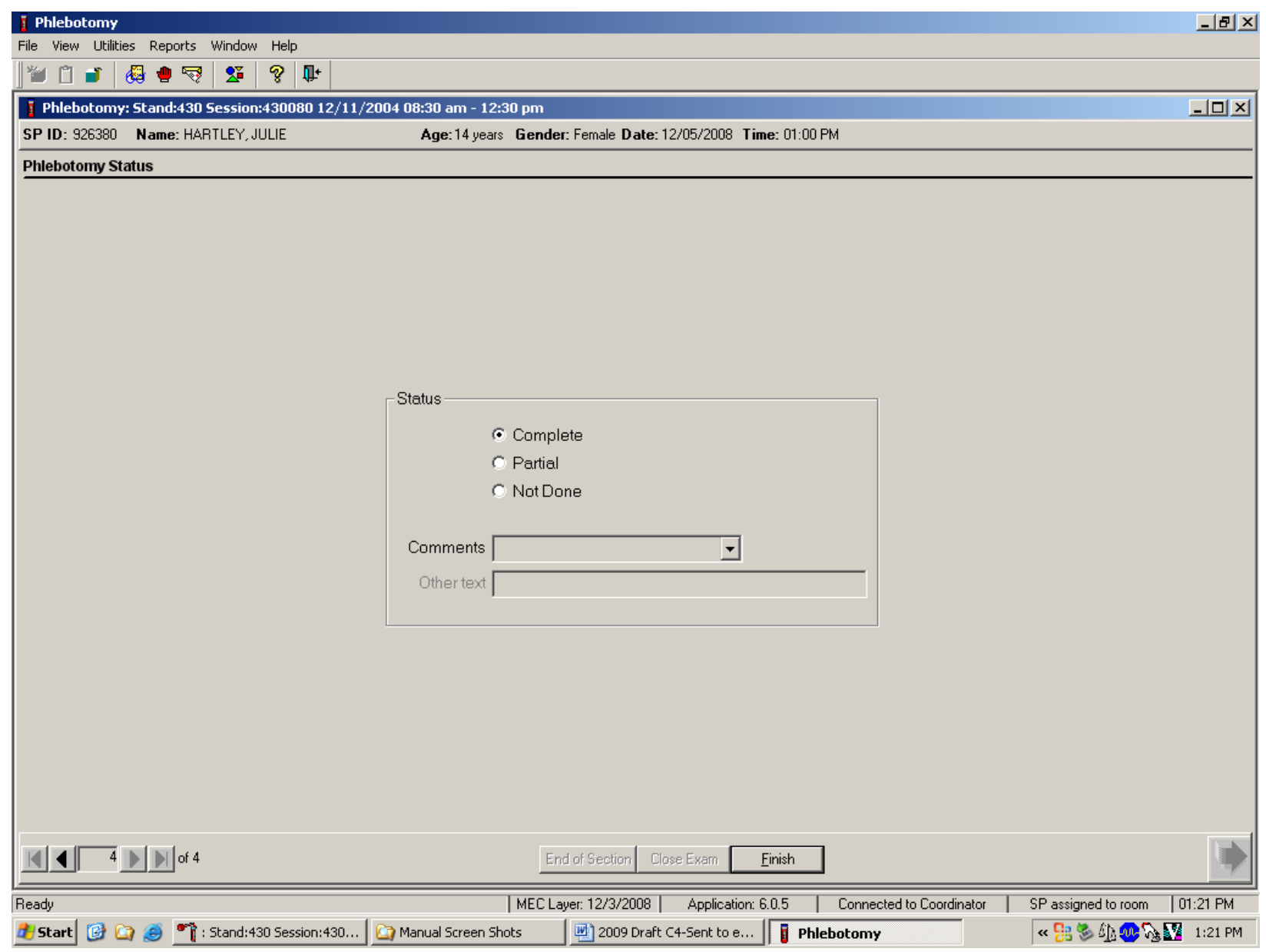

The venipuncture status is complete if all tubes were collected. 
Comment codes are used to explain Partial and Not Done status codes.

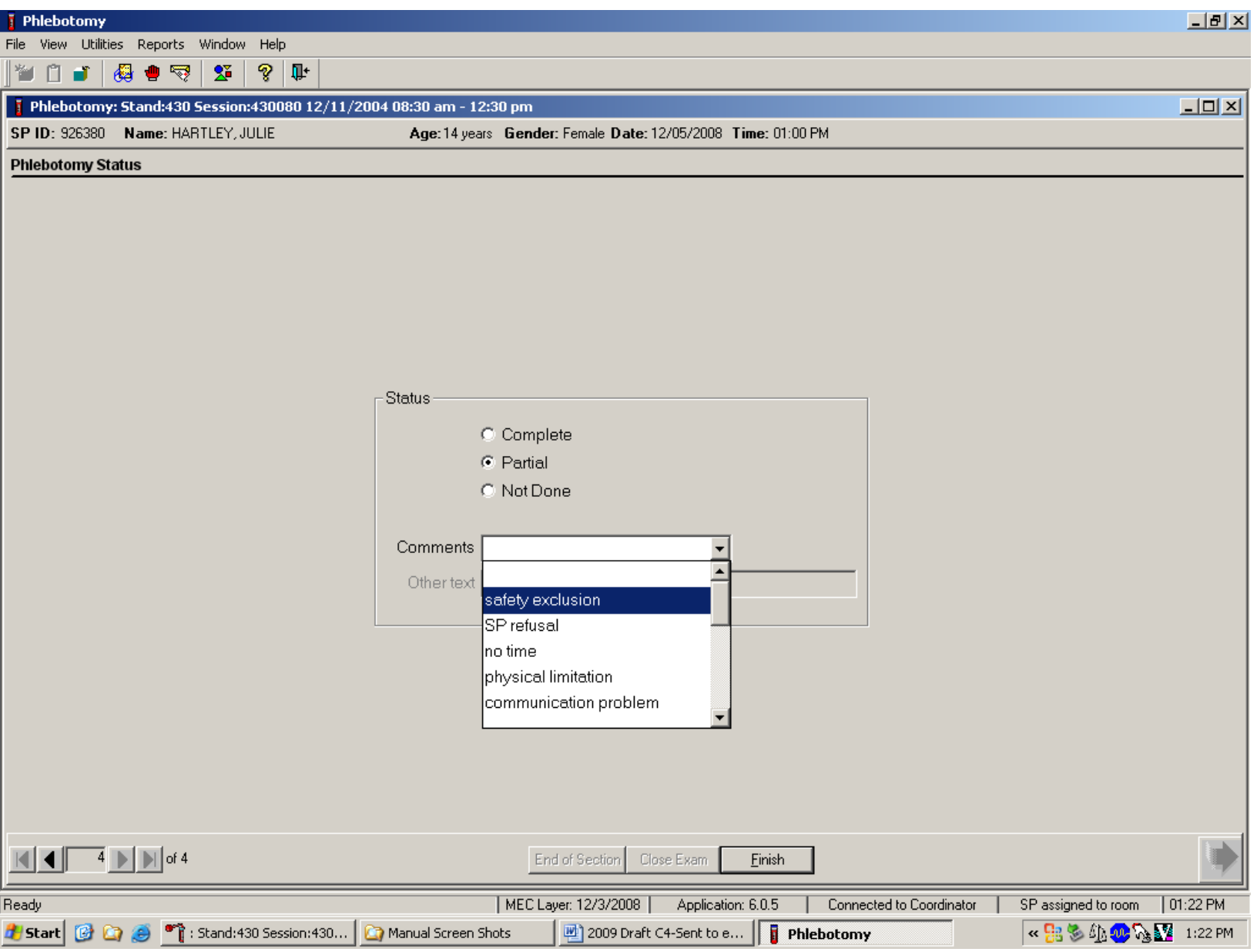

Choose and enter the appropriate comment code when the venipuncture section status is Partial or Not Done. To record a comment in the Comment text box, use the mouse to direct the mouse arrow to the scroll arrow on the drop-down list, left click, drag the mouse arrow to the desired choice, and left click. Use the scroll bar to view all choices. Alternatively, use the up and down keyboard arrows to scroll through the choices or type the first letter of the desired comment code. 


\begin{tabular}{|c|c|}
\hline Comment code & Use when: \\
\hline Safety exclusion & $\begin{array}{l}\text { Not applicable. } \\
\text { This is reserved for positive responses to the hemophilia and chemotherapy } \\
\text { exclusion questions and it is automatically coded by the application. } \\
\text { The coordinator may code exams using this comment. }\end{array}$ \\
\hline SP refusal & $\begin{array}{l}\text { The SP refuses to have his or her blood collected. This is an SP-initiated } \\
\text { nonresponse due to refusal. The SP refuses the component for any reason other } \\
\text { than an illness or emergency. If the SP refuses in the reception area, the } \\
\text { coordinator codes the exam. If the SP refuses after starting the exam, the } \\
\text { examiner codes the exam. Use this comment to code partial exams when the } \\
\text { SP refuses after one tube has been drawn, or the blood flow stops after one } \\
\text { tube but before all tubes have been successfully drawn. }\end{array}$ \\
\hline No time & Not applicable. \\
\hline Physical limitation & Not applicable. \\
\hline $\begin{array}{l}\text { Communication } \\
\text { problem }\end{array}$ & Not applicable. \\
\hline Equipment failure & Not applicable. \\
\hline SP ill/emergency & Not applicable. \\
\hline Interrupted & Not applicable. \\
\hline $\begin{array}{l}\text { Error } \\
\text { (technician/software/ } \\
\text { supply) }\end{array}$ & $\begin{array}{l}\text { Use this comment to code Partial and Not Done exams when there are } \\
\text { phlebotomist errors, or software or supply issues. }\end{array}$ \\
\hline Other, specify & $\begin{array}{l}\text { If the above reason for a status code of Not Done is not explained by one of the } \\
\text { comment codes, the examiner must choose Other, specify and record a } \\
\text { comment in the text field. }\end{array}$ \\
\hline No suitable vein & $\begin{array}{l}\text { Use this comment to code exams as Not Done (no tubes drawn) when the SP } \\
\text { does not have an accessible vein, any arms available, casts on both arms, there } \\
\text { is a rash over the entire area, or the blood draw was started but there was no } \\
\text { blood in the tubing. }\end{array}$ \\
\hline Vein collapsed & $\begin{array}{l}\text { Use this comment to code exams as Partial when one or more tubes are } \\
\text { successfully drawn but blood flow stops before all tubes are drawn or when the } \\
\text { vein collapses. }\end{array}$ \\
\hline SP not feeling well & $\begin{array}{l}\text { Use this comment to code Not Done or Partial when the SP is about to faint or } \\
\text { the SP became ill and the test could not be performed on the SP. }\end{array}$ \\
\hline Fainting episode & $\begin{array}{l}\text { Use this comment to code Not Done or Partial when the SP faints and the test } \\
\text { could not be performed on the SP. }\end{array}$ \\
\hline No tubes drawn & Use this comment to code exams as Partial when no tubes are drawn. \\
\hline Language barrier & Not applicable. \\
\hline
\end{tabular}

When finished, use the mouse to direct the mouse arrow to the bright blue arrow in the bottom right corner and left click to exit or select [Enter] when this blue arrow is highlighted. 
Escort the SP to the location indicated in the Message Center text box.

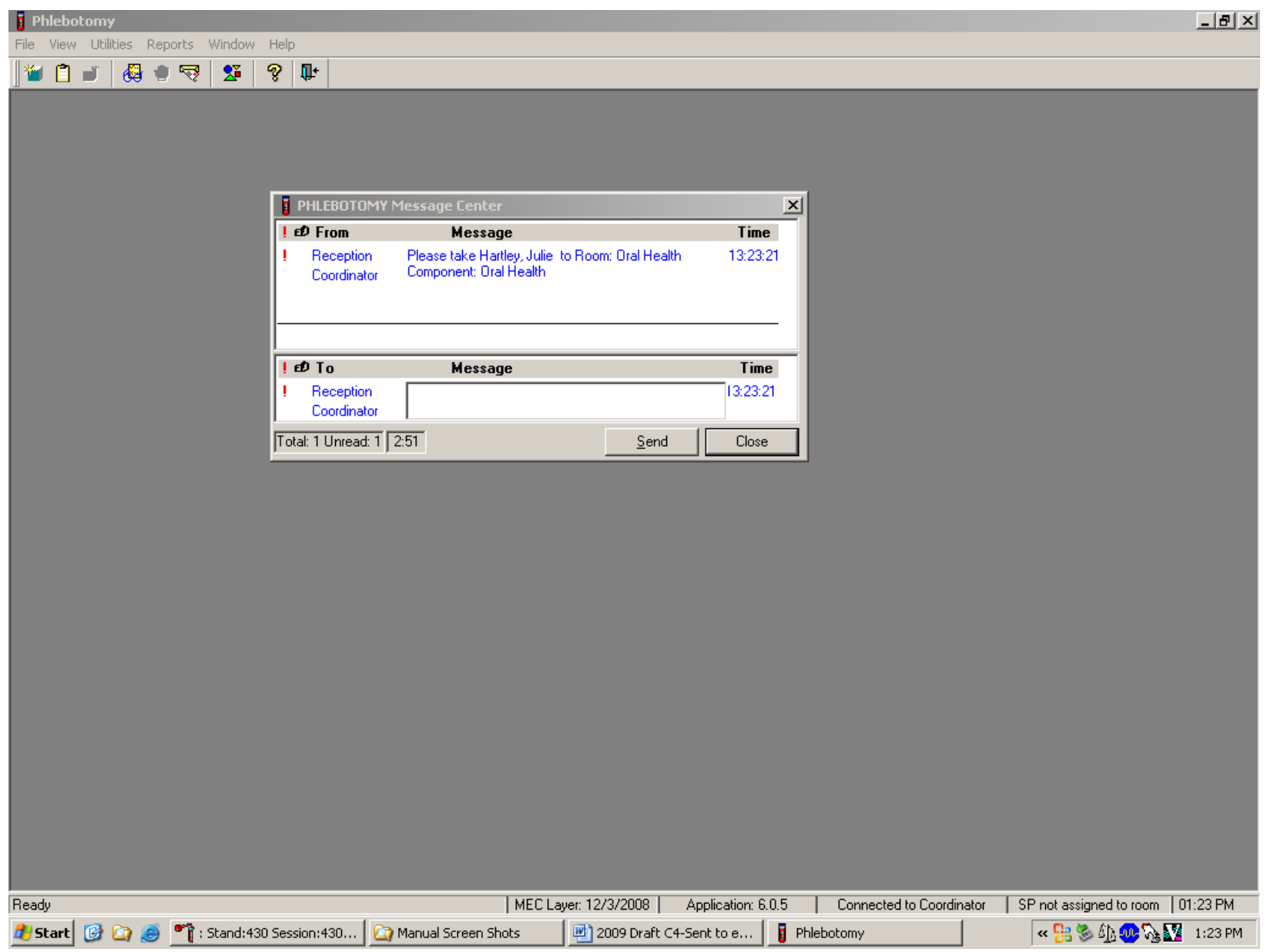

Review the instructions in the Message Center text box and follow the directions. 


\subsection{Repeat Blood Draw}

In rare circumstances a result from the complete blood count may be an extreme value that would be considered a "panic" value in clinical practice. An example is severe thrombocytopenia. The physician may repeat the complete blood count if the participant reports no history of nosebleeds or prolonged bleeding and the physician verifies that the site of the first venipuncture clotted normally. The second complete blood count will overwrite the first results in ISIS and will be used for reporting and referrals.

\section{Problem Identification}

Rare occurrences of critical CBC results, particularly low platelet counts, have been observed that resulted in an awkward medical referral for the physicians on the MEC. While the med techs and physician may suspect a laboratory instrument or blood collection effect in the reported results, it is not standard practice to repeat the test with a new blood specimen from a second phlebotomy. There is nothing in the phlebotomy, laboratory, and physician protocols that requires a second blood draw on an SP when critical results are obtained.

False low platelet results (low) are most likely the result of micro clots that cannot be observed. The techs do visually look for clots but they will not see micro clots. Many false low platelet results are caused by cold agglutinins. The techs are able to recognize cold agglutinins because the platelet number will decrease each time the blood is run. In this instance they warm the blood and rerun the sample.

The NHANES protocol is unusual because it requires the techs to run all samples in duplicate (whenever possible), whereas a clinical lab would only rerun a sample if the result was abnormal.

\section{Recommended Action}

If the physician feels a repeat blood count is needed, then he/she has the discretion to discuss this with the SP and have the phlebotomist draw a 3-mL EDTA tube for a repeat CBC if the SP agrees. 
Prior to a second phlebotomy, the physician should visually inspect the site of the first venipuncture to rule out prolonged bleeding. If the puncture site looks normal, then a second phlebotomy can be requested. The SP always has the right to refuse the blood draw. The need for this action should be rare as evidenced by the four total low platelet counts in the last 2 years.

After the physician reviews the findings with the SP, and the SP agrees to a repeat CBC phlebotomy:

- The physician should consult with the MEC manager and chief med tech to arrange to have the blood drawn at the next earliest opportunity.

- If the SP is eligible for the second GTT blood draw, we currently require the phlebotomist to draw any tubes that might not have been collected during the first blood draw. The second 3-mL EDTA tube could be drawn at that time but the phlebotomist will need to be alerted because the tube will not display on the GTT blood draw protocol screen.

- If the SP is not eligible for the GTT blood draw then he/she will need to be walked to phlebotomy to have the blood drawn.

- This additional blood draw will not be supported by the laboratory and phlebotomy applications.

- As long as the SP has not checked out of the MEC or if the Report of Findings has not been printed, the lab application will allow the med techs to overwrite the existing $\mathrm{CBC}$ data with the new data and the new data will become the final result of record.

- The physician will document this event under observations in the physician application. 
Perform phlebotomy quality control at the end of the second session even if there are no SPs scheduled into either session. Record the room temperature reading and the action that the counters were disinfected using the End of Session tab. Record the supply lot numbers and expiration dates by using the Supply Use tab.

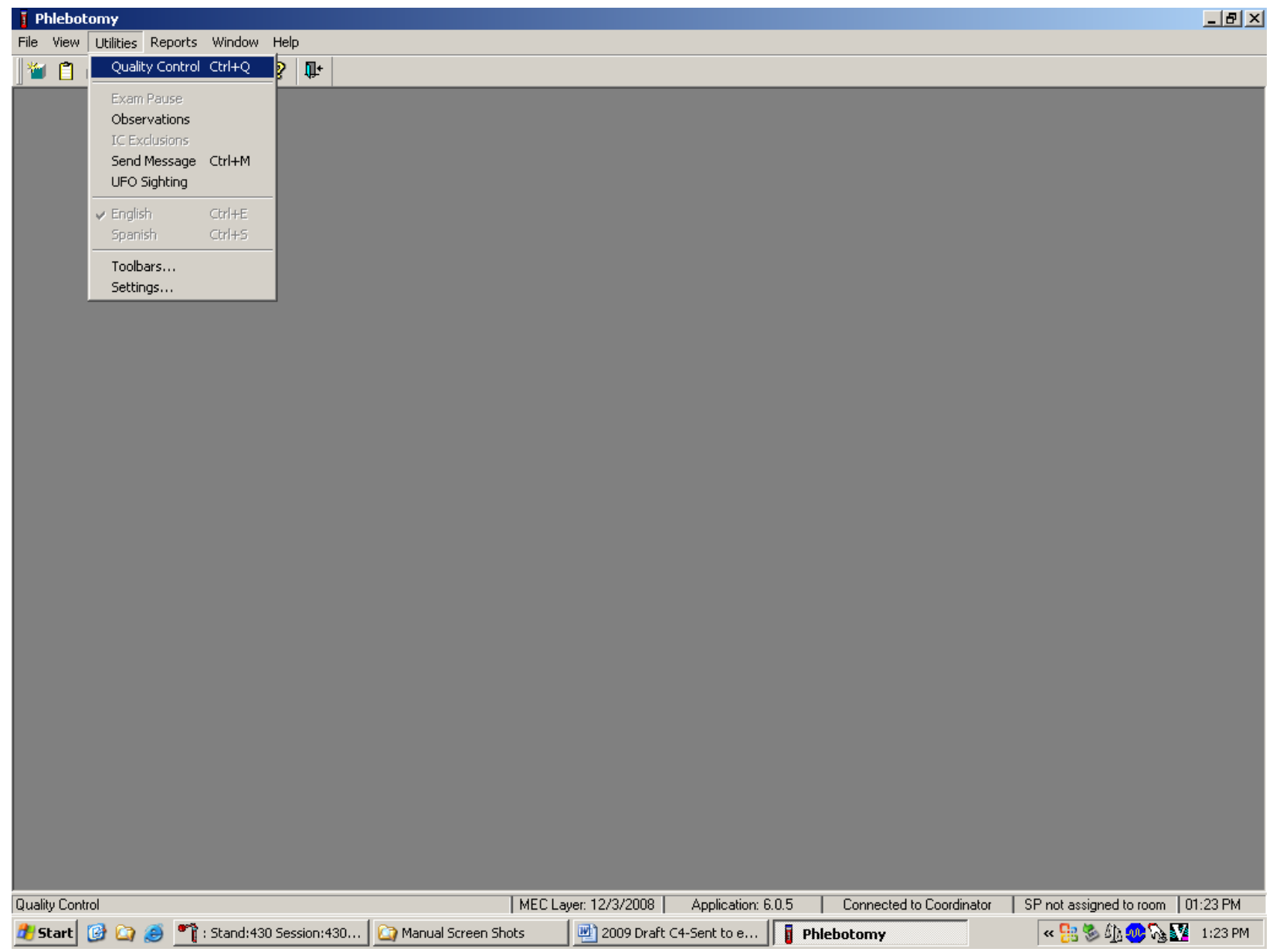

To access the phlebotomy Quality Control module, use the mouse to direct the mouse arrow to $\{$ Utilities $\}$ in the menu bar, left click, drag the arrow to \{Quality Control\} and left click, or select [Alt] $[\mathrm{U} / \mathrm{u}],[\mathrm{Q} / \mathrm{q}]$ or $[\mathrm{Ctrl}][\mathrm{Q} / \mathrm{q}]$. 


\subsubsection{Daily QC}

Record the daily quality control activities at the end of the second session. Mark each Done check box with a check mark.

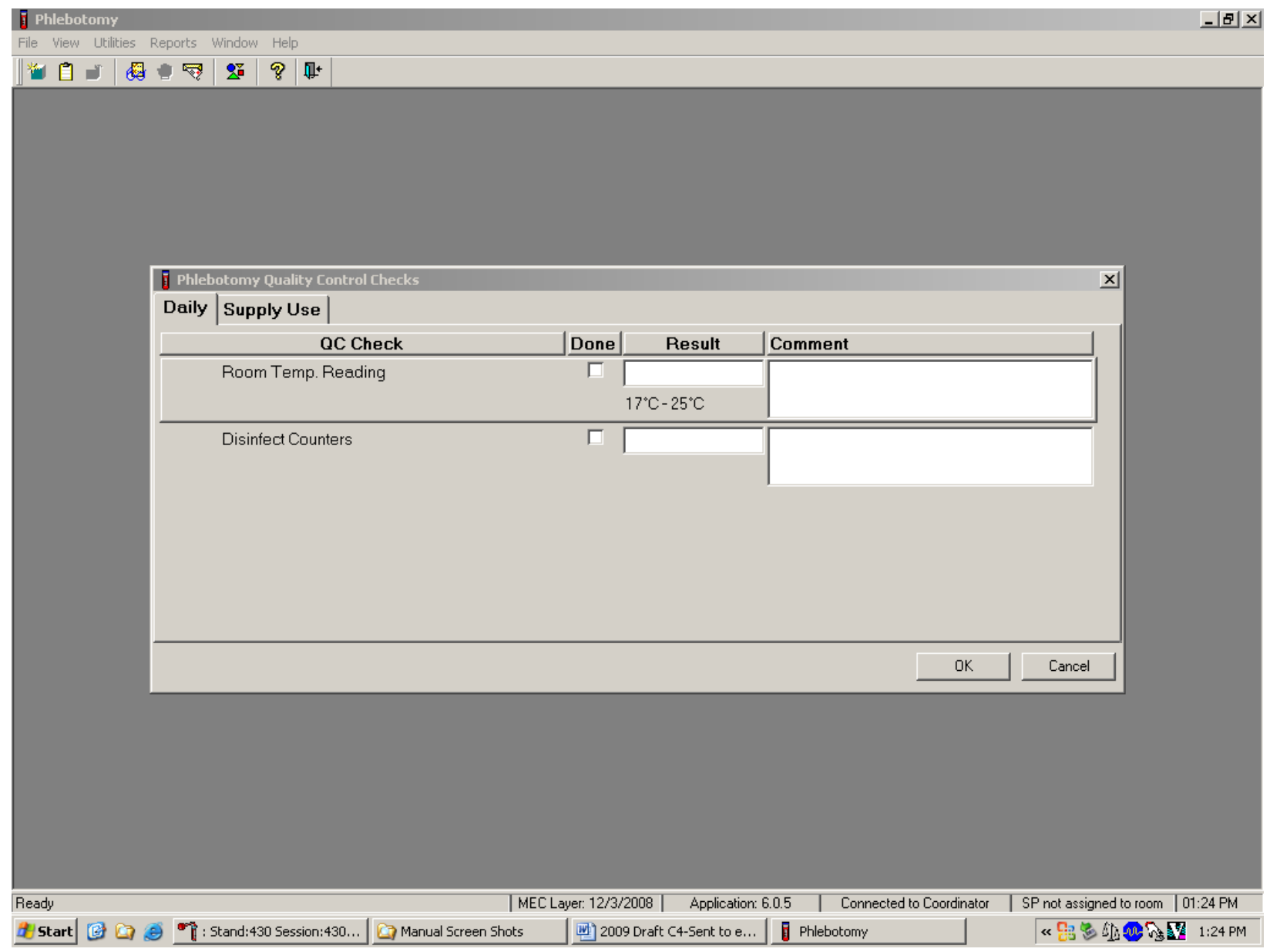

To record a check mark in the Room Temp. Reading check box, use the mouse to direct the mouse arrow to the check box in the Done column and left click, then select [Tab] to move to the Result text box. Enter the temperature in the Result text box and select [Tab] to move to the Comment text box. If the reading is outside the established range $\left(17-25^{\circ} \mathrm{C}\right)$, document the actions taken to resolve the situation in the Comment text box. Select [Tab] to move to the next QC item. 
Continue entering the QC.

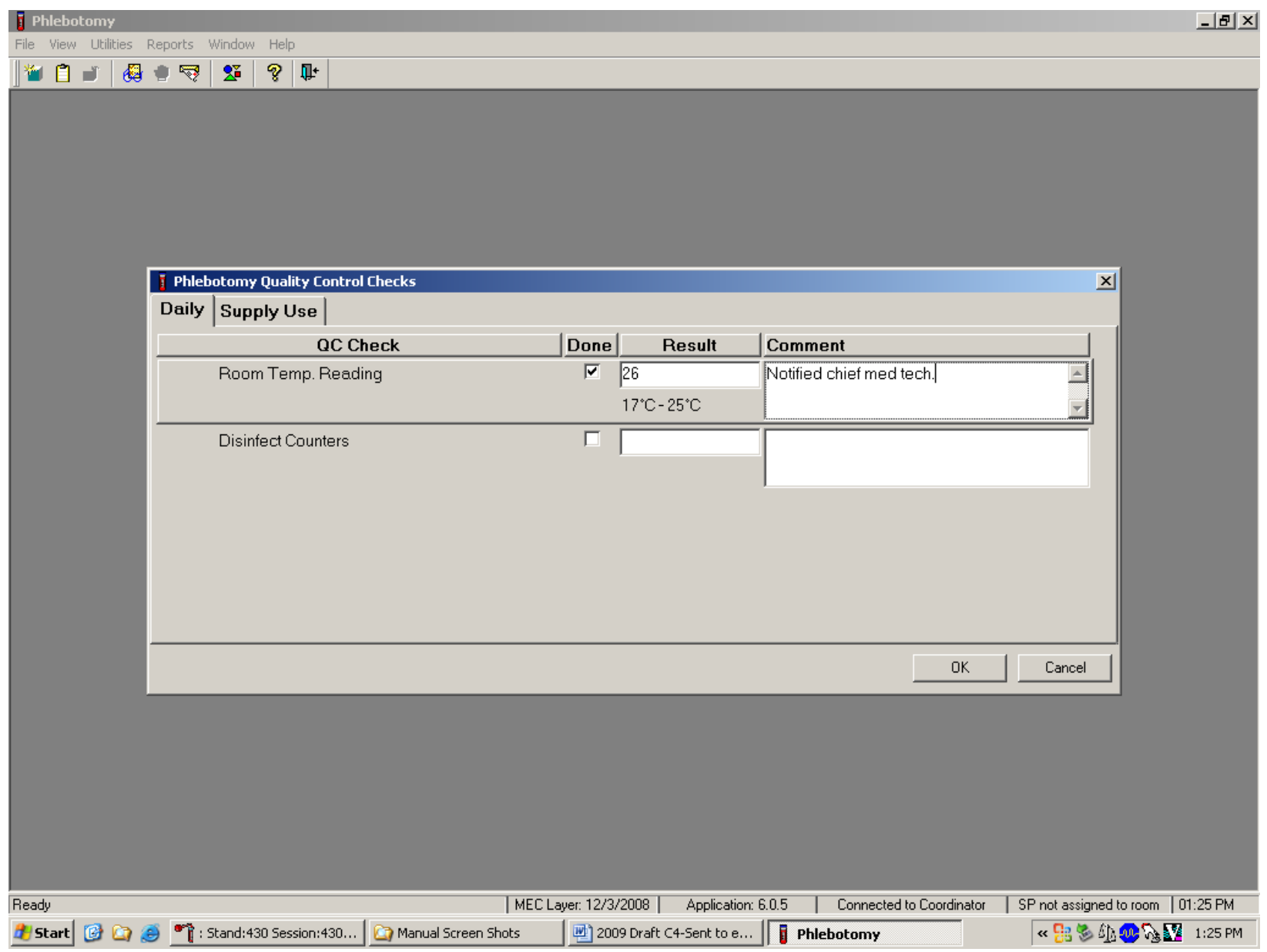

To record a check mark in the Disinfect Counters check box, use the mouse to direct the mouse arrow to the check box and left click, then select [Tab] to move to the Result box. Type "Yes" or "Not Done” in the Disinfect Counters Comment text box and then [Tab] to add free form text explaining why the counters were not disinfected for any "Not Done” response. 


\subsubsection{Supply Use Control Log}

Many items have specific lot numbers and expiration dates. Enter the lot numbers and expiration dates in the Supply Use module. This module lists every inventory item associated with the phlebotomy component that includes a lot number and expiration date.

\begin{tabular}{lcc}
\hline \multicolumn{1}{c}{ Item } & Lot Number & Expiration Date \\
\hline Butterfly Needle 19G Saf-T EZ Set & Yes & Yes \\
\hline Luer Adapter & Yes & No \\
\hline Vacutainer 21G Multi Sample Needle & No & Yes \\
\hline Vacutainer Safety-Lok 21G Needle & Yes & Yes \\
\hline Vacutainer Safety-Lok 23G Needle & Yes & Yes \\
\hline Band-Aid Liquid Bandage & Yes & Yes \\
\hline BD Vacutainer 3-mL EDTA Blood Tube & Yes & Yes \\
\hline BD Vacutainer 4-mL EDTA Blood Tube & Yes & Yes \\
\hline BD Vacutainer 2-mL Gray Blood Tube & Yes & Yes \\
\hline BD Vacutainer 3-mL Red Blood Tube & Yes & Yes \\
\hline BD Vacutainer 7-mL Red Blood Tube & Yes & Yes \\
\hline BD Vacutainer 10-mL Red Blood Tube & Yes & Yes \\
\hline Sherwood Kendall 15-mL Red Blood Tube & Yes & Yes \\
\hline BD Vacutainer 7-mL EDTA Blood Tube & Yes & Yes \\
\hline BD Vacutainer 10-mL EDTA Blood Tube & Yes & Yes \\
\hline VOC 10-mL Vacutainer Blood Tube & Yes & Yes \\
\hline Trutol Glucose Drink - Fruit Punch & Yes & Yes \\
\hline Trutol Glucose Drink - Lemon/Lime & Yes & Yes \\
\hline Trutol Glucose Drink - Orange & Yes & Yes \\
\hline Apple Juice & No & Yes \\
\hline Orange Juice & No & Yes \\
\hline Peanut Butter Crackers & No & Yes \\
\hline Solar Thermometer & No & Yes \\
\hline Ammonia Ampules & & \\
\hline
\end{tabular}


Use the Supply Use tab to enter lot numbers and expiration dates.

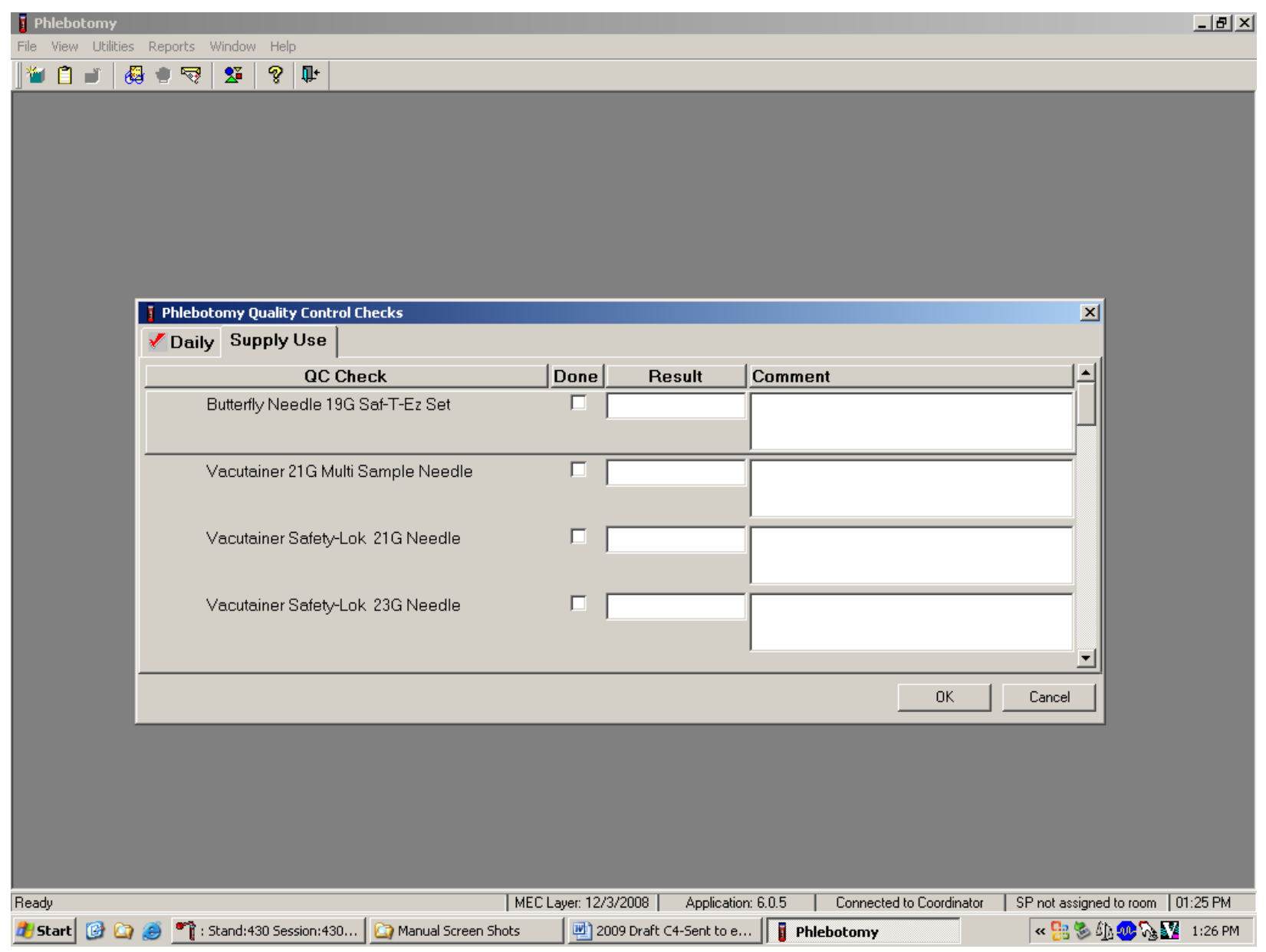

Select the correct activity (Supply Use) by using the mouse to direct the mouse arrow to the correct heading on the top of the file and left click. Mark each check box and record the lot and expiration date of each item. Perform this activity at the start of the stand and whenever a new lot is put into use. 
Mark activities as done and enter the lot number and expiration date in the Comment text box.

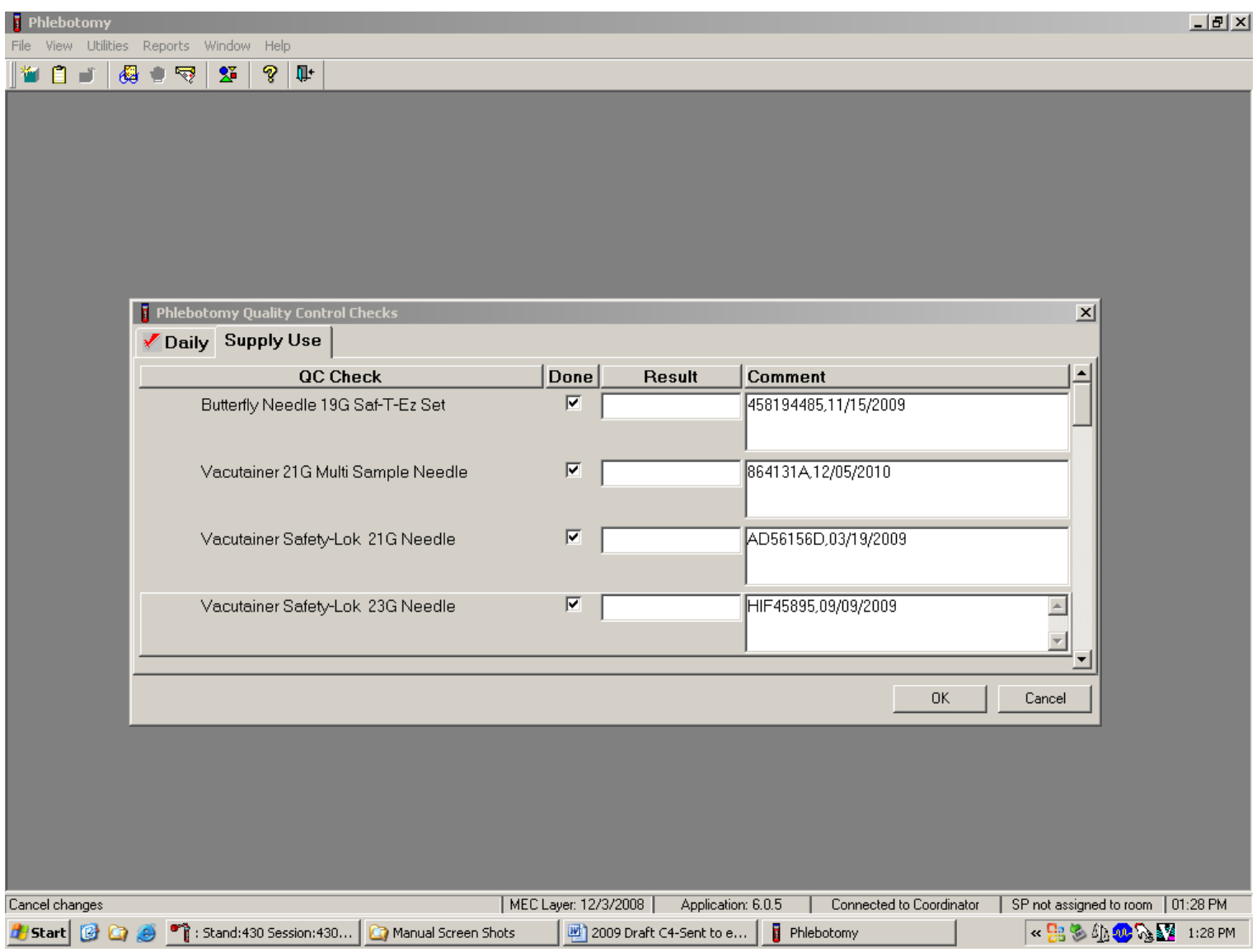

To record an activity as complete or done, use the mouse to direct the mouse arrow to the check box in the "Done" column and left click to insert a check mark. Select [Tab] or [Enter] to move to the Result text box. Do not enter the response or reading in the Result text box. Select [Tab] or [Enter] to move to the Comment text box.

\section{For each item:}

- Record the lot number (if the item does not have a lot number, then record "no lot";

- Add a comma;

- Add a space; and

- Record the expiration date using the MM/DD/YY format. 
There is no frequency requirement for this module within the QC application. Update the information each time a new lot is put into use.

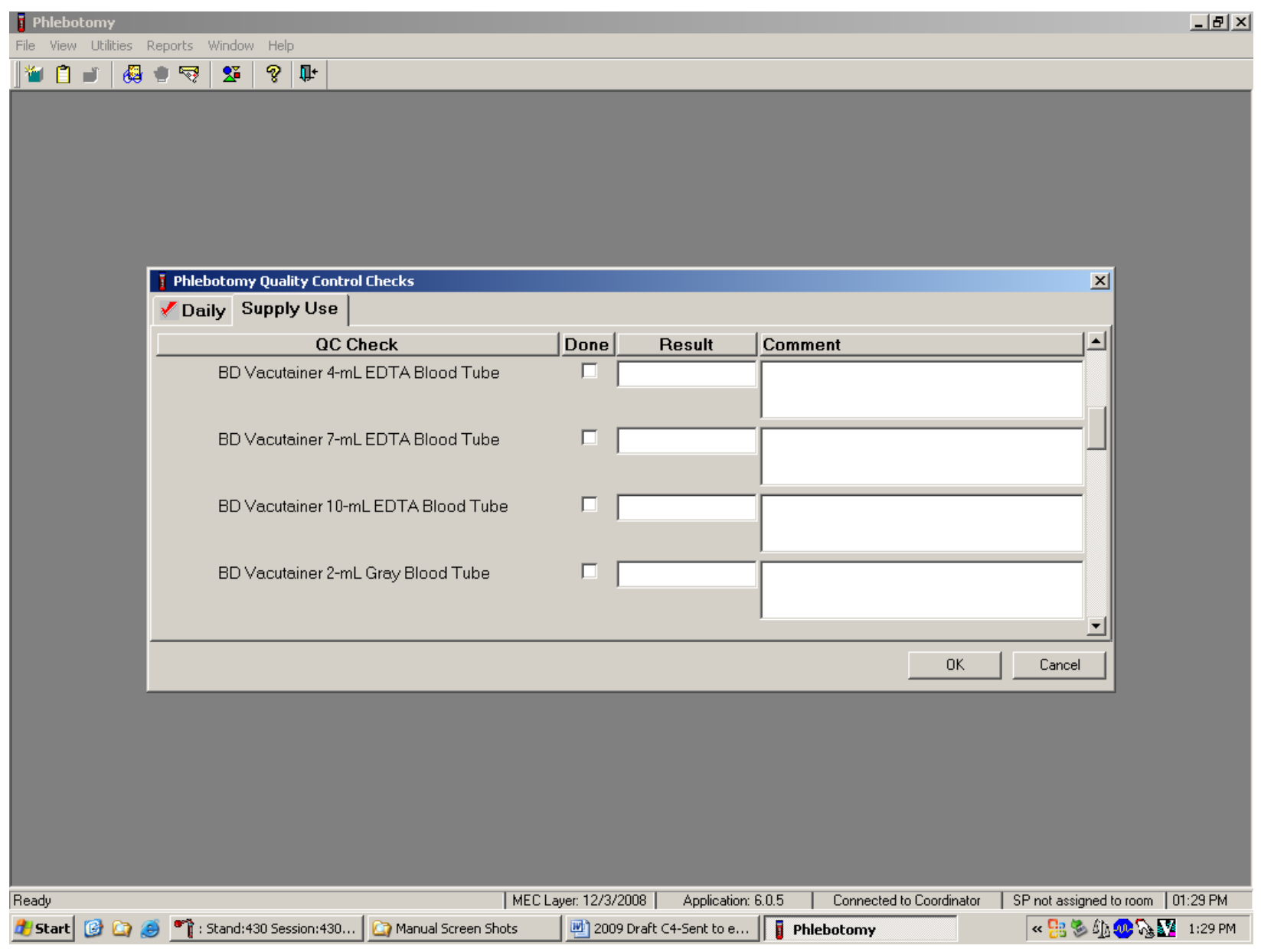

To save these results in the database, use the mouse to direct the mouse arrow to the "OK" button and left click, or select [Enter]. To exit the QC module without saving these actions in the database, use the mouse to direct the mouse arrow to the "Cancel” button and left click.

\subsection{Red Cross Procedures for Handling Fainting (Syncope)}

"Fainting” is a partial or complete loss of consciousness. This is due to a reduced supply of blood to the brain for a short time. Occasionally, a person collapses without warning. Recovery of consciousness usually occurs when the victim falls. Injury may occur from the fall. To prevent a fainting 
attack, a person who feels weak and dizzy should lie down or bend over with his or her head at the level of the knees.

- Signs and symptoms include:
1. Extreme paleness
2. Sweating
3. Coldness of the skin
4. Dizziness
5. Numbness and tingling of the hands and feet
6. Nausea
7. Possible disturbance of vision

If at any time during the venipuncture procedure the SP exhibits any of the manifestations listed above, conclude the venipuncture immediately and perform the first aid procedures listed below.

- Place the SP in a supine position.

- Loosen any tight clothing and keep crowds away.

Provide a disposable airsickness bag if the SP vomits and either roll the SP onto his or her side or turn the SP's head to the side.

- Maintain an open airway.

- Do not pour water over the SP's face because of the danger of aspiration; instead, provide a cool wet cloth to the forehead.

- Do not give any liquid unless the SP has revived.

- Examine the SP to determine if he or she has suffered injury from falling.

- Seek medical assistance. Do not leave the SP. Call for assistance or use the intercom to speak to the coordinator. Observe the SP carefully afterward because fainting might be a brief episode in the development of a serious underlying illness. ${ }^{1}$

\footnotetext{
${ }^{1}$ From Standard First Aid and Personal Safety, Second Edition, the American Red Cross, New York: Doubleday and Company, Inc., 1981, pp. 173-174.
} 


\subsection{How to Deal With System Failure}

If the computer system fails, record results on a preprinted Phlebotomy Worksheet (Exhibits 4-6 and 4-7). Complete a Phlebotomy worksheet for each SP while conducting the exam. Enter the results after the system is operational. 
Exhibit 4-6. Phlebotomy worksheet - English Primary

\section{PHLEBOTOMY INTERVIEW, FASTING INTERVIEW, and PROTOCOL PRIMARY SP}

SP ID

\begin{tabular}{|c|c|c|}
\hline Phlebotomy Interview & \multicolumn{2}{|c|}{$\begin{array}{l}\text { Responses } \\
\text { Yes, No, Refused, Don't Know }\end{array}$} \\
\hline \multicolumn{3}{|l|}{ Do you have hemophilia? } \\
\hline \multicolumn{3}{|l|}{$\begin{array}{l}\text { Have you received cancer chemotherapy in the past four } \\
\text { weeks? }\end{array}$} \\
\hline Fasting Questionnaire & \multicolumn{2}{|c|}{ Record time and date } \\
\hline \multicolumn{3}{|l|}{$\begin{array}{l}\text { Q1. When was the last time you ate or drank anything other } \\
\text { than plain water? Do not include diet soda or black coffee } \\
\text { or tea with artificial sweeteners like Sweet'N Low, } \\
\text { NutraSweet, Equal, or Splenda. }\end{array}$} \\
\hline $\begin{array}{l}\text { Confirmation Question: } \\
\text { Q2. Have you had any of the following since \{insert time from } \\
\text { Q1 here\}? }\end{array}$ & $\begin{array}{l}\text { Responses } \\
\underline{\text { Yes, No }}\end{array}$ & $\begin{array}{l}\text { If Yes, record time } \\
\text { and date }\end{array}$ \\
\hline \multicolumn{3}{|l|}{$\begin{array}{l}\text { Coffee or tea with cream or sugar? [Include milk or non- } \\
\text { dairy creamers.] }\end{array}$} \\
\hline \multicolumn{3}{|l|}{ Alcohol, such as beer, wine, or liquor? } \\
\hline \multicolumn{3}{|l|}{$\begin{array}{l}\text { Gum, breath mints, lozenges, or cough drops, or other } \\
\text { cough or cold remedies? }\end{array}$} \\
\hline \multicolumn{3}{|l|}{ Antacids, laxatives, or anti-diarrheals? } \\
\hline \multicolumn{3}{|l|}{$\begin{array}{l}\text { Dietary supplements such as vitamins and minerals? } \\
\text { [Include multivitamins and single nutrient supplements.] }\end{array}$} \\
\hline $\begin{array}{c}\text { Primary SP Protocol } \\
\end{array}$ & \# Filled & Not Filled \\
\hline \multicolumn{3}{|l|}{$\begin{array}{l}3 \text { mL lavender (1 age 1-2, } 2 \text { age 3-11, } 1 \text { age 6-11, } 2 \text { age } 12+, 1 \\
\text { age } 20+\text { ) }\end{array}$} \\
\hline \multicolumn{3}{|l|}{4 mL lavender (1 age 6-11, 1 age $12+, 2$ age $20+12+)$} \\
\hline \multicolumn{3}{|l|}{2 mL gray (morning only 1 age $12+$ ) } \\
\hline \multicolumn{3}{|l|}{$3 \mathrm{~mL}$ red (2 age $1-2,1$ age $6-11)$} \\
\hline \multicolumn{3}{|l|}{$7 \mathrm{~mL}$ red (2 age $3-5)$} \\
\hline \multicolumn{3}{|l|}{$15 \mathrm{~mL}$ red $(3$ age $12+, 3$ age $20+)$} \\
\hline \multicolumn{3}{|l|}{$\begin{array}{l}10 \mathrm{~mL} \text { red ( } 2 \text { age } 6-11,2 \text { age } 12+, 3 \text { age } 20+\text { ) } \\
\text { Plus ( } 2 \text { age } 6+\text { if eligible for Persistent Pesticides or BFR } \\
\text { subsamples) }\end{array}$} \\
\hline \multicolumn{3}{|l|}{$\begin{array}{l}10 \mathrm{~mL} \text { red ( } 1 \text { age } 12+\text { if eligible for Perfluoronated and Srm } \\
\text { Pesticide subsamples) }\end{array}$} \\
\hline \multicolumn{3}{|l|}{$\begin{array}{l}7 \text { mL EDTA (1 age 12+ if eligible for Perfluoronated and Srm } \\
\text { Pesticide subsamples) }\end{array}$} \\
\hline \multicolumn{3}{|l|}{10 mL EDTA (2 age 20+ if not IC excluded) } \\
\hline $10 \mathrm{ml}$ gray (1 age $12+$ if eligible for VOC subsample) & & \\
\hline \multicolumn{3}{|l|}{ Comments: } \\
\hline
\end{tabular}

${ }^{2}$ Genetics tubes; must meet IC Exclusion criteria 
Exhibit 4-7. Phlebotomy worksheet - Spanish Primary

\section{PHLEBOTOMY INTERVIEW, FASTING INTERVIEW, and PROTOCOL}

PRIMARY SP

SP ID

\begin{tabular}{|c|c|c|}
\hline Phlebotomy Interview & \multicolumn{2}{|c|}{$\begin{array}{l}\text { Responses } \\
\text { Yes, No, Refused, Don't Know }\end{array}$} \\
\hline \multicolumn{3}{|l|}{ ¿Tiene hemofilia? } \\
\hline \multicolumn{3}{|l|}{$\begin{array}{l}\text { ¿Ha recibido algún tratamiento para el cáncer en las cuatro } \\
\text { semanas pasadas?? }\end{array}$} \\
\hline Fasting Questionnaire & \multicolumn{2}{|c|}{ Record time and date } \\
\hline \multicolumn{3}{|l|}{$\begin{array}{l}\text { Q1. ¿Cuándo fue la última vez que comió o tomó alguna cosa } \\
\text { aparte de agua sola? No incluya soda de dieta o café o té } \\
\text { solo endulzado con endulzadores artificiales como } \\
\text { Sweet'N Low, NutraSweet, Equal o Splenda. }\end{array}$} \\
\hline $\begin{array}{l}\text { Confirmation Question: } \\
\text { Q2. ¿Ha tomado o comido algo de lo siguiente desde ayer a las } \\
\quad \text { insert time from Q1 here }\} \text { ? }\end{array}$ & $\begin{array}{l}\text { Responses } \\
\text { Yes, № }\end{array}$ & $\begin{array}{l}\text { If Yes, record time } \\
\text { and date }\end{array}$ \\
\hline \multicolumn{3}{|l|}{$\begin{array}{l}\text { ¿Café o té con crema o azúcar? } \\
\text { [Incluya leche o cremas que no sean productos lácteos.] }\end{array}$} \\
\hline \multicolumn{3}{|l|}{ ¿Alcohol, tal como cerveza, vino o licor? } \\
\hline \multicolumn{3}{|l|}{$\begin{array}{l}\text { ¿Chicle, mentas para el aliento, tabletas o pastillas para la } \\
\text { tos, u otra medicina para la tos o el resfriado? }\end{array}$} \\
\hline \multicolumn{3}{|l|}{ ¿Antiácidos, laxantes, o antidiarréicos? } \\
\hline \multicolumn{3}{|l|}{$\begin{array}{l}\text { ¿Suplementos para la dieta tales como vitaminas y } \\
\text { minerales? [Incluya multivitaminas y suplementos } \\
\text { nutritivos individuales.] }\end{array}$} \\
\hline Primary SP Protocol & \# Filled & Not Filled \\
\hline \multicolumn{3}{|l|}{$\begin{array}{l}3 \text { mL lavender ( } 1 \text { age } 1-2,2 \text { age } 3-11,1 \text { age } 6-11,2 \text { age } 12+, 1 \\
\text { age } 20+\text { ) }\end{array}$} \\
\hline \multicolumn{3}{|l|}{4 mL lavender (1 age 6-11, 1 age $12+, 2$ age $20+12+$ ) } \\
\hline \multicolumn{3}{|l|}{$2 \mathrm{~mL}$ gray (morning only 1 age $12+$ ) } \\
\hline \multicolumn{3}{|l|}{$3 \mathrm{~mL}$ red (2 age $1-2,1$ age $6-11)$} \\
\hline \multicolumn{3}{|l|}{$7 \mathrm{~mL}$ red $(2$ age $3-5)$} \\
\hline \multicolumn{3}{|l|}{$15 \mathrm{~mL}$ red $(3$ age $12+, 3$ age $20+)$} \\
\hline \multicolumn{3}{|l|}{$\begin{array}{l}10 \mathrm{~mL} \text { red ( } 2 \text { age } 6-11,2 \text { age } 12+, 3 \text { age } 20+) \\
\text { Plus ( } 2 \text { age } 6+\text { if eligible for Persistent Pesticides or BFR } \\
\text { subsamples) }\end{array}$} \\
\hline \multicolumn{3}{|l|}{$\begin{array}{l}10 \mathrm{~mL} \text { red ( } 1 \text { age } 12+\text { if eligible for Perfluoronated and Srm } \\
\text { Pesticide subsamples) }\end{array}$} \\
\hline \multicolumn{3}{|l|}{$\begin{array}{l}7 \text { mL EDTA (1 age 12+ if eligible for Perfluoronated and Srm } \\
\text { Pesticide subsamples) }\end{array}$} \\
\hline \multicolumn{3}{|l|}{10 mL EDTA (2 age 20+ if not IC excluded) } \\
\hline $10 \mathrm{ml}$ gray (1 age $12+$ if eligible for VOC subsample) & & \\
\hline Comments: & & \\
\hline
\end{tabular}


The following four screenshots document the English text and official Spanish translations for the phlebotomy component:

- Phlebotomy Interview English,

- Phlebotomy Interview Spanish,

- Fasting Questionnaire English, and

- Fasting Questionnaire Spanish. 
Phlebotomy Interview English

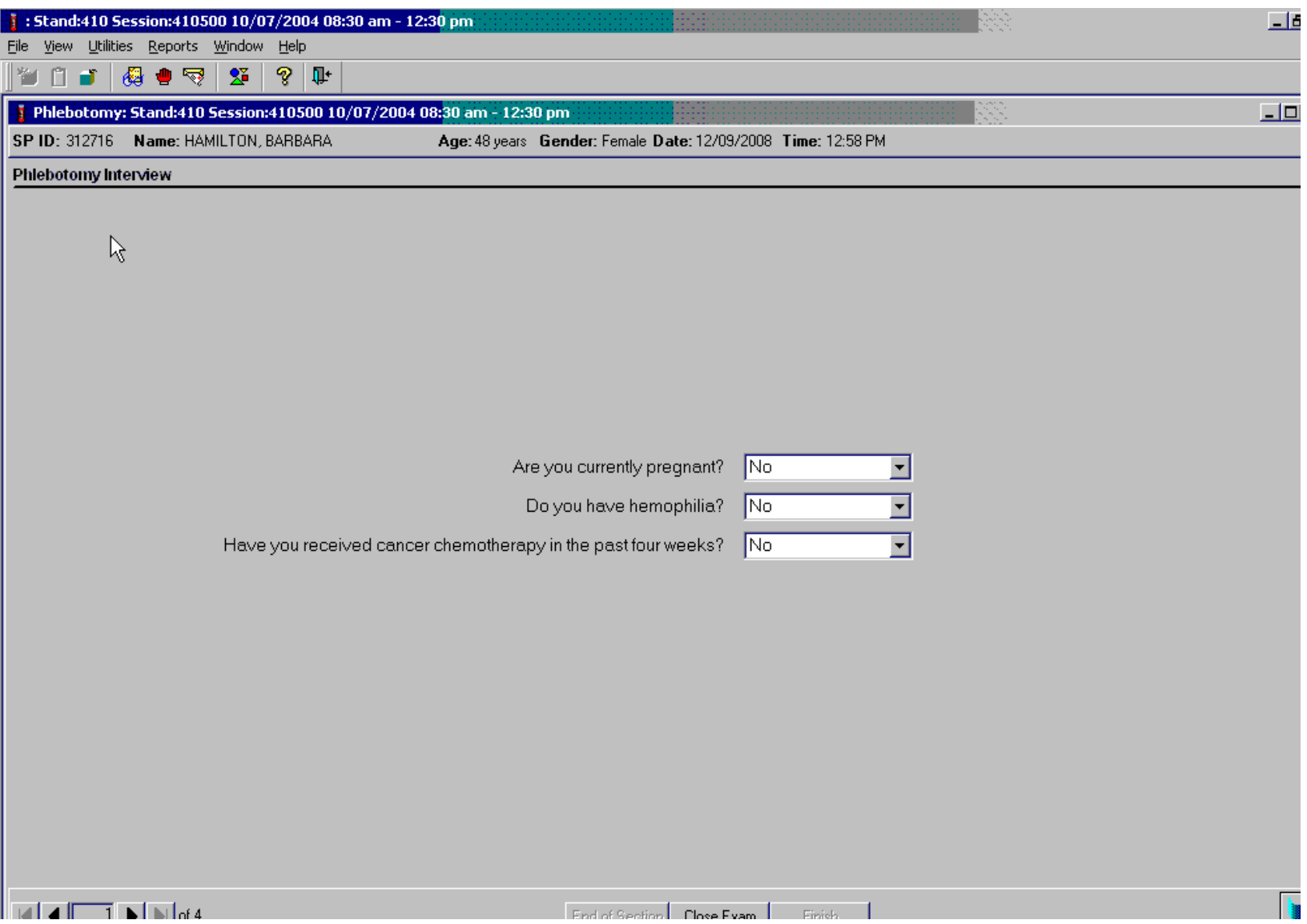


Phlebotomy Interview Spanish

: Stand:410 Session:410500 10/07/2004 08:30 am - 12:30 pm

$-15$

File View untilities Reports Window Help

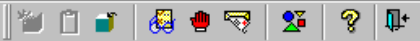

Phlebotomy: Stand:410 Session:410500 10/07/2004 08:30 am-12:30 pm

Age: 48 years Gender: Female Date: 12/09/2008 Time: 12:58 PM

SP ID: 312716 Name: HAMILTON, BARBARA

Phlebotomy Interview

4

$\begin{aligned}$\[ \text { ¿Está usted actualmente embarazada? } \]$ & \text { No } \\ \text { ¿Tiene hemofilia? } & \text { No recibido tratamiento de quimioterapia para el cáncer en las } \\ \text { cuatro semanas pasadas? } & \text { No }\end{aligned}$

cuatro semanas pasadas?

$4 \sqrt{1}+1 \times 1+4$

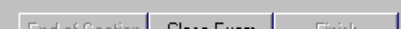




\section{Fasting Questionnaire English}

\section{Phlebotomy}

File View untilities Reports Window Help

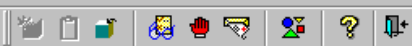

Thlebotomy: Stand:410 Session:410500 10/07/2004 08:30 am -12:30 pm

SP ID: 306325 Name: KAIGA, NEWTON

Age: 13 years Gender: Ma

Date: 12/09/2008 Time: 12:54 PM

Fasting Questionnaire

When was the last time you ate or drank anything other than plain water? Do not $09: 30$ PM 12/08/2008

NutraSweet. Equal, or Splenda

Have you had any of the following since yesterday at 9:30 PM?

$$
\text { Coffee or tea with cream or sugar? [Include milk or non-dairy } \begin{gathered}
\text { creamers.] } \\
\text { No }
\end{gathered}
$$

Alcohol, such as beer, wine, or liquor? No

Gum, breath mints, lozenges or cough drops, or other cough or cold $\begin{gathered}\text { Nomedies? } \\ \text { reme }\end{gathered}$

Antacids, laxatives, or anti-diarrheals? No $\quad$ N

Dietary supplements such as vitamins and minerals? [Include No N multivitamins and single nutrient supplements.]
Fasting
15 Hours
26 Minutes
4 


\section{Fasting Questionnaire Spanish}

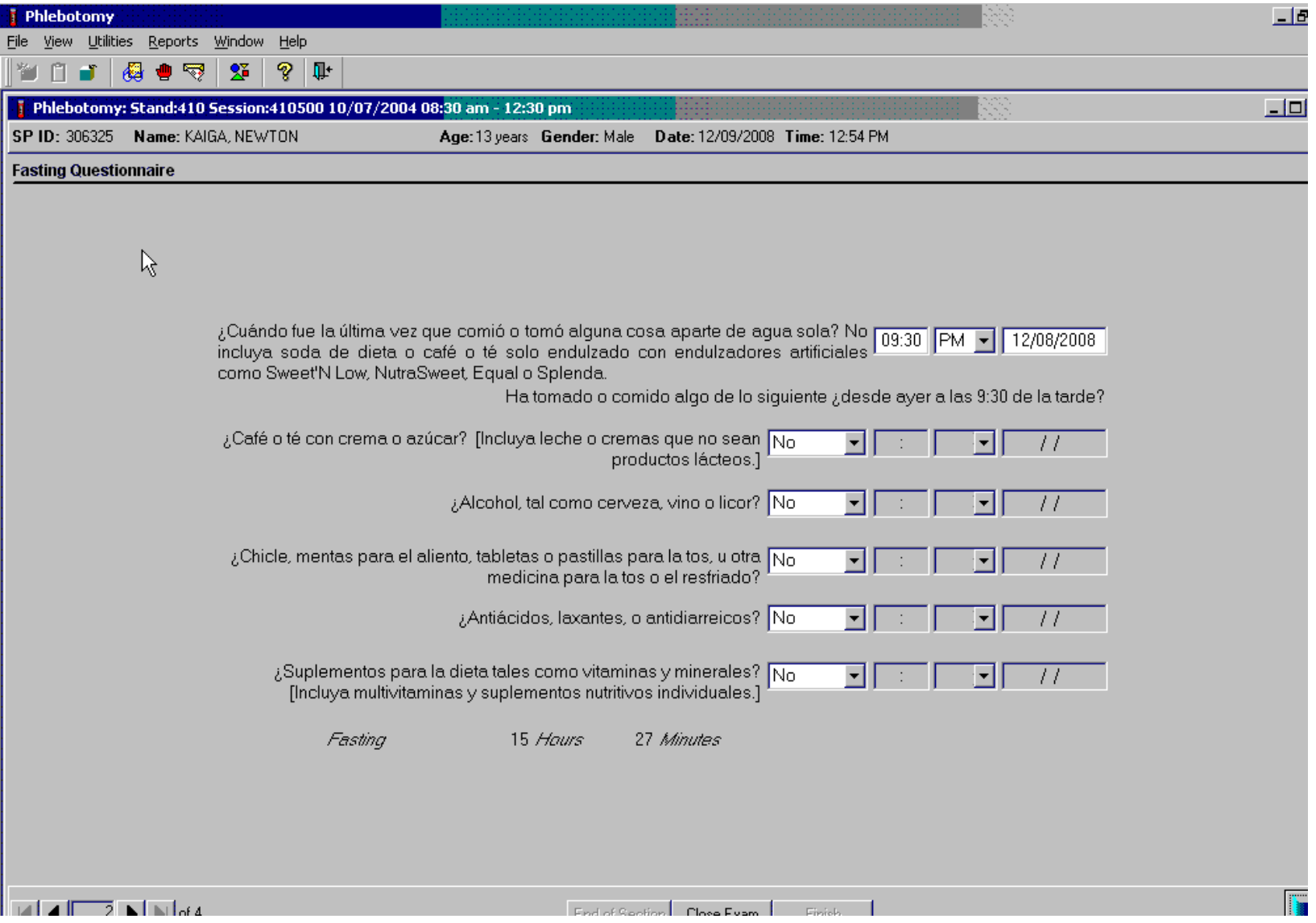




\section{URINE SPECIMEN COLLECTION AND PROCESSING}

\subsection{Introduction}

The purpose of urine collection and processing is to collect sufficient urine from participants aged 6 years and older to be able to (1) collect a date, time, and volume of urine collection for analysis of urine flow rate; (2) perform a pregnancy test on selected females aged 8 to 17 years and all females aged 18 to 59 years to exclude pregnant participants from other components that could put the participant at risk; (3) perform a test for osmolality; (4) allocate urine into vessels for storage and transport to multiple government and contact laboratories for analysis; and (5) allocate urine into a vessel for future studies.

Collect a urine specimen from all SPs who are 6 years of age and older. The volume of urine required to complete each SP's protocol is displayed in the urine collection module. Place the urine container on the Mettler Toledo scale and then scan the label on the bar code. The weight is converted to volume and transferred to the urine collection module. Visually compare the volume in the urine container to the volume displayed in the urine collection screen. Accept or correct the weight. Perform the pregnancy test if indicated, perform the osmolality test, process the urine protocol, and store the vessels. At least 5-mL of urine is required on VIP guest and guest appointment types.

The MEC coordinator instructs each SP aged 6 years and older to provide a urine sample as soon as possible when he or she enters the MEC. Ideally, this sample would be collected at the same time that the SP changes into the exam outfit. If the SP cannot provide a urine sample at that time, the coordinator offers the SP a glass of water and asks the SP to provide a sample as soon as possible. The SP is instructed to collect a complete void by emptying the bladder into the urine collection cup. The coordinator, assistant coordinator, or any MEC staff member is responsible for transporting the urine specimen to the laboratory.

The coordinator explains the following instructions to the subject before urine collection:

- It is important to collect ALL urine by COMPLETELY emptying your bladder into the cup.

- Wash hands with soap and water.

- Take the cap off the cup.

- Urinate directly into the cup until it is full or until you have finished urinating. 
- DO NOT OVERFILL THE CUP.

- The inside of the cup and cap should not touch or come into contact with any part of your body, clothing, or external surfaces.

- Recap the collection cup and seal tightly.

- Please remember to wash your hands.

\section{$5.2 \quad$ Supplies}

The supplies needed for urine collection and urine processing and storage are listed in Exhibits 5-1 and 5-2.

Exhibit 5-1. Urine collection equipment

\begin{tabular}{|c|}
\hline Blue Scale Carrying Case \\
\hline Mettler Toledo PL601-S Scale \\
Cone protector \\
Weigh pan \\
One Troemner 100g weight (start of stand QC) \\
Two Troemner 300g weights (start of stand QC) \\
Care Pac M \\
\hline Cleaning cloth \\
Two gloves \\
Weight transfer tool for 500g weight \\
Weight transfer forceps for 10g weight \\
Cleaning brush \\
One Ohaus 500g weight (daily QC) \\
One Ohaus 10g weight (daily QC) \\
\hline Clear Acrylic Scale Cover \\
\hline Bar Code Scanner \\
\hline
\end{tabular}


Exhibit 5-2. Urine collection supplies - urine processing and storage

\begin{tabular}{|l|l|}
\hline 5-mL cryovial sterile & 14-mL Falcon snap cap test tube \\
\hline 2-mL cryovial & Cube rack - 4 way flipper rack \\
\hline $\begin{array}{l}\text { 10-mL VWR screwcap vial with colored dot (Urn } \\
\text { Merc) }\end{array}$ & 10 -mL VWR screwcap vial \\
\hline Bench Kote paper & Kimwipes \\
\hline $10 x 10$ three inch box & $\begin{array}{l}5.25 \times 5.25 \times 3.0 \text { inch cardboard box with } 9 \times 9 \\
\text { cardboard grid }\end{array}$ \\
\hline 5.25 x 5.25 x 3.0 inch cardboard box without grid & Three inch 5 x 5 cardboard grid \\
\hline 3.5 mL Starstedt Transfer pipette & $\begin{array}{l}\text { VWR 15mL narrow stem, large bulb disposable } \\
\text { transfer pipette }\end{array}$ \\
\hline $\begin{array}{l}\text { Nonsterile, powder-free, gloves-small, medium, } \\
\text { large }\end{array}$ & Windex for scale cleaning \\
\hline
\end{tabular}

\subsection{Urine Flow Rate Background}

The NHANES protocol includes many measures of environmental toxicants or their metabolites in urine. It is difficult to quantify the level of exposure based on measurements in urine because concentrations will vary by the amount of urine produced. Currently, NHANES urine measurements are adjusted by the urinary creatinine concentration to account for variations in urinary dilution. However, creatinine excretion into the urine varies by many factors other than the urinary dilution (e.g., age, gender, muscle mass, and diet). Also, the amount of toxicant excreted in a 24-hour period is often needed to quantify exposures for risk and exposure assessment purposes. Ideally, this requires 24-hour urine collections but this is impractical in NHANES for logistical reasons. However, it is possible to estimate the mass of analyte excreted in the urine in a 24-hour period by estimating the urine flow for the spot urine specimen collection (i.e., equivalent to a single urine specimen as collected in the current NHANES protocol).

The date and time of last urine void, along with the volume of the urine specimen and the date and time of urine collection captured during urine collection will provide NCHS with a measurement of urine flow rate. These items are captured during the SP's visit to the MEC. All SPs aged 6+ will be eligible for urine flow rate. Urine specimen volume and date and time of collection will be captured only on SPs who submit a urine specimen.

The MEC appointment reminder letter will include the reminder "Before arriving at the exam center, please make a note of the time you last went to the bathroom and urinated (peed). Please 
write the time on the appointment card you were given. You will be asked for this time when you get to the exam center," and will provide a space for the SP to write a response. When the SP arrives at the MEC, the coordinator will prompt the SP to write down the time of his or her last urine void on the verification form. The coordinator will record this time. If there is any discrepancy between the time on the reminder letter and the time reported at the MEC, the coordinator should use the time reported at the MEC.

\subsection{Using the Scale for Urine Collection}

The Mettler Toledo scale is located in the clean laboratory area near workstation 2. Check to make sure the scale's weighing pan is free of any objects or debris. Turn on the Mettler Toledo scale by pressing the "On” button. After the display briefly lights up all segments, the LED will display "0.0 g." This indicates that the scale is ready to weigh samples. The scale should be used only when set to measure in grams. Cover the scale with the clear acrylic scale cover until a sample is available for weighing.

Ensure that computer workstation 2 (Urine/Sink) is turned on and a technician is logged into the Laboratory application. Open the Urine Scale application by double clicking on the Urine Scale icon on the desktop. The Urine Scale icon looks like this:

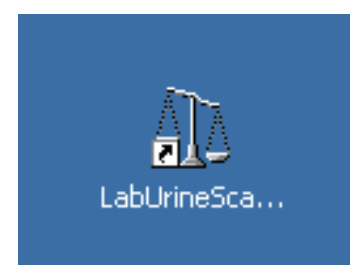

The Urine Scale application icon will appear in the system tray, similar to the Coulter application. This application captures data from the scale and logs it into the database. The Urine Scale application must be opened and in the system tray in order to capture any urine collection readings from the scale.

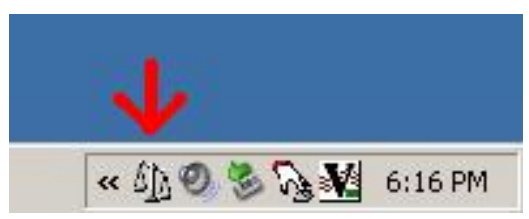


Place the urine collection cup on the Mettler Toledo scale. Cover the scale with the clear box cover to protect the scale from draft. Look at the scale's LED display. In the bottom left corner of the display the stability detector "o" will appear briefly. Once the stability indicator goes away, the weight result is valid. Scan the bar code on the cup using the bar-code scanner next to the scale.

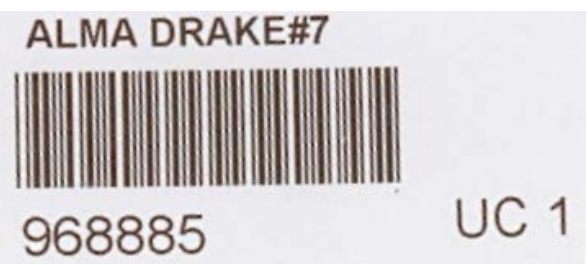

Look at the scale's LED screen to ensure that the SP ID displays. Checking for the SP ID display on the LED screen is verification that the collection was recorded in the database.

Once the urine cup has been successfully scanned, move it to workstation 2. 


\section{5}

\section{Document Urine Collection}

Once a urine sample has been scanned in, the UC circle on the heads-up screen for that SP will turn blue ( $\odot$ for QNS or $\bigcirc$ for Yes). The blue color indicates that there is a urine sample in the lab ready to be validated by the laboratory technician.

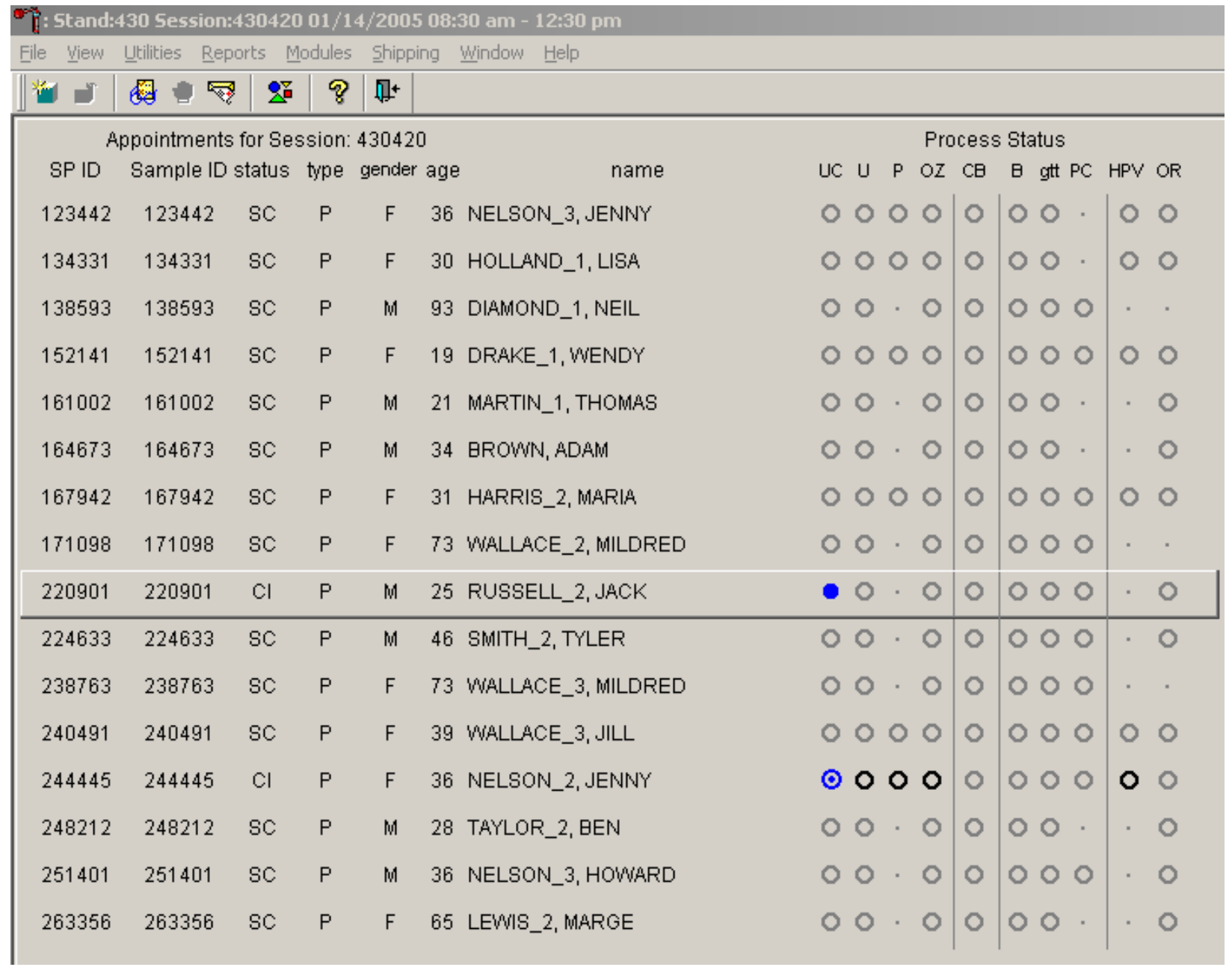


Select an SP with a blue UC circle and access the Urine Collection module.

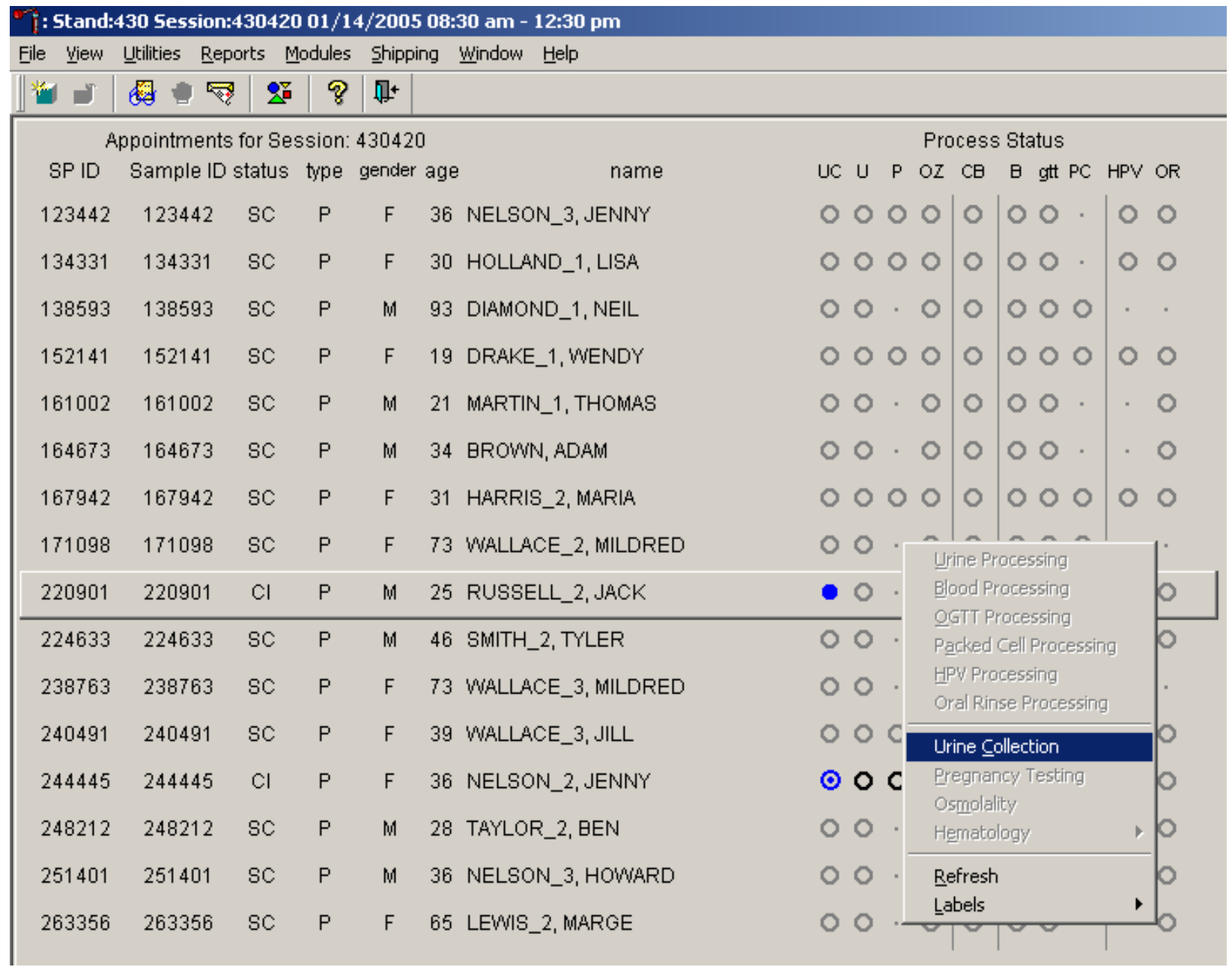

To select an SP, use the up and down keys to move up and down the list until the correct SP is highlighted, or drag the mouse arrow to the correct SP and right click. To access the Urine Collection module, drag the mouse arrow to \{Urine Collection\}, and left click or left click and type [C/c]. 
The urine collection window displays.

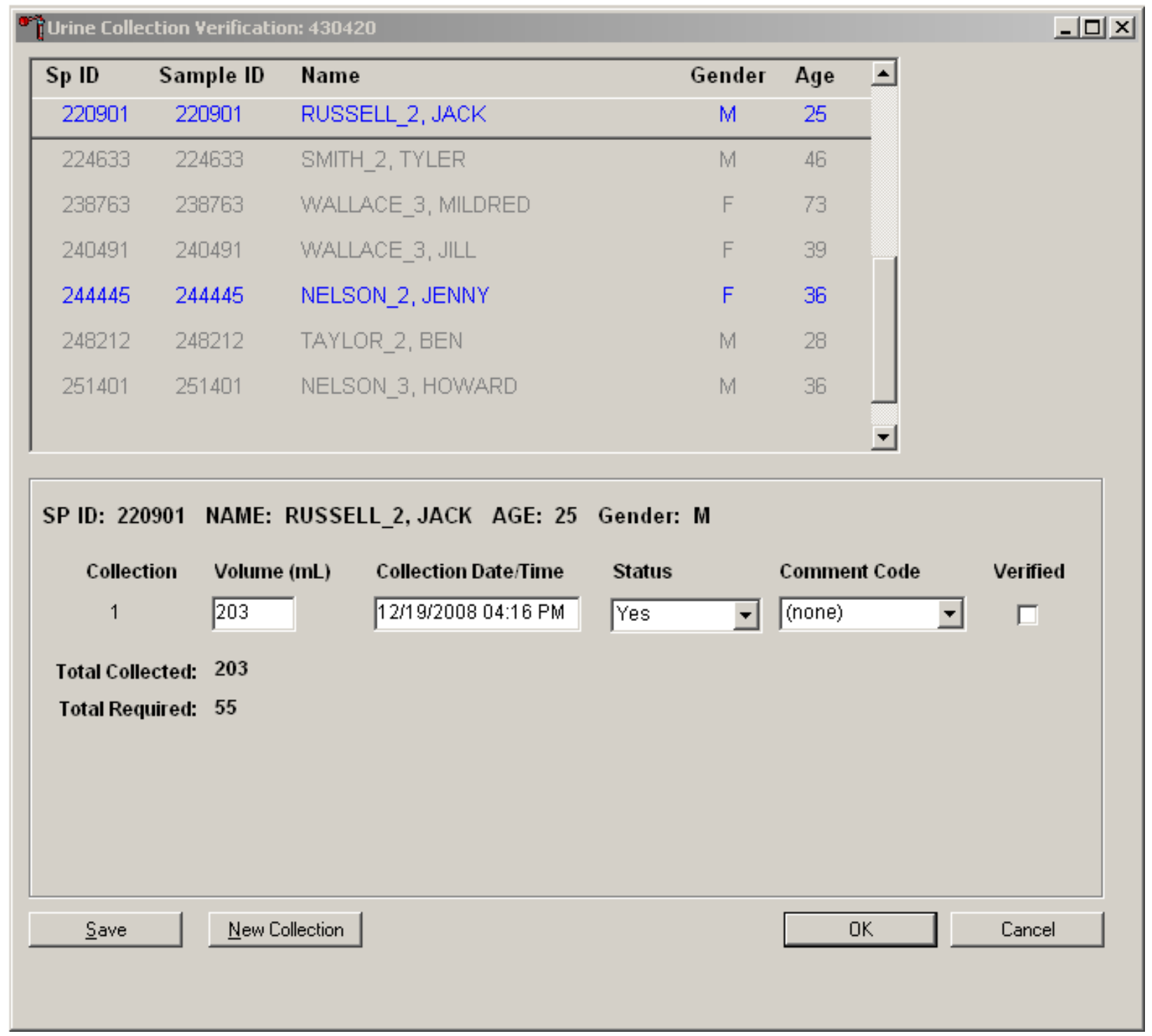

The top of the urine collection window contains columns for the SP ID, Person Name (last, first), Gender, and Age. The row for checked-in SPs is indicated in black, while SPs who have not checked into the MEC session are gray. SPs who have a urine collection ready for verification display in blue. Use the scroll bar to view all SPs scheduled into the session. To select an SP, use the up and down keys to move up and down the list until the correct SP is highlighted, or drag the mouse arrow to the correct SP and right click. 
The lower portion of the urine collection window displays the selected SP ID, Person Name (last, first), Gender, and Age. Below that are columns for Collection instance, Volume (mL) in whole numbers, Collection date/time, Collection status, Comment code, and a check box for Verify. The bottom of the screen displays the Total Collected (sum in $\mathrm{mL}$ of all urine collections) and Total Required (total $\mathrm{mL}$ required to meet the SP's urine protocol). The total volume includes 2-mL for pregnancy testing and osmolality for eligible female SPs, and 1-mL for osmolality testing only on pregnancy test ineligible female SPs and all male SPs.

One gram of urine is equal to one $\mathrm{mL}$ of urine. The application records the weight of the urine in whole numbers following standard rounding procedures.

If the SP has submitted a urine collection that has been weighed and scanned into the database, the volume, collection status, and collection date and time will automatically display in the lower portion of the Urine Collection screen. If the collection did not meet the minimum requirement for the protocol, the system will automatically select "QNS" with the comment code "quantity not sufficient." A QNS result immediately notifies the coordinator to prompt for an additional urine collection. A second instance of urine collection for the first QNS sample or third instance of urine collection for the second QNS sample will appear below the current sample. If the quantity of urine collection does meet the requirement of the urine protocol, the system will automatically select "Yes" and no additional collections will appear. 
Evaluate the quantity of urine received in the laboratory.

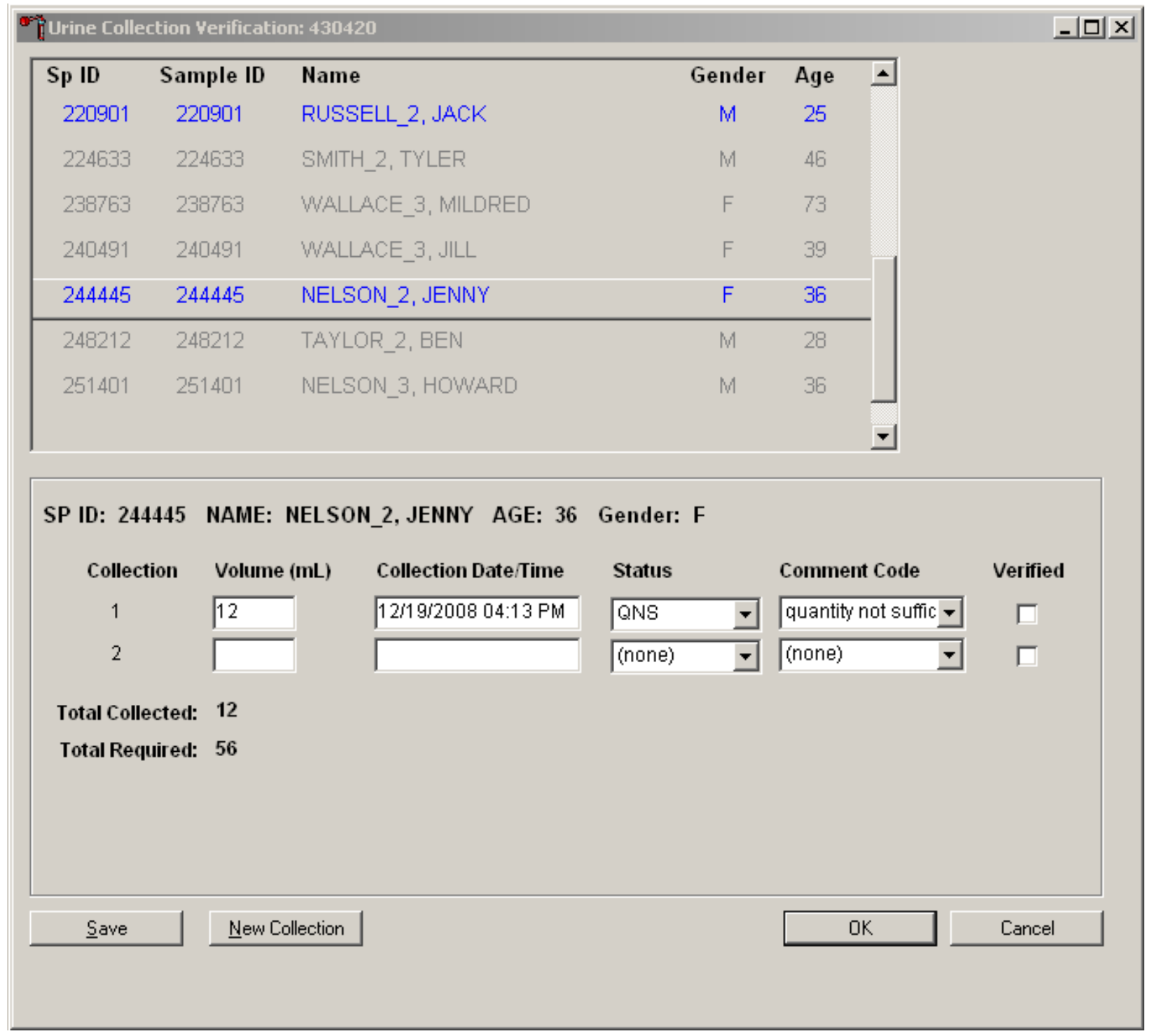

Compare the number displayed in the Total Collected column and the actual volume of urine in the cup. Adjust the Volume (mL) if necessary by using the mouse to direct the mouse arrow to the text box under the Volume (mL) column. Right click on the text box to enable the cursor in the box. Make changes using the number pad on the keyboard. Once any necessary adjustments have been made, use the mouse to direct the mouse arrow to the box under the Collection Date/Time column or press the Tab key. A prompt will pop up asking if you would like to subtract the weight of the cup. If the volume of the 
sample was transmitted from the scale and you have made a manual adjustment to that reading, select "No."

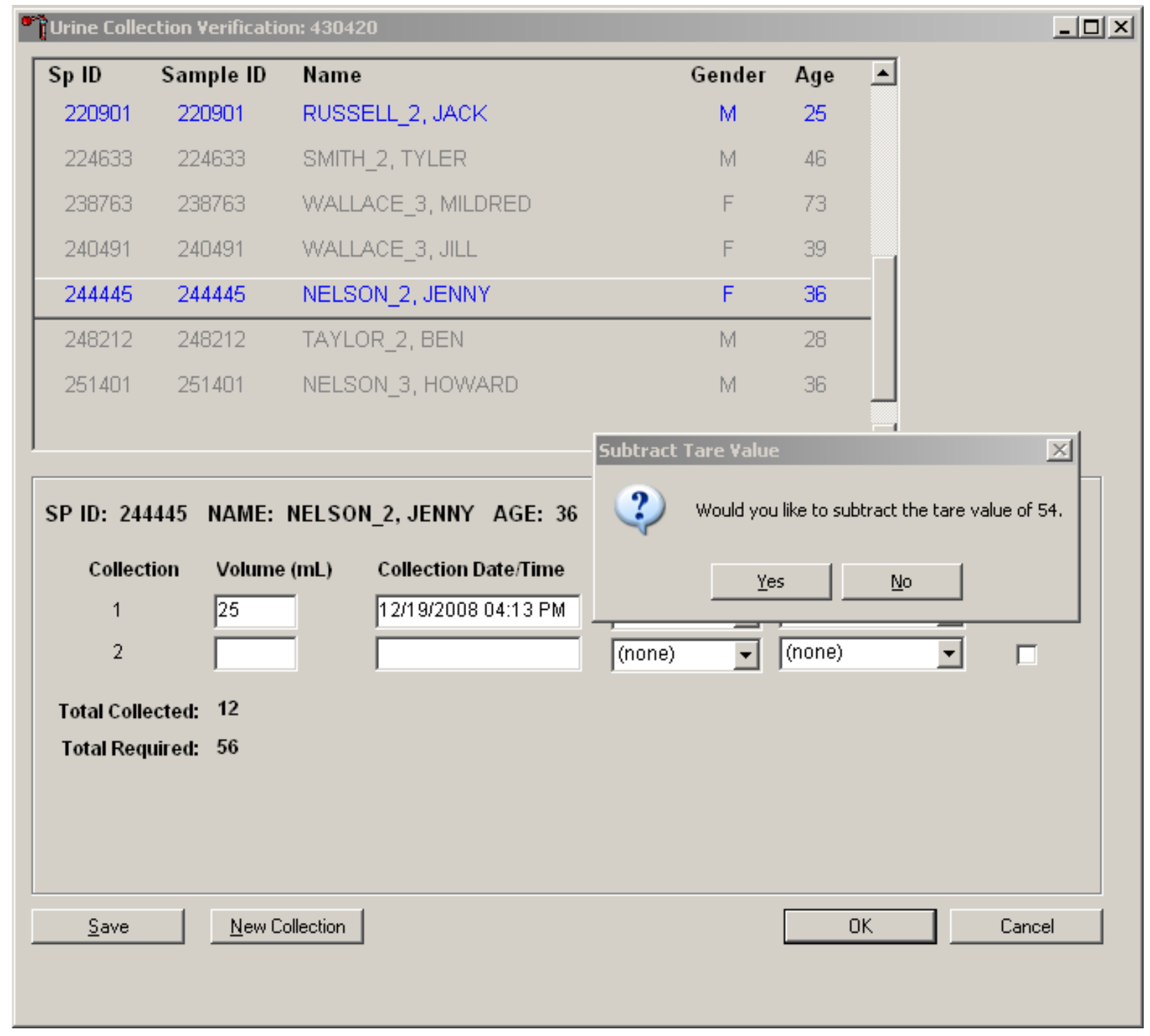

In the Collection Date/Time box, right click on the text box to enable the cursor inside the box. Adjust the date and time by entering the date in the format “MMDDYYYY \#\#:\#\#” using the number pad on the keyboard. Adjust "am/pm” by using the up or down arrows. Once any necessary adjustments have been made, use the mouse to direct the mouse arrow to the box under the Status column or press the Tab key. Record the urine collection result for each SP by typing [Y/y] for "Yes,” [N/n] for "No," or $[\mathrm{Y} / \mathrm{y}]$ a second time for "QNS." Alternatively, use the mouse to direct the mouse arrow to the drop-down 
list, left click to display the responses, drag the mouse arrow to "Yes," "No," or "QNS” and left click. A "QNS” result automatically turns the lab progress bar yellow on the Coordinator screen. This yellow progress bar alerts the coordinator, who then asks the SP to provide an additional urine specimen.

Refrigerate all insufficient urine samples. When additional urine is obtained and collected, pool the urine into the most recently collected cup (the cup with the highest urine collection instance number on the cup label), mix, and process.

Do not enter a result until urine arrives in the laboratory. At the end of each session, review the results for each SP. Enter "No" in the result field for all SPs who have not produced a urine sample. Record a result other than "(none)" for all SPs. The coordinator also has the ability to mark urine as "No" with a comment code. The Laboratory application will not be able to modify any entries made by the coordinator. 


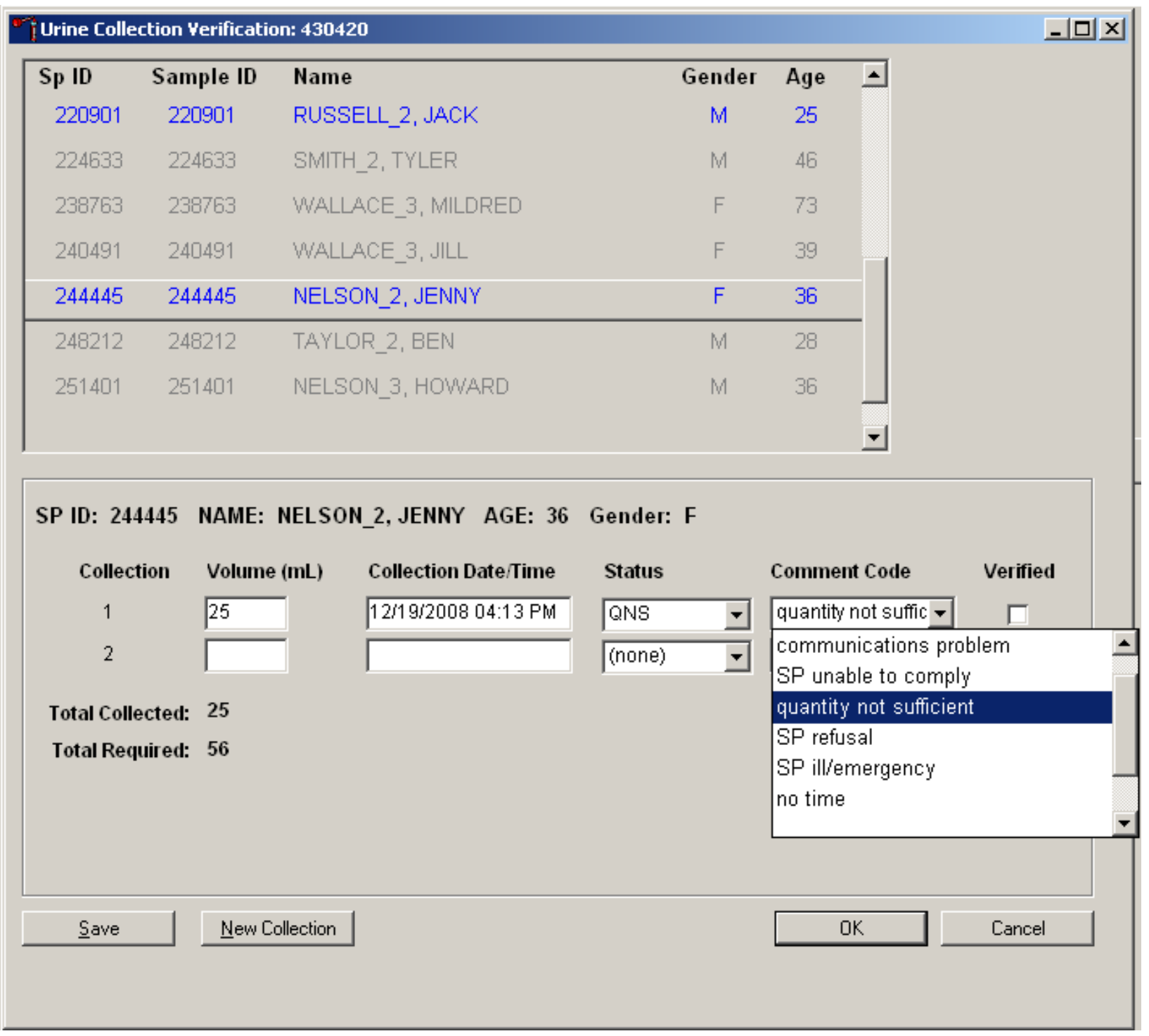

The urine colletion cup capacity may contain a sample that weighs more than the scale weight capacity of 610 grams. Look at the scale display for full specimens. If the scale displays dashes when it should display a weight, the reading is over the scale limit: $\ulcorner-ー-ー\urcorner$. When the bar code for the sample is scanned in, instead of the bar-code number displaying on the LCD screen, the words "over Lnt” display.

When the scale is over limit it does not transmit data to the ISIS application. Manually enter the volume $610 \mathrm{~mL}$ in the volume column. 


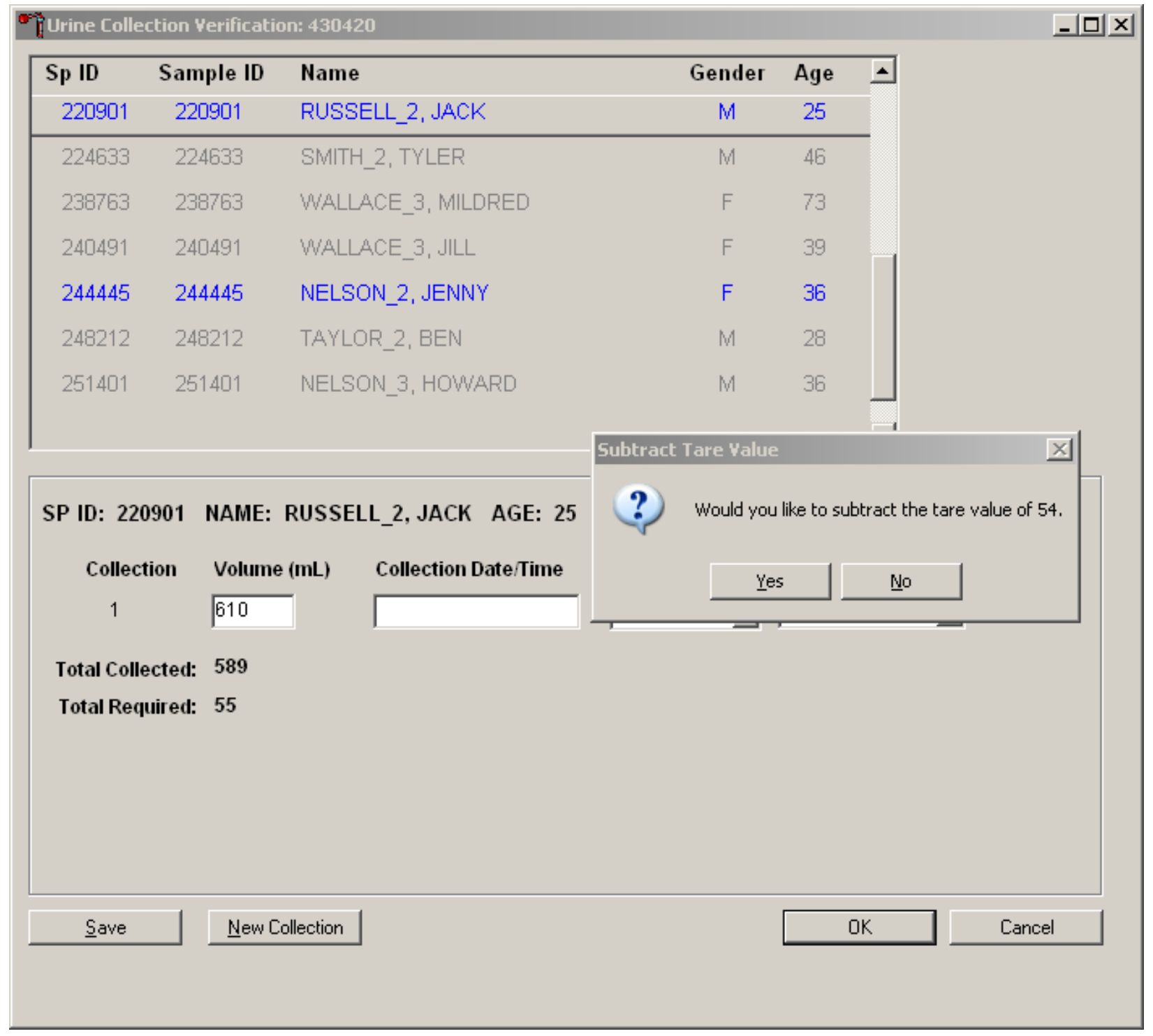

Press “Tab" to move to the Collection Date/Time field. The application will ask "Would you like to subtract the tare value of 54"? Select OK. Enter the date and time of specimen collection. Press tab to continue on to the Status field. 


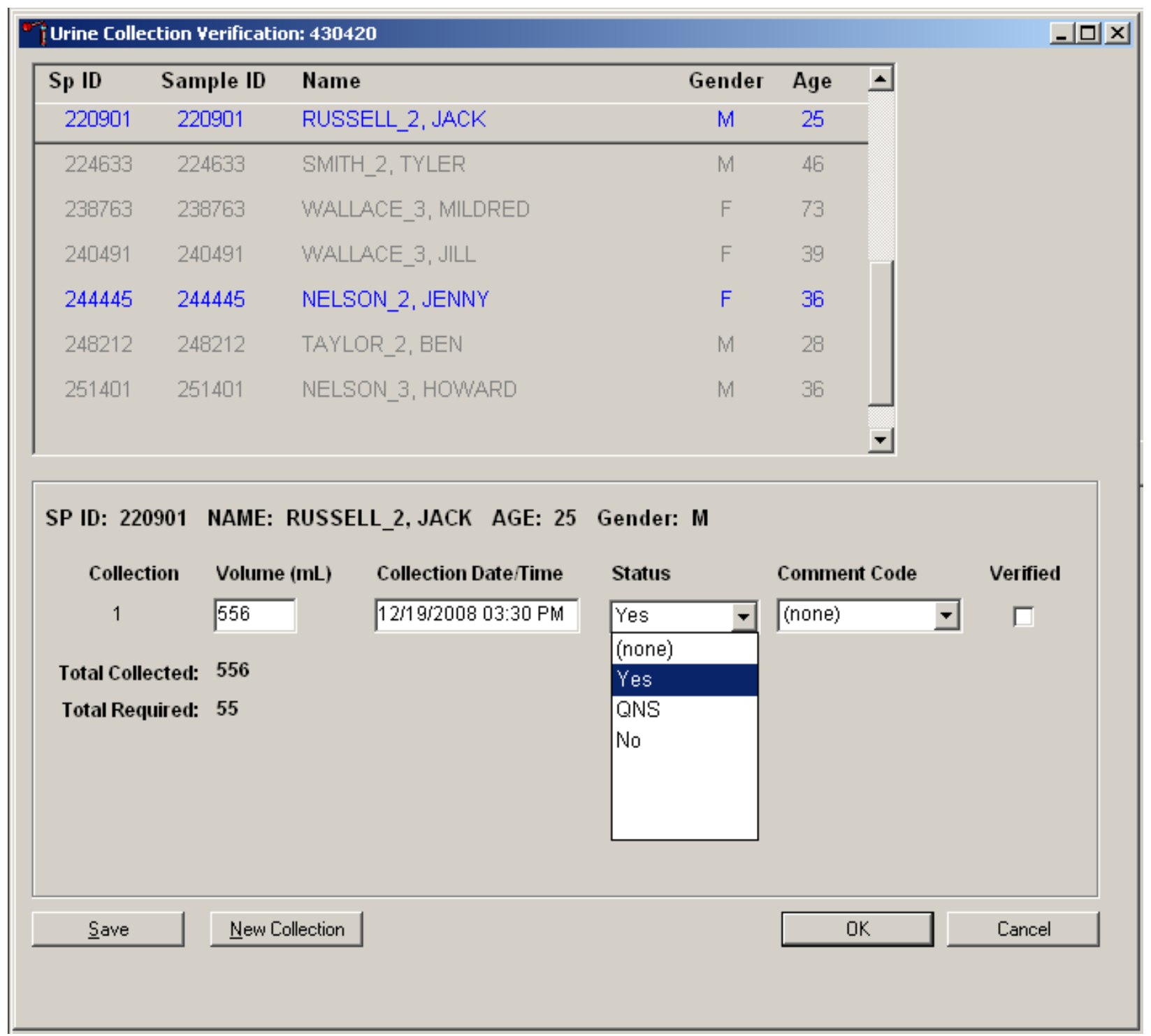

Record urine collections that exceed the scale as "Yes." To mark the urine collection status, direct the mouse arrow to the drop-down list, click to display the different status, and select "Yes" by clicking once on "Yes" with the mouse arrow. Use the Tab key to proceed on to the Comment Code field.

Record a comment for sufficient urine collections if the weight of the urine exceeds the scale weight capacity of 610 grams. 


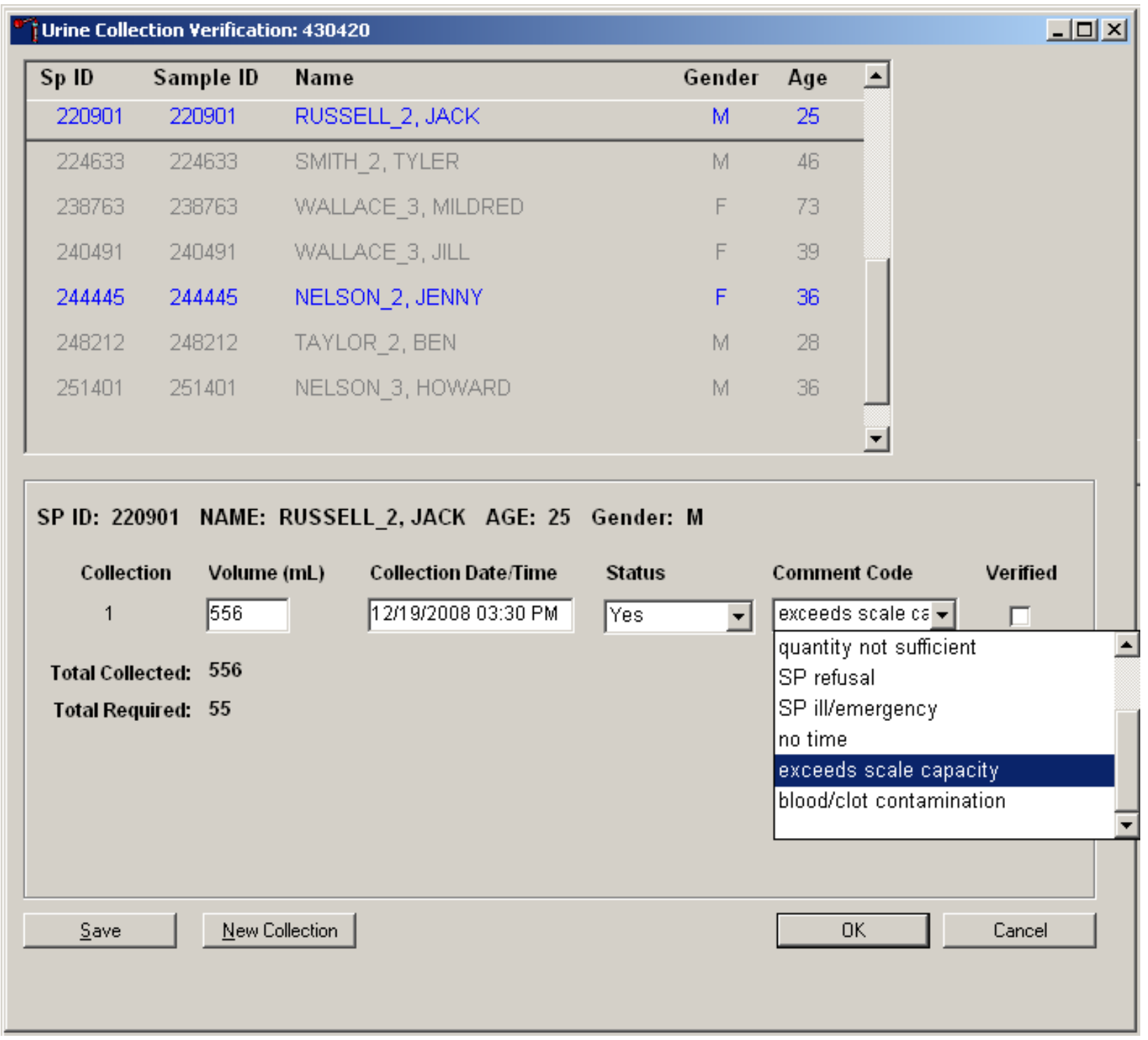

To record a comment, use the mouse to direct the mouse arrow to the drop-down list, click to display the comment codes, and select “exceeds scale capacity” from the drop-down list. Alternatively, use the up and down keyboard arrows to scroll through the choices or type the first letter of the desired comment code and, when the correct choice is highlighted, left click. 
Visually evaluate the urine sample for blood contamination. Record a comment for sufficient and QNS urine collections if the sample is contaminated with blood.

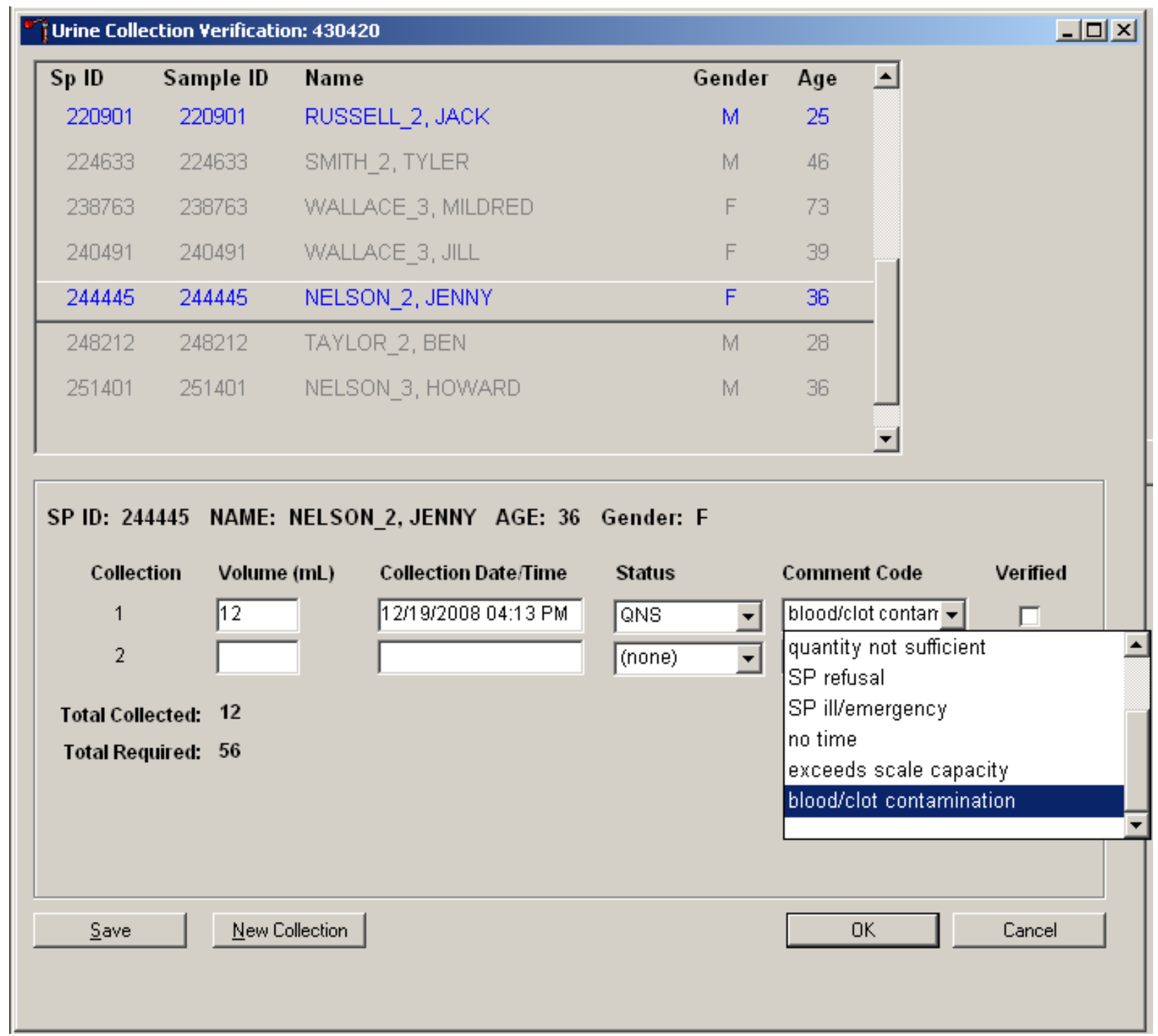

The "blood/clot contamination" comment should supersede the "quantity not sufficient" comment code when blood is present in a QNS specimen. To record a comment, use the mouse to direct the mouse arrow to the drop-down list, click to display the comment codes, and select "blood/clot contamination” from the drop-down list. Alternatively, use the up and down keyboard arrows to scroll through the choices or type the first letter of the desired comment code and when the correct choice is highlighted, left click. 
Record a comment for all insufficient urine collections. The laboratory application will automatically assign the comment code "quantity not sufficient" for all urine collections that are calculated as QNS by the scale application. The laboratory technician may also assign this comment code to all samples that are changed to QNS during the verification process.

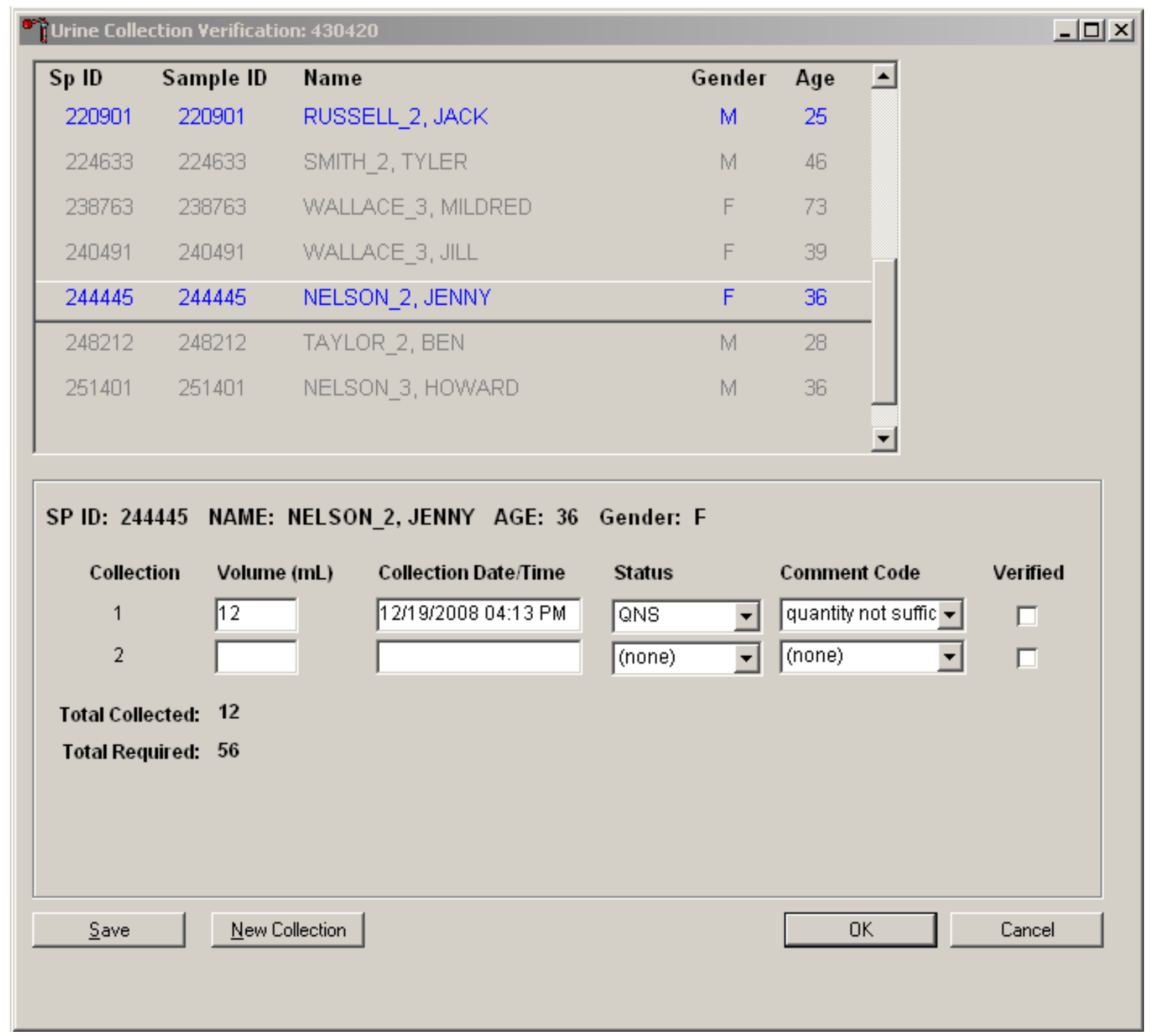

To record a comment, use the mouse to direct the mouse arrow to the drop-down list, click to display the comment codes, and select the most appropriate choice. Alternatively, use the up and down keyboard arrows to scroll through the choices or type the first letter of the desired comment code (Exhibit 5-3) and when the correct choice is highlighted, left click. 
Exhibit 5-3. Comment codes for insufficient urine collection

\begin{tabular}{|l|l|}
\hline Comment Code & Use when: \\
\hline $\begin{array}{l}\text { communication } \\
\text { problem }\end{array}$ & $\begin{array}{l}\text { SP is unable to understand and follow instructions for the component due to } \\
\text { language, cognitive impairment, or other problem, and is unable to complete } \\
\text { the test. }\end{array}$ \\
\hline SP unable to comply & $\begin{array}{l}\text { SP has a physical limitation that prohibits him or her from producing a urine } \\
\text { specimen or the SP is unable to produce urine during the session. }\end{array}$ \\
\hline quantity not sufficient & The quantity of urine is insufficient to process any or all urine vessel(s). \\
\hline SP refusal & SP refuses to provide urine during the session. \\
\hline SP ill/emergency & Not applicable. \\
\hline no time & Not applicable. \\
\hline exceeds scale capacity & The weight of the urine exceeds the scale weight capacity of 610 grams. \\
\hline $\begin{array}{l}\text { blood/clot } \\
\text { contamination }\end{array}$ & Blood or clots are present in the urine and may affect the volume capture. \\
\hline
\end{tabular}


Verify the results of the urine collection. To verify, use the mouse to direct the mouse arrow to the "Verify" check box. Left click on the check box and a check mark will appear in the box. Verifying the results will save the results to the database.

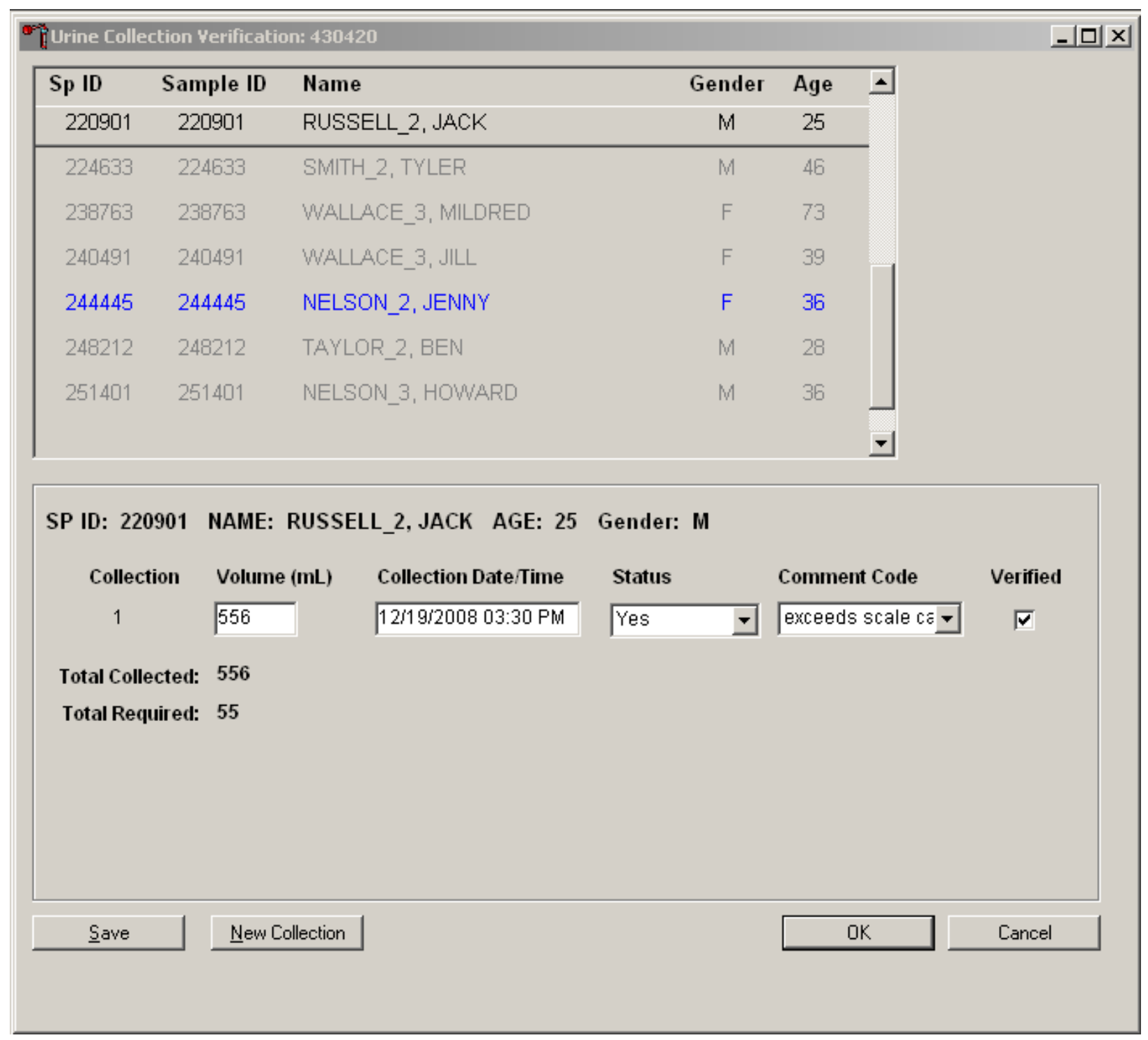

Continue recording and verifying urine collection results as specimens are received in the laboratory. Make sure to use the scroll bar to view all SPs in the top part of the Urine Collection screen. SPs who need their urine verified will appear in blue on the top screen. 
An SP will be prompted for additional urine only if (1) the total of his or her urine collection was insufficient to meet the SP's protocol requirement and (2) the SP has submitted less than three collection samples. The Urine Collection screen will display up to three instances of urine collection. Urine collection is complete regardless of the amount of urine collected after three attempts. When urine is collected as QNS, a message is sent to the coordinator to prompt the SP for more urine.

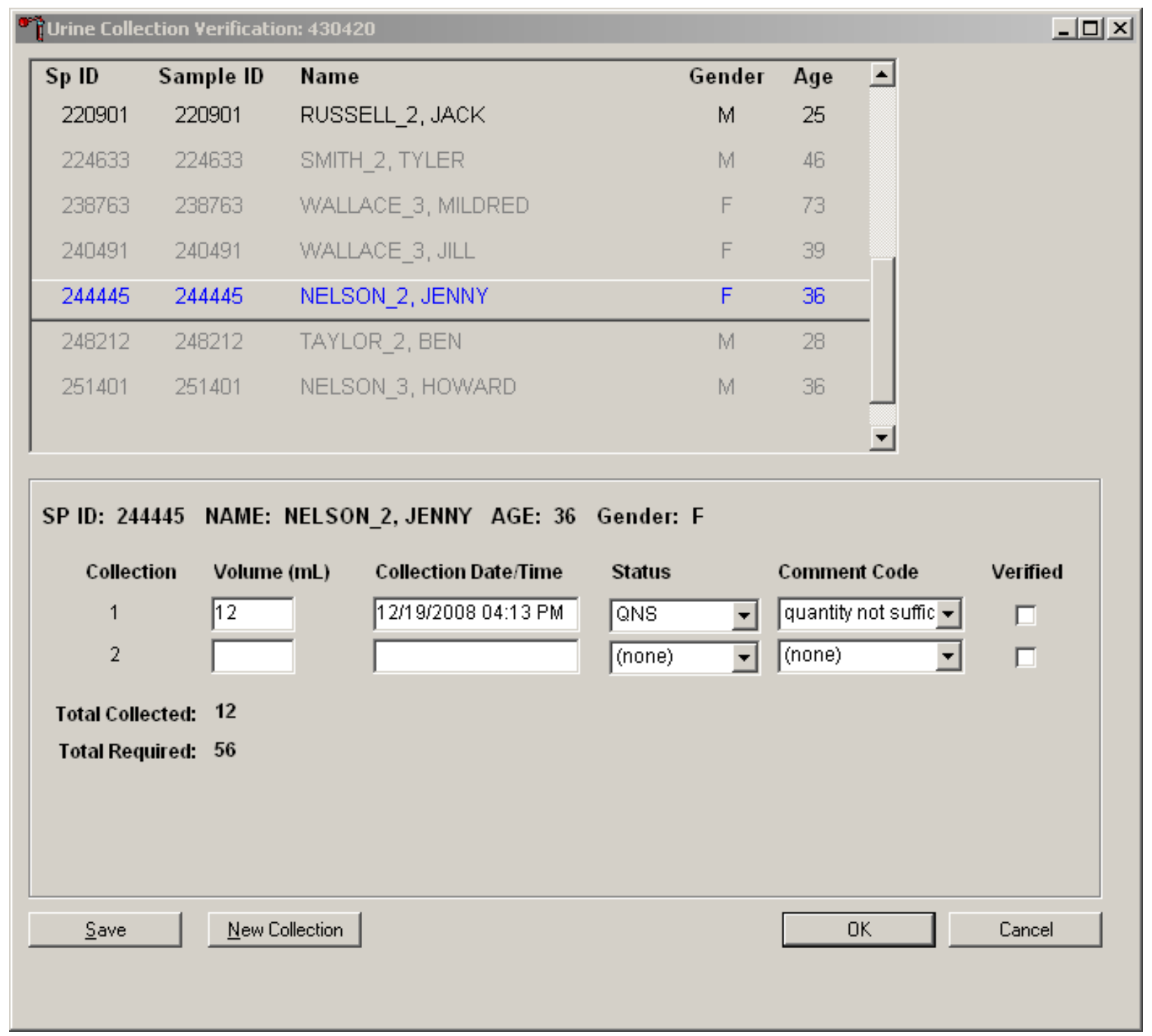

If the urine collection is the first or second collection and the status is QNS, a record for the second and third instances of urine collection will automatically be added by the Laboratory application. 
Second and third collections will display as empty rows after the last urine collection. The instance number is displayed under the "Collection" column.

Verify the urine collection by checking the "Verify" box. Use "Save" to save changes or select the $\mathrm{X}$ in the upper right corner to exit out of the Urine Collection screen.

Once a urine collection has been recorded for an SP, the circle on the heads-up screen will turn black ( $\odot$ for QNS or $\odot$ for Yes). A black urine collection circle indicates that no additional action needs to be taken on that SP for urine collection at that time.

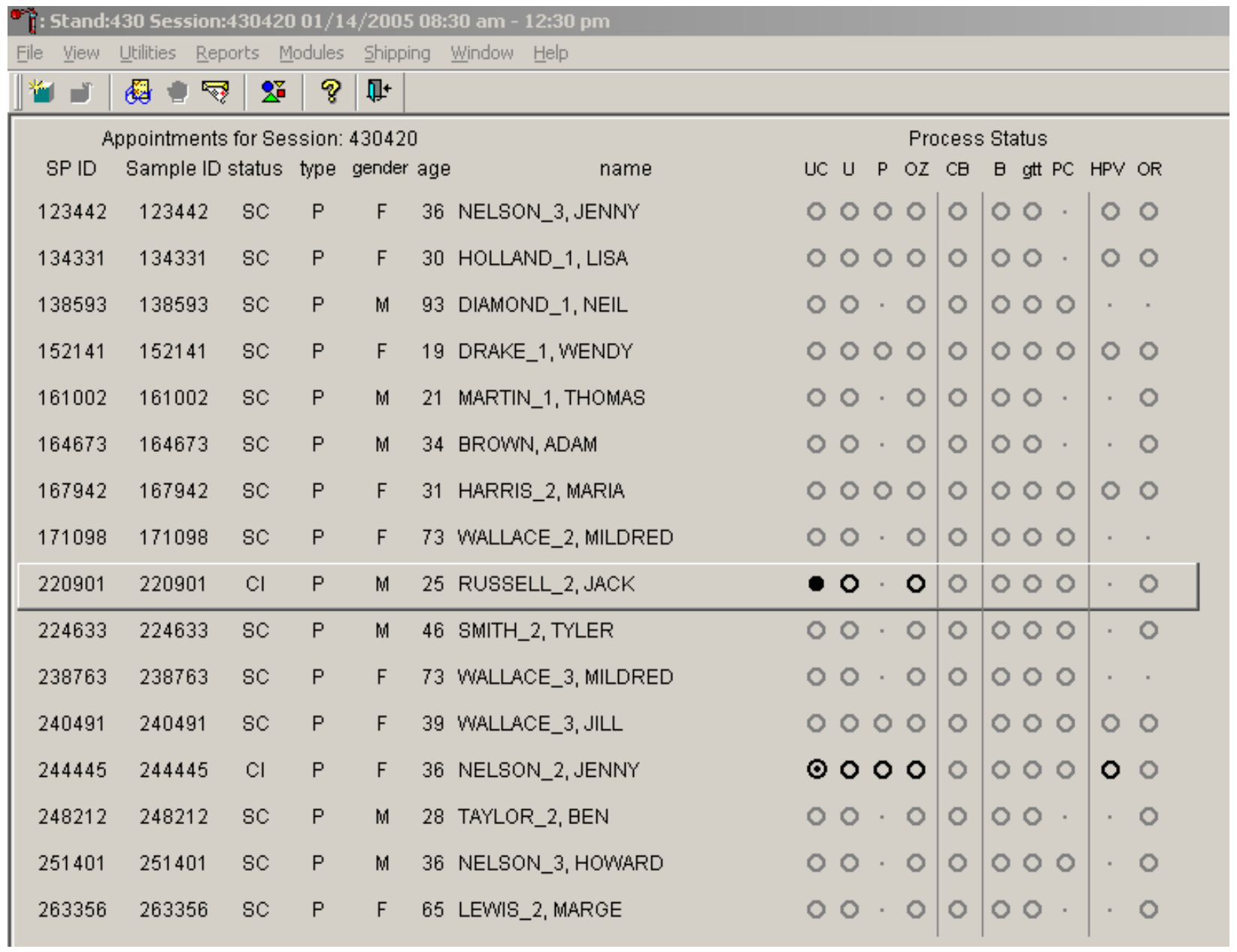




\subsubsection{Manually Document Urine Collection With Barcode Scanning}

The technician is able to manually record the volume, status, and collection date and time in the urine collection module within the laboratory application. Place the urine collection cup on the scale and cover with the clear acrylic scale cover. Scan the barcode on the urine cup.

Open the Urine Collection screen from the heads-up as described in Section 3.3. The urine collection window displays. At this point, the technician has the ability to modify the volume of the urine and the collection date/time.

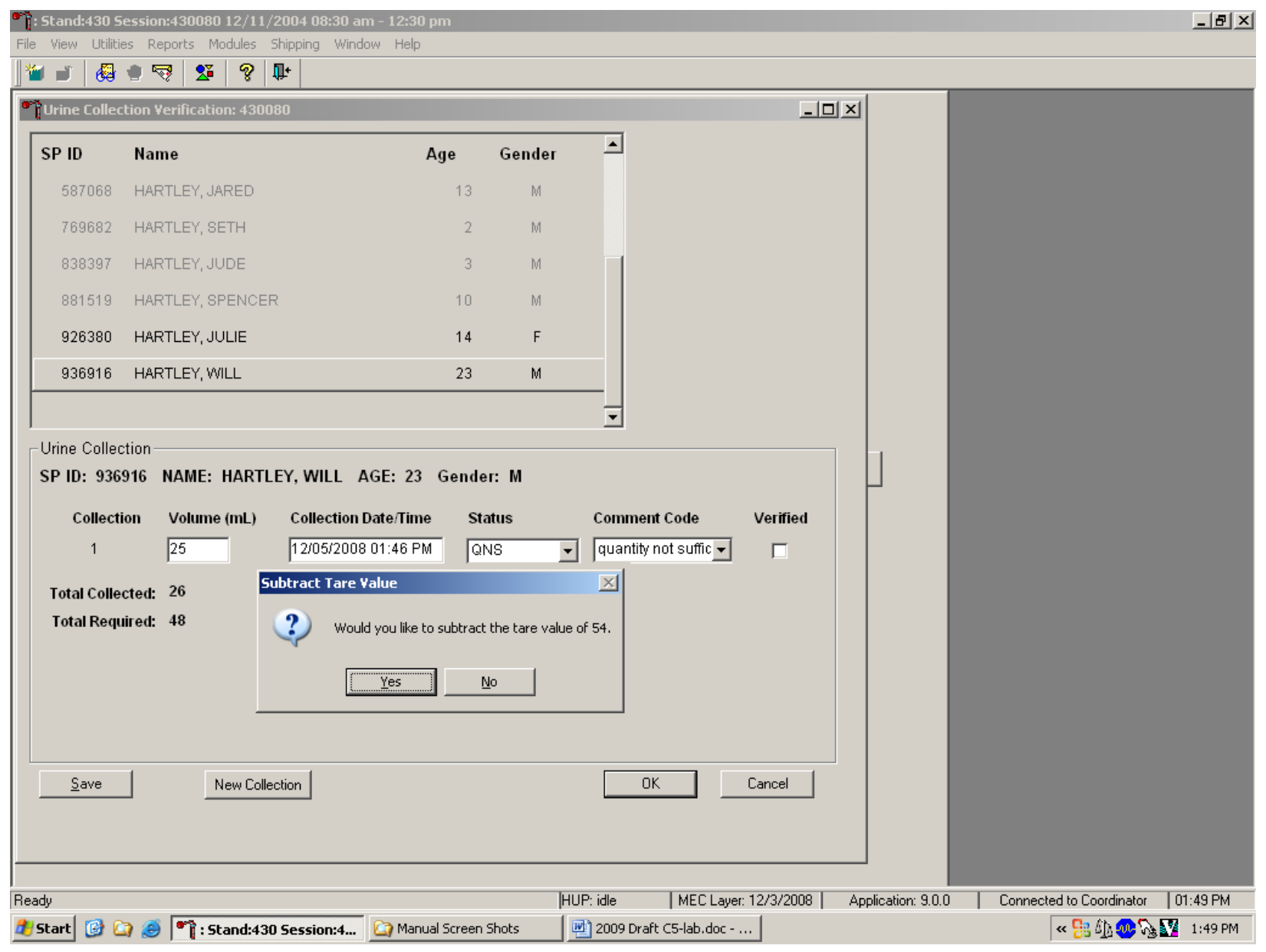


Continue to enter the collection date/time, selecting the appropriate urine collection status, and a comment code if applicable. Verify the urine collection by checking the "Verify" box.

If the urine collection is the first or second collection and is QNS, second and third instances of urine collection may be added by using the "New Collection” button at the bottom of the screen.

\section{Save}

\section{New Collection}

OK

OK Cancel

Add a second or third collection only if the previous collection was QNS. Enter the second or third urine collection results when the sample is submitted to the MEC laboratory by typing in the boxes for volume and collection date/time, then selecting the appropriate urine collection status and a comment code if applicable.

Verify the urine collection by checking the "Verify" box. Use "Save" to save changes or select the $\mathrm{X}$ in the upper right corner to exit out of the Urine Collection screen.

\subsubsection{Manually Document Urine Collection Without Barcode Scanning}

If the connection between the scale and workstation 2 is down, the technician is able to manually record the volume, status, and collection date and time in the urine collection module within the laboratory application. Place the urine collection cup on the scale and cover with the clear acrylic scale cover. Note the weight reading from the LCD.

Open the Urine Collection screen from the heads-up as described in Section 3.3. The urine collection window displays. Double click in the area underneath Volume or Collection Date/Time to open the boxes where the values can be entered. Enter the urine collection results by typing in the weight reading from the scale's display in the box for volume. 


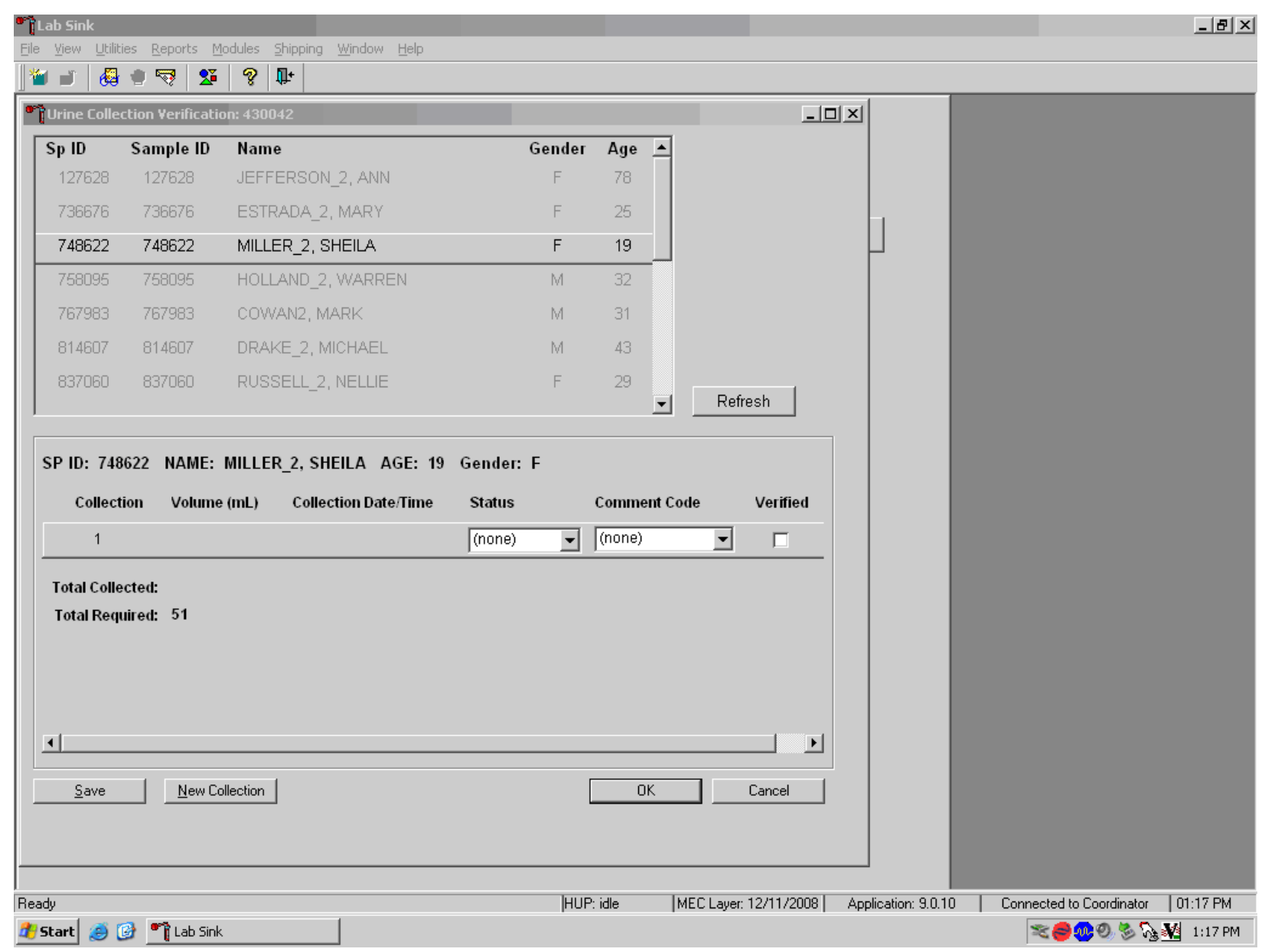

If the next box is selected by pressing the "tab" button or selected by the mouse, a prompt will pop up asking if you would like to subtract the weight of the urine cup from the result entered. Select "Yes." 


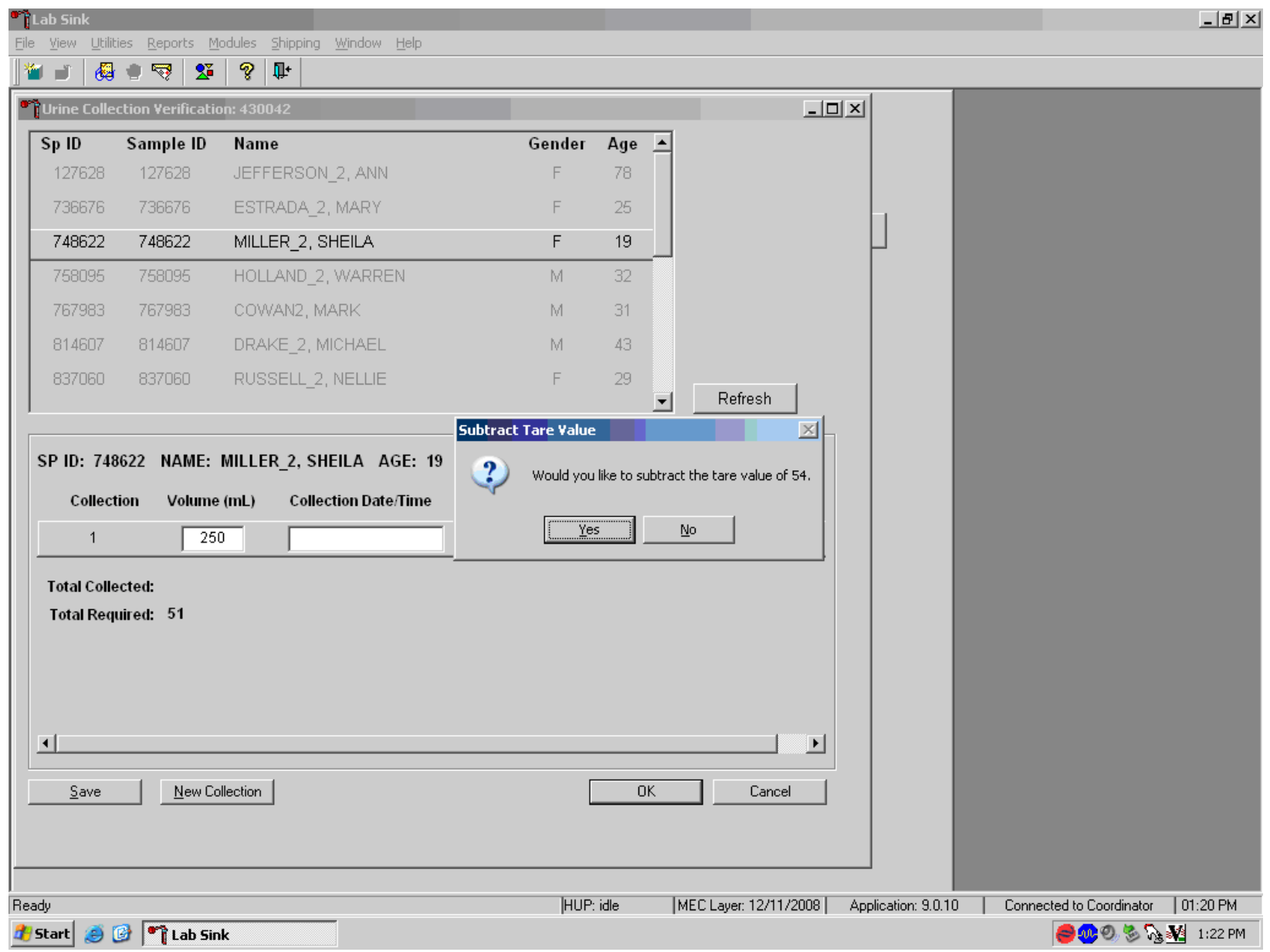

Continue to enter the collection date/time, selecting the appropriate urine collection status, and a comment code if applicable. Verify the urine collection by checking the "Verify" box.

If the urine collection is the first or second collection and is QNS, second and third instances of urine collection may be added by using the "New Collection” button at the bottom of the screen.

Save $\quad$ New Collection

Add a second or third collection only if the previous collection was QNS. Enter the second or third urine collection results when the sample is submitted to the MEC laboratory by typing in the boxes for volume and collection date/time, then selecting the appropriate urine collection status and a comment code if applicable. 
Verify the urine collection by checking the "Verify" box. Use "Save" to save changes or select the $\mathrm{X}$ in the upper right corner to exit out of the Urine Collection screen.

\subsection{Urine Specimen Assays}

Process urine specimens for the following tests:

- Pregnancy test for selected females aged 8-11 and all females aged 12 to 59 years

- Albumin/Creatinine (Alb/Creat) on primary SPs and VIP guests aged 6+ years

- Chlamydia (Chlam) on primary SPs 14-39 years old

- Speciated Arsenic (Spc Arsenic) on selected SPs aged 6+ years

- Priority Pesticides (Prior Pest) on selected SPs aged 6+ years

- Organophosphates (Organophos) on selected SPs aged 6+ years

- Urine Mercury/Iodine (Urin Merc) on selected SPs aged 6+ years

- Heavy Metal on selected SPs aged 6+ years

- Phytoestrogens (Urn Phytoes) on selected SPs aged 6+ years

- Polyaeromatic Hydrocarbons (PAH) on selected SPs aged 6+ years

- Phthalates on selected SPs aged 6+ years

- Perchlorate on selected SPs aged 6+ years

- Caffeine on selected SPs aged 6+ years

- NNAL (a tobacco-specific nitrosamine) on SPs aged 6+ years

- $\quad$ Extra urine (Xtra Urine) on SPs aged 6+ years

\subsection{Urine Specimen Protocols}

Table 5-1 summarizes the urine processing protocol for primary SPs. Process urine in priority order with vessel 45 filled first, 46 second, etc. 
Table 5-1. NHANES urine processing protocol - primary

\begin{tabular}{cllcl}
\hline $\begin{array}{c}\text { Vessel } \\
\text { no. }\end{array}$ & Assay & $\begin{array}{c}\text { Age in } \\
\text { years }\end{array}$ & $\begin{array}{c}\text { Sample } \\
\text { size mL }\end{array}$ & Vessel \\
\hline 45 & Alb/Creat & $6+$ & 3 & $5 \mathrm{~mL}$ cryovial \\
46 & Chlam & $14-39$ & 12 & $14 \mathrm{~mL}$ Falcon \\
86 & Spc Arsenic & $6+$ & 3 & $5 \mathrm{~mL}$ cryovial \\
48 & Prior Pest & $6+$ & 8 & $10 \mathrm{~mL}$ VWR screwcap \\
49 & Organophos & $6+$ & 8 & $10 \mathrm{~mL}$ VWR screwcap \\
62 & Urin Merc & $6+$ & 5 & $10 \mathrm{~mL}$ VWR screwcap \\
50 & Heavy Metal & $6+$ & 8 & $10 \mathrm{~mL}$ VWR screwcap \\
65 & Urn Phytoes & $6+$ & 3 & $5 \mathrm{~mL}$ cryovial \\
66 & PAH & $6+$ & 3 & $5 \mathrm{~mL}$ cryovial \\
67 & Phthalates & $6+$ & 3 & $5 \mathrm{~mL}$ cryovial \\
102 & Perchlorate & $6+$ & 5 & $5 \mathrm{~mL}$ cryovial \\
121 & NNAL & $6+$ & 10 & $10 \mathrm{~mL}$ VWR screwcap \\
128 & Caffeine & $6+$ & 1 & $2 \mathrm{~mL}$ cryovial \\
52 & Xtra Urine & $6+$ & 5 & $5 \mathrm{~mL}$ cryovial \\
\hline
\end{tabular}

Table 5-2 summarizes the urine processing protocol for the VIP guest appointment type.

Table 5-2. NHANES urine processing protocol - VIP guest

\begin{tabular}{ccccc}
\hline $\begin{array}{c}\text { Vessel } \\
\text { No. }\end{array}$ & Assay & $\begin{array}{c}\text { Age in } \\
\text { years }\end{array}$ & $\begin{array}{c}\text { Sample } \\
\text { size mL }\end{array}$ & Vessel \\
\hline 45 & Alb/Creat & $6+$ & 3 & $5-m L$ Cryovial \\
\hline
\end{tabular}




\subsection{Labeling Urine Processing Vessels}

The appropriate barcode labels automatically print for each SP based on the SP's age, sex, appointment type, and subsample selection. The label/ship technologist at workstation 3 prints the labels at least one session in advance. The printing procedure is described in Chapter 9. Place labels at workstation 2.

Process urine on one SP at a time. Set up each urine processing rack using the preprinted labels. Label each vessel with the appropriate vessel label, according to the protocol. Place the label on the vessel, wrapping it around the vessel horizontally making sure the label wraps onto itself. Place the label so the first digit of the vessel number is at the top of the vessel. Set the vessels in the cube racks in priority order.

One hour into the session, if an SP has not checked in, separate the labels for SPs who have checked in from SPs who have not checked in. Set the labels aside for SPs who have not checked in. If the SP does not show up for the session, then discard the labels at the end of the session.

\subsection{Urine Specimen Processing}

- Perform pregnancy test and record results before aliquoting the urine specimen.

- Perform the osmolality test and record results.

- Label all urine aliquot vessels for the SP and place in priority order in the rack.

Using the large 15-mL plastic transfer pipette for all but vessel 62, allocate the urine sample for each of these tests using the following procedure:

45. Aliquot 3-mL of the specimen for Alb/Creat into a 5-mL vessel.

46. Aliquot 12-mL of the specimen for Chlam into 14-mL vessel.

86. Aliquot 3-mL of the specimen for Spc Arsenic into a 5-mL vessel.

48. Aliquot 8-mL of specimen for Prior Pest into 10-mL vessel.

49. Aliquot 8-mL of specimen for Organophos into 10-mL vessel. 
62. Urin Merc - Use a 3.5-mL plastic transfer pipette to transfer exactly 5-mL of urine into the 10-mL VWR screwcap vessel, close the cap, and mix gently. Keep vessel 62 upright at all times; do not invert the tube because the preservative could drain into the cap and spill when opened. If the vessel does become inverted, stand it upright for at least several hours or overnight, then open the cap, and pipette the urine into the 10$\mathrm{mL}$ vessel. Tap the bottom of the capped vessel against a hard surface before opening. Do not discard the prepared tubes even if the preservative crystallizes. If the preservative crystallizes, add 5-ml of urine with the transfer pipette and mix extremely well. Do not use these tubes for any other purpose since they contain a preservative.

50. Aliquot 8-mL of the specimen for Heavy Metal into a 10-mL vessel.

65. Aliquot 3-mL of the specimen for Urn Phytoes into a 5-mL vessel.

66. Aliquot 3-mL of the specimen for PAH into a 5-mL vessel.

67. Aliquot 3-mL of the specimen for Phthalates into a 5-mL vessel.

102. Aliquot 5-mL of the specimen for Perchlorate into a 5-mL vessel.

121. Aliquot $10-\mathrm{mL}$ of the specimen for NNAL into a $10-\mathrm{mL}$ vessel.

128 Aliquot 1-mL of the specimen for caffeine into a 2-mL vessel.

52. Aliquot 5-mL of the specimen for Xtra Urine into a 5-mL vessel.

Discard any remaining urine specimen at the end of each session. Pour urine down the drain in the laboratory sink that is connected to the sewer. Flush the sink with water. Discard urine cup in the biohazard trash.

\section{Do not process urine on more than one SP at a time.}

\subsection{Record the Results of Urine Specimen Processing}

After filling the urine specimen vessels for each SP, use the Urine Processing module to enter the results of urine processing. 
Access the Urine Processing module.

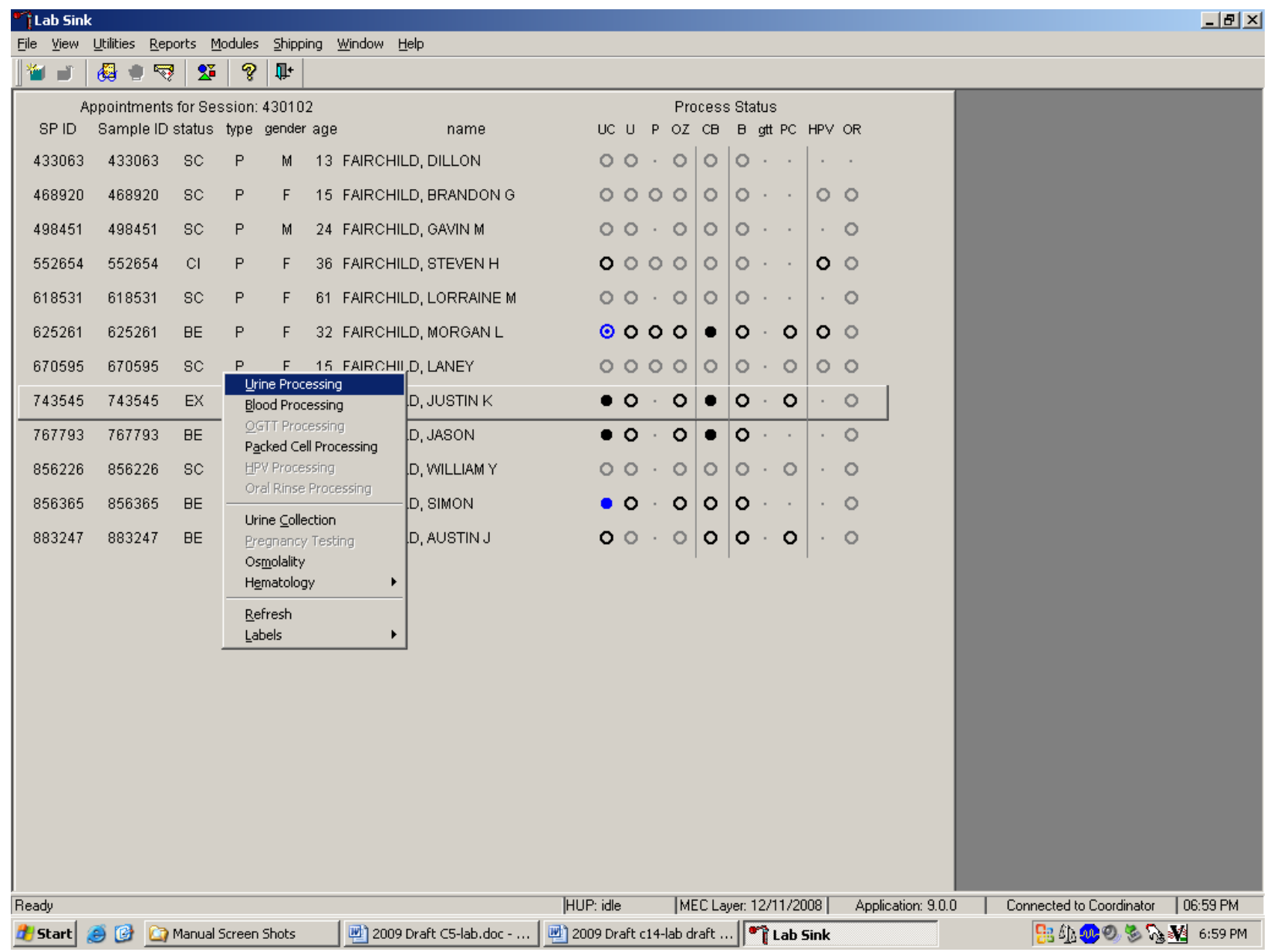

To access the Urine Processing module, use the mouse to direct the mouse arrow to the correct SP, right click, drag the mouse arrow to \{Urine Processing\}, and right click or right click and type [U/u]. Alternatively, use the up and down keys to move up and down the list until the correct SP is highlighted, right click, drag the mouse arrow to \{Urine Processing\}, and right click or right click and type $[\mathrm{U} / \mathrm{u}]$. 
The SP's urine processing window displays.

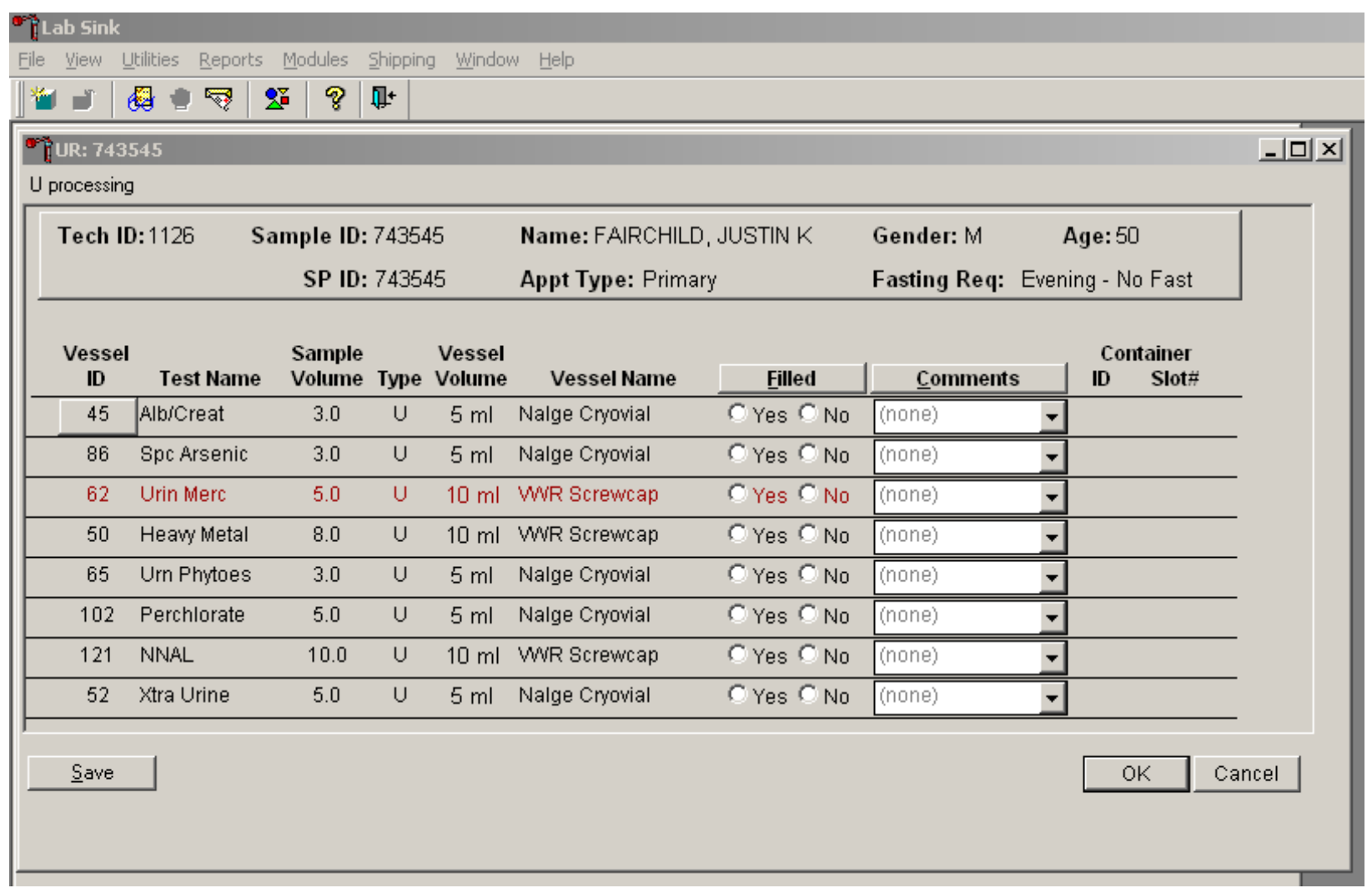

The urine processing window contains the following information for SPs: Tech ID, Sample ID, SP ID, Name (last, first), Gender, Age, Appt (appointment) Type, and Fasting Req. There are columns that list the Vessel ID, Test Name, Sample Volume, Sample Type (U = urine), Vessel Volume, Vessel Name, Filled (Yes or No) radio buttons, Comment drop-down box, and Container ID and Container Slot\# column. 
Individually mark each vessel as Filled -’Yes.”

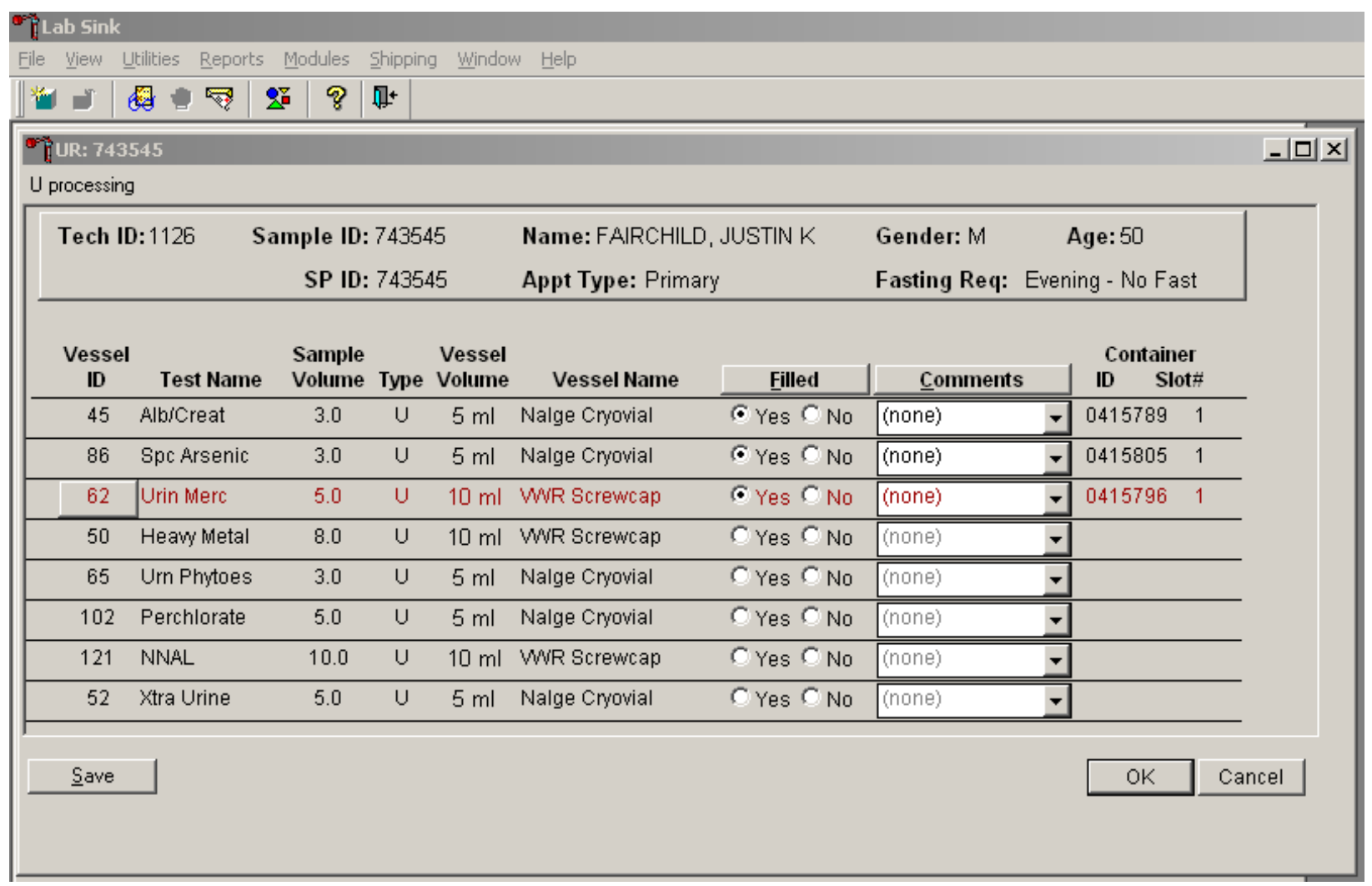

To mark an individual urine vessel as collected or Filled -"Yes," use the mouse to direct the mouse arrow to the center of the "Yes" radio button and left click. As each vessel is marked as Filled "Yes," it is automatically assigned to a slot in an existing (open) container and the next open slot number. 
Individually mark each vessel as Filled -”No.”

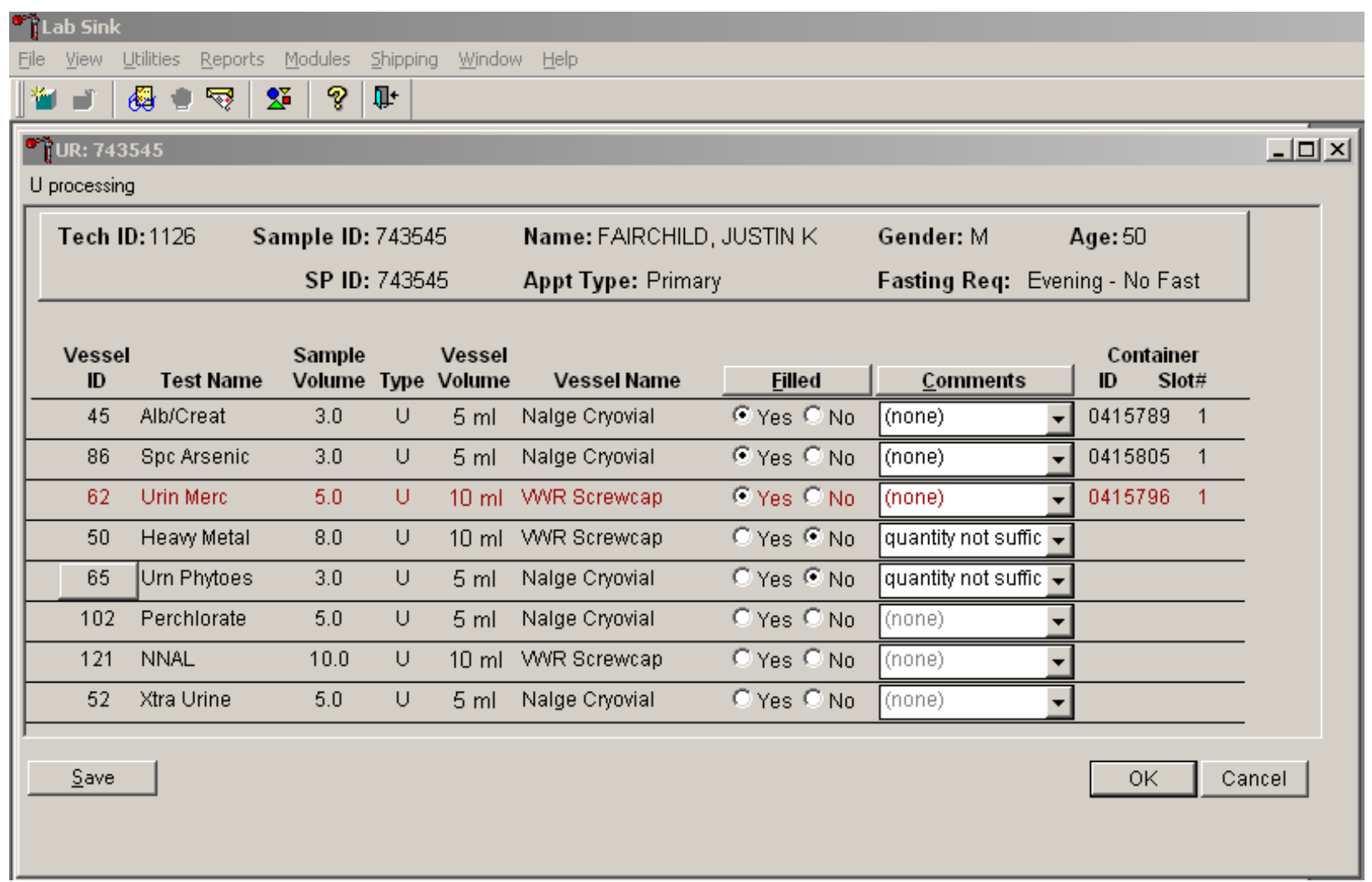

To mark an individual urine vessel as not collected or Filled -"No,” use the mouse to direct the mouse arrow to the center of the "No" radio button and left click. As each vessel is marked as Filled "No,” the comment, "quantity not sufficient” is automatically entered in the Comments column. 
Collectively mark all vessels as Filled-"Yes.”

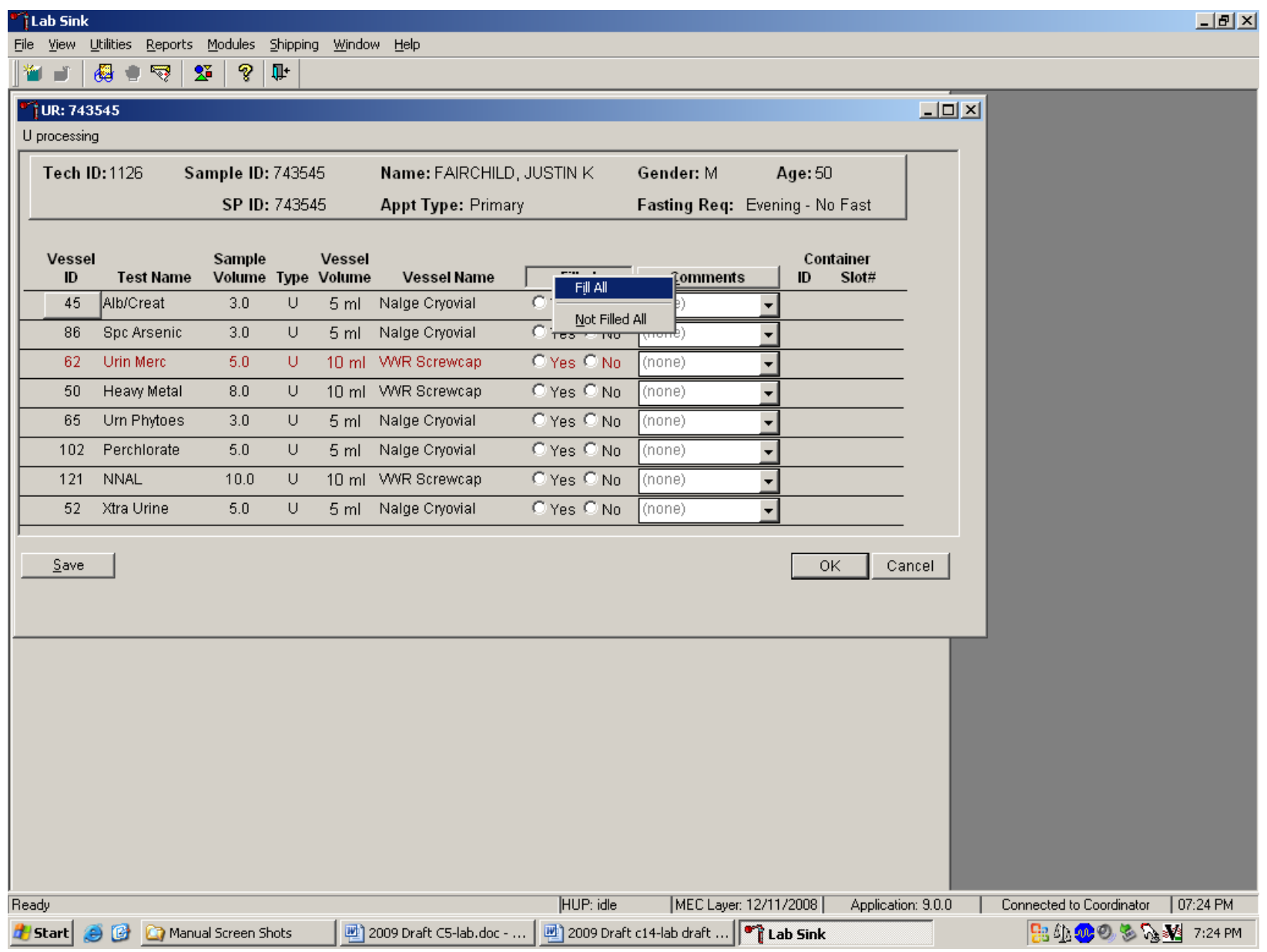

To collectively mark all vessels as Filled -’Yes,” use the mouse to direct the mouse arrow to the "Filled" button on the top of the radio buttons, left click, and drag the arrow to \{Fill All $\}$ and left click or type [Shift] [F/f], [I/i.] 
Collectively mark all vessels as Filled -”No.”

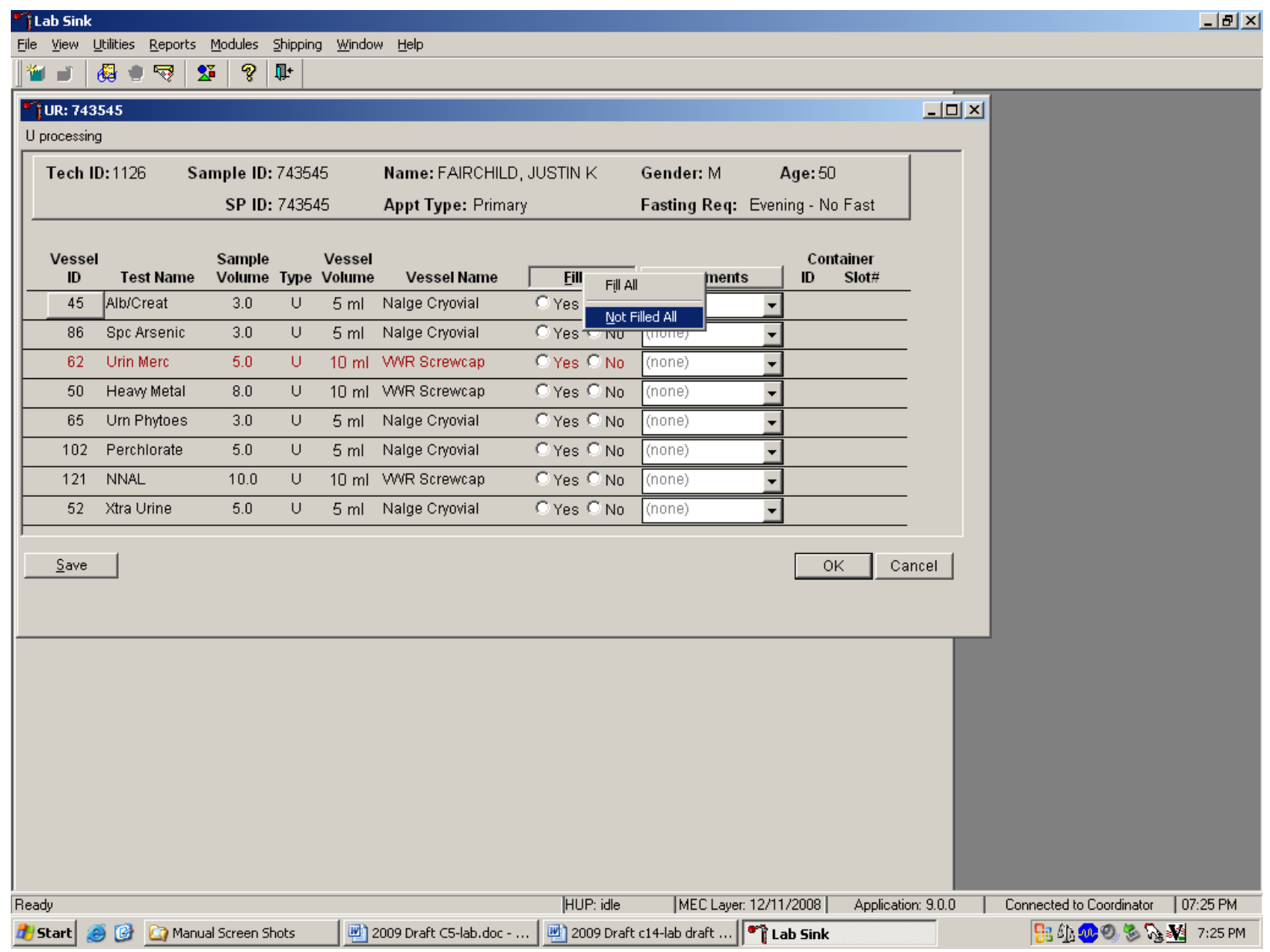

To mark all vessels as Filled-”No,” use the mouse to direct the mouse arrow to the Filled box, left click, drag the mouse arrow to \{Not Filled All , and left click or type [Shift] [F/f], [N/n.] 
Use the quality comment code to indicate if blood is present.

\begin{tabular}{|l|l|}
\hline Comment Code & Use when: \\
\hline \hline Blood present & Blood is visible in the specimen. \\
\hline
\end{tabular}

Attach the quality comment code to indicate if blood is present.

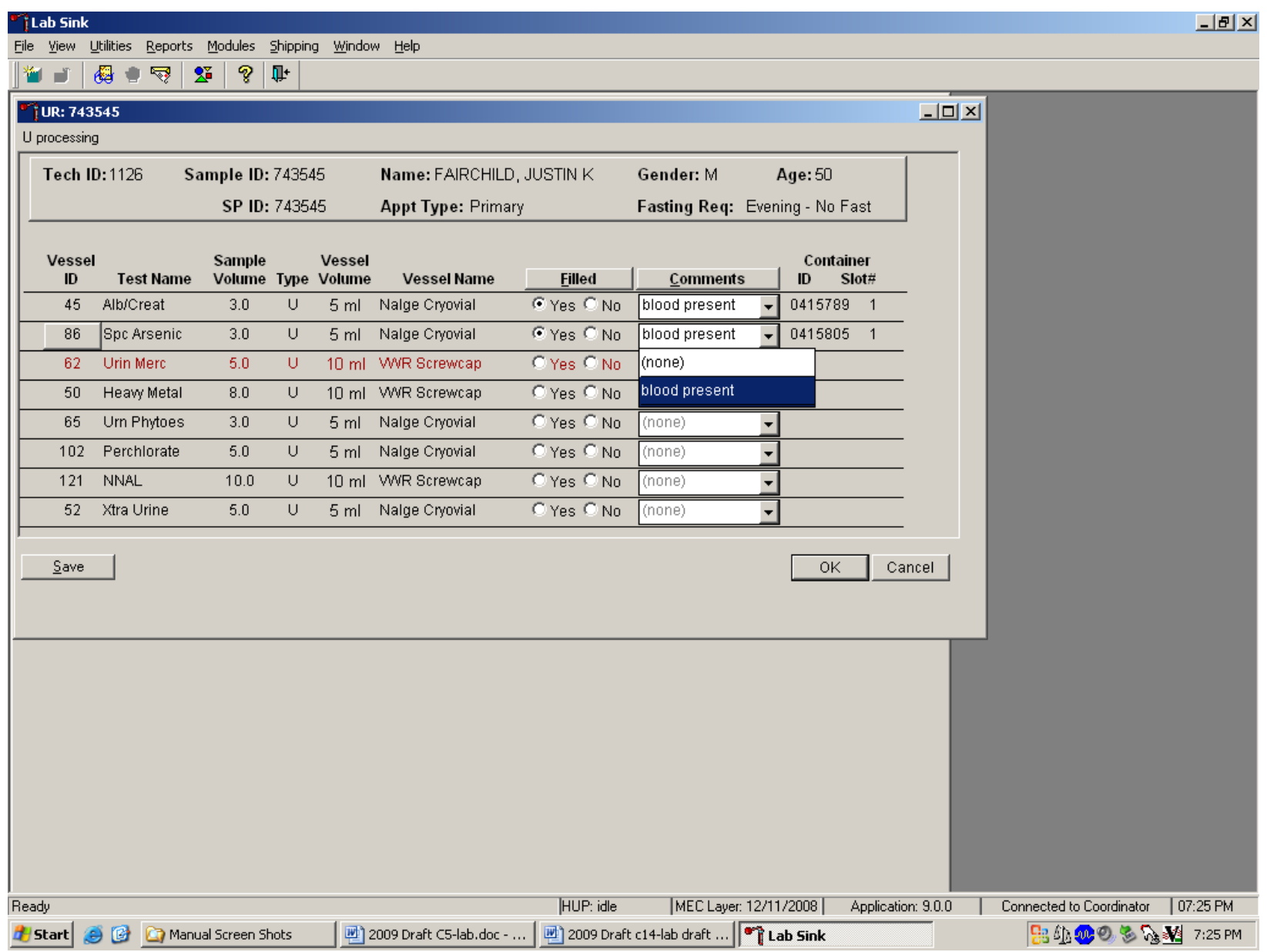

To enter the quality control comment "blood present" for a filled vessel, use the mouse to direct the mouse arrow to the drop-down list in the Comments column, left click to display the choices, drag the mouse arrow to \{blood present , and left click. 
If a vessel is marked as Filled-"No," the comment "quantity not sufficient” is automatically entered. Review the comment for all insufficient urine collections and correct the comment, when necessary.

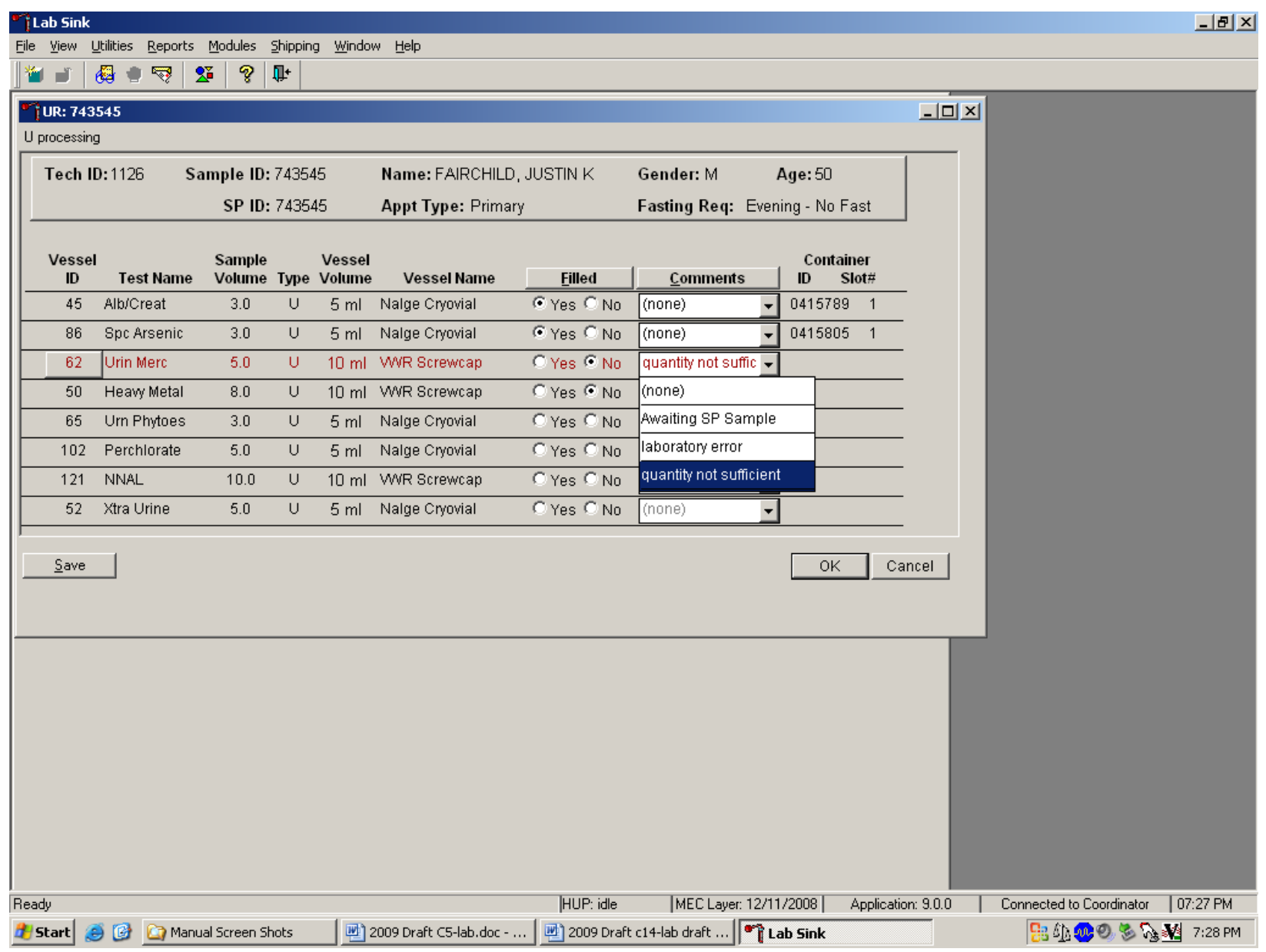

\begin{tabular}{|l|l|}
\hline Comment Code & Use when: \\
\hline \hline laboratory error & A technologist makes an inadvertent error when processing \\
\hline quantity not sufficient & The amount of urine received in the lab is insufficient to fill this vessel \\
\hline
\end{tabular}

Continue recording and updating urine collection results as specimens are delivered to the laboratory. 
Review the information in the urine-processing window and save the data to the database.

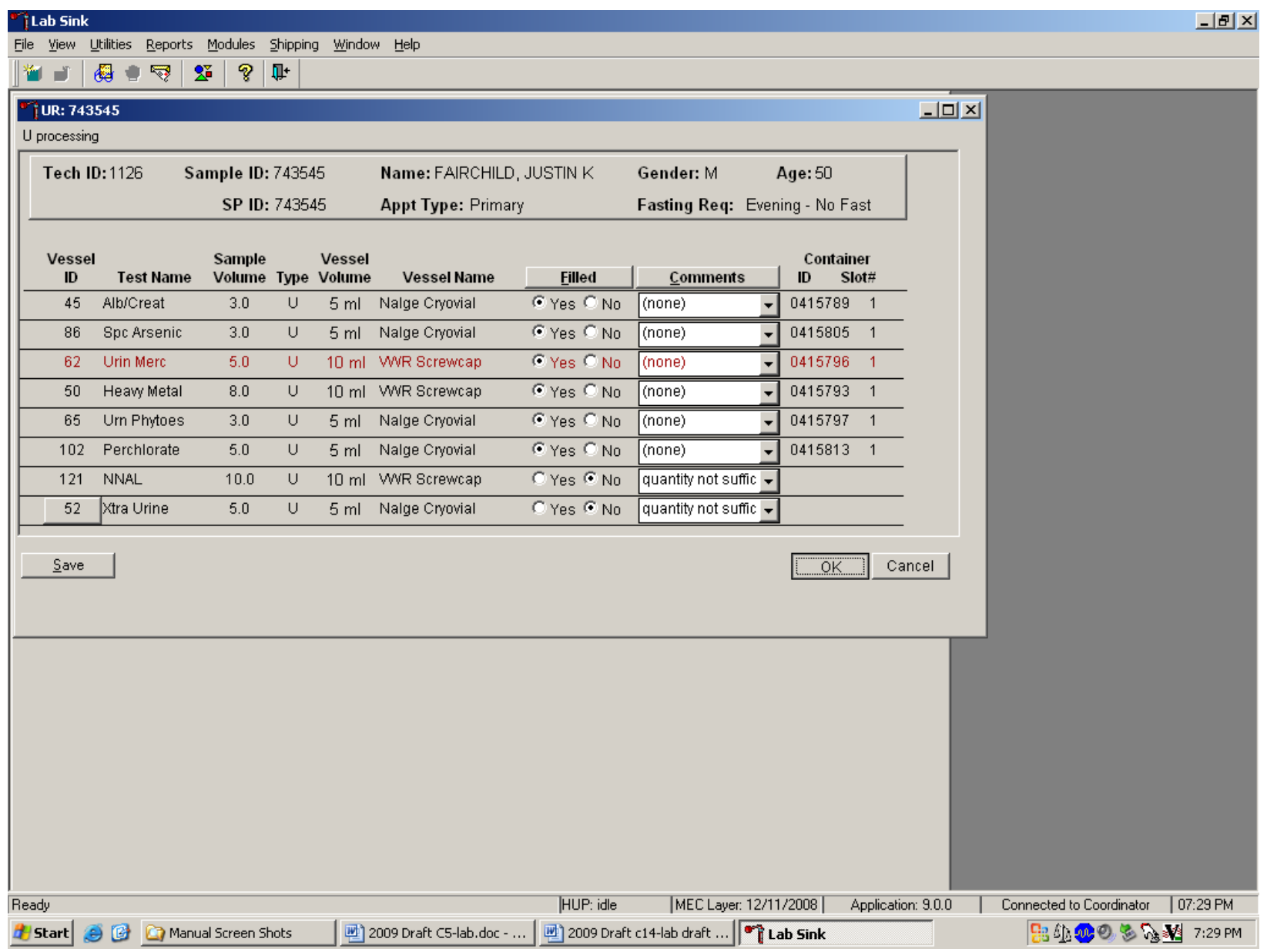

Store each filled vessel in the assigned slot in the assigned container. To record this action or to save this data to the database, use the mouse to direct the mouse arrow to the "Save" button and left click. To record this action or to save the data to the database and exit the module, use the mouse to direct the mouse arrow to the "OK" button and left click. To close the window without saving any data in the database, use the mouse to direct the mouse arrow to the "Cancel” button and left click. 


\subsection{Second and Third Urine Samples}

If the initial urine sample does not meet the minimum volume requirement, refrigerate the initial urine until enough additional urine is received in the second or third urine collection to meet the minimum requirements to complete the protocol or the session ends. If a subsequent sample collection fulfills the protocol requirement, pool the initial and subsequent urine samples into the most recently collected specimen urine cup, update the Urine Collection module, access the Urine Processing module, fill the vessels, and record the results. If the session ends and no additional urine has been collected, access the Urine Processing module, fill as many vessels as possible in priority order, mark the remaining vessels as filled "No," with the comment code "quantity not sufficient," and save the results.

\subsection{Specimen Storage}

Fill the urine vessels, enter the urine processing results, and prepare to store the vessels. Store vessels in numbered storage boxes according to test as indicated in Table 5-3. Check the slot assignment of each urine processing container at the end of each processing session by accessing the Container Map Report. Verify the contents of each container against the container map.

At the beginning of each stand, generate and print a series of bar-coded, numbered labels for storage containers as described in Chapter 9. Use the shipping module to assign a bar-code label to a specific storage container (test). This process “opens” a storage box. Each vessel is assigned to a specific slot in a specific container as processing results are entered. Slots in containers are assigned according to a standard left to right, top to bottom procedure. Store each vessel in the appropriate slot in the correct container immediately after processing. 
Table 5-3. Storage and shipping protocol for urine

\begin{tabular}{|c|c|c|c|c|c|}
\hline $\begin{array}{c}\text { Lab } \\
\#\end{array}$ & Shipping location & Vessels & Conditions & Frequency & Vessel storage \\
\hline 16 & $\begin{array}{l}\text { University of } \\
\text { Minnesota }\end{array}$ & 45 Alb/Creat & Frozen & Weekly & 9x9 three inch box \\
\hline 5 & CDC/NCID NCHSTD & 46 Chlam & Frozen & Weekly & $5 \times 5$ three inch box \\
\hline 61 & CASPIR & 86 Spc Arsenic & Frozen & Weekly & 9x9 three inch box \\
\hline 26 & $\begin{array}{l}\text { CDC/NCEH Pesticide } \\
\text { Laboratory }\end{array}$ & 48 Prior Pest & Frozen & End of stand & $5 \times 5$ three inch box \\
\hline 26 & $\begin{array}{l}\text { CDC/NCEH Pesticide } \\
\text { Laboratory }\end{array}$ & 49 Organophos & Frozen & End of stand & $5 \times 5$ three inch box \\
\hline 61 & CASPIR & 62 Urin Merc & Frozen & Weekly & $5 \times 5$ three inch box \\
\hline 61 & CASPIR & 50 Heavy Metal & Frozen & Weekly & 5x5 three inch box \\
\hline 54 & CASPIR & 65 Urn Phytoes & Frozen & End of stand & 10x10 three inch box \\
\hline 31 & CASPIR & $66 \mathrm{PAH}$ & Frozen & End of stand & 9x9 three inch box \\
\hline 24 & $\begin{array}{l}\mathrm{CDC} / \mathrm{NCEH} \\
\text { Environmental Health } \\
\text { Laboratory }\end{array}$ & 67 Phthalates & Frozen & End of stand & 9x9 three inch box \\
\hline 62 & CASPIR & 102 Perchlorate & Frozen & End of stand & 9x9 three inch box \\
\hline 59 & CASPIR & $121 \mathrm{NNAL}$ & Frozen & End of stand & $5 \times 5$ three inch box \\
\hline 54 & CASPIR & 128 Caffeine & Frozen & Weekly & 10x10 two inch box \\
\hline 6 & CASPIR & 52 Xtra Urine & Frozen & End of stand & 9x9 three inch box \\
\hline
\end{tabular}

\subsection{How to Deal With System Failure}

If the computer system fails, record results on a preprinted workstation 2 processing worksheet (Exhibit 5-3). Complete a workstation 2 worksheet for each SP after performing the pregnancy test, and while processing the urine specimens. Enter the results after the system is operational. Send the worksheets to the home office at the end of the stand. 
Exhibit 5-4. Workstation 2 processing worksheet

URINE COLLECTION, PREGNANCY TEST, URINE PROCESSING

SP ID

\begin{tabular}{|c|c|c|c|c|c|c|c|}
\hline & \multicolumn{2}{|c|}{$\begin{array}{l}\text { Urine Collected } \\
\text { Age } 6+\end{array}$} & $\begin{array}{l}\text { Yes } \\
\checkmark\end{array}$ & $\begin{array}{c}\text { Yes/QNS } \\
\checkmark\end{array}$ & $\begin{array}{l}\text { No } \\
\checkmark\end{array}$ & \multicolumn{2}{|c|}{$\begin{array}{l}\text { Comments: } \\
\text { Communication problem } \\
\text { No time } \\
\text { Quantity not sufficient } \\
\text { SP ill/emergency } \\
\text { SP refusal } \\
\text { SP unable to comply }\end{array}$} \\
\hline \multicolumn{8}{|c|}{ Result } \\
\hline & \multicolumn{2}{|c|}{$\begin{array}{l}\text { Pregnancy Test } \\
\text { Age 8-17 }\end{array}$} & Negative & $\begin{array}{l}\text { Invalid } \\
\checkmark\end{array}$ & $\begin{array}{l}\text { Positive } \\
\checkmark\end{array}$ & \multicolumn{2}{|c|}{$\begin{array}{l}\text { Serum Confirmation Result } \\
\text { (Send observation to physician) }\end{array}$} \\
\hline \multicolumn{8}{|c|}{ Result } \\
\hline & \multicolumn{2}{|c|}{$\begin{array}{l}\text { Pregnancy Test } \\
\text { Age 18-59 }\end{array}$} & Negative & $\stackrel{\text { Invalid }}{\checkmark}$ & Positive & & \\
\hline \multicolumn{8}{|c|}{ Result } \\
\hline ID & Name & Ages & Sample $\mathrm{mL}$ & $\begin{array}{l}\text { Sample } \\
\text { Type }\end{array}$ & $\begin{array}{l}\text { Filled } \\
\checkmark\end{array}$ & $\begin{array}{l}\text { Comments } \\
\frac{\text { QNS }}{\text { Blood Present }}\end{array}$ & Slot \# \\
\hline 45 & Alb/Creat & $6+$ & 3 & Urine & & & \\
\hline 46 & Chlam & $14-39$ & 10 & Urine & & & \\
\hline 86 & Spc Arsenic & $6+$ & 3 & Urine & & & \\
\hline 48 & Prior Pest & $6+$ & 8 & Urine & & & \\
\hline 49 & Organophos & $6+$ & 7 & Urine & & & \\
\hline 62 & Urn Merc & $6+$ & 5 & Urine & & & \\
\hline 50 & Heavy Metal & $6+$ & 8 & Urine & & & \\
\hline 65 & Urn Phytoes & $6+$ & 3 & Urine & & & \\
\hline 66 & PAH & $6+$ & 3 & Urine & & & \\
\hline 67 & Phthalates & $6+$ & 3 & Urine & & & \\
\hline 102 & Perchlorate & $6+$ & 5 & Urine & & & \\
\hline 121 & NNAL & $6+$ & 10 & Urine & & & \\
\hline 128 & Caffeine & $6+$ & 1 & Urine & & & \\
\hline 52 & Xtra Urine & $6+$ & 5 & Urine & & & \\
\hline
\end{tabular}




\section{National Health and Nutrition Examination Survey National Center for Health Statistics \\ United States Public Health Service \\ Procedure: Pregnancy Testing \\ January 2009}

Prepared by

Date Adopted

Approved by

Director

Signed

Director

Dated

Director

Reviewed by

Date Reviewed

Reviewed by

Date Reviewed

Reviewed by

Date Reviewed

Reviewed by

Date Reviewed

Reviewed by

Date Reviewed

Reviewed by

Date Reviewed

Reviewed by

Date Reviewed

Reviewed by

Date Reviewed

Reviewed by

Date Reviewed 
THIS PAGE INTENTIONALLY LEFT BLANK. 


\section{URINE PREGNANCY TEST}

I. Purpose and Principle of the Test

Perform a pregnancy test on females of all appointment types who are aged 12-59 years, and girls 8-11 who report that they are menstruating when asked during the home interview. A positive test result excludes pregnant women aged 8-59 from participating in the dual-energy x-ray absorptiometry (DXA) section of the body composition component of the MEC exam. Report test results immediately since these components are not assigned until the results of the pregnancy test are documented. If a urine pregnancy test is positive on any female SP aged 8-17 years, the result is confirmed using a serum test. If no blood is drawn, repeat the urine test. Notify the physician of the second positive or negative confirmatory test or the inability to perform a confirmation test using the observation function.

Tests for confirming pregnancy are based on detecting elevated levels of human chorionic gonadotropin (hCG), a hormone that the placenta begins to produce in increasing amounts shortly after fertilization. The Icon ${ }^{\circledR} 25$ hCG (Urine/Serum) test kit is a rapid chromatographic immunoassay for the qualitative detection of human chorionic gonadotropin (hCG) in urine or serum to aid in the early detection of pregnancy.

In normal pregnancy, hCG can be detected in both urine and serum as early as 7 to 10 days after conception. The hCG levels continue to rise very rapidly, frequently exceeding $100 \mathrm{mIU} / \mathrm{mL}$ by the first missed menstrual period, and peaking in the 100,000 to $200,000 \mathrm{mIU} / \mathrm{mL}$ range about $10-12$ weeks into pregnancy. The appearance of hCG in both urine and serum soon after conception, and its subsequent rapid raise in concentration during early gestational growth, make an excellent marker for the detection of pregnancy.

The Icon ${ }^{\circledR} 25$ hCG test is a rapid test that qualitatively detects the presence of hCG in urine or serum sample at the sensitivity of $25 \mathrm{mIU} / \mathrm{mL}$. The test utilizes a combination of monoclonal and polyclonal antibodies to selectively detect elevated levels of hCG in urine or serum. At the level of claimed sensitivity, the ICON ${ }^{\circledR} 25$ hCG test shows no cross-reactivity interference from the structurally related glycoprotein hormones hFSH, hLH, and hTSH at high physiological levels. 
The assay is conducted by adding urine or serum sample to the sample well of the test device. The sample migrates via capillary action along the membrane to react with colored conjugate. Positive samples react with the specific antibody-hCG-colored conjugate to form a colored line at the test region of the membrane. Absence of this colored line suggests a negative result. To serve as a procedural control, a colored line will always appear at the control line region if the test has been performed properly.

II. Special Safety Precautions

ICON® 25 hCG Test (Urine or Serum)

- This test is for professional in vitro diagnostic use only.

- Do not use the kit beyond the expiration date.

- The test device should remain in the sealed pouch until use.

- Observe standard guidelines for handling biological hazards.

- Wear gloves while handling specimens. After use, dispose of gloves and other contaminated materials appropriately in a proper biohazard container and wash hands.

Sure-Vue ${ }^{\mathrm{TM}}$ hCG Urine and Serum Control Sets

- Sure-Vue ${ }^{\mathrm{TM}}$ hCG urine and serum controls are intended to be used to monitor the performance and accuracy of pregnancy test kits. These controls are for professional use only.

- The use of known controls in the laboratory is invaluable. It is important to verify testing procedures to confirm the validity of the results reported. Testing Sure-Vue ${ }^{\mathrm{TM}}$ hCG Urine and Serum Controls will provide assurance that the pregnancy test kit is performing properly.

- Reagents in these kits contain 0.2 percent of sodium azide, which may react with lead or copper plumbing to form potentially explosive metal azides. When disposing of such reagents, always flush with large volumes of water to prevent azide buildup. This reagent is harmful if swallowed. Contact with acids liberates very toxic gas. After contact with skin, wash immediately with plenty of water.

- Do not use the controls beyond the expiration date. 


\section{Computerization: Integrated Survey and Information System (ISIS)}

The MEC-automated ISIS system captures all data and stores it electronically at Westat's home office in Rockville, Maryland. Access the Pregnancy QC module to enter the quality control results. The session number, technologist ID, and run number are automatically captured; enter the kit lot number and expiration date, and the urine and serum control lot and expiration dates. Record control results and save them to the database. All pregnancy quality control results are immediately available electronically at the home office. All data are backed up and stored at Westat's home office.

\section{Specimen Collection and Preparation}

Collect a urine specimen on all SPs 6 years and older into a clean and dry plastic container and document the collection in the urine collection module as described in Chapter 5. Perform a urine pregnancy test on females of all person types who are aged 12-59 years and girls aged 8-11 who report that they are menstruating when asked during the home interview. If a urine pregnancy test is positive on any female SP aged 8-17, confirm the result using serum. If no blood is drawn, repeat the urine test. Notify the physician of the second positive or negative confirmatory test or the inability to perform a confirmation test using the observation function. Assay all specimens immediately and record results in the Laboratory Pregnancy Testing module.

V. Procedure for Microscopic Examination

Not applicable. 
VI. Reagents and Supplies

A. Reagents

ICON® 25 hCG Urine/Serum test kit

Product Number 43025 (25)

Beckman Coulter

4300 N. Harbor Blvd

Fullerton, CA 92834-3100

$1-800-877-6242$ or $650-845-3526$

- Components

- $\quad$ One test device. (25) - Contains anti-hCG particles and anti-hCG coating on the membrane.

- $\quad$ Disposable sample droppers - Plastic pipettes for measuring and dispensing patient samples. (Packaged together with the test device in a white foil pouch.)

- $\quad$ Zip closable bag with two extra sample droppers

- $\quad$ Product instructions

- Storage and Stability

- $\quad$ Store the ICON 25 hCG test kit at $2-30^{\circ} \mathrm{C},\left(59-83^{\circ} \mathrm{F}\right)$. The test device is stable up to the expiration date printed on the sealed pouch. Keep the test device in the sealed pouch until use. DO NOT FREEZE. Do not use beyond expiration date. Date and initial the kit when putting it into use.

Sure-Vue ${ }^{\mathrm{TM}}$ hCG Control Sets - Urine and Serum

Sure-Vue ${ }^{\mathrm{TM}}$ hCG Control Sets - Urine

Product Number SA087413-F

Fisher Scientific Company

1-888-727-3315 (Technical assistance)

- Components - Sure-Vue ${ }^{\mathrm{TM}}$ hCG Control Set

- $\quad$ Urine is a stable human urine-based material for use as a quality control material. Use controls in the same manner as SP specimens in accordance with the protocol provided in the Sure-Vue ${ }^{\mathrm{TM}}$ hCG Control Sets directional insert. 
- $\quad$ Low Positive Control: Human urine containing approximately 25 mIU hCG/mL and $0.2 \%$ sodium azide as a preservative. ( $1 \times 5 \mathrm{~mL}$ )

- High Positive Control: Human urine containing approximately $250 \mathrm{mIU}$ hCG $/ \mathrm{mL}$ and $0.2 \%$ sodium azide as a preservative. ( 1 x $5 \mathrm{~mL}$ )

- $\quad$ Negative Control: Contains $0.2 \%$ sodium azide as a preservative. ( 1 x $5 \mathrm{~mL}$ )

- $\quad$ Storage and Stability - Sure-Vue ${ }^{\mathrm{TM}}$ hCG Control Set

- $\quad$ Store the urine control set between 2 to $8^{\circ} \mathrm{C}\left(35\right.$ to $\left.46^{\circ} \mathrm{F}\right)$ at which temperatures these controls are stable until the expiration date printed on the label.

Sure-Vue ${ }^{\mathrm{TM}}$ hCG Serum Control Set

Product Number 087712-F

Fisher Scientific Company

1-888-727-3315 (Technical assistance)

- Components - Sure-Vue ${ }^{\mathrm{TM}}$ hCG Serum Control

- $\quad$ The Sure-Vue ${ }^{\mathrm{TM}}$ hCG Serum Control is a stable human serum-based material for use as a quality control material. Use controls in the same manner as SP specimens in accordance with the protocol provided in the Sure-Vue ${ }^{\mathrm{TM}}$ hCG Control Set directional insert.

- Low Positive Control: Human serum containing approximately $25 \mathrm{mIU}$ hCG $/ \mathrm{mL}$ and $0.2 \%$ sodium azide as a preservative. ( 1 x $5 \mathrm{~mL}$ )

- High Positive Control: Human serum containing approximately $250 \mathrm{mIU}$ $\mathrm{hCG} / \mathrm{mL}$ and $0.2 \%$ sodium azide as a preservative. ( 1 x $5 \mathrm{~mL}$ )

- $\quad$ Negative Control: Contains $0.2 \%$ sodium azide as a preservative. ( 1 x $5 \mathrm{~mL}$ )

- Storage and Stability - Sure-Vue ${ }^{\mathrm{TM}}$ hCG Serum Control

- $\quad$ Store the Sure-Vue ${ }^{\mathrm{TM}}$ hCG Serum Control Set between 2 to $8^{\circ} \mathrm{C}$ ( 35 to $46^{\circ} \mathrm{F}$ ) at which temperatures these controls are stable until the expiration date printed on the label.

B. Supplies

- Timer 
VII. Calibration

Not applicable

VIII. Assay Procedure

ICON® 25 hCG Test kit

- Label each test device with the preprinted label or write the SP ID on a clear section of the test device using a felt tip pen.

- Use a new transfer dropper for each specimen.

Sure-Vue ${ }^{\mathrm{TM}}$ hCG Urine and Serum Control Sets

- The Sure-Vue ${ }^{\mathrm{TM}}$ urine and serum controls have their own droppers. The use of the control droppers to add three drops of sample has been validated; do not use the ICON 25 hCG plastic pipette to add controls.

- Allow controls to reach room temperature before testing. The controls are ready to use. No dilution is required.

- The controls are used in place of the specimen and should be tested according to the ICON® 25 hCG test procedure.

- Discard the high positive urine and serum vials $(250 \mathrm{mIU} / \mathrm{mL})$.

Urine or Serum Testing Procedure

Perform the test procedure for ICON® 25 hCG Urine/Serum test at room temperature (15 $\left.30^{\circ} \mathrm{C}, 59-86^{\circ} \mathrm{F}\right)$. Before proceeding, carefully read Section XII titled "Limitations of Method: Specimen Rejection, Interfering Substances, and Conditions.”

1. Bring the pouch to room temperature before opening it. Remove the test device from the sealed pouch and use it as soon as possible.

2. Place the test device on a clean and level surface. Hold the dropper vertically and transfer three full drops of urine or serum (approximately $100 \mu \mathrm{L}$ ) to the sample well 
(S) of the test device, and then start the timer. Avoid trapping air bubbles in the sample well.

3. Wait for the red line(s) to appear. Read the results at 3 minutes when testing a urine sample or 5 minutes when testing a serum sample. It is important that the background is clear before the result is read.

NOTE: A low hCG concentration might result in a weak line appearing in the test region (T) after an extended period; therefore, do not interpret the result after 3 minutes when testing a urine sample or after 5 minutes when testing a serum sample.

IX. Reportable Range of Results

Report test results as Positive, Negative, or Invalid.

X. Quality Control

Run negative and low positive urine controls containing hCG at concentrations of 0 and 25 $\mathrm{mIU} / \mathrm{mL}$, respectively, just prior to or concurrently with the first examinee pregnancy test each exam day, and when putting a new lot into use. Run negative and low positive serum controls with each serum pregnancy test. The pregnancy QC module contains the following information:

- The session ID number links to the stand number, stand location, date, including day, month, and year. The technologist ID is automatically captured.

- Kit lot number and expiration date fill in automatically from the prior QC report. Update or manually enter this information if necessary.

- The control lot number and expiration date for both the urine and serum control fills in automatically from the prior QC report. Update or manually enter this information if necessary.

- The Control QC Type is either urine or serum. The default type is set as urine.

- Negative Control result.

- Positive Control result. 


\section{CAP Proficiency Testing}

For purposes of this document, proficiency testing is one method of external quality control in which the analytical performance of a method is evaluated using specimens provided on a periodic basis (usually every 3 months).

Participation in the College of American Pathologist (CAP) EXCEL proficiency-testing program is part of the comprehensive quality control program. Each MEC submits results for CAP specimens for evaluation. CAP compares the results to established values and issues a report.

CAP sends samples three times a year for qualitative serum hCG and urine hCG. Each serum survey includes five lyophilized sera specimens and each urine survey includes 5 liquid specimens. Handle and analyze these samples in a manner identical to SP samples. Record results on the CAP forms and send to the address specified by CAP Surveys Program Support. Send a copy of the CAP form to the home office at the end of the stand. 
Open the Pregnancy QC module.

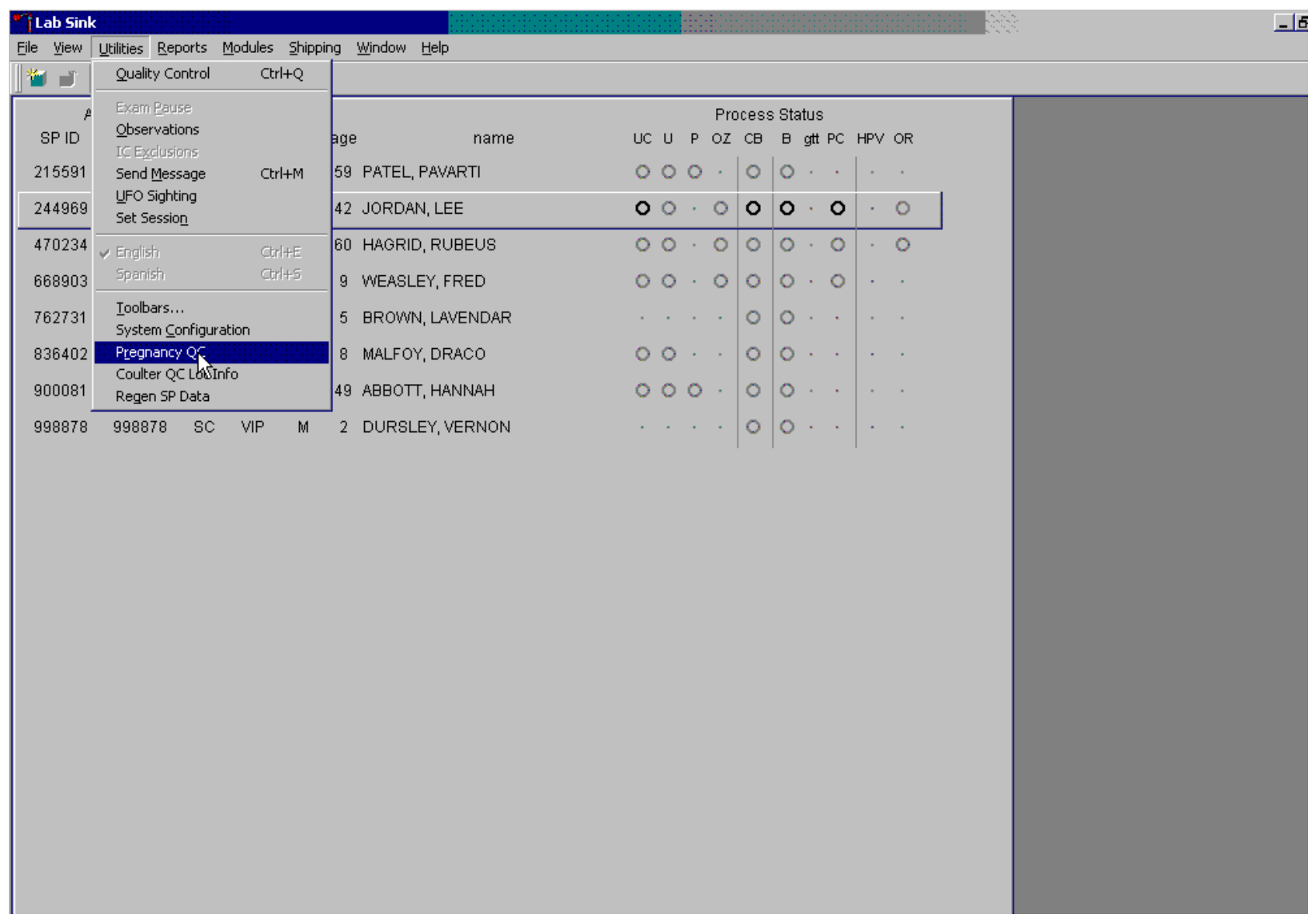

To open the pregnancy QC module, use the mouse to direct the mouse arrow to \{Utilities\} in the top menu bar, drag the arrow to \{Pregnancy QC , and left click or type [Alt] [U/u], [R/r]. 
Alternatively, open the Pregnancy QC module from the heads-up screen.

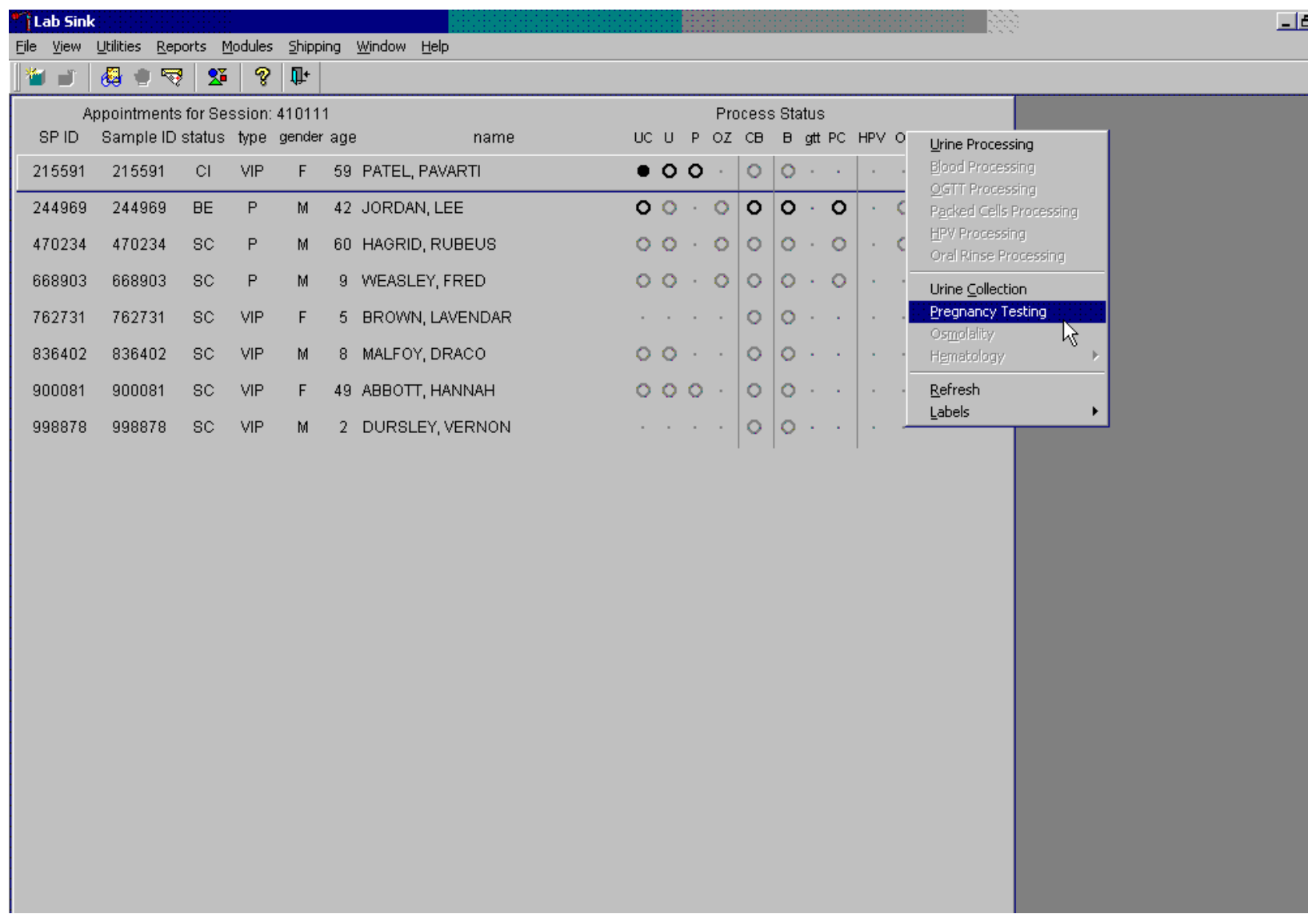

To access the Pregnancy QC module from the heads-up screen, use the up and down keys to move up and down the list until the correct SP is highlighted or use the mouse to direct the mouse arrow to the correct SP, right click, drag the mouse arrow to \{Pregnancy Testing\}, and left click or left click and type $[\mathrm{P} / \mathrm{p}]$. 
The pregnancy QC window displays.

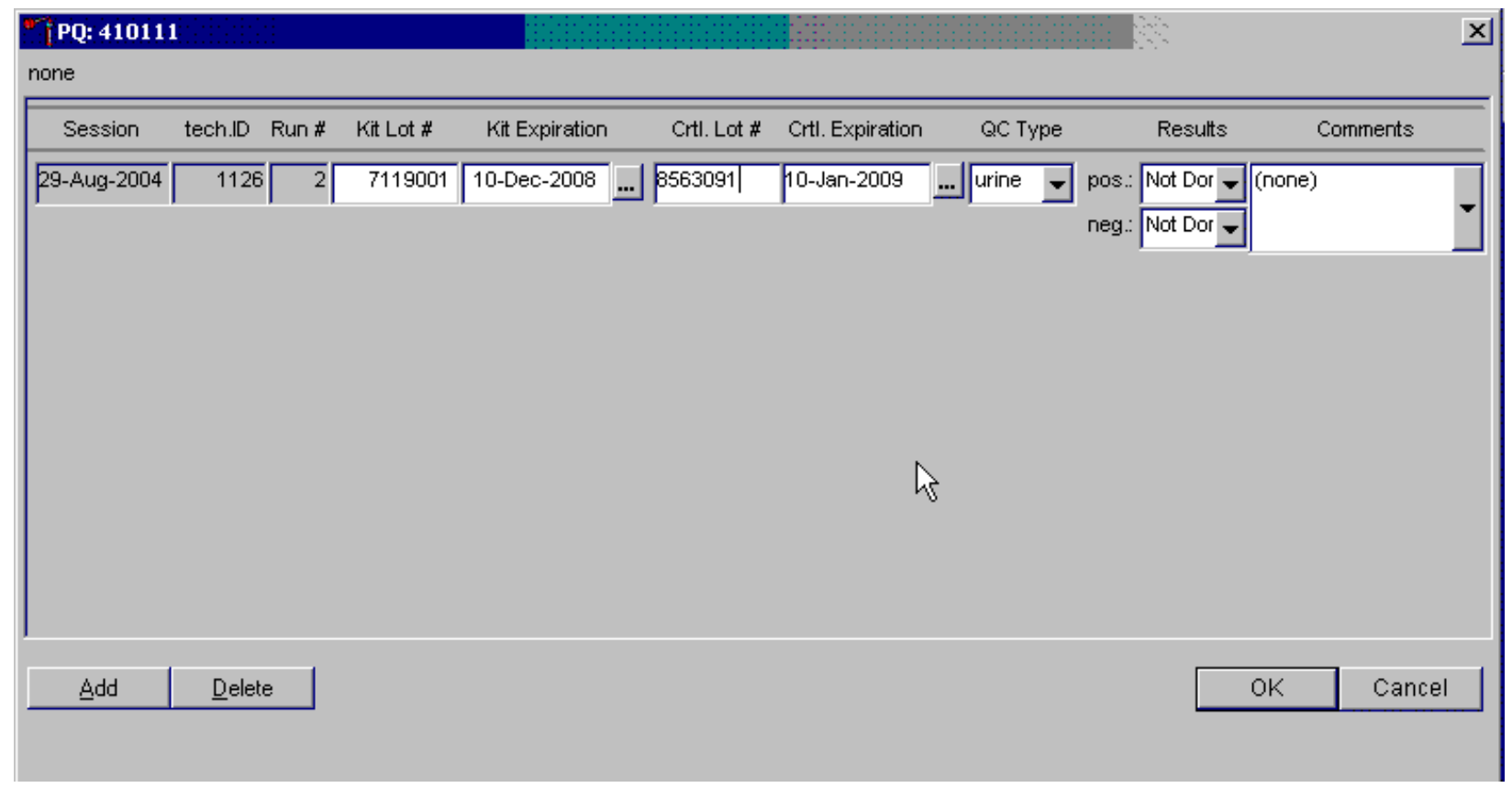

The pregnancy QC module automatically captures the session date and technologist ID and assigns the run number. The module defaults to the last kit lot number, kit expiration date, Ctrl (control) lot number, Ctrl expiration date, and the QC type - urine. Verify the existing kit lot number and expiration date. If the lot number is incorrect, type in the new kit lot number using the keyboard's numeric keys and select [Tab] to progress to the Kit Expiration blank. Use the calendar to correct the Kit Expiration date. If the screen defaults to the current Ctrl lot number, verify the existing lot number and expiration date. If the lot number is incorrect, type in the new control lot number using the keyboard numeric keys and select [Tab] to progress to the Ctrl Expiration blank. Use the calendar to correct either the Kit or the Ctrl Expiration date. 
Update the Kit or Ctrl Expiration date using the calendar function.

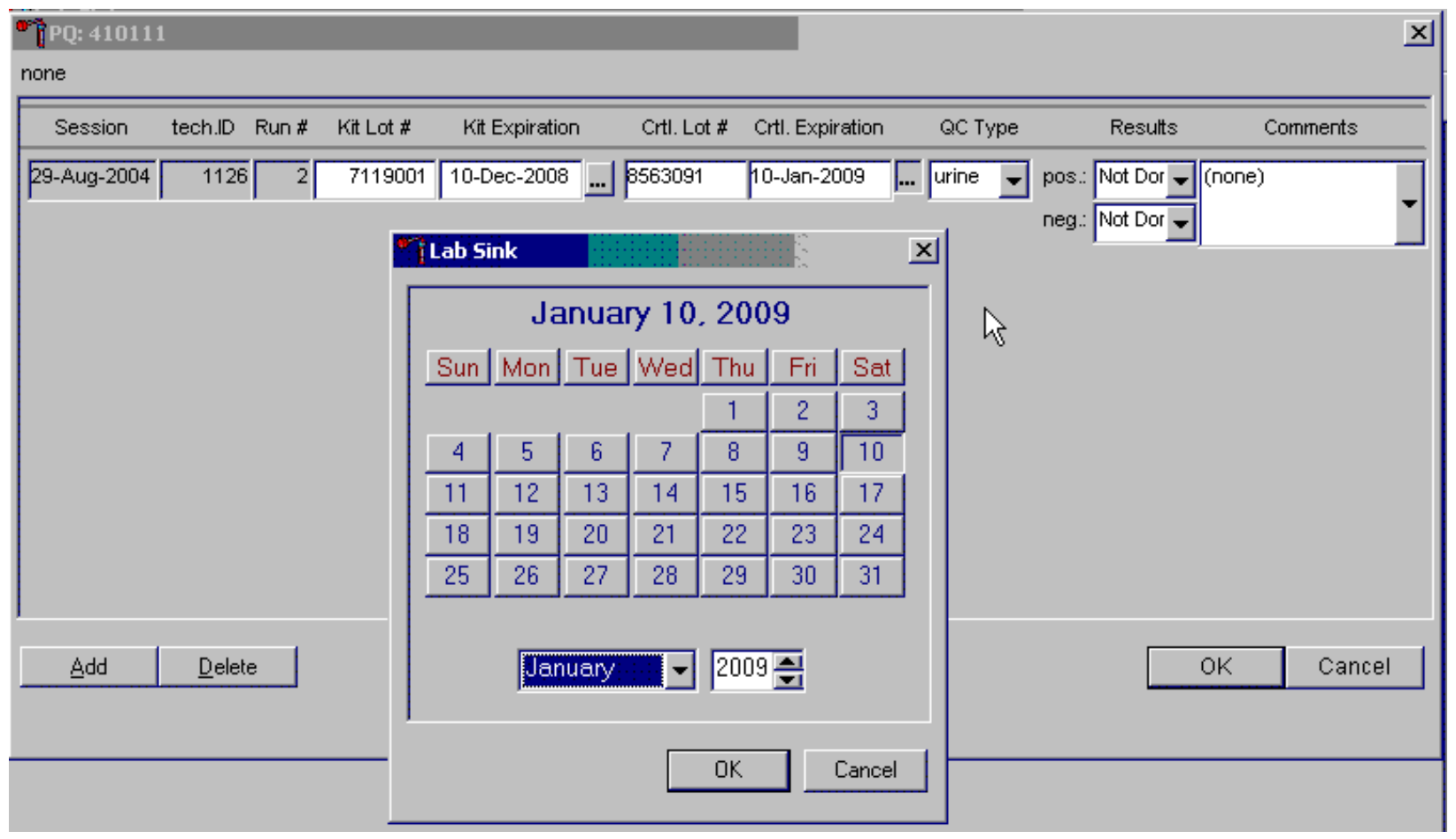

Type in the date using the keyboard's numeric keys and the $\mathrm{dd} / \mathrm{mm} / \mathrm{yyyy}$ format and select [Tab] or use the calendar to enter the date. To access the calendar, use the mouse to direct the mouse arrow to the ellipsis and left click. To select the correct month, use the mouse to direct the mouse arrow to the drop-down list, drag the arrow to the correct month (use the scroll bar if necessary) and left click. To select the correct day, use the mouse to direct the mouse arrow to the correct day on the displayed month and left click. To correct the year, use the mouse to direct the mouse arrow to up/down controls on the spin box and toggle the year up and down. To transfer this date into the text box, use the mouse to direct the mouse arrow to the OK button and left click; to exit the calendar function, use the mouse to direct the mouse arrow to the Cancel button and left click. 
Select the control QC type.

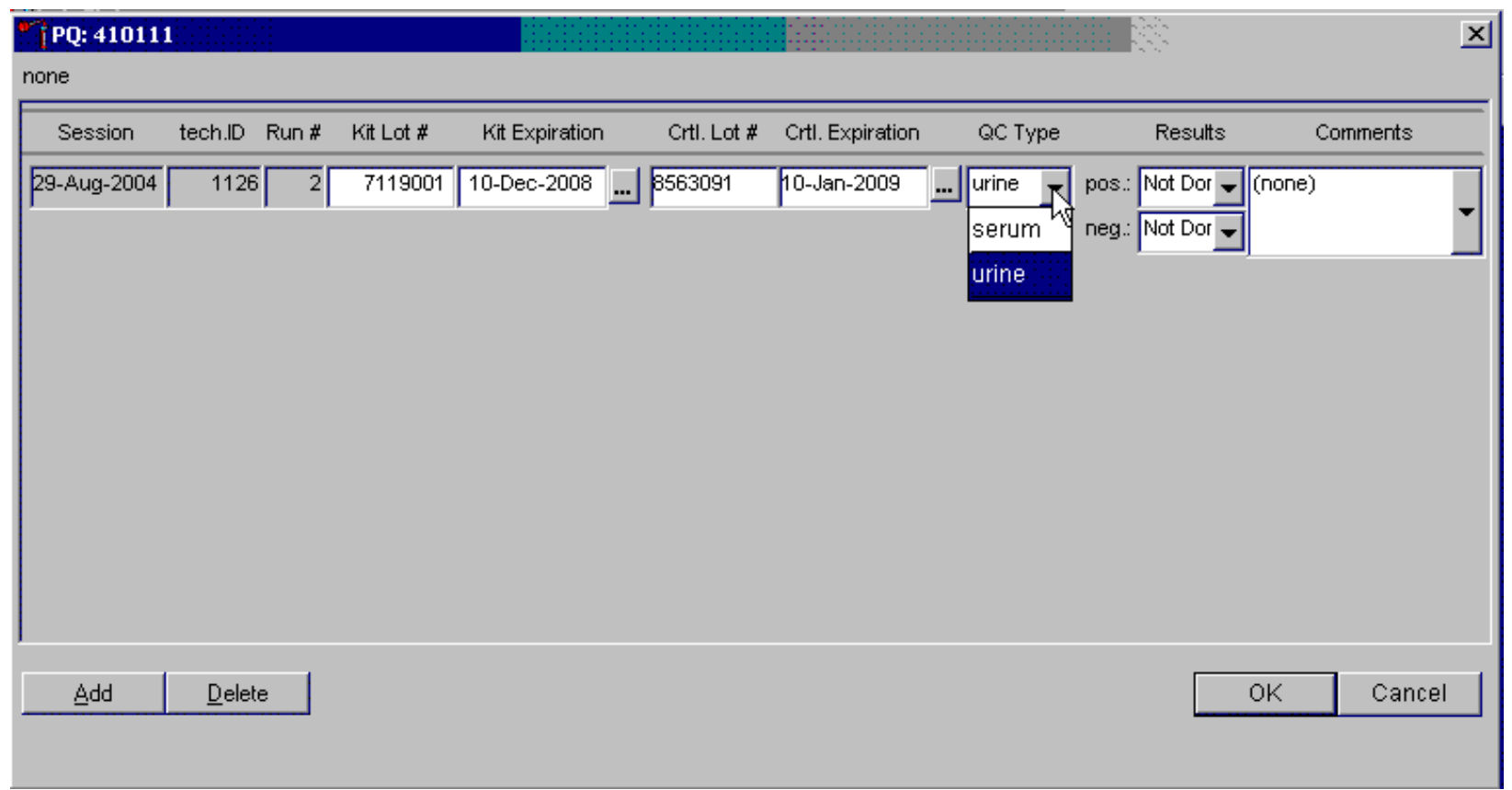

The QC control type default is “Urine.” To change the QC type to serum or to select urine, use the mouse to direct the mouse arrow to the drop-down list, drag the mouse arrow to either serum or urine and left click. Alternatively, highlight the QC type text box and type [S/s] for serum or [U/u] for urine and [Tab] to move to the result test boxes. 
Record the positive and negative control results.

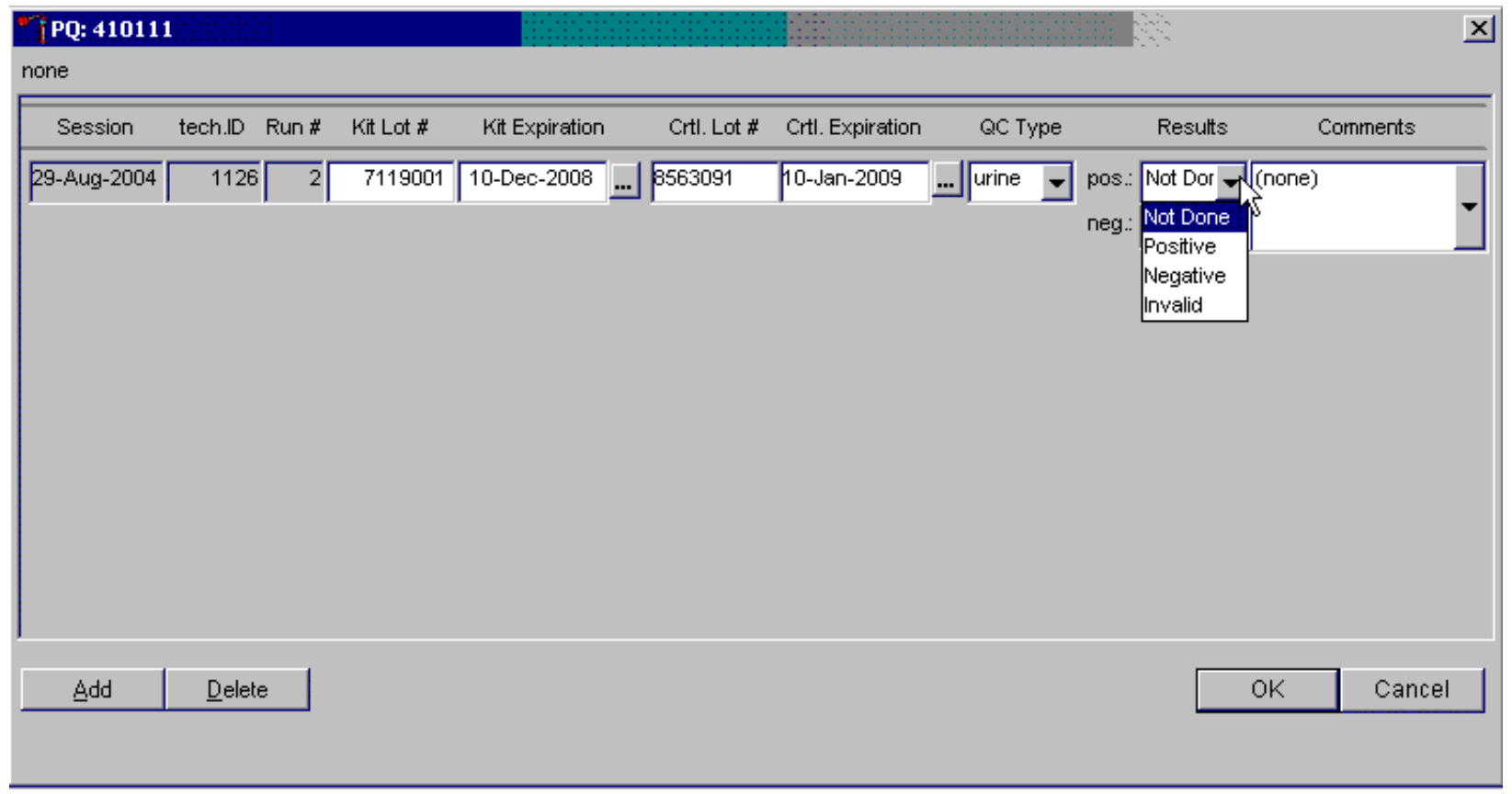

To enter a result in the positive result text box, type [P/p] for "Positive," [N/n] for "Negative," or [I/i] for "Invalid" or use the up and down arrow keys to toggle between the three choices: Positive, Negative, and Invalid and select [Tab]. Alternatively, use the mouse to direct the mouse arrow to the drop-down list, drag the mouse arrow to "Positive," "Negative," or "Invalid" and left click. If "Positive" is entered, the Negative response box is highlighted. Type [Tab] to move to the negative result text box. 
Enter a result in the negative result text box.

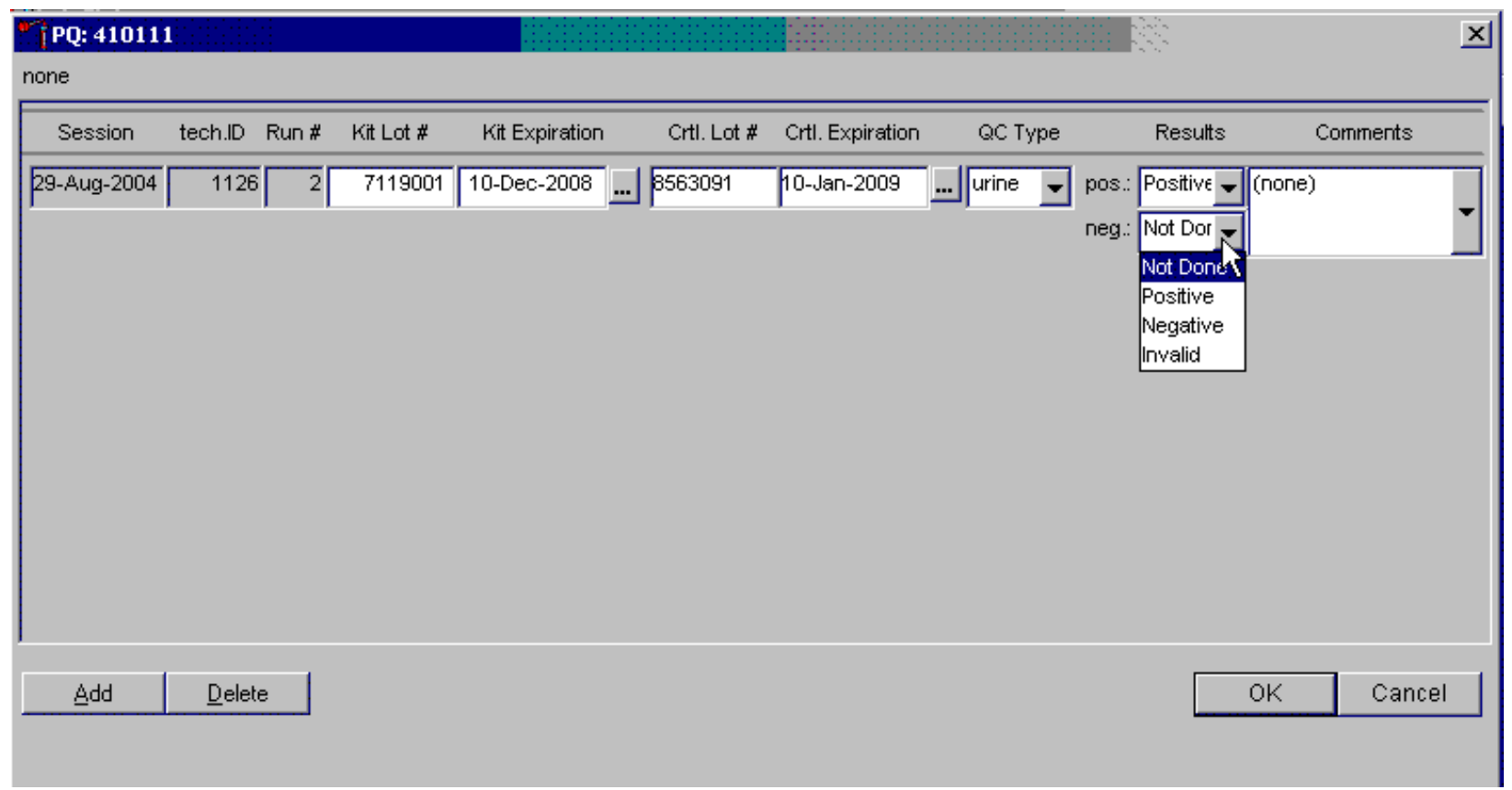

To enter a result in the negative result text box, type [P/p] for "Positive," [N/n] for "Negative," or [I/i] for "Invalid” or use the up and down arrow keys to toggle between the three choices: Positive, Negative, and Invalid and [Tab]. Alternatively, use the mouse to direct the mouse arrow to the drop-down list, drag the mouse arrow to "Positive," "Negative,” or "Invalid,” and left click. 
Save results when positive and negative controls demonstrate the expected result.

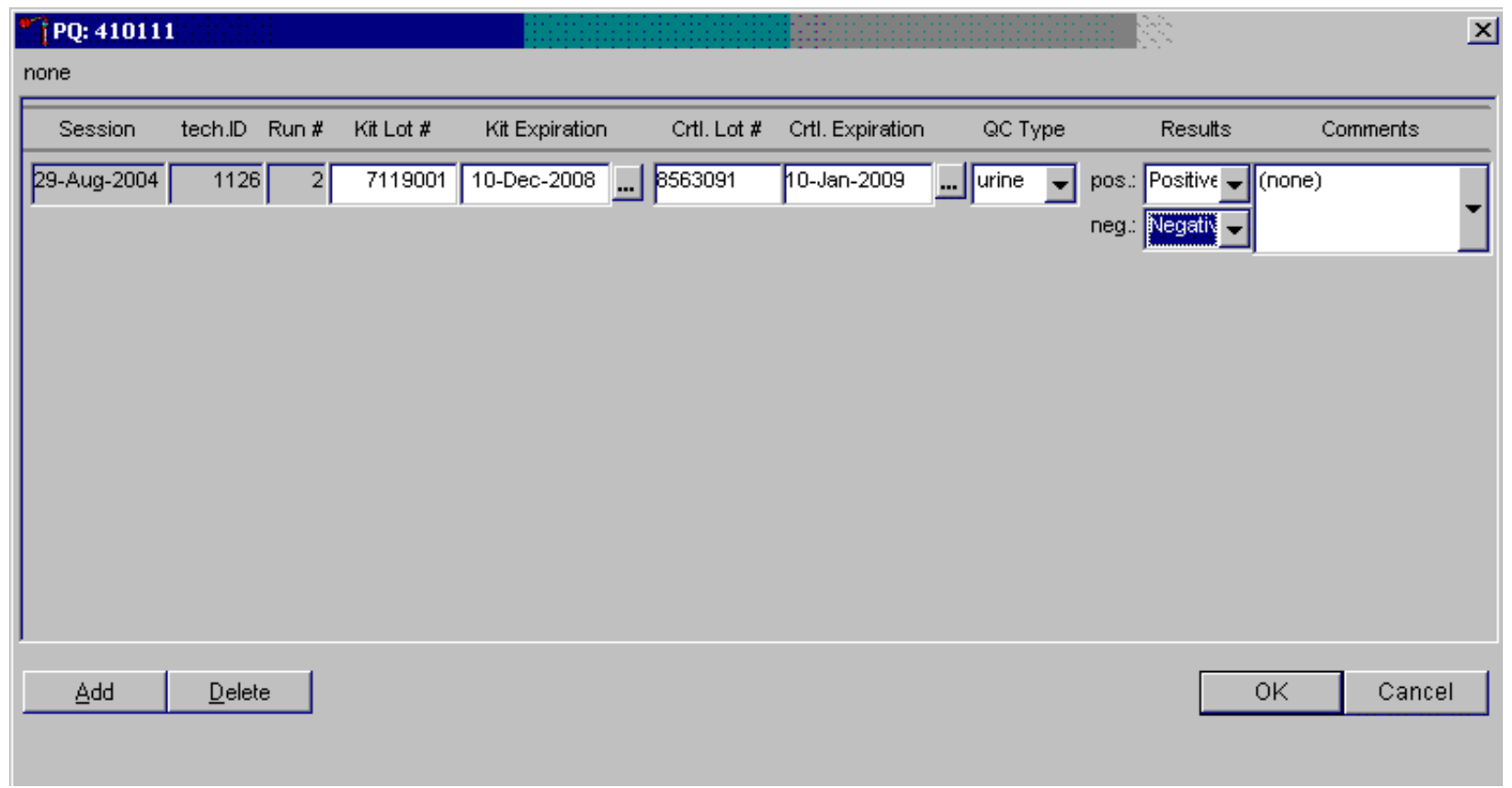

If the positive and negative controls demonstrate the expected result, use the mouse to direct the mouse arrow to the OK button and left click to save these results and to proceed with pregnancy testing. To cancel and exit the module without recording or saving any QC results to the database, use the mouse to direct the mouse arrow to the Cancel button and left click. To delete the information in the window and exit the module without recording or saving any QC results to the database, use the mouse to direct the mouse arrow to the Delete button and left click or type [Shift] [D/d]. 
Enter a comment for any result that is inconsistent with expected results. If the OK button is selected before a comment is entered, the ERROR informational message box displays. Review the text in this box.

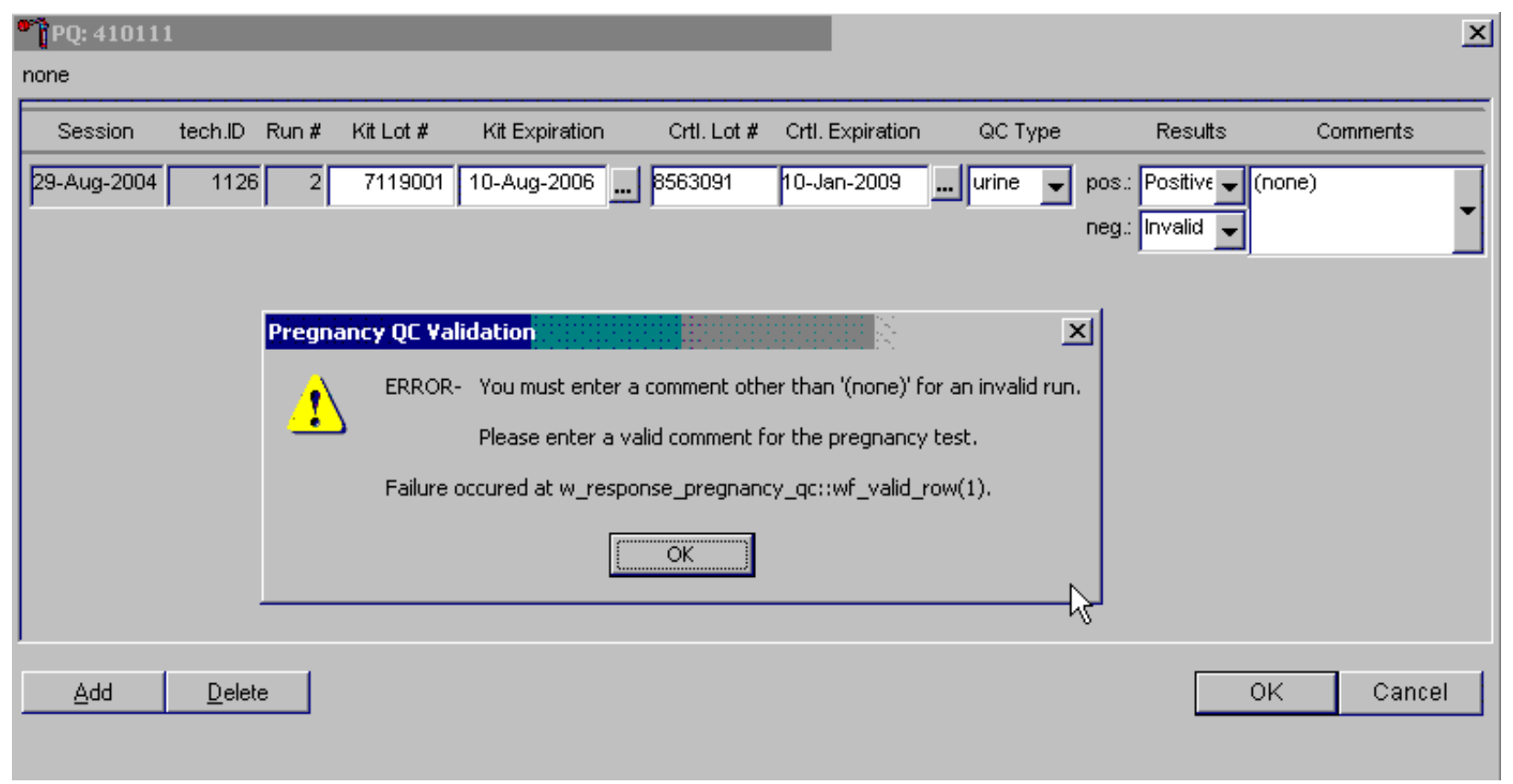

To close the box, use the mouse to direct the mouse arrow to the OK button and left click or select [Enter]. 
Enter a comment for any result that is inconsistent with expected results.

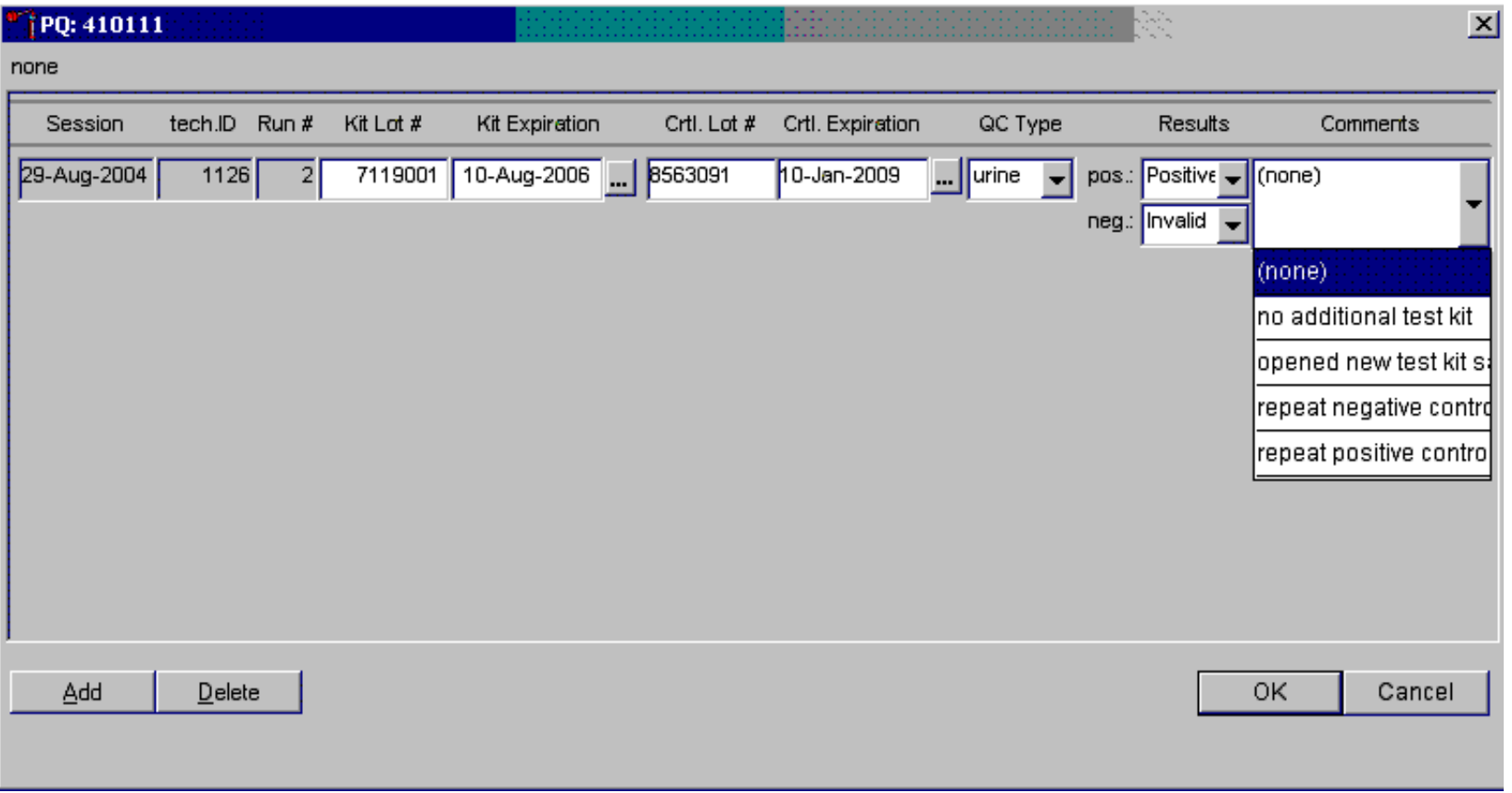

Enter the appropriate comment in the "Comments" column by using the mouse to direct the mouse arrow to the drop-down list, left click to display the choices, drag the mouse arrow to the most appropriate choice, and left click when the correct choice is highlighted. Alternatively, use the up and down keyboard arrows to scroll through the choices or type the first letter of the desired comment code. 
Review the comment.

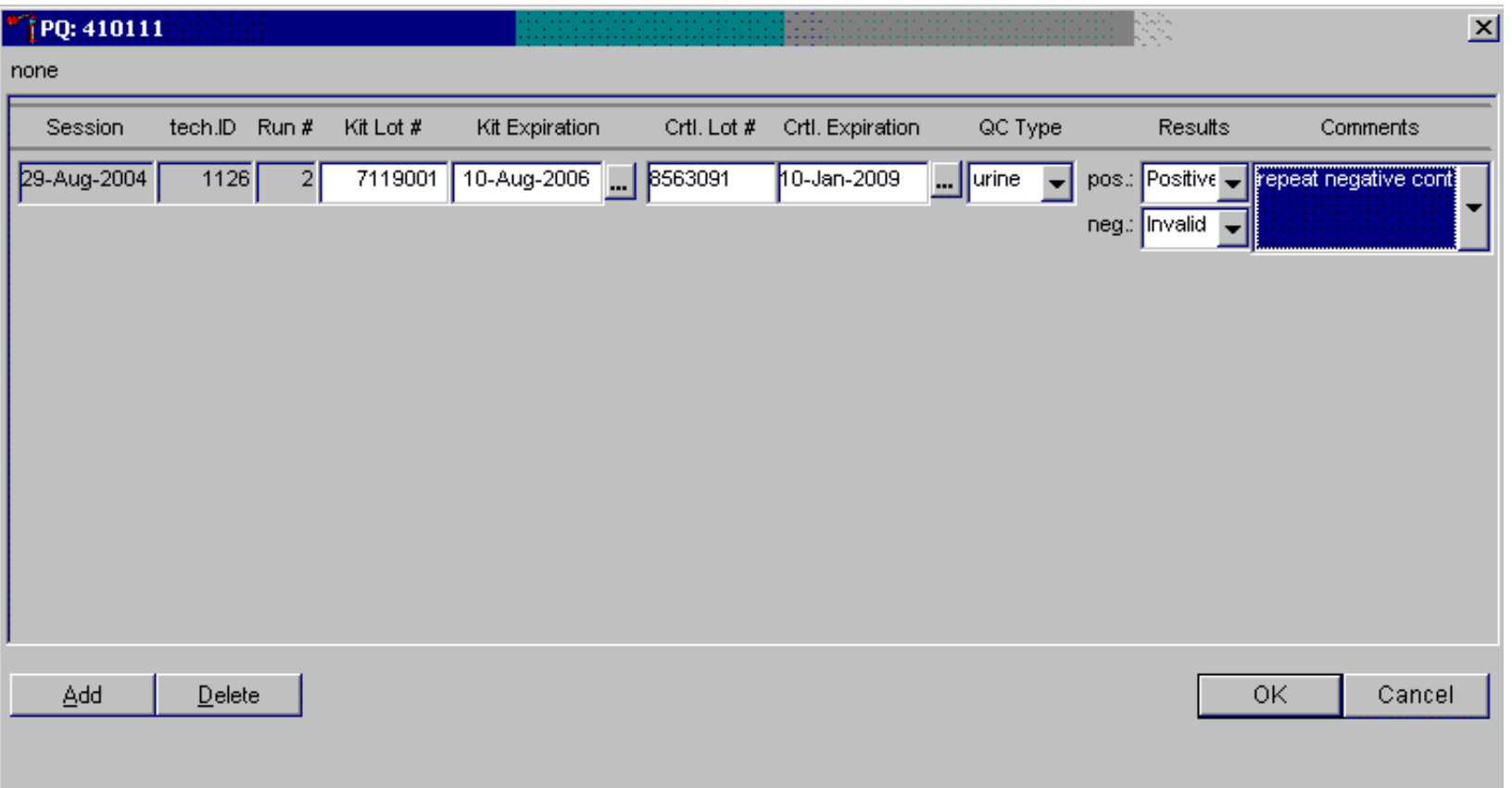

Review all the information in the QC window to verify its accuracy. 
Add a new QC record and repeat the control for invalid or unexpected results.

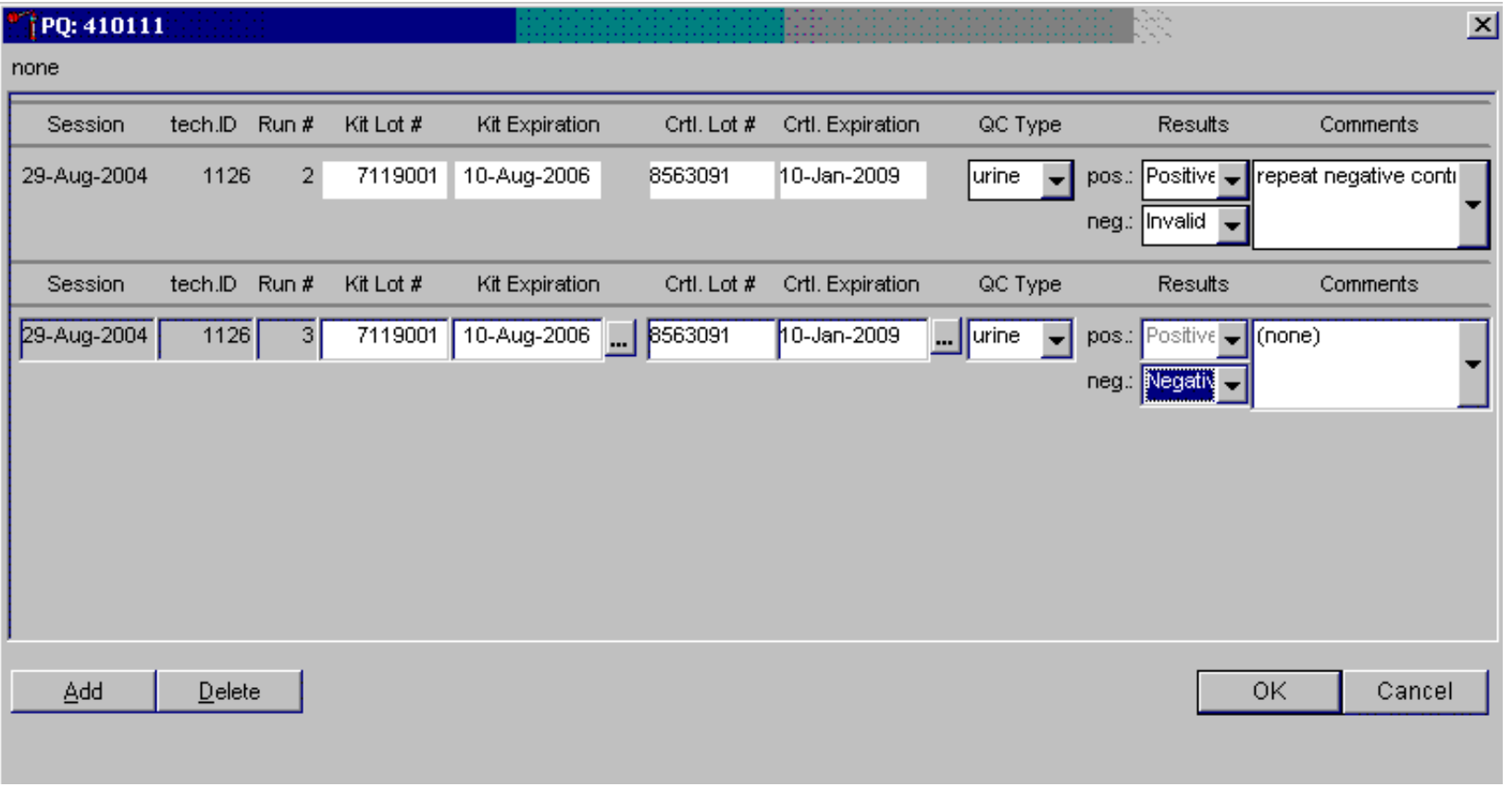

Rerun or repeat the control that demonstrated the unexpected result. Add a new test line to record the new control results. To add a new QC test line, use the mouse to direct the mouse arrow to the Add button and left click or type [Shift] [A/a]. Record the new results. If this resolves the inconsistency, use the mouse to direct the mouse arrow to the OK button and left click to save these results and to proceed with pregnancy testing.

If this does not resolve the situation, repeat the procedure with a different lot of controls. If this fails to resolve the problem, discard the entire lot of pregnancy test kits and test a new lot. 
Add a new test line each time a control is run and remember to record the serum QC each time it is performed.

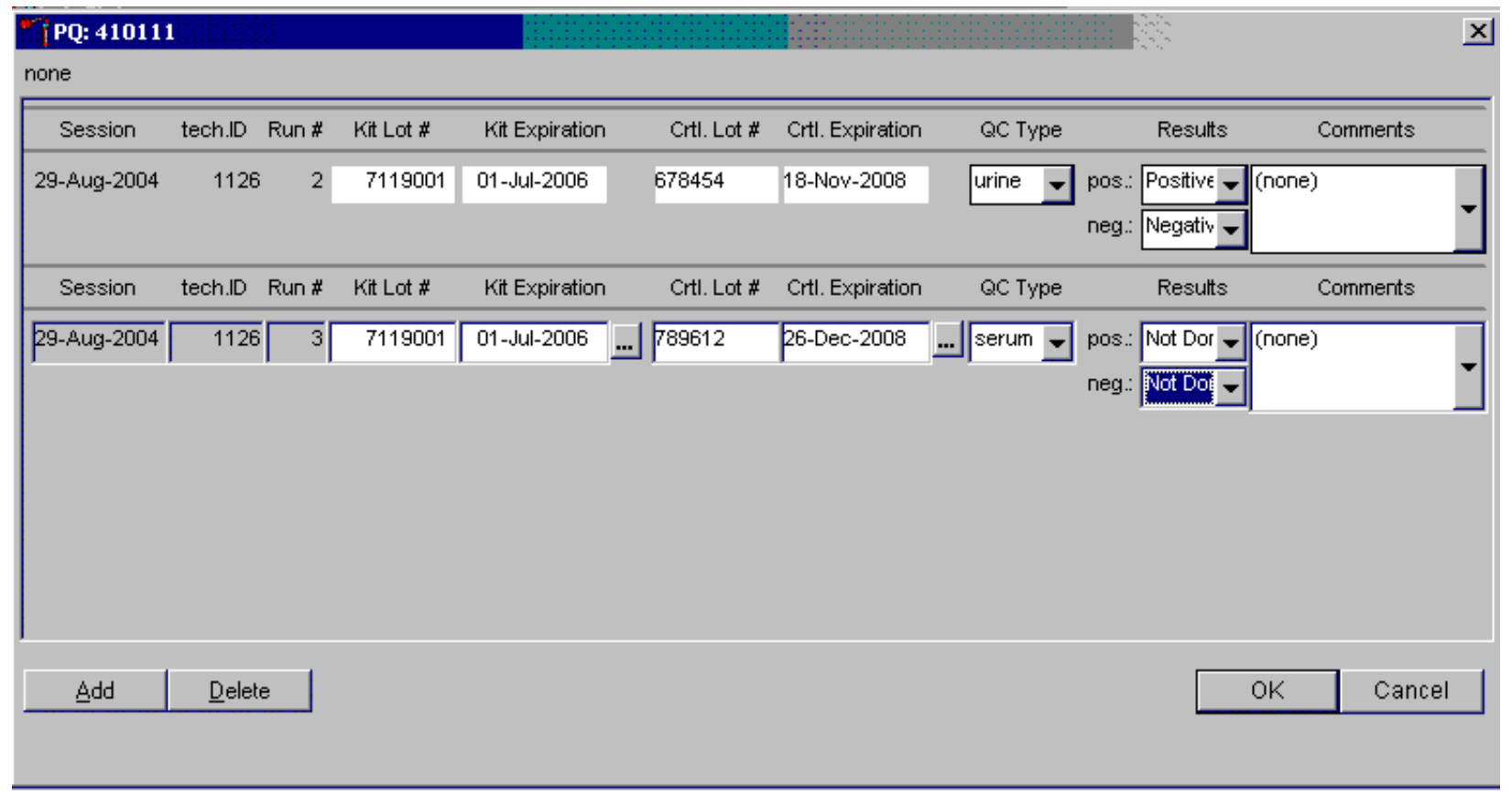

To add a new QC test line, use the mouse to direct the mouse arrow to the Add button and left click or type [Shift] [A/a]. The QC Type defaults to "Urine." Correct the control lot number and expiration date. To change the QC type to serum, use the mouse to direct the mouse arrow to the dropdown list, drag the mouse arrow to serum and left click. 
Record the serum control results.

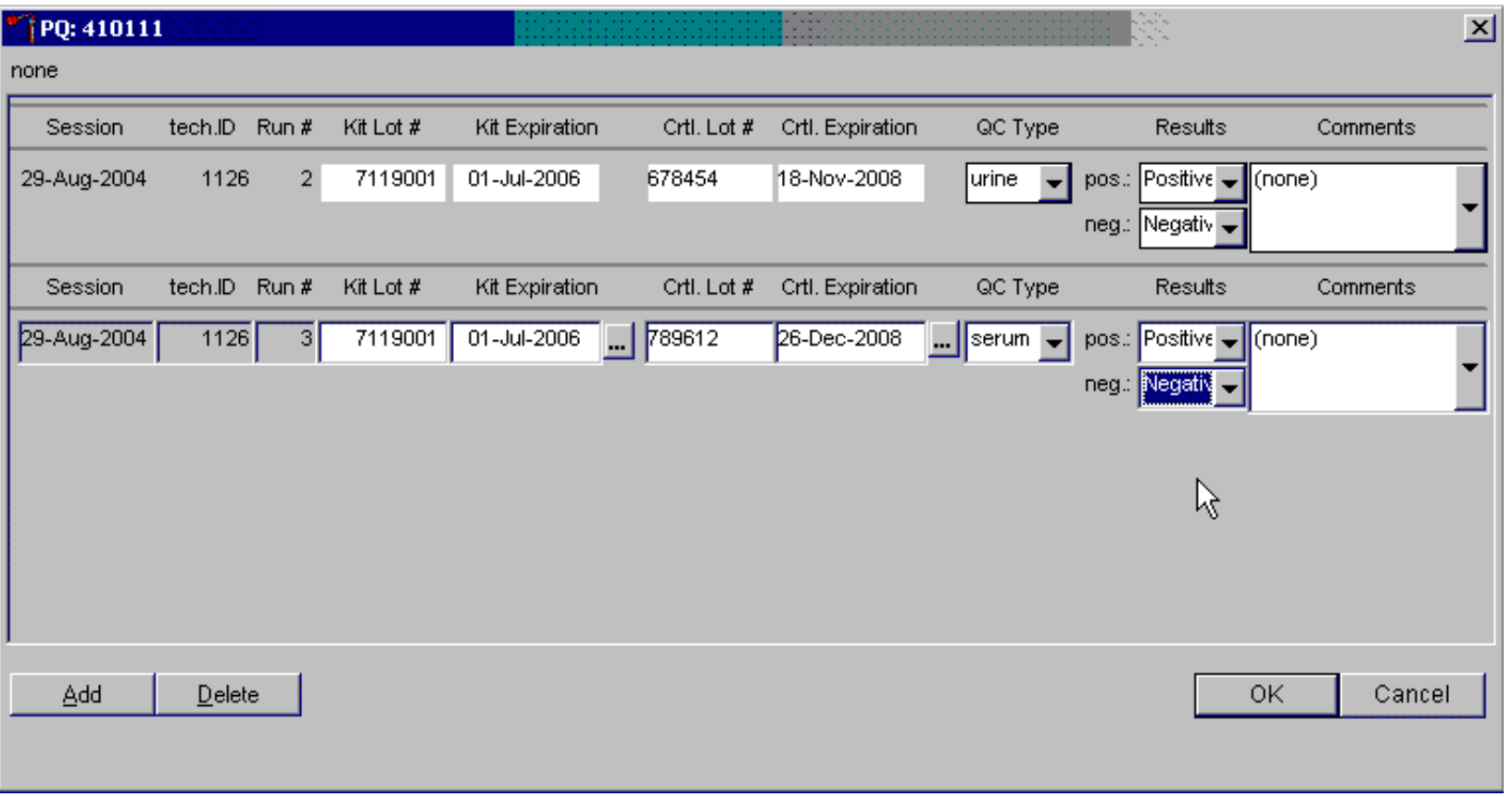

If the positive and negative serum controls demonstrate the expected result, use the mouse to direct the mouse arrow to the OK button and left click to save these results and to proceed with pregnancy testing. If the positive and/or the negative serum controls fail to demonstrate the expected result(s), add a new QC record, and repeat the control. 
XI. Interpretation of Results and Remedial Action

ICON® 25 hCG Test

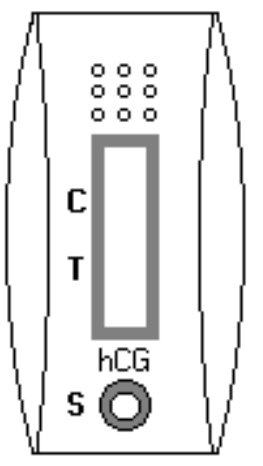

Figure 1.

ICON 25 test device

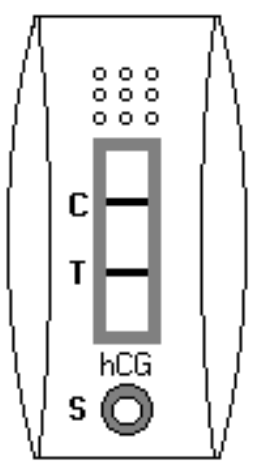

Figure 2.

Positive Result

Two distinct red lines appear. One line should be in the control region (C) and another line should be in the test region (T). 


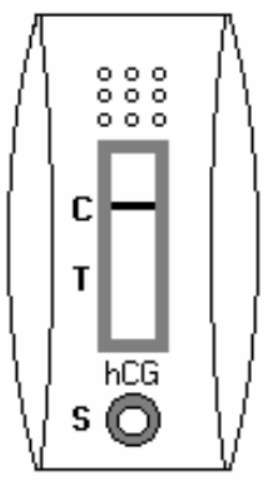

Figure 3.

Negative Result

One red line appears in the control

region (C). No apparent red or pink line

appears in the test region.
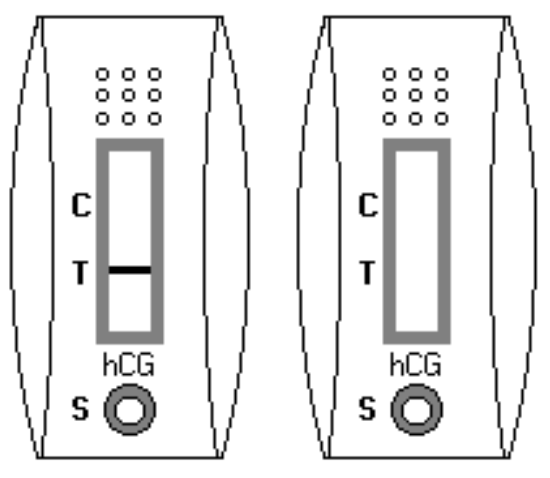

Figure 4.

Invalid Result

Control line fails to appear.

\section{Positive Result:}

A specimen that produces two distinct red lines is positive for the presence of hCG. One line should be in the control region (C) and another line should be in the test region (T).

\section{Negative Result:}

A specimen that does produce a red line appears in the control region (C) or no apparent red or pink line in the test region is negative for the presence of hCG. 


\section{Invalid Result (any of the following):}

For the test to be valid, a red line must appear in the control region (C). Insufficient sample volume or incorrect procedural techniques are the most likely reasons for control line failure. Review the procedure and repeat the test with a new test device. If the problem persists, discontinue using the test kit and immediately contact the technical supervisor.

If an invalid test result occurs repeatedly, or for technical assistance, contact the Technical Support Department, 1-800-877-6242.

\section{Sure-Vue ${ }^{\mathrm{TM}}$ hCG Urine and Serum Control Sets}

\section{Positive Result:}

The $25 \mathrm{mIU} / \mathrm{mL}$ hCG positive urine and serum controls should produce positive results.

\section{Negative Result:}

The negative urine and serum control should produce a negative result. 
XII.

Record Results

Use the heads-up display and the Pregnancy Testing module to enter the results of the ICON® 25 hCG test kit pregnancy test result for each SP.

Use the heads-up display to access the Pregnancy Testing module. Note that the first urine collection must be marked as verified for the pregnancy testing module to enable on the heads-up display and on the right click menu.

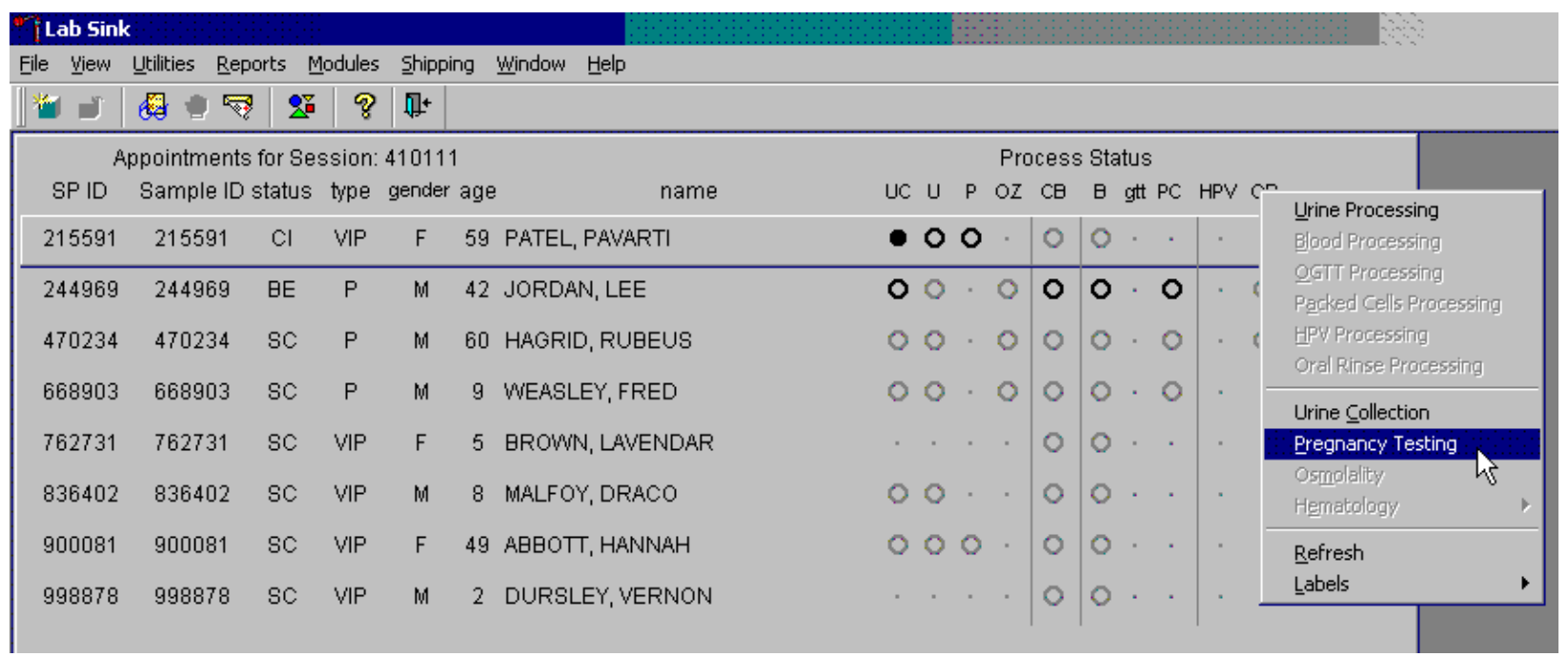

To access the Pregnancy Testing module, use the mouse to direct the mouse arrow to the correct SP, right click, drag the mouse arrow to \{Pregnancy Testing\}, and left click, or left click and type $[\mathrm{P} / \mathrm{p}]$. Alternatively, use the up and down keys to move up and down the list until the correct SP is highlighted, right click, drag the mouse arrow to \{Pregnancy Testing\}, and left click, or left click and type $[\mathrm{P} / \mathrm{p}]$. 
The Pregnancy Testing window displays.

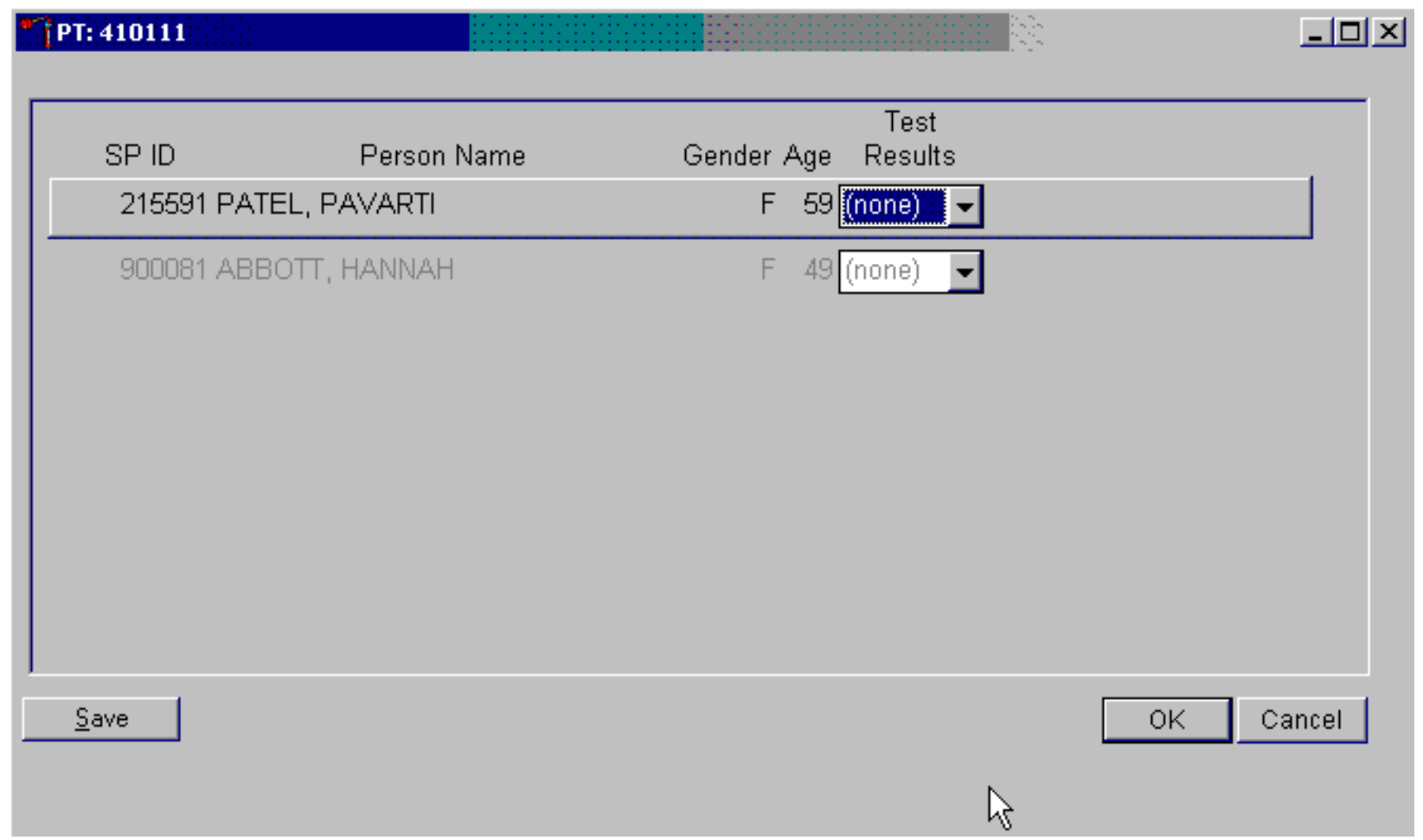

The text is black for SPs who have urine collection results recorded as "Yes" or "Yes/QNS." The text is gray for SPs who do not have urine collection results recorded. The window lists the SP ID, Person Name, Gender, Age, and includes a Test Results column (text box). 
Report the results for each SP as positive, negative, not done, or invalid.

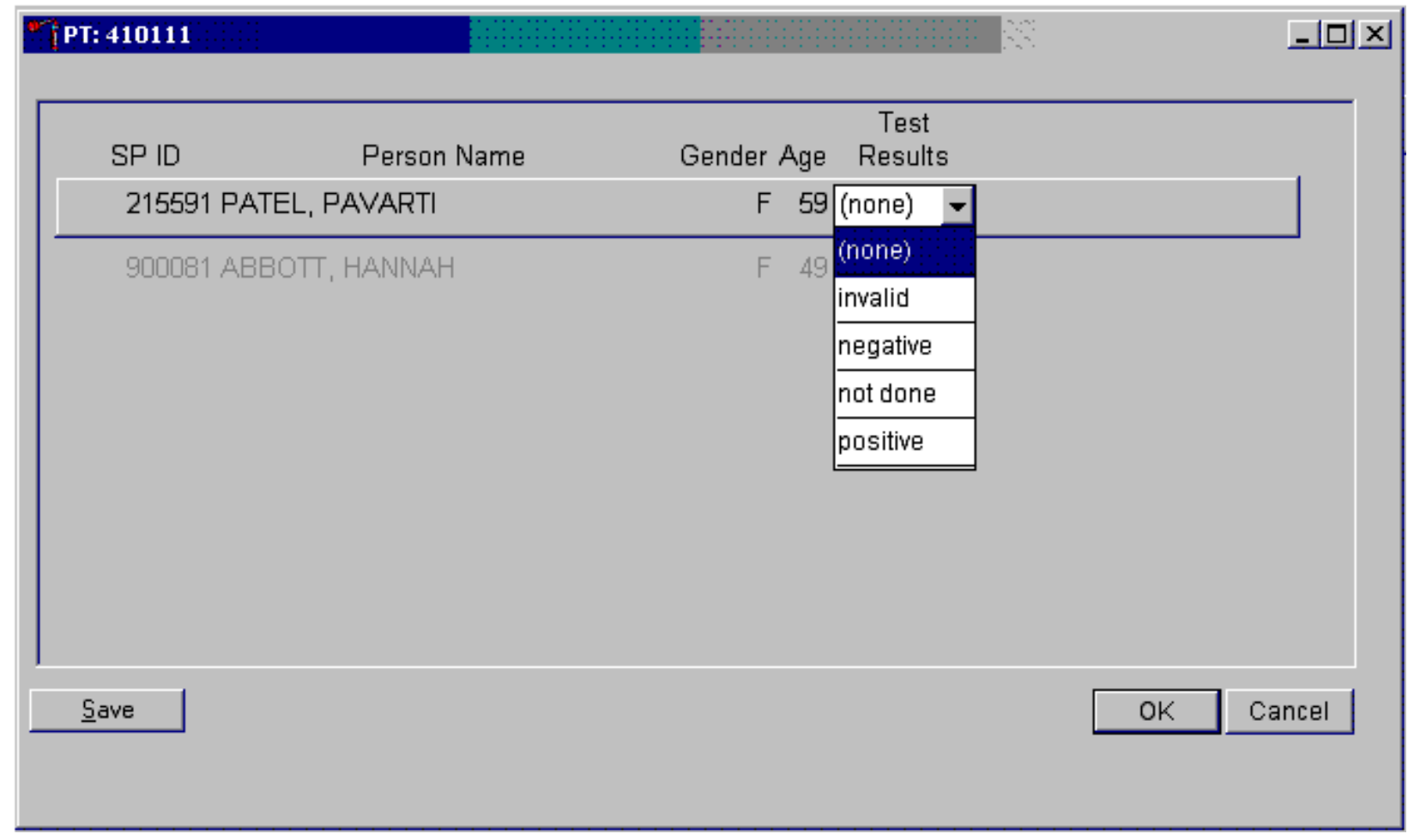

If the Test Result field is black or highlighted, record the result by typing [P/p] for positive, $[\mathrm{N} / \mathrm{n}]$ for negative, or $[\mathrm{I} / \mathrm{i}]$ for an invalid result. Enter $[\mathrm{N} / \mathrm{n}]$ not done when the test is not performed. Alternatively, to record a result, use the mouse to direct the mouse arrow to the drop-down list, drag the arrow to the correct choice and left click. To update the results without exiting the window, use the mouse to direct the mouse arrow to the Save button and left click or type [ALT] [S/s]. To update the results and exit the window, use the mouse to direct the mouse arrow to the OK button and left click or select [Enter]. To exit the module without saving any result, use the mouse to direct the mouse arrow to the Cancel button and left click. 
Continue performing pregnancy tests and recording the results. Save the test results after entering each positive, negative, or invalid result. To update the results without exiting the window, use the mouse to direct the mouse arrow to the Save button and left click or type [Shift] [S/s]. To update the results and exit the window, use the mouse to direct the mouse arrow to the OK button and left click or select [Enter]. To exit the module without saving any result, use the mouse to direct the mouse arrow to the Cancel button and left click.

A series of message boxes display to inform that an SP has been excluded (blocked) or is eligible (unblocked.) After each negative result is saved to the database, an unblocking message box displays.

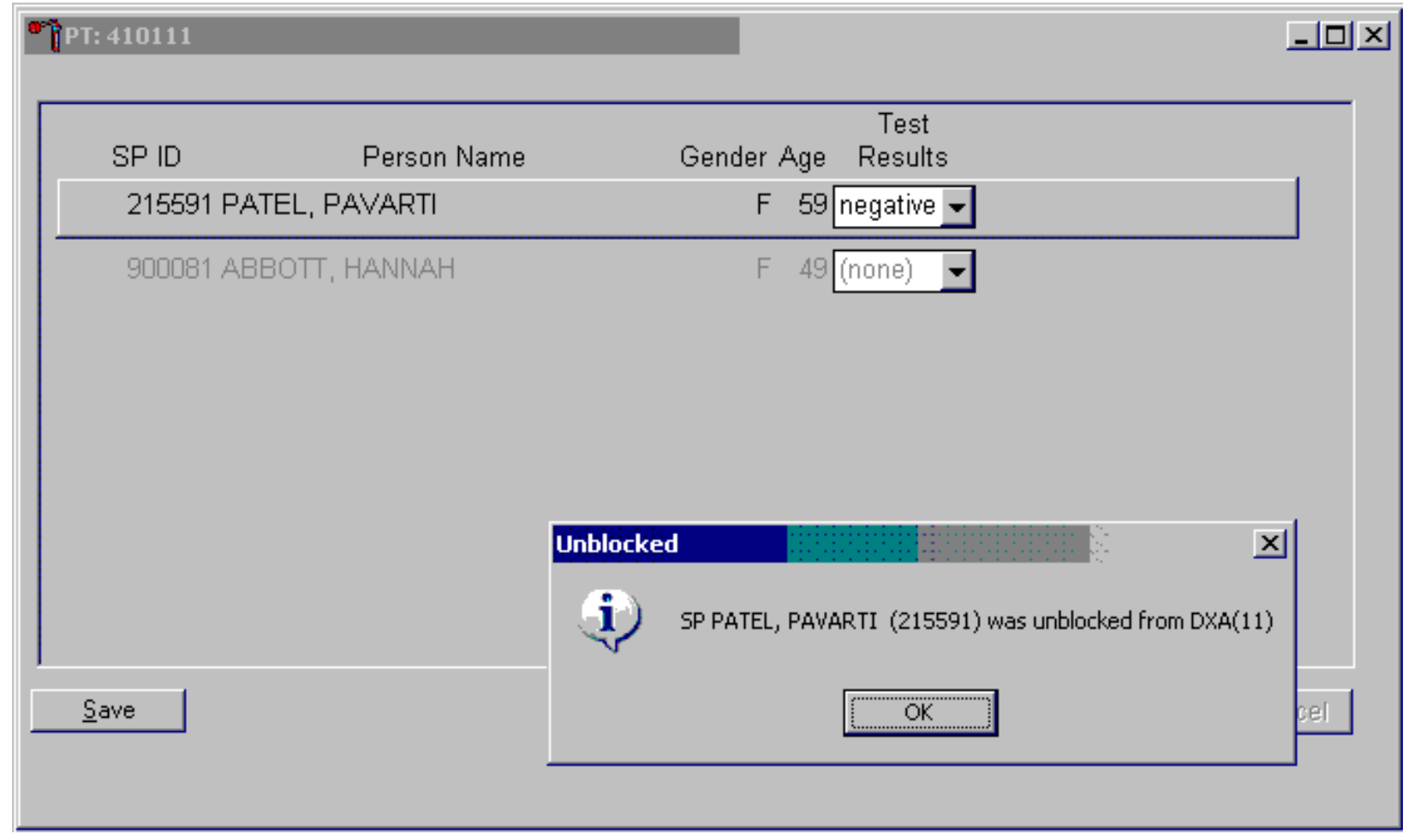

To remove the informational message box, use the mouse to direct the mouse arrow to the OK button and left click, or select [Enter]. 
As each positive result is saved to the database a blocking message displays.

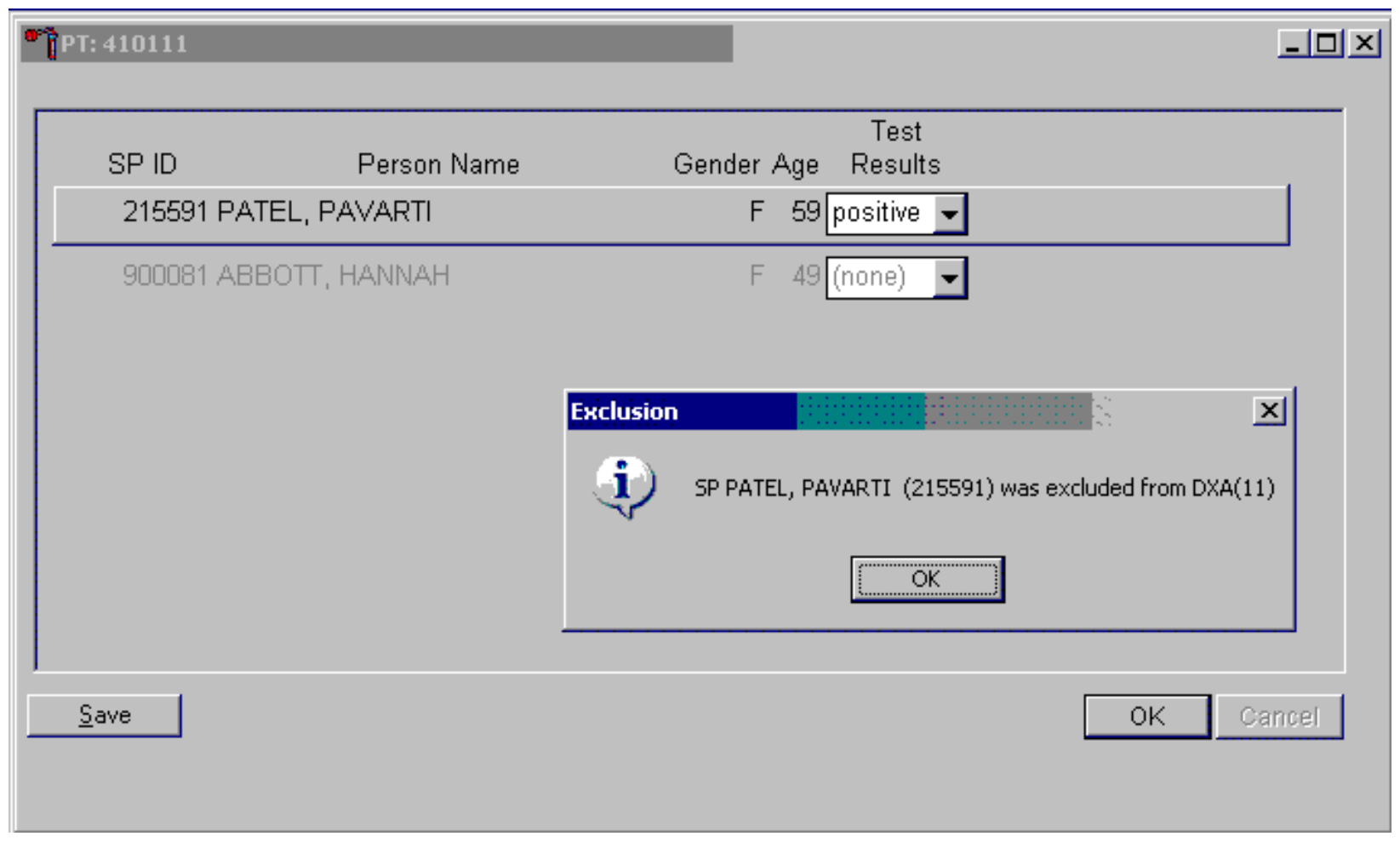

To remove the informational message box, use the mouse to direct the mouse arrow to the OK button and left click, or select [Enter]. 
If a urine pregnancy test is positive on any female SPs aged 8-17 years, confirm the result using serum. An informational pop-up box displays as a reminder to perform the confirmation test.

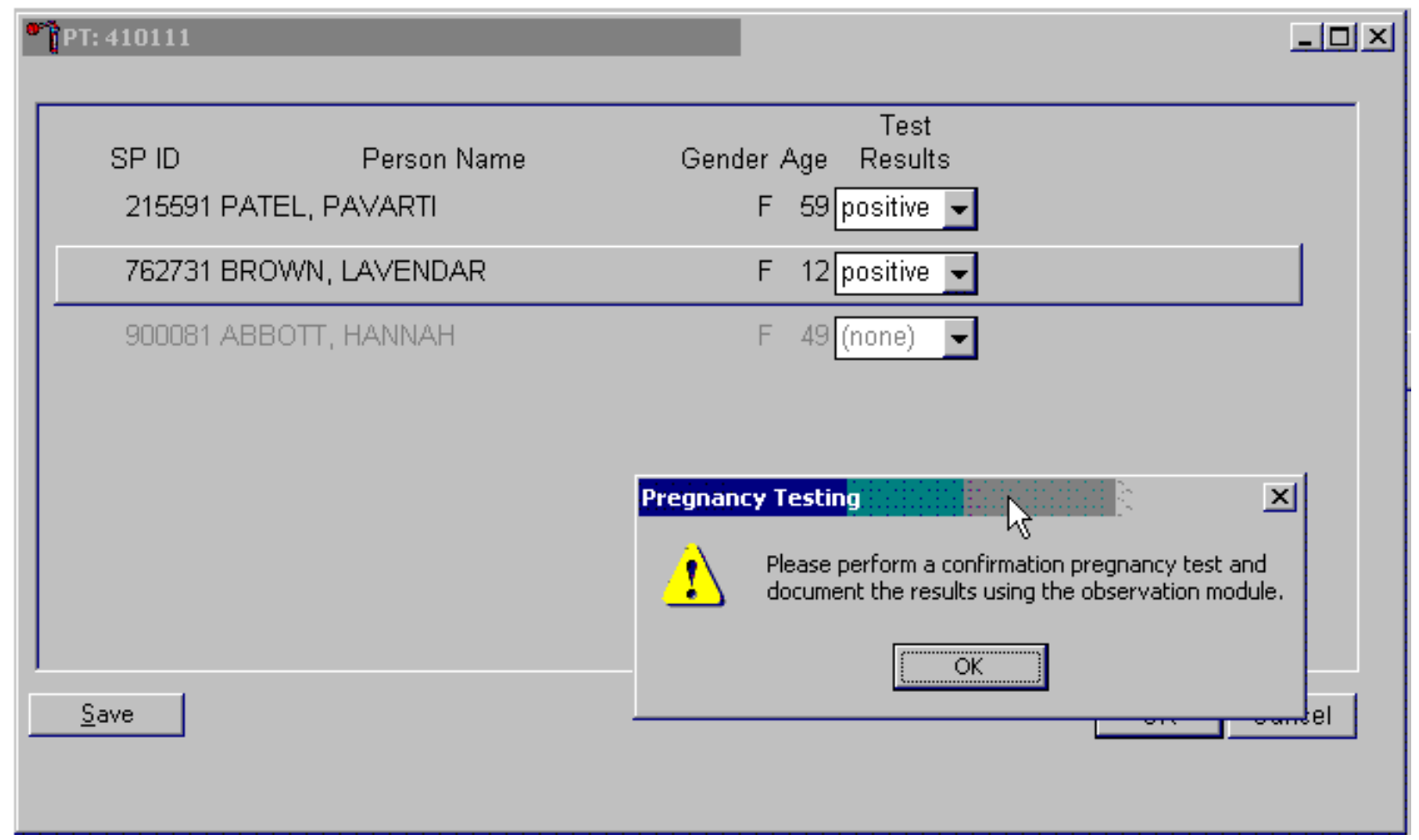

To remove the informational message box, use the mouse to direct the mouse arrow to the OK button and left click, or select [Enter].

If no blood is drawn, repeat the urine test. Notify the physician of the second positive or negative confirmatory test or the inability to perform a confirmation test using the observation function. 
Access the observation function to send an observation to the physician.

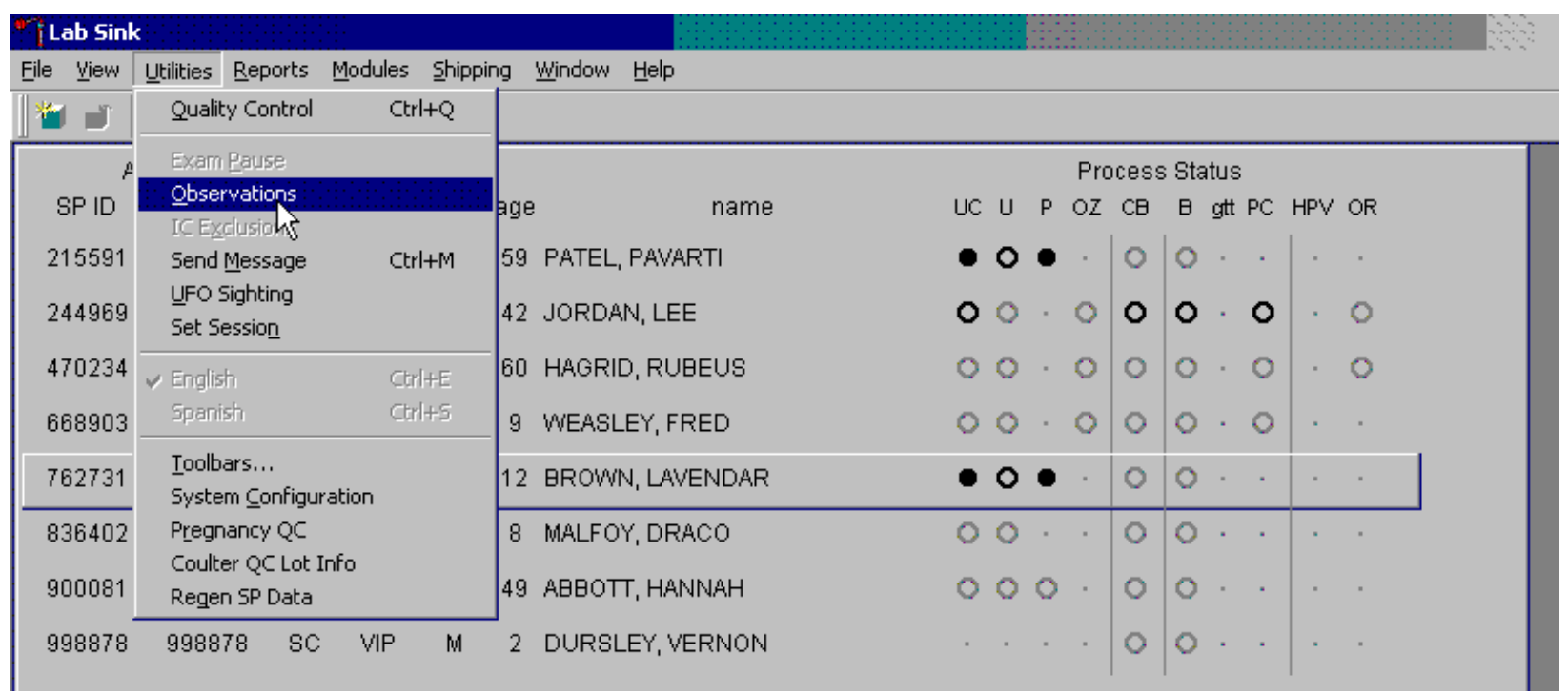

To send an observation to the physician, use the mouse to direct the mouse arrow to \{Utilities\} in the top menu bar, drag the mouse arrow to \{Observations $\}$ and left click or type [Alt] [U/u], [O/o]. 
Select or highlight the correct SP.

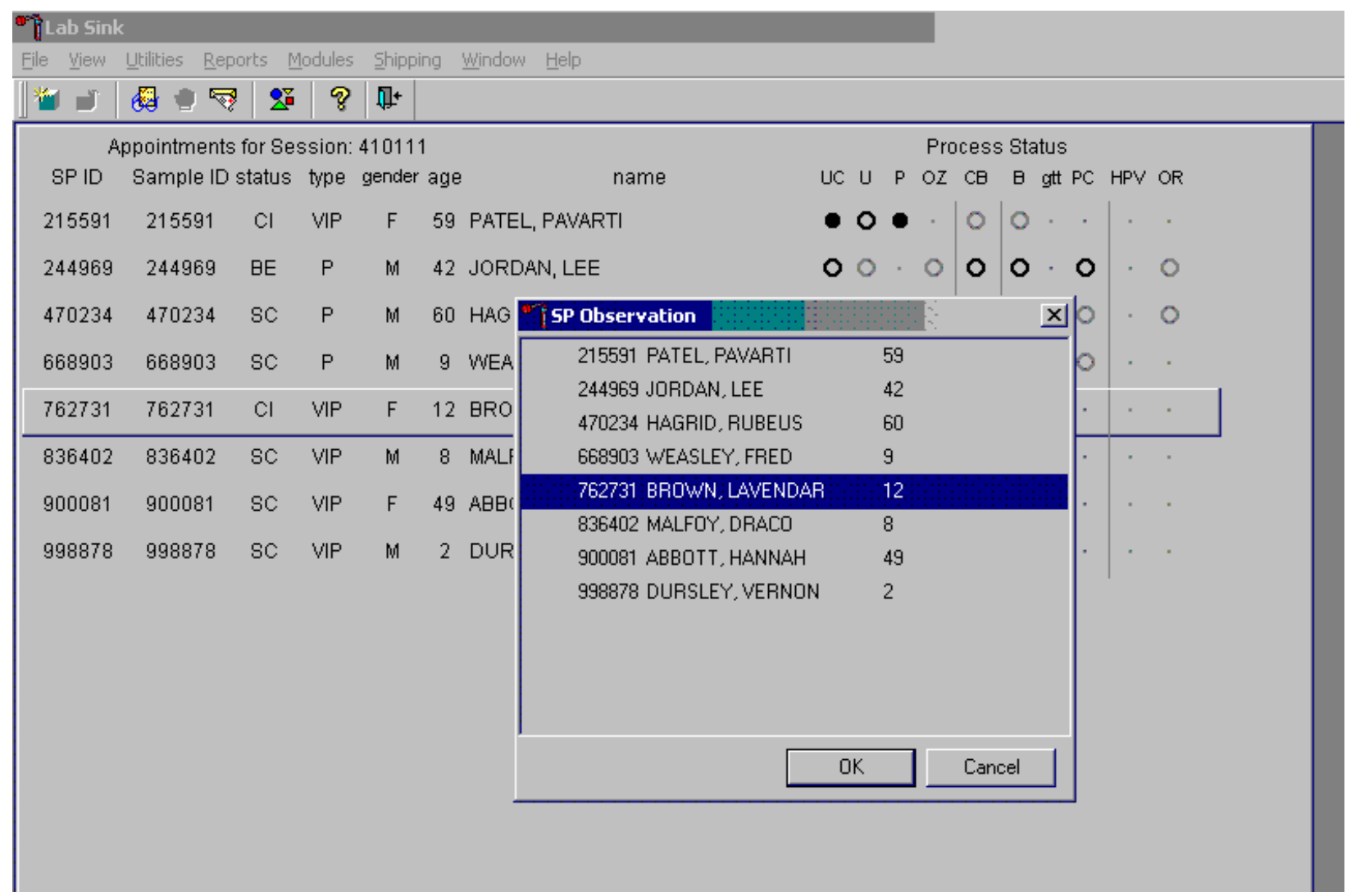

To select or highlight an SP, drag the mouse arrow to the correct SP and left click. Verify that the SP ID, name, and age are correct. Use the vertical scroll bar to view the complete list of SPs. To continue, use the mouse to direct the mouse arrow to the OK button and left click or select [Enter]. To cancel these actions and exit the observation function, use the mouse to direct the mouse arrow to the Cancel button and left click. 
The observation text window displays.

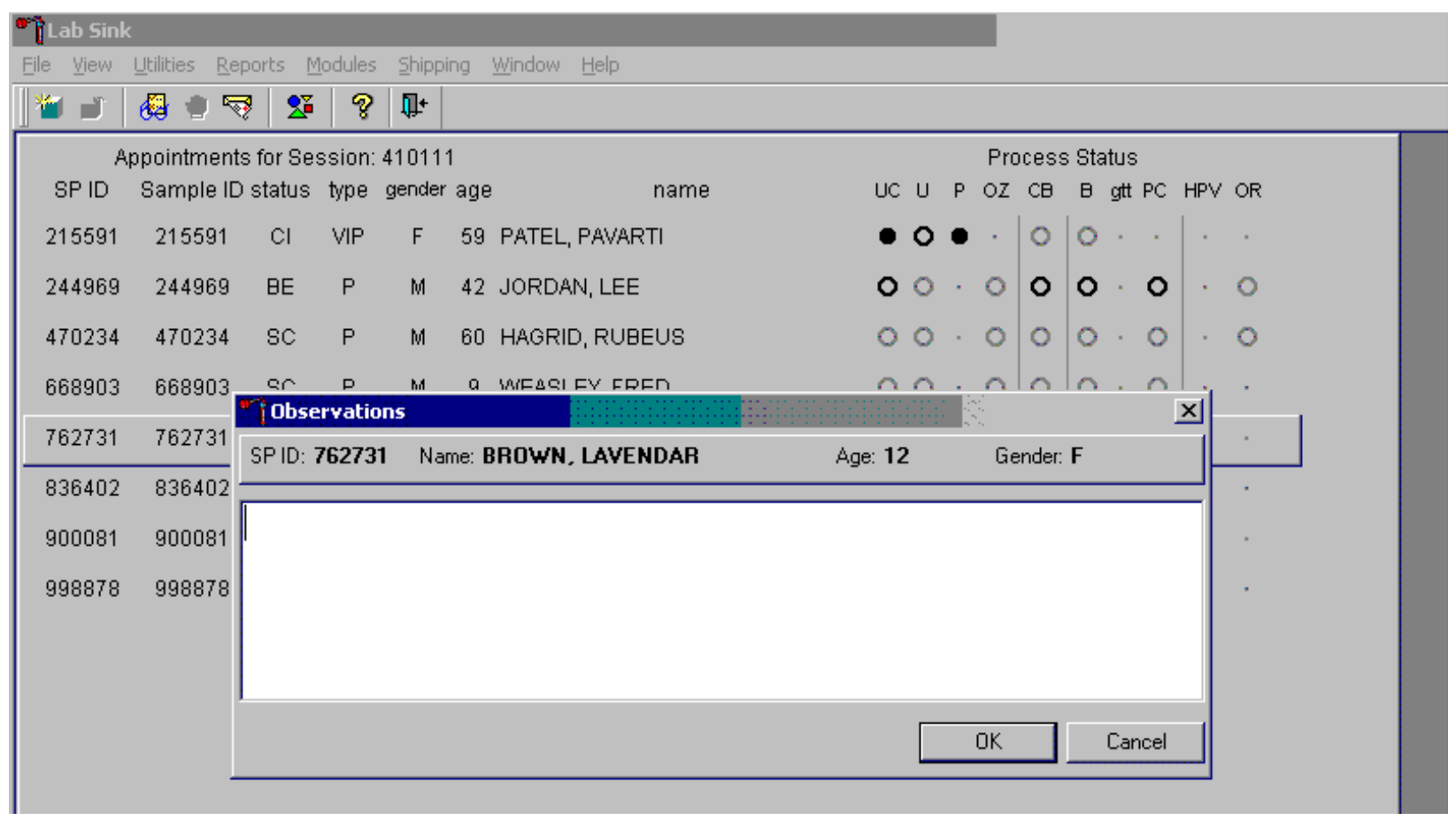

The observation text window contains the SP ID, name, age, and gender. Type the observation using the keyboard's keys. To send the observation to the physician, use the mouse to direct the mouse arrow to the OK button and left click or select [Enter]. To cancel these actions or to exit the observation window without entering an observation, use the mouse to direct the mouse arrow to the Cancel button and left click. 
Send an observation to report a negative serum test result on SPs aged 8-17 years.

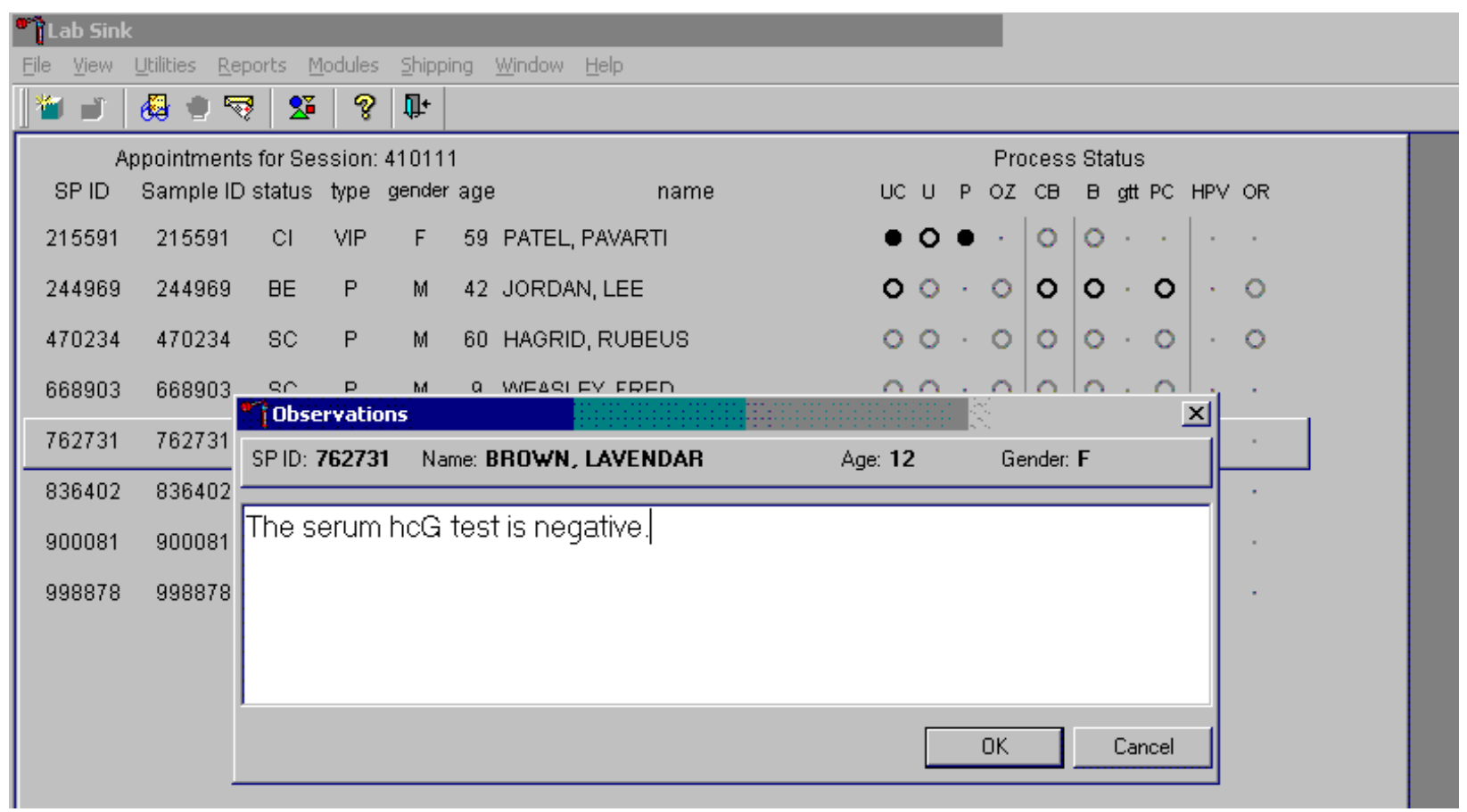

The observation window contains the SP ID, name, age, and gender. Type the observation, “The serum hCG test is negative” using the keyboard keys. To send the observation to the physician, use the mouse to direct the mouse arrow to the OK button and left click or select [Enter]. To cancel these actions or to exit the observation window without entering an observation, use the mouse to direct the mouse arrow to the Cancel button and left click. 
Send an observation to report a positive serum test result on SPs aged 8-17 years.

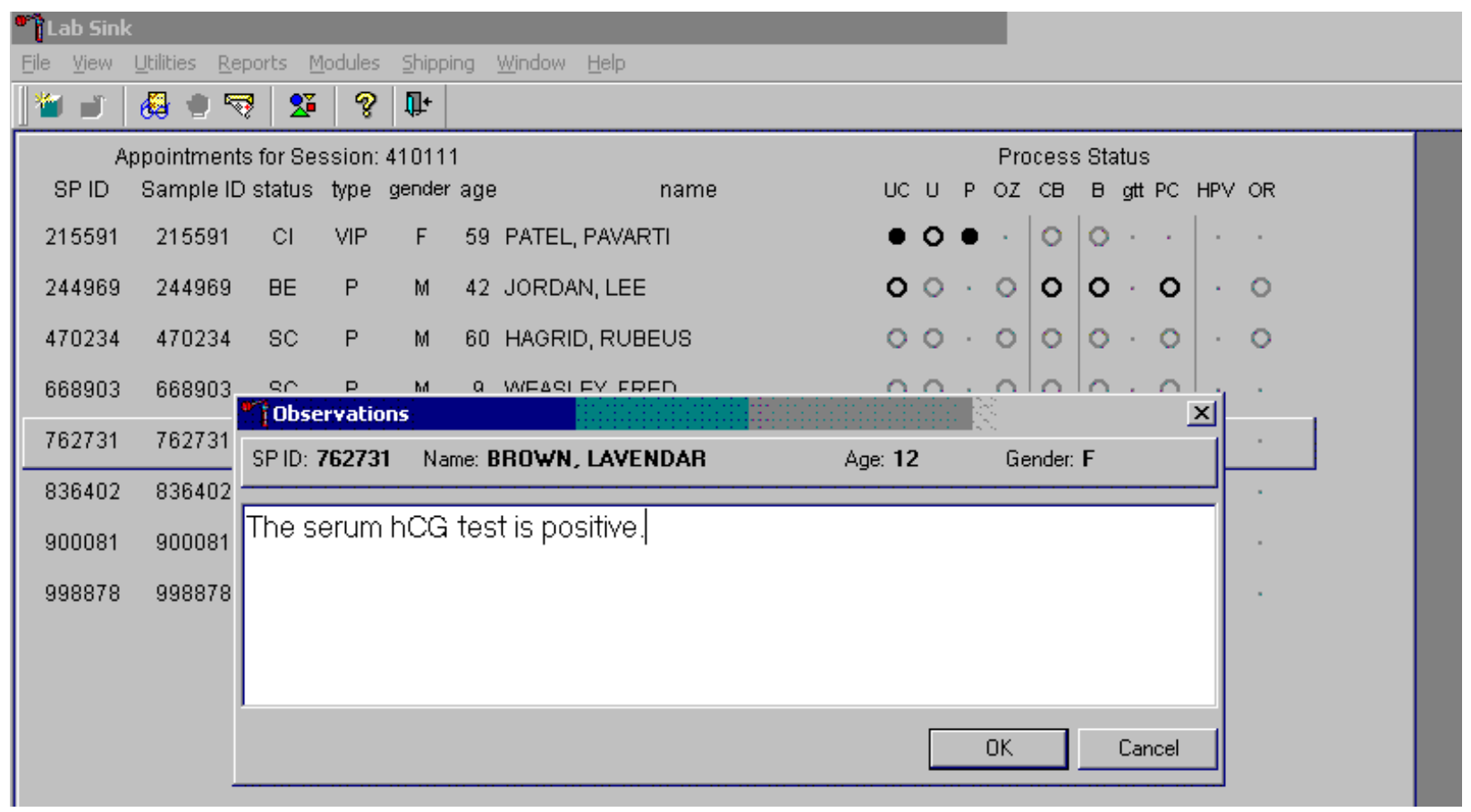

The observation window contains the SP ID, name, age, and gender. Type the observation, "The serum hCG test is positive" using the keyboard keys. To send the observation to the physician, use the mouse to direct the mouse arrow to the OK button and left click or select [Enter]. To cancel these actions or to exit the observation window without entering an observation, use the mouse to direct the mouse arrow to the Cancel button and left click. 
Send an observation to report the inability to perform the serum confirmation test and to report the results of a repeat negative urine test on female SPs aged 8-17 years.

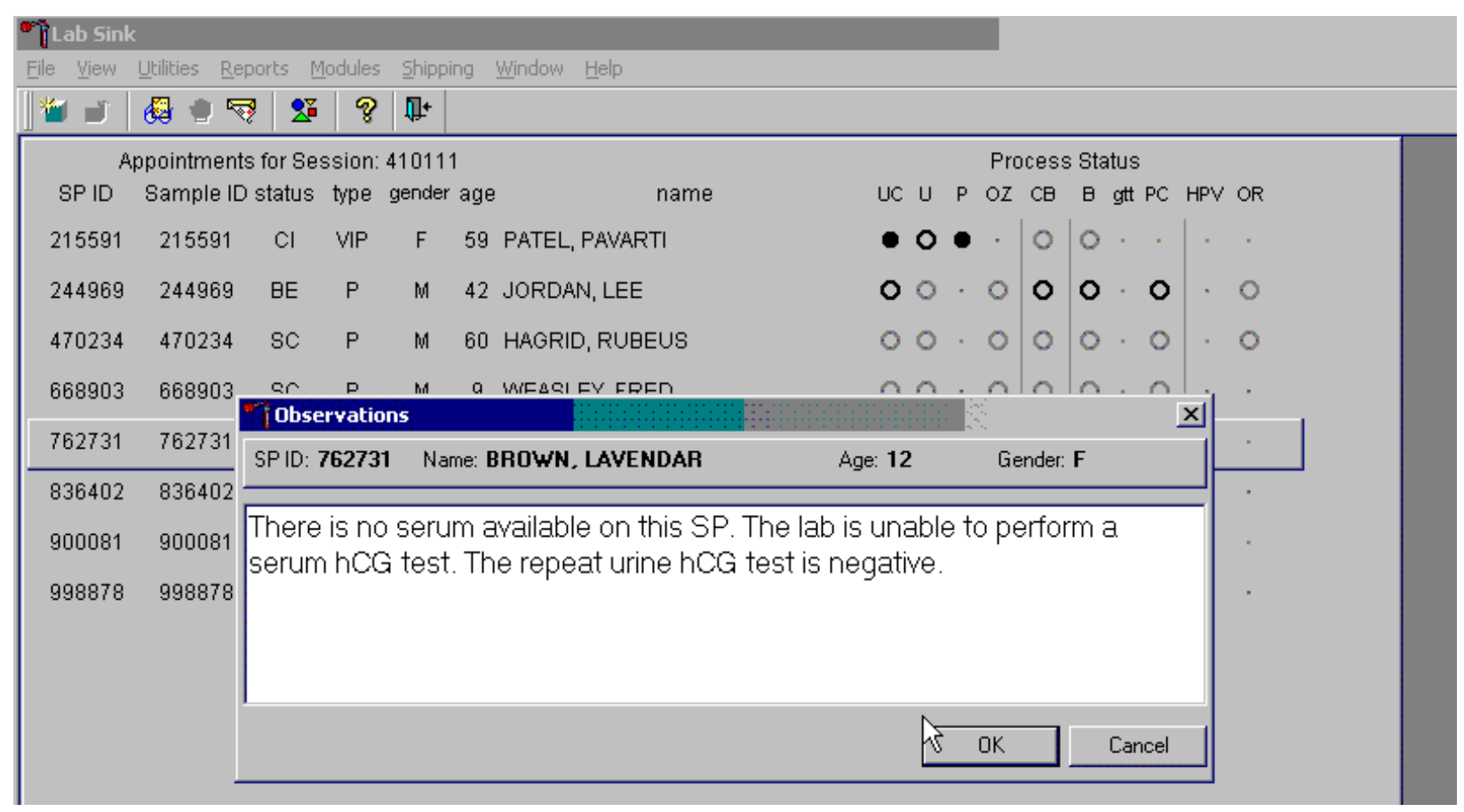

The observation window contains the SP ID, name, age, and gender. Type the observation, "There is no serum available on this SP. The lab is unable to perform a serum hCG test. The repeat urine hCG test is negative.” using the keyboard keys. To send the observation to the physician, use the mouse to direct the mouse arrow to the OK button and left click or select [Enter]. To cancel these actions or to exit the observation window without entering an observation, use the mouse to direct the mouse arrow to the Cancel button and left click. 
Send an observation to report the inability to perform the serum confirmation test and to report the results of a repeat positive urine test on female SPs aged 8-17 years.

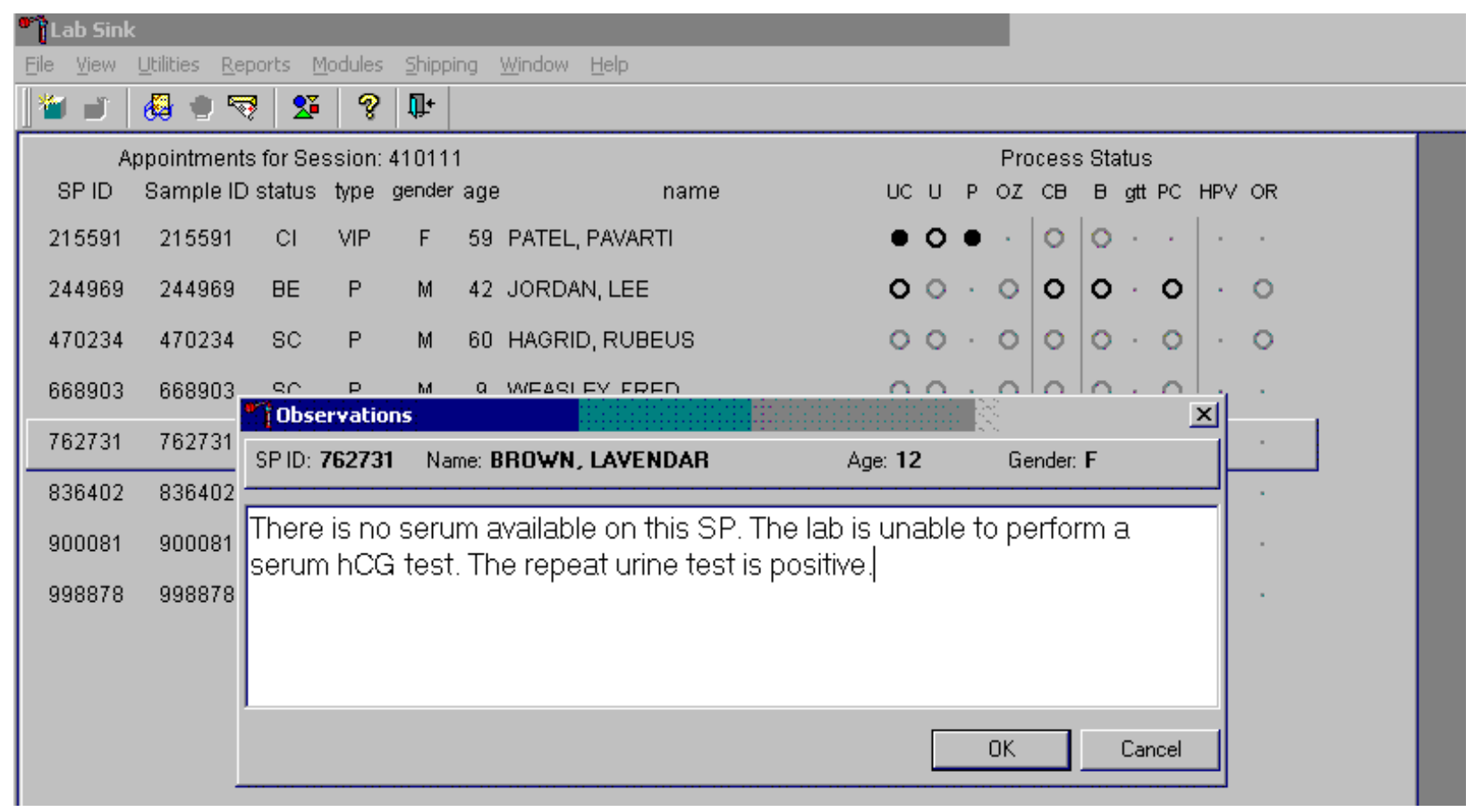

The observation window contains the SP ID, name, age, and gender. Type the observation, "There is no serum available on this SP. The lab is unable to perform a serum hCG test. The repeat urine test is positive." using the keyboard keys. To send the observation to the physician, use the mouse to direct the mouse arrow to the OK button and left click or select [Enter]. To cancel these actions or to exit the observation window without entering an observation, use the mouse to direct the mouse arrow to the Cancel button and left click. 
Send an observation to report the inability either to perform the serum confirmation test or to repeat the urine test on female SPs aged 8-17 years.

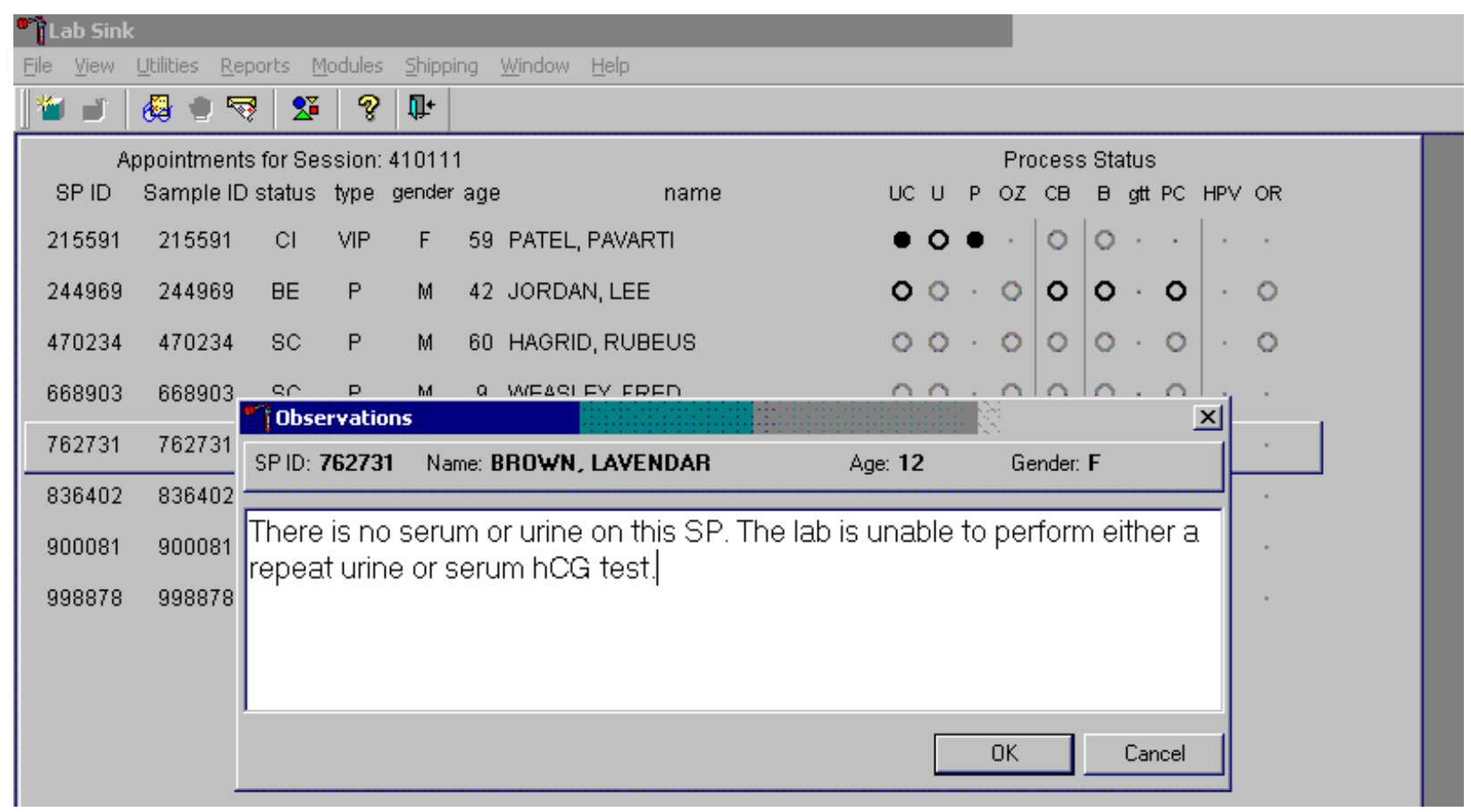

The observation window contains the SP ID, name, age, and gender. Type the observation, "There is no serum or urine on this SP. The lab is unable to perform either a repeat urine or serum hCG test.” using the keyboard keys. To send the observation to the physician, use the mouse to direct the mouse arrow to the OK button and left click or select [Enter]. To cancel these actions or to exit the observation window without entering an observation, use the mouse to direct the mouse arrow to the Cancel button and left click. 
The physician reviews all observations.

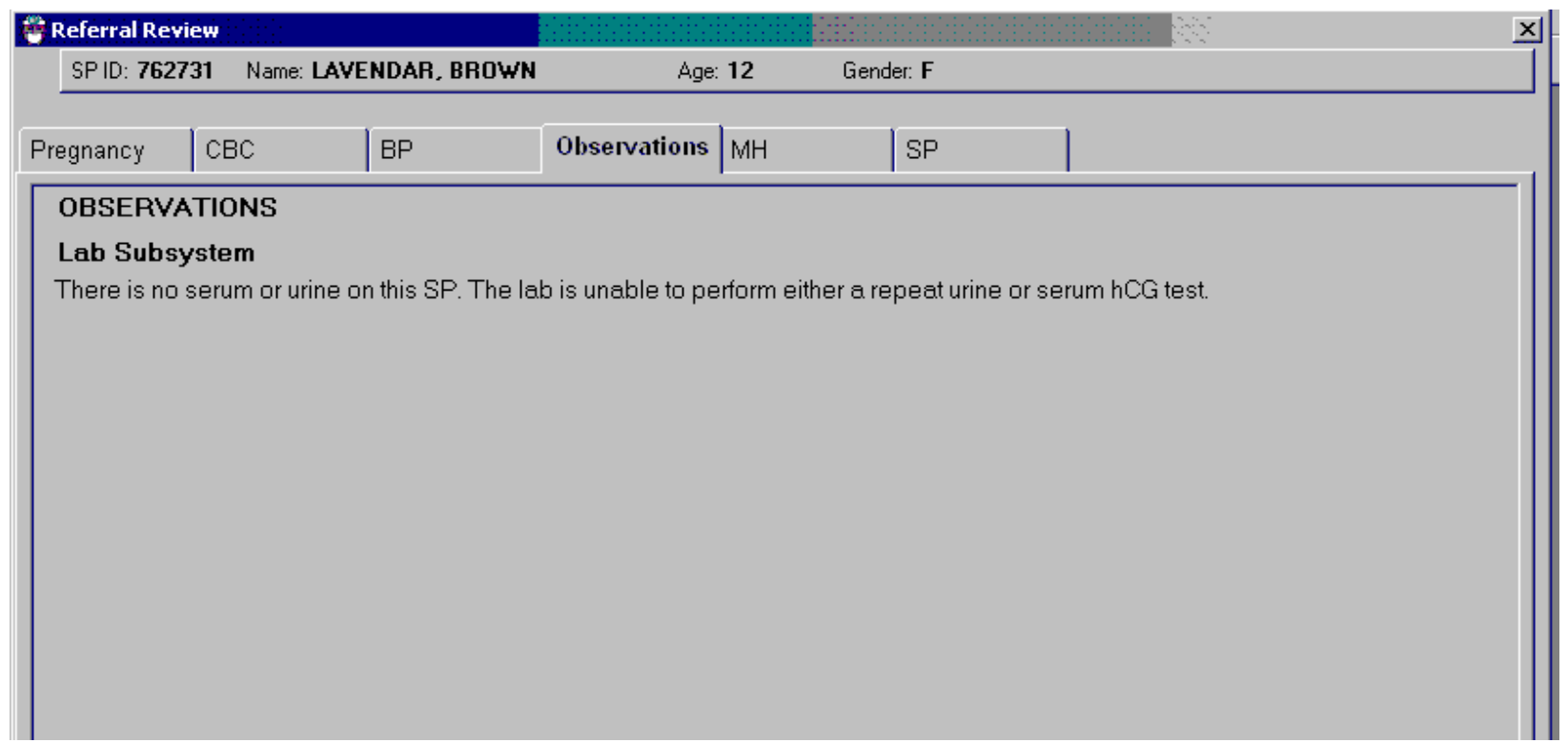

The physician accesses the observations using the Review in Box Details.

XIII. Limitations of Method: Specimen Rejection, Interfering Substances, and Conditions

Read the following procedural notes to ensure the best results with the ICON 25 hCG test kit. 


\section{ICON® 25 hCG Test}

- $\quad$ Dispensing Specimen - Do not reuse a test device or use a test device for multiple patient samples or controls, as this will produce inaccurate results.

- Incubation Times - The intensity of the red color in the test line region (T) will vary depending on the concentration of hCG present in the sample. However, neither the qualitative value nor the rate of increase in hCG can be determined by this qualitative test.

- Batch Processing - If several specimens are to be tested during one run, perform each test step for all specimens and controls at timed intervals before proceeding to the next test step. It is recommended that no more than ten (10) test devices be run in one batch.

- Specimens Containing Particulate Matter - Urine samples exhibiting visible precipitates should be centrifuged or allowed to settle to obtain a clear sample for testing.

- Very dilute urine samples, as indicated by a low specific gravity, may not contain representative levels of hCG.

- False negative results may occur when the levels of hCG are below the sensitivity level of the test.

- Very low levels of hCG (less than $50 \mathrm{mIL} / \mathrm{mL}$ ) are present in urine and serum samples shortly after implantation. However, because a significant number of first trimester pregnancies terminate for natural reasons, a test result that is weakly positive should be confirmed.

- A number of conditions other than pregnancy, including trophoblastic disease and certain nontrophoblastic neoplasms including testicular tumors, prostate cancer, breast cancer, and lung cancer, cause elevated levels of hCG. Therefore, the presence of hCG in urine or serum should not be used to diagnose pregnancy unless these conditions have been ruled out.

- This test provides a presumptive diagnosis for pregnancy. A physician should only make a confirmed pregnancy diagnosis after all clinical and laboratory findings have been evaluated.

- Negative results are expected in healthy nonpregnant women and healthy men. Healthy pregnant women have hCG in their urine and serum samples. The amount of hCG will vary greatly with gestational age and between individuals. Concentrations of hCG in pregnant women are generally between 10 and $30 \mathrm{mIU}$ hCG/mL in the 7-10 days following implantation or 3 weeks after the last menstrual period (LMP). During the latter part of the first trimester of pregnancy, the hCG concentration reaches a maximum level of greater than 100,000 $\mathrm{mIL} / \mathrm{mL}$. 
- The ICON 25 hCG test has a stated sensitivity of $25 \mathrm{mIU} / \mathrm{mL}$ and is capable of detecting pregnancy as early as 1 day after the first missed menses.

- Do not use urine samples containing grossly hemolyzed blood since they may give inaccurate or erratic results.

\section{Sure-Vue ${ }^{\mathrm{TM}}$ hCG Urine and Serum Control Sets}

- These controls are formulated for use as quality control specimens in the reagent verification of pregnancy test kits. 


\section{Accuracy, Sensitivity, and Specificity \\ ICON® 25 hCG Test}

Accuracy

A multicenter clinical evaluation was conducted comparing the results obtained using the ICON® 25 hCG test and another commercially available urine/serum membrane hCG test. The urine study included 159 samples and both tests identified 88 negative and 71 positive results. The serum study included 73 samples and both assays identified 51 negative and 21 positive and 1 inconclusive result. The results demonstrated a 100 percent overall agreement (for an accuracy of $>99 \%$ ) of the ICON® 25 hCG test when compared to the other urine/serum membrane hCG test.

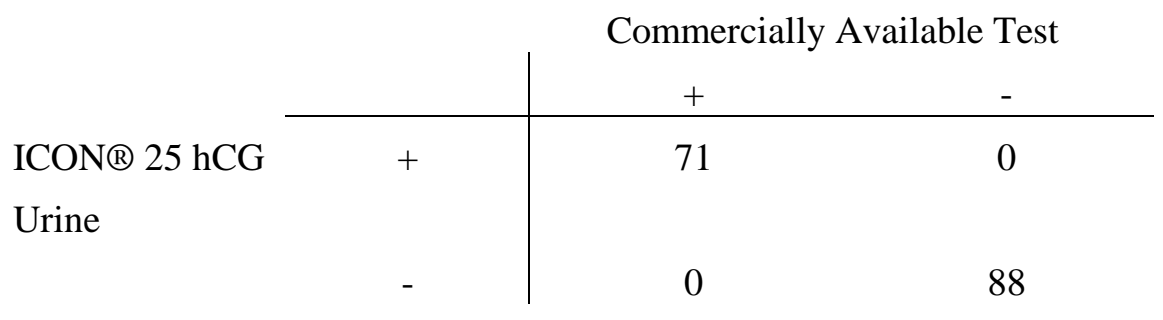

\begin{tabular}{|c|c|c|c|}
\hline & & Comm & Test \\
\hline & & + & - \\
\hline $\begin{array}{l}\text { ICON® } 25 \text { hCG } \\
\text { Serum }\end{array}$ & + & 21 & 0 \\
\hline & - & 0 & 51 \\
\hline
\end{tabular}

Sensitivity

- The ICON® 25 hCG test detects hCG at concentrations of $25 \mathrm{mIU}$ hCG/mL or greater. The test has been standardized to the W.H.O. Third International Standard. The addition of $300 \mathrm{mIU} / \mathrm{mL}$ of luteinizing hormone, (LH), $1000 \mathrm{mIU} / \mathrm{mL}$ of follicle stimulating hormone (FSH), and $1000 \mathrm{mIU} / \mathrm{mL}$ of thyroid stimulating hormone (TSH) to negative $(0 \mathrm{mIU} / \mathrm{mL}$ hCG) urine/serum specimens and to positive $(25 \mathrm{mIU} / \mathrm{mL}$ hCG) urine/serum specimens did not exhibit cross-reactivity in the assay. 


\section{Specificity}

- The following potentially interfering substances were added to hCG negative and positive samples.

- None of the substances at the concentrations tested interfered in the test.

Interfering Substances

\begin{tabular}{lc}
\hline Substance Added & Concentration \\
\hline Acetaminophen & $20 \mathrm{mg} / \mathrm{dL}$ \\
Ascorbic Acid & $20 \mathrm{mg} / \mathrm{dL}$ \\
Caffeine & $20 \mathrm{mg} / \mathrm{dL}$ \\
Gentisic Acid & $20 \mathrm{mg} / \mathrm{dL}$ \\
Acetylsalicylic Acid & $20 \mathrm{mg} / \mathrm{dL}$ \\
Atropine & $20 \mathrm{mg} / \mathrm{dL}$ \\
Bilirubin (serum) & $40 \mathrm{mg} / \mathrm{dL}$ \\
Glucose & $2 \mathrm{~g} / \mathrm{dL}$ \\
Hemoglobin & $1 \mathrm{mg} / \mathrm{dL}$ \\
Bilirubin (Urine) & $2 \mathrm{mg} / \mathrm{dL}$ \\
Triglycerides & $1200 \mathrm{mg} / \mathrm{dL}$ \\
\hline
\end{tabular}

\section{Sure-Vue ${ }^{\mathrm{TM}}$ hCG Urine and Serum Controls}

These controls have been designed to produce correct results in pregnancy test kits. These controls have been tested with the ICON ${ }^{\circledR} 25$ hCG test and were found to produce satisfactory results.

XIV. Reference Ranges

Not applicable.

XV. $\quad$ Action Limits

Not applicable. 
XVI. Specimen Storage and Handling During Testing

Serum

Serum is required; do not use plasma. Specimens may be stored for 48 hours at $2^{\circ} \mathrm{C}-8^{\circ} \mathrm{C}$ $\left(36^{\circ} \mathrm{F}-46^{\circ} \mathrm{F}\right)$ before testing. Specimens held for longer times should be frozen at $-20^{\circ} \mathrm{C}\left(-4^{\circ} \mathrm{F}\right)$ before testing. Bring all specimens to room temperature before beginning the assay procedure and mix before testing. Use clear nonhemolyzed samples when possible.

Urine

A urine sample must be collected in a clean and dry container. Centrifuge very turbid urine specimens at 2500 RPM for 5 minutes before use. Do not shake or disturb specimens containing particulate matter, such as salts that have settled out of solution; pipette samples from the clear supernatant of such specimens.

XVII. Alternative Method for Performing Test or Storing Specimens if Test System Fails

There is no alternative method for testing.

XVIII. Test Results Reporting System Protocol for Reporting Action Limits

Not applicable.

XIX. Specimen Accountability and Tracking

All records, including QA/QC data are maintained for 6 years. Use only numerical identifiers for SP results.

XX. Quality Control Summary Statistics and Graphs

Quality control reports are monitored by stand and maintained for 6 years. 
XXI. References

Batzer, F.R. (1980). Hormonal evaluation of early pregnancy, Fertility \& Sterility, 34(1), 1-13.

Catt, K.J., Dafau, M.L., and Vaitukaitis, J.L. (1975). Appearance of hCG in pregnancy plasma following the initiation of implantation of the blastocyte, J. Clin. Endocrinol. Metab, 40(3), 537-540.

Braunstein, G.D., Rasor, J., Adler, J., and Danzer, H. (1976). ME Wade Serum human chorionic gonadotropin levels throughout normal pregnancy, AM. J. Obstet. Gynecol., 126(6), 678-681.

Lenton, E.A., Neal, L.M., and Sulaiman, R. (1982). Plasma concentrations of human chorionic gonadotropin from the time of implantation until the second week of pregnancy, Fertil. Steril., 37(6), 773-778.

Steier, J.A., Bergsjo, P., and Myking, O.L. (1984). Human chorionic gonadotropin in maternal plasma after induced abortion, spontaneous abortion and removed ectopic pregnancy, Obstet. Gynecol., 64(3), 391-394.

Dawood, M.Y., Saxeba, B.B., and Landersman, R. (1977). Human chorionic gonadotropin and its subunits in hydatiform mole and choriocarcinoma, Obstet. Gynecol., 5(2), 172-181.

Braunstein, G.D., Vaitukatitis, J.L., Carbone, P.P., and Ross, G.T. (1973). Ectopic production of human chorionic gonadotropin by neoplasms, Ann. Inern. Med., 78(1), 39-45. 


\section{National Health and Nutrition Examination Survey National Center for Health Statistics \\ United States Public Health Service \\ Procedure: Complete Blood Count (CBC) \\ January 2009}

Prepared by

Date Adopted

Approved by

Director

Signed

Director

Dated

Director

Reviewed by

Date Reviewed

Reviewed by

Date Reviewed

Reviewed by

Date Reviewed

Reviewed by

Date Reviewed

Reviewed by

Date Reviewed

Reviewed by

Date Reviewed

Reviewed by

Date Reviewed

Reviewed by

Date Reviewed

Reviewed by

Date Reviewed 
THIS PAGE INTENTIONALLY LEFT BLANK. 


\section{COMPLETE BLOOD COUNT (CBC)}

Introduction

Perform a complete blood count (CBC) in duplicate on all Sample Persons (SPs) aged 1 and older. Perform the CBC on the Coulter ${ }^{\circledR}$ HMX. Run a CBC on the SP's EDTA blood tubes.

The three Coulter HMXs serial numbers and system IDs are:

HMX \#3 Serial \# AJ47275 System ID \# 493955

HMX \#2 Serial \# AK04023 System ID \# 495613

HMX \#1 Serial \# AK04024 System ID \# 495616

I. Purpose and Principle of Test

CBC Analysis

The Coulter ${ }^{\circledR}$ method accurately counts and sizes cells by detecting and measuring changes in electrical resistance when a particle (such as a cell) in a conductive liquid passes through a small aperture.

Each cell suspended in a conductive liquid (diluent) acts as an insulator. As each cell goes through the aperture, it momentarily increases the resistance of the electrical path between the submerged electrodes on either side of the aperture. This causes a measurable electronic pulse. For counting, the vacuum used to pull the diluted suspension of cells through the aperture must be at a regulated volume.

The number of pulses correlates to the number of particles. The height of the electrical pulse is proportional to the cell volume.

\section{Differential Analysis}

As the sample, prepared for differential analysis, streams through the flow cell these three measurements occur simultaneously on each individual white cell to classify it:

- Low-frequency current measures volume.

- High-frequency current senses cellular internal content through measuring changes in conductivity.

- Light from the laser bouncing off the individual WBC cells characterizes cellular surface, shape, and reflectivity. 
The Coulter HMX Hematology Analyzer is a quantitative, automated hematology analyzer and leukocyte differential cell counter for In Vitro Diagnostic use in clinical laboratories. The purpose of the HMX Hematology Analyzer is to separate the normal participant, with all normal systemgenerated parameters, from the participant who needs additional studies. These studies include further measurements of cell size and cell distribution, biochemical investigation, or any other test that helps diagnose the abnormality.

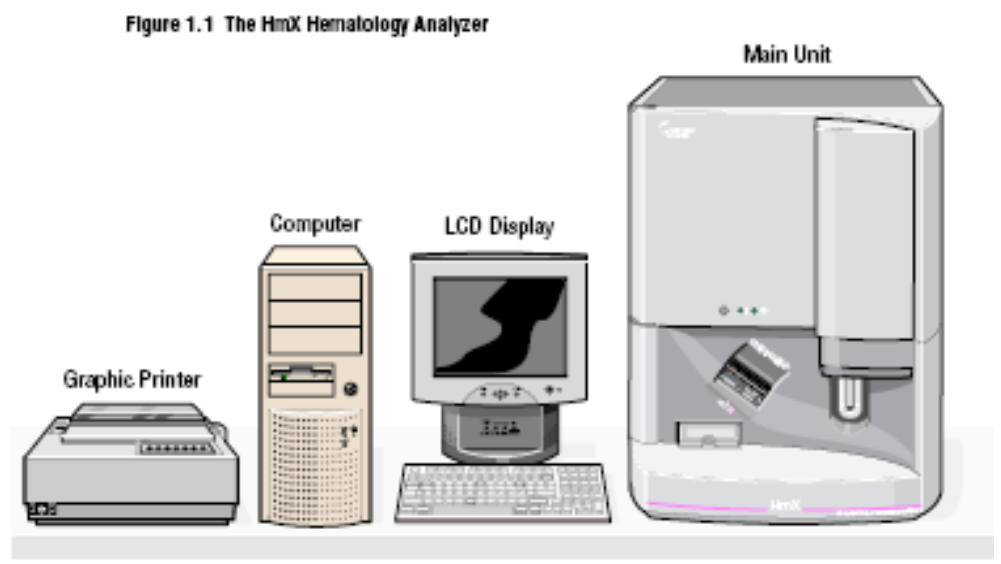

The HMX measures these parameters in whole blood:

\begin{tabular}{|c|c|c|c|c|}
\hline Cell & Parameter & Measured & $\begin{array}{l}\text { Pulse size } \\
\text { Wavelength } \\
\text { Calculation }\end{array}$ & Reported Units \\
\hline WBC & $\begin{array}{l}\text { White Blood Cell Count } \\
\text { This is the number of } \\
\text { leukocytes measured } \\
\text { directly, multiplied by } \\
\text { the calibration constant, } \\
\text { and expressed as: } \\
\mathrm{n} \times 10^{3} \text { cells/ } \mu \mathrm{L}\end{array}$ & WBC bath & $\geq 35 \mathrm{fL}$ & $\mathrm{n} \times 10^{3}$ cells $/ \mu \mathrm{L}$ \\
\hline RBC & $\begin{array}{l}\text { Red Blood Cell Count } \\
\text { This is the number of } \\
\text { erythrocytes measured } \\
\text { directly, multiplied by } \\
\text { the calibration constant, } \\
\text { and expressed as: } \\
\mathrm{n} \times 10^{6} \text { cells/ } \mathrm{\mu L}\end{array}$ & RBC bath & 36 to $360 \mathrm{fL}$ & $\mathrm{n} \times 10^{6}$ cells $/ \mu \mathrm{L}$ \\
\hline
\end{tabular}




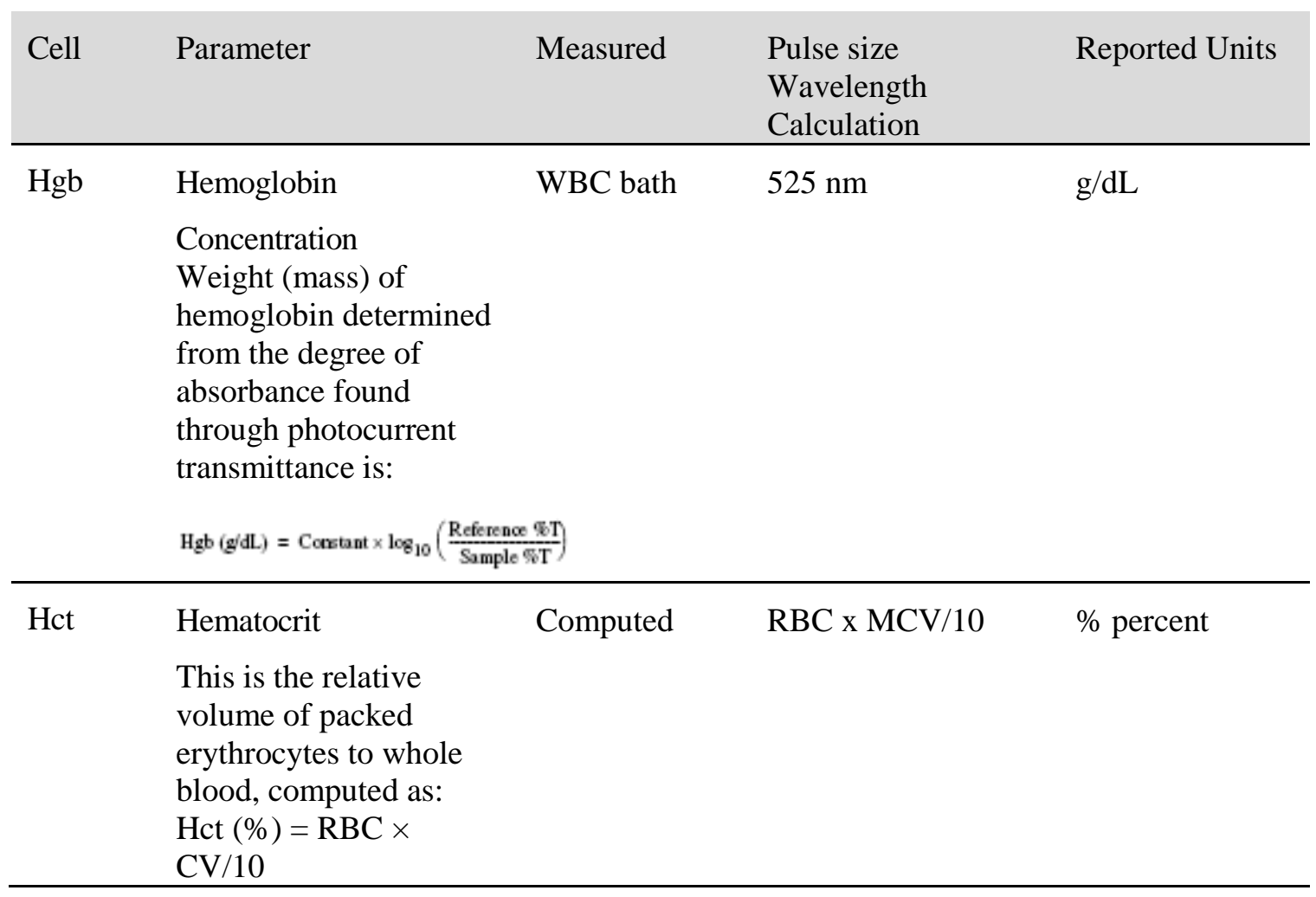




\begin{tabular}{|c|c|c|c|c|}
\hline Cell & Parameter & Measured & $\begin{array}{l}\text { Pulse size } \\
\text { Wavelength } \\
\text { Calculation }\end{array}$ & Reported Units \\
\hline MCV & $\begin{array}{l}\text { Mean Cell Volume } \\
\text { This is the average } \\
\text { volume of individual } \\
\text { erythrocytes derived } \\
\text { from the RBC } \\
\text { histogram. The } \\
\text { system: } \\
\text { - Multiplies the } \\
\text { number of } \\
\text { RBCs in each } \\
\text { channel by the } \\
\text { size of the } \\
\text { RBCs in that } \\
\text { channel. } \\
\text { Adds the } \\
\text { products of } \\
\text { each channel } \\
\text { between } 36 \mathrm{fL} \\
\text { and } 360 \mathrm{fL} . \\
\text { Divides that } \\
\text { sum by the } \\
\text { total number } \\
\text { of RBCs } \\
\text { between } 36 \mathrm{fL} \\
\text { and } 360 \mathrm{fL} \text {. } \\
\text { Multiplies by } \\
\text { a calibration } \\
\text { constant and } \\
\text { expresses MCV } \\
\text { in femtoliters. }\end{array}$ & $\begin{array}{l}\text { Derived from } \\
\text { RBC } \\
\text { histogram }\end{array}$ & $\begin{array}{l}\text { \# } x \text { size of } R B C / \\
\text { Total RBC }\end{array}$ & $\mathrm{fL}$ \\
\hline $\mathrm{MCH}$ & $\begin{array}{l}\text { Mean Cell Hemoglobin } \\
\text { This is the weight of } \\
\text { hemoglobin in the } \\
\text { average erythrocyte } \\
\text { count, computed as: } \\
\text { Hgb/RBC x } 10\end{array}$ & Computer & Hgb/RBC x 10 & pg \\
\hline
\end{tabular}




\begin{tabular}{|c|c|c|c|c|}
\hline Cell & Parameter & Measured & $\begin{array}{l}\text { Pulse size } \\
\text { Wavelength } \\
\text { Calculation }\end{array}$ & Reported Units \\
\hline \multirow[t]{2}{*}{ MCHC } & $\begin{array}{l}\text { Mean Cell Hemoglobin } \\
\text { Concentration }\end{array}$ & \multirow[t]{2}{*}{ Computed } & \multirow[t]{2}{*}{ Hgb/Hct x 100} & \multirow[t]{2}{*}{ g/dL } \\
\hline & $\begin{array}{l}\text { This is the average } \\
\text { weight of hemoglobin in } \\
\text { a measured dilution, } \\
\text { computed as: } \\
\text { Hgb/Hct x } 100\end{array}$ & & & \\
\hline \multirow[t]{2}{*}{ RDW } & $\begin{array}{l}\text { Red Cell Distribution } \\
\text { Width }\end{array}$ & \multirow{2}{*}{$\begin{array}{l}\text { Derived from } \\
\text { RBC } \\
\text { histogram }\end{array}$} & \multirow{2}{*}{$\begin{array}{l}\text { CV expressed in \% } \\
\text { of the RBC size } \\
\text { distribution }\end{array}$} & \multirow[t]{2}{*}{$\%$ percent } \\
\hline & $\begin{array}{l}\text { RDW represents the } \\
\text { size distribution spread } \\
\text { of the erythrocyte } \\
\text { population derived from } \\
\text { the RBC histogram. It is } \\
\text { the coefficient of } \\
\text { variation (CV), } \\
\text { expressed in percent, of } \\
\text { the RBC size } \\
\text { distribution. }\end{array}$ & & & \\
\hline \multirow[t]{2}{*}{ Plt } & Platelet Count & \multirow[t]{2}{*}{ RBC bath } & \multirow[t]{2}{*}{2 to $20 \mathrm{fL}$} & \multirow[t]{2}{*}{$\mathrm{n} \times 10^{3}$ cells $/ \mu \mathrm{L}$} \\
\hline & $\begin{array}{l}\text { This is the number of } \\
\text { thrombocytes derived } \\
\text { from the Plt histogram } \\
\text { and multiplied by a } \\
\text { calibration constant. } \\
\text { This number is } \\
\text { expressed as: } \\
\mathrm{n} \times 10^{3} \text { cells } / \mu \mathrm{L}\end{array}$ & & & \\
\hline \multirow[t]{2}{*}{ MPV } & Mean Platelet Volume & \multirow{2}{*}{$\begin{array}{l}\text { Derived from } \\
\text { Plt histogram }\end{array}$} & \multirow{2}{*}{$\begin{array}{l}\text { Mean volume of Plt } \\
\text { population under the } \\
\text { fitted curve } x \\
\text { constant }\end{array}$} & \multirow[t]{2}{*}{$\mathrm{fL}$} \\
\hline & $\begin{array}{l}\text { MPV is the average } \\
\text { volume of individual } \\
\text { platelets derived from } \\
\text { the Plt histogram. It } \\
\text { represents the mean } \\
\text { volume of the Plt } \\
\text { population under the } \\
\text { fitted Plt curve } \\
\text { multiplied by a } \\
\text { calibration constant, and } \\
\text { expressed in femtoliters. }\end{array}$ & & & \\
\hline
\end{tabular}




\begin{tabular}{|c|c|c|c|c|}
\hline Cell & Parameter & Measured & $\begin{array}{l}\text { Pulse size } \\
\text { Wavelength } \\
\text { Calculation }\end{array}$ & Reported Units \\
\hline NE\% & $\begin{array}{l}\text { Neutrophil Percent } \\
\text { The percentages of } \\
\text { leukocytes from each } \\
\text { category are derived } \\
\text { from the scatterplot. }\end{array}$ & $\begin{array}{l}\text { Derived from } \\
\text { scatterplot }\end{array}$ & $\begin{array}{l}\text { \# cells inside NE } \\
\text { area/\# cells inside } \\
\text { total cell area x } 100\end{array}$ & \% percent \\
\hline NE \# & $\begin{array}{l}\text { Neutrophil Number } \\
\text { The absolute numbers } \\
\text { of leukocytes in each } \\
\text { category are computed } \\
\text { from the WBC count } \\
\text { and the differential } \\
\text { percentage parameters. }\end{array}$ & $\begin{array}{l}\text { Absolute } \\
\text { number }\end{array}$ & $\begin{array}{l}\text { NE\%/100 x WBC } \\
\text { count }\end{array}$ & $10^{3}$ cells $/ \mu \mathrm{L}$ \\
\hline LY\% & $\begin{array}{l}\text { Lymphocyte Percent } \\
\text { The percentages of } \\
\text { leukocytes from each } \\
\text { category are derived } \\
\text { from the scatterplot. }\end{array}$ & $\begin{array}{l}\text { Derived from } \\
\text { scatterplot }\end{array}$ & $\begin{array}{l}\text { \# cells inside LY } \\
\text { area/\# cells inside } \\
\text { total cell area x } 100\end{array}$ & \% percent \\
\hline LY\# & $\begin{array}{l}\text { Lymphocyte Number } \\
\text { The absolute numbers } \\
\text { of leukocytes in each } \\
\text { category are computed } \\
\text { from the WBC count } \\
\text { and the differential } \\
\text { percentage parameters. }\end{array}$ & $\begin{array}{l}\text { Absolute } \\
\text { number }\end{array}$ & $\begin{array}{l}\text { Ly\%/100 x WBC } \\
\text { count }\end{array}$ & $10^{3}$ cells $/ \mu \mathrm{L}$ \\
\hline $\mathrm{MO} \%$ & $\begin{array}{l}\text { Monocyte Percent } \\
\text { The percentages of } \\
\text { leukocytes from each } \\
\text { category are derived } \\
\text { from the scatterplot. }\end{array}$ & $\begin{array}{l}\text { Derived from } \\
\text { scatterplot }\end{array}$ & $\begin{array}{l}\text { \# cells inside MO } \\
\text { area/\# cells inside } \\
\text { total cell area x } 100\end{array}$ & \% percent \\
\hline MO\# & $\begin{array}{l}\text { Monocyte Number } \\
\text { The absolute numbers } \\
\text { of leukocytes in each } \\
\text { category are computed } \\
\text { from the WBC count } \\
\text { and the differential } \\
\text { percentage parameters. }\end{array}$ & $\begin{array}{l}\text { Absolute } \\
\text { number }\end{array}$ & $\begin{array}{l}\text { MO\%/100 x WBC } \\
\text { count }\end{array}$ & $10^{3}$ cells $/ \mu \mathrm{L}$ \\
\hline
\end{tabular}




\begin{tabular}{|c|c|c|c|c|}
\hline Cell & Parameter & Measured & $\begin{array}{l}\text { Pulse size } \\
\text { Wavelength } \\
\text { Calculation } \\
\end{array}$ & Reported Units \\
\hline $\mathrm{EO} \%$ & $\begin{array}{l}\text { Eosinophil Percent } \\
\text { The percentages of } \\
\text { leukocytes from each } \\
\text { category are derived } \\
\text { from the scatterplot. }\end{array}$ & $\begin{array}{l}\text { Derived from } \\
\text { scatterplot }\end{array}$ & $\begin{array}{l}\text { \# cells inside EO } \\
\text { area/\# cells inside } \\
\text { total cell area x } 100\end{array}$ & $\%$ percent \\
\hline EO\# & $\begin{array}{l}\text { Eosinophil Number } \\
\text { The absolute numbers } \\
\text { of leukocytes in each } \\
\text { category are computed } \\
\text { from the WBC count } \\
\text { and the differential } \\
\text { percentage parameters. }\end{array}$ & $\begin{array}{l}\text { Absolute } \\
\text { number }\end{array}$ & $\begin{array}{l}\mathrm{EO} \% / 100 \times \mathrm{WBC} \\
\text { count }\end{array}$ & $10^{3}$ cells $/ \mu \mathrm{L}$ \\
\hline BA\% & $\begin{array}{l}\text { Basophil Percent } \\
\text { The percentages of } \\
\text { leukocytes from each } \\
\text { category are derived } \\
\text { from the scatterplot. }\end{array}$ & $\begin{array}{l}\text { Derived from } \\
\text { scatterplot }\end{array}$ & $\begin{array}{l}\text { \# cells inside BA } \\
\text { area/\# cells inside } \\
\text { total cell area x } 100\end{array}$ & $\%$ percent \\
\hline BA\# & $\begin{array}{l}\text { Basophil Number } \\
\text { The absolute numbers } \\
\text { of leukocytes in each } \\
\text { category are computed } \\
\text { from the WBC count } \\
\text { and the differential } \\
\text { percentage parameters. }\end{array}$ & $\begin{array}{l}\text { Absolute } \\
\text { number }\end{array}$ & $\begin{array}{l}\text { BA\%/100 x WBC } \\
\text { count }\end{array}$ & $10^{3}$ cells $/ \mu \mathrm{L}$ \\
\hline
\end{tabular}

\section{*PDW -- Platelet Distribution Width and Pct -- Platelet Crit are NOT for diagnostic use and do not print. Coulter uses the value for PDW as an internal check on the reported platelet parameters, Pct and MPV.}

Methodology: The methods used to derive CBC parameters are based on the Coulter ${ }^{\circledR}$ method of counting and sizing, in combination with an automatic diluting and mixing device for sample processing, and a single beam photometer for hemoglobinometry. The WBC differential uses VCS technology. Analysis and classification of WBCs use three simultaneous measurements of individual cell volume (V), high frequency conductivity (C), and laser light scatter (S). The scattergram plots the cells based upon the measurements of these three parameters. 


\section{A. Access Screen}

The Access screen provides quick access to the most commonly used areas of the software. It is the first screen to appear after a system reset or power up. If you go to any of these areas using the Access screen, you will automatically return to the Access screen upon exit. The Access screen is also available from the Main Menu by using the F1 key.

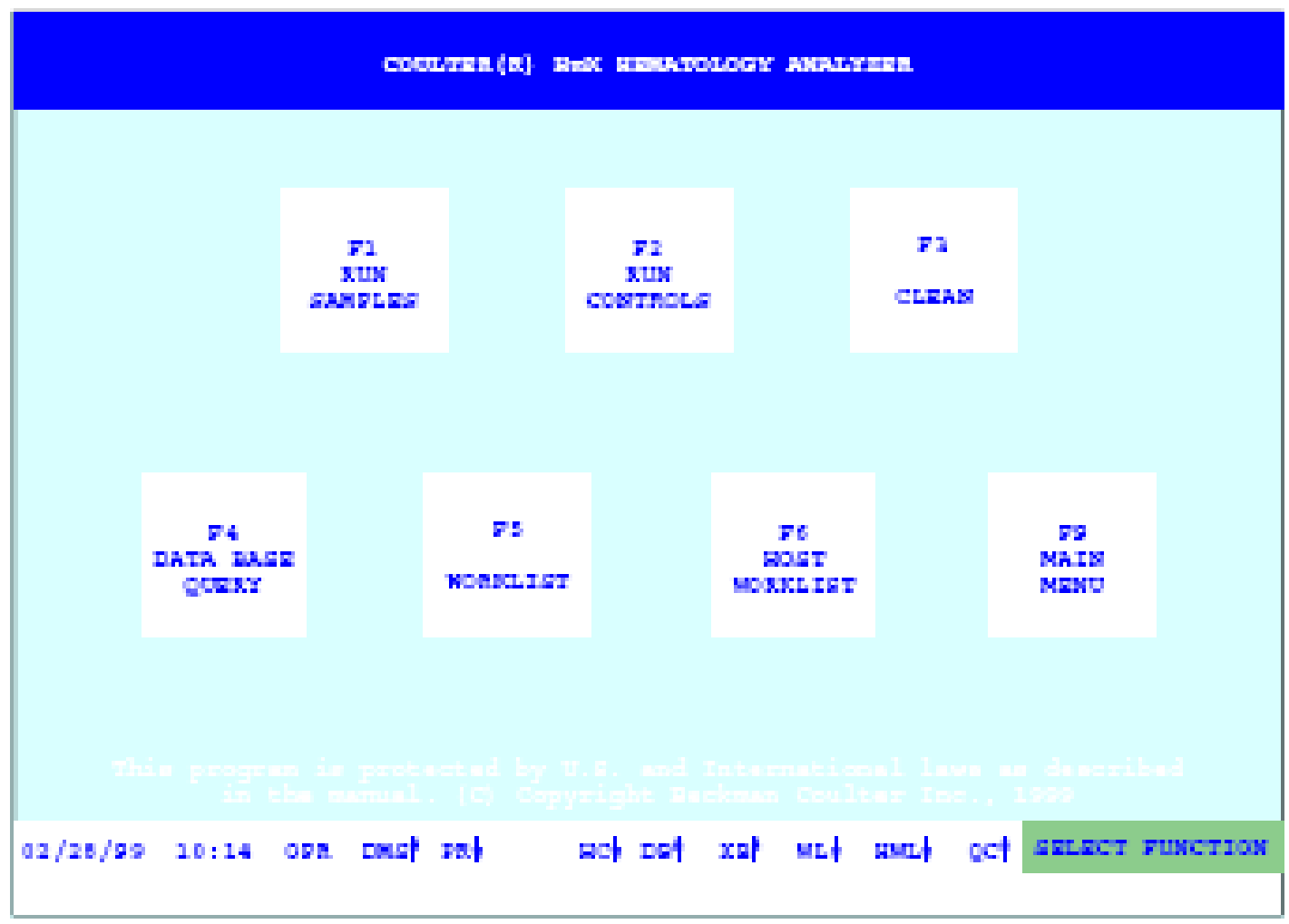


B. Software Menu Tree

The Main Menu consists of the four items listed across the top of the menu tree: Sample Analysis, Controls, Diluter Functions, and Special Functions.

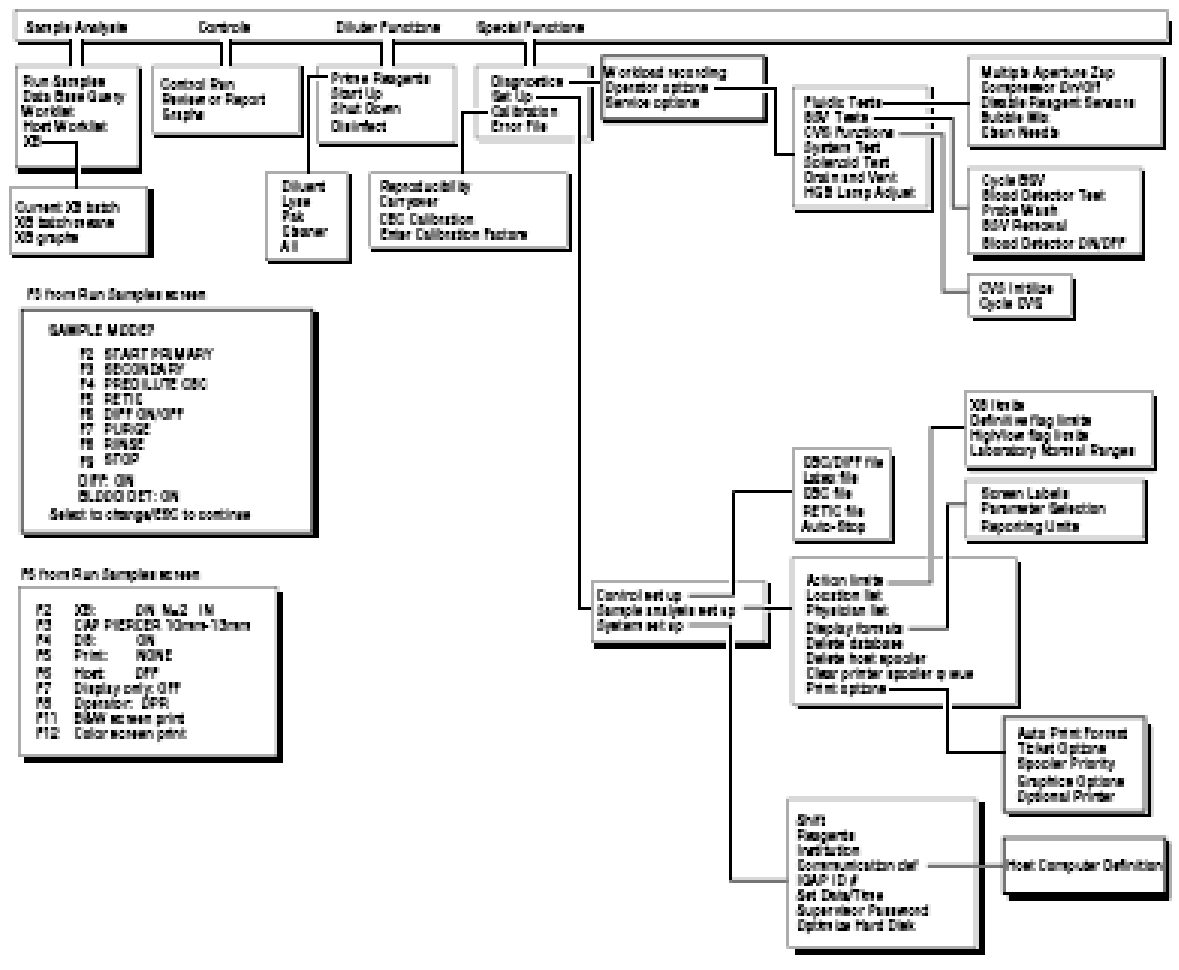


C. Run Sample Screen Options

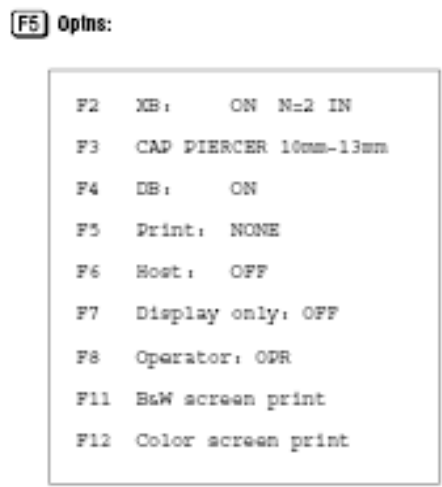

Description of Run Screen Options

\begin{tabular}{|l|l|}
\hline F2 XB: & $\begin{array}{l}\text { Turns XB ON and OFF. N is the number of } \\
\text { samples stored in the current batch. Also displays } \\
\text { the status of the last completed batch (IN or OUT). }\end{array}$ \\
\hline F3 CAP PIERCER 10mm-13mm & $\begin{array}{l}\text { Alternate between this and 16mm. Select the } \\
\text { correct carousel slot based on the size of the tube } \\
\text { you run. }\end{array}$ \\
\hline F4 DB: ON & $\begin{array}{l}\text { Turns the data base ON and OFF. Default setting is } \\
\text { ON. }\end{array}$ \\
\hline F5 Print: NONE & $\begin{array}{l}\text { Sets the automatic printing of samples to the } \\
\text { graphic printer. Choose between NONE, } \\
\text { NORMALS, ABNORMALS, or ALL. Default } \\
\text { setting is NONE. }\end{array}$ \\
\hline F6 Host: OFF & $\begin{array}{l}\text { Turns the automatic host transmission ON and } \\
\text { OFF. Default setting is OFF. }\end{array}$ \\
\hline F7 Display only: OFF & $\begin{array}{l}\text { If ON, then XB, DB, and HOST turn OFF. Default } \\
\text { setting is OFF. }\end{array}$ \\
\hline F8 Operator: OPR & $\begin{array}{l}\text { Enter up to three alphanumeric characters for an } \\
\text { Operator ID. Default setting is OPR. }\end{array}$ \\
\hline F11 B\&W screen print & $\begin{array}{l}\text { Initiates a large black and white screen print of the } \\
\text { current sample. }\end{array}$ \\
\hline F12 Color screen print & $\begin{array}{l}\text { Initiates a large color screen print of the current } \\
\text { sample if your printer can print in color. }\end{array}$ \\
\hline
\end{tabular}

NOTE: After a system reset, these options return to their default settings. Be sure to set them up again according to your laboratory's protocol before running patient samples.

NOTE: Print, Host, and Operator can also be set up from the Main Menu using [F5]-Options. 
D. Status Line

The status line at the bottom of your screen indicates the current operating status of the HMX Hematology Analyzer.

\begin{tabular}{|c|c|c|c|c|c|c|}
\hline Symbol & Refers to & $\uparrow$ & $\downarrow$ & Red & Yellow & White \\
\hline $\begin{array}{l}\text { DMS } \\
\uparrow\end{array}$ & $\begin{array}{l}\text { Data } \\
\text { Management } \\
\text { System }\end{array}$ & $\begin{array}{l}\text { Connected } \\
\text { to Analyzer. }\end{array}$ & $\begin{array}{l}\text { Not } \\
\text { connected to } \\
\text { Analyzer. }\end{array}$ & $\begin{array}{l}\text { Not } \\
\text { communicating } \\
\text { with Analyzer. }\end{array}$ & $\begin{array}{l}\text { DMS } \\
\text { busy or } \\
\text { receiving } \\
\text { data. }\end{array}$ & $\begin{array}{l}\text { DMS } \\
\text { is OK. }\end{array}$ \\
\hline $\begin{array}{l}\mathrm{PR} \\
\downarrow\end{array}$ & $\begin{array}{l}\text { Graphics } \\
\text { Printer }\end{array}$ & $\begin{array}{l}\text { Autoprint is } \\
\text { set to ALL, } \\
\text { ABNORMALS, } \\
\text { or NORMALS. }\end{array}$ & $\begin{array}{l}\text { Autoprint is } \\
\text { set to } \\
\text { NONE. }\end{array}$ & $\begin{array}{l}\text { Printer is off- } \\
\text { line, or printer is } \\
\text { out of paper. }\end{array}$ & $\begin{array}{l}\text { Printer is } \\
\text { printing. }\end{array}$ & $\begin{array}{l}\text { Printer and } \\
\text { DMS are } \\
\text { connected. }\end{array}$ \\
\hline $\begin{array}{l}\mathrm{HC} \\
\uparrow\end{array}$ & $\begin{array}{l}\text { Host } \\
\text { Computer }\end{array}$ & $\begin{array}{l}\text { Auto } \\
\text { transmission } \\
\text { ON. }\end{array}$ & $\begin{array}{l}\text { Auto } \\
\text { transmission } \\
\text { OFF. }\end{array}$ & $\begin{array}{l}\text { Not connected } \\
\text { to host. }\end{array}$ & $\begin{array}{l}\text { Sending } \\
\text { data to } \\
\text { host } \\
\text { computer. }\end{array}$ & $\begin{array}{l}\text { Host and } \\
\text { DMS are } \\
\text { connected. }\end{array}$ \\
\hline $\begin{array}{l}\mathrm{DB} \\
\uparrow\end{array}$ & Data Base & $\begin{array}{l}\text { Store is } \\
\text { ON. }\end{array}$ & $\begin{array}{l}\text { Store is } \\
\text { OFF. }\end{array}$ & $\begin{array}{l}\text { Data Base is not } \\
\text { functional. } \\
\text { System stops. } \\
\text { Reset the system } \\
\text { and rerun last } 2 \\
\text { samples. }\end{array}$ & $\begin{array}{l}\text { Data Base } \\
\text { is storing } \\
\text { data. }\end{array}$ & $\begin{array}{l}\text { Data Base } \\
\text { is OK. }\end{array}$ \\
\hline $\begin{array}{l}\mathrm{XB} \\
\downarrow\end{array}$ & $\mathrm{X}_{\mathrm{B}}$ Analysis & $\mathrm{XB}$ is $\mathrm{ON}$. & $\mathrm{XB}$ is OFF. & $\begin{array}{l}\text { Last completed } \\
\text { batch was OUT. }\end{array}$ & N/A & $\begin{array}{l}\text { Last } \\
\text { completed } \\
\text { batch } \\
\text { was IN. }\end{array}$ \\
\hline $\begin{array}{l}\text { WL } \\
\downarrow\end{array}$ & Worklist & $\begin{array}{l}\text { Preassigned } \\
\text { entries pending } \\
\text { on Worklist. }\end{array}$ & $\begin{array}{l}\text { No } \\
\text { preassigned } \\
\text { entries on } \\
\text { Worklist. }\end{array}$ & $\begin{array}{l}\text { Three } \\
\text { consecutive or } \\
10 \text { total error } \\
\text { messages are in } \\
\text { the status field. }\end{array}$ & $\begin{array}{l}\text { The } \\
\text { Worklist } \\
\text { is full. } \\
\text { (There are } \\
300 \\
\text { preassigned } \\
\text { samples.) }\end{array}$ & $\begin{array}{l}\text { Worklist } \\
\text { is OK. }\end{array}$ \\
\hline $\begin{array}{l}\text { HWL } \\
\downarrow\end{array}$ & Host Worklist & $\begin{array}{l}\text { Preassigned } \\
\text { entries pending } \\
\text { on the Host } \\
\text { Worklist. }\end{array}$ & $\begin{array}{l}\text { No } \\
\text { preassigned } \\
\text { entries on } \\
\text { Worklist. }\end{array}$ & $\begin{array}{l}\text { Host Worklist } \\
\text { is full. }\end{array}$ & $\begin{array}{l}\text { DMS is } \\
\text { receiving } \\
\text { preassigned } \\
\text { samples } \\
\text { from the } \\
\text { host } \\
\text { computer. }\end{array}$ & $\begin{array}{l}\text { Host } \\
\text { Worklist } \\
\text { is OK. }\end{array}$ \\
\hline $\begin{array}{l}\text { QC } \\
\uparrow\end{array}$ & $\begin{array}{l}\text { Quality } \\
\text { Control }\end{array}$ & $\begin{array}{l}\text { Auto-Stop is } \\
\text { ON. }\end{array}$ & $\begin{array}{l}\text { Auto-Stop } \\
\text { is OFF. }\end{array}$ & $\begin{array}{l}\text { Last control run } \\
\text { had an error } \\
\text { message. }\end{array}$ & $\begin{array}{l}\text { Receiving } \\
\text { a control } \\
\text { ru. }\end{array}$ & $\begin{array}{l}\text { Results of } \\
\text { last control } \\
\text { run are OK. }\end{array}$ \\
\hline
\end{tabular}

*Changes to MA for manual printing, BA for batch printing, and AU for auto-printing. 


\title{
II. Special Safety Precautions
}

\begin{abstract}
All specimens may be potentially positive for infectious agents including HIV and the hepatitis B and $\mathrm{C}$ viruses. Observe universal precautions. It is mandatory to wear gloves and lab coat when handling all human blood products and Coulter ${ }^{\circledR}$ controls. Wear safety glasses whenever operating the instrument in SECONDARY mode. Dispose of all biological samples in a biohazard container and wipe down all work surfaces with $10 \%$ bleach solution at the end of each session.
\end{abstract}

The mobile examination center (MEC) Working Safely with Hazardous Chemicals manual contains all Coulter material safety data sheets (MSDS).

III. Computerization: Integrated Survey and Information System (ISIS)

The HMX Data Management System (DMS) transmits individual SP results to the MEC automated ISIS system. Review all SP results at the Coulter DMS monitor. The hematology module in the laboratory application automatically receives the results or transmits them manually to the hematology module. The laboratory application evaluates the data for completeness and accuracy. The final decision to accept or reject a result is the responsibility of the medical technologist.

All data are backed up and stored at Westat's home office.

IV. Specimen Collection and Preparation

A. Specimen Collection

1. The phlebotomist collects a 3 or $4-\mathrm{mL}_{3}$ EDTA tube on all SP's aged 1+ following established venipuncture protocol and procedures. (A 1-2\% dilution effect occurs in this liquid EDTA tube.) 
2. Sample volume is $185 \mu \mathrm{L}$ of whole blood in the closed-vial mode. The minimum sample volume per tube in the closed-vial mode is 1-mL with the proper proportion of blood to anticoagulant.

\section{B. Specimen Preparation}

1. The blood specimen-processing technologist initially processes the tube by taking off whole blood for various tests. The blood specimen-processing technologist places the specimen on a rocker until the hematology technologist can perform the $\mathrm{CBC}$. Run the $\mathrm{CBC}$ as soon as possible; there is no requirement to wait any length of time between drawing the blood and running the CBC.

V. Procedure for Microscopic Examination

Not Applicable - Do not prepare differential microscopic slides.

VI. Reagents and Supplies

The HMX DMS stores and maintains the lot numbers and expiration dates.

A. Reagents, Controls, and Calibrators

1. $\quad$ Isoton III ${ }^{\circledR}$ (diluent) - PN 8546733 (20 L) - an isotonic electrolyte

- Dilutes the whole-blood samples.

- Stabilizes cell membranes for accurate counting and sizing.

- Conducts aperture current.

- Carries and focuses the sample stream in the flow cell to enable the WBC differential measurements.

- Rinses the system between samples.

- Expires on expiration date printed on container. 
2. $\quad$ COULTER CLENZ ${ }^{\circledR}$ - PN 8546931 (10L) - a cleaning agent that cleans and rinses the internal surfaces of the Diluter components. Daily use prevents protein buildup and eliminates routine aperture bleaching.

- Expires 90 days after being opened and installed on the instrument.

3. $\quad$ LYSE S® III - PN 8546983 (1L diff lytic reagent is a lytic reagent used for the CBC mode.)

- Rapidly lyses erythrocytes (RBCs), freeing hemoglobin (Hgb), and reducing the size of cellular debris to a level that does not interfere with the leukocyte (WBC) count.

- Causes a substantial conversion of the Hgb to a stable pigment, the absorbance of which is directly proportional to the Hgb concentration over the clinical range.

- NOTE: If you use LYSE S III diff lytic reagent you must use ISOTON III diluent.

- Expires 60 days after being opened and installed on the instrument.

4. HMX Pak®-PN 8547166 - The HMX Pak contains the PAK LYSE (Erythrolyse $^{\mathrm{TM}}$ II erythrocyte lytic reagent) and the PAK PRESERVE (StabiLyse ${ }^{\mathrm{TM}}$ leukocyte preservative) used for the differential measurement.

- The PAK LYSE (also called the diff lytic reagent), while maintaining leukocytes (WBCs) in near-native state.

- The PAK PRESERVE preserves the leukocytes (WBCs) in near-native state. It allows the leukocytes to be differentiated into their subpopulations through the volume, conductivity, and light-scatter measurements.

- Expires 60 days after being opened and installed on the instrument.

5. $\quad$ Latron ${ }^{\circledR}$ Controls - PN 7547116 (5 x 16-mL)

- LATRON control monitors the performance of the volume, conductivity, and light scatter measurements. 
- Expires 30 days after being opened.

6. Latron ${ }^{\circledR}$ Primer - PN 7546915 (5 x 16-mL)

- LATRONTM primer prepares the tubing and instrument components for the LATRON control.

- Expires 30 days after being opened.

7. $\quad 5 C \AA$ Cell Controls Tri Pack contains Normal, Abnormal I, Abnormal II - PN $7547001(9 \times 3.3-\mathrm{mL})$

- COULTER 5C® cell control monitors the CBC and differential parameters.

- $\quad$ Expires 13 days or 13 events after being opened.

8. $\quad$ Calibration S-CAL ${ }^{\circledR}-$ PN $7546808(2 \times 6-m L)$

- Use at the start of each stand.

- The S-CAL ${ }^{\circledR}$ calibrator kit calibrates Primary mode CBC parameters and is an acceptable alternative to the whole-blood reference method of calibration.

- $\quad$ Expires 1 hour after being opened.

9. $\quad$ 5C® Cell Control Normal - PN $7546923(9$ x 3.3-mL)

- Use when calibrating with S-CAL for reproducibility study.

10. Lin-C - PN 6605374

- LIN-C ${ }^{\circledR}$ linearity control verifies the reportable range of the instrument's CBC parameters. 
B. Supplies

1. 3-mL $\mathrm{K}_{2}$ EDTA Becton Dickinson Hemogard Vacutainer ${ }^{\circledR}$ tube (367856)

2. 4-mL $\mathrm{K}_{2}$ EDTA Becton Dickinson Hemogard Vacutainer ${ }^{\circledR}$ tube (367861)

3. Tube rocker

4. Bleach, 5.25\% Sodium Hypochlorite

5. Bottled distilled water

6. Three 30-mL plastic containers with lid

7. Two 1-liter containers with lid

8. Plastic squirt bottle

9. $\quad$ Cotton gauze pads

10. Three-hole paper punch

11. Notebook

12. Flashlight

13. $10-\mathrm{mL}$ syringe with plastic tubing

14. Precision screwdriver set

15. Distilled water bottle

16. Disposable lab jacket - 48 inches long

17. Diskettes

C. Notes

1. If reagents become frozen in transit, mix thoroughly by inversion and let bubbles settle before use. 
2. To avoid electrical interference, do not place reagents on or near electric cords or lines.

VII. Calibration

S-CAL ${ }^{\circledR}\left(\right.$ PN 7546808 - 2 x 6-mL) - The S-CAL ${ }^{\circledR}$ calibrator kit determines the adjustment factors for the calibration of the Coulter ${ }^{\circledR}$ HMX. Calibration is a procedure to standardize the instrument by determining its deviation from calibration references and to apply any necessary correction factors. Perform calibration in the close-vial mode at ambient room temperature range $\left(16-32^{\circ} \mathrm{C}, 60-90^{\circ} \mathrm{F}\right)$, using S-CAL $\AA$ as an alternative to whole blood.

A. Perform Calibration

- At the start of each stand, before you begin analyzing samples;

- After you replace any component dealing with dilution preparation, such as the BSV primary measurement, such as an aperture; and

- If your Beckman Coulter Representative suggests you calibrate.

B. Verify the Calibration of your Instrument

- If controls show unusual trends or are outside limits; and

- When room temperature varies more than $10^{\circ} \mathrm{F}\left(5.5^{\circ} \mathrm{C}\right)$ from the room temperature during the last calibration.

Principle - The HMX uses the S-CAL ${ }^{\circledR}$ kit that requires a calibrator to convert electronic measurements of each sample into accurate results expressed in clinical terms. S-CAL ${ }^{\circledR}$ calibrates the WBC, RBC, Hgb, Plt, and MPV parameters. It is a stabilized humanblood preparation. S-CAL $®$ is an acceptable alternative to whole blood calibration.

The calibration procedure uses replicate measurements of S-CAL® calibrator. The S-CAL ${ }^{\circledR}$ divides the average result into the calibrator Assigned Value to 
give the Adjustment Factor. Then, it obtains and adjusts an instrument reading according to the Adjustment Factor.

Hct, MCH, MCHC, RDW, and the DIFF parameters do not require calibration.

1. Reagents - S-CAL $\AA$ consists of treated, stabilized, human erythrocytes and platelet sized components in an isotonic bacteriostatic medium. Fixed erythrocytes simulate leukocytes.

Materials required - Before calibrating, assemble the following materials: S-CAL ${ }^{\circledR}$ kit containing two 6-mL vials of S-CAL ${ }^{\circledR}$ calibrator.

2. Storage, handling, and stability - Sealed vials are stable through the expiration date when stored at $2-8^{\circ} \mathrm{C}\left(35-46^{\circ} \mathrm{F}\right)$. Open vials are stable for 1 hour.

Potential biohazard - Each human donor used in preparation of this material was tested by an FDA-approved method for the presence of the antibodies to Human Immunodeficiency Virus (HIV-1 and HIV-2) and Hepatitis C (HCV), as well as for hepatitis B surface antigen, and found to be negative (were not repeatedly reactive). Handle these reagents at Biosafety Level 2 because no test method can offer complete assurance that these and other infectious agents are absent.

This product contains $<0.1 \%$ Sodium Azide. Sodium Azide preservative may form explosive compounds in metal drain lines. Discard this product in biohazardous waste containers.

3. Indications of instability or deterioration - Inability to obtain expected values in the absence of known instrument problems or gross hemolysis (darkly-colored supernatant) is indicative of product deterioration. However, a slight pink color to the supernatant is normal and should not be confused with deterioration of the product. 
C. Pre-calibration, Reproducibility, and Carryover Check

Perform a calibration after the instrument has been "cleaned" for at least 30 minutes. From the Access screen, press [F3] Clean.

1. Pre-calibration procedure

Bleach the aspiration system using the Clean Needle procedure.

a) Prepare a fresh bleach solution. Put 1-mL bleach and 1-mL distilled water into 5-mL lavender top Vacutainer ${ }^{\circledR}$ tube.

b) Stop the system before it performs the Clean Needle procedure. If status message is not Select Function or Compressor Off, go to SAMPLE ANALYSIS, RUN SAMPLE, [F3], Run. Press [F9] Stop.

c) Select SPECIAL FUNCTIONS, DIAGNOSTICS, OPERATOR OPTIONS, FLUIDIC TESTS, and CLEAN NEEDLE. Press [Enter] and follow the screen instructions. Wait for green light before placing tube into the carousel.

To rinse the system, perform daily Start Up. Select DILUTOR FUNCTIONS; START UP [Enter].

Run 5C® cell controls. 
2. Reproducibility check

a) Select SAMPLE ANALYSIS, RUN SAMPLES. Press [Enter]. Select [F5] to access options. Change [F5] Print: to none and [F6] Host to Off.

b) Use an unopened, normal-level, 5C® cell control for reproducibility studies. Label the control vial with date and initial. Use of expired controls for this procedure is acceptable.

c) Select SPECIAL FUNCTIONS, CALIBRATION, REPRODUCIBILITY [Enter].

d) Is Sample Mode? displayed? If YES, go to step 3. If NO, press [F9] Stop.

e) Press [F6]. Press space bar to turn the DIFF ON. Press [Enter]. Press [F2] START PRIMARY.

f) Does the following message appear? "MODE REQUIRES EXISTING RUNS TO BE DELETED. ARE YOU SURE?: NO.” If the message appears, press the space bar to answer "YES.” Press [Enter]. This deletes the old data.

If there are runs present in the table and the message does not appear, press [F8] Delete. Press the space bar to answer "YES.” Press [Enter]. This deletes the old data.

(1) Follow the directions listed in Section $\mathrm{X}$ for preparation and mixing the $5 \mathrm{C}{ }^{\circledR}$ cell control.

(2) Cycle the sample 11 times. Do not use the bar code reader. Mix gently before each cycle. Insert the tube into the carousel when the green light appears. The first sample is automatically marked DEL and its results are not included in the calculations. 
(3) Check the results. Verify the average \%CV does not exceed these limits:

$\begin{array}{lll}\text { - } & \text { WBC } & 2.5 \% \\ \text { - } & \text { RBC } & 2.0 \% \\ \text { - } & \text { Hgb } & 1.5 \% \\ \text { - } & \text { MCV } & 2.0 \% \\ \text { - } & \text { Plt } & 5.0 \% \\ \text { - } & \text { MPV } & 3.0 \%\end{array}$

Check the low to high difference (bottom line, right side) for the diff parameters with these limits:

$\begin{array}{lll}\text { - } & \mathrm{LY} \% & \leq 4.8 \\ \text { - } & \mathrm{MO} \% & \leq 3.2 \\ \text { - } & \mathrm{NE} \% & \leq 4.8 \\ \text { - } & \mathrm{EO} \% & \leq 1.6 \\ \text { - } & \mathrm{BA} \% & \leq 1.6\end{array}$

If the results are outside the limits, call the Beckman Coulter ${ }^{\circledR}$ representative.

(4) Press [F4] to print the screen for the logbook. Press [F3] Run [F9] STOP. Press [Esc]. Proceed with a Carryover Check.

\section{g) Notes}

(1) Be sure the PR $\downarrow$ is on the status line before starting the reproducibility study.

(2) Be sure to delete any data on the Reproducibility screen before starting a new study.

(3) On the Reproducibility screen, use the \%CV line for CBC parameters only and the Diff line for the DIFF parameters only. 
3. Carryover check (Draw two 4-mL EDTA tubes of normal blood.)

a) Select SPECIAL FUNCTIONS, CALIBRATION, and CARRYOVER. Press [F2] START PRIMARY. Does the following message appear? "MODE REQUIRES EXISTING RUNS TO BE DELETED. ARE YOU SURE?: NO.” If the message appears, press the space bar to answer "Yes.” Press [Enter]. This deletes the old data.

(1) Use a routine 4-mL Vacutainer ${ }^{\circledR}$ EDTA for the carryover check clean vials. Fill three separate vials with diluent, then cap.

(2) Follow the screen directions. Cycle two vials of normal blood and three vials of diluent. Do not use the bar code reader. Insert the tube into the carousel when the green light appears.

(3) Check the lower right corner of the screen for CARRYOVER ACCEPTABLE. If the result is not acceptable and the $\mathrm{H}$ flags appear next to the carryover value results, repeat the procedure. If results are still OUT, call the Beckman Coulter® representative.

(4) Press [F4] to print the carryover screen for the logbook. Press [F9] to return to the main menu.

b) Notes

(1) Choose a sample with a WBC count of 10,000 $\pm 1,000$ if possible. All other parameters should be within normal ranges.

(2) Ignore count period (CP) voteouts on diluent runs.

(3) Percent carryover is calculated by the formula.

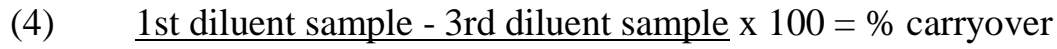
2nd sample. 


\section{CBC Calibration with S-CAL ${ }^{\circledR}$ Calibrator}

Discard the Calibrator Disks from the S-Cal Calibrator Kit. Locate the hard copy Table of Expected Results that contains 5 gray boxes labeled 1 through 5. Locate box 2 where it lists ISOTON III, Isoton 3E or LH Series Diluent / Lyse S III diff Lytic Reagent------------- HMX system. Locate the corresponding gray \#2 box in the table.

Take the S-CAL ${ }^{\circledR}$ calibrator from the refrigerator. Remove one vial of S-Cal from the kit. Return the unopened second vial to the refrigerator. Let the vial warm to ambient temperature for 15 minutes. Meanwhile, continue with the following steps:

1. Select Special Functions $\rightarrow$ Calibration $\rightarrow$ CBC Calibration. Press [F2] Start Primary. Delete any data in the table by pressing [F8]. Press the space bar to answer "YES." Press [Enter].

2. Prepare the CBC calibration screen.

a) Enter the S-CAL ${ }^{\circledR}$ calibrator assigned values from the Table of Expected Results on the package insert on the REF: VALUES line. The Hct is not calibrated although the value is listed on the package insert.

b) Enter the expiration date and the lot number.

3. Prepare and cycle the calibrator.

a) Mix by hand as follows: Roll the tube slowly between the palms of the hands eight times in an upright position. Invert the tube and slowly roll it again eight times. Gently invert the tube eight times. Repeat these actions again.

b) Determine that all cells are uniformly distributed by inspecting the vial contents. If the cells are not totally distributed, repeat the mixing procedure. 
c) Cycle the first cap-pierceable calibrator 11 times. Do not use the bar code reader. Invert the vial and mix the S-CAL ${ }^{\circledR}$ calibrator before each cycle. Insert the tube into the carousel when the green light appears. The first sample is automatically marked DEL and its results are not included in the calculations.

d) After the cycles are complete, press [F4] Print to print the screen for the logbook. Press [F3] Run, [F9] Stop. Press [Esc].

4. Assess the run.

a) Check for trending. If trending is present, stop and contact a Coulter ${ }^{\circledR}$ representative.

b) Check the \%CV values against these precision \%CV limits. If any parameter exceeds the limit, STOP and contact a Coulter ${ }^{\circledR}$ representative.

$\begin{array}{cc}\text { Parameter } & \text { Precision (CV\%) } \\ \text { WBC } & \leq 2.5 \\ \text { RBC } & \leq 2.0 \\ \text { Hgb } & \leq 1.5 \\ \text { MCV } & \leq 2.0 \\ \text { Plt } & \leq 5.0 \\ \text { MPV } & \leq 3.0\end{array}$


c) Determine which calibration factors (if any) should be changed by checking the FAC\%DIFF and DELTA DIFF values against these ranges. NOTE: Disregard minus signs. The values are absolute numbers.

$\begin{array}{ccc}\text { Parameter } & \begin{array}{c}\text { Calibrate if } \\ \text { FAC\%DIFF is: }\end{array} & \begin{array}{c}\text { Calibrate if } \\ \text { DELTA DIFF is: }\end{array} \\ \text { WBC } & >1.25 \text { and } \leq 5.00 & >0.10 \text { and } \leq 0.40 \\ \text { RBC } & >0.70 \text { and } \leq 2.00 & >0.03 \text { and } \leq 0.09 \\ \text { Hgb } & >0.78 \text { and } \leq 3.00 & >0.10 \text { and } \leq 0.40 \\ \text { MCV } & >1.18 \text { and } \leq 2.50 & >1.00 \text { and } \leq 2.00 \\ \text { Plt } & >2.70 \text { and } \leq 9.00 & >6.00 \text { and } \leq 20.00 \\ \text { MPV } & >5.00 \text { and } \leq 20.00 & >0.50 \text { and } \leq 2.00\end{array}$

d) If both the FAC\%DIFF and DELTA DIFF values of a parameter fall below their lower limits, that parameter does not need to be calibrated. NOTE: If all parameter values fall below the lower limits of both ranges, you are finished. Resume normal operation. Press [F4] to print the screen for the logbook.

e) If either the FAC\%DIFF or DELTA DIFF value of a parameter exceeds its upper limit, STOP. There could be an instrument problem. Call your Beckman Coulter Representative.

f) If either the FAC\%DIFF or DELTA DIFF value of a parameter falls between its lower and upper limits, that parameter should be calibrated. Continue with step 5 (below).

5. Verify calibration.

a) Determine which calibration factors to change. Check the FAC \% DIFF and DELTA DIFF values against these ranges. 


$\begin{array}{ccc}\text { Parameter } & \begin{array}{c}\text { Calibrate if } \\ \text { FAC\%DIFF is: }\end{array} & \begin{array}{c}\text { Calibrate if } \\ \text { DELTA DIFF is: }\end{array} \\ \text { WBC } & >1.25 \text { but } \leq 5.00 & >0.10 \text { but } \leq 0.40 \\ \text { RBC } & >0.70 \text { but } \leq 2.00 & >0.03 \text { but } \leq 0.09 \\ \text { Hgb } & >0.78 \text { but } \leq 3.00 & >0.10 \text { but } \leq 0.40 \\ \text { MCV } & >1.18 \text { but } \leq 2.50 & >1.00 \text { but } \leq 2.00 \\ \text { Plt } & >2.70 \text { but } \leq 9.00 & >6.0 \text { but } \leq 20.0 \\ \text { MPV } & >5.00 \text { but } \leq 20.00 & >0.50 \text { but } \leq 2.00\end{array}$

b) Is Select Function displayed? If NO, press [F3] Run [F9] Stop [Esc]. If YES, Press [F5] Optns. Choose SELECT PARAMETERS. Use the space bar to select YES for parameters that need adjusting, NO for others. Press [Esc].

c) Select TRANSMIT FACTORS. The following message appears: "WARNING - DATA WILL BE CLEARED AFTER TRANSMISSION. DO YOU WANT TO PRINT DATA? Y/N” If you did not print the Calibration screen press $[\mathrm{Y}]$. Select $[\mathrm{N}]$ if the values have already been printed.

d) Press [F4] Print to print this final calibration screen for the logbook. It reflects the new calibration factors for parameters you adjusted.

e) Verify calibration by cycling the $5 \mathrm{C}$ cell controls in the primary mode. If any of the control level's results are outside the expected range, run a second sample of the control. If the second sample is also outside of the expected range, call your Beckman Coulter representative.

6. Calibration terms and formulas

a) MEAN

- The average of the $10 \mathrm{~S}-\mathrm{CAL}$ calibrator runs 
b) NEW CAL FAC

- The calibration factor needed to obtain the S-CAL Calibrator Reference value. The instrument calculates and displays it whether or not it needs to be changed.

- $\quad$ Reference Value $x$ Old Cal Factor $=$ NEW CAL FAC

$\mathrm{S}-\mathrm{CAL}$ mean value $(\mathrm{n}=10)$

c) OLD CAL FAC

- The current calibration factor

d) $\quad \% \mathrm{CV}$

- Indicates the reproducibility of the S-CAL calibrator run. Check to ensure that the data being used are valid when making decisions to recalibrate or not.

e) $\quad$ FAC $\%$ DIFF

- The difference between the OLD CAL FAC and the NEW CAL FAC expressed as a percentage.

- $\quad$ NEW CAL FAC - OLD CAL FAC x 100 = FAC \%DIFF OLD CAL FAC

f) DELTA DIFF

- The absolute difference between the reference value and the SCAL calibrator MEAN. 


\section{g) REF VALUES}

- The assigned value for each parameter.

\section{Assay Procedure}

A. Run Start Up at the Beginning of the First Session Each Operational Day

1. Leave the instrument on and operate the HMX with all panels closed. Check to make sure the date and time displayed are correct. To set date and time select Special Functions, Set Up, System Set Up, Set Date/Time. Move the cursor and enter the correct date or time and press [Enter] or the arrow keys. Press [F10] to save and exit.

2. Are Start Up results already displayed as the result of a Clean cycle?
a) If No, go to step 2 .
b) If Yes, go to step 3 .

NOTE: The Clean cycle consists of 30 minutes in Shut Down followed by an automatic Start Up.

3. To begin Start Up, select Diluter Functions, and Start Up. Press [Enter]. Follow the instructions on the screen.

4. After Start Up is complete, evaluate the display. Expired reagents and failed checks appear in red. Press [F2] to view detailed results. Make sure the Background and other Start Up results are within limits. Results automatically print. Results outside limits turn red. File in the logbook. 
5. Review and verify sample analysis set up.

a) Select Sample Analysis, Run Samples [Enter]. Press [F5] for options. Press the corresponding function key to enter specific options.

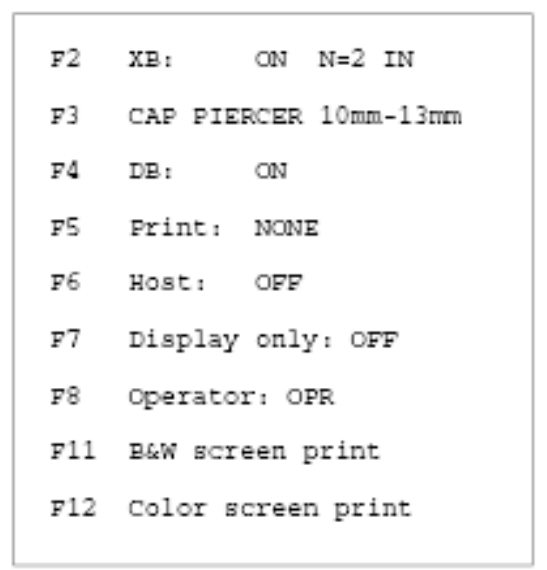

Settings

\begin{tabular}{|l|l|l|}
\hline F2 & XB & Off \\
\hline F3 & CAP PIERCER & 10mm-13mm \\
\hline F4 & DB & On \\
\hline F5 & Print & None \\
\hline F6 & Host & On \\
\hline F7 & Display only & Off \\
\hline F8 & Operator & Tech initials \\
\hline F11 & B \& W screen print & \\
\hline F12 & Color screen print & \\
\hline
\end{tabular}

Use the corresponding [F] key to change the setting. For example, press [F6] to turn the Diff ON. Press [Esc] to exit. 


\section{B. Run Controls}

Run Coulter ${ }^{\circledR}$ LATRON primer and latex control, and all three levels, normal (blue), abnormal I (yellow), and abnormal II (pink), of Coulter ${ }^{\circledR} 5$ C ${ }^{\circledR}$ cell control daily at the beginning of the first session. Run all three levels (normal, abnormal I, and abnormal II) of Coulter ${ }^{\circledR} 5 C \AA$ cell control at the beginning of the second session.

\section{Run Sample Persons}

1. Review the placement of the bar code on the sample tube. Place the end of the label flush with the stopper. The bars on the label must be parallel to the stopper. If the label is skewed more than $5^{\circ}$, the scanner may not read it. Do not cover the bottom of the tube with the bar-code label. The tube may jam in the carousel.

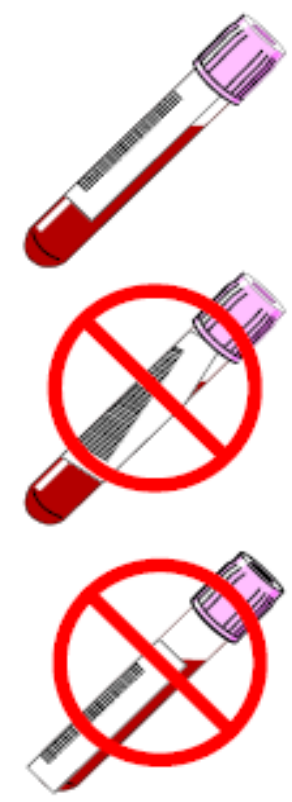

2. Run all samples in duplicate.

3. Run samples in the Primary mode.

In Primary mode, the system checks each sample aspiration using dual sensors called blood detectors, which monitor the blood before and after it passes through the Blood Sampling Valve (BSV). These blood detectors optically sense air 
bubbles, diluent, and blood. As an indication of a good aspiration, the system looks for blood in both detectors. If the detectors optically identify bubbles in the sample, the instrument pierces the tube a second time. If the second aspiration contains bubbles, the instrument reports a partial aspiration. Bubbles or air may be present for various reasons, such as short sample aspirations or blockages in the aspiration pathway. Single dots $\left(\bullet^{\cdots} \bullet\right)$ and $P A R T$. ASP are reported instead of numeric results when a partial aspiration occurs. Samples that generate multiple partial aspiration messages should be evaluated for specimen quality according to the laboratory's protocol. Samples with very low hemoglobin results may give partial aspirations when run in the Primary mode because the blood detectors do not recognize the sample as being blood. To obtain results, cycle the sample in the Secondary mode.

Check to make sure the printer is working properly. Operate the Analyzer with the doors and panels closed. Monitor reagent levels.

a) Does SELECT FUNCTION or S/A $1^{\circ}$ MODE ON appear at the lower right corner of the DMS monitor?

If not, then access the Run Samples screen. At the Access screen, press [F1] RUN SAMPLES or at the Main Menu, select Sample Analysis $\rightarrow$ Run Samples.

b) Press [F3] Run. 
c) The instrument automatically prepares itself to run in the Primary mode, DIFF ON. Press [F6] DIFF ON/OFF to change the DIFF setting. Note, if SAMPLE MODE is not displayed, press [F9] Stop first.

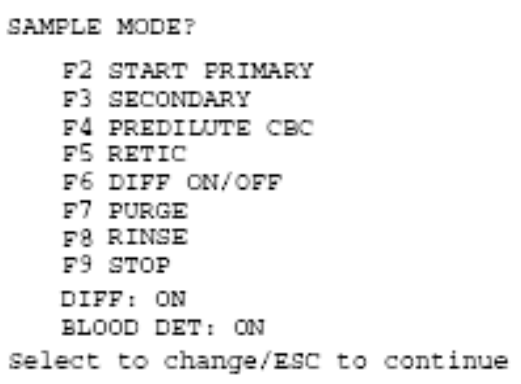

d) Does the top of the F3-Run window display PRIMARY: SAMPLE ANALYSIS? If Yes, press [Esc]. If No, press [F2] START PRIMARY.

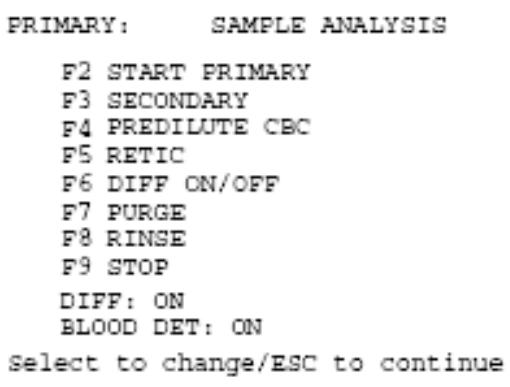


e) Identify the sample by holding the bar code label on the tube in front of the reader. Green light will beep; place the tube in the carousel. If the red light appears, wait and try reading the bar code label again. Cycle the sample within 10 seconds of reading the bar code. After 10 seconds, the system deletes the identification. If necessary, enter 1 to 16 alphanumeric characters then press [Enter].

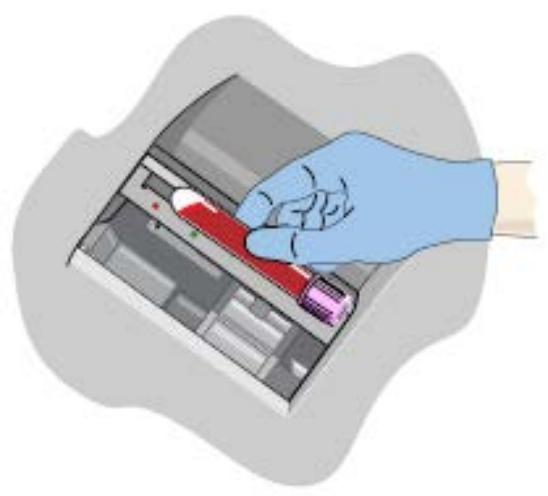

4. Alternatively, cycle samples in the Secondary mode.

Blood detectors are inactive in the Secondary mode. This mode does not check sample and aspiration integrity. Run samples in the Secondary mode only if the Primary mode is unavailable.

a) Access the Run Samples screen. At the Access screen press [F1] RUN SAMPLES or at the Main Menu, select Sample Analysis $\rightarrow$ Run Samples.

b) Press [F3] Run.

c) The instrument automatically prepares itself to run in the Primary mode, DIFF ON. Press [F6] DIFF ON/OFF to change the DIFF setting. Note, if SAMPLE MODE or S/A $1^{\circ}$ MODE ON is not displayed, press [F9] Stop first. 
d) Press [F3] SECONDARY.

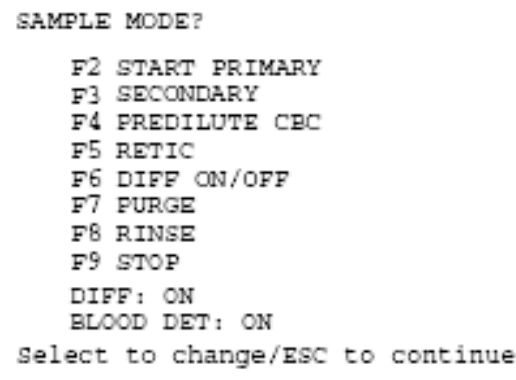

e) Identify the sample by holding the bar code label on the tube in front of the reader. Green light will beep. If the red light appears, wait and try reading the bar code label again. Cycle the sample within 10 seconds of reading the bar code. After 10 seconds, the system deletes the identification. If necessary, enter 1 to 16 alphanumeric characters then press [Enter].

f) Cycle the sample in the Secondary mode:

(1) Mix the sample gently.

(2) Open the tube and immerse the aspirator tip in the sample.

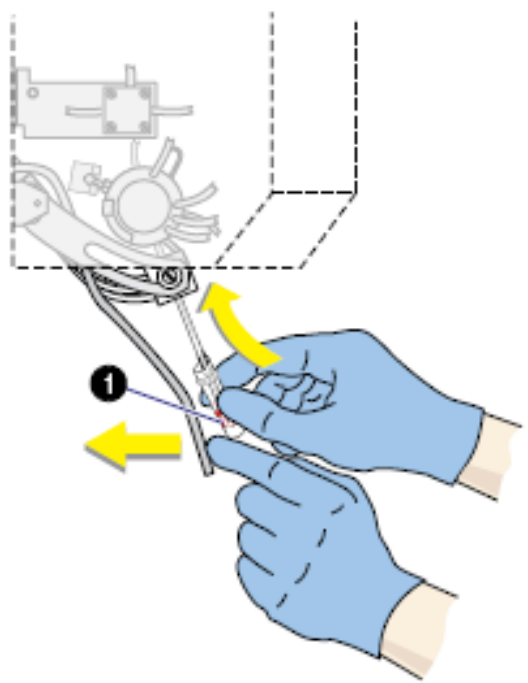


(3) Press and release the sample bar.

(4) Remove the tube when the instrument beeps.

5. Review data and transmit

a) Review the data using the criteria described in Section X.

b) Set host computer to $\Uparrow$. Data from the Coulter DMS is automatically transmitted to the ISIS system.

c) Use the Data Base Query to sort, retrieve, transmit, and archive data to diskette. Transmit data from the Coulter DMS to ISIS as necessary. Archive all data at the end of each stand.

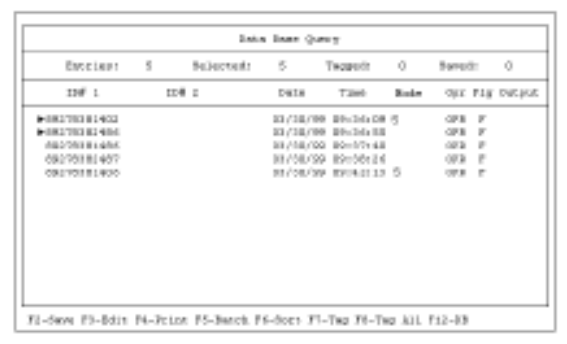

To access the Data Base Query screen, at the Access screen, press [F4] DATA BASE QUERY or at the Main Menu, select Sample Analysis $\rightarrow$ Data Base Query.

Use the Sort feature to define the criteria for the data. Sort by date, time, or ID. If you do not choose any sort criteria, the samples in the database are sorted chronologically by date and time.

To transit data from the DMS to ISIS, select Sample Analysis $\rightarrow$ Data Base Query, [Enter.] When you access this option, you see what was selected by the last Sort criteria. To review other samples, change the sort criteria. If the last sorting process resulted in no entries displayed here, then when you access this option, the sort window appears. 
To access the Sort Criteria window, press [F6] Sort. Enter sort criteria by TIME, DATE, ID \#1, or ID \#2. Select [F8] to execute the sorting process, [F7] Tag to tag or untag a highlighted individual sample or [F8] to Tag All to tag or untag all samples for batch processing, and [F5] Batch to display the Batch Process window.

At the Batch Process window, use the up and down arrows to move through the choices. Use the space bar to toggle between Yes and No. Set Print: to No and Host to Yes. Select [F8] Execute.

D. Daily Shut Down

1. Shut down the instrument for at least 30 minutes but less than 48 hours each day it is in use.

2. To begin shut down make sure the status line displays Select Function. Select Diluter Functions $\rightarrow$ Shut Down. Press [Enter]. Allow the cleaning agent to remain in the instrument for a minimum of 30 minutes.

3. Perform Start Up before running samples or controls. Results must be within tolerance.

E. Clean Cycle

1. The Clean Cycle consists of a Shut Down cycle followed 30 minutes later by a Start Up cycle. 
a) To initiate the Clean Cycle: Go to the Access screen [F1] from the Main Menu. Press [F3] Clean. Press [Enter] to begin. After the Shut Down portion of the cycle finishes, a window displays. Your options are: (1) Do nothing and allow the Clean Cycle to complete. (2) Press [F4] to abort the Clean Cycle. Cleaning agent remains in the system until you perform Start Up. (3) Press [F5] to begin the Start Up cycle immediately.

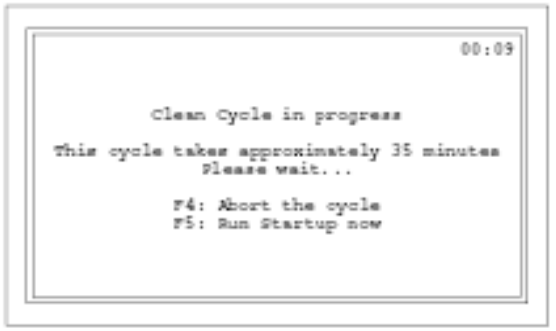

\section{F. Prolonged Shut Down}

1. If the instrument is going to be idle for 48-72 hours, perform the following procedure:

a) Go to the Access screen and press [F3] Clean.

b) Once the cycle is complete, turn OFF the instrument using the On/Off switch on the back of the main unit.

c) When it is time to use the instrument:

(1) Turn power ON.

(2) Prime the HMX PAK.

(3) Perform Start Up.

d) Perform and verify QC checks according to laboratory protocol.

e) Operate as usual. 
2. If the instrument is going to be shut down for more than 72 hours, perform the following steps:

a) Place all reagent pickup tubes into distilled water.

b) Repeatedly perform Shut Down and Start Up procedures throughout the day.

c) Let distilled water remain in the reagent lines.

d) Turn off the power.

e) Place reagent caps on all open containers.

f) When it is time to use the instrument, turn on the power and place the reagent pickup tubes into the reagent containers, prime all reagents, perform a shut down and then perform a Start Up. Results must be within tolerance.

g) Perform daily QC and operate as usual.

\section{G. Autopurge Cycle}

1. After 23 hours in Shut Down, with the power ON and the pneumatics OFF, the system automatically:

a) Turns ON the pneumatics.

b) Purges the flow cell and sample lines with diluent.

c) Turns OFF the pneumatics.

d) Repeats this cycle every 24 hours until a Start Up is performed. 


\section{H. Beginning and End of Stand Operations}

1. Beginning of stand:

a) Obtain one urine collection container or a $50-\mathrm{mL}$ conical tube, one 3 or 4-mL EDTA tubes (1 clean aspiration system), enough supplies to draw two 4-mL EDTA blood tubes, and a few plastic transfer pipettes.

b) Using all new containers of reagents, carefully unwrap and place all reagent pickup tubes in their appropriate container. Handle reagent tubes by the collar only to avoid contamination. Turn the power ON. Prime reagents through all the lines by selecting Diluter Functions, Prime Reagents, and choose All.

c) Perform the Start Up procedure. This should take approximately 5-6 minutes. Results should be within tolerance.

d) Contact the local Coulter ${ }^{\circledR}$ representative to perform an instrument verification procedure on setup day or as soon as possible.

e) Calibrate, perform, and verify QC checks according to procedure.

f) Review and verify system set up. Select Special Functions $\rightarrow$ Set Up $\rightarrow$ System Set Up:

\section{(1) Select Shift [Enter]}

(a) Move the cursor and set up the starting times for each shift and press [Enter] or the arrow keys. The system automatically calculates the end of the shift to prevent overlap.

(b) Set shift 1 "Time” at 0800.

Set shift 2 "Time” at 1230.

Set shift 3 “Time” at 2230. 
(c) Press [F10] to save and return to the previous screen.

(2) Select Reagents [Enter] - Update reagent logs

(a) To record new reagent information - Key in the new reagent information: lot number, date reagent opened (pressing [Enter] automatically gives today's date), and the expiration date. Do not forget to enter revised expiration dates where appropriate. Press [Enter] after each item. Press [F10] to save the data and leave the reagent screen.

(3) Select Institution [Enter]

(a) Enter the "SUPER" password.

(b) Type in:

\begin{tabular}{|c|c|c|}
\hline & & $\begin{array}{l}\text { Printed on } \\
\text { graphic report }\end{array}$ \\
\hline Name & NHANES & Yes \\
\hline Lab Director & & No \\
\hline Address & $\begin{array}{l}\text { NCHS } \\
\text { Maryland }\end{array}$ & No \\
\hline Phone & & No \\
\hline Instrument & $\begin{array}{l}\text { AJ47275 } \\
\text { AK04023 } \\
\text { AK04024 }\end{array}$ & No \\
\hline
\end{tabular}

(4) Select Communication Def. Enter the SUPERVISOR PASSWORD, “super.” Select Host Computer Definition.

(a) Use [Enter] or arrow keys to move to the appropriate field. 
(b) Type in the following information where necessary and use the space bar to choose between the responses. Press [F10] to save and [Esc] to return to previous screen.

$\begin{array}{llcl}\text { STKS 2A Host Mode } & \text { Yes } & \text { Retics transmission } & \text { No } \\ \text { Timeout (secs) } & 8 & \text { Overall Retics } & \text { No } \\ \text { Baud rate } & 9600 & \text { Graphics } & \text { No } \\ \text { Parity } & \text { none } & \text { DF5 } & \text { No } \\ \text { Stop bits } & 1 & \text { DF6 } & \text { No } \\ \text { Handshake } & \text { Yes } & & \\ \text { Block size } & & \text { Enable Spooler } & \text { Yes } \\ & & \text { Replace NULL by SP } & \text { No }\end{array}$

$\begin{array}{ll}\text { Graphic transmission } & \text { No } \\ \text { DF1 } & \text { No } \\ \text { DF2 } & \text { No } \\ \text { VCS histograms } & \text { No } \\ \text { RBC histogram } & \text { No } \\ \text { PLT histogram } & \text { No }\end{array}$

\section{(5) Select IQAP ID\#}

Type in the IQAP number.

Enter $329791 \mathrm{H} 1$ for instrument AJ47275

$329791 \mathrm{H} 2$ for instrument AK04023

329791 H3 for instrument AK04024

Press [F10] to save and escape.

(6) Select Set Date/Time

Move the cursor and enter the correct date or time and press

[Enter] or use the arrow keys. Press [F10] to save and exit.

\section{(7) Select Supervisor Password}

Do NOT change the password under any circumstances.

\section{(8) Select Optimize Hard Disk}

Enter the password "super." Respond to the question, "Do you want to automatically optimize the Hard Disk during Powerup?” Verify that YES is displayed. Press [F10] to save and escape. 
g) Review sample analysis set up. Select Special Functions, Set Up, and Sample Analysis Set Up. Type in the password "super."

(1) Select Action limits [Enter], XB limits [Enter].

Enter target and limit percent values.

XB Limits

\begin{tabular}{lcc} 
& Target & Limit \% \\
MCV & 88.5 & 3 \\
MCH & 29.5 & 3 \\
MCHC & 33.5 & 3 \\
\multicolumn{2}{l}{ Press [F10] to save and exit. }
\end{tabular}


(2) Select Action limits [Enter], Definitive flag limits [Enter].

Disable definitive flag limits. Set low limit for all parameter values to zero except for PCT. Set lower limit for PCT to 0.000 . Set high limit to 99.9 for all parameters except RBC, PLT, and PCT. Set RBC upper limit to 9.9, PLT to 999 (with no decimal) and PCT to 0.990. Press [F10] and [Enter] to save and escape.

(3) Select Action limits [Enter], High/low flag limits [Enter].

Disable High/Low flag limits. Set low limit for all parameter values to zero except for PCT. Set lower limit for PCT to 0.000 . Set high limit to 99.9 for all parameters except RBC, PLT, and PCT. Set RBC upper limit to 9.9, PLT to 999 (with no decimal) and PCT to 0.990. Press [F10] and [Enter] to save and exit.

(4) Select Action limits [Enter], Laboratory Normal Ranges [Enter].

Disable High/Low flag limits. Set low limit for all parameter values to zero except for PCT. Set lower limit for PCT to 0.000 . Set high limit to 99.9 for all parameters except RBC, PLT, and PCT. Set RBC upper limit to 9.9, PLT to 999 (with no decimal) and PCT to 0.990. Press [F10] and [Enter] to save and exit.

(5) Location list N/A Do not access

(6) Physician list N/A Do not access

(7) Display formats [Enter] Screen Labels N/A Do not access 
(8)

\section{Select Display formats [Enter] Parameter Selection}

Use the space bar to select No for each of these parameters.

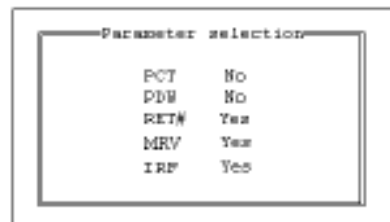

Press [F10] to save and escape.

(9) Select Display formats [Enter], Reporting Units

Use the space bar to select US 1. Press [F10] to save and escape.

(10) Select Delete database [Enter]

Delete database at the end of each stand after archiving the data to a diskette. The question, "You have asked to delete the ENTIRE database. Are you sure you want to delete?” Use the space bar to toggle between Yes and No then press [Enter].

(11) Select Delete host spooler [Enter]

Use this feature to clear the buffer of results waiting to be transmitted to the host computer. "Do you want to delete the host spooler?” Press the space bar to answer Yes or No to the displayed question. Press [Enter].

\section{(12) Clear printer spooler queue}

Use this feature to stop a print job and clear the DMS printer spooler of all data not yet sent to the printer. Move the cursor to the appropriate option. Press the space bar to toggle between Yes and No. Press [F10].

$\begin{array}{ll}\text { Auto Print: } & \text { No } \\ \text { Manual: } & \text { No } \\ \text { Batch: } & \text { No }\end{array}$


(13)

Select Print options [Enter]

\section{Auto Print Format}

Use the space bar to select [GRAPHIC FORMAT]. Press [F10]

to save and escape.

\section{Ticket Options}

Verify the following settings.

$\begin{array}{ll}\text { PRINT UNITS: } & \mathrm{Y} \\ \text { PRINT NORMAL RANGES: } & \mathrm{N} \\ \text { PRINT PARAMETER LABELS: } & \mathrm{Y} \\ \text { S/D FLAGS: } & \mathrm{N} \\ \text { DIFF ORDER: } & \text { Standard } \\ \text { TICKET FORMAT START OF } & \\ \text { NEXT FORM } & \text { Continuous }\end{array}$

Spooler Priority [Enter]

Leave [AUTO PRINT] as the default.

Graphic Options [Enter]

Verify the following settings.

$\begin{array}{ll}\text { Page Format } & \text { Width/Font } \\ \text { Wide/Large } \\ \text { DF 1. RBC, PLT } & \text { Yes } \\ \text { DF2, DF3 } & \text { No } \\ \text { VCS Histograms } & \text { No } \\ \text { Demographics } & \text { No } \\ \text { S/D flags } & \text { No } \\ \text { Print Units } & \text { Yes } \\ \text { Print Diff Box } & \text { No } \\ \text { Print Normal Ranges } & \text { No }\end{array}$

\section{Optional Printer}

Select “N." 


\section{2. $\quad$ End of stand}

a) Obtain three urine collection cups and three 4-mL EDTA blood tubes.

b) Review, print, and clear error file.

(1) Select Special Functions, Error File [Enter]. Review data and print [F4]. Document any relevant information on the printout and file in the logbook. Delete file [F8] - "Do you want to delete error file; NO” message will appear. Use the space bar to change No to Yes [Enter]. Return to main menu [F9].

c) Download IQAP files to diskette following procedure described on the screen.

d) Download or archive all stand result data to disk.

The DMS Archive feature lets you copy result data from the DMS onto a diskette and retrieve it on another computer in a spreadsheet format. Use a spreadsheet program that is compatible with the WKS format. Archive data at the end of each stand. 
To Archive data, select Sample Analysis $\rightarrow$ Data Base Query. Perform a sort that includes all samples run during the stand. Use [F7] or [F8] to tag the samples you want to archive. Press [F5] Batch to display the Batch Process window. Move the cursor to the Archive field then press [F2] Choice List. Use the space bar to highlight your choice then press [Enter]. If you select New, all tagged samples that have not yet been archived will be processed. If you select All, all tagged samples will be processed, even if they have already been archived. If you select No, Archive is inactive. Move the cursor to the Filename: field and enter a file name of your choice. Type A: $\backslash$ then up to eight characters. An extension is not required. Example: A:Istand210 could be the file name for sample results archived at the end of stand 210. Insert a formatted diskette into the DMS diskette drive. Press [F8] Execute. NOTE: If a power failure occurs during the archiving process, the samples from this archiving session are incorrectly marked as archived but the data file is empty. Reselect the samples from the session and select All to ensure all of the samples in process are correctly archived. Wait until the Batch is Inactive message appears, and then remove the diskette from the diskette drive. NOTE: If the space on the diskette is insufficient for archiving all of the tagged samples, the DMS displays the error DISK FULL ARCHIVING DISCONTINUED. Remove the full diskette from the DMS diskette drive and insert an empty formatted diskette. Ensure the Archive option selected is New, then press [F8] Execute. Any samples tagged but not archived yet are copied onto the new diskette.

e) Perform daily Shut Down. Let cleaning agent remain in the instrument for at least 30 minutes.

f) Perform Start-up.

g) Disable the reagent sensors. Select SPECIAL FUNCTIONS, DIAGNOSTIC, OPERATOR OPTIONS, FLUIDIC TESTS, and DISABLE REAGENT SENSORS. Toggle through each reagent line using the space bar to change "ON” to "OFF." 
h) Bleach the reagent lines.

(1) Remove all reagent pick-up tubes from the reagent containers. Dispose of all open containers. Wash and save the reagent collars.

(2) Prepare a $25 \%$ solution of bleach (250-mL) and distilled water (750-mL) in one liter bottle. Place all reagent pickup tubes into the $25 \%$ bleach/distilled water solution. Select Diluter Functions, Prime Reagents, select ALL. Press [Enter]. Repeat this procedure two more times.

i) Place all reagent pickup tubes into containers of distilled water.

j) Bleach the apertures and flow cell.

(1) Mix 15-mL bleach and 15-mL distilled water in a plastic container.

(2) Put 30-mL of distilled water in a second plastic container.

(3) Open the front panel.

(4) Select Diluter Functions, and Disinfect. Press [Enter].

(5) The instrument defaults to 15 minutes; change the number to 03 or stop the procedure after 3 minutes. Press [Enter].

(6) Immerse the bleach probe in the bleach solution when the screen displays, "PRESS ANY KEY WHEN READY TO ASPIRATE BLEACH.” Press any key. Aspirate all the bleach solution. 
(7) Immerse the bleach probe in the distilled water when the screen displays, "PRESS ANY KEY WHEN READY TO ASPIRATE WATER.” Press any key. Aspirate all the distilled water. Stop the cycle after 3 minutes by pressing [F4].

(8) Wait until the screen displays Select Function before touching any keys. When Select Function is displayed, continue with the following procedure.

k) Disinfect the needle. Bleach the aspiration system using the Clean Needle procedure.

(1) Prepare a fresh bleach solution. Put 1-mL bleach and 1-mL distilled water into 4-mL lavender top Vacutainer ${ }^{\circledR}$ tube.

(2) Stop the system before beginning the Clean Needle procedure. If status message is not Select Function or Compressor Off, go to Sample Analysis, Run Sample, [F3], Run. Press [F9] Stop.

\section{Select Special Functions, Diagnostics, Operator Options,} Fluidic Tests, and Clean Needle. Press [Enter] and follow the screen instructions. Wait for green light before placing tube into the carousel.

l) Perform reproducibility in Primary and Secondary mode using this $1 \%$ bleach solution. Prepare a 1\% bleach solution and fill one 4-mL EDTA blood tube with this solution. Turn the diff off. Is Sample Mode? displayed? If Yes, go to step 3. If No, press [F9] Stop. Press [F6]. Press space bar to turn the DIFF OFF. Press [Enter]. Press [F2] Start Primary. Select Special Functions, Calibration, and Reproducibility. Select Primary and aspirate three samples of 1\% bleach. Enter [F3] Sample

Mode. Enter [F9] Stop. Select Secondary mode and aspirate three samples of $1 \%$ bleach solution. 
m) Rinse the reagent lines. Make sure all reagent lines are immersed in containers of distilled water. Select Diluter Functions, Prime Reagents, select ALL. Press [Enter]. Repeat this procedure two more times.

n) Perform a Start Up. Make sure containers are full.

o) Perform reproducibility in Primary and Secondary mode by using distilled water. Fill one 4-mL EDTA blood tube with this distilled water. Turn the diff off. Is Sample Mode? displayed? If Yes, go to step 3. If No, press [F9] Stop. Press [F6]. Press space bar to turn the DIFF OFF. Press [Enter]. Press [F2] START PRIMARY. Select Special Functions, Calibration, and Reproducibility. Select Primary and aspirate three samples of distilled water. Enter [F3] Sample Mode. Enter [F9] Stop. Select Secondary mode and aspirate three samples of distilled water. Press [Enter].

p) Select Diluter Functions, Start Up. Press [Enter]. Perform one additional Start-Up procedure with the lines in the distilled water containers.

q) Cycle the instrument dry.

(1) Remove all reagent pickup tubes from the distilled water containers.

(2) Continue to cycle the instrument dry by priming air through all the lines by selecting Diluter Functions, Prime Reagents, and choose ALL. Repeat these actions at least three times. 
(3) Open front cover. Using hemostats, seal tubing with check valve coming out of the bottom of the sheath tank. Loosen the four large screws that hold the panel containing the sheath tank. Gently pull panel away from instrument. Trace two red wires from top of sheath tank to plastic electrical connection. Disconnect the junction. Insert paper clip into junction so that a circuit is formed on the instrument side of the connector. Do this by bending paper clip into a $U$ and placing one end of paper clip into each of the open sockets on the instrument side of the junction. This will allow the instrument to Drain, Vent, and Shut Down without reagent. Select Special Functions, Diagnostics, Operator options, and Drain and Vent. Press [Enter.]

(4) Select Diluter Functions, and Shut Down. Press [Enter].

(5) After cycles are complete, remove paper clip, reconnect junction, put panel back into instrument and tighten screws, then remove hemostats.

r) Enable the reagent sensors. Select Special Functions, Diagnostics, Fluidic Tests, and Disable Reagent Sensors. Toggle through each reagent line using the space bar to change "No" to "Yes."

s) To protect the reagent pickup tubes from contamination, wrap them securely in paper towels. Allow air to continue to dry the tubes.

t) Turn off the power.

u) Rinse exterior of BSV with distilled water to remove built up Isoton and blue Clenz agent. Blot the BSV dry with lint free tissue. Do not use gauze.

v) Wash exterior of instrument with distilled water. 
w) Empty waste and rinse container.

x) Blow air through the flow cell at the end of each stand. This is referred to as the Triple Transducer Module (TTM). Address questions to the service representative. Remove the 6-inch sample line to the flow cell at the mixing chamber. Connect a $10-\mathrm{mL}$ syringe with tubing to the 6 -inch line and force air into the line to remove ALL remaining liquid from the flow cell and the 6-inch sample line. Reconnect the sample line.

IX. Coulter ${ }^{\circledR}$ Reportable Range of Results

\begin{tabular}{lll} 
Parameter & Linearity & Limits: The greater of \\
\hline WBC $\times 10^{3}$ cells/ $\mu \mathrm{L}$ & 0 to 99.9 & 0.2 or $3.0 \%$ \\
\hline RBC $\times 10^{6}$ cells $/ \mu \mathrm{L}$ & 0 to 7.00 & 0.05 or $2.0 \%$ \\
\hline Hgb g/dL & 0 to 25.0 & 0.2 or $3.0 \%$ \\
\hline MCV fL & 50.0 to 150.0 & 2.0 or $3.0 \%$ \\
\hline Plt $\times 10^{3}$ cells $/ \mu \mathrm{L}$ & 0 to 999 & 10.0 or $7.0 \%$ \\
\hline MPV fL & 5.0 to 20.0 & $5.0 \%$ \\
\hline
\end{tabular}


X. Quality Control

A. Latron ${ }^{\circledR}$ Primer and Latex Controls (PN 7546914 - 5 x 16-mL) - The Latron ${ }^{\circledR}$ control is for use in monitoring the volume, conductivity, and light scatter (VCS) parameters on the HMX. Use the Latron ${ }^{\circledR}$ control immediately following the Latron ${ }^{\circledR}$ primer. Run this control at the beginning of each day. Date and initial the vials when opened.

1. Principle - The Latron control is a ready-to-use suspension of latex particles. These particles pass through the flow cell and produce characteristic electrical signals. It measures these signals as volume, conductivity, and light scatter. The Latron Primer is supplied as a ready-to-use solution that prepares the sample line by eliminating interfering particles.

2. Reagents - The Latron control consists of latex particles suspended in a buffered bacteriostatic and fungistatic medium containing a surfactant. The Latron primer consists of a buffered bacteriostatic and fungistatic medium containing a surfactant.

3. Storage, handling, and stability - Sealed vials are stable until their expiration date when stored and used at $2-30^{\circ} \mathrm{C}\left(35-86^{\circ} \mathrm{F}\right)$. Once open, date and initial vials. Opened vials are stable for 30 days when stored at recommended temperatures. Keep vials tightly capped when not in use. Do not freeze.

4. Indications of instability or deterioration - Inability to recover expected results might indicate product instability or deterioration due to improper storage, handling, or contamination. Discard vial if debris is visible and put new vial into use.

5. Instructions for use - Before running control, verify that a Startup has been performed. 
a) Run Latron ${ }^{\circledR}$ primer in the secondary mode. Access the Latex Control Run screen at the Access screen press [F2] Run Controls or at the Main Menu, select Controls $\rightarrow$ Control Run. If the LATEX FILE does not appear, press [F2] File. Select the Latex File. Press [Enter]. Press [F3] Run [F4] Primer. Cycle the primer by immersing the aspirator tip completely in the primer. Press and release the sample bar. The aspirate probe for open vials will begin to aspirate the primer. Remove vial when the audible "beep” occurs. Evaluate primer results. Results should be less than 500. Press [Esc] to remove the primer run.

b) Run the latex control. Mix a room-temperature control vial gently by inverting five (5) to eight (8) times. Be careful to avoid foaming. When Select Function appears on the status line, press [F3] Run, [F3] Control (Secondary). Cycle the control by immersing the aspirator tip completely in the vial. Press and release the sample bar. The aspirate probe for open vials will begin to aspirate the control. Remove vial when the audible "beep” occurs.

c) Examine the Mean Channel and Coefficient of Variation for volume, conductivity, and light scatter for the Diff Mode only. Compare the results to the Assigned Mean Values and Expected Coefficient of Variation. Check for $\mathbf{H}$ (high) or $\mathbf{L}$ (low) beside the results. If there are no H's or L's, results are within range. Press [F4] to print results for the logbook. If $\mathrm{H}$ or $\mathrm{L}$ displays, do the following:

(1) Assigned value or range is incorrect - Be sure the assigned values match the ones on the Latron control package insert. If in error, correct them by selecting Special Functions $\rightarrow$ Set Up $\rightarrow$

\section{Control Set Up.}

(2) Bubbles in the flow cell or improper vial handling - Rerun the primer and control. 
(3) Contaminated control, improperly mixed, or past open vial expiration date - Wipe the aspirator tip. Try a new vial of Latron control. Mix gently according to directions.

(4)

“:::::" System detects a clog in the flow cell - Press [F3]. Press [F7] to PURGE the flow cell. Press [F4]. Cycle the Latron ${ }^{\circledR}$ primer again. Press [Esc] [F3] [F3]. Cycle the Latron ${ }^{\circledR}$ latex control again. If the control is still "out" of range, repeat the actions 2 or 3 more times. If the problem remains, either perform a ShutDown or turn the DIFF OFF and run CBCs only. If problem continues, call a Coulter representative for assistance.

(5) Assigned value or range is incorrect - Be sure the assigned values and ranges match the ones on the Latron package insert. If in error, correct them by selecting SPECIAL FUNCTIONS, SET-UP, CONTROL SET UP.

6. Setting up a Latex control file

Set up a file each time a new lot number is received. Assigned channel, expected range, and \%CV are automatically entered; change them to assigned values on assay sheet, if necessary.

\section{Choose SPECIAL FUNCTIONS $\rightarrow$ SET UP $\rightarrow$ CONTROL SET-UP $\rightarrow$}

LATEX FILE. Select a NOT SET-UP or an inactive file. Manually enter the name of the file (Latron), Lot \#, expiration date, and operator initials. The system automatically enters the assigned values, expected ranges, and expected \%CVs. Check to make sure that the HOST: is set to ON. This means that the control run results are transmitted to the host computer at the time of the run. Use the space bar to toggle between ON and OFF. Check all entries to make sure they are correct. Press [F10] to save and escape. 
B. 5 C ${ }^{\circledR}$ Cell Controls Tri Pack contains Normal (blue), Abnormal I (yellow), Abnormal II (pink) (PN 7547001 - 9 x 3.3-mL.) The 5C cell control is a hematology reference control used to monitor the performance of instruments with complete CBC and VCS differential technology. Run all three levels of control at the beginning of each session. Date and initial the open vials.

1. Principle - The 5C cell control is a reference product prepared from stabilized human blood. 5C cell controls confirms and monitors instrument accuracy and precision performance by providing measurements for counting, sizing, hemoglobin determination, and white blood cell differentiation using CVS technology.

2. Reagents - 5C cell control consists of treated, stabilized human erythrocytes in an isotonic bacteriostatic medium. 5C cell control also contains a stabilized, platelet-sized component, and fixed erythrocytes to simulate leukocytes.

3. Storage, handling, and stability - Store $5 \mathrm{C}$ cell control at $2-8^{\circ} \mathrm{C}$. When stored at $2-8^{\circ} \mathrm{C}$, sealed vials are stable at least until the expiration date shown on the Table of Expected Results. NOTE: The MCV and/or RDW parameters may show trending through the shelf life of the product. This is inherent to the product and is not an indicator of product stability. Date and initial open vials. Opened vials are stable for 13 days or 13 events when stored at $2-8^{\circ} \mathrm{C}$.

Potential biohazard - Each human donor used in preparation of this material was tested by an FDA-approved method for the presence of the antibodies to Human Immunodeficiency Virus (HIV-1 and HIV-2) and Hepatitis C (HCV) as well as for hepatitis B surface antigen and found to be negative (were not repeatedly reactive). Handle these reagents at Biosafety Level 2 because no test method can offer complete assurance that these and other infectious agents are absent.

This product contains $<0.1 \%$ Sodium Azide. Sodium Azide preservative may form explosive compounds in metal drain lines. Discard this product in biohazardous waste containers. 
4. Indications of instability or deterioration - Inability to obtain expected values without known instrument problems or gross hemolysis (darkly colored supernatant) indicate product deterioration. However, a slight pink color to the supernatant is normal. Do not confuse this pink color with deterioration of the product.

5. Prepare the instrument - Insert the Control Disk when a new lot of 5C cell controls is put into use. Follow the instructions on the Workstation/computer screen.

6. Instructions for use - Remove 5C cell control tubes from refrigerator and warm to ambient temperature for 10-15 minutes. After warming, mix by hand as follows:

Do not use a rotator, rocker, or mechanical mixer. Roll the tube slowly between the palms of the hands eight full rotations in an upright position. Invert the tube and slowly roll it again eight times. Gently invert the tube eight times. Repeat these actions again.

a) Run the 5C cell control in the Primary Mode. Does SELECT FUNCTION appear at the lower right corner of the DMS screen? If No, Access the Run Samples screen: at the Access screen, press [F1] Run Samples or at the Main Menu, select Sample Analysis $\rightarrow$ Run Samples. If yes, access the Run Samples screen: at the Access screen, press [F1] Run Samples or at the Main Menu, select Sample Analysis $\rightarrow$ Run Samples. Press [F3] Run. The instrument automatically prepares itself for Primary mode, DIFF ON. Make sure the DIFF is ON. If it is OFF, press [F6] DIFF ON/OFF. NOTE: If SAMPLE MODE? is not displayed, press [F9] Stop first. Does the top of the F3-Run window display PRIMARY: SAMPLE ANALYSIS? If yes, press [Esc]. If No, press [F2] Start Primary. 
b) Complete the entire procedure and return the controls to the refrigerator within 30 minutes.

c) Identify the sample by holding the bar code label in front of the reader. Place the control in the carousel. Repeat for other levels of controls.

d) Check the results of each control. Coulter has established control limits for each parameter for each of the three levels of 5C cell controls. The limits are set at +/- two standard deviations. NCHS does not want QC results that are out of range to be included in the NCHS analytical database. Delete any individual 5C cell control run that includes any results that are flagged out of range. Print the results before deleting and document on the printout what action was taken to correct it. Send all documentation to the home office at the end of each stand.

Select CONTROLS $\rightarrow$ REVIEW, OR REPORT and [F2] to select a file for review. Choose one control file and [Enter]. Check for $\mathbf{H}$ (high) or $\mathbf{L}$ (low) beside the results. If there are no H's or L's, results are within range. To print results for the logbook for the last control run, press [F4] Print. Use [F2] to select another file and repeat actions. If $\mathbf{H}$ or $\mathbf{L}$ displays, consider the following reasons and perform and document the following actions:

(1) Improper mixing - Follow instructions and rerun control.

(2) Control files set-up incorrectly - Make sure the assigned values and ranges match those on the control package insert. If in error, correct them by selecting, SPECIAL FUNCTIONS, SET UP, CONTROL SET UP.

(3) Chance (statistical outliner) - Rerun the control. If it is still out, continue with the following actions.

(4) Change in the control - Try another vial or level of control. 
(5) Instrument change - Watch for normal sample flow. Call Coulter ${ }^{\circledR}$ representative to help troubleshoot abnormal operation.

7. Setting up a CBC/Diff control file

Set up a file whenever receiving a new lot number of control. Enter control package insert information for control with differential. Enter information using the bar code wand. Enter information manually if necessary.

Select Special Functions $\rightarrow$ Set Up $\rightarrow$ Control set up $\rightarrow$ CBC/DIFF file. Select a file to set up. Insert the $5 \mathrm{C}$ cell control diskette into the diskette drive of the computer. Press [F5] Upload Assay Values. Press the function key for the desired level of control: [F1] for Normal, [F2] for Abnormal I, [F3] for Abnormal II. Manually enter Shift and Operator ID. Check that HOST: is set according to $\uparrow$. ON means that control run results are transmitted to your host computer at the time of the run. Space bar toggles between ON and OFF. Check all entries to make sure they are correct then press [F10] to save and escape. Repeat these steps for the other levels of control. Once you are finished, remove the 5C cell control diskette from the diskette drive of the computer.

a) Manual entry

If the 5C cell control diskette fails to upload, you can enter all data manually. Refer to the package insert for lot specific information and assigned values. The system automatically enters the level and expected ranges based on the first two digits of the lot number. Press [Enter] after each entry. Press $[\downarrow]$ at the end of each row of assigned values unless you are also entering your own expected ranges. 


\section{Control Statistics and Graphs}

1. Use to review and print:

Control results, cumulative statistics and histograms for LATEX Files;

Control results, cumulative statistics and graphs for CBC/DIFF files; or

Check cumulative results for trends or shifts as necessary for troubleshooting.

Print, review, and delete cumulative statistics and graphs for all CBC/DIFF and LATEX files at the end of each stand after transferring information to the IQAP disk and file in the logbook.

2. Latex control review and report

a) Use this report to review and print control results and cumulative statistics and histograms for LATEX files. Check cumulative results to look for trends, shifts, or, if necessary, troubleshooting.

b) Screen-specific function keys:

[F2] File Displays all available files. Use $[\downarrow]$ and $[\uparrow]$ to select the correct file. Press [Enter].

[F3] Transmit Transmits the data of the entire control file to a host computer.

[F4] Print Prints the entire file in a line list format.

[F5] Histo Displays the volume, conductivity, and scatter (CVS) histograms screen. Use [F4] to print the screen and [F6] Additional Histo to switch between Diff and RETIC histograms.

[F6] Rem/Res Removes a highlighted run from the calculations. A DEL appears in place of the run number. The statistics recalculate. Pressing [F6] again restores the run and original statistics. 
[F8] Delete File Deletes the current control file. Displays a message "You have asked to delete ENTIRE control file. Are you sure you want to delete?" Press the space bar to select Yes or No then press [Enter] to confirm the choice.

[F9] Menu Exits to the main menu.

$[\leftarrow][\rightarrow] \quad$ Press $[\leftarrow]$ or $[\rightarrow]$ to go back and forth between the Diff

Latex Control Review screen and the Retic Latex Control Review screen. 
3. CBC/DIFF control review and report

a) Use this report to review and print control results and cumulative statistics for CBC files. Can also be used to transmit the data of the entire control file to a host computer. Select CONTROLS $\rightarrow$ REVIEW OR REPORT, and [Enter]. Press [F2] to select one of the following files: Normal, Abnormal I, Abnormal II, and Latex [Enter]. Press [F4] to print. Review and file in logbook. Continue to select each file in turn, print, and file.

Screen-specific function keys:

[F2] File $\quad$ Displays all available files. Use $[\downarrow]$ and $[\uparrow]$ to select the correct file. Press [Enter].

[F3] Transmit Transmits the data of the entire control file to a host computer.

[F4] Print Prints the entire file in a line list format.

[F6] Rem/Res Removes a highlighted run from the calculations. A DEL appears in place of the run number. The statistics recalculate. Pressing [F6] again restores the run and original statistics.

[F8] Delete File Deletes the current control file. Displays a message "You have asked to delete ENTIRE control file. Are you sure you want to delete?” Press the space bar to select Yes or No then press [Enter] to confirm the choice.

[F9] Menu Exits to the main menu.

[F12] Graphics Displays scatterplots, histograms, and numeric results.

$[\leftarrow][\rightarrow]$ More Press $[\leftarrow]$ or $[\rightarrow]$ to see additional parameters not displayed on the screen. 
D. Record 5C Cell and Latron Lot Numbers in ISIS

The ISIS maintains the capability to download all 5C cell and Latron control data. The data are used to monitor quality control results. Upload or enter data for each lot number each time a new lot number is put into use. The ISIS uses the same 5C cell control disk as the $\mathrm{HmX}$. Manually enter the Latron data using information contained in the package insert.

Access the Coulter QC Lot Info module.

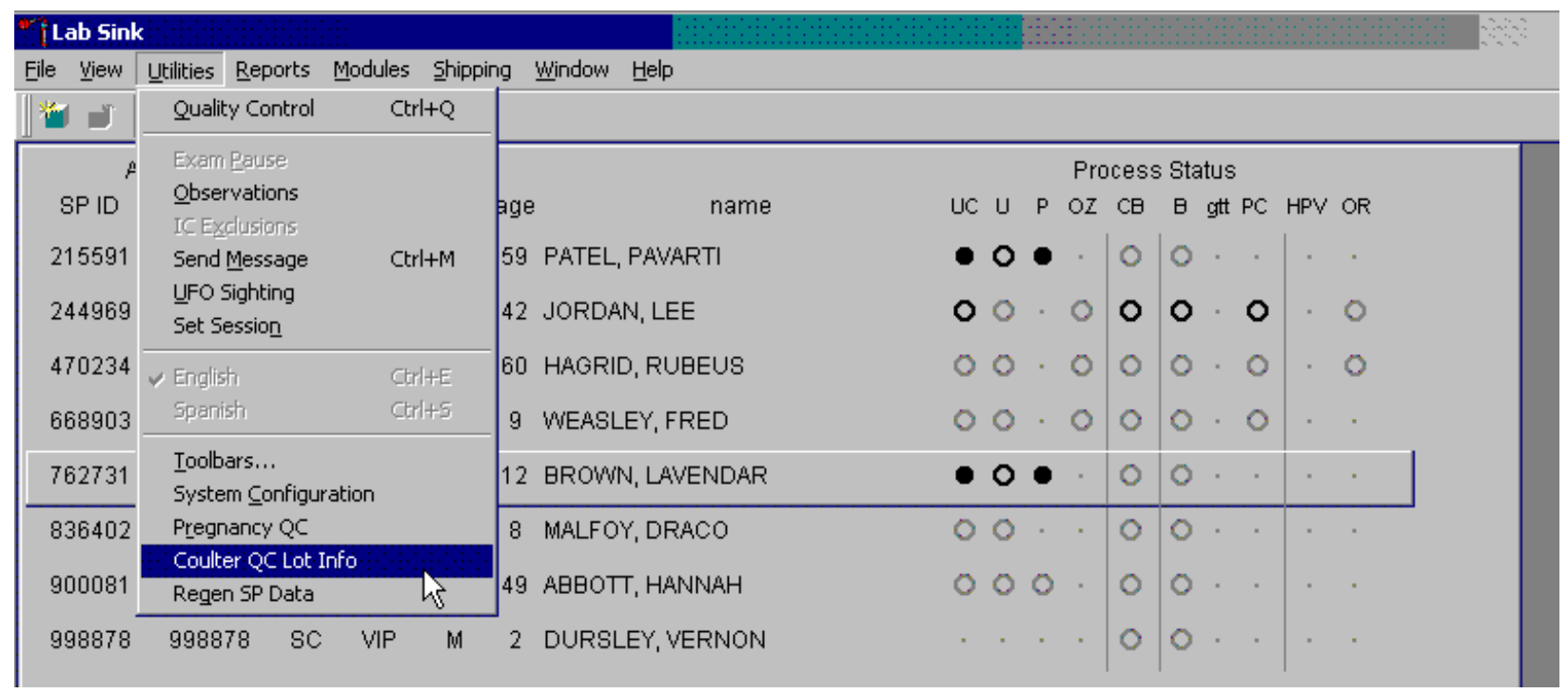

To open the Coulter QC Lot Info module, use the mouse to direct the mouse arrow to \{Utilities\}, in the top menu bar, left click, drag the mouse arrow to \{Coulter QC Lot Info\}, and right click. 
The Lab Hood or data entry window displays.

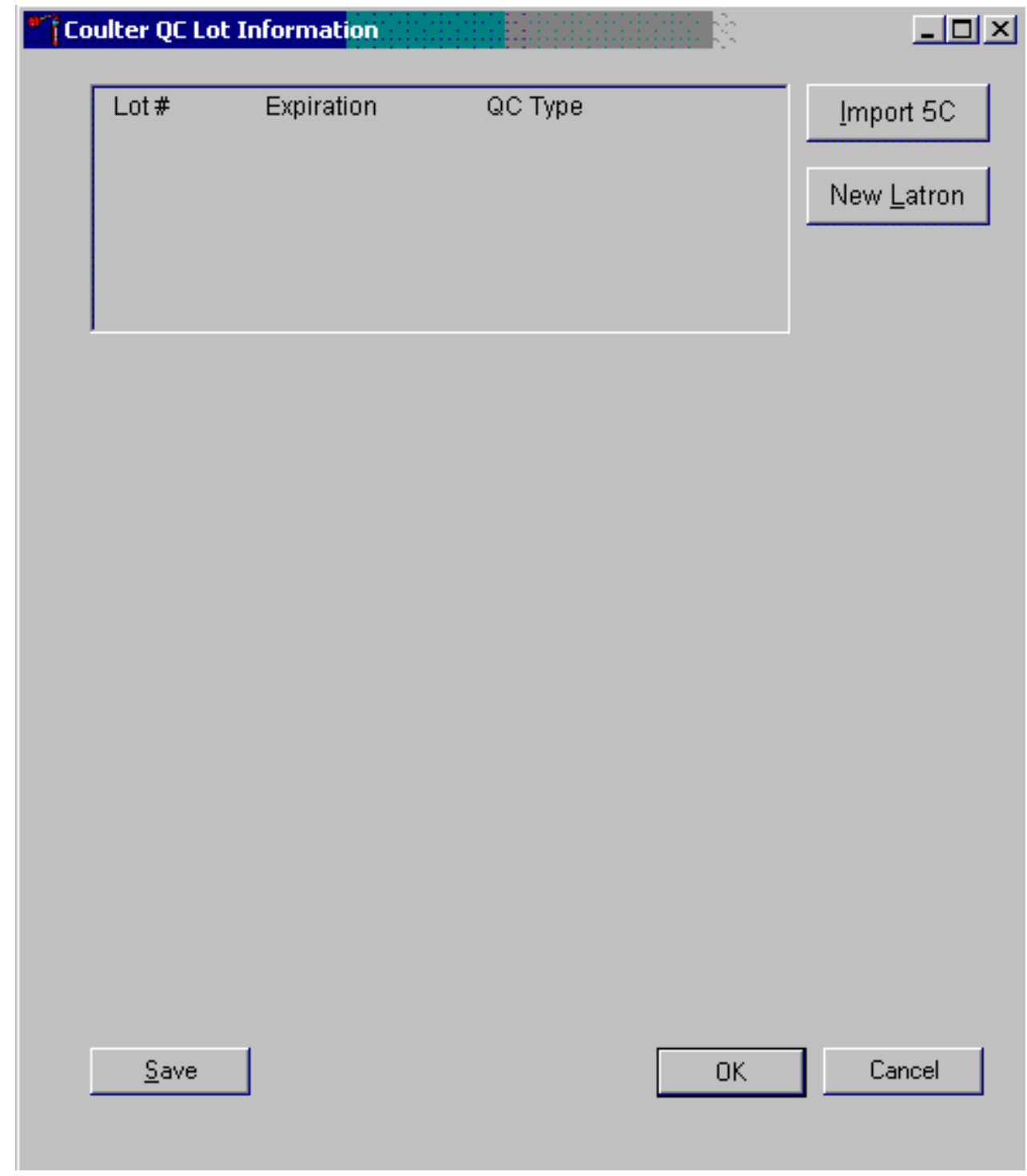

The data entry window contains Import 5C and New Latron buttons. The window also contains Save, OK, and Cancel buttons. After the data are entered, there are possible actions: save, save and exit, or exit without saving the data. To save these data to the database, use the mouse to direct the mouse arrow to the Save button and left click. To record this action or to save these data to the database and to exit the module, use the mouse to direct the mouse arrow to the $\mathbf{O K}$ button and left click. To close the window without saving any data in the database, use the mouse to direct the mouse arrow to the Cancel button and left click. 
Make sure the separate floppy drive is connected to a USB port on the sink hard dive. Insert the $\mathrm{HmX} 5 \mathrm{C}$ cell control disk into the floppy drive attached to this computer.

Select the Import 5C button or type [Alt] [I/i] to begin the process of entering $5 \mathrm{C}$ cell lot information.

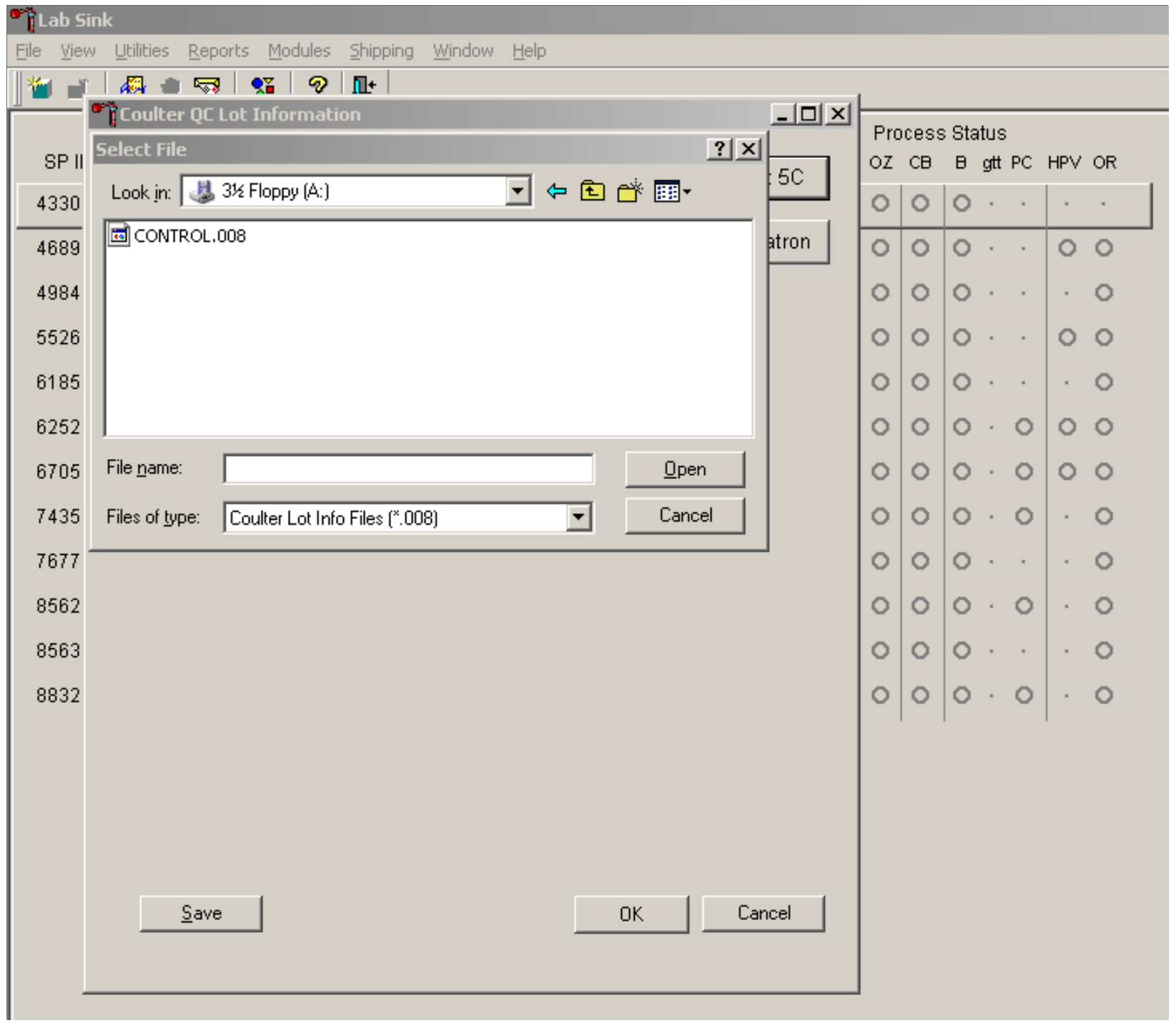

The Select File window displays. 
Identify the location of the disk containing the 5C cell control data.

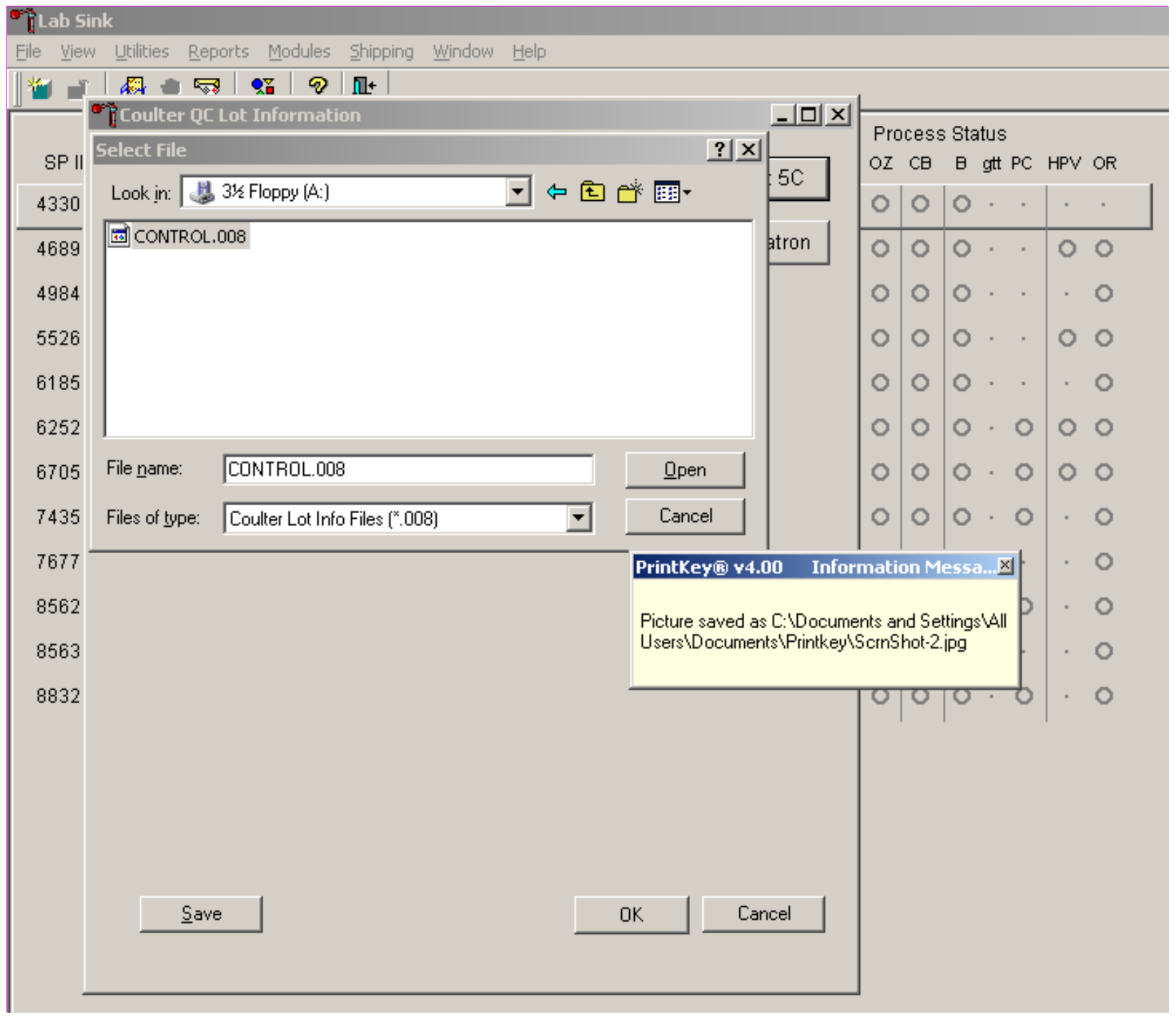

Highlight the CONTROL.008 file, select the Open button and left click. To close the window without identifying a file, use the mouse to direct the mouse arrow to the Cancel button and left click. 
Once the Open button is selected, the data are uploaded and the Lab Hood window reappears.

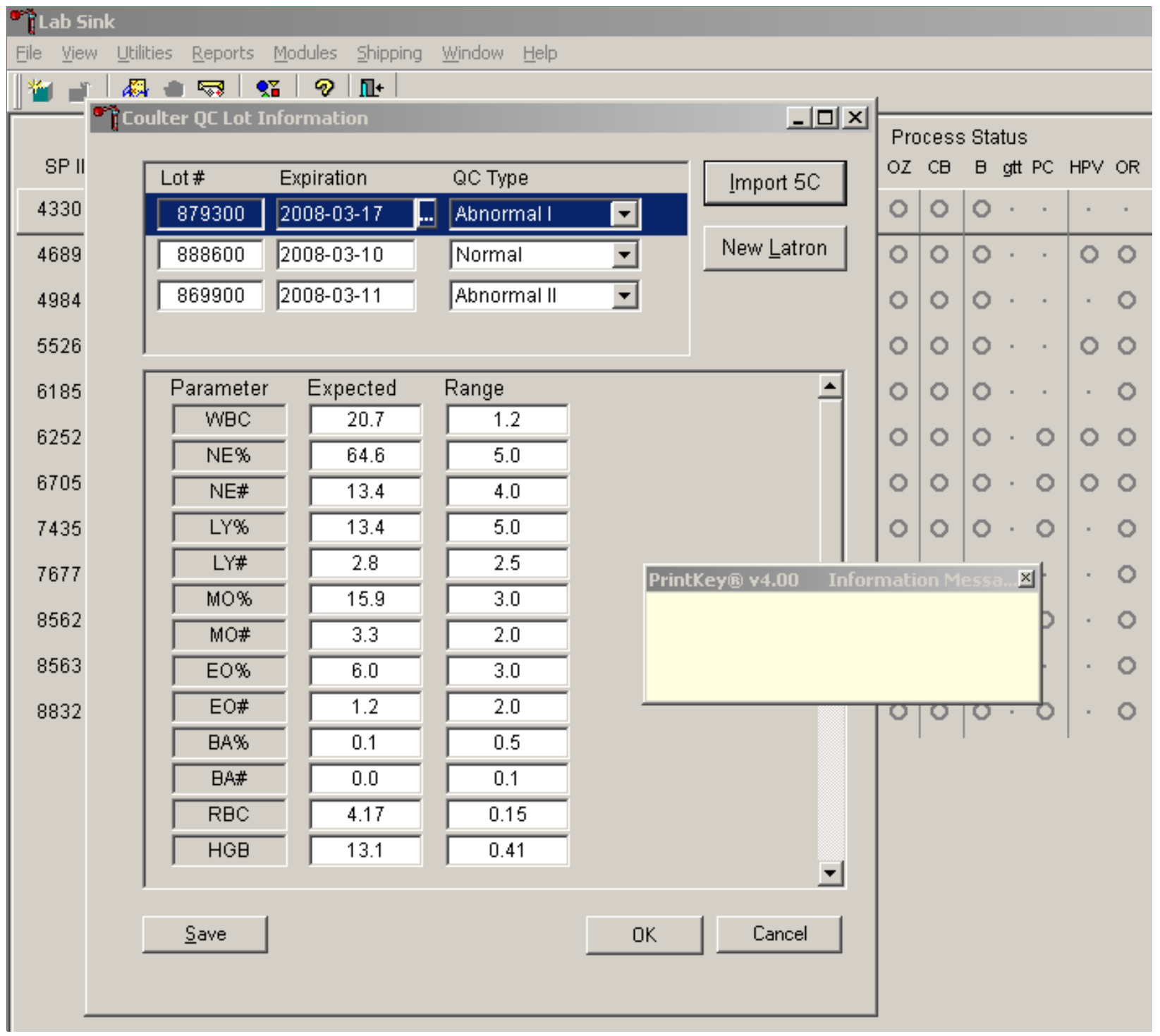

The data fields display the uploaded data for the three levels of 5C controls: Abnormal I, Normal, and Abnormal II. 
Stretch the window to view all parameters and values.

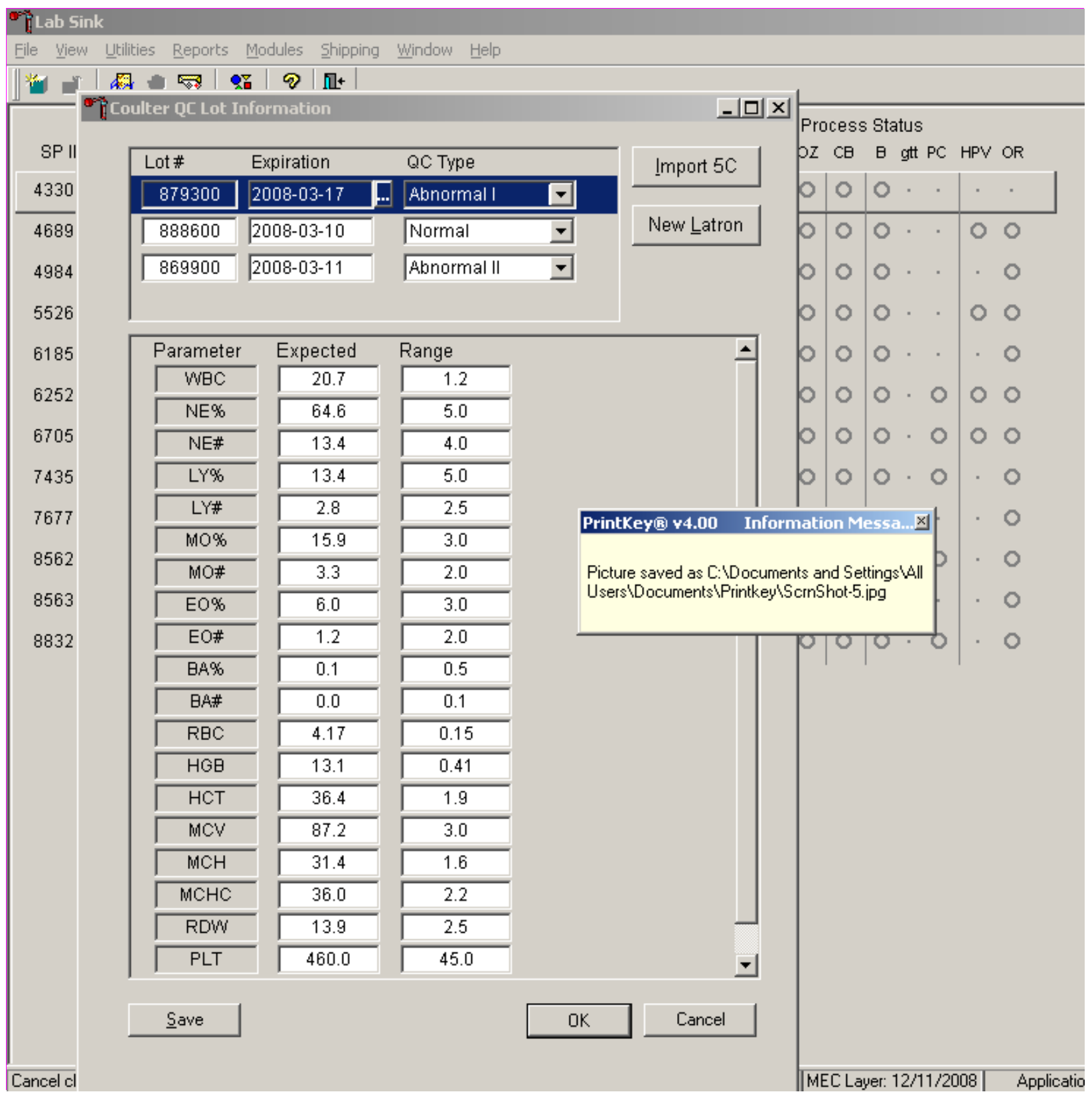

To stretch the window, use the mouse to direct the mouse arrow to the bottom right corner and drag the mouse arrow so that the window displays the entire data window. 
Review the data in the window.

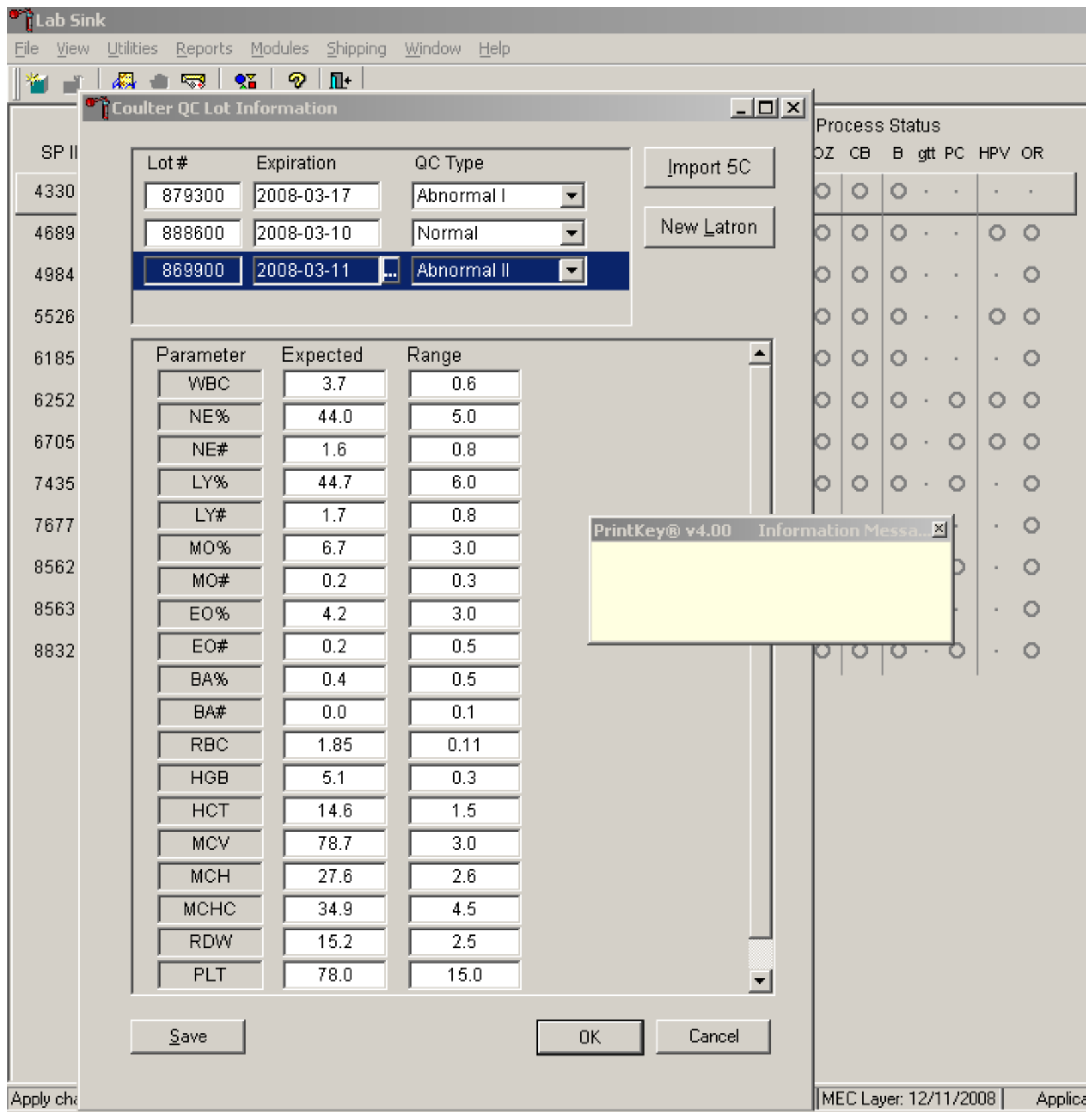

Compare the data in the window to the data on the package insert. Verify the lot number and expiration date. Validate the Expected and Range values for each parameter. Confirm this information for each QC Type. To select another QC type use the mouse to direct the mouse arrow to the drop-down 
arrow, left click, drag the mouse arrow to the correct choice, and left click. Save the data by selecting the Save button.

Enter the Latron data by hand entering the data from the package insert. To access the Latron data entry fields, select the "New Latron” button.

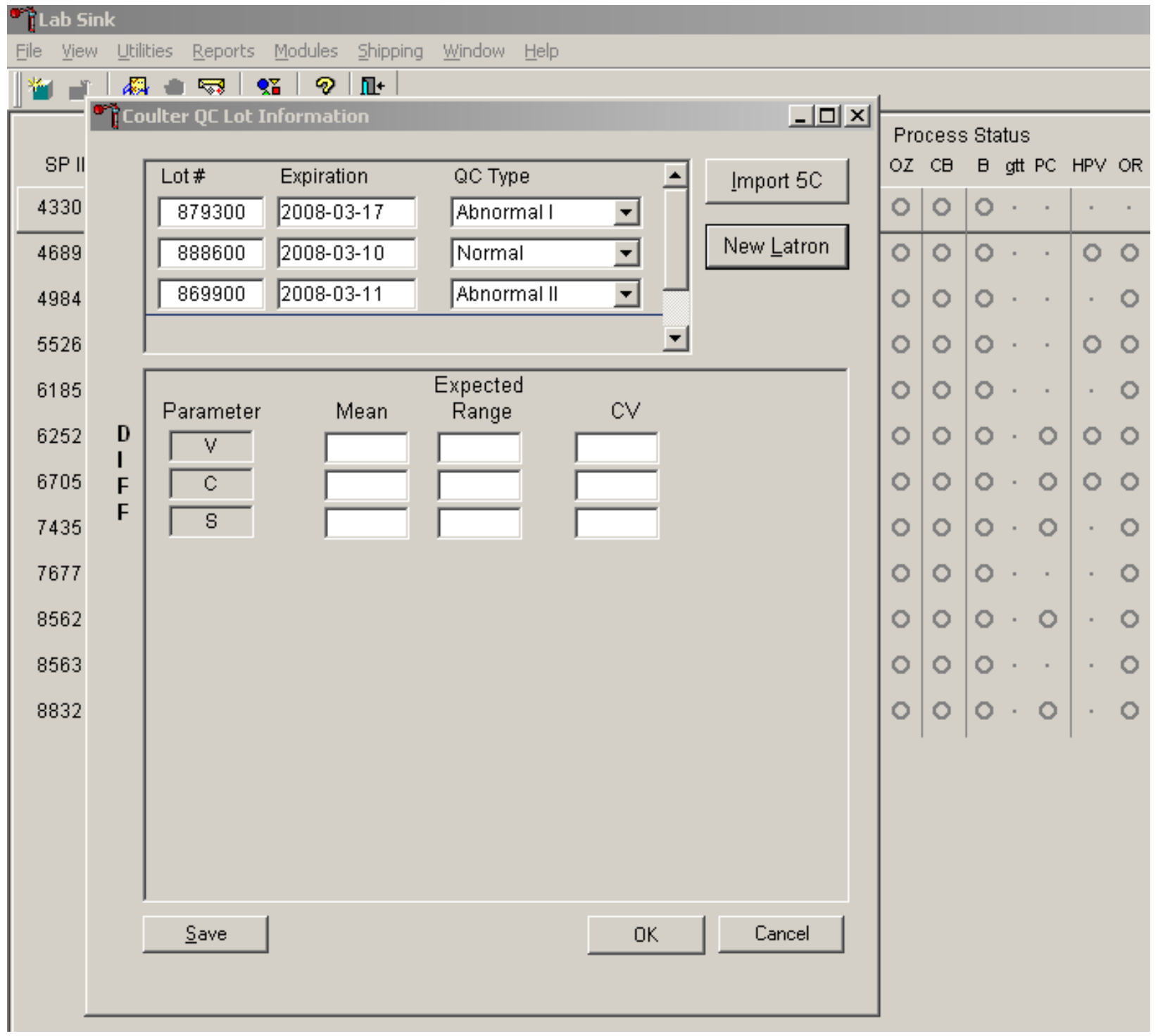

The lower portion of the window contains 9 text boxes, three each for Volume (V), Conductivity (C), and Scatter (S). 
Enter the lot number and expiration date.

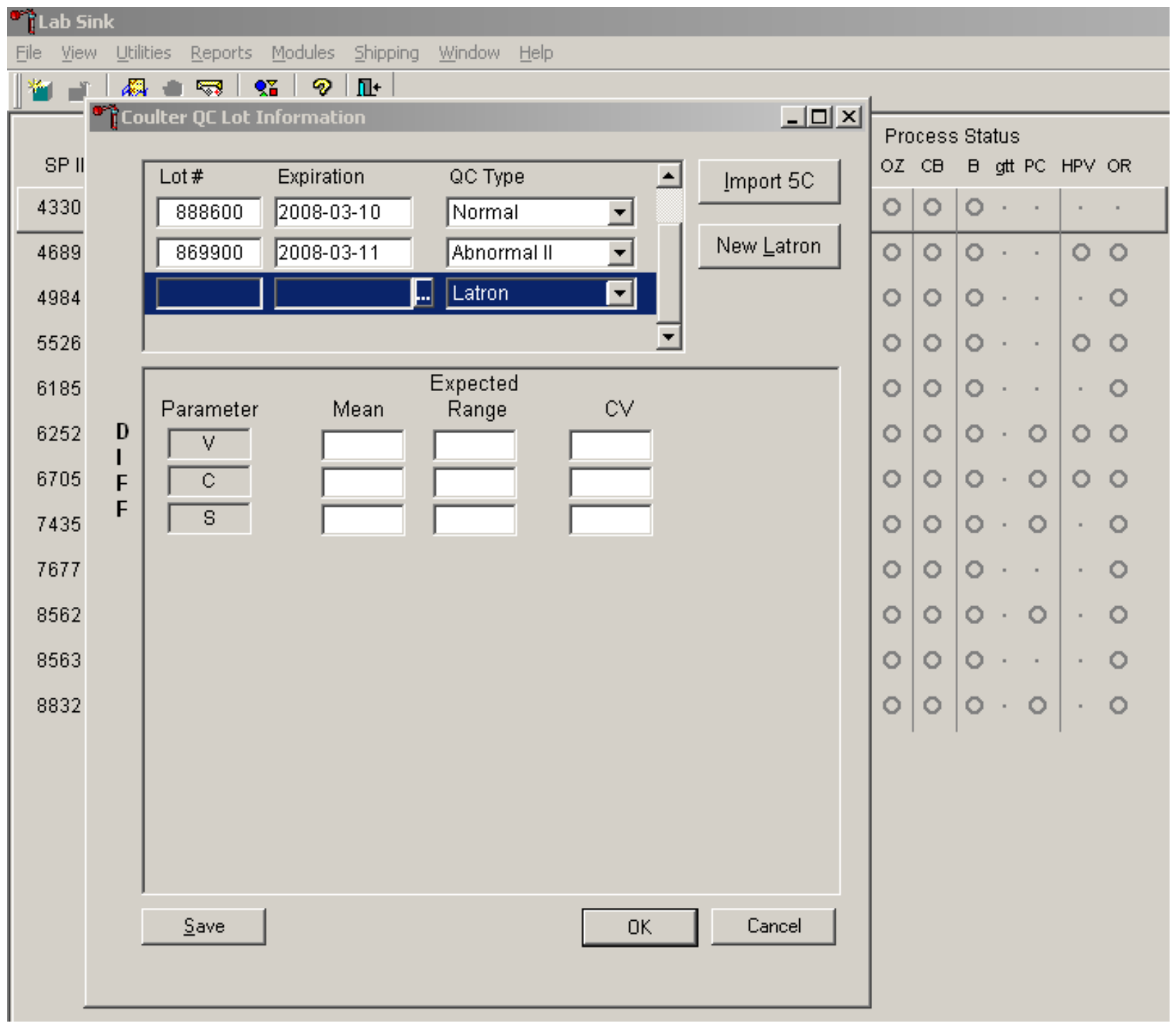

The Lot\# and Expiration text boxes are located in the top portion of the window. Use the scroll bar to view the row. 
Type in the Lot number using the keyboard numbers and use the calendar to enter the expiration date.

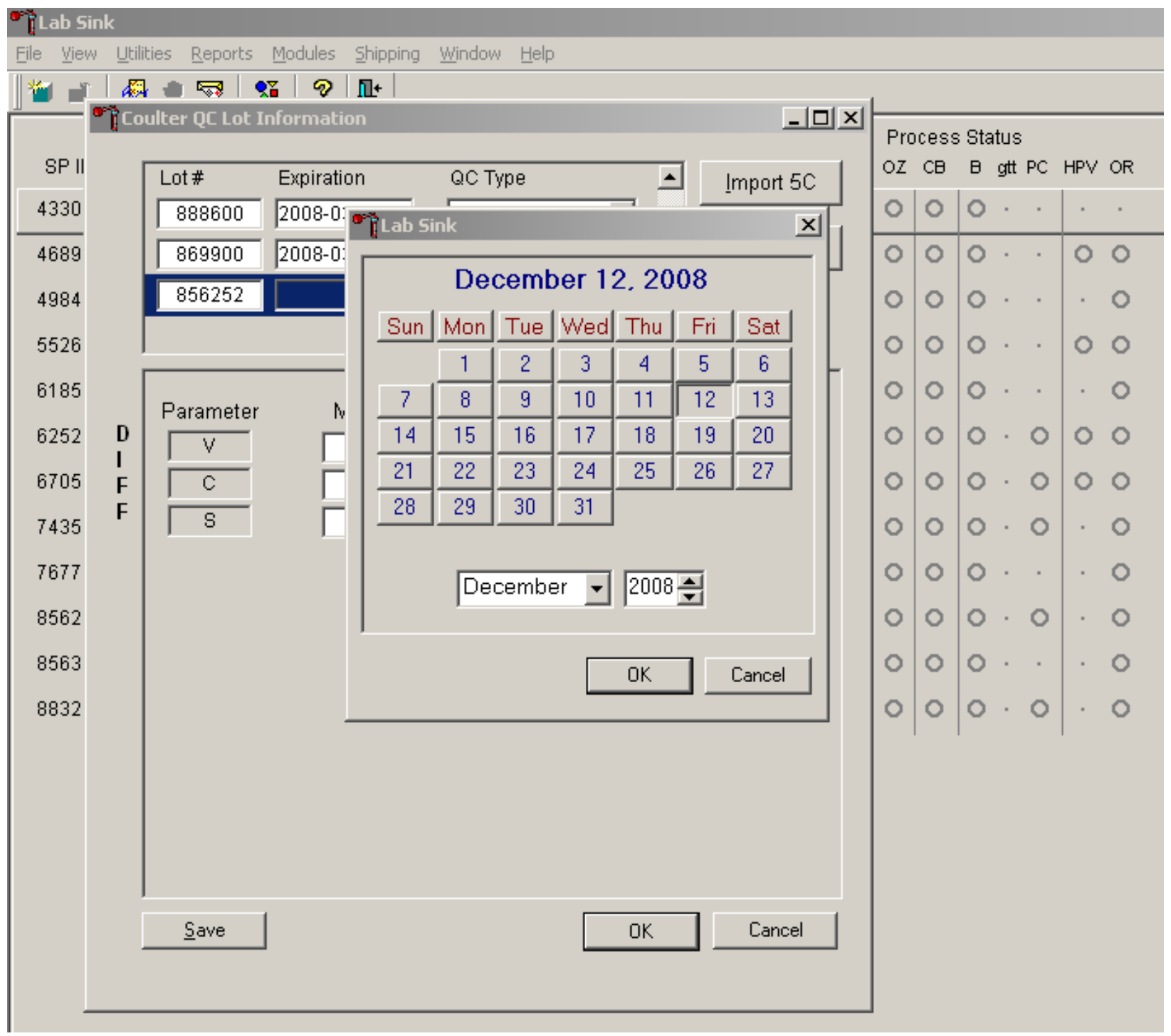

Use the keyboard number keys to enter the lot number then select [Tab] to move to the expiration date field. Use the mouse to direct the mouse arrow to the Ellipsis symbol located on the right side of the text box and left click. The calendar displays. Select the correct expiration date and, when finished, select the "OK" button to insert the date into the field. 
Use the keyboard number keys to enter the Latron data. The window is identical to the layout of the data on the package insert.

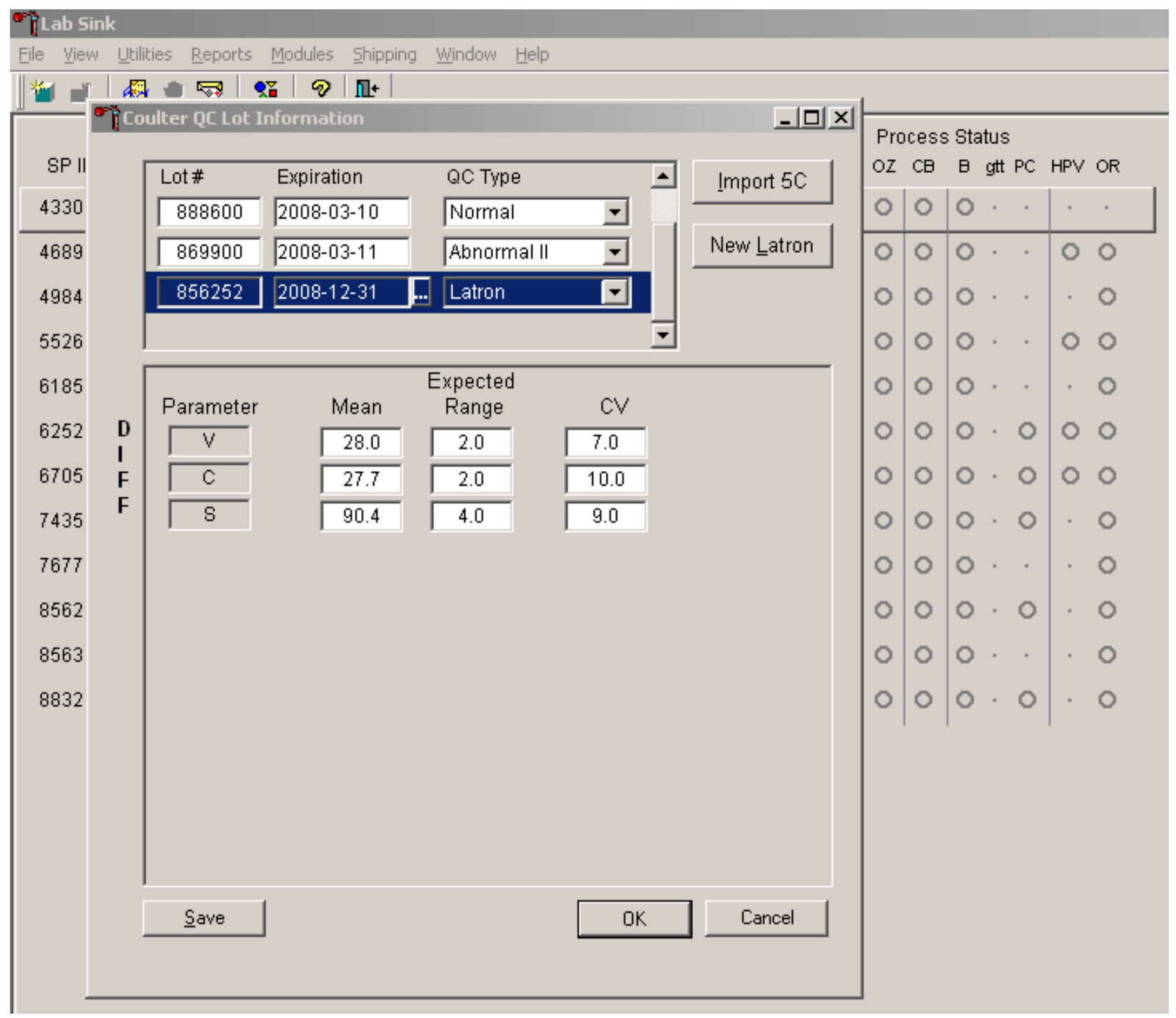

Use the keyboard number keys to enter each value for Mean, Expected Range, and CV. Select [Tab] to move to the next data field. When finished, select the Save button to save the data to the database. 
It is possible to enter Latron data for a new lot without entering $5 \mathrm{C}$ cell control data.

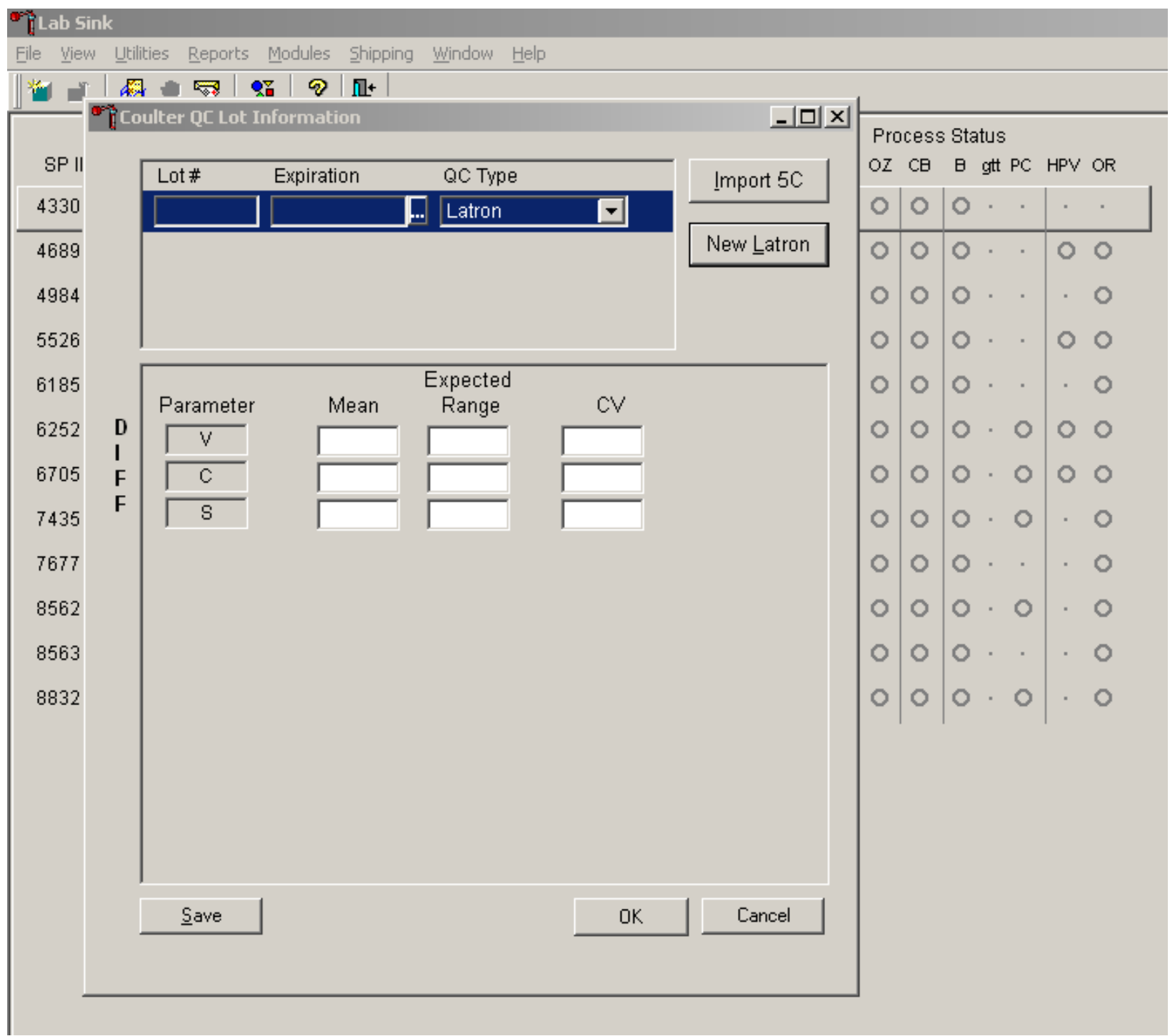

Select the "New Latron" button to access the module. The window only contains text boxes for the Latron control. Enter the data and save the information to the database.

E. Interlaboratory Quality Assurance Program (IQAP)

All instruments participate in Coulter's IQAP program. The IQAP program includes saving results of 5C cell controls, transferring them to a disk, and sending them to 
Coulter who compares the results to other laboratories. Coulter issues a report that contains a statistical analysis to evaluate performance. Perform this procedure at the end of each stand, or when a lot expires.

Follow procedure for CBC/DIFF and Latex control review and report. After downloading and printing results, delete control files [F8].

1. Procedure to download IQAP files to diskette.

a) Slide the diskette into the drive with the arrow side up and pointed toward the drive in the Coulter ${ }^{\circledR}$ Data Management System. Gently push the diskette until it clicks into place.

b) Turn the computer OFF. Wait 15 seconds, and then turn the computer back ON. A screen will appear showing the Coulter ${ }^{\circledR}$ Corporation logo. To exit this screen, follow the instructions on the bottom left portion of the screen, and press [Esc].

c) The screen will indicate the process the system is performing. Read the instructions on the screen. When the process is complete, the DMS displays the list of possible files on the screen for review and selection. If some of the values are incorrect, edit or delete the fields on this screen. Any alteration to this screen will affect only data on the IQAP diskette, not in the HMX computer. 
d) FILE_NAME indicates the files by file number for downloading to the diskette.

- LOT_\# indicates the type of control. Prefixes 86, 87, and 88 refer to $5 \mathrm{C}$ cell controls.

- SHIFT refers to the shift designation selected for running the controls. Edit this field if necessary. Use arrow keys to position.

- $\quad$ START and END indicate beginning and end dates for each file. These fields are not editable.

- REPORT indicates whether IQAP will issue a report on the lot number.

- N_REC indicates the number of records found in the file.

e) Press [Esc] to leave this screen. If the IQAP\# and Serial\# are present and valid, the following screen message will appear, "Updating the IQAP Disk.” After writing the control files to the diskette, a screen message will give instructions to follow. Follow these instructions and the computer will reboot to the routine program. To remove the diskette, press the eject button on the lower right of the diskette drive opening. Do not remove the diskette until the drive indicator light is off.

f) Label the diskette with the supplied IQAP ID label. Place the diskette in the preaddressed mailer provided and mail. Do not place the label over the drive spindle or high-density hole.

\section{F. Proficiency Testing}

Evaluation and participation in the College of American Pathologist (CAP) proficiencytesting program is part of the comprehensive quality control program. These survey materials are shipped three times per year and consist of 5 3-mL whole blood specimens. Follow all CAP instruction in preparing the materials before performing the test. Run specimens in a manner identical to routine specimens. Fill out the CAP result form, make a copy for the logbook, and send results to CAP. 
G. Linearity for WBC, RBC, Hgb, and Plt Parameters

Lin-CTM (PN 7547065 - 5 x 3.3-mL) - The Lin-C ${ }^{\text {TM }}$ linearity controls verifies the reportable range of Coulter ${ }^{\circledR}$ hematology systems that use both Isoton ${ }^{\circledR}$ III diluent and Lyse S® III diff lytic reagent.

OR

CAP Hematology Calibration Verification/Linearity Survey (LN9) - These materials are shipped twice per year and consist of 18 3-mL liquid specimens. Follow all CAP instruction in preparing and running the materials before performing the test. Fill out the CAP result form, make a copy for the logbook, and send results to CAP.

Run either the Lin-C ${ }^{\mathrm{TM}}$ or CAP LN9 survey material:

- At installation

- At least yearly

- Whenever experiencing an altitude change of 1 mile or more between stands

1. Principle - Lin-C ${ }^{\mathrm{TM}}$ linearity controls are human blood components from which repeated measurements verify the reportable range of Coulter ${ }^{\circledR}$ hematology systems. Controls contain one each of Ultra Low Range, Low Range, Mid Range, High Range, and Ultra High Range. Lin-C ${ }^{\mathrm{TM}}$ verifies ranges for the following parameters: WBC, RBC, Hgb, and Plt.

To ensure the accuracy of linearity control ranges, Coulter system calibrates with S-CAL ${ }^{\circledR}$ calibrator.

2. Reagents - Lin-C ${ }^{\mathrm{TM}}$ controls consist of treated, stabilized, human erythrocytes in an isotonic bacteriostatic medium. Linearity controls also contain a stabilized platelet-sized component, and fixed erythrocytes to simulate leukocytes. 
3. Storage, handling, and stability - Ship Lin-C controls in thermally insulated containers designed to keep kits cool. Store Lin-C linearity controls between 2$8^{\circ} \mathrm{C}\left(35-46^{\circ} \mathrm{F}\right)$. Store vials in an upright position to achieve maximum product yield. Storage of the control product in a cap down position might require additional mixing or complete resuspension of cellular components.

Potential biohazard - Each human donor used in preparation of this material was tested by an FDA approved method for the presence of the antibodies to human immunodeficiency virus (HIV) and hepatitis C (HCV) as well as for hepatitis B surface antigen and found to be negative (were not repeatedly reactive.) Handle these reagents at Biosafety Level 2 because no test method can offer complete assurance that these and other infectious agents are absent.

4. Indications of instability or deterioration - Inability to obtain expected values without known instrument problems or gross hemolysis (dark colored supernatant) indicates product deterioration. A slight pink color to the supernatant is normal. Do not confuse this pink color with deterioration of the product.

5. Instructions for use - Remove Lin-C controls from refrigerator and warm to ambient temperature for 10-15 minutes. After warming, mix by hand as follows:

Do not use a rotator, rocker, or mechanical mixer. Roll the tube slowly between the palms of the hands eight full rotations in an upright position. Invert the tube and slowly roll it again eight times. Gently invert the tube eight times. Repeat these actions again. Controls expire 7 days after opening.

a) Turn the blood detectors off to analyze the Ultra Low Range. Select SPECIAL FUNCTIONS, DIAGNOSTICS, OPERATOR OPTIONS, BSV TESTS, and BLOOD DETECTOR ON/OFF. Press [Enter]. Use space bar to select On or Off. Press [Enter]. 
b) Disable the differential before analyzing Lin-C linearity controls. Is Sample Mode displayed? If no, press [F9] Stop. Make sure the DIFF is off. If it is on, press [F6].

c) When analyzing Lin-C linearity controls, flags such as L, LL, H, HH, R, $\mathrm{RR}, * * \mathrm{R}$, and $* \mathrm{~V}$ will occur. Ignore these flags if a numerical result is obtained. When ---- (voteout), ..... (incomplete computation), or ++++ (over range) flags occur, the sample should be repeated. Ignore Coulter histogram differential flags and results.

d) Run the Lin-C controls in the Primary mode of the instrument. Select SPECIAL FUNCTIONS, CALIBRATION, and REPRODUCIBILITY. Press [Enter]. Press [F2] Start Primary. Analyze controls six times and record the results for WBC, RBC, Hgb, and Plt parameters for each sample on the worksheet. Repeat flagged samples with non-numeric results.

e) Coulter recommends that five sample results should be used to calculate the mean value. Use a minimum of three samples to calculate the mean when repetitive results with non-numeric values occur. Delete the first sample result. Plot mean recovered values on the Lin-C linearity control graphs.

f) Compare the mean value to the linearity control ranges listed on the Table of Expected Results on the package insert. Mean values should recover within the ranges. Use the ranges established by Coulter Corporation as a guideline. 
g) Plot the instrument background count as a zero value to extend the reportable range. Coulter will prepare tabular summaries and graphic presentations of the data. Submit the top copy of the worksheet to IQAP at the following address:

Coulter Corporation

IQAP (M/C 31 B04)

PO BOX 169015

Miami, FL 33116-9015

XI. Interpretation of Results and Remedial Action

A. Sample Person Hemoglobin and Hematocrit Review and Remedial Actions

1. Review all results to make sure the hemoglobin and hematocrit are acceptable. The hematocrit should be approximately three times the hemoglobin.

B. Sample Person Parameter Value Review and Remedial Actions

1. Access the Hematology module or reject a clotted blood tube.

Open the Hematology module.

The Hematology module does not need to be open before running SP samples on the HMX. Note the red icon in the lower right corner of the system tray.

«ent证 11:35 AM 
This is the NHANES Coulter Monitor icon. It must be open and running at the start of each session. This icon stores all HMX runs in the ISIS database. Open the icon by double-clicking on the Coulter icon on the desktop. The Coulter icon looks like this:

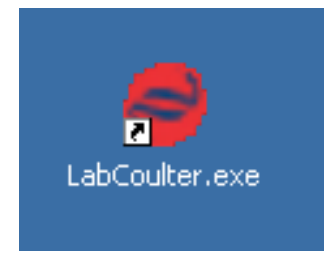

Open the Hematology module.

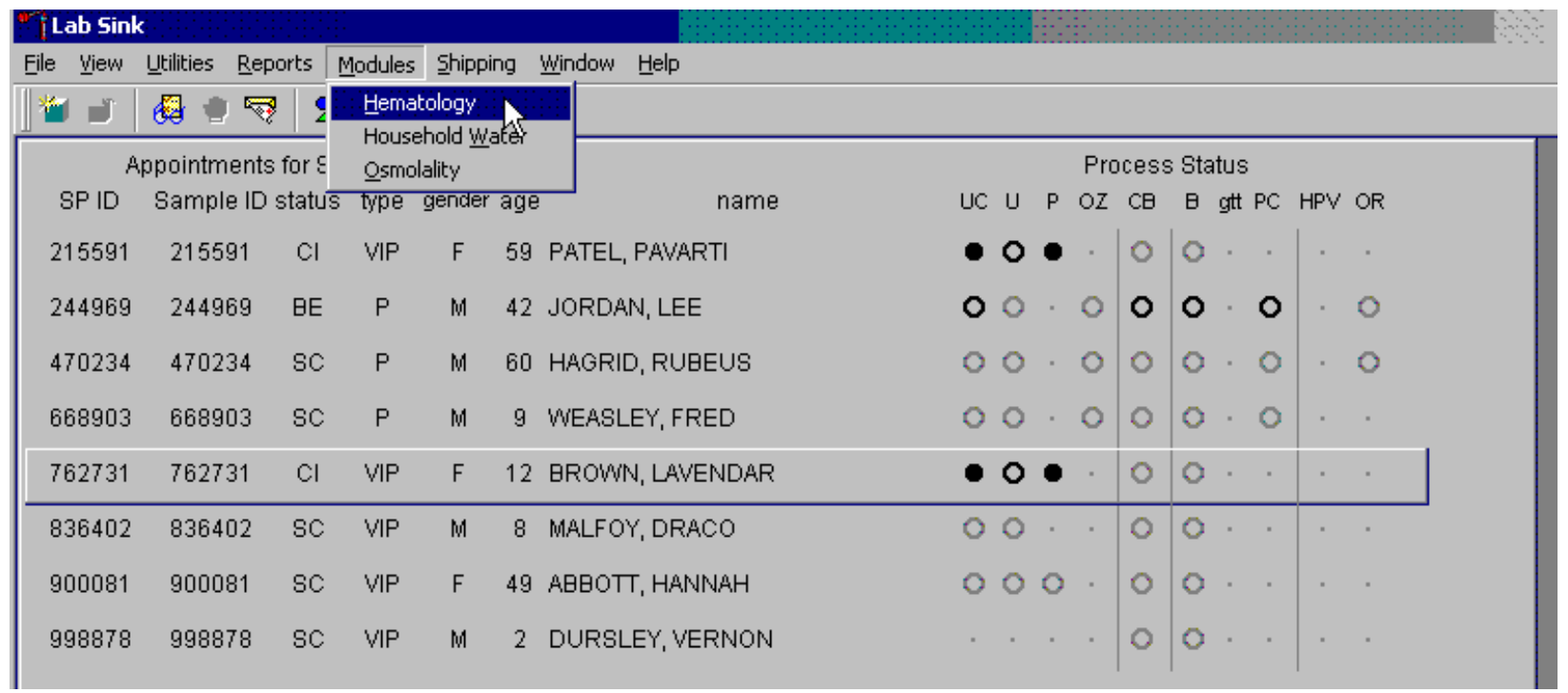


To open the Hematology module, use the mouse to direct the mouse arrow to \{Modules\}, in the top menu bar, left click, drag the mouse arrow to $\{$ Hematology $\}$, and right click, or type $[\mathrm{Alt}][\mathrm{M} / \mathrm{m}]$, $[\mathrm{H} / \mathrm{h}]$.

Alternatively, open the Hematology module from the heads-up display.

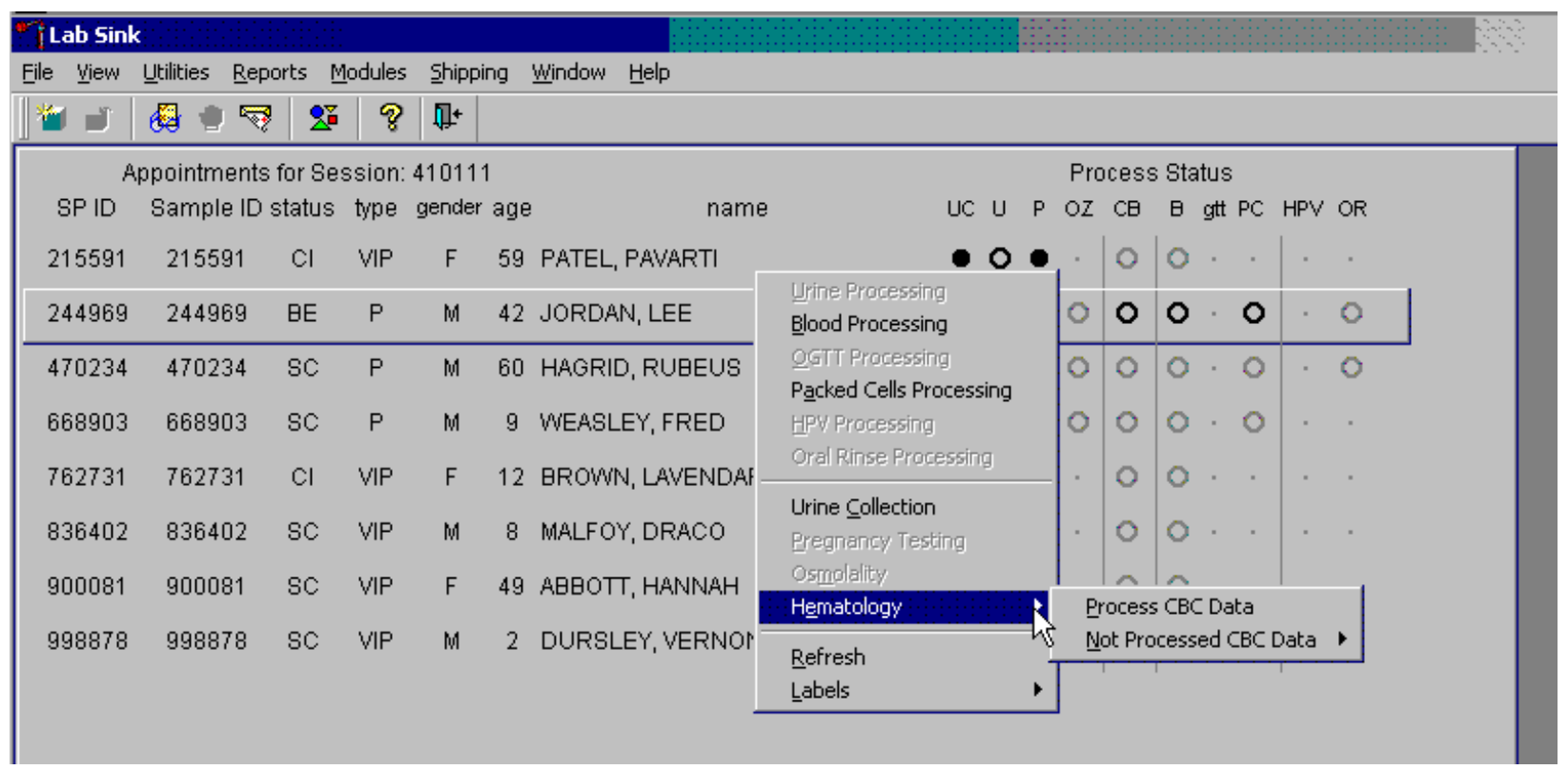

To access the Hematology module, use the mouse to direct the mouse arrow to any SP, right click, drag the mouse arrow to $\{$ Hematology\} then to \{Process CBC Data\}, and right click or right click and type [Shift] [E/e] [P/p]. Alternatively, use the up and down keys to move up and down the list until a particular SP is highlighted, right click, drag the mouse arrow to $\{$ Hematology $\}$ then to $\{$ Process CBC Data, and right click or right click and type [Shift] [E/e] [P/p]. 
Either open the module or reject the specimen and add a reason or comment for every CB record where blood was drawn in phlebotomy AND there are no CBC results.

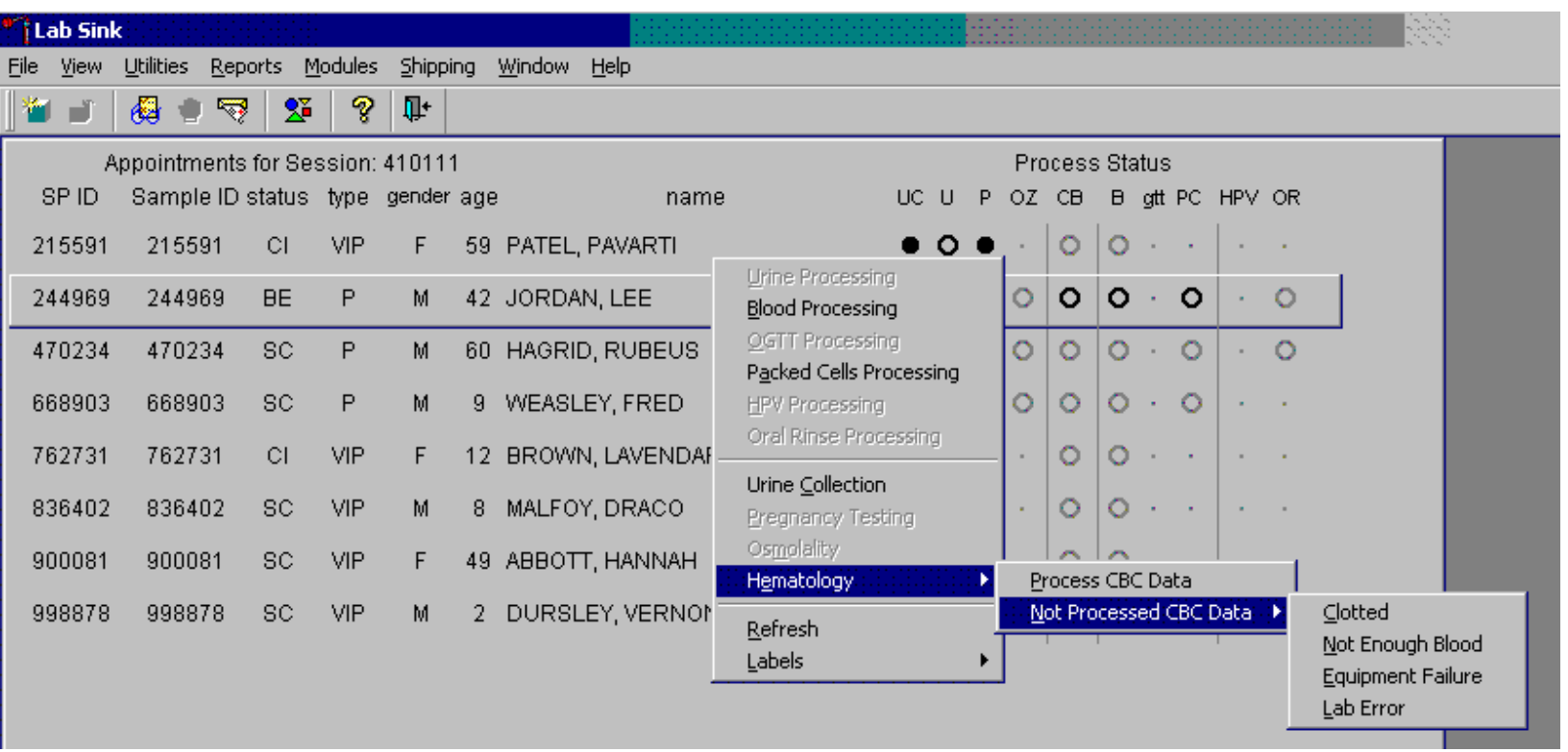

Open the module \{Process CBC Data\} or record a reason why the CBC is not being run \{Not Processed CBC Data\}. Select or record a comment for every CBC that is not run. To complete opening or accessing the Hematology module, use the mouse to direct the mouse arrow to Process CBC Data and right click or type $[\mathrm{P} / \mathrm{p}$.] To record a reason why a CBC is not being run, use the mouse to direct the mouse arrow to the exact SP's line on the heads-up display, right click, drag the mouse arrow to \{Not Processed CBC Data\}, and drag the mouse arrow to the correct reason and right click. Alternatively, type [Shift] [E/e] [N/n] and [C/c] for Clotted, [N/n] for Not Enough Blood, [E/e] for Equipment Failure, or [L/l] for Lab Error. 
A pop-up window will display. Confirm the selection.

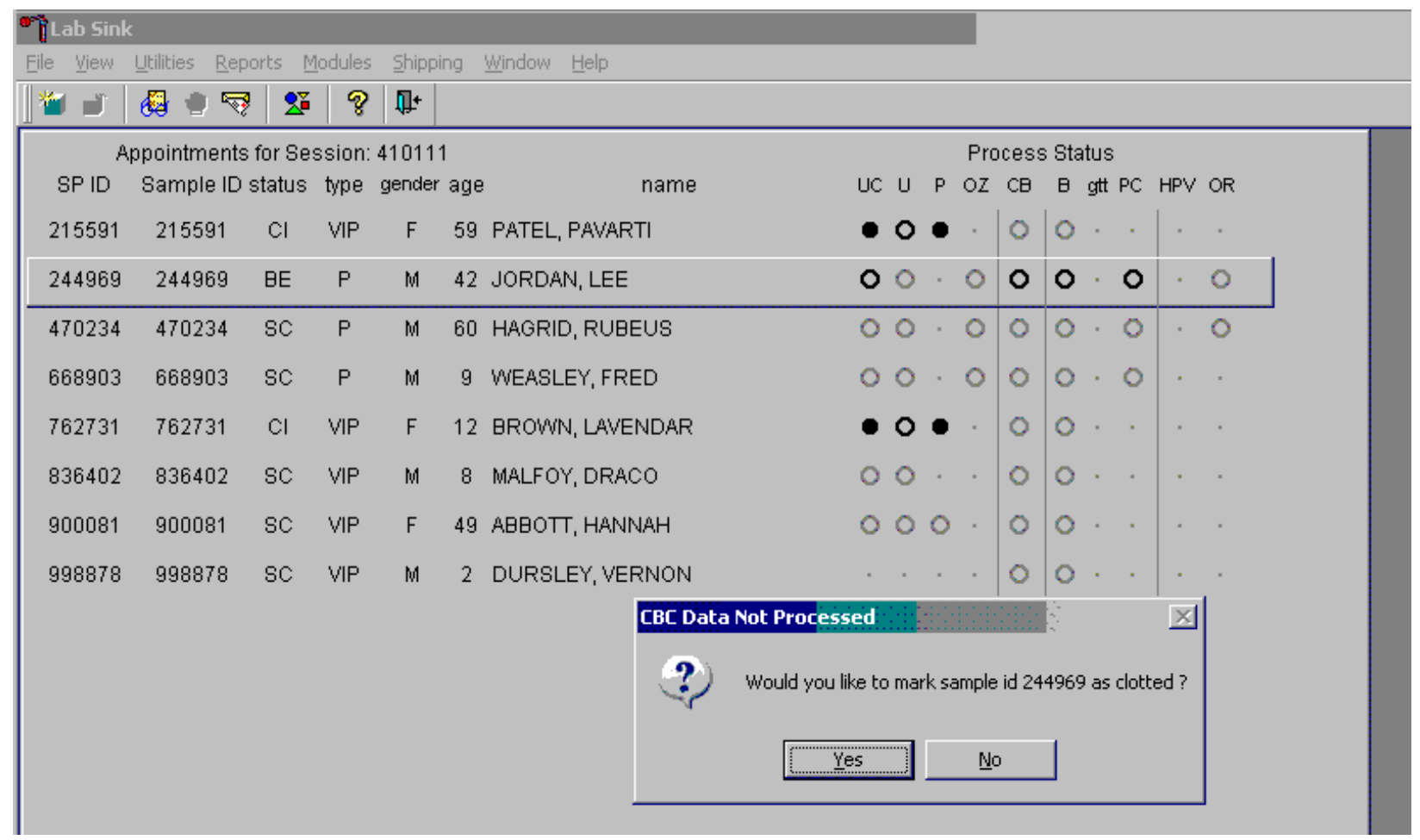

A CBC Data Not Processed message text box displays that asks, "Would you like to mark sample id XXXXXX as Clotted/Not Enough Blood/Equipment Failure/Lab Error?” To record a "Yes" response, use the mouse to direct the mouse arrow to the Yes button and left click, type [Y/y], or press [Enter]. To record a "No" response, use the mouse to direct the mouse arrow to the No button and left click, or type [N/n]. If a Yes response is recorded, the comment is saved to the database. If a No response is recorded, no comment is saved to the database. If the record is marked with the selection in the database, then the heads-up display updates to complete (the CB circle fills in black). 


\section{Hematology module overview.}

Coulter does not automatically transmit results to the Laboratory application. Use the Retrieve button to send the results from the DMS to the Hematology module. Select after each run. Make sure the Coulter DMS host computer icon (HC) is displaying an up arrow ( $\uparrow$ ) in the DMS bottom tile bar.

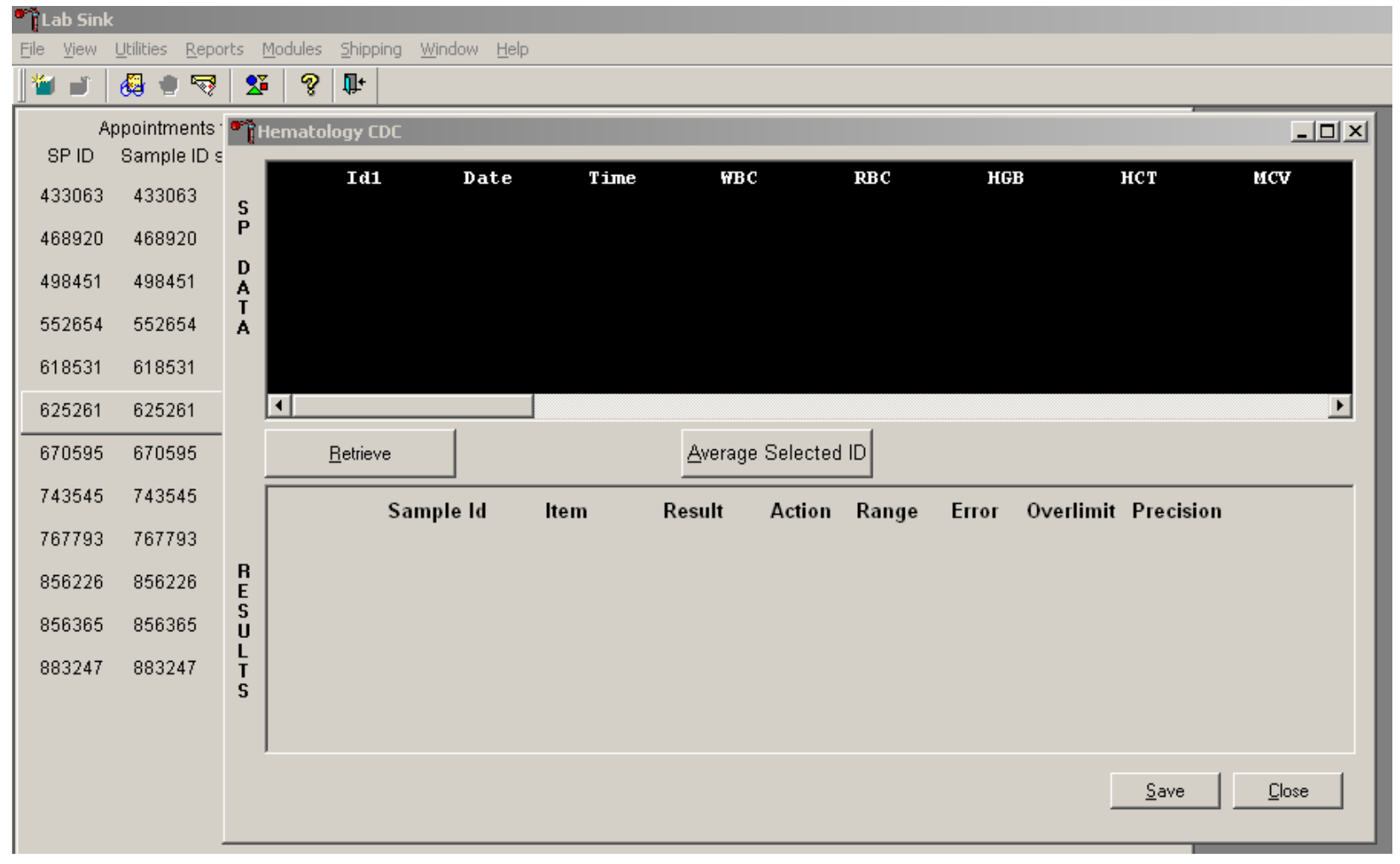

After data are retrieved, it displays in the top portion of the window, the SP Data section. This section includes the SP ID, the date and time the CBC was run, and columns for each individual parameter. The bottom portion of the window is the Results section. Results display after the Average Selected ID button is selected. 
Review all Coulter data in the SP Data section after it has been retrieved.

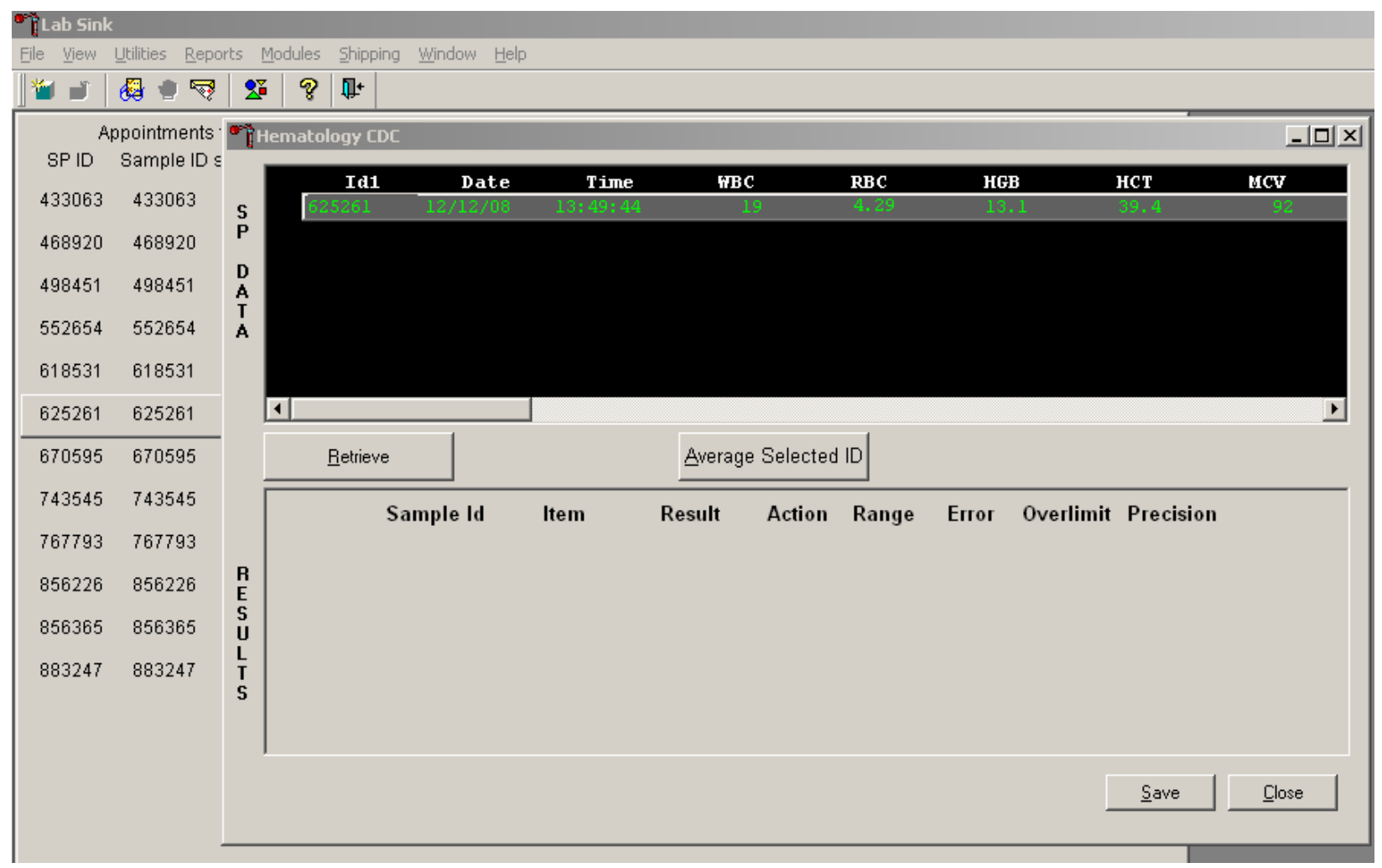

To view SP data for all parameters, use the mouse to direct the mouse arrow to the horizontal scroll bar at the bottom of the SP Data section and drag the scroll bar to the left or right. Alternatively, to view the SP data for all parameters, use the mouse to direct the mouse arrow to the small scroll arrows on the left and right edges of the horizontal scroll bar, and left click. 
The Hematology Results section overview.

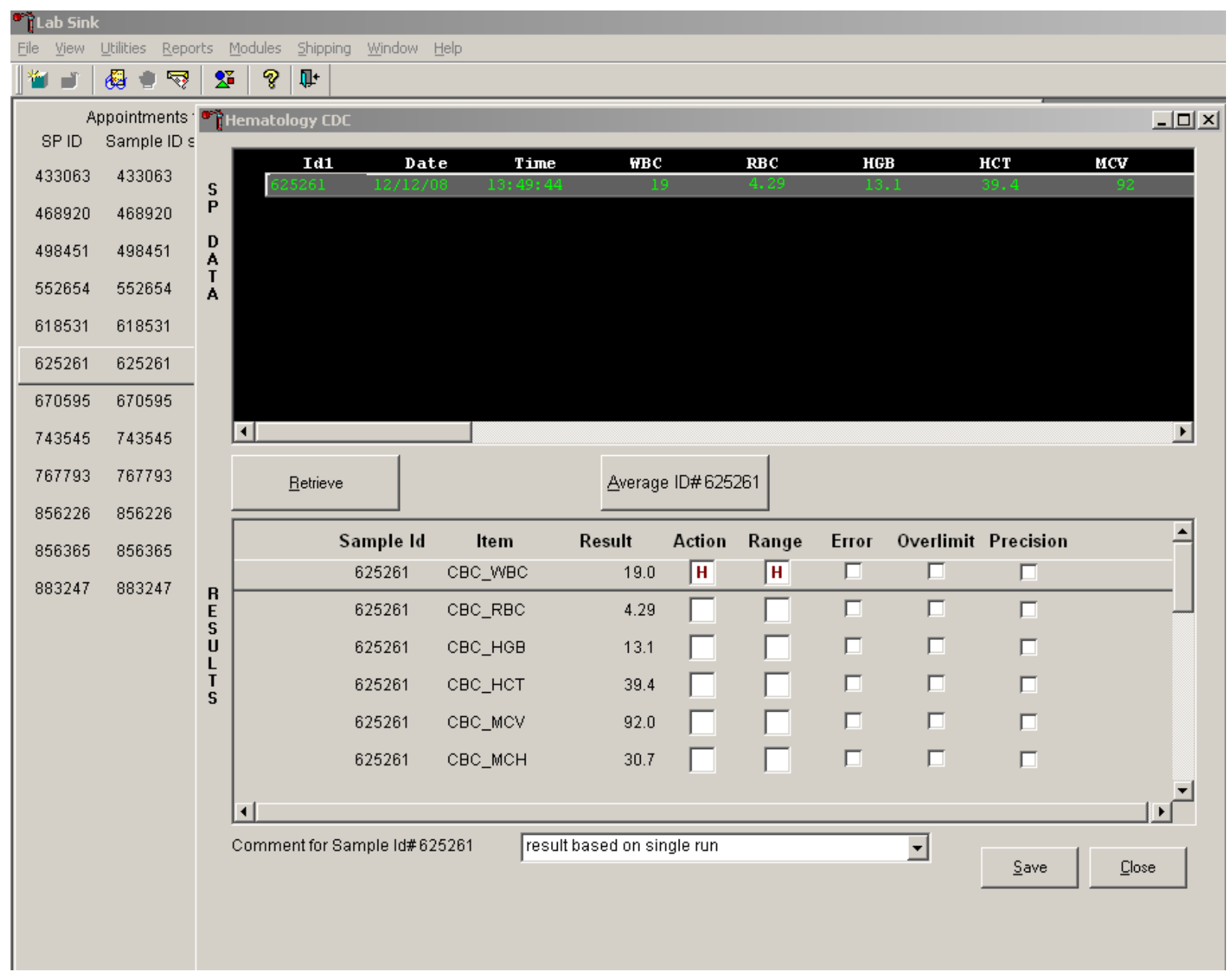

The Result columns include: Sample ID, Item (CBC parameter), Result (Coulter data for a single run or the ISIS averaged result for multiple runs), Action (CDC established critical limits), Range (CDC established reference ranges for both genders and four age groups), Error (Coulter transmitted instrument errors), Overlimit (Coulter transmitted result that exceeds the instrument's linearity limit), and Precision (CDC established values for the difference between any two runs.) Checkmarks display in boxes for parameters that have errors, are overlimit, or for those that exceed precision limits. An " $H$ " for "high" and "L" for "low" display for parameters that exceed action limits or reference ranges.

Average, evaluate, and save results for all parameters. The SP's results display in the bottom portion of the window after the Average Selected ID button is selected. To view results for all parameters, 
use the mouse to direct the mouse arrow to the vertical scroll bar at the right side of the Results section and drag the bar up or down. Alternatively, to view all the results, use the mouse to direct the mouse arrow to the small scroll arrows on the top and bottom edges of the vertical scroll bar, and left click. Evaluate each parameter for error, overlimit, and precision checkmarks, and " $\mathrm{H}$ " or " $\mathrm{L}$ " action limit and reference range flags. To save the result to the database, use the mouse to direct the mouse arrow to the Save button and left click or type [Shift] [S/s]. After results are saved, they are erased from the screen. To exit without saving the result to the database, use the mouse to direct the mouse arrow to the Cancel button and left click or type [Shift] [C/c].

A warning text box displays if the Close button is selected before the results have been saved.

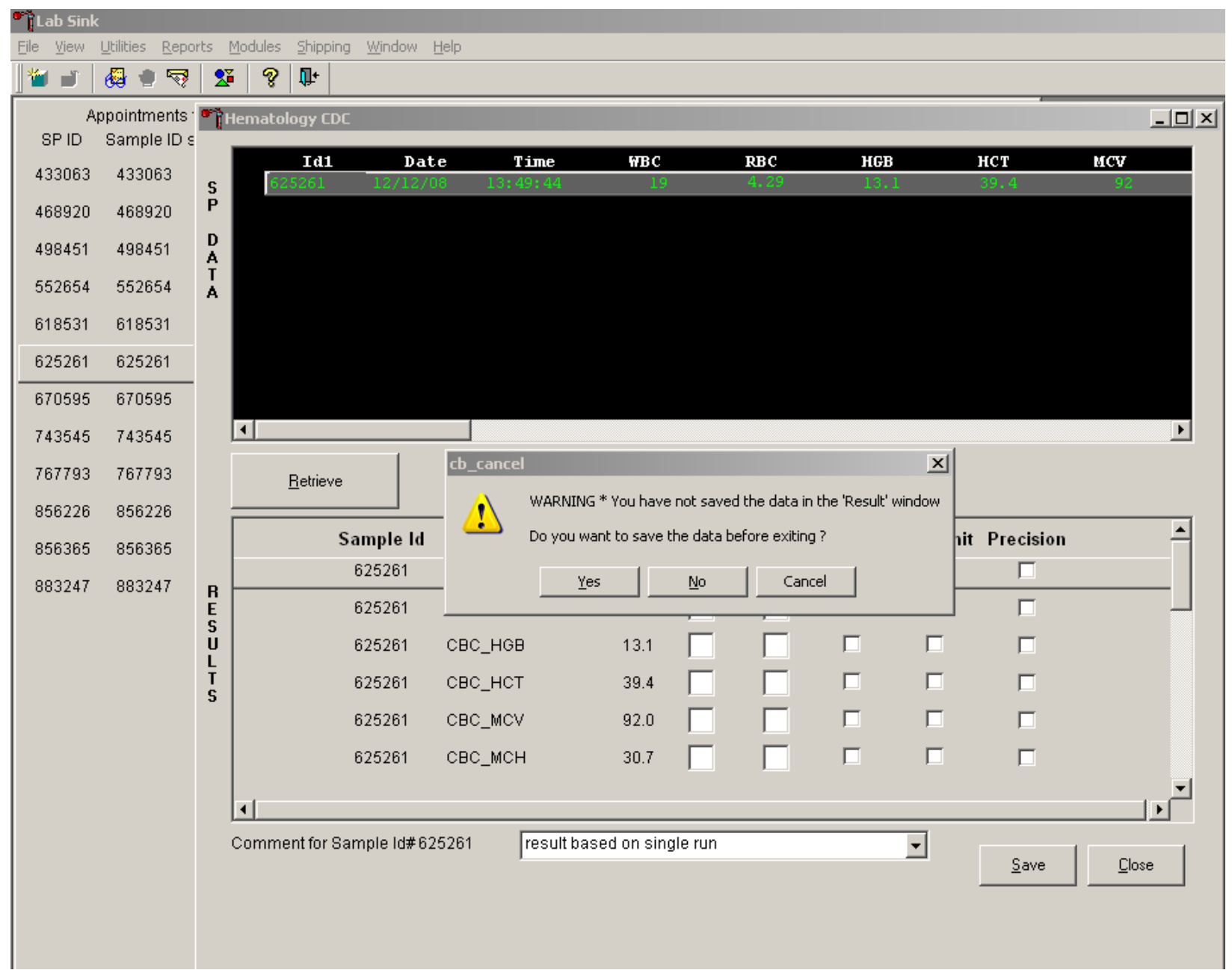


If the Close button is selected before the results are saved to the database, a warning message text box displays that states, "WARNING *You have not saved the data in the 'Result' window" and asks, "Do you want to save the data before exiting?" To record a "Yes" response, use the mouse to direct the mouse arrow to the Yes button and left click, type [Y/y], or press [Enter]. To record a "No" response, use the mouse to direct the mouse arrow to the No button and left click, or type [N/n]. To cancel the action, use the mouse to direct the mouse arrow to the Cancel button and left click. If a Yes response is recorded, the CBC results are saved to the database and erased. If a No response is recorded, no results are saved to the database and the data in the Results section is erased. A Cancel response returns the screen to its previous state. 
3. Running samples when only one run is possible.

Save results where only one run was possible.

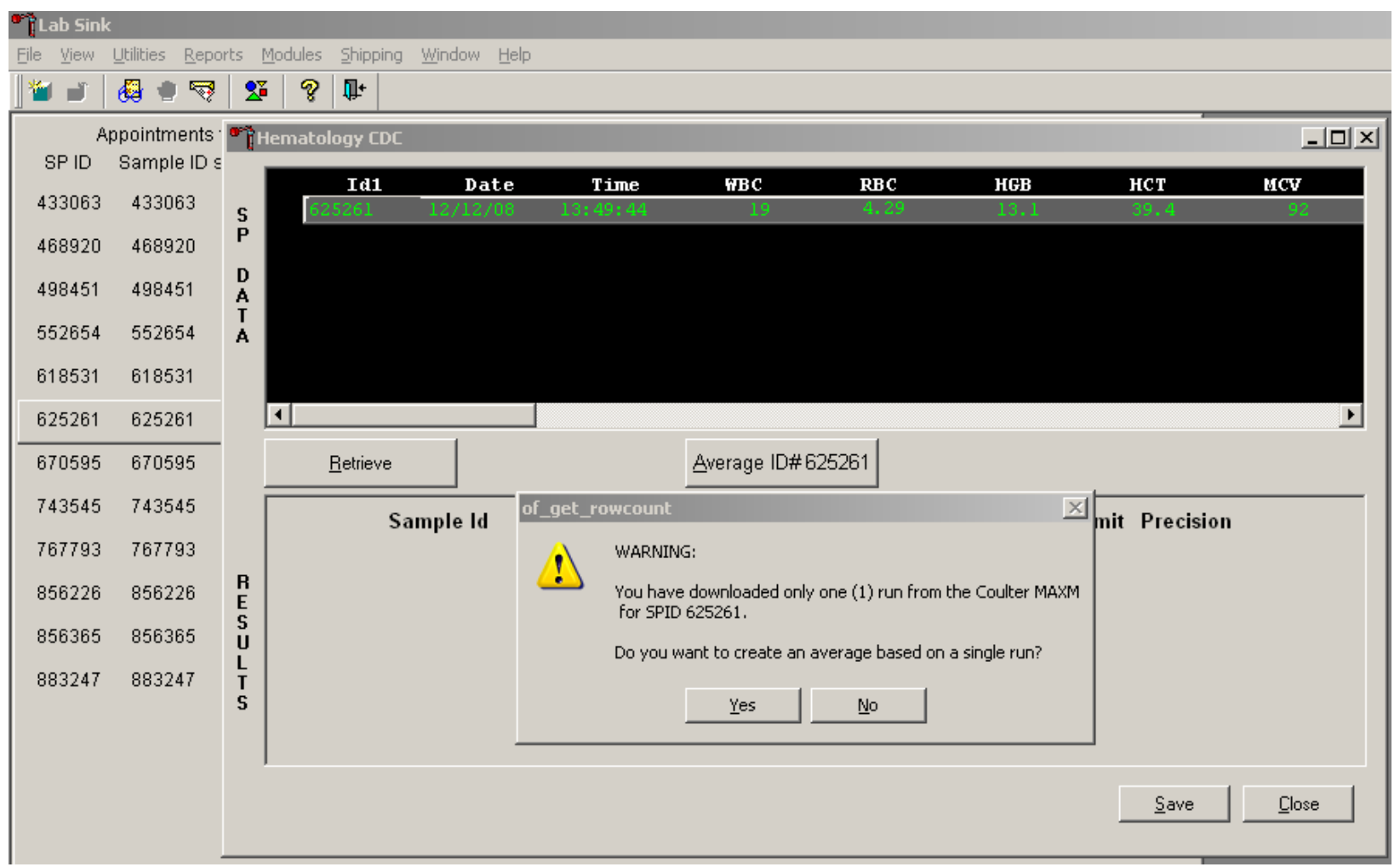

Run samples in duplicate whenever possible. If the whole blood is insufficient, it is acceptable to average and save only one run. To save results where only one run is possible, use the mouse to direct the mouse arrow to the Average Selected ID box and left click. A warning message text box displays that states, "You have downloaded only one (1) run from the Coulter HMX for SPID $X X X X X X$ " and asks, "Do you want to create an average based on a single run?" To record a "Yes" response, use the mouse to direct the mouse arrow to the Yes button and left click or type [Y/y]. To record a "No" response, use the mouse to direct the mouse arrow to the No button and left click, or type $[\mathrm{N} / \mathrm{n}]$, or press [Enter]. If a Yes response is recorded, the CBC results display in the bottom Results section of the window. A "No" response cancels the action and returns the window to its previous state. 
For results where only one run was possible, the Comment box at the bottom of the window defaults to "result based on single run."

4. Running samples in duplicate.

Run all samples in duplicate and average the data.

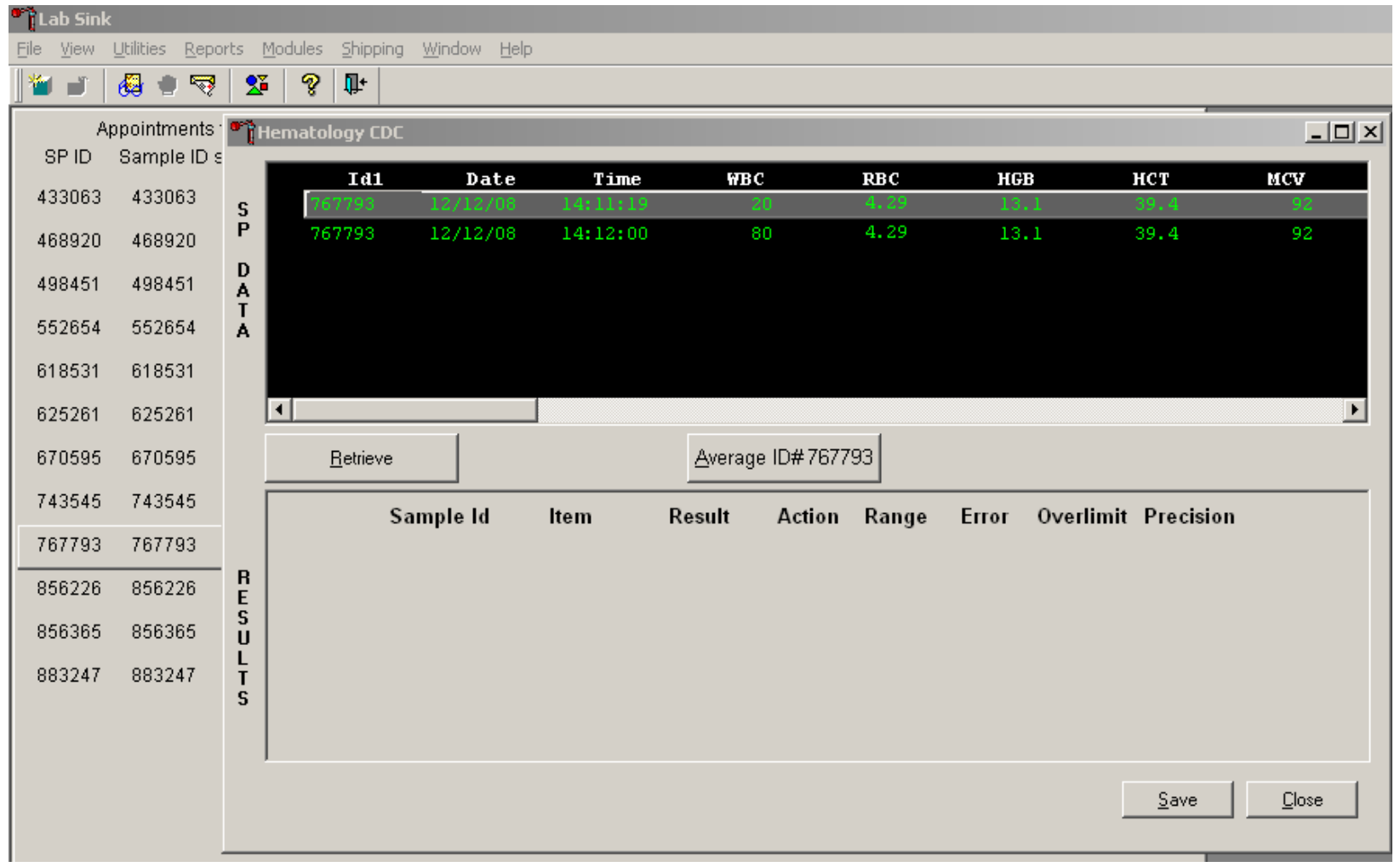

Whenever a sample is run in duplicate, both results display in the SP Data section. To average these two results, use the mouse to direct the mouse arrow to Average ID\#XXXXXX button and left click. 
Results display in the lower section of the window.

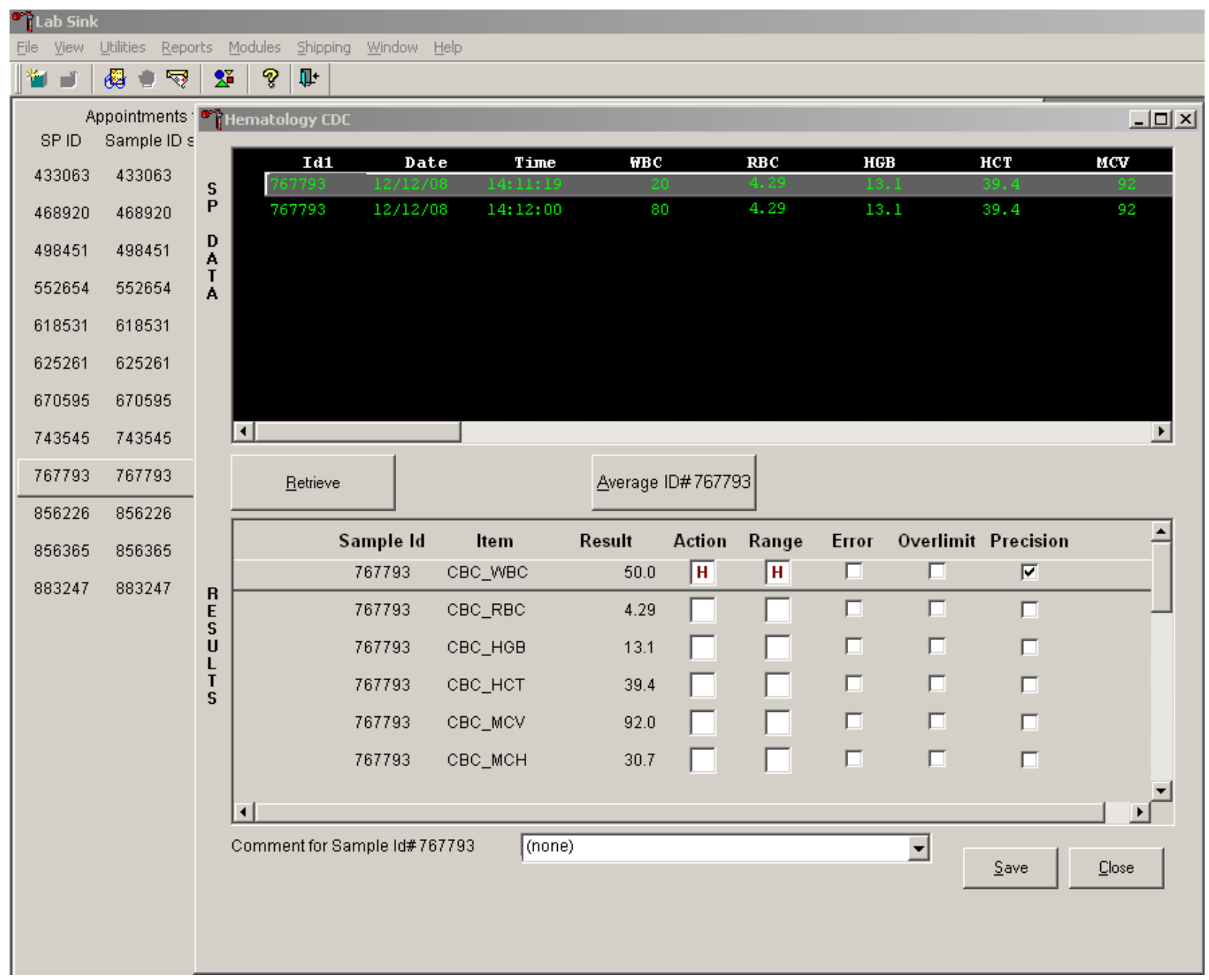

The difference between duplicate values for WBC, RBC, Hgb, MCV, Plt, NE\#, LY\#, MO\#, EO\# and BA\# are calculated and evaluated to determine if the differences are within the following CDC established precision limits. 
CDC established precision limits:

$\begin{array}{ll}\text { Test } & \text { Precision Limits } \\ \text { WBC } & 0.4 \times 10^{3} \\ \text { RBC } & 0.1 \times 10^{6} \\ \text { Hgb } & 0.4 \mathrm{~g} / \mathrm{dL} \\ \text { MCV } & 2.2 \mathrm{fL} \\ \text { Plt } & 23 \times 10^{3} \\ \text { NE\# } & 0.4 \times 10^{3} \\ \text { LY\# } & 0.2 \times 10^{3} \\ \text { MO\# } & 0.2 \times 10^{3} \\ \text { EO\# } & 0.2 \times 10^{3} \\ \text { BA\# } & 0.2 \times 10^{3}\end{array}$

If RBC parameters are out of range, evaluate the data for drift. If drift is evident, evaluate the possibility of an instrument malfunction. If any WBC differential absolute number is out of range, check the WBC scattergram for abnormal cell population(s). 
Evaluate and save results when precision limits are not exceeded.

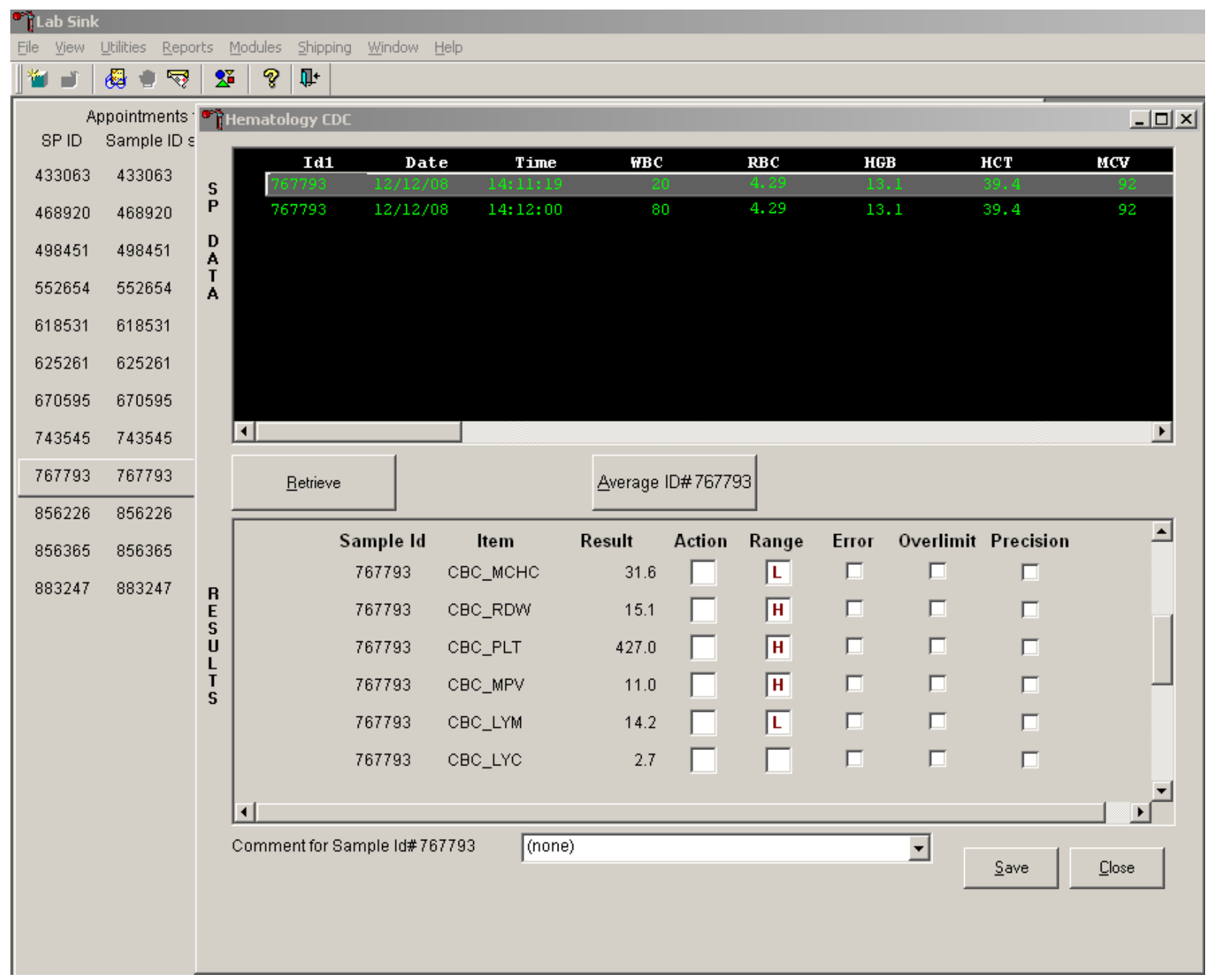

Evaluate results for all parameters. Evaluate each parameter for error, overlimit, and precision checkmarks, and " $\mathrm{H}$ " or " $\mathrm{L}$ " action limit and reference range flags. If there are no checkmarks in the Precision column (no precision limit was exceeded), save the results to the database. To save the results to the database, use the mouse to direct the mouse arrow to the Save button and left click or type [Shift] [S/s]. After results are saved, they are erased from the screen. To exit without saving the result to the database, use the mouse to direct the mouse arrow to the Close button and left click or type [Shift] $[\mathrm{C} / \mathrm{c}]$. 
Average, evaluate, and rerun specimens that exceed precision limits.

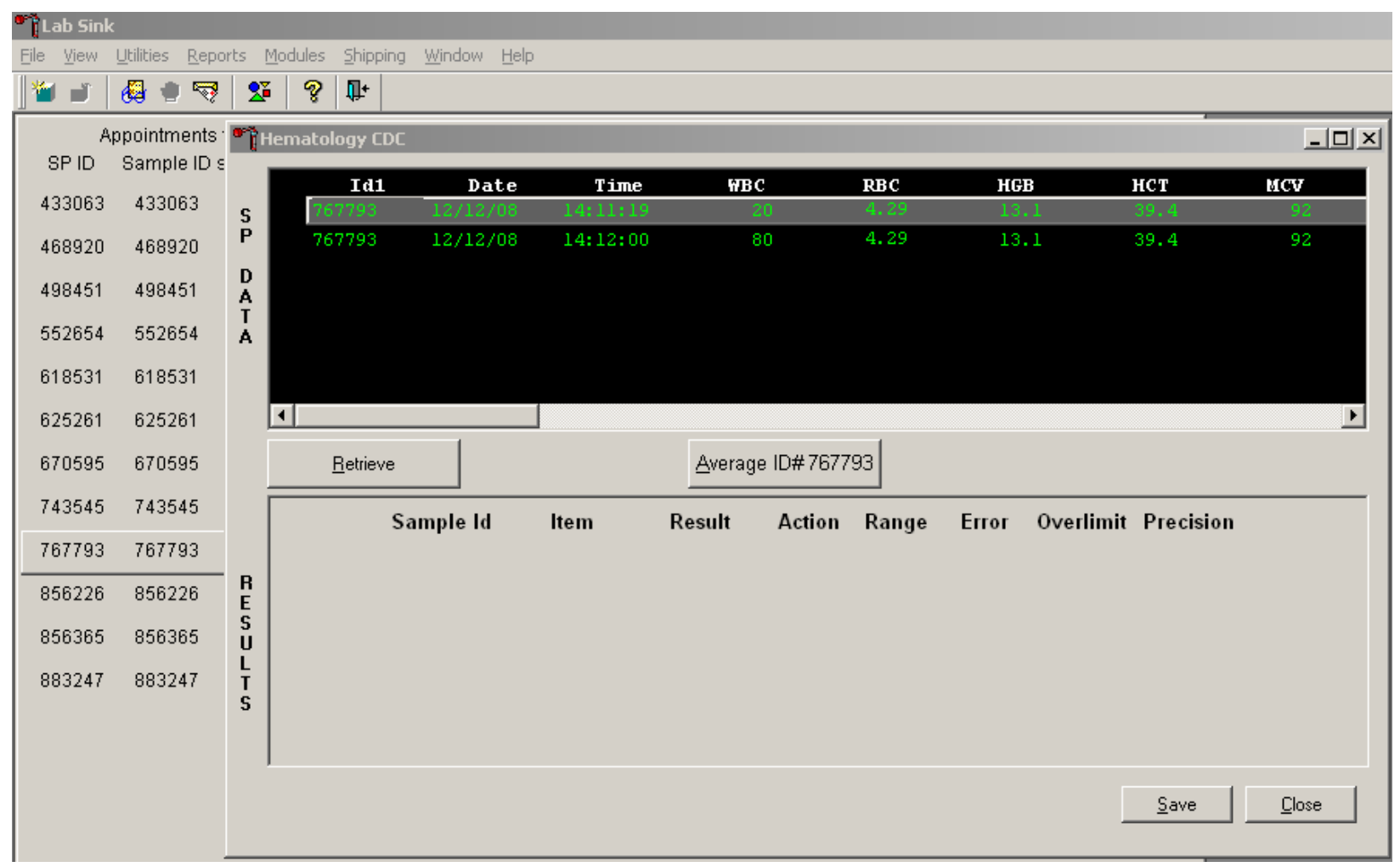

Average the two results by using the mouse to direct the mouse arrow to the Average ID\#XXXXXX button and left click. If at this point, precision limits are exceeded for any individual parameter, the technologist is prompted to run a control and evaluate the control to determine if all control values fall within the control's established range. 
If any parameter exceeds its precision limit, the Hematology Control Run window displays.

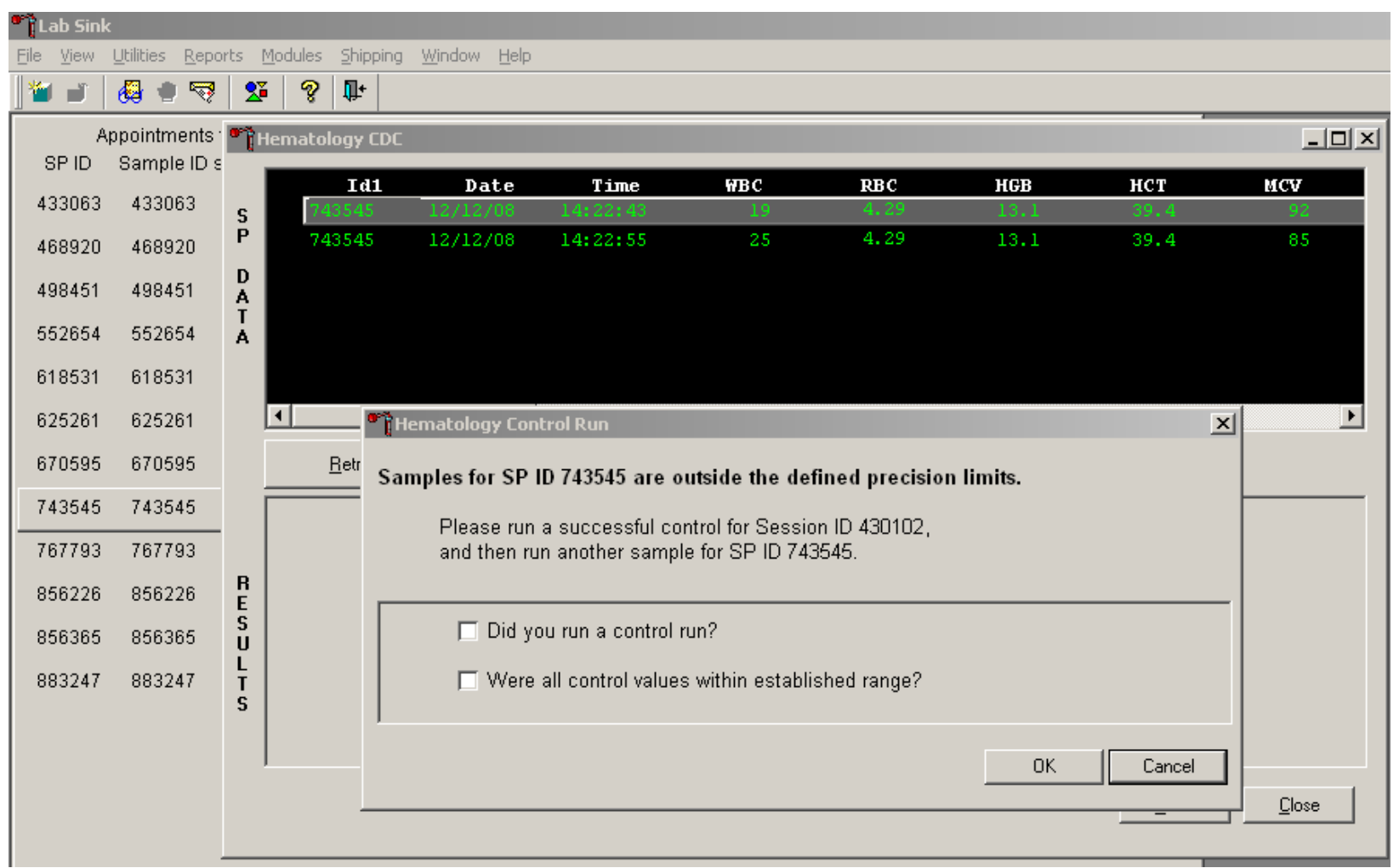

The Hematology Control Run window indicates that the "Samples for SP ID $X X X X X X$ are outside the defined precision limits." It instructs the technologist to "Please run a successful control for Session ID $X X X X X X$, and then run another sample for SP ID $X X X X X X$.” Run any one level of 5C cell control and evaluate the results. Respond to the two questions, "Did you run a control run?” and "Were all control values within established range?” To confirm these actions, use the mouse to direct the mouse arrow to each of the two check boxes and left click to record the check marks. 
Save the responses to the check box questions.

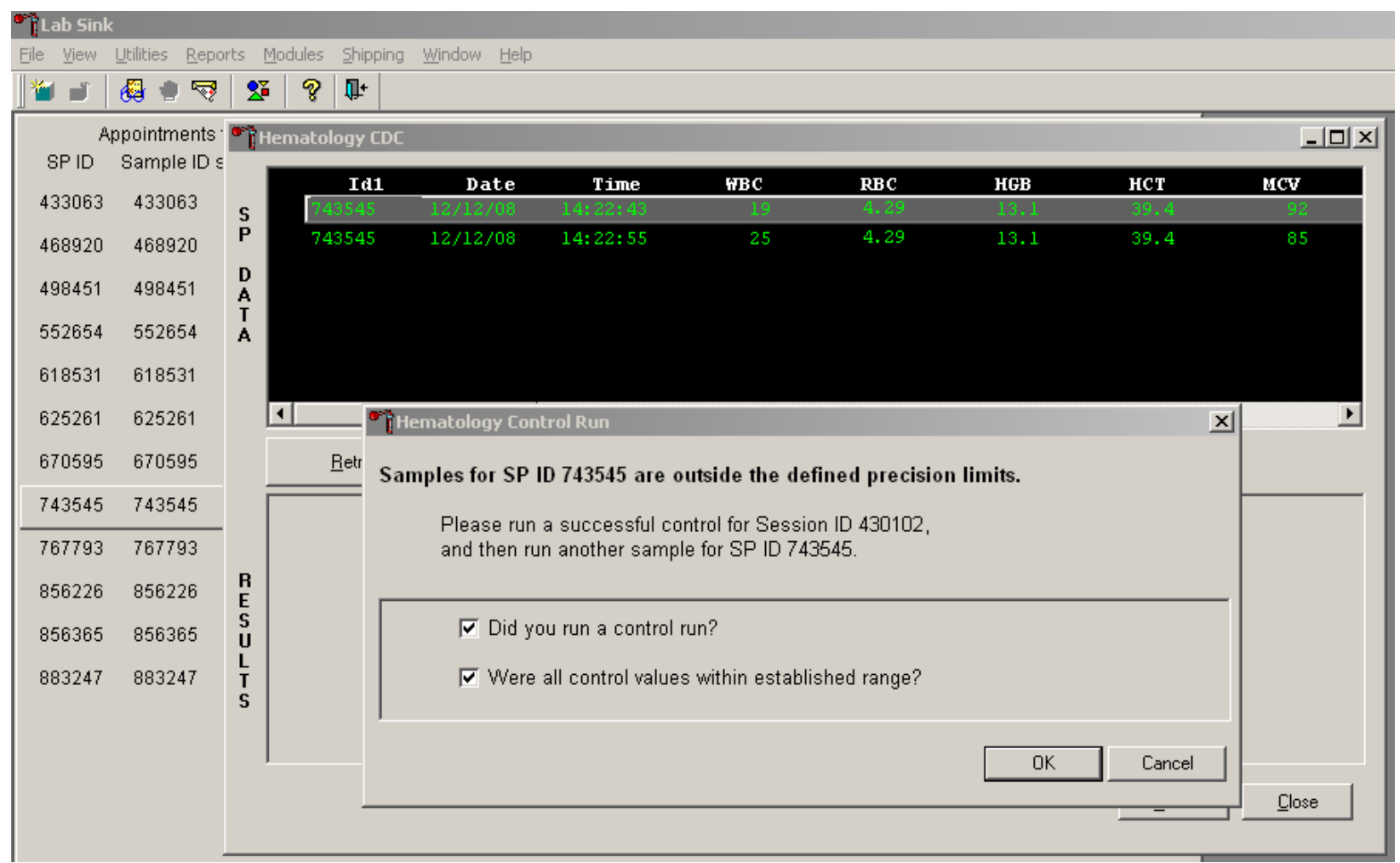

To save these responses to the database, use the mouse to direct the mouse arrow to the OK button and left click or type [Enter], or to exit the Hematology Control Run window without saving the responses, use the mouse to direct the mouse arrow to the Cancel button and left click. As soon as the OK button is selected, the averages display in the Results section of the window. 
Evaluate the results displayed in the Results section of the window.

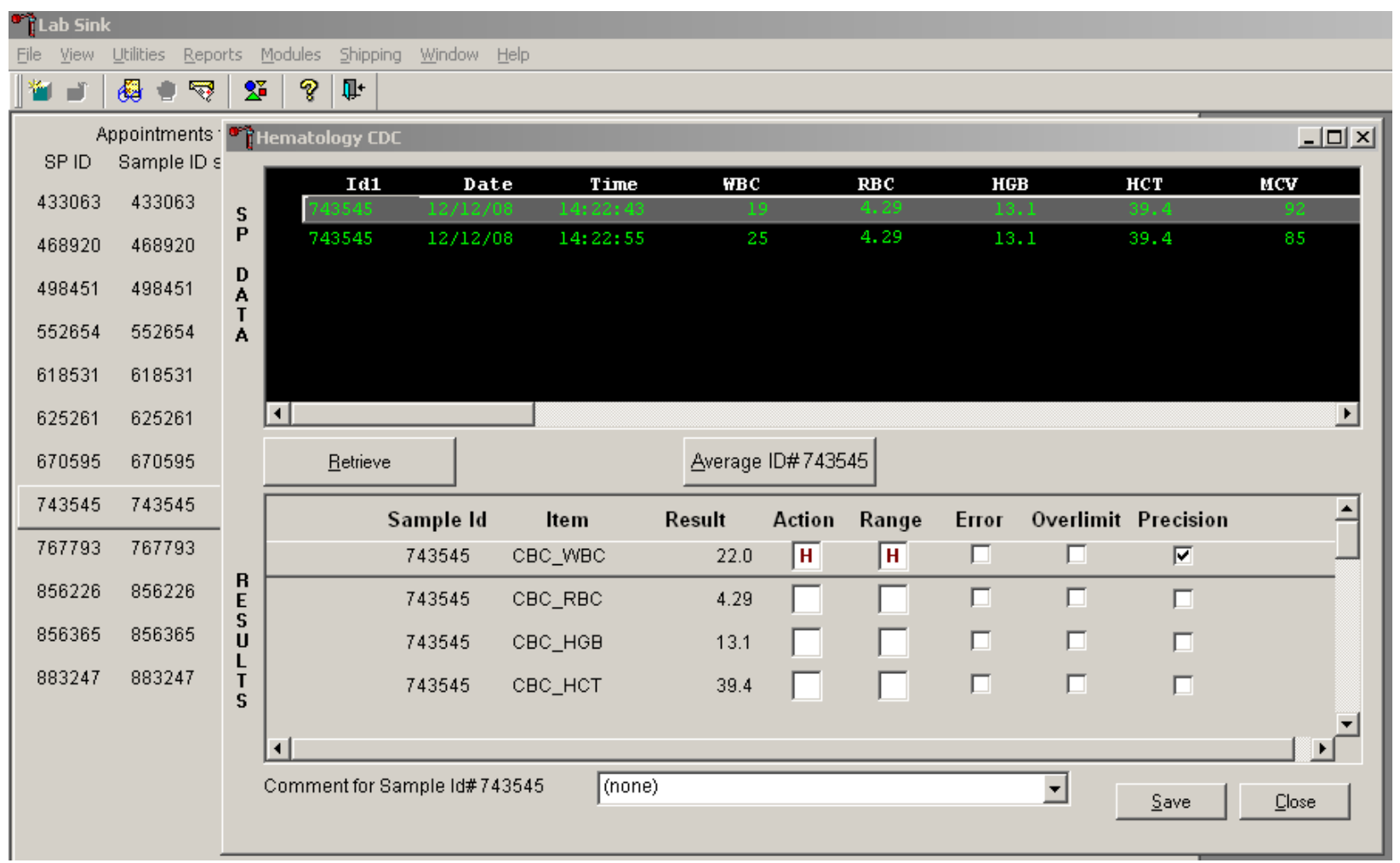

Evaluate each parameter for error, overlimit, and precision check marks, and " $\mathrm{H}$ " or "L" action limit and reference range flags. Use the scroll bar to view all results. When there are checkmarks in any of the Precision columns, meaning that the precision limit was exceeded, rerun the blood sample a third time. If there is insufficient blood to run the CBC a third time, save the result to the database. To save the results to the database, use the mouse to direct the mouse arrow to the Save button and left click or type [Shift] [S/s]. After results are saved, they are erased from the screen. To exit without saving the result to the database, use the mouse to direct the mouse arrow to the Close button and left click or type [Shift] [C/c]. 
Run the specimen a third time when any precision limit is exceeded.

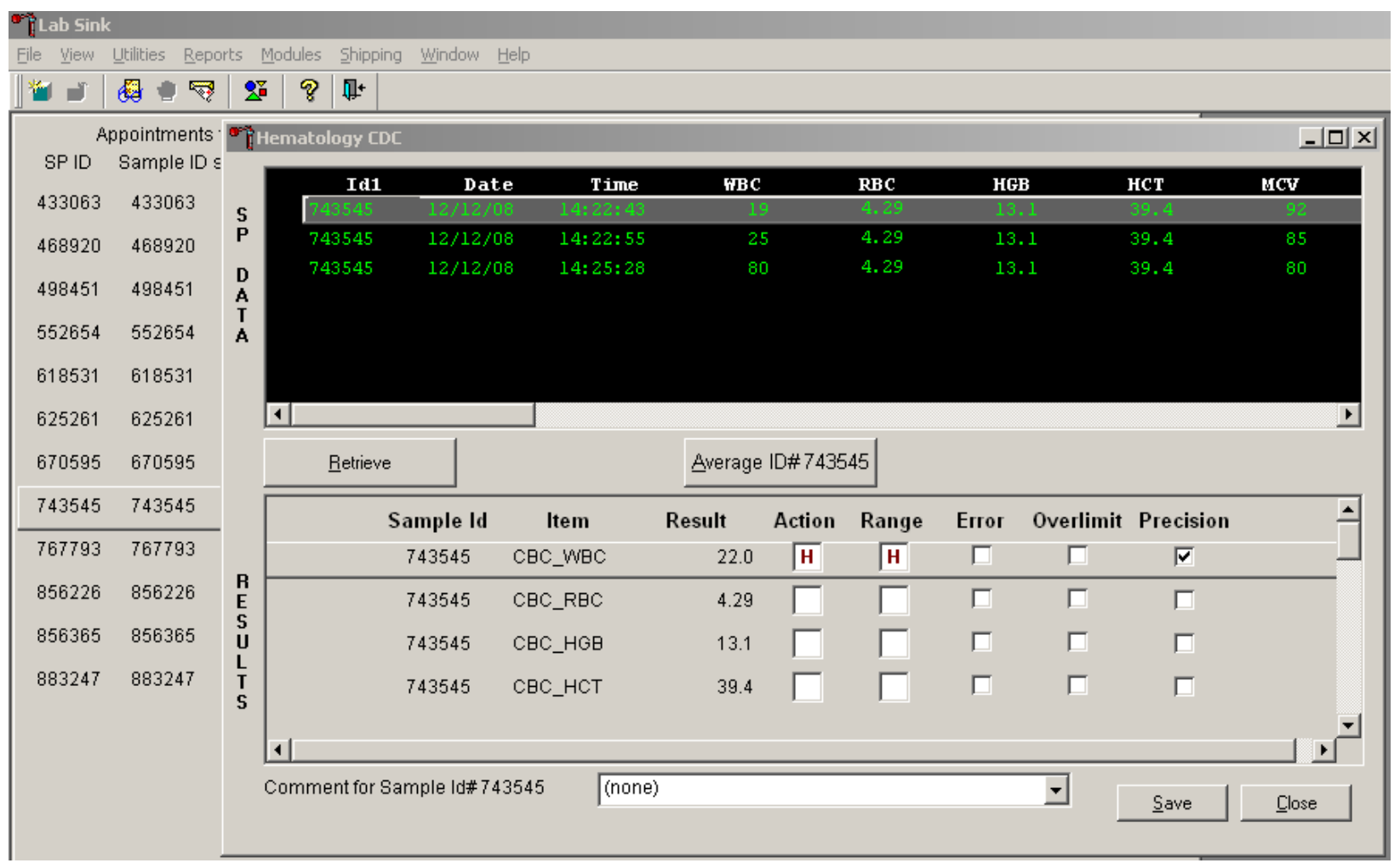

Run the blood sample a third time. Whenever a sample is run more than once, all results in the SP Data section are displayed. To re-average these results, use the mouse to direct the mouse arrow to “Average ID\#XXXXXX" and left click. The initial averaged results for parameters that do not exceed precision limits are fixed and are not recalculated. 
Override the current averaged results for parameters that exceeded the precision limit with a new average.

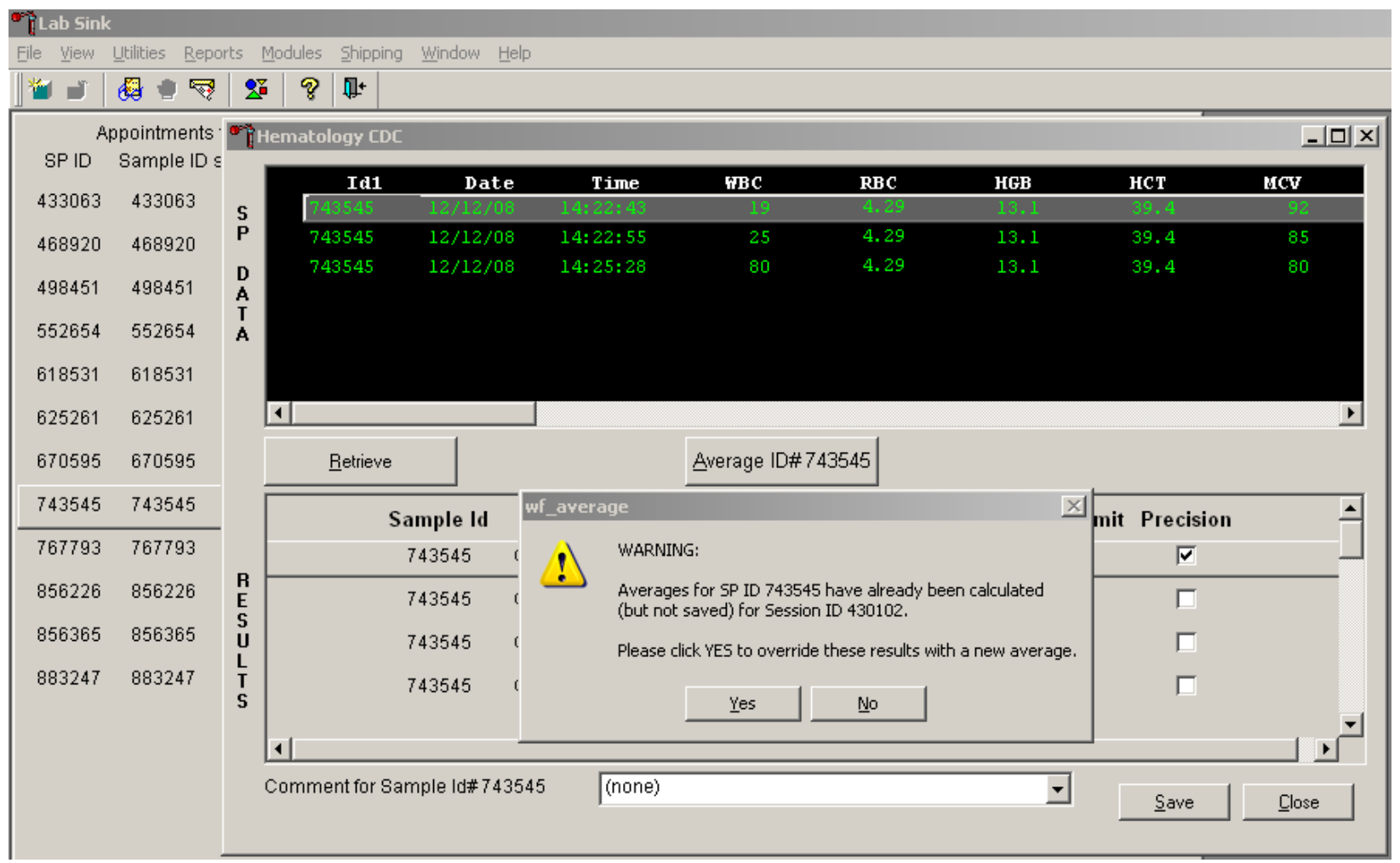

Whenever a sample has previously been averaged, and a new average is calculated, a warning message text box displays stating, "Averages for SP ID $X X X X X X$ have already been calculated (but not saved) for session ID $X X X X X X$." The text box instructs, "Please click YES to override these results with a new average.” To override the current average with a new average (but for only those parameters that exceeded precision limits), use the mouse to direct the mouse arrow to the Yes button and left click or type [Shift] [Y/y], or press [Enter]. To exit or return to the previous screen containing the previous averages, use the mouse to direct the mouse arrow to the No button and left click, or type [Shift] $[\mathrm{N} / \mathrm{n}]$. 
Evaluate the new results.

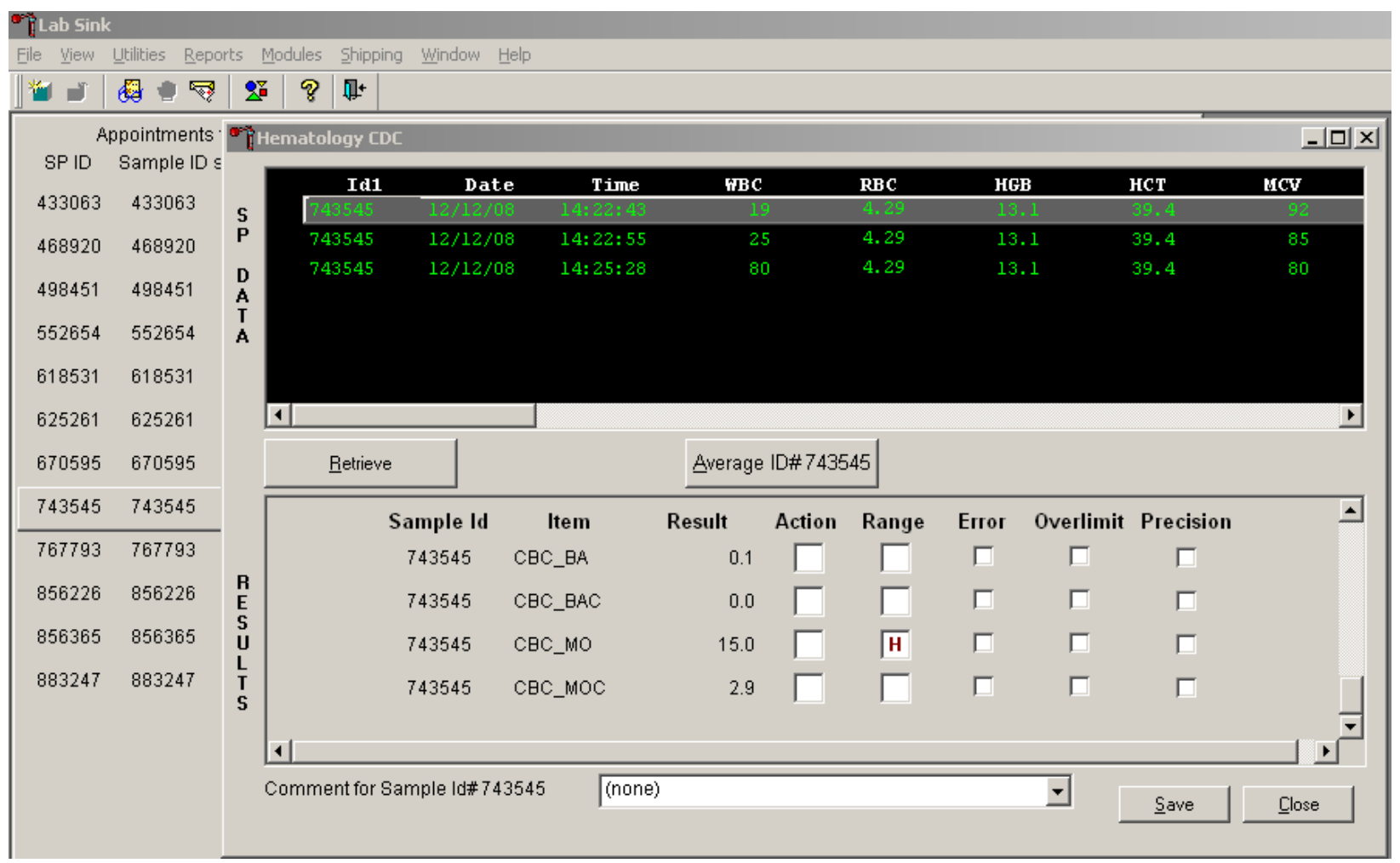

The first three runs are recalculated to find the closest two results for parameters where the difference between any two results exceeded its precision limits. If all parameters now meet precision limits (there are no checkmarks in the Precision column), save the results to the database. To save the result to the database, use the mouse to direct the mouse arrow to the Save button and left click or type [Shift] [S/s]. After results are saved, they are erased from the screen. To exit without saving the result to the database, use the mouse to direct the mouse arrow to the Close button and left click or type [Shift] $[\mathrm{C} / \mathrm{c}]$.

If necessary, run the specimen a fourth time and evaluate.

If precision limits are still not met for any parameter, run the blood tube a fourth time. Reaverage, evaluate, and save the results. If after four runs precision limits are still not met for any parameter, a comment is automatically attached to the results. Do not run a specimen more than four times. 
5. Attaching comments to the results.

Enter a comment for any run where results were repeated and confirmed, the equipment failed, or there was a laboratory error.

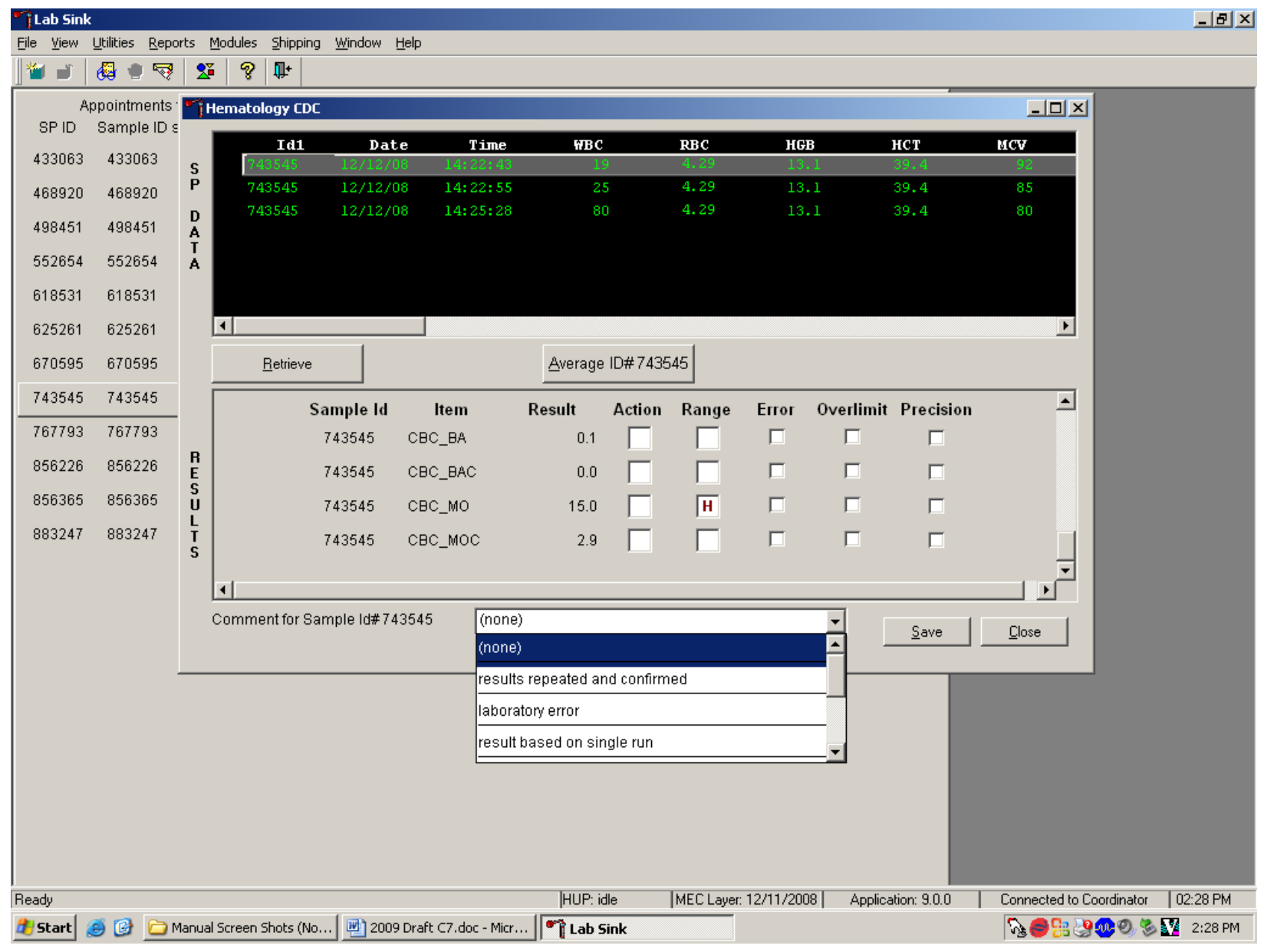

To attach a comment to the results in the database, use the mouse to direct the mouse arrow to the drop-down list on the Comment box, left click to display the codes, drag the mouse arrow to the most appropriate choice, and left click. Alternatively, to record a comment, use the mouse to direct the mouse arrow to the drop-down list on the Comment box, click to display the codes, use the up and down keyboard arrows to scroll through the choices or type the first letter of the desired comment code, and when the correct choice is highlighted, left click. 


\begin{tabular}{|c|c|}
\hline Comment & Use when: \\
\hline results repeated and confirmed & $\begin{array}{l}\text { Any parameter exceeded an action, range, or } \\
\text { precision limit and the specimen (blood tube) } \\
\text { was run more than once. }\end{array}$ \\
\hline laboratory error & There was a technologist error. \\
\hline result based on single run & $\begin{array}{l}\text { The quantity of specimen is insufficient to run it } \\
\text { more than once. }\end{array}$ \\
\hline equipment failure & The Coulter malfunctioned. \\
\hline no diff results/diff voteout & $\begin{array}{l}\text { The Coulter does not report parameter results for } \\
\text { any part of the differential or the instrument } \\
\text { reported a voteout for any of the differential } \\
\text { parameters. }\end{array}$ \\
\hline clotted & The blood is clotted. \\
\hline not enough blood & $\begin{array}{l}\text { There is insufficient blood to perform at least one } \\
\text { complete aspiration. }\end{array}$ \\
\hline
\end{tabular}


Save the result after attaching a comment.

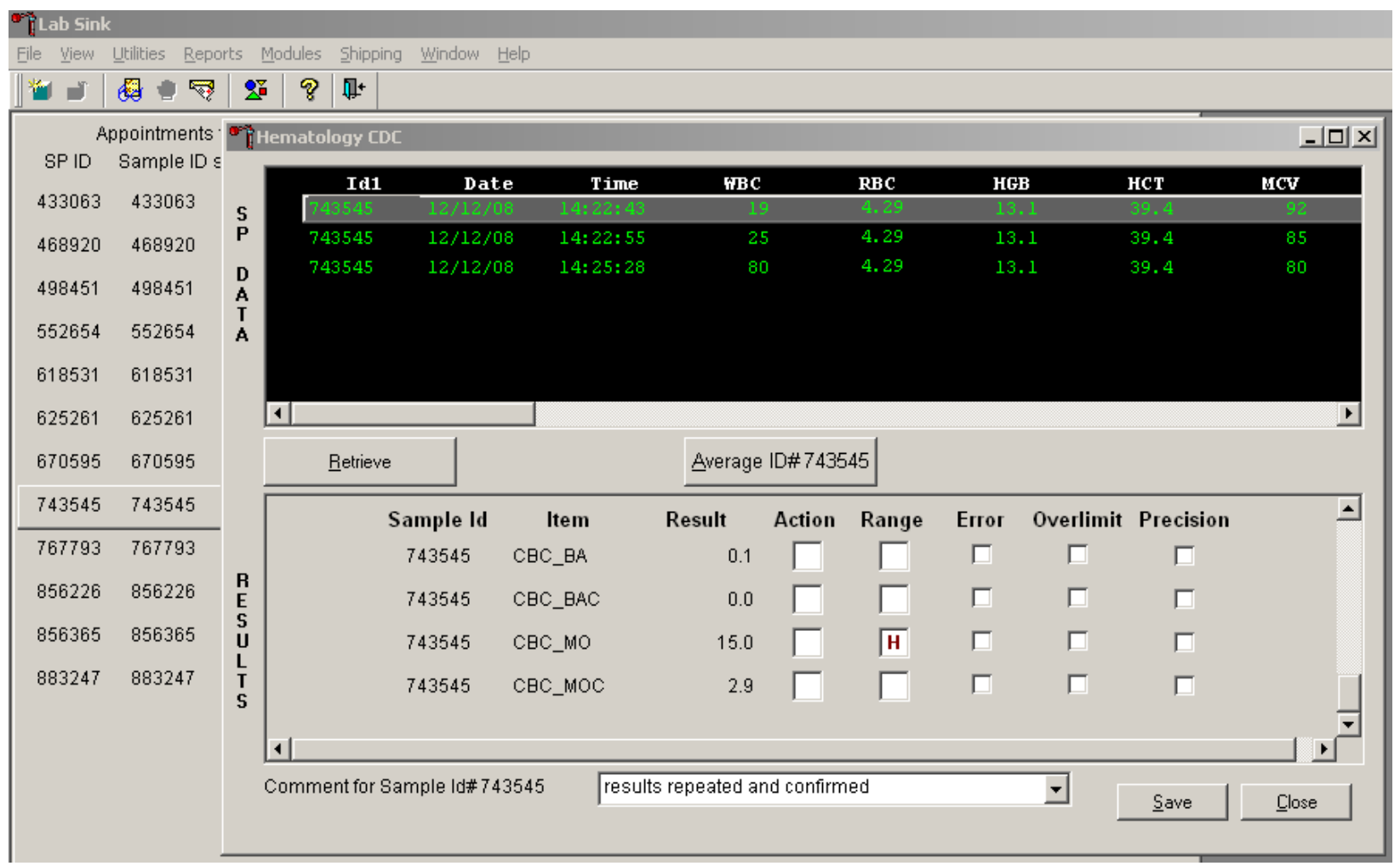

Save the averaged result and update the comment, by using the mouse to direct the mouse arrow to the Save button and left click or type [Shift] [S/s]. To exit without saving any data to the database, use the mouse to direct the mouse arrow to the Close button and left click or type [Shift] [C/c]. 
6. Using alternative data manipulation choices.

Retain data for multiple SPs in the SP Data section of the window.

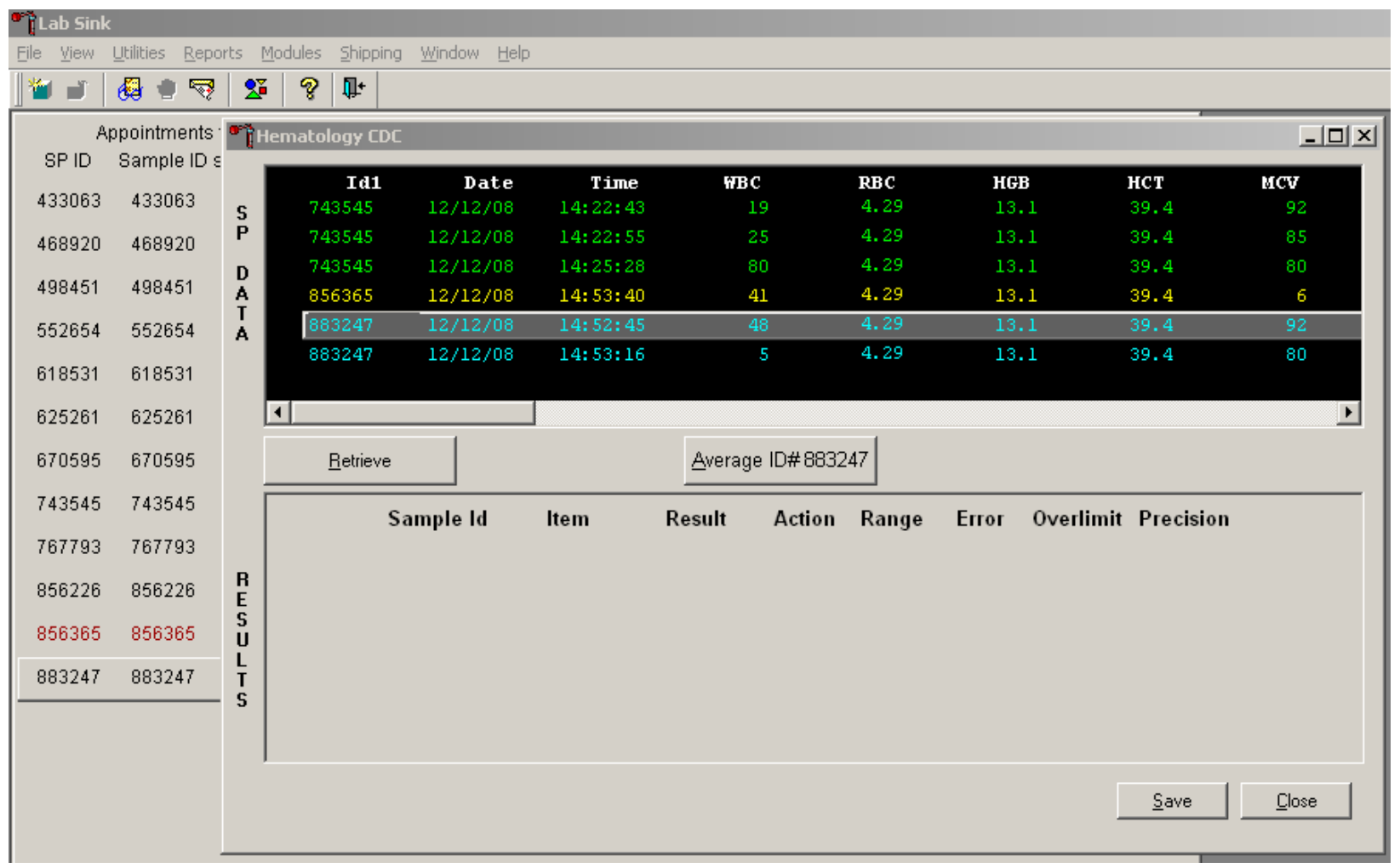

In general, run one SP in duplicate through the Coulter, retrieve the data, average the result, and save the data to the database. The Hematology module will display data for multiple SPs in the SP Data section. Each individual SP's results or pairs of results are displayed in a different color. To select or highlight any one SP, use the mouse to direct the mouse arrow to the row containing the correct SP ID and left click. 
Average the results for one SP at a time.

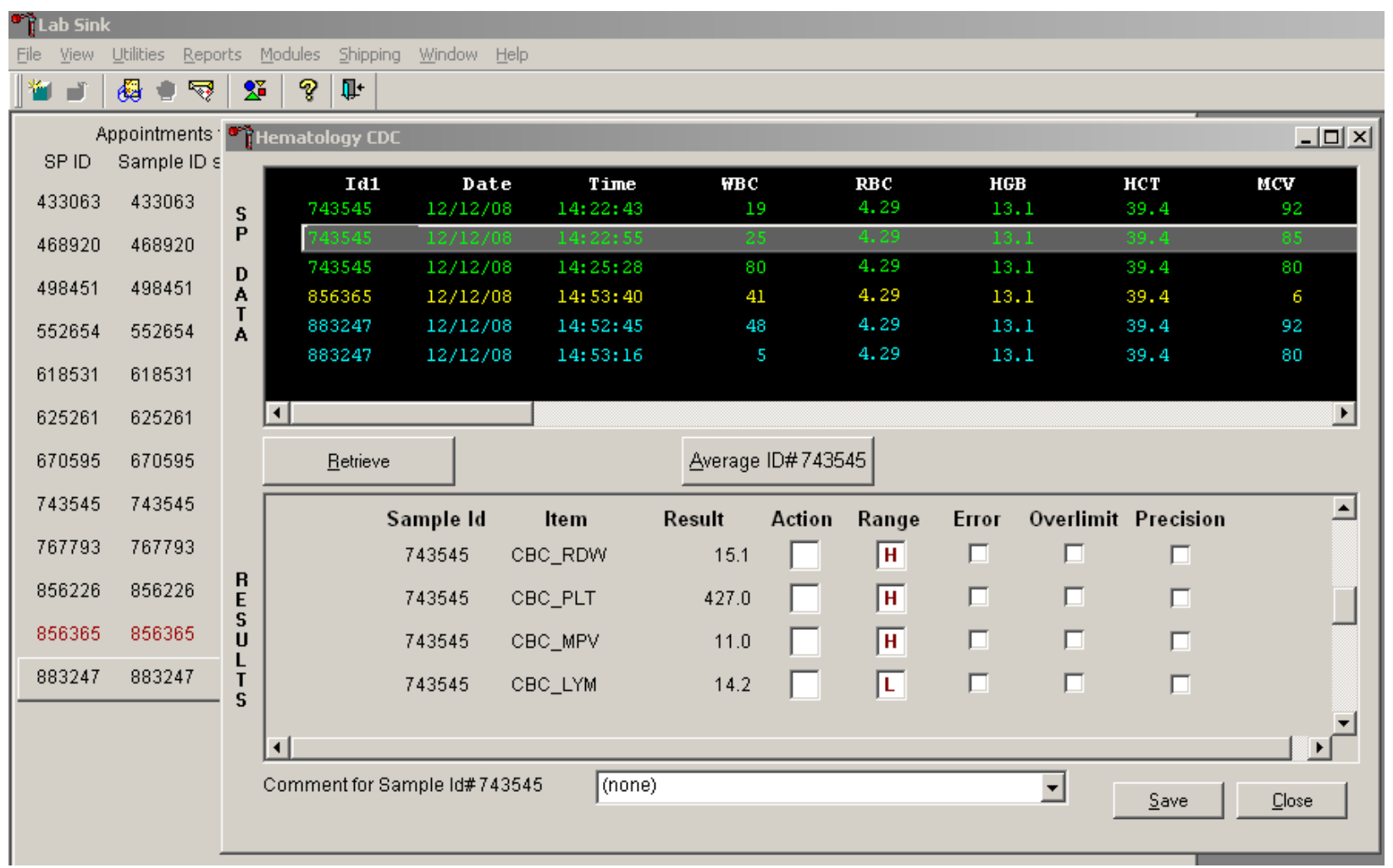

Evaluate each parameter for error, overlimit, and precision checkmarks, and " $H$ " or "L" action limit and reference range flags. If there are no checkmarks in the Precision column (no precision limit was exceeded), save the result to the database. To save the result to the database, use the mouse to direct the mouse arrow to the Save button and left click or type [Shift] [S/s]. To exit without saving the result to the database, use the mouse to direct the mouse arrow to the Close button and left click or type [Shift] [C/c]. If there are any Precision checkmarks, run a 5C cell control, evaluate the control results, rerun the blood tube a third time, re-average, evaluate, and save the results. 
Use alternative data manipulation choices as desired.

Use the "Clear All Downloads" function to delete all data for all SPs in the SP Data section.

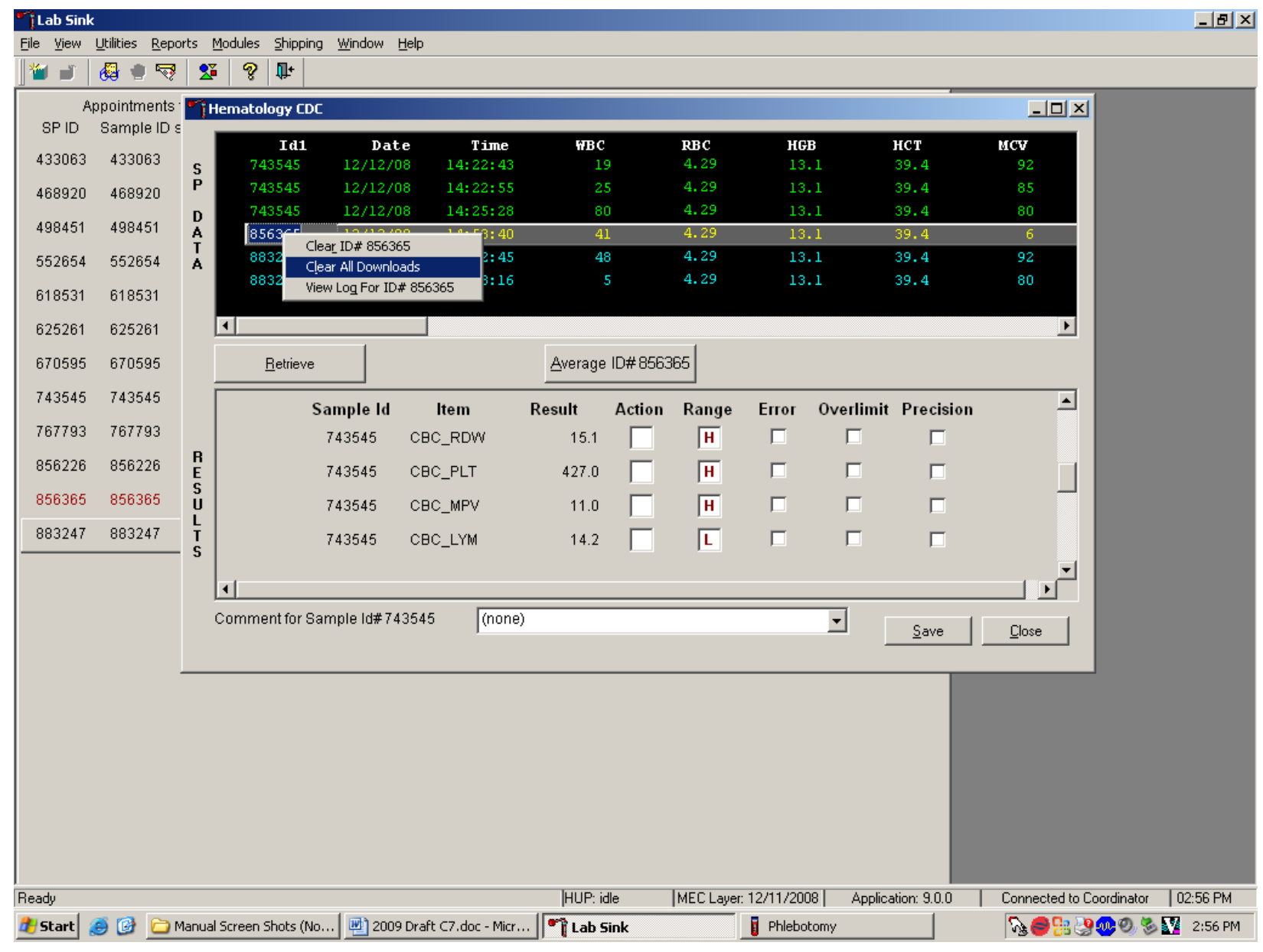

To clear all downloads from the SP Data section, use the mouse to direct the mouse arrow to any individual row in the SP Data section and right click to display the alternative data manipulation choices. To select “Clear All Downloads," drag the mouse arrow to \{Clear All Downloads\} and left click or type [Shift] [L/l]. 
Release the mouse button to clear all downloads.

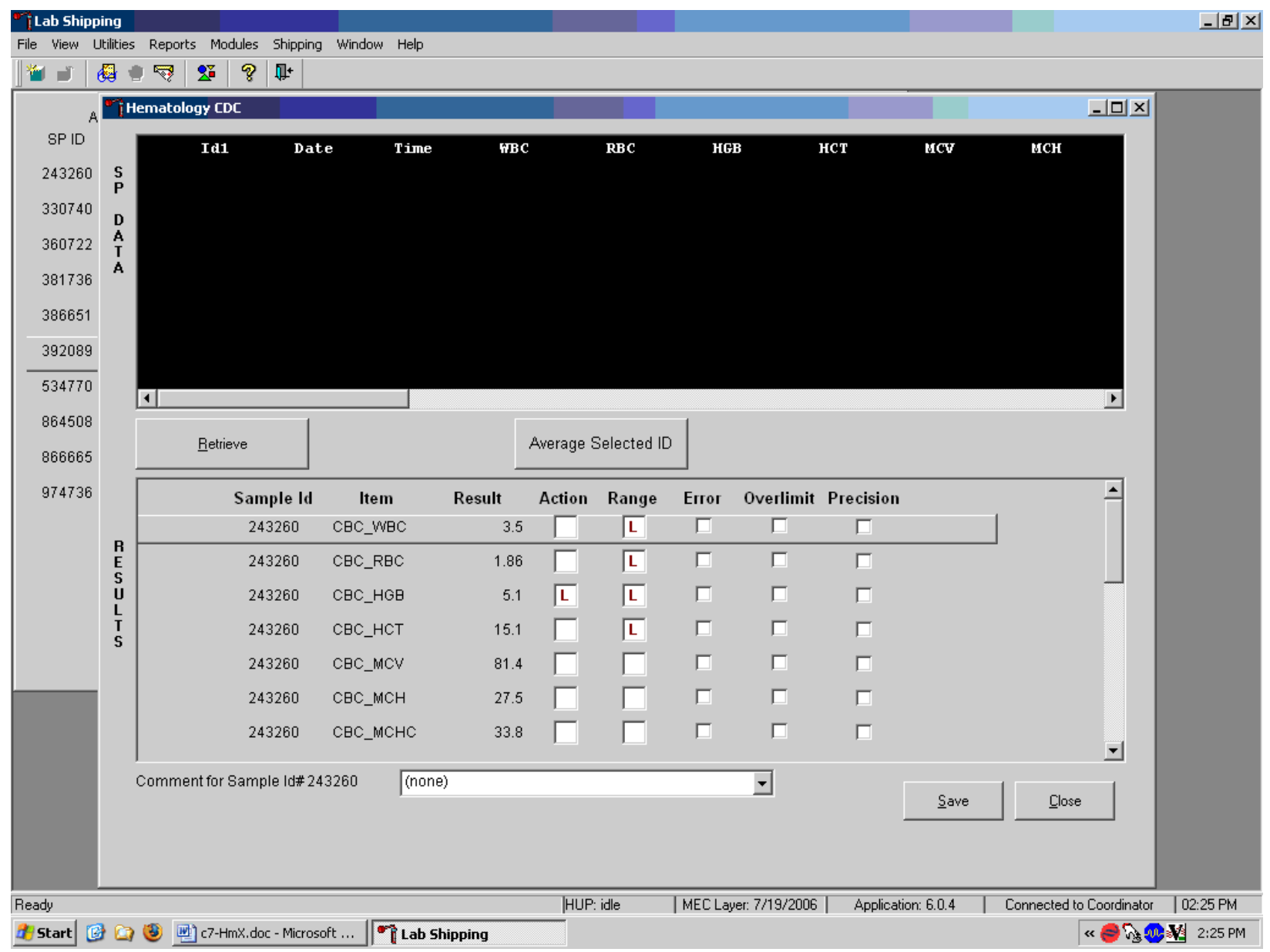

All data in the SP Data section is erased but any averaged result remains in the Results section of the window. To clear the result section, close the Hematology module. To close the hematology module, use the mouse to direct the mouse arrow to the Close button and left click or type [Shift] [C/c]. 
Use the data manipulation functions to erase or delete one SP's data in the SP Data section.

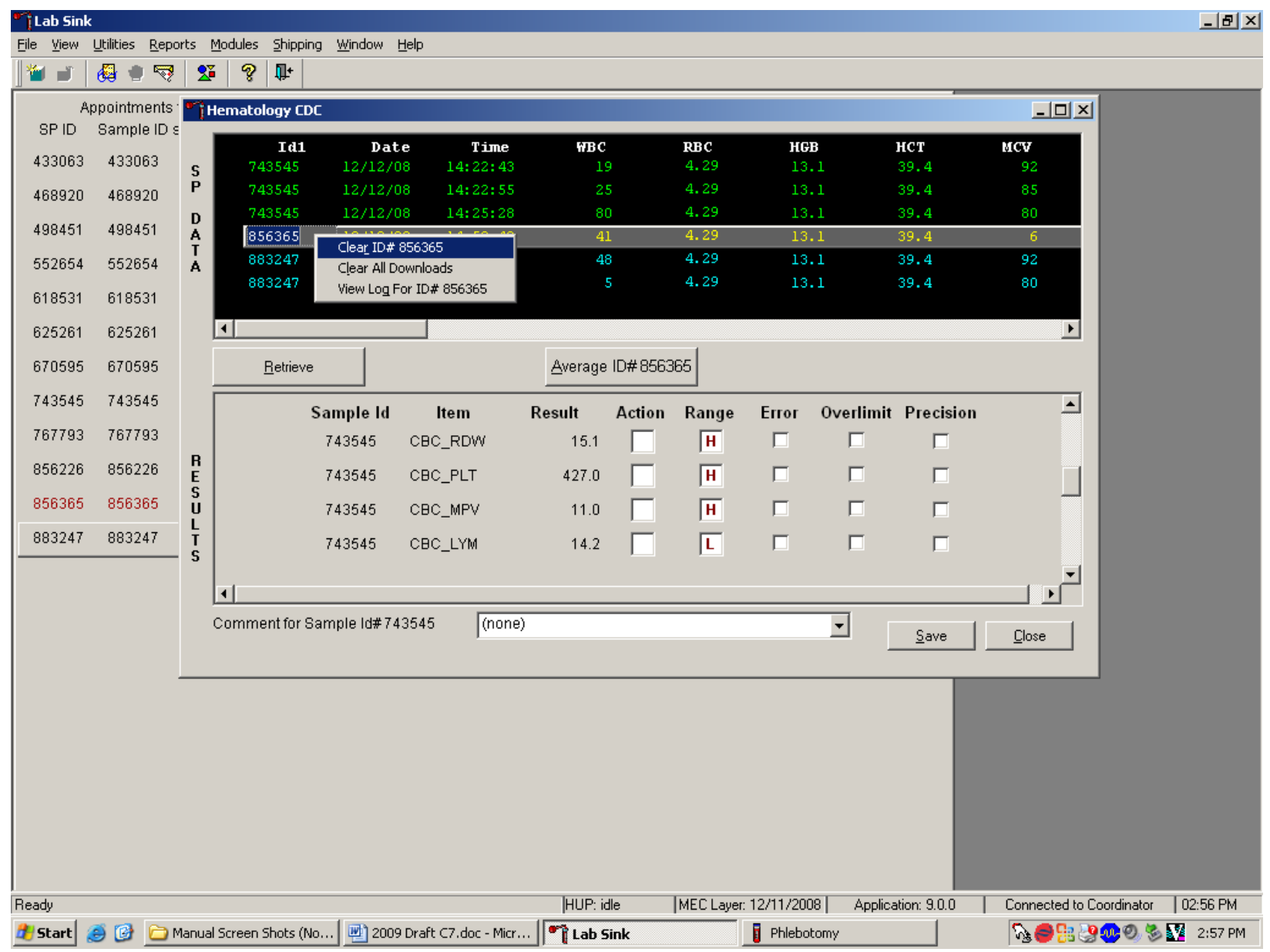

To clear the Coulter downloads for any one SP, use the mouse to direct the mouse arrow to the individual row in the SP Data section, right click to display the alternative data manipulation choices, drag the mouse arrow to \{Clear ID\#XXXXXX\}, and right or left click or type [Shift] [R/r]. Release the mouse button to clear this download. Retransmit, retrieve, average, and save the data. 
Use the "View Log for ID\# $X X X X X X$ " choice to view all Coulter data, averages, and precision limit values for any SP.

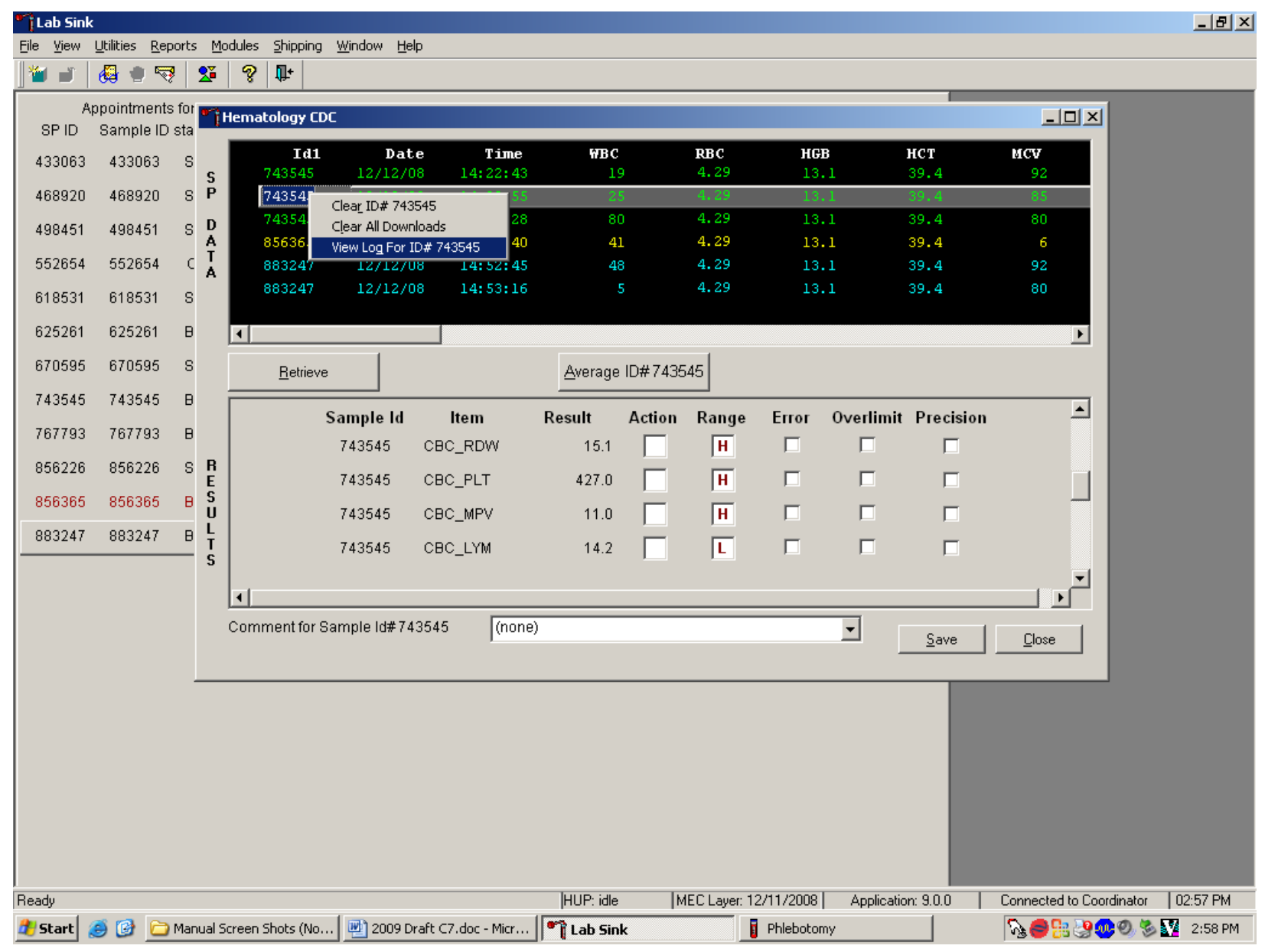

To view the detailed log for any one SP's result, use the mouse to direct the mouse arrow to the individual row in the SP Data section and right click to display the alternative data manipulation choices. Drag the mouse arrow to $\{$ View Log for ID $X X X X X X\}$ and release the mouse button to view the detailed log for this SP's results. 
Review the SP's log.

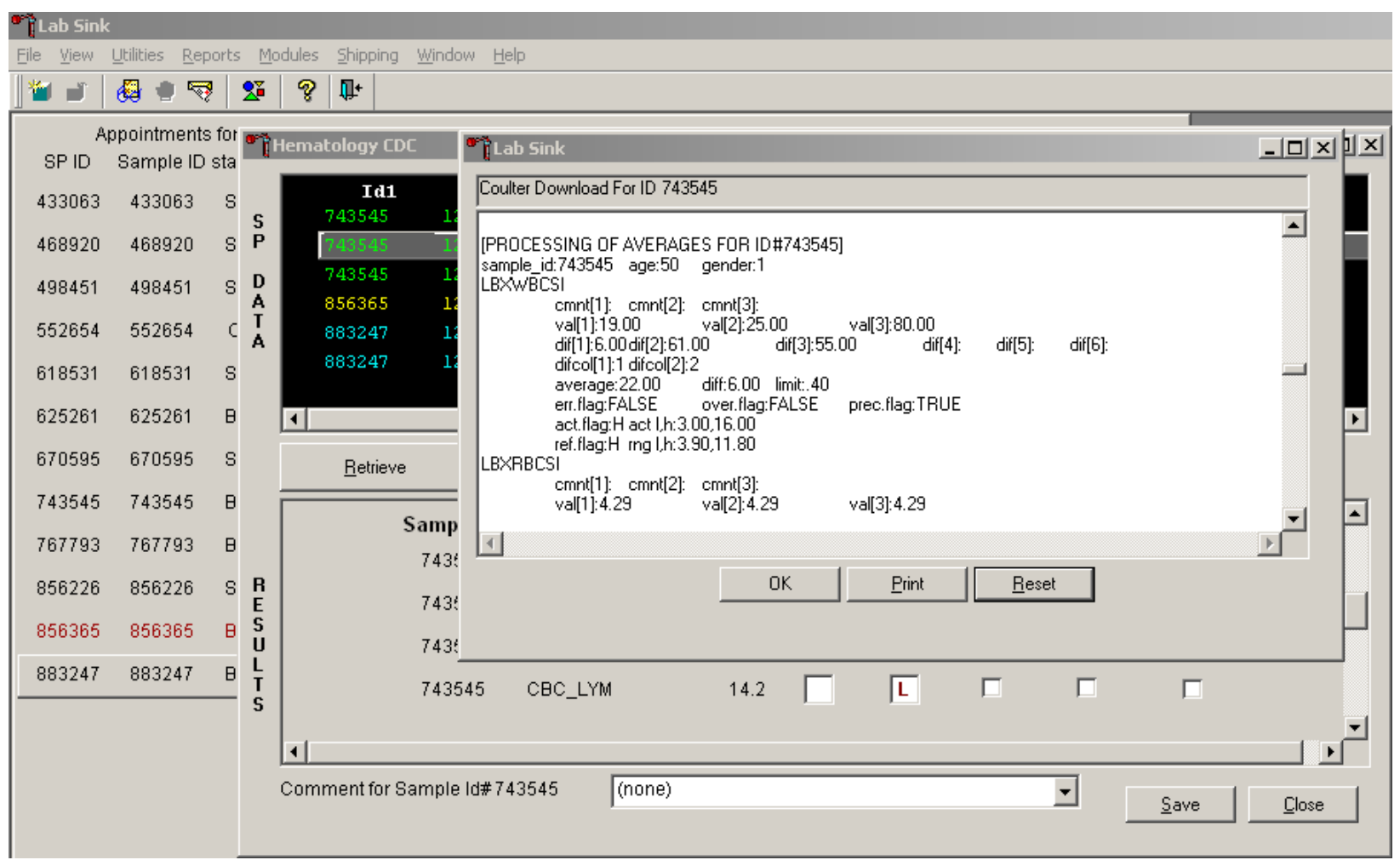

The SP's log contains detailed information for each parameter and each run. When finished reviewing the information, use the mouse to direct the mouse arrow to the OK button to close the window. To print the log, drag the mouse arrow to the Print button and left click or type [Shift] [P/p]. To clear the log and close the screen, use the mouse to direct the mouse arrow to the Reset button and left click, or type [Shift] [R/r], or select [Enter]. 
7. $\quad$ Re-averaging results.

Re-average a result that has or has not previously been saved to the database.

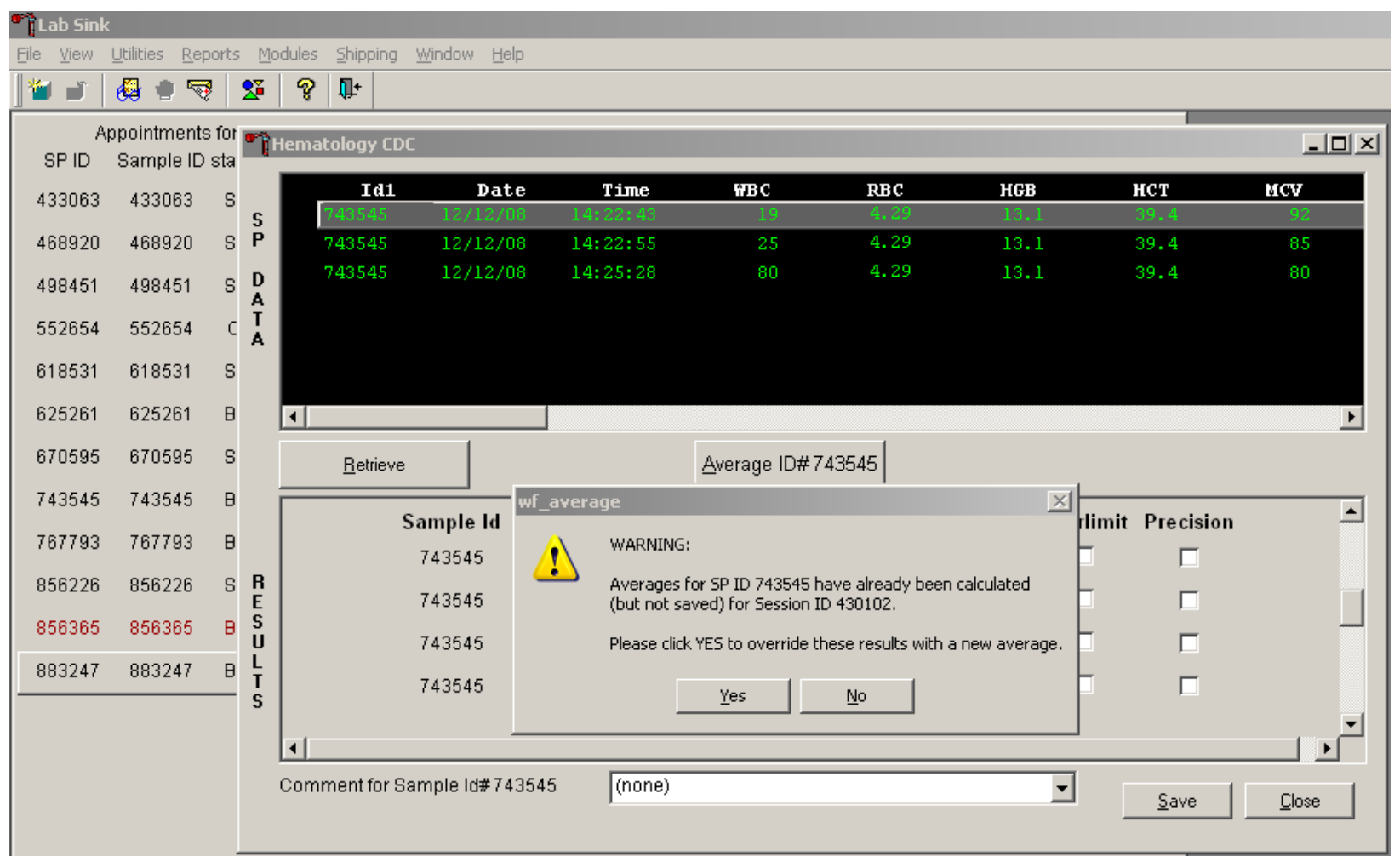

If a SP has not exited the MEC and the Report of Findings has not been printed, it is possible to retransmit and re-average the results for a SP and overwrite (save) the results in the database. A warning message text box displays when an attempt is made to save the data on a SP who has not exited the MEC or for whom a Report of Findings has not been printed. This text states, "Averages for SP ID $X X X X X X$ have already been calculated (but not saved) for Session ID $X X X X X$. Please click "Yes" to override these results with a new average." To override the previous results, use the mouse to direct the mouse arrow to the "Yes" button and left click or select [Enter] or type [Shift] [Y.] To remove the text box without overriding the previous results, use the mouse to direct the mouse arrow to the "No" button and left click, or type [Shift] [N].

If the SP has exited the MEC or the Report of Findings has been printed, it is impossible to overwrite (save) the results in the database. 


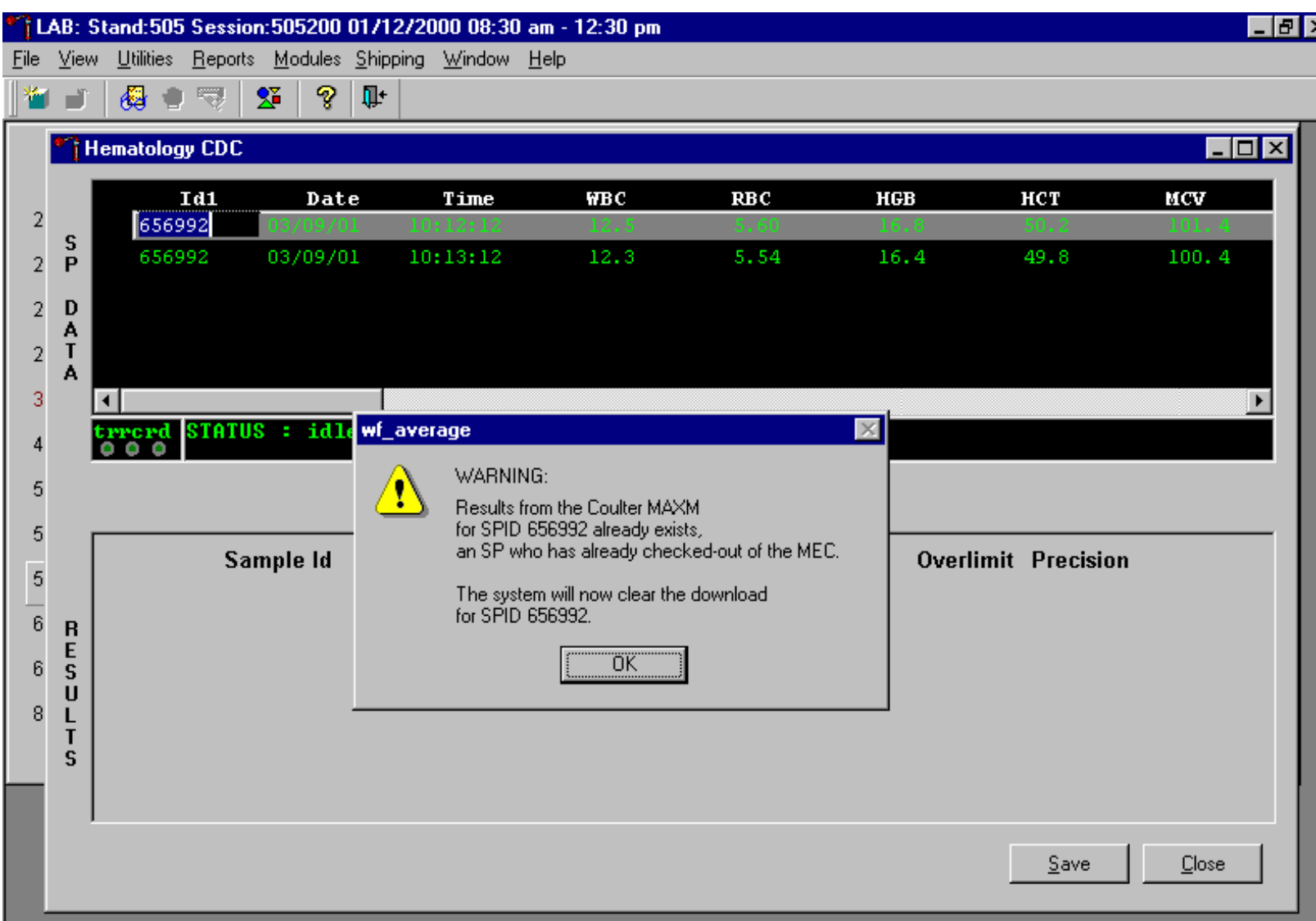

A warning message text box displays when an attempt is made to save the data on a SP who has exited the MEC or for whom a Report of Findings has been printed. This text states, "Results from the Coulter HMX for SPID $X X X X X X$ already exists, an SP who has already checked-out of the MEC. The system will now clear the download for SPID $X X X X X X$." To remove the text box, use the mouse to direct the mouse arrow to the OK button and left click or type [Enter]. The downloaded data, including the results, are automatically erased. 


\section{HMX Parameter Codes}

1. Review the message

The Coulter HMX uses triplicate counting with strict voting criteria. It has proprietary flagging algorithms to confirm parameter results before reporting. The instrument may not detect a transient or partial aperture blockage by any of these processes. A partial aperture blockage may cause erroneous results, such as WBC count lower than what is present. Monitor the aperture-viewing screen when cycling specimens that are likely to contain fibrin or debris.

The HMX displays abnormal parameter results for all cell populations and values. The following table describes the display and cause for the parameter codes.

\begin{tabular}{|c|c|}
\hline Display & Cause \\
\hline \multicolumn{2}{|c|}{ All Parameters } \\
\hline 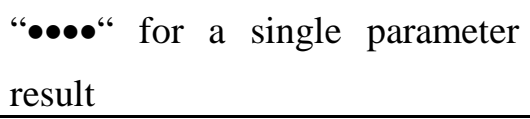 & Incomplete or abnormal computation. \\
\hline 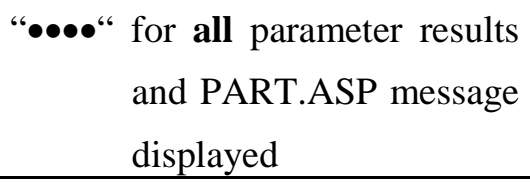 & $\begin{array}{l}\text { Sample integrity check failed, short sample } \\
\text { or clot. }\end{array}$ \\
\hline 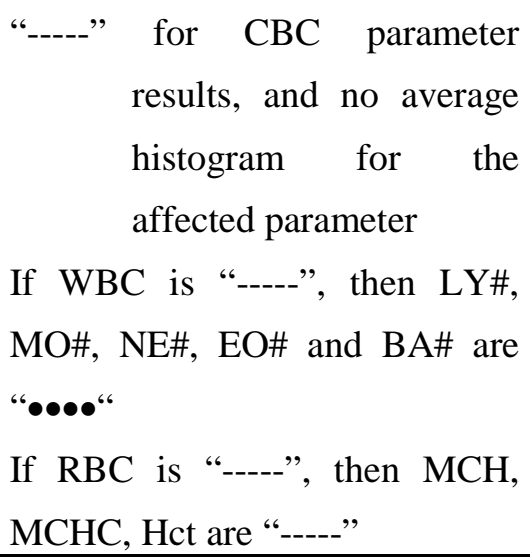 & $\begin{array}{l}\text { Total voteout (none of the three counts } \\
\text { agreed); Diff parameters do not vote out. }\end{array}$ \\
\hline “*V" & $\begin{array}{l}\text { Single-count period voteout; May indicate } \\
\text { an erroneous result due to aperture blockage } \\
\text { or lack of mixing bubble. }\end{array}$ \\
\hline
\end{tabular}




\begin{tabular}{|c|c|}
\hline "V" & $\begin{array}{l}\text { Appears next to parameter derived from the } \\
\text { parameter with the single count period } \\
\text { voteout. }\end{array}$ \\
\hline \multirow[t]{2}{*}{ “+++++” for parameter results } & Result exceeds: \\
\hline & WBC $\geq 99.9 \times 10^{3}$ cells $/ \mu \mathrm{L}$ \\
\hline IMPORTANT & $\mathrm{RBC} \geq 9.99 \times 10^{6}$ cellss $\mu \mathrm{L}$ \\
\hline \multirow{9}{*}{$\begin{array}{l}\text { If the WBC, RBC, Hgb, or Plt } \\
\text { result is }+++++ \text {, run a blank } \\
\text { cycle to prevent carryover to } \\
\text { the next sample. }\end{array}$} & Plt $\geq 999 \times 10^{3}$ cells $/ \mu \mathrm{L}$ \\
\hline & If $\mathrm{WBC}$ is over range \\
\hline & \\
\hline & $\begin{array}{l}\text { MCV, MCH, MCHC, RDW, MPV, Plt } \\
\text { and Diff\% results. Results for Diff\% are } \\
\text { dots (.......). }\end{array}$ \\
\hline & $\begin{array}{l}\text { If RBC is overrange } \\
\text { an } \mathrm{R} \text { appears next to Hct, } \mathrm{MCH} \text {, and } \\
\text { MCHC results. }\end{array}$ \\
\hline & If Plt is overrange \\
\hline & $\begin{array}{l}\text { an R appears next to WBC, Hgb, } \mathrm{MCH} \text {, } \\
\text { MCHC, diff\# and Pct results. }\end{array}$ \\
\hline & $\begin{array}{l}\text { Data Base Query displays average values } \\
\text { for over range WBC, RBC, or Plt. [F3] } \\
\text { Results or [F3] Edit, beneath the definitive } \\
\text { flag section. These values are for review } \\
\text { only; do not report them. }\end{array}$ \\
\hline & $\begin{array}{l}\qquad \text { IMPORTANT } \\
\text { Sample dilution may result in erroneous } \\
\text { differential results. } \\
\text { Set DIFF to OFF before cycling the diluted } \\
\text { sample when WBC is over range. }\end{array}$ \\
\hline “????” for Diff results & DMS has received questionable data \\
\hline \multicolumn{2}{|c|}{ Plt Parameter } \\
\hline “R” next to Plt and MPV result & $\begin{array}{l}\text { PDW }>20 \% \text { mode not between } 3 \text { and } 15 \mathrm{fL} \text {, } \\
\text { or non-positive curve detected, or } \\
\text { Plt }<20,000 \text {, or } \\
\text { Total voteout of fitted curve, or } \\
\text { WBC is over range. }\end{array}$ \\
\hline
\end{tabular}




\begin{tabular}{|c|c|}
\hline \multicolumn{2}{|c|}{ RBC Parameters } \\
\hline “R” next to RDW result & $\begin{array}{l}\text { Excessive asymmetry in RBC histogram, or } \\
\text { WBC or MCV overrange. }\end{array}$ \\
\hline $\begin{array}{l}\text { “* R" next to MCV; also “R” } \\
\text { next to RBC, Hct, MCH, RDW, } \\
\text { Plt, and MPV }\end{array}$ & MCV below lower limit of $<50 \mathrm{fL}$. \\
\hline \multicolumn{2}{|c|}{ WBC Parameters } \\
\hline $\begin{array}{l}\text { “* R" next to WBC; also "R" } \\
\text { next to Diff numbers }\end{array}$ & $\begin{array}{l}\text { Check of WBC lower threshold failed. } \\
\text { Particles are present at the } 35 \mathrm{fL} \text { threshold } \\
\text { (Plt clump, giant Plt, nrbc, lyse resistant } \\
\text { RBC, malarial parasites). }\end{array}$ \\
\hline $\begin{array}{l}\text { " } \mathrm{R} \text { ” next to Diff percentages and } \\
\text { numbers }\end{array}$ & $\begin{array}{l}\text { Low differential count statistics. These } \\
\text { messages occur also: } \\
\text { Population message: ABNORMAL } \\
\text { WBC POP } \\
\text { Suspect message: REVIEW SLIDE }\end{array}$ \\
\hline “:::::” for Diff results & $\begin{array}{l}\text { System detects a clog in the flow cell. There } \\
\text { are three types of clogs: } \\
\text { FC = Full Clog } \\
\text { PC1 = Partial Clog } 1 \\
\text { PC2 = Partial Clog } 2\end{array}$ \\
\hline
\end{tabular}

2. Remedial action

Review the result for the affected parameter. Rerun the specimen if any of the parameter flags occurs.

\section{HMX Suspect Messages}

1. Suspect messages flag an abnormal cell distribution or population. The system generates these messages according to an internal algorithm. Specific suspect messages indicate some abnormalities that exhibit characteristic cluster patterns. Suspect messages indicate the possibility of a particular abnormality. Not every atypical scatterplot has a corresponding suspect message. 
2. Remedial action - Rerun the specimen.

\section{E. Physician Review}

1. The MEC physician reviews and interprets all CBC results. Results outside action limits flash and transmit to the physician immediately. The physician determines if referral for the SP for treatment is necessary.

2. The medical technologist sends an observation to the physician whenever a critical or action limit is detected for any CBC parameter. This observation includes the date, time, responsible laboratory individual, person notified, and test results. 
Send an observation on any SP scheduled into the MEC session. Access the observation function.

\begin{tabular}{|c|c|c|c|c|c|c|c|c|c|c|c|c|}
\hline \multicolumn{13}{|l|}{ Lab Sink } \\
\hline \multirow{2}{*}{ 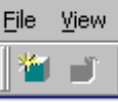 } & \multicolumn{12}{|c|}{ ULtilities Reports Modules Shipping Window Help } \\
\hline & \multicolumn{12}{|c|}{\begin{tabular}{ll|l} 
Quality Control & Ctrl+Q & \\
\end{tabular}} \\
\hline & \multicolumn{2}{|c|}{ Exam Pause } & \multirow[b]{2}{*}{ name } & \multicolumn{8}{|c|}{ Process Status } & \\
\hline SPID & Observations & & & UC & $u$ & $\mathrm{P}$ & $O Z$ & $\mathrm{CB}$ & $\theta g$ & gtt PC & $\mathrm{CH}$ HV & $\checkmark \mathrm{OR}$ \\
\hline 215591 & Send Message & $\mathrm{Ctrl}+\mathrm{M}$ & L, PAVARTI & - & 0 & e & $\cdot$ & 0 & 0 & $\cdot$ & . & • \\
\hline 244969 & $\begin{array}{l}\text { Uro slgnting } \\
\text { Set Session }\end{array}$ & & PAN, LEE & 0 & 0 & • & 0 & 0 & 0 & .0 & & 0 \\
\hline 470234 & $\checkmark$ English & $C t r l+E$ & IID, RUEEUS & 0 & 0 & · & 0 & 0 & 0 & $\cdot 0$ & & 0 \\
\hline 668903 & Spanishi & Ctril+5 & PLEY, FRED & 0 & 0 & . & 0 & 0 & 0 & $\cdot 0$ & . & . \\
\hline 762731 & $\begin{array}{l}\text { Toolbars... } \\
\text { System Configuration }\end{array}$ & & WN, LAVENDAR & - & 0 & $\bullet$ & . & 0 & 0 & . & . & . \\
\hline 836402 & Pregnancy QC & & OY, DRACO & 0 & 0 & . & - & 0 & 0 & $\cdot$ & . & - \\
\hline 900081 & $\begin{array}{l}\text { Coulter QC Lot Info } \\
\text { Regen SP Data }\end{array}$ & & TT, HANNAH & 0 & 0 & 0 & $\cdot$ & 0 & 0 & $\cdot$ & & . \\
\hline 998878 & $\checkmark \underline{1}$ Heads-Up for Session: 41011 & & PLEY, VERNON & - & · & . & . & 0 & 0 & · & & . \\
\hline
\end{tabular}

To access the observation function, use the mouse to direct the mouse arrow to \{Utilities $\}$ in the menu bar, left click, drag the mouse arrow to \{Observations\}, and left click, or type [Alt] [U/u], [O/o]. 
Select or highlight the correct SP.

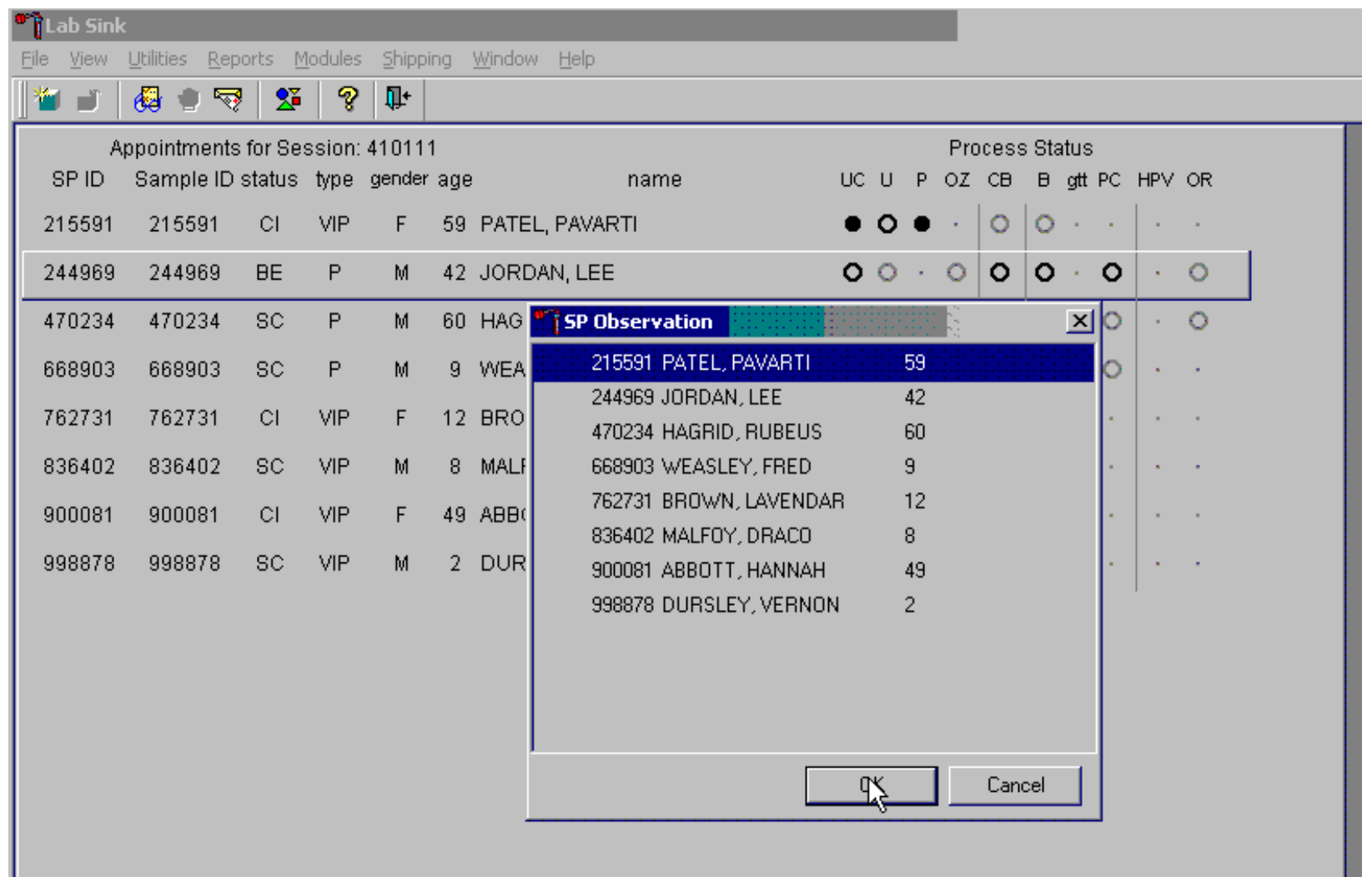

To select or highlight an SP, drag the mouse arrow to the correct SP and left click or use the up and down arrows to move up and down the list. Verify that the SP ID, name, and age are correct. Use the scroll bar to view the complete list of SPs. To continue, use the mouse to direct the mouse arrow to the OK button and left click, or select [Enter]. To cancel these actions and exit the observation function, use the mouse to direct the mouse arrow to the Cancel button and left click. 
The observation window displays.

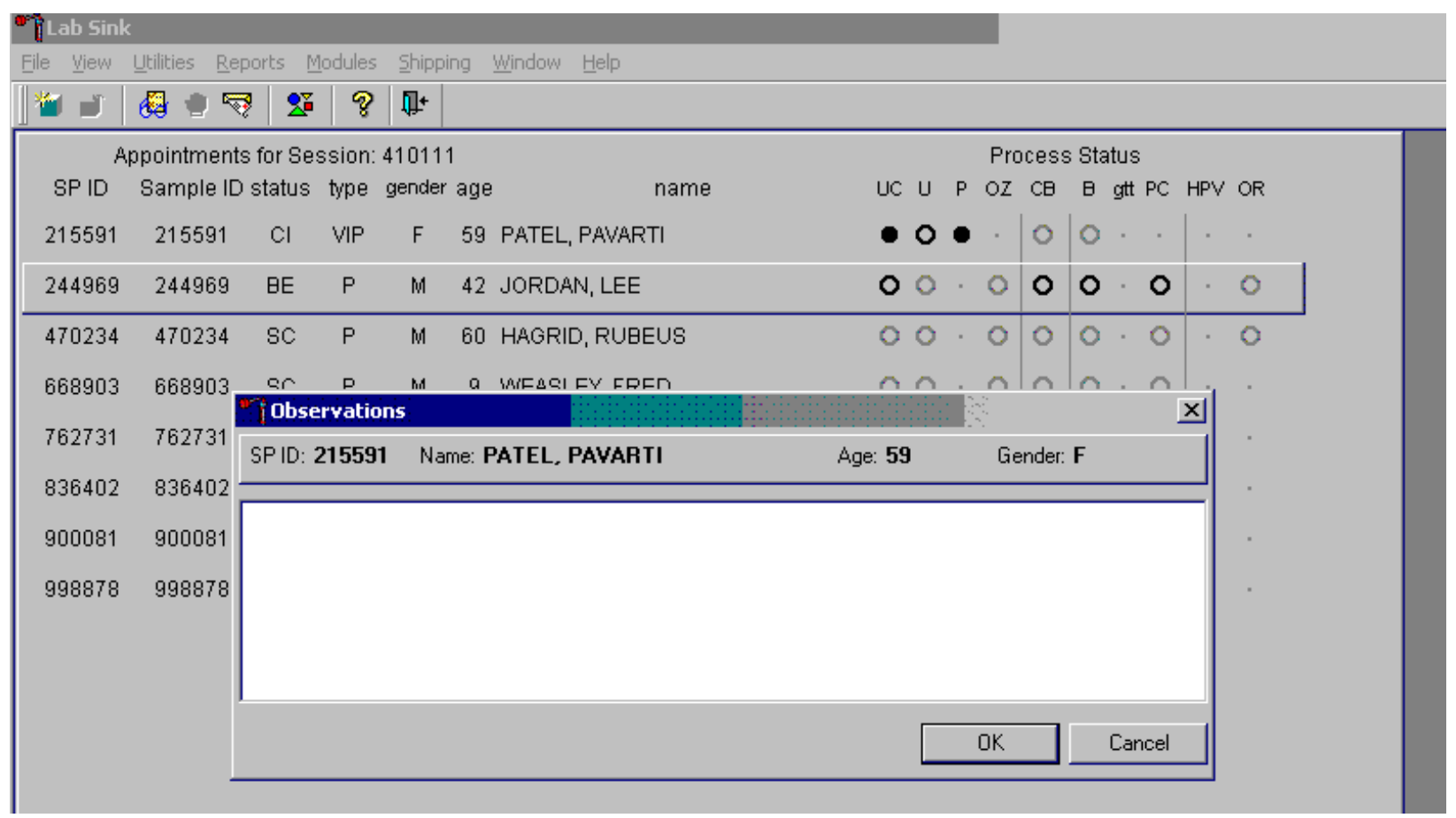

The observation window contains the SP ID, name, age, and gender. Type the observation using the keyboard. To send the observation to the physician, use the mouse to direct the mouse arrow to the OK button and left click, or select [Enter]. To cancel these actions or to exit the observation window without entering an observation, use the mouse to direct the mouse arrow to the Cancel button and left click. 
Enter the observation on a SP.

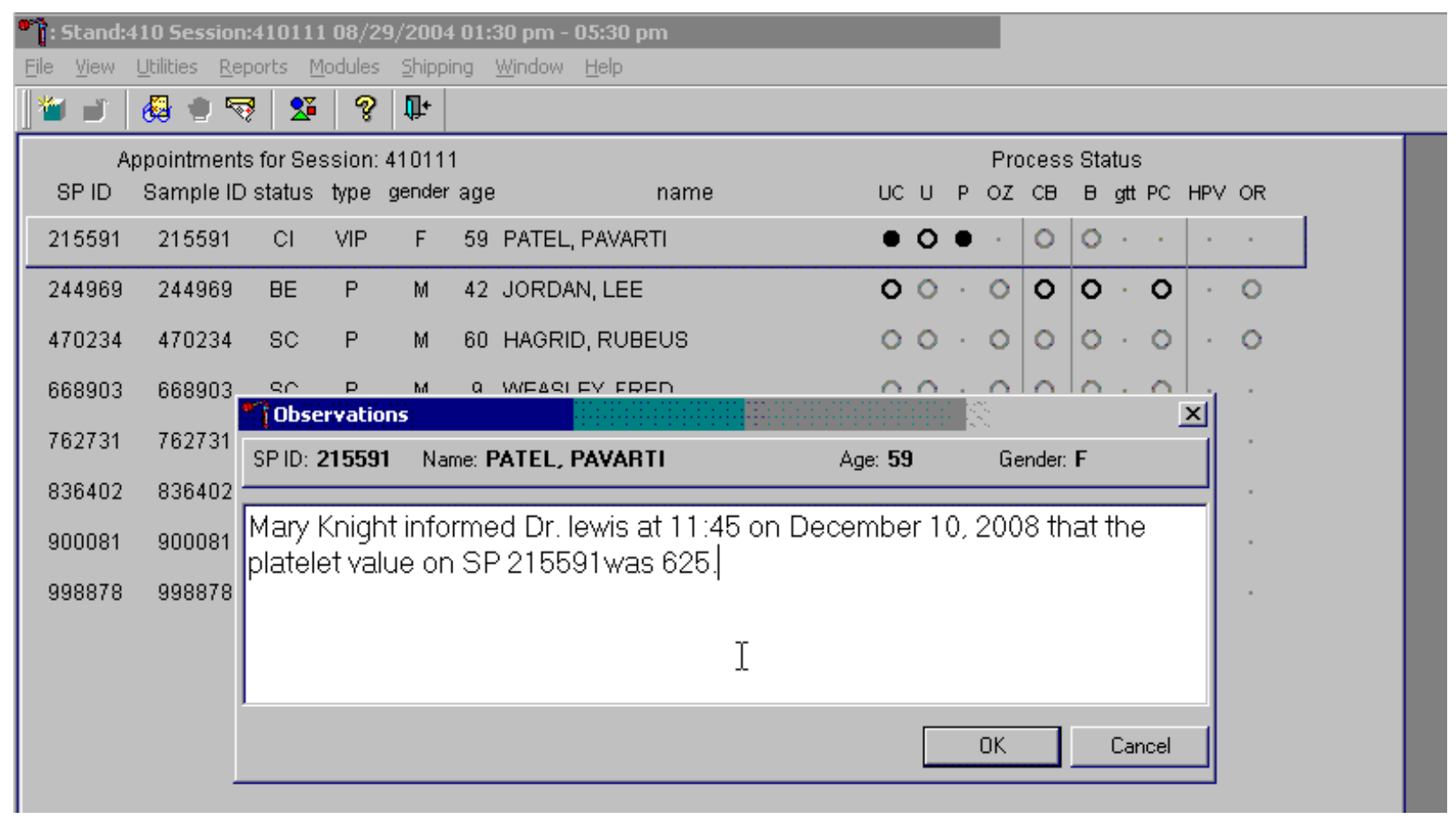

Document the date, time, responsible laboratory individual, person notified, and test results, including the parameter.

XII. Limitations of Method: Specimen Rejection, Interfering Substances, and Conditions

This method limits samples to human whole blood.

A. Specimen Rejection

1. Reject clotted specimens and recollect. 


\section{B. Interfering Substances and Conditions}

Because the Coulter directly measures RBC, WBC, Hgb, and Diff \%, it is most important to concentrate on analytes and substances that interfere with these parameters. The Coulter calculates HCT, MCH, MCHC, and DIFF \# parameters. The Coulter derives MCV, RDW, PLT, and MPV from RBC or platelet histograms. The following are possible interfering substances or conditions:

- Abnormal BUN, glucose, or sodium levels could affect the MCV.

- Abnormal WBCs could affect lymphocytes, monocytes, and granulocytes.

- Abnormally small WBCs could affect white count, lymphocytes, monocytes, and granulocytes.

- Clumped platelets could affect white count, lymphocytes, monocytes, granulocytes, RBC, MCV, RDW, platelet count, and MPV.

- Cryofibrinogen and cryoglobulin crystals could affect white count, lymphocytes, monocytes, granulocytes, RBC, hemoglobin, platelet count, and MPV.

- An elevated WBC count could affect RBC, hemoglobin, MCV, RDW, platelet count, and MPV parameters.

- Fragile WBCs could affect white count, lymphocytes, monocytes, granulocytes, platelet count, and MPV.

- Giant platelets could affect white count, lymphocytes, monocytes, granulocytes, RBC, MCV, RDW, platelet count, and MPV.

- Hemolyzed specimens could affect RBC, hemoglobin, platelet count, and MPV.

- Lipemic specimens could affect MCV.

- Severely icteric plasma causes increased hemoglobin. Evaluate CBC result carefully and report all parameters except the hemoglobin result.

- Nucleated RBCs could affect the white count, lymphocytes, monocytes, granulocytes, and hemoglobin values.

- WBC - Certain unusual RBC abnormalities that resist lysing, nucleated RBCs, fragmented WBCs, agglutinated WBCs, any unlysed particles greater than $35 \mathrm{fL}$, very large or aggregated platelets as when anticoagulated with oxalate or heparin, specimens containing fibrin, cell fragments, or other debris such as pediatric and oncology specimens. 
- RBC - Very high WBC count, high concentration of very large platelets, agglutinated RBCs, RBCs smaller than $36 \mathrm{fL}$, specimens containing fibrin, cell fragments, or other debris such as pediatric and oncology specimens.

- Hgb - Very high WBC count, severe lipemia, heparin, certain unusual RBC abnormalities that resist lysing, or anything that increases the turbidity of the sample such as elevated levels of triglycerides.

- MCV - Very high WBC count, high concentration of very large platelets, agglutinated RBCs, RBC fragments that fall below the 36-fL threshold, or rigid RBCs.

- RDW - Very high WBC count, high concentration of very large or clumped platelets as in blood anticoagulated with oxalate or heparin, RBCs below the 36fL threshold, two distinct populations of RBCs, RBC agglutinates, or rigid RBCs.

- Plt - Very small red blood cells near the upper threshold, cell fragments, clumped platelets as with oxalate or heparin, platelet fragments, or cellular debris near the lower platelet threshold.

- MPV - Known factors that interfere with the Plt count and shape of the histogram or known effects of EDTA.

- Hct - Known factors that interfere with the parameters used for computation: RBC and MCV.

- $\mathrm{MCH}$ - Known factors that interfere with the parameters used for computation: Hgb and RBC.

- MCHC - Known factors that interfere with the parameters used for computation: Hgb, RBC, and MCV.

- Diff Parameters - Known factors that affect the WBC count as listed above or high triglycerides that affect lysing. 
XIII. Reference Ranges

\section{Males}

\begin{tabular}{|l|cc|cc|cc|cc|}
\hline \multirow{2}{*}{ Age in years } & \multicolumn{2}{|c|}{$1-5$} & \multicolumn{2}{c|}{$6-18$} & \multicolumn{2}{c|}{$19-65$} & \multicolumn{2}{c|}{$66+$} \\
& 2.5 & 97.5 & 2.5 & 97.5 & 2.5 & 97.5 & 2.5 & 97.5 \\
\hline White blood cell count (SI) & 4.3 & 14.6 & 3.6 & 11.5 & 3.9 & 11.8 & 3.8 & 12.1 \\
Red cell count (SI) & 3.98 & 5.3 & 4.14 & 5.78 & 4.18 & 5.86 & 3.57 & 5.67 \\
Hemoglobin (g/dL) & 10.7 & 14.2 & 11.9 & 16.9 & 13.1 & 17.5 & 11.4 & 17.1 \\
Hematocrit (\%) & 32.1 & 41.7 & 35.3 & 49.9 & 38.7 & 51.4 & 33.9 & 50.9 \\
Mean cell volume (fL) & 68.2 & 88.8 & 75.6 & 94.6 & 79.8 & 99.1 & 81.4 & 102.7 \\
Mean cell hemoglobin (pg) & 22.3 & 30.6 & 25.0 & 32.3 & 26.3 & 34.0 & 26.3 & 35.0 \\
MCHC (g/dL) & 32.3 & 35.6 & 32.3 & 35.3 & 32.3 & 35.3 & 32.1 & 35.1 \\
Red cell distribution width & & & & & & & & \\
(\%) & 11.4 & 15.8 & 11.4 & 14.0 & 11.4 & 14.5 & 11.8 & 16.2 \\
Platelet count (\%) SI & 212 & 546 & 179 & 439 & 152 & 386 & 124 & 384 \\
Mean platelet volume (fL) & 6.1 & 8.9 & 6.6 & 10 & 6.8 & 10.1 & 6.6 & 10.2 \\
Lymphocyte percent (\%) & 22.8 & 68.4 & 17.5 & 54.3 & 16.1 & 47.9 & 12.3 & 46.4 \\
Monocyte percent (\%) & 4.6 & 15.2 & 4.8 & 13.7 & 4.4 & 13.5 & 4.6 & 14.0 \\
Segmented neutrophils percent & & & & & & & & \\
(\%) & 17.6 & 67.1 & 30.3 & 72.8 & 37.8 & 74.6 & 39.5 & 78.1 \\
Eosinophils percent (\%) & 0.7 & 11.3 & 0.7 & 11.5 & 0.7 & 8.5 & 0.6 & 8.8 \\
Basophils percent (\%) & 0.1 & 2.5 & 0.1 & 1.6 & 0.1 & 1.6 & 0.1 & 1.6 \\
\hline
\end{tabular}




\section{Females}

\begin{tabular}{|l|cc|cc|cc|cc|}
\hline \multirow{2}{*}{ Age in years } & \multicolumn{2}{|c|}{$1-5$} & \multicolumn{2}{c|}{$6-18$} & \multicolumn{2}{c|}{$19-65$} & \multicolumn{2}{c|}{$66+$} \\
& 2.5 & 97.5 & 2.5 & 97.5 & 2.5 & 97.5 & 2.5 & 97.5 \\
\hline White blood cell count (SI) & 4.3 & 14.0 & 3.9 & 12.2 & 4.1 & 12.9 & 4.0 & 11.6 \\
Red cell count (SI) & 3.96 & 5.28 & 3.84 & 5.24 & 3.64 & 5.2 & 3.51 & 5.34 \\
Hemoglobin (g/dL) & 11.0 & 14.2 & 11.2 & 15.1 & 10.6 & 15.6 & 10.9 & 15.9 \\
Hematocrit (\%) & 32.5 & 41.9 & 33.5 & 44.6 & 32.0 & 45.9 & 32.8 & 47.0 \\
Mean cell volume (fL) & 70.2 & 89.1 & 74.7 & 94.9 & 74.6 & 98.2 & 80.3 & 100.6 \\
Mean cell hemoglobin (pg) & 23.3 & 30.8 & 24.5 & 32.6 & 24.3 & 33.8 & 26.4 & 34.5 \\
MCHC (g/dL) & 32.4 & 35.5 & 32.3 & 35.3 & 32.1 & 35.3 & 32.3 & 35.1 \\
Red cell distribution width & & & & & & & & \\
(\%) & 11.3 & 15.4 & 11.3 & 14.8 & 11.4 & 16.3 & 11.6 & 16.3 \\
Platelet count (\%) SI & 215 & 547 & 190 & 446 & 168 & 441 & 155 & 428 \\
Mean platelet volume (fL) & 6.1 & 8.9 & 6.6 & 10.0 & 6.8 & 10.2 & 6.7 & 10.5 \\
Lymphocyte percent (\%) & 21.6 & 68.8 & 17.2 & 54.7 & 14.1 & 47.6 & 13.7 & 46.9 \\
Monocyte percent (\%) & 4.2 & 14.4 & 4.3 & 12.7 & 3.8 & 11.6 & 4.4 & 12.8 \\
Segmented neutrophils percent & & & & & & & & \\
(\%) & 19.4 & 69.5 & 31.9 & 74.3 & 39.8 & 78.1 & 40.9 & 78.1 \\
Eosinophils percent (\%) & 0.6 & 9.9 & 0.6 & 9.9 & 0.6 & 7.3 & 0.6 & 7.5 \\
Basophils percent (\%) & 0.1 & 2.5 & 0.1 & 1.6 & 0.1 & 1.7 & 0.1 & 1.7 \\
\hline
\end{tabular}

3. Reference ranges for normal values were calculated from the NHANES data set (1999-2004) using 95\% reference interval(s) determined nonparametrically, through ranking the observations and determining the lower (2.5th percentile) and the upper (97.5th percentile) reference limits. The nonparametric (ranking) method was used because most measured hematology parameters have a skewed, non-Gaussian distribution.

XIV. Action Limits

Action limits are a guide to inform the physician that a CBC result(s) is/are abnormal. Since all specimens are run in duplicate, there is no reason to retest the sample.

- WBC male and female (all ages) $<$ or $=$ to $3 \times 10^{3} \mu \mathrm{L}$ or $>$ or $=$ to $16.0 \times 10^{3} \mu \mathrm{L}$

- Hgb male and female ( $<6$ years) $<6.5 \mathrm{~g} / \mathrm{dL}$ or $>14.5 \mathrm{~g} / \mathrm{dL}$ 
- $\quad$ Hgb female (>6 years)

- $\quad$ Hgb male ( $>6$ years)

- $\quad$ PLT male and female (all ages)
$<6.5 \mathrm{~g} / \mathrm{dL}$ or $>16.0 \mathrm{~g} / \mathrm{dL}$

$<6.5 \mathrm{~g} / \mathrm{dL}$ or $>18.0 \mathrm{~g} / \mathrm{dL}$

$<50 \times 10^{3} \mu \mathrm{L}$ or $>800 \times 10^{3} \mu \mathrm{L}$

Possible causes of abnormal parameters:

- High RBC, Hgb, or HCT - dehydration, polycythemia, shock, chronic hypoxia

- Low RBC, Hgb, or HCT - anemia, thalassemia, and other hemoglobinopathies

- Low MCV - microcytic anemia

- High MCV - macrocytic anemia, liver disease

- Low WBC - sepsis, marrow hypoplasia

- High WBC - acute stress, infection, malignancies

- Low platelets - risk of bleeding

- High platelets - risk of thrombosis

XV. Specimen Storage and Handling During Testing

A. Specimen Storage

1. Store specimens capped and place on a rocker at room temperature until processed.

2. Run within 24 hours of drawing.

XVI. Alternative Method for Performing Test or Storing Specimens if Test System Fails

There is no alternative method for this test. Store EDTA tube at room temperature for no more than 24 hours. Restore the instrument to functionality and then run the specimen. 
XVII. Test Results Reporting System: Protocol for Reporting Action Limits

Results outside the action limits are automatically brought to the physician's attention for a decision as to "course-to-follow."

All records, including QA/QC data will be maintained for 6 years. Use only numerical identifiers for SP results.

XVIII. Quality Control Summary Statistics and Graphs

Chapter 14 includes a separate detailed description of the comprehensive quality control plan. Monitor $5 C \AA$ cell control results for bias and maintain results for the entire study period. Compare all three instruments using the CAP proficiency results.

XIX. References

1. Coulter ${ }^{\circledR}$ HMX Analyzer Operator’s Guide. (1992, January 21). PN 4235935J.

2. Coulter ${ }^{\circledR}$ HMX Analyzer Use and Function. (1995, January). PN 4235933H.

3. Coulter ${ }^{\circledR}$ HMX Analyzer Special Procedures and Troubleshooting. (1995, December). PN 4235934J.

4. Corash, L. (1983). Platelet Sizing: Techniques, Biological Significance, and Clinical Applications. Current Topics in Hematology. New York: Alan R. Wise, Inc.

5. Geigy Scientific Tables. (1984). Vol. 3, 210.

6. Jones, A.R., Twedl, D., Swaim, W., and Gottfried E. (1996). Diurnal change of blood count analytes in normal subjects. Am J Clin Path, 106, 723-7.

7. NCCLS document H15-A. (1984). Reference procedure for the quantitative determination of hemoglobin in blood. National Committee for Clinical Laboratory Standards, Villanova, PA.

8. NCCLS document H7-A. (1985). Procedure for determining packed cell volume by the micro hematocrit method. National Committee for Clinical Laboratory Standards, Villanova, PA.

9. NCCLS document H18-A. (1990). Procedures for the handling and processing of blood specimens. National Committee for Clinical Laboratory Standards, Villanova, PA.

10. NCCLS document H3-A3. (1991). Procedures for the collection of diagnostic blood specimens by venipuncture. National Committee for Clinical Laboratory Standards, Villanova, PA. 
11. NCCLS document H4-A3. (1991). Procedures for the collection of diagnostic blood specimens by skin puncture. National Committee for Clinical Laboratory Standards, Villanova, PA.

12. NCCLS document H26-A. (1996). Performance goals for the internal quality control of multichannel hematology analyzers; Approved Standard. NCCLS, Wayne, PA.

13. Standard 493-1211. Federal Register, 57: 40, (1992, February 28). Rules and Regulations, p. 7164.

14. Threatte, G.A., Andrados, C., Ebbe, S., and Brecher, G. (1984). Mean platelet volume: The need for a reference method. AJCP, 81, 769-772. 


\section{BLOOD PROCESSING}

\subsection{Introduction}

The purpose of blood processing is to (1) perform a complete blood count; (2) allocate blood into 48 vessels for storage and transport to 24 laboratories across the United States for analysis; (3) allocate plasma and serum into vessels for future studies and storage; and (4) ship tubes for genetic analysis. The purpose of packed cell processing is to allocate washed packed cells for storage and transport to two separate analysis laboratories. Processing includes centrifuging and storing blood collection tubes; aliquoting whole blood into storage vessels; centrifuging, separating, and aliquoting plasma and serum into storage vessels; performing dilutions; washing and lysing whole blood cells; and storing the specimens.

\subsection{Equipment and Supplies}

The blood processing area includes two under-counter refrigerators, four under-counter freezers, two refrigerated centrifuges, two data terminals, a laminar flow hood, and two specimen rockers. This area also includes counter space for processing blood. Store extra processing supplies in cabinets and/or the trailer compartments. Store the sterile saline in the refrigerator. Exhibits 8-1 and 8-2 list nonconsumable and consumable supplies for blood processing.

Exhibit 8-1. Nonconsumable supplies for blood processing

\begin{tabular}{|ll|}
\hline Blood processing template $(6-11 / 12+$ and 1-2/3-5) & Nalge cryovial racks \\
\hline One Eppendorf pipette $100-5,000 \mu \mathrm{L}$ & Two Eppendorf pipettes $50-1,000 \mu \mathrm{L}$ \\
\hline One Eppendorf pipette $5-100 \mu$ & Pipette charging stand \\
\hline One pipette battery & 50-mL graduated cylinder \\
\hline $13 \mathrm{~mm}$ half rack & Cube rack - 4-way flipper rack \\
\hline $16 \mathrm{~mm}$ full rack & Floor mat \\
\hline $16 \mathrm{~mm}$ half rack & Protective eyewear \\
\hline Deionized water bottle & Saline squeeze bottle \\
\hline Phosphate Buffered Saline & \\
\hline
\end{tabular}


Exhibit 8-2. Consumable supplies for blood processing

\begin{tabular}{|c|c|}
\hline Cotton swabs & Eight gallon biohazard sharps container \\
\hline Biohazard bags 25 x 35 & Biohazard bags $19 \times 23$ \\
\hline Kimwipes & Bench Kote paper \\
\hline Pipette tips $1,000 \mu \mathrm{L}$ & Pipette tips $200 \mu \mathrm{L}$ \\
\hline Pipette tips 5-mL & Wooden applicator sticks \\
\hline Zip closable bags & 3.5-mL transfer pipette nonsterile \\
\hline 5-mL cryovial sterile & 14-mL snap cap test tube \\
\hline Conical tube $50-\mathrm{mL}$ & 2-mL cryovial sterile \\
\hline 2-mL cryovial non-sterile & Caps for 2-mL cryovial \\
\hline Serum filters $13 \mathrm{~mm} \times \mathrm{x} 4$ inches & Serum filters $16 \mathrm{~mm}$ x 6 inches \\
\hline $\begin{array}{l}5.25 \times 5.25 \times 2.0 \text { inch cardboard box with } \\
9 \times 9 \text { cardboard grid }\end{array}$ & $\begin{array}{l}5.25 \times 5.25 \times 3.0 \text { inch cardboard box with } \\
9 \times 9 \text { cardboard grid }\end{array}$ \\
\hline $\begin{array}{l}5.25 \times 5.25 \times 3.0 \text { inch cardboard box } \\
\text { without grid }\end{array}$ & Three inch 5 x 5 cardboard grid \\
\hline $\begin{array}{l}5.25 \times 5.25 \times 5.0 \text { inch cardboard box with } \\
5 \times 5 \text { foam insert }\end{array}$ & $\begin{array}{l}5.25 \times 5.25 \times 3.0 \text { inch cardboard box with } 5 \times 5 \\
\text { foam insert }\end{array}$ \\
\hline \multicolumn{2}{|l|}{$10 \times 10$ two-inch box } \\
\hline Ascorbic acid 0.5 gm per vessel & $\begin{array}{l}\text { Nonsterile, powder-free, latex (nonlatex for use } \\
\text { by latex allergic staff) gloves-small, medium, } \\
\text { large }\end{array}$ \\
\hline Sterile saline & Deionized water \\
\hline Sharpie pens & Lab marker - fine \\
\hline
\end{tabular}

\subsection{Protocols}

Exhibits 8-3 through 8-6 illustrate the primary SP's protocol for blood processing. It is extremely important to follow the processing procedures outlined in these protocols and in this manual. Specimen loss or biased results will occur if the exact procedures are not followed. 
Exhibit 8-3. Primary SP's whole blood processing protocol

\begin{tabular}{|c|c|c|c|c|c|c|c|}
\hline ID & Test name & Ages & $\begin{array}{l}\text { Sample } \\
(\mathrm{mL})\end{array}$ & Sample type & $\begin{array}{l}\text { Collection } \\
\text { type }\end{array}$ & $\begin{array}{l}\text { Vessel } \\
\text { type }\end{array}$ & Remarks \\
\hline 119 & Genetics & $20+$ & 10.0 & Whole blood & EDTA & 10-mL & Ship twice per week \\
\hline 120 & Genetics & $20+$ & 10.0 & Whole blood & EDTA & 10-mL & Ship twice per week \\
\hline 54 & VOC Blood & $12+$ & 10.0 & Whole blood & $\begin{array}{c}\text { 10-mL } \\
\text { Grey }\end{array}$ & 10-mL & $\begin{array}{l}1 / 2 \text { subsample } \\
1,2,4,5,10,11\end{array}$ \\
\hline 1 & Lead/Cad/HG & $1+$ & 0.4 & Whole blood & EDTA & 2-mL & \\
\hline 3 & RBC Folate & $1+$ & 0.1 & Whole blood & EDTA & 2-mL & Dilute with 1-mL AA \\
\hline 106 & RBC Fol1 & $1+$ & 0.1 & Whole blood & EDTA & 2-mL & $\begin{array}{l}\text { Dilute with } 1-\mathrm{mL} \text { AA } \\
1 / 3 \text { subsample } \\
1,2,5,11\end{array}$ \\
\hline 4 & Glycohem & $12+$ & 0.4 & Whole blood & EDTA & 2-mL & Refrigerate \\
\hline 5 & Mercury & $1+$ & 0.5 & Whole blood & EDTA & 2-mL & \\
\hline 125 & HLA-B27 & $20-69$ & 1.0 & Whole blood & EDTA & 2-mL & Refrigerate \\
\hline
\end{tabular}

Exhibit 8-4. Primary SP’s plasma processing protocol

\begin{tabular}{|c|l|c|c|c|c|c|l|}
\hline ID & Test name & Ages & $\begin{array}{c}\text { Sample } \\
(\mathrm{mL})\end{array}$ & Sample type & $\begin{array}{c}\text { Collection } \\
\text { type }\end{array}$ & $\begin{array}{c}\text { Vessel } \\
\text { type }\end{array}$ & \multicolumn{1}{|c|}{ Remarks } \\
\hline 109 & PUFA/TFA & $3+$ & 0.7 & Plasma & EDTA & 2-mL & $\begin{array}{l}\text { Process SPs ages 3-11 } \\
\text { all sessions and SPs 12+ } \\
\text { morning sessions only } \\
\text { Add cap insert to 12+ } \\
\text { morning only }\end{array}$ \\
\hline 8 & Xtra Plasma & $6+$ & 0.5 & Plasma & EDTA & 2-mL & \\
\hline 9 & Glucose & $12+$ & 0.5 & Plasma & Grey & 2-mL & Morning SPs only \\
\hline 91 & SRM Pesticide & $12+$ & 4.0 & Plasma & $\begin{array}{c}\text { 7-mL } \\
\text { EDTA }\end{array}$ & 5-mL & $\begin{array}{l}\text { 1/3 subsample } \\
\text { 4, 6, 8, 9 }\end{array}$ \\
\hline
\end{tabular}

Exhibit 8-5. Primary SP’s packed cells processing protocol

\begin{tabular}{|c|c|c|c|c|c|c|c|}
\hline ID & Test name & Ages & $\begin{array}{c}\text { Sample } \\
\text { (mL) }\end{array}$ & Sample type & $\begin{array}{c}\text { Collection } \\
\text { type }\end{array}$ & $\begin{array}{l}\text { Vessel } \\
\text { type }\end{array}$ & Remarks \\
\hline 88 & Acrylamide & $6+$ & 1.0 & Packed cells & EDTA & $2-m L$ & $\begin{array}{l}\text { Wash } 3 \text { times, lyse } \\
1 / 3 \text { subsample } \\
1,2,5,11\end{array}$ \\
\hline 126 & Xtra RBCs & $12+$ & 1.0 & Packed cells & EDTA & $2-m L$ & $\begin{array}{l}\text { Wash } 3 \text { times, lyse } \\
\text { stabilize } \\
1 / 3 \text { subsample } \\
1,2,5,11\end{array}$ \\
\hline
\end{tabular}


Exhibit 8-6. Primary SP's serum processing protocol

\begin{tabular}{|c|c|c|c|c|c|c|c|}
\hline ID & Test name & Ages & $\begin{array}{l}\text { Sample } \\
\text { (mL) }\end{array}$ & $\begin{array}{l}\text { Sample } \\
\text { type }\end{array}$ & $\begin{array}{l}\text { Collection } \\
\text { type }\end{array}$ & $\begin{array}{l}\text { Vessel } \\
\text { type }\end{array}$ & Remarks \\
\hline 81 & Vit B6/Fol & $1+$ & 0.5 & Serum & Red & $2-\mathrm{mL}$ & \\
\hline 70 & Vitamin D & $\begin{array}{l}1-5 \\
6+\end{array}$ & $\begin{array}{l}0.5 \\
0.7\end{array}$ & Serum & Red & 2-mL & \\
\hline 13 & CRP & $3+$ & 0.3 & Serum & Red & 2-mL & \\
\hline 14 & Hep Profile & $20+$ & 1.5 & Serum & Red & 2-mL & \\
\hline 122 & HAV & $6-19$ & 1.5 & Serum & Red & 2-mL & \\
\hline 87 & Hepatit ab & $\begin{array}{c}2 \\
3-5 \\
\end{array}$ & $\begin{array}{l}0.3 \\
0.5 \\
\end{array}$ & Serum & Red & 2-mL & Age 2-5 only \\
\hline 99 & Fer/TfR & $\begin{array}{c}1-2 \\
3-5 \\
12-49\end{array}$ & $\begin{array}{l}0.3 \\
0.5 \\
0.5 \\
\end{array}$ & Serum & Red & 2-mL & 12-49 females only \\
\hline 17 & Cotinine & $\begin{array}{c}3-5 \\
6-11 \\
12+ \\
\end{array}$ & $\begin{array}{l}0.8 \\
1.1 \\
1.8 \\
\end{array}$ & Serum & Red & 2-mL & \\
\hline 21 & Lipids & $\begin{array}{l}6-11 \\
12+\end{array}$ & $\begin{array}{l}1.0 \\
1.5\end{array}$ & Serum & Red & 2-mL & \\
\hline 77 & HPV Serum & $14-59$ & 0.5 & Serum & Red & 2-mL & 14-59 only \\
\hline 18 & Biochem & $12+$ & 0.8 & Serum & Red & 2-mL & \\
\hline 23 & Insulin & $12+$ & 1.0 & Serum & Red & 2-mL & Morning SPs only \\
\hline 22 & HIV & $18-59$ & 0.8 & Serum & Red & 2-mL & $18-59$ only \\
\hline 24 & HSV & 14-49 & 0.2 & Serum & Red & 2-mL & $14-49$ only \\
\hline 75 & PSA & $40+$ & 1.0 & Serum & Red & 2-mL & Males $40+$ only \\
\hline 118 & Thyroid & $12+$ & 1.0 & Serum & Red & 2-mL & $\begin{array}{l}1 / 3 \text { subsample } \\
1,2,5,11\end{array}$ \\
\hline 127 & IgA-TTG/EMA & $6+$ & 0.5 & Serum & Red & 2-mL & \\
\hline 16 & MMRV & $6-49$ & 1.5 & Serum & Red & 2-mL & \\
\hline 110 & Folate1 & $1+$ & 0.7 & Serum & Red & 2-mL & $\begin{array}{l}1 / 3 \text { subsample } \\
1,2,5,11\end{array}$ \\
\hline 32 & Xtra Sera1 & $3+$ & 0.5 & Serum & Red & 2-mL & \\
\hline 33 & Xtra Sera2 & $3+$ & 0.5 & Serum & Red & 2-mL & \\
\hline 34 & Xtra Sera3 & $3+$ & 0.5 & Serum & Red & 2-mL & \\
\hline 35 & Xtra Sera4 & $12+$ & 0.5 & Serum & Red & 2-mL & \\
\hline 36 & Xtra Sera5 & $12+$ & 1.0 & Serum & Red & 2-mL & \\
\hline 37 & Xtra Sera6 & $12+$ & 1.0 & Serum & Red & 2-mL & \\
\hline 38 & Xtra Sera7 & $12+$ & 1.0 & Serum & Red & 2-mL & \\
\hline 39 & Xtra Sera8 & $12+$ & 1.0 & Serum & Red & 2-mL & \\
\hline
\end{tabular}


Exhibit 8-6. Primary SP's serum processing protocol (continued)

\begin{tabular}{|l|l|l|c|c|c|c|l|}
\hline ID & Test name & Ages & $\begin{array}{c}\text { Sample } \\
(\mathrm{mL})\end{array}$ & Sample type & $\begin{array}{c}\text { Collection } \\
\text { type }\end{array}$ & $\begin{array}{c}\text { Vessel } \\
\text { type }\end{array}$ & \multicolumn{1}{|c|}{ Remarks } \\
\hline 29 & Persis Pest1 & $12+$ & 4.0 & Serum & 10 -mL Red & 5 -mL & $\begin{array}{l}1 / 3 \text { Subsample } \\
1,2,5,11\end{array}$ \\
\hline 89 & Persis Pest2 & $12+$ & 4.0 & Serum & 10-mL Red & 5 -mL & $\begin{array}{l}1 / 3 \text { Subsample } \\
1,2,5,11\end{array}$ \\
\hline 90 & Perfluor & $12+$ & 4.0 & Serum & 10 -mL Red & 5 -mL & $\begin{array}{l}1 / 3 \text { subsample } \\
4,6,8,9\end{array}$ \\
\hline 92 & BFR1 & $12+$ & 4.0 & Serum & 10-mL Red & 5 -mL & $\begin{array}{l}1 / 3 \text { Subsample } \\
0,3,7,10\end{array}$ \\
\hline 93 & BFR2 & $12+$ & 4.0 & Serum & 10-mL Red & $5-\mathrm{mL}$ & $\begin{array}{l}1 / 3 \text { Subsample } \\
0,3,7,10\end{array}$ \\
\hline
\end{tabular}

Exhibits 8-7 through 8-9 illustrate the VIP guest's protocol for blood processing. It is extremely important to follow the processing procedures outlined in these protocols and in this manual. Specimen loss or biased results will occur if the exact procedures are not followed.

Exhibit 8-7. VIP Guest's blood whole blood processing protocol

\begin{tabular}{|l|l|c|c|c|c|c|c|}
\hline ID & \multicolumn{1}{|c|}{ Test name } & $\begin{array}{c}\text { Ag } \\
\text { es }\end{array}$ & $\begin{array}{c}\text { Sample } \\
(\mathrm{mL})\end{array}$ & Sample type & $\begin{array}{c}\text { Collection } \\
\text { type }\end{array}$ & $\begin{array}{c}\text { Vessel } \\
\text { type }\end{array}$ & Remarks \\
\hline 1 & Lead/Cad/HG & $1+$ & 0.4 & Whole blood & EDTA & $2-\mathrm{mL}$ & \\
\hline 3 & RBC Folate & $1+$ & 0.1 & Whole blood & EDTA & $2-\mathrm{mL}$ & Dilute with 1-mL AA \\
\hline 106 & RBC Fol1 & $1+$ & 0.1 & Whole blood & EDTA & $2-\mathrm{mL}$ & Dilute with 1-mL AA \\
\hline 5 & Mercury & $1+$ & 0.5 & Whole blood & EDTA & $2-\mathrm{mL}$ & \\
\hline
\end{tabular}

Exhibit 8-8. VIP Guest’s plasma processing protocol

\begin{tabular}{|l|l|l|c|c|c|c|c|}
\hline ID & Test name & Ages & $\begin{array}{c}\text { Sample } \\
(\mathrm{mL})\end{array}$ & Sample type & $\begin{array}{c}\text { Collection } \\
\text { type }\end{array}$ & $\begin{array}{c}\text { Vessel } \\
\text { type }\end{array}$ & Remarks \\
\hline 9 & Glucose & $12+$ & 0.5 & Plasma & Grey & 2-mL & Morning SPs only \\
\hline
\end{tabular}


Exhibit 8-9. VIP Guest’s serum processing protocol

\begin{tabular}{|c|c|c|c|c|c|c|c|}
\hline ID & Test name & Ages & $\begin{array}{c}\text { Sample } \\
\text { (mL) }\end{array}$ & Sample type & $\begin{array}{l}\text { Collection } \\
\text { type }\end{array}$ & $\begin{array}{l}\text { Vessel } \\
\text { type }\end{array}$ & Remarks \\
\hline 81 & Vit B6/Fol & $1+$ & 0.5 & Serum & Red & $2-\mathrm{mL}$ & \\
\hline 99 & Fer/TfR & $\begin{array}{c}1-2 \\
3-5 \\
12-49 \\
\end{array}$ & $\begin{array}{l}0.3 \\
0.5 \\
0.5 \\
\end{array}$ & Serum & Red & 2-mL & 12-49 females only \\
\hline 21 & Lipids & $6+$ & 1.5 & Serum & Red & 2-mL & \\
\hline 23 & Biochem & $12+$ & 0.8 & Serum & Red & 2-mL & \\
\hline
\end{tabular}

Exhibit 8-10 illustrates the guest and surplus protocol for blood processing. It is extremely important to follow the processing procedures outlined in these protocols and in this manual. Specimen loss or biased results will occur if the exact procedures are not followed.

Exhibit 8-10. Guest serum processing protocol

\begin{tabular}{|l|l|c|c|c|c|c|c|}
\hline ID & Test name & Ages & $\begin{array}{c}\text { Sample } \\
(\mathrm{mL})\end{array}$ & $\begin{array}{c}\text { Sample } \\
\text { type }\end{array}$ & $\begin{array}{c}\text { Collection } \\
\text { type }\end{array}$ & $\begin{array}{c}\text { Vessel } \\
\text { type }\end{array}$ & Remarks \\
\hline 18 & Biochem & $12+$ & 0.8 & Serum & Red & $2-m L$ & \\
\hline
\end{tabular}




\subsection{Blood Processing Age-Specific Protocols}

Exhibits 8-11 through 8-15 illustrate the blood processing vessels for each primary SP by age group.

Exhibit 8-11. Blood processing protocol for primary SPs aged 1-2 years

\begin{tabular}{|c|l|c|c|c|c|c|}
\hline ID & \multicolumn{1}{|c|}{ Test name } & Ages & $\begin{array}{c}\text { Sample } \\
(\mathrm{mL})\end{array}$ & Sample type & Collection type & $\begin{array}{c}\text { Vessel } \\
\text { type }\end{array}$ \\
\hline 1 & Lead/Cad/HG & $1-2$ & 0.4 & Whole blood & 3-mL EDTA & 2-mL \\
\hline 3 & RBC Folate & $1-2$ & 0.1 & Whole blood & 3-mL EDTA & 2-mL \\
\hline 106 & RBC Fol1 & $1-2$ & 0.1 & Whole blood & 3-mL EDTA & 2-mL \\
\hline 5 & Mercury & $1-2$ & 0.5 & Whole blood & 3-mL EDTA & $2-\mathrm{mL}$ \\
\hline 81 & Vit B6/Fol & $1-2$ & 0.5 & Serum & 3-mL Red & 2-mL \\
\hline 70 & Vitamin D & $1-2$ & 0.5 & Serum & 3-mL Red & 2-mL \\
\hline 87 & Hepatitis ab & 2 & 0.3 & Serum & 3-mL Red & $2-\mathrm{mL}$ \\
\hline 99 & Fer/TfR & $1-2$ & 0.3 & Serum & 3-mL Red & 2-mL \\
\hline 110 & Folate1 & $1-2$ & 0.7 & Serum & 3-mL Red & 2-mL \\
\hline
\end{tabular}

Exhibit 8-12. Blood processing protocol for primary SPs aged 3-5 years

\begin{tabular}{|c|l|c|c|c|l|c|}
\hline ID & \multicolumn{1}{|c|}{ Test name } & Ages & $\begin{array}{c}\text { Sample } \\
(\mathrm{mL})\end{array}$ & Sample type & \multicolumn{1}{|c|}{ Collection type } & $\begin{array}{c}\text { Vessel } \\
\text { type }\end{array}$ \\
\hline 1 & Lead/Cad/HG & $3-5$ & 0.4 & Whole blood & 3-mL EDTA & 2-mL \\
\hline 3 & RBC Folate & $3-5$ & 0.1 & Whole blood & 3-mL EDTA & 2 -mL \\
\hline 106 & RBC Fol1 & $3-5$ & 0.1 & Whole blood & 3-mL EDTA & 2-mL \\
\hline 5 & Mercury & $3-5$ & 0.5 & Whole blood & 3-mL EDTA & 2-mL \\
\hline 109 & PUFA/TFA & $3-5$ & 0.7 & Plasma & 3-mL EDTA & 2-mL \\
\hline 81 & Vit B6/Fol & $3-5$ & 0.5 & Serum & 7-mL Red & 2-mL \\
\hline 70 & Vitamin D & $3-5$ & 0.5 & Serum & 7-mL Red & 2-mL \\
\hline 13 & CRP & $3-5$ & 0.3 & Serum & 7-mL Red & 2-mL \\
\hline 87 & Hepatitis ab & $3-5$ & 0.5 & Serum & 7-mL Red & 2-mL \\
\hline 99 & Fer/TfR & $3-5$ & 0.5 & Serum & 7-mL Red & 2-mL \\
\hline 17 & Cotinine & $3-5$ & 0.8 & Serum & 7-mL Red & 2-mL \\
\hline 110 & Folate1 & $3-5$ & 0.7 & Serum & 7-mL Red & 2-mL \\
\hline 32 & Extra Sera1 & $3-5$ & 0.5 & Serum & 7-mL Red & 2-mL \\
\hline 33 & Extra Sera2 & $3-5$ & 0.5 & Serum & 7-mL Red & 2-mL \\
\hline 34 & Extra Sera3 & $3-5$ & 0.5 & Serum & 7-mL Red & 2-mL \\
\hline
\end{tabular}


Exhibit 8-13. Blood processing protocol for primary SPs aged 6-11 years

\begin{tabular}{|c|c|c|c|c|c|c|}
\hline ID & Test name & Ages & $\begin{array}{c}\text { Sample } \\
\text { (mL) }\end{array}$ & Sample type & Collection type & $\begin{array}{l}\text { Vessel } \\
\text { type }\end{array}$ \\
\hline 1 & Lead/Cad/HG & $6-11$ & 0.4 & Whole blood & 3-mL EDTA & $2-\mathrm{mL}$ \\
\hline 3 & RBC Folate & $6-11$ & 0.1 & Whole blood & 3-mL EDTA & $2-m L$ \\
\hline 106 & RBC Fol1 & 6-11 & 0.1 & Whole blood & 3-mL EDTA & 2-mL \\
\hline 5 & Mercury & $6-11$ & 0.5 & Whole blood & 3-mL EDTA & 2-mL \\
\hline 109 & PUFA/TFA & $6-11$ & 0.7 & Plasma & 4-mL EDTA & $2-\mathrm{mL}$ \\
\hline 8 & Xtra Plasma & $6-11$ & 0.5 & Plasma & 4-mL EDTA & $2-\mathrm{mL}$ \\
\hline 88 & Acrylamide & $6-11$ & 1.0 & Washed packed cells & 3 or $4-m L$ EDTA & $2-m L$ \\
\hline 81 & Vit B6/Fol & $6-11$ & 0.5 & Serum & 10 and 3-mL Red & $2-m L$ \\
\hline 70 & Vitamin D & $6-11$ & 0.7 & Serum & 10 and 3-mL Red & 2-mL \\
\hline 13 & CRP & $6-11$ & 0.3 & Serum & 10 and 3-mL Red & $2-m L$ \\
\hline 122 & HAV & $6-19$ & 1.5 & Serum & 10 and 3-mL Red & 2-mL \\
\hline 17 & Cotinine & $6-11$ & 1.1 & Serum & 10 and 3-mL Red & $2-m L$ \\
\hline 21 & Lipids & $6-11$ & 1.0 & Serum & 10 and 3-mL Red & $2-\mathrm{mL}$ \\
\hline 127 & IGA-TTG/EMA & $6-11$ & 0.5 & Serum & 10 and 3-mL Red & 2-mL \\
\hline 16 & MMRV & $6-11$ & 1.5 & Serum & 10 and 3-mL Red & 2-mL \\
\hline 110 & Folate1 & $6-11$ & 0.7 & Serum & 10 and 3-mL Red & 2-mL \\
\hline 32 & Xtra Sera1 & $6-11$ & 0.5 & Serum & 10 and 3-mL Red & $2-m L$ \\
\hline 33 & Xtra Sera2 & $6-11$ & 0.5 & Serum & 10 and 3-mL Red & 2-mL \\
\hline 34 & Xtra Sera3 & $6-11$ & 0.5 & Serum & 10 and 3-mL Red & $2-\mathrm{mL}$ \\
\hline
\end{tabular}


Exhibit 8-14. Blood processing protocol for primary SPs aged 12-19 years

\begin{tabular}{|c|c|c|c|c|c|c|}
\hline ID & Test name & Ages & $\begin{array}{c}\text { Sample } \\
\text { (mL) }\end{array}$ & Sample type & Collection type & $\begin{array}{l}\text { Vessel } \\
\text { type }\end{array}$ \\
\hline 54 & VOC Blood & $12-19$ & 10.0 & Whole blood & 10-mL grey & 10-mL \\
\hline 1 & Lead/Cad/HG & $12-19$ & 0.4 & Whole blood & 3 -mL EDTA & $2-\mathrm{mL}$ \\
\hline 3 & RBC Folate & $12-19$ & 0.1 & Whole blood & 3-mL EDTA & $2-\mathrm{mL}$ \\
\hline 106 & RBC Fol1 & $12-19$ & 0.1 & Whole blood & 3-mL EDTA & 2-mL \\
\hline 4 & Glycohem & $12-19$ & 0.4 & Whole blood & 3-mL EDTA & $2-\mathrm{mL}$ \\
\hline 5 & Mercury & $12-19$ & 0.5 & Whole blood & 3-mL EDTA & $2-\mathrm{mL}$ \\
\hline 109 & PUFA/TFA & $12-19$ & 0.7 & Plasma & 4-mL EDTA & $2-\mathrm{mL}$ \\
\hline 8 & Xtra Plasma & $12-19$ & 0.5 & Plasma & 4-mL EDTA & 2-mL \\
\hline 9 & Glucose & $12-19$ & 0.5 & Plasma & 2-mL Gray & 2-mL \\
\hline 88 & Acrylamide & $12-19$ & 1.0 & Washed packed cells & 4-mL EDTA & $2-m L$ \\
\hline 126 & Xtra RBCs & $12-19$ & 1.0 & Washed packed cells & 4-mL EDTA & 2-mL \\
\hline 91 & Srm Pest & $12-19$ & 4.0 & Plasma & 7-mL EDTA & 5-mL \\
\hline 98 & OGTT & $12-19$ & 0.5 & Plasma & 2-mL Gray & $2-\mathrm{mL}$ \\
\hline 81 & Vit B6/Fol & $12-19$ & 0.5 & Serum & 15 and $10-\mathrm{mL}$ Red & $2-\mathrm{mL}$ \\
\hline 70 & Vitamin D & $12-19$ & 0.7 & Serum & 15 and $10-m L$ Red & 2-mL \\
\hline 13 & CRP & $12-19$ & 0.3 & Serum & 15 and $10-\mathrm{mL}$ Red & $2-\mathrm{mL}$ \\
\hline 122 & HAV & $12-19$ & 1.5 & Serum & 15 and $10-m L$ Red & 2-mL \\
\hline 99 & Fer/TfR & $12-19$ & 0.5 & Serum & 15 and $10-m L$ Red & $2-\mathrm{mL}$ \\
\hline 17 & Cotinine & $12-19$ & 1.8 & Serum & 15 and $10-m L$ Red & 2-mL \\
\hline 21 & Lipids & $12-19$ & 1.5 & Serum & 15 and $10-m L$ Red & $2-\mathrm{mL}$ \\
\hline 77 & HPV Serum & $14-19$ & 0.5 & Serum & 15 and $10-m L$ Red & 2-mL \\
\hline 18 & Biochem & $12-19$ & 0.8 & Serum & 15 and 10-mL Red & 2-mL \\
\hline 23 & Insulin & $12-19$ & 1.0 & Serum & 15 and $10-\mathrm{mL}$ Red & $2-\mathrm{mL}$ \\
\hline 22 & HIV & $18-19$ & 0.8 & Serum & 15 and $10-m L$ Red & 2-mL \\
\hline 24 & HSV & $14-49$ & 0.2 & Serum & 15 and $10-m L$ Red & $2-\mathrm{mL}$ \\
\hline 118 & Tyyroid & $12-19$ & 1.0 & Serum & 15 and $10-m L$ Red & 2-mL \\
\hline 127 & IgA-TTG/EMA & $12-19$ & 0.5 & Serum & 15 and $10-m L$ Red & 2-mL \\
\hline 16 & MMRV & $12-19$ & 1.5 & Serum & 15 and $10-m L$ Red & 2-mL \\
\hline 110 & Folate1 & $12-19$ & 0.7 & Serum & 15 and $10-\mathrm{mL}$ Red & 2-mL \\
\hline 32 & Xtra Sera1 & $12-19$ & 0.5 & Serum & 15 and $10-m L$ Red & 2-mL \\
\hline 33 & Xtra Sera2 & $12-19$ & 0.5 & Serum & 15 and $10-m L$ Red & 2-mL \\
\hline 34 & Xtra Sera3 & $12-19$ & 0.5 & Serum & 15 and $10-m L$ Red & 2-mL \\
\hline 35 & Xtra Sera4 & $12-19$ & 0.5 & Serum & 15 and $10-m L$ Red & 2-mL \\
\hline 36 & Xtra Sera5 & $12-19$ & 1.0 & Serum & 15 and $10-m L$ Red & 2-mL \\
\hline 37 & Xtra Sera6 & $12-19$ & 1.0 & Serum & 15 and $10-m L$ Red & 2-mL \\
\hline 38 & Xtra Sera7 & $12-19$ & 1.0 & Serum & 15 and $10-\mathrm{mL}$ Red & 2-mL \\
\hline 39 & Xtra Sera8 & $12-19$ & 1.0 & Serum & 15 and $10-m L$ Red & 2-mL \\
\hline 29 & Persis Pest & $12-19$ & 4.0 & Serum & 10-mL Red & 5-mL \\
\hline 89 & Persis Pest2 & $12-19$ & 4.0 & Serum & 10-mL Red & 5-mL \\
\hline 90 & Perflour1 & $12-19$ & 4.0 & Serum & 10-mL Red & 5-mL \\
\hline 92 & BFR1 & $12-19$ & 4.0 & Serum & 10-mL Red & 5-mL \\
\hline 93 & BFR2 & $12-19$ & 4.0 & Serum & 10-mL Red & 5-mL \\
\hline
\end{tabular}


Exhibit 8-15. Blood processing protocol for primary SPs aged 20+ years

\begin{tabular}{|c|c|c|c|c|c|c|}
\hline ID & Test name & Ages & $\begin{array}{c}\text { Sample } \\
\text { (mL) }\end{array}$ & Sample type & Collection type & $\begin{array}{l}\text { Vessel } \\
\text { type }\end{array}$ \\
\hline 119 & Genetics & $20+$ & 10.0 & Whole Blood & 10-mL EDTA & $\begin{array}{l}\text { 10-mL } \\
\text { EDTA }\end{array}$ \\
\hline 120 & Genetics & $20+$ & 10.0 & Whole Blood & 10-mL EDTA & $\begin{array}{l}10-\mathrm{mL} \\
\text { EDTA }\end{array}$ \\
\hline 54 & VOC Blood & $20+$ & 10.0 & Whole blood & 10-mL gray & $\begin{array}{c}10-\mathrm{mL} \\
\text { Gray }\end{array}$ \\
\hline 1 & Lead/Cad/HG & $20+$ & 0.4 & Whole blood & 3 or $4-m L$ EDTA & $2-\mathrm{mL}$ \\
\hline 3 & RBC Folate & $20+$ & 0.1 & Whole blood & 3 or 4-mL EDTA & 2-mL \\
\hline 106 & RBC Fol1 & $20+$ & 0.1 & Whole blood & 3 or 4-mL EDTA & 2-mL \\
\hline 4 & Glycohem & $20+$ & 0.4 & Whole blood & 3 or 4 -mL EDTA & 2-mL \\
\hline 5 & Mercury & $20+$ & 0.5 & Whole blood & 3 or $4-m L$ EDTA & 2-mL \\
\hline 109 & PUFA/TFA & $20+$ & 0.7 & Plasma & 4-mL EDTA & 2-mL \\
\hline 8 & Xtra Plasma & $20+$ & 0.5 & Plasma & 4-mL EDTA & 2-mL \\
\hline 9 & Glucose & $20+$ & 0.5 & Plasma & 2-mL Gray & 2-mL \\
\hline 88 & Acrylamide & $20+$ & 1.0 & Washed packed cells & 3 or $4-m L$ EDTA & 2-mL \\
\hline 126 & Xtra RBCs & $20+$ & 1.0 & Washed packed cells & 3 or 4 -mL EDTA & 2-mL \\
\hline 91 & Srm Pest & $20+$ & 4.0 & Plasma & 7-mL EDTA & 5-mL \\
\hline 98 & OGTT & $20+$ & 0.5 & Plasma & 2-mL Gray & 2-mL \\
\hline 81 & Vit B6/Fol & $20+$ & 0.5 & Serum & 15 and $10-\mathrm{mL}$ Red & 2-mL \\
\hline 70 & Vitamin D & $20+$ & 0.7 & Serum & 15 and $10-\mathrm{mL}$ Red & 2-mL \\
\hline 13 & CRP & $20+$ & 0.3 & Serum & 15 and $10-\mathrm{mL}$ Red & 2-mL \\
\hline 14 & Hep Profile & $20+$ & 1.5 & Serum & 15 and $10-\mathrm{mL}$ Red & 2-mL \\
\hline 99 & Fer/TfR & $20-49$ & 0.5 & Serum & 15 and $10-\mathrm{mL}$ Red & 2-mL \\
\hline 17 & Cotinine & $20+$ & 1.8 & Serum & 15 and $10-\mathrm{mL}$ Red & 2-mL \\
\hline 21 & Lipids & $20+$ & 1.5 & Serum & 15 and $10-m L$ Red & $2-\mathrm{mL}$ \\
\hline 77 & HPV Serum & $20-59$ & 0.5 & Serum & 15 and $10-\mathrm{mL}$ Red & 2-mL \\
\hline 18 & Biochem & $20+$ & 0.8 & Serum & 15 and $10-\mathrm{mL}$ Red & 2-mL \\
\hline 23 & Insulin & $20+$ & 1.0 & Serum & 15 and $10-\mathrm{mL}$ Red & 2-mL \\
\hline 22 & HIV & $20-59$ & 0.8 & Serum & 15 and $10-\mathrm{mL}$ Red & 2-mL \\
\hline 24 & HSV & $20-49$ & 0.2 & Serum & 15 and $10-\mathrm{mL}$ Red & 2-mL \\
\hline 75 & PSA & $40+$ & 1.0 & Serum & 15 and 10-mL Red & 2-mL \\
\hline 118 & Thyroid & $20+$ & 1.0 & Serum & 15 and $10-\mathrm{mL}$ Red & 2-mL \\
\hline 127 & IgA-TTG/EMA & $20+$ & 0.5 & Serum & 15 and $10-\mathrm{mL}$ Red & 2-mL \\
\hline
\end{tabular}


Exhibit 8-15. Blood processing protocol for primary SPs aged 20+ years (continued)

\begin{tabular}{|c|c|c|c|c|c|c|}
\hline ID & Test name & Ages & $\begin{array}{c}\text { Sample } \\
\text { (mL) }\end{array}$ & Sample type & Collection type & $\begin{array}{l}\text { Vessel } \\
\text { type }\end{array}$ \\
\hline 16 & MMRV & $20-49$ & 1.5 & Serum & 15 and $10-m L$ Red & 2-mL \\
\hline 110 & Folate1 & $20+$ & 0.7 & Serum & 15 and $10-\mathrm{mL}$ Red & 2-mL \\
\hline 32 & Xtra Sera1 & $20+$ & 0.5 & Serum & 15 and $10-\mathrm{mL}$ Red & 2-mL \\
\hline 33 & Xtra Sera2 & $20+$ & 0.5 & Serum & 15 and $10-\mathrm{mL}$ Red & 2-mL \\
\hline 34 & Xtra Sera3 & $20+$ & 0.5 & Serum & 15 and $10-\mathrm{mL}$ Red & 2-mL \\
\hline 35 & Xtra Sera4 & $20+$ & 0.5 & Serum & 15 and $10-\mathrm{mL}$ Red & 2-mL \\
\hline 36 & Xtra Sera5 & $20+$ & 1.0 & Serum & 15 and $10-\mathrm{mL}$ Red & 2-mL \\
\hline 37 & Xtra Sera6 & $20+$ & 1.0 & Serum & 15 and $10-\mathrm{mL}$ Red & 2-mL \\
\hline 38 & Xtra Sera7 & $20+$ & 1.0 & Serum & 15 and $10-\mathrm{mL}$ Red & 2-mL \\
\hline 39 & Xtra Sera8 & $20+$ & 1.0 & Serum & 15 and $10-\mathrm{mL}$ Red & 2-mL \\
\hline 29 & Persis Pest & $20+$ & 4.0 & Serum & 10-mL Red & 5-mL \\
\hline 89 & Persis Pest2 & $20+$ & 4.0 & Serum & 10-mL Red & 5-mL \\
\hline 90 & Perflour1 & $20+$ & 4.0 & Serum & 10-mL Red & 5-mL \\
\hline 92 & BFR1 & $20+$ & 4.0 & Serum & 10-mL Red & 5-mL \\
\hline 93 & BFR2 & $20+$ & 4.0 & Serum & 10-mL Red & 5-mL \\
\hline
\end{tabular}

Exhibits 8-16 through 8-19 illustrate the blood processing vessels for each VIP guest.

Exhibit 8-16. Blood processing protocol for VIP guest aged 1-2 years

\begin{tabular}{|c|l|c|c|c|c|c|}
\hline ID & \multicolumn{1}{|c|}{ Test name } & Ages & $\begin{array}{c}\text { Sample } \\
(\mathrm{mL})\end{array}$ & Sample type & Collection type & $\begin{array}{c}\text { Vessel } \\
\text { type }\end{array}$ \\
\hline 1 & Lead/Cad/HG & $1-2$ & 0.4 & Whole blood & 3-mL EDTA & 2-mL \\
\hline 3 & RBC Folate & $1-2$ & 0.1 & Whole blood & 3-mL EDTA & 2-mL \\
\hline 106 & RBC Fol1 & $1-2$ & 0.1 & Whole Blood & 3-mL EDTA & 2-mL \\
\hline 5 & Mercury & $1-2$ & 0.5 & Whole blood & 3-mL EDTA & 2-mL \\
\hline 81 & Vit B6/Fol & $1-2$ & 0.5 & Serum & 3-mL Red & 2-mL \\
\hline 99 & Fer/TfR & $1-2$ & 0.3 & Serum & 3-mL Red & 2-mL \\
\hline
\end{tabular}

Exhibit 8-17. Blood processing protocol for VIP guest aged 3-5 years

\begin{tabular}{|c|l|c|c|c|c|c|}
\hline ID & \multicolumn{1}{|c|}{ Test name } & Ages & $\begin{array}{c}\text { Sample } \\
(\mathrm{mL})\end{array}$ & Sample type & Collection type & $\begin{array}{c}\text { Vessel } \\
\text { type }\end{array}$ \\
\hline 1 & Lead/Cad/HG & $3-5$ & 0.4 & Whole blood & 3-mL EDTA & $2-\mathrm{mL}$ \\
\hline 3 & RBC Folate & $3-5$ & 0.1 & Whole blood & 3-mL EDTA & 2-mL \\
\hline 106 & RBC Fol1 & $3-5$ & 0.1 & Whole blood & 3-mL EDTA & 2-mL \\
\hline 5 & Mercury & $3-5$ & 0.5 & Whole blood & 3-mL EDTA & $2-\mathrm{mL}$ \\
\hline 81 & Vit B6/Fol & $3-5$ & 0.5 & Serum & 3-mL Red & 2-mL \\
\hline 99 & Fer/TfR & $3-5$ & 0.5 & Serum & 3-mL Red & 2-mL \\
\hline
\end{tabular}


Exhibit 8-18. Blood processing protocol for VIP guest aged 6-11 years

\begin{tabular}{|c|l|c|c|c|c|c|}
\hline ID & \multicolumn{1}{|c|}{ Test name } & Ages & $\begin{array}{c}\text { Sample } \\
(\mathrm{mL})\end{array}$ & Sample type & Collection type & $\begin{array}{c}\text { Vessel } \\
\text { type }\end{array}$ \\
\hline 1 & Lead/Cad/HG & $6-11$ & 0.4 & Whole blood & 3-mL EDTA & 2-mL \\
\hline 3 & RBC Folate & $6-11$ & 0.1 & Whole blood & 3-mL EDTA & 2-mL \\
\hline 106 & RBC Fol1 & $6-11$ & 0.1 & Whole blood & 3-mL EDTA & 2-mL \\
\hline 5 & Mercury & $6-11$ & 0.5 & Whole blood & 3-mL EDTA & 2-mL \\
\hline 81 & Vit B6/Fol & $6-11$ & 0.5 & Serum & 7-mL Red & 2-mL \\
\hline 21 & Lipids & $6-11$ & 1.5 & Serum & 7-mL Red & 2-mL \\
\hline
\end{tabular}

Exhibit 8-19. Blood processing protocol for VIP guest aged 12+ years

\begin{tabular}{|c|l|c|c|c|c|c|}
\hline ID & \multicolumn{1}{|c|}{ Test name } & Ages & $\begin{array}{c}\text { Sample } \\
(\mathrm{mL})\end{array}$ & Sample type & Collection type & $\begin{array}{c}\text { Vessel } \\
\text { type }\end{array}$ \\
\hline 1 & Lead/Cad/HG & $12+$ & 0.4 & Whole blood & 3-mL EDTA & 2 -mL \\
\hline 3 & RBC Folate & $12+$ & 0.1 & Whole blood & 3-mL EDTA & $2-\mathrm{mL}$ \\
\hline 106 & RBC Fol1 & $12+$ & 0.1 & Whole blood & 3-mL EDTA & $2-\mathrm{mL}$ \\
\hline 5 & Mercury & $12+$ & 0.5 & Whole blood & 3-mL EDTA & $2-\mathrm{mL}$ \\
\hline 9 & Glucose & $12+$ & 0.5 & Plasma & 2-mL Grey & $2-\mathrm{mL}$ \\
\hline 81 & Vit B6/Fol & $12+$ & 0.5 & Serum & $10-\mathrm{mL} \mathrm{Red}$ & $2-\mathrm{mL}$ \\
\hline 99 & Fer/TfR & $12-49$ & 0.5 & Serum & $10-\mathrm{mL}$ Red & $2-\mathrm{mL}$ \\
\hline 21 & Lipids & $12+$ & 1.5 & Serum & $10-\mathrm{mL}$ Red & $2-\mathrm{mL}$ \\
\hline 18 & Biochem & $12+$ & 0.8 & Serum & $10-\mathrm{mL}$ Red & $2-\mathrm{mL}$ \\
\hline
\end{tabular}

Exhibit 8-20 illustrates the blood processing vessel for each guest.

Exhibit 8-20. Blood processing protocol for guest aged 12+ years

\begin{tabular}{|c|l|c|c|c|c|c|}
\hline ID & Test name & Ages & $\begin{array}{c}\text { Sample } \\
(\mathrm{mL})\end{array}$ & Sample type & Collection type & $\begin{array}{c}\text { Vessel } \\
\text { type }\end{array}$ \\
\hline 18 & Biochem & $12+$ & 0.8 & Serum & 3-mL Red & 2 -mL \\
\hline
\end{tabular}




\subsection{Labeling Vessels}

The phlebotomist and the technologist assigned to shipping are responsible for labeling vessels and assembling the blood processing racks. Use extreme care in setting up the blood processing racks and in labeling the vessels with the appropriate bar-code label.

Use the following materials to set up and label blood racks:

- Rack

- Age-appropriate processing template

- Storage vessels of the following sizes

- $\quad$ 2.0-mL vessel

- $\quad$ 5.0-mL vessel

- $\quad$ 50-mL conical tube

- Bar-coded SP ID labels, each containing the unique 6-digit SP ID number concatenated with the vessel ID

Prepare one blood processing rack for each SP. The tubes for which each SP is eligible are determined by their sex, appointment type, and inclusion into certain subsamples so each SP's blood processing rack may be unique. When possible, prepare the racks at least 1 day before the SP arrives for the MEC examination.

Before each session, the technologist assigned to workstation 3 prints all labels for all SPs, distributes the labels, and labels the blood processing racks. The correct bar-code labels automatically print for each SP based on the SP's unique profile.

While the technologist assigned to shipping is responsible for labeling the vessels and setting up the blood processing racks, it is the blood processing technologist's responsibility to verify that each vessel has the correct label before filling. Do not rely only upon memory or the positioning of the vessels in a rack to indicate which vessels to fill. 
Always double check:

- The SP's ID number on the bar-code label;

- The vessel number on the label;

- The adhesion quality of the labels; and

- To ensure that specimens are not missing labels or incorrectly labeled.

Save and recycle unused vessels:

- If the SP does not show up for the MEC exam at the appointed date and time, then it is possible that he or she will reschedule;

- Store the SP's labeled vials in a zip closable bag until the end of the stand; and

- If the SP has not shown up by the end of the stand, discard the unused vessels.

\subsection{Equipment}

\subsubsection{Benchtop Centrifuges}

Each laboratory contains two benchtop refrigerated centrifuges.

\section{Beckman GS-6R}

The centrifuge has the following safety features:

- An imbalance detector triggers an automatic shutdown if rotor loads are severely out of balance.

- A safety-lock mechanism and secondary manual latch prohibit opening the centrifuge door during a run until the rotor is virtually stopped.

- A lockout system requires the door to be closed and latched before the centrifuge will start.

- An anchoring system secures the centrifuge in place. Anchoring systems reduce the possibility of injury or damage that could result from centrifuge movement in case of a major rotor mishap. 
The control panel is mounted at an angle on the centrifuge front for easy visibility and access. The control panel has the following features:

- $\quad$ Power - This rocker switch, labeled I (ON), and O (OFF), controls electrical power to the centrifuge. The chamber door cannot be opened unless the power is turned ON.

- Door - When pressed to the OPEN position, this switch releases the latch mechanism and allows the door to be opened when the secondary manual latch lever is switched to UNLOCK (the rotor must be stopped and the power switched on).

- Brake - This switch is used to select the brake mode used during deceleration. Set the position to HIGH or LOW. The HIGH brake position activates the dynamic and the electromechanical disk braking. Dynamic braking starts immediately after the timer turns the rotor off. At 1,600 rpm the disk brake engages and remains on until the speed reaches $400 \mathrm{rpm}$, followed by dynamic braking alone to $50 \mathrm{RPM}$. At $50 \mathrm{rpm}$, all braking stops and the rotor coasts to a stop.

- Time - This knob may be set for any run time between 2 and 30 minutes in 1-minute increments. During a run, the timer rotates back to zero, at which point the centrifuge begins declaration. A HOLD setting enables longer-duration runs - the centrifuge will run until turned off. Stop runs at any time by turning the knob to OFF.

For plasma and serum - set appropriate time and temperature for each tube as follows:

\begin{tabular}{|c|c|c|}
\hline 3-mL, 4-mL, or 7-mL EDTA & 10 minutes & $17-25^{\circ} \mathrm{C}$ or $4-8^{\circ} \mathrm{C}$ \\
\hline 2-mL gray & 10 minutes & $17-25^{\circ} \mathrm{C}$ or $4-8^{\circ} \mathrm{C}$ \\
\hline 3-mL, 7-mL, $10-\mathrm{mL}$ or $15-\mathrm{mL}$ red & 15 minutes & $17-25^{\circ} \mathrm{C}$ \\
\hline
\end{tabular}

For washed packed cells - set appropriate time and temperature for each tube as follows:

\begin{tabular}{|c|c|c|}
\hline Vessel 88 & 3 or 4 -mL EDTA & 5 minutes \\
\hline Vessel 126 & 3 or $4-m L$ EDTA & 5 minutes \\
\hline
\end{tabular}

- Speed and ACCU-SET - Use this knob to set the drive speed, in 200-rpm increments, up to $8,000 \mathrm{rpm}$. For precise adjustments, the ACCU-SET button can be pressed as the speed knob is turned until the desired speed appears on the RPM display. ACCU-SET can be adjusted at any time during a run.

For plasma and serum - set the speed at 2,900 rpm.

For washed packed cells vessel 88 - set the speed at 1,985 rpm.

For washed packed cells vessel 126 - set the speed at 3,700 rpm. 
- RPM - During the run, the display indicates the actual rotor speed to the nearest increment of $10 \mathrm{rpm}$.

- Temperature - Use this knob to set operating temperature. The dial is marked from -10 to $+40^{\circ} \mathrm{C}$ in $1^{\circ} \mathrm{C}$ increments. Set the refrigerated centrifuge at $4^{\circ} \mathrm{C}$ and the room temperature centrifuge at $22^{\circ} \mathrm{C}$. The display indicates the temperature $\left(+/-2^{\circ} \mathrm{C}\right)$ within the bowl in increments of $1^{\circ} \mathrm{C}$.

\section{Run Procedure:}

1. Press the POWER to switch to ON.

2. Move the door latch lever to UNLOCK. Press the DOOR switch to OPEN and lift the centrifuge door up; it will remain in the open position.

3. Load the Aerosolve canisters symmetrically. If only two canisters are being run, place the canisters in opposite buckets; place two empty buckets for balance in the other two buckets. If only three canisters are required, balance tubes in an empty canister across from third canister.

4. Close the centrifuge door and push firmly down on it until hearing a clicking or latching sound. Move the secondary latch lock to LOCK position.

5. Set the TEMPERATURE knob to the required run temperature.

6. Set the run speed using the following procedure:

- $\quad$ Press the small ACCU-SET button in; rotate the SPEED dial until the required speed shows on the display.

- While holding the ACCU-SET button in, rotate the SPEED dial until the required speed shows on the display.

- $\quad$ When the selected speed is displayed, release the ACCU-SET button.

7. Select the HIGH brake position for the EDTA, gray, and blue top tubes and select the LOW brake for the red top tubes.

8. Turn the timer to the required time. The centrifuge will start. As the run begins, acceleration control protects delicate gradients; when the rotor speed reaches about $200 \mathrm{rpm}$, full acceleration to set speed occurs.

9. To end a run in progress for any reason: Turn the timer knob to OFF. The door interlock system keeps the door latched until the rotor has virtually stopped. 
10. After the rotor stops spinning, move the secondary latch lever to UNLOCK and press OPEN switch on the control panel to open the door.

11. After completing a run, unload the canisters.

Leave the door open to prevent chamber icing.

\section{Allegra X15R}

The centrifuge has the following safety features:

- An imbalance detector triggers an automatic shutdown if rotor loads are severely out of balance.

- A safety-lock mechanism and secondary manual latch prohibit opening the centrifuge door during a run until the rotor is virtually stopped.

- A lockout system requires the door to be closed and latched before the centrifuge will start.

- An anchoring system secures the centrifuge in place. Anchoring systems reduce the possibility of injury or damage that could result from centrifuge movement in case of a major rotor mishap.

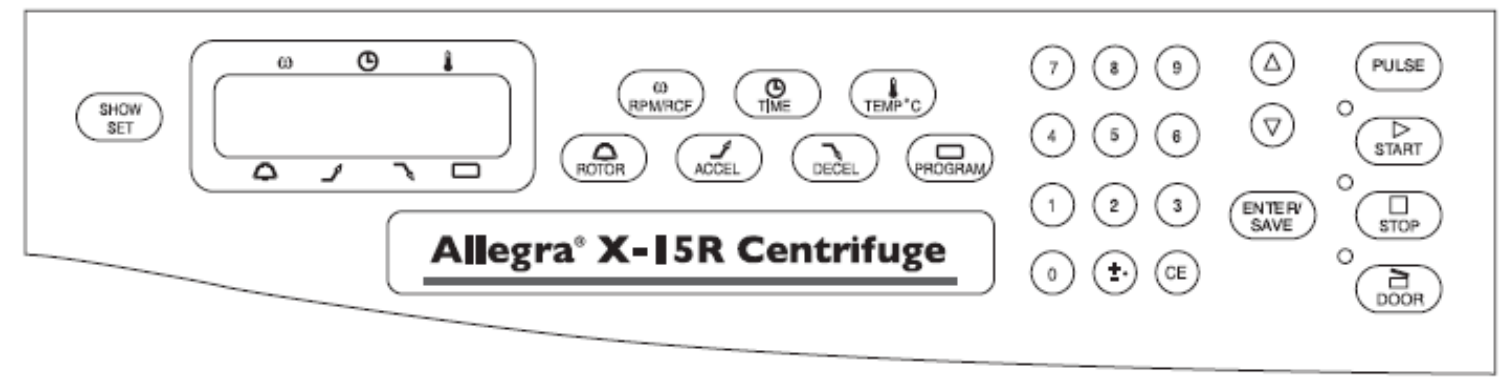




\section{Control Panel:}

The control panel is mounted at an angle on the centrifuge front for easy visibility and access. The control panel has the following features:

\section{Digital Displays}

The displays provide run information, user messages, and diagnostics. During a run, they show the actual (real-time) operating conditions.

$\boldsymbol{\omega}$ (SPEED)

() (TIME)

(TEMPERATURE)

$\Delta$ (ROTOR)

$\boldsymbol{\rho}(\mathrm{ACCEL})$

$\checkmark(\mathrm{DECEL})$

$\square($ PROGRAM)

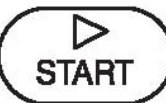

STOP

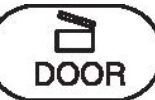

Indicates rotor speed in revolutions per minute (rpm)

In a timed run, indicates the remaining run time in hours and minutes.

Indicates rotor temperature in degrees $\mathrm{C}$ at thermoequilibrium.

Displays the name of the selected rotor.

When the ACCEL key is pressed, one of ten available acceleration rates can be entered. Displays the selected acceleration rate throughout the run.

When the (DECEL) key is pressed, one of eleven available deceleration rates can be entered. Displays the selected deceleration rate throughout the run.

During setup-when the PROGRAM) key is pressed, a program number (one of ten possible) can be selected and program parameters entered.

During centrifugation — the number of the program being run is displayed.

Pressing ENTER/SAVE, then START) begins the run. (START) must be pressed within 5 seconds of pressing ENTER/SAVE.) This key combination can also be used to abort a deceleration process and restart the centrifuge.

Can be pressed at any time while the rotor is spinning to terminate a run. The centrifuge decelerates to a complete stop according to the deceleration setting. The green light next to the key flashes while the rotor decelerates.

Unlocks and opens the door. (The instrument will not accept the unlock command if the rotor is spinning.) 


\section{Time}

For plasma and serum - set appropriate time and temperature for each tube as follows:

- $\quad$ 3-mL, 4-mL, or 7-mL EDTA

- $\quad$ 2-mL gray

- $\quad$ 3-mL, 7-mL, 10-mL or 15-mL red
10 minutes $\quad 17-25^{\circ} \mathrm{C}$ or $4-8^{\circ} \mathrm{C}$

10 minutes $\quad 17-25^{\circ} \mathrm{C}$ or $4-8^{\circ} \mathrm{C}$

15 minutes $\quad 17-25^{\circ} \mathrm{C}$

For washed packed cells - set appropriate time and temperature for each tube as follows:

- $\quad$ Vessel $88 \quad 3$ or 4-mL EDTA 5 minutes $\quad 4-8^{\circ} \mathrm{C}$

- $\quad$ Vessel $126 \quad 3$ or 4-mL EDTA 5 minutes $\quad 4-8^{\circ} \mathrm{C}$

\section{Speed}

For plasma and serum - set the speed at 2,900 rpm.

For washed packed cells vessel 88 - set the speed at 1,985 rpm.

For washed packed cells vessel 126 - set the speed at 3,700 rpm.

\section{Run Procedure:}

\section{MANUAL RUN}

\begin{tabular}{|c|c|c|}
\hline 1 & POWER & Turn the power switch on (I). \\
\hline 2 & (DOOR) & Press (DOOR) to open the chamber door; lift the door open. \\
\hline 3 & & $\begin{array}{l}\text { Install the rotor according to the applicable rotor manual, then close } \\
\text { the chamber door. }\end{array}$ \\
\hline 4 & ROTOR & $\begin{array}{l}\text { Press ROTOR, then press ROTOR again or use the arrow keys to } \\
\text { select the rotor. }\end{array}$ \\
\hline 5 & RPM/RCF & $\begin{array}{l}\text { Press } R P M / R C F \text {, then use the keypad to enter the run speed. Press } \\
\text { RPM/RCF a second time to toggle between RPM and RCF modes, } \\
\text { RPM/RCF RPM/RCF. }\end{array}$ \\
\hline
\end{tabular}




\begin{tabular}{|c|c|c|}
\hline 6 & (TIME) & $\begin{array}{l}\text { Press (TIME), then use the keypad to enter the run time (to } 99 \text { hrs, } \\
59 \text { minutes); or press (TIME) twice to toggle between hold } \\
\text { (continuous) run and set time mode. }\end{array}$ \\
\hline 7 & TEMP ${ }^{\circ} \mathrm{C}$ & $\begin{array}{l}\text { Press } \text { TEMP }^{\circ} \mathrm{C} \text {, then use the keypad to enter the required run } \\
\text { temperature }-10 \text { to }+40^{\circ} \mathrm{C} \text {. TEMP }{ }^{\circ} \mathrm{C}\end{array}$ \\
\hline 8 & ACCEL & $\begin{array}{l}\text { Press ACCEL), then use the keypad or arrow keys to enter the } \\
\text { selected acceleration rate number, } 1 \text { (SLOW) through } 10 \text { (MAX). }\end{array}$ \\
\hline 9 & (DECED & $\begin{array}{l}\text { Press (DECEL), then use the keypad or arrow keys to enter the } \\
\text { selected deceleration rate number, } 0 \text { (OFF) through } 10 \text { (MAX). }\end{array}$ \\
\hline 10 & $\begin{array}{l}\text { ENTER/SAVE } \\
\text { START }\end{array}$ & $\begin{array}{l}\text { Check that all parameters are correct and that the door is closed } \\
\text { (door LED will be lit). Press ENTER/SAVE, then press START) } \\
\text { (within } 5 \text { seconds). ENTER/SAVE }\end{array}$ \\
\hline 11 & STOP & $\begin{array}{l}\text { Wait for the set time to count down to zero, or end the run by } \\
\text { pressing STOP. }\end{array}$ \\
\hline 12 & (DOOR) & $\begin{array}{l}\text { When the rotor stops (a tone sounds and the (DOOR) LED lights), } \\
\text { press (DOOR) to open the chamber door; lift the door. }\end{array}$ \\
\hline
\end{tabular}

\section{PROGRAMMED RUN}

\begin{tabular}{|c|c|c|}
\hline 1 & POWER & Turn the power switch on (I). \\
\hline 2 & (DOOR) & Press (DOOR) to open the chamber door. \\
\hline 3 & & $\begin{array}{l}\text { Install the rotor according to the applicable rotor manual, then } \\
\text { close the chamber door. }\end{array}$ \\
\hline 4 & PROGRAM & $\begin{array}{l}\text { Press PROGRAM, use the keypad or arrow keys to enter the } \\
\text { required program number, then press ENTER/SAVE. }\end{array}$ \\
\hline 5 & $\begin{array}{l}\text { ENTER/SAVE } \\
\text { START }\end{array}$ & $\begin{array}{l}\text { Check that all parameters are correct and that the door is closed } \\
\text { (door LED will be lit). Press ENTER/SAVE, then press (START) } \\
\text { (within } 5 \text { seconds). }\end{array}$ \\
\hline 6 & STOP & Wait for the run to end, or end the run by pressing (STOP). \\
\hline 7 & (DOOR) & $\begin{array}{l}\text { When the rotor stops (a tone sounds and the (DOOR) LED } \\
\text { lights), press (DOOR) to open the chamber door; lift the door. }\end{array}$ \\
\hline
\end{tabular}

\subsubsection{Laminar Flow Biological Safety Cabinet Operating Sequence}

- Turn on the cabinet blower and lights, and check the air intake and exhaust portals of the cabinet to make sure they are unobstructed.

- Check the pressure on the magnehelic gauge. If the pressure has increased more than 0.1 w.g. since the previous reading, notify the biomedical engineer. Record the pressure reading and any comments in the Laboratory QC module. 
- Allow the blowers to operate for a minimum of 15 minutes before beginning work in the cabinet.

- Disinfect the interior of the workspace by wiping it down with a 10 percent solution of bleach and water (1:10).

- Place a disposable paper blotter on the work surface. Set up the equipment for processing. Do not place any equipment or supplies over the front intake grills.

- Arrange and separate clean and contaminated materials.

- Wait 2 to 3 minutes after placing the materials and supplies in the cabinet before beginning work. This will rid the area of all "loose" contamination.

- Wear a long-sleeved laboratory coat and gloves. This will minimize the shedding of skin flora into the work area and concurrently protect hands and arms from viable agent contamination. Wash hands with soap and water before and after working in the cabinet.

- After completing work, leave the blower on for 2 to 3 minutes to purge the unit.

- Decontaminate interior surfaces after removal of all specimens and biohazard waste by wiping them with a 10 percent solution of bleach and water (1:10).

- Check the grills and diffuser vents for spills or splashes and clean using appropriate techniques and materials.

\subsection{Blood Processing Procedures}

Centrifuge all blood specimens unopened, then transport to, and uncap and process in the laminar flow hood. The laminar flow hood provides a HEPA filtered, recirculated mass airflow within the workspace, and protects from any spattering that may occur when opening the Vacutainer ${ }^{\circledR}$ tubes or when pouring or pipetting the specimens. It also prevents drawing contaminated air or dust over the specimens. It is mandatory to use the blower.

Always wear gloves when handling specimens. Check gloves for small holes or tears. Change gloves if they become visibly contaminated with blood. Immediately soak visibly contaminated racks in 1:100 bleach solution.

Decontaminate racks and templates by soaking them in 10 percent bleach solution at the end of each session. Place on blotter to air dry. 


\subsubsection{Ependorf Research Pro Pipettes}

Pipette whole blood and pooled serum and make dilutions using the Eppendorf Research pro pipettes. Pipette plasma using plastic transfer pipettes.

The MEC laboratory has four Eppendorf Research pro electronic pipettes: one 5-100 $\mu \mathrm{L}$, two 50-1,000 $\mu \mathrm{L}$, and one 100-5,000 $\mu \mathrm{L}$. The lab also uses $100 \mu \mathrm{L}$ and 1,000 $\mu \mathrm{L}$ Biopur pipette tips and 5-mL bulk pipette tips. Charge the pipettes in the 4-stand charger.

- Use the one 5-100 $\mu \mathrm{L}$ pipette in the standard (PIP) mode and set the volume to 100 $\mu \mathrm{L}$. Use this dedicated pipette to aliquot the whole blood for RBC Folate - vessel 3 and RBC Fol1 - vessel 106.

- Use one of the two $50-1,000 \mu \mathrm{L}$ pipettes in the standard (PIP) mode and set the volume to $1,000 \mu \mathrm{L}$. Use this dedicated pipette to aliquot the ascorbic acid for RBC Folate - vessel 3 and RBC Fol1 - vessel 106.

- Use the second 50-1,000 $\mu \mathrm{L}$ pipette in the FIX mode. Set the five fixed volumes to $300 \mu \mathrm{L}, 400 \mu \mathrm{L}, 500 \mu \mathrm{L}, 600 \mu \mathrm{L}$, and $1,000 \mu \mathrm{L}$. Use this pipette to aliquot all whole blood tests, except RBC Folate - vessel 3 and RBC Fol1 - vessel 106 and pooled serum volumes that match the five volume settings.

- Use the second $50-1,000 \mu \mathrm{L}$ pipette and the $100-5,000 \mu \mathrm{L}$ pipette in the DIS mode. Set the DIS volume on the $50-1,000 \mu \mathrm{L}$ to $100 \mu \mathrm{L}$. Set the DIS volume on the 100$5,000 \mu \mathrm{L}$ to $500 \mu \mathrm{L}$. Use these pipettes to aliquot pooled serum.

- Use the $100-5,000 \mu \mathrm{L}$ pipette in the FIX mode. Set the five fixed volumes to 1,000 $\mu \mathrm{L}, 2,000 \mu \mathrm{L}, 3,000 \mu \mathrm{L}, 4,000 \mu \mathrm{L}$, and $5,000 \mu \mathrm{L}$. Use this pipette to aliquot pooled serum volumes that match the five volume settings.

- Use the $100-5,000 \mu \mathrm{L}$ pipette in the ADS mode. Program this mode in slot 2. Set the volume to $500 \mu \mathrm{L}$ and the interval to 1 second. Use this pipette to aliquot pooled serum.

- Use the $100-5,000 \mu \mathrm{L}$ pipette in the SD mode. Set the volumes to match the agespecific processing protocols. Set program 1 to match the adult profile for vessels 8175. Set program 3 to match the child's profile ages $1-2$. Set program 4 to match the child's profile ages 3-5. Set program 5 to match the child's profile ages 6-11. Use this pipette to aliquot pooled serum. 


\begin{tabular}{|c|c|c|c|c|}
\hline Pipette & Tip & Mode & Volume & Matrix \\
\hline *5-100 $\mu \mathrm{L}$ & $200 \mu L$ & $P I P$ & $100 \mu \mathrm{L}$ & Whole blood \\
\hline$* 50-1,000 \mu L$ & $1,000 \mu \mathrm{L}$ & $P I P$ & $1,000 \mu \mathrm{L}$ & Ascorbic acid \\
\hline $50-1,000 \mu L$ & $1,000 \mu L$ & FIX & $\begin{array}{l}300 \mu \mathrm{L} \\
400 \mu \mathrm{L} \\
500 \mu \mathrm{L} \\
600 \mu \mathrm{L} \\
1,000 \mu \mathrm{L}\end{array}$ & $\begin{array}{l}\text { Whole blood } \\
\text { Pooled serum }\end{array}$ \\
\hline $50-1,000 \mu \mathrm{L}$ & $1,000 \mu \mathrm{L}$ & DIS & $100 \mu \mathrm{L}$ & $\begin{array}{l}\text { Whole blood } \\
\text { Pooled serum }\end{array}$ \\
\hline $100-5,000 \mu \mathrm{L}$ & $5,000 \mu \mathrm{L}$ & FIX & $\begin{array}{l}1,000 \mu \mathrm{L} \\
2,000 \mu \mathrm{L} \\
3,000 \mu \mathrm{L} \\
4,000 \mu \mathrm{L} \\
5,000 \mu \mathrm{L}\end{array}$ & Pooled serum \\
\hline $100-5,000 \mu \mathrm{L}$ & $5,000 \mu \mathrm{L}$ & DIS & $500 \mu \mathrm{L}$ & Pooled serum \\
\hline $100-5,000 \mu \mathrm{L}$ & $5,000 \mu \mathrm{L}$ & $A D S$ & $\begin{array}{l}500 \mu \mathrm{L} \\
1 \text { second }\end{array}$ & Pooled serum \\
\hline $100-5,000 \mu L$ & $5,000 \mu L$ & $S D$ & $\begin{array}{l}\text { Program } 1-12+ \\
\text { Program } 3-\text { Child 1-2 } \\
\text { Program } 4-\text { Child 3-5 } \\
\text { Program } 5 \text { - Child 6-11 }\end{array}$ & Pooled serum \\
\hline
\end{tabular}

Italic $=$ Pipette's primary program

* Dedicated pipette for RBC Folate dilutions

\subsubsection{Safety Precautions, Handling, Care, Maintenance, and Battery for the Research Pro}

Before using the Research pro for the first time, please read the entire Eppendorf Research Pro Instruction Manual. Observe the following points:

\section{Handling}

- Use the Research pro only when a pipette tip is attached.

- Do not lay down the Research pro when a filled pipette tip is attached.

- After liquid has been aspirated, press the Actuate key only when you are sure the liquid will be dispensed correctly. 
- When the pipette is switched on and when the Reset key is pressed, the piston may move. Please ensure beforehand that the liquid in the pipette tip will be dispensed correctly.

- Emergency stop: pressing the Reset key can stop a moving piston.

- Operate the Research pro at temperatures between $15^{\circ} \mathrm{C}$ and $40^{\circ} \mathrm{C}$. Operate at a maximum humidity of 80 percent at a temperature up to $31^{\circ} \mathrm{C}$.

\section{Care and Maintenance}

- Do not clean the display or any of the labeling using acetone or aggressive solutions.

- Do not allow any liquid to enter the device.

- Only authorized service personnel may carry out repairs.

- Use original spare parts and accessories (battery, pipette tips) only.

\section{Battery}

- Charge the battery before operating the device for the first time.

- Charge the battery in the pipette only, using the charging stand and the original power unit.

- If a flashing battery symbol appears in the display, stop dispensing and charge the battery.

- When charging the battery, do not allow the battery to be exposed to any metallic surfaces.

- Dispose of used batteries as special waste.

- Never charge the Research pro without a battery.

- Before storing the Research pro for a long period, remove the battery. 


\subsubsection{Technical Data for the Research Pro}

Single Channel Pipette

\begin{tabular}{|c|c|c|c|c|c|c|}
\hline $\begin{array}{c}\text { Volume } \\
\text { Range } \mu \mathrm{L}\end{array}$ & $\begin{array}{c}\text { Step } \\
\text { size } \mu \mathrm{L}\end{array}$ & $\begin{array}{l}\text { Color } \\
\text { code }\end{array}$ & $\begin{array}{l}\text { Pipette } \\
\text { tip }\end{array}$ & $\begin{array}{c}\text { Test } \\
\text { volume }\end{array}$ & $\begin{array}{c}\text { Inaccuracy } \\
\%\end{array}$ & $\begin{array}{c}\text { Imprecision } \\
\%\end{array}$ \\
\hline 5-100 & 0.1 & Yellow & $200 \mu \mathrm{L}$ & $100 \mu \mathrm{L}$ & \pm 0.8 & $\leq 0.2$ \\
\hline $50-1,000$ & 1 & Blue & $\begin{array}{c}1,000 \\
\mu \mathrm{L}\end{array}$ & $\begin{array}{c}1,000 \\
\mu \mathrm{L}\end{array}$ & \pm 0.6 & $\leq 0.2$ \\
\hline $100-5,000$ & 10 & Violet & 5-mL & $\begin{array}{c}500 \mu \mathrm{L} \\
5,000 \\
\mu \mathrm{L}\end{array}$ & $\begin{array}{l} \pm 3.0 \\
\pm 0.6\end{array}$ & $\begin{array}{l}\leq 0.6 \\
\leq 0.15\end{array}$ \\
\hline
\end{tabular}

The technical data are valid only when the Research pro is used with Eppendorf pipette tips.

- Test conditions in accordance with DIN 12650 for piston-stroke pipettes with an air cushion by means of a standardized fine balance with a moisture trap

- Number of determination: 10

- Degassed distilled water, $20^{\circ} \mathrm{C}-25^{\circ} \mathrm{C} \pm 0.5^{\circ} \mathrm{C}$

- Maximum speed

- Standard operation

- Prewetted pipette tip, dispensing onto inside of tube due to possible evaporation

- Check volumes $\leq 10 \mu \mathrm{L}$ immediately after aspiration

\section{Battery}

- Nickel-metal hydride battery pack, $1,200 \mathrm{mAh} / 2.4 \mathrm{~V}$ with reversible overcurrent and overtemperature protection

- Charging time: approximately 9.5 hours for a fully discharged battery; overcharging protection with the pipette 


\section{Power Unit}

- Input voltage, country-specific: $230 \mathrm{~V} \pm 10 \%, 50 / 60 \mathrm{~Hz} ; 115 \mathrm{~V} \pm 10 \%, 60 \mathrm{~Hz} ; 100 \mathrm{~V}$ $\pm 10 \%, 50 / 60 \mathrm{~Hz}$.

- $\quad$ Output voltage: 9 V DC; 200 mA (1.8 VA)

\section{Delivery Package}

The Research pro is supplied as a single-channel pipette for different volume ranges. The pipette is delivered without a charging adapter, but each lab is supplied with a charging stand. Each package contains:

- Charging stand with separate power unit;

- Special tool(s) according to pipette type;

- Operating manual with measuring protocol and CE conformity assurance document; and

- Ni-MH battery pack.

\section{Inserting the Battery}

Charge the battery in the pipette. Charging may only be carried out using the charging stand with the original power unit. Do not try to recharge a pipette if the battery is not installed. A new battery does not attain its full capacity until two or three complete charging/discharging cycles have been completed.

For charging purposes, place the pipette in the charging stand.

During the start and termination of the charging process, "CON" appears briefly on the left side of the display. The display is switched on during the charging process. The battery symbol appears in a rolling form. During charging, the dispensing function is frozen. When the charging process has finished, the battery symbol appears in the display without moving. When the pipette has been fully charged, it may remain in the charging stand. To increase the charging capacity, the battery should be 
discharged until the flashing battery symbol appears. To avoid accidental discharge, remove the battery from the Research pro during extended periods of nonuse. In this case, all data remain stored.

\subsubsection{Operating Principle}

Display and Keypad

- To switch on: Press any key firmly.

- To switch off: Unit does this automatically after 10 minutes of nonuse.
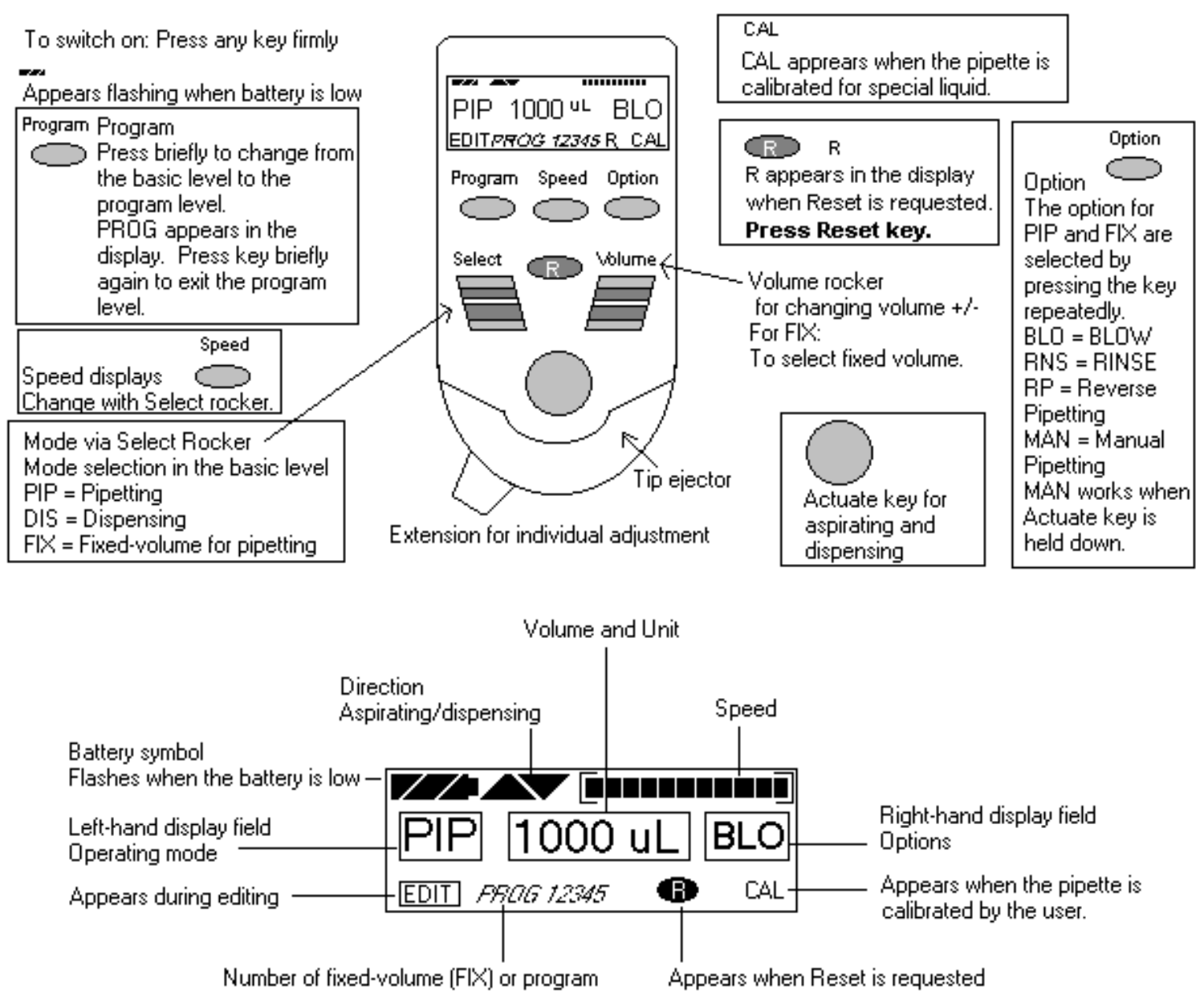


\subsubsection{Functions of the Unit}

The Research pro is a microprocessor-controlled pipette, which executes the piston movement with the aid of a stepper motor. The power supply is a rechargeable nickel-metal hydride battery.

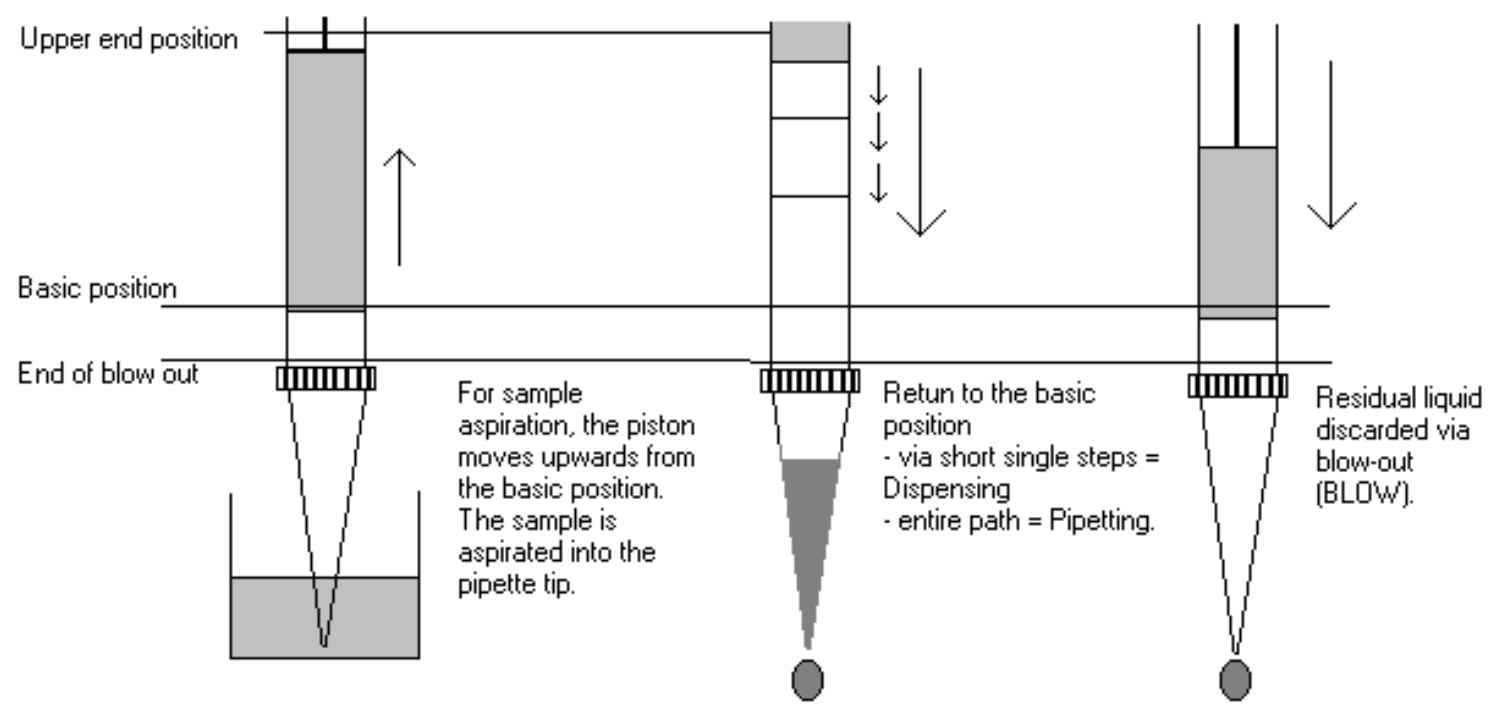

Dispensing and reverse pipetting require a different basic position than that for pipetting. The request to change the basic position is accomplished by selecting the Reset button.

\section{Ejector with Extension}

The force translation of the ejector for pipette tips ensures that the tips are ejected very easily. The extension can be individually adjusted to accommodate left- and right-handed users as well as different hand sizes. Select the desired setting by simply sliding the extension into the position as required. 


\section{Hook on the Hand Rest}

Loosening the screw enables the hook on the rear of the pipette to be adjusted to suit all hand sizes. This can be done easily with a small Phillips screwdriver.

\section{Contact Surface on the Read of the Pipette}

Both upper surfaces are required for charging the nickel-metal hydride battery.

The lower surfaces are the data interfaces for the service department.

The contact to the charging adapter or the charging stand cannot be confused with any other contacts.

\section{Acoustic Signals}

Certain signals assist the user for different operations:

- Short signal to acknowledge that the keypad has been pressed;

- Slightly higher tone to indicate that the function for which the key has been pressed cannot be executed;

- Louder tone to indicate that the piston movement has ended;

- Louder tone to indicate that a specific sequence of steps (e.g., dispensing) has ended; and

- Louder tone to indicate an error message.

The acoustic signals are a great help when you are familiarizing yourself with the operating procedure of the Research pro. They can be switched off if required. 


\subsubsection{Mode of Operation}

If the display of the Research pro is dark, activate the pipette by pressing any key firmly. If the pipette has not been used for a long period, a motor reset is executed after it has been activated.

- Attach an Eppendorf pipette tip to the Research pro. The color coding of the Actuate key corresponds to the color code of the racks for the pipette tips. The dispensing liquid is aspirated into the tip.

- When aspirating liquid, immerse the pipette tip vertically - and as little as possible into the liquid. Following aspiration, remove the pipette tip from the liquid after the acoustic signal has been emitted. If the air bubbles have been aspirated, repeat this process.

- Never lay the pipette down when the tip is filled.

- To dispense the liquid, position the pipette tip against the side of the aspirating vessel.

- Discard the pipette tip by pressing the Eject key, attach a new tip, and reaspirate liquid.

\section{Essential Operating Information}

The following sections contain systematic explanations of the operating procedure. It is essential to work through these sections with the pipette in your hand.

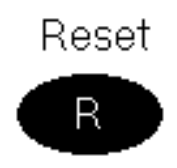

If a Reset is requested in the display, a piston movement always accompanies this Reset request. For this reason, please ensure that any residual liquid in the pipette tip is dispensed beforehand. A Reset can also be used to empty the pipette tip and to end an operating process. If you quit an input field during programming (e.g., changing the speed) using Reset, the changes that have been made are not stored. The Reset button can also act as an emergency stop function.

If the Reset key $(\mathbf{R})$ is held down until the piston movement begins and $\mathrm{R}$ that the piston movement is correct. This reset lasts for approximately 10 seconds. 
Please refer to the following illustrations when going through each operation.

The Research pro has two operating levels. The basic modes for pipetting (PIP and FIX) and (DIS) are accessed in the first level by pressing the Select rocker. PROG appears in the display by pressing the "Program" key. You are now in the second level. Using the Select rocker, select from the five available program slots. To quit this level, press the "Program" key briefly and PROG will disappear from the display.

If the piston movements appear in the display without any volumes, this indicates a process that is not connected to liquid aspiration or dispensing.

\section{Aspirating and Dispensing Speeds}

The selected speed can be viewed by pressing the Speed key several times. The speed is changed as follows:
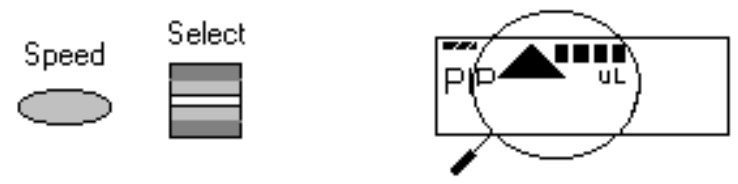

Display and selection of aspirating speed.

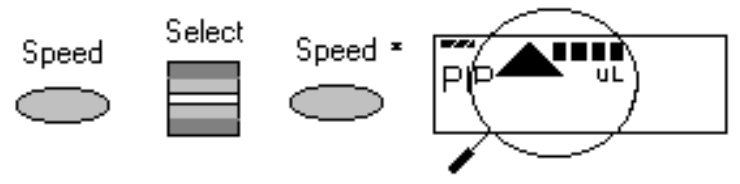

Display and selection of dispensing speed.

- This procedure does not have to be completed using the Speed key. Any key, with the exception of Reset, may be used. At the same time, pressing the key executes the respective key function.

- The direction of the arrow for speed flashes in this input field. 
- The speed can be altered before every dispensing or aspiration operation.

- Important - High speeds have a much lower current consumption than low speeds.

8.7.1.6 Pipetting in the Standard Mode (PIP) (Use this mode for whole blood RBC folate and Vitamin C [5-100 $\mu \mathrm{L}$ pipette] and ascorbic acid [50-1,000 $\mu \mathrm{L}$ pipette].)

In the standard mode, blowout is executed automatically when liquid is dispensed. The standard mode is recommended for rapid series pipetting with aqueous solutions.

\begin{tabular}{|c|c|}
\hline \multicolumn{2}{|c|}{ Programming - PIP } \\
\hline Step 1 & $\begin{array}{l}\text { Select the pipetting mode "PIP." Use the Select rocker switch to move up } \\
\text { and down through the three modes (PIP, DIS, and FIX) until the "PIP" } \\
\text { choice appears in the left side of the display. } \\
\text { (If PROG appears in the display: Press the Program key briefly until PROG } \\
\text { disappears.) }\end{array}$ \\
\hline Step 2 & $\begin{array}{l}\text { Select the volume. Use the Volume rocker to move the number up or down } \\
\text { until the number (volume) } 100 \text { for the } 5-100-\mu \mathrm{L} \text { pipette and } 1,000 \mu \mathrm{L} \text { for the } \\
50-1,000-\mu \mathrm{L} \text { pipette is reached. If necessary, press the Option key several } \\
\text { times until the right side of the display is blank. (Do not use the BLO, RNS, } \\
\text { RP, or MAN options.) }\end{array}$ \\
\hline \multicolumn{2}{|c|}{ Pipetting Procedure } \\
\hline Step 1 & $\begin{array}{l}\text { Aspirate the liquid. Make sure the Aspirating arrow in the display points up. } \\
\text { Immerse the tip of the pipette into the liquid and press the Actuate key to } \\
\text { draw the liquid into the pipette tip. }\end{array}$ \\
\hline Step 2 & $\begin{array}{l}\text { Place the pipette tip against the side of the receiving vessel. Make sure the } \\
\text { Dispensing arrow in the display points down. To dispense the liquid with a } \\
\text { blowout, press the Aspirate button. }\end{array}$ \\
\hline Step 3 & $\begin{array}{l}\text { The pipette is ready to aspirate again if the Aspirating arrow in the display } \\
\text { points up. }\end{array}$ \\
\hline
\end{tabular}




\subsubsection{Pipetting with a Preselected Fixed (FIX) Volume (Use this mode for aliquoting whole}

blood volumes 300, 400, 500, 600, and 1,000 $\mu \mathrm{L}$ and for $400 \mu \mathrm{L} M P A-50-1,000 \mu L$ pipette, and for pipetting pooled serum volumes 1,000, 2,000, 3,000, 4000, and 5,000 $\mu \mathrm{L}-100$ $5,000 \mu L$.)

FIX is used to rapidly set and recall five frequently used pipette volumes. Five FIX volumes are predefined in the pipette. FIX volumes can be changed via programming.

\begin{tabular}{|c|c|}
\hline \multicolumn{2}{|c|}{ Programming FIX fixed volumes } \\
\hline Step 1 & $\begin{array}{l}\text { Select the pipetting mode "FIX." Use the Select rocker switch to move up } \\
\text { and down through the three modes (PIP, DIS, FIX) until the "FIX" choice } \\
\text { appears in the display. } \\
\text { (If PROG appears in the display: Press the Program key briefly until PROG } \\
\text { disappears.) }\end{array}$ \\
\hline Step 2 & $\begin{array}{l}\text { Hold down the Program key until EDIT appears in the lower left corner of } \\
\text { the display. The volume flashes. }\end{array}$ \\
\hline Step 3 & $\begin{array}{l}\text { Select the first memory slot using the Select rocker. Use the Select rocker to } \\
\text { move the memory slot number up and down until the \#1 is displayed. }\end{array}$ \\
\hline Step 4 & $\begin{array}{l}\text { Select the volume for memory slot } \# 1 \text {. Use the Volume rocker to move the } \\
\text { number (volume) up and down until the number (volume) } 300 \mu \mathrm{L} \text { for the } 5 \text { - } \\
1,000 \text { - } \mu \mathrm{L} \text { pipette or } 1,000 \mu \mathrm{L} \text { for the } 100-5,000 \mu \mathrm{L} \text { pipette is reached. }\end{array}$ \\
\hline Step 5 & $\begin{array}{l}\text { Select the next (second) memory slot for purposes of changing the volume. } \\
\text { Select the second memory slot using the Select rocker. Use the Select rocker } \\
\text { to move the memory slot number up and down until the } \# 2 \text { is displayed. }\end{array}$ \\
\hline Step 6 & $\begin{array}{l}\text { Select the volume for memory slot } \# 2 \text {. Use the Volume rocker to move the } \\
\text { number (volume) up and down until the number (volume) } 400 \mu \mathrm{L} \text { for the } 5 \text { - } \\
1,000-\mu \mathrm{L} \text { pipette or } 2,000 \mu \mathrm{L} \text { for the } 100-5,000 \mu \mathrm{L} \text { pipette is reached. }\end{array}$ \\
\hline Step 7 & $\begin{array}{l}\text { Select the next (third) memory slot for purposes of changing the volume. } \\
\text { Select the third memory slot using the Select rocker. Use the Select rocker to } \\
\text { move the memory slot number up and down until the \#3 is displayed. }\end{array}$ \\
\hline Step 8 & $\begin{array}{l}\text { Select the volume for memory slot \#3. Use the Volume rocker to move the } \\
\text { number (volume) up and down until the number (volume) } 500 \mu \mathrm{L} \text { for the } 5 \text { - } \\
1,000 \text { - } \mu \mathrm{L} \text { pipette or } 3,000 \mu \mathrm{L} \text { for the } 100-5,000 \mu \mathrm{L} \text { pipette is reached. }\end{array}$ \\
\hline
\end{tabular}




\begin{tabular}{|c|c|}
\hline Step 9 & $\begin{array}{l}\text { Select the next (fourth) memory slot for purposes of changing the volume. } \\
\text { Select the fourth memory slot using the Select rocker. Use the Select rocker } \\
\text { to move the memory slot number up and down until the \#4 is displayed. }\end{array}$ \\
\hline Step 10 & $\begin{array}{l}\text { Select the volume for memory slot \#4. Use the Volume rocker to move the } \\
\text { number (volume) up and down until the number (volume) } 600 \mu \mathrm{L} \text { for the } 5 \text { - } \\
1,000 \text { - } \mu \mathrm{L} \text { pipette or } 4000 \mu \mathrm{L} \text { for the } 100-5,000 \mu \mathrm{L} \text { pipette is reached. }\end{array}$ \\
\hline Step 11 & $\begin{array}{l}\text { Select the next (fifth) memory slot for purposes of changing the volume. } \\
\text { Select the fifth memory slot using the Select rocker. Use the Select rocker to } \\
\text { move the memory slot number up and down until the } \# 5 \text { is displayed. }\end{array}$ \\
\hline Step 12 & $\begin{array}{l}\text { Select the volume for memory slot \#5. Use the Volume rocker to move the } \\
\text { number (volume) up and down until the number (volume) } 1,000 \mu \mathrm{L} \text { for the } 5 \text { - } \\
1,000 \text { - } \mu \mathrm{L} \text { pipette or } 5,000 \mu \mathrm{L} \text { for the } 100-5,000 \mu \mathrm{L} \text { pipette is reached. }\end{array}$ \\
\hline Step 13 & $\begin{array}{l}\text { End the programming. Press the Program key to stop programming. } \\
\text { Alternatively, to stop programming and aspirate the volume of liquid in the } \\
\text { display, press the Aspirate button. EDIT and the flashing volume disappear } \\
\text { from the display. }\end{array}$ \\
\hline \multicolumn{2}{|c|}{ Pipetting Procedure } \\
\hline Step 1 & $\begin{array}{l}\text { Select the correct mode. Use the Select rocker to move up and down through } \\
\text { the three mode choices (PIP, DIS, FIX) until FIX displays. }\end{array}$ \\
\hline Step 2 & $\begin{array}{l}\text { Select one of the five programmed fixed volumes. Select the correct memory } \\
\text { slot. Use the Volume rocker to move the volume number up and down until } \\
\text { the correct choice displays. }\end{array}$ \\
\hline Step 3 & $\begin{array}{l}\text { Aspirate the liquid. Make sure the Aspirating arrow in the display points up. } \\
\text { Immerse the tip of the pipette into the liquid and press the Actuate key to } \\
\text { draw the liquid into the pipette tip. }\end{array}$ \\
\hline Step 4 & $\begin{array}{l}\text { Place the pipette tip against the side of the receiving vessel. Make sure the } \\
\text { Dispensing arrow in the display points down. To dispense the liquid with a } \\
\text { blowout, press the Aspirate button. }\end{array}$ \\
\hline Step 5 & $\begin{array}{l}\text { The pipette is ready to aspirate again if the Aspirating arrow in the display } \\
\text { points up. }\end{array}$ \\
\hline
\end{tabular}


8.7.1.8 Dispensing (DIS) (Use this mode to pipette $100 \mu \mathrm{L}$ whole blood and/or pooled serum - 501,000 $\mu \mathrm{L}$ pipette and $500 \mu \mathrm{L}$ pooled serum 100-5,000 $\mu \mathrm{L}$ pipette.)

During this procedure, the aspirating liquid is dispensed in partial steps. As with "Reverse Pipetting," slightly more liquid is aspirated than is necessary for the sum of the dispensing steps. The maximum number of dispensing steps per filling of the pipette tip is displayed on the right side of the display. The maximum number of dispensing steps is calculated by the pipette and is based on the dispensing volume and the pipette tip. For example, if the dispensing volume is set at $100 \mu \mathrm{L}$ and the pipette tip is $1,000 \mu \mathrm{L}$ then there are 10 dispensing steps. If the dispensing volume is set at $500 \mu \mathrm{L}$ and the pipette tip is $5,000 \mu \mathrm{L}$, then there are also 10 dispensing steps.

\begin{tabular}{|c|c|}
\hline \multicolumn{2}{|c|}{ Programming DIS } \\
\hline Step 1 & $\begin{array}{l}\text { Select the pipetting mode "DIS." Use the Select rocker switch to move up } \\
\text { and down through the three modes (PIP, DIS, FIX) until the "DIS" choice } \\
\text { appears in the display. } \\
\text { (If PROG appears in the display: Press the Program key briefly until PROG } \\
\text { disappears.) }\end{array}$ \\
\hline Step 2 & $\begin{array}{l}\text { Select the volume per dispensing step. Use the Volume rocker to move the } \\
\text { number up or down until the number (volume) } 100 \text { for the } 50-1,000-\mu \mathrm{L} \\
\text { pipette or } 500 \mu \mathrm{L} \text { for the } 100-5,000 \mu \mathrm{L} \text { pipette is reached. }\end{array}$ \\
\hline Step 3 & $\begin{array}{l}\text { Select the aspirating speed using the Speed key and the Select rocker. Press } \\
\text { the Speed key, then use the Select rocker to move the speed indicator all the } \\
\text { way to the right. When finished, press the Speed key. The pipette is ready for } \\
\text { entry of the dispensing speed. }\end{array}$ \\
\hline Step 4 & $\begin{array}{l}\text { Select the dispensing speed. Use the Select rocker to move the speed } \\
\text { indicator all the way to the right. Press the Speed key to exit. }\end{array}$ \\
\hline \multicolumn{2}{|c|}{ Pipetting Procedure } \\
\hline Step 1 & $\begin{array}{l}\text { Select the correct mode. Use the Select rocker to move up and down through } \\
\text { the three mode choices (PIP, DIS, FIX) until DIS displays. }\end{array}$ \\
\hline Step 2 & $\begin{array}{l}\text { Aspirate the liquid. Make sure the Aspirating arrow in the display points up. } \\
\text { Immerse the tip of the pipette into the liquid and press the Actuate key to } \\
\text { draw the liquid into the pipette tip. (If } \mathbf{R} \text { appears in the display: Press the } \mathrm{R} \\
\text { key briefly.) }\end{array}$ \\
\hline
\end{tabular}




\begin{tabular}{|l|l|}
\hline Step 3 & $\begin{array}{l}\text { Place the pipette tip against the side of the first receiving vessel. Make sure } \\
\text { the Dispensing arrow in the display points down. To dispense the first } \\
\text { aliquot, press the Aspirate button. The dispensing step counter (number) on } \\
\text { the right-hand side of the display counts down one number. }\end{array}$ \\
\hline Step 4 & $\begin{array}{l}\text { Place the pipette tip against the side of the second receiving vessel. Make } \\
\text { sure the Dispensing arrow in the display points down. To dispense the second } \\
\text { aliquot, press the Aspirate button. The dispensing step counter (number) on } \\
\text { the right side of the display counts down one more number. }\end{array}$ \\
\hline Step 5 & $\begin{array}{l}\text { Continue dispensing until the dispensing step number reaches 0. A special } \\
\text { acoustic signal is emitted. }\end{array}$ \\
\hline Step 6 & $\begin{array}{l}\text { Complete the "blank" step. Press the Actuate key to perform the blank step. } \\
\text { This is not a dispensing step. A special acoustic signal is emitted. }\end{array}$ \\
\hline Step 7 & $\begin{array}{l}\text { Discard the last drop. Press the Actuate key to blow out the last drop. This is } \\
\text { not a dispensing step. Dashes replace the volume number in the display. If } \\
\text { the Actuate key is held down during the final blowout, the device does not } \\
\text { return the Aspiration arrow to the up position until the key is released. }\end{array}$ \\
\hline Step 8 & $\begin{array}{l}\text { The pipette is ready to aspirate again if the Aspirating arrow in the display } \\
\text { points up and the volume number and dispensing step display. }\end{array}$ \\
\hline
\end{tabular}




\subsubsection{Notes on the Programs}

- To reach the programming level, press the Program key briefly.

- $\quad$ PROG appears in the display together with a memory slot number from 1 to 5 .

- With the aid of the Select rocker, five programs can be shown on the left side of the display.

- To program the five memory slots, it is possible to select from seven different program sequences:

1. $\quad$ PIP $=$ Pipetting

As per PIP outside program level. The complete sequence with volume, option, and speeds is programmed in one memory slot.

2. $\quad \mathrm{SP}=$ Sequential Pipetting

Up to five different pipette steps can be linked to each other. Volume, option, and speeds are defined for each pipetting sequence.

3. $\quad$ DIS $=$ Dispensing

As per DIS outside the program level. The complete procedure is programmed in one memory slot with dispensing volume, dispensing steps, and speeds.

4. $\quad \mathrm{SD}=$ Sequential Dispensing

Up to 20 dispensing steps can be defined. Each dispensing step may have a different partial volume. The total volume may exceed the filling level of a pipette tip. This means that new liquid for the pipette tip must be aspirated during the dispensing procedure. Only one aspirating and dispensing speed is defined for all dispensing steps.

5. $\quad$ ADS $=$ Automatic Dispensing

With the Actuate key held down, all dispensing strokes are executed automatically with the same volume and in a user-defined rhythm (.01-10.0 seconds). Automatic dispensing may be interrupted by releasing the Actuate key. Apart from this, the procedure is identical to the DIS function.

6. $\quad$ DIL $=$ Diluting

During the diluting process, a diluent, then an air bubble, and finally a sample are aspirated into the pipette tip. The user defines the diluent volume and the sample volume. The air bubble is dependent on the sample volume and is always specified by the program. Selecting a pipetting option can dispense the entire 
contents of the pipette tip. Depending on the pipette used, the dilution ratio is between approximately 1:2 and approximately 1:20.

7. SDI $=$ Serial Diluting

For serial diluting, a defined volume of liquid is aspirated into the pipette tip. This liquid is dispensed and then mixed with a specific liquid volume by means of a programmable mixing process (volume, mixing cycles). Pressing the Actuate key separately starts the mixing process (MIX).

\section{EDIT}

1. If PROG is in the display, holding down the Program key can open the programming level. The Program key must be held down until the word EDIT appears in the display in front of PROG 1-5. Systematically programming is explained in the following subsection.

2. If the programming of a program sequence is quit by pressing Reset, the entries for the program sequence are not stored.

3. The same program sequence - with, for example, different volumes or options - can be stored in several memory slots. It would, therefore, be possible for different pipetting sequences to be stored permanently in the program level.

4. Programming sequences can be overwritten at any time under EDIT (hold down the Program key). During the programming process, the entry that is to be defined flashes in the display. Selections are made using the Select rocker. Volumes are selected using the Volume rocker.

5. Data are stored by pressing the Actuate key (ENTER function).

6. Options and speeds can be selected in the programming level by using the Select rocker or by using the Option and Speed key. We recommend that you read these sections before you start programming. The following sections on programming for pipetting contain only a description of operations using the rockers. Programming and execution of the other programs are contained in Part B of the Research pro manual.

\subsubsection{Introduction - Additional Program Sequences}

During programming (EDIT and PROG in display), the Speed and Option keys and the Volume rocker can also be used. This enables users to skip backward and forward during the program sequence. 
When the programs are executed (only PROG is in the display), the Speed and Option keys and the Volume rocker are blocked.

If only parts of the program have been changed, programming can be ended prematurely by pressing the Program key briefly.

If a memory slot is quit using Reset before programming has been completed, the changes that have not been carried out for this memory slot are not saved. EDIT disappears from the display.

\subsubsection{SD $=$ Sequential Dispensing (Use this mode to pipette the 4 age-specific (1-2, 3-5, 6-11 and} $12+$ ) serum protocols - 100-5,000 $\mu$ L pipette.)

The sequential dispensing program can be used when different volumes of a solution have to be dispensed consecutively. Up to 20 steps can be defined. If the sum of the dispensing steps is larger than the volume of the pipette tip, the procedure for normal dispensing is applicable when a liquid is aspirated repeatedly.

\begin{tabular}{|l|l|}
\hline Programming SD \\
\hline Step 1 & $\begin{array}{l}\text { Select the programming mode. Press the Program key. PROG appears in the } \\
\text { display. }\end{array}$ \\
\hline Step 2 & $\begin{array}{l}\text { Select the memory slot number } 1 \text {. Use the Select rocker to move up and } \\
\text { down through the } 5 \text { program numbers until "1" is selected. }\end{array}$ \\
\hline Step 3 & $\begin{array}{l}\text { Hold down the Program key until EDIT appears in the display. } \\
\text { programming choices until "SD" appears in the display. Press the Actuate } \\
\text { key. } 4\end{array}$ \\
\hline Step 5 & $\begin{array}{l}\text { Determine the dispensing steps } 1 \text {-20. Use the Select rocker to move the } \\
\text { number up or down until the number } 19 \text { is displayed. Press the Actuate key } \\
\text { to store the number of steps in the program. }\end{array}$ \\
\hline Step 6 & $\begin{array}{l}\text { Select and store the volume for the first dispensing step. Use the Select or } \\
\text { Volume rocker to move the number up or down until the number } 1,000 \text { is } \\
\text { displayed. Press the Actuate key to store the volume for step 1. }\end{array}$ \\
\hline
\end{tabular}




\begin{tabular}{|c|c|}
\hline Step 7 & $\begin{array}{l}\text { Continue programming the remaining } 18 \text { steps and volumes for the } 12+ \\
\text { protocol. }\end{array}$ \\
\hline Step 8 & $\begin{array}{l}\text { Select the aspirating and dispensing speed using the Speed key and the Select } \\
\text { rocker. Press the Speed key, then use the Select rocker to move the speed } \\
\text { indicator all the way to the right. Press the Actuate key to store the speed in } \\
\text { the program. }\end{array}$ \\
\hline Step 9 & $\begin{array}{l}\text { Programming is complete. The display should show: } \\
\text { "SD } 500 \mu \mathrm{L} 19 \text { " }\end{array}$ \\
\hline \multicolumn{2}{|c|}{ Pipetting Procedure } \\
\hline Step 1 & $\begin{array}{l}\text { The necessary or largest possible amount of serum is aspirated. Aspirate the } \\
\text { serum. Make sure the direction of the aspirating arrow is pointed up. Press } \\
\text { the Actuate key once. The pipette will aspirate } 1,000 \mu \mathrm{L} \text {. }\end{array}$ \\
\hline Step 2 & Dispense the serum. Make sure the aspirating arrow is pointed downward. \\
\hline Step 3 & $\begin{array}{l}\text { Place the pipette tip against the side of the first receiving vessel. To dispense } \\
\text { the first aliquot, press the Actuate key once to dispense one } 1,000 \mu \mathrm{L} \text { aliquot. } \\
\text { The dispensing step counter (number) on the right side of the display counts } \\
\text { down one number. }\end{array}$ \\
\hline Step 4 & Place the pipette tip against the side of the second receiving vessel. \\
\hline Step 5 & $\begin{array}{l}\text { Continue dispensing the remaining aliquots until the (number) on the right } \\
\text { side of the display reaches } 0 \text {. If a slot in the processing rack is empty, } \\
\text { dispense the aliquot back into the polled serum vessel. }\end{array}$ \\
\hline Step 6 & $\begin{array}{l}\text { Complete the "blank" step. Press the Actuate key to perform the blank step. } \\
\text { This is not a dispensing step. A special acoustic signal is emitted. }\end{array}$ \\
\hline Step 7 & $\begin{array}{l}\text { Discard the last drop. Press the Actuate key to blow out the last drop. This is } \\
\text { not a dispensing step. Dashes replace the volume number in the display. If } \\
\text { the Actuate key is held down during the final blowout, the device does not } \\
\text { return the Aspiration arrow to the up position until the key is released. }\end{array}$ \\
\hline Step 8 & $\begin{array}{l}\text { The pipette is ready to aspirate again if the Aspirating arrow in the display } \\
\text { points up and the volume number and dispensing step display. }\end{array}$ \\
\hline
\end{tabular}

Continue programming the remaining program slots, age-specific protocols, and volumes. 
8.7.1.12 ADS = Automatic Dispensing (Use this mode to repetitively pipette $500 \mu \mathrm{L}$ pooled serum 100-5,000 $\mu$ L pipette.)

The automatic dispensing function allows a single volume to be dispensed repetitively with a predetermined time interval of between 0 and 10 seconds.

\begin{tabular}{|c|c|}
\hline \multicolumn{2}{|c|}{ Programming ADS } \\
\hline Step 1 & $\begin{array}{l}\text { Select the programming mode. Press the Program key. PROG appears in the } \\
\text { display. }\end{array}$ \\
\hline Step 2 & $\begin{array}{l}\text { Select the memory slot number } 2 \text {. Use the Select rocker to move up and } \\
\text { down through the } 5 \text { program numbers until " } 2 \text { " is selected. }\end{array}$ \\
\hline Step 3 & Hold down the Program key until EDIT appears in the display. \\
\hline Step 4 & $\begin{array}{l}\text { Select the ADS program. Use the Select rocker to move through the } \\
\text { programming choices until "ADS" appears in the display. Press the Actuate } \\
\text { key. }\end{array}$ \\
\hline Step 5 & $\begin{array}{l}\text { Select and store the dispensing volume. Use the Select or Volume rocker to } \\
\text { move the number until } 500 \text { is displayed. Press the Actuate key to store the } \\
\text { volume in the program. }\end{array}$ \\
\hline Step 6 & $\begin{array}{l}\text { Select the aspirating and dispensing speed using the Speed key and the Select } \\
\text { rocker. Press the Speed key, and then use the Select rocker to move the speed } \\
\text { indicator all the way to the right. Press the Actuate key to store the speed in } \\
\text { the program. }\end{array}$ \\
\hline Step 7 & $\begin{array}{l}\text { Reduction of the dispensing steps. The total number of dispensing steps } \\
\text { displays on the right side of the display. Do not use the Select rocker to } \\
\text { change this number. The pipette has calculated the number of dispensing } \\
\text { steps based on the aspirating volume and the size of the pipette tip. Press the } \\
\text { Actuate key to store the number of calculated dispensing steps and to move } \\
\text { to the final programming step. }\end{array}$ \\
\hline Step 8 & $\begin{array}{l}\text { Set the dispensing frequency. Use the Select rocker to move the number of } \\
\text { seconds (SEC) up or down until } 1 \text { is displayed before SEC in the display. } \\
\text { Press the Actuate key to store this frequency in the program. }\end{array}$ \\
\hline Step 9 & $\begin{array}{l}\text { Programming is complete. The display should show: } \\
\text { "ADS } 500 \mu \mathrm{L} 10 \text { " }\end{array}$ \\
\hline
\end{tabular}




\begin{tabular}{|c|c|}
\hline \multicolumn{2}{|c|}{ Pipetting Procedure } \\
\hline Step 1 & $\begin{array}{l}\text { Aspirate the serum. Make sure the aspirating arrow is pointed upward. Press } \\
\text { the Actuate key once. The pipette will aspirate } 5,000 \mu \mathrm{L} \text {. }\end{array}$ \\
\hline Step 2 & $\begin{array}{l}\text { Dispense the serum. Make sure the aspirating arrow is pointed downward. } \\
\text { Press the Actuate key once. Dispensing is carried out while the Actuate key } \\
\text { is held down. An acoustic signal is emitted after the dispensing step. To } \\
\text { interrupt the action, release the Actuate key. }\end{array}$ \\
\hline Step 3 & $\begin{array}{l}\text { Place the pipette tip against the side of the first receiving vessel. To dispense } \\
\text { the first aliquot, press the Actuate key once to dispense one } 500 \mu \mathrm{L} \text { aliquot, } \\
\text { or to automatically pipette sequential } 500 \mu \mathrm{L} \text { aliquots, hold the actuate key } \\
\text { down. The dispensing step counter (number) on the right side of the display } \\
\text { counts down one number. As soon as you hear the acoustic signal, move the } \\
\text { pipette tip to the next vessel. }\end{array}$ \\
\hline Step 4 & Place the pipette tip against the side of the second receiving vessel. \\
\hline Step 5 & $\begin{array}{l}\text { Continue dispensing } 500 \mu \mathrm{L} \text { aliquots until the (number) on the right side of } \\
\text { the display reaches } 0 \text {. }\end{array}$ \\
\hline Step 6 & $\begin{array}{l}\text { Complete the "blank" step. Press the Actuate key to perform the blank step. } \\
\text { This is not a dispensing step. A special acoustic signal is emitted. }\end{array}$ \\
\hline Step 7 & $\begin{array}{l}\text { Discard the last drop. Press the Actuate key to blow out the last drop. This is } \\
\text { not a dispensing step. Dashes replace the volume number in the display. If } \\
\text { the Actuate key is held down during the final blowout, the device does not } \\
\text { return the Aspiration arrow to the up position until the key is released. }\end{array}$ \\
\hline Step 8 & $\begin{array}{l}\text { The pipette is ready to aspirate again if the Aspirating arrow in the display } \\
\text { points up and the volume number and dispensing step display. }\end{array}$ \\
\hline
\end{tabular}




\subsubsection{Device Parameters}

Trained personnel may change device parameters only after a thorough inspection.

Device parameters are made up of the following:

BE1 To switch on/off the acoustic signal for the keypad (short beep).

BE2 To switch on/off the second acoustic signal for the end position of the dispensing piston, for warning, and for confirmation (different beeps).

CAL To alter the calibration data $\mu \mathrm{L}$, axle section (b) and gradient (m).

OPT Option for volume restriction:

When the $200 \mu \mathrm{L}$ tips are used with the $300 \mu \mathrm{L}$ pipettes (limit: $200 \mu \mathrm{L}$ ).

With "RP” and "DIS" when filter tips are used.

INI To initialize the original pipette data upon delivery.

The device parameters can all be found on one list. The Actuate key functions as an Enter key. After Enter has been pressed, the next line appears in the display.

\begin{tabular}{|l|l|}
\hline $\begin{array}{l}\text { To access the device } \\
\text { parameters }\end{array}$ & $\begin{array}{l}\text { With the pipette switched on, press both the Speed and } \\
\text { Option keys briefly at the same time. }\end{array}$ \\
\hline $\begin{array}{l}\text { To switch off/on acoustic } \\
\text { signal for the keypad }\end{array}$ & $\begin{array}{l}\text { Use the Select rocker to move through the selections until } \\
\text { "BE 1" is displayed in the left side of the display window. } \\
\text { Press the Actuate key. }\end{array}$ \\
\hline $\begin{array}{l}\text { To switch on/off (on), } \\
\text { second acoustic signal }\end{array}$ & $\begin{array}{l}\text { Use the Select rocker to move through the selections until } \\
\text { "BE 2" is displayed in the left side of the display window. } \\
\text { Press the Actuate key. }\end{array}$ \\
\hline $\begin{array}{l}\text { To exit the device } \\
\text { parameters }\end{array}$ & $\begin{array}{l}\text { Press the Actuate key when "INI" is displayed in the left side } \\
\text { of the display window. }\end{array}$ \\
\hline $\begin{array}{l}\text { To initialize the pipette } \\
\text { and to erase all settings }\end{array}$ & $\begin{array}{l}\text { Using "ENTER, access "INI" in device parameters. Hold } \\
\text { down the Program key. Initialization is completed when the } \\
\text { device parameter "INI” disappears from the display. }\end{array}$ \\
\hline
\end{tabular}




\subsubsection{Care, Sterilization, Maintenance, and Ordering Information}

The outside of the pipette can be wiped with a moist cloth. The use of water with a wetting agent is also permitted.

DO NOT ALLOW ANY LIQUID TO ENTER THE PIPETTE.

Only the lower part of the pipette should be rinsed with distilled water and dried. The pipette may be wiped clean with 60 percent isopropanol.

If the pipette is very contaminated, or if very aggressive chemicals are dispensed, the lower half of the Research pro should be disassembled. The individual parts are rinsed in distilled water and then dried. The piston is then lightly lubricated using silicone grease.

After opening the pipette, check that the piston is correctly positioned. A motor reset must also be executed. The motor reset is triggered by holding down the Reset key until $\mathbf{R}$ appears in the display.

\section{Sterilization}

Only the lower half of the pipette can be disassembled. The individual parts of the lower part must be disassembled as separate pieces. Please review the diagrams on page 27 of the Eppendorf Research Pro Instruction Manual before attempting to disassemble any pipette.

To loosen the lower part of the pipette for all pipettes except the $5,000 \mu \mathrm{L}$ :

1. Hold down the ejector and pull off the ejector sleeve. Force may be required.

2. Unscrew the lower part using the key. During disassembly, do not damage the piston!

3. Unscrew the piston. If necessary, first loosen the piston at the upper end using flat nose pliers. The piston is moved to the lowest position. 
To loosen the lower part of the 5,000 $\mu \mathrm{L}$ pipette:

1. Hold down the ejector and pull off the ejector sleeve. Force may be required.

2. Unscrew the cylinder. The piston is held in place magnetically. During reassembly, do not damage the piston!

3. Pull the piston out of the upper part.

Assemble in reverse order. Engage the magnetic coupling of the 5,000 $\mu \mathrm{L}$ piston as shown in the diagram on page 27 of the instruction manual. Check that the dispensing function of the pipette is working correctly.

\section{Maintenance}

Apart from general care and occasional discharging (when the battery symbol starts flashing) and recharging of the pipette, no special maintenance is necessary if the pipette is used correctly. The lower part of the pipette may be replaced completely. The process for replacing the seals in the lower part is described in Part B of the Research pro manual: "Ordering information/service parts." The Service Department may carry out maintenance on the Research pro. Contact Brinkmann Instruments at 800-645-3050 Ext. 404.

A complete list of troubleshooting information is included on pages 28 and 29 of the Eppendorf Research Pro Instruction Manual. 


\section{Ordering Information}

\begin{tabular}{|l|l|l|}
\hline \multicolumn{1}{|c|}{ Item } & \multicolumn{1}{c|}{ Size } & \multicolumn{1}{c|}{ Part number } \\
\hline Eppendorf Research pro & $5-100 \mu \mathrm{L}$ & $2246-136-2$ \\
\hline Eppendorf Research pro & $50-1,000 \mu \mathrm{L}$ & $2246-138-9$ \\
\hline Eppendorf Research pro & $100-5,000 \mu \mathrm{L}$ & $2246-139-7$ \\
\hline Charging stand & Four pipettes & $2246-155-9$ \\
\hline Pipette Tips & $5-\mathrm{mL}$ & $2235-081-1$ \\
\hline Bulk-packaged, in bags & $200 \mu \mathrm{L}$ & $2249-002-8$ \\
\hline $\begin{array}{l}\text { Eppendorf Biopur, colorless, sterile, } \\
\text { pyrogen-free, DNA-free, Rnase-free } \\
\text { In Boxes 5 x 96 = 480 tips }\end{array}$ & \multicolumn{2}{|l}{} \\
\hline $\begin{array}{l}\text { Eppendorf Biopur, colorless, sterile, } \\
\text { pyrogen-free, DNA-free, Rnase-free } \\
\text { In Boxes 5 x 96 = 480 tips }\end{array}$ & $1,000 \mu \mathrm{L}$ & $2249-006-1$ \\
\hline \begin{tabular}{l} 
Additional accessories \\
\hline \begin{tabular}{l} 
Spare Ni-MH battery, 1,200 mAh \\
\hline
\end{tabular}
\end{tabular} & $2246-701-8$ \\
\hline
\end{tabular}

Page 102 in the instruction manual contains a complete list of additional parts and accessories.

- Use the dedicated 5-100 $\mu \mathrm{L}$ and the dedicated 50-1,000 $\mu \mathrm{L}$ Eppendorf Research pro pipettes and trace metal free pipette tips to aliquot whole blood and perform the dilution for RBC Folate - vessel 3 and RBC Fol1 - vessel 106 using 1.0-uL ascorbic acid.

Pipetting Procedure

1. Using the pipette tip dispenser tray, press the pipette nozzle firmly into a fresh tip.

2. Aspirate and dispense the whole blood, serum, and ascorbic acid using procedures described in Section 8.7.

3. Place the tip against the inner wall of the cryovial as close to the bottom as possible. 
4. Discard the tip in the Sharps container.

5. Cover the pipette tips at the end of each session to prevent contamination.

\subsubsection{Process the 3-mL. 4-mL, 7-mL, and 10-mL EDTA Tubes}

The EDTA (tripotassium salts of ethylene diamine-tetracetic acid) acts as a chelating agent to combine with calcium in the blood to prevent coagulation. Use the well-mixed lavender 3-mL and 4-mL EDTA tubes for the hematology profile (CBC) and certain whole blood and plasma blood tests. Use the 3- and/or 4-mL (age 12+) EDTA tubes for washed packed cells. Use the dedicated 7-mL EDTA tube as the source tube for the plasma for vessel 91 - SRM Pesticide. Use the two 10-mL EDTA tubes for genetic testing - vessels 119 and 120 .

\subsubsection{Process the 3-mL or 4-mL EDTA Tube for Lead/Cad/HG, RBC Folate, RBC Fol1, Glycohem, Mercury, and HLA-B27}

The hematology technologist runs the CBC on the room temperature 3-mL (age 1+) EDTA tube after the blood-processing technologist verifies proper identification and labeling, and processes the tube for the Lead/Cad/HG, RBC Folate, RBC Fol1, Glycohem, Mercury, and HLA-B27. If the EDTA tube is clotted, do not process vessel 1 (Lead/Cad/HG), vessel 3 (RBC Folate), vessel 106 (RBC Fol1), vessel 4 (Glycohem), or vessel 5 (Mercury). If the CBC was accidentally run before the whole blood for vessels 1 (Lead/Cad/HG) and 5 (Mercury) was pipetted, do not process these two vessels and add the comment “laboratory error” to vessel 1 and vessel 5.

Complete the following process for all SPs. Use the following tubes for the CBC and whole blood protocol:

1. Ages 1-2

a. $\quad$ Process the 3-mL EDTA tube for CBC and all whole blood tests.

2. Ages 3-5

a. $\quad$ Process the first 3-mL EDTA tube for CBC and all whole blood tests.

b. Process the second 3-mL EDTA tube for plasma tests. 
3. Ages 6-11

a. $\quad$ Process the 3-mL EDTA tube for the CBC and all whole blood tests.

b. Process the 4-mL EDTA tube for plasma tests (vessels 109 and 8) and the washed packed cells for vessel 88.

4. Ages 12-19

a. Process one of the 3-mL EDTA tubes for the CBC and all whole blood tests.

b. $\quad$ Process the second 3-mL EDTA tubes for washed packed cells vessel 126.

c. $\quad$ Process the 4-mL EDTA tube for plasma tests (vessels 109 and 8) and washed packed cells for vessel 88.

5. Ages $20+$

a. $\quad$ Process the first 4-mL EDTA tube for the CBC and all whole blood tests.

b. $\quad$ Process the 3-mL EDTA tubes for washed packed cells for vessel 126.

c. $\quad$ Process the second 4-mL EDTA tube for plasma tests (vessels 109 and 8) and washed packed cells for vessel 88.

- The blood-processing technologist initially processes the 3- or 4-mL EDTA tube for the whole blood tests.

- $\quad$ Invert the appropriate EDTA tube several times to remix contents thoroughly.

- $\quad$ Refrigerate L-ascorbic acid diluent when not in use. Discard unused diluent at end of each stand.

\section{Vessel 1}

- $\quad$ Use trace metal free pipette tips and the 50-1,000 $\mu \mathrm{L}$ Eppendorf pipette to aliquot $0.4-\mathrm{mL}$ whole blood for vessel 1 (Lead/Cad/HG) on primary SPs and VIP guests aged 1+ into a 2-mL vessel.

\section{Vessel 3}

- $\quad$ Use trace metal free pipette tips and the dedicated 5-100 $\mu \mathrm{L}$ Eppendorf pipette to pipette $0.1-\mathrm{mL}$ whole blood for vessel 3 (RBC Folate) on primary SPs and VIP guests aged 1+ into a 2-mL vessel. Do not prewet the pipette tips. Dilute the whole blood with ascorbic acid. Use trace metal free pipette tips and the dedicated 50-1,000 $\mathrm{LL}$ Eppendorf pipette to immediately pipette 1.0-mL ascorbic acid into the 2-mL vessel. Mix well. 


\section{Vessel 106}

- $\quad$ Use trace metal free pipette tips and the dedicated 5-100 $\mu \mathrm{L}$ Eppendorf pipette to pipette $0.1-\mathrm{mL}$ whole blood for vessel 106 (RBC Fol1) on selected (1/3 subsample 1, 2, 5, 11) primary SPs and VIP guests aged 1+ into a 2-mL vessel. Do not prewet the pipette tips. Dilute the whole blood with ascorbic acid. Use trace metal free pipette tips and the dedicated 50-1,000 $\mu \mathrm{L}$ Eppendorf pipette to immediately pipette $1.0-\mathrm{mL}$ ascorbic acid into the $2-\mathrm{mL}$ vessel. Mix well.

\section{Vessel 4}

- $\quad$ Use trace metal free pipette tips and the 50-1,000 $\mu \mathrm{L}$ Eppendorf pipette to aliquot $0.4-\mathrm{mL}$ whole blood for vessel 4 (Glycohem) on primary SPs aged 12+ into a 2-mL vessel.

\section{Vessel 5}

- $\quad$ Use trace metal free pipette tips and the 50-1,000 $\mu \mathrm{L}$ Eppendorf pipette to aliquot 0.5-mL whole blood for vessel 5 (Mercury) on primary SPs and VIP guests aged $1+$ into a $2-\mathrm{mL}$ vessel.

\section{Vessel 127}

- $\quad$ Use trace metal free pipette tips and the 50-1,000 $\mu \mathrm{L}$ Eppendorf pipette to aliquot 1.0-mL whole blood for vessel 127 (HLA-B27) on primary SPs aged 20-69 into a 2-mL vessel.

- Securely close all vessels to prevent leakage and evaporation.

- $\quad$ Record results using the blood-processing module as described in Section 8.8.

- Store all specimens immediately as outlined in Section 8.10.

- Place the EDTA tube on a hematology rocker until the hematology technologist performs the CBC.

Run the CBC within 2 hours. If unable to run the CBC within 2 hours, store the 2-mL EDTA blood tube at room temperature for no more than 24 hours. After 24 hours, the blood will undergo changes that will render the WBC, RBC, HgB, and HCT results inaccurate. 


\subsubsection{Process the 3- or 4-mL EDTA Tube for Plasma for PUFA/FTA and Xtra Plasma}

Process the EDTA tube for PUTA/FTA and Xtra Plasma and centrifuge at $17-25^{\circ} \mathrm{C}$ or $4-8^{\circ} \mathrm{C}$.

Use the following tubes for the plasma protocol:

1. Ages 3-5

- $\quad$ Process the second 3-mL EDTA tube on primary SPs aged 3-5 for vessel 109.

2. Ages 6+

- $\quad$ Process the dedicated 4-mL EDTA tube on SPs aged 6+ and save the cells from this tube on SPs aged 6+ for the washed packed cells vessel 88.

Complete the following process for all SPs:

- Place the tube in the centrifuge carrier of the refrigerated tabletop centrifuge. Balance the tubes in the centrifuge by using water-filled tubes if necessary. Immediately centrifuge the whole blood from the 3- or 4-mL EDTA tube in the $17-25^{\circ} \mathrm{C}$ or $4-8^{\circ} \mathrm{C}$ centrifuge at 2,900 rpm for 10 minutes to yield plasma for vessel 109 (PUFA/TFA) and vessel 8 (Xtra Plasma.)

\section{Vessel 109}

- $\quad$ Use a calibrated plastic transfer pipette to aliquot at least 0.7-mL plasma for vessel 109 (PUFA/TFA) on primary SPs aged 3+ into a 2-mL vessel.

\section{Vessel 8}

- $\quad$ Use a calibrated plastic transfer pipette to aliquot at least $0.5-\mathrm{mL}$ plasma for vessel 8 (Xtra Plasma) on primary SPs aged 6+ into a 2-mL vessel.

- If there is any excess plasma remaining after pipetting the protocol, then add any remainder to vessel 8. Save the cells from the 4-mL EDTA tube processed on SPs aged $6+$.

- Determine if the plasma is hemolyzed, turbid, lipemic, or icteric. If so, enter a comment to describe the plasma when entering the processing results.

- Securely close all vessels to prevent leakage and evaporation. 
- $\quad$ Record results using the blood-processing module as described in Section 8.8.

- Store all specimens immediately as outlined in Section 8.10.

\subsubsection{Process the 3- or 4-mL EDTA Tube for Washed Packed Cells for Acrylamide (vessel 88) and Xtra RBCs (vessel 126)}

Centrifuge the EDTA tube and remove the plasma as described in Section 8.7.2.2. Leave the cells in the original blood collection tube. Wash the remaining red blood cells (erythrocytes) with sterile saline three times and then lyse the cells with distilled water. In the case of vessel 126, after the last wash cycle, add an equal amount (to the packed cells) of phosphate buffer saline (PBS) solution, $\mathrm{pH}$ 7, to return the cells to their physiological $\mathrm{pH}$. Store the saline in the refrigerator and use it cold. Pour saline and deionized water into squeeze bottles. It is acceptable to batch the samples as long as the samples and reagents are refrigerated.

Use the following tubes for the washed packed cells protocol for vessel 88:

- $\quad$ Process the cells from the 4-mL EDTA tube on SPs aged 6+.

- $\quad$ Place the tube(s) in the centrifuge carrier of the refrigerated tabletop centrifuge. Balance the tube(s) in the centrifuge by using water-filled tubes if necessary. Take care not to disturb the pellet of red blood cells when removing the plasma.

\section{Vessel 88}

1. Centrifuge the 4-mL EDTA tube at $4-8^{\circ} \mathrm{C}$. Centrifuge at $1,985 \mathrm{rpm}$ for 5 minutes to separate the plasma from the cells.

2. Use a disposable transfer pipette to remove the plasma, which is the upper clear layer, and add it to vessels 109 and 8.

3. Use the refrigerated squeeze bottle of saline to add an equal amount (to the packed cells) of 0.9 percent cold saline to the packed cells.

4. Close vial and mix by gently shaking.

5. Centrifuge at $1,985 \mathrm{rpm}$ and $4^{\circ} \mathrm{C}$ for $5 \mathrm{~min}$.

6. Remove and discard the clear upper layer.

7. Repeat steps 3 to 6 two more times (total of 3 wash cycles). 
8. After the last wash cycle use the squeeze bottle of deionized water to add an equal amount of deionized water to the packed cells. (The final volume should be at least 1.0-mL of washed lysed packed cells.)

9. Close vial and mix by gently shaking.

10. Transfer the lysed cells to a 2-mL cryovial.

Use the following tubes for the washed packed cells protocol for vessel 126:

- Process the cells from the 3-mL EDTA tube on SPs aged 12-19 and second 4-mL EDTA tube on SPs aged 20+.

- $\quad$ Place the tube(s) in the centrifuge carrier of the refrigerated tabletop centrifuge. Balance the tube(s) in the centrifuge by using water-filled tubes if necessary. Take care not to disturb the pellet of red blood cells when removing the plasma.

\section{Vessel 126}

1. Centrifuge the 4-mL EDTA tube at $4^{\circ} \mathbf{C}$. Centrifuge at 2,900 rpm for 5 minutes to separate the plasma from the cells.

2. Use the refrigerated squeeze bottle of saline to add an equal amount (to the packed cells) of 0.9 percent cold saline to the packed cells.

3. Close vial and mix by gently shaking.

4. Centrifuge at $4,000 \mathrm{rpm}$ and $4^{\circ} \mathrm{C}$ for $5 \mathrm{~min}$.

5. Remove and discard the clear upper layer.

6. Repeat step 3 to 6 two more times (total of 3 wash cycles).

7. After the last wash cycle, add an equal amount (to the packed cells) of phosphate buffer saline (PBS) solution, $\mathrm{pH}$ 7, to return the cells to their physiological $\mathrm{pH}$.

8. Close vial and mix by gently shaking.

9. Centrifuge at $4,000 \mathrm{rpm}$ and $4^{\circ} \mathrm{C}$ for $5 \mathrm{~min}$.

10. Remove and discard the clear upper layer.

11. Use the squeeze bottle of deionized water to add an equal amount of deionized water to the packed cells.

12. Close vial and mix by gently shaking.

13. Transfer the lysed cells to a 2-mL cryovial. 
- Close all vessels secured to prevent leakage and evaporation.

- $\quad$ Record results using the blood-processing module as described in Section 8.8.

- Store all specimens immediately as outlined in Section 8.10.

\subsubsection{Process the 7-mL EDTA Tube for SRM Pesticide}

Centrifuge and separate the plasma from the 7-mL EDTA tube as soon as possible. Process the specimen even if the contents of the EDTA tube clot.

- Place the tube in the centrifuge carrier of either tabletop centrifuge. Balance the tube in the centrifuge by using water-filled tubes if necessary. Centrifuge the 7-mL EDTA tube at $17-25^{\circ} \mathrm{C}$ or $4-8^{\circ} \mathrm{C}$ and $2,900 \mathrm{rpm}$ for 10 minutes to yield plasma for vessel 91 (SRM Pesticide.)

\section{Vessel 91}

- $\quad$ Use a calibrated plastic transfer pipette to aliquot all the plasma for vessel 91 (SRM Pesticide) on selected primary SPs aged 12+ into a 5-mL vessel.

- Determine if the plasma is hemolyzed, turbid, lipemic, or icteric. If so, enter a comment to describe the plasma when entering the processing results.

- Securely close all vessels to prevent leakage and evaporation.

- $\quad$ Record results using the blood-processing module as described in Section 8.8.

- Store all specimens immediately as outlined in Section 8.10.

\subsubsection{Process the 10-mL EDTA Tubes for Genetics}

- Mix blood tubes 119 and 120 well.

- Check to ensure that the tubes are securely closed.

- $\quad$ Record results using the blood-processing module as described in Section 8.8.

- Store all specimens immediately as outlined in Section 8.10. 


\subsubsection{Process the 2-mL Gray Tube for Glucose and OGTT}

Centrifuge and separate the plasma from the 2-mL gray top tube as soon as possible. Process the specimen even if the contents of the gray tube clot.

- Place the tube in the centrifuge carrier of either tabletop centrifuge. Balance the tube in the centrifuge by using water-filled tubes if necessary. Centrifuge the 2-mL gray tube at $17-25^{\circ} \mathrm{C}$ or $4-8^{\circ} \mathrm{C}$ and 2,900 rpm for 10 minutes to yield plasma for vessel 9 (Glucose) or vessel 98 (OGTT.)

\section{Vessel 9 or 98}

- $\quad$ Use a calibrated plastic transfer pipette to transfer all plasma from this tube but at least 0.5 -mL plasma for vessel 9 (Glucose) or vessel 98 (OGTT) on primary SPs aged $12+$ into a 2-mL vessel.

- Determine if the plasma is hemolyzed, turbid, lipemic, or icteric. If so, enter a comment to describe the plasma when entering the processing results.

- Close all vessels secured to prevent leakage and evaporation.

- $\quad$ Record results using the blood-processing module as described in Section 8.8.

- Store all specimens immediately as outlined in Section 8.10.

\subsubsection{Process the 3-mL, 7-mL, 10-mL, and 15-mL Red Top Tubes for Pooled Sera}

- Gently invert the tubes 5-6 times as soon as possible after receiving them in the laboratory.

- Allow the blood in each 3-mL, 7-mL, 10-mL, and 15-mL red top tube to clot for 3045 minutes at room temperature. Do not let clot for more than 1 hour.

- $\quad$ Place the tubes in the room temperature centrifuge carrier of either tabletop centrifuge. Balance the tubes in the centrifuge by using water-filled tubes if necessary. Centrifuge the red top tubes at $17-25^{\circ} \mathrm{C}$ and $2,900 \mathrm{rpm}$ for 15 minutes to yield serum.

- $\quad$ Determine if the serum is hemolyzed, turbid, lipemic, or icteric. If so, enter a comment to describe the serum when entering the processing results.

If the serum in any one red top tube from a SP is grossly hemolyzed, do not pool it with serum from the remaining tubes. Reserve it for the storage vessels 32-39. 
If the serum in all red top tubes for a SP is turbid, lipemic, or icteric, pool it and allocate as usual.

- Vessels 81, 70, 13, 14, 122, 87, 99, 17, 21, 77, 18, 23, 22, 24, 75, 118, 127, 16, 110 and 32-39.

- $\quad$ Use a serum separator to remove serum from all the red top tubes by pushing the serum separator to the clot barrier gently to ensure cell-free separation. Do not push the separator too hard. Do not touch the serum separator to the clot barrier.

- $\quad$ Label the 50-mL conical tube with SP ID \# label. Pool all clear sera. For each SP pool the serum from all red top tubes by decanting the separated serum into the 50-mL polypropylene tube. Hold the tube and the separator level while decanting the serum. Be careful to avoid introducing any cellular debris. Discard all tubes and separators in a sharps container.

- $\quad$ Securely close the 50-mL polypropylene tube and mix its contents by gentle inversion.

- $\quad$ Confirm that all vessels have the correct information with the appropriate SP's bar-code ID label before filling them.

- $\quad$ Use the 100-5,000 Eppendorf ${ }^{\circledR}$ pipette and 5-mL pipette tips to aliquot the serum for all vessels.

- $\quad$ Using clear serum only, fill as many vessels as possible in the priority order shown in Section 8.3. Exhibits 8.10 - 8.18 describe the order of allocation and specific procedures to follow for each person type and vessel.

- $\quad$ If excess serum remains after completing the entire protocol, add an additional 1.5-mL serum beginning with vessel 32 and continuing through vessel 39 until the serum is exhausted. If extra serum remains, continue to top off the other serum vessels, beginning with vessel 81 , until the serum is exhausted.

- Securely close all vessels to prevent leakage and evaporation.

- $\quad$ Record results using the blood processing module as described in Section 8.8.

- $\quad$ Store all specimens immediately as outlined in Section 8.10.

- If the serum cannot be aliquoted immediately, refrigerate it at $4^{\circ} \mathrm{C}$ for no more than 4 hours. 


\subsubsection{Process the 10-mL Red Top Tubes for Environmental Subsamples}

The environmental subsamples represent pairs of $1 / 3$ nonoverlapping subsamples of SPs aged $12+$. An SP is eligible for vessels 29 and 89 or vessel 90 or vessels 92 and 93.

- Do not substitute any other red top tube for these vessels; the 10-ml red top tube is prescreened by the CDC for these tests.

- Gently invert the tubes 5-6 times as soon as possible after receiving them in the laboratory.

- Allow the blood in the $10-\mathrm{mL}$ red top tubes to clot for 30-45 minutes at room temperature. Do not let clot for more than 1 hour.

- $\quad$ Place the tubes in the room temperature centrifuge carrier of either tabletop centrifuge. Balance the tubes in the centrifuge by using water-filled tubes if necessary. Centrifuge the red top tubes at $17-25^{\circ} \mathrm{C}$ and $2,900 \mathrm{rpm}$ for 15 minutes to yield serum.

\section{Vessel 29/Vessel 90/Vessel 92}

- $\quad$ Determine if the serum is hemolyzed, turbid, lipemic, or icteric. If so, enter a comment to describe the serum when entering the processing results.

- $\quad$ Use a serum separator to carefully remove serum from the first 10-mL red top tube by pushing the serum separator to the clot barrier gently to ensure cell-free separation. Do not push the separator too hard or some flow through may occur. Do not touch the serum separator to the clot barrier.

- $\quad$ Aliquot the serum immediately by pouring the serum for vessel 29 (Persis Pest), vessel 90 (Perfluor1) or vessel 92 (BFR1) on primary SPs aged 12+ into a 5.0-mL vessel.

\section{Vessel 89/ Vessel 93}

- $\quad$ Determine if the serum is hemolyzed, turbid, lipemic, or icteric. If so, enter a comment to describe the serum when entering the processing results.

- $\quad$ Use a serum separator to carefully remove serum from the second 10-mL red top tube by pushing the serum separator to the clot barrier gently to ensure cellfree separation. Do not push the separator too hard or some flow through may occur. Do not touch the serum separator to the clot barrier.

- $\quad$ Aliquot the serum immediately by pouring the serum for vessel 89 (Persis Pest2), or vessel 93 (BFR2) on primary SPs age 12+ into a 5.0-mL vessel. 
- Securely close all vessels to prevent leakage and evaporation.

- $\quad$ Record results using the blood-processing module described in Section 8.8.

- Store all specimens immediately as outlined in Section 8.10.

\subsubsection{Reagent Processing and Preparation}

The Centers for Disease Control and Prevention supplies premeasured 1 percent ascorbic acid (AA) for vessel 3 (RBC Folate), and vessel 106 (RBC Fol1). Prepare this reagent before processing the blood samples. Allow the reagent to equilibrate to room temperature before pipetting and performing the dilution. Use the Eppendorf Research pro pipettes to make the dilution. Add 1.0-mL ascorbic acid to 0.1-mL whole blood for vessel 3 (RBC Folate) and vessel 106 (RBC Fol1). Mix by gentle inversion.

- The ascorbic acid powder is in a 50-mL Falcon tube. Dissolve the powder in 50-mL of distilled water using a graduated cylinder to measure the water and mix gently. Prepare this reagent weekly. Date and label the Falcon tube. If the powder appears discolored or the solution appears cloudy, discard and prepare fresh reagent. If no bottled distilled water is available, use tap water. Record the reagent in the Supply Use Control Log each time it is prepared.

- Refrigerate the prepared reagent at the end of each day to maintain its stability and bring the reagent back to room temperature the next day.

\subsection{Record the Results of Specimen Processing}

After filling or processing the whole blood, plasma, serum, and/or the EDTA or gray top tubes for each SP, use the Blood Processing module to enter the blood processing results. 
Open and log onto the Laboratory application. Use the heads-up display to view the SPs, the modules for which they are eligible, and their current process status.

Select the correct SP and access the Blood Processing module.

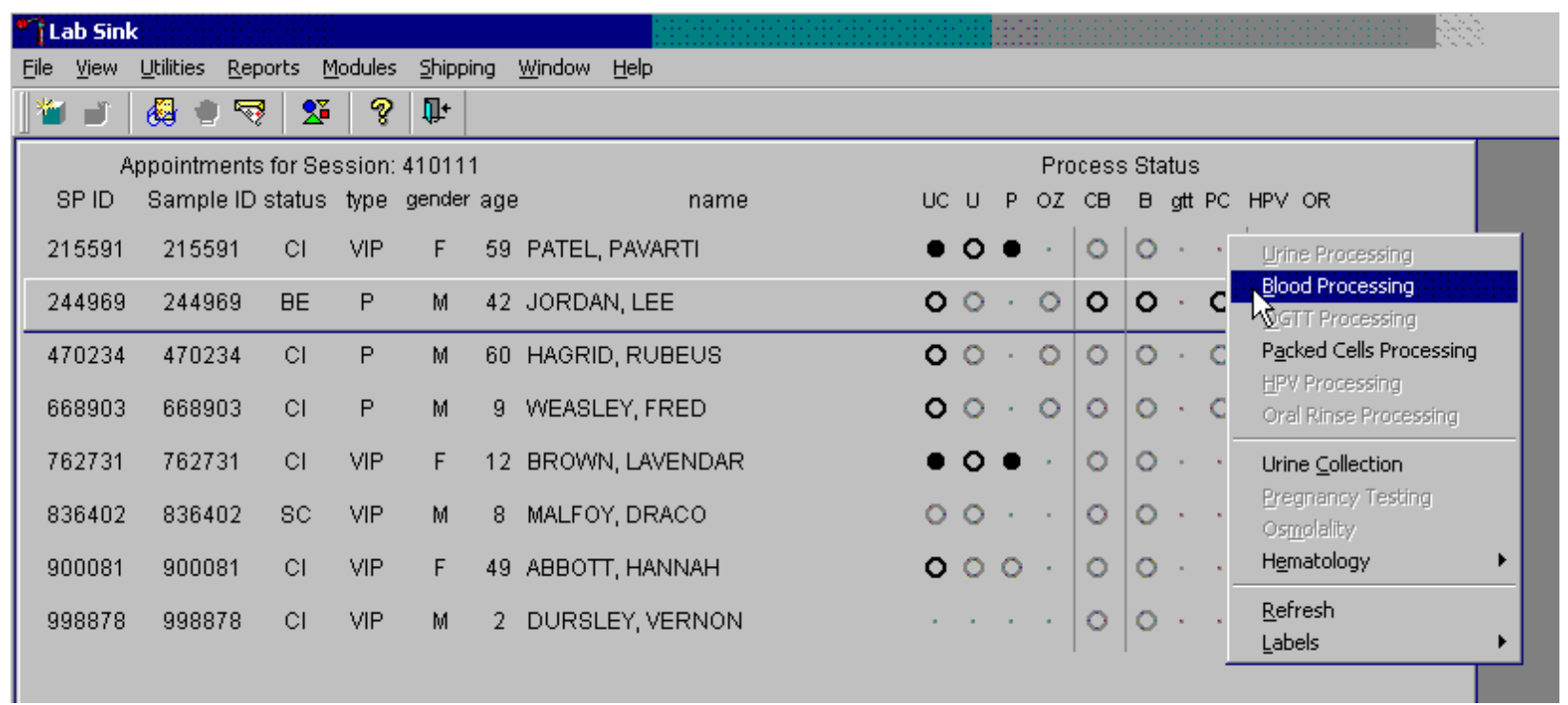

If labels have not been printed in advance, a Blood Processing message text box displays. To access the blood processing module, highlight the SP, right click, drag the mouse arrow to B Blood Processing\}, and left click or type [Alt] [B/b]. 
The SP's blood processing screen displays.

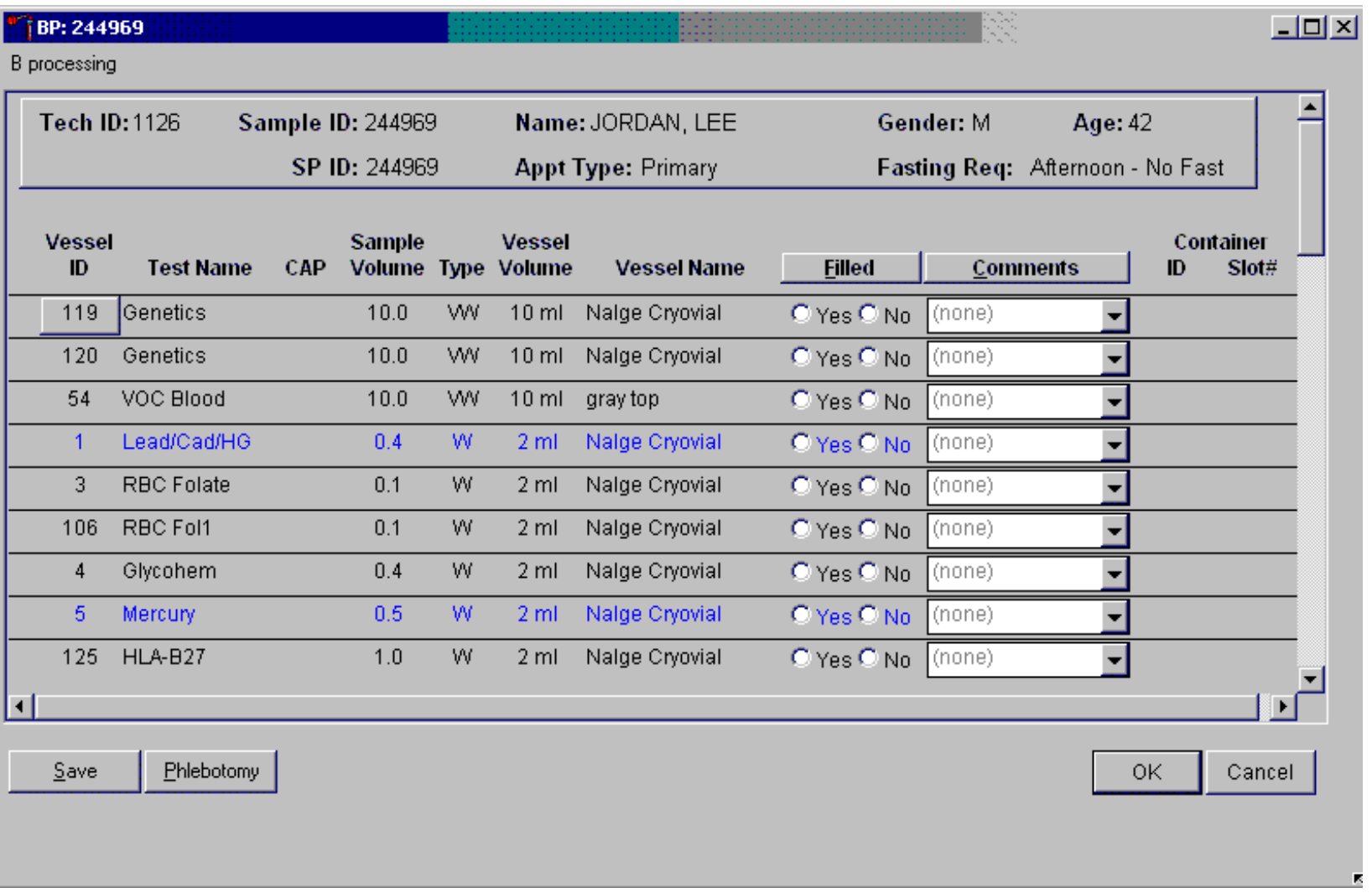

The blood processing window for an SP contains the following information: Tech ID, Sample ID, SP ID, Name (last, first), Gender, Age, Appt (appointment) Type, Fasting Req, columns for Vessel ID, Test Name, Sample Volume, Type (whole blood, plasma, serum), Vessel Volume, Vessel Name, Filled radio buttons, Comments text box, and a Container ID/Slot\# column. Vessels that require sterile 2-mL cryovials are displayed in blue. 
Inspect the SPs Vacutainer ${ }^{\circledR}$ tubes. Compare the Sample ID on the tubes to the bar-code label on the vessels. Verify that the number of tubes and the recorded amount of blood collected are correct.

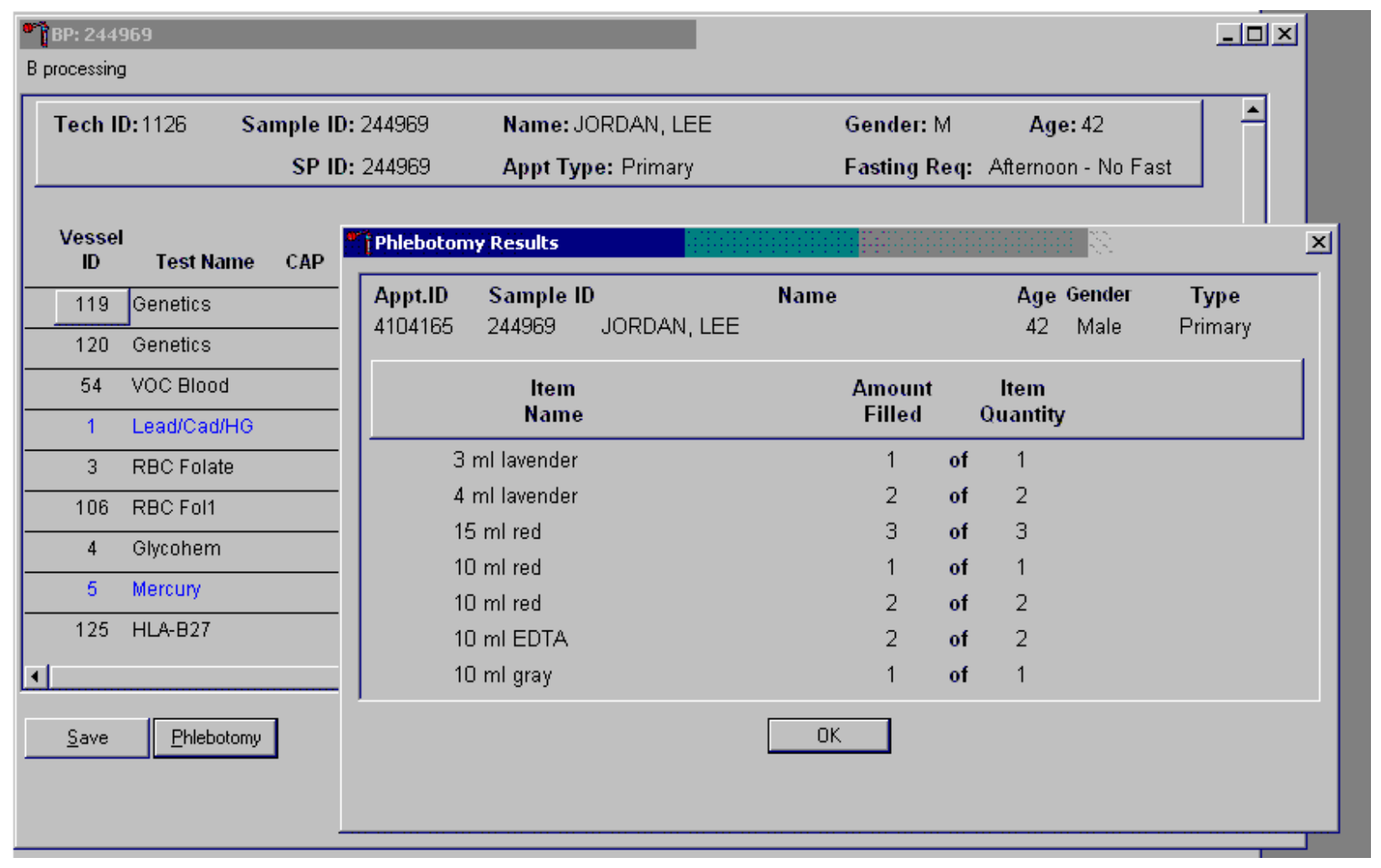

To review the phlebotomy results, use the mouse to direct the mouse arrow to the "Phlebotomy" button on the blood processing screen and left click or type [Shift] [P/p.] The phlebotomy protocol and number of tubes successfully collected displays. To close this screen, use the mouse to direct the mouse arrow to the "OK” button and left click. 
Record blood processing results for each SP. Individually mark each vessel as Filled-’Yes.”

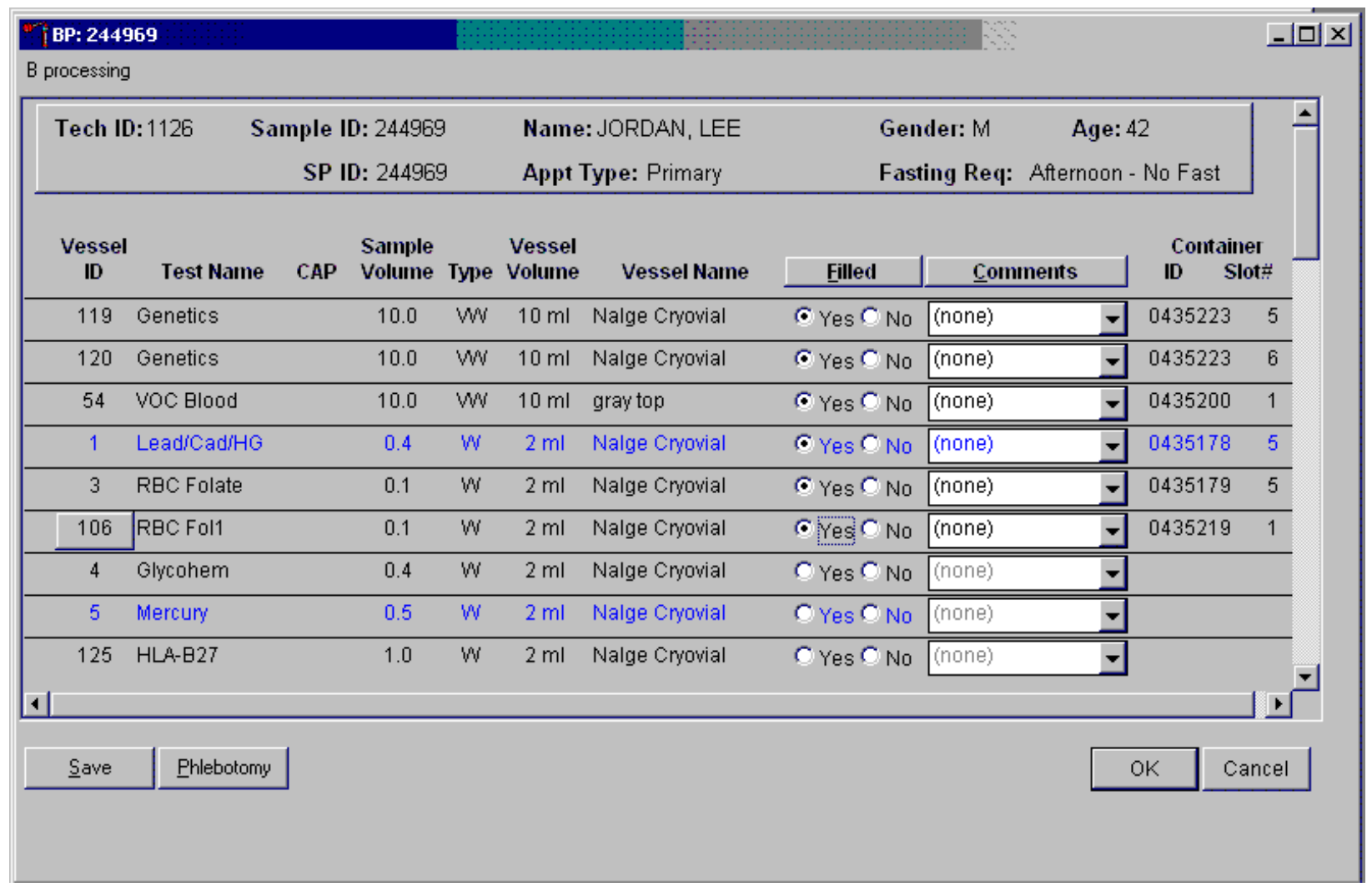

To mark an individual blood vessel as collected or Filled-”Yes” use the mouse to direct the mouse arrow to the center of the "Yes" radio button and left click. As each vessel is marked as Filled"Yes," it is automatically assigned to a slot in an existing (open) container. 
Individually mark each vessel as Filled-'No.”

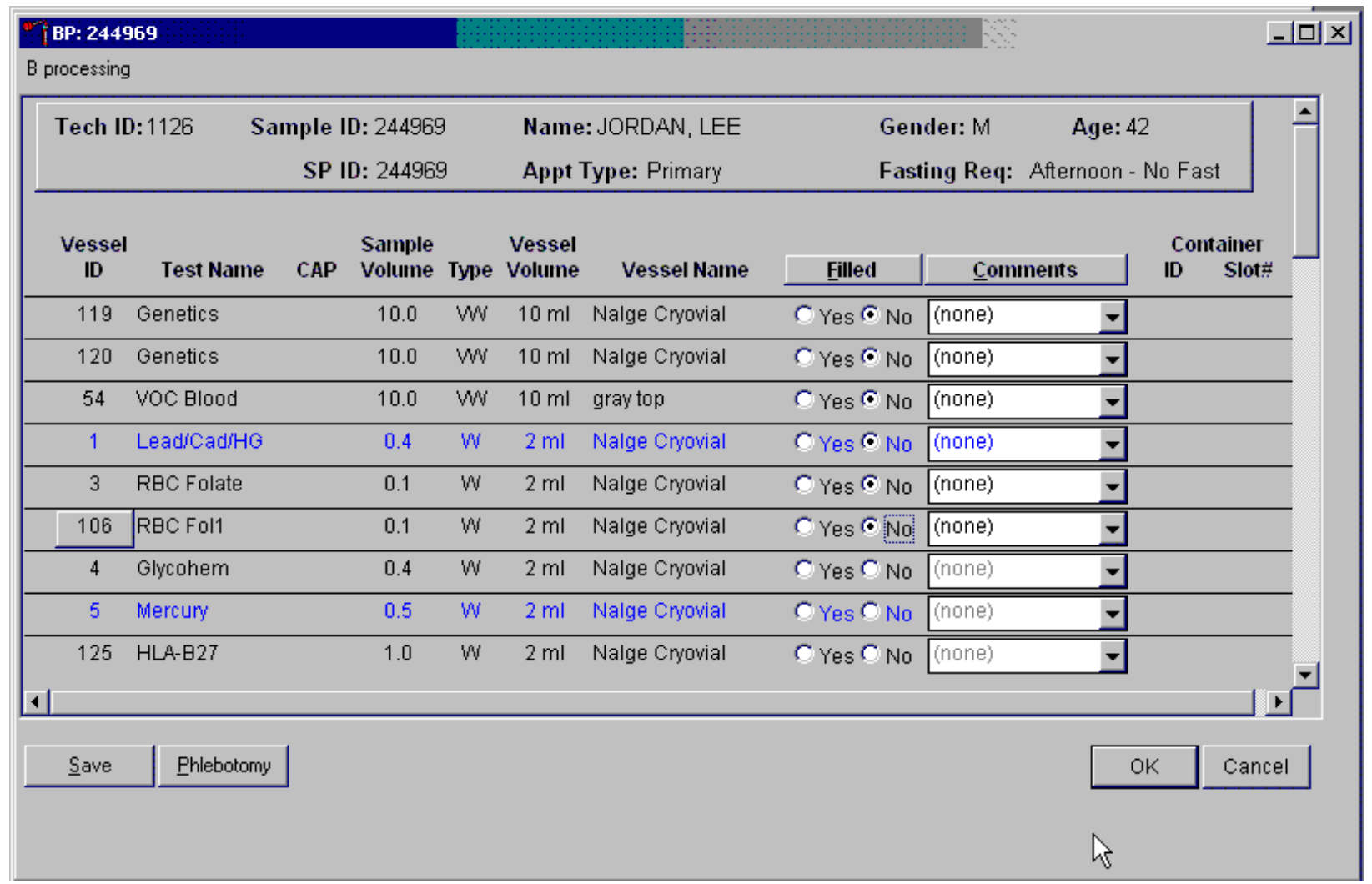

To mark an individual blood vessel as not collected or Filled-”No," use the mouse to direct the mouse arrow to the center of the "No" radio button and left click. Vessels marked as "No" require a comment. 
Collectively mark all vessels as Filled-"Yes.”

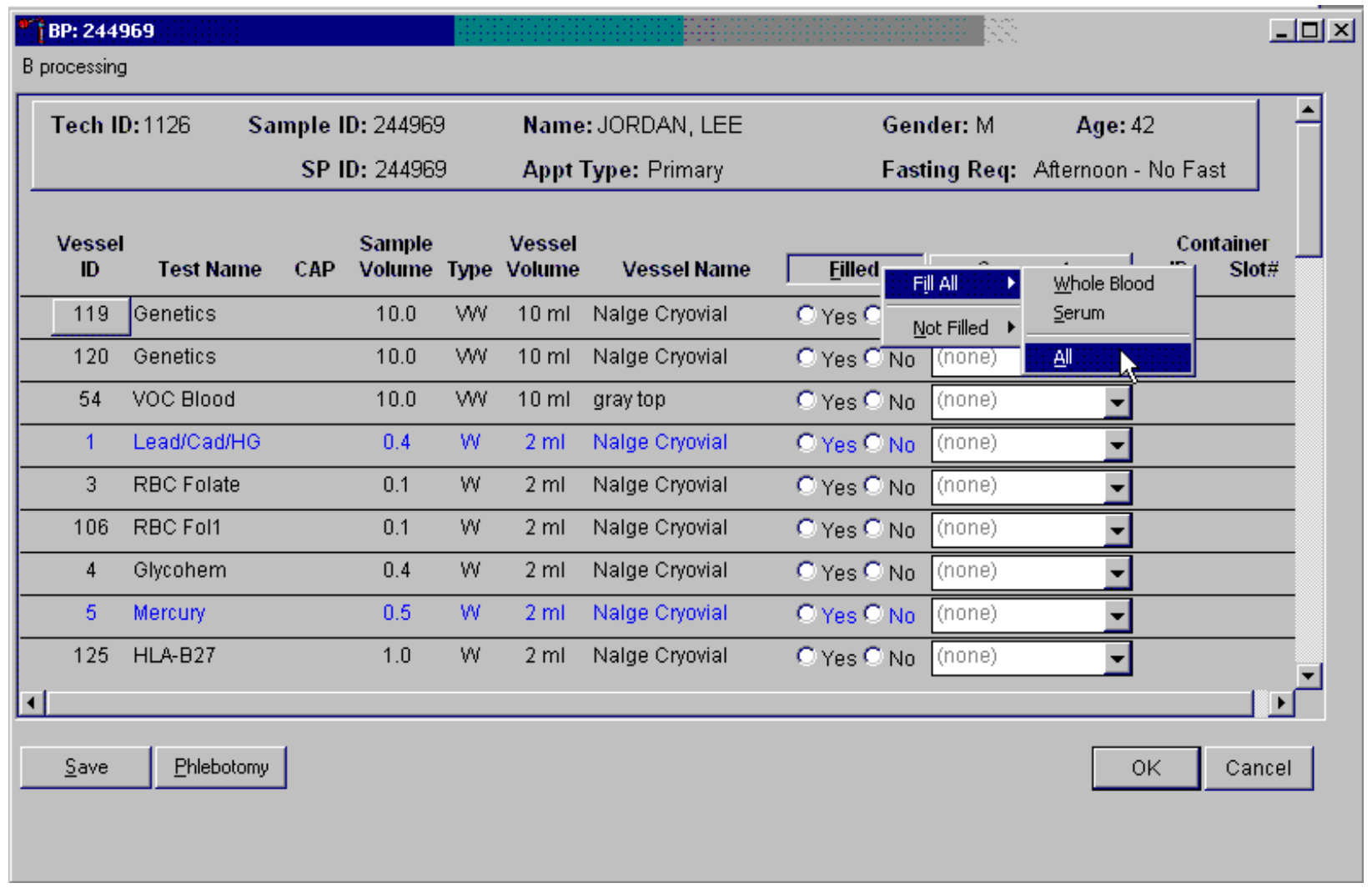

To collectively mark all vessels as Filled-"Yes,” use the mouse to direct the mouse arrow to the "Filled" button on the top of the radio buttons, left click, drag the arrow to \{Fill All\}, drag the mouse arrow to $\{$ All $\}$, and left click. Alternatively, to collectively mark all vessels as Filled-”Yes,” type [Shift] [F/f], [I/i], and [A/a.] 
Selectively mark all whole blood or serum vessels as Filled-”Yes.”

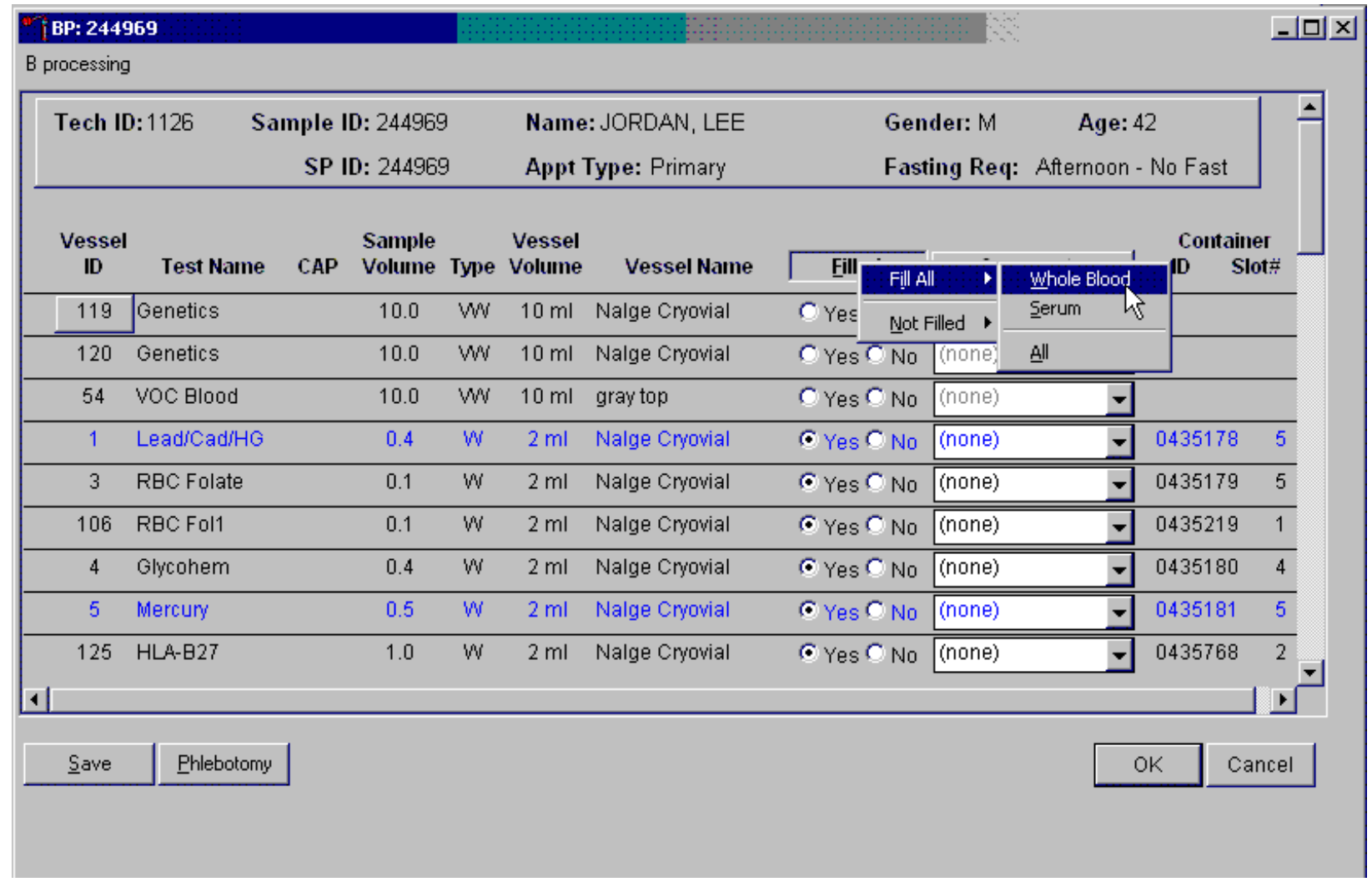

To collectively mark all whole blood vessels as Filled-”Yes," use the mouse to direct the mouse arrow to the "Filled" button on the top of the radio buttons, left click, drag the arrow to \{Fill All\}, drag the mouse arrow to \{Whole Blood\}, and left click. To collectively mark all serum vessels as Filled"Yes," use the mouse to direct the mouse arrow to the "Filled" button on the top of the radio buttons, left click, drag the arrow to \{Fill All\}, drag the mouse arrow to \{Serum\}, and left click. As each vessel is marked as "Filled," it is automatically assigned to a slot in an existing (open) container. Alternatively, to collectively mark all whole blood vessels as Filled-"Yes," type [Shift] [F/f], [I/i] and [W/w] and to collectively mark all serum vessels as Filled-’Yes,” type [Shift] [F/f], [I/i], and [S/s.] 
Quality comment codes are available to describe the quality of the specimen.

\begin{tabular}{|c|c|}
\hline Comment for filled vessels & Use when: \\
\hline Clotted & $\begin{array}{l}\text { The whole blood sample contains a fibrin formation. This is due to } \\
\text { inadequate mixing or inadequate clotting. }\end{array}$ \\
\hline Equipment failure & Pipettes are malfunctioning. \\
\hline Hemolyzed & $\begin{array}{l}\text { There is red appearance to the serum or plasma. Hemolysis refers to the } \\
\text { abnormal lysis of erythrocytes. Hemolysis can occur before venipuncture } \\
\text { (in vivo hemolysis) or during the analytic procedure (in vitro hemolysis). If } \\
\text { in vivo, hemolysis will give plasma a red color. Use when describing visible } \\
\text { hemolysis, which occurs when the concentration of hemoglobin exceeds } \\
200 \mathrm{mg} / \mathrm{L} \text {. }\end{array}$ \\
\hline Icteric & $\begin{array}{l}\text { There is visible orange color to the serum or plasma. Icteric refers to orange } \\
\text { color imparted to a sample because of the presence of bilirubin. Bilirubin } \\
\text { present in serum results in visible "jaundice" color when the concentration } \\
\text { of bilirubin is above } 430 \mathrm{umol} / \mathrm{L} \text {. }\end{array}$ \\
\hline Lipemic & $\begin{array}{l}\text { There is turbid appearance to the serum or plasma. Lipemia refers to the } \\
\text { presence of lipid particles (usually very low-density lipoprotein) in a } \\
\text { sample, which gives the sample a turbid appearance. Plasma appears visibly } \\
\text { lactescent or lipemic when the concentration of triglycerides exceeds } 4.6 \\
\text { mmol/L. }\end{array}$ \\
\hline Post OGTT draw & $\begin{array}{l}\text { During the OGTT blood draw ( } 2 \text { hours after the initial venipuncture and } \\
\text { after the SP has consumed the Trutol), the phlebotomist will draw any tubes } \\
\text { that were missed during the first venipuncture. If any vessel is filled from } \\
\text { one of the tubes drawn during the OGTT blood draw, then add this } \\
\text { comment. }\end{array}$ \\
\hline
\end{tabular}


Attach a comment to an individual filled vessel.

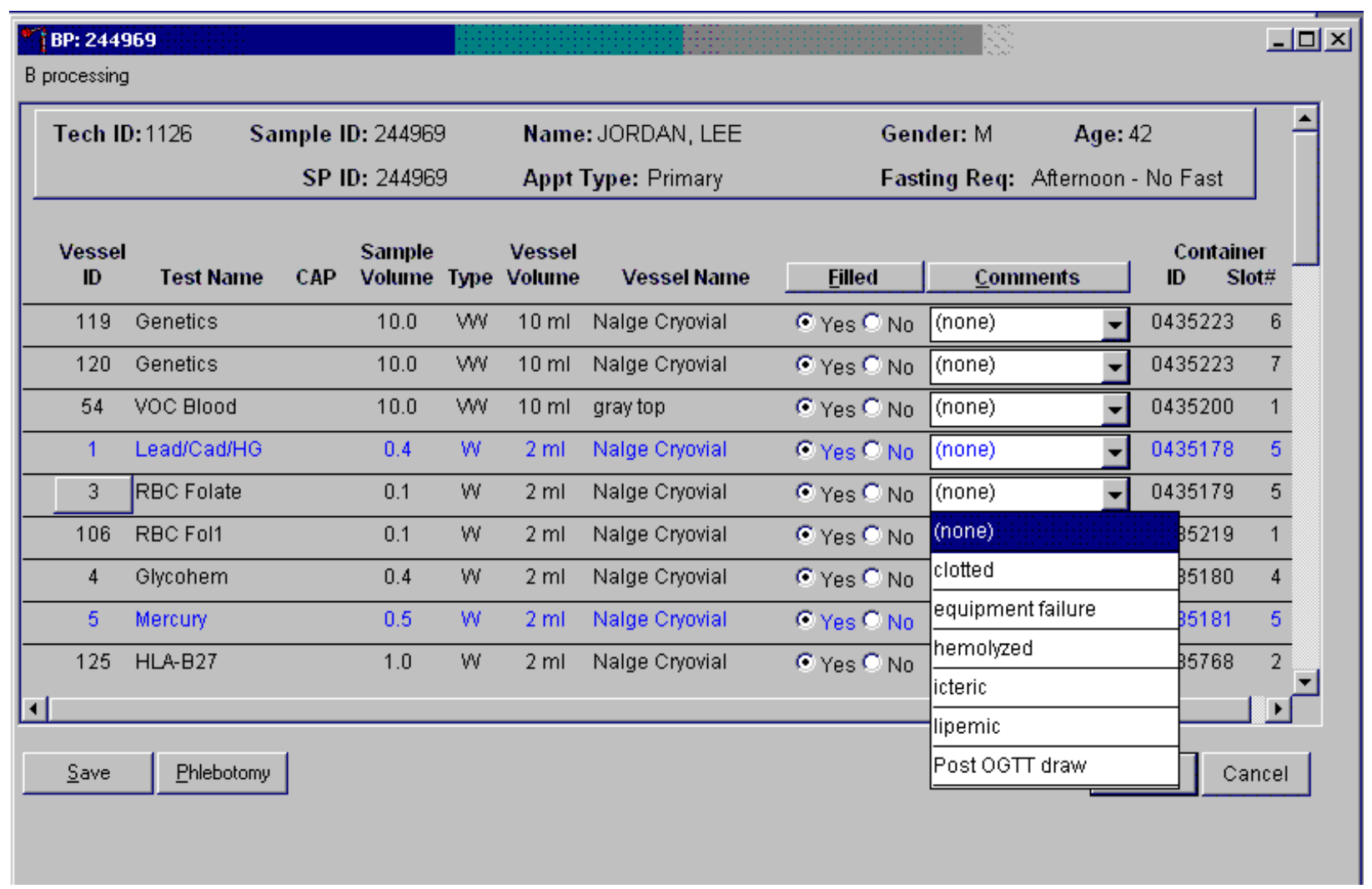

To attach a quality control comment to an individual filled vessel, use the mouse to direct the mouse arrow to the drop-down list under the "Comments" button to display the choices, drag the arrow to the correct choice, and left click or highlight the Comment text box and type the first letter of the desired choice (c, e, h, i, l, p.) 
Use the following procedure when all filled vessels require the same comment.

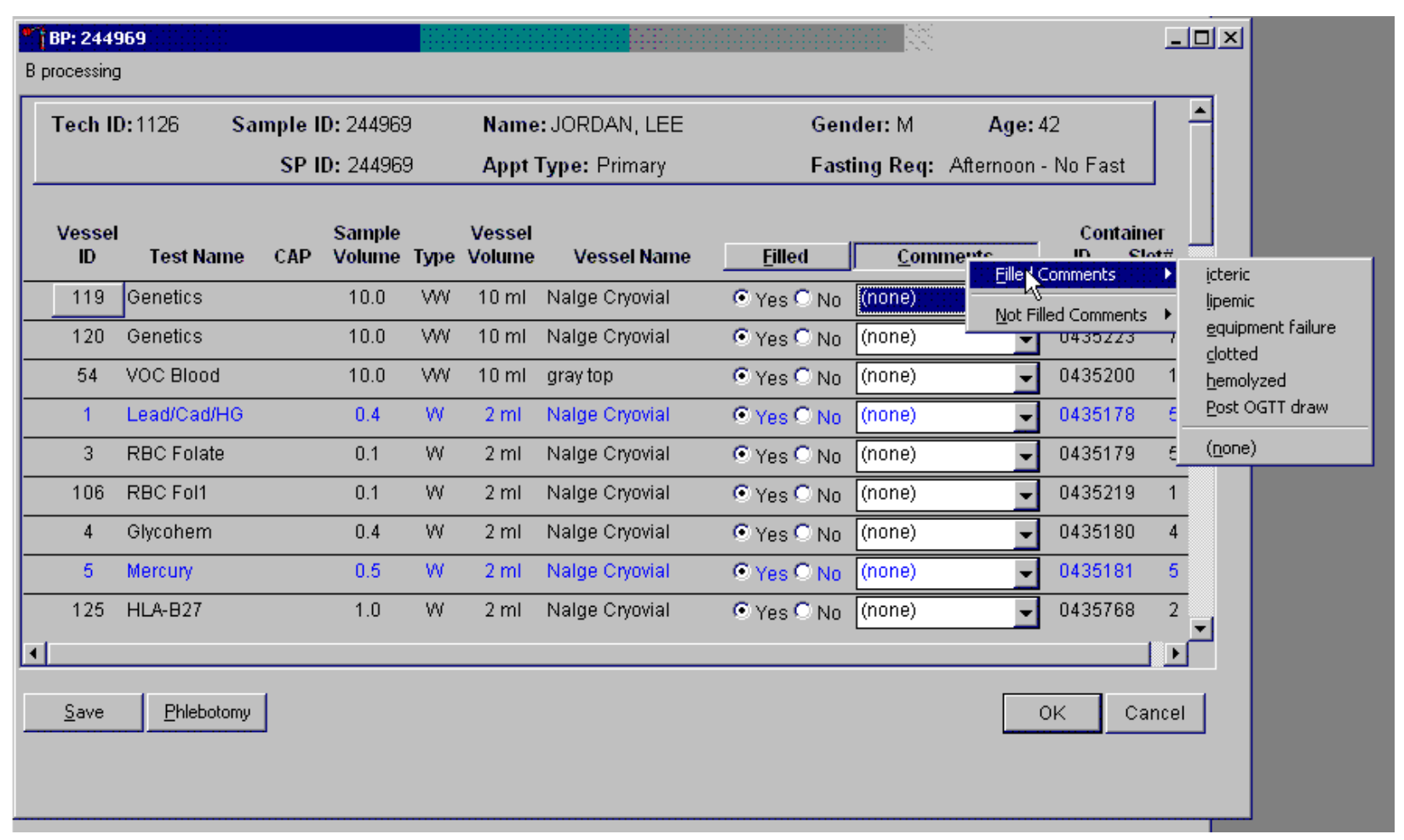

To collectively assign the same comment to all vessels, use the mouse to direct the mouse arrow to the "Comments" button on the top of the radio buttons, left click, drag the arrow to \{Filled Comments\}, drag the mouse arrow to the correct choice, and left click. Alternatively, to assign the same comment to all filled vessels type [Shift] [C/c], and the first letter of the correct choice (i, l, e, c, h, p.) 
All blood tubes (vessels 119/120/54) and plasma vessels (109 and 8) must be marked individually.

All vessels marked as Filled-”No” require a comment or explanation.

Enter comment codes to describe the reasons why a vessel is marked as Filled-”No," or why the protocol is incomplete.

\begin{tabular}{|l|l|}
\hline Comment for not filled vessels & Use when: \\
\hline Laboratory error & $\begin{array}{l}\text { A tube breaks in the centrifuge, contents spill, or other technologist } \\
\text { error. }\end{array}$ \\
\hline Quantity not sufficient & Quantity of blood is not sufficient to complete the protocol. \\
\hline SP refusal & The SP does not provide consent and consequently, excluded from HIV. \\
\hline
\end{tabular}


Attach a comment to an individual Filled-“No” vessel.

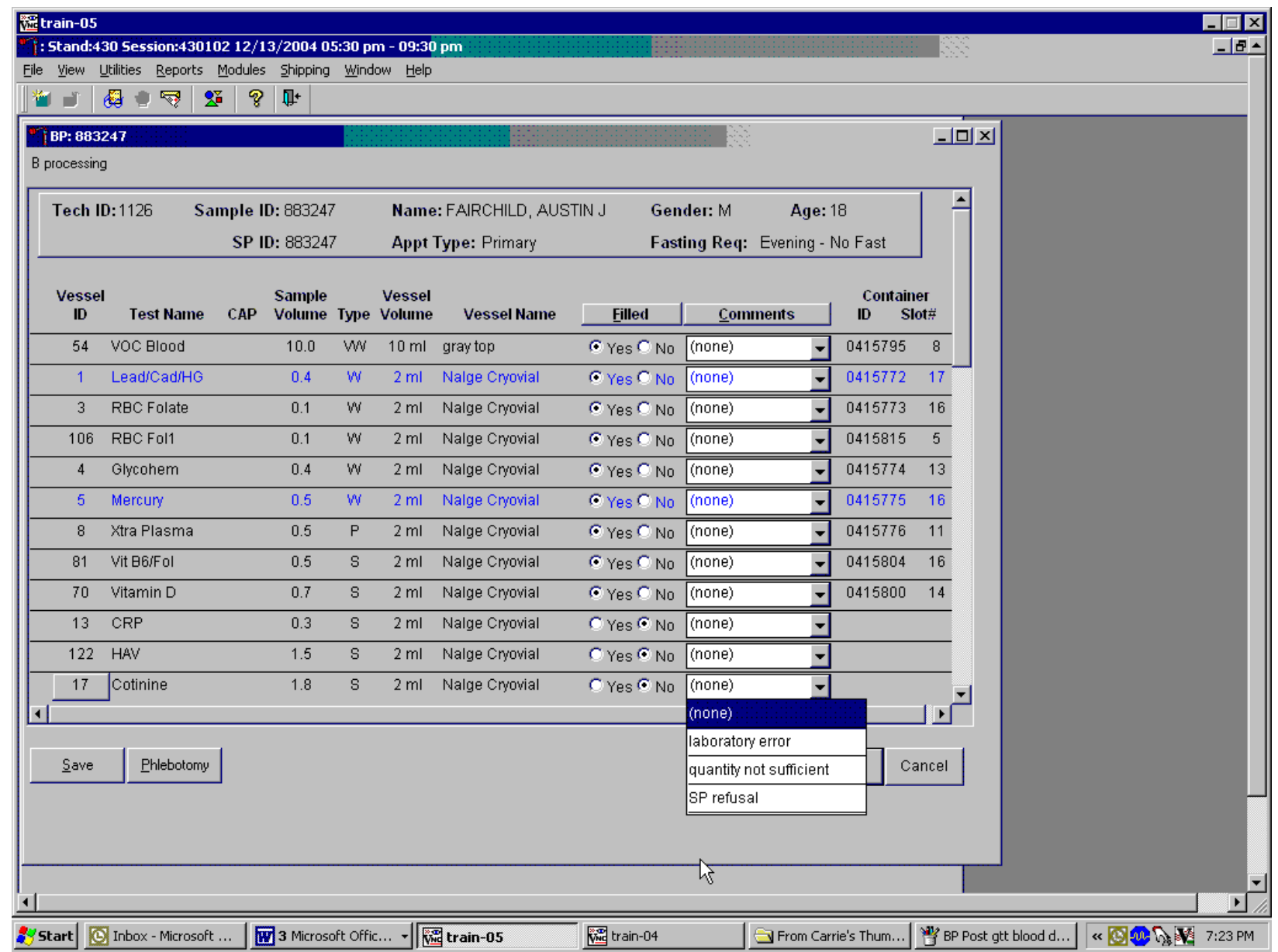

To attach a Filled-“No" comment to an individual vessel, use the mouse to direct the mouse arrow to the drop-down list under the "Comments" button to display the choices, drag the arrow to the correct choice, and left click. Alternatively, highlight the Comment text box and type the first letter of the desired choice. 
Use the following procedure when all Filled - "No" vessels require the same comment.

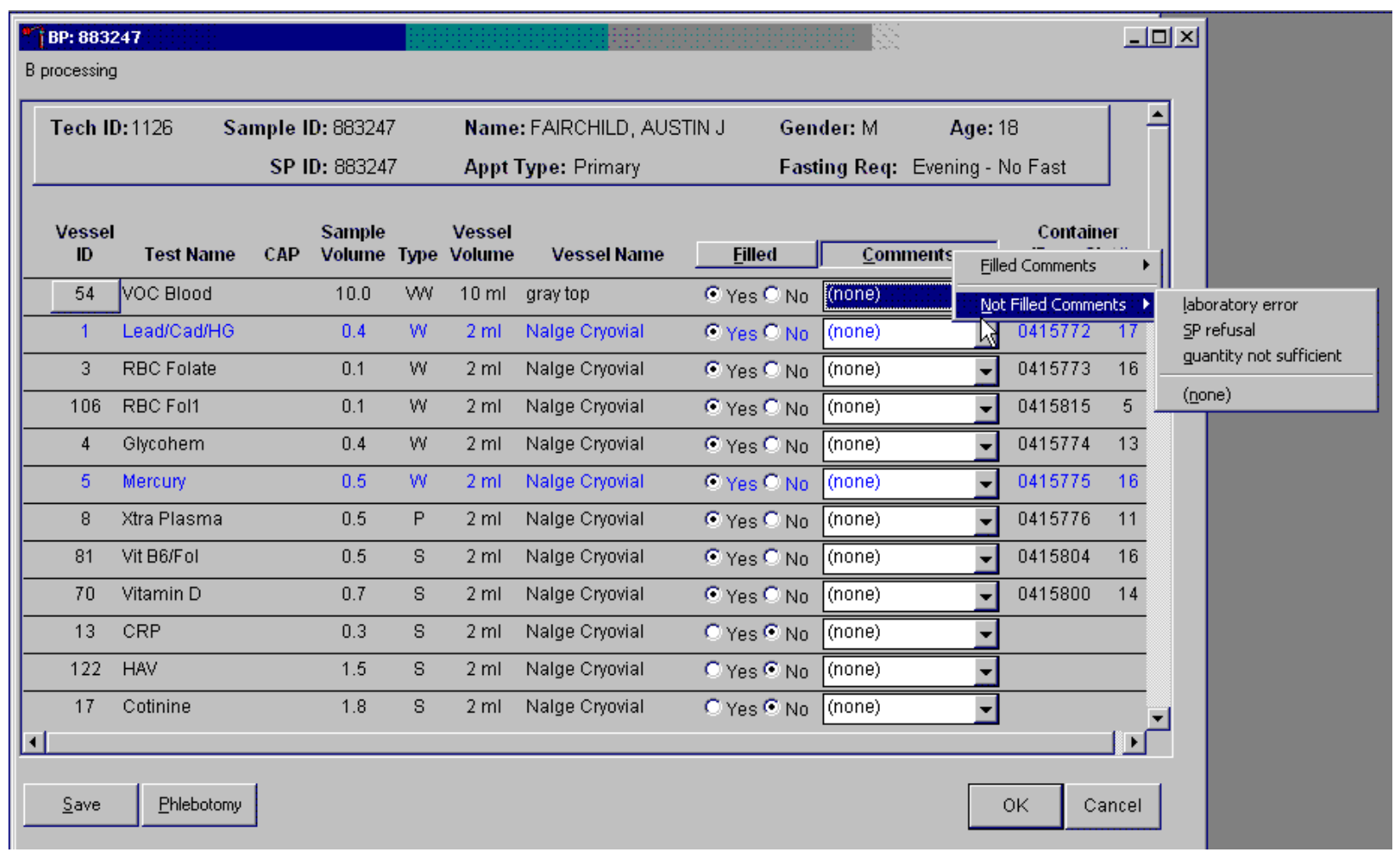

To collectively attach the same comment to all Filled-“No" vessels, use the mouse to direct the mouse arrow to the "Filled" button on the top of the radio buttons, left click, drag the arrow to Not Filled All\}, drag the mouse arrow to the desired choice, and left click. Alternatively, to collectively attach the same comment to all vessels marked as Filled - "No" type [Shift] [L/l], [N/n], and the first letter of the desired choice (l=laboratory error, s= SP refusal, q=quantity not sufficient.). 
If the EDTA tube is clotted, do not process vessel 1 (Lead/Cad), vessel 3 (RBC Folate), vessel 106, vessel 4 (Glycohem) or vessel 5 (Mercury).

Attach the "Laboratory error" comment to a Filled-No result for vessels 1 and 5, if the $\mathrm{CBC}$ was run before the vessels were processed.

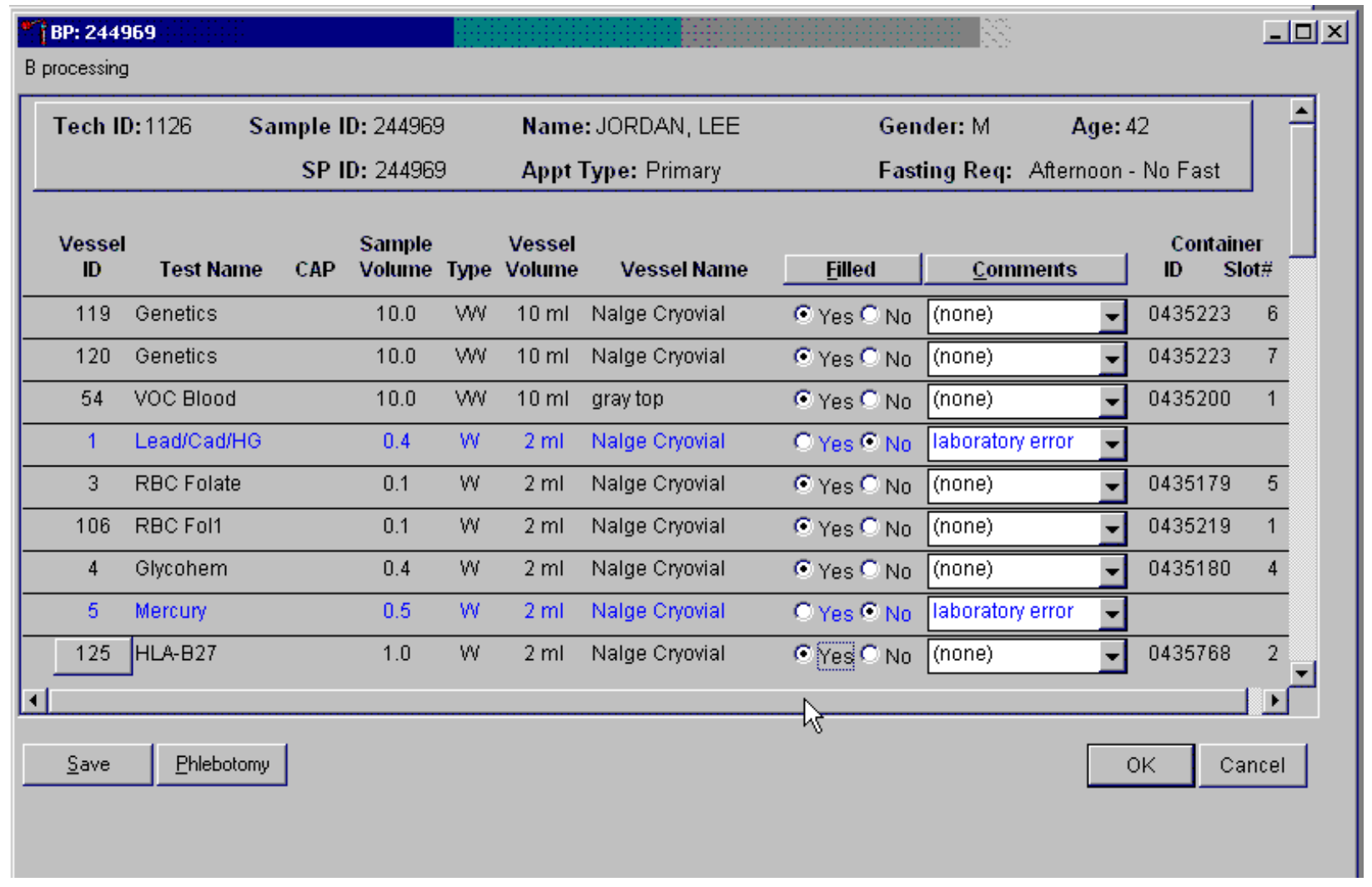

If the CBC was accidentally run before the whole blood for vessels 1 (Lead/Cad) and 5 (Mercury) was pipetted, then do not process these two vessels. Mark vessel 1 and 5 as Filled-“No" and attach the comment "laboratory error." Continue to pipette the whole blood for vessels 3, 106, and 4 even if the CBC was run before the whole blood was pipetted. 
Continue to enter results as vessels are processed. Review the information in the bloodprocessing window and save the data to the database.

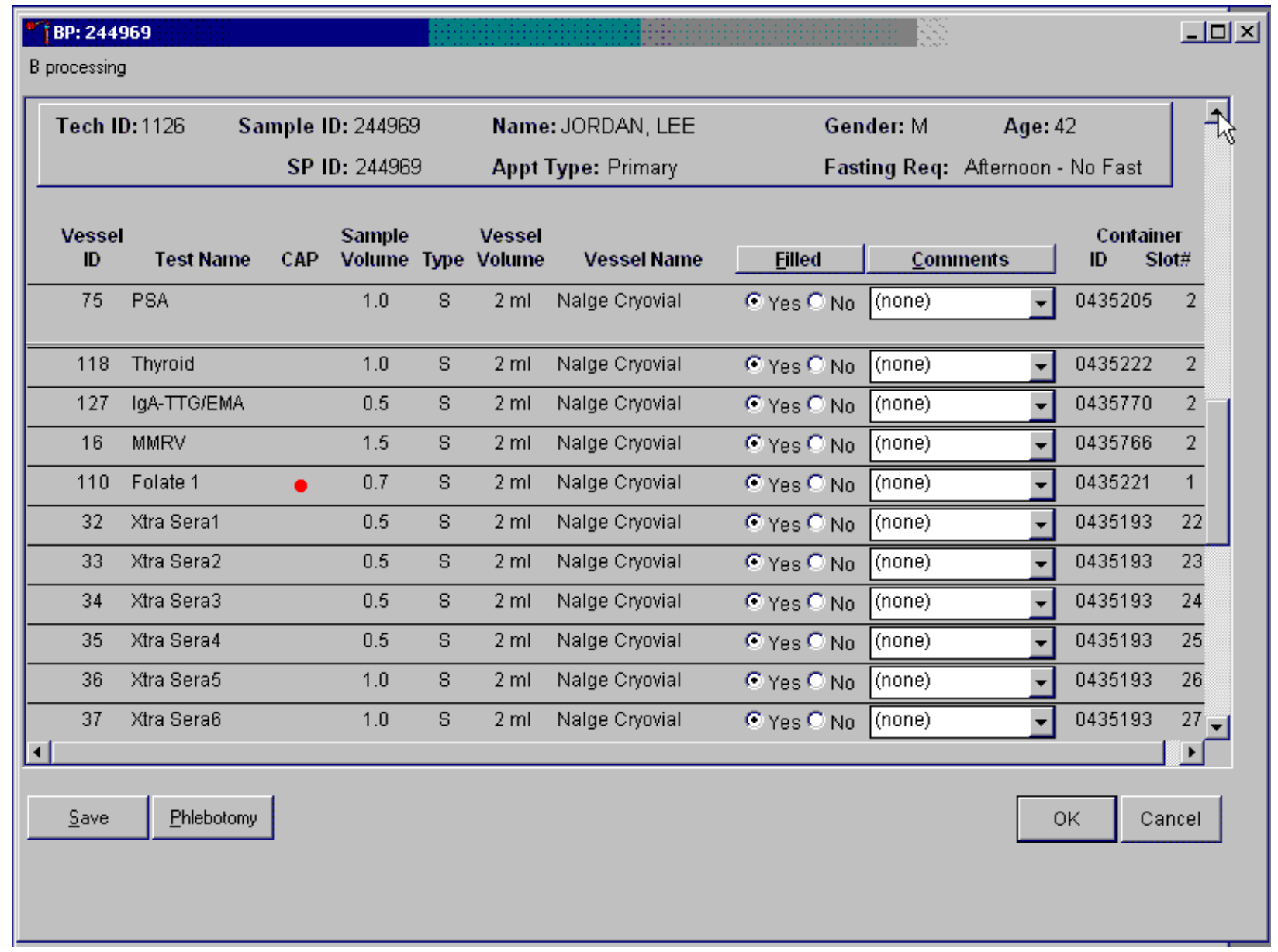

Store each filled vessel in the assigned slot in the assigned container. To record this action or to save this data to the database, use the mouse to direct the mouse arrow to the "Save" button and left click. To record this action or to save this data to the database and to exit the module, use the mouse to direct the mouse arrow to the "OK" button and left click. To close the window without saving any data in the database, use the mouse to direct the mouse arrow to the "Cancel" button and left click. 


\subsection{Washed Packed Cell Processing}

The washed packed cell module (PC) is located on the far right side of the laboratory headsup display. Use this module to enter processing results for vessels 88 and 126. It functions in the same way that the gtt module functions; all SPs are listed on the same screen. The module is enabled but individual SPs cannot be accessed and processing results cannot be entered until the SP has blood collected in phlebotomy.

\begin{tabular}{|c|c|c|c|c|c|c|c|c|c|c|c|c|c|c|c|c|}
\hline \multicolumn{17}{|c|}{ Lab Sink } \\
\hline \multirow{2}{*}{\multicolumn{16}{|c|}{ 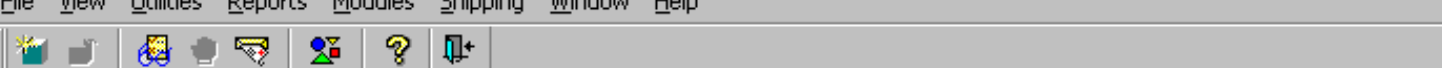 }} & \\
\hline & & & & & & & & & & & & & & & & \\
\hline \multicolumn{7}{|c|}{ Appointments for Session: 410111} & \multicolumn{10}{|c|}{ Process Status } \\
\hline SPID & Sample ID st & tatus & type & gender & age & name & uC & $\mathrm{u}$ & $\mathrm{P}$ & $\mathrm{OZ}$ & $\mathrm{CB}$ & $\mathrm{B}$ & gtt $P C$ & ${ }^{\circ} \mathrm{C}$ & HPV & OR \\
\hline 215591 & 215591 & $\mathrm{Cl}$ & VIP & $\mathrm{F}$ & 59 & PATEL, PAVARTI & $\bullet$ & 0 & $\bullet$ & · & 0 & 0 & · & . & . & . \\
\hline 244969 & 244969 & $\mathrm{BE}$ & $\mathrm{P}$ & M & 42 & JORDAN, LEE & 0 & 0 & . & 0 & 0 & $\bullet$ & .0 & & & 0 \\
\hline 470234 & 470234 & $\mathrm{Cl}$ & $P$ & $M$ & 60 & HAGRID, RUBEUS & 0 & 0 & $\cdot$ & 0 & 0 & 0 & . $\mathrm{c}$ & & & 0 \\
\hline 668903 & 668903 & $\mathrm{Cl}$ & $P$ & M & 9 & WEASLEY, FRED & 0 & 0 & $\cdot$ & 0 & 0 & 0 & $\cdot 0$ & & . & . \\
\hline 762731 & 762731 & $\mathrm{Cl}$ & VIP & $\mathrm{F}$ & 12 & BROWN, LAVENDAR & $\bullet$ & 0 & $\bullet$ & . & 0 & 0 & . & . & . & . \\
\hline 836402 & 836402 & $\mathrm{sc}$ & VIP & $M$ & 8 & MALFOY, DRACO & 0 & 0 & . & . & 0 & 0 & . & . & . & . \\
\hline 900081 & 900081 & $\mathrm{Cl}$ & VIP & $\mathrm{F}$ & 49 & ABEOTT, HANNAH & 0 & 0 & 0 & . & 0 & 0 & . . & . & . & . \\
\hline 998878 & 998878 & $\mathrm{Cl}$ & VIP & $M$ & 2 & DURSLEY, VERNON & . & . & . & . & 0 & 0 & . & . & . & . \\
\hline
\end{tabular}


Record blood processing results for vessels 88 and 126 using the "PC" module. Select the correct SP and access the Packed Cell Processing module.

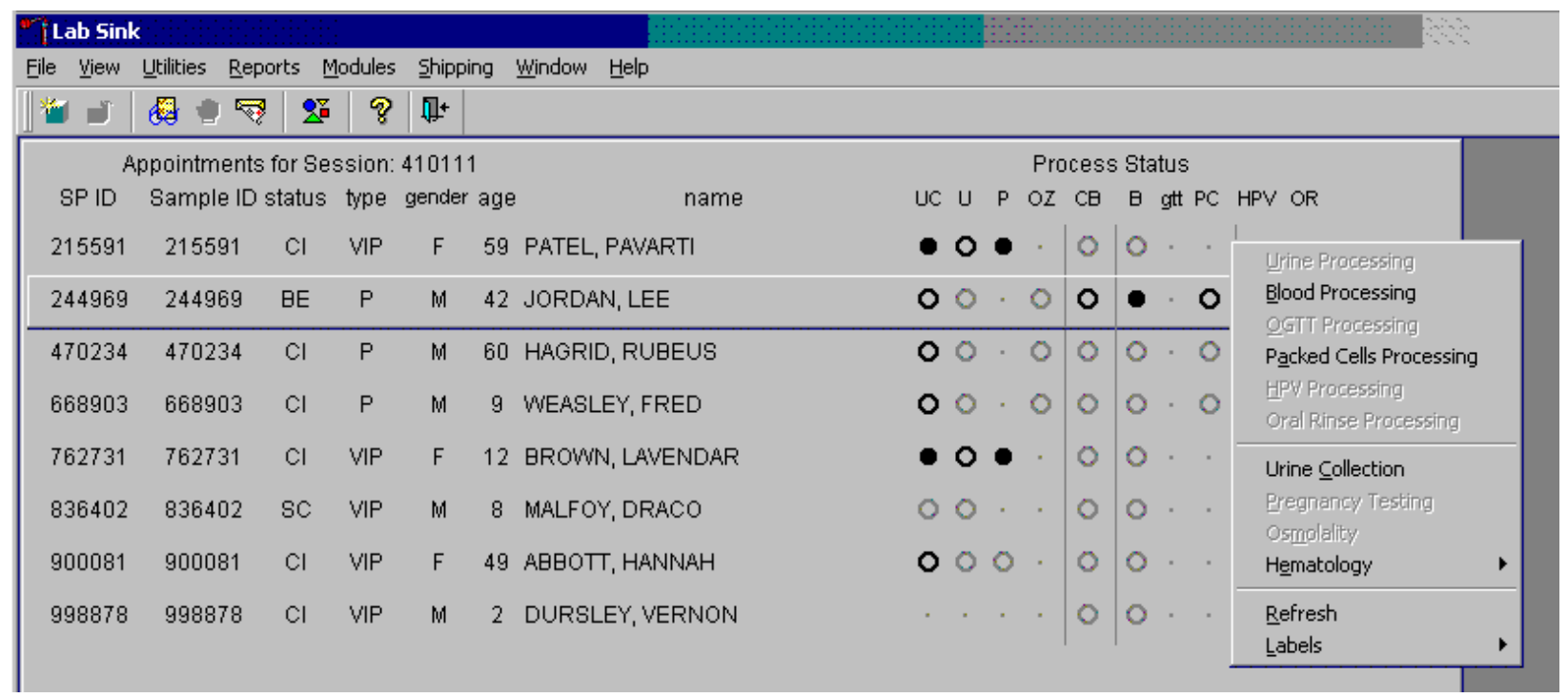

To access the Packed Cell Processing module, highlight the SP, right click, drag the mouse arrow to \{Packed Cell Processing\}, and left click or type [Alt] [A/a]. 
The Packed Cell Processing screen displays. Record the vessel 126 and vessel 88 processing results in the Packed Cell Processing screen. There are two sections to the screen. The top sections lists the SP ID, Sample ID, Name, Gender and Age for all eligible SPs. Once the venipuncture is complete, the SP's row becomes enabled (black). A refresh button has been placed to the right of the heads-up pane on the Packed Cell Processing Screen.

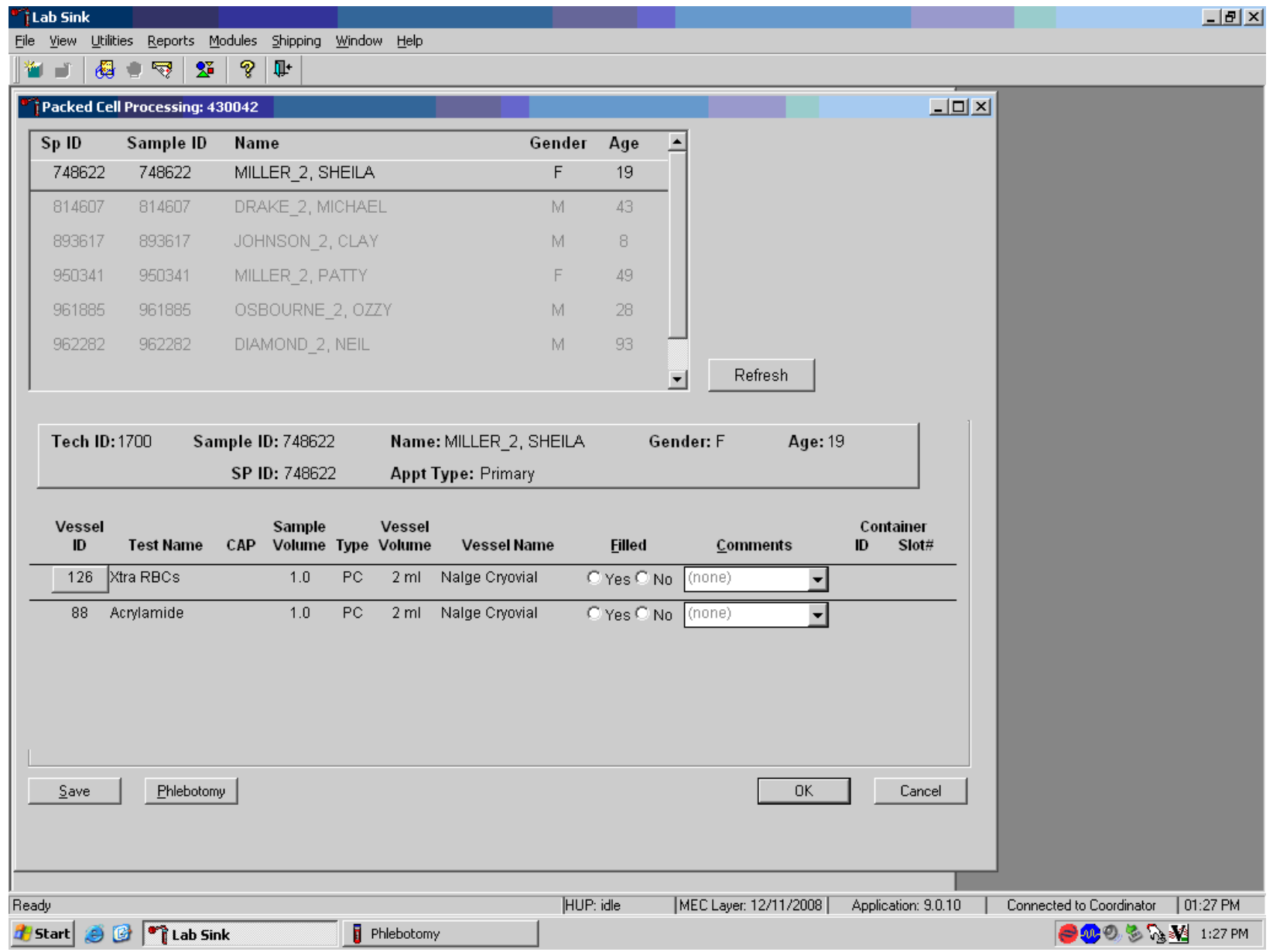

The bottom section of the Packed Cell Processing window for an SP contains the following information: Tech ID, Sample ID, SP ID, Name (last, first), Gender, Age, Appt (appointment) Type, Fasting Req, columns for Vessel ID, Test Name, CAP, Sample Volume, Type (PC), Vessel Volume, Vessel Name, Filled radio buttons, Comments text box, and a Container ID/Slot\# column. 
Use the "Phlebotomy" button to view the SP's original venipuncture protocol and updated results.

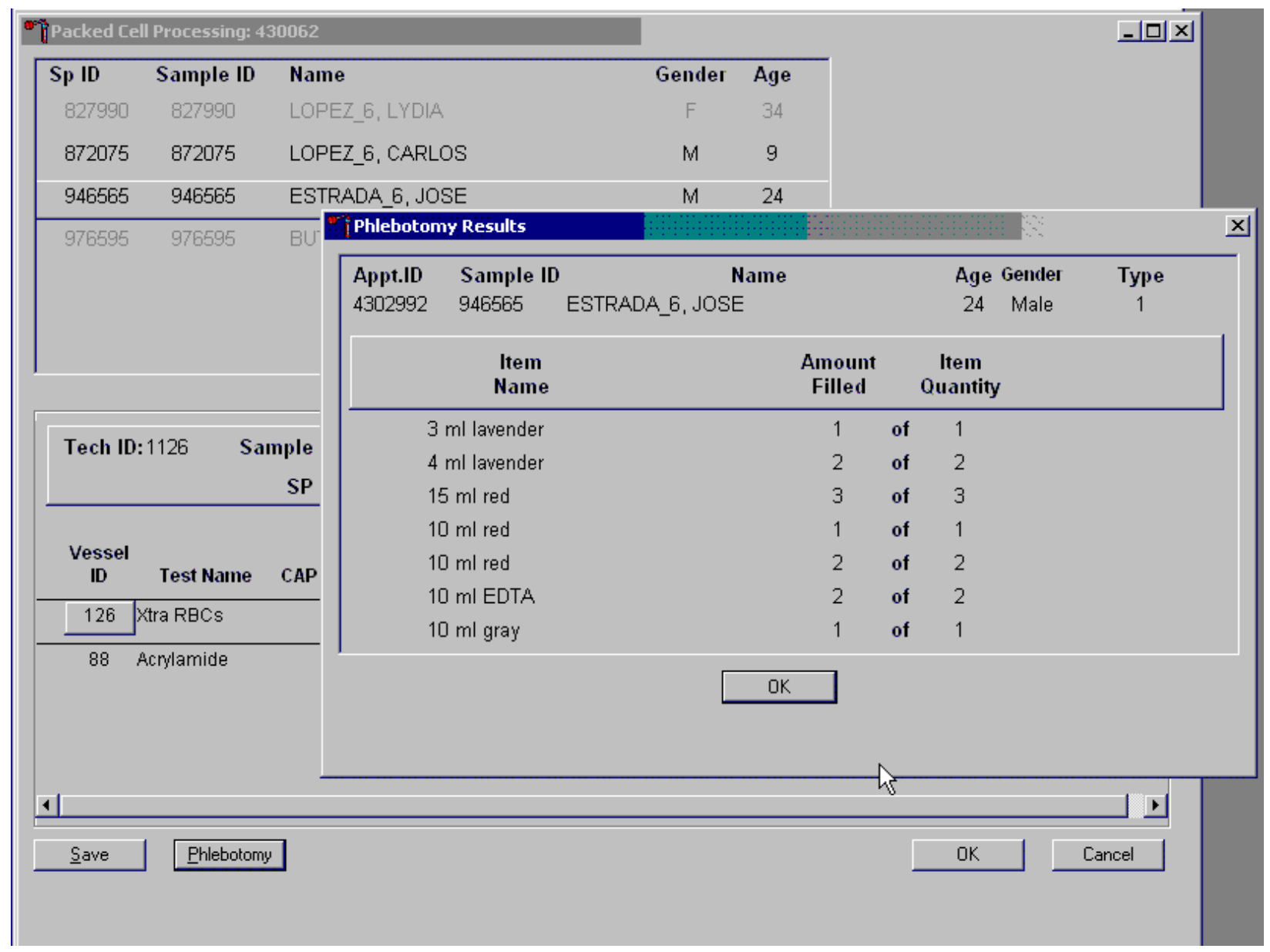

Select the OK button to remove the Phlebotomy Results window. 
Record the packed cell processing results using procedures described in Section 8.8.

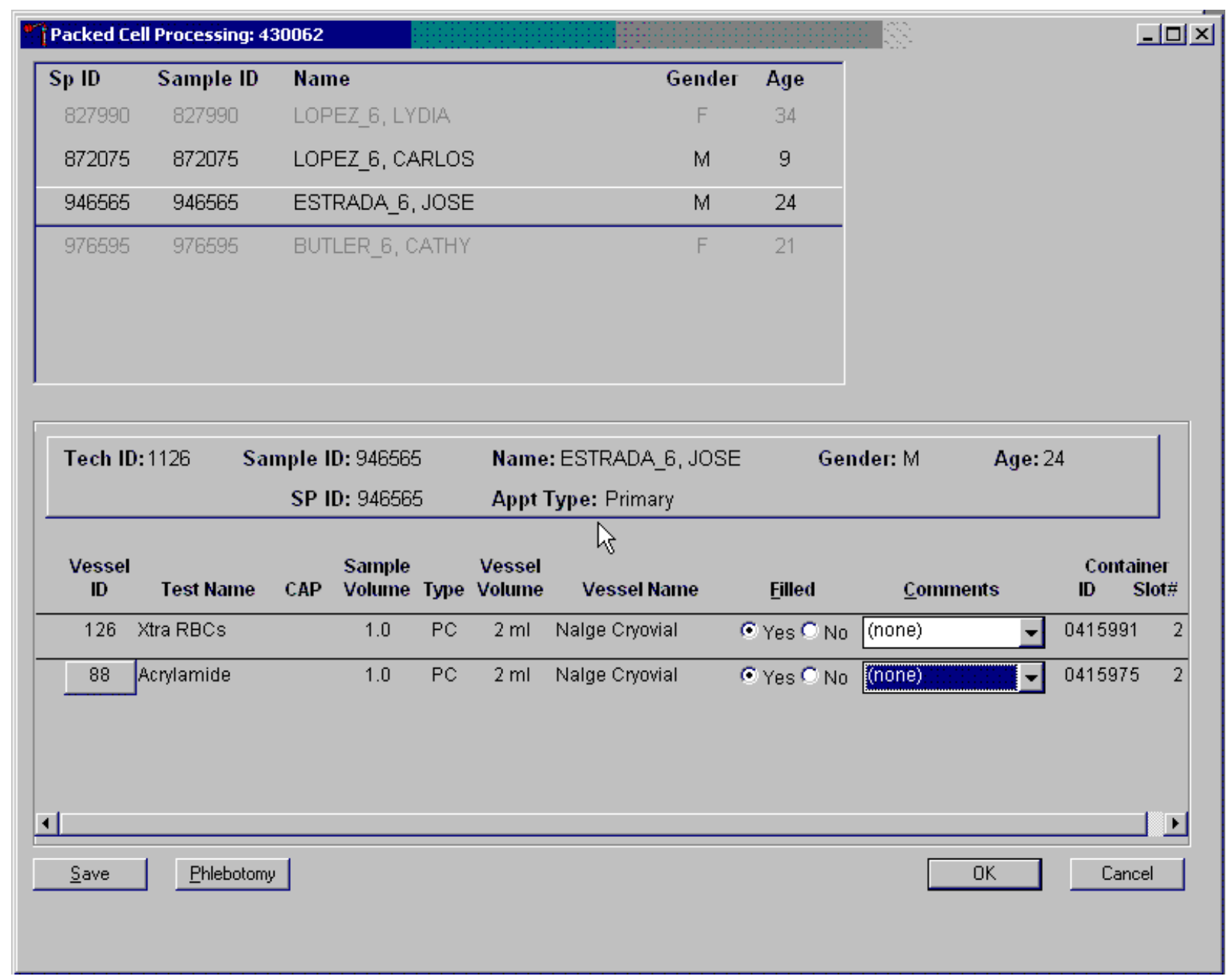

Use the radio button to mark the vessels as filled "Yes" or "No" and attach any appropriate comments. Use the "Save" button to save the results to the database. This does not close the window. 
Select another SP by using the up and down arrows to identify another SP listed in the top section.

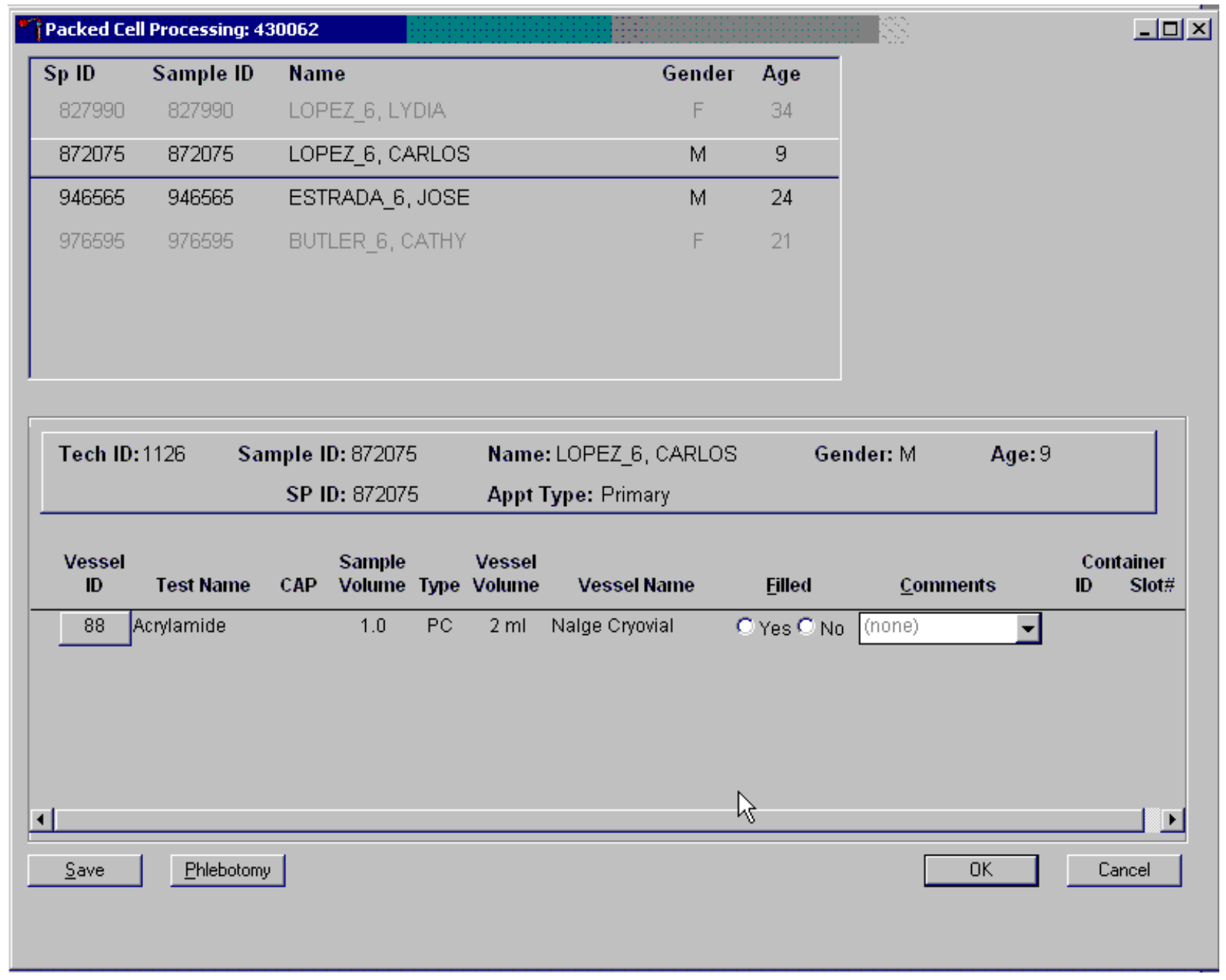

The active SP is contained in a rectangular box. The information in the bottom section will update with the SP's Sample ID, SP ID, Name, Appt Type, Gender and Age. If the SP is grayed out, the vessels will display but it’s impossible to mark them as filled "Yes" or "No." 
Use the "OK" button to save the data and close the screen. Use the "Cancel" button to close the screen without saving any data to the database. If another SP is selected from the top part of the window before the Save button has been selected, then an Unsaved Changes informational text box displays.

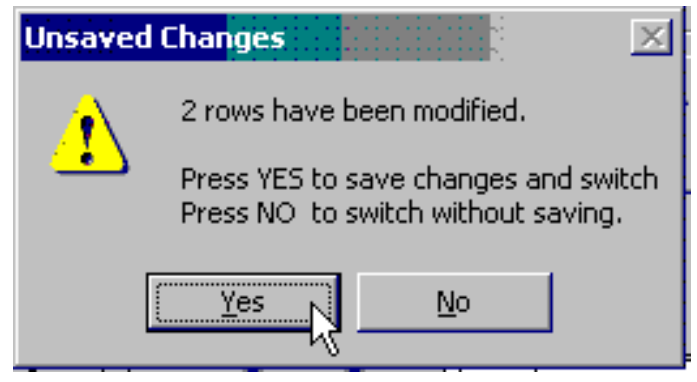

Select the "Yes" button to save the changes and switch to the new SP or select the "No" button to switch without saving the data. 
Continue to process all SPs.

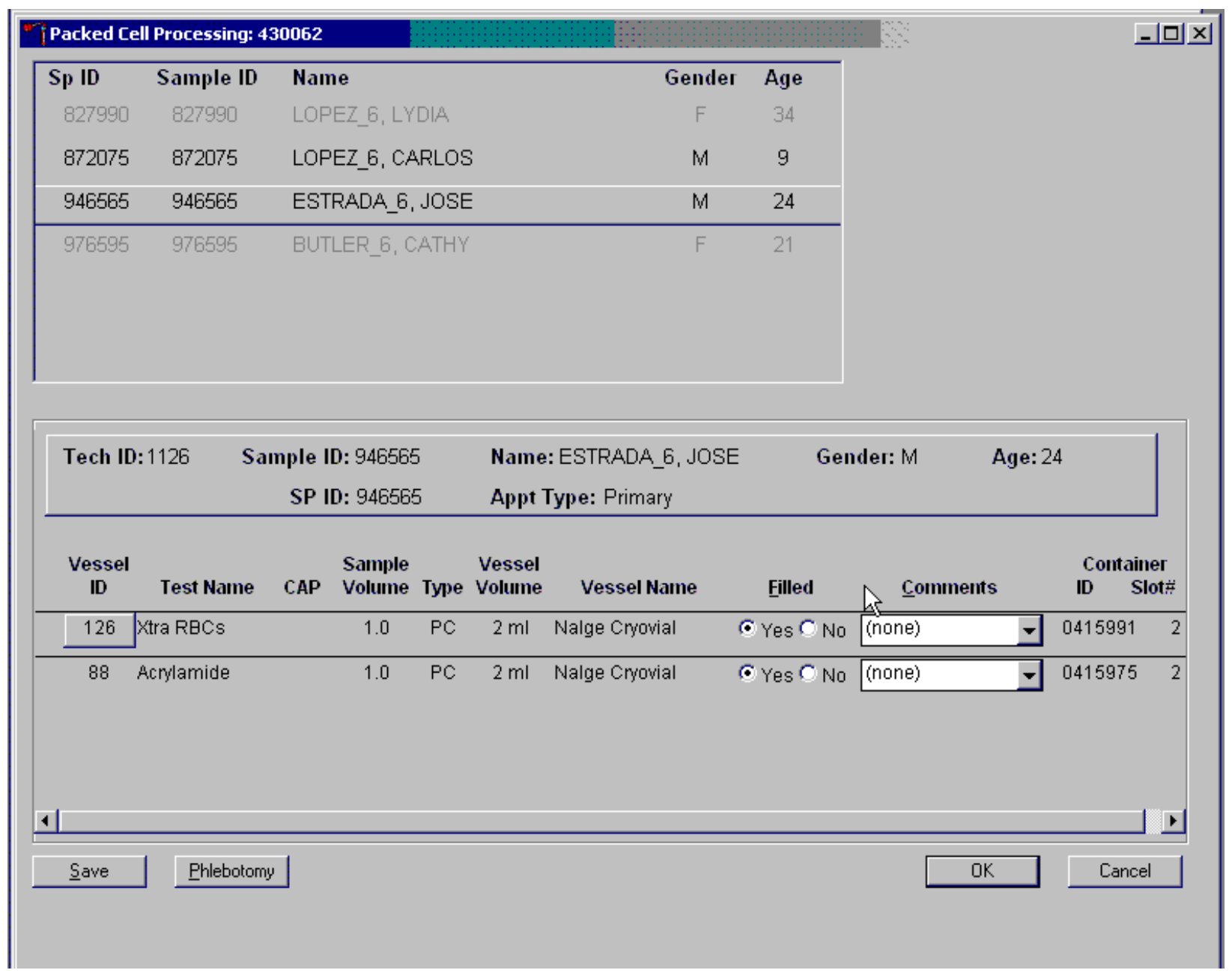

To exit the module, select the OK button. 


\subsection{Blood Specimen Storage}

\subsubsection{Blood Specimen Storage Protocol}

Fill the blood vessels, enter the blood processing results, and prepare to store the vessels. Store vessels in numbered storage boxes according to test as indicated in Table 8-1.

At the beginning of each stand, generate and print a series of bar-coded, numbered labels for storage containers as described in Chapter 9. Use the shipping module to assign a bar-code label to a specific storage container (test). This process “opens” a storage box. Each vessel is assigned to a specific slot in a specific container as processing results are entered. Slots in containers are assigned according to a standard left to right, top to bottom procedure. Store each vessel in the appropriate slot in the correct container immediately after processing. 
Exhibit 8-21. Storage protocol for blood

\begin{tabular}{|c|c|c|c|c|}
\hline Shipping Location & Vessels & Conditions & Vessel Storage & $\begin{array}{c}\text { Ship } \\
\text { Frequency } \\
\end{array}$ \\
\hline $\begin{array}{l}\text { CDC/NCEH Molecular Biology } \\
\text { Branch }\end{array}$ & 119/120 Genetics & Refrigerated & $\begin{array}{l}5 \times 5 \text { five inch foam } \\
\text { box }\end{array}$ & Twice week \\
\hline $\begin{array}{l}\text { CDC/NCEH Air Toxicant } \\
\text { Branch }\end{array}$ & 54 VOC Blood & Refrigerated & $\begin{array}{l}5 \times 5 \text { five inch foam } \\
\text { box }\end{array}$ & Weekly \\
\hline CASPIR & $1 \mathrm{Lead} / \mathrm{Cad} / \mathrm{HG}$ & Frozen & $9 \times 9$ two inch box & Weekly \\
\hline CASPIR & 3 RBC Folate & Frozen & 10x10 two inch box & Weekly \\
\hline CASPIR & 106 RBC Fol1 & Frozen & 10x10 two inch box & Weekly \\
\hline NHANES Diabetes Laboratory & 4 Glycohem & Refrigerated & $9 \times 9$ two inch box & Weekly \\
\hline CASPIR & 5 Mercury & Frozen & $9 \times 9$ two inch box & Weekly \\
\hline Esoterix Clinical Trials Services & 125 HLA-B27 & Refrigerated & $9 \times 9$ two inch box & Weekly \\
\hline CASPIR & 109 PUFA/TFA & Frozen & 10x10 two inch box & Weekly \\
\hline CASPIR & 8 Xtra Plasma & Frozen & $9 \times 9$ two inch box & End of stand \\
\hline NHANES Diabetes Laboratory & 9 Glucose & Frozen & $9 \times 9$ two inch box & Weekly \\
\hline NHANES Diabetes Laboratory & 98 OGTT & Frozen & $9 \times 9$ two inch box & Weekly \\
\hline CASPIR & 88 Acrylamide & Frozen & $9 \times 9$ two inch box & Weekly \\
\hline CASPIR & 126 Xtra RBCs & Frozen & $9 \times 9$ three inch box & Weekly \\
\hline CASPIR & 81 Vit B6/Fol & Frozen & 10x10 two inch box & Weekly \\
\hline CASPIR & 70 Vitamin D & Frozen & 10x10 two inch box & Weekly \\
\hline University of Washington & 13 CRP & Frozen & 9 x 9 two inch box & Weekly \\
\hline CDC/NCID Hepatitis Ref Lab & 14 Hep Profile & Frozen & $9 \times 9$ two inch box & Weekly \\
\hline CDC/NCID Hepatitis Ref Lab & $122 \mathrm{HAV}$ & Frozen & $9 \times 9$ two inch box & Weekly \\
\hline CDC/NCID Hepatitis Ref Lab & 87 Hepatitis ab & Frozen & $9 \times 9$ two inch box & Weekly \\
\hline CASPIR & $99 \mathrm{Fer} / \mathrm{TfR}$ & Frozen & 10x10 two inch box & Weekly \\
\hline CASPIR & 17 Cotinine & Frozen & $9 \times 9$ two inch box & Weekly \\
\hline NHANES Diabetes Laboratory & 21 Lipids & Frozen & $9 \times 9$ two inch box & Weekly \\
\hline CDC /DVRD HPV & 77 HPV Serum & Frozen & $9 \times 9$ two inch box & Weekly \\
\hline $\begin{array}{l}\text { Collaborative Laboratory } \\
\text { Services }\end{array}$ & 18 Biochem & Refrigerated & $9 \times 9$ two inch box & Weekly \\
\hline NHANES Diabetes Laboratory & 23 Insulin & Frozen & $9 \times 9$ two inch box & Weekly \\
\hline CDC NCID AIDS Division & $22 \mathrm{HIV}$ & Frozen & $9 \times 9$ two inch box & Weekly \\
\hline Emory University & $24 \mathrm{HSV}$ & Frozen & $9 \times 9$ two inch box & Weekly \\
\hline University of Washington & 75 PSA & Frozen & $9 \times 9$ two inch box & Weekly \\
\hline University of Washington & 118 Thyroid & Frozen & $9 \times 9$ two inch box & Weekly \\
\hline Mayo Clinic & $\begin{array}{l}127 \text { IgA- } \\
\text { TTG/EMA }\end{array}$ & Frozen & $9 \times 9$ two inch box & Weekly \\
\hline CDC/NCID DASH & $16 \mathrm{MMRV}$ & Frozen & $9 \times 9$ two inch box & Weekly \\
\hline CASPIR & 110 Folate1 & Frozen & $10 \times 10$ two inch box & Weekly \\
\hline
\end{tabular}


Exhibit 8-21. Storage protocol for blood (continued)

\begin{tabular}{|l|l|l|l|l|}
\hline \multicolumn{1}{|c|}{ Shipping Location } & \multicolumn{1}{c|}{ Vessels } & \multicolumn{1}{c|}{ Conditions } & \multicolumn{1}{c|}{ Vessel Storage } & \multicolumn{1}{c|}{$\begin{array}{c}\text { Ship } \\
\text { Frequency }\end{array}$} \\
\hline CASPIR & 32-39 Xtra Sera & Frozen & $9 \times 9$ two inch box & End of stand \\
\hline $\begin{array}{l}\text { CDC/NCEH Pesticide } \\
\text { Laboratory }\end{array}$ & 91 Srm Pest & Frozen & $9 \times 9$ three inch box & End of stand \\
\hline CASPIR & $\begin{array}{l}\text { 29 Persis Pest1 } \\
\text { 89 Persis Pest2 }\end{array}$ & Frozen & $9 \times 9$ three inch box & End of stand \\
\hline $\begin{array}{l}\text { CDC/NCEH Environmental } \\
\text { Health Laboratory }\end{array}$ & 90 Perfluor & Frozen & $9 \times 9$ three inch box & End of stand \\
\hline CASPIR & $\begin{array}{l}92 \text { BFR } 1 \\
93 \text { BFR } 2\end{array}$ & Frozen & $9 \times 9$ three inch box & End of stand \\
\hline
\end{tabular}

\subsection{How to Deal With System Failure}

If the computer system fails, record results on a preprinted workstation 1 processing worksheet (Exhibit 8-21). Complete a Blood Processing worksheet for each SP while processing the blood specimens. Enter the results after the system is operational. 
Exhibit 8-22. Workstation 1 processing worksheet

SP ID

BLOOD PROCESSING

\begin{tabular}{|c|c|c|c|c|c|c|c|c|}
\hline ID & Name & Ages & $\begin{array}{c}\text { Sample } \\
\text { mL }\end{array}$ & $\begin{array}{c}\text { Sample } \\
\text { Type }\end{array}$ & $\underset{\checkmark}{\text { Filled }}$ & $\begin{array}{c}\text { Filled } \\
\text { Comments } \\
\text { IctericLipemic } \\
\text { Equip Failure } \\
\text { Clotted } \\
\text { Hemolyzed }\end{array}$ & $\begin{array}{l}\text { Not Filled } \\
\text { Comments } \\
\text { QNSSP } \\
\underline{R} e f u s a l \\
\text { Lab Error }\end{array}$ & Slot \# \\
\hline 119 & Genetics & $20+$ & 10.0 & WB & & & & \\
\hline 120 & Genetics & $20+$ & 10.0 & WB & & & & \\
\hline 54 & VOC Blood & $12+$ & 10.0 & WB & & & & \\
\hline 1 & Lead/Cad/HG & $1+$ & 0.4 & WB & & & & \\
\hline 3 & RBC Folate & $1+$ & 0.1 & WB & & & & \\
\hline 106 & RBC Fol1 & $1+$ & 0.1 & WB & & & & \\
\hline 4 & Glycohem & $12+$ & 0.4 & WB & & & & \\
\hline 5 & Mercury & $1+$ & 0.5 & WB & & & & \\
\hline 125 & HLA-B27 & $20-69$ & 1.0 & WB & & & & \\
\hline 109 & PUFA/TFA & $3+$ & 0.7 & $\mathrm{P}$ & & & & \\
\hline 8 & Xtra Plasma & $6+$ & 0.5 & $\mathrm{P}$ & & & & \\
\hline 9 & Glucose & $12+$ & 0.5 & $\mathrm{P}$ & & & & \\
\hline 98 & OGTT & $12+$ & 0.5 & $\mathrm{P}$ & & & & \\
\hline 88 & Acrylamide & $6+$ & 1.0 & $\mathrm{PC}$ & & & & \\
\hline 126 & Xtra RBCs & $12+$ & 1.0 & $\mathrm{PC}$ & & & & \\
\hline 81 & Vit B6/Fol & $1+$ & 0.5 & $\mathrm{~S}$ & & & & \\
\hline 70 & Vitamin D & $\begin{array}{l}1-5 \\
6+ \\
\end{array}$ & $\begin{array}{l}0.5 \\
0.7 \\
\end{array}$ & $S$ & & & & \\
\hline 13 & CRP & $3+$ & 0.3 & $S$ & & & & \\
\hline 14 & Hep Profile & $20+$ & 1.5 & $\mathrm{~S}$ & & & & \\
\hline 122 & HAV & $6-19$ & 1.5 & $\mathrm{~S}$ & & & & \\
\hline 87 & Hepatitis ab & $\begin{array}{c}2 \\
3-5 \\
\end{array}$ & $\begin{array}{l}0.3 \\
0.5\end{array}$ & $\mathrm{~S}$ & & & & \\
\hline 99 & Fer/TfR & $\begin{array}{c}1-2 \\
3-5 \\
12-49\end{array}$ & $\begin{array}{l}0.3 \\
0.5 \\
0.5 \\
\end{array}$ & $S$ & & & & \\
\hline 17 & Cotinine & $\begin{array}{c}3-5 \\
6-11 \\
12+ \\
\end{array}$ & $\begin{array}{l}0.8 \\
1.1 \\
1.8 \\
\end{array}$ & $S$ & & & & \\
\hline 21 & Lipids & $\begin{array}{l}6-11 \\
12+\end{array}$ & $\begin{array}{l}1.0 \\
1.5 \\
\end{array}$ & $S$ & & & & \\
\hline
\end{tabular}


Exhibit 8-22. Workstation 1 processing worksheet (continued)

\begin{tabular}{|c|c|c|c|c|c|c|c|c|}
\hline ID & Name & Ages & $\begin{array}{c}\text { Sample } \\
\text { mL }\end{array}$ & $\begin{array}{c}\text { Sample } \\
\text { Type }\end{array}$ & $\underset{\checkmark}{\text { Filled }}$ & $\begin{array}{c}\text { Filled } \\
\text { Comments } \\
\text { ÍctericLipemic } \\
\text { Equip Failure } \\
\text { Clotted } \\
\text { Hemolyzed } \\
\end{array}$ & $\begin{array}{c}\text { Not Filled } \\
\text { Comments } \\
\text { QNSSP } \\
\underline{\text { Refusal }} \\
\underline{\text { Lab Error }}\end{array}$ & Slot \# \\
\hline 77 & HPV Serum & $14-59$ & 0.5 & $\mathrm{~S}$ & & & & \\
\hline 18 & Biochem & $12+$ & 0.8 & $S$ & & & & \\
\hline 23 & Insulin & $12+$ & 1.0 & $S$ & & & & \\
\hline 22 & HIV & 18-59 & 0.8 & $S$ & & & & \\
\hline 24 & HSV & 14-49 & 0.2 & $S$ & & & & \\
\hline 75 & PSA & $40+$ & 1.0 & $S$ & & & & \\
\hline 118 & Thyroid & $12+$ & 1.0 & $S$ & & & & \\
\hline 127 & $\begin{array}{l}\text { IgA-TTG/ } \\
\text { EMA }\end{array}$ & $6+$ & 1.0 & $S$ & & & & \\
\hline 16 & MMRV & $6+$ & 1.5 & $S$ & & & & \\
\hline 110 & Folate1 & $1+$ & 0.7 & $S$ & & & & \\
\hline 32 & Xtra Sera1 & $3+$ & 0.5 & $S$ & & & & \\
\hline 33 & Xtra Sera2 & $3+$ & 0.5 & $S$ & & & & \\
\hline 34 & Xtra Sera3 & $3+$ & 0.5 & $S$ & & & & \\
\hline 35 & Xtra Sera4 & $12+$ & 0.5 & $S$ & & & & \\
\hline 36 & Xtra Sera5 & $12+$ & 1.0 & $S$ & & & & \\
\hline 37 & Xtra Sera6 & $12+$ & 1.0 & $S$ & & & & \\
\hline 38 & Xtra Sera7 & $12+$ & 1.0 & $\mathrm{~S}$ & & & & \\
\hline 39 & Xtra Sera8 & $12+$ & 1.0 & $S$ & & & & \\
\hline 29 & Persis Pest1 & $12+$ & 4.0 & $S$ & & & & \\
\hline 89 & Persis Pest2 & $12+$ & 4.0 & $\mathrm{~S}$ & & & & \\
\hline 90 & Perfluor1 & $12+$ & 4.0 & $S$ & & & & \\
\hline 92 & BFR1 & $12+$ & 4.0 & $S$ & & & & \\
\hline 93 & BFR2 & $12+$ & 4.0 & S & & & & \\
\hline 91 & Srm Pest & $12+$ & 4.0 & $\mathrm{P}$ & & & & \\
\hline
\end{tabular}

WB $=$ Whole Blood $\quad \mathrm{P}=$ Plasma $\quad \mathrm{PC}=$ Packed cells $\quad \mathrm{S}=$ Serum 


\section{PRINTING LABELS AND SPECIMEN SHIPMENT}

\section{$9.1 \quad$ Introduction}

Labels are essential to the laboratory component. Labels support each sample person's (SP's) unique processing protocol. The laboratory record is created when the medical technologist accesses a session for the first time. This record forms the basis for each SP's data in the database. Container labels are generated and assigned to vessel storage containers and allow a processed sample to be tracked from mobile examination center (MEC) processing through contract laboratory receipt.

Biological specimens are shipped to a variety of contract laboratories in double-sided Styrofoam and corrugated cardboard shippers or hard-sided shippers with Styrofoam inserts using dry ice or wet ice (refrigerant packs). A copy of the shipping manifest is included with each shipment that contains a list of the items contained in the shipment and certain demographic information. Exhibit 9-1, the Blood and urine shipping protocol, illustrates the specifications for the shipment of blood and urine specimens to each contract laboratory.

Packages are shipped using FedEx. The chief medical technologist is responsible for contacting FedEx at the start of each stand to determine availability. The facility and equipment specialist contacts the dry ice company at the start of each stand to establish a contact but the chief medical technologist arranges for the delivery of dry ice on shipping days. It is the chief medical technologist's responsibility to confirm the arrangements for shipping and dry ice delivery with the facility and the equipment specialist at the beginning of each stand.

The chief medical technologist works with the MEC manager and the home office to determine exactly which days to ship specimens to the contract laboratories. As a rule, pack and ship most specimens once a week during the MEC split examination sessions. Ship vessels 119/120 twice per week. Hold the containers for vessels 8, 32-39, 29/89, 90, 91, 92/93, 48, 49, 65, 66, 67, 102, 121, and 52 and ship once they are full, or hold and ship at the end of the stand. All specimens should arrive at the destination the day after shipping. The next-day limitation may conflict with off days, holidays, and vacations. If a conflict with the shipping schedule, working schedule, or the contract laboratory schedule occurs, consult the MEC manager and the home office technical supervisor. 
Exhibit 9-1. Blood and urine shipping protocol

\begin{tabular}{|c|c|c|c|c|}
\hline Location & Vessels & Conditions & Vessel storage & $\begin{array}{l}\text { Shipping } \\
\text { frequency }\end{array}$ \\
\hline \multicolumn{5}{|l|}{ Whole Blood } \\
\hline $\begin{array}{l}\text { CDC/NCEH Molecular } \\
\text { Biology Branch } \\
\text { Vessels 119/120 }\end{array}$ & Genetics & Refrigerated & $\begin{array}{l}5 \times 5 \text { five inch box } \\
\text { with foam insert }\end{array}$ & $\begin{array}{l}\text { Twice } \\
\text { weekly }\end{array}$ \\
\hline $\begin{array}{l}\text { CDC/NCEH Air Toxicant } \\
\text { Branch } \\
\text { Vessel } 54\end{array}$ & VOC Blood & Refrigerated & $\begin{array}{l}5 \times 5 \text { five inch box } \\
\text { with foam insert }\end{array}$ & Weekly \\
\hline $\begin{array}{l}\text { CASPIR } \\
\text { Vessel } 1 \\
\end{array}$ & Lead/Cad/HG & Frozen & $9 \times 9$ two inch box & Weekly \\
\hline $\begin{array}{l}\text { CASPIR } \\
\text { Vessel } 3 \\
\end{array}$ & RBC Folate & Frozen & $10 \times 10$ two inch box & Weekly \\
\hline $\begin{array}{l}\text { CASPIR } \\
\text { Vessel } 106\end{array}$ & RBC Fol1 & Frozen & 10 x 10 two inch box & Weekly \\
\hline $\begin{array}{l}\text { NHANES Diabetes Laboratory } \\
\text { Vessel } 4\end{array}$ & Glycohem & Refrigerated & $9 \times 9$ two inch box & Weekly \\
\hline $\begin{array}{l}\text { CASPIR } \\
\text { Vessel } 5\end{array}$ & Mercury & Frozen & $9 \times 9$ two inch box & Weekly \\
\hline $\begin{array}{l}\text { Esoterix Clinincal Trials } \\
\text { Services } \\
\text { Vessel } 125\end{array}$ & HLA-B27 & Refrigerated & $9 \times 9$ two inch box & Weekly \\
\hline \multicolumn{5}{|l|}{ Plasma } \\
\hline $\begin{array}{l}\text { CASPIR } \\
\text { Vessel } 109\end{array}$ & PUFA/TFA & Frozen & $10 \times 10$ two inch box & Weekly \\
\hline $\begin{array}{l}\text { CASPIR } \\
\text { Vessel } 8\end{array}$ & Xtra Plasma & Frozen & $9 \times 9$ two inch box & $\begin{array}{l}\text { Full or end } \\
\text { of stand }\end{array}$ \\
\hline $\begin{array}{l}\text { NHANES Diabetes Laboratory } \\
\text { Vessel } 9\end{array}$ & Glucose & Frozen & $9 \times 9$ two inch box & Weekly \\
\hline $\begin{array}{l}\text { NHANES Diabetes Laboratory } \\
\text { Vessel } 98\end{array}$ & OGTT & Frozen & $9 \times 9$ two inch box & Weekly \\
\hline $\begin{array}{l}\text { CDC/NCEH Pesticide } \\
\text { Laboratory } \\
\text { Vessel } 91 \\
\end{array}$ & Srm Pest & Frozen & $9 \times 9$ three inch box & $\begin{array}{l}\text { Full or end } \\
\text { of stand }\end{array}$ \\
\hline \multicolumn{5}{|l|}{ Packed Cells } \\
\hline $\begin{array}{l}\text { CASPIR } \\
\text { Vessel } 88\end{array}$ & Arcylamide & Frozen & $9 \times 9$ two inch box & Weekly \\
\hline $\begin{array}{l}\text { CASPIR } \\
\text { Vessel } 126 \\
\end{array}$ & Xtra RBCs & Frozen & $9 \times 9$ two inch box & Weekly \\
\hline \multicolumn{5}{|l|}{ Serum } \\
\hline $\begin{array}{l}\text { CASPIR } \\
\text { Vessel } 81 \\
\end{array}$ & Vit B6/Fol & Frozen & $10 \times 10$ two inch box & Weekly \\
\hline $\begin{array}{l}\text { CASPIR } \\
\text { Vessel } 70\end{array}$ & Vitamin D & Frozen & $10 \times 10$ two inch box & Weekly \\
\hline
\end{tabular}


Exhibit 9-1. Blood and urine shipping protocol (continued)

\begin{tabular}{|c|c|c|c|c|}
\hline Location & Vessels & Conditions & Vessel storage & $\begin{array}{l}\text { Shipping } \\
\text { frequency }\end{array}$ \\
\hline \multicolumn{5}{|l|}{ Serum } \\
\hline $\begin{array}{l}\text { University of Washington } \\
\text { Vessel } 13\end{array}$ & CRP & Frozen & $9 \times 9$ two inch box & Weekly \\
\hline $\begin{array}{l}\text { CDC/NCID Hepatitis Ref Lab } \\
\text { Vessel } 14\end{array}$ & Hep Profile & Frozen & $9 \times 9$ two inch box & Weekly \\
\hline $\begin{array}{l}\text { CDC/NCID Hepatitis Ref Lab } \\
\text { Vessel } 122\end{array}$ & HAV & Frozen & $9 \times 9$ two inch box & Weekly \\
\hline $\begin{array}{l}\text { CDC NCID Hepatitis Ref Lab } \\
\text { Vessel } 87\end{array}$ & Hepatitis ab & Frozen & $9 \times 9$ two inch box & Weekly \\
\hline $\begin{array}{l}\text { CASPIR } \\
\text { Vessel } 99\end{array}$ & Fer/TfR & Frozen & $10 \times 10$ two inch box & Weekly \\
\hline $\begin{array}{l}\text { CASPIR } \\
\text { Vessel } 17\end{array}$ & Cotinine & Frozen & $9 \times 9$ two inch box & Weekly \\
\hline $\begin{array}{l}\text { NHANES Diabetes Laboratory } \\
\text { Vessel } 21\end{array}$ & Lipids & Frozen & $9 \times 9$ two inch box & Weekly \\
\hline $\begin{array}{l}\text { CDC /DVRD/Molecular } \\
\text { Epidemiology } \\
\text { Vessel } 77\end{array}$ & HPV Serum & Frozen & $9 \times 9$ two inch box & Weekly \\
\hline $\begin{array}{l}\text { Collaborative Laboratory } \\
\text { Services } \\
\text { Vessel } 18\end{array}$ & Biochem & Refrigerated & $9 \times 9$ two inch box & Weekly \\
\hline $\begin{array}{l}\text { NHANES Diabetes Laboratory } \\
\text { Vessel } 23\end{array}$ & Insulin & Frozen & $9 \times 9$ two inch box & Weekly \\
\hline $\begin{array}{l}\text { CDC/NCID AIDS Division } \\
\text { Vessel } 22\end{array}$ & HIV & Frozen & $9 \times 9$ two inch box & Weekly \\
\hline $\begin{array}{l}\text { Emory University } \\
\text { Vessel } 24\end{array}$ & HSV & Frozen & $9 \times 9$ two inch box & Weekly \\
\hline $\begin{array}{l}\text { University of Washington } \\
\text { Vessel } 75\end{array}$ & PSA & Frozen & $9 \times 9$ two inch box & Weekly \\
\hline $\begin{array}{l}\text { University of Washington } \\
\text { Vessel } 118\end{array}$ & Thyroid & Frozen & $9 \times 9$ two inch box & Weekly \\
\hline $\begin{array}{l}\text { Mayo Clinic } \\
\text { Vessel } 127\end{array}$ & IgA-TTG/EMA & Frozen & $9 \times 9$ two inch box & Weekly \\
\hline $\begin{array}{l}\text { CDC/NCID DASH } \\
\text { Vessel } 16\end{array}$ & MMRV & Frozen & $9 \times 9$ two inch box & Weekly \\
\hline $\begin{array}{l}\text { CASPIR } \\
\text { Vessel } 110\end{array}$ & Folate1 & Frozen & 10x10 two inch box & Weekly \\
\hline $\begin{array}{l}\text { CASPIR } \\
\text { Vessels 32-39 } \\
\end{array}$ & Xtra Sera & Frozen & $9 \times 9$ two inch box & $\begin{array}{l}\text { Full or end } \\
\text { of stand }\end{array}$ \\
\hline $\begin{array}{l}\text { CASPIR } \\
\text { Vessels } 29 \text { and } 89\end{array}$ & $\begin{array}{l}\text { Persis Pest1 } \\
\text { Persis Pest2 }\end{array}$ & Frozen & $9 \times 9$ three inch box & $\begin{array}{l}\text { Full or end } \\
\text { of stand }\end{array}$ \\
\hline $\begin{array}{l}\text { CDC/NCEH Environmental } \\
\text { Health Laboratory } \\
\text { Vessel } 90\end{array}$ & Perfluor1 & Frozen & $9 \times 9$ three inch box & $\begin{array}{l}\text { Full or end } \\
\text { of stand }\end{array}$ \\
\hline
\end{tabular}


Exhibit 9-1. Blood and urine shipping protocol (continued)

\begin{tabular}{|c|c|c|c|c|}
\hline Location & Vessels & Conditions & Vessel storage & $\begin{array}{l}\text { Shipping } \\
\text { frequency }\end{array}$ \\
\hline $\begin{array}{l}\text { CASPIR } \\
\text { Vessels } 92 \text { and } 93\end{array}$ & $\begin{array}{l}\text { BFR1 } \\
\text { BFR2 }\end{array}$ & Frozen & $9 \times 9$ three inch box & $\begin{array}{l}\text { Full or end } \\
\text { of stand }\end{array}$ \\
\hline \multicolumn{5}{|l|}{ Urine } \\
\hline $\begin{array}{l}\text { University of Minnesota } \\
\text { Vessel } 45\end{array}$ & Alb/Creat & Frozen & $9 \times 9$ three inch box & Weekly \\
\hline $\begin{array}{l}\text { CDC/NCID NCHSTD } \\
\text { Vessel } 46\end{array}$ & Chlam & Frozen & $5 \times 5$ three inch box & Weekly \\
\hline $\begin{array}{l}\text { CASPIR } \\
\text { Vessel } 86 \\
\end{array}$ & Spc Arsenic & Frozen & $9 \times 9$ three inch box & Weekly \\
\hline $\begin{array}{l}\text { CDC/NCEH Pesticde } \\
\text { Laboratory } \\
\text { Vessel } 48 \\
\end{array}$ & Prior Pest & Frozen & $5 \times 5$ three inch box & $\begin{array}{l}\text { Full or end } \\
\text { of stand }\end{array}$ \\
\hline $\begin{array}{l}\text { CDC/NCEH Pesticde } \\
\text { Laboratory } \\
\text { Vessel } 49 \\
\end{array}$ & Organophos & Frozen & $5 \times 5$ three inch box & $\begin{array}{l}\text { Full or end } \\
\text { of stand }\end{array}$ \\
\hline $\begin{array}{l}\text { CASPIR } \\
\text { Vessel } 62 \\
\end{array}$ & Urn Merc & Frozen & $5 \times 5$ three inch box & Weekly \\
\hline $\begin{array}{l}\text { CASPIR } \\
\text { Vessel } 50\end{array}$ & Heavy Metal & Frozen & $5 \times 5$ three inch box & Weekly \\
\hline $\begin{array}{l}\text { CASPIR } \\
\text { Vessel } 65 \\
\end{array}$ & Urn Phytoes & Frozen & $10 \times 10$ three inch box & $\begin{array}{l}\text { Full or end } \\
\text { of stand }\end{array}$ \\
\hline $\begin{array}{l}\text { CASPIR } \\
\text { Vessel } 66\end{array}$ & PAH & Frozen & $9 \times 9$ three inch box & $\begin{array}{l}\text { Full or end } \\
\text { of stand }\end{array}$ \\
\hline $\begin{array}{l}\text { CDC/NCEH Environmental } \\
\text { Health Laboratory } \\
\text { Vessel } 67\end{array}$ & Phthalates & Frozen & $9 \times 9$ three inch box & $\begin{array}{l}\text { Full or end } \\
\text { of stand }\end{array}$ \\
\hline $\begin{array}{l}\text { CDC/NCEH Air Toxicant } \\
\text { Branch } \\
\text { Vessel } 102\end{array}$ & Perchlorate & Frozen & $9 \times 9$ three inch box & $\begin{array}{l}\text { End of } \\
\text { stand }\end{array}$ \\
\hline $\begin{array}{l}\text { CASPIR } \\
\text { Vessel } 121 \\
\end{array}$ & NNAL & Frozen & $5 \times 5$ three inch box & $\begin{array}{l}\text { End of } \\
\text { stand }\end{array}$ \\
\hline $\begin{array}{l}\text { CASPIR } \\
\text { Vessel } 128 \\
\end{array}$ & Caffeine & Frozen & $10 \times 10$ two inch box & Weekly \\
\hline $\begin{array}{l}\text { CASPIR } \\
\text { Vessel } 52\end{array}$ & Xtra Urine & Frozen & $9 \times 9$ three inch box & $\begin{array}{l}\text { Full or end } \\
\text { of stand }\end{array}$ \\
\hline
\end{tabular}




\section{$9.2 \quad$ Equipment and Supplies}

Exhibit 9-2 contains a list of label/shipping supplies.

Exhibit 9-2. Supplies for labels/shipping

\begin{tabular}{l|l}
\hline Small Styrofoam shipper (14 x 14 x 12) & Medium Styrofoam shippers (16 x16 x 15.5) \\
\hline 5-mL cryovial sterile & 2-mL cryovial sterile \\
\hline 14-mL snap cap test tube & 2-mL cryovial nonsterile \\
\hline Conical tube 50 -mL & Caps for 2-mL cryovial \\
\hline $\begin{array}{l}5.25 \text { x } 5.25 \text { x } 2.0 \text { inch cardboard box with } \\
9 \text { x } 9 \text { cardboard grid }\end{array}$ & $\begin{array}{l}5.25 \text { x } 5.25 \text { x } 3.0 \text { inch cardboard box with } 9 \text { x } 9 \\
\text { cardboard grid }\end{array}$ \\
\hline 5.25 x 5.25 x 5.0 inch cardboard box with & Three-inch 5 x 5 cardboard grid \\
5 x 5 foam insert & \\
\hline 5.25 x 5.25 x 3.0 inch cardboard box \\
without grid & Scale \\
\hline 10 x 10 two-inch box & 10 x 10 three-inch box \\
\hline STP-710 Pressure vessel & STP-310 Pressure vessel \\
\hline STP-740 Pressure vessel & U-TEK Reusable Refrigerant Packs Large \\
\hline Plastic bag 9 x 12 & Zip closable bags - quart size \\
\hline Absorbent sheets (2 x 2) & Rubber bands \\
\hline Large padded envelope & Newsprint paper \\
\hline Cryo gloves & Full face shield \\
\hline Hammer & Dry ice scoop \\
\hline Tape gun & Packaging tape 2” \\
\hline Overpack label & Address labels for FedEx class 9 sticker \\
\hline UN 3373 label & Refrigerate Do Not Freeze label \\
\hline Keep Frozen label & Avery 5160 labels \\
\hline Label Datamax (1.125”) & Printer ribbon, Datamax (thermal transfer) \\
\hline Sheet-fed laser labels & Wipes, Terri 13” x 18” \\
\hline FedEx large boxes & FedEx Class 9 label \\
\hline Clear FedEx label pouches & Dymo Labels \\
\hline FedEx form 0215 & FedEx preprinted return air bill - stand \\
\hline Toner cartridge (HP Laser Jet 4000) & Lab jackets (Petite, Lg., X-Lg.) \\
\hline
\end{tabular}

\subsection{Print Vessel Labels and Label Blood Processing Racks}

Before each session, the technologist assigned to workstation 3 prints all labels for all SPs assigned to a session. The correct bar-coded labels automatically print for each SP based on the SP's age, sex, appointment type, and selection into one or more subsamples. Print pregnancy testing, urine processing, blood processing, packed cell processing, pool blood tube, OGTT processing labels, and HPV 
oral rinse for all SPs scheduled into a MEC session. The phlebotomist and the technologist assigned to shipping are responsible for labeling vessels and assembling the blood processing racks. The MEC physician prints the HPV swab labels.

\section{Distribute labels}

- Place urine processing, pregnancy testing, pack cell processing, and HPV oral rinse labels at workstation 2 in the laboratory.

- Save and recycle unused vessels.

- If the SP does not show up for the appointment, it is possible he or she will reschedule.

- Store the SP's vessels in a zip-closable bag until the end of the stand.

- If the SP has not shown up by the end of the stand, recycle the unused vessels.

\section{Assemble blood-processing racks}

The phlebotomist and the medical technologist assigned to the shipping workstation are responsible for labeling vessels and assembling the blood processing racks. Use extreme care in setting up the blood vessel racks and in labeling the vessels with the appropriate bar-code label.

Label each vessel with the appropriate vessel label, according to the protocol. Place the label on the vessel, wrapping it around the vessel horizontally and making sure the label wraps onto itself. Place the label so the first digit of the vessel number is at the top of the vessel. Set the vessels in the cube racks in priority order.

Use the following materials to set up and label blood racks:

- Plastic rack

- Template for processing 
- Vessels stored of the following sizes:

- $\quad 2.0-\mathrm{mL}$ vessel

- $\quad 5.0-\mathrm{mL}$ vessel

- $\quad$ 14.0-mL tube (HPV oral rinse only)

- $\quad$ 50-mL conical tube

- Bar-coded SP ID labels each containing the unique 6-digit SP ID number concatenated with the vessel ID

Prepare one blood processing rack for each SP. The set up of the rack depends on the subject's age, sex, appointment type, and inclusion in selected subsamples. When possible, prepare the racks at least 1 day before the SP arrives for the MEC examination.

- Place the OGTT labels for vessel 98 at workstation 1 at the start of each session.

- Label the acrylamide labels for vessel 88, place them in a separate rack, and place the rack at workstation 1 at the start of each session.

- Label the Xtra RBCs labels for vessel 126, place them in a separate rack, and place the rack at workstation 1 at the start of each session.

- Place the prelabeled blood processing racks in the laboratory at the start of each session.

\subsubsection{Print Vessel Labels Using the Print Labels Module}

Open the Laboratory application. Log onto the computer terminal. If the laboratory is conducting a MEC session, the heads-up display will default to the current session. If the MEC is not conducting a session, then use the Set Session utility to access a different session. 
If the MEC is not conducting a session, then the Session PickUp list displays. Select the correct session from the pick list.

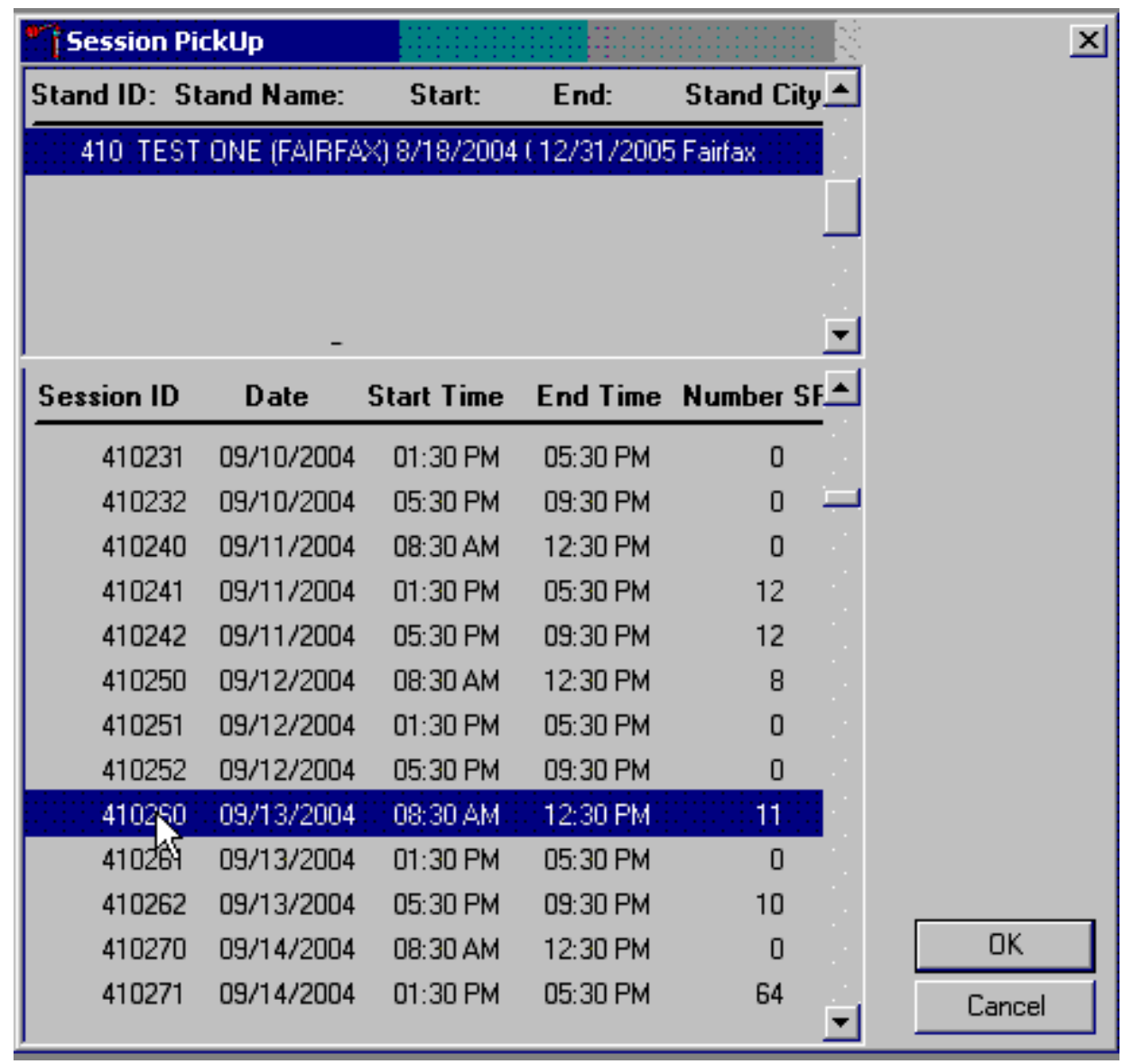

The Session PickUp window displays if the MEC is not conducting a session. The current stand is displayed in the top section of the window. To select or highlight the correct MEC session, use the mouse to direct the mouse arrow to the correct session, date, and time, and right click. Alternatively, to select or highlight a session, use the up and down keys to move up and down the list until the correct session is highlighted and left click. Use the lower vertical scroll bar to view all sessions. To proceed, use the mouse to direct the mouse arrow to the "OK" button and right click or press [Enter]. To cancel, use the mouse to direct the mouse arrow to the "Cancel” button and right click. 
After selecting a session from the Session PickUp list and selecting OK, the laboratory heads-up screen displays. If the staff is conducting a MEC session, the laboratory application defaults to the current MEC session.

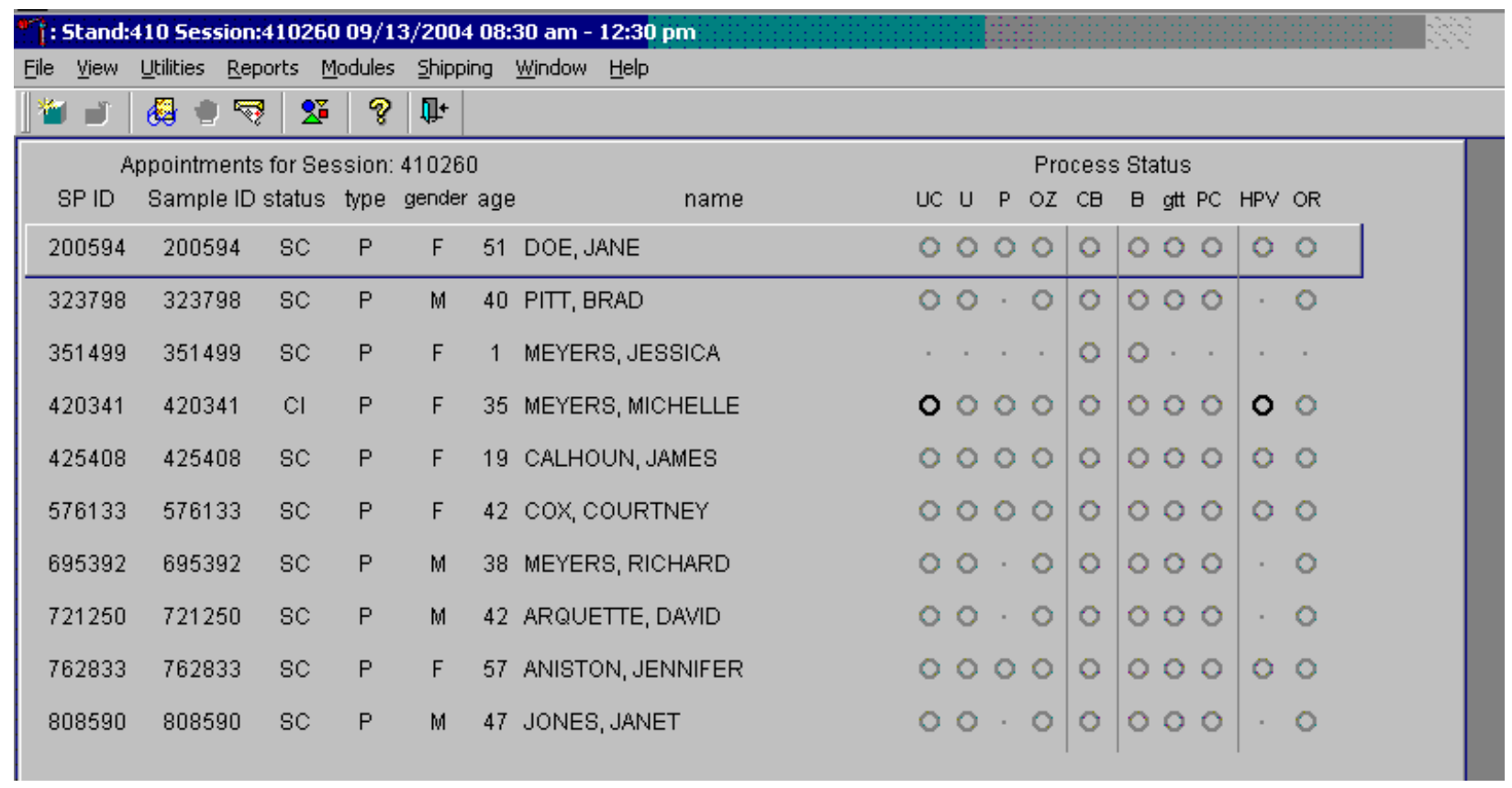


Use the Set Session utility to access a different MEC session when the staff is conducting a MEC session.

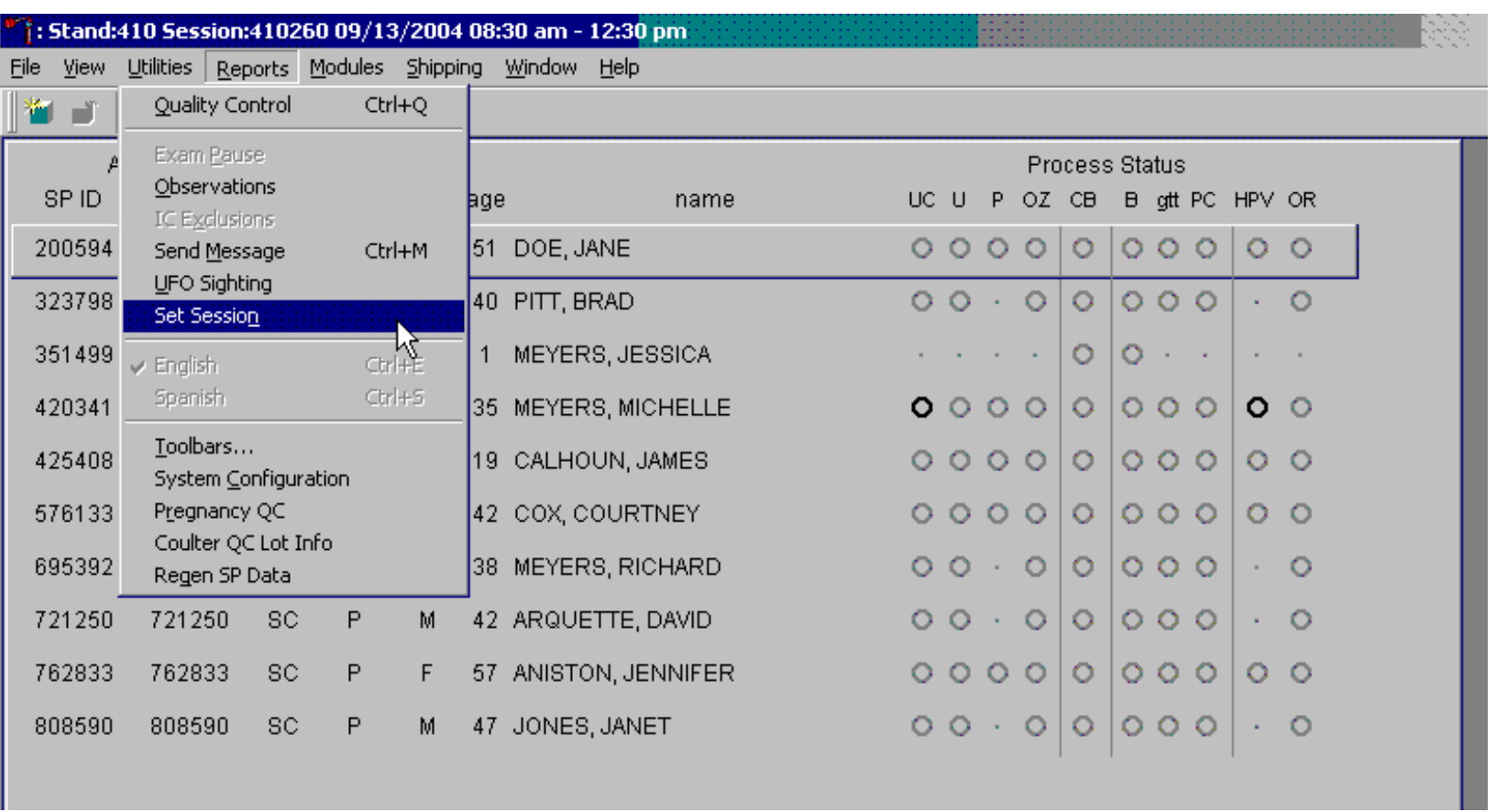

To access a different MEC session when the staff is conducting a MEC session, use the mouse to direct the mouse arrow to the $\{$ Utilities $\}$ module in the top menu bar, drag the mouse arrow to \{Set Session\}, and left click or type [ALT] [U/u], [N/n]. 
Print vessel labels using a variety of options. It is possible to print labels for all modules (blood, urine, pregnancy, and OGTT) or for individual modules. It is also possible to print labels for all SPs in a session, an individual SP, or print labels for ALL appointments, appointments NOT previously printed, or appointments previously printed.

Print vessel labels for all modules.

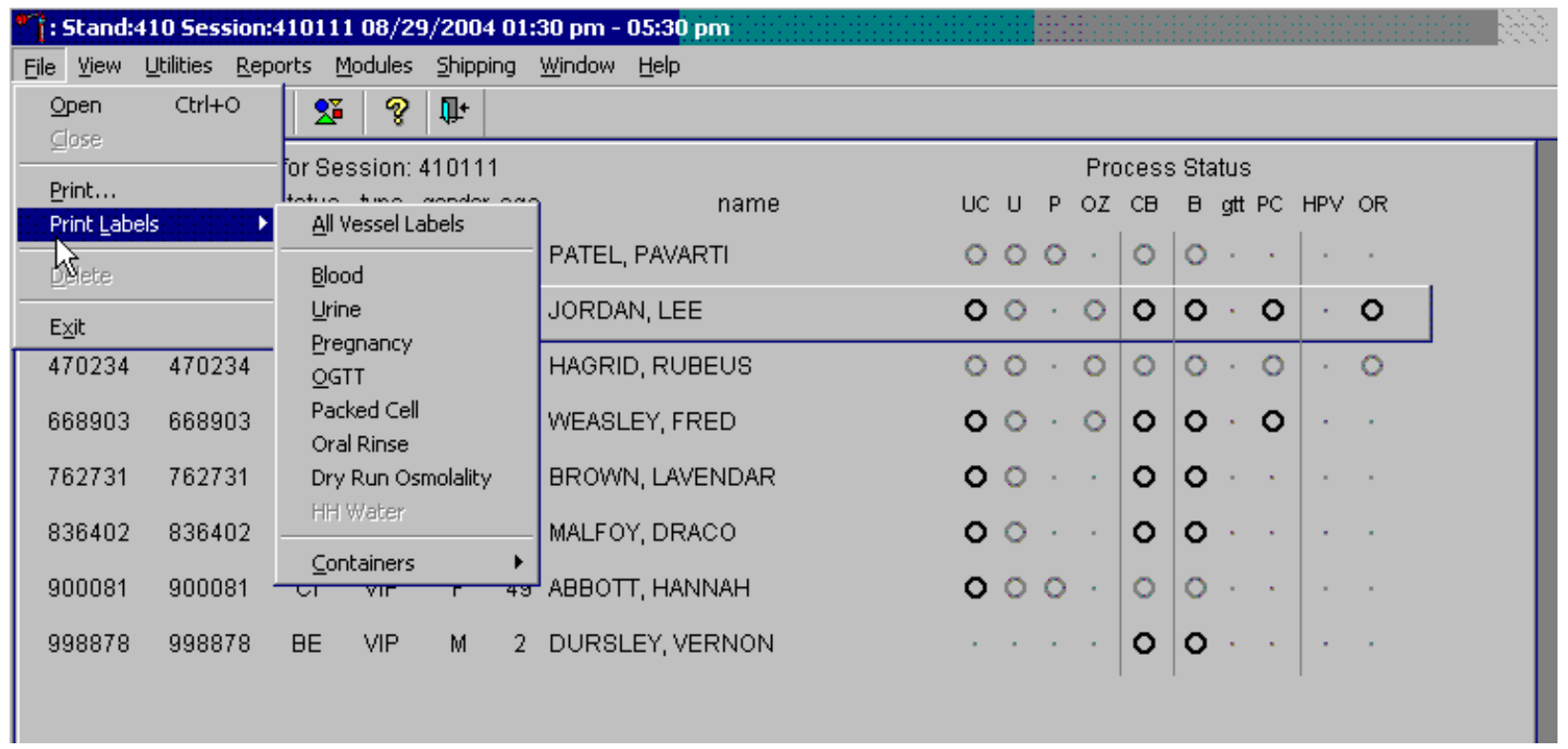

To print vessel labels for all modules, use the mouse to direct the mouse arrow to \{File $\}$ in the top menu bar, drag the arrow to \{Print Labels\}, then to \{All Vessel Labels\} and left click, or type [Alt] [F/f], [L/l], [A/a]. Selecting \{All Vessel Labels $\}$ prints a complete set of blood processing, urine processing, pregnancy testing, OGTT processing, packed cell processing, oral rinse processing and pool tube labels. 
The Print ALL Labels window displays. Use the Print ALL Labels window to print vessel labels for all modules for all SPs in a MEC session.

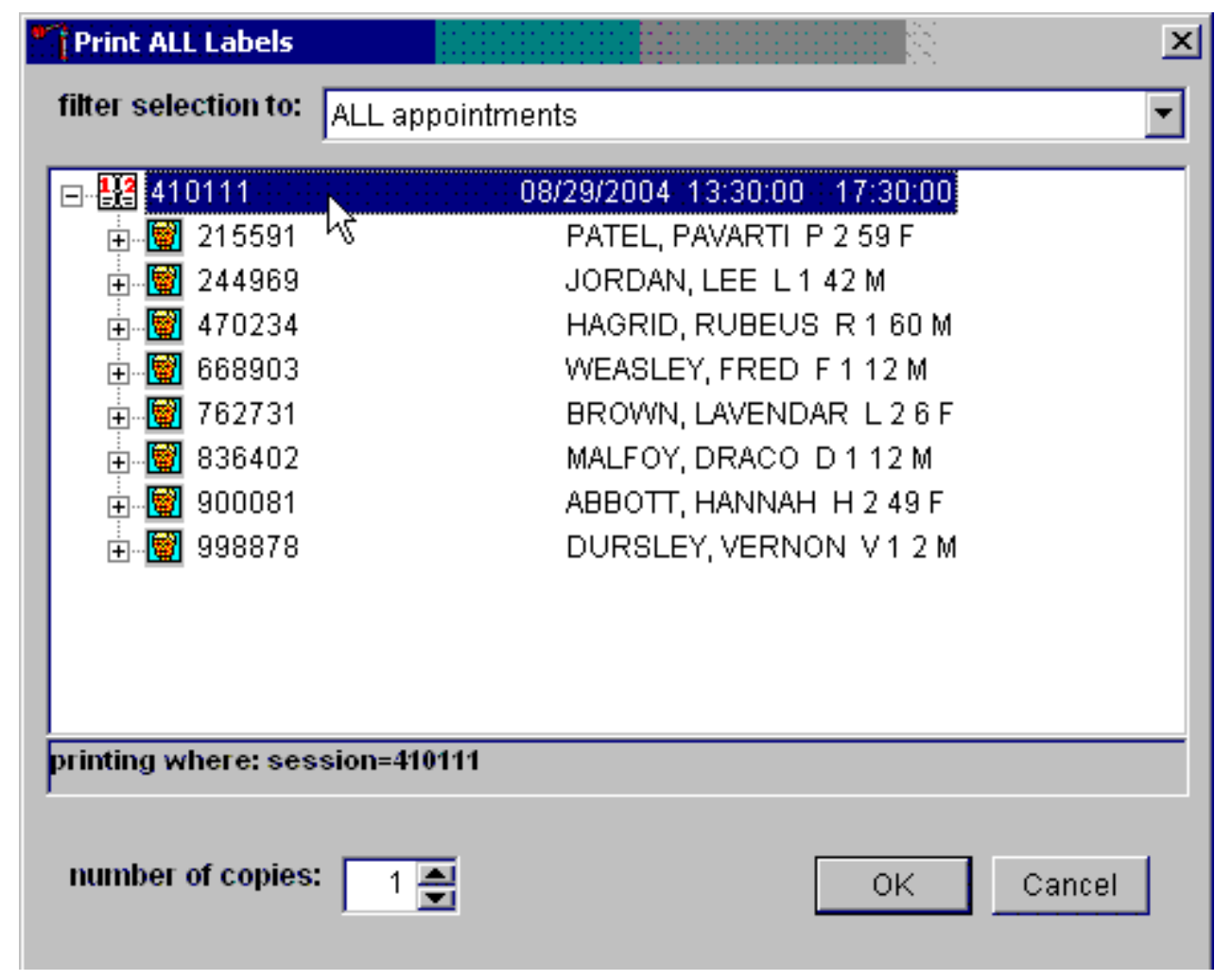

To print vessel labels for all modules for all SPs in a MEC session, use the mouse to direct the mouse arrow to the session number or use the up and down arrows to move up and down the list and left click when the correct session is highlighted. All SPs scheduled for the session are listed below the session number. Select the number of copies (number of sets of labels) by using the mouse to direct the mouse arrow to the up/down controls on the "number of copies" spin box to toggle the number up and down. To print the labels, use the mouse to direct the mouse arrow to the "OK" button and left click or select [Enter]. To exit without printing any labels, use the mouse to direct the mouse arrow to the “Cancel” button and left click. 
Print vessel labels for all modules for one SP in a MEC session.

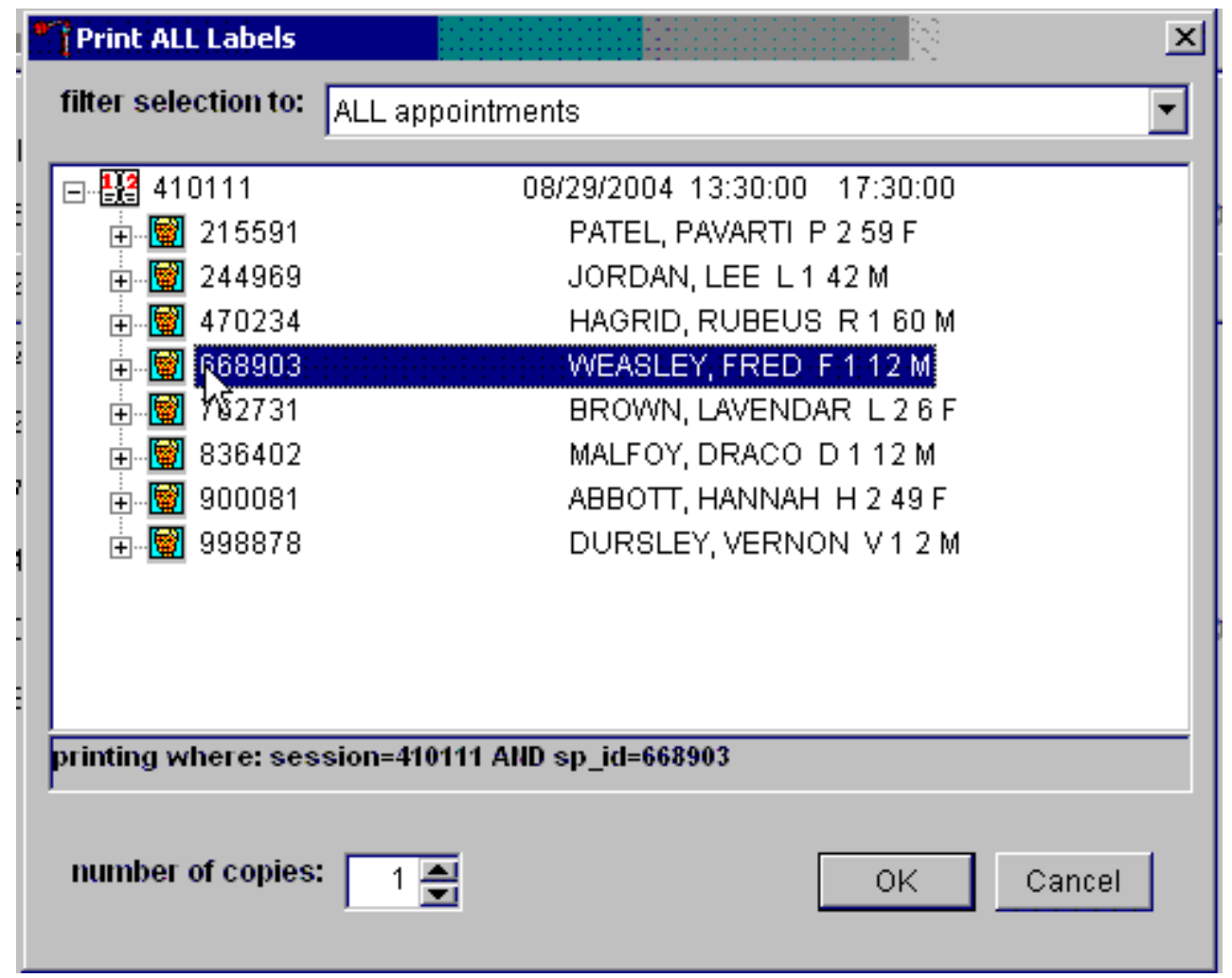

To print vessel labels for all modules for one SP in a MEC session, use the mouse to direct the mouse arrow to the session number or use the up and down arrows to move up and down the list and left click when the correct session is highlighted. All SPs scheduled into the session are listed below the session number. To select or highlight one SP, use the mouse to direct the mouse arrow to the line containing the SP and right click. Select the number of copies (number of sets of labels) by using the mouse to direct the mouse arrow to the up/down controls on the "number of copies" spin box to toggle the number up and down. To print the labels, use the mouse to direct the mouse arrow to the "OK" button and left click or select [Enter]. To exit without printing any labels, use the mouse to direct the mouse arrow to the "Cancel” button and left click. 
Print vessel labels for one specific module for all SPs in a MEC session.

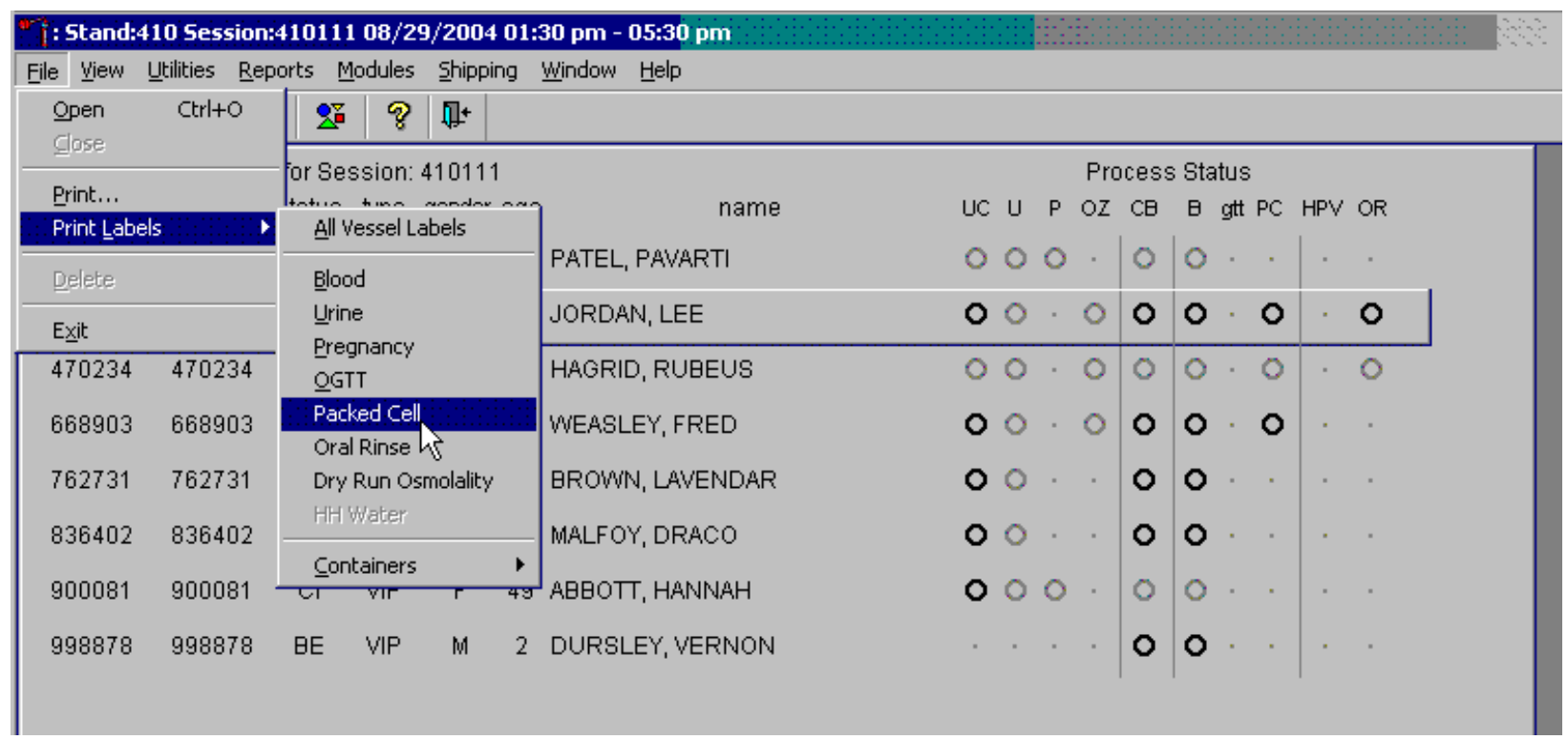

To print vessel labels for one specific module for all SPs in a MEC session, use the mouse to direct the mouse arrow $\{$ File $\}$ in the top menu bar, drag the mouse arrow to \{Print Labels\}, then to the specific module and left click or type [Alt] [F/f], [L/l], and the first letter of the specific module. 
The Print \{Module\} Labels window displays.

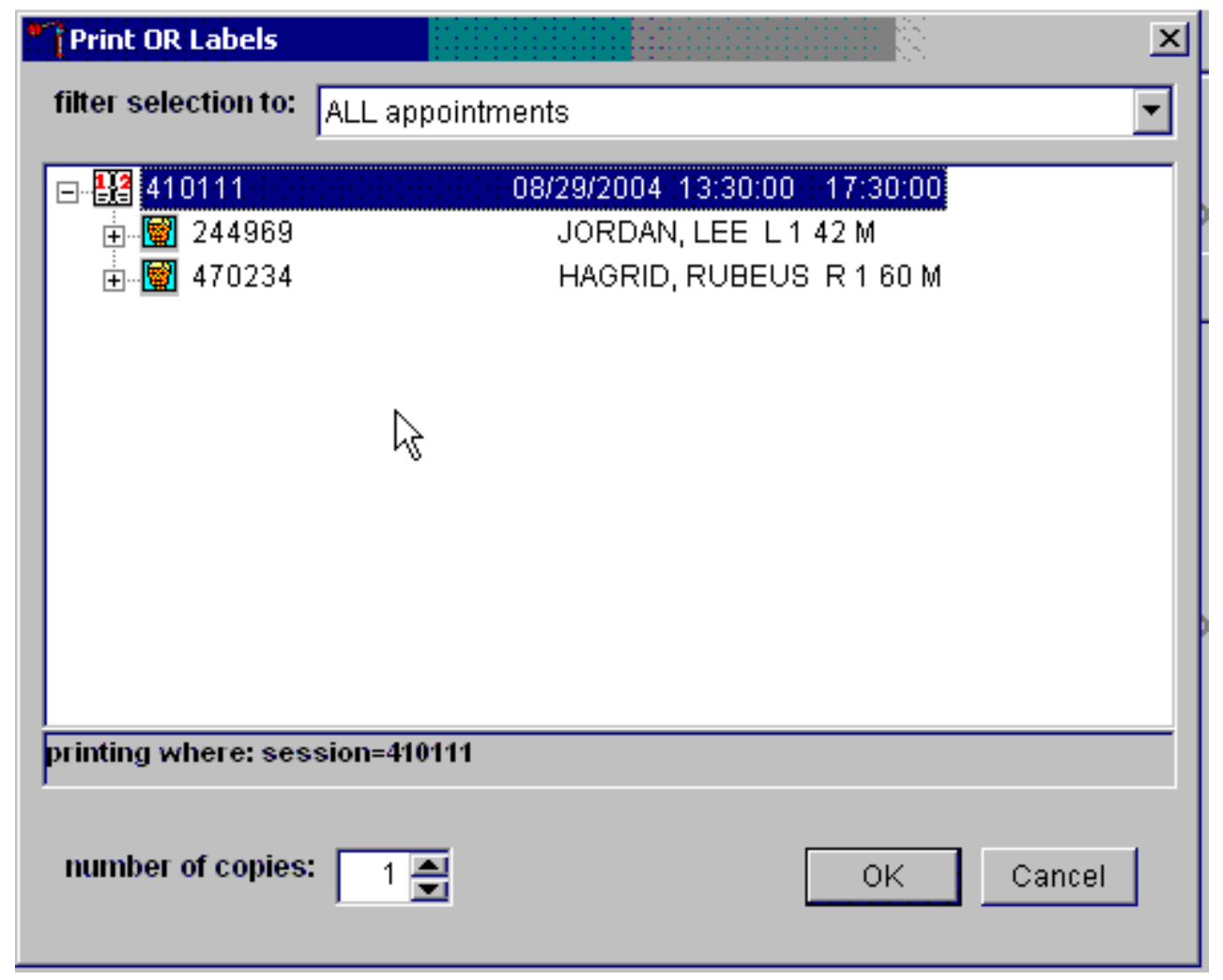

To print vessel labels for one module for all SPs in a MEC session, use the mouse to direct the mouse arrow to the session number or use the up and down arrows to move up and down the list and left click when the correct session is highlighted. All SPs scheduled for the session are listed below the session number. Select the number of copies (number of sets of labels) by using the mouse to direct the mouse arrow to the up/down controls on the "number of copies" spin box to toggle the number up and down. To print the labels, use the mouse to direct the mouse arrow to the "OK" button and left click or select [Enter]. To exit without printing any labels, use the mouse to direct the mouse arrow to the "Cancel” button and left click. 
Print vessel labels for one specific module for one SP in a MEC session.

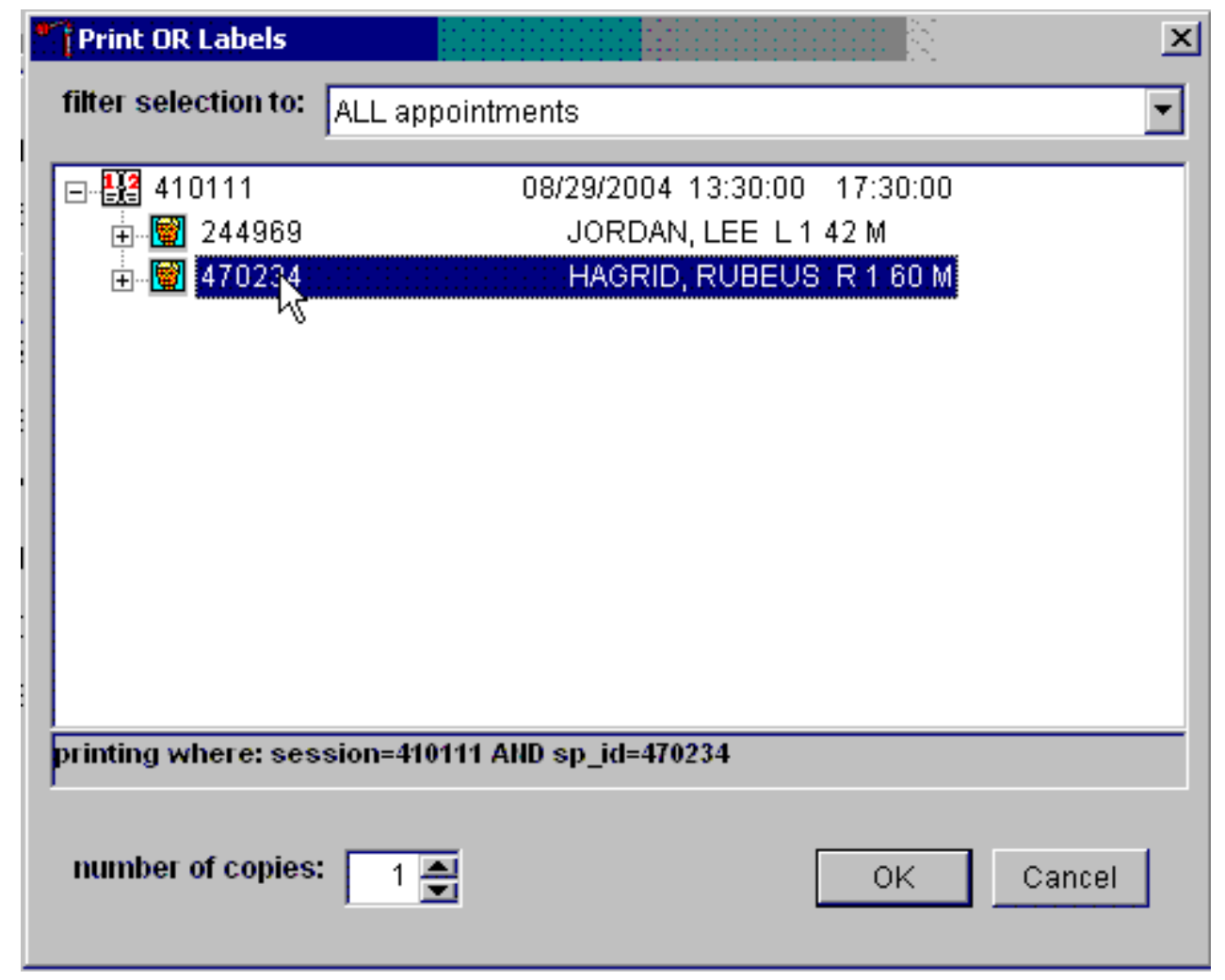

To print vessel labels for one specific module for one SP in a MEC session, use the mouse to direct the mouse arrow to the session number or use the up and down arrows to move up and down the list and left click when the correct session is highlighted. All SPs scheduled into the session are listed below the session number. To select or highlight one SP, use the mouse to direct the mouse arrow to the line containing the SP and right click. Select the number of copies (number of sets of labels) by using the mouse to direct the mouse arrow to the up/down controls on the "number of copies" spin box to toggle the number up and down. To print the labels, use the mouse to direct the mouse arrow to the "OK" button and left click or select [Enter]. To exit without printing any labels, use the mouse to direct the mouse arrow to the "Cancel” button and left click. 
It is also possible to print labels for specific SPs using criteria such as "ALL appointments," “appointments NOT previously printed,” or “appointments PREViously printed” by using a filter.

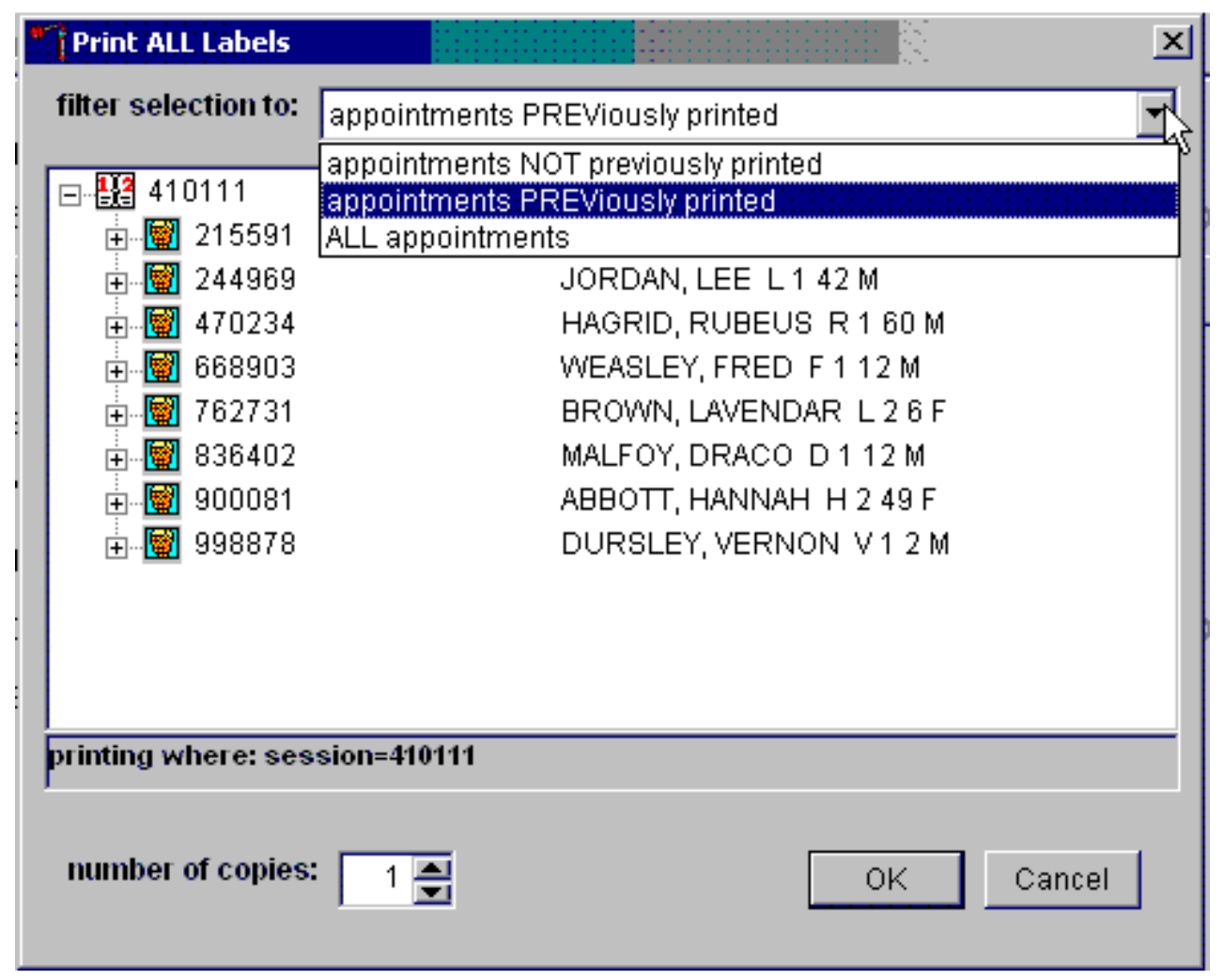

The default filter selection is "ALL appointments." To select or change a filter, use the mouse to direct the mouse arrow to the drop-down list on the "filter selection to:" box and drag the mouse arrow to “appointments NOT previously printed," “appointments PREViously printed,” or “ALL appointments” and right click. Select the number of copies (number of sets of labels) by using the mouse to direct the mouse arrow to the up/down controls on the "number of copies" spin box to toggle the number up and down. To print the labels, use the mouse to direct the mouse arrow to the "OK" button and left click or select [Enter]. To exit without printing any labels, use the mouse to direct the mouse arrow to the "Cancel” button and left click. 


\subsection{2}

Print Vessel Labels Using the Heads-up Display

Print labels for a particular SP using the heads-up display. Print labels for all modules or for a specific module.

Print vessel labels for all modules for one SP.

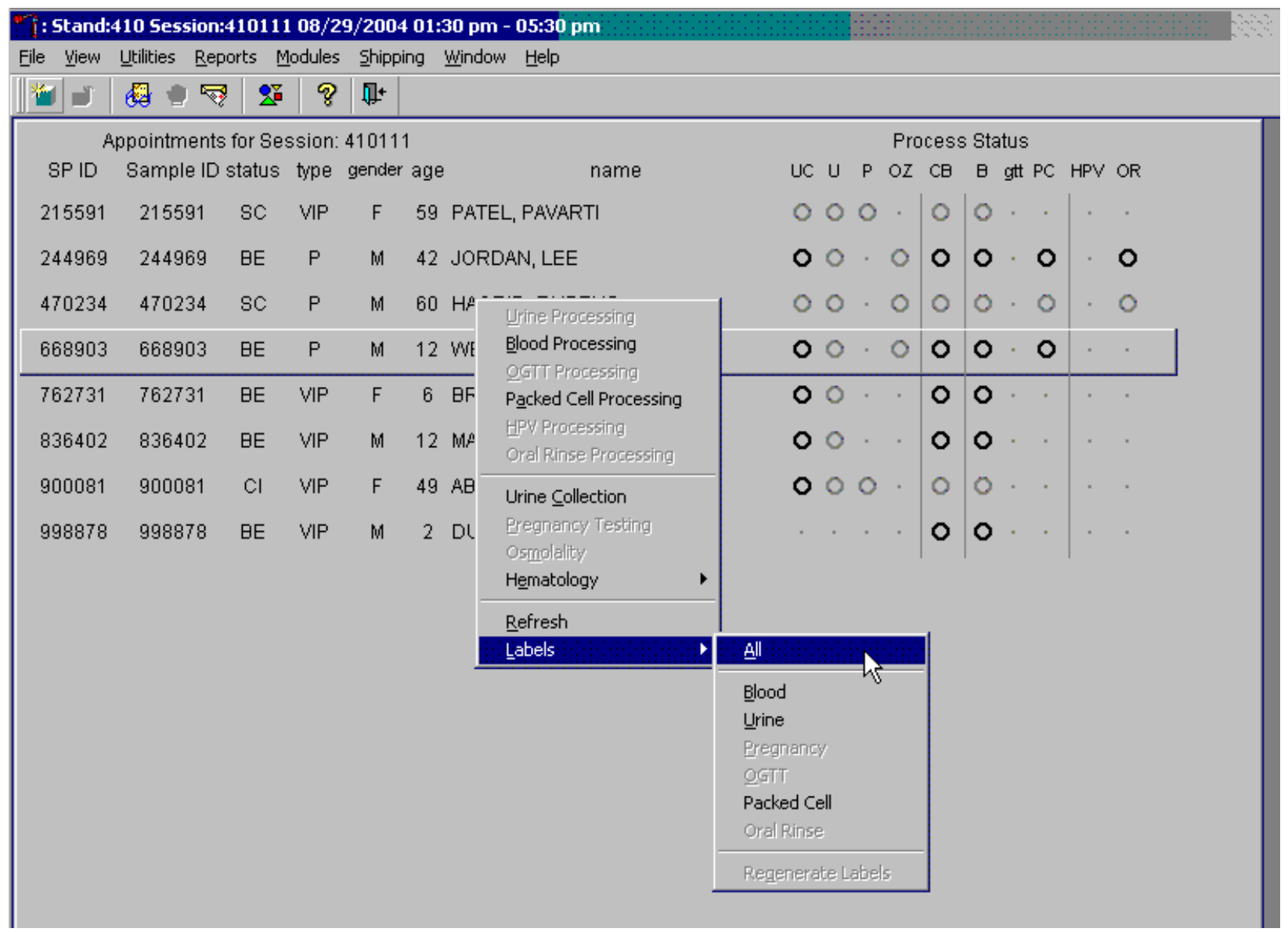

To print labels for all modules for a particular SP, use the mouse to direct the mouse arrow to the correct SP, right click, drag the mouse arrow to \{Labels\}, then to $\{$ All $\}$ and left click or use the mouse to direct the mouse arrow to the correct SP, right click and type [L/l], [A/a]. Labels print in the label/ship area. 
Print labels for one specific module for one SP in a MEC session using the heads-up display.

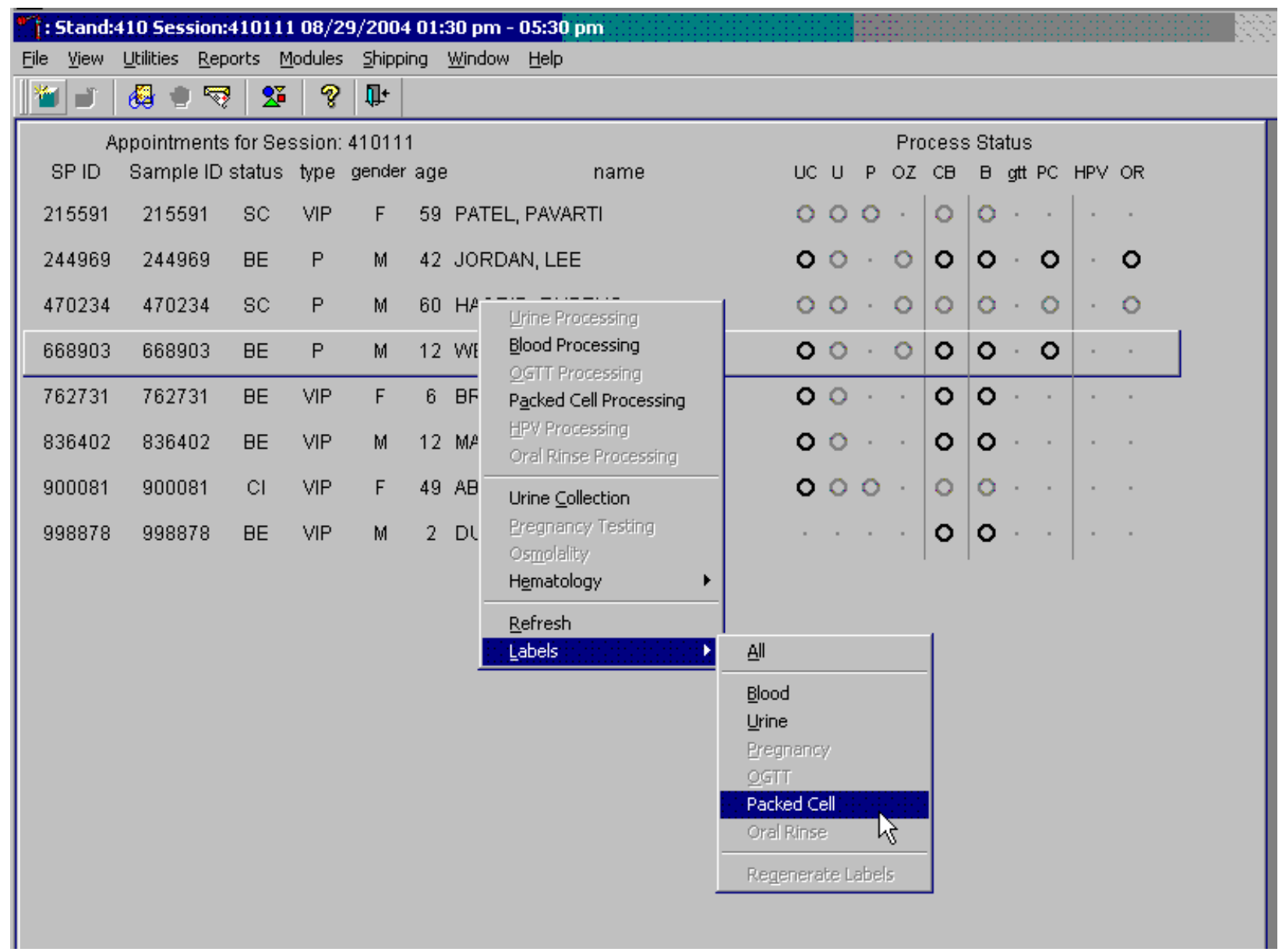

To print additional vessel labels for a particular SP, use the mouse to direct the mouse arrow to the correct SP, right click, drag the mouse arrow to $\{$ Labels $\}$, then drag the arrow to $\{$ Blood $\}$, $\{$ Urine), \{Pregnancy\}, \{OGTT\} or $\{$ Packed Cell $\}$, \{Oral Rinse $\}$, and right click. Alternatively, to print additional labels, use the mouse to direct the mouse arrow to the correct SP, right click, and type [L/l] and the appropriate letter of the module of interest. The labels print in the Label/Ship area. 


\subsubsection{Rescheduled Appointments Report}

If the SP does not show up for a MEC exam, it is possible they will reschedule. Previously labeled vessels may be stored in individual zip-closable bags. Do not store unused labels. At the beginning of each session, use the Rescheduled Appointments report to view a list of rescheduled SPs.

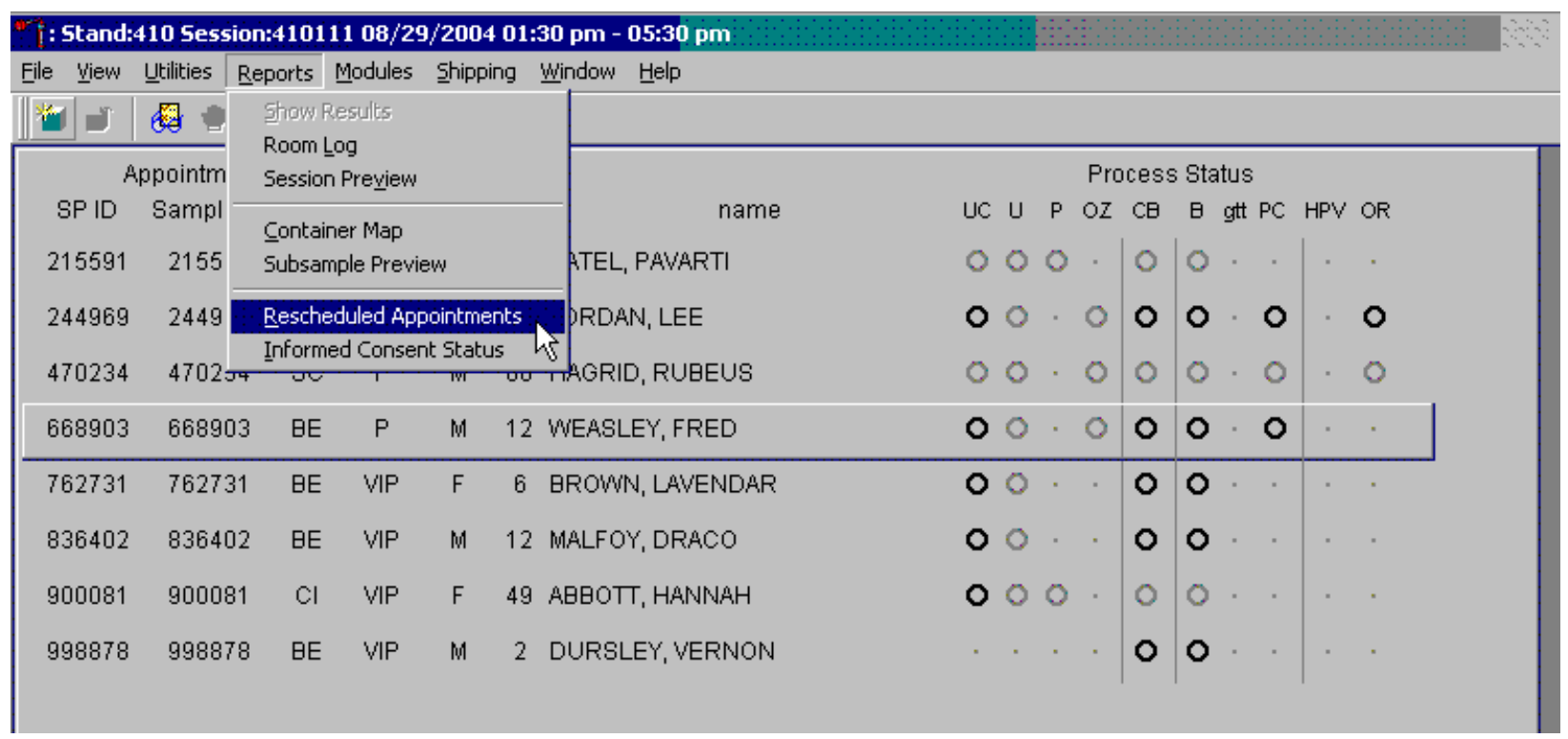

To access the Rescheduled Appointments report, use the mouse to direct the mouse arrow to \{Reports\} in the top menu bar, left click, drag the mouse arrow to \{Rescheduled Appointments\}, and left click or type [Alt] [R/r], [[R/r]. 
The Rescheduled Appointments report lists the rescheduled SPs.

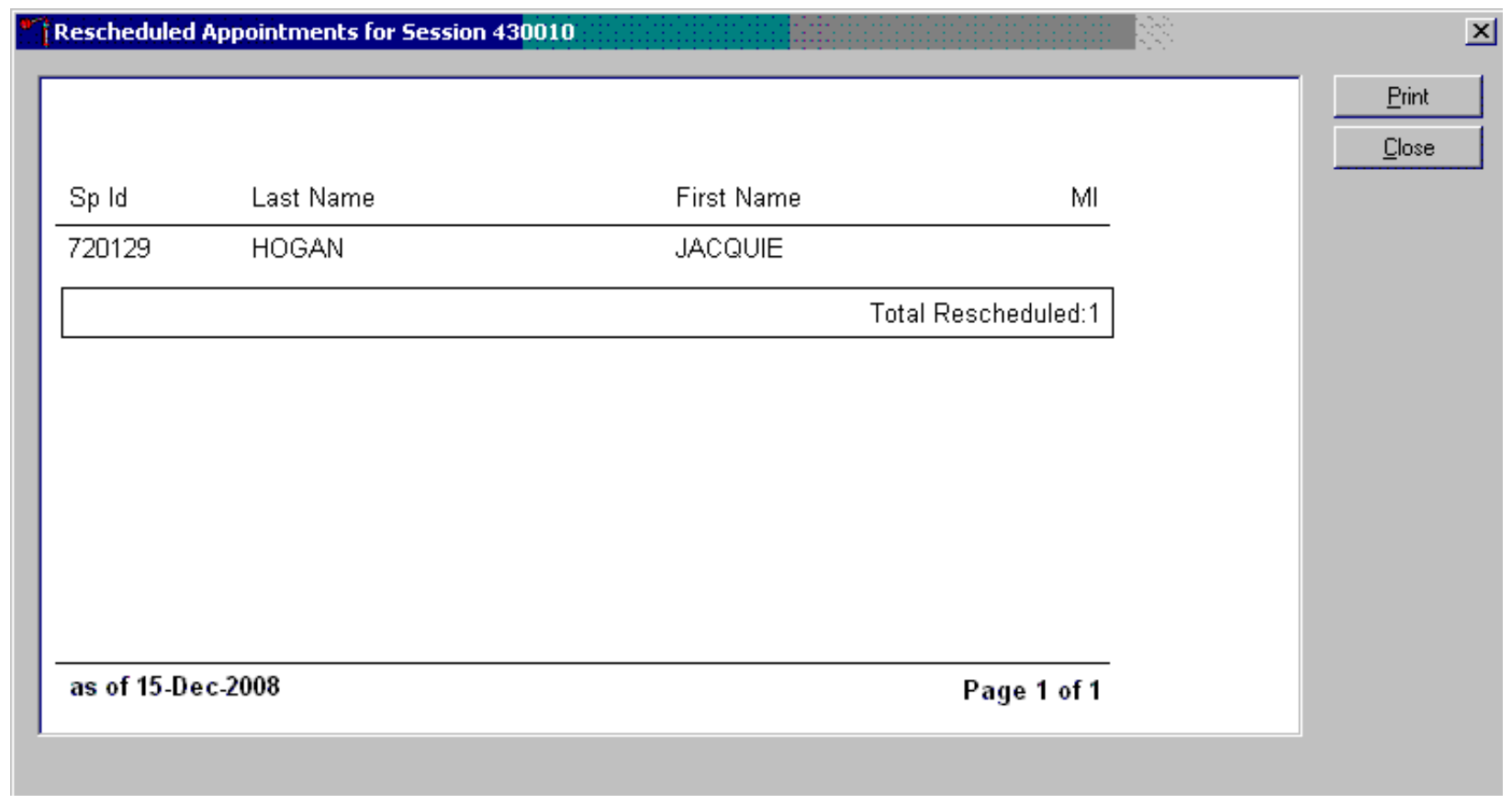

To close the report, use the mouse to direct the mouse arrow to the "Close" button and left click. To print the report, use the mouse to direct the mouse arrow to the "Print" button and left click.

The report may display a message text box indicating that no appointments have been rescheduled for the current session.

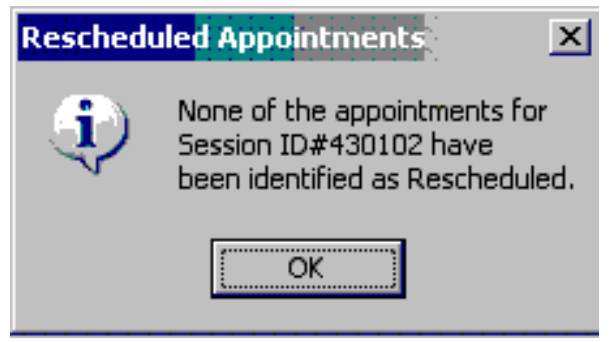

If no appointments have been rescheduled for the current session, the Rescheduled Appointments message text box displays the following message, "None of the appointments for Session ID XXXXXX have been identified as Rescheduled.” To remove the message text box, use the mouse to direct the mouse arrow to the "OK" button and left click, or select [Enter]. 


\section{4}

\section{Regeneration of Data}

Processing protocols are SP specific and are based on the age and gender information obtained during the home interview. Protocols are also dependent on session times and appointment type. Labels are based on the SP's unique protocol. When a session is accessed for the first time, the laboratory record is generated. The application compares the profile for each SP when a session is subsequently opened by the coordinator.

If the age or gender information obtained during the home interview is incorrect, or the session time or appointment type changes, the processing protocol may be affected. If the incorrect information is corrected before labels are generated, no action is necessary. If the incorrect information is corrected after the labels have been printed and the SP's profile changes, the color of the SP's row on the heads-up display turns red. The SP's data must be regenerated to correct the protocol and the record in the database.

If the coordinator changes an SP's age and/or the gender, a message center text box displays on all three laboratory terminals.

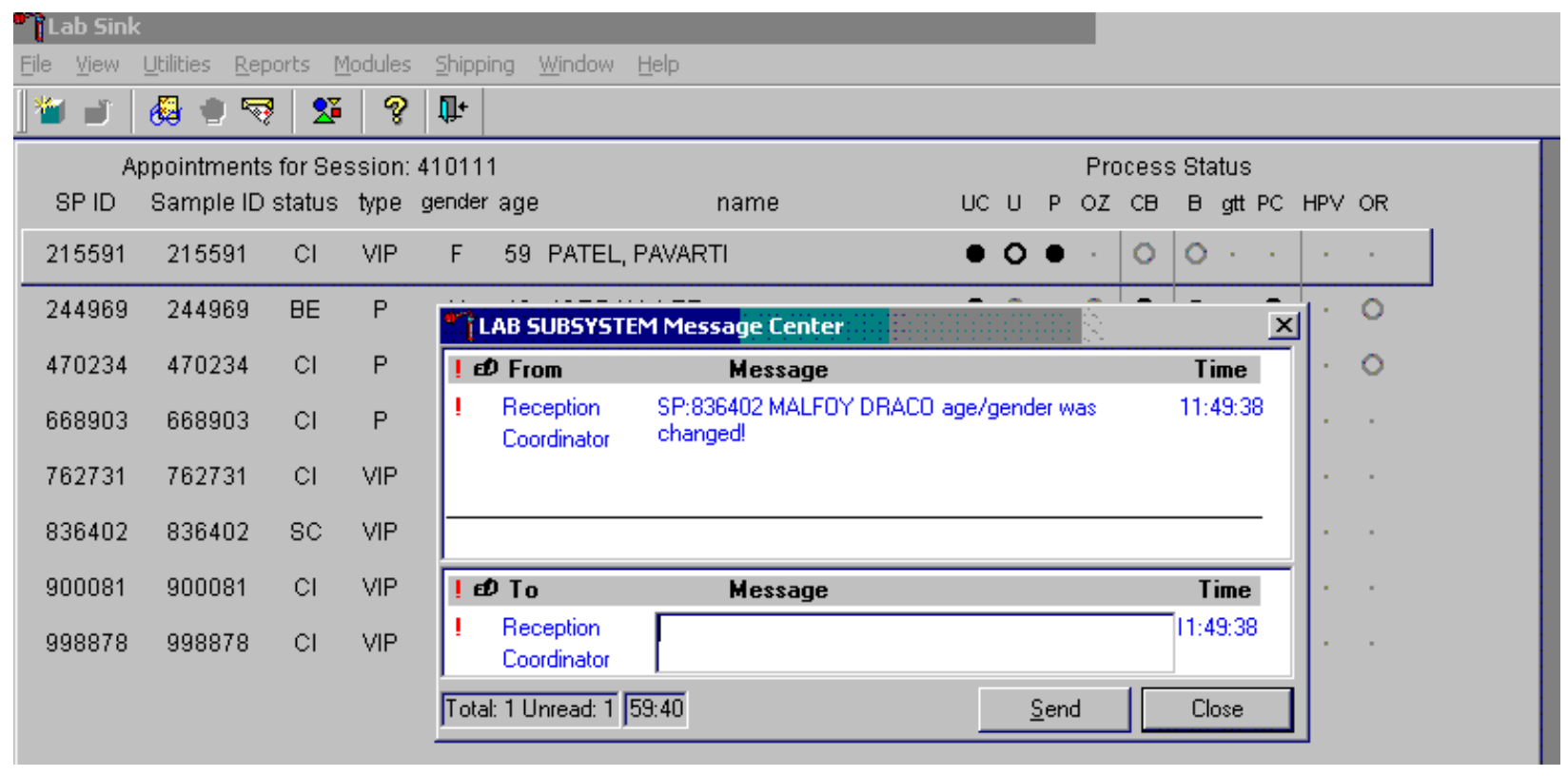

Review the information. To send a response to the coordinator, type the text of the response into the lower Message text box, use the mouse to direct the mouse arrow to the "Send" button, and left click, or type $[\mathrm{S} / \mathrm{s}]$. To close the Message Center text box without responding to the message, use the mouse to direct the mouse arrow to the "Close” button and left click, type [C/c], or select [Enter]. 
Refresh the SP to initiate the regeneration process.

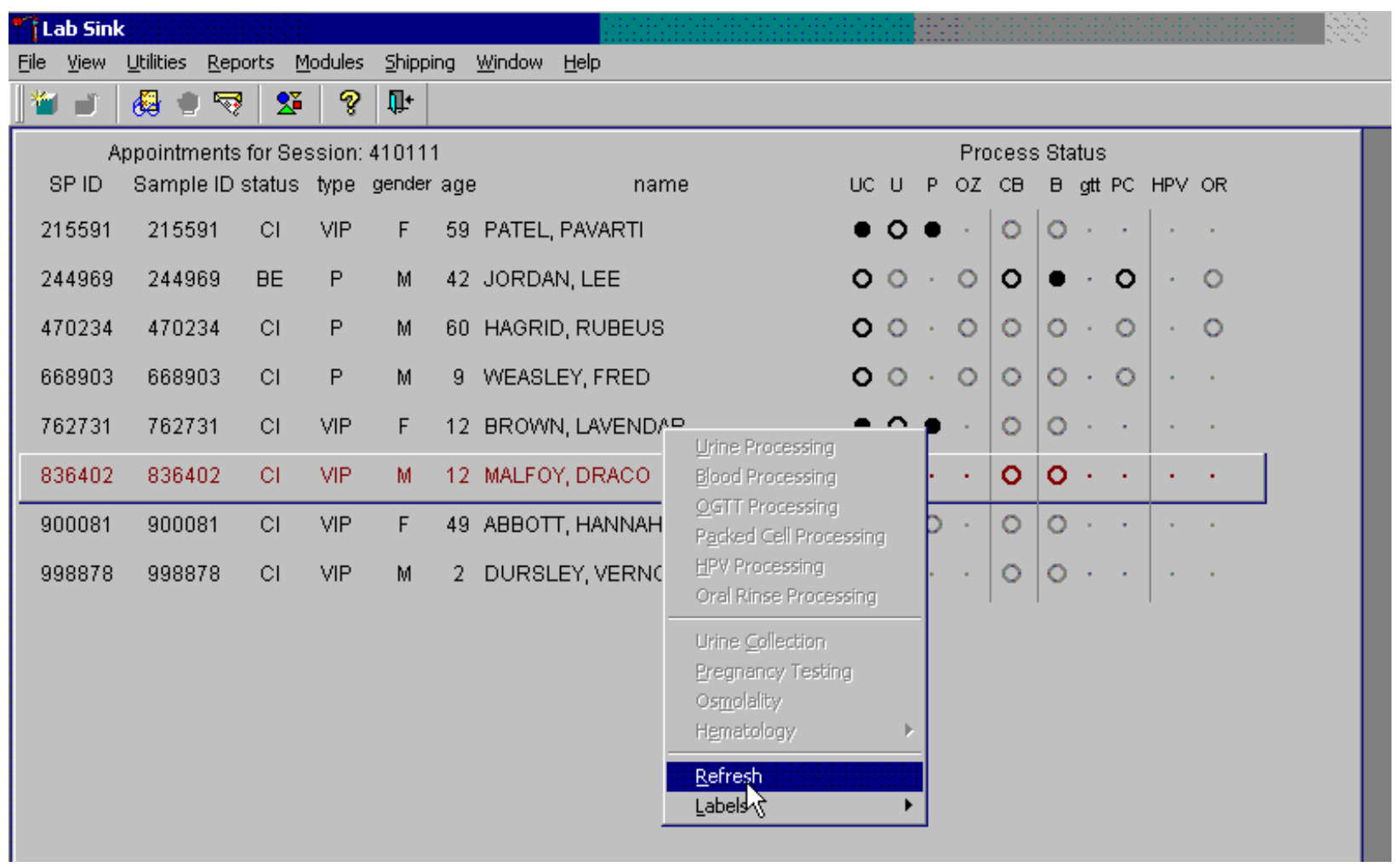

To refresh the SP, use the mouse to direct the mouse arrow to the SP's row on the heads-up display, right click, drag the mouse arrow to $\{$ Refresh $\}$, and right click. 
Regenerate the data for each MEC SP indicated in red on the heads-up display.

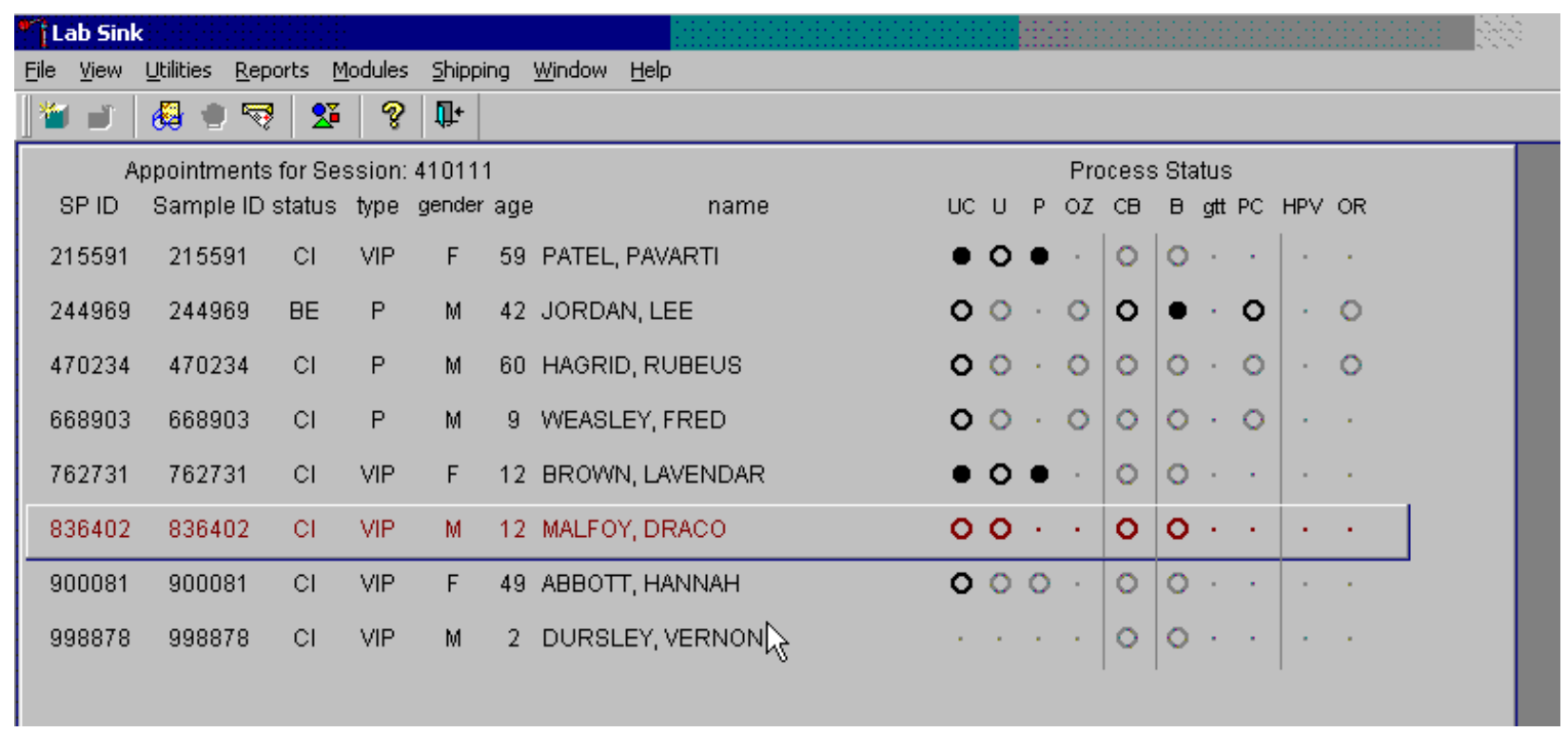

Do not process the SP's specimens if the line on the heads-up display is red. 
Select the correct SP and regenerate the data.

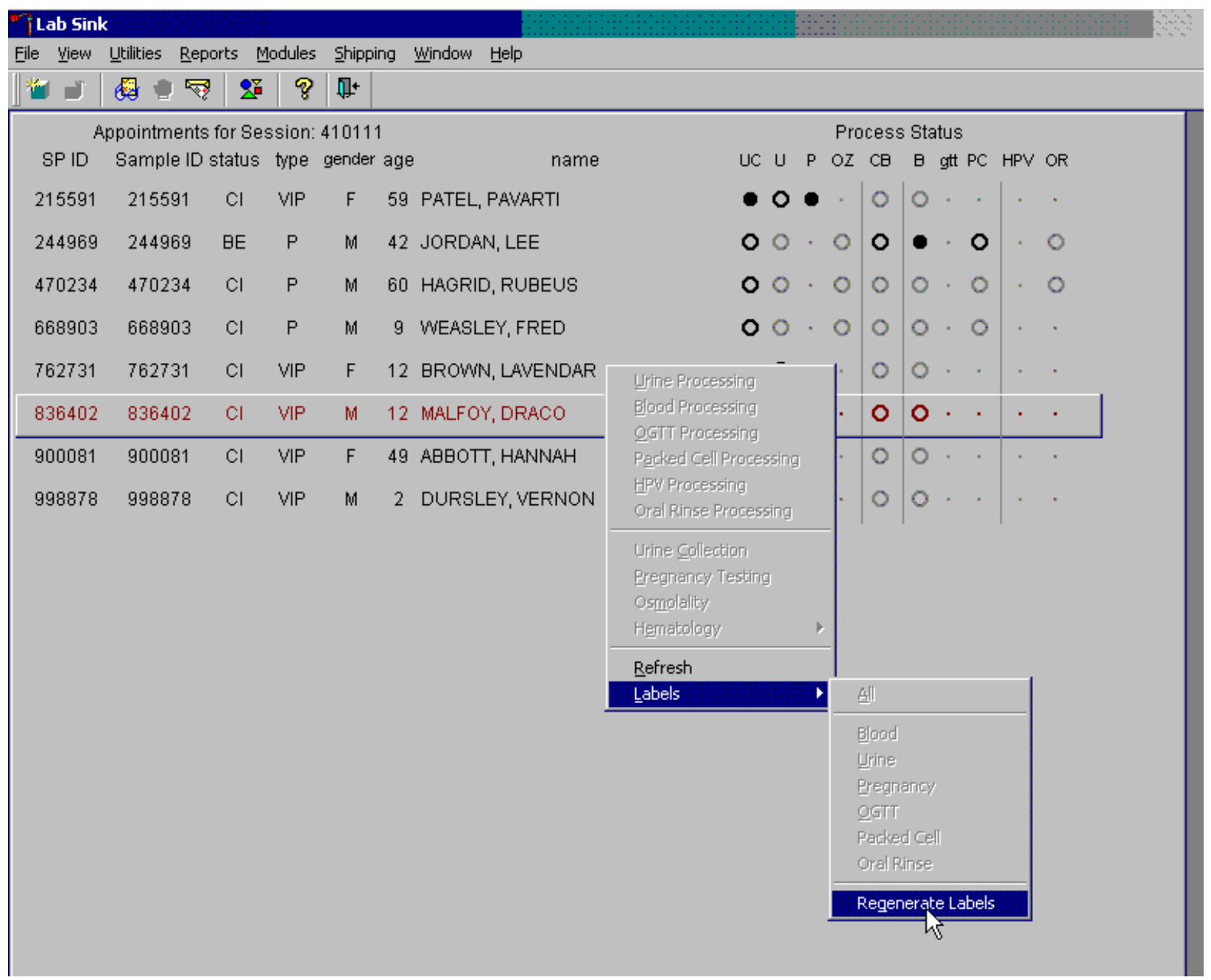

To regenerate the data for the SP, drag the mouse arrow to the SP's row, right click, drag the mouse arrow to \{Labels\}, then to \{Regenerate Labels\}, and left click. Alternatively, use the up and down keys to move up and down the list until the correct SP is selected or highlighted, right click, drag the mouse arrow to $\{$ Labels\}, then to \{Regenerate Labels\}, and right click. 
Respond to the question in the window.

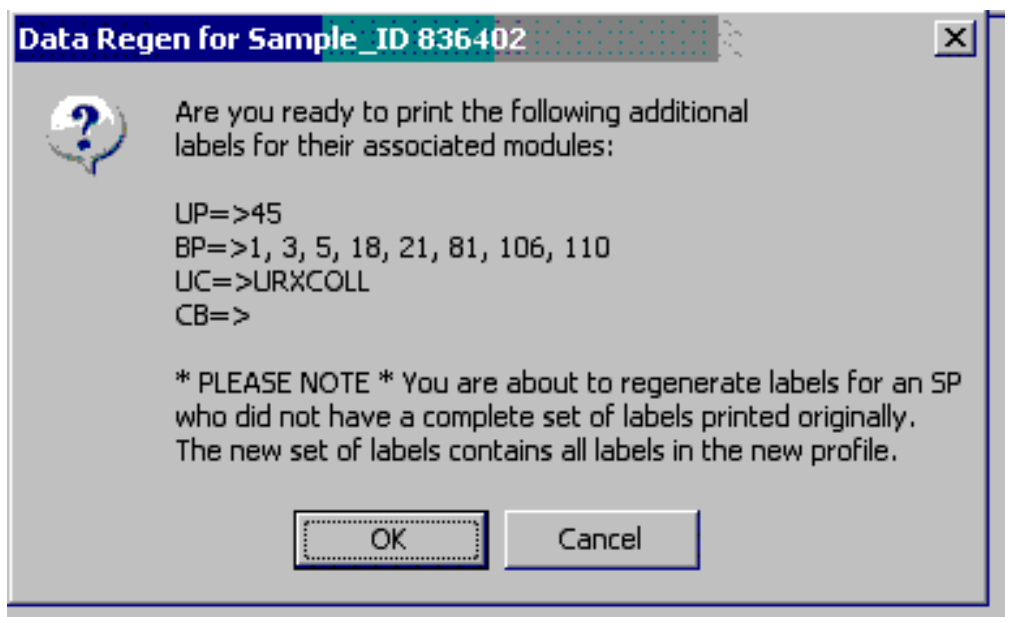

Review the question, follow the instructions, and respond to the question. To respond affirmatively to the message, use the mouse to direct the mouse arrow to the "OK" button, and left click or select [Enter]. To cancel the action, use the mouse to direct the mouse arrow to the "Cancel" button and left click. 
New labels print for vessels that are in addition to the existing vessels. If an incomplete set of labels has been printed, then a complete set of all labels print. A series of informational messages display.

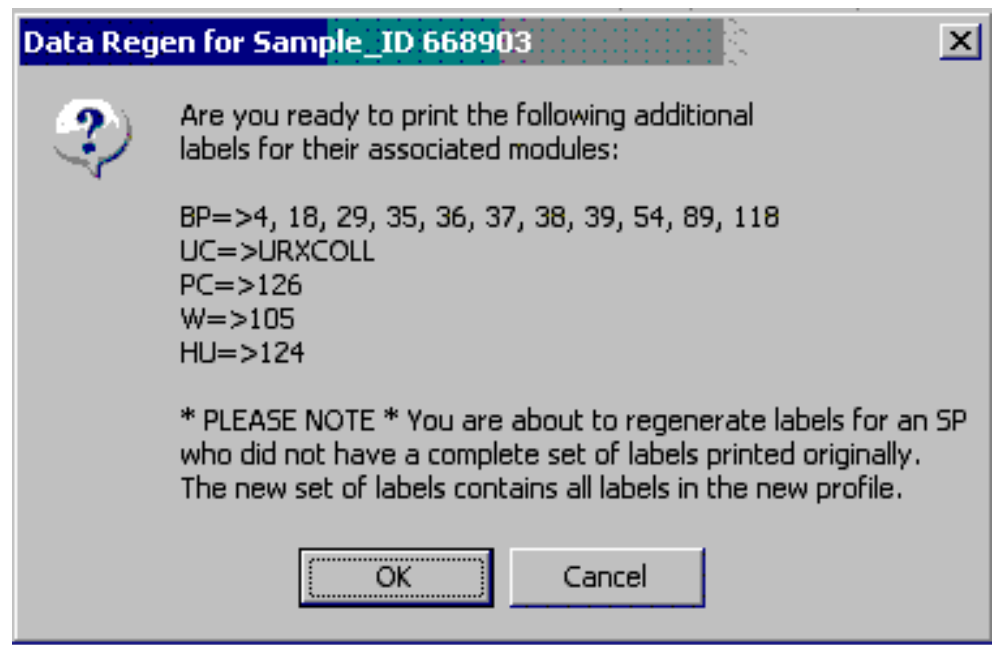

Review the text in each message and follow the instructions.

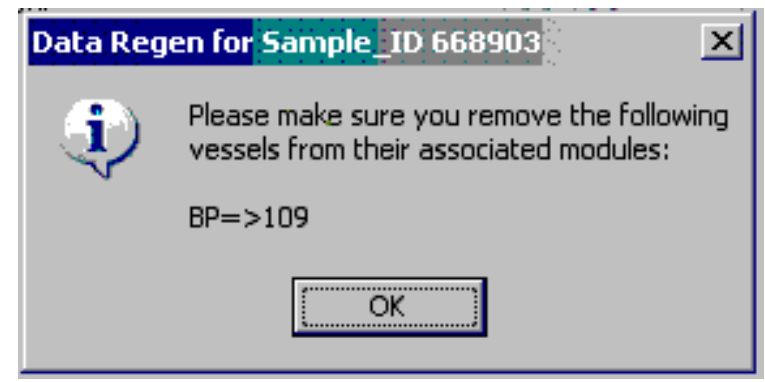

The informational message text box displays and states, "Please make sure you remove the following vessels from their associated modules:” Discard (destroy) the vessels and labels indicated in the message. To respond affirmatively to the message, use the mouse to direct the mouse arrow to the "OK" button and left click or type [Enter]. 
Allow the heads-up screen to update.

\begin{tabular}{|c|c|c|c|c|c|c|c|c|c|c|c|c|c|c|c|}
\hline \multicolumn{16}{|c|}{ Lab Sink } \\
\hline File View & \multirow{2}{*}{$\begin{array}{l}\text { utilities Repor } \\
\text { 橉 }\end{array}$} & \multirow{2}{*}{ 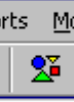 } & \multirow{2}{*}{$\begin{array}{l}\text { Modules } \\
8\end{array}$} & \multirow{2}{*}{\begin{tabular}{|l|}
\multicolumn{2}{|c|}{ Shippir } \\
$7]+$ \\
\end{tabular}} & \multicolumn{2}{|c|}{ ing Window Help } & & & & & & & & & \\
\hline$\|$ 为 v & & & & & & & & & & & & & & & \\
\hline & ppointments $f$ & for Ses & ssion: & 410111 & & & & & & Pro & cess & Stat & & & \\
\hline$S P I D$ & Sample ID s & tatus & type & gender & age & name & uc & $\mathrm{u}$ & $\mathrm{P}$ & $\mathrm{OZ}$ & $\mathrm{CB}$ & $\mathrm{Bg}$ & $\mathrm{gtt} P C$ & $\mathrm{CHPV}$ & OR \\
\hline 215591 & 215591 & $\mathrm{Cl}$ & VIP & $\mathrm{F}$ & 59 & PATEL, PAVARTI & - & 0 & - & . & 0 & 0 & . & . & . \\
\hline 244969 & 244969 & $\mathrm{BE}$ & $P$ & m & 42 & JORDAN, LEE & 0 & 0 & . & 0 & 0 & - & .0 & . & 0 \\
\hline 470234 & 470234 & $\mathrm{Cl}$ & $P$ & $\mathrm{M}$ & 601 & HAGRID, RUBEUS & 0 & 0 & . & 0 & 0 & 0 & .0 & & 0 \\
\hline 668903 & 668903 & $\mathrm{Cl}$ & $P$ & $m$ & 9 & WEASLEY, FRED & 0 & 0 & . & 0 & 0 & 0 & . 0 & . & . \\
\hline 762731 & 762731 & $\mathrm{Cl}$ & VIP & $\mathrm{F}$ & 12 & BROWN, LAVENDAR & & & - & . & 0 & & . . & . & . \\
\hline 836402 & 836402 & $\mathrm{Cl}$ & VIP & M & $12 n$ & MALFOY, DRACO & 0 & 0 & . & . & 0 & 0 . & . & . & . \\
\hline 900081 & 900081 & $\mathrm{Cl}$ & VIP & $\mathrm{F}$ & $49 A$ & ABEOTT, HANNAH & & 0 & 0 & . & 0 & 0 & . & 3 & . \\
\hline 998878 & 998878 & $\mathrm{Cl}$ & VIP & $M$ & 2 & DURSLEY, VERNON & . & . & . & . & 0 & & .. & . & . \\
\hline
\end{tabular}

Allow the heads-up display to update before proceeding. After the screen updates, the color of the SP's row changes from red to black. 


\subsection{Create New Container Labels}

Store processed vessels and biological samples in boxes and bags (containers). Print and assign container labels using the \{Create New Container Labels\} module. Once a label has been created, the container is designated as "open” in the database; it is available for slot assignments as vessels are marked as filled.

At the beginning of each stand, print and assign one new container ID for each vessel using either the $\{$ Print \} module or the \{Shipping\} module. Print additional labels as needed. Each stand is allocated a maximum of 999 labels. The actual container label will include the vessel name, vessel ID, the size of the container (9x9 2-inch box, etc.), and the container number.

Chlam/GC (46)

$5 \times 53$ inch box |||||||||||||||||||||||||||||||||||||||||||
Prior Pest (48)

$5 \times 53$ inch box

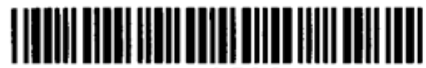


Use one of the two locations within the laboratory application to print and assign new container ID numbers or labels. Print and assign new container ID numbers using the \{Print Labels module.

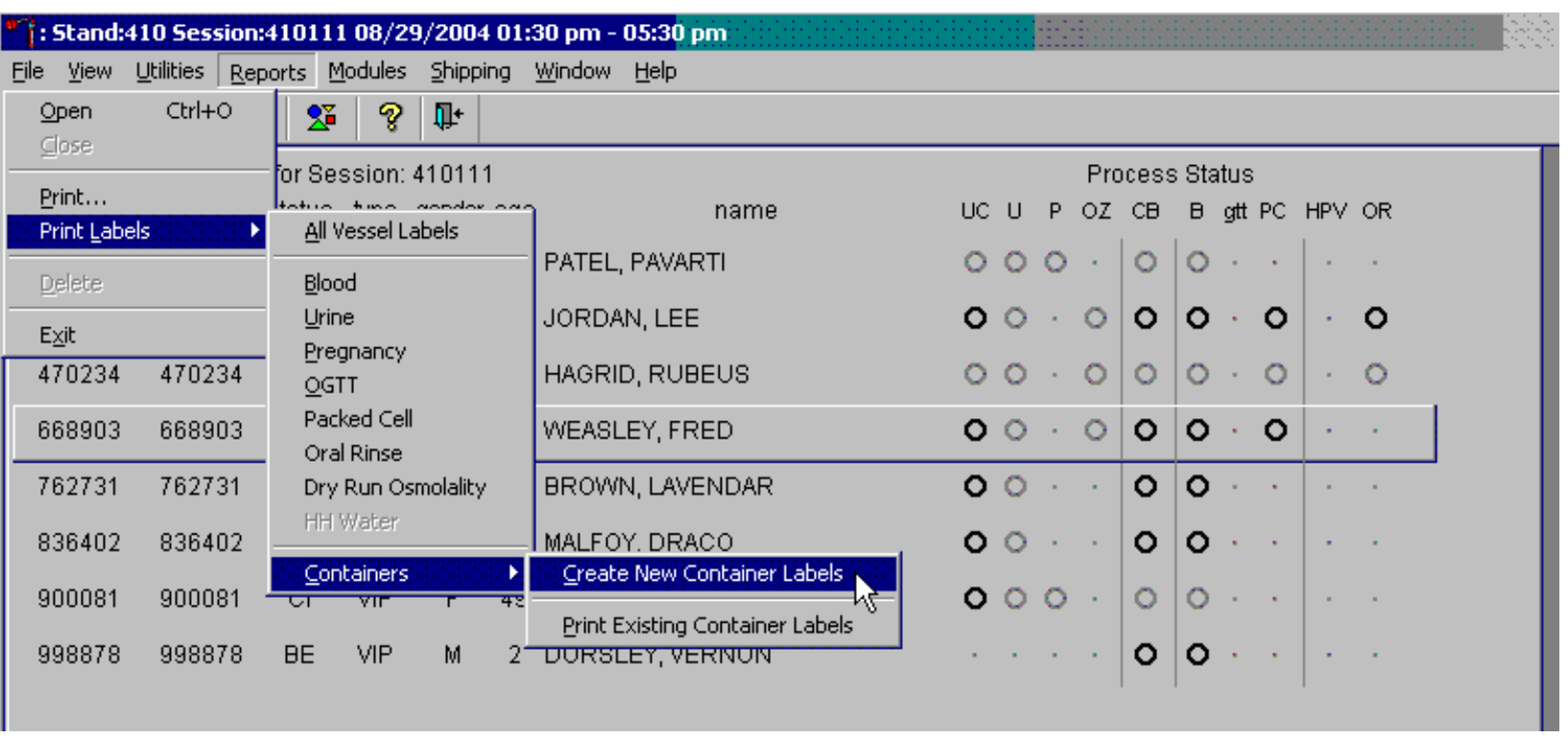

To print and assign new container ID numbers or labels using the (Print Labels) module, use the mouse to direct the mouse arrow to \{File\} in the top menu bar, left click, then drag the mouse arrow to $\{$ Print Labels\}, \{Containers\}, \{Create New Container Labels\}, and left click or type [Alt] [F/f], [L/l], $[\mathrm{C} / \mathrm{c}][\mathrm{C} / \mathrm{c}]$. 
Print and assign new container ID numbers using the \{Shipping $\}$ module.

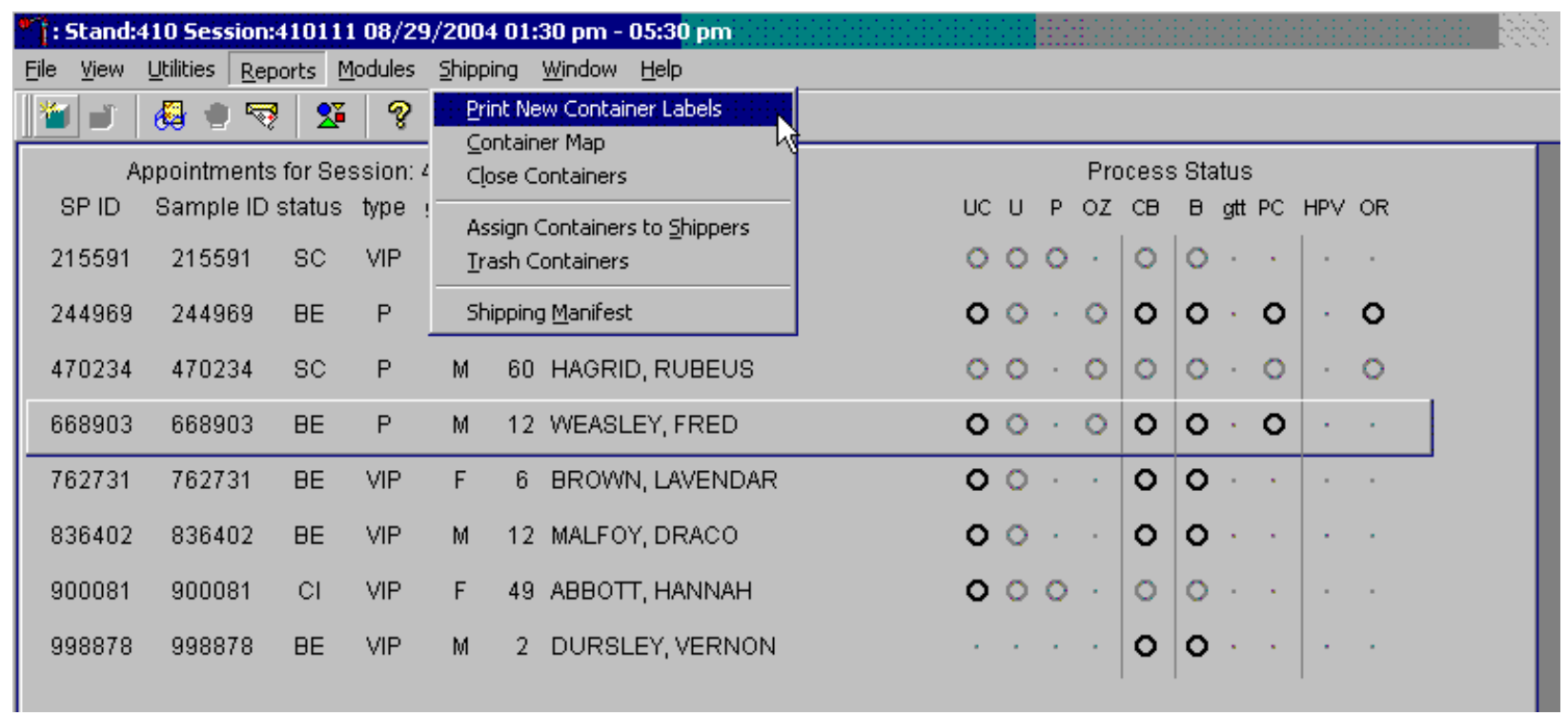

To access the \{Print New Container Labels module, use the mouse to direct the mouse arrow to \{Shipping\} in the menu bar and left click, drag the mouse arrow to \{Print New Container Labels\}, and left click or type [Alt] [S/s], [P/p]. 
There are three instances when it is appropriate to use this module. At the start of each stand, create one new container label for each vessel ID or "group." Use the same module to create a new label for a vessel after the last slot in an open container is filled. Use this module to print additional labels at any time.

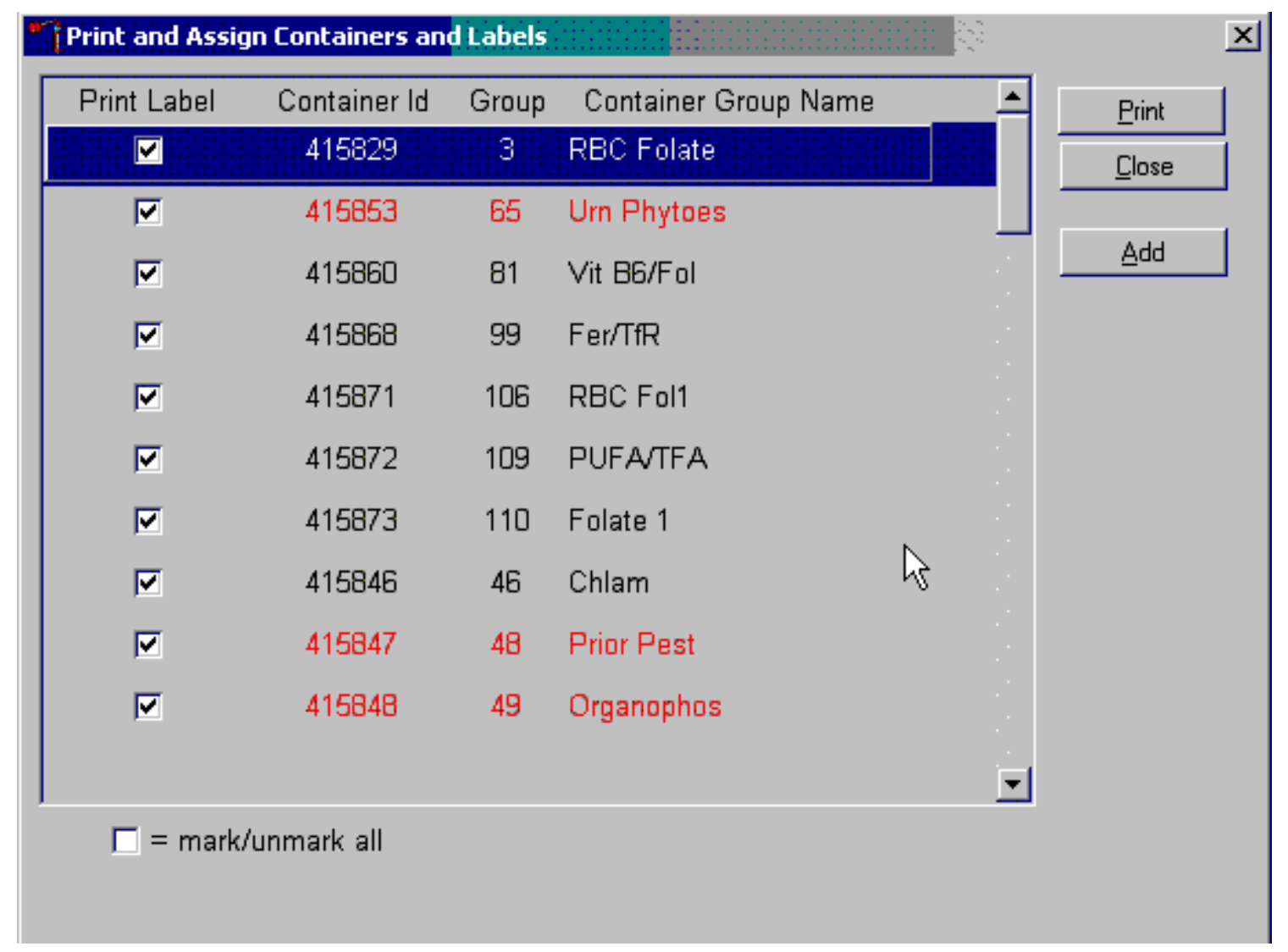

The Print and Assign Containers and Labels window displays once the PPrint New Container m module is accessed. The window includes check boxes to mark or identify specific groups, new Container Ids that are assigned to the Groups, the number of the Group, and the Container Group Name or vessel name. The Group and corresponding Container Group Name listed in this screen represent the Group where the container has been "closed" and a new label has not been created. This screen also includes a "mark/unmark all" check box and three buttons: Print, Close, and Add. Use the "Print" button to print the container labels, use the "Close" button to exit the module without printing any labels or assigning Container IDs to the Groups, and use the "Add” button to open another window that allows the user to add additional or multiple labels for any vessel. 
Individually mark the Print Label check box to identify vessels that require labels.

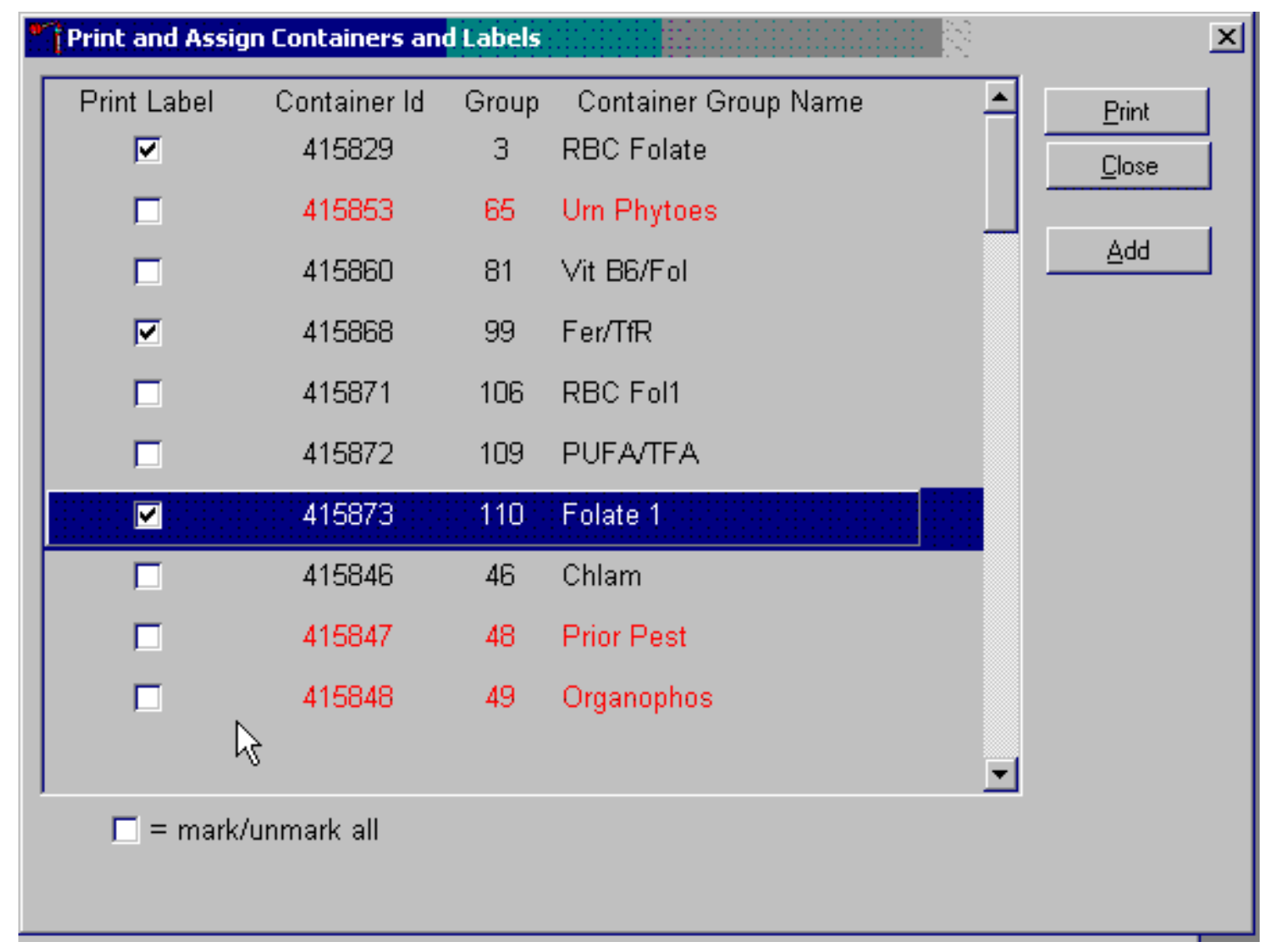

To begin the process of printing and assigning new Container IDs, mark the individual check boxes that correspond to the Group (vessel) of interest. To add a check mark to the Print Label check box, use the mouse to direct the mouse arrow to the Print Label check box and left click. 
Collectively mark all the Print Label check boxes for all the Groups listed in the window.

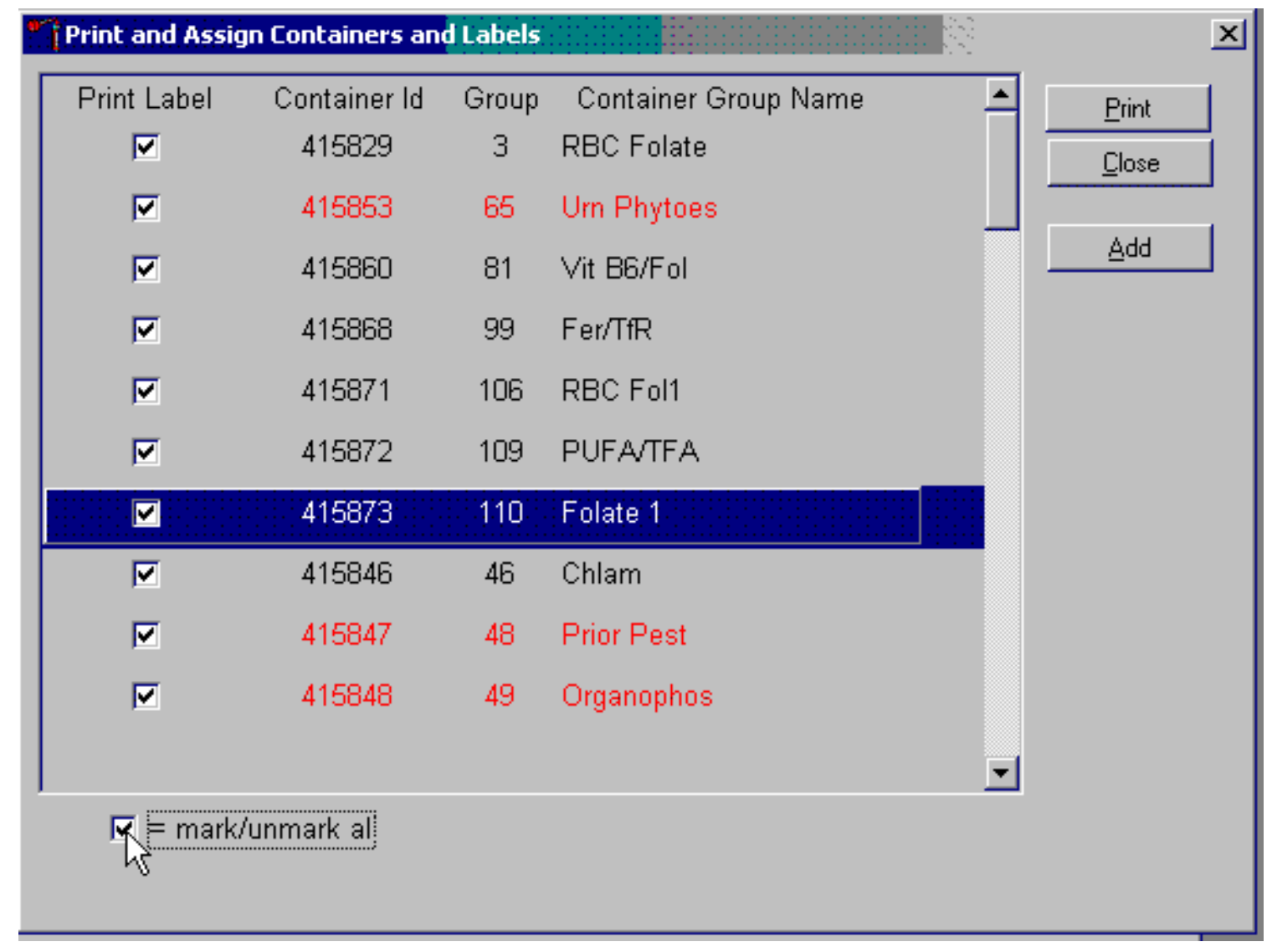

To collectively mark all the check boxes, use the mouse to direct the mouse arrow to the mark/unmark all check box and left click. To collectively unmark all the check boxes, use the mouse to direct the mouse arrow to the mark/unmark all check boxes and left click. 
Access the Generate Additional Containers window to print additional labels for any Group at any time.

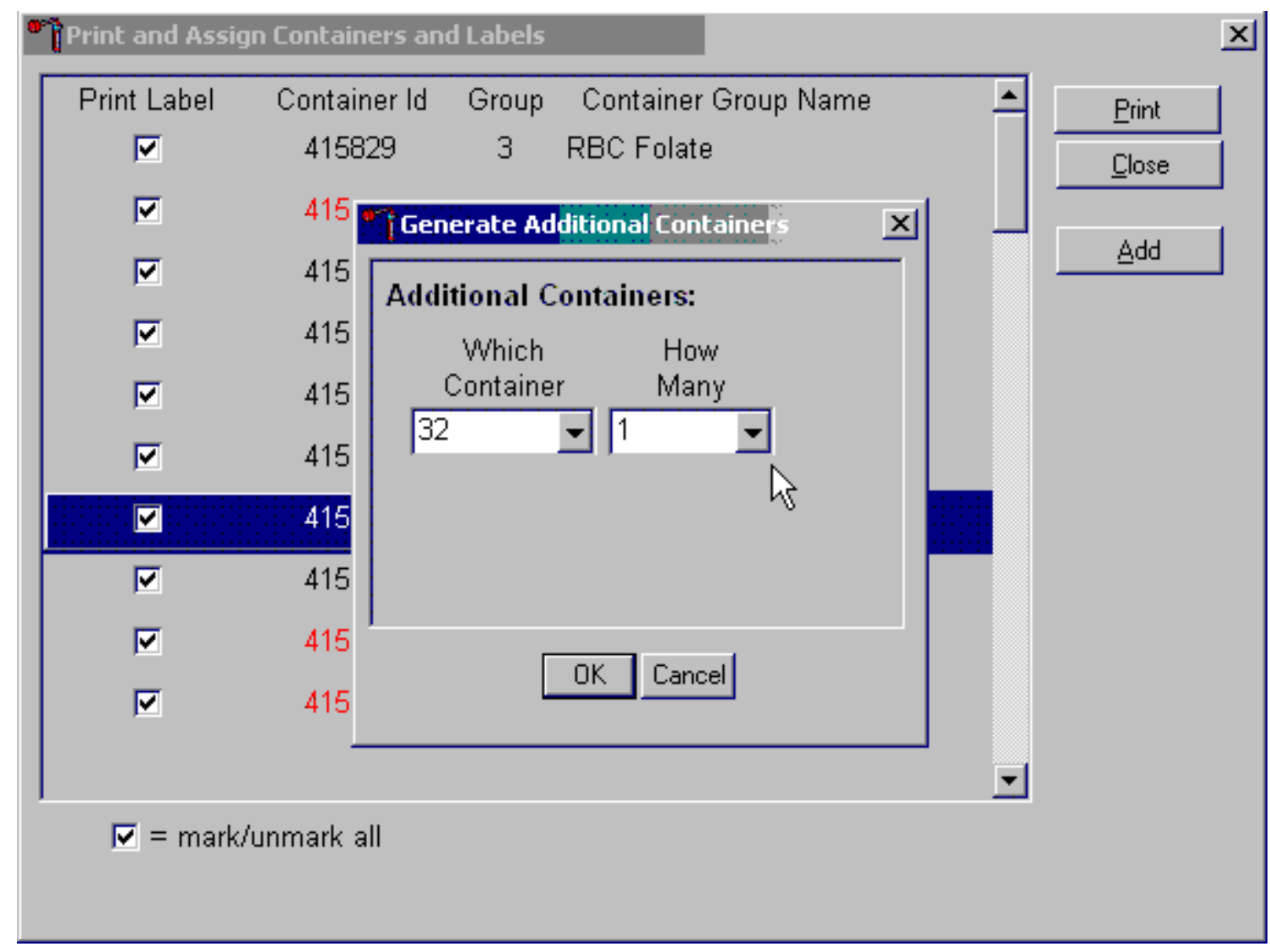

To assess the Generate Additional Containers window, use the mouse to direct the mouse arrow to the "Add" button and left click, or type [Alt] [A]. 
The Generate Additional Containers window displays.

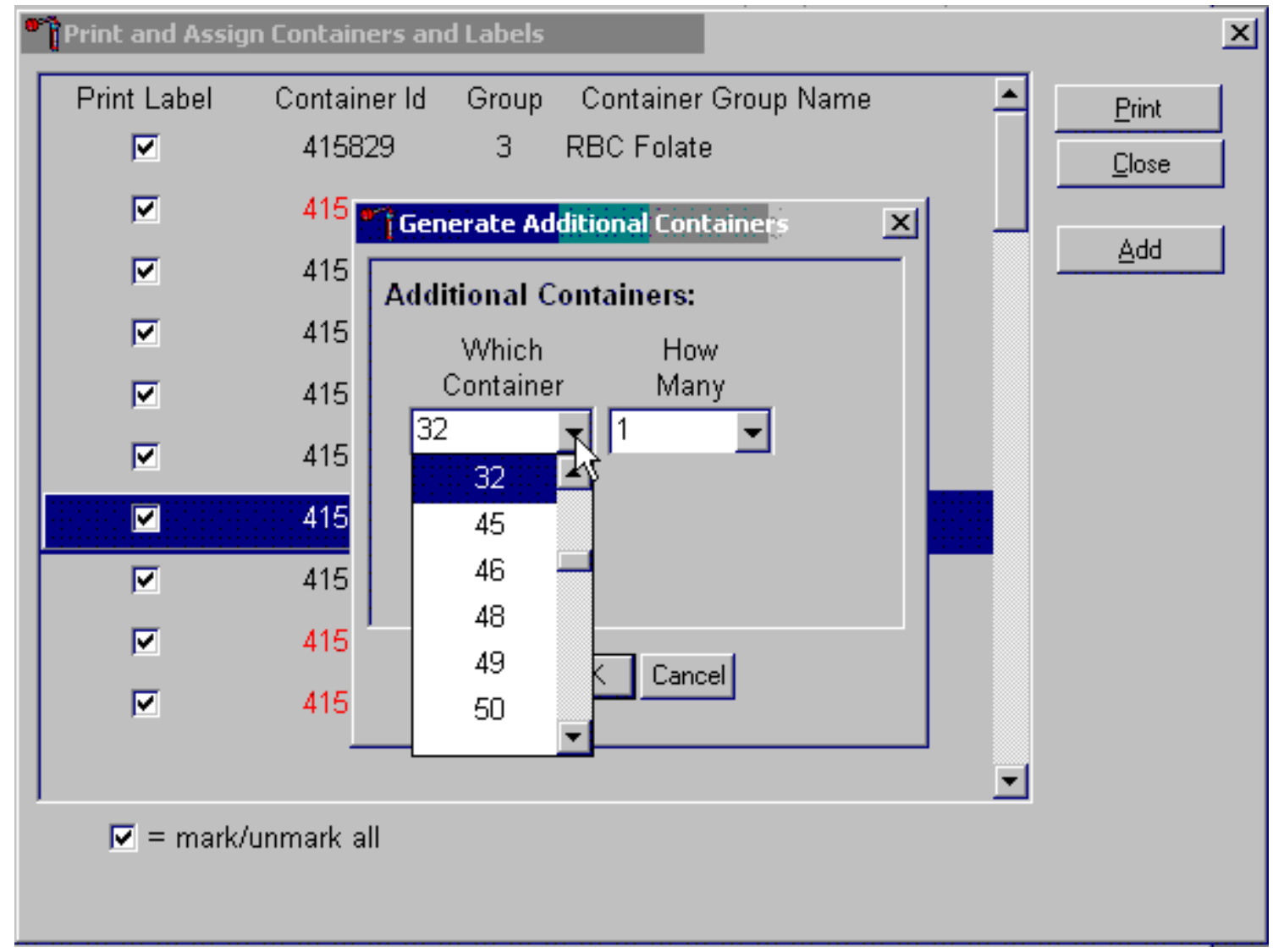

The Generate Additional Containers window includes two text boxes with drop-down lists: Which Container and How Many. The Which Container text box defaults to vessel 32. To select a vessel other than 32, use the mouse to direct the mouse arrow to the drop-down arrow and left click, drag the arrow to the desired choice and left click. Use the scroll bar to view all the choices. 
Select the number (1-5) of additional container labels.

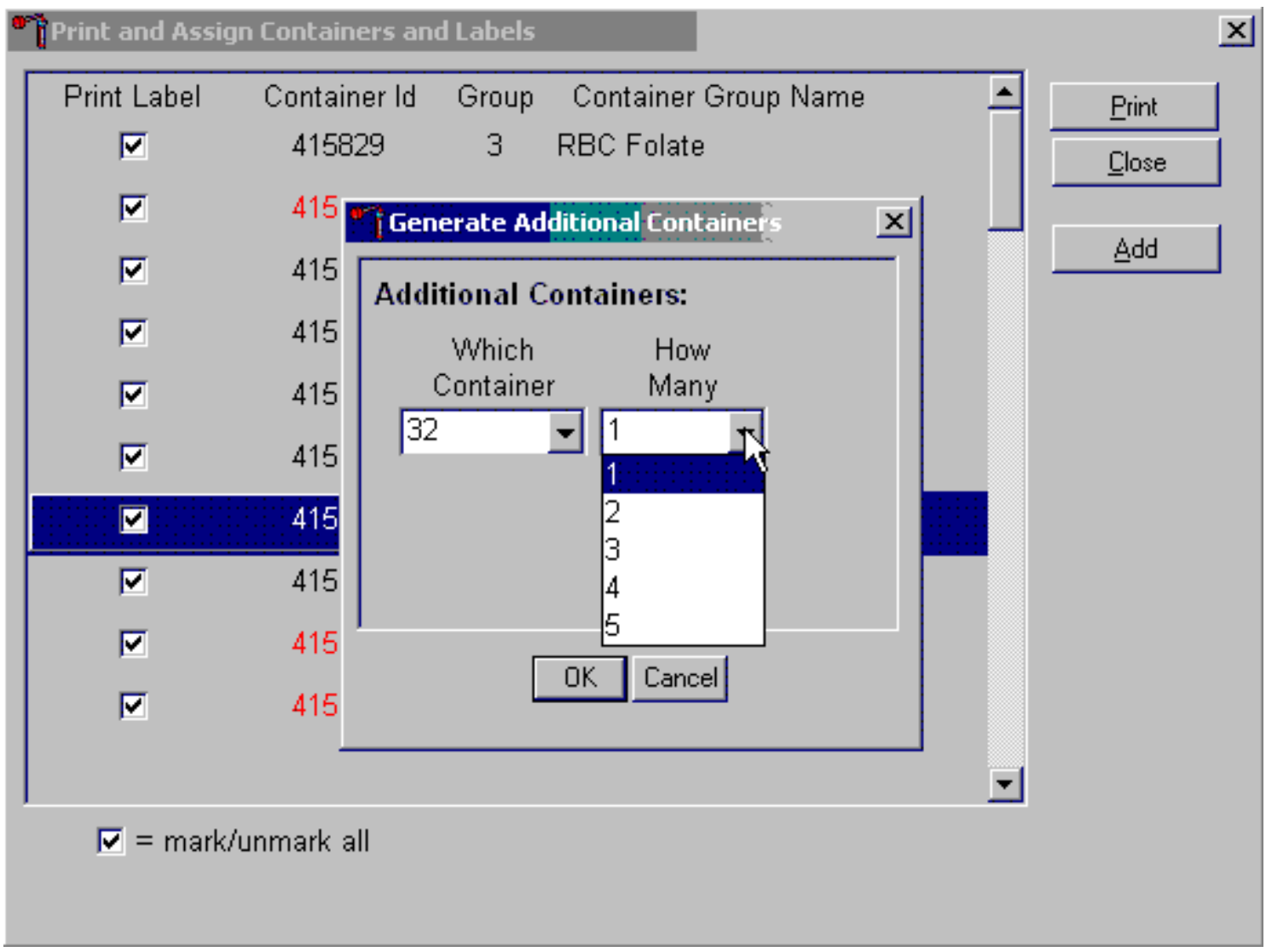

The How Many text box defaults to vessel 1 . To select 2, 3, 4, or 5, use the mouse to direct the mouse arrow to the drop-down arrow and left click, then drag the arrow to the desired choice and left click. Use the scroll bar to view all the choices. 
Add these vessels to the Print and Assign Containers and Labels window or cancel the action.

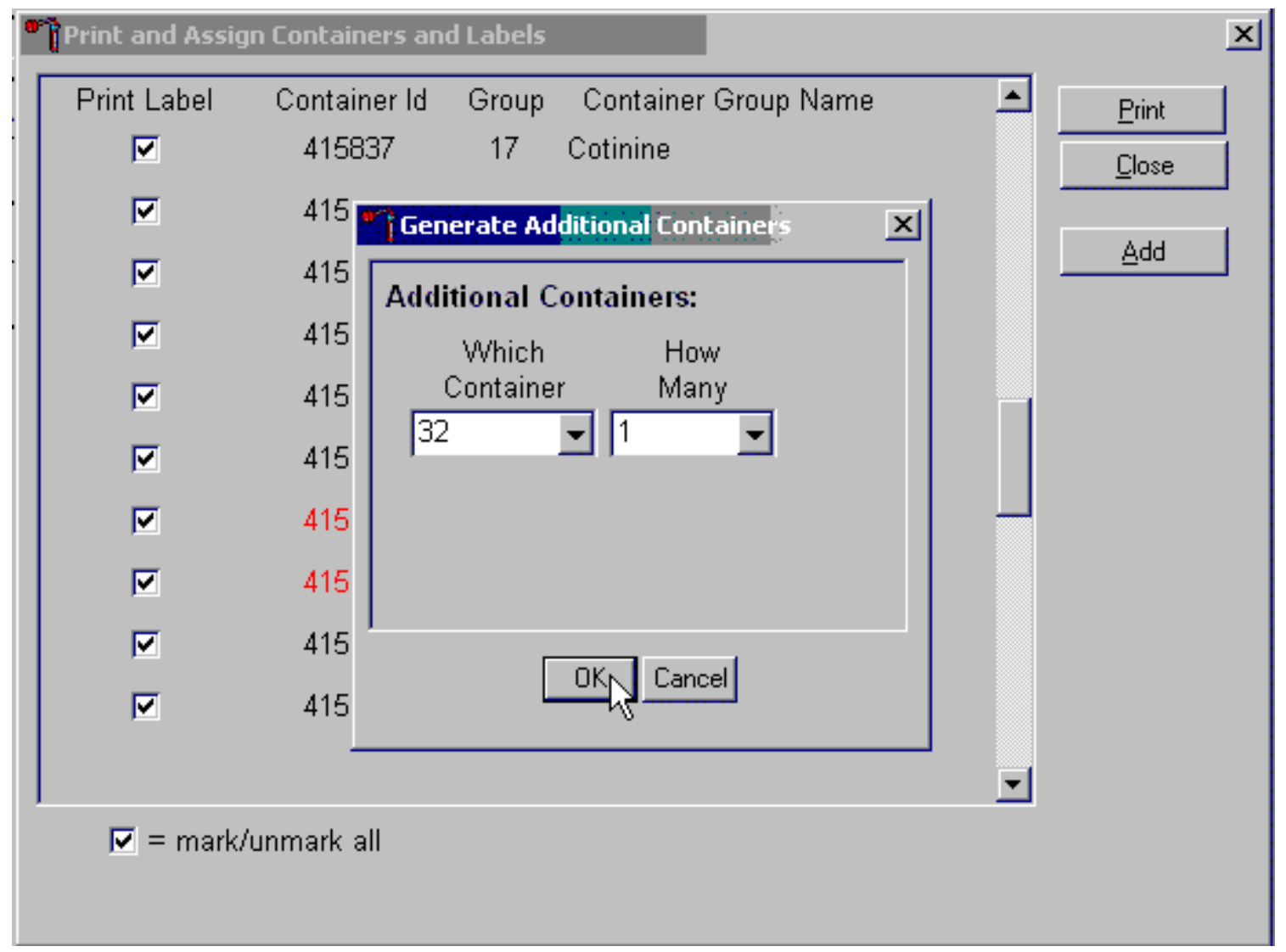

To accept the choices displayed in the Generate Additional Containers window, use the mouse to direct the mouse arrow to the "OK" button and left click or type [Enter]. To close the window without creating a new container, use the mouse to direct the mouse arrow to the "Cancel" button and left click. 
If the "OK" button is selected, then the containers are added to the Print and Assign Containers and Labels window.

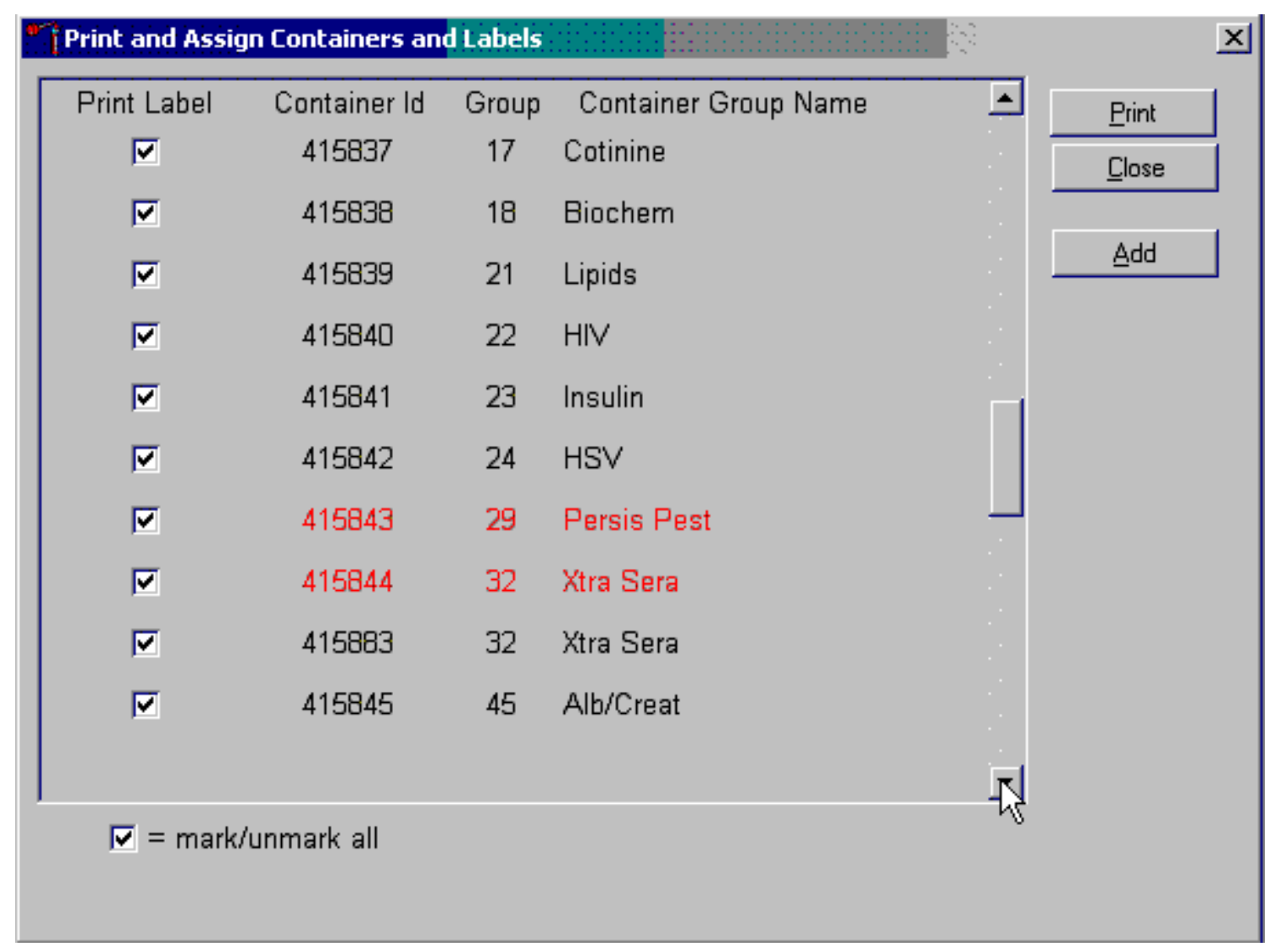

Review the information on the screen. 
Complete the assignment process and print the labels.

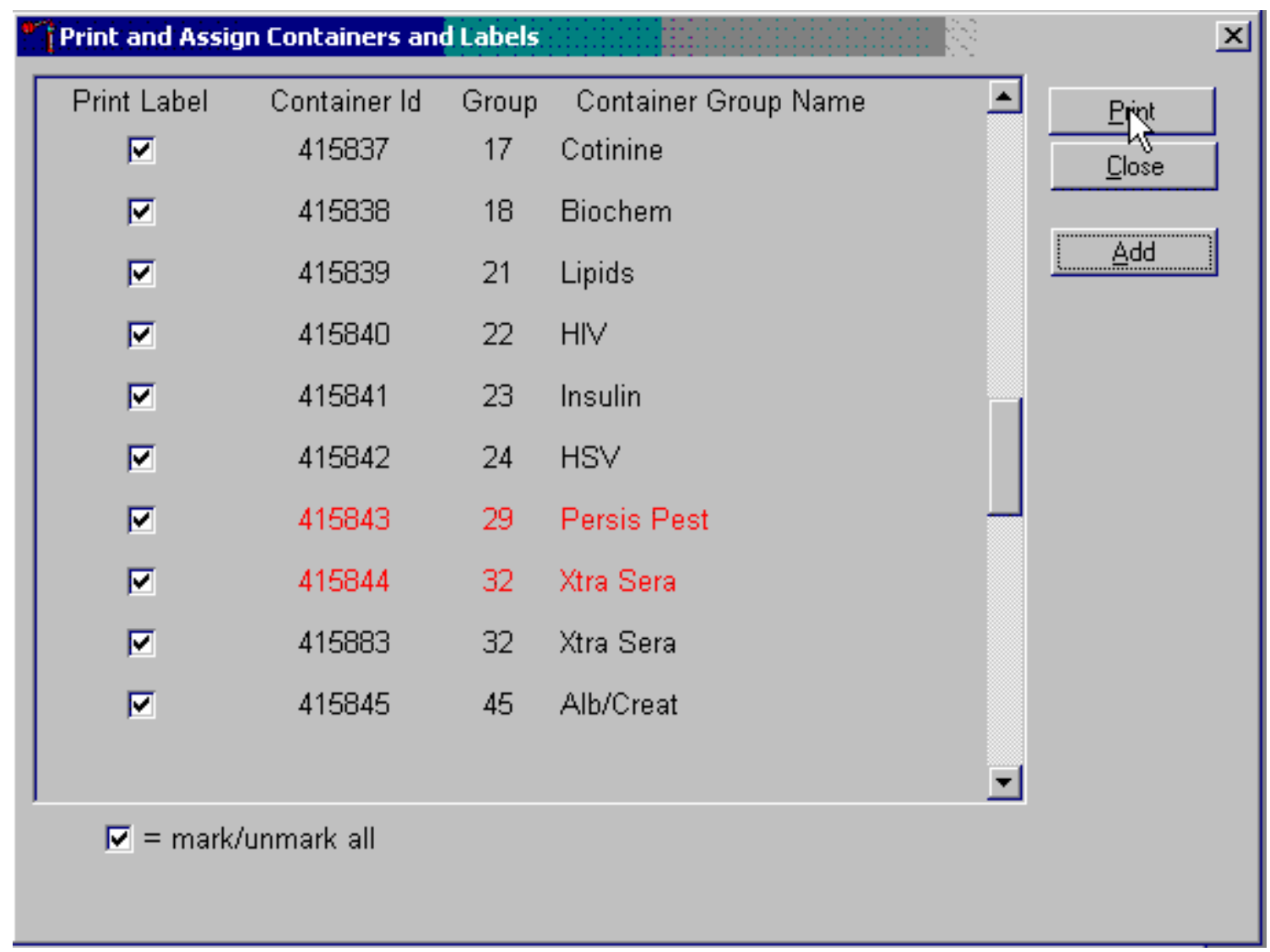

After reviewing the screen, print the labels. To print the labels, use the mouse to direct the mouse arrow to the "Print" button and left click, or type [Alt] [P/p]. To exit the window without printing any labels, use the mouse to direct the mouse arrow to the "Close" button and left click, or type [Alt] $[\mathrm{C} / \mathrm{c}]$.

Affix the container ID label(s) to the container(s) of the correct type. 
If the "Close" button is selected before the labels have been printed, then a Print and Assign Containers and Labels window displays.

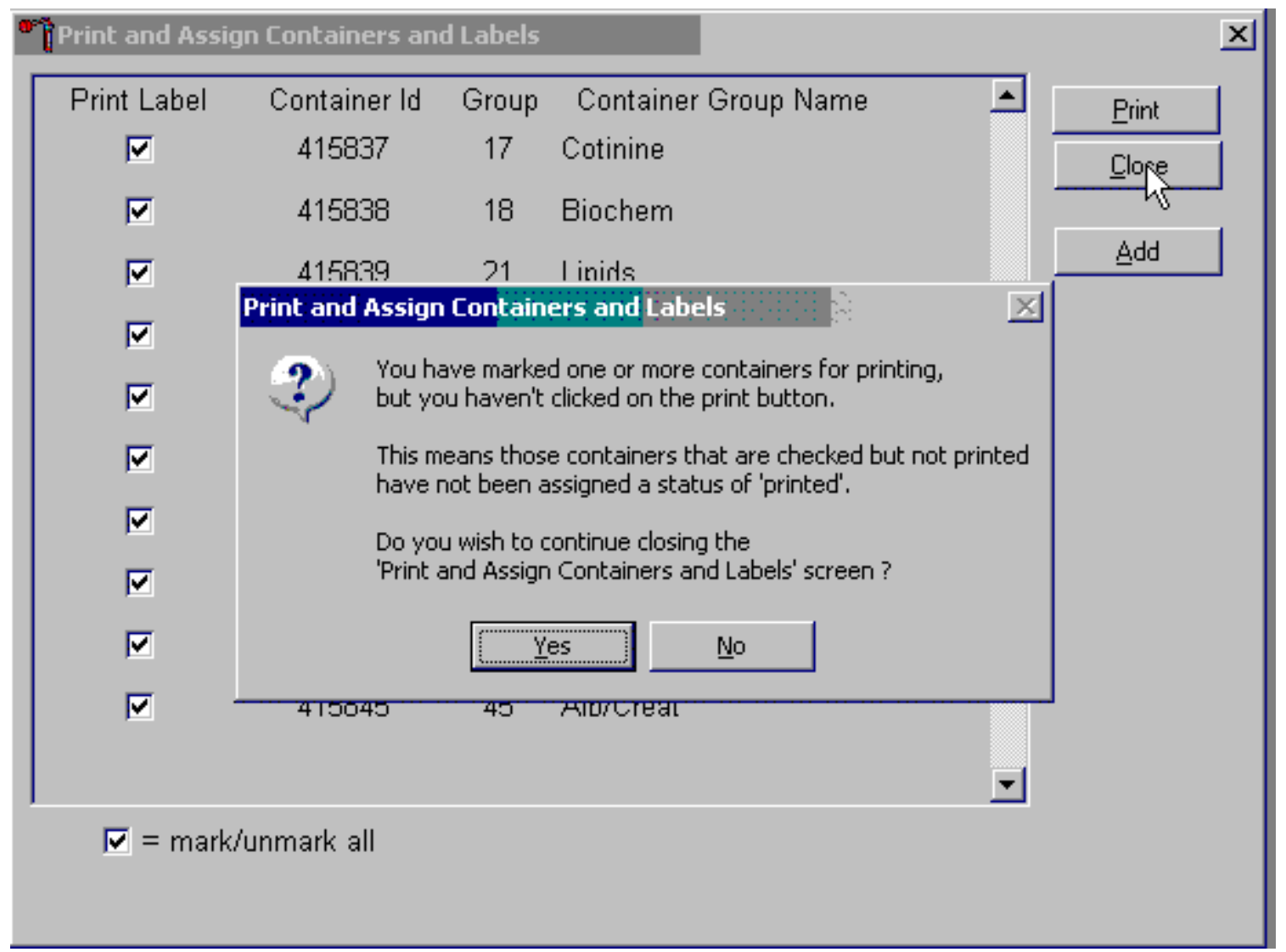

Review the text. To close the Print and Assign Containers and Labels screen, select the "Yes" button. To return to the Print and Assign Containers and Labels screen, select the "No" button. 
When a container is full, the container will automatically "close" and the Print and Assign Containers and Labels window opens with that vessel displayed and the check mark prefilled. Alternatively, open a new container to replace the closed container by using the Print and Assign Containers and Labels window.

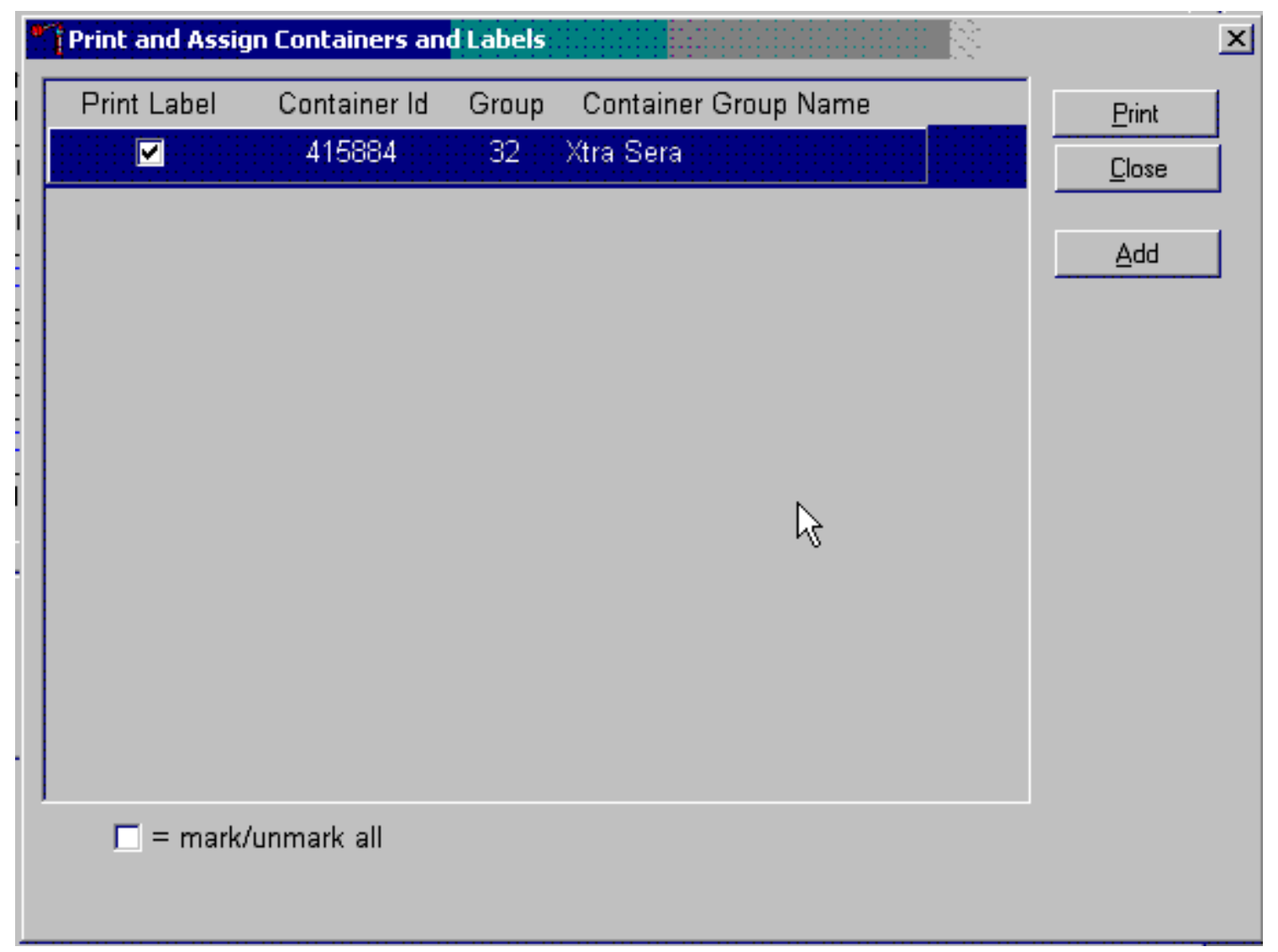

When a container is full, an opportunity to open a new container is automatically displayed on the "Print and Assign Container and Labels" window. Repeat the process of marking the check box and selecting the "Print" button. 
Each stand is allocated a maximum of 999 container IDs.

If unused container labels become lost or damaged, use the \{Trash Containers\} module to delete the container IDs. Access the \{Trash Containers $\}$ module.

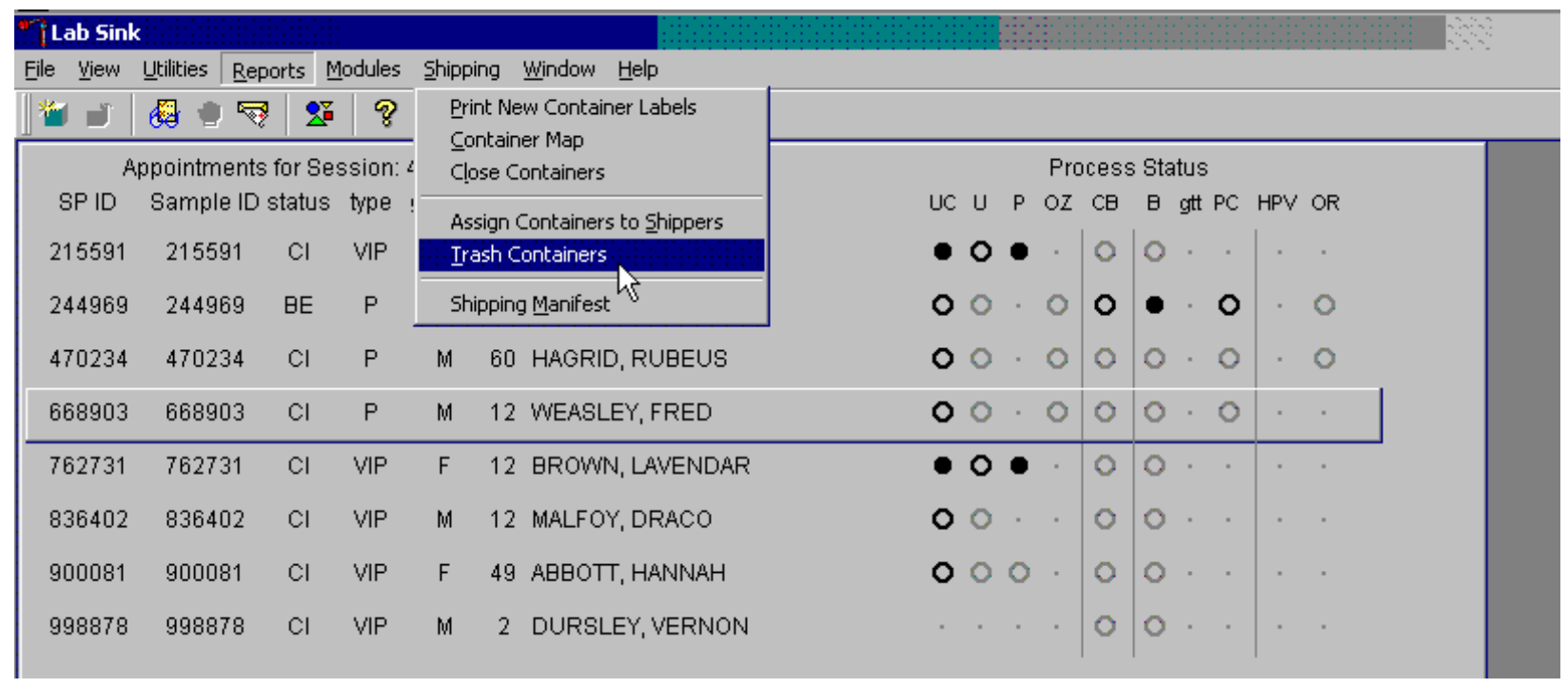

To access the \{Trash Containers $\}$ module, use the mouse to direct the mouse arrow to \{Shipping\}, left click, drag the arrow to \{Trash Containers\}, and left click, or type [Alt] [S/s], [T/t]. 
The Trash Unused Containers window displays.

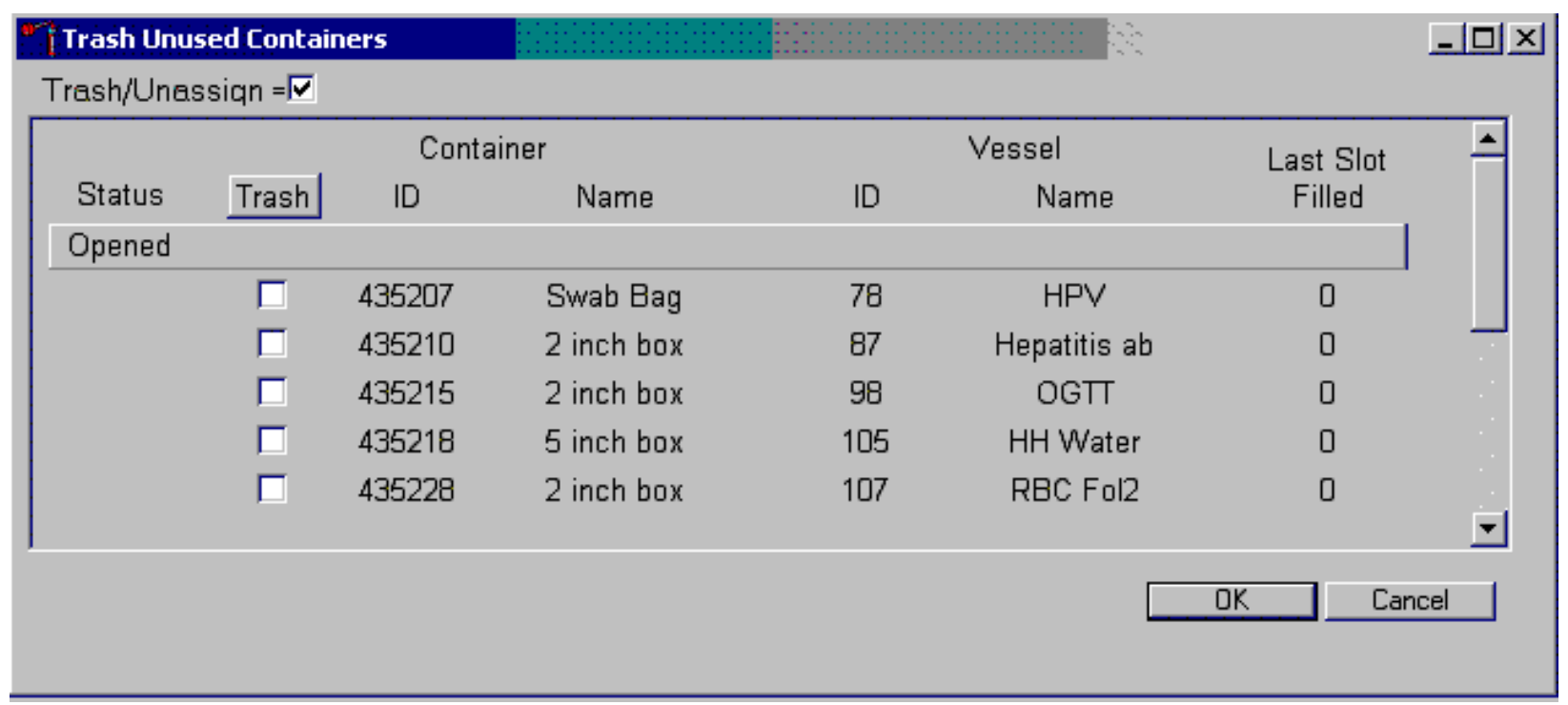

The status of each container ID is "Opened." To individually mark the container IDs for deletion, use the mouse to direct the mouse arrow to the check boxes and individually left click on specific boxes or, to mark all outstanding container IDs for deletion, use the mouse to direct the mouse arrow to the "Trash" button and left click. To collectively mark ALL containers, use the mouse to direct the mouse arrow to the Trash header and left click. 
As the container IDs are marked for deletion, the status changes from "Opened" to “Trashed.”

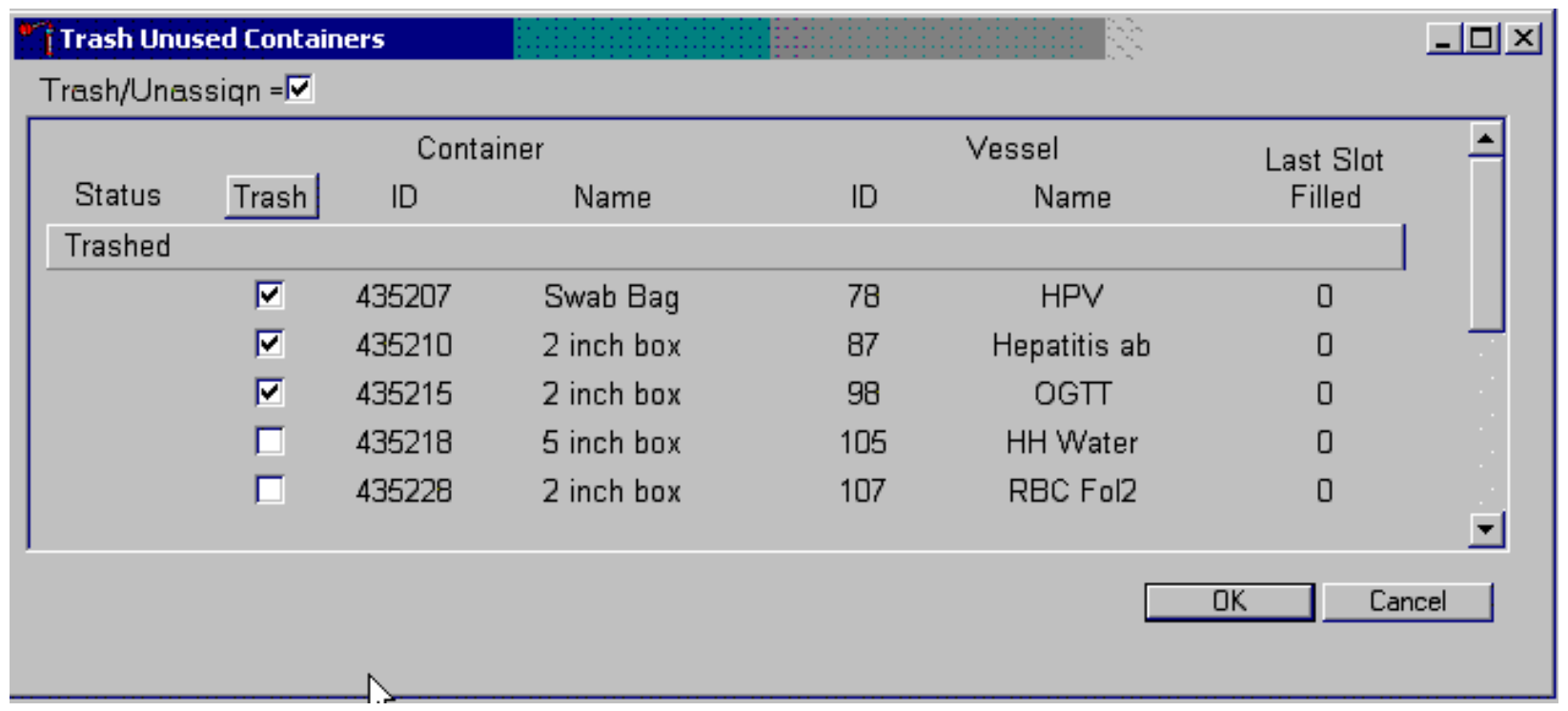

To record these actions and execute the Trash Unused Containers function, use the mouse to direct the mouse arrow to the "OK" button and left click, or select [Enter]. To exit the \{Trash Unused Containers $\}$ module without recording or executing the Trash Containers function, use the mouse to direct the mouse arrow to the "Cancel” button and left click. 


\subsubsection{Reprint Container Labels for Existing Containers}

Reprint labels for existing, not shipped containers.

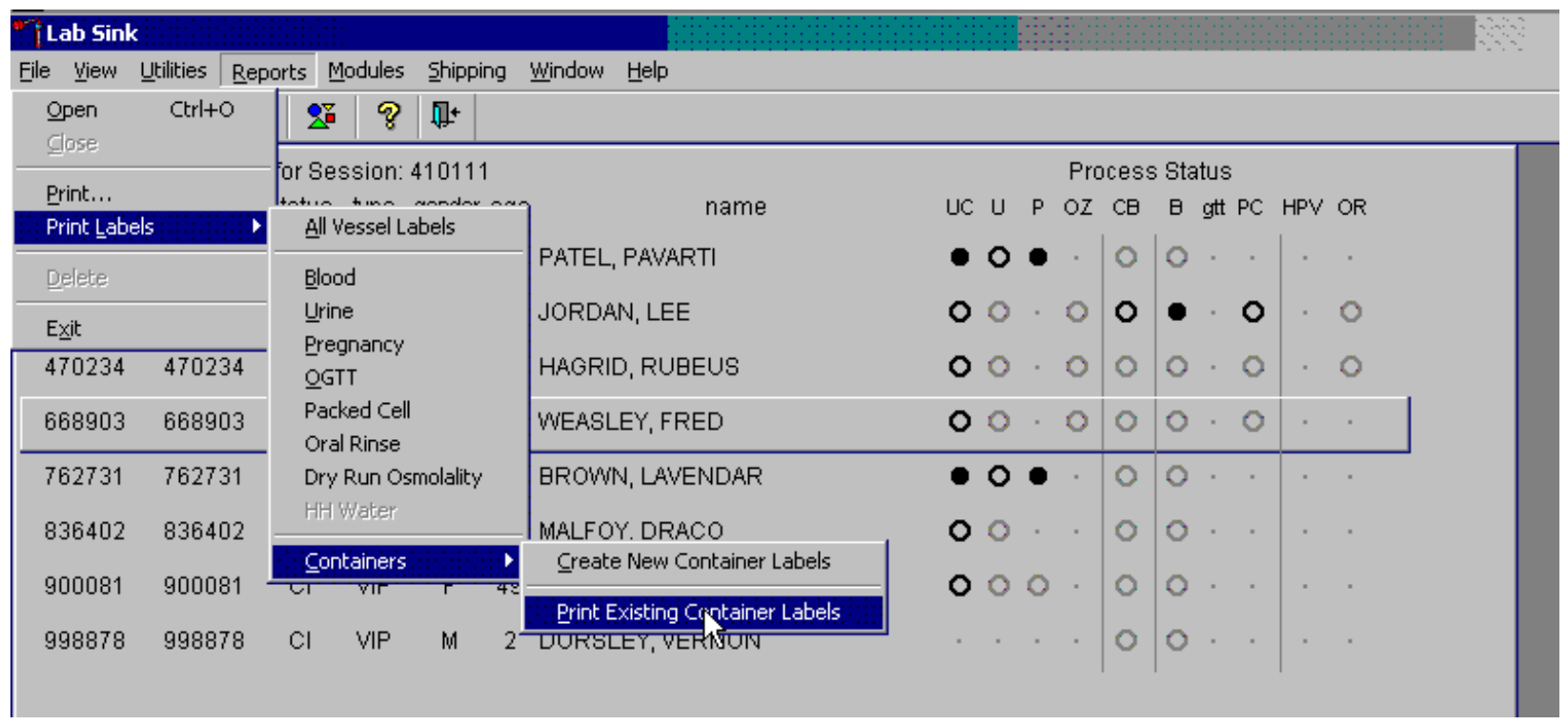

To reprint labels for an existing (not shipped) container, use the mouse to direct the mouse arrow to $\{$ File\} in the top menu bar, left click, then drag the mouse arrow to \{Print Labels\}, \{Containers\}, \{Print Existing Container Labels\} and left click, or type [Alt] [F/f], [L/l], [C/c] [P/p]. 
A Print Existing Container Labels screen displays.

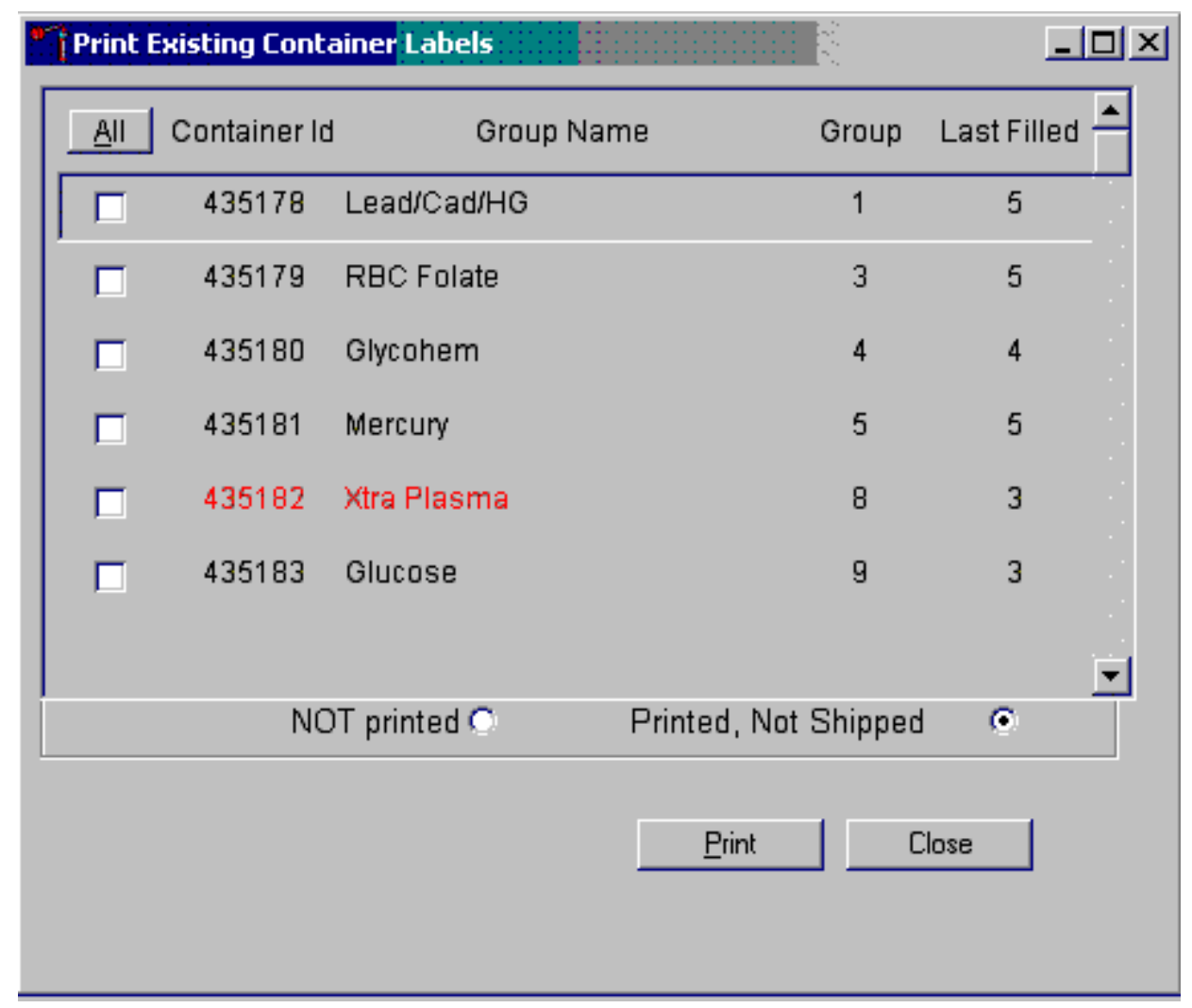

The Print Existing Container Labels screen includes a check box associated with each open (not shipped) container, columns for container IDs (or labels), Group Name, Group number, Last Filled, an "ALL" selection button, radio buttons or filters for "Not printed” and "Printed, not Shipped," and Print and "Close" buttons. The window will default to display containers where the labels have not been printed. 
A list of container IDs displays if the radio button is set to Printed, Not Shipped.

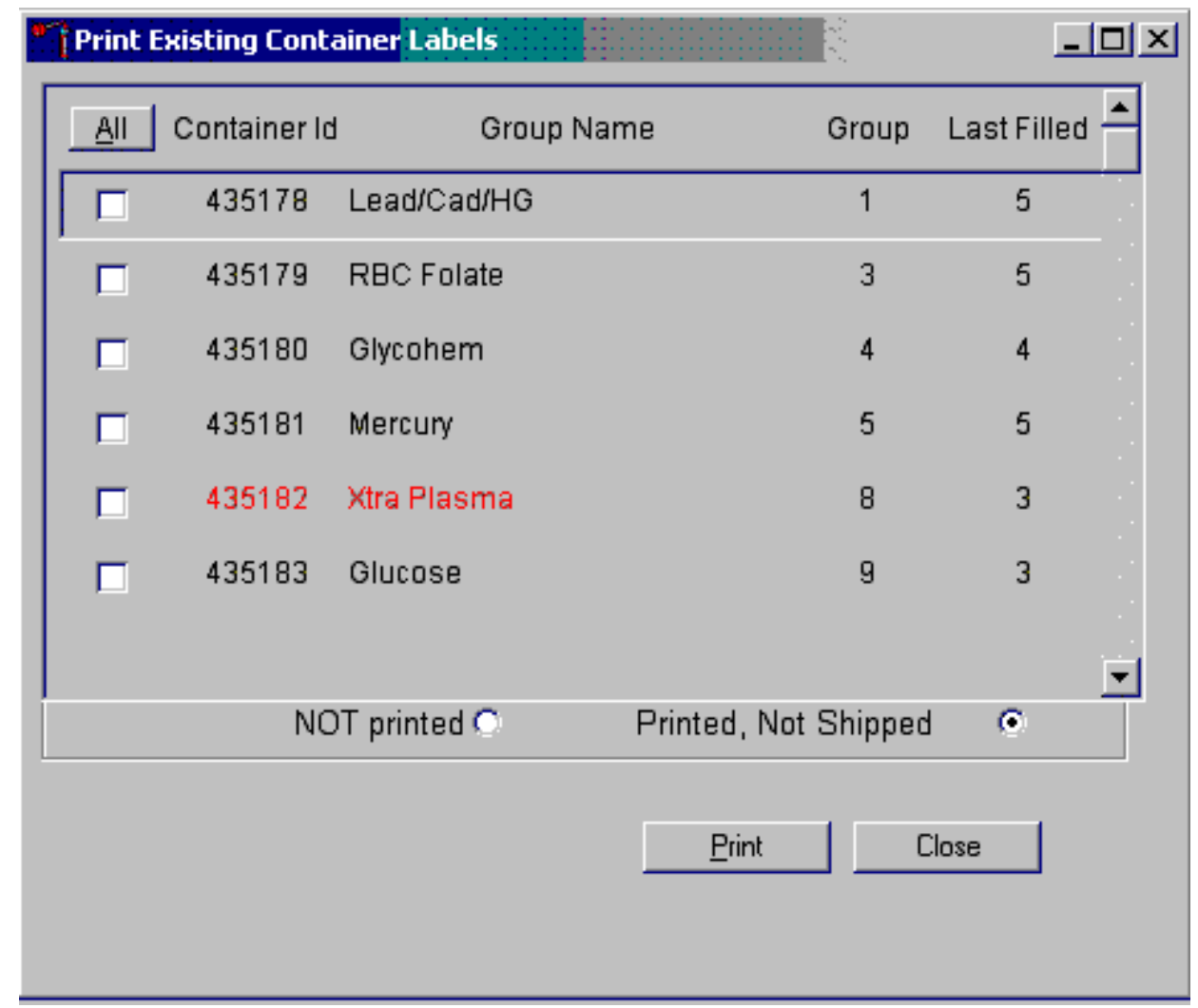

To individually mark or identify a Container ID to be reprinted, use the mouse to direct the mouse arrow to an individual check box and left click. To mark all Container IDs to be reprinted, use the mouse to direct the mouse arrow to the "All" button and left click. Check marks fill in all the check boxes. To unmark all container IDs, use the mouse to direct the mouse arrow to the "All” button a second time and left click.

Select the "Print" button to print the labels. To select the "Print" button, use the mouse to direct the mouse arrow to the "Print" button and left click. To exit the screen without printing the labels, use the mouse to direct the mouse arrow to the "Close" button and left click. 
The window will default to "NOT printed." Select "Printed, not shipped” to display existing containers.

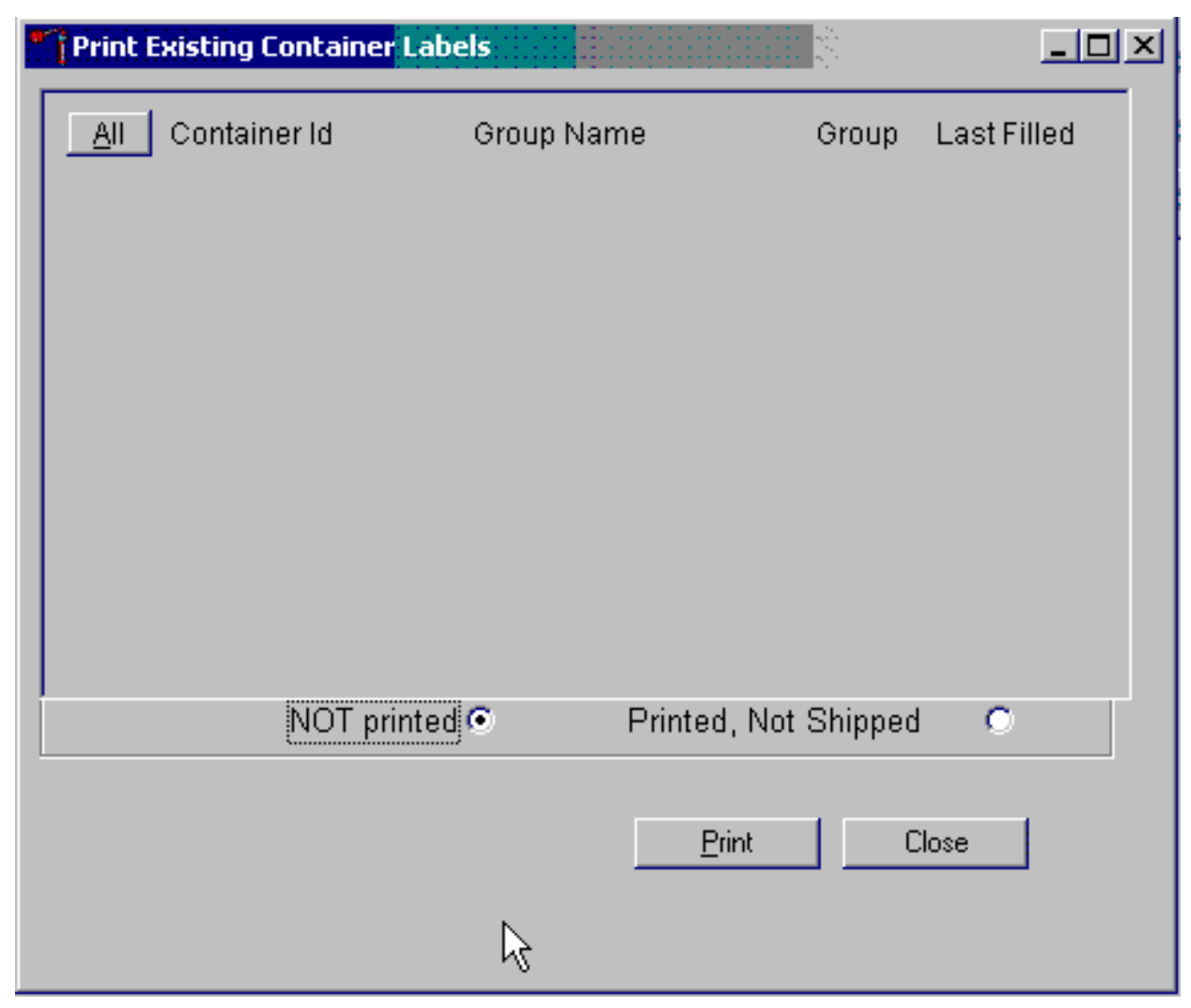

When the "NOT Printed" radio button is selected, the Print Existing Container Labels window displays all containers where the Container ID has been generated but the label has not been printed. These labels are in the queue waiting to be printed. To individually mark or identify a Container ID to be reprinted, use the mouse to direct the mouse arrow to an individual check box and left click. To mark all Container IDs to be reprinted, use the mouse to direct the mouse arrow to the "All” button and left click. Check marks fill in all the check boxes. To unmark all container IDs, use the mouse to direct the mouse arrow to the "All” button a second time and left click.

Select the "Print" button to print the labels. To select the "Print" button, use the mouse to direct the mouse arrow to the "Print" button and left click. To exit the screen without printing the labels, use the mouse to direct the mouse arrow to the "Close" button and left click. 


\subsection{Shipping Overview}

The integrity of laboratory specimens is critical to NHANES. Storage temperatures have been established for each analyte in consultation with the National Center for Health Statistics (NCHS) and contract laboratories. It is critical to maintain these established conditions throughout specimen storage and shipping and until their ultimate receipt by the contract laboratories. Maintain these conditions by using documented procedures, which include using sufficient dry ice, refrigerant packs, and sending specimens by FedEx priority overnight.

FedEx Priority Overnight guarantees next-business-day delivery by 10:30 a.m. to most U.S addresses and by noon, 4:30 p.m., or 5 p.m. in remote areas. Delivery by FedEx can be delayed due to a weekend or holiday. This extraordinary circumstance usually occurs at the end of a stand or just before vacation. In any case, ship all specimens before vacations. The following actions are intended to mitigate unforeseen delay:

- Consider using medium shippers instead of small shippers for all laboratories east of the Mississippi when shipping from areas west of the Mississippi, and for all laboratories west of the Mississippi when shipping from areas east of the Mississippi. Substitute a larger shipper when shipping specimens in extreme summer heat or whenever professional judgment dictates added precautions.

- Use a medium shipper for all international shipments.

- $\quad$ Use dry ice in blocks instead of pellets since blocks sublimate at a slower rate.

- Increase the number of refrigerant packs from four to at least six per shipper.

The shipping technologist is primarily responsible for packing and shipping the specimens to various contract laboratories on a designated day. As necessary, other medical technologists assist in performing each of the steps listed below to complete the shipping procedures.

At the start of each stand, the laboratory staff creates address labels with the current MEC address to use on the Class 9 labels.

Shipping procedures:

- On the day before or the morning of a designated shipping day, create and print shipper labels (air bills) using FedEx Ship Manager. 
- $\quad$ Prepare the Class 9 labels for shipments that use dry ice.

- On the morning of a shipping day, close all containers, assign containers to a shipper, print the paper shipping manifest for each shipper, and open all new containers.

- Assemble the paper shipping manifest, prepared FedEx label, FedEx preprinted return air bills, and assorted "warning” labels for each shipper. Clip all materials together until ready to pack the shippers.

- Collect the shippers from the compartments under the MEC.

- $\quad$ Pack the shippers with the containers, absorbent material, newsprint (padding), plastic bags, and a sufficient supply of dry ice or refrigerant packs as appropriate.

- $\quad$ Place the appropriate paper manifest and the preprinted FedEx return air bill in a $9 \times 12$ plastic bag and place the plastic bag on top of the Styrofoam shipper lid.

- Label each shipper with the appropriate FedEx label and warning labels.

- When the contract laboratory personnel receive the shippers, they unpack the specimens and return the shippers and refrigerant packs to the warehouse.

\subsection{Create Air Bills using FedEx Ship Manager}

Create air bills using the Ship Manager application at FedEx.com. Each physical stand location will be designated as a department in the system. The department is set up by home office staff. The name of the department shows up as the department designation in all of the screens within Ship Manager. The department designation is "stand\#\#\#” where \#\#\# is the stand number. The default point of contact for the department is Renee Storandt. The department address is the address of the MEC for the stand the department represents. The main contact telephone number for the department is 301-738-8324. The FedEx account number assigned to the department is 129015748. The service option for the department has been restricted to FedEx Priority Overnight and FedEx International Priority. Each department is assigned a default reference number for each package shipped; this is the project charge code for each stand. The User ID is designated "stand $x x x$ " where " $x x x$ ” represents the stand number. The password is "mec $x x x$ " where $x x x$ is the stand number.

Access the FedEx web site using Explorer and logon to the application. Create one air bill for each domestic shipment. Print the air bill, fold it in half, and insert it into a FedEx pouch. Attach the pouch to the shipper. 


\subsection{1}

Create Air Bills for Domestic Shipments

Use Internet Explorer to access the FedEx web site.

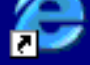

Internet

Explorer

To access the Internet Explorer application, use the mouse to direct the mouse arrow to the Explorer icon on the desktop and double click.

Select FedEx from the Favorites menu. Select the Ship header or tab, then drag the mouse arrow to Prepare Online Shipment and left click.

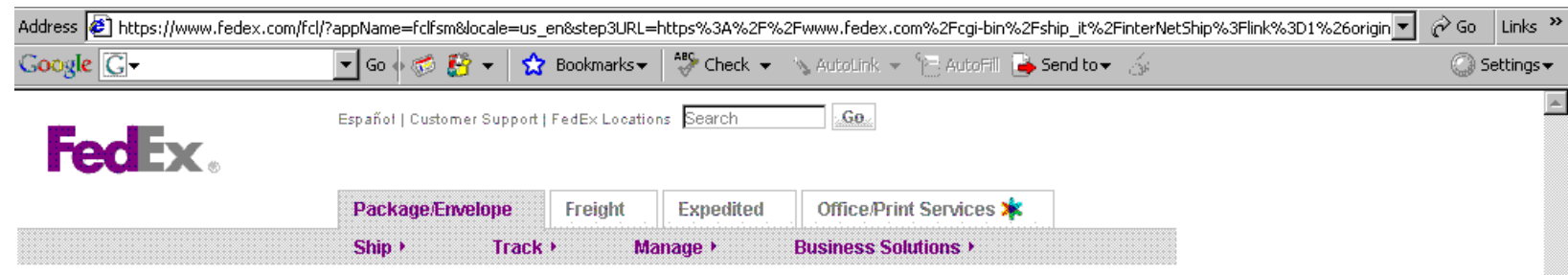

fedex.com Login

for access to FedEx Ship Manager@ at fedex.com

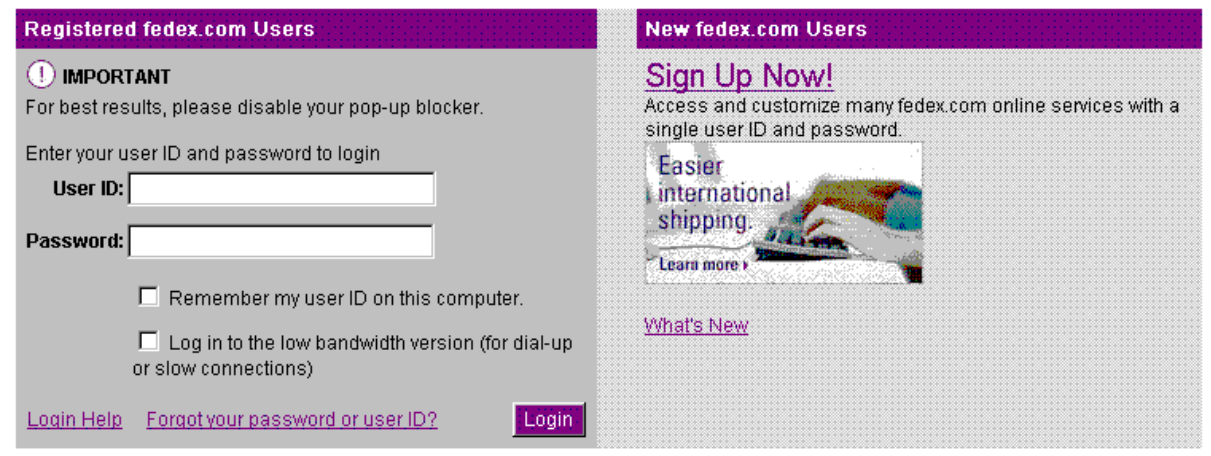

Global Home | FedEx Mobile | Service Info | About FedEx| Investor Relations | Careers | fedex.com Terms of Use | Privacy Policy | Site Map This site is protected by copyright and trademark laws under US and International law. All rights reserved. (1995-2008 FedEx

The FedEx.com Login window displays. 
Enter the User ID and password.

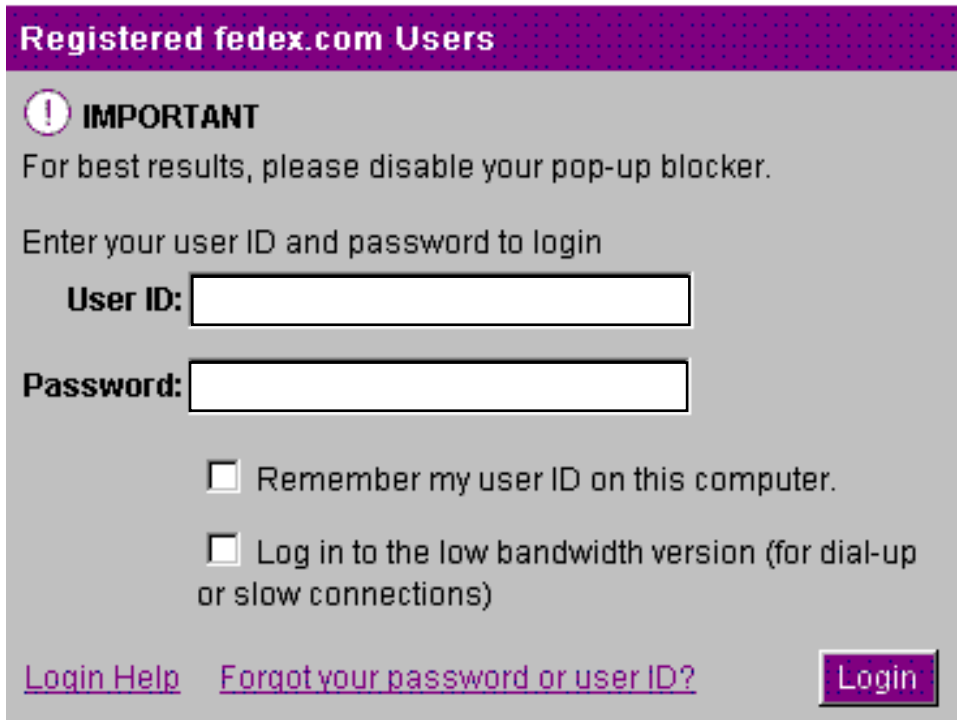

To enter the User ID, use the mouse to direct the mouse arrow to the User ID text box. To move to the Password text box, use the mouse to insert the cursor in the Password text. Enter the Password by using the keyboard keys. Both text boxes are case sensitive. To move to the shipping window, use the mouse to direct the mouse arrow to the "Login" button and left click or select [Enter]. 
The Create a Shipment window should display; if it does not, select Ship, Prepare

\section{Shipment.}

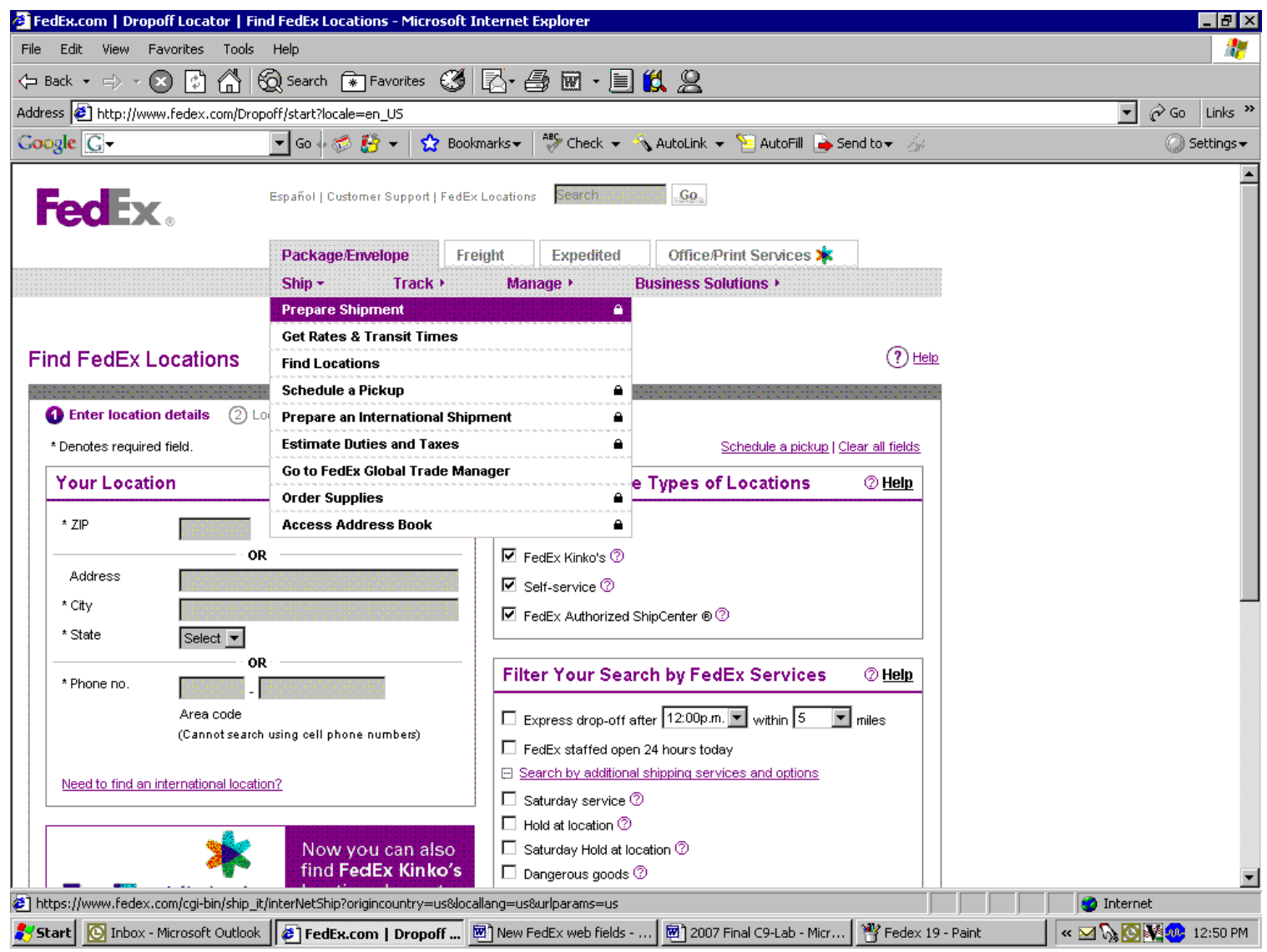


The Create a Shipment window displays. There are major tabs for Prepare Shipment, Ship History, My Lists, Reports and My Profile.

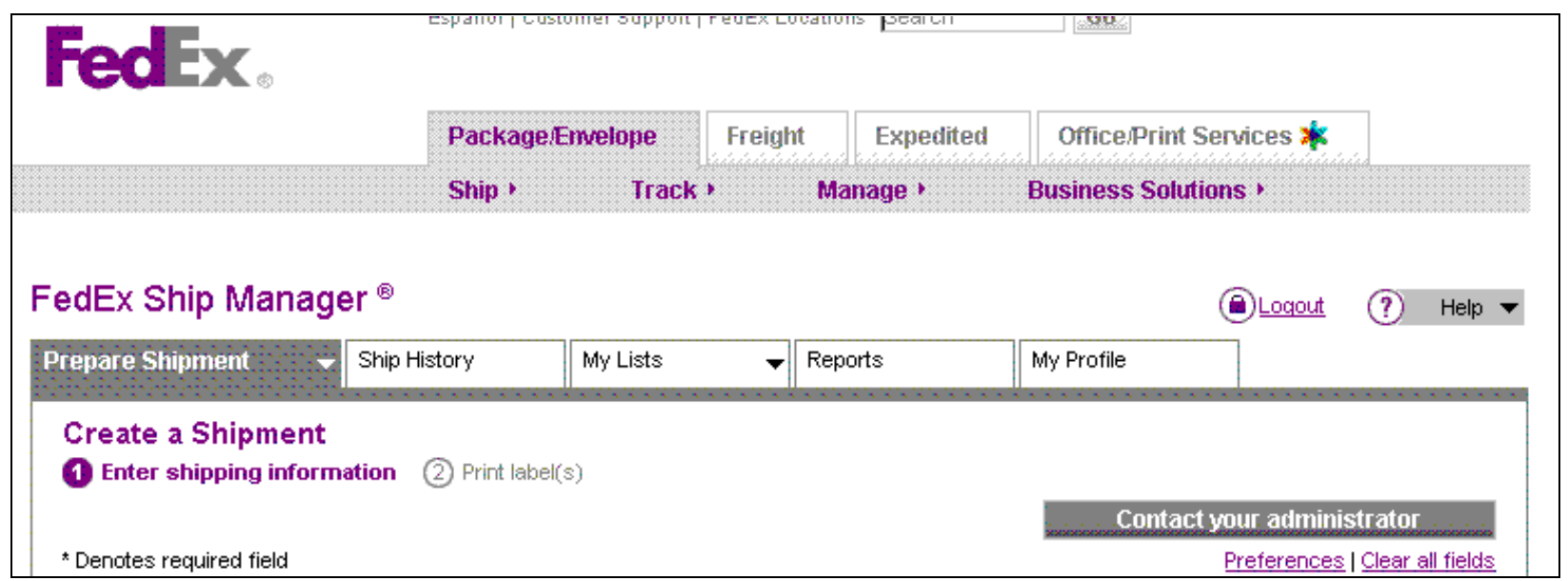

Prepare Shipment contains all the fields necessary to create an air bill.

Prepare Shipment
Create a Shipment
View Pending Shipments


Ship History includes filters to search for specific air bills by date or air bills for previous date ranges. Buttons on the bottom of the list allow the user to cancel, download, track, view details, and reprint selected air bills.

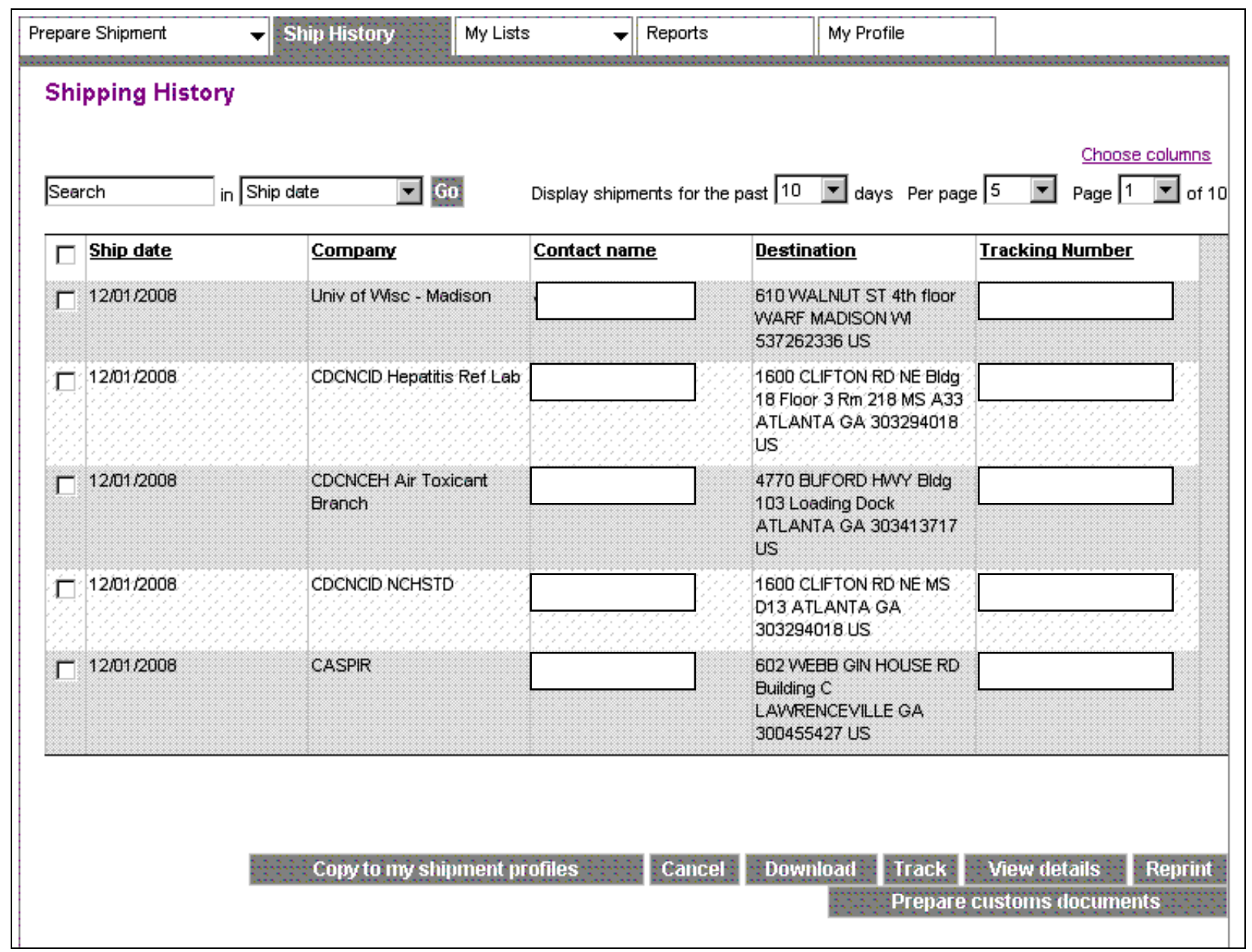

The bottom of the page provides buttons for End of Day Close and Print Manifest. Separate Track another shipment and Additional tracking options windows provide additional tracking options. 
Finished shipping FedEx Ground for the day?

\section{End of day close Print Manifest}

\section{Track another shipment}

Enter any combination of up to 30 FedEx tracking numbers (one per line). To track by reference number or RMA number, enter a reference number or an RMA number for shipments processed on your FedEx Ship Manager at fedex.com user ID.

Track by

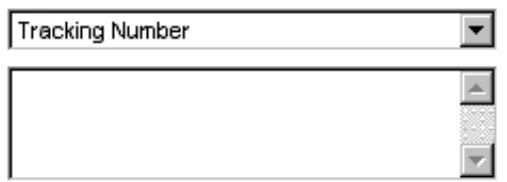

\section{Additional tracking options}

Start date 11/30/2008 (团

End date 11/30/2008 (团)

Track by recipient

Г Track exceptions only

Please Note :

Reprint function is not available for FedEx Ground shipments.

Do not use My Shipment Profiles when you want to use a special feature like Saturday Pickup, Saturday Delivery, Dangerous Goods, or Dry Ice. For a complete list, see the My Shipment Profiles Help section.

The Track another Shipment window allows the user to track shipments by Tracking Number, Your reference, Invoice number, P.O. number, Department number and RMA Number.

\section{Track another shipment}

Enter any combination of up to 30 FedEx tracking numbers (one per line). To track by reference number or RMA number, enter a reference number or an RMA number for shipments processed on your FedEx Ship Manager at fedex.com user ID.

Track by

Tracking Number

Tracking Number

Your reference

Invoice number

P.O. number

Department number

RMA Number 
The Additional tracking options window allows the user to track by start and end dates.

\begin{tabular}{|lll|}
\hline \multicolumn{2}{|l|}{ Additional tracking options } \\
\hline Start date & $11 / 30 / 2008$ & \\
End date & $11 / 30 / 2008$ & \\
& $\square$ Track by recipient & \\
& $\square$ Track exceptions only & \\
& & Track \\
\hline
\end{tabular}

The Access Address Book option is located at the bottom of the list under the Ship tab.

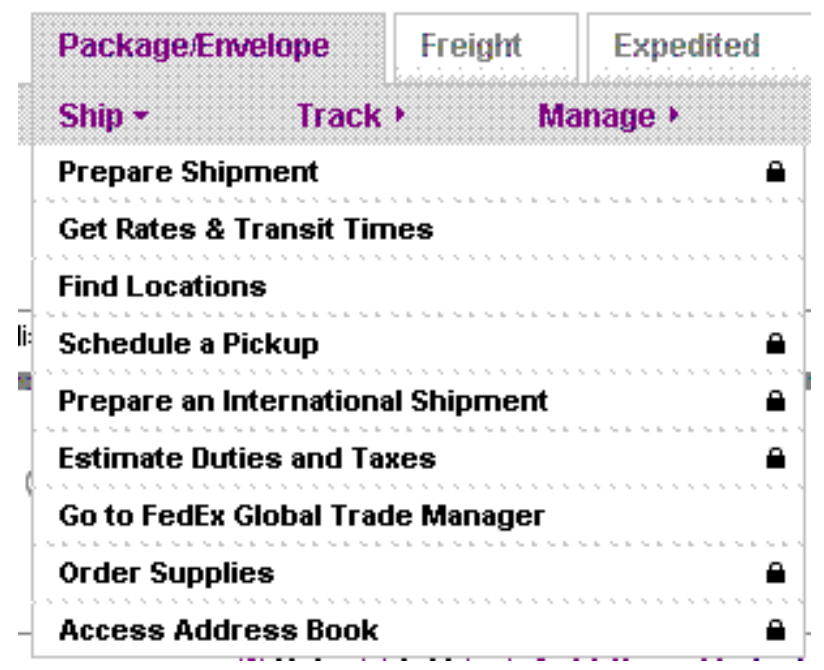

The Address Book is also under the My Lists tab.

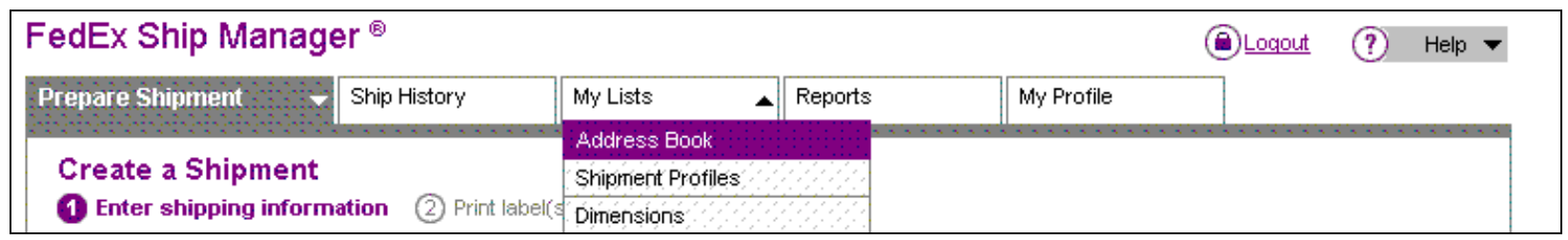


The Reports header provides access to the Report Manager page. Use this page to create and print reports for any shipment that has been processed using FedEx Ship Manager at fedex.com in the past 45 days.

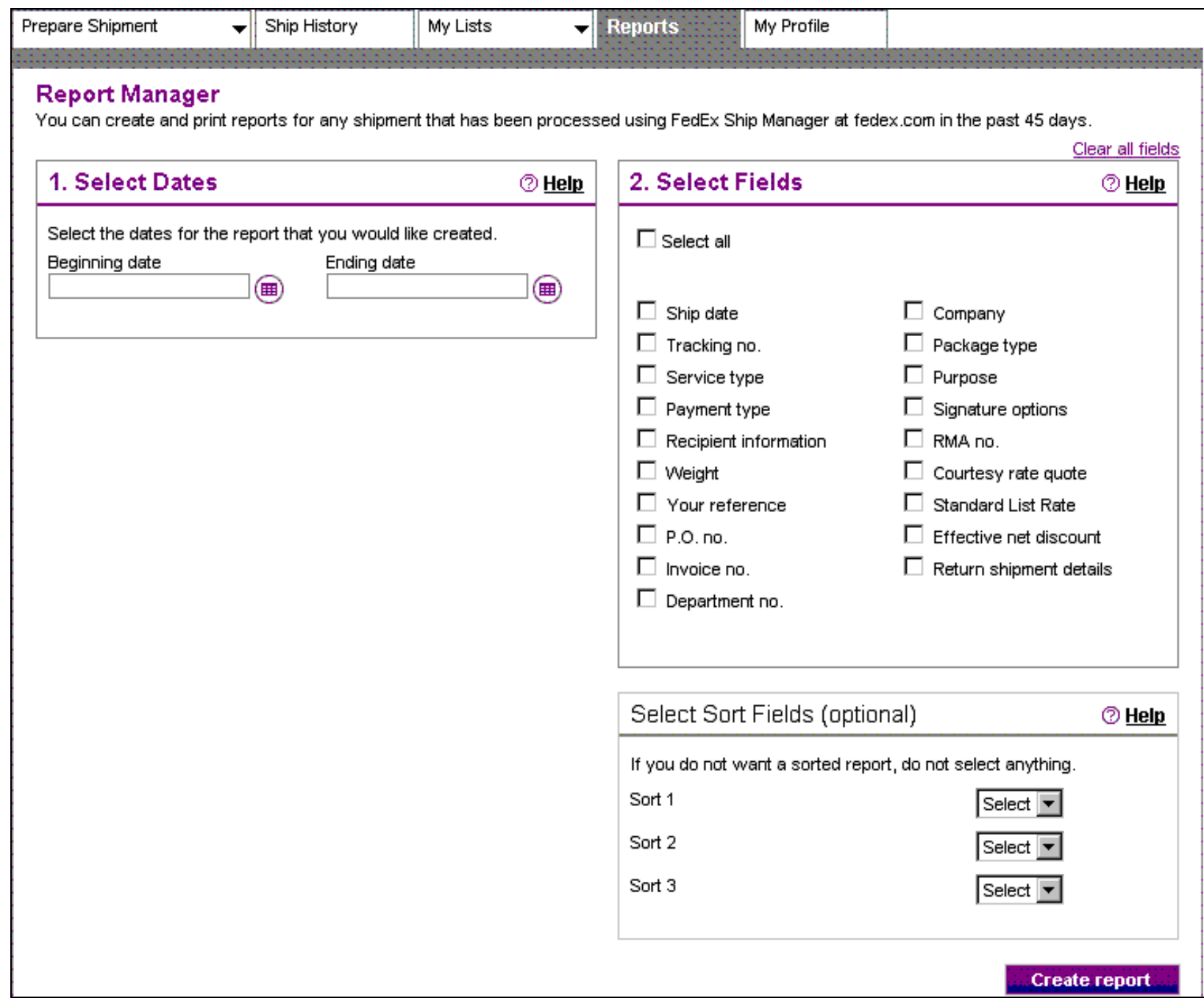


The My Profile header provides a summary of the services available to the user.

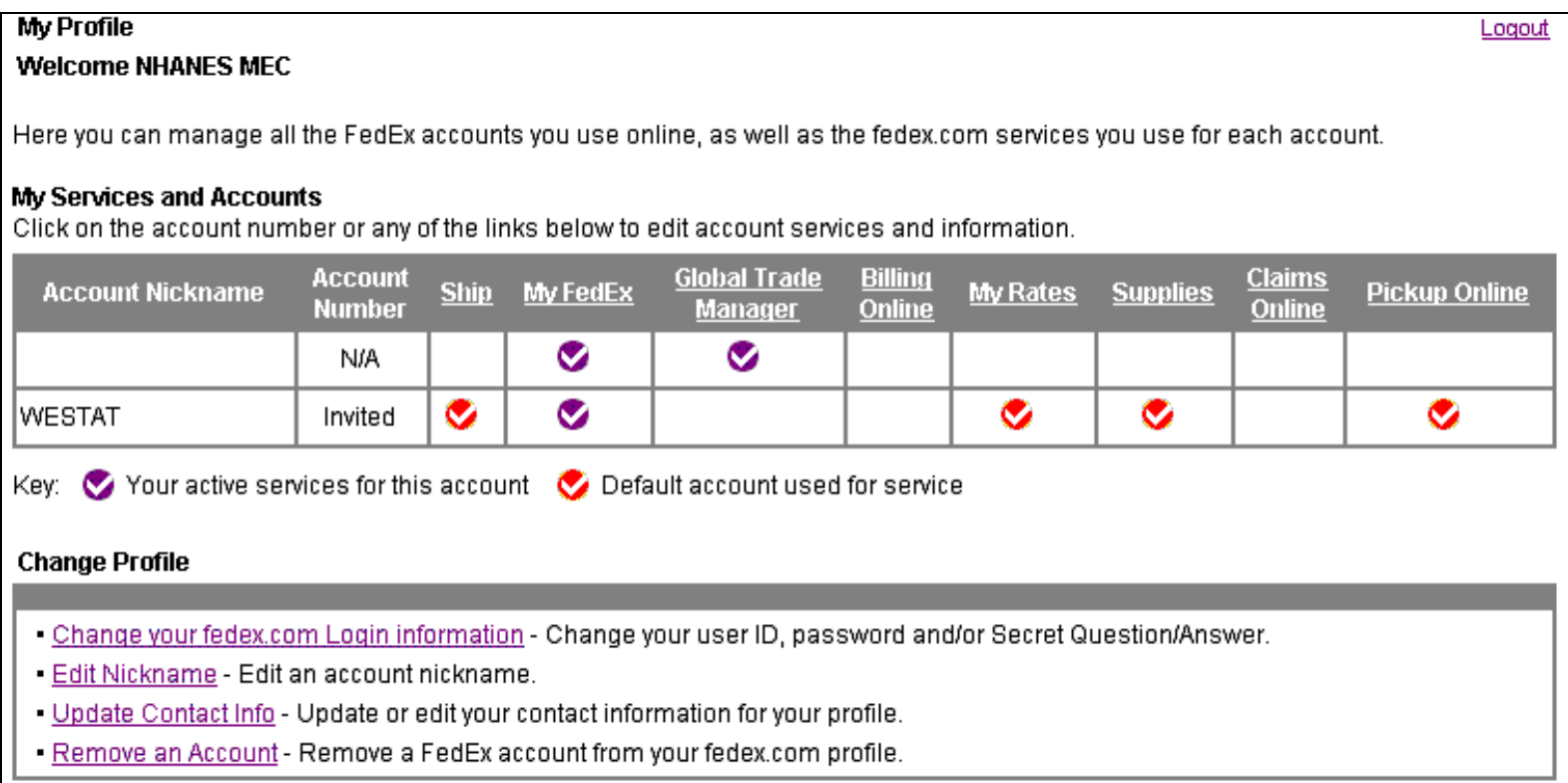


Select Prepare Shipment and then Create a Shipment to create a new air bill. FedEx displays the number of pages required to create an air bill under the Create a Shipment text in the window. The current page location is indicated by a full purple circle.

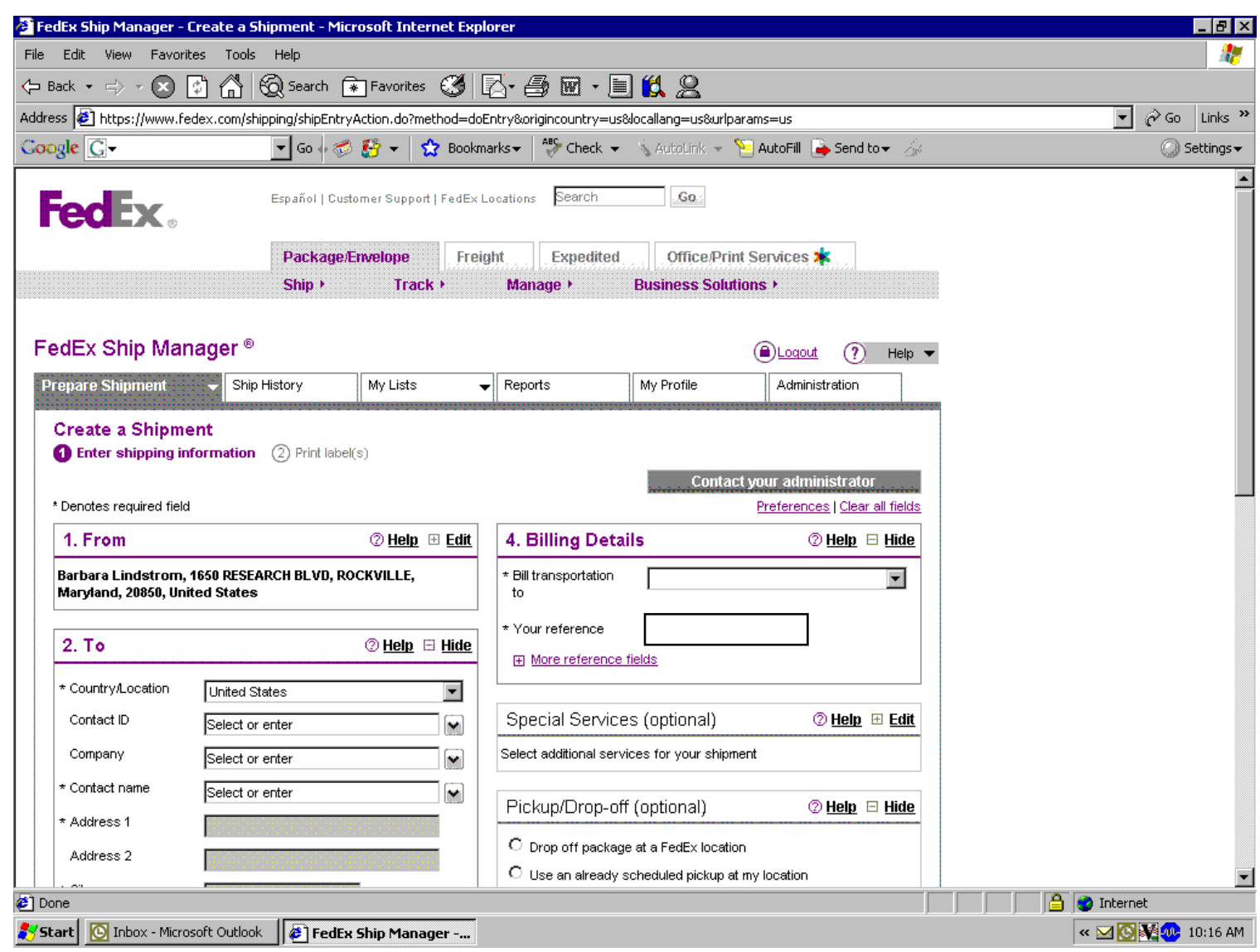


There are five boxes to complete on page one: From, To, Package \& Shipment Details, Billing Details, and Continue your Shipment.

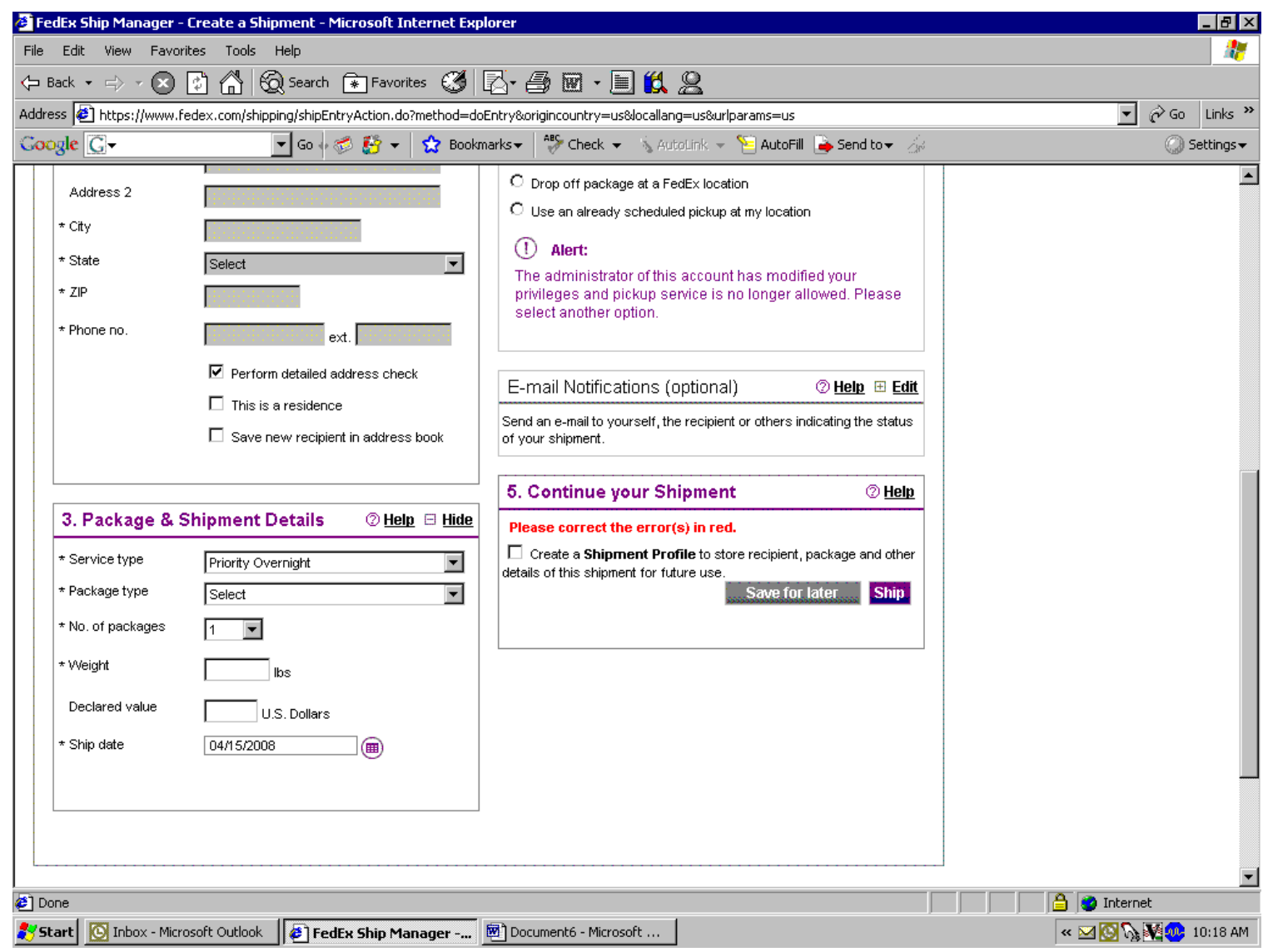

This page contains all of the information necessary to create an air bill. The recipient information is contained in the Address Book.

Note that items marked with an asterisk are required fields. 
Complete each of the five boxes.

1. From

\begin{tabular}{|l|l|}
\hline 1. From & (2) Help $\oplus$ Edit \\
\hline NHANES MEC, & \\
\hline & \\
\hline States & United \\
\hline
\end{tabular}

Select Edit to modify the information.

\begin{tabular}{|ll|}
\hline 1. From & (2) Help E Hide \\
\hline * Countryhocation & United States \\
Company & \\
* Contact name & NHANES MEC \\
* Address 1 & \\
Address 2 & \\
* City & \\
* State & \\
* ZIP & \\
* Phone no. & \\
& \\
\hline
\end{tabular}

To change this information on a one time basis, type the new information in the shaded text boxes. To make the change permanent, add a checkmark to the Save as my default address checkbox. Select the Hide link to go back to the previous screen. 
2. To

\begin{tabular}{|ll|}
\hline 2. To & \\
\hline * CountryLocation & United States \\
Contact ID & Select or enter \\
Company & Select or enter \\
* Contact name & Select or enter \\
* Address 1 & \\
Address 2 & \\
* City & \\
* State & \\
* ZIP & \\
* Phone no. & $\square$ \\
& $\square$ This is a residence \\
& $\square$ Save new recipient in address book \\
& \\
& $\square$ \\
& $\square$
\end{tabular}


Select the correct Contact ID from the drop-down list.

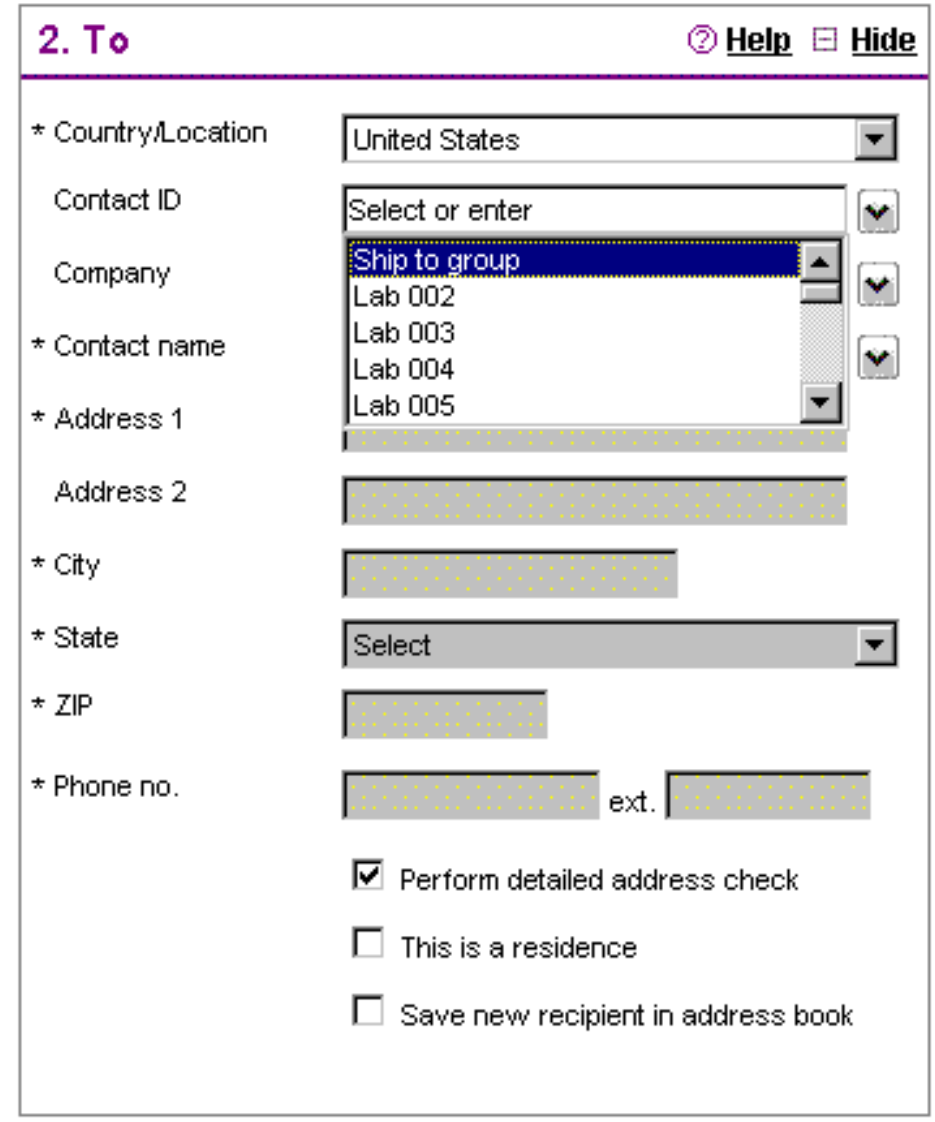


Once the contact ID has been selected, the shaded text boxes will fill in with the information contained in the address book.

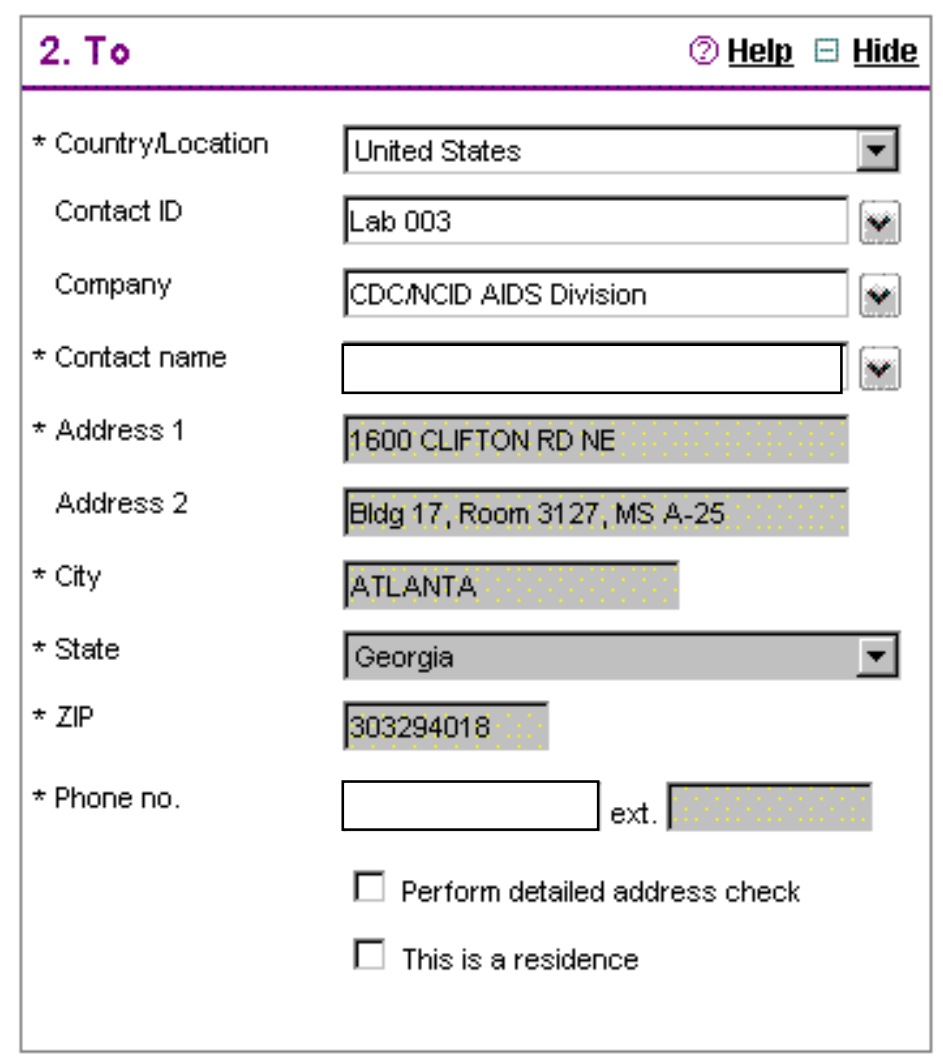

Review the information for accuracy. If it is incorrect, then contact the laboratory supervisor.

Do not place a check mark in the "Perform a detailed address check" or "This is a residence" check mark boxes. 
3. Package and Shipment Details

Review the default information contained in the Package and Shipment Details section of the air bill.

\begin{tabular}{|ll|}
\hline 3. Package \& Shipment Details & (3) Help \\
\hline * Service type & Hide \\
* Package type & Your Packaging \\
* No. of packages & 1 \\
* Weight & \\
Dirnensions & Select \\
Declared value & \\
* Ship date & $04 / 15 / 2008$ \\
& \\
\end{tabular}

Package and shipment details

- The Service type should default to Priority Overnight.

- The Package type should default to Your Packaging.

- The No. of packages should default to " 1 ."

- The Weight text box is blank. Enter the estimated weight of the package in the Estimated weight text box. To enter the weight, use the mouse to direct the mouse arrow to the weight text box and left click. Use the keyboard to enter the correct weight in pounds. Use whole numbers.

- Do not change the default (Select) option in the Dimensions text box.

- Do not enter a declared value in the Declared value text box.

- The Ship date will default to today's date. 


\section{Service type}

\begin{tabular}{|ll|}
\hline 3. Package \& Shipment Details & (2) Help $\square$ Hide \\
\hline * Service type & Priority Overnight \\
* Package type & Select \\
* No. of packages & Sriority Overnight \\
\hline Standard Overnight
\end{tabular}

The service type will default to Priority Overnight. The other option is Standard Overnight. Use Priority Overnight for all temperature dependent lab shipments.

\section{Package Type}

\begin{tabular}{|c|c|c|}
\hline \multicolumn{2}{|c|}{ 3. Package \& Shipment Details } & \multirow{2}{*}{$\frac{\text { (2) Help } \square \text { Hide }}{\nabla}$} \\
\hline * Service type & Priority Overnight & \\
\hline * Package type & Your Packaging & $\nabla$ \\
\hline $\begin{array}{l}\text { * No. of packages } \\
\text { * Weight }\end{array}$ & $\begin{array}{l}\text { Select } \\
\text { FedEx Envelope } \\
\text { FedEx Pak } \\
\text { FedEx Box } \\
\text { FedEx Tube }\end{array}$ & \\
\hline Dimensions & Your Packaging & 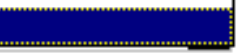 \\
\hline
\end{tabular}

The Package type will default to Your Packaging. Change the package type when necessary. 


\section{Ship Date}

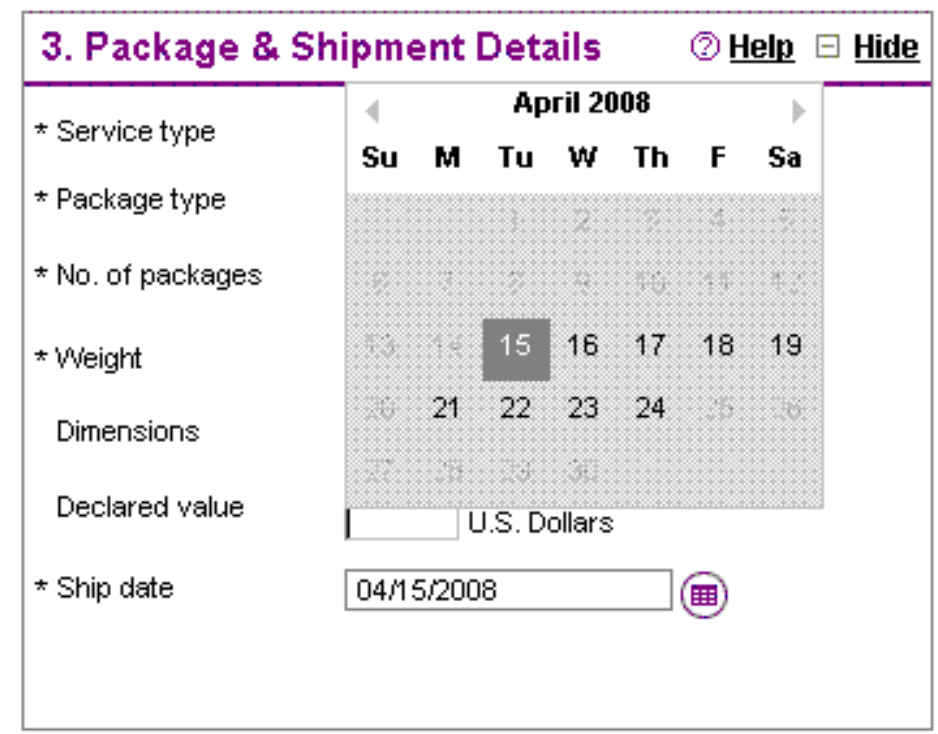

To change the date to tomorrow or any of the next 7 days, select the calendar icon to the right of the text box. Once selected, a calendar w1ll display. Use the cursor to highlight the correct date and left click. 
4. Billing Details

\begin{tabular}{|l|l|}
\hline 4. Billing Details & (2) Help $\square$ Hide \\
\hline * Bill transportation \\
to \\
* Your reference \\
MESTAT More reference fields \\
\hline
\end{tabular}

The Bill transportation to should default to WESTAT.

Your reference should default to the project number for the stand.

\section{Special Services}

\begin{tabular}{|l|}
\hline Special Services (optional) \\
\hline Select additional services for your shipment
\end{tabular}

To expand the Special Services window select the Edit link.

\begin{tabular}{|l|}
\hline Special Services (optional) \\
$\square$ Saturday delivery \\
$\square$ Hold at FedEx location $\boxplus$ \\
$\square$ Dry ice $⿴$ \\
$\square$ Dangerous goods $\boxplus$ \\
\hline FedEx@ Delivery Signature Options \\
Signature type Select \\
\hline
\end{tabular}

The Special Services window includes check boxes for Saturday delivery, Hold at FedEx location, Dry ice, and Dangerous goods. Use the separate Signature type drop-down list under the 
FedEx Delivery Signature Options to designate the type of signature. Do not use the Saturday delivery, Hold at FedEx location or Dangerous goods options.

Dry ice

Select the plus icon to the right of Dry ice” check box to access the data entry text box.

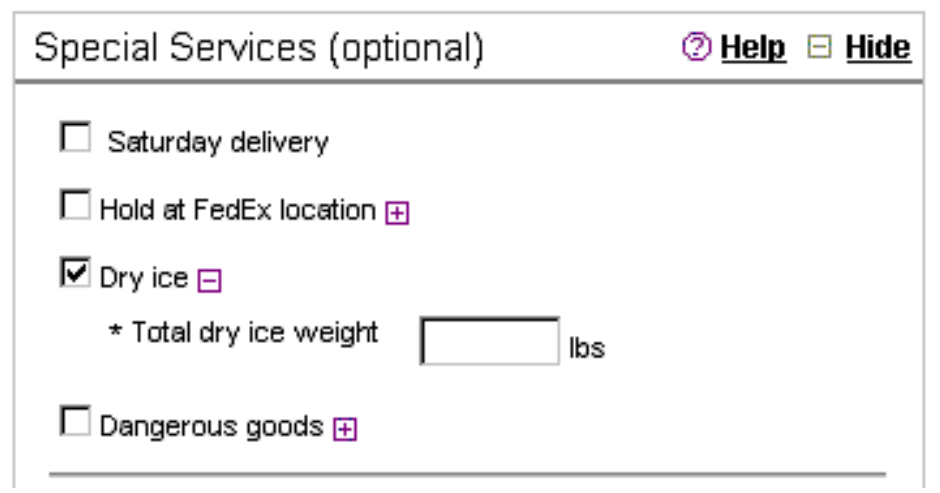

Enter weight of the dry ice, use the mouse to go to the text box and left click. Type the weight, in pounds using the keyboard keys. Use whole numbers.

\section{Signature type}

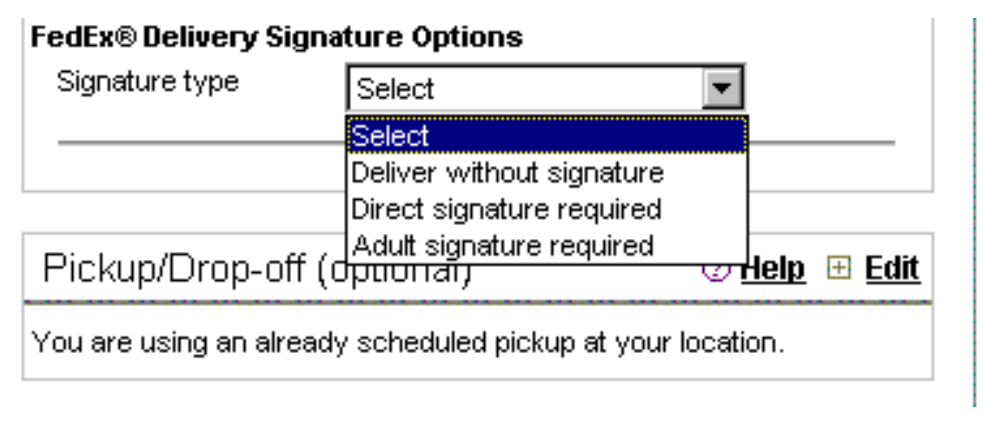

Access the drop-down list to select the Direct signature required option. 


\section{Pickup/Drop-off}

\begin{tabular}{|l|}
\hline Pickup/Drop-off (optional) \\
\hline You are using an already scheduled pickup at your location. \\
\hline
\end{tabular}

The Pickup/Drop-off option will default to "You are using an already scheduled pickup at your location.”

To expand the Pickup/Drop-off window select the $\underline{\text { Edit link. }}$

\begin{tabular}{|l|}
\hline Pickup/Drop-off (optional) \\
\hline Drop off package at a FedEx location \\
$\odot$ Use an already scheduled pickup at my location \\
\hline
\end{tabular}

The second option is "Drop off package at a FedEx location." Change the option as necessary.

\section{E-mail Notifications}

E-mail Notifications (optional)
$\begin{aligned} & \text { Send an e-mail to yourself, the recipient or others indicating the status } \\ & \text { of your shipment. }\end{aligned}$

This option is not required. 
5. Continue your Shipment

\begin{tabular}{|l|}
\hline 5. Continue your Shipment \\
\hline Create a Shipment Profile to store recipient, package and other \\
details of this shipment for future use. Ship \\
\hline
\end{tabular}

It is possible to perform two actions. If a check mark is placed in the check box, the "Save for later" button disappears. If the "Save for later" button is selected, a "Pending shipments" window displays.

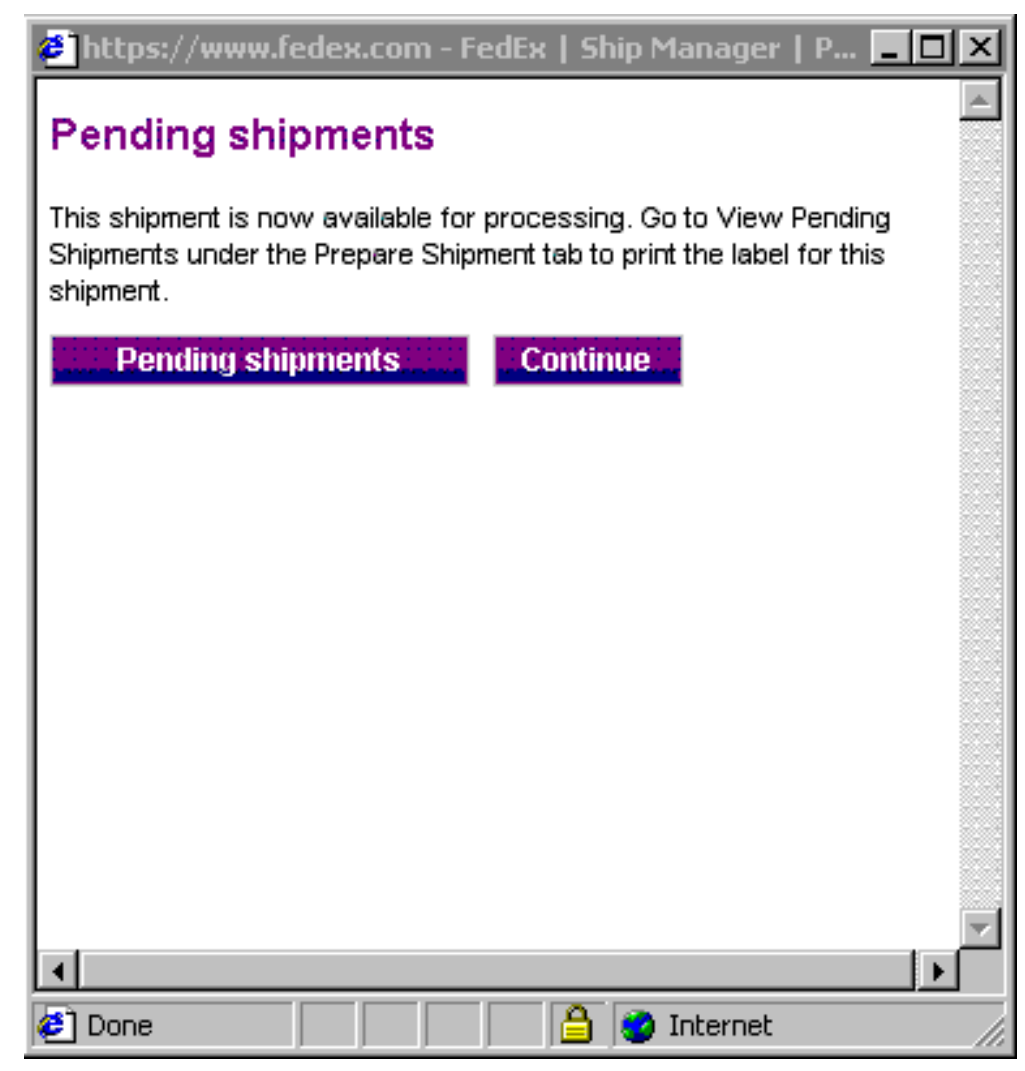

Follow the instruction in the window to continue. 
Select the Ship button to go to the next screen.

The Print Label(s) window displays.

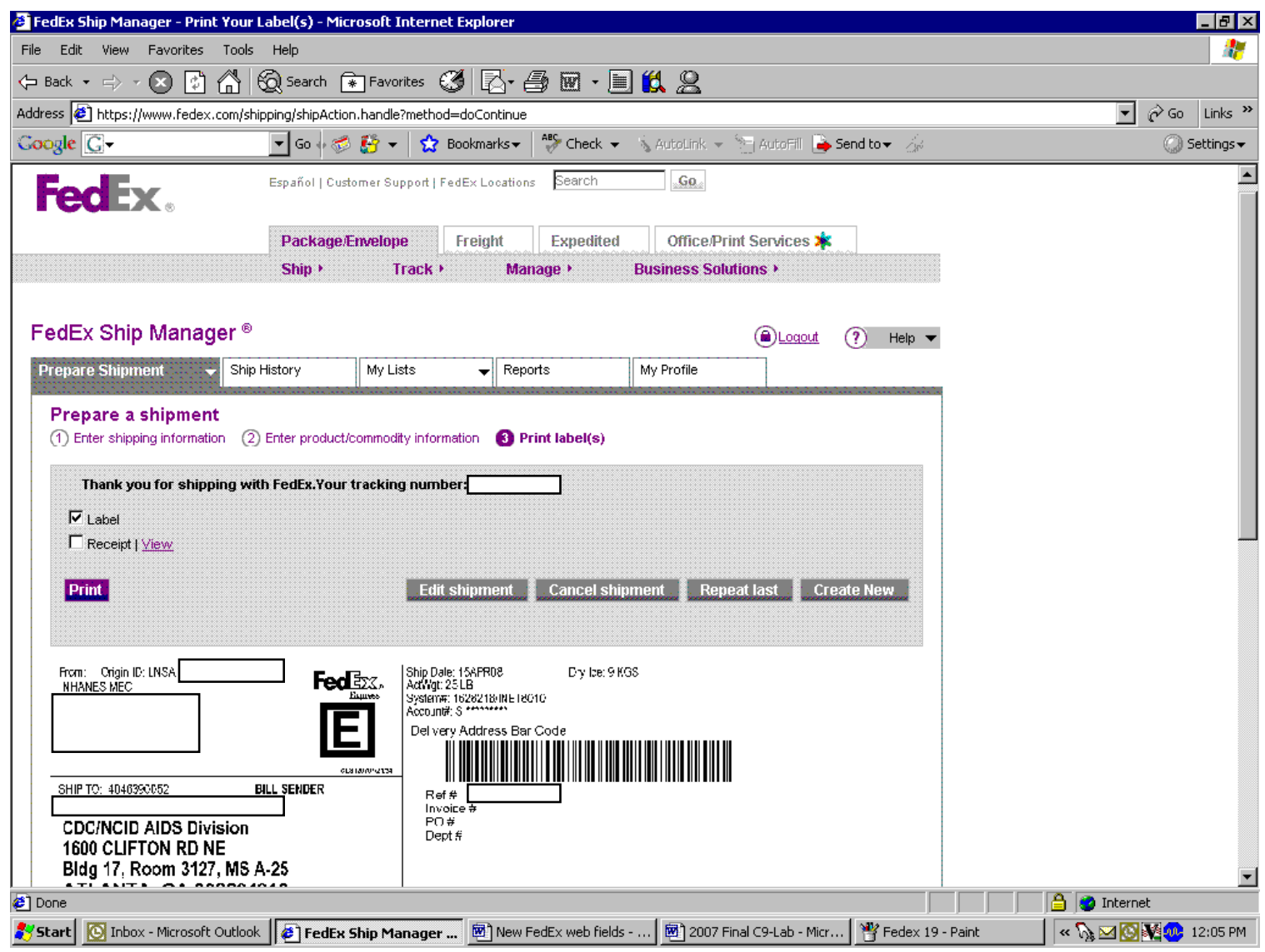

The top section of the window includes two check boxes and four buttons.

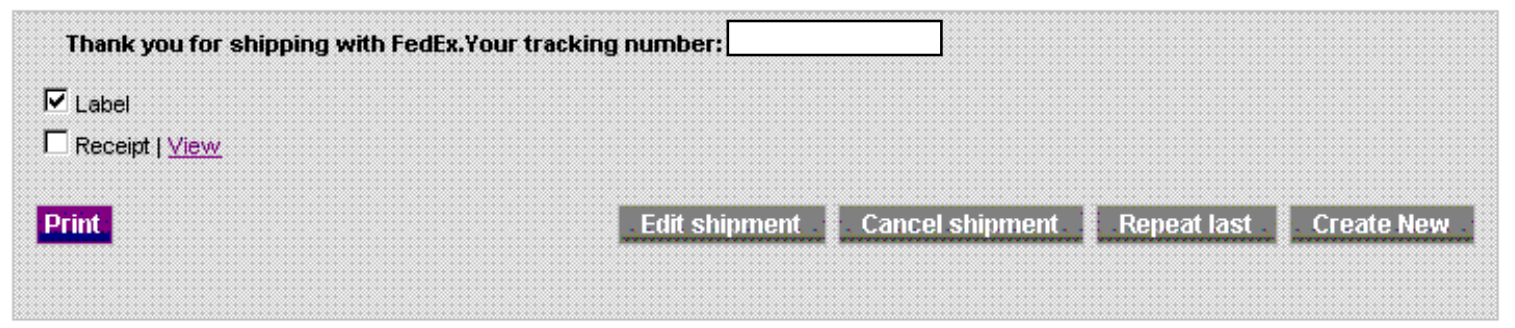

The two check boxes are Label and Receipt. The Label check box will default to checked.

Do not add a check mark to the Receipt check box.

To print the air bill, select the Print button. 
Select one of the four buttons to proceed. The choices are as follows:

- Edit shipment - Use this option to go back to the Create a Shipment window.

- Cancel shipment - Use this option to cancel the shipment. Alert: Are you sure you want to cancel the shipment? Click Yes to cancel the shipment. Select No to remove the text.

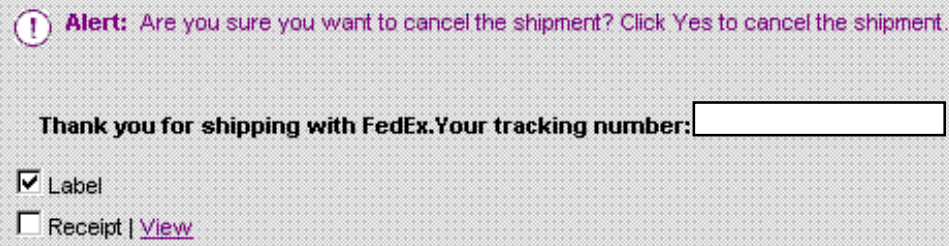

- Repeat last - Moves back to the Create a Shipment window. All previously entered data are saved and displayed.

- Create new - Moves back to the Create a Shipment window. All fields are blank or set with the standard defaults. 
The bottom half of the window contains instructions to complete the shipment.

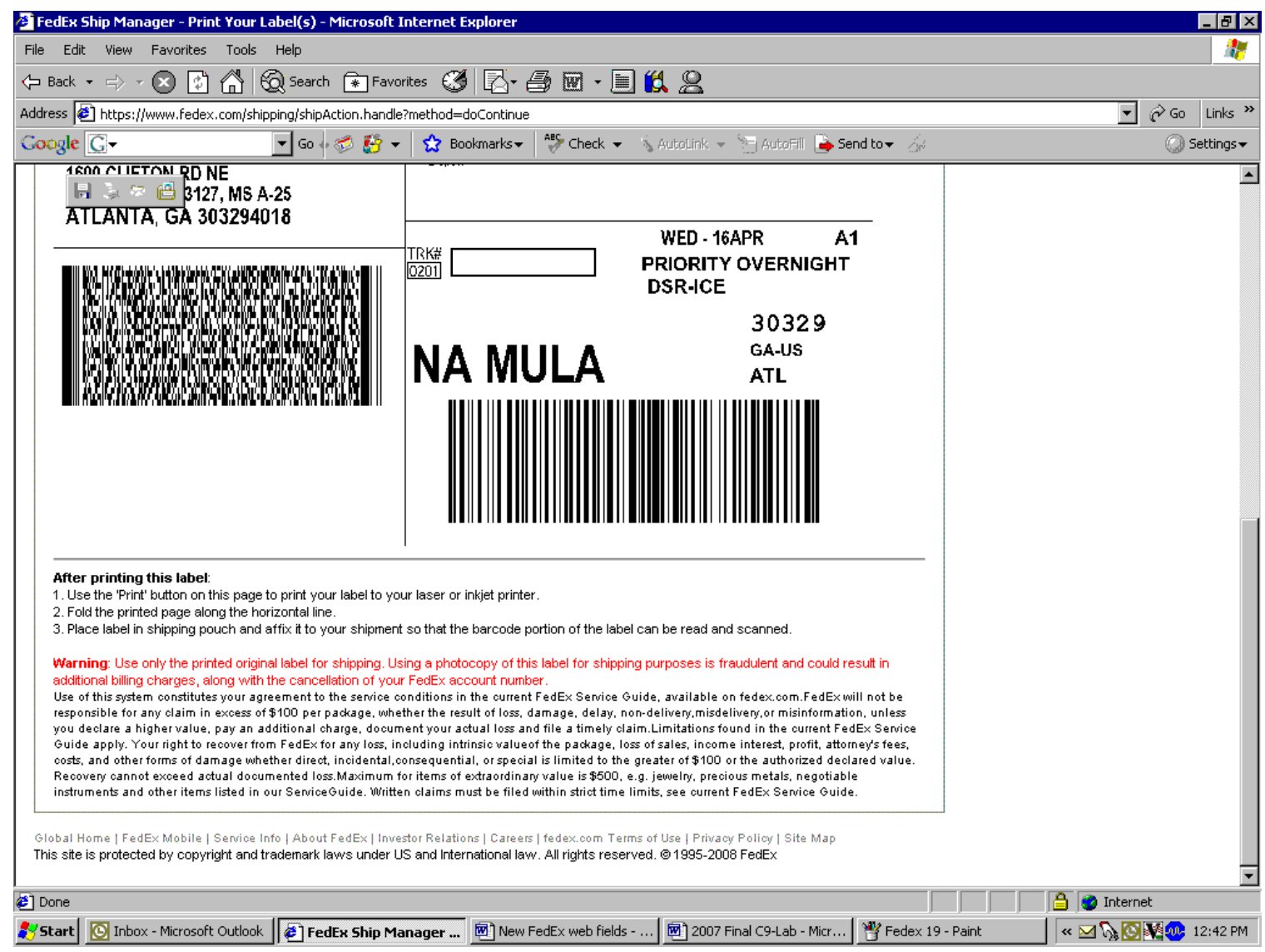

After printing this label:

1. Use the "Print" button on this page to print your label to your laser or inkjet printer.

2. Fold the printed page along the horizontal line.

3. Place label in shipping pouch and affix it to your shipment so that the bar-code portion of the label can be read and scanned. 
The Logout link is located in the upper right hand corner of the main window. Select the Logout link to exit the application. Select the Contract your administrator button to send an email to the home office component specialist.

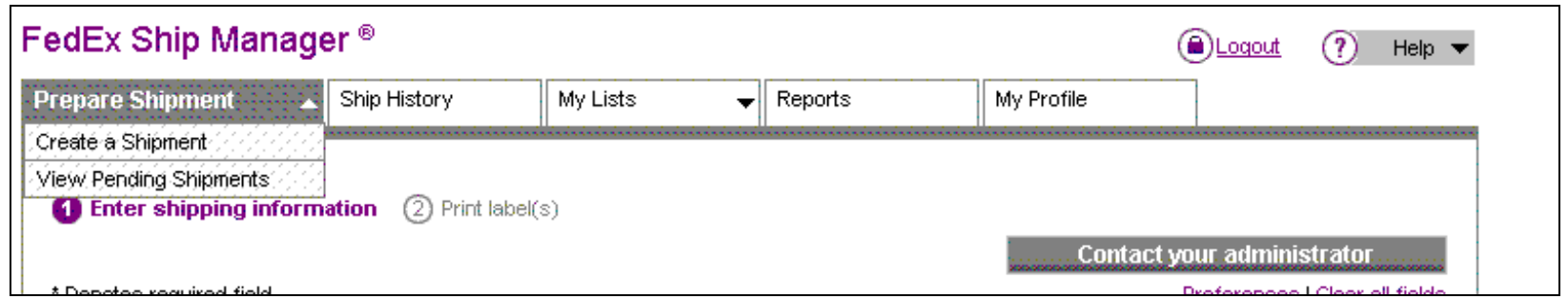

9.9

Close Containers

After a container is full, it is automatically closed. All open containers must be virtually and manually closed before shipping.

Access the $\{$ Close Containers $\}$ module.

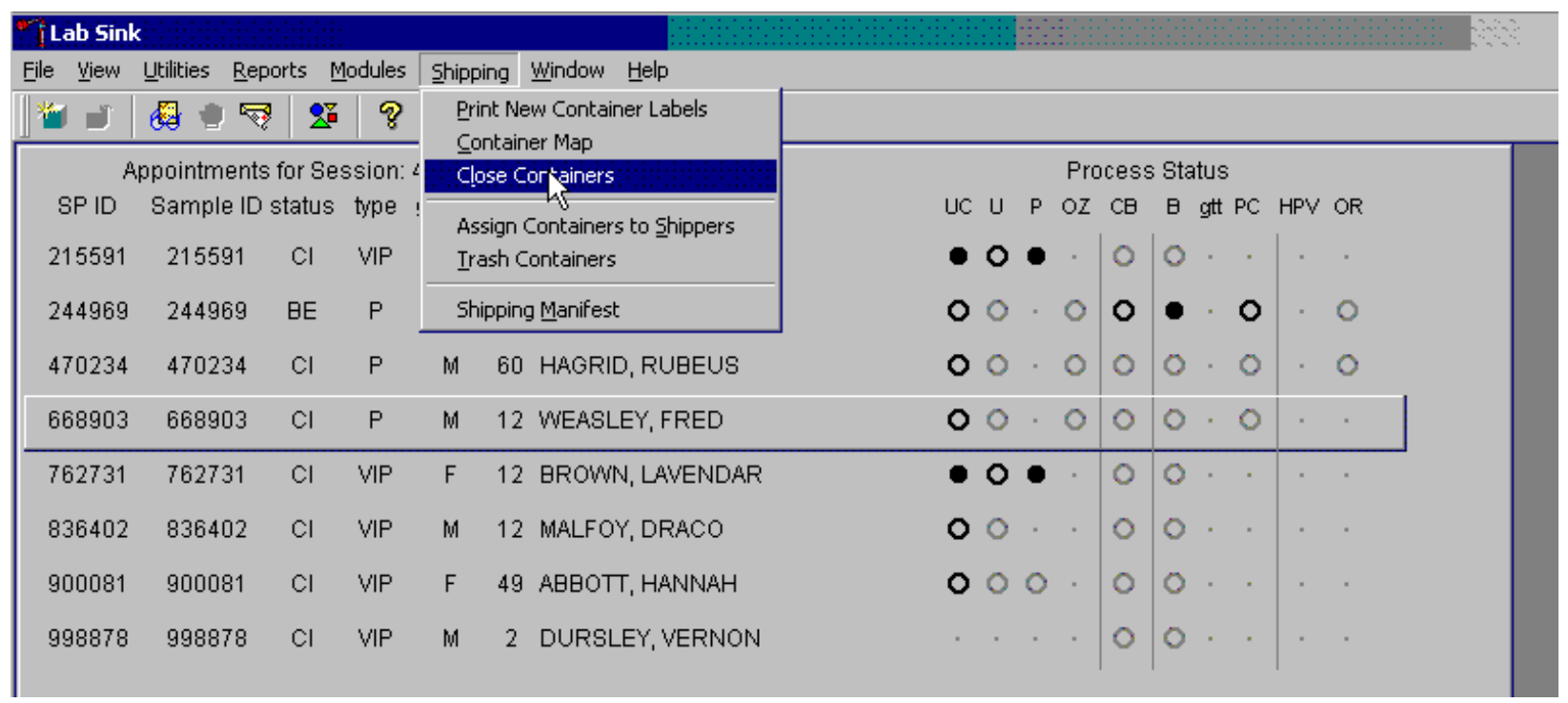

To access the \{Close Containers \}odule, use the mouse to direct the mouse arrow to \{Shipping\}, left click, drag the mouse arrow to \{Close Containers\}, and left click, or type [Alt] [S/s], $[\mathrm{L} / \mathrm{l}]$. 
Individually close open containers or collectively close all open containers.

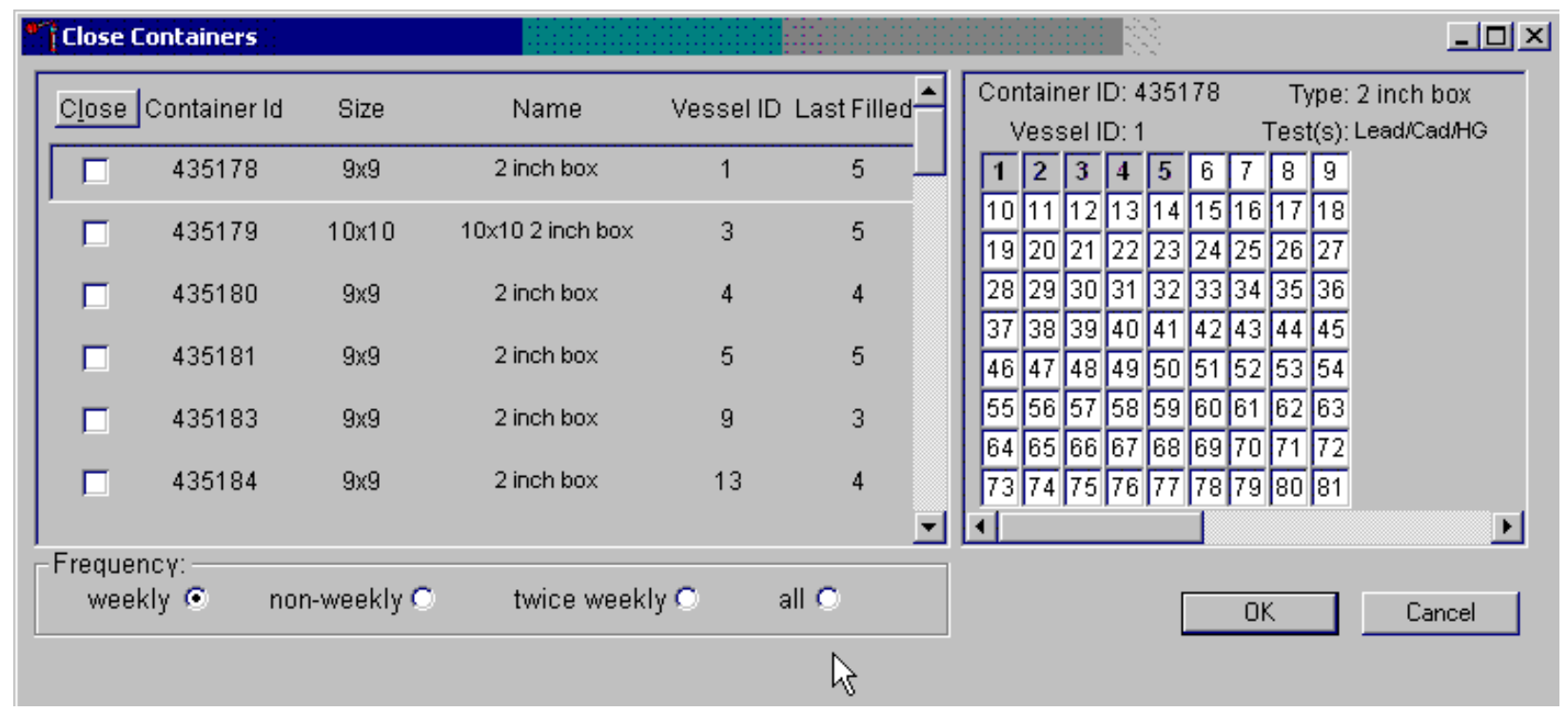

The left side of the Close Containers screen includes a check box for each open container and columns for the Container ID, Size, Name, Vessel ID, and number of the Last Filled slot. The container line in the rectangular box is the active container. The right side of the screen displays the corresponding container map for the active container. Containers displayed in red indicate containers that can be shipped at the end of the stand or when they are full. The bottom of the screen includes three filters: weekly, non-weekly, and both.

To move up and down the list, use the up and down keyboard arrows or use the mouse to direct the mouse arrow to any individual line. Use the vertical scroll bar to view all containers. To sort the lines by Container ID, Size, Name, Vessel ID or Last Filled, use the mouse to direct the mouse arrow to the column title and left click. The window will update and display the rows in the desired priority. 
Use the filters to display containers that are shipped weekly, non-weekly, or both.

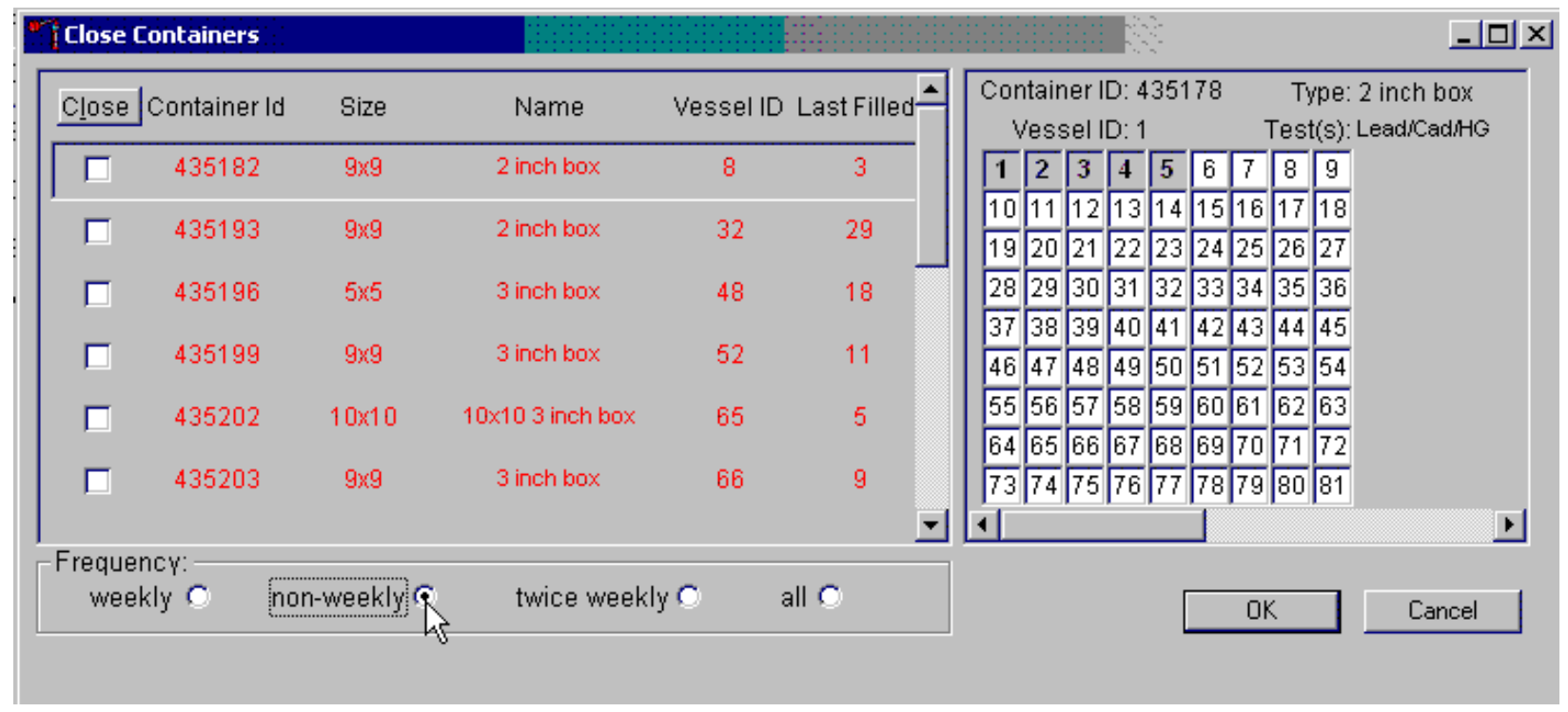

To select one of the four filters, use the mouse to direct the mouse arrow to the correct radio button and left click. The window updates to display those containers that meet the filter criteria. 
Individually identify containers or select all containers.

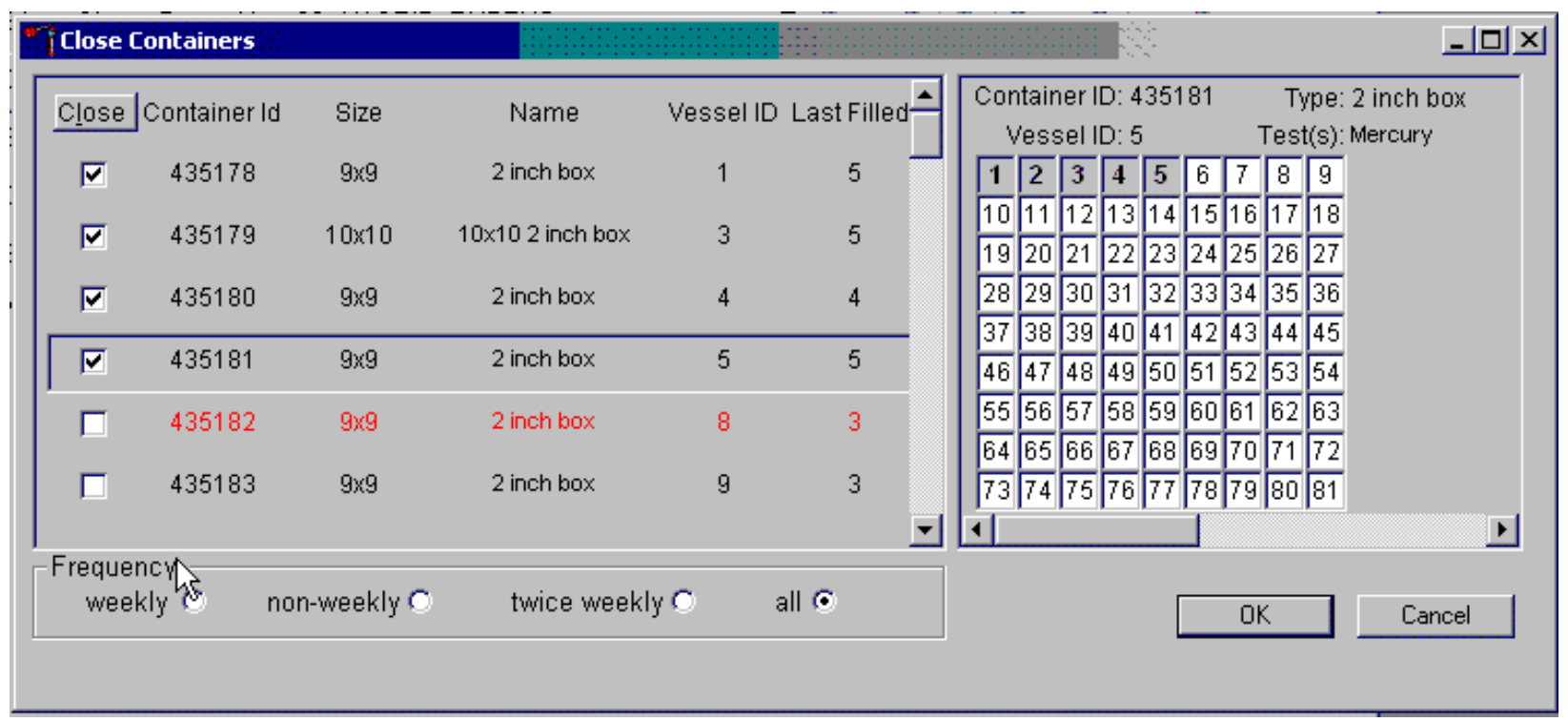

To mark individual containers, use the mouse to direct the mouse arrow to individual “Close” button check boxes and left click. To close all open containers, use the mouse to direct the mouse arrow to the "Close" button and left click. To remove the check marks, use the mouse to direct the mouse arrow to the "Close" button and left click a second time.

Verify the contents of each closed box by visually matching the contents of the box to the map. To record these actions, use the mouse to direct the mouse arrow to the "OK" button and left click, or select [Enter]. To exit the \{Close Containers\} module without recording these actions, use the mouse to direct the mouse arrow to the "Cancel” button and left click. 


\subsection{0}

\section{Assign Containers to Shippers}

Assign closed containers to or associate closed containers with a specific air bill. To associate a specific closed container to the air bill for that laboratory, use the Assign Container to Shippers\} module.

Access the $\{$ Assign Containers to Shippers $\}$ module.

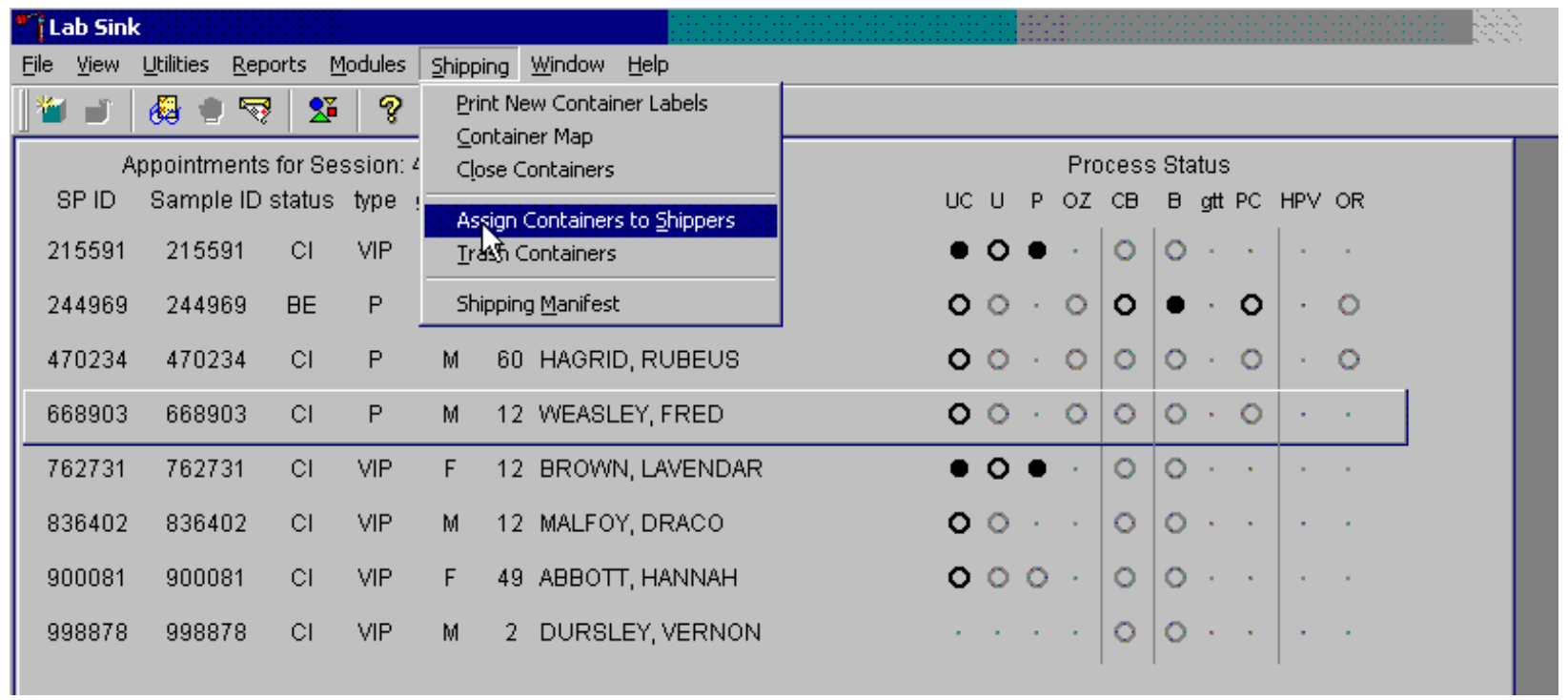

To access the \{Assign Containers to Shippers \} module, use the mouse to direct the mouse arrow to \{Shipping\} in the top menu bar, left click, drag the mouse arrow to A Assign Container to Shippers $\}$, and left click or type [Alt] [S/s], [S/s]. 
The Assign Containers to Shippers window displays.

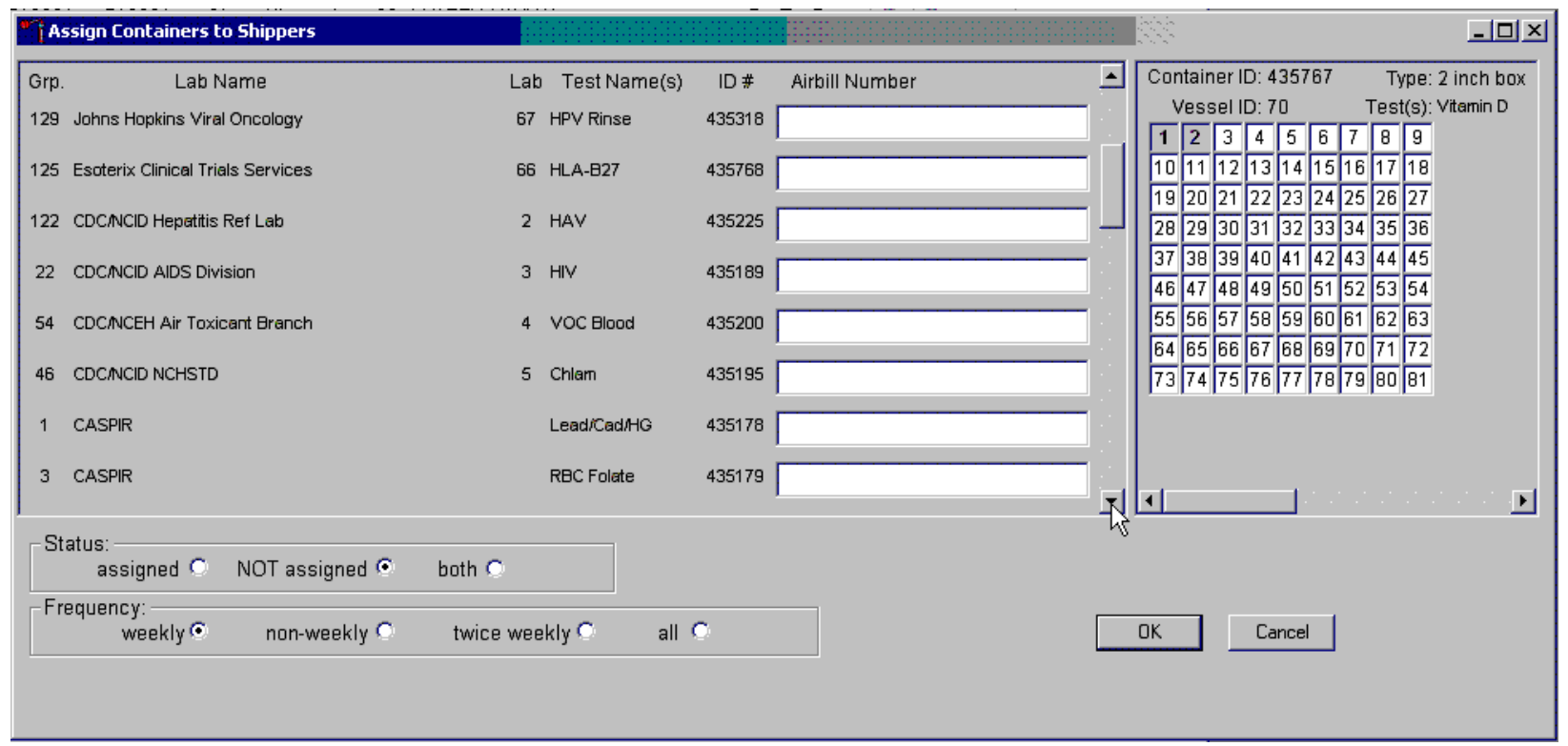

This left side of the screen includes columns for a group designation (which represents a number), Type of container, Lab designation, Test Name(s), Container ID number, and Air bill Number. The container line within the rectangular box is the active container. The right side of the screen displays the corresponding container map for the active container. The left side of the bottom of the window contains three "Status” filters (radio buttons): "assigned," "NOT assigned,” and "both.” The right side of the bottom of the window contains three "Frequency" filters (radio buttons): "weekly," "non-weekly," "twice weekly," and "all." Use the column titles to sort the rows. To sort the rows, use the mouse to direct the mouse arrow to the desired column title and left click. The window updates based on the selection. 
Enter an air bill number into each Air bill Number text box. There are three ways to enter the air bill number.

- Use the bar-code gun to scan the bar code from the FedEx Ship Manager air bill. The bar code is located in the bottom of the top half of the folded air bill. It is the tallest bar code on the air bill. Hold the gun in the right hand, squeeze the trigger, and move the gun up and down to adjust the laser light so that it covers the width of the bar code. Once the gun successfully scans the bar code, an audible beep is emitted.

- Use the bar-code gun to scan the bar code on the second page (Recipient's Copy) of the two-part Form ID 0215. This bar code is located on the lower left-hand side toward the bottom of the form. Hold the gun in the right hand, squeeze the trigger, and move the gun up and down to adjust the laser light so that it covers the width of the bar code. Once the gun successfully scans the bar code, an audible beep is emitted.

- Use the keyboard keys to type the air bill number from either the FedEx Ship Manager air bill or the FedEx air bill form 0215.

To assign a container to the corresponding air bill:

- $\quad$ Set the Status filter to: "NOT assigned";

- Set the Frequency to: weekly, non-weekly, or both, depending on the shipping situation; and

- Sort the laboratories by Lab number. 
Set the Status and Frequency filters.

\begin{tabular}{|c|c|c|c|c|c|c|c|}
\hline Assign Containers to Shippers & & & & & & & $-|\square| x$ \\
\hline Lab Name & Lab & Test Name(s) & ID\# & Airbill Number & $\Delta$ & Container ID: 435767 & Type: 2 inch box \\
\hline 129 Johns Hopkins Viral Oncology & 67 & HPV Rinse & 435318 & & & $\begin{array}{l}\text { Vessel ID: } 70 \\
\begin{array}{|l|l|l|l|l|l|l|}\mathbf{1} & 2 & 3 & 4 & 5 & 6 & 7\end{array}\end{array}$ & $\begin{array}{l}\text { Test(s): Vitamin D } \\
8\end{array}$ \\
\hline 125 Esoterix Clinical Trials Services & 66 & HLA-B27 & 435768 & & & 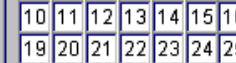 & \begin{tabular}{l|l|l|}
6 & 17 & 18 \\
26 & 27
\end{tabular} \\
\hline 122 CDCNCID Hepattitis Ref Lab & 2 & HAV & 435225 & & - & $\begin{array}{llllllll}28 & 29 & 30 & 31 & 32 & 33 & 3\end{array}$ & $4 \sqrt{35} 36$ \\
\hline 22 CDCNCID AIDS Division & 3 & HIV & 435189 & & & \begin{tabular}{|l|l|l|l|l|l|l|l|l|l|}
37 & 38 & 39 & 41 & 42 & 4 \\
46 & 47 & 48 & 49 & 50 & 51 & 5 \\
\end{tabular} & \begin{tabular}{l|l|l|}
3 & 44 & 45 \\
2 & 53 & 54
\end{tabular} \\
\hline 54 CDCNCEH Air Toxicant Eranch & 4 & VOC Elood & 435200 & & & \begin{tabular}{|l|l|l|l|l|l|l|}
55 & 56 & 57 & 58 & 59 & 60 & 6 \\
64 & 65 & 66 & 67 & 68 & 69 & 7 \\
\end{tabular} & \begin{tabular}{l|l|l|}
1 & 62 & 63 \\
0 & 71 & 72
\end{tabular} \\
\hline 46 CDCNCID NCHSTD & 5 & Chlam & 435195 & & & \begin{tabular}{|l|l|l|l|l|l|l|}
73 & 74 & 75 & 76 & 77 & 78 & 7 \\
\end{tabular} & \begin{tabular}{l|l|l}
9 & 80 & 81 \\
\end{tabular} \\
\hline 1 CASPIR & & Lead/CadMG & 435178 & & & & \\
\hline 3 CASPIR & & RBC Folate & 435179 & & & 11 & $\perp$ \\
\hline Status: - assigned $C$ NOT assigned 6 & both $C$ & & & & & & \\
\hline $\begin{array}{l}\text { Frequency: } \\
\text { weekly } \odot\end{array}$ & twice weel & $\mathrm{kly} C$ & & & & Cancel & \\
\hline
\end{tabular}

To select the Status filter, use the mouse to direct the mouse arrow to the "assigned," "NOT assigned," or "both,” radio button and left click. To select the shipping Frequency, use the mouse to direct the mouse arrow to weekly, non-weekly, twice weekly, or all, and left click. 
Sort the rows by Lab Name.

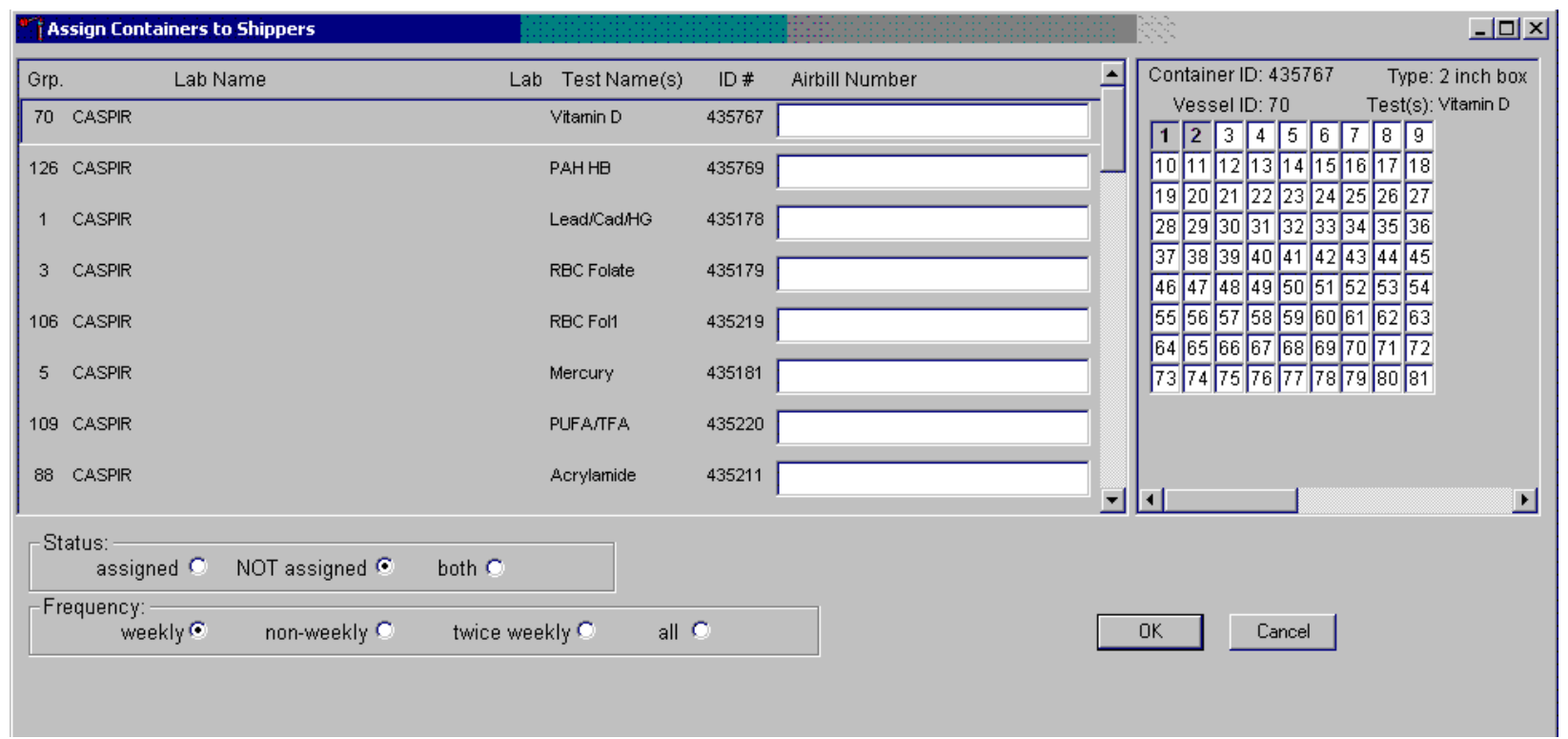

To sort the rows by lab Name, use the mouse to direct the mouse arrow to the top header "Lab Name” and left click. All containers associated with a specific lab will be grouped together in ascending order. 
Enter the air bill number into the Airbill Number text box.

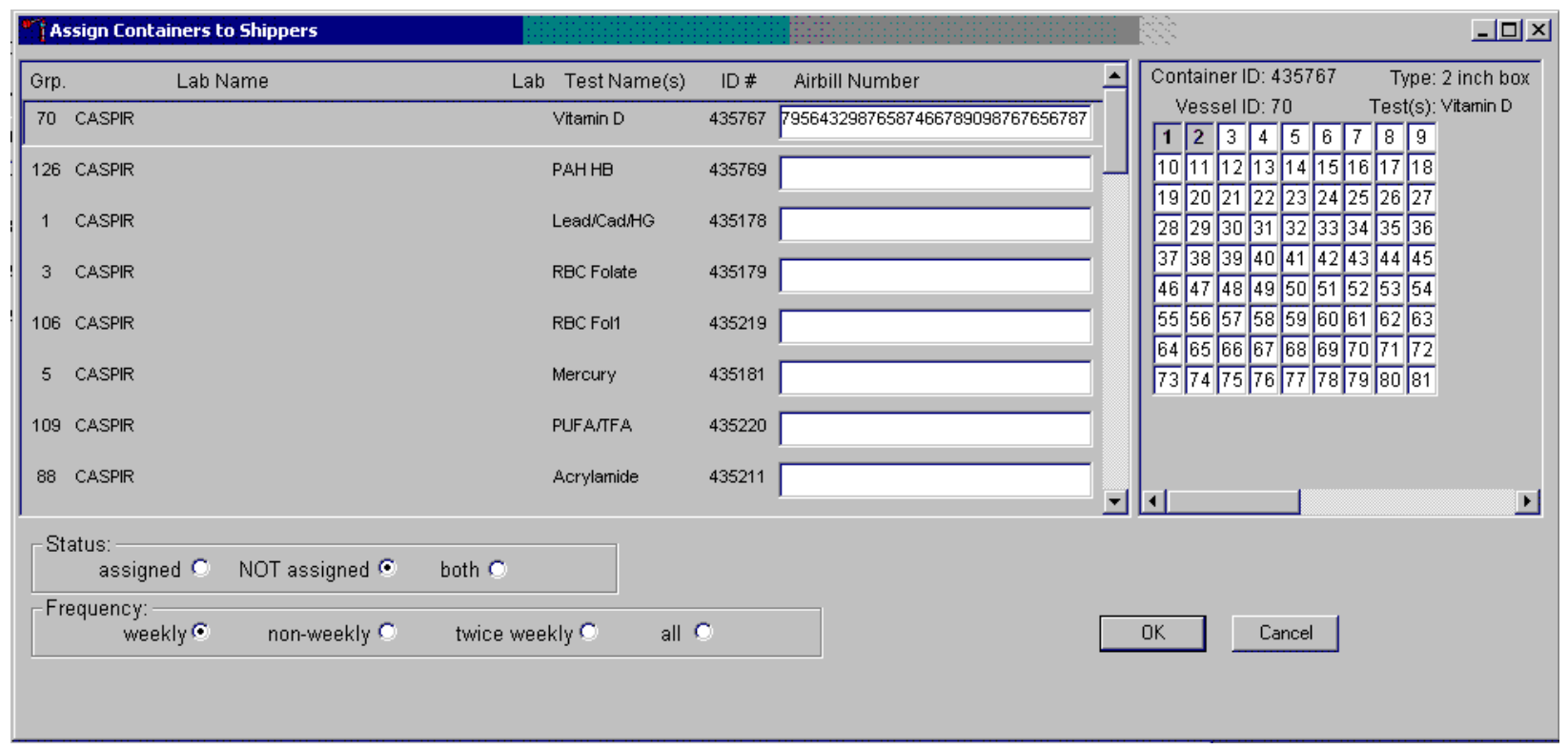

Place the cursor in the first Airbill Number text box. Use the bar-code gun to scan the air bill number. The scanned number contains 20 extra digits. The actual 12-digit air bill number is embedded in the 32-digit scanned number. Select the [Tab] key to move to the next text box. Do not select the [Tab] key until the application has been allowed to enter the entire number into the text box. Once the [Tab] key is selected, the program edits the first air bill number to delete the extra numbers. 
Continue the assignment process.

\begin{tabular}{|c|c|c|c|c|c|}
\hline Assign Containers to Shippers & & & & & $-|\square| x$ \\
\hline Grp. Lab Name & Lab TestName(s) & ID \# Airbill Number & $\Delta$ & $\begin{array}{l}\text { Container ID: } 435187 \\
\text { Vessel } 18\end{array}$ & $\begin{array}{l}\text { Type: } 2 \text { inch box } \\
\text { Test's) Biochem }\end{array}$ \\
\hline 22 CDCNCID AIDS Division & $3 \mathrm{HIV}$ & $4 3 5 1 8 9 \longdiv { 7 9 6 1 7 0 3 8 9 7 9 6 }$ & & \begin{tabular}{|c|c|c|c|c|} 
& 3 & 5 & 6 \\
\end{tabular} & $\begin{array}{l}\text { Test(s): Blochem } \\
789\end{array}$ \\
\hline 16 CDCNCID DASH & 64 MMRV & $4 3 5 7 6 6 \longdiv { 7 9 6 1 7 0 3 8 9 7 5 4 }$ & & \begin{tabular}{|l|l|l|l|l|l|l|l|}
10 & 11 & 12 & 13 & 14 & 15 \\
19 & 20 & 21 & 22 & 23 & 24 \\
\end{tabular} & \begin{tabular}{l|l|l|}
16 & 17 & 18 \\
25 & 26 & 27
\end{tabular} \\
\hline 14 CDCNCID Hepattis Ref Lab & 2 Hep Profile & $4 3 5 1 8 5 \longdiv { 7 9 6 1 7 0 3 8 8 7 6 5 }$ & & $28 \quad 29 \quad 30 \quad 32 \quad 33$ & 343536 \\
\hline 122 CDCNCID Hepattis Ref Lab & $2 \mathrm{HAV}$ & $4 3 5 2 2 5 \longdiv { 7 9 6 1 7 9 3 8 8 7 6 5 }$ & & \begin{tabular}{|l|l|l|l|l|l|l|}
37 & 38 & 39 & 40 & 42 & 42 \\
46 & 47 & 48 & 49 & 50 & 51 \\
\end{tabular} & \begin{tabular}{l|l|l}
43 & 44 & 45 \\
52 & 53 & 54
\end{tabular} \\
\hline 46 CDCNCID NCHSTD & 5 Chlam & $4 3 5 1 9 5 \longdiv { 7 9 6 1 7 0 3 7 8 7 9 0 }$ & & \begin{tabular}{|l|l|l|l|l|l|}
55 & 56 & 57 & 58 & 59 & 60 \\
64 & 65 & 66 & 67 & 68 & 69 \\
\end{tabular} & \begin{tabular}{l|l|l|}
61 & 62 & 63 \\
70 & 71 & 72
\end{tabular} \\
\hline 18 Collaborative Laboratory Services & 40 Biochem & 435187 & & 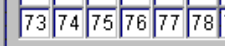 & $\begin{array}{ll}79 & 81 \\
\end{array}$ \\
\hline 24 Emory University & $9 \mathrm{HSV}$ & 435191 & & & \\
\hline 125 Esoterix Clinical Trials Services & 66 HLA-B27 & 435768 & $\nabla$ & 4 & $\perp$ \\
\hline Status: $\quad$ assigned $C$ NOT assigned $\odot$ & both $C$ & & & & \\
\hline Frequency: $\quad$ weekly $\odot \quad$ non-weekly $C$ & twice weekly $\mathrm{C}$ & $\mathrm{c}$ & & Cancel & \\
\hline
\end{tabular}

Continue scanning the bar codes and selecting the [Tab] key until all closed containers are assigned to air bills. 
Complete the assignment process.

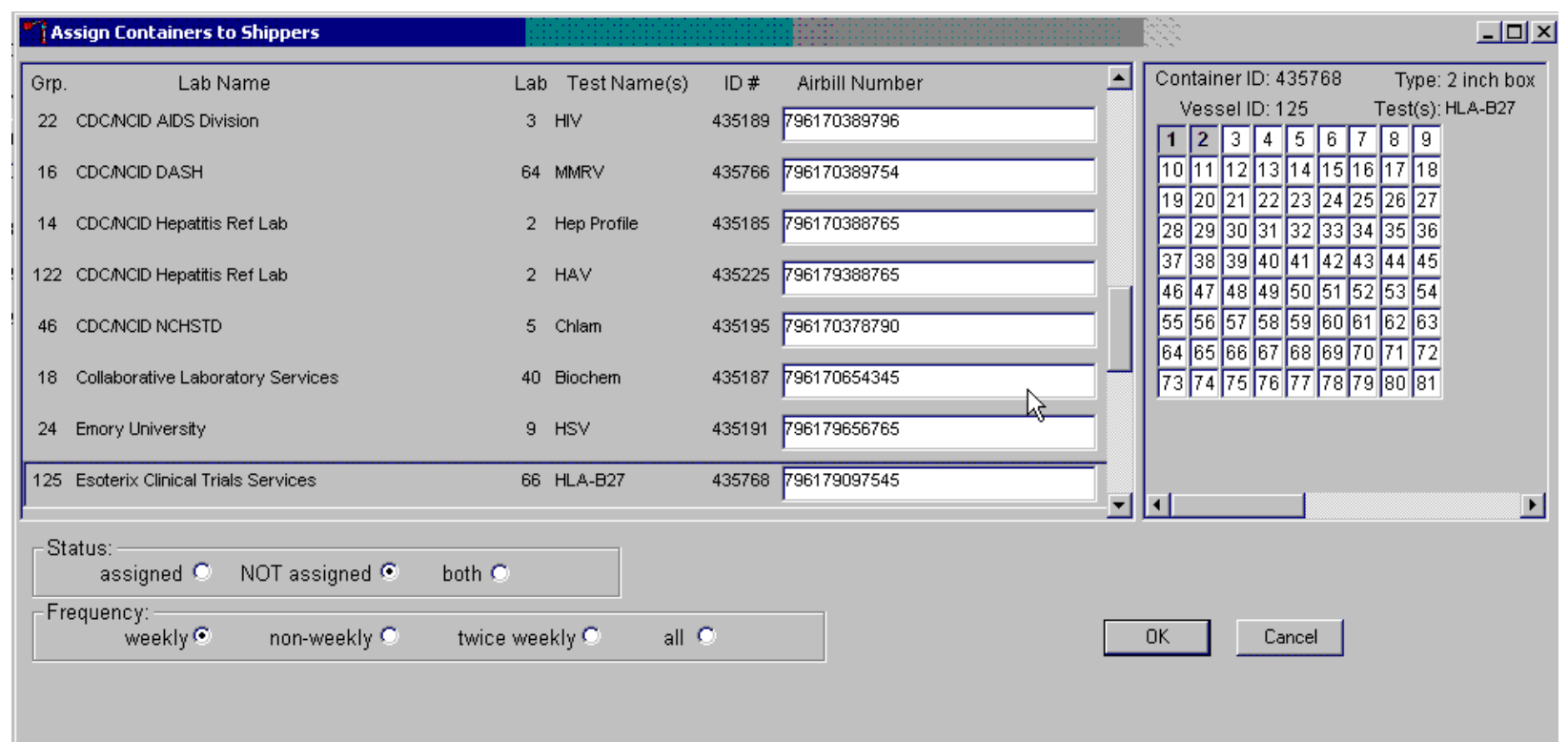

To record the assignment of containers to shippers, use the mouse to direct the mouse arrow to the "OK" button and left click. This step is required to save the information. To exit the A Assign Containers to Shippers\} module without recording any assignments, use the mouse to direct the mouse arrow to the "Cancel" button and left click. 


\subsection{Create Shipping Manifests}

Each shipper must include a paper manifest, which lists the inventory of the specimens in the shipping boxes. In addition, each laboratory receives an identical electronic file. Follow the steps listed below to prepare the paper manifest and to send the electronic file.

Access the $\{$ Shipping Manifest $\}$ module.

\begin{tabular}{|c|c|c|c|c|c|c|c|c|c|c|c|c|}
\hline \multicolumn{13}{|c|}{ ILab Sink } \\
\hline File View & Uيtilities Repo & orts $\underline{M}$ & Modules | & Shhipping Window Help & & & & & & & & \\
\hline 散 & 圆 & 28 & Q & \multirow{2}{*}{$\begin{array}{l}\text { Print New Container Labels } \\
\text { Container Map } \\
\text { Close Containers }\end{array}$} & & & & & & & & \\
\hline \multicolumn{4}{|c|}{ Appointments for Session: 2} & & \multicolumn{8}{|c|}{ Process Status } \\
\hline SPID & \multicolumn{2}{|c|}{ Sample ID status } & type : & \multirow{2}{*}{$\begin{array}{l}\text { Assign Containers to Shhippers } \\
\text { Irash Containers }\end{array}$} & UC U & $\mathrm{P}$ & $\mathrm{OZ}$ & $\mathrm{CB}$ & $\mathrm{B}$ gtt $\mathrm{PC}$ & & $\mathrm{HPV}$ of & \\
\hline 215591 & 215591 & $\mathrm{Cl}$ & VIP & & - 0 & $\bullet$ & . & 0 & 0. & & . & \\
\hline 244969 & 244969 & $\mathrm{BE}$ & $\mathrm{P}$ & Shipping Manifest & 00 & $\cdot$ & 0 & 0 & - 0 & & . & \\
\hline 470234 & 470234 & $\mathrm{Cl}$ & $P$ & M 60 HAGRID, RUBEUS & 00 & . & 0 & 0 & $0 \cdot 0$ & & & \\
\hline 668903 & 668903 & $\mathrm{Cl}$ & $P$ & 12 WEASLEY, FRED & 00 & $\cdot$ & 0 & 0 & $0 \cdot 0$ & 0 & & \\
\hline 762731 & 762731 & $\mathrm{Cl}$ & VIP & F 12 BROWN, LAVENDAR & - 0 & $\bullet$ & . & 0 & 0. & & & \\
\hline 836402 & 836402 & $\mathrm{Cl}$ & VIP & M 12 MALFOY, DRACO & 00 & . & . & 0 & $0 .$. & - & & \\
\hline 900081 & 900081 & $\mathrm{Cl}$ & VIP & F 49 ABEOTT, HANNAH & 00 & 0 & . & 0 & 0. & - & . & \\
\hline 998878 & 998878 & $\mathrm{Cl}$ & VIP & M 2 DURSLEY, VERNON & . & . & . & 0 & $0 .$. & . & . & \\
\hline
\end{tabular}

To access the \{Shipping Manifest\} module, use the mouse to direct the mouse arrow to \{Shipping\} in the top menu bar, left click, drag the mouse arrow to \{Shipping Manifest\}, and left click or type $[\mathrm{Alt}][\mathrm{R} / \mathrm{r}],[\mathrm{M} / \mathrm{m}]$. 
A Shipping Manifest window displays either after the \{Shipping Manifest module is accessed or after a positive response is recorded to the IC Informed Consent Exclusion window.

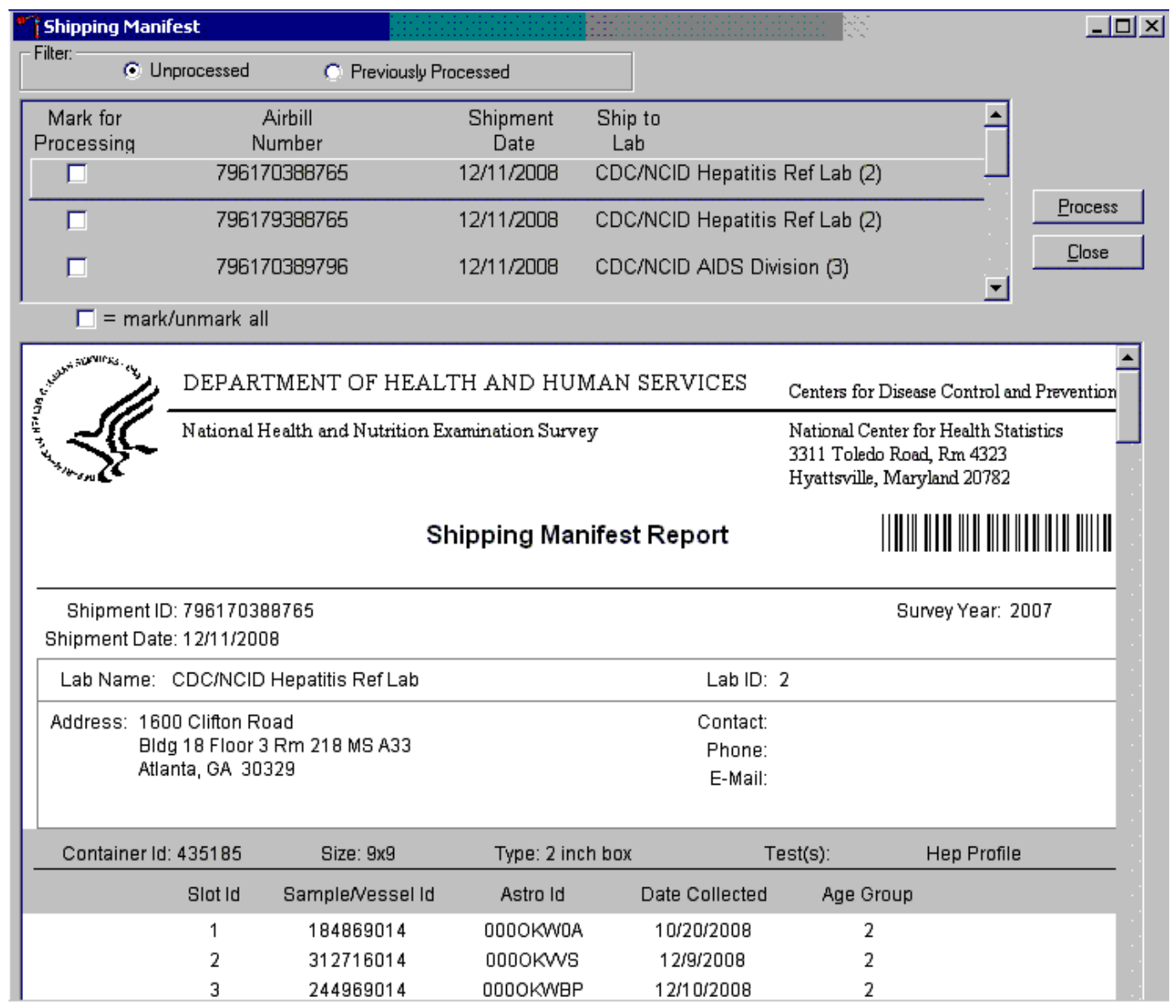

The upper portion of the Shipping Manifest Report contains two filters (Unprocessed and Previously Processed) and one row for each air bill assigned in the \{Assign Container to Shipper\} module. The row includes a Mark for Processing check box, the Airbill Number, Shipment Date, and Ship to Lab ID, and one check box to mark or unmark all air bills.

The lower portion of the window includes the Shipment ID or air bill number, Shipment Date, Lab Name, Lab ID number, Address, Contact personnel, Survey Year, Container ID associated with the shipment ID, container Size, container Type, Test(s) associated with the container, and a list by Slot ID of the Sample/Vessel ID, Astro ID, and Date Collected. 
Select one of the filters.

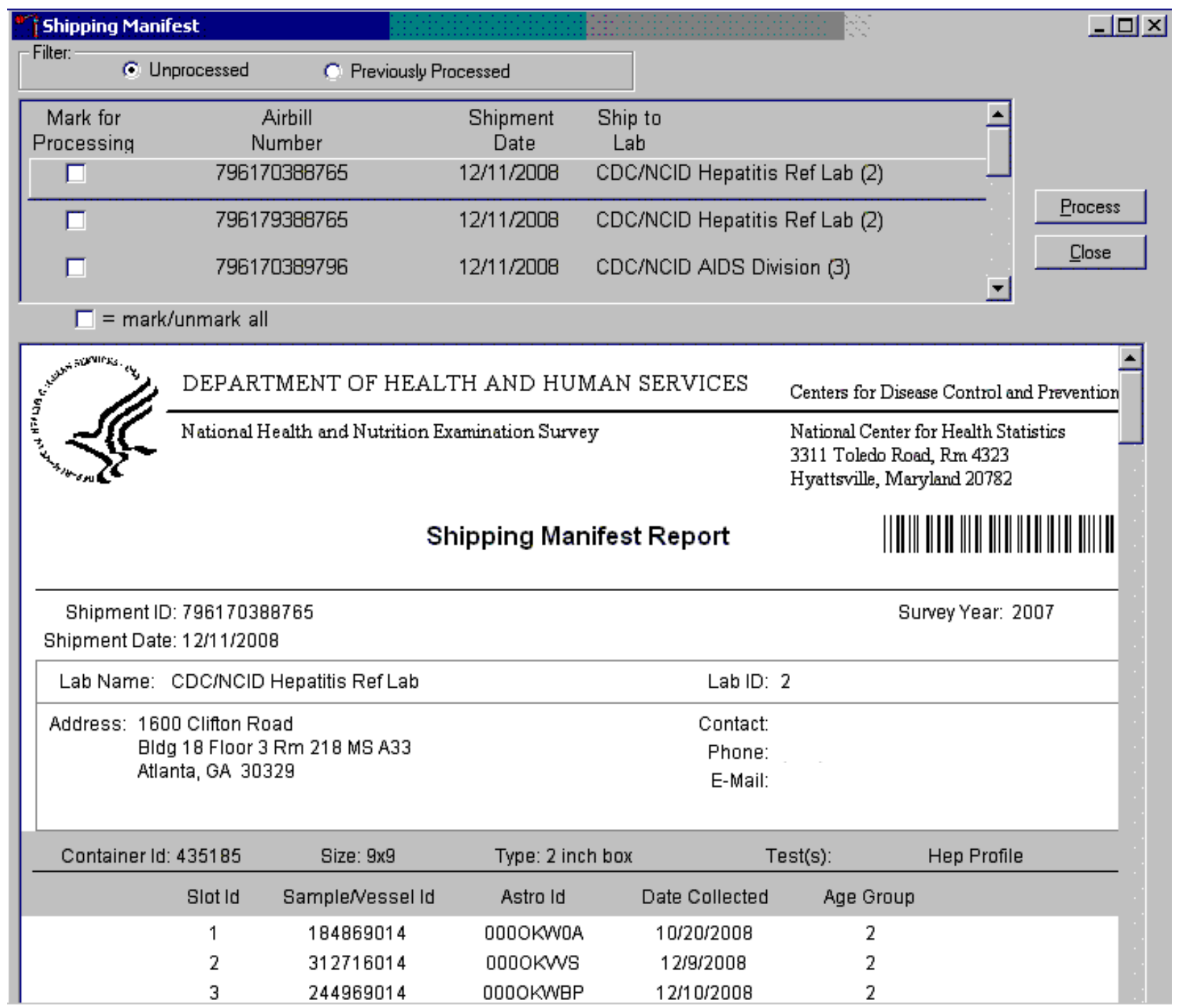

Set the filter to "Unprocessed" to print manifests and generate email files for shipments created but not previously processed using this module. Set the filter to "Previously Processed" to reprint an air bill or to recreate an electronic file for shipments previously processed using this module. To filter on unprocessed air bills, use the mouse to direct the mouse arrow to the "Unprocessed" radio button and left click. To filter on previously processed air bills, use the mouse to direct the mouse arrow to the "Previously Processed” radio button and left click. 
Select one or more manifests by placing a check mark in the Mark for Processing check boxes.

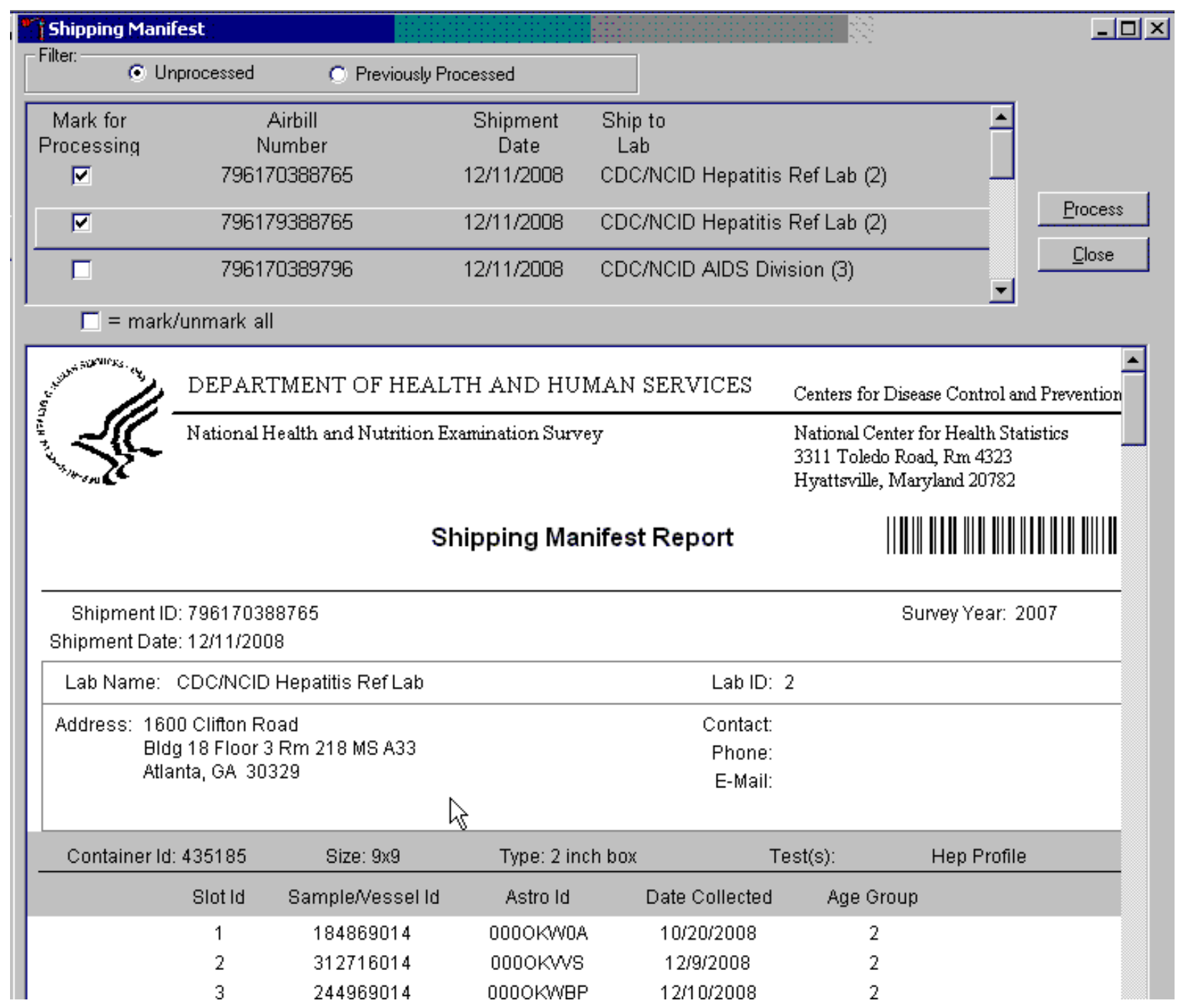

To select or mark one manifest for printing and email, use the mouse to direct the mouse arrow to the check box and left click. Repeat this process to include other air bills. Use the scroll bar to display all existing air bills. The lower portion of the window updates to display only marked air bills. 
Collectively mark or unmark all the air bills.

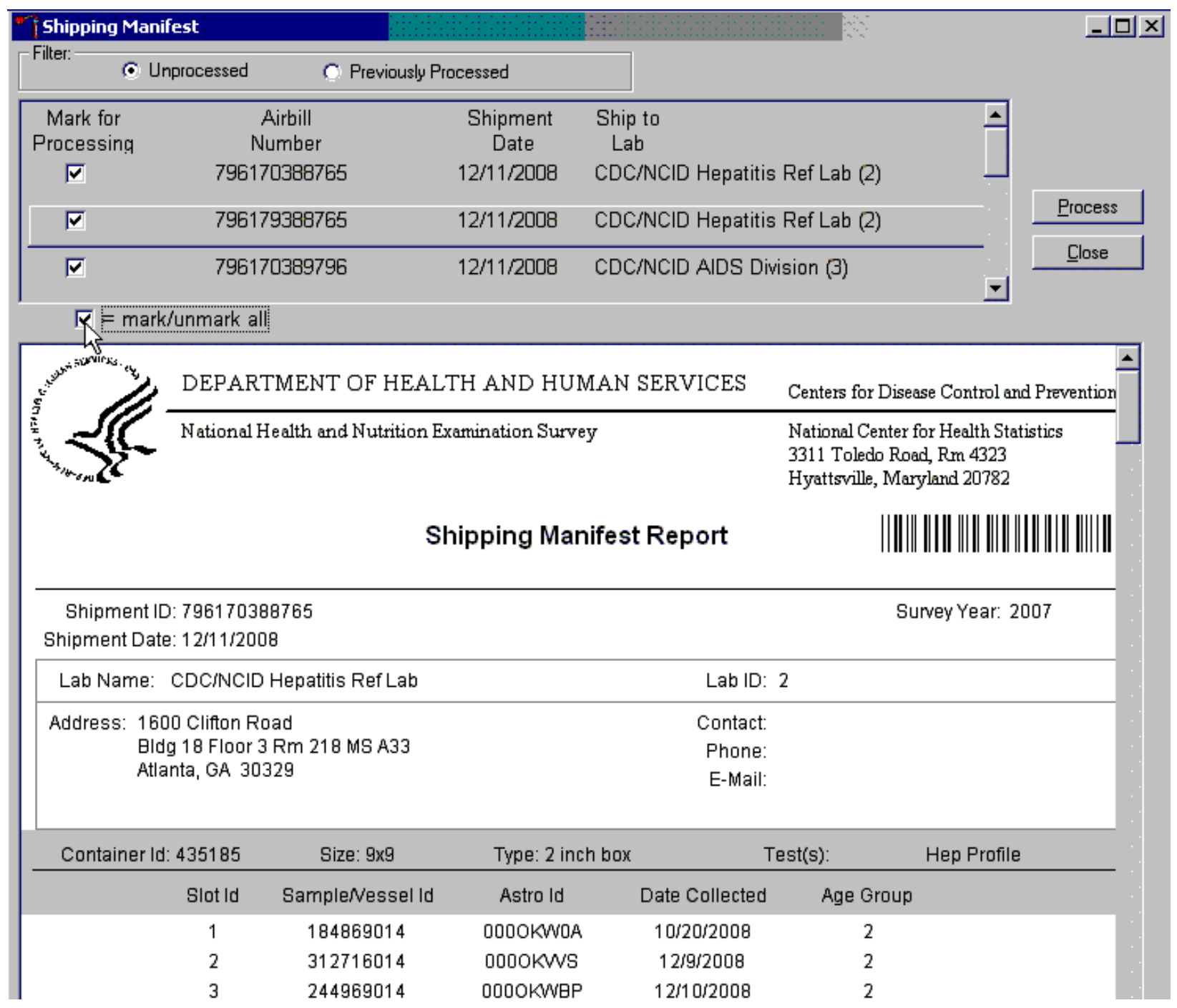

To collectively mark all the air bills, use the mouse to direct the mouse arrow to the mark/unmark all check box and left click. To collectively unmark all the air bills, use the mouse to direct the mouse arrow to the mark/unmark all check box and left click a second time. The lower portion of the screen will update to display the marked air bills. 
The right side of the window includes two buttons: Process and Close. Process the shipping manifests using the "Process" button.

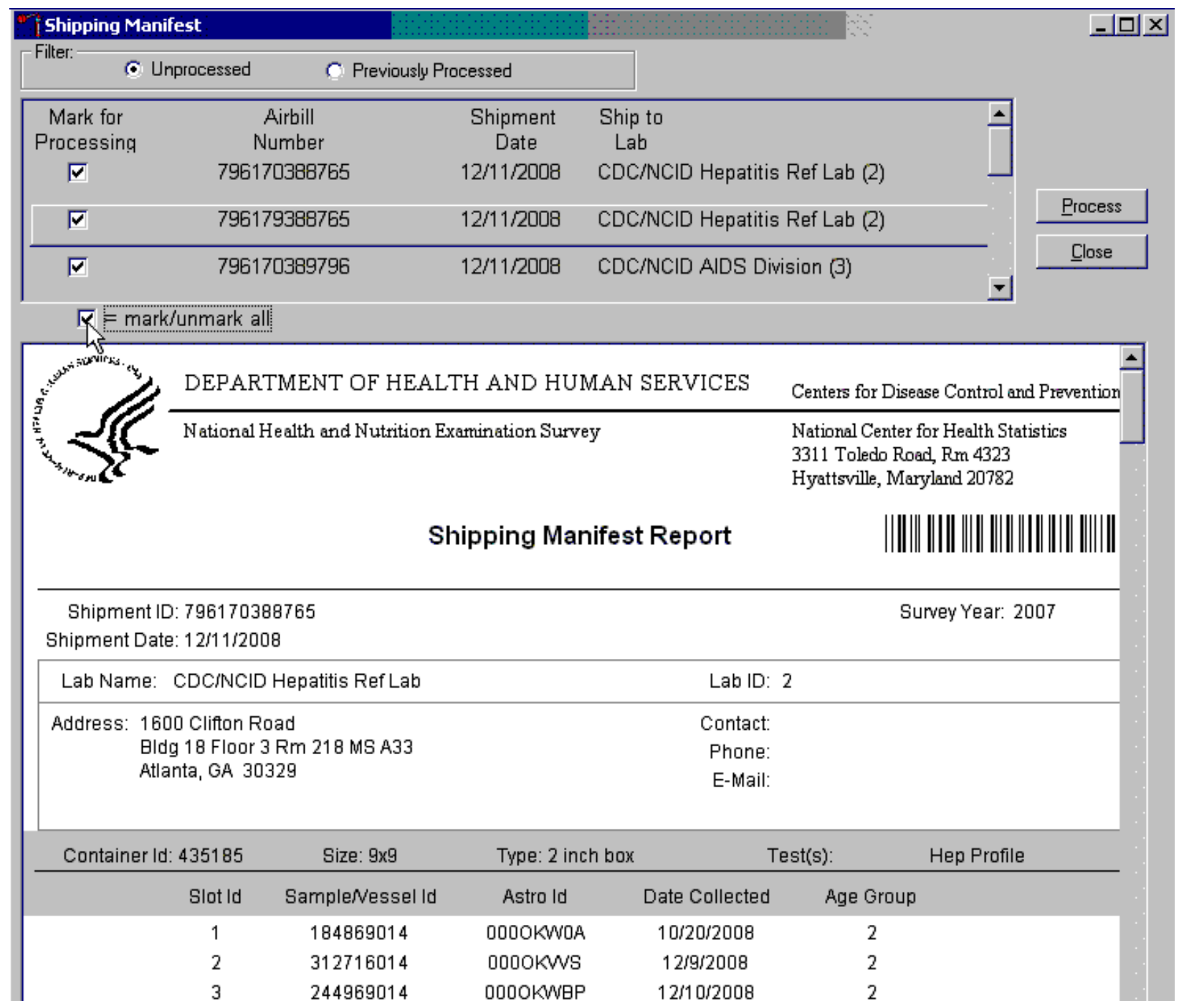

Selecting the "Process" button will generate and print the Shipping Manifest Report(s). To print the manifests use the mouse to direct the mouse arrow to the Process button and left click, or type [Shift] [P/p]. To exit the module without printing the manifest use the mouse to direct the mouse arrow to the "Close” button and left click, or type [Shift] [C/c.] 
The Informed Consent Exclusions window displays either when the \{Close Container\} or \{Shipping Manifest $\}$ module is accessed. It displays when the following conditions have been met:

1. SPs initially signed their consent form thereby agreeing to have their samples tested for HIV, HPV, PSA, STD testing; and/or genetics;

2. They consent to have their vessels stored for future research; and

3. They subsequently revoke either consent; and

4. The associated vessels have already been processed.

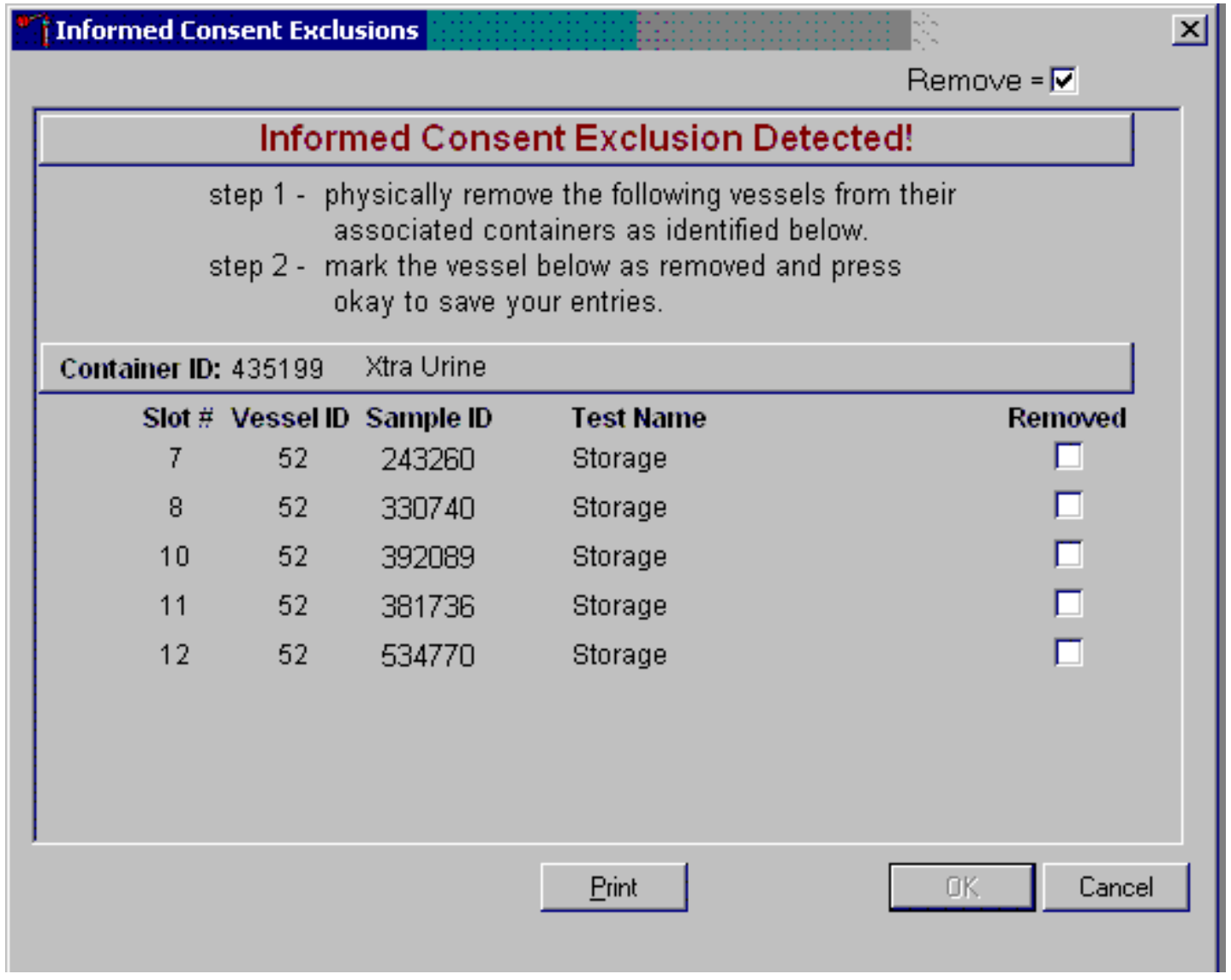

The window contains two steps that must be taken in order to resolve the inconsistencies. It includes the specific container ID and slot number within the container where the vessels are located, the sample ID, and the test name. Each row represents a vessel that must be marked as removed by placing a check mark in the check box. A "Print" button on the bottom of the screen allows the list to be printed. 
These vessels must be removed from the container and destroyed before the container is shipped. Follow the exact instructions indicated in the window-"Physically remove the following vessels from their associated containers as identified below.”

Affirm the actions taken to resolve these exclusions.

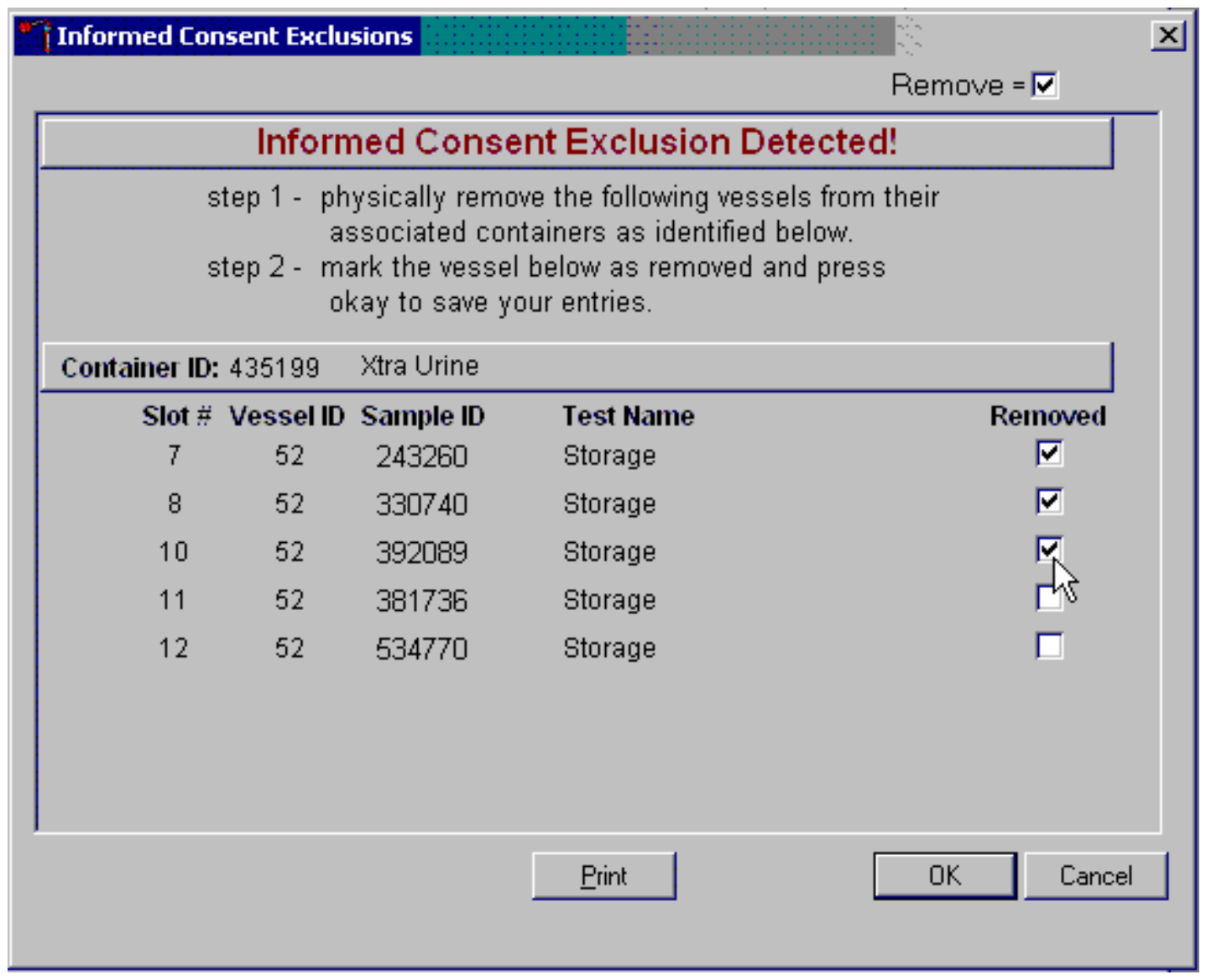

After following the exact instructions displayed in the window, record the actions taken to resolve the exclusions. To record these actions, use the mouse to direct the mouse arrow to the Removed check boxes and left click to insert a check mark. To record these actions in the database and to close the Informed Consent Exclusion window, use the mouse to direct the mouse arrow to the "OK" button and left click, or select [Enter]. To remove the window and exit without saving the actions to the database, use the mouse to direct the mouse arrow to the "Cancel” button and left click. 
If all the vessels are not marked, then an Informed Consent Exclusions for Stand XXX warning pop-up box displays.

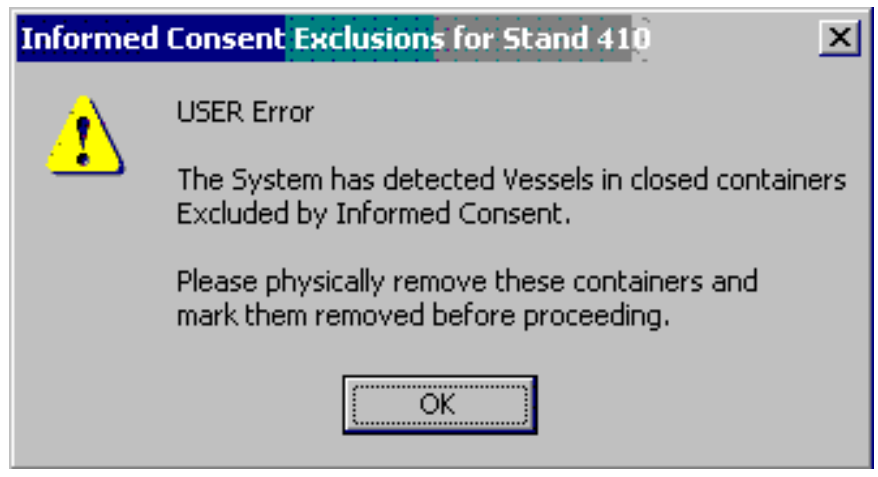

The system tracks each vessel in the Informed Consent Exclusion\} module to ensure that every vessel is marked as removed. Follow the instructions in the pop-up box, "Please physically remove these containers and mark them removed before proceeding." To acknowledge the message, use the mouse to direct the mouse arrow to the "OK" button and left click, or type [Enter]. 
If all the vessels were not marked, then access the \{Informed Consent Exclusion $\}$ module a second time.

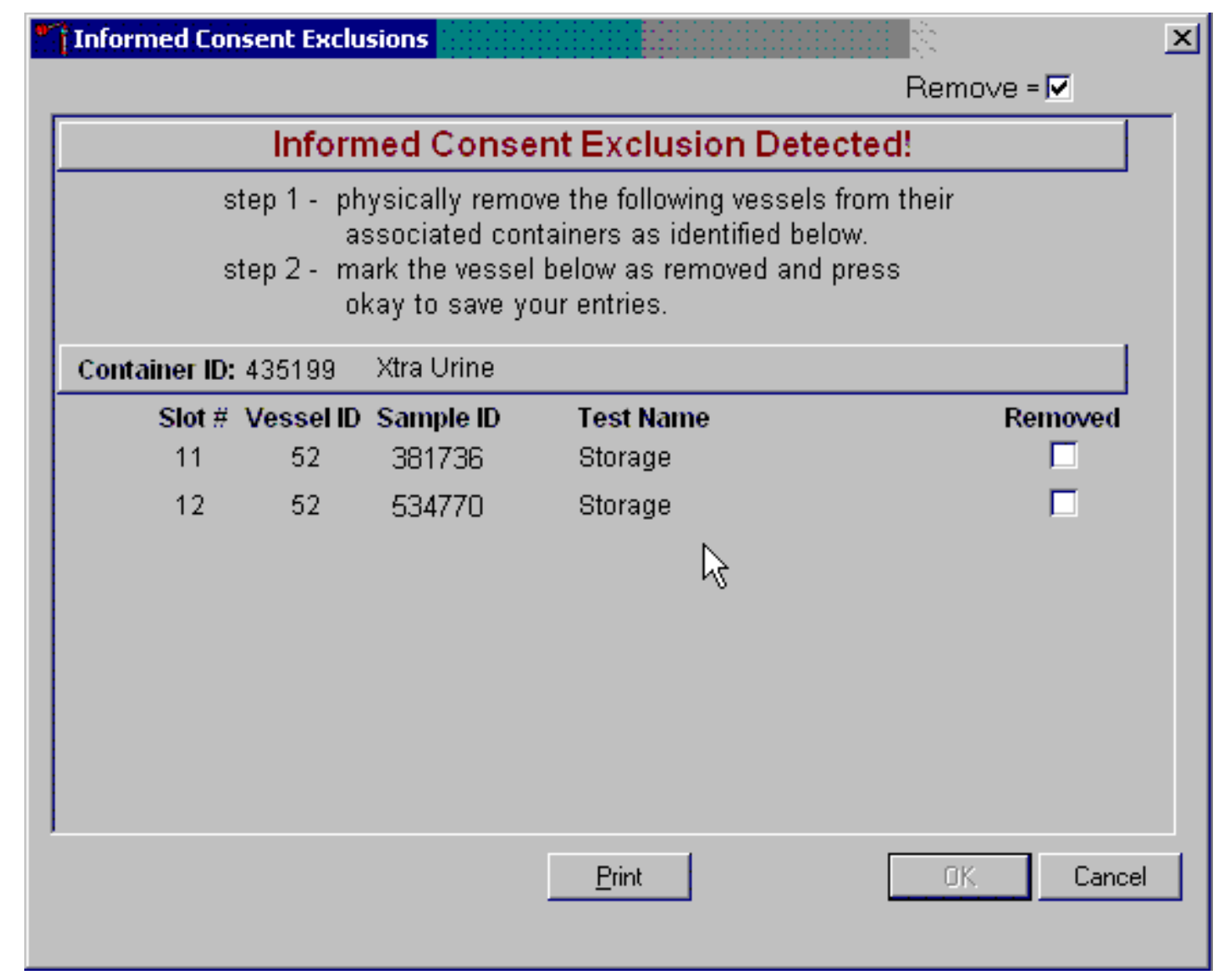

Follow the exact instructions displayed in the window, and then record the actions taken to resolve the exclusions. To record these actions, use the mouse to direct the mouse arrow to the Removed check boxes and left click to insert a check mark. To record these actions in the database and to close the Informed Consent Exclusions window, use the mouse to direct the mouse arrow to the "OK" button and left click, or select [Enter]. Once all vessels have been marked correctly, the close container window will automatically display. 


\subsection{Packing Procedure for the Shippers}

Most of the shipments require dry ice to keep the specimens frozen during shipment. Some shipments require reusable refrigerant packs to keep specimens cold but not frozen. Store reusable refrigerant packs in the freezers. (Distribute the refrigerant packs across all freezers.) It is the chief medical technologist's responsibility to arrange for delivery of dry ice pellets or blocks for shipping specimens. Use sliced blocks whenever possible since these sublimate less than pellets. Well in advance of a scheduled shipment, the shipping technologist estimates how much dry ice to order for the shipment and informs the MEC manager. Store the dry ice in extra shipping containers. When handling dry ice, wear cryo gloves to avoid burning hands. Also, wear an eye shield when filling bags with ice chips or breaking blocks into chunks. Use the scale to ensure that each shipper has the required amount of dry ice. Exhibit 9-3 is a list of all contract laboratory addresses.

Federal regulations require packaging all blood, urine, and other liquid diagnostic specimens according to certain regulations and packaging procedures. A complete description of these regulations is contained in Appendix A.

Diagnostic specimens must be packed in good quality packaging, which must be strong enough to withstand the shocks and loading normally encountered during transport, including transshipment between transport units and warehouses as well as removal from a pallet or overpack for subsequent manual or mechanical handling. Packaging must be constructed and closed to prevent any loss of contents when prepared for transport, which might be caused under normal conditions of transport, by vibration, or by changes in temperature, humidity, or pressure.

The packaging must consist of three components:

1. A primary receptacle(s);

2. A secondary packaging; and

3. A rigid outer packaging.

Primary receptacles must be packed in secondary packaging in such a way that, under normal conditions of transport, they cannot break, be punctured, or leak their contents into secondary packaging. Secondary packaging must be secured in outer packaging with suitable cushioning material. Any leakage of the contents must not substantially impair the protection properties of the cushioning material or of the outer packaging. 
Exhibit 9-3. Contract laboratory addresses

\begin{tabular}{|c|c|c|c|}
\hline $\begin{array}{c}\text { Laboratory/destination } \\
\text { specimen shipment address }\end{array}$ & Vessels/assays & Conditions & $\begin{array}{c}\text { Contact name } \\
\text { Telephone number }\end{array}$ \\
\hline $\begin{array}{l}\text { Lab } 2 \\
\text { CDC/NCID Hepatitis Ref Lab } \\
\text { Attn: } \\
\text { Code NH4 } \\
\text { 1600 CLIFTON RD NE } \\
\text { Bldg } 18 \text { Floor } 3 \text { Rm } 218 \text { MS } \\
\text { A33 } \\
\text { Atlanta, GA 30329-4018 }\end{array}$ & $\begin{array}{l}14 \text { - Hep profile } \\
122 \text { - HAV } \\
87 \text { - Hepatitis ab }\end{array}$ & Frozen & \\
\hline $\begin{array}{l}\text { Lab } 3 \\
\text { CDC/NCID AIDS Division } \\
\text { Attn: } 1600 \text { Clifton Road NE } \\
\text { Bldg 17, Room 3127, MS A-25 } \\
\text { Atlanta, GA 30329-4018 }\end{array}$ & $22-\mathrm{HIV}$ & Frozen & \\
\hline $\begin{array}{l}\text { Lab } 4 \\
\text { CDC/NCEH Air Toxicant } \\
\text { Branch } \\
\text { Attn: Dr. Ben Blount } \\
\text { 4770 Buford Highway } \\
\text { Bldg 103 Loading Dock } \\
\text { Atlanta, GA 30341-3717 } \\
\text { 770-488-4312 }\end{array}$ & $\begin{array}{l}54-\text { VOC Blood } \\
105-\text { HH Water }\end{array}$ & Refrigerated & $\begin{array}{l}\text { Dr. Ben Blount } \\
770-488-4312\end{array}$ \\
\hline $\begin{array}{l}\text { Lab } 5 \\
\text { CDC/NCID NCHSTD } \\
\text { Attn: Carol Farshy } \\
1600 \text { Clifton Road NE } \\
\text { MS D13 } \\
\text { Atlanta, GA 30329-4018 } \\
\text { 404-639-2870 }\end{array}$ & 46 - Chlam & Frozen & $\begin{array}{l}\text { Carol Farshy } \\
404-639-2870\end{array}$ \\
\hline
\end{tabular}


Exhibit 9-3. Contract laboratory addresses (continued)

\begin{tabular}{|c|c|c|c|}
\hline $\begin{array}{l}\text { Lab } 6 \\
\text { CASPIR } \\
\text { Attn: Robert White } \\
602 \text { Webb Gin House Road } \\
\text { Building C } \\
\text { Lawrenceville, GA 30045-5427 } \\
\text { 770-339-5942 }\end{array}$ & $\begin{array}{l}1 \text { - Lead/Cad/HG } \\
3 \text { - RBC Folate } \\
106 \text { - RBC Fol1 } \\
5 \text { - Mercury } \\
109 \text { - PUFA/FTA } \\
\mathbf{8} \text { - Xtra Plasma } \\
88 \text { - Acrylamide } \\
126 \text { - Xtra RBCs } \\
81 \text { - Vit B6/Fol } \\
70 \text { - Vitamin D } \\
110 \text { - VB6/Fol1 } \\
99 \text { - Fer/TFR } \\
17 \text { - Cotinine } \\
110 \text { - Folate } 1 \\
\mathbf{3 2} \text { - Xtra Sera } \\
\mathbf{2 9 / 8 9} \text { - Persis Pest } \\
\mathbf{9 2 / 9 3} \text { - BFR } \\
86 \text { - Spc Arsenic } \\
62 \text { - Urn Merc } \\
50 \text { - Heavy Metal } \\
\mathbf{6 5} \text { - Urn Phytoes } \\
\mathbf{6 6} \text { - PAH } \\
\mathbf{1 0 2} \text { - Perchlorate } \\
\mathbf{1 2 1} \text { - NNAL } \\
128 \text { - Caffeine } \\
\mathbf{5 2} \text { - Extra urine }\end{array}$ & Frozen & $\begin{array}{l}\text { Robert White } \\
770-339-5942\end{array}$ \\
\hline $\begin{array}{l}\text { Lab } 9 \\
\text { Emory University } \\
\text { Dr. Francis Lee } \\
2015 \text { UPPERGATE DR. } \\
\text { ROOM 560 } \\
\text { Atlanta, GA 30322-0001 } \\
\text { 404-727-4652 }\end{array}$ & $24-\mathrm{HSV}$ & Frozen & $\begin{array}{l}\text { Dr. Francis Lee } \\
\text { 404-616-6229 }\end{array}$ \\
\hline
\end{tabular}


Exhibit 9-3. Contract laboratory addresses (continued)

\begin{tabular}{|c|c|c|c|}
\hline $\begin{array}{l}\text { Laboratory/destination } \\
\text { specimen shipment address }\end{array}$ & Vessels/assays & Conditions & $\begin{array}{c}\text { Contact name } \\
\text { Telephone number }\end{array}$ \\
\hline $\begin{array}{l}\text { Lab } 11 \\
\text { University of Washington } \\
\text { Attn: Kathy Hutchinson } \\
\text { 1959 NE PACIFIC ST } \\
\text { Room NW 176, Box } 357110 \\
\text { Seattle, WA 98195-0001 } \\
\text { 206-548-6149 }\end{array}$ & $\begin{array}{l}13-\text { CRP } \\
75-\text { PSA } \\
118 \text { - Thyroid }\end{array}$ & Frozen & $\begin{array}{l}\text { Kathy Hutchinson } \\
\text { 206-548-4181 }\end{array}$ \\
\hline $\begin{array}{l}\text { Lab } 16 \\
\text { University of Minnesota } \\
\text { Attn: Susan Kupcho } \\
515 \text { Delaware Street SE } \\
\text { Room 13-219 - MOOS Tower } \\
\text { Minneapolis, MN 55455-0357 } \\
\text { 612-624-6153 }\end{array}$ & 45 - Alb/Creat & Frozen & $\begin{array}{l}\text { Susan Kupcho } \\
612-624-6153\end{array}$ \\
\hline $\begin{array}{l}\text { Lab } 24 \\
\text { CDC/Environmental Health } \\
\text { Laboratory } \\
\text { Attn: Antonio Calafat } \\
4770 \text { Buford Highway } \\
\text { Building 110, Loading Dock } \\
\text { Atlanta, GA 30341-3717 } \\
\text { 770-488-7886 }\end{array}$ & $\begin{array}{l}90 \text { - Perfluor1 } \\
67 \text { - Phthalates }\end{array}$ & Frozen & $\begin{array}{l}\text { Dr. Antonia Calafat } \\
\text { 770-488-7891 }\end{array}$ \\
\hline $\begin{array}{l}\text { Lab } 26 \\
\text { CDC/ NCEH Pesticide } \\
\text { Labortory } \\
4770 \text { Buford Highway } \\
\text { Building 103, Loading Dock } \\
\text { Atlanta, GA 30341-3717 } \\
\text { 770-488-4598 }\end{array}$ & $\begin{array}{l}91 \text { - Srm Pest } \\
48 \text { - Prior Pest } \\
49 \text { - Organophos }\end{array}$ & Frozen & $\begin{array}{l}\text { Dr. Dana Barr } \\
\text { 770-488-4598 }\end{array}$ \\
\hline $\begin{array}{l}\text { Lab } 37 \\
\text { CDC/Human Papillomavirus } \\
\text { Laboratory } \\
\text { Attn: Sonya Patel } \\
\text { 1600 Clifton Road NE } \\
\text { MS G41 Bldg/Rm 1/1355 } \\
\text { Atlanta, GA 30329-4018 } \\
\text { 404-639-2187 } \\
\end{array}$ & $78-\mathrm{HPV}$ & Room Temperature & $\begin{array}{l}\text { Sonya Patel } \\
404-639-2187\end{array}$ \\
\hline $\begin{array}{l}\text { Lab } 40 \\
\text { Collaborative Laboratory } \\
\text { Services } \\
\text { Attn: Sue Vanness } \\
1005 \text { Pennsylvania } \\
\text { Suite } 102 \\
\text { Ottumwa, Iowa 52501-6408 } \\
641 \text { 684-4621 }\end{array}$ & 18 - Biochem & Refrigerated & $\begin{array}{l}\text { Sue Vanness } \\
641 \text { 684-4621 }\end{array}$ \\
\hline
\end{tabular}


Exhibit 9-3. Contract laboratory addresses (continued)

\begin{tabular}{|c|c|c|c|}
\hline $\begin{array}{l}\text { Laboratory/destination } \\
\text { specimen shipment address }\end{array}$ & Vessels/assays & Conditions & $\begin{array}{c}\text { Contact name } \\
\text { Telephone number }\end{array}$ \\
\hline $\begin{array}{l}\text { Lab 51 } \\
\text { NHANES Diabetes Laboratory } \\
\text { Attn: Jean Bucksa } \\
420 \text { Delaware St SE } \\
\text { Mayo L275 } \\
\text { Minneapolis, MN 55455-0341 } \\
612-273-3645\end{array}$ & $\begin{array}{l}9-\text { Glucose } \\
98-\text { OGTT } \\
23 \text { - Insulin } \\
21-\text { Lipids }\end{array}$ & Frozen & $\begin{array}{l}\text { Jean Bucksa } \\
612-273-3645\end{array}$ \\
\hline $\begin{array}{l}\text { Lab 51 } \\
\text { NHANES Diabetes Laboratory } \\
\text { Attn: Jean Bucksa } \\
\text { 420 Delaware St SE } \\
\text { Mayo L275 } \\
\text { Minneapolis, MN 55455-0341 } \\
612-273-3645\end{array}$ & 4-Glycohem & Refrigerated & $\begin{array}{l}\text { Jean Bucksa } \\
612-273-3645\end{array}$ \\
\hline $\begin{array}{l}\text { Lab } 52 \\
\text { CDC/DVRD HPV } \\
\text { Attn: Kristi Singletary Meadows } \\
\text { 1600 Clifton Rd NE } \\
\text { MS G41 Bldg/Rm 7/116 } \\
\text { Atlanta, GA 30329-4018 } \\
\text { 404-639-2183 }\end{array}$ & 77 - HPV Serum & Frozen & $\begin{array}{l}\text { Kristi Singletary } \\
\text { Meadows } \\
\text { 404-639-2183 }\end{array}$ \\
\hline $\begin{array}{l}\text { Lab 58 } \\
\text { CDC/NCEH Molecular Biology } \\
\text { Branch } \\
\text { Attn: Peg Gallagher } \\
\text { 4770 BUFORD HWY } \\
\text { F24 Room 3204 } \\
\text { Atlanta, GA 30341-3717 } \\
\text { 770-488-4313 } \\
\end{array}$ & 119/120 Genetics & Refrigerated & $\begin{array}{l}\text { Cindy Sturco } \\
\text { (770) 488-4313 }\end{array}$ \\
\hline $\begin{array}{l}\text { Lab 63 } \\
\text { CDC/NCEH PAH Adducts } \\
\text { Attn: Angela Ragin } \\
\text { 4770 BUFORD HWY } \\
\text { Building 110, Room } 2202 \\
\text { Atlanta, GA 30341-3717 } \\
\text { 770-488-7494 }\end{array}$ & $126-\mathrm{PAH} \mathrm{HB}$ & Refrigerated & $\begin{array}{l}\text { James Grainger } \\
770-488-4445\end{array}$ \\
\hline $\begin{array}{l}\text { Lab 64 } \\
\text { CDC/NCEH DASH } \\
\text { Attn: Dr. William Bellini } \\
\text { 1600 CLIFTON RD NE } \\
\text { Group 81 MS C22 } \\
\text { Atlanta, GA 30329-4018 } \\
\text { 404-639-4183 }\end{array}$ & $16-\mathrm{MMRV}$ & Frozen & $\begin{array}{l}\text { Dr. William Bellini } \\
\text { 404-639-4183 }\end{array}$ \\
\hline
\end{tabular}


Exhibit 9-3. Contract laboratory addresses (continued)

\begin{tabular}{|c|c|c|c|}
\hline $\begin{array}{l}\text { Laboratory/destination } \\
\text { specimen shipment address }\end{array}$ & Vessels/assays & Conditions & $\begin{array}{c}\text { Contact name } \\
\text { Telephone number }\end{array}$ \\
\hline $\begin{array}{l}\text { Lab } 65 \\
\text { Mayo Clinic } \\
\text { Attn: Tricia Brantner } \\
2214^{\text {th }} \text { Avenue SW } \\
\text { Guggenheim 10-02 } \\
\text { ROCHESTER, MN } 55902 \\
\text { 507-538-4128 }\end{array}$ & 127 - IgA-TTG/EMA & Frozen & $\begin{array}{l}\text { Tricia Brantner } \\
\text { 507-538-4128 }\end{array}$ \\
\hline $\begin{array}{l}\text { Lab } 66 \\
\text { Esoterix Clinical Trials Services } \\
1904 \text { Alexander Dr } \\
\text { Attn: Melanie Little } \\
\text { Research Triangle Park, NC } \\
27709 \\
\text { (919) } 572-6900 \\
\end{array}$ & 125 - HLA-B27 & Refrigerated & $\begin{array}{l}\text { Melanie Little } \\
\text { (919) 572-6900 }\end{array}$ \\
\hline $\begin{array}{l}\text { Lab } 67 \\
\text { Gillison Laboratory } \\
2001 \text { Polaris Pkwy \# } 1320 \\
\text { OSU - Pathology Core Facility } \\
\text { Columbus, OH } \\
\text { 43240-2000 } \\
614-293-5942\end{array}$ & 129 - HPV Rinse & Frozen & $\begin{array}{l}\text { Dr. Maura Gillison } \\
\text { 614-293-5942 }\end{array}$ \\
\hline
\end{tabular}


Packages must be prepared as follows:

\section{0(a) For Liquids}

- The primary receptacle(s) must be leakproof and must not contain more than $1 \mathrm{~L}$;

- The secondary packaging must be leakproof;

- If multiple fragile primary receptacles are placed in a single secondary packaging, they must be individually wrapped or separated to prevent contact between them.

- Absorbent material must be placed between the primary receptacle and the secondary packaging. The absorbent material, such as cotton wool, must be in sufficient quantity to absorb the entire contents of the primary receptacle (so that any release of the liquid substance will not compromise the integrity of the cushioning material or of the outer packaging.

- The primary receptacle or the secondary packaging must be capable of withstanding, without leakage, an internal pressure producing a pressure differential of not less than $95 \mathrm{kPa}$ in the range of $-40^{\circ} \mathrm{C}$ to $+55^{\circ} \mathrm{C}\left(-40^{\circ} \mathrm{F}\right.$ to $\left.130^{\circ} \mathrm{F}\right)$.

- The outer packaging must not contain more than $4 \mathrm{~L}$.

Specific Requirements - refrigerated or frozen specimens: ice, dry ice, and

\section{liquid nitrogen}

- When dry ice or liquid nitrogen is used to keep specimens cold, all applicable requirements of these Regulations must be met. When used, ice or dry ice must be placed outside the secondary packaging or in the outer packaging or an overpack. Interior supports must be provided to secure the secondary packaging in the original position after the ice or dry ice (solid carbon dioxide) has been dissipated. If ice is used, the packaging or overpack must be leakproof. If dry ice (solid carbon dioxide) is used, the outside packaging or overpack must be designed and constructed to permit the release of carbon dioxide gas to prevent a buildup of pressure that could rupture the packaging.

- The primary receptacle and the secondary packaging must maintain their integrity at the temperature of the refrigerant used as well as the temperatures and the pressures, which could result if refrigeration were to be lost. 
Infectious substances assigned to UN 3373 which are packed and marked in accordance with this packing instruction are not subject to any other requirement of these regulations except for the following:

a. The name, address, and telephone number of a responsible person must be provided on the waybill or on the package.

\section{Packing Instruction 904 - Dry Ice}

Dry ice (solid carbon dioxide), when offered for transport by air, must be in packaging designed and constructed to permit the release of carbon dioxide gas and to prevent a buildup of pressure that could rupture the packaging.

The net weight of dry ice must be marked on the outside of the package.

Arrangements between the shipper and operator(s) must be made for each shipment to ensure that ventilation and safety procedures are followed.

When a Shipper's Declaration is not required, the following information as required by 8.2.3 for dry ice (solid carbon dioxide) must be contained in the "Nature and Quantity of Dangerous Goods" box on the air waybill:

- $\quad$ Proper shipping name (Dry Ice or Carbon Dioxide, solid);

- UN 1845;

- The number of packages; and

- The net weight of dry ice in each package.

The net weight of the dry ice must be marked on the outside of the package. 


\subsubsection{Pack Boxed Specimens}

Store the blood and urine vessels in 2-, 3-, and 5-inch storage boxes. Separate the vessels with cardboard grids or foam inserts. Secure the top of the box to the bottom with a rubber band. Place the box in a pressure vessel, add one absorbent paper, and seal the pressure vessel.

Ship the vessels to the addresses listed in Exhibit 9-3, Contract Laboratory addresses, shown earlier.

\subsubsection{Pack Medium Shipping Containers for Frozen Shipment}

Pack the specimen storage boxes tightly in the Styrofoam container to prevent movement.

- $\quad$ Place the shipper on the scale and tare.

- Place a layer 3-inches thick or at least 15 pounds of chipped dry ice or dry ice slabs in the bottom of a medium Styrofoam shipper.

- $\quad$ Place a single layer of newsprint between the box and the dry ice.

- Place the sealed pressure vessels containing the boxes on top of the dry ice.

- Pack the sides with crumbled newsprint.

- Tare the scale.

- Place enough chipped dry ice or dry ice slabs on top so that the Styrofoam container is filled with dry ice to $1 / 4$ " below the rim or add a minimum of another 15 pounds of dry ice.

- Place the Styrofoam lid on top of the Styrofoam bottom.

- Place a plastic envelope containing the appropriate shipping manifest and return preprinted label on top of the Styrofoam lid.

- Weigh the shipper on the scale or assume the medium shipper weighs approximately 35 pounds.

- Record the weight of the dry ice (30 pounds for a medium shipper) on the FedEx Class 9 label. 
- Secure the shipper with tape.

- Attach the air bill.

\subsubsection{Pack Small Shipping Containers for Frozen Shipment}

Pack the specimen storage boxes tightly in the Styrofoam container to prevent movement.

- Place the shipper on the scale and tare.

- Place a layer 3-inches thick or at least 10 pounds of chipped dry ice or dry ice slabs in the bottom of a medium Styrofoam shipper.

- $\quad$ Place a single layer of newsprint between the box and the dry ice.

- $\quad$ Place the sealed pressure vessels containing the boxes on top of the dry ice.

- $\quad$ Pack the sides with crumbled newsprint.

- Tare the scale.

- $\quad$ Place enough chipped dry ice or dry ice slabs on top so that the Styrofoam container is filled with dry ice to $1 / 4$ " below the rim or add a minimum of another 10 pounds of dry ice.

- $\quad$ Place the Styrofoam lid on top of the Styrofoam bottom.

- Place a plastic envelope containing the appropriate shipping manifest and return preprinted label on top of the Styrofoam lid.

- Weigh the shipper on the scale or assume the medium shipper weighs approximately 25 pounds.

- Record the weight of the dry ice (20 pounds for a small shipper) on the FedEx Class 9 label.

- Secure the shipper with tape.

- Attach the air bill. 


\subsubsection{Pack Medium Shipping Container for Refrigerated Shipment}

Some shipments require a coolant to keep the specimens cold but not frozen during shipment. For these shipments, use reusable refrigerant packs. Add enough frozen refrigerant packs to the shipper to keep specimens cold for at least 48-72 hours. Keep approximately 30 refrigerant packs in the freezers at all times, replacing as necessary. Pack all refrigerated containers in Styrofoam shippers.

Pack the specimen storage boxes tightly in the Styrofoam container to prevent movement.

- Seal the 9 x 12 zip closable plastic bag or the pressure vessel containing the boxes.

- Place at least two large frozen refrigerant packs in the bottom of a shipper.

- Place the sealed plastic bag containing the swabs or the pressure vessel containing the boxes on top of the refrigerant pack.

- Pack the sides with crumbled newsprint.

- $\quad$ Place two additional refrigerant packs on top of the container(s).

- Place the Styrofoam lid on top of the Styrofoam bottom.

- Place the appropriate shipping manifest and one return FedEx preprinted air bill into a $9 \times 12$ plastic bag and seal.

- Place the plastic envelope containing the appropriate shipping manifest and one FedEx preprinted air bill on top of the Styrofoam lid.

- Weigh the shipper on the scale or assume that the package weighs 10 pounds.

- Record the total weight of the shipper (10 pounds) on the appropriate FedEx label. 


\subsubsection{Label Shippers}

For all destinations, include a preprinted FedEx return air bill containing the warehouse address.

All shippers must contain a FedEx bar-coded label and appropriate orientation and warning labels. Label all shippers with the appropriate warning labels as follows:

1. A “Keep Frozen” label and a FedEx Class 9 - Miscellaneous Dangerous Goods label on shippers containing dry ice.

2. A "Refrigerate" label on shippers containing refrigerant packs.

3. A UN 3373: - "BIOLOGICAL SUBSTANCE, CATEGORY B label on all shippers.

4. An “overpack” label on all shippers

5. Complete the section of the FedEx label that requests the weight of the contents of the shipper, and if applicable, the section that requests the weight of the dry ice contained in the shipper.

6. Place the FedEx label with the appropriate contract laboratory address in a plastic FedEx pouch and attach the window to the cardboard lid of the shipper.

7. Add one additional strip of tape across the FedEx pouch.

\subsection{Container Protocol and Weekly Shipping List}

Exhibit 9-4 illustrates the Container protocol; Exhibit 9-5 illustrates the FedEx shipping list by lab name; and Exhibit 9-6 illustrates the shipping list by lab number. Use the container protocol as an aid at the start of each stand when opening new containers. Use the FedEx shipping lists as an aid when creating air bills in FedEx Ship Manager. 
Exhibit 9-4. Container protocol

2009 Container Protocol

\begin{tabular}{|c|c|c|c|c|c|}
\hline Lab & Vessel & Test & Lab & Vessel & Container \\
\hline 61 & 1 & Lead/Cad/HG & CASPIR & $2 \mathrm{~mL}$ cryovial & $9 \times 9$ two inch box \\
\hline 54 & 3 & RBC Folate & CASPIR & $2 \mathrm{~mL}$ cryovial & $10 \times 10$ two inch box \\
\hline 54 & 106 & RBC Fol1 & CASPIR & $2 \mathrm{~mL}$ cryovial & $10 \times 10$ two inch box \\
\hline 51 & 4 & Glycohem & $\begin{array}{l}\text { NHANES Diabetes } \\
\text { Laboratory }\end{array}$ & $2 \mathrm{~mL}$ cryovial & $9 \times 9$ two inch box \\
\hline 61 & 5 & Mercury & CASPIR & $2 \mathrm{~mL}$ cryovial & $9 \times 9$ two inch box \\
\hline 66 & 125 & HLA-B27 & $\begin{array}{l}\text { Esoterix Clinical Trials } \\
\text { Services }\end{array}$ & $2 \mathrm{~mL}$ cryovial & $9 \times 9$ two inch box \\
\hline $54 / 60$ & 109 & PUFA/TFA & CASPIR & $2 \mathrm{~mL}$ cryovial & $10 \times 10$ two inch box \\
\hline 6 & 8 & Xtra Plasma & CASPI R & $\begin{array}{l}2 \mathrm{~mL} \\
\text { cryovial }\end{array}$ & $9 \times 9$ two inch box \\
\hline 51 & 9 & Glucose & $\begin{array}{l}\text { NHANES Diabetes } \\
\text { Minnesota }\end{array}$ & $2 \mathrm{~mL}$ cryovial & $9 \times 9$ two inch box \\
\hline 51 & 98 & OGTT & $\begin{array}{l}\text { NHANES Diabetes } \\
\text { Minnesota }\end{array}$ & $2 \mathrm{~mL}$ cryovial & $9 \times 9$ two inch box \\
\hline 26 & 91 & SRM Pesticide & $\begin{array}{l}\text { CDC/ NCEH Pesticide } \\
\text { Laboratory }\end{array}$ & $\begin{array}{l}5 \mathrm{~mL} \\
\text { cryovial }\end{array}$ & $9 \times 9$ three inch box \\
\hline 60 & 88 & Acrylamide & CASPIR & $2 \mathrm{~mL}$ cryovial & $9 \times 9$ two inch box \\
\hline 63 & 126 & Xtra RBCs & CASPIR & $2 \mathrm{~mL}$ cryovial & $9 \times 9$ three inch box \\
\hline 54 & 81 & Vit B6/Fol & CASPIR & $2 \mathrm{~mL}$ cryovial & $10 \times 10$ two inch box \\
\hline 54 & 70 & Vitamin D & CASPIR & $2 \mathrm{~mL}$ cryovial & $10 \times 10$ two inch box \\
\hline 11 & 13 & CRP & $\begin{array}{l}\text { University of } \\
\text { Washington }\end{array}$ & $2 \mathrm{~mL}$ cryovial & $9 \times 9$ two inch box \\
\hline 2 & 14 & Hep profile & $\begin{array}{l}\text { CDC/NCID Hepatitis Ref } \\
\text { Lab }\end{array}$ & $2 \mathrm{~mL}$ cryovial & $9 \times 9$ two inch box \\
\hline 2 & 122 & HAV & $\begin{array}{l}\text { CDC/NCID Hepatitis Ref } \\
\text { Lab }\end{array}$ & $2 \mathrm{~mL}$ cryovial & $9 \times 9$ two inch box \\
\hline 2 & 87 & Hepatit ab & $\begin{array}{l}\text { CDC/NCID Hepatitis Ref } \\
\text { Lab }\end{array}$ & $2 \mathrm{~mL}$ cryovial & $9 \times 9$ two inch box \\
\hline 54 & 99 & Fer/TfR & CASPIR & $2 \mathrm{~mL}$ cryovial & $10 \times 10$ two inch box \\
\hline 59 & 17 & Cotinine & CASPIR & $2 \mathrm{~mL}$ cryovial & $9 \times 9$ two inch box \\
\hline 51 & 21 & Lipids & $\begin{array}{l}\text { NHANES Diabetes } \\
\text { Laboratory }\end{array}$ & $2 \mathrm{~mL}$ cryovial & $9 \times 9$ two inch box \\
\hline 52 & 77 & HPV Serum & CDC/DVRD HPV & $2 \mathrm{~mL}$ cryovial & $9 \times 9$ two inch box \\
\hline 40 & 18 & Biochem & $\begin{array}{l}\text { Collaborative Laboratory } \\
\text { Services }\end{array}$ & $2 \mathrm{~mL}$ cryovial & $9 \times 9$ two inch box \\
\hline 51 & 23 & Insulin & $\begin{array}{l}\text { NHANES Diabetes } \\
\text { Laboratory }\end{array}$ & $2 \mathrm{~mL}$ cryovial & $9 \times 9$ two inch box \\
\hline 3 & 22 & $\mathrm{HIV}$ & CDC/NCID AIDS Division & $2 \mathrm{~mL}$ cryovial & $9 \times 9$ two inch box \\
\hline 9 & 24 & HSV & Emory University & $2 \mathrm{~mL}$ cryovial & $9 \times 9$ two inch box \\
\hline 11 & 75 & PSA & $\begin{array}{l}\text { University of } \\
\text { Washington }\end{array}$ & $2 \mathrm{~mL}$ cryovial & $9 \times 9$ two inch box \\
\hline 11 & 118 & Thyroid & $\begin{array}{l}\text { University of } \\
\text { Washington }\end{array}$ & $2 \mathrm{~mL}$ cryovial & $9 \times 9$ two inch box \\
\hline
\end{tabular}


Exhibit 9-4. Container protocol (continued)

\begin{tabular}{|c|c|c|c|c|c|}
\hline & & & $\begin{array}{l}2009 \text { Container } \\
\text { Protocol }\end{array}$ & & \\
\hline Lab & Vessel & Test & Lab & Vessel & Container \\
\hline 65 & 127 & IgA-TTG/EMA & Mayo Clinic & $2 \mathrm{~mL}$ cryovial & $9 \times 9$ two inch box \\
\hline 64 & 16 & MMRV & CDC/NCEH DASH & $2 \mathrm{~mL}$ cryovial & $9 \times 9$ two inch box \\
\hline 54 & 110 & Folatel & CASPIR & $2 \mathrm{~mL}$ cryovial & $10 \times 10$ two inch box \\
\hline 6 & 32-39 & Xtra sera1 & CASPI R & $\begin{array}{l}2 \mathrm{~mL} \\
\text { cryovial }\end{array}$ & $9 \times 9$ two inch box \\
\hline 28 & $29 / 89$ & Persis Pest1 & CASPIR & $\begin{array}{l}5 \mathrm{~mL} \\
\text { cryovial }\end{array}$ & $9 \times 9$ three inch box \\
\hline 24 & 90 & Perfluor & $\begin{array}{l}\mathrm{CDC} / \mathrm{NCEH} \\
\text { Environmental Health } \\
\text { Laboratory }\end{array}$ & $\begin{array}{l}5 \mathrm{~mL} \\
\text { cryovial }\end{array}$ & 9x9 three inch box \\
\hline 28 & $92 / 93$ & BFR1 & CASPIR & $\begin{array}{l}5 \mathrm{~mL} \\
\text { cryovial }\end{array}$ & $9 \times 9$ three inch box \\
\hline 16 & 45 & Alb/Creat & University of Minnesota & $5 \mathrm{~mL}$ cryovial & 9x9 three inch box \\
\hline 5 & 46 & Chlam & CDC/NCID NCHSTD & 14 mL Falcon & $5 \times 5$ three inch box \\
\hline 61 & 86 & Spc Arsenic & CASPIR & $5 \mathrm{~mL}$ cryovial & $9 \times 9$ three inch box \\
\hline 26 & 48 & Prior Pest & $\begin{array}{l}\text { CDC/ NCEH Pesticide } \\
\text { Laboratory }\end{array}$ & $\begin{array}{l}10 \mathrm{~mL} \text { VWR } \\
\text { screwcap }\end{array}$ & $5 \times 5$ three inch box \\
\hline 26 & 49 & Organophos & $\begin{array}{l}\text { CDC/ NCEH Pesticide } \\
\text { Laboratory }\end{array}$ & $\begin{array}{l}10 \mathrm{~mL} \text { VWR } \\
\text { screwcap }\end{array}$ & $5 \times 5$ three inch box \\
\hline 61 & 62 & Urn Merc & CASPIR & $\begin{array}{l}10 \mathrm{~mL} \text { VWR } \\
\text { screwcap }\end{array}$ & $5 \times 5$ three inch box \\
\hline 61 & 50 & Heavy Metal & CASPIR & $\begin{array}{l}10 \mathrm{~mL} \text { VWR } \\
\text { screwcap }\end{array}$ & $5 \times 5$ three inch box \\
\hline 54 & 65 & Urn Phytoes & CASPI R & $\begin{array}{l}5 \mathrm{~mL} \\
\text { cryovial }\end{array}$ & $\begin{array}{l}10 \times 10 \text { three inch } \\
\text { box }\end{array}$ \\
\hline 31 & 66 & PAH & CASPIR & $\begin{array}{l}5 \mathrm{~mL} \\
\text { cryovial }\end{array}$ & $9 \times 9$ three inch box \\
\hline 24 & 67 & Phthalates & $\begin{array}{l}\text { CDC/ NCEH } \\
\text { Environmental Health } \\
\text { Laboratory }\end{array}$ & $\begin{array}{l}5 \mathrm{~mL} \\
\text { cryoviall }\end{array}$ & 9x9 three inch box \\
\hline 62 & 102 & Perchlorate & CASPI R & $\begin{array}{l}5 \mathrm{~mL} \\
\text { cryoviall }\end{array}$ & 9x9 three inch box \\
\hline 59 & 121 & NNAL & CASPI R & $\begin{array}{l}10 \mathrm{~mL} \text { VWR } \\
\text { screwcap }\end{array}$ & $5 \times 5$ three inch box \\
\hline 54 & 128 & Caffeine & CASPIR & $2 \mathrm{~mL}$ cryovial & $10 \times 10$ two inch box \\
\hline 6 & 52 & Xtra Urine & CASPIR & $\begin{array}{l}5 \mathrm{~mL} \\
\text { cryoviall }\end{array}$ & $9 \times 9$ three inch box \\
\hline 4 & 54 & VOC Blood & $\begin{array}{l}\text { CDC/NCEH Air Toxicant } \\
\text { Branch }\end{array}$ & $\begin{array}{l}10 \mathrm{~mL} \text { Gray } \\
\text { tube }\end{array}$ & $\begin{array}{l}5 \times 5 \text { five inch with } \\
\text { foam box }\end{array}$ \\
\hline 4 & 105 & HH Water & $\begin{array}{l}\text { CDC/NCEH Air Toxicant } \\
\text { Branch }\end{array}$ & $\begin{array}{l}10 \mathrm{~mL} \\
\text { Wheaton vial }\end{array}$ & $\begin{array}{l}5 \times 5 \text { three inch with } \\
\text { foam insert }\end{array}$ \\
\hline 58 & $119 / 120$ & Genetics & $\begin{array}{l}\text { CDC/NCEH Molecular } \\
\text { Biology Branch }\end{array}$ & $\begin{array}{l}10 \mathrm{~mL} \text { EDTA } \\
\text { tube }\end{array}$ & $\begin{array}{l}5 \times 5 \text { five inch foam } \\
\text { box }\end{array}$ \\
\hline 37 & 78 & HPV & $\begin{array}{l}\text { CDC/Human } \\
\text { Papillomavirus } \\
\text { Laboratory }\end{array}$ & Swab & 9x12 Ziplock bag \\
\hline 67 & 129 & HPV Rinse & Gillison Laboratory & $\begin{array}{l}14 \mathrm{~mL} \text { Falcon } \\
\text { tube }\end{array}$ & $5 \times 5$ three inch box \\
\hline
\end{tabular}


Exhibit 9-5. FedEx shipping list by lab name

\section{FedEx by Lab Name}

\begin{tabular}{|c|c|c|c|c|c|c|c|}
\hline Lab & Vessel & Test & Lab & Conditions & $\begin{array}{l}\text { Dry } \\
\text { I ce } \\
\text { LBS }\end{array}$ & $\begin{array}{l}\text { Dry } \\
\text { Ice } \\
\text { KG } \\
\end{array}$ & Weight \\
\hline \multirow[t]{25}{*}{6} & 1 & Lead/Cad/HG & CASPIR & Frozen & 30 & 14 & 35 \\
\hline & 3 & RBC Folate & & & & & \\
\hline & 5 & Mercury & & & & & \\
\hline & 8 & Xtra Plasma & & & & & \\
\hline & 17 & Cotinine & & & & & \\
\hline & 29/89 & Persis Pest & & & & & \\
\hline & 50 & Heavy Metal & & & & & \\
\hline & 52 & Xtra Urine & & & & & \\
\hline & 62 & Urn Merc & & & & & \\
\hline & 65 & Urn Phytoes & & & & & \\
\hline & 66 & PAH & & & & & \\
\hline & 70 & Vitamin D & & & & & \\
\hline & 81 & Vit B6/Fol & & & & & \\
\hline & 86 & Spc Arsenic & & & & & \\
\hline & 88 & Acrylamide & & & & & \\
\hline & 126 & Xtra RBCs & & & & & \\
\hline & $92 / 93$ & BFR & & & & & \\
\hline & 99 & Fer/TfR & & & & & \\
\hline & 102 & Perchlorate & & & & & \\
\hline & 106 & RBC Fol1 & & & & & \\
\hline & 109 & PUFA/TFA & & & & & \\
\hline & 110 & Folate1 & & & & & \\
\hline & 121 & NNAL & & & & & \\
\hline & 128 & Caffeine & & & & & \\
\hline & $32-39$ & Xtra sera & & & & & \\
\hline 52 & 77 & HPV Serum & CDC/DVRD HPV & Frozen & 20 & 9 & 25 \\
\hline 37 & 78 & HPV & $\begin{array}{l}\text { CDC/Human Papillomavirus } \\
\text { Laboratory }\end{array}$ & Room Temp & & & 1 \\
\hline \multirow[t]{2}{*}{4} & 54 & VOC Blood & CDC/NCEH Air Toxicant Branch & Refrigerated & 20 & & 10 \\
\hline & 105 & $\mathrm{HH}$ Water & & & & & \\
\hline 64 & 16 & MMRV & CDC/NCEH DASH & Frozen & 20 & 9 & 25 \\
\hline \multirow[t]{2}{*}{24} & 90 & Perfluor & $\begin{array}{l}\text { CDC/ NCEH Environmental Health } \\
\text { Laboratory }\end{array}$ & Frozen & 30 & 14 & 35 \\
\hline & 67 & Phthalates & & & & & \\
\hline 58 & $119 / 120$ & Genetics & CDC/NCEH Molecular Biology Branch & Refrigerated & 20 & & 10 \\
\hline \multirow[t]{4}{*}{26} & 91 & SRM Pesticide & CDC/ NCEH Pesticide Laboratory & Frozen & 30 & 14 & 35 \\
\hline & 48 & Prior Pest & & & & & \\
\hline & & & 2009 FedEx by Lab Name & & & & \\
\hline & 49 & Organophos & & & & & \\
\hline
\end{tabular}


Exhibit 9-5. FedEx shipping list by lab name (continued)

2009 FedEx by Lab Name

\begin{tabular}{|c|c|c|c|c|c|c|c|}
\hline Lab & Vessel & Test & Lab & Conditions & $\begin{array}{l}\text { Dry } \\
\text { I ce } \\
\text { LBS }\end{array}$ & $\begin{array}{l}\text { Dry } \\
\text { Ice } \\
\text { KG } \\
\end{array}$ & Weight \\
\hline 3 & 22 & HIV & CDC/NCID AIDS Division & Frozen & 20 & 9 & 25 \\
\hline \multirow[t]{3}{*}{2} & 14 & Hep profile & CDC/NCID Hepatitis Ref Lab & Frozen & 30 & 14 & 35 \\
\hline & 122 & HAV & & & & & \\
\hline & 87 & Hepatit $a b$ & & & & & \\
\hline 5 & 46 & Chlam & CDC/NCID NCHSTD & Frozen & 20 & 9 & 25 \\
\hline 40 & 18 & Biochem & Collaborative Laboratory Services & Refrigerated & 20 & & 10 \\
\hline 9 & 24 & HSV & Emory University & Frozen & 20 & 9 & 25 \\
\hline 66 & 126 & HLA-B27 & Esoterix Clinical Trials Services & Refrigerated & 20 & & 10 \\
\hline 67 & 129 & HPV Rinse & Johns Hopkins Viral Oncology & Refrigerated & 20 & & 10 \\
\hline 65 & 127 & IgA-TTG/EMA & Mayo Clinic & Frozen & 20 & 9 & 25 \\
\hline 51 & 4 & Glycohem & NHANES Diabetes Laboratory & Refrigerated & 20 & & 10 \\
\hline \multirow[t]{4}{*}{51} & 21 & Lipids & NHANES Diabetes Laboratory & Frozen & 30 & 14 & 35 \\
\hline & 23 & Insulin & & & & & \\
\hline & 9 & Glucose & & & & & \\
\hline & 98 & OGTT & & & & & \\
\hline 16 & 45 & Alb/Creat & University of Minnesota & Frozen & 20 & 9 & 25 \\
\hline \multirow[t]{3}{*}{11} & 13 & CRP & University of Washington & Frozen & 30 & 14 & 35 \\
\hline & 75 & PSA & & & & & \\
\hline & 118 & Thyroid & & & & & \\
\hline
\end{tabular}


Exhibit 9-6. FedEx shipping list by lab number

2009 FedEx by Lab Number

\begin{tabular}{|c|c|c|c|c|c|c|c|}
\hline Lab & Vessel & Test & Lab & Conditions & $\begin{array}{l}\text { Dry } \\
\text { I ce } \\
\text { LBS }\end{array}$ & $\begin{array}{l}\text { Dry } \\
\text { Ice } \\
\text { KG }\end{array}$ & Weight \\
\hline 2 & $\begin{array}{l}14 \\
122 \\
87 \\
\end{array}$ & $\begin{array}{l}\text { Hep profile } \\
\text { HAV } \\
\text { Hepatit ab }\end{array}$ & CDC/NCID Hepatitis Ref Lab & Frozen & 30 & 14 & 35 \\
\hline 3 & 22 & HIV & CDC/NCID AIDS Division & Frozen & 20 & 9 & 25 \\
\hline 4 & $\begin{array}{l}54 \\
105 \\
\end{array}$ & $\begin{array}{l}\text { VOC Blood } \\
\text { HH Water }\end{array}$ & CDC/NCEH Air Toxicant Branch & Refrigerated & 20 & & 10 \\
\hline 5 & 46 & Chlam & CDC/NCID NCHSTD & Frozen & 20 & 9 & 25 \\
\hline 6 & $\begin{array}{l}1 \\
3 \\
5 \\
\mathbf{8} \\
17 \\
\mathbf{2 9 / 8 9} \\
\mathbf{3 2 - 3 9} \\
50 \\
\mathbf{5 2} \\
62 \\
65 \\
\mathbf{6 6} \\
70 \\
81 \\
86 \\
88 \\
126 \\
\mathbf{9 2 / 9 3} \\
99 \\
\mathbf{1 0 2} \\
106 \\
109 \\
110 \\
\mathbf{1 2 1} \\
128 \\
\end{array}$ & $\begin{array}{l}\text { Lead/Cad/HG } \\
\text { RBC Folate } \\
\text { Mercury } \\
\text { Xtra Plasma } \\
\text { Cotinine } \\
\text { Persis Pest1 } \\
\text { Xtra sera } \\
\text { Heavy Metal } \\
\text { Xtra Urine } \\
\text { Urn Merc } \\
\text { Urn Phytoes } \\
\text { PAH } \\
\text { Vitamin D } \\
\text { Vit B6/Fol } \\
\text { Spc Arsenic } \\
\text { Acrylamide } \\
\text { Xtra RBCs } \\
\text { BFR1 } \\
\text { Fer/TfR } \\
\text { Perchlorate } \\
\text { RBC Fol1 } \\
\text { PUFA/TFA } \\
\text { Folate1 } \\
\text { NNAL } \\
\text { Caffeine }\end{array}$ & CASPIR & Frozen & 30 & 14 & 35 \\
\hline 9 & 24 & HSV & Emory University & Frozen & 20 & 9 & 25 \\
\hline 11 & $\begin{array}{l}13 \\
75 \\
118 \\
\end{array}$ & $\begin{array}{l}\text { CRP } \\
\text { PSA } \\
\text { Thyroid }\end{array}$ & University of Washington & Frozen & 30 & 14 & 35 \\
\hline 16 & 45 & Alb/Creat & University of Minnesota & Frozen & 20 & 9 & 25 \\
\hline 24 & $\begin{array}{l}90 \\
67 \\
\end{array}$ & $\begin{array}{l}\text { Perfluor } \\
\text { Phthalates }\end{array}$ & $\begin{array}{l}\text { CDC/ NCEH Environmental Health } \\
\text { Laboratory }\end{array}$ & Frozen & 30 & 14 & 35 \\
\hline 26 & 91 & SRM Pesticide & CDC/ NCEH Pesticide Laboratory & Frozen & 30 & 14 & 35 \\
\hline
\end{tabular}


Exhibit 9-6. FedEx shipping list by lab number (continued)

2009 FedEx by Lab Number

\begin{tabular}{|c|c|c|c|c|c|c|c|}
\hline Lab & Vessel & Test & Lab & Conditions & $\begin{array}{l}\text { Dry } \\
\text { I ce } \\
\text { LBS }\end{array}$ & $\begin{array}{l}\text { Dry } \\
\text { Ice } \\
\text { KG } \\
\end{array}$ & Weight \\
\hline & 48 & Prior Pest & & & & & \\
\hline & 49 & Organophos & & & & & \\
\hline 37 & 78 & HPV & $\begin{array}{l}\text { CDC/Human Papillomavirus } \\
\text { Laboratory }\end{array}$ & Room Temp & & & 1 \\
\hline 40 & 18 & Biochem & Collaborative Laboratory Services & Refrigerated & 20 & & 10 \\
\hline 51 & 4 & Glycohem & NHANES Diabetes Laboratory & Refrigerated & 20 & & 10 \\
\hline 51 & 21 & Lipids & NHANES Diabetes Laboratory & Frozen & 30 & 14 & 35 \\
\hline 51 & 23 & Insulin & & & & & \\
\hline 51 & 9 & Glucose & & & & & \\
\hline 51 & 98 & OGT & & & & & \\
\hline 52 & 77 & HPV Serum & CDC/DVRD HPV & Frozen & 20 & 9 & 25 \\
\hline 58 & $119 / 120$ & Genetics & CDC/NCEH Molecular Biology Branch & Refrigerated & 20 & & 10 \\
\hline 64 & 16 & MMRV & $\mathrm{CDC} / \mathrm{NCEH} \mathrm{DASH}$ & Frozen & 20 & 9 & 25 \\
\hline 65 & 127 & IgA-TTG/EMA & Mayo Clinic & Frozen & 20 & 9 & 25 \\
\hline 66 & 125 & HLA-B27 & Esoterix Clinical Trail Services & Refrigerated & 20 & & 10 \\
\hline 67 & 129 & HPV Rinse & Gillison Laboratory & Frozen & 20 & & 10 \\
\hline
\end{tabular}




\section{HPV SWAB PROCESSING}

\subsection{Introduction}

The purpose of collecting vaginal swabs is to obtain suitable biological specimens to test for human papillomavirus (HPV). Human papillomavirus infection is one of the most common sexually transmitted infections in the United States. Cervical infection with certain types of HPV is a major risk factor for cervical cancer in women. The "high-risk" types of HPV (e.g., HPV 16, 18, 31, 33, 35, 39, 45, 51, 52, 56, 58, 59, 68) are associated with cervical cancer and the "low-risk” types (e.g., HPV 6, 11, 42. $43,44)$ with genital warts. No national surveillance system exists to measure the full burden of HPV infection, and no reliable national population estimate of HPV exists. NHANES offers a unique opportunity to assess the prevalence of HPV infection in the general population.

Reducing the prevalence of HPV infection is a Developmental Healthy People 2010 objective: "Reducing the number of new HPV cases can help minimize the overall number of cases of high risk subtypes associated with cervical cancer in females.” Detection and typing of HPV DNA in vaginal swabs (in conjunction with testing of NHANES sera for HPV 16 antibody) will allow evaluation of trends in prevalence of type-specific HPV infection by age, sexual behavior, and race/ethnicity. HPV vaccine development is underway, and knowledge of the national prevalence of HPV infection will be critical for planning vaccination strategies in the United States. Studies have demonstrated that recovery of HPV from self-collected swabs is comparable to that from physician-collected cervical samples. The HPV Hybrid Capture ${ }^{\circledR}$ II DNA results will be reported to survey participants via the same reporting system as for STD/HIV testing. This system is a respondent-initiated toll-free phone call to NCHS. Results are given to persons tested after a password is provided to verify identity. The HPV-16 serology and the HPV DNA typology will not be reported.

Primary female SPs aged 14-59 collect the HPV swab. The physician prints the appropriate label. Each label includes the test name, 6-digit SP ID concatenated with the vessel ID, and the corresponding bar code. The physician counsels each eligible SP and introduces her to the vaginal collection technique using a prepared collection kit. The collection kit is designed for SPs aged 14-59 and it includes illustrated collection instructions and one Epicentre ${ }^{\circledR}$ Dacron swab with foam tip. The physician removes the appropriate swab from the collection kit and labels the Epicentre ${ }^{\circledR}$ Dacron swab 
with the foam tip with the label displaying the vessel ID \#78, The physician then places the swab(s) back in the collection kit and gives the collection kit to the eligible SP.

The SP self-collects the appropriate swab in the mobile examination center (MEC) bathroom, places the swab back in its original container, and places the swab back in the plastic bag. The SP hands the plastic bag containing the swab(s) to the assistant coordinator. The assistant coordinator delivers the plastic bag to the laboratory.

The laboratory staff verifies the SP ID and the vessel ID (\#78) on the label of the Epicentre ${ }^{\circledR}$ Dacron swab with the foam tip, records the collection in the HPV module, and stores the swab at room temperature. The laboratory staff ships the specimens to the CDC laboratory weekly.

The laboratory staff also processes serum for vessel 77 (HPV Serum) on SPs aged 14-59 as part of an SP's blood processing profile. The vessels are stored at $-20^{\circ} \mathrm{C}$ and shipped to the $\mathrm{CDC} / \mathrm{Human}$ Papillomavirus Laboratory weekly.

\subsection{Supplies}

The supplies needed for HPV processing and storage are listed in Exhibit 10-1.

Exhibit 10-1. Equipment and supplies - HPV processing and storage

\begin{tabular}{|l|l}
\hline Large padded envelope & Zip closable bag (9” x 12” clear) \\
\hline
\end{tabular}

\subsection{Protocol}

Exhibit 10-2 illustrates the SP's protocol for HPV processing. It is extremely important to follow the processing procedures outlined in these protocols and in this manual. Specimen loss or bias results will occur if the exact procedures are not followed. 
Exhibit 10-2. HPV processing protocol

\begin{tabular}{|c|l|c|c|c|c|c|}
\hline ID & Test name & Ages & Sample & Sample type & Vessel type & Appointment type \\
\hline 78 & HPV & $14-59$ females & 1 swab & $\begin{array}{c}\text { Vaginal } \\
\text { swab }\end{array}$ & $\begin{array}{c}\text { Epicentre }{ }^{\circledR} \\
\text { Dacron swab }\end{array}$ & Primary only \\
\hline
\end{tabular}

\subsection{Labeling Vessels}

The physician prints the vessel label. It is possible to reprint the label in the lab using the Ad Hoc functionality.

\subsection{HPV Protocol}

Process the Epicentre ${ }^{\circledR}$ Dacron swab with the foam for HPV. Verify that the swab is labeled correctly and that the label is legible.

Access the HPV Processing module for all SPs.

- $\quad$ Vessel 78 - HPV

- $\quad$ Verify that the tip of the Dacron swab in the clear plastic tube is foam and that the top of the tube is marked with a green dot. Verify that the SP ID on the label matches the SP ID on the processing screen. Record the processing results in the HPV module and store the swab at room temperature.

\subsection{Record Processing Results}

The protocol includes a serum vessel (HPV Serum \#77) for primary SPs aged 14-59. The volume requirement is $0.5-\mathrm{mL}$ of serum; the storage vessel is a 2-mL cryovial, and it is stored in a 2-inch box with a 9 x 9 grid. The vessel is processed after the Lipids vessel (\#21) and before the Biochem vessel (\#18.) The serum vessel is displayed on the blood-processing screen in the correct priority and is recorded in the same manner as the other vessels. This vessel is frozen and shipped to CDC/DVRD HPV weekly. 
Select the correct SP and access the Blood Processing module.

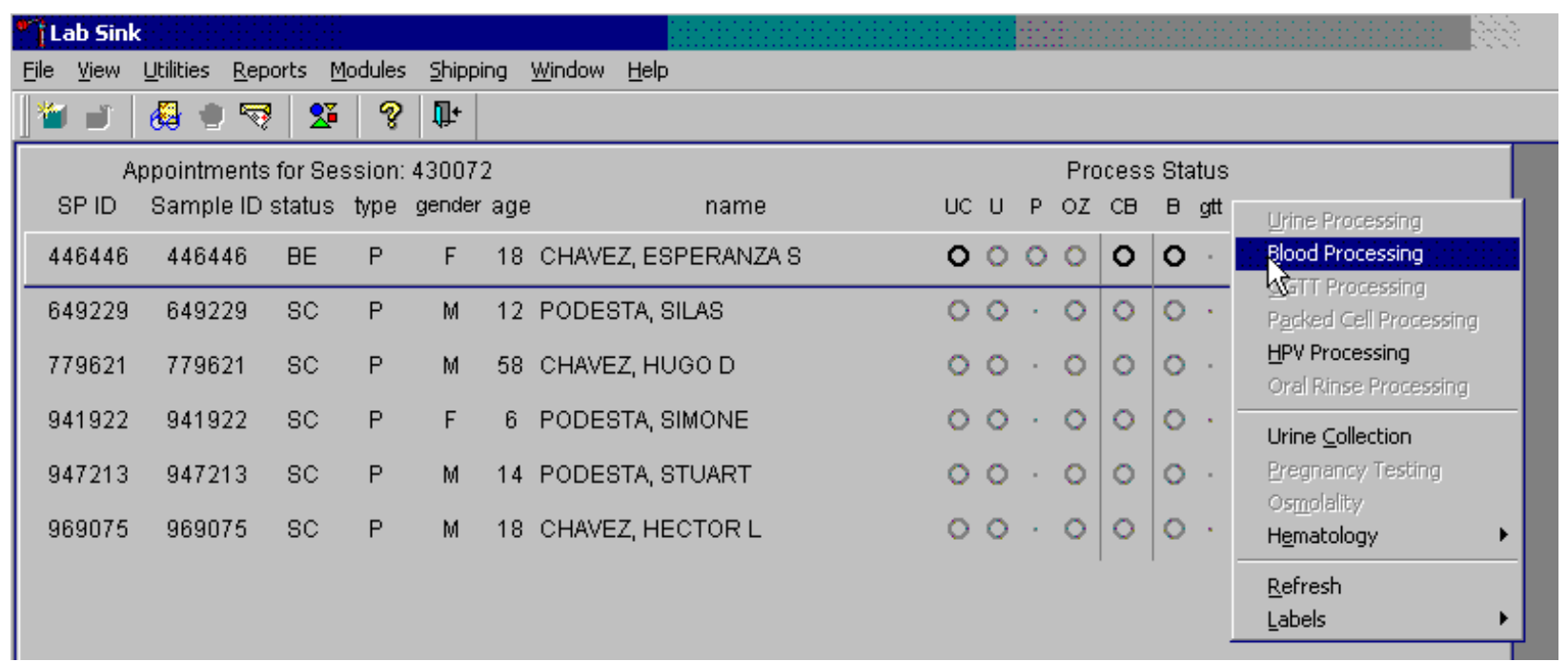

To select an SP, use the up and down keys to move up and down the list until the correct SP is highlighted or drag the mouse arrow to the correct SP and right click. To access the Blood Processing module, drag the mouse arrow to \{Blood Processing\}, and left click or left click and type [B/b]. 
Record processing results for vessel 77 (HPV Serum) in the Blood Processing module.

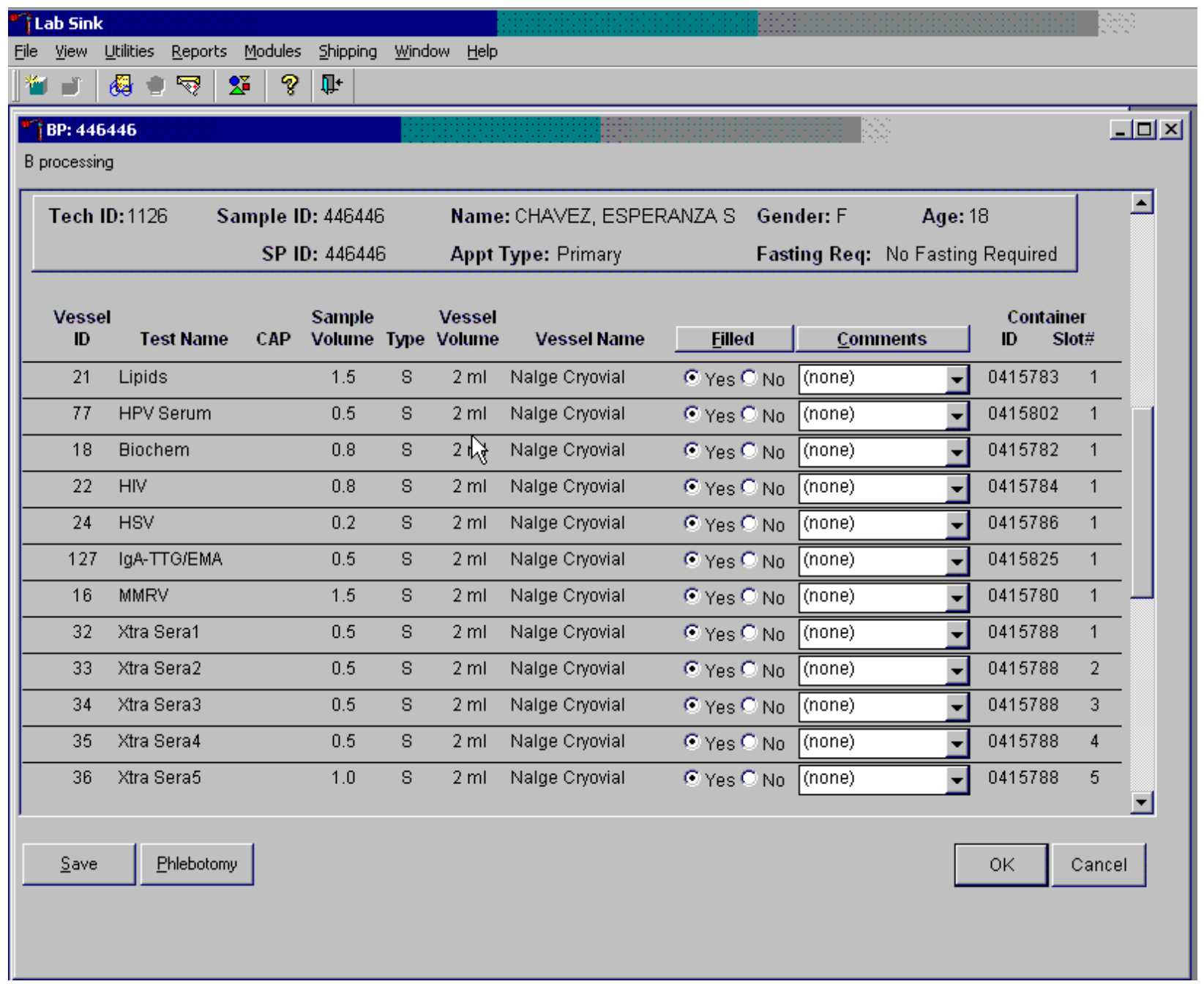

Primary SPs aged 14-59 are eligible for the HPV serum test. Pool the serum and aliquot 0.5$\mathrm{mL}$ into a prelabeled 2.0-mL cryovial. Mark the vessel as Filled -’Yes” or Filled -’No” using procedures described in Chapter 8. Store the vessels in a 2-inch tall container with a 9 x 9 grid. Pack the container in a small shipper, add sufficient dry ice, and ship weekly to CDC/DVRD HPV. Use existing procedures described in Chapter 9 to pack, label, and ship the shipper. 
Select the correct SP and access the HPV Processing module.

\begin{tabular}{|c|c|c|c|c|c|c|c|c|c|c|}
\hline \multicolumn{11}{|c|}{ Lab Sink } \\
\hline \multicolumn{11}{|c|}{ File View Uيtilities Reports Modules shipping Wwindow Help } \\
\hline$\|$ 为 & 娄 & 2 & 8 & 䅦 & & & & & & \\
\hline \multicolumn{7}{|c|}{ Appointments for Session: 430072} & \multicolumn{4}{|l|}{ Process Status } \\
\hline SPID & Sample ID s & status & type & gender & age & name & \multirow{3}{*}{$\begin{array}{l}\text { UC U P OZ CB B att } \\
\text { Urine Processing } \\
\text { Blood Processing } \\
\text { OgGTा Processing } \\
\text { Packed Cell Processing }\end{array}$} & \multicolumn{3}{|c|}{ PC HPV OR } \\
\hline 446446 & 446446 & $\mathrm{BE}$ & $\mathrm{P}$ & $\mathrm{F}$ & 18 & CHAVEZ, ESPERANZA S & & . & 0 & 0 \\
\hline 649229 & 649229 & $\mathrm{sc}$ & $\mathrm{P}$ & M & 12 & PODESTA, SILAS & & 0 & . & \\
\hline 779621 & 779621 & Sc & $\mathrm{P}$ & M & 58 & CHAVEZ, HUGO D & HPV Frocessing & 0 & $\cdot$ & 0 \\
\hline 941922 & 941922 & $\mathrm{sc}$ & $\mathrm{P}$ & $\mathrm{F}$ & & PODESTA, SIMONE & Oral Ahse Processing & . & & . \\
\hline 947213 & 947213 & $\mathrm{sc}$ & $P$ & $M$ & 14 & PODESTA, STUART & $\begin{array}{l}\text { Urine Gollection } \\
\text { Pregnancy Testing }\end{array}$ & 0 & & 0 \\
\hline \multirow[t]{2}{*}{969075} & \multirow[t]{2}{*}{969075} & \multirow[t]{2}{*}{$\mathrm{sc}$} & & \multirow{2}{*}{\multicolumn{3}{|c|}{ M 18 CHAVEZ, HECTOR L }} & $\begin{array}{l}\text { Stmolality } \\
\text { Hematology }\end{array}$ & \multirow{2}{*}{\multicolumn{3}{|c|}{$\cdot 0$}} \\
\hline & & & & & & & $\begin{array}{l}\text { Refresh } \\
\text { Labels }\end{array}$ & & & \\
\hline
\end{tabular}

Use the scroll bar to view all SPs scheduled into the session. The active SP is contained in a rectangular box. Use the heads-up display to select the correct SP. Select a different SP if the active SP is not the correct SP.

To select an SP, use the up and down keys to move up and down the list until the correct SP is highlighted or drag the mouse arrow to the correct SP and right click. To access the HPV Processing module, drag the mouse arrow to \{HPV Processing\} and left click or right click and type [S/s]. Alternatively, use the up and down keys to move up and down the list until the correct SP is highlighted, right click, drag the mouse arrow to the correct module, and left click or type [S/s]. 
Document processing results.

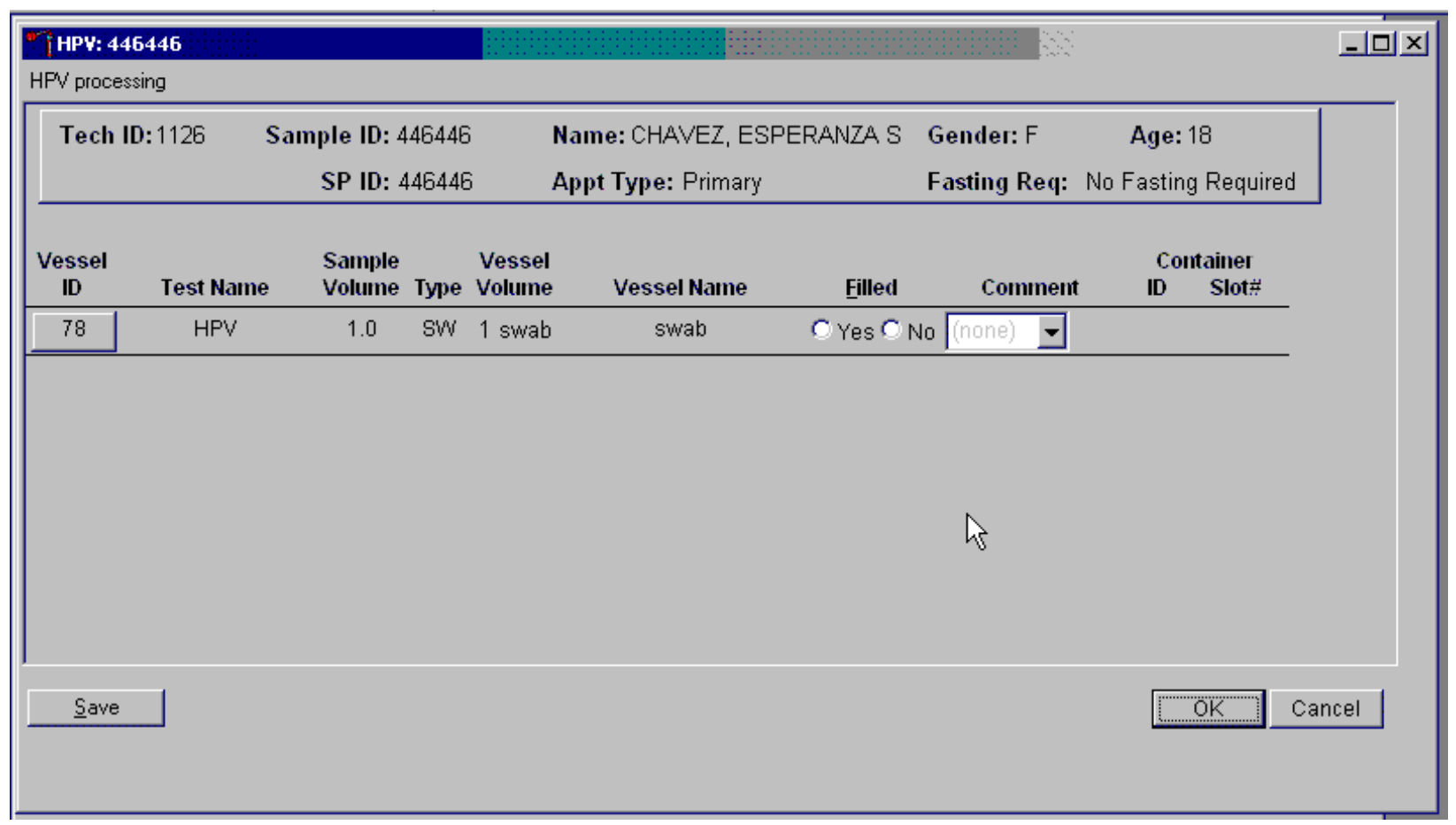

The HPV Processing window contains the following information for SPs: Tech ID, Sample ID, SP ID, Name (last, first), Gender, Age, App (appointment) type, and Fasting_Req. There are columns that list the Vessel ID, Test Name, Sample Volume, Sample Type (SW = swab), Vessel Volume, Vessel Name, Filled (Yes or No) radio buttons, Comment drop-down box, and a Container ID and Container Slot\# column. There is one row associated with vessel 78. Use radio buttons to record the processing results, Filled -"Yes” or Filled -"No.” If necessary, attach a comment using the Comment drop-down box, and store the vessel in the assigned Container ID and Slot\#. 
Mark vessel 78 as Filled-”Yes” if the swab was collected.

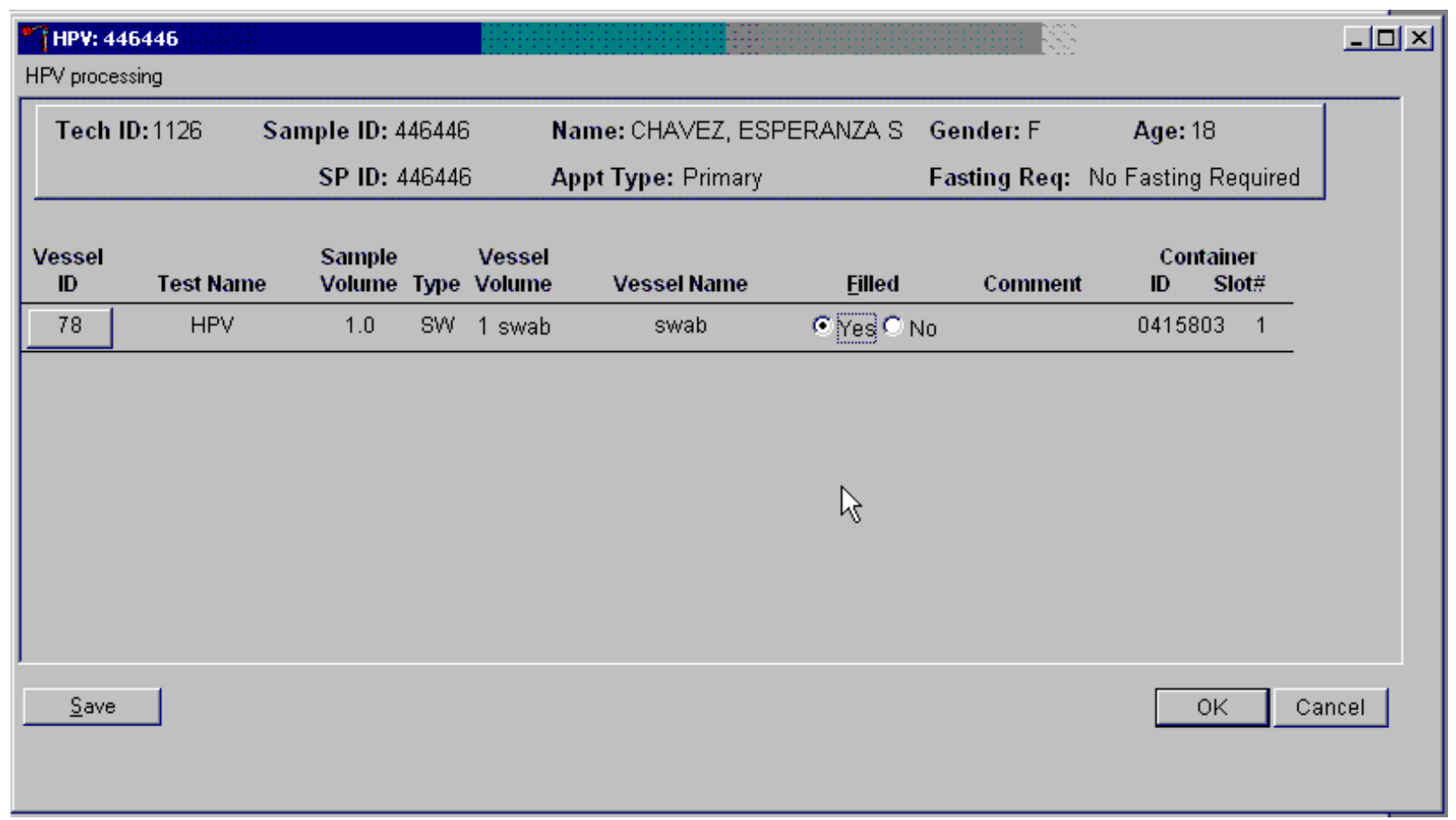

To mark vessel 78 as collected or Filled-"Yes" use the mouse to direct the mouse arrow to the "Yes" radio button and left click. As a vessel is marked as Filled -"Yes," it is automatically assigned to a slot in an existing (open) container. 
Mark vessel 78 as Filled -”No” if the swab was not collected.

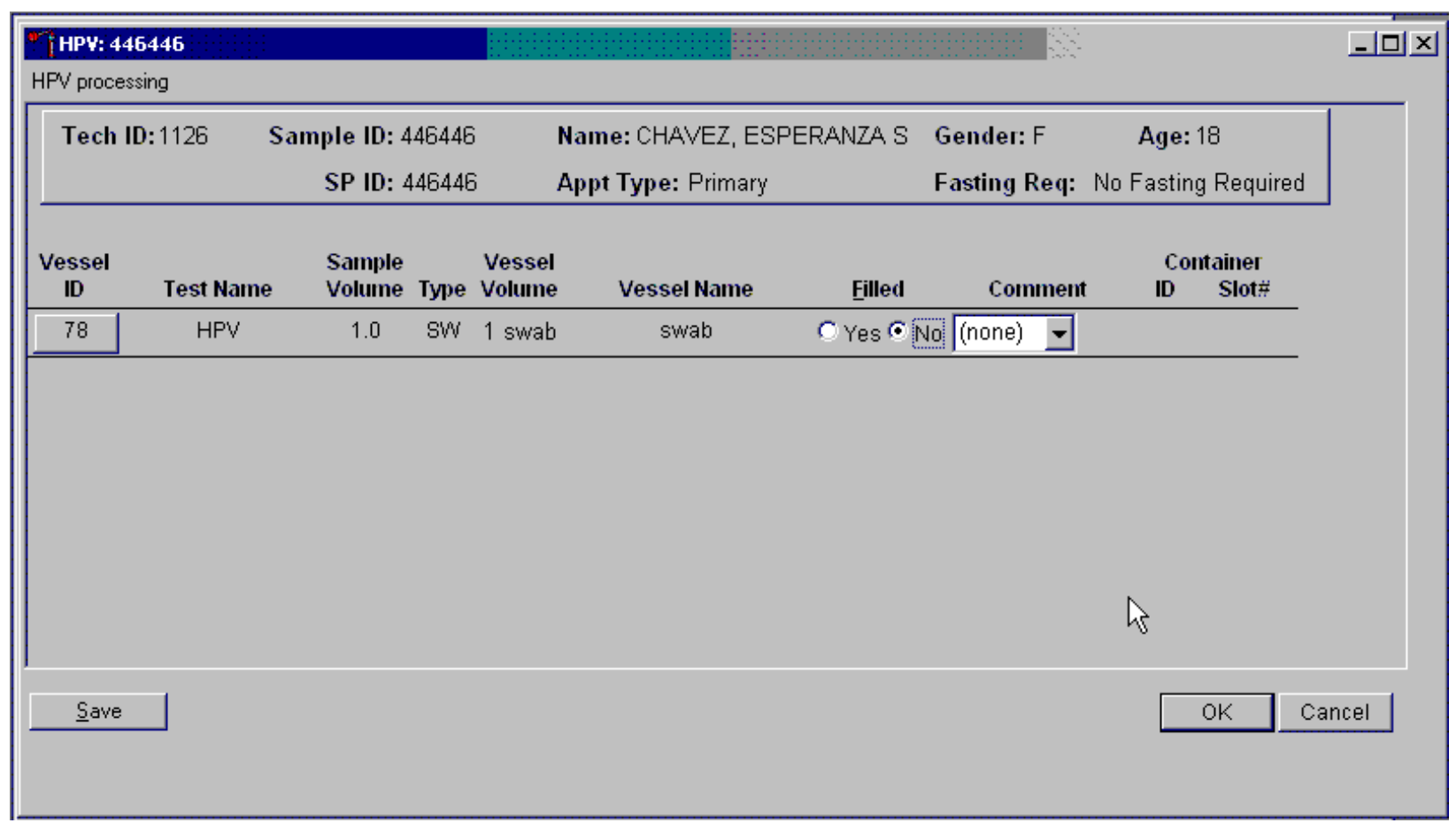

To mark vessel 78 as not collected or Filled -"No" use the mouse to direct the mouse arrow to the "No" radio button and left click. 
Enter a quality control comment for vessel 78, when the swab is dry and the judgment is that the SP did not attempt to collect the sample(s).

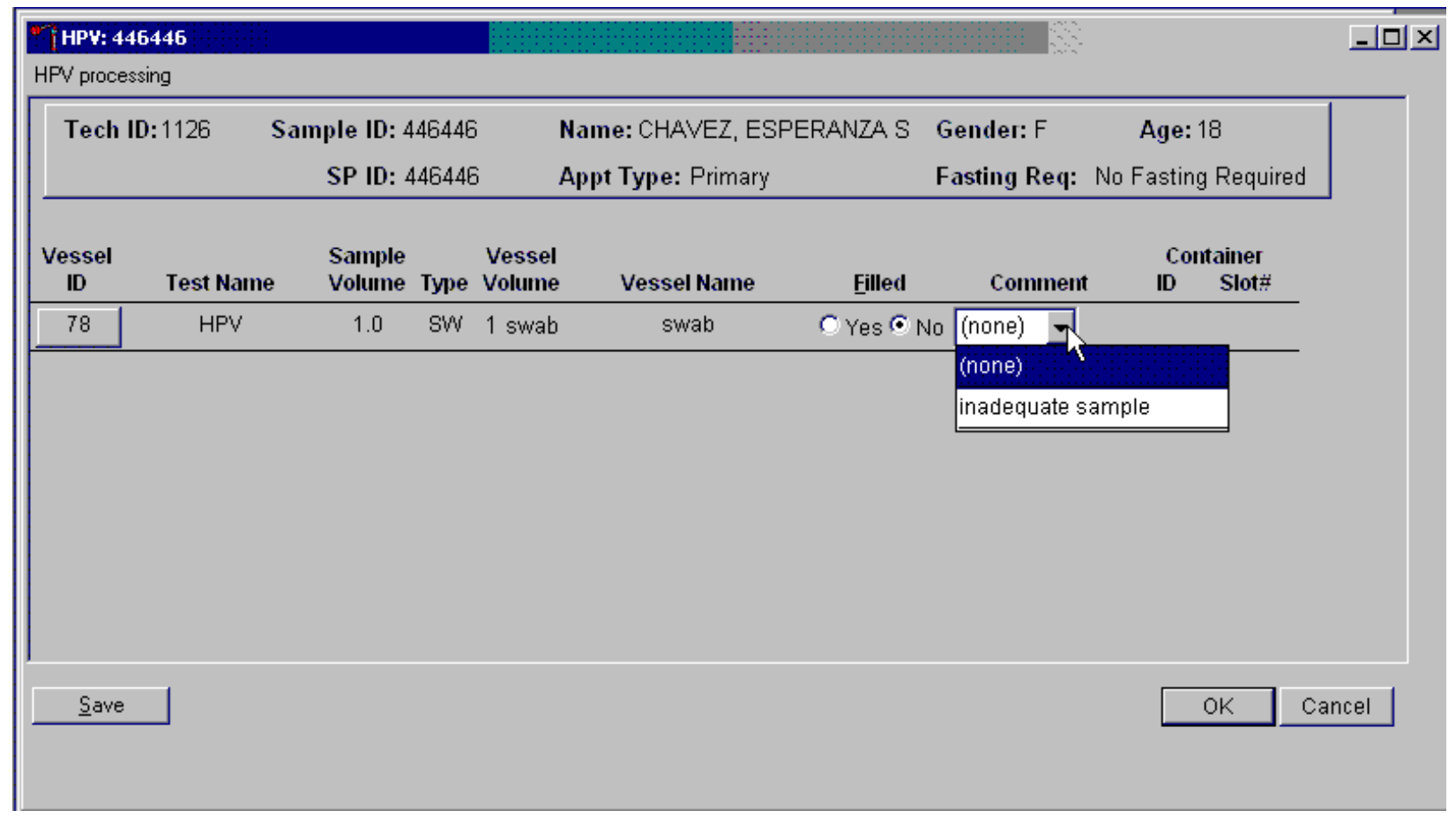

To record an "inadequate sample" comment, use the mouse to direct the mouse arrow to the drop-down list, click to display the comment codes, drag the mouse arrow to "inadequate sample" and left click. Alternatively, to record the comment "inadequate sample," [Tab] to advance to the Comment text box and type [I/I] and [Enter]. 
Store the Epicentre ${ }^{\circledR}$ Dacron swab in the assigned 9” x 12” plastic bag at room temperature; review the information in the HPV-Processing window, and save the data to the database.

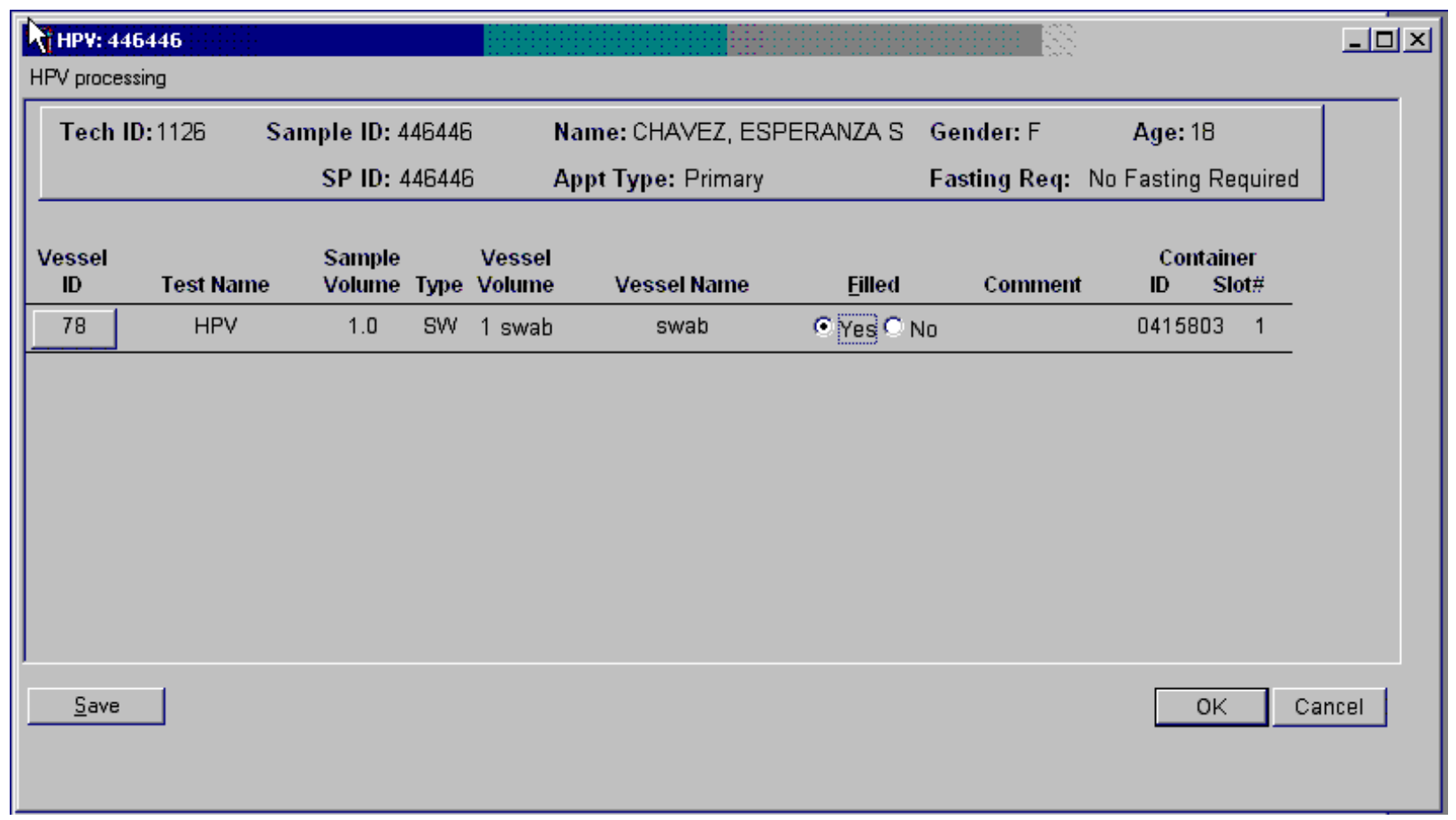

Store each filled vessel in the assigned slot in the assigned container. To record this action or to save the data to the database, use the mouse to direct the mouse arrow to the "Save" button and left click or type $[\mathrm{S} / \mathrm{s}]$. To record this action or to save the data to the database and to exit the module, use the mouse to direct the mouse arrow to the "OK" button and left click. To close the window without saving any data in the database, use the mouse to direct the mouse arrow to the "Cancel" button and left click. 
The process status circle in the heads-up display fills in completely for SPs whose vessel 78 recorded as collected or Filled -"Yes.” Do not enter a result until the swab arrives in the laboratory. At the end of each session, review the results for each SP. Access each record and enter the Filled -"No" result for all vessels for SPs for whom no swab was received.

\subsection{Specimen Storage}

Record the processing result for the Epicentre ${ }^{\circledR}$ Dacron swab and store the swab in a 9” $\mathrm{x}$ 12” plastic bag at room temperature. Store vessels in numbered storage containers according to each vessel as indicated in Exhibit 10-3.

Store each vessel in the appropriate slot in the correct container immediately after processing. Check the slot assignment of containers associated with vessel 78 at the end of each session by accessing the Container Map report. Verify the contents of each container against the container map.

Exhibit 10-3. Storage protocol for HPV

\begin{tabular}{l|l|l|l}
\hline Shipping Location & Vessel & Condition & Vessel Storage \\
\hline CDC/DVRD HPV & 77 HPV Serum & Frozen & $9 \times 9$ two-inch box \\
\hline $\begin{array}{l}\text { CDC/Human Papillomavirus } \\
\text { Laboratory }\end{array}$ & $78 \mathrm{HPV}$ & Room temperature & 9 x 12” plastic bag \\
\hline
\end{tabular}

\subsection{Shipping Procedures}

Ship vessel 77 according to procedures described in Section 9.13. Ship vessel 78 in a padded envelope at room temperature. Include a copy of a shipping manifest, which contains a list of the items contained in the shipment and certain demographic information with each shipment. Exhibit 10-4, the Shipping protocol, illustrates the specifications for the shipment of biological specimens to each contract laboratory. 
Exhibit 10-4. Shipping protocol

\begin{tabular}{l|l|l|l|l}
\hline Laboratory & Vessel & Test Name & Vessel Storage & Shipping Frequency \\
\hline CDC/DVRD HPV & 77 & HPV Serum & Frozen & Weekly \\
\hline $\begin{array}{l}\text { CDC/Human Papillomavirus } \\
\text { Laboratory }\end{array}$ & 78 & HPV & $\begin{array}{l}\text { Room } \\
\text { Temperature }\end{array}$ & Weekly \\
\hline
\end{tabular}

\subsubsection{Pack and Ship Vessel 78 at Room Temperature}

Ship the container (9” x 12” plastic bag) for vessel 78 (HPV swab) at room temperature.

- Place the plastic bag containing the specimens in a large padded envelope.

- Place the appropriate shipping manifest in a 9” x 12” plastic bag and seal.

- Place a plastic envelope containing the appropriate shipping manifest in the padded envelope and seal.

- Assume that the package weighs 1 pound.

- Record the total weight of the envelope (1 pound) on the appropriate FedEx label.

\subsubsection{Label Shippers}

All shippers must contain a FedEx bar-coded label and appropriate orientation and warning labels. Label all shippers with the appropriate warning labels as follows:

- Complete the section of the FedEx label that requests the weight of the contents of the shipper.

- Place the FedEx label with the appropriate contract laboratory address in a plastic FedEx pouch and attach the window to the cardboard lid of the shipper.

- Add one additional strip of tape across the FedEx pouch. 
If the computer system fails, record results on a preprinted HPV Processing worksheet (Exhibit 10-5). Complete a workstation 2 worksheet for each SP while processing the HPV specimens. Enter the results after the system is operational. Send the worksheets to the home office at the end of the stand.

Exhibit 10-5. HPV Processing worksheet

HPV PROCESSING

SP ID

\begin{tabular}{|c|c|c|c|c|c|c|c|}
\hline ID & Name & Ages Gender & Sample mL & Sample Type & pH & $\begin{array}{l}\text { Filled } \\
\checkmark\end{array}$ & Slot \# \\
\hline 78 & $\mathrm{HPV}$ & 14-59 females & 1 swab & $\begin{array}{l}\text { Epicentre }{ }^{\circledR} \\
\text { Dacron Swab }\end{array}$ & & & \\
\hline
\end{tabular}




\section{HPV ORAL RINSE PROCESSING}

\subsection{Introduction}

The overall goal of collecting an oral rinse is to perform the first population-based study of oral HPV infection to determine the prevalence and type distribution of infection and to investigate the demographic and behavioral factors associated with infection.

Testing for 37 types of HPV will be accomplished by means of a multiples polymerase-chain reaction (PCR) assay targeted to the L1 region of the viral genome, using PGMY09/11 L1 primer pools and primers for Beta globulin followed by hybridization to a linear probe array (Roche Molecular.)

The HPV oral rinse is collected by the dental hygienist during the oral health exam on primary SPs aged $14-69$. The SP is asked to swish a $10 \mathrm{~mL}$ sample of Scope mouthwash (or a sterile saline solution if preferred by the SP) in their mouth and then expectorate the sample into a sterile collection cup. The cap is secured on the cup, a label is attached, and the sample is then taken to the MEC laboratory for processing.

The hygienist places the cup in the MEC laboratory in the same area as the urine collection samples. The HPV oral rinse samples are easily distinguished from the urine collection samples by the color of the sample (blue-green unless the clear sterile saline solution is used) and the collection cup (clear and cylindrical with a screw on metal cap) which is much smaller than the urine collection cup.

The MEC laboratory technologists process the sample by transferring the HPV oral rinse from the collection cup to a $14 \mathrm{~mL}$ Falcon snap cap tube. It is critical to transfer the entire sample from the collection cup to the processing vessel. The technologists use the OR (oral rinse) module to document collection status. All SPs are listed on one screen so the samples can be batch processed. They make sure that the snap cap is pressed down fully to prevent leakage during shipment.

The sample is stored frozen at -20 degrees Celsius and shipped weekly to Lab 67, Gillison Laboratory 2001 Polaris PKWY \# 1320 OSU - Pathology Core Facility Columbis, OH 43240-2000 (614)293-5942 


\section{$11.2 \quad$ Supplies}

The supplies needed for HPV Rinse processing and storage are listed in Exhibit 11-1.

Exhibit 11-1. Equipment and supplies - HPV processing and storage

\begin{tabular}{|l|l|}
\hline 14-mL snap cap test tube & Small Styrofoam shipper (14 x 14 x 12) \\
\hline Three-inch 5 x 5 cardboard grid & STP-310 Pressure vessel \\
\hline Absorbent sheets $(2 \times 2)$ & \\
\hline Packaging tape 2” & Plastic bag 9” x 12” \\
\hline Overpack label & \\
\hline Rubber bands & Dry ice \\
\hline
\end{tabular}

\subsection{Protocol}

Exhibit 11-2 illustrates the SP's protocol for HPV Rinse processing. It is extremely important to follow the processing procedures outlined in these protocols and in this manual. Specimen loss or bias results will occur if the exact procedures are not followed.

Exhibit 11-2. HPV Rinse processing protocol

\begin{tabular}{|c|c|c|c|c|c|c|}
\hline ID & Test name & Ages & Sample & Sample type & Vessel type & Appointment type \\
\hline 129 & HPV Rinse & $14-69$ & 1 & Oral rinse & 14-mL snap cap tube & Primary only \\
\hline
\end{tabular}




\subsection{Labeling Vessels}

The dental hygienist prints the label for the specimen cup. The medical technologist assigned to workstation 3 prints the vessel labels for all eligible SPs and places these labels at workstation 2. It is possible to reprint the vessel label in the lab using the ad hoc functionality.

\subsection{HPV Rinse Protocol}

Process the oral rinse for HPV Rinse. Verify that the specimen container is labeled correctly and that the label is legible.

Access the OR Processing module for all SPs.

- Vessel 129 - HPV Rinse

- $\quad$ Verify that the SP ID on the label matches the SP ID on the processing screen.

- $\quad$ Label the 14-mL Falcon snap cap tube.

- $\quad$ Pour the oral rinse from the specimen container into the Falcon tube.

- $\quad$ Snap the cap firmly on the Falcon tube.

- $\quad$ Record the processing results in the OR module.

- $\quad$ Store the vessel in a three inch tall box with a $5 \mathrm{x} 5$ grid.

- $\quad$ Store the container in the freezer.

- $\quad$ Ship weekly frozen. 


\subsection{Record Processing Results}

Open and log onto the Laboratory application. Use the heads-up display to view the SPs, the modules for which they are eligible, and their current process status. Record oral rinse processing results for vessel 129 using the “OR” module.

\begin{tabular}{|c|c|c|c|c|c|c|c|c|c|c|c|c|c|c|c|c|}
\hline \multicolumn{17}{|c|}{ Lab Sink } \\
\hline File view & 느tilities Repc & orts $\underline{M}$ & lodules & Shippir & ing & Window Help & & & & & & & & & & \\
\hline$\| x \in$ & 国 & 8 & 8 & 1) + & & & & & & & & & & & & \\
\hline \multicolumn{7}{|c|}{ Appointments for Session: 410111} & \multicolumn{10}{|c|}{ Process Status } \\
\hline SPID & Sample ID $s$ & status & type & gender & age & name & UC & $\mathrm{U}$ & $\mathrm{P}$ & $O Z$ & $\mathrm{CB}$ & 日 & gtt $\mathrm{PC}$ & $\mathrm{CH}$ & HPV & OR \\
\hline 215591 & 215591 & $\mathrm{SC}$ & VIP & $\mathrm{F}$ & 59 & PATEL, PAVARTI & 0 & 0 & 0 & $\cdot$ & 0 & 0 & $\cdot$ & - & & - \\
\hline 244969 & 244969 & $\mathrm{BE}$ & $P$ & M & 42 & JORDAN, LEE & 0 & 0 & . & 0 & 0 & 0 & $\cdot c$ & 0 & & 0 \\
\hline 470234 & 470234 & $\mathrm{SC}$ & $\mathrm{P}$ & M & 60 & HAGRID, RUBEUS & 0 & 0 & . & 0 & 0 & 0 & $\cdot \mathrm{C}$ & & & 0 \\
\hline 668903 & 668903 & $\mathrm{Cl}$ & $\mathrm{P}$ & M & 12 & WEASLEY, FRED & 0 & 0 & - & 0 & 0 & 0 & $\cdot \mathrm{C}$ & & & - \\
\hline 762731 & 762731 & $\mathrm{Cl}$ & VIP & $\mathrm{F}$ & & BROWN, LAVENDAR & 0 & 0 & . & . & 0 & 0 & · & - & & . \\
\hline 836402 & 836402 & $\mathrm{SC}$ & VIP & M & 12 & MALFOY, DRACO & 0 & 0 & . & . & 0 & 0 & . & - & & . \\
\hline 900081 & 900081 & $\mathrm{SC}$ & VIP & $\mathrm{F}$ & 49 & ABBOTT, HANNAH & 0 & 0 & 0 & · & 0 & 0 & $\cdot$ & - & & · \\
\hline 998878 & 998878 & $\mathrm{Cl}$ & VIP & M & 2 & DURSLEY, VERNON & $\cdot$ & - & · & - & 0 & 0 & . & - & & - \\
\hline
\end{tabular}

The "OR" module is grayed out until at least one SP has the oral rinse collected in the oral health component. Once one SP has the oral rinse marked as collected, the processing circle for that SP turns black. 
Select the correct SP and access the Oral Rinse Processing module.

\begin{tabular}{|c|c|c|c|c|c|c|c|c|c|c|c|c|c|c|c|}
\hline \multicolumn{6}{|c|}{ Appointments for Session: 410111} & & \multicolumn{9}{|c|}{ Process Status } \\
\hline SPID & Sample ID & status & type & gender & age & name & UC & U & $\mathrm{P} O Z$ & $\mathrm{CB}$ & 日 & gtt $P$ & PC & HPV & OR \\
\hline 215591 & 215591 & $\mathrm{SC}$ & VIP & $F$ & 59 & PATEL, PAVARTI & 08 & of & $P$. & 0 & 0 & . & $\cdot$ & . & . \\
\hline 244969 & 244969 & $\mathrm{BE}$ & $\mathrm{P}$ & M & 42 & JORDAN, LEE & Blood Processing & & 0 & 0 & 0 & . & 0 & - & 0 \\
\hline 470234 & 470234 & $\mathrm{SC}$ & $P$ & M & 60 & HAGRID, RUBEUE & $\begin{array}{l}\text { Packed Cell Processing } \\
\text { Pack Prossing }\end{array}$ & & 0 & 0 & 0 & . & 0 & . & 0 \\
\hline 668903 & 668903 & $\mathrm{Cl}$ & $P$ & $M$ & 12 & WEASLEY, FRED & HPy Processing & & 0 & 0 & 0 & . & 0 & $\cdot$ & - \\
\hline 762731 & 762731 & $\mathrm{Cl}$ & VIP & $F$ & 6 & BROWN, LAVEND, & $\begin{array}{l}\text { Oral Rinse Processing } \\
\text { Urine Collection }\end{array}$ & & - & 0 & 0 & · & & $\cdot$ & $\cdot$ \\
\hline 836402 & 836402 & $\mathrm{SC}$ & VIP & M & 12 & MALFOY, DRACO & Bregnancy Testing & & · & 0 & 0 & . & . & $\cdot$ & - \\
\hline 900081 & 900081 & $\mathrm{SC}$ & VIP & $\mathrm{F}$ & 49 & ABEOTT, HANNAH & $\begin{array}{l}\text { Osmolality } \\
\text { Hematology }\end{array}$ &. & p & 0 & 0 & · & - & - & - \\
\hline 998878 & 998878 & $\mathrm{Cl}$ & VIP & M & 2 & DURSLEY, VERNC & $\begin{array}{l}\text { Refresh } \\
\text { Labels }\end{array}$ & - & - & 0 & 0 & · & • & - & $\cdot$ \\
\hline
\end{tabular}

To access the OR module, use the mouse to direct the mouse arrow to the SP on the headsup display, left click, drag the mouse arrow to Oral Rinse Processing, and right click. 
Record the vessel 129 processing results in the Oral Rinse processing window. All SPs are included in the same window but are listed separately by row.

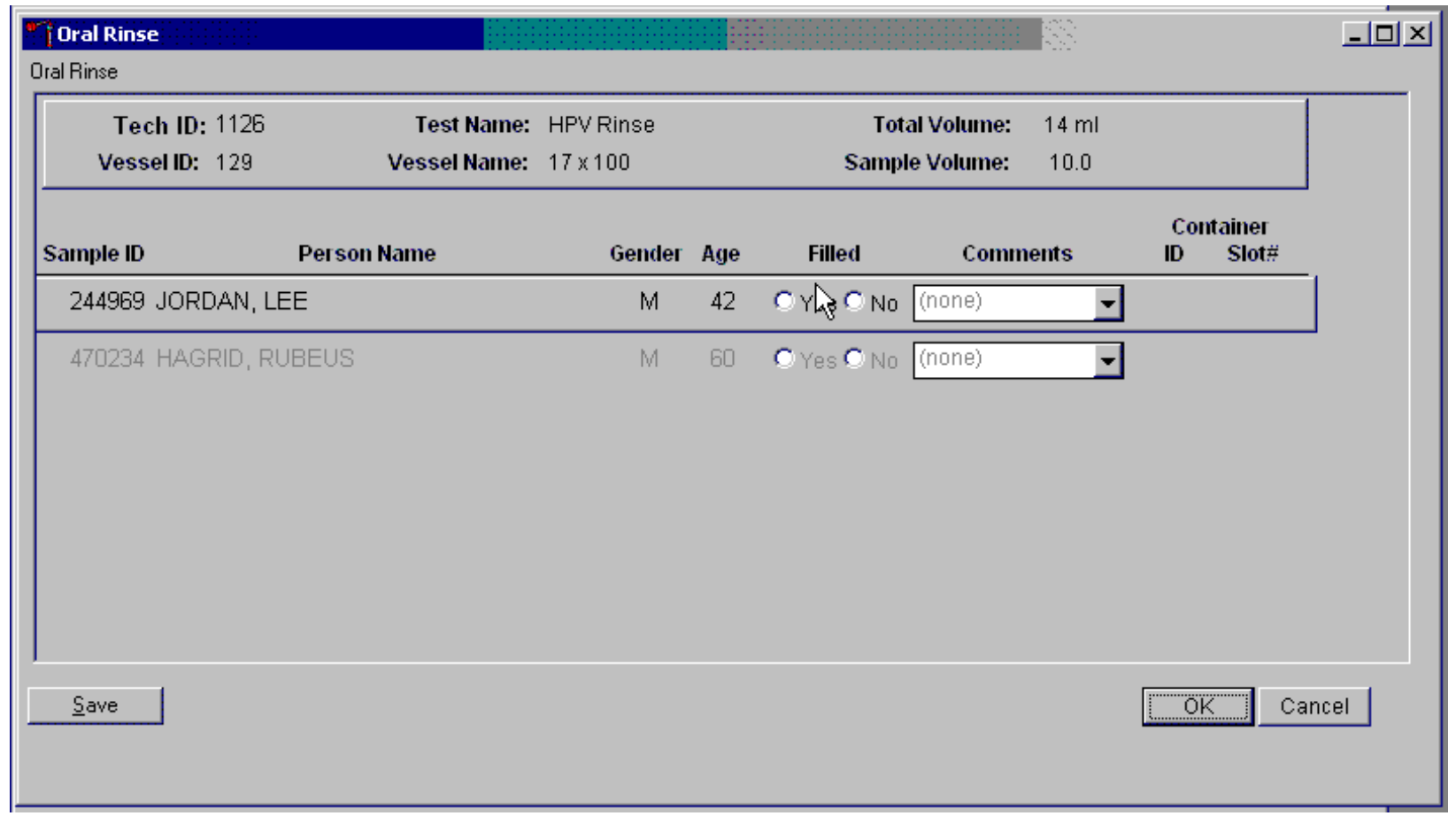

The Oral Rinse processing window lists the vessel requirements across the top. SPs are listed separately in rows. The upper section of the Oral Rinse window contains the following information: Tech ID, Vessel ID (129), Test Name (HPV Rinse), Total Volume (14 ml), Vessel Name (17 x 100), and Sample Volume (10.0.) The lower section of the window contains columns for Sample ID, Person Name (Last, First), Gender, Age, Filled Yes and No radio buttons, Comments text box, and a Container ID/Slot \# column N. Use the Save button to save the results to the database. Use the OK button to save the results to the database and close the module. Use the Cancel button to close the window without saving any data to the database. 
Record oral rinse processing results for each SP. Individually mark each vessel as Filled"Yes."

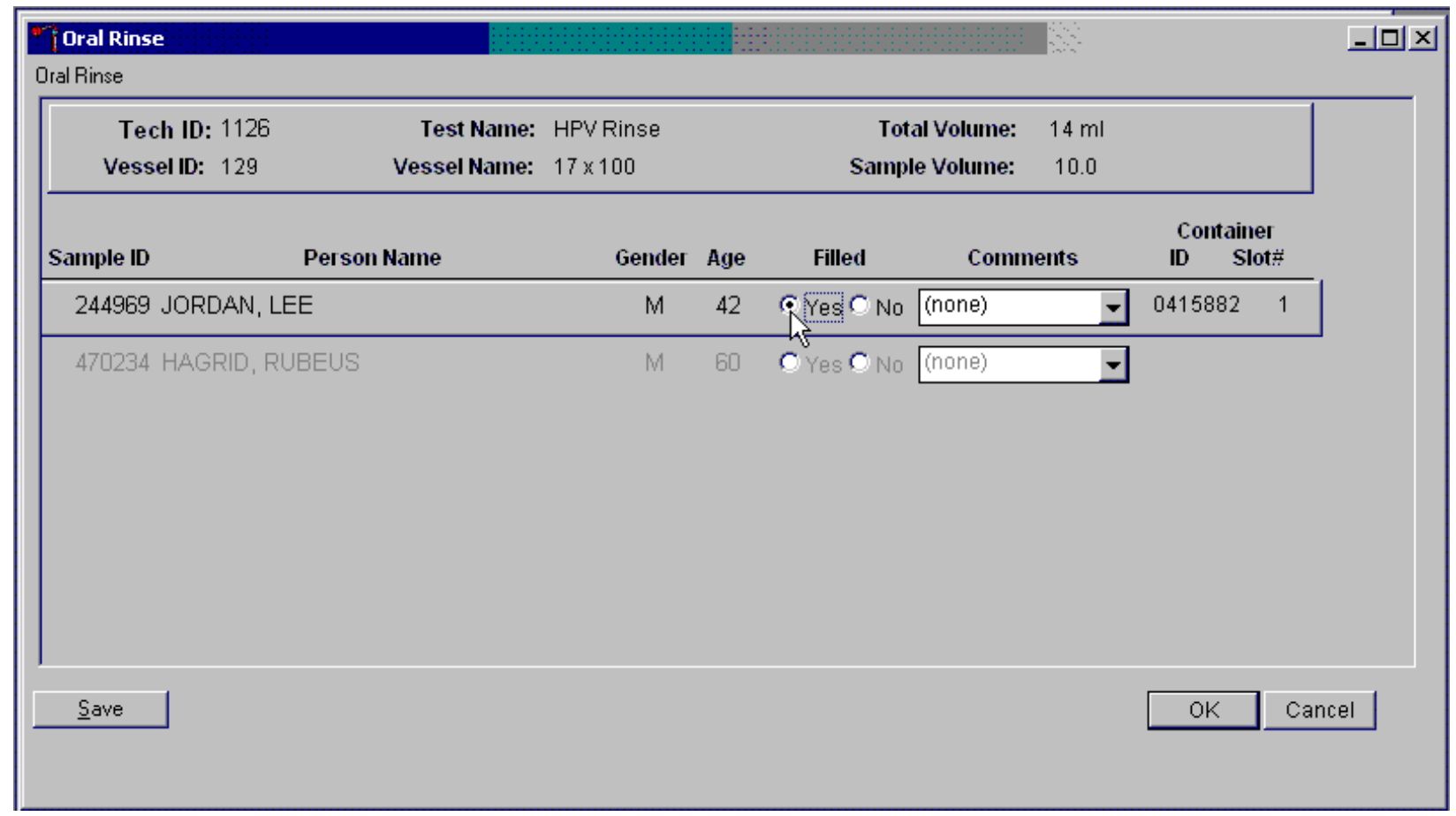

Only SPs who have had the oral rinse marked as collected in the Oral Health application are eligible to be processed. Once the sample is collected, the row changes from gray to black, indicating that the SP is ready to be marked as processed. To mark an individual vessel as collected or Filled-“Yes" use the mouse to direct the mouse arrow to the center of the "Yes" radio button and left click. As each vessel is marked as Filled-“Yes," it is automatically assigned to a slot in an existing (open) container. 
There are no quality comment codes available to describe the quality of the specimen or to explain why a vessel is marked as not filled.

Record oral rinse processing results for each SP. Individually mark each vessel as Filled“No.”

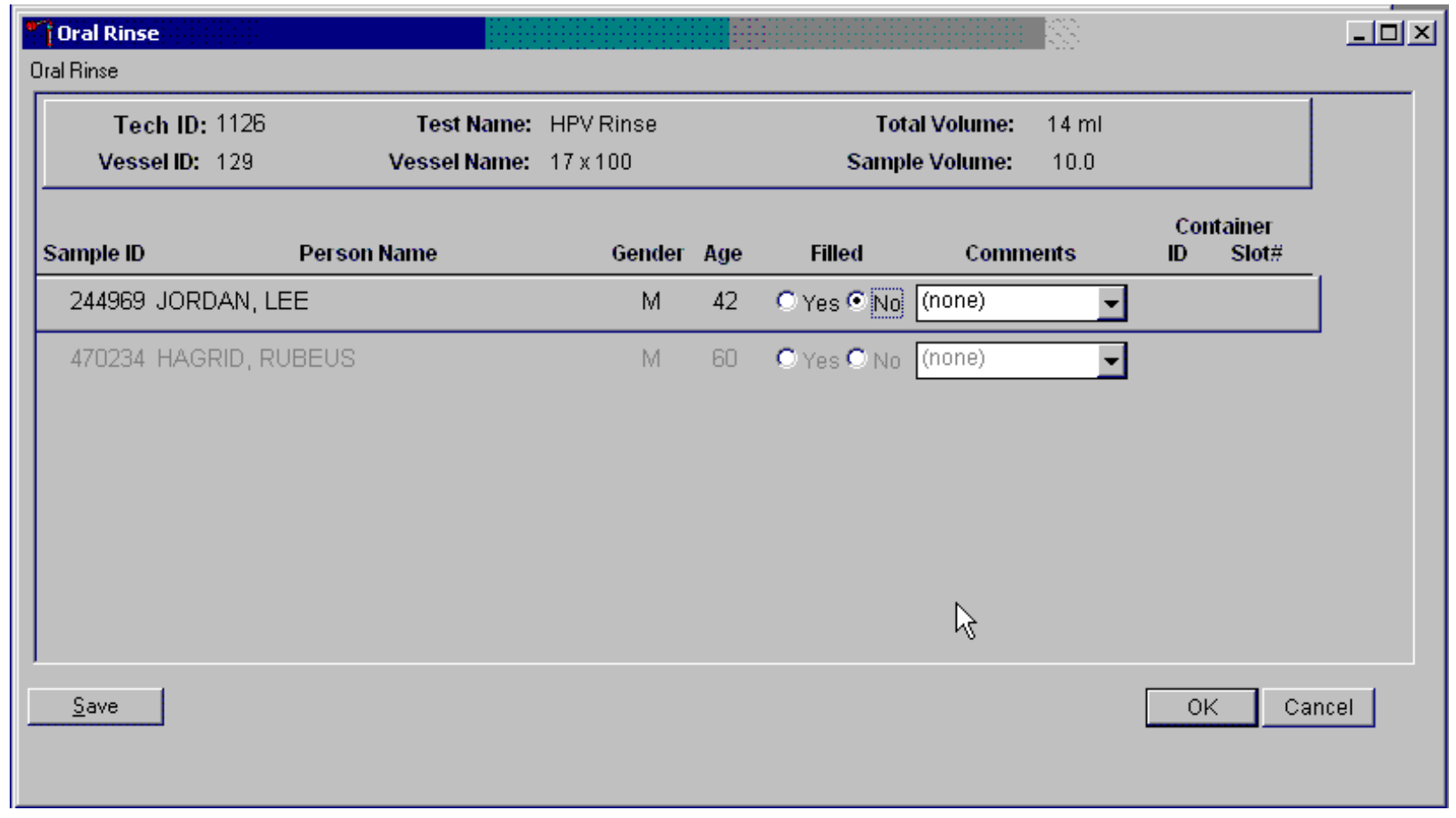

To mark vessel 129 as not collected or Filled-“No," use the mouse to direct the mouse arrow to the center of the "No" radio button and left click. Vessels marked as "No" do not require a comment.

Continue to enter results as vessels are processed. Review the information in the Oral Rinse processing window and save the data to the database.

Store each filled vessel in the assigned slot in the assigned container. To record this action or to save this data to the database, use the mouse to direct the mouse arrow to the Save button and left click. To record this action or to save this data to the database and to exit the module, use the mouse to direct the mouse arrow to the OK button, and left click. To close the window without saving any data in the database, use the mouse to direct the mouse arrow to the Cancel button, and left click. 


\subsection{Specimen Storage}

Record the processing result for the HPV Rinse and store the vessel in a 3-inch tall container with a 5 x 5 grid. Store the container in the refrigerator.

Check the slot assignment of containers associated with vessel 129 at the end of each session by accessing the Container Map report. Verify the contents of each container against the container map.

Exhibit 11-3. Storage protocol for HPV

\begin{tabular}{l|l|l|l}
\hline Shipping Location & Vessel & Condition & Vessel Storage \\
\hline Lab 67 & 129 HPV Rinse & Frozen & 5 x 5 three-inch box \\
Gillison Laboratory & & & \\
2001 Polaris PKWY \# 1320 & & & \\
OSU - Pathology Core Facility & & & \\
Columbis, OH 43240-2000 & & & \\
(614)293-5942 & & \\
\hline
\end{tabular}

\subsection{Shipping Procedures}

Ship vessel 129 according to procedures described in Section 9.13. Exhibit 9-4, the Shipping protocol, illustrates the specifications for the shipment of biological specimens to each contract laboratory.

Exhibit 11-4. Shipping protocol

\begin{tabular}{l|l|l|l|l}
\hline Laboratory & Vessel & Test Name & Vessel Storage & Shipping Frequency \\
\hline Gillison Laboratory & 129 & HPV Rinse & Frozen & Weekly \\
2001 Polaris PKWY \# 1320 & & & & \\
OSU - Pathology Core Facility & & & & \\
Columbis, OH 43240-2000 & & & & \\
$(614) 293-5942$ & & & & \\
\hline
\end{tabular}

Pack shipper according to the procedure described in Section 9.13 
If the computer system fails, record results on a preprinted HPV Oral Rinse Processing worksheet (Exhibit 11-5). Complete a workstation 2 worksheet for each SP while processing the HPV specimens. Enter the results after the system is operational. Send the worksheets to the home office at the end of the stand.

Exhibit 11-5. HPV Oral Rinse Processing worksheet

HPV ORAL RINSE PROCESSING

SP ID

\begin{tabular}{|c|c|c|c|c|c|c|}
\hline ID & Name & Ages Gender & Sample mL & Sample Type & $\begin{array}{l}\text { Filled } \\
\checkmark\end{array}$ & Slot \# \\
\hline 129 & HPV Rinse & $14-69$ & 1 oral rinse & 10-ml Scope & & \\
\hline
\end{tabular}




\section{National Health and Nutrition Examination Survey National Center for Health Statistics \\ United States Public Health Service \\ Procedure: Osmolality Testing \\ January 2009}

Prepared by

Date Adopted

Approved by

Director

Signed

Director

Dated

Director

Reviewed by

Date Reviewed

Reviewed by

Date Reviewed

Reviewed by

Date Reviewed

Reviewed by

Date Reviewed

Reviewed by

Date Reviewed

Reviewed by

Date Reviewed

Reviewed by

Date Reviewed

Reviewed by

Date Reviewed

Reviewed by

Date Reviewed 
THIS PAGE INTENTIONALLY LEFT BLANK. 


\section{OSMOLALITY TESTING}

Introduction

Perform Urine Osmolality Determination on primary sample participants (SPs) who are 6 years and older and dry run odd and even volunteers aged 12+. Perform the test using the random urine specimens collected at the MEC. There are no SP safety exclusions for this test. Perform the test in the MEC laboratory using the Osmette II osmometer instrument.

I. Purpose and Principle of Test

A. Purpose

The osmolality test is a determination of the number of osmotically active solutes present in the urine. It is performed to help evaluate the body's water balance and its ability to produce and concentrate urine. Increased urine output may be due to increased fluid intake, lack of appropriate amounts of $\mathrm{ADH}$, or diabetes mellitus (increased glucose levels leading to increased urine output). Decreased urine output may be due to a variety of causes including decreased blood flow to the kidneys, an appropriate response to dehydration, or damage to tubular cells in the kidneys. Urine sodium and creatinine are often performed along with urine osmolality. Sometimes a urine osmotic gap is calculated and used to help evaluate the kidney's ability to excrete acid and reabsorb bicarbonate, to detect the presence of osmotically active molecules, and to compare with the plasma osmotic gap.

Urine osmolality is dynamic and will fluctuate as the body responds to and corrects temporary water imbalances. Urine osmolality tests must be evaluated in the context of the participant's clinical presentation and along with the findings of other tests, such as sodium, glucose, and BUN. When participants have increased urine output and a low osmolality, then they are either ridding their body of excess fluids or unable to concentrate urine appropriately (which may be due to diabetes insipidus, a lack of $\mathrm{ADH}$ ). If they have increased urine output and a high osmolality, then it may be due to diabetes mellitus. If participants have decreased urine output and high osmolality, they may be dehydrated; if they have low or normal osmolality, they may have kidney damage. Osmolality results are not diagnostic; 
they suggest that a participant has an imbalance but they do not pinpoint the cause. Urine osmolality may be increased with:

- $\quad$ congestive heart failure

- hypernatremia

- inappropriate ADH secretion

- $\quad$ liver damage

- shock

Urine osmolality may be decreased with:

- diabetes insipidus

- excess fluid intake

- $\quad$ hypercalcemia

- $\quad$ hypokalemia

- kidney tubular damage

Within NHANES the osmolality test will be used in conjunction with the urine flow rate. The urine flow rate evaluates the weight of solids in water. Osmolality and urine flow rate usually change in parallel to each other. When large and heavy molecules (such as glucose and protein) are present in the urine, however, the results will diverge. The flow rate will be increased more, due to the weight of the molecules, while urine osmolality will be increased less, reflecting the number of molecules.

\section{B. Principle}

The urine osmolality determination is based upon the principle that increased concentration of a solute in a solution causes lowering of its freezing point. This method is referred to as freezing point depression osmometry. A sample of the specimen to be analyzed is aspirated into the sample tube, which is then placed in the cooling chamber of the osmometer. The sample is super cooled below the freezing point. Then crystallization is initiated by rapidly vibrating the sample to seed it with air bubbles. After seeding, the sample temperature rises because of the heat of fusion released during the freezing process. 
The temperature rises until the equilibrium plateau is reached (Figure 1-2). During the equilibrium plateau only a small fraction of the water is frozen. The sample continues to freeze as the temperature begins to decrease again because of the colder environment.

\section{TYPICAL COOLING CURVE}

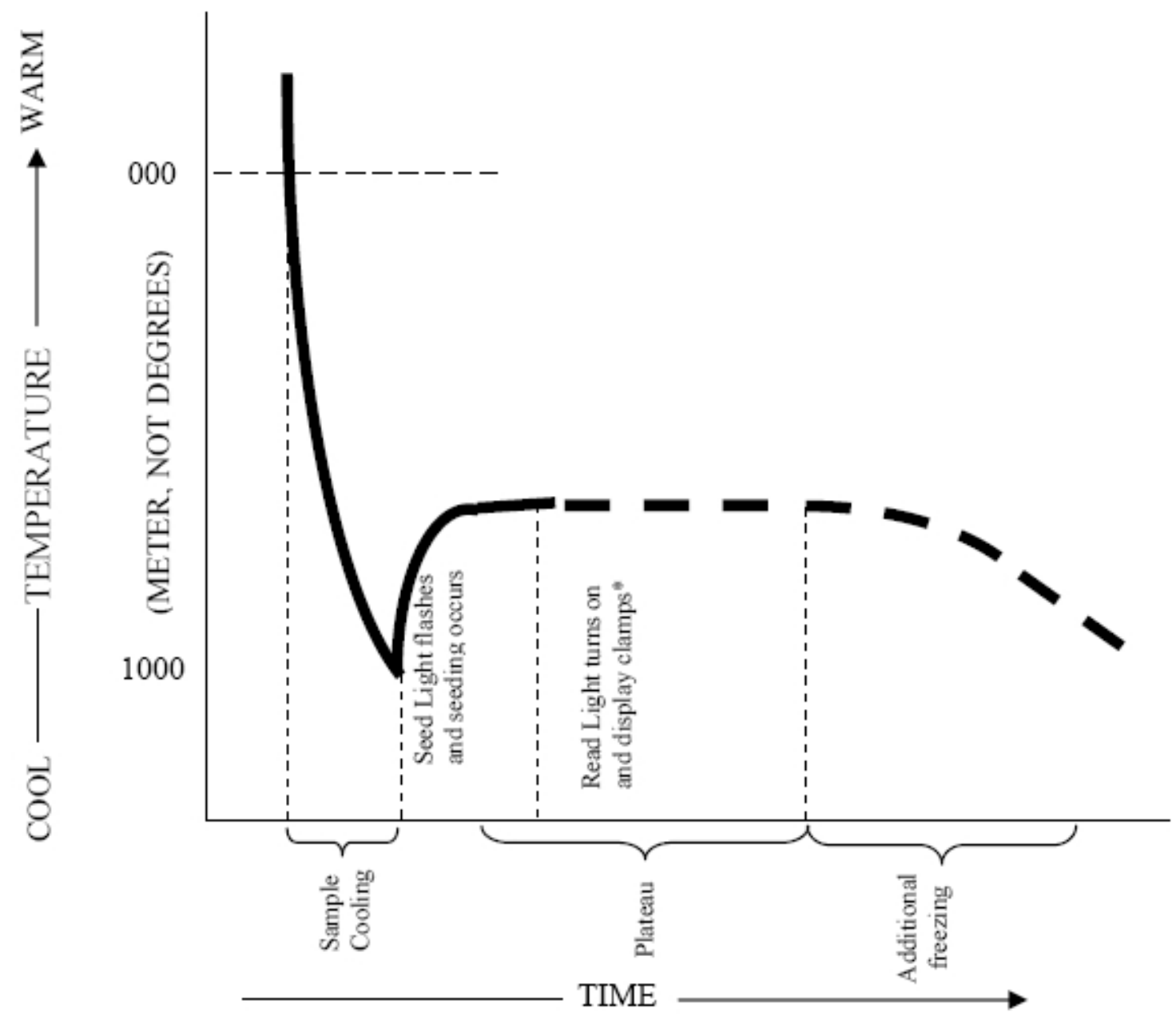

Figure 12-1. Typical cooling curve

Crystallization causes the release of the heat of fusion of water causing a constant temperature or a plateau. This temperature plateau is the freezing point of the urine specimen and is measured by the use of thermistors. Osmometers read directly in mOsm/kg (milli osmoles per kilogram of water) water by converting the thermistor probe (a precise temperature sensor) readings and directly comparing it with readings obtained using standard aqueous salt solutions of known osmolality (Standards). 
The temperature at the plateau is the freezing point of the sample and can be converted to units of osmolality (osmotic concentration) by observing that 1.0 osmole depresses the freezing point of water by $1.858 \mathrm{oC}$, where 1.0 osmole $=1.0$ mole of osmotically active particles.

The most commonly used technique for converting the reading to units of mOsm $/ \mathrm{kg} \mathrm{H} 2 \mathrm{O}$ is direct comparison with Standards of known osmolality. Osmolality is expressed in units of milliOsmoles (mOsm) per $\mathrm{kg}$ of water or $\mathrm{mOsm} / \mathrm{kg}$ where one mOsm is equivalent to one millimole of dissolved solute particles. A solution containing 1 osmole (1000 mOsm) of dissolved solute per kg of water lowers the freezing point of water by $1.585 \mathrm{oC}$. Thus, the freezing point depression of the urine specimen can be converted to units of osmolality or osmotic concentration by dividing it by 1.858 .

II. Special Safety Precautions

All specimens may be potentially positive for infectious agents including HIV and the hepatitis B and C viruses. Observe universal precautions. It is mandatory to wear gloves and a lab coat when handling all human urine products and Quantimetrix ${ }^{\circledR}$ controls. Dispose of all biological samples in a biohazard container and wipe down all work surfaces with 10 percent bleach solution at the end of each session.

The mobile examination center (MEC) Working Safely with Hazardous Chemicals manual contains the material safety data sheets (MSDS) for both the Precision Systems Standards and CONTROL solutions and the Quantimetrix Human Urine Controls.

Neither the Precision Systems Standards nor CON-TROL solutions, nor the Quantimetrix Human Urine controls contain any ingredients that have been determined to be health hazards pursuant to Federal OSHA standards.

The Quantimetrix Human Urine Controls contains human urine. It is recommended that such samples be handled at the Centers for Disease Control Biosafety Level 2. 
III. Computerization: Integrated Survey and Information System (ISIS)

The Osmette II automatically transmits successfully completed SP results to the MEC automated ISIS system. The session number and technologist ID are automatically captured. The medical technologist reviews all SP results in the Osmolality module within the ISIS Laboratory application. The Osmolality QC module captures the QC results from the osmometer. The medical technologist enters the control lot number and expiration date. The Laboratory application evaluates the osmolality data for accuracy based on a preset precision limit. The final decision to accept or reject a result is the responsibility of the medical technologist.

All data are backed up and stored at Westat's home office.

IV. Specimen Collection and Preparation

A. Specimen Collection

The urine sample is collected as a clean catch in a sterile 500 -mL capacity container. The volume of the collection is calculated and the medical technologist records the sample as collected in the ISIS Laboratory application.

\section{B. Specimen Preparation}

The technologist prepares for the urine osmolality test by recording urine collection as either complete or quantity not sufficient (QNS). Once at least 2-mL of urine has been collected on female SPs eligible for pregnancy tests, or 1-mL on all other SPs eligible for urine collection, the urine osmolality test may be completed on the SP. The urine osmolality test requires at least 1-mL of urine. Do not perform osmolality on urines that are cloudy or bloody. Do not spin urine prior to performing the osmolality.

QNS urine samples should be held at ambient temperature until enough urine is obtained from the SP and pooled with the previous specimen(s). This pooling process results in the urine having the same concentration as the environmental samples. 
V. Procedure for Microscopic Examination

Not Applicable - Do not prepare differential microscopic slides.

VI. Equipment, Reagents, and Supplies

The ISIS Laboratory application stores and maintains the lot numbers and expiration dates of QC controls and calibration standards.

A. Equipment

1. OSMETTE II ${ }^{\mathrm{TM}}$ Model 5005, Automatic Osmometer (Precision Systems, Inc). The four Osmette II serial numbers and locations are:

\begin{tabular}{|c|c|c|c|}
\hline Osmette & Location & Serial Number & NCHS Property Tag \\
\hline 1 & MEC 1 & LL0809C & 174145 \\
\hline 2 & MEC 2 & LL0807C & 17412 \\
\hline 3 & MEC 3 & LL0808C & 174144 \\
\hline 4 & Westat (backup) & LL0806C & 174113 \\
\hline
\end{tabular}

2. $10 \mu \mathrm{l}$ Pipette (Precision Systems, Inc.). Use one pipette in each MEC. Keep one backup pipette in each MEC and two backup pipettes in the NHANES warehouse. 


\section{B. Controls and Calibrators}

Use the Precision Systems Osmometry Standards to calibrate the Osmette II machines and validate the calibration. Use the Precision Systems CON-TROL reference standard glass ampules to validate the Standards. Both are manufactured by Precision Systems:

Precision Systems, Inc.

16 Tech Circle

Natick, MA 01760-1029

508-655-7010

1. Precision Systems Osmometry Standards are aqueous solutions of known osmolality containing high purity sodium chloride for the calibration of osmometers. Standard osmolalities are listed on the bottle label. Acceptable ranges are used for quality control only.

- 100 mOsm, 125-mL bottle (catalogue \#2101) - acceptable range 96-104 mOsm

- 500 mOsm, 125-mL bottle (catalogue \#2105) - acceptable range 496-504 mOsm

- $\quad$ 1,500 mOsm, 125-mL bottle (catalogue \#2115) - acceptable range 1485-1515 mOsm

- 2,000 mOsm, 125-mL bottle (catalogue \#2120) - acceptable range 1980-2020 mOsm

2. Precision Systems CON-TROL reference standards are aqueous solutions of known osmolality containing high purity sodium chloride for validation of calibration standards. CON-TROL osmolalities are listed on the glass ampule.

- CON-TROL 100, 100 mOsm reference solution, 5mL ampule (catalogue \#2201) - acceptable range 98-102 mOsm

- CON-TROL 500, 500 mOsm reference solution, 5mL ampule (catalogue \#2203) - acceptable range 496-504 mOsm 


\section{Controls}

The Quantimetrix Human Urine Controls are intended as a means of monitoring human urine assay methods to validate quantification of patient samples. Control materials having known component concentrations are an integral part of diagnostic procedures. Daily monitoring of control values establishes intralaboratory parameters for accuracy and precision of the test method. The controls are manufactured by the Quantimetrix Corporation.

Quantimetrix

2005 Manhattan Beach Blvd.

Redondo Beach, CA 90278-1205

(310) 563-0006

3. Quantimetrix Human Urine Control, Level 1 and 2 are supplied as a ready-to-use liquid requiring no reconstitution. They are prepared from human urine. The Human Urine Controls are fortified to target levels with human proteins and reagent grade chemicals. Preservatives have been added to inhibit microbial growth.

- Human Urine Control, Level 1 Catalog \#1431-31, three 10-mL bottles

- $\quad$ Human Urine Control, Level 2 Catalog \#1432-31, three 10-mL bottles

C. Supplies

1. 50-ml Precision Systems sample tubes (Precision Systems catalog \#2023)

2. Teflon pipette tip piston replacements (Precision Systems catalog \#2036)

3. $10 \mu \mathrm{l}$ disposable pipette tips (Precision Systems catalog \#2048)

4. Cleanettes (Precision Systems catalog \#2048)

5. Distilled water

6. Kimwipes

7. Latex or nitrile gloves

8. Disposable lab jacket -48 inches long

9. Dow 311 Valve Lubricant 
VII. Calibration

Calibration is a procedure to standardize the instrument by determining its deviation from calibration references and to apply any necessary correction factors. The Osmette II stores calibrations. All subsequent valid calibration values are added and averaged into previous calibrations.

A. Precalibration

Perform a calibration after the instrument has been turned on and running for at least 10 to 15 minutes to allow for the Osmette temperature to completely equilibriate.

1. Precalibration procedure

a. Run a 100 mOsm standard through the machine to clean the well and ensure that the electronics and cooler are cycling correctly.

B. Osmette Total Calibration with Standards

Use the Precision Systems 100, 500, 1,500, and 2,000 standards to complete a total calibration. Perform a total calibration:

- At the start of each stand before analyzing samples.

- After cleaning or doing any maintenance on the equipment.

- If the error message “OUTSIDE CALIBRATION” displays.

- If the Precision Systems Representative suggests a calibration.

- Acceptable ranges for Standards used in the calibration procedure:

- Water and 100 Standard - acceptable range +/-1

- 500 Standard - acceptable range $+/-2$

- 1500 Standard - acceptable range $+/-4$

- 2000 Standard - acceptable range $+/-6$

- Note: Run each standard three times. Each standard can be run up to six times as the instrument uses each standard result as a running average. If the standards are out of range, the calibration must be started over by exiting the calibration mode and then reentering the calibration mode. 


\section{Calibration Procedure}

1. Press “CALIBRATE” button.

2. Wipe the well with a Cleanette.

3. Run two distilled water samples. Record the result reading of each in the Lab Start of Stand QC Module.

4. Run two 100 mOsm Standards. Record the result reading of each in the Lab Start of Stand QC Module. Wipe the sample well with a Cleanette.

5. Run three 500 mOsm Standards. Record the result reading of each in the Lab Start of Stand QC Module. Wipe the sample well with a Cleanette.

6. Run three 1,500 mOsm Standards. Record the result reading of each in the Lab Start of Stand QC Module. Wipe the sample well with a Cleanette.

7. Run three 2,000 mOsm Standards. Record the result reading of each in the Lab Start of Stand QC Module. Wipe the sample well with a Cleanette.

8. Press the "CALIBRATE" button again to complete the calibration process. If the "CALIBRATE" button is not pressed again at the end of calibration, the calibration will not be saved.

C. Osmette Troubleshooting Calibration with Standards When QC is Not Within the Acceptable Range

- Note: Run each standard three times. Each standard can be run up to six times as the instrument uses each standard result as a running average. If the standards are out of range, the calibration must be started over by exiting the calibration mode and then re-entering the calibration mode.

- Acceptable ranges for Standards used in the calibration procedure:

- Water and 100 Standard - acceptable range +/-1

- 500 Standards - acceptable range $+/-2$

- 1500 Standard - acceptable range +/-4

- 2000 Standard - acceptable range +/-6 
1. Run a 100 mOsm standard through the machine to clean the well and ensure the electronics and cooler are cycling correctly.

2. Press "CAL” button.

3. Run two100 mOsm reference standards. Wipe the sample well with a Cleanette.

4. Run three 500 mOsm reference standards. Wipe the sample well with a Cleanette.

5. Run three 1500 mOsm Standards. Record the result reading of each in the Lab Start of Stand QC Module. Wipe the sample well with a Cleanette.

6. Run three 2000 mOsm Standards. Record the result reading of each in the Lab Start of Stand QC Module. Wipe the sample well with a Cleanette.

7. Press the "CALIBRATE” button again to complete the calibration process. If you do not press the "CALIBRATE" button again at the end of calibration, the calibration will not be saved.

VIII. Assay Procedure

Run SP urine collection samples in duplicate and average the two results. If the difference between the two values exceeds 40 (four times the SD of 10\%), then run the sample a third time. Average the closest two of the three results. If the final result is based on a single run, then a comment "results based on single run” will automatically be appended to the result. Results are saved in whole numbers.

Osmolality test results will not be reported back to the participant. There are no panic or action limits for this test. If the measured value is $>2,000 \mathrm{mOsm} / \mathrm{kg}$, then the result will be reported as “2,000” with “out-of-range” comment. 


\section{A. Osmette II Instrument Placement}

Allow at least 3 inches on both sides and back of the instrument for placement of the Osmette. The Osmette should not be placed near a heat source or in direct sunlight. Do not block the air outlet (at rear of instrument) and the air intake (at front right side of instrument). Any blockage of the air intake or outlet will slow the cooling of the Cooler Well, so that it will take longer to reach operating temperature. The instrument work area must be free of vibration (avoid benches with centrifuges).

\section{B. Instrument Buttons and Functions}

1. Plug the OSMETTE II into a grounded AC outlet of the proper voltage (115 v AC).

2. The ON/OFF button is located on the rear of the machine. It is a black rocker button. Always make sure the button is in the OFF position when plugging in the instrument.

3. The SPEC button is an unlabeled button on the rear of the machine. The button is a thin white unlabeled stick projecting from the back of the instrument. This button frequently acts as a cancel button. Stops both stabilizing, reading, digit entry, or any special function: acts as an escape key.

4. The CALIBRATE button initiates instrument calibration. The CAL button is located on the right front console of the instrument.

5. The RUN button initiates running a reading, whether calibrating or running an unknown. The RUN button is located on the right front console of the instrument.

6. The shutter is a metal cover that will automatically close and protect the cooler well. The shutter opens when the instrument is turned on. It will close to clamp around the pipette when a sample is run. It will close after approximately 10 minutes of inactivity to protect the cooler well from dust and debris. Always check to ensure the shutter is open before wiping the cooler well or running a sample. To open the shutter after it has automatically closed, the instrument operator must manually open the shutter:

- $\quad$ Press the SPEC button on the back of the machine.

- $\quad$ Press the run key twice to display "SPEC2” on the Osmette screen.

- $\quad$ Press the CALIBRATE key. The shutter will open.

The shutter closes after 10 minutes of inactivity regardless of the pipette or Cleanette sitting in the cooler well. The medical technologist should avoid leaving the pipette in the instrument after a sample has been run. The medical technologist should also not leave Cleanettes in the cooler well between samples. If a sample (or Cleanette) is left sitting in the cooler well and the shutter has clamped around it, follow the above instructions to open the shutter. Do not manually force it open or pull the pipette or Cleanette out. 


\section{Proper Pipetting Technique}

Proper pipetting technique is critical to accurate osmolality results. Take several steps to ensure that the pipette is functioning properly:

1. Check the pipette calibration daily as described in VIII. F and Section 15.10.

2. Wipe the pipette wire and Teflon tip with a Kimwipe before attaching a disposable pipette tip. This will absorb any residual moisture from the previous sample and prevent inadvertently drawing the sample too far up into the pipette tip from residual liquid attraction.

3. When placing a disposable pipette tip on the pipette, ensure that the pipette tip snaps onto the pipette. If it is not properly seated, the entire pipette tip will come off when the piston is depressed. Check this by depressing the pipette piston once when a new pipette tip is attached and before drawing the sample. If the pipette tip immediately comes off, firmly reattach it. If that does not correct the problem, discard the pipette tip and affix another one to the pipette. Recheck.

4. If the disposable pipette tip is properly seated on the pipette and the Teflon tip does not give any resistance against the pipette tip when depressing the pipette piston, change the Teflon tip as described in Section 15.10.

5. Ensure that, when the pipette is placed in the sample, that the pipette tip is fully submerged to avoid accidentally drawing air bubbles in the sample.

6. Wipe the end of the pipette tip with a Kimwipe after drawing the sample. Make sure that the end of the drawn sample is flush with the very tip of the pipette. Over wiping the sample may draw some of the sample out of the end of the pipette and lead to premature sample freezing from air bubbles. If the sample is not flush with the end of the pipette, discard the sample and pipette tip and redraw the sample.

7. Check to make sure there are no other air bubbles in the sample. If there are other air bubbles visible in the sample, discard the sample and pipette tip and redraw the sample.

8. Always check the air bubble between the sample and the Teflon tip. Familiarity with the correct air bubble size is critical to recognizing a properly functioning pipette. The correct air bubble size is shown in Exhibit 12-1. If the air bubble appears smaller or larger than the picture, dispose of the plastic pipette tip and drawn sample and check the pipette calibration. Check to make sure sufficient resistance is felt from the Teflon tip when a new disposable pipette tip is inserted on the instrument. Inspect the pipette for any undetected damage, such as a bent pipette wire. Ensure that there is enough sample for a full sample draw and that the disposable pipette tip is properly seated on the instrument. 
Exhibit 12-1. Note the air bubble between the blue-green liquid sample and the white Teflon pipette tip

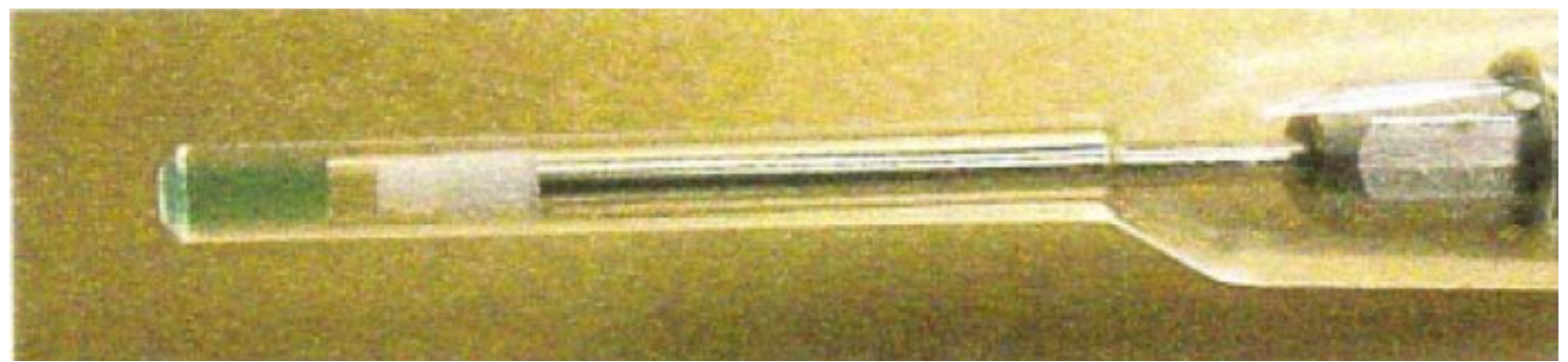

9. When placing the pipette into the Osmette II, ensure that the pipette is placed in with the thumb and forefingers (the same finger position as gripping a dart) as opposed to the same hand position used for pipetting. This is to prevent against the thumb accidentally bumping or depressing the piston, even just a tiny bit, while the pipette is in the osmometer.

D. Running SP Samples

It is critical that the Osmo application is running on the ISIS computer before running any SP samples. This application captures results from the Osmette II. Any samples run without the Osmo application running will be lost and will need to be rerun. To open the Osmo application, double click on the icon of a pipette on the desktop:

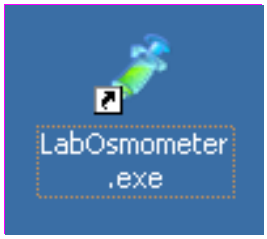

The application icon will display in the system tray when the application is running. It is very faint, but will turn green when data are transmitted from the Osmette to the ISIS computer. Double check to make sure this icon is visible before running SP samples.

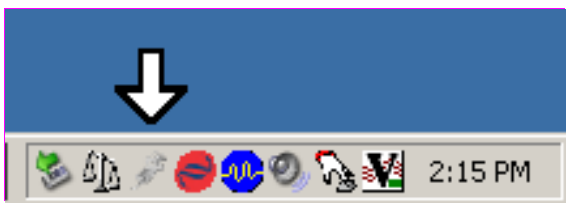

1. Switch (at rear of instrument) to ON, and allow the cooler well to cool down. Allow the instrument to run for 15 minutes to warm up to equilibrate all the circuitry before running SP samples or QC.

2. Notice that the green display turns on, displaying "COOLER STABILIZING." 
3. In a few minutes, the display will change to "WIPE BEFORE NEXT SAMPLE," indicating that the refrigeration system has reached operation temperature and the instrument is ready to use. Do not attempt to use the OSMETTE until this change, as the refrigeration system will be too warm to give satisfactory results.

4. Push a Cleanette all the way to the bottom of the cooler well. The Cleanette is placed in the cooler well and twisted to soak up moisture from the previous samples. The tip of the Cleanette will cover the probe when inserted all the way into the well. Rotate it to remove any moisture. Be careful to insert the Cleanette in a straight down motion. Twist the Cleanette in the cooler well by spinning the Cleanette between thumb and forefingers - do not bend the Cleanette while wiping the well. Remove it by pulling it straight out to avoid bending the probe. Remove and dispose of the Cleanette after use. A fresh Cleanette is used with each sample.

\section{Do not place a sample in an unwiped cooler well.}

5. Examine the urine collection sample. If blood is present, do not run the sample. This is to protect the probe from additional protein. Mark the sample as not done with comment code "blood present." If particulate matter is present, centrifuge the sample before running as described in Section IV.B.

6. Attach a clean, dry pipette tip to the pipette. Verify the function of the pipette as described in Section VII.B.

7. Scan the bar code (Figure 12-2) on the urine collection cup.

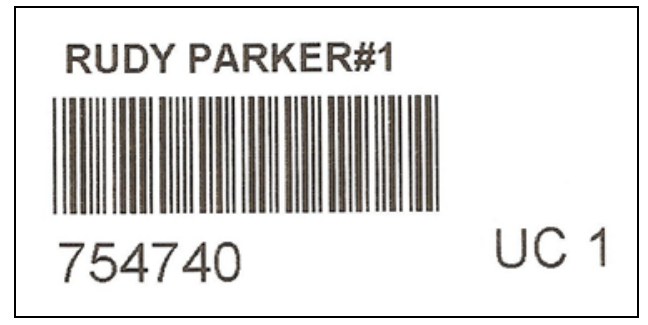

Figure 12-2. Example of a urine collection bar-code label

8. Using the Osmette II pipette draw $10 \mathrm{uL}$ of sample directly out of the urine collection cup. Carefully pipette samples to avoid capturing air in the bottom of the pipette tip. Dry the outside of the pipette tip with a Kimwipe tissue. Do not wipe the pipette with any other material.

9. Place the pipette tip in the cooler well. The Thermistor Probe is located at the bottom center of the cooler well. The pipette places the pipette tip with the sample into the bottom of the cooler well, surrounding the Thermistor Probe with the sample. The sample remains in the pipette tip, with the pipette attached, when it is placed in the cooler well. 
10. Press RUN to initiate the osmolality test. The instrument screen will display the sample ID of the specimen. If no sample ID is displayed, use the SPEC button to cancel the test. Scan in the bar code for the sample before rerunning the test.

11. Observe the Display: The number displayed is an indication of the approach to the seeding temperature. The sample is fully cooled when this number decreases to 0 . During this process the Osmette shutter will clamp onto the pipette to secure the pipette for seeding.

\section{Do not attempt to remove the pipette while the shutter is clamped to the pipette.}

12. When the display reaches approximately 0 , the sample is automatically seeded by a vibration of the shutter. If the display does not reach 0 , and vibration does not occur, READ will not be displayed, and no reading can be made. Rerun the sample.

13. After a few seconds, READ will be displayed, together with the result in mOsm. The shutter will then open to allow for removal of the pipette and for cleaning the cooler well.

14. The osmolality measurement is complete. Before leaving the instrument or placing a new sample in the cooler well, use a fresh Cleanette to wipe the cooler well.

\section{Do not leave the Cleanette in the cooler well.}

15. Eject the used pipette tip into the biohazards container and wipe the Teflon tip of the pipette with a Kimwipe before attaching a new disposable pipette tip for the next sample.

E. Daily Shut-down Procedures

1. Wipe cooler well with a Cleanette before turning off the instrument. This is to ensure that there is no residual moisture in the cooler well before shutting off the instrument.

2. Turn off the instrument using the rocker button located on the back of the instrument. Turn the instrument off after each session. There is no standby mode for the instrument and turning it off when not in use will prolong the instrument's life.

F. Verifying the Pipette Calibration

The pipette requires two small instruments to verify that it is calibrated correctly and will draw the proper amount of solution into the disposable pipette tip. One calibrator resides in the clear red box with the replacement Teflon tips. This calibrator is made of a hard black plastic and is about an inch long. It has a nib on one end. This calibrator measures the size of the bubble that is held between the Teflon tip and the sample when in the disposable pipette tip. To ensure the bubble is the proper size place a clean disposable pipette tip on the pipette. Depress the 
plunger. Insert the nib into the end of the disposable pipette tip. The end of the nib should just touch the end of the Teflon tip; the tip should not push the tool out, and oppositely there should be no space gap between the tool and the Teflon tip.

If the bubble is not properly calibrated, remove the disposable pipette tip and unscrew the lock nut at the base of the pipette wire. Hold the plunger firmly with one hand while unscrewing the lock nut with the other. Use a pair of pliers if necessary. Unscrew the lock nut until it is loose. The pipette wire will also feel loose. Be careful not to turn the pipette wire accidentally when unscrewing the lock nut. Controlled, small turns will adjust the pipette wire to change the calibration setting. Turn the pipette wire in a clockwise direction (if looking at the end of the pipette tip head on) to pull the pipette tip up into the pipette and therefore make the bubble size larger. Turn the wire clockwise to adjust the pipette so the calibration tool will insert further up into the end of the clean pipette tip. Turning the wire counterclockwise adjusts the pipette so that the calibration tool will close the gap between the calibration tool and the Teflon tip. The tool and the tip should just touch when properly calibrated. When the desired adjustment has been made, tighten the lock nut down with your fingers.

Place a clean disposable pipette tip on the pipette. Use the black calibration tool again to check the bubble size. Readjust if needed by following the above procedure. Once the calibration is successful, tighten the lock nut so that it will not come loose during regular use. Be careful not to damage the pipette.

The second instrument required to calibrate the pipette resides on the pipette itself. The end of the plunger opposite the tip has a tool that reads "Calib Gage \& Key." This tool can be unscrewed from the end. The length of the tool measures the correct draw amount for the pipette. To measure the draw amount place a clean disposable pipette tip on the pipette. Without depressing the plunger, insert the calibration gage into the end of the disposable pipette tip. The end of the calibration gage should just touch the end of the Teflon tip.

If the draw amount is not properly calibrated, twist the pipette plunger around until the nut on the inside of the plunger aligns with the hole in the side of the pipette body. Use the tiny hex wrench on the end of the calibration gauge to carefully turn - usually not more than a quarter turn - the nut inside the hole to loosen plunger. Use the slack in plunger to move wire with Teflon tip towards end of pipette tip. Use the hex wrench as a guage to push the wire with 
Teflon tip back so it's calibrated. Immediately tighten the hex nut. Verify that the pipette is calibrated by depressing the piston and use the calibration gage to see if the draw amount is now correct. Readjust if needed by following the above procedure. Be sure to replace the calibration gage in the end of the pipette when calibration is complete.

\section{Changing the Osmette II Pipette Teflon Tip}

Change the Teflon tip if any of the below are encountered:

- The beginning of each stand.

- The resistance of the tip when depressing the pipette appears to be too loose.

- The air bubble separation above the sample is gone after a reading.

- The readings are erratic.

Replace the Teflon pipette tip. Remove the pipette tip from the disposable pipette tip or the protective cover. The Teflon tip is the tiny white plastic on the end of the pipette wire. The tip can be removed several ways. Any method is acceptable as long as care is taken to not bend the pipette wire or damage the pipette in any other way. A razor blade can be used to carefully cut along the side of the tip to cause it to release from the end of the wire. The tip will also come off by using a fingernail and some patience to work a space between the tip and wire and then push the tip off.

Locate the small clear red box labeled “Osmette Teflon Tips and Calibrator.” Use one of the new Teflon tips inside the box as the replacement. The Teflon tip has one end that is solid and one end with a hole. The end with the hole should be placed on the end of the pipette wire. Ensure that the wire is fully inserted into the new tip. There should be no gap between the white tip and the pipette wire.

\section{G. Cleaning, Opening, and Oiling the Osmette II Probe}

The Osmette II may need to have the probe cleaned for several reasons:

- The instrument displays a large amount of "NO FREEZE” errors.

- The instrument displays a large amount of "PRE FREEZE" errors.

- The readings are erratic. 
Clean the Osmette II probe. Wet the end of a Cleanette with and alcohol prep pad. Use as many pads as needed to ensure the end of the Cleanette is wet. Insert the wet end of the Cleanette into the probe well and wipe by rotating the Cleanette left and right. Remove the Cleanette. Wet the end of a Cleanette with distilled water. Insert the wet end of the Cleanette into the probe well and wipe by rotating the Cleanette left and right. Remove the Cleanette. Insert a dry Cleanette into the probe well and wipe by rotating the Cleanette left and right. Remove the Cleanette. This process should clean any residual proteins left in the well.

Over time some sample may leak out into the probe chamber because of leakage due to loss of surface tension (as in the case of proteins in the sample or improperly drawn samples). The only way to clean this residue is to open the probe chamber and wipe off the entire probe, base, and chamber. Open the Osmette II for cleaning the probe chamber. Ensure that the power button on the back of the Osmette is switched to "Off." Unplug the instrument from both the electrical outlet and the computer. Very gently turn the instrument over and lay it on its top so that the underside is tilted towards you. The bottom of the instrument is exposed. There is a black plastic piece on the underside of the instrument known as the "coffin" for its shape. The coffin acts as a cover for the probe. Open the coffin by using a Phillips head screwdriver to loosen the single screw on the left hand, broad end of the coffin. Carefully remove the loose screw and place it somewhere secure so it will not get lost. The loose end of the coffin can now swing up to reveal an opening underneath.

Inside the opening is a large black piece with a white circle in the middle. The white circle is made out of a rubbery, softer material. In the white circle is a white hard plastic donut with a white wire coming out. Using gloved hands, use your fingertips to gently unscrew the white donut by turning it to the left. Once the donut is loose, gently pull it straight out of the probe chamber, being careful not to touch the probe tip to the sides of the chamber. The probe is on the end of the white plastic donut. It is made of a fine metal wire, much like a pin. At its base is a flat surface with a black circle surrounded by a metal circle.

To clean the Osmette II, use only a Cleanette to wipe around the base of the probe (black circle and metal circle) to remove any residual sample not picked up by normal cleaning. Look down into the probe chamber on the instrument. On each side of the chamber are threads to fasten the donut back in place. Use a cleanette to wipe around the threads and the ring at the bottom of the chamber to absorb any residual sample. 
On a rare occasion the probe base may need to be oiled. Only oil the probe if recommended by the Precision Systems technician. To oil the probe use a tiny dab of Dow 311 industrial lubricant on the end of a Cleanette. Wipe the lubricant around the base of the probe (black and metal circles). The lubricant should be spread very thinly. The purpose of the lubricant is to ensure that no sample seeps down into the break between the black circle and the metal circle at the base of the probe. The lubricant also helps to create surface tension to hold the sample in the pipette tip. Use a clean Cleanette to wipe off any globs of lubricant on the base of the probe.

Once cleaning and/or oiling is complete, put the Osmette II back together. Carefully direct the probe back down into the probe chamber. Use your fingers to gently tighten the white plastic donut into the white circle. The donut should only be finger tight; do not overtighten. Swing the coffin back down into place so that the hole in the coffin for the screw lines up with the hole in the osmette, and the probe opening is completely covered. Use the screw and Phillips head screwdriver to fasten in place. Do not over fasten.

IX. Reportable Range of Results

Not applicable.

X. Quality Control

A. Standards and CON-TROL Principle

Both the Precision Systems Standards and CON-TROL Standards are aqueous solutions of known osmolality. CON-TROL Standards are used to verify the accuracy of the Standards by equal comparison. The Standards verify the accuracy of the Osmette II instrument by running a solution of known osmolality as an unknown.

B. Standards and CON-TROL Storage and Handling

Precision Systems Standards should be stored in upright bottles at room temperature. They should not be diluted. 
Always:

- Squeeze a small amount of the Standards solution into a disposable container or into the sink. This is to clear out the pour spout and remove any salt crystals that may have formed.

- Pour (never pipette) the Standards into the Precision Systems sample tubes and immediately snap the cap shut on the sample tubes. Clearly label the tubes with the Standards level using a permanent marker.

- Use only the amount of solution needed to gain acceptable pipetting technique and results. Under normal circumstances, this will be about two to five drops of Standards solution.

- $\quad$ Recap or snap down the pour spout on the Standards bottle quickly after dispensing. This is to prevent evaporation, which will change the osmolality of the solution, and to prevent microbiological contamination.

- Discard the sample tubes containing Standards after calibration or a QC process. This is to ensure fresh standards are used for every procedure. Never return a Standard to the bottle.

Discard Standards bottles when:

- There is evidence of turbidity or other microbiological contamination.

- Crystals appear on the outside of the bottle or ampule.

- The current date is past the expiration date on the bottle.

- Only 30 percent of the contents of the bottle remain.

Open the Precision Systems CON-TROL Standards glass ampules carefully. To use, pour directly from the ampule into the Precision Systems disposable test tube. Never pipette directly from the ampule. Discard the ampule after one use; never save and reuse an open ampule.

\section{Quantimetrix Controls Principle}

The Quantimetrix Human Urine Controls are intended as a means of monitoring human urine osmolality results to validate quantiation of patient samples. Control materials having known component concentrations are an integral part of diagnostic procedures. Daily monitoring of control values establishes intralaboratory parameters for accuracy and precision of the test method. 


\section{Quantimetrix Controls Storage and Handling}

The Quantimetrix Human Urine Controls are supplied as a ready-to-use liquid requiring no reconstitution. They are prepared from human urine. The Human Urine Controls are fortified to target levels with human proteins and reagent grade chemicals. Preservatives have been added to inhibit microbial growth.

Though the Quantimetrix Human Urine Controls have been tested nonreactive for Hepatitis and HIV antibodies, no known test product can assure that a product derived from human blood does not contain these viruses. Therefore, the controls should be handled according to the Centers for Disease Control's Biosafety Level 2 recommendations.

Always:

- Treat the controls as an SP sample.

- Store the controls refrigerated at $2-8$ 


\section{E. Running Quality Control Samples}

It is critical that the ISIS Osmo application is running on the ISIS computer before running Quantimetrix control QC samples. This application captures results from the Osmette II. Any Quantimetrix QC samples run without the Osmo application running will be lost and will need to be rerun. Precision Systems Standards and CON-TROL results are recorded manually and do not require the Osmo application to be running.

To open the Osmo application, double click on the icon of a pipette on the desktop:

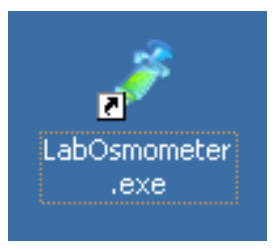

The application icon will display in the system tray when the application is running. It is very faint, but will turn green when data are transmitted from the Osmette to the ISIS computer. Double check to make sure this icon is visible before running Quantimetrix samples.

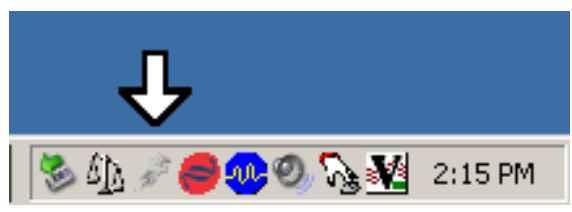

Start the Osmette II instrument and run samples.

1. Switch (at rear of instrument) to ON, and allow the Cooler Well to cool down. Allow the instrument to run for 15 minutes to warm up to equilibrate all the circuitry before QC samples.

2. Note that the green display turns on, displaying "COOLER STABILIZING."

3. In a few minutes, the display will change to "WIPE BEFORE NEXT SAMPLE," indicating that the refrigeration system has reached operation temperature and the instrument is ready to use. Do not attempt to use the OSMETTE until this change, as the refrigeration system will be too warm to give satisfactory results.

4. Push a Cleanette all the way to the bottom of the cooler well. The Cleanette is placed in the cooler well and twisted to soak up moisture from the previous samples. The tip of the Cleanette will cover the probe when inserted all the way into the well. Rotate it to remove any moisture. Be careful to insert the Cleanette in a straight down motion. Twist the Cleanette in the cooler well by spinning the Cleanette between thumb and forefingers - do not bend the Cleanette while wiping the well. Remove it by pulling it straight out to avoid bending the probe. Remove and dispose of the Cleanette after use. A fresh Cleanette is used with each sample. 


\section{Do not place a sample in an unwiped cooler well.}

5. Attach a clean, dry pipette tip to the pipette. Verify the function of the pipette as described in Section VII.B.

6. For Quantimetrix QC samples, scan the bar code for the appropriate QC level:
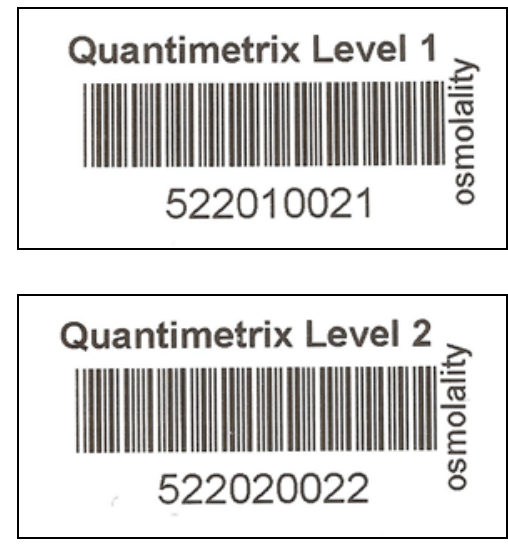

Figure 12-3. Example of Quantimetrix bar-code labels

This step is not required for Precision Systems Standards and CON-TROL samples.

7. Using the Osmette II pipette draw $10 \mathrm{uL}$ of sample directly out of the Precision Systems sample tube. Carefully pipette samples to avoid capturing air in the bottom of the pipette tip. Dry the outside of the pipette tip with a Kimwipe tissue. Do not wipe the pipette with any other material.

8. Place the pipette tip into the cooler well. The Thermistor Probe is located at the bottom center of the cooler well. The pipette places the pipette tip with the sample into the bottom of the cooler well, surrounding the Thermistor Probe with the sample. The sample remains in the pipette tip, with the pipette attached, when it is placed in the cooler well.

9. Press RUN to initiate the osmolality test.

10. Observe the Display: The number displayed is an indication of the approach to the seeding temperature. The sample is fully cooled when this number decreases to 0 . During this process the Osmette shutter will clamp onto the pipette to secure the pipette for seeding.

\section{Do not attempt to remove the pipette while the shutter is clamped to the pipette.}

11. When the display reaches approximately 0 , the sample is automatically seeded by a vibration of the shutter. If the display does not reach 0 , and vibration does not occur, READ will not be displayed, and no reading can be made. Rerun the sample. 
12. After a few seconds, READ will be displayed, together with the result in mOsm. The shutter will then open to allow for removal of the pipette and for cleaning the cooler well.

13. The osmolality measurement is complete. Before leaving the instrument or placing a new QC sample in the cooler well, use a fresh Cleanette to wipe the cooler well.

\section{Do not leave the Cleanette in the cooler well.}

14. Eject the used pipette tip into the biohazards container and wipe the Teflon tip of the pipette with a Kimwipe before attaching a new disposable pipette tip for the next QC sample.

15. Run each Quantimetrix Human Urine Control twice, adding a third run if needed.

16. Run each Standard or CON-TROL according to the run iterations defined in the QC module, repeating runs if necessary. 


\section{F. Record Precision Systems Standards and CON-TROLs in ISIS}

Access the ISIS QC module. Manually enter the QC results.

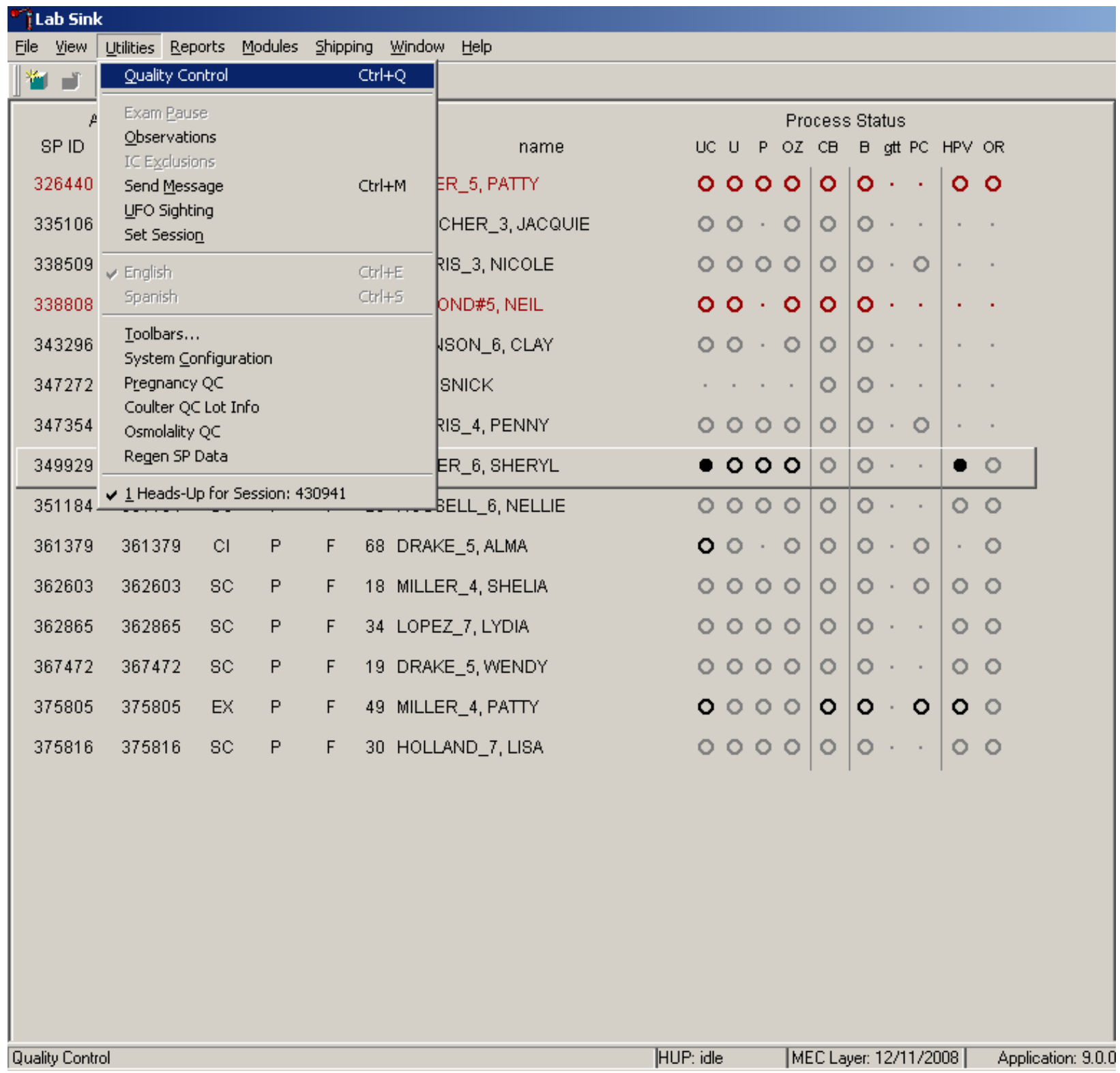

Standards and CON-TROL QC are found under the Lab Quality Control module. 


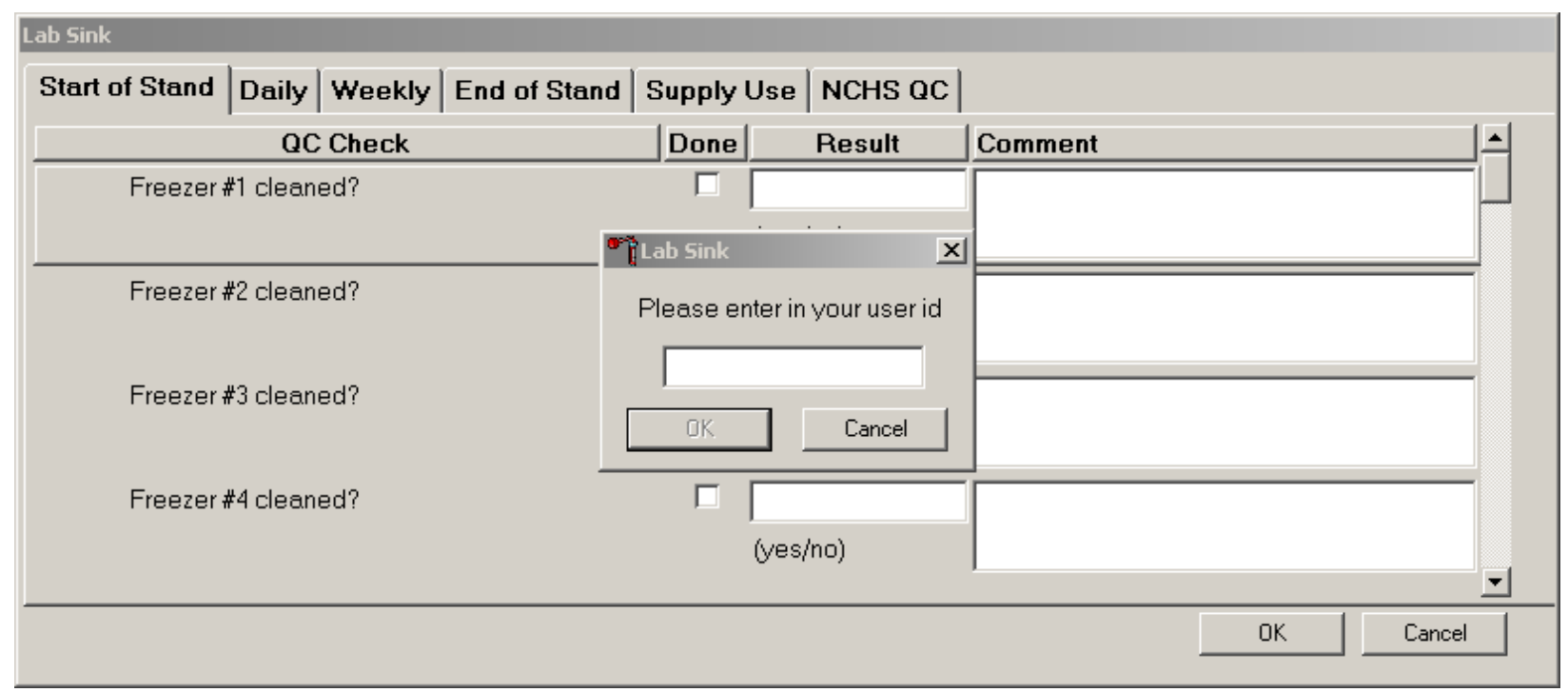

Enter the user ID of the tech running the QC samples.

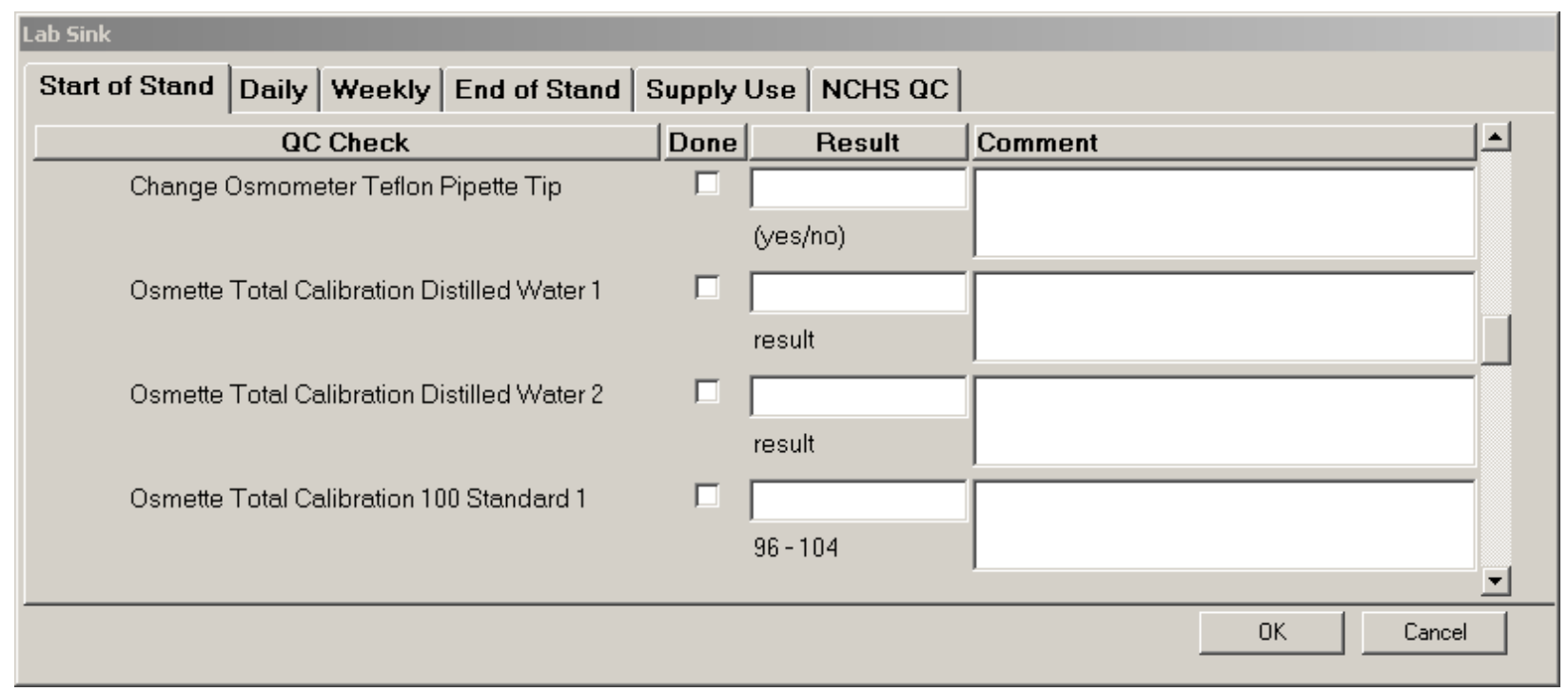

Under the Start of Stand tab, use the scroll bar on the right side of the screen to scroll down to the beginning of the Osmette Start of Stand QC tasks. 


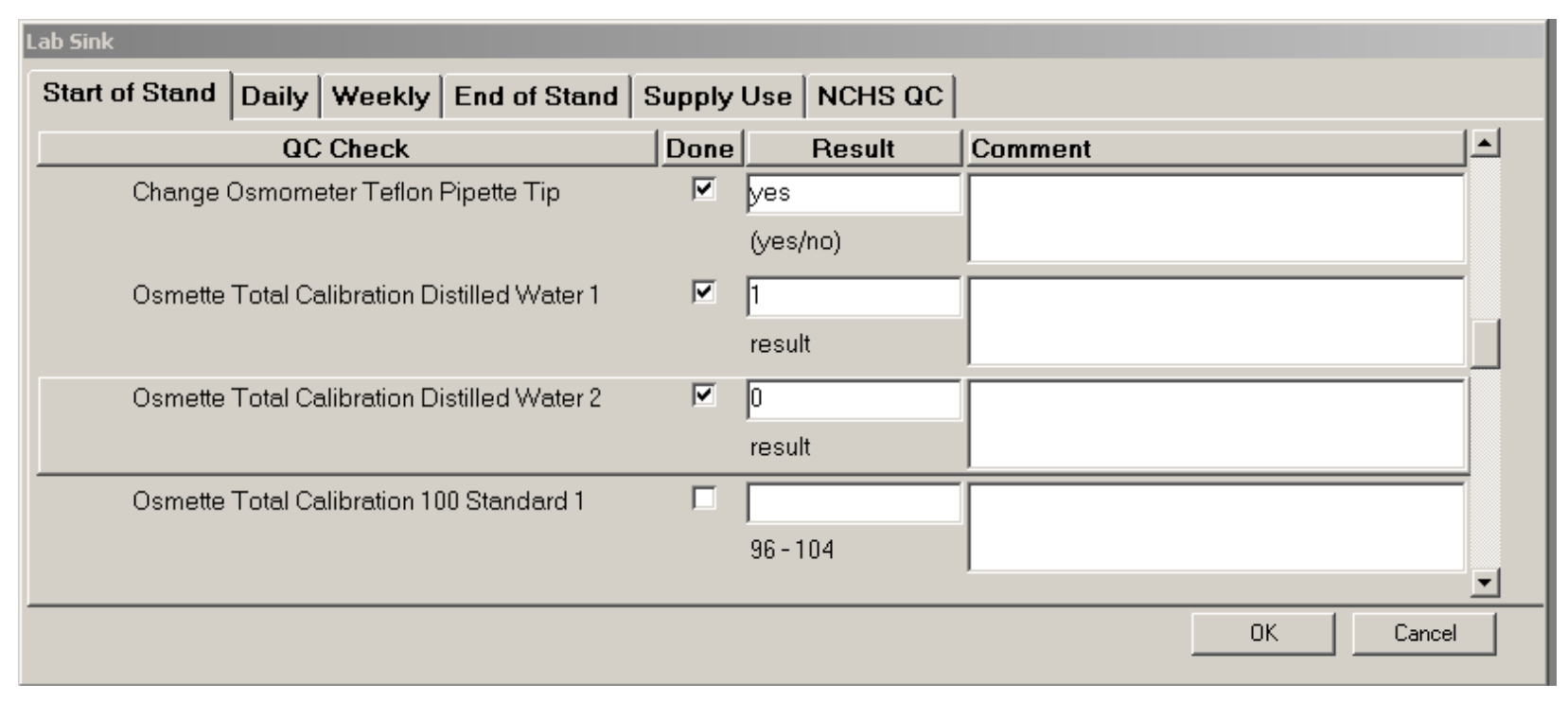

The first task is to change the Osmometer Teflon Pipette tip. As each task is completed, direct the mouse to the box under the "Done" column and right click. This will place a check mark in the box to verify that the task was completed. Note the desired entry under each box in the "Result" column. This is the type of result that should be entered in the Result column. A result (yes/no) indicates a response to verify that the task was completed. Some tasks require a result entry. If there is a valid range displayed below the box, make sure that the result entered is within that range. If the result is out of range, make a note in the comment column.

For Osmolality QC, each required run of a QC is listed. If additional runs for a type of QC are required, note the results in the Comment column.

Osmolality QC appears under the Daily QC tab. 


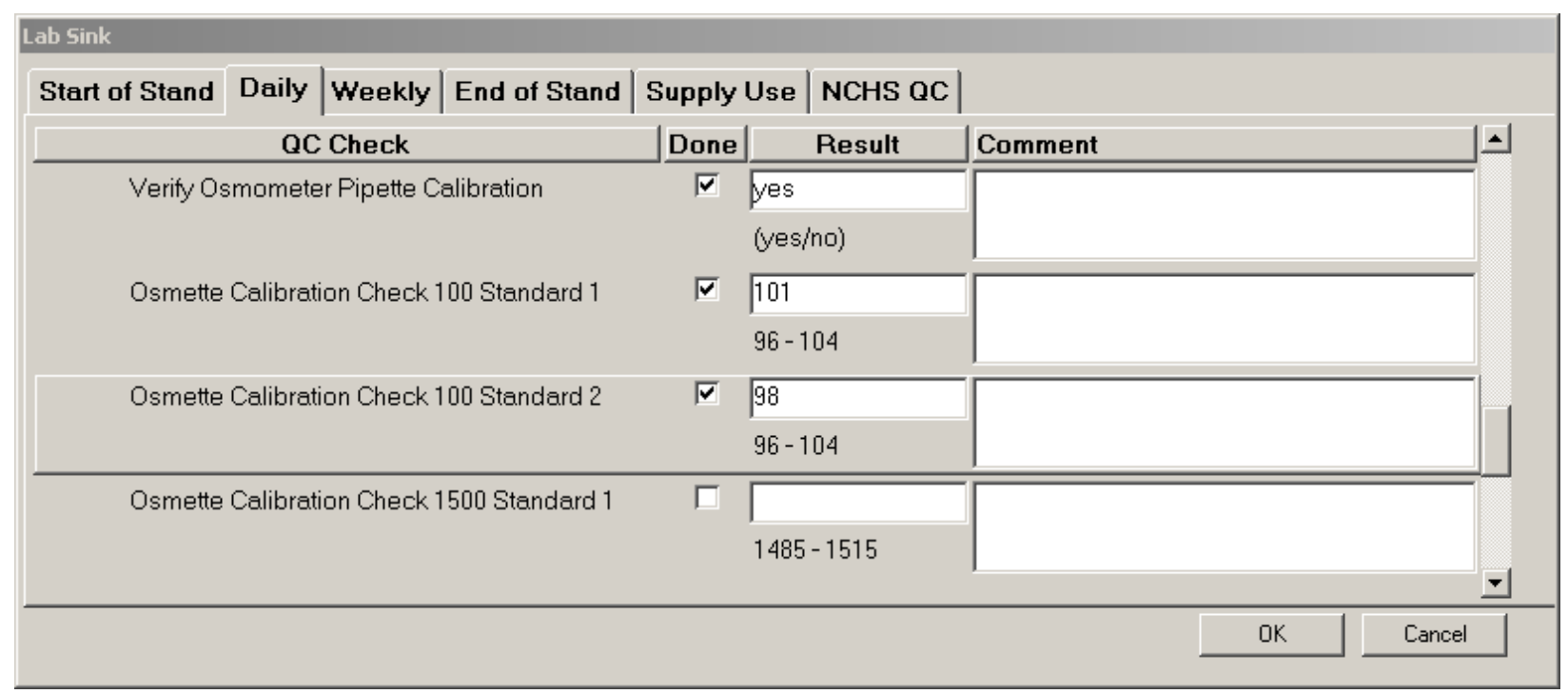

Osmolality QC appears under the Weekly QC tab.

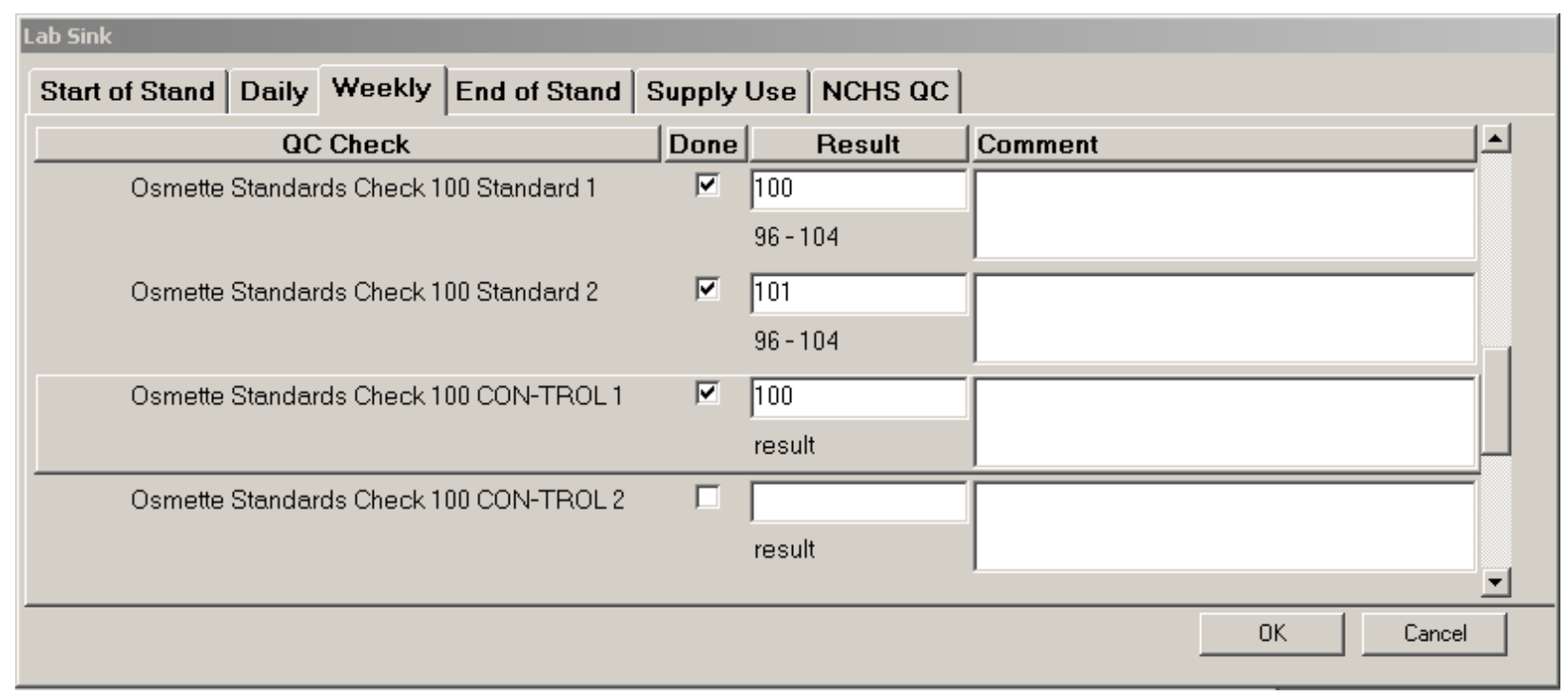

Enter lot and expiration dates under the Supply Use tab. 


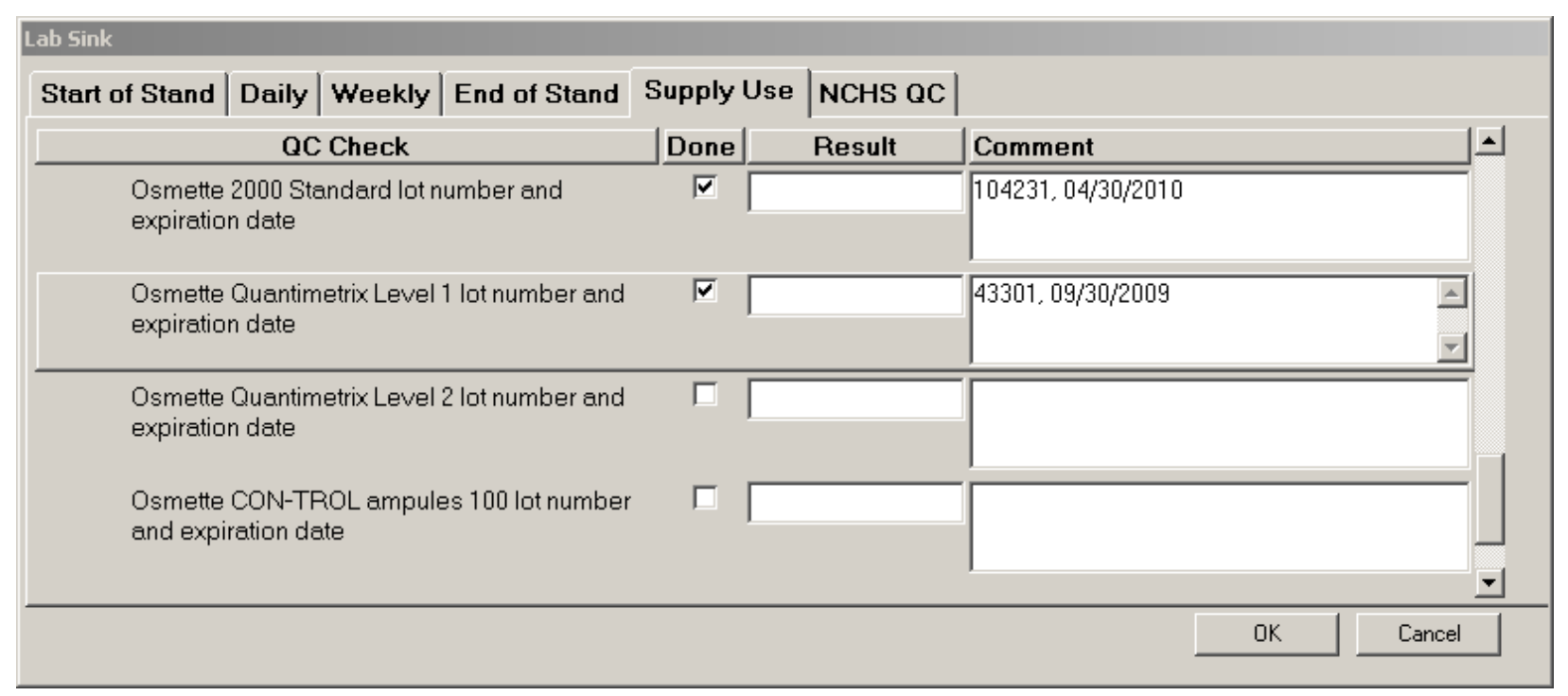

G. Record Quantimetrix Human Urine Controls in ISIS

\section{Enter Quantimetrix QC Lot Information.}

The ISIS maintains the capability to record all Quantimetrix Human Urine Control data and results. The data are used to monitor quality control results. Enter data for each lot number each time a new lot number is put into use. Manually enter the Quantimetrix data using information contained in the package insert and the predefined mean and range from NCHS.

Access the Osmolality QC module from the Utilities menu. 


\begin{tabular}{|c|c|c|c|c|c|c|c|c|c|c|c|c|c|c|c|c|}
\hline Eile view & ㄴtilities $\mathrm{Re}$ & & lodul & Shhip & g g & window Help & & & & & & & & & & \\
\hline X'in & \multicolumn{4}{|c|}{ Quality Control $\quad$ Ctrl+Q } & & & & & & & & & & & & \\
\hline A & \multirow{4}{*}{\multicolumn{4}{|c|}{$\begin{array}{l}\text { Exam Pause } \\
\text { Observations } \\
\text { IC Exclusions } \\
\text { Send Message } \\
\text { UFF Sighting } \\
\text { Set Session }\end{array}$}} & & & \multicolumn{10}{|c|}{ Process Status } \\
\hline SPID & & & & & $\mathrm{age}$ & name & UC & $\mathrm{U}$ & $P$ & $O Z$ & $\mathrm{CB}$ & $\mathrm{B}$ & gtt & PC & HPV & OR \\
\hline 724352 & & & & & & MILLER_1, HARVEY & 0 & 0 & - & 0 & 0 & 0 & & & & 0 \\
\hline 728152 & & & & & & HOLLAND_1, BLAKE & 0 & 0 & . & 0 & 0 & 0 & & - & - & $\cdot$ \\
\hline 728291 & \multirow{2}{*}{\multicolumn{2}{|c|}{$\begin{array}{l}\checkmark \text { English } \\
\text { Spanish }\end{array}$}} & \multirow{2}{*}{\multicolumn{2}{|c|}{$\begin{array}{l}C t r l+E \\
C t r l+5\end{array}$}} & 8 & JOHNSON_1,CLAY & $\odot$ & 0 & · & 0 & 0 & 0 & - & 0 & $\cdot$ & - \\
\hline \multirow{2}{*}{$\begin{array}{l}754740 \\
778058\end{array}$} & & & & & & PARKER_1, RUDY & - & 0 & . & 0 & 0 & 0 & . & - & & 0 \\
\hline & \multicolumn{4}{|c|}{$\begin{array}{l}\text { Ioolbars... } \\
\text { System Configuration }\end{array}$} & & RODENHAUSEN, KAREN & 0 & 0 & 0 & 0 & 0 & 0 & . & 0 & 0 & 0 \\
\hline 789290 & \multicolumn{4}{|c|}{$\begin{array}{l}\text { Pregnancy QC } \\
\text { Coulter QC Lot Info }\end{array}$} & & WALLACE_1, JILL & 0 & 0 & 0 & 0 & 0 & 0 & - & 0 & & \\
\hline 797474 & \multicolumn{4}{|c|}{ Osmolality QC } & & HARRIS_1, MARIA & 0 & 0 & 0 & 0 & 0 & 0 & . & 0 & 0 & 0 \\
\hline 814480 & \multicolumn{4}{|c|}{ Regen SP Data } & 68 & DRAKE_1, ALMA & 0 & 0 & . & 0 & 0 & 0 & . & $\cdot$ & & 0 \\
\hline 820803 & 820803 & $\mathrm{Cl}$ & $\mathrm{P}$ & $\mathrm{F}$ & 46 & SMITH_1,TYLER & 0 & 0 & 0 & 0 & 0 & 0 & · & · & & 0 \\
\hline 828086 & 828086 & $\mathrm{SC}$ & $\mathrm{P}$ & M & 28 & TAYLOR_1, BEN & 0 & 0 & - & 0 & 0 & 0 & - & 0 & & 0 \\
\hline 866059 & 866059 & $\mathrm{SC}$ & $\mathrm{P}$ & M & 18 & CLEAVER_1, WALLY & 0 & 0 & - & 0 & 0 & 0 & . & . & & 0 \\
\hline 870408 & 870408 & $\mathrm{SC}$ & $\mathrm{P}$ & $M$ & 5 & ESTRADA_1,PACO & . & - & - & . & 0 & 0 & . & - & - & . \\
\hline 885421 & 885421 & $\mathrm{SC}$ & $\mathrm{P}$ & M & & WALLACE_3,KEVIN & 0 & 0 & - & 0 & 0 & 0 & . & 0 & & 0 \\
\hline 951940 & 951940 & $\mathrm{SC}$ & $\mathrm{P}$ & M & & RIDDLE, PAUL & 0 & 0 & . & 0 & 0 & 0 & . & . & & 0 \\
\hline
\end{tabular}

Direct the mouse arrow to the "Utilities" menu option near the top of the screen. Left click and drag the mouse down to "Osmolality QC" without releasing the left mouse button. Once "Osmolality QC”is highlighted, release the mouse button.

The Osmolality QC window will appear. 


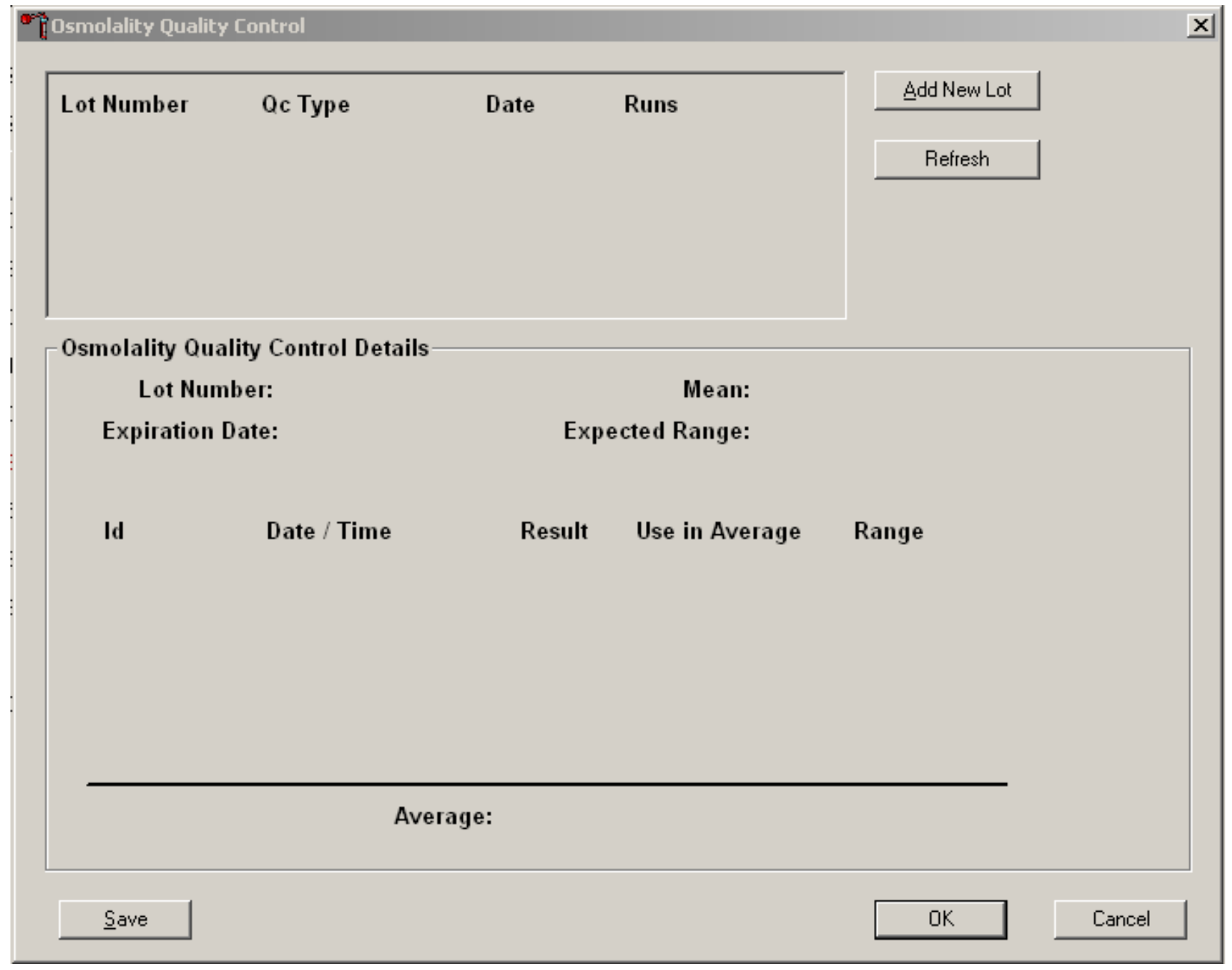

Add new Quantimetrix QC lot information. 


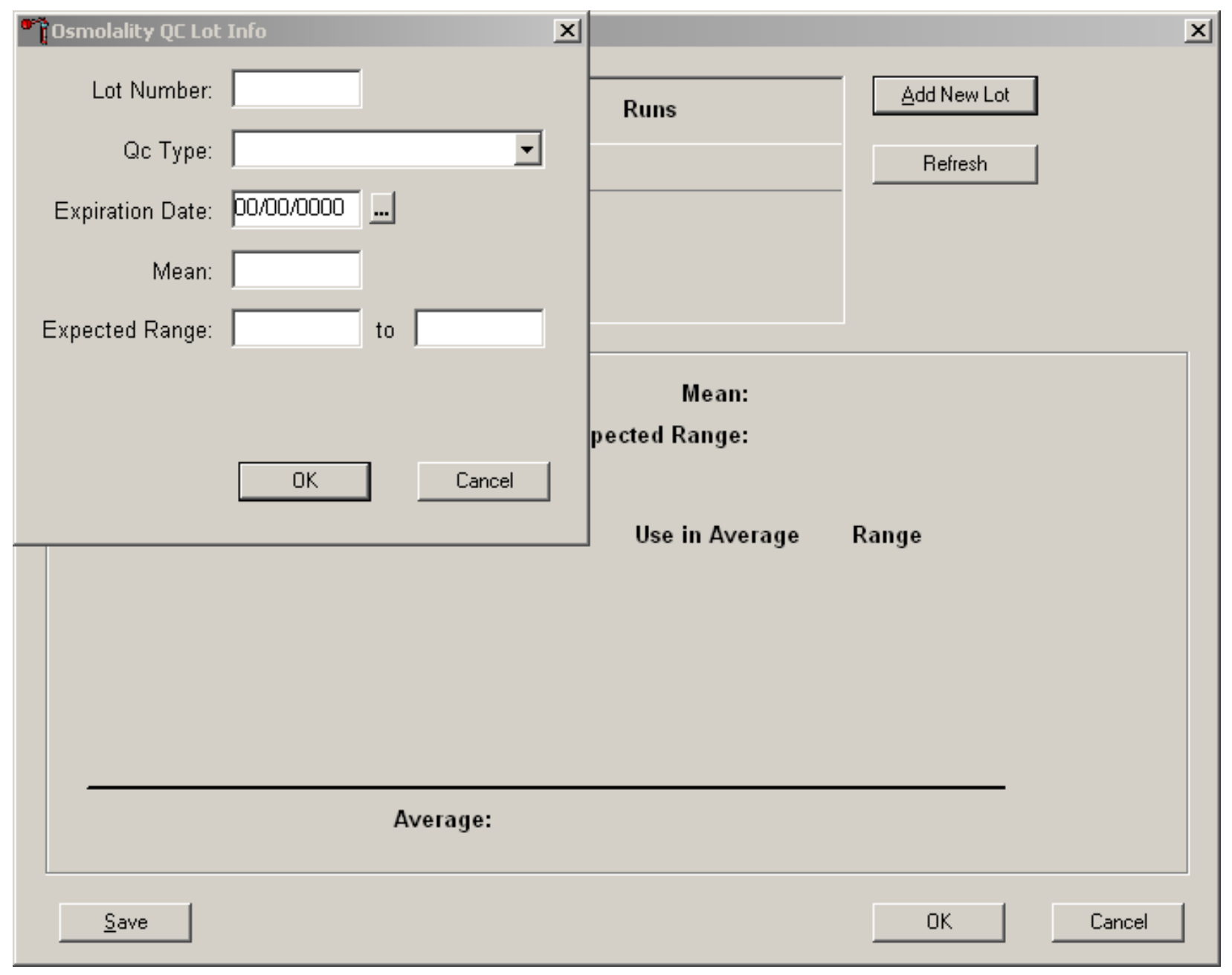

Direct the mouse over the "Add New Lot" button and left click. A small window, “Osmolality QC Lot Info,” will appear. Enter the Quantimetrix QC information in this window. The data entry values are found in the package insert of each box of Quantimetrix Human Urine Control. 
Enter the lot number in the entry box "Lot Number:"

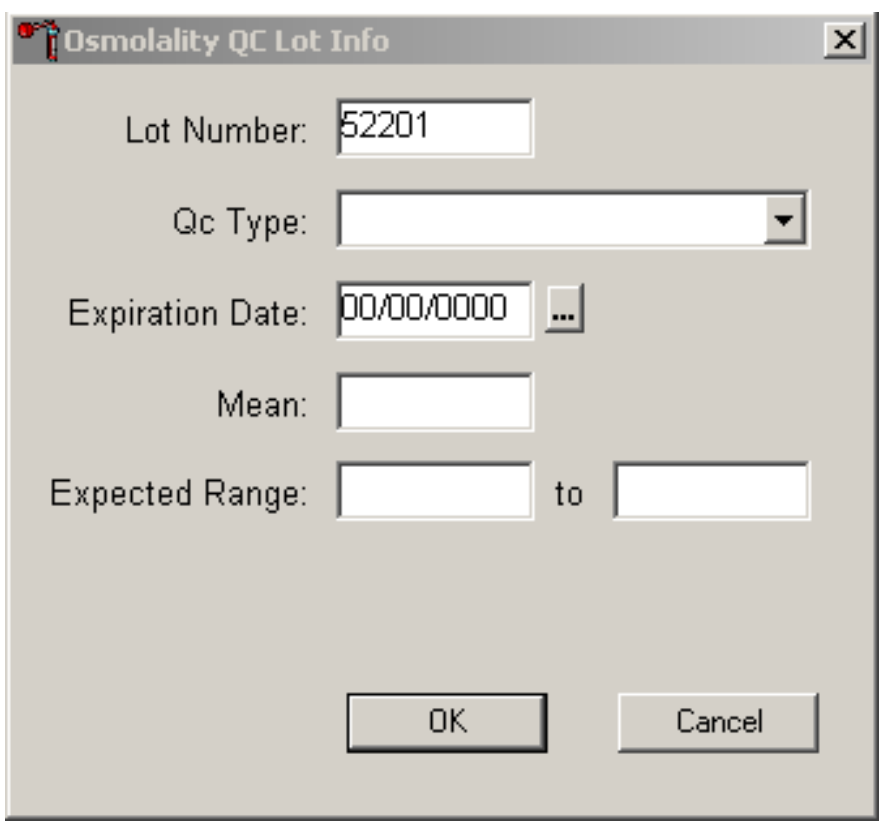

Direct the mouse over the Lot Number field and left click. Use the number pad on the keyboard to enter the lot number on the package insert. 
Each set of QC has a high and low control defined by the lot number. The value of each QC can be determined by looking at the last digit of the QC Lot Number. "01” is Level 1 and "02” is Level 2. Select the QC control type from the drop-down list.

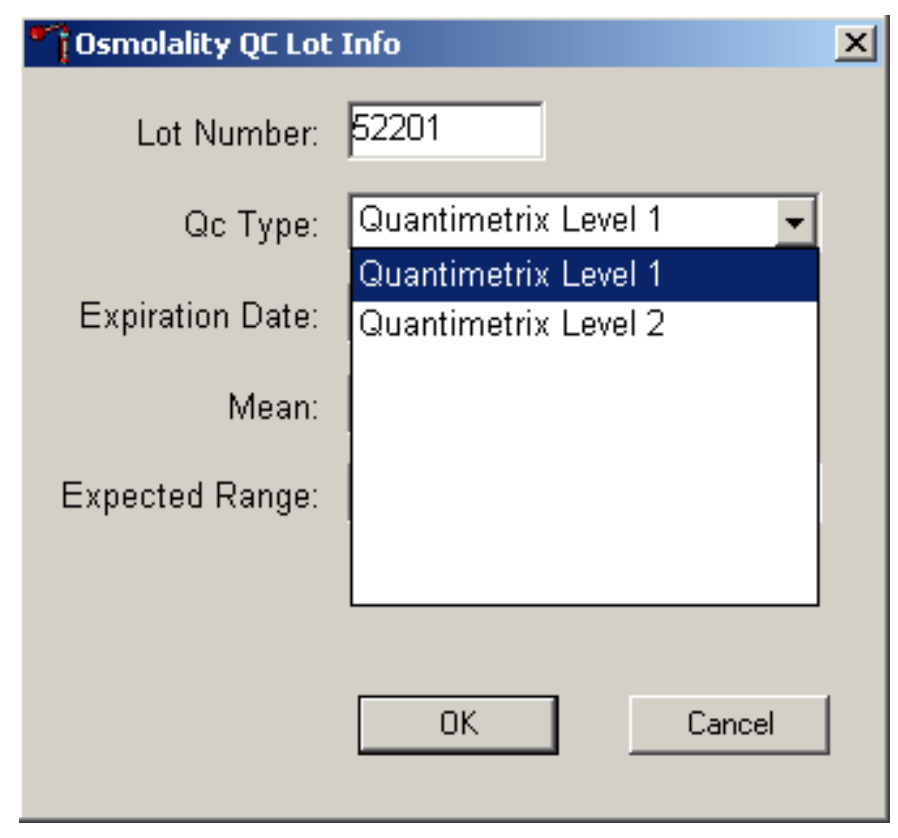

Direct the mouse arrow over the down arrow and left click to view the drop-down selections for Quantimetrix levels. Direct the mouse arrow so the desired Quantimetrix Level is highlighted and left click. 
Enter the expiration date of the human urine controls.

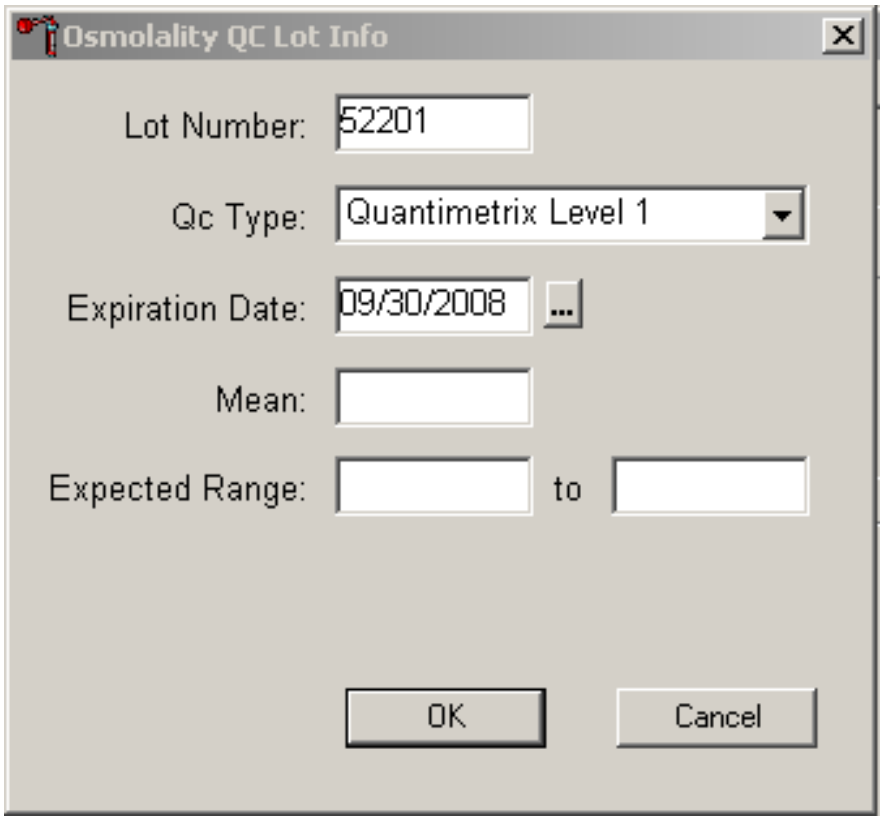

Direct the mouse to the Expiration Date field and left click. Alternatively, press the [Tab] key to move from the QC Type field to the Expiration Date field. Enter the date in MM/DD/YYYY format. Alternatively, direct the mouse over to the ellipsis [...] button and left click to select the calendar function. 


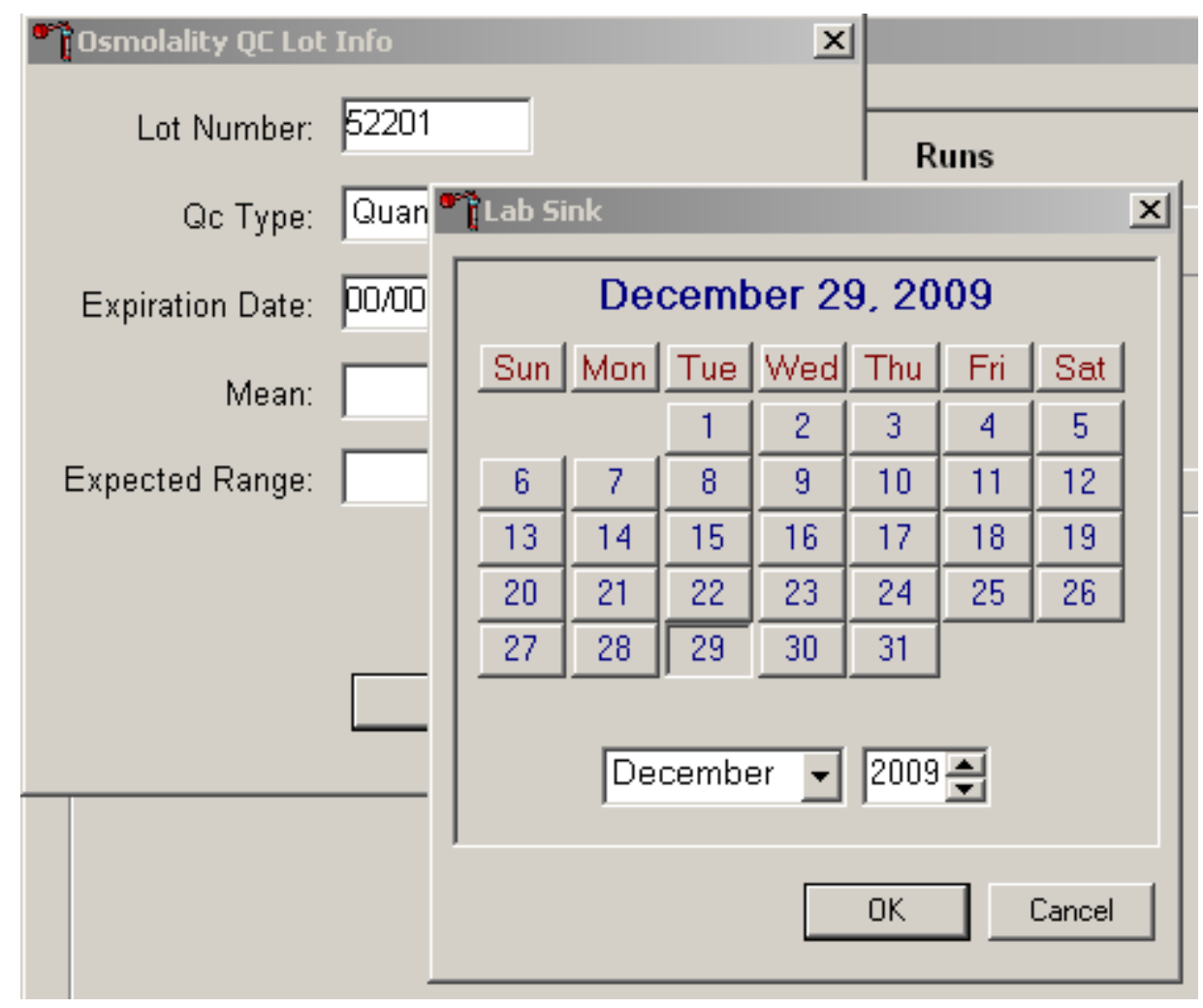

Use the up and down arrow buttons to scroll to the correct year of the expiration date.

Use the drop-down list to select the correct month. 


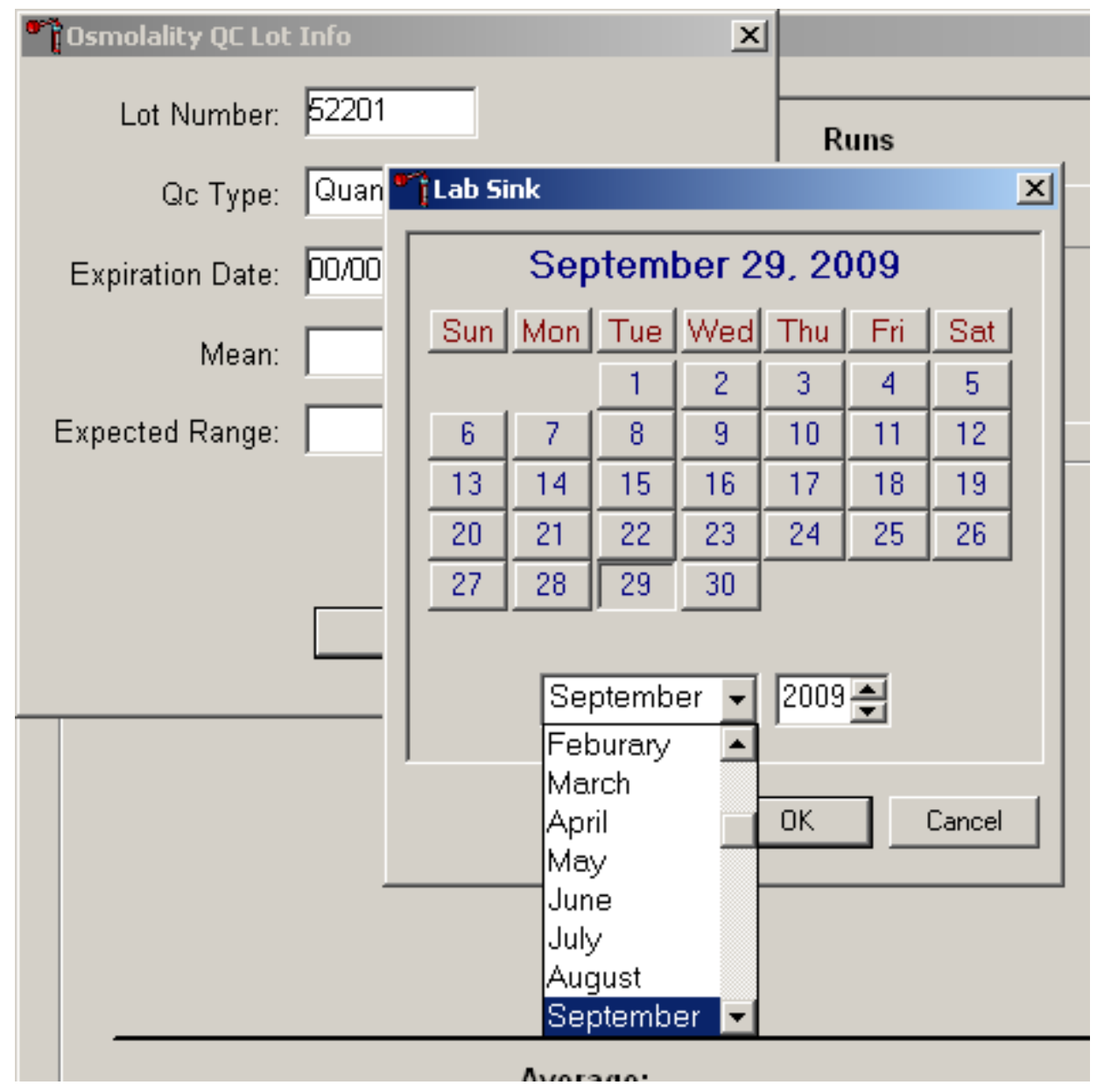

Direct the mouse arrow to the down arrow and left click to view the drop-down menu months. Use the scroll bar to view all month selections. To select the month, direct the mouse arrow to the month and left click.

Select the last day of the expiration month by clicking on the day in the calendar. 


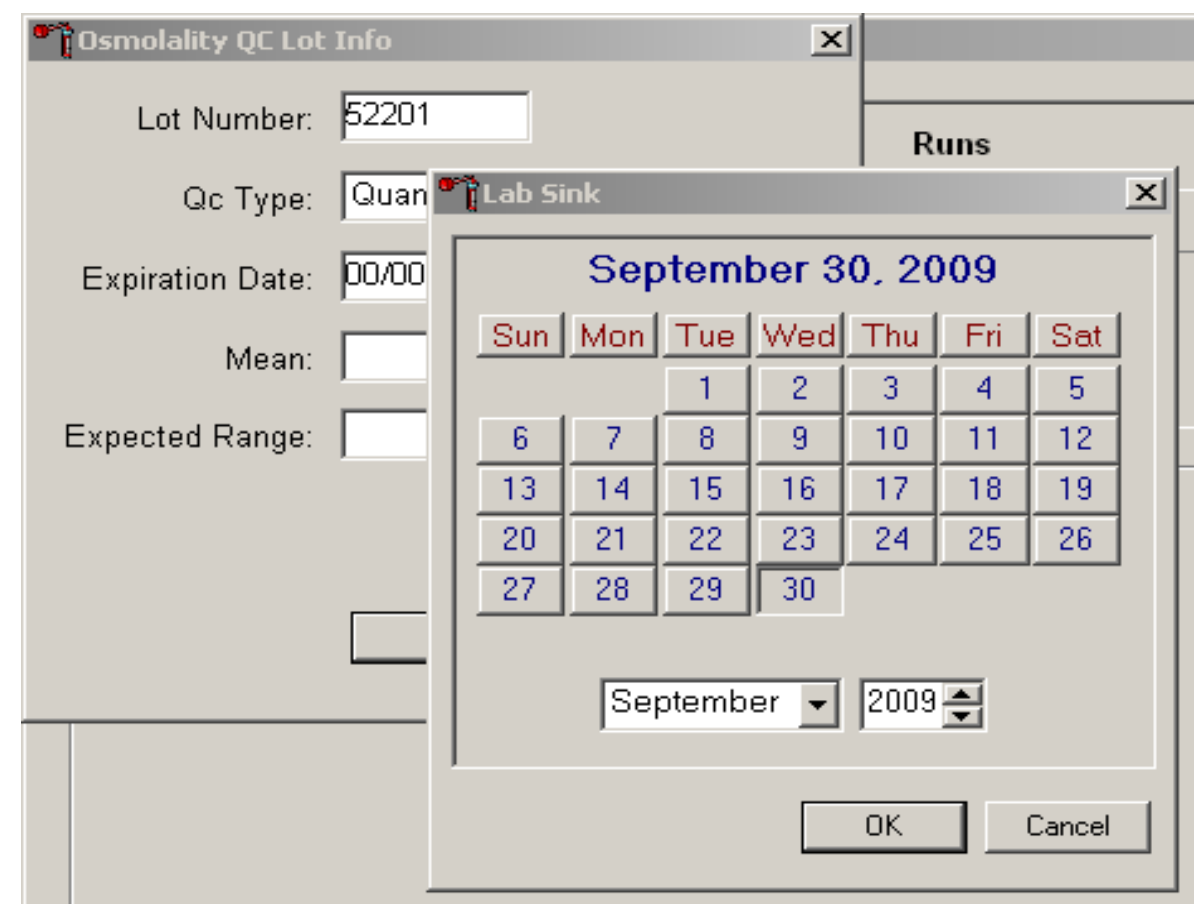

Direct the mouse arrow over the "OK” button and left click.

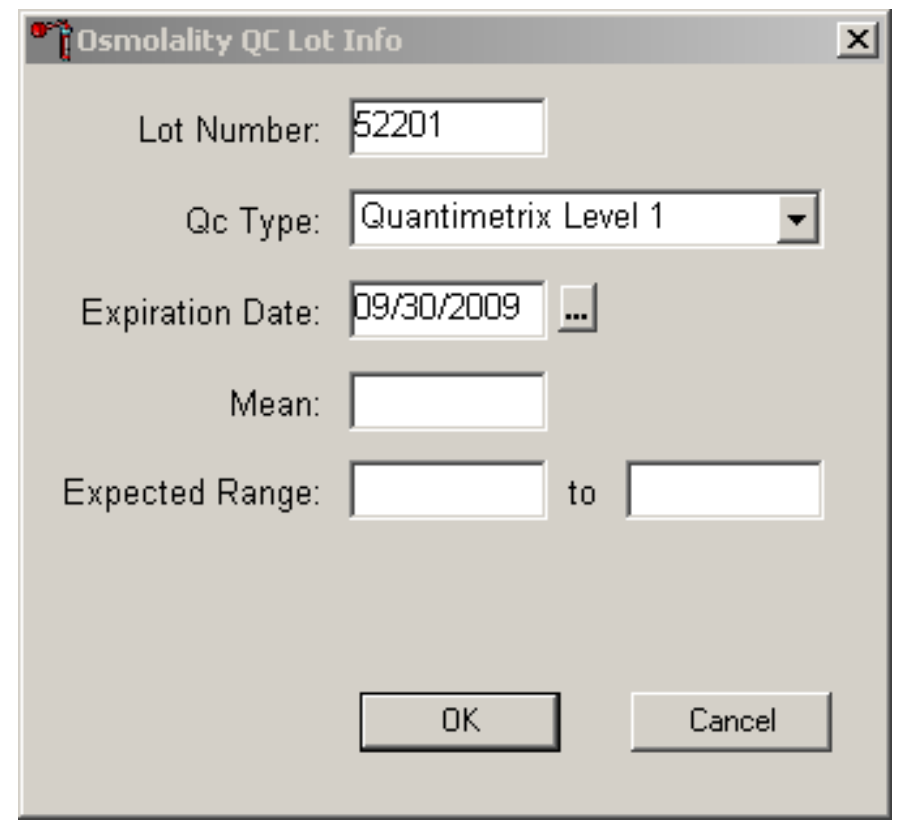

The calendar widow will disappear and the selected date will automatically fill in the Expiration Date field. 
Enter the predefined mean and range for each lot number. These numbers are defined by NCHS based on a test of each new lot number. Do not use the information included in the package insert for these fields.

Enter the mean.

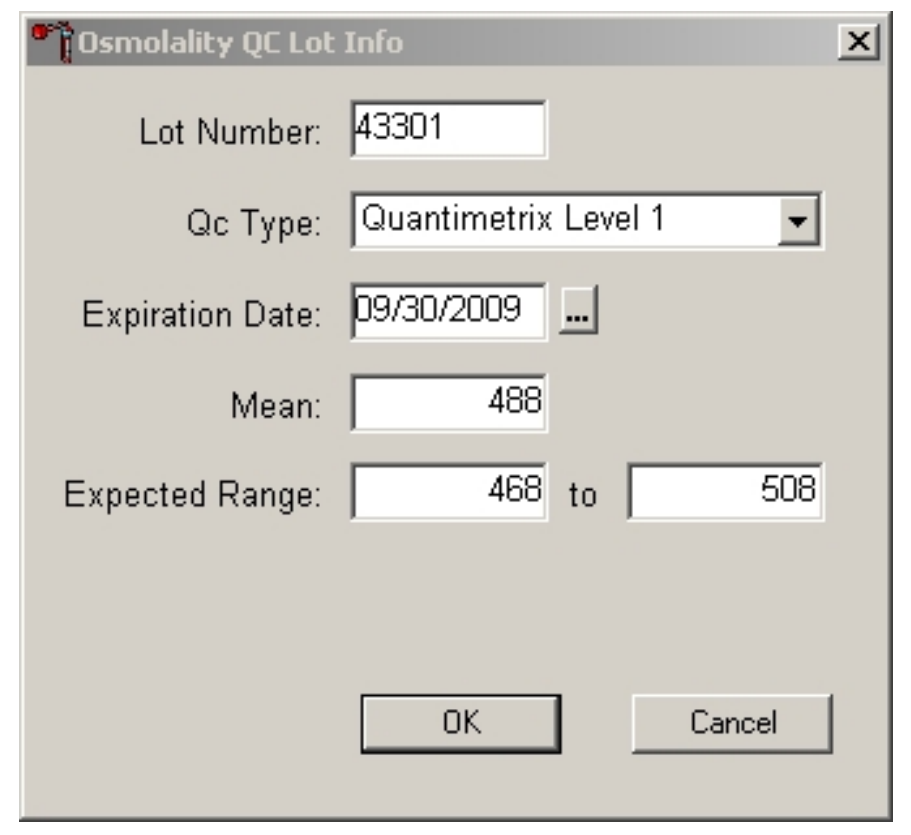

Direct the mouse arrow over the Mean entry field and left click. Alternatively, press the [Tab] key to move to the Mean field from the Expiration Date field. Enter the mean in the Mean field.

Enter the expected range. 


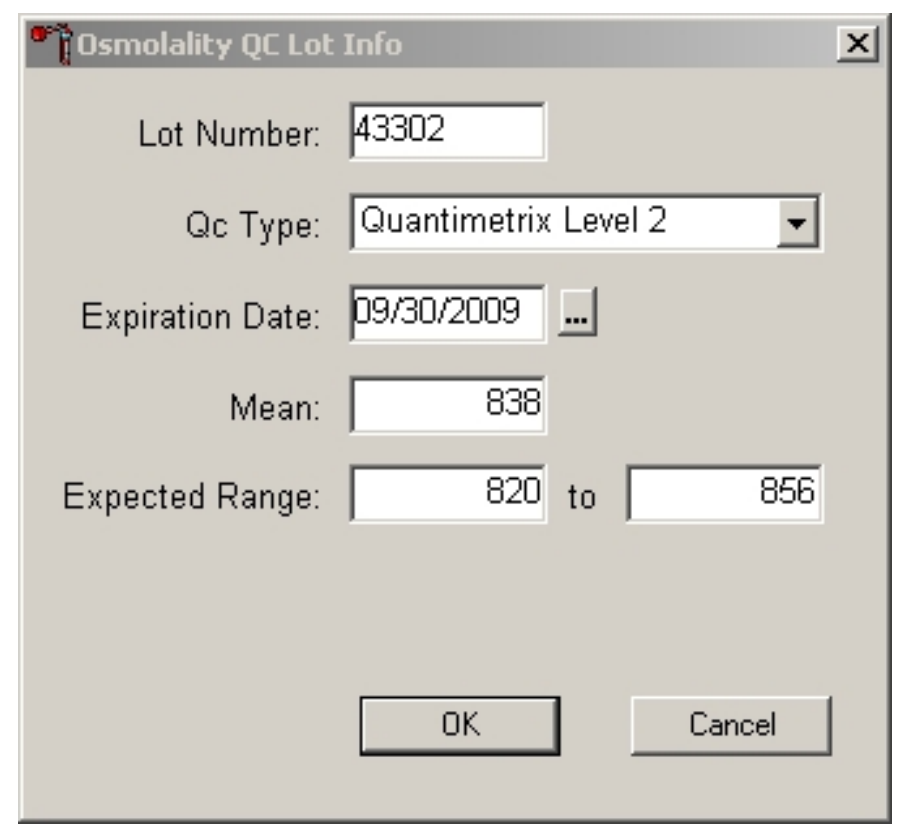

Direct the mouse arrow over the first Expected Range entry field and left click. Alternatively, press the [Tab] key to move to the Expected Range field from the Mean field. Enter the low range value in the first entry field. Direct the mouse arrow over the second Expected Range field and left click. Alternatively, press the [Tab] key to move to the second Expected Range field. Using the keyboard number pad, type the high range value in the second entry field.

Direct the mouse arrow to the OK button and left click. Alternatively, press the [Enter] key. 


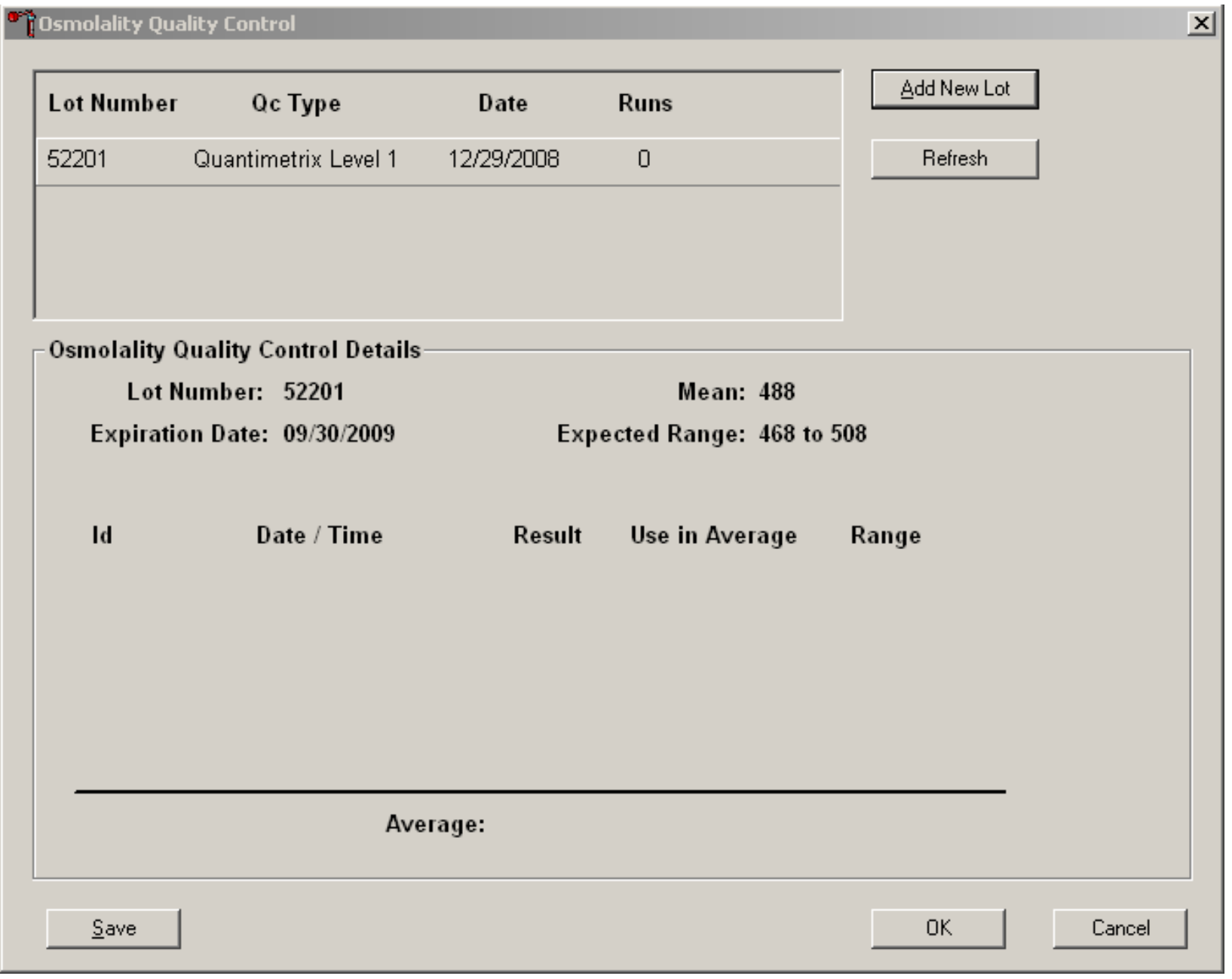

The Osmolality QC Lot Info window disappears. The entered Lot displays in the top of the QC window. A label for Quantimetrix Level 1 prints on the Dymo label printer.

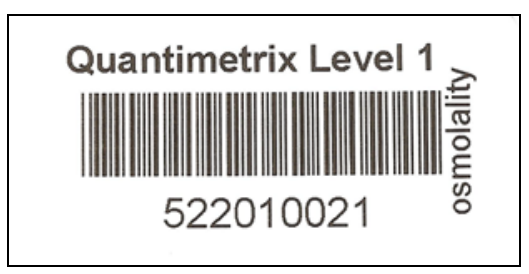

Save this label near the Osmette II instrument. It is required in order to run the Quantimetrix Human Urine Control QC. 
Add the Level 2 lot information by directing the mouse to "Add New Lot” and left clicking. Enter the information in the Osmolality QC Lot Info window using the same steps as the first lot above. Be sure to enter the correct mean and range for the Level 2.

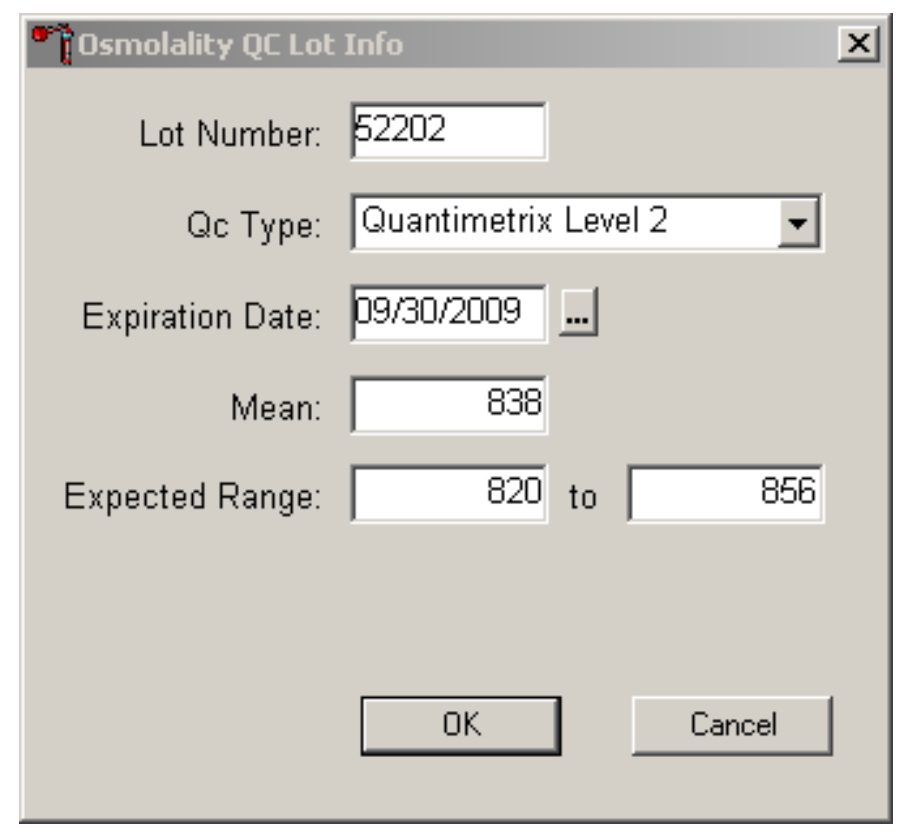

Direct the mouse arrow to the OK button and left click. Alternatively, press the [Enter] key. 


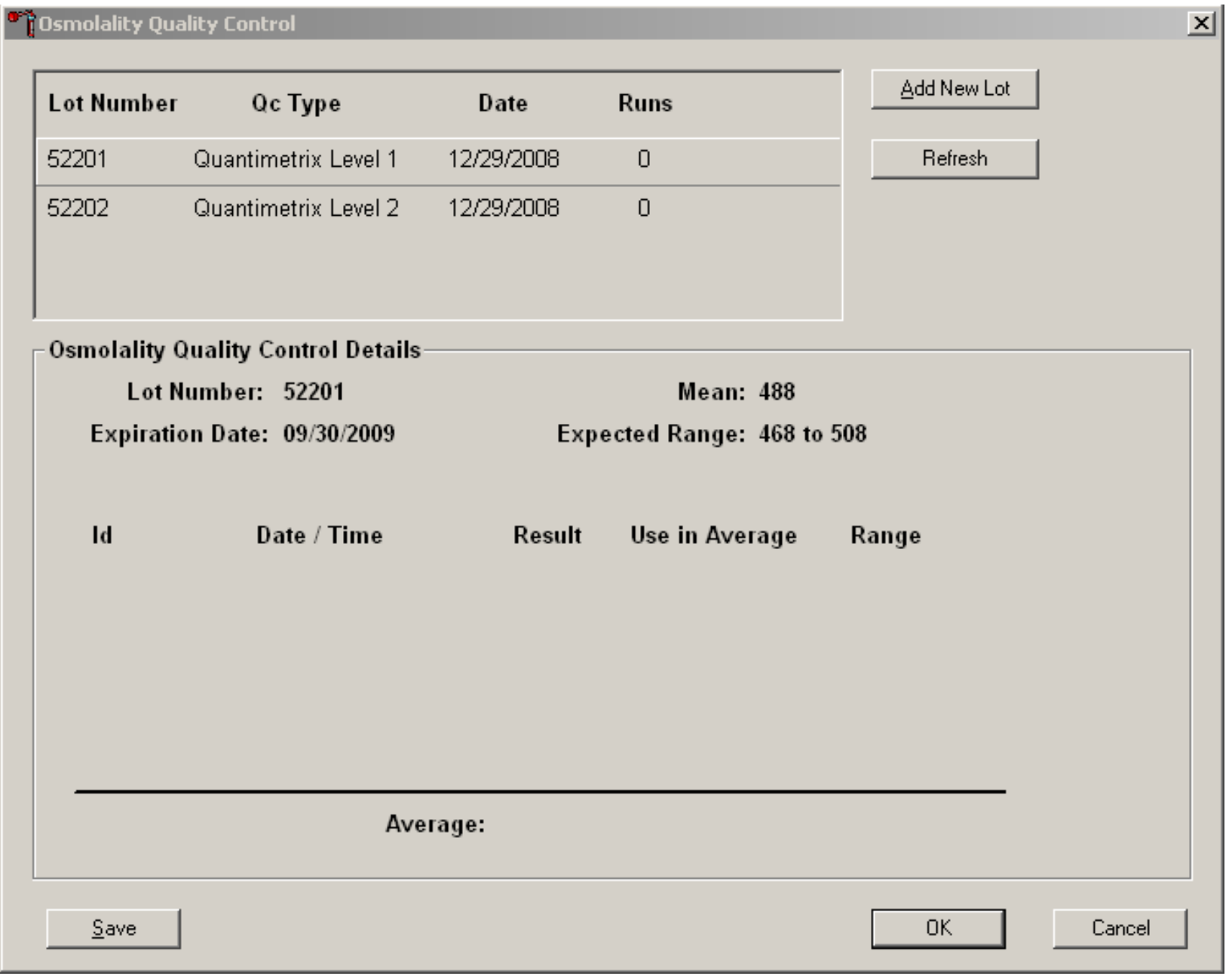

The Osmolality QC Lot Info window disappears. The entered Lot displays in the top of the QC window. A label for Quantimetrix Level 2 prints on the Dymo label printer.

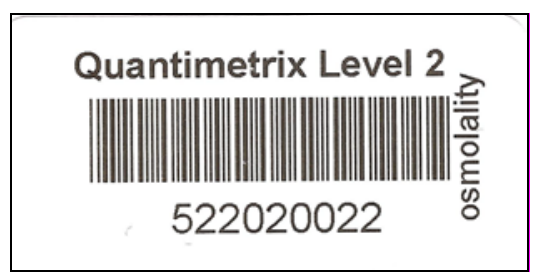

Save this label near the Osmette II instrument. It is required in order to run the Quantimetrix Human Urine Control QC. 


\section{Record Quantimetrix Human Urine Control Samples}

Scan the Quantimetrix Level 1 bar code label for the Quantimetrix Lot before running the Level 1 samples as described in Section E. Refresh the window if necessary to make the results of the run appear in the bottom of the window.

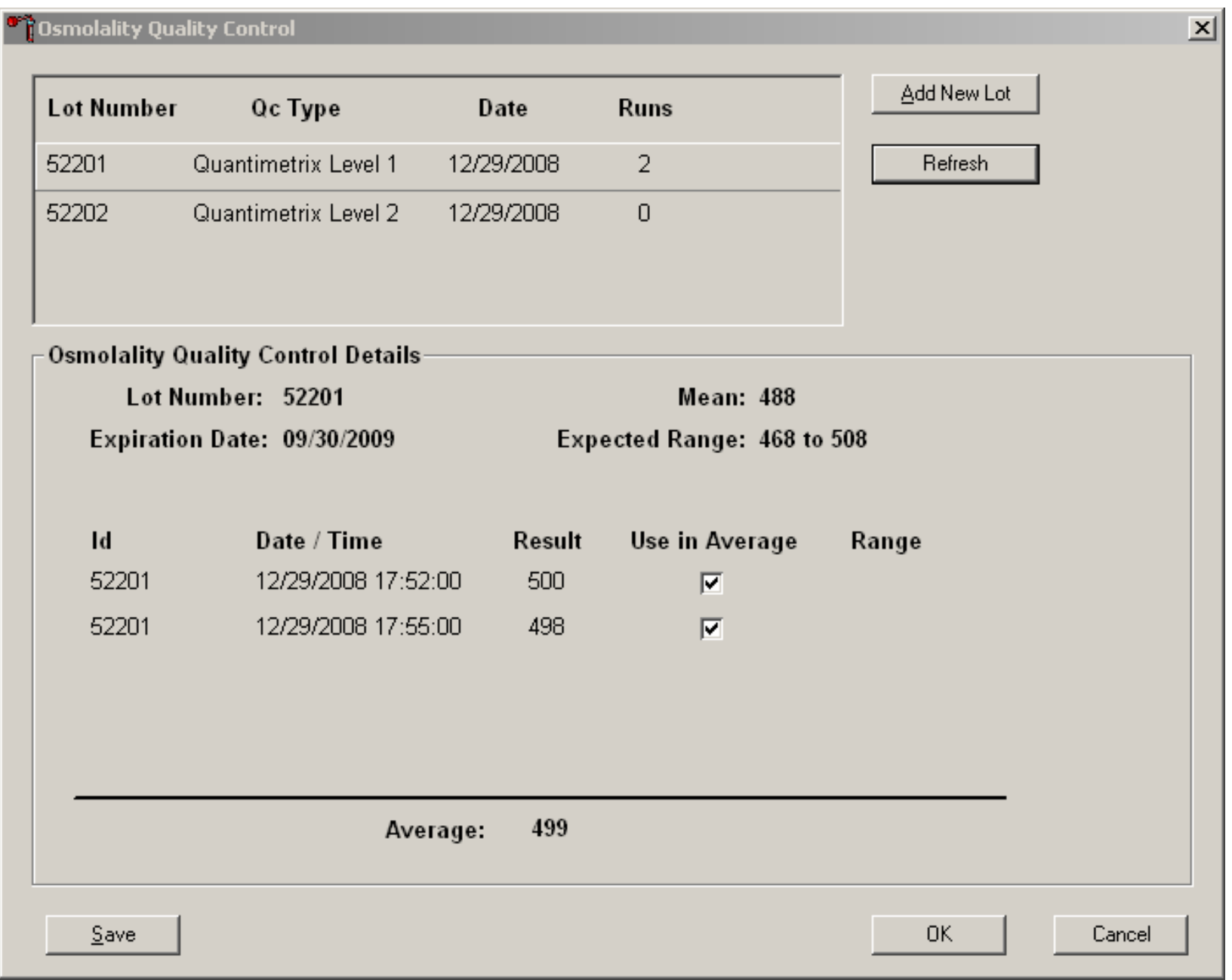

To refresh, direct the mouse arrow over the "Refresh" button and left click. The results should appear in the bottom of the window under "Osmolality Quality Control Details.” Note that the ID number is the same as the Lot Number. The date and time are captured. The result as shown on the Osmette display appears in the Result column. The Mean and Expected Range entered in the QC Lot Info screen now display at the top for reference. 
Two QC runs are required to complete the QC. The average of the two QC runs should fall within the expected range. At least one of the two individual runs should fall within the expected range. The computer application will automatically select runs that it evaluates to meet the criteria for saving to the database. However, it is ultimately the responsibility of the medical technologist to evaluate each run and determine whether the result should be used in the average and save to the database. The application will not prevent any result from being used in an average if selected by the medical technologist, or any two results selected by the medical technologist from being saved to the database.

The application automatically selects two runs to be saved as an average. These runs are noted by the check mark in the box under the "Use in Average" column. Runs will be automatically selected if they fall within the range defined in the QC Lot Info window.

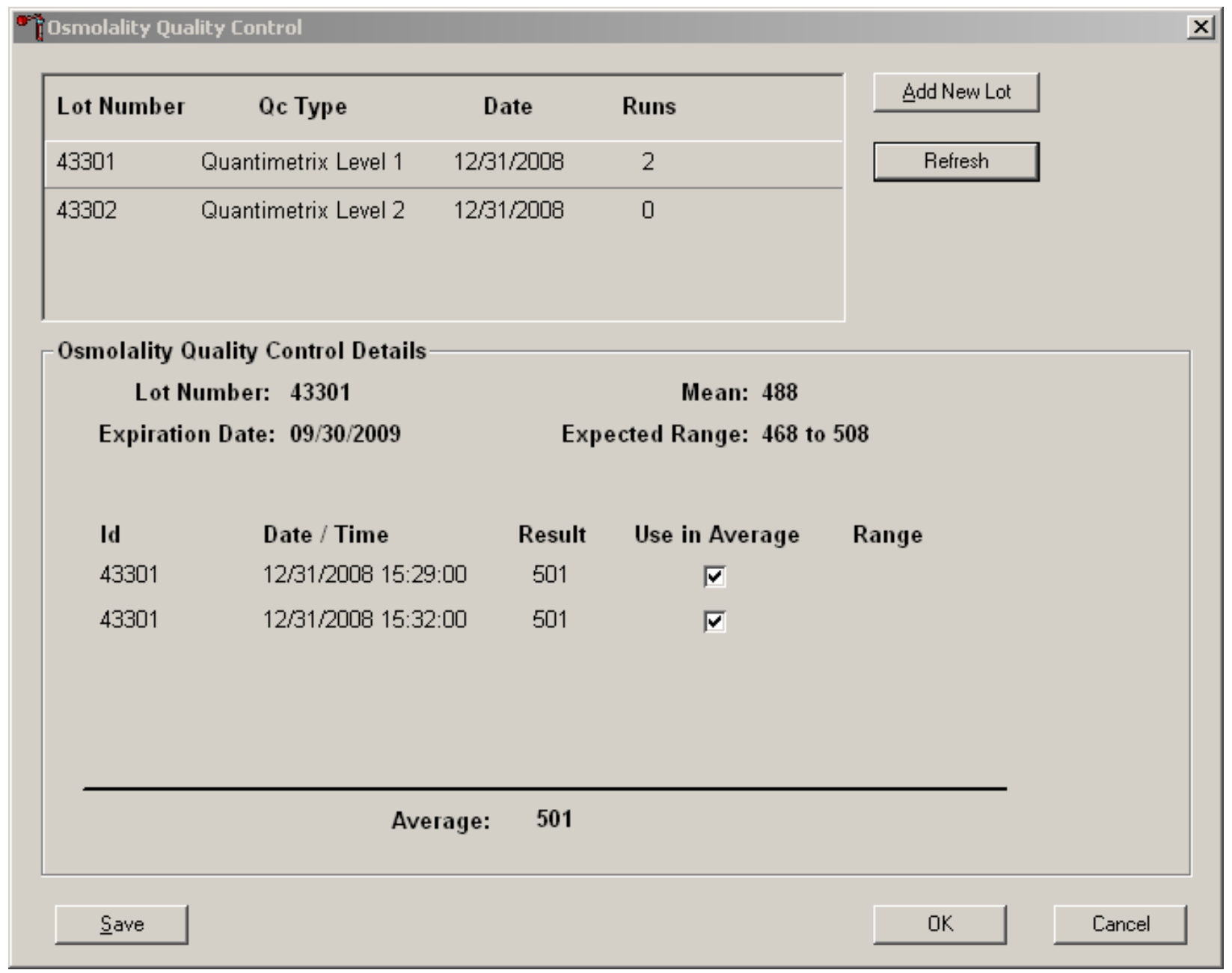


The runs can be unchecked manually if desired by directing the mouse over the check mark and left clicking. The run is deselected when no check mark appears in the "Use in Average" column. To select the run again, direct the mouse over the box and left click to display the check mark.

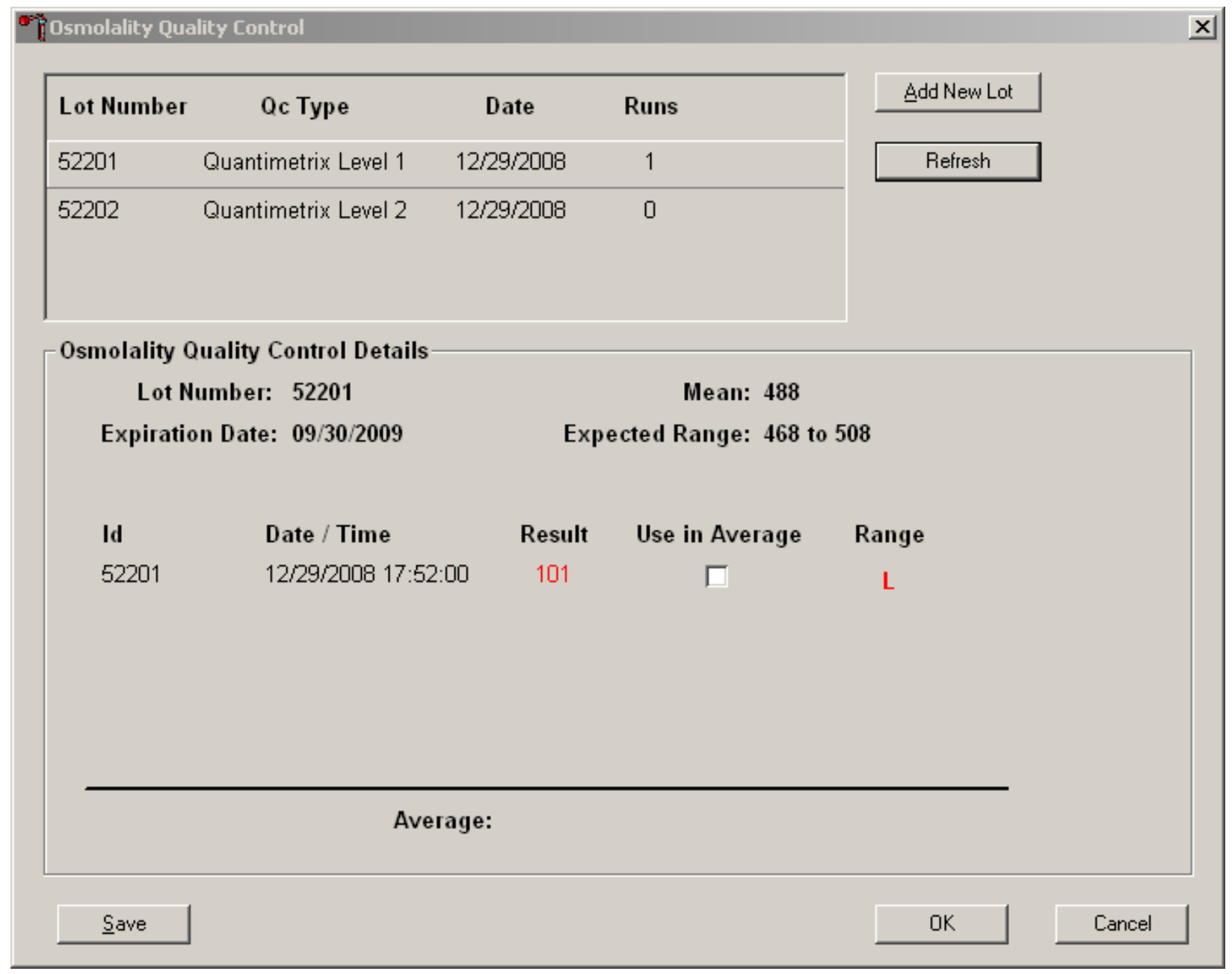

If the result is out of the Expected Range, it will appear in red and a flag will appear in the Range column. The red letter "L" appears for results less than the lower limit of the range. 


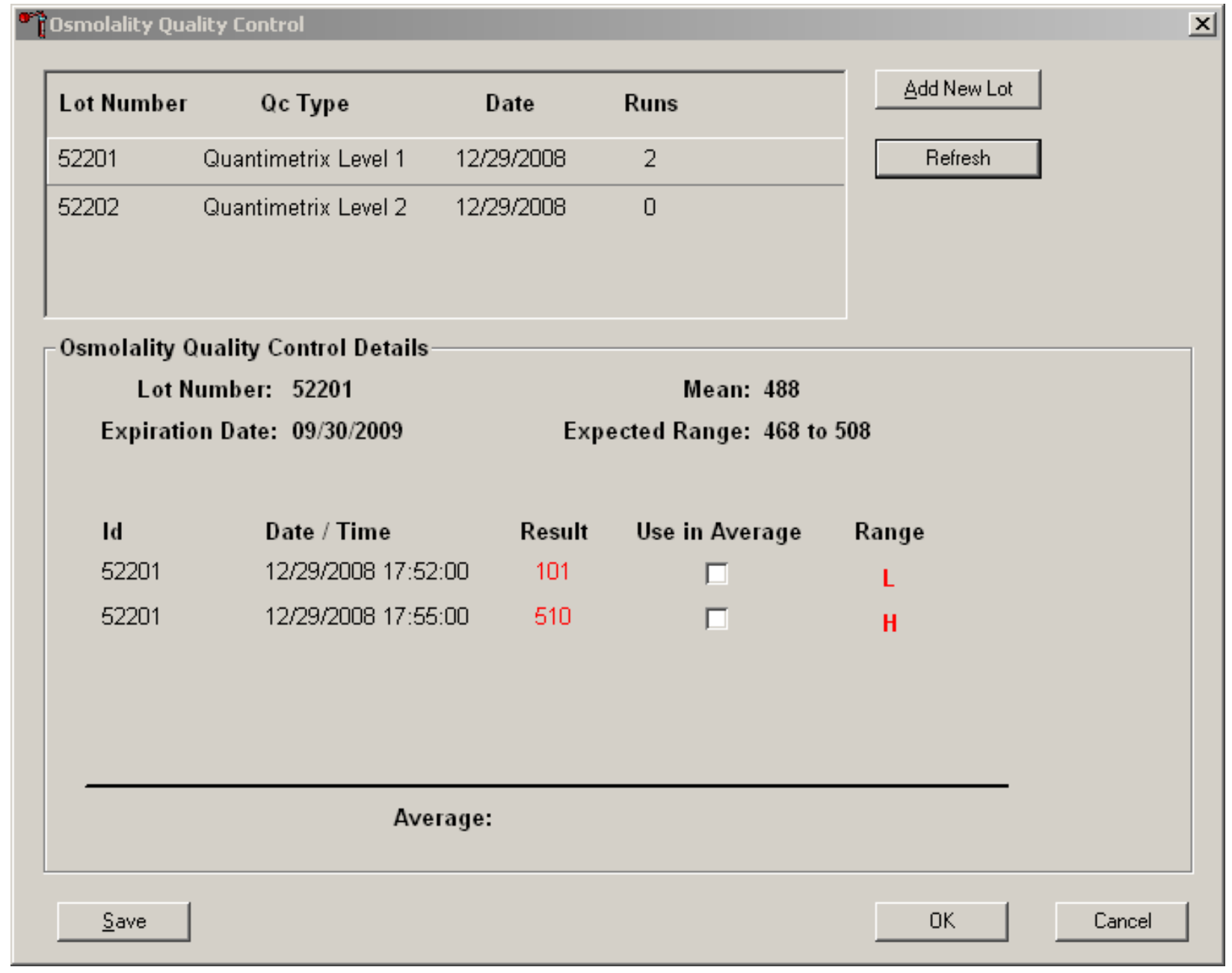

If the Result is greater than the upper limit of the range, the result will display in red and a red " $\mathrm{H}$ " will display in the Range column. In either case, the result will not automatically be marked for use in average unless another run creates an average that falls within the predefined range and the run itself falls within the range. 


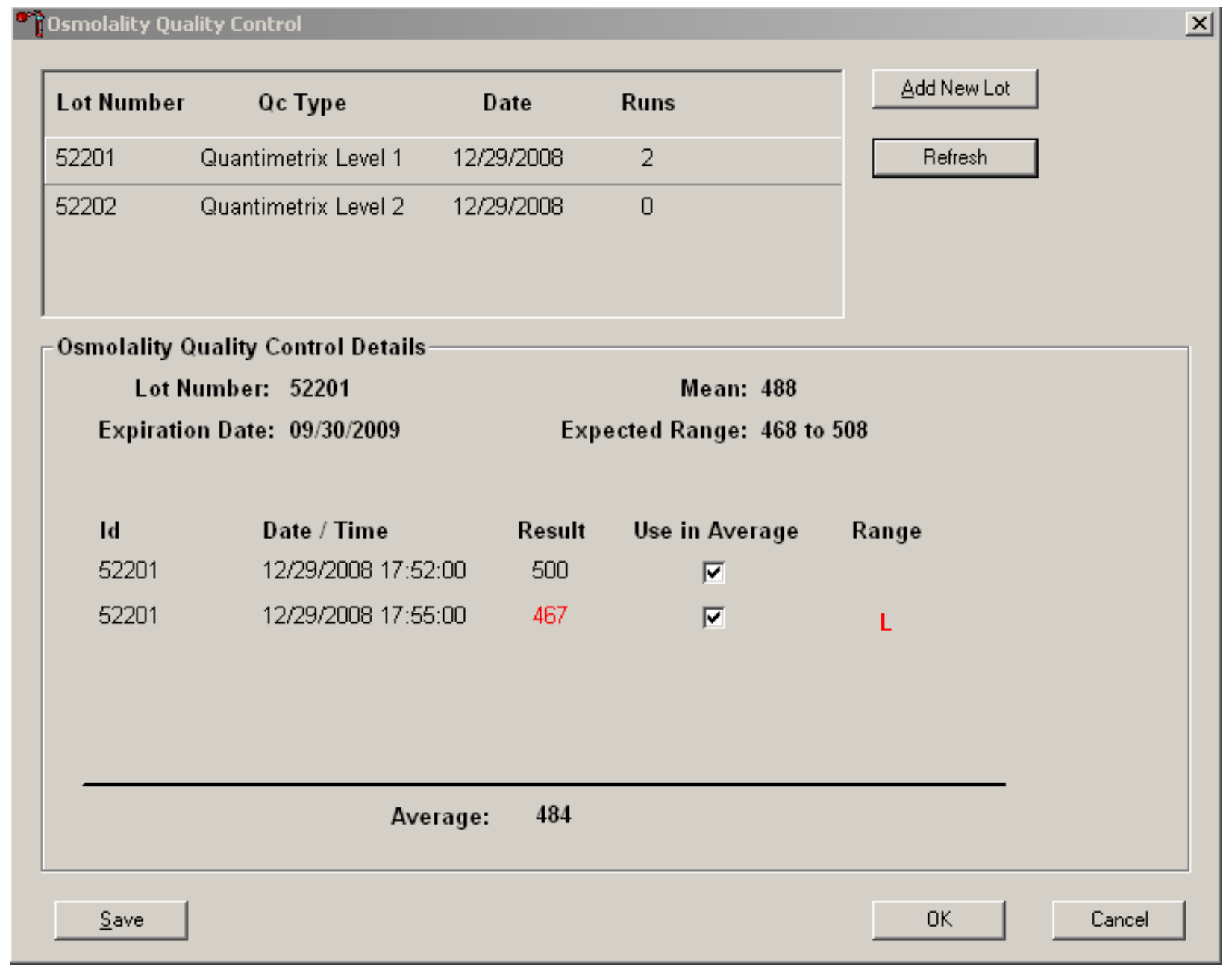

The above screen displays two runs. The first run falls within the expected range. The second run is low, but the average still falls within the expected range. These two runs will be automatically selected for the saved average. No further runs are necessary to save the averaged result from a system perspective; however, each run should be evaluated by the medical technologist. It is the technologist's responsibility to determine if additional runs are necessary. 


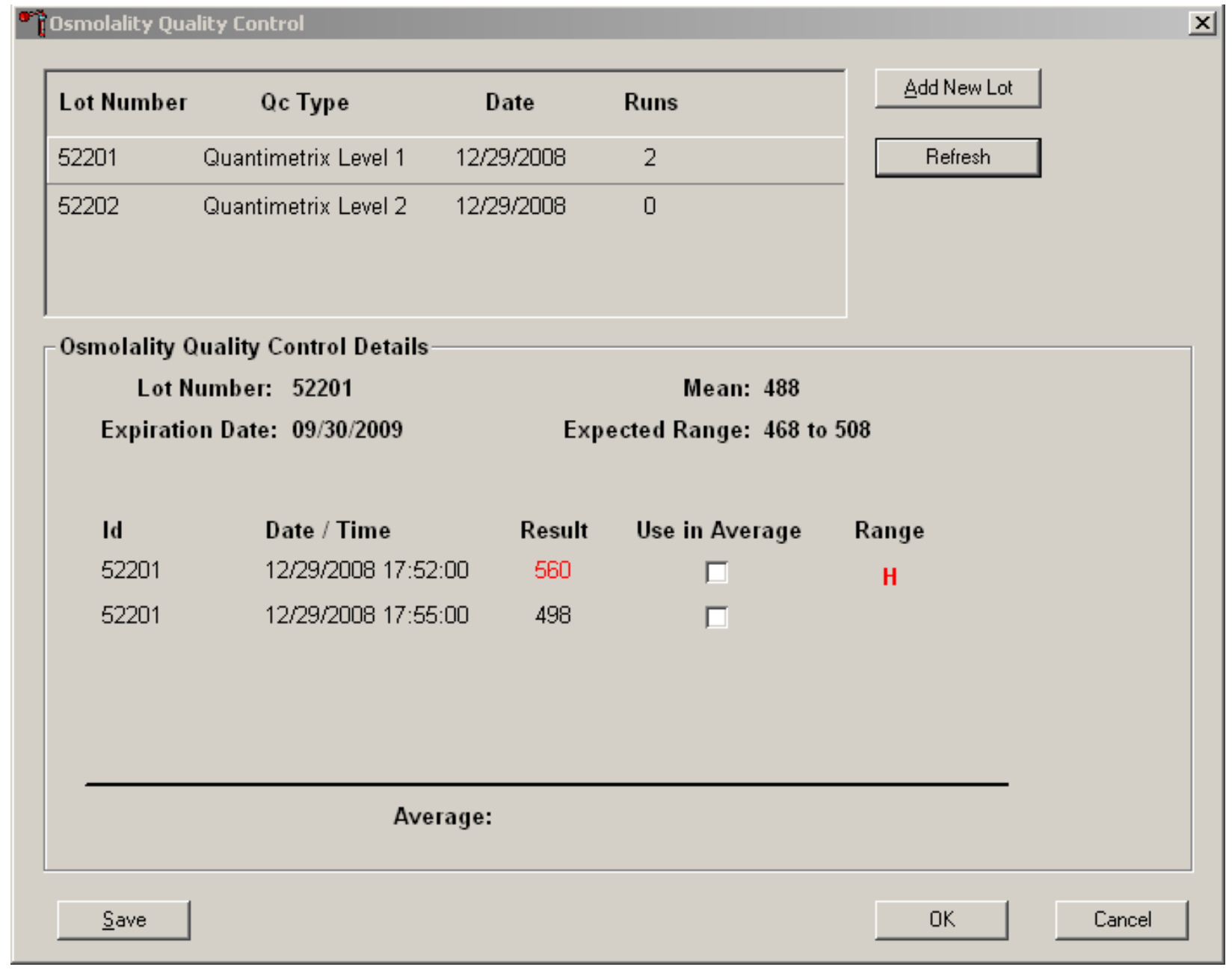

The first of the above runs is far out of the expected range. The second run is within range; however, the average of the two (529) falls out of the expected range. Neither of these two runs will be automatically selected for the result average. This is an indication that the medical technologist should run the calibration procedure described in Section VII.D and then run the sample a third time. 


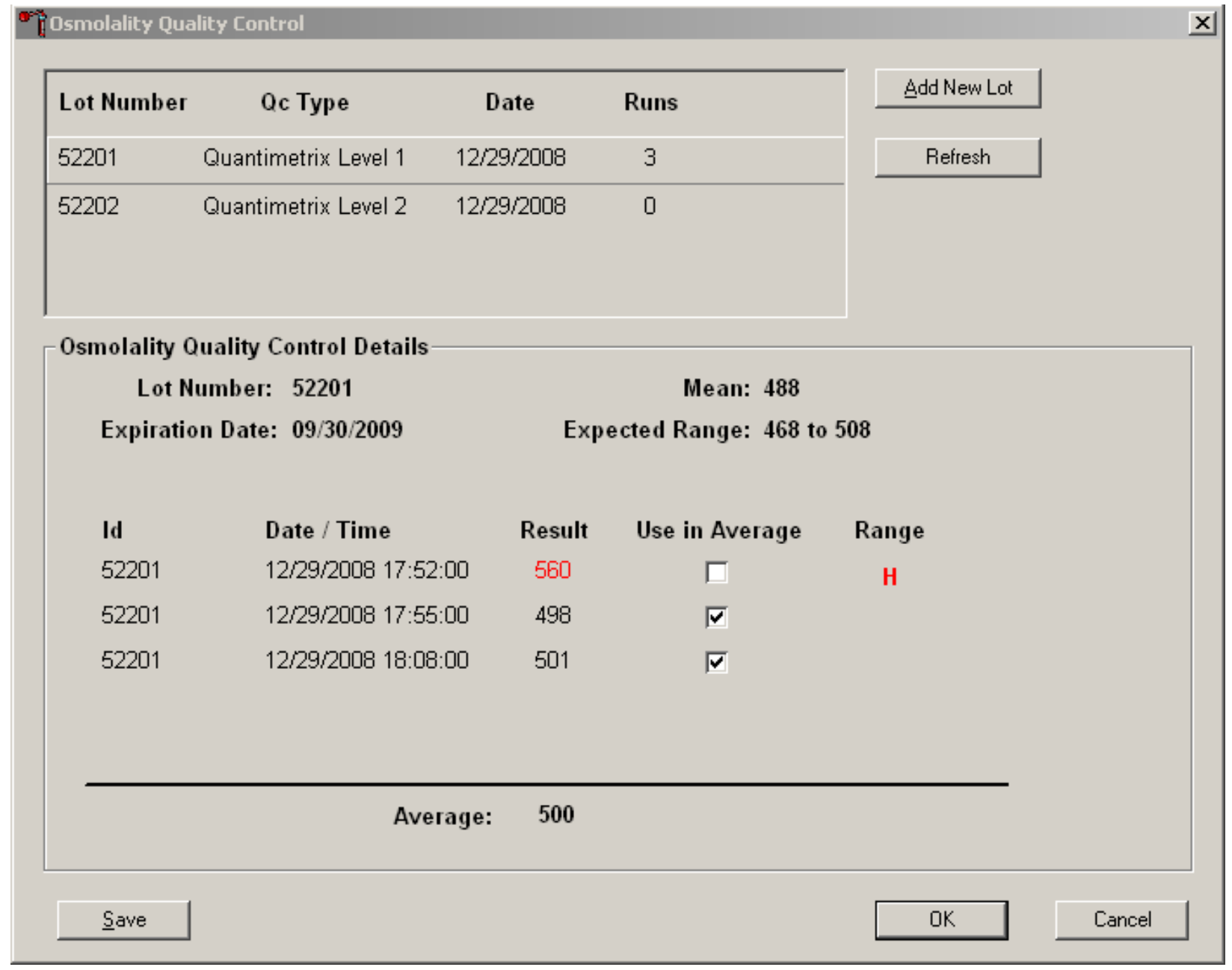

After the calibration, the third run is transmitted from the Osmette and falls within the expected range. The application automatically selects the two runs that fall within range to average their results.

To save the average result of the selected runs without exiting the window, direct the mouse arrow to the "Save" button and left click. To save the average result of the selected runs and exit the window, direct the mouse arrow to the "OK" button and left click. To cancel any changes made in the screen, direct the mouse arrow to the "Cancel” button and left click. 


\section{H. Proficiency Testing}

Evaluation and participation in the College of American Pathologists (CAP) proficiencytesting program is part of the comprehensive quality control program. The CAP samples for osmolality are shipped three times per year. Each Urine Chemistry (General) "U” shipment includes six 15.0-mL urine specimens. Follow all CAP instructions in preparing the materials before performing the test. Run specimens in a manner identical to routine specimens. Fill out the CAP result form, make a copy for the logbook, and send results to CAP.

\section{Linearity for Osmolality}

CAP LN6 verifies the reportable range of the Precision Osmette II instrument. Survey LN6 corresponds to CAP Urine Chemistry (General) Survey U. Each shipment of Survey LN6 includes 15 4.0-mL liquid specimens. Linearity was performed at installation and will be repeated in November of each year. The medical technologist follows all CAP instructions in preparing and running the materials before performing the test. They fill out the CAP result form, make a copy for the logbook, send results to CAP, and send the copy back to the home office at the end of the stand.

XI. Interpretation of Results and Remedial Action

\section{A. Sample Person Parameter Value Review and Remedial Actions.}

If a sufficient, acceptable quality sample is submitted for osmolality testing, the medical technologist should run the sample twice. The two runs should have a difference no greater than 40 , and the average of the two runs will be saved to the database as the osmolality result. The computer application will automatically select runs that it evaluates to meet the criteria for saving to the database. However, it is ultimately the responsibility of the medical technologist to evaluate each run and determine whether the result should be used in the average and save to the database. The application will not prevent any result from being used in an average if selected by the medical technologist, or any two results selected by the medical technologist from being saved to the database. 
1. Access the Osmolality module, reject a blood contaminated sample, or record a reason for no osmolality results.

Open the Osmolality module.

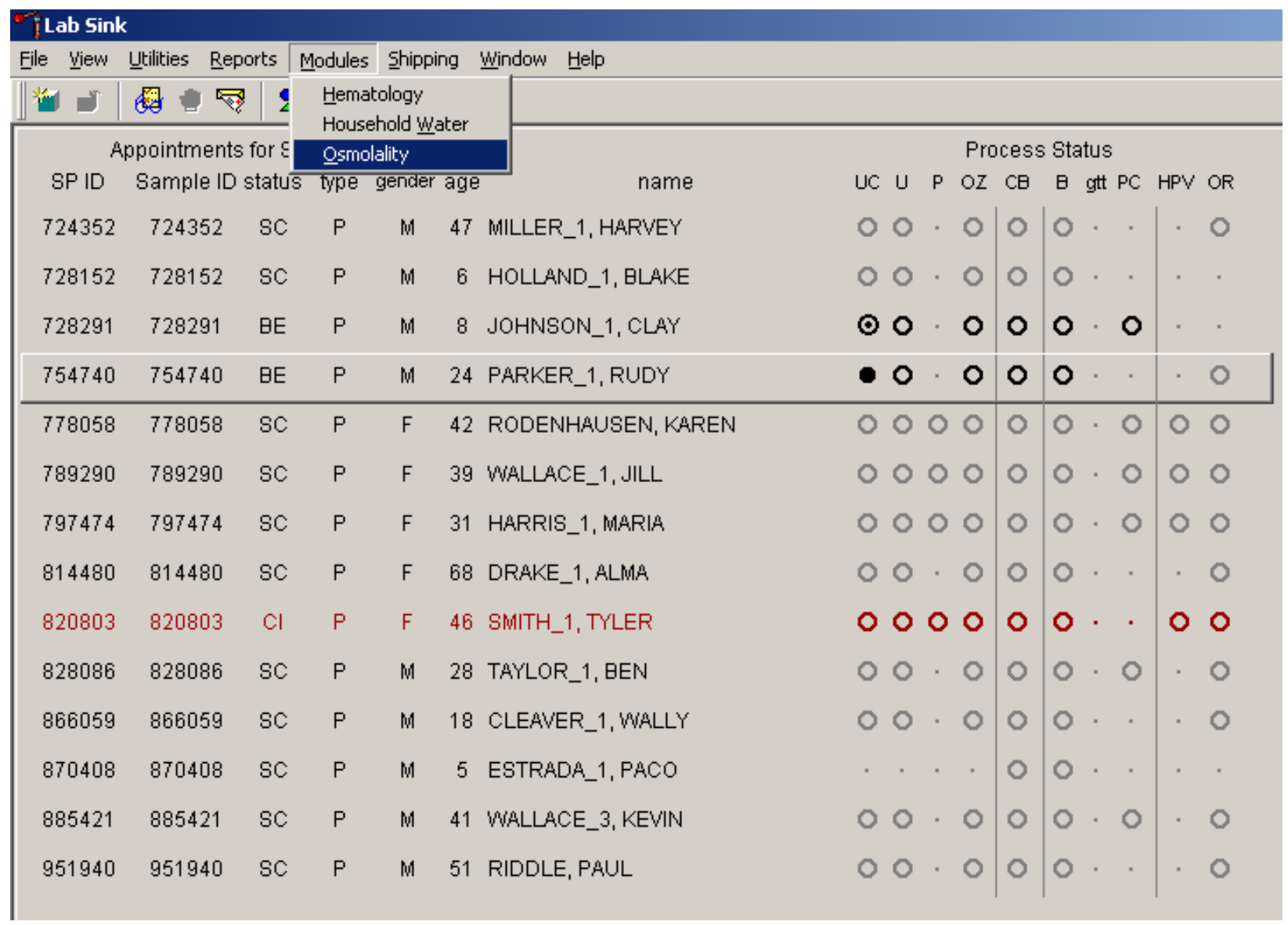

To open the Osmolality module, use the mouse to direct the mouse arrow to \{Modules\}, in the top menu bar, left click, drag the mouse arrow to \{Osmolality\}, and right click, or type [Alt][M/m], [O/o].

Alternatively, open the Osmolality module from the heads-up display. 


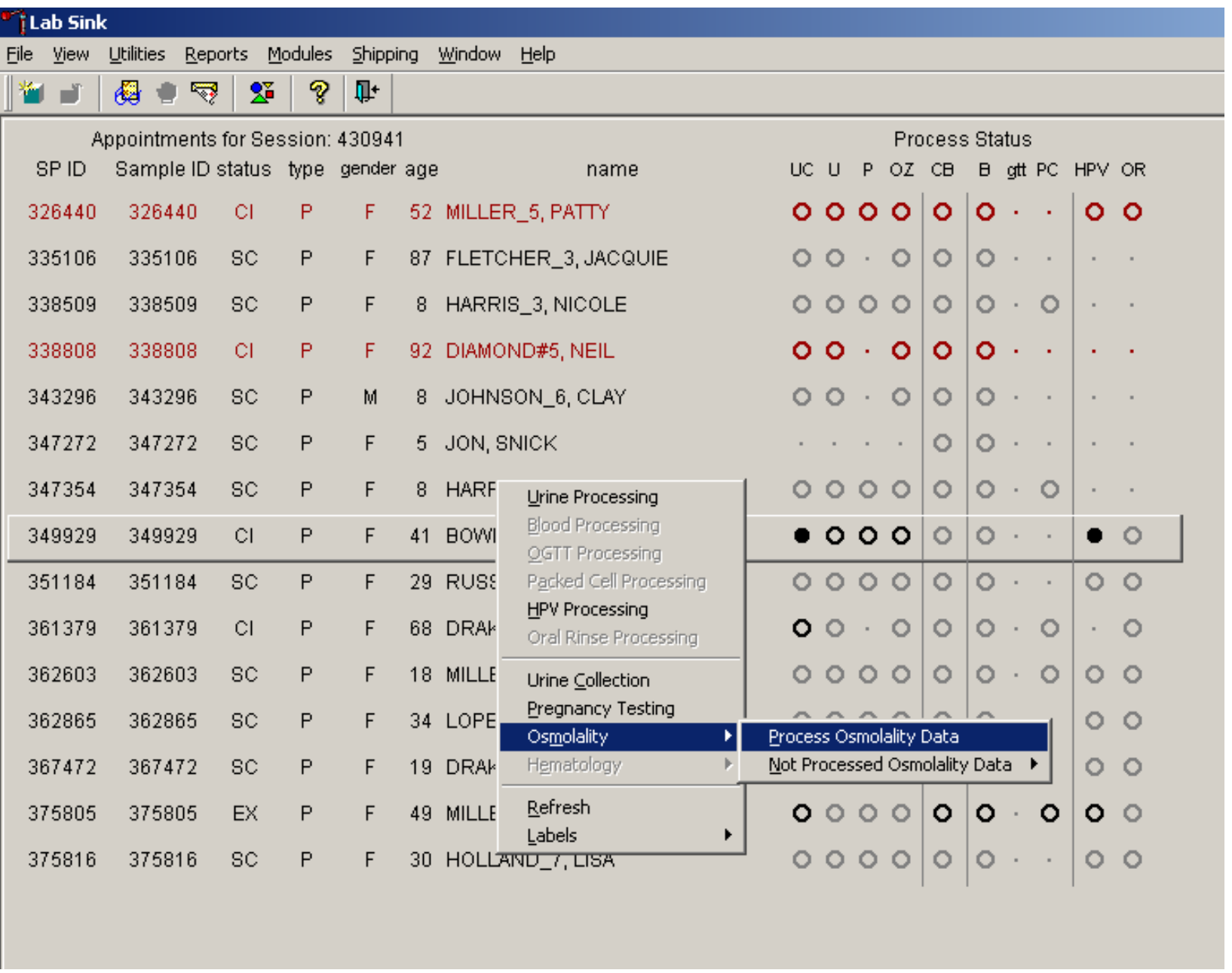

To access the Osmolality module, use the mouse to direct the mouse arrow to any SP, right click, drag the mouse arrow to $\{$ Osmolality\} then to \{Process Osmolality Data\}, and right click or right click and type [Shift] [M/m] [P/p]. Alternatively, use the up and down keys to move up and down the list until a particular SP is highlighted, right click, drag the mouse arrow to \{Osmolality then to $\{$ Process Osmolality Data\}, and right click or right click and type [Shift] [M/m] [P/p].

Either open the module or reject the specimen or add a reason or comment for every Osmolality record where urine was collected AND there are no Osmolality results. 


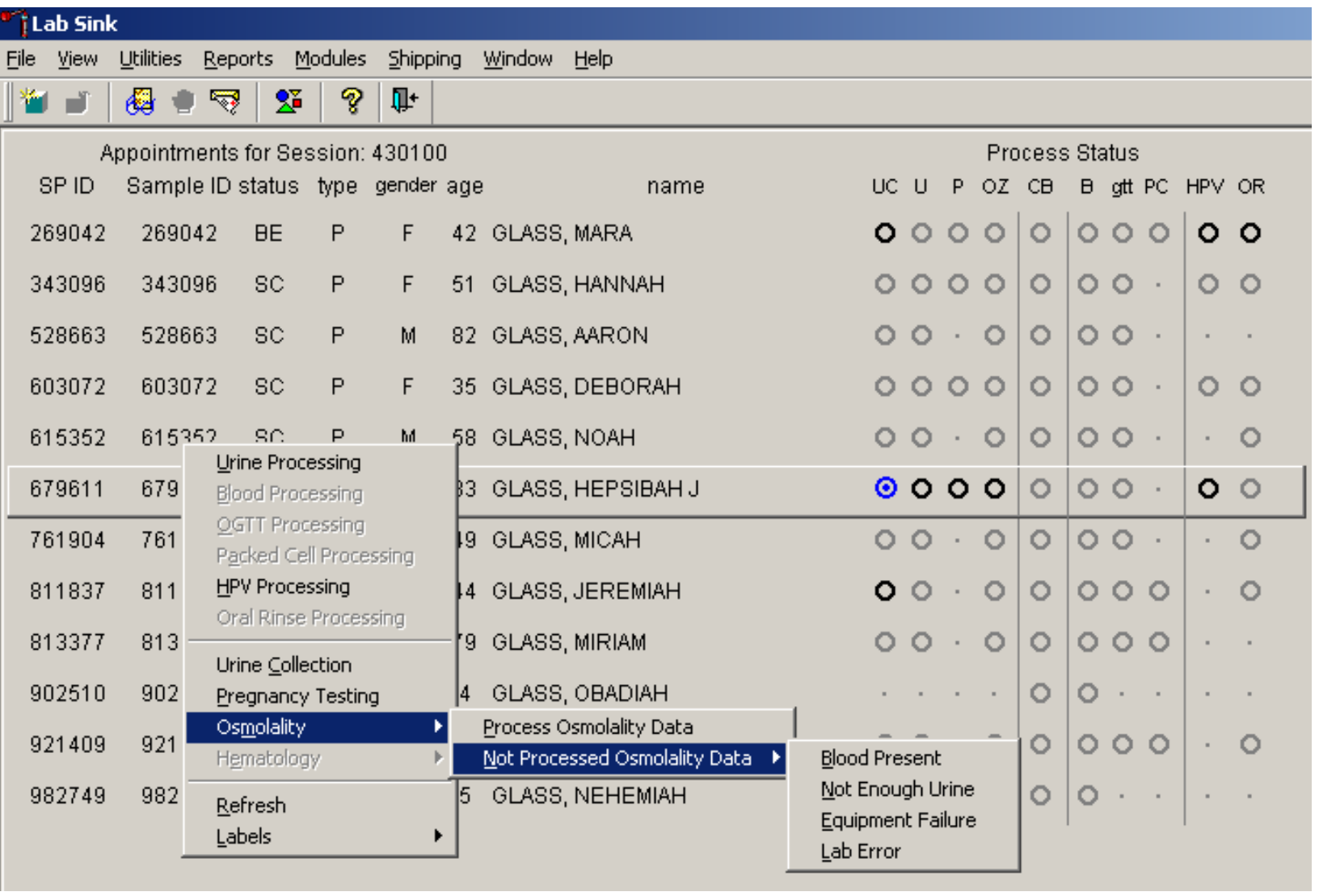

Open the module \{Process Osmolality Data\} or record a reason why Osmolality is not being run $\{$ Not Processed Osmolality Data\}. Select or record a comment for every Osmolality that is not run. To complete opening or accessing the Osmolality module, use the mouse to direct the mouse arrow to \{Process Osmolality Data\} and right click or type [P/p.] To record a reason why Osmolality is not being run, use the mouse to direct the mouse arrow to the exact SP's line on the heads-up display, right click, drag the mouse arrow to \{Not Processed Osmolality Data\}, and drag the mouse arrow to the correct reason and right click. Alternatively, type $[$ Shift] $[\mathrm{M} / \mathrm{m}][\mathrm{N} / \mathrm{n}]$ and $[\mathrm{B} / \mathrm{b}]$ for Blood Present, [N/n] for Not Enough Urine, [E/e] for Equipment Failure, or [L/l] for Lab Error. 


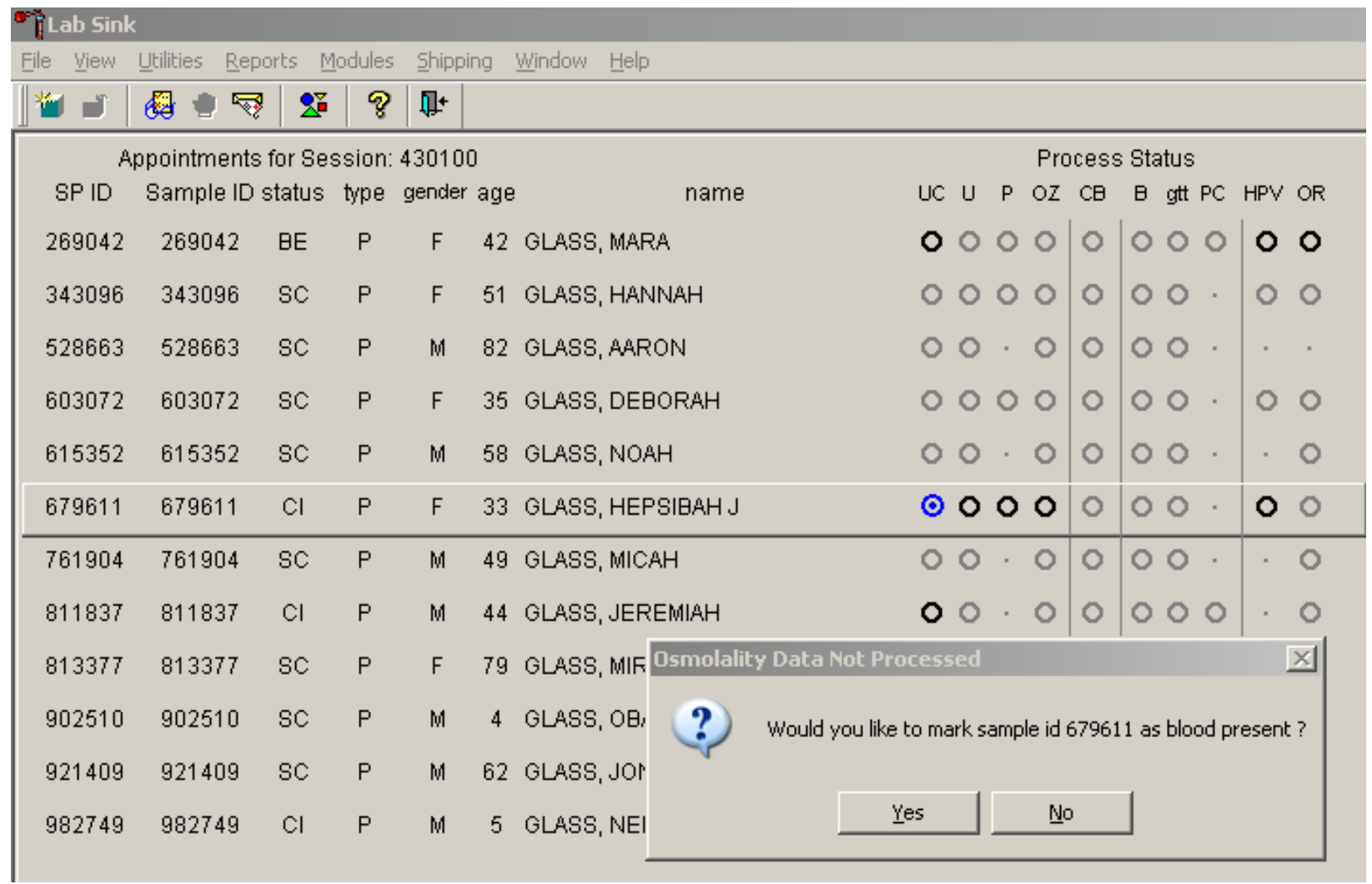

The system will ask for verification that the sample id should be marked as "not done" for the selected reason. To verify the reason, direct the mouse arrow over the Yes button and left click. Alternatively, type [Shift] [Y/y]. To cancel the selection, direct the mouse arrow over the No button and left click. Alternatively, type [Shift] [N/n]. 


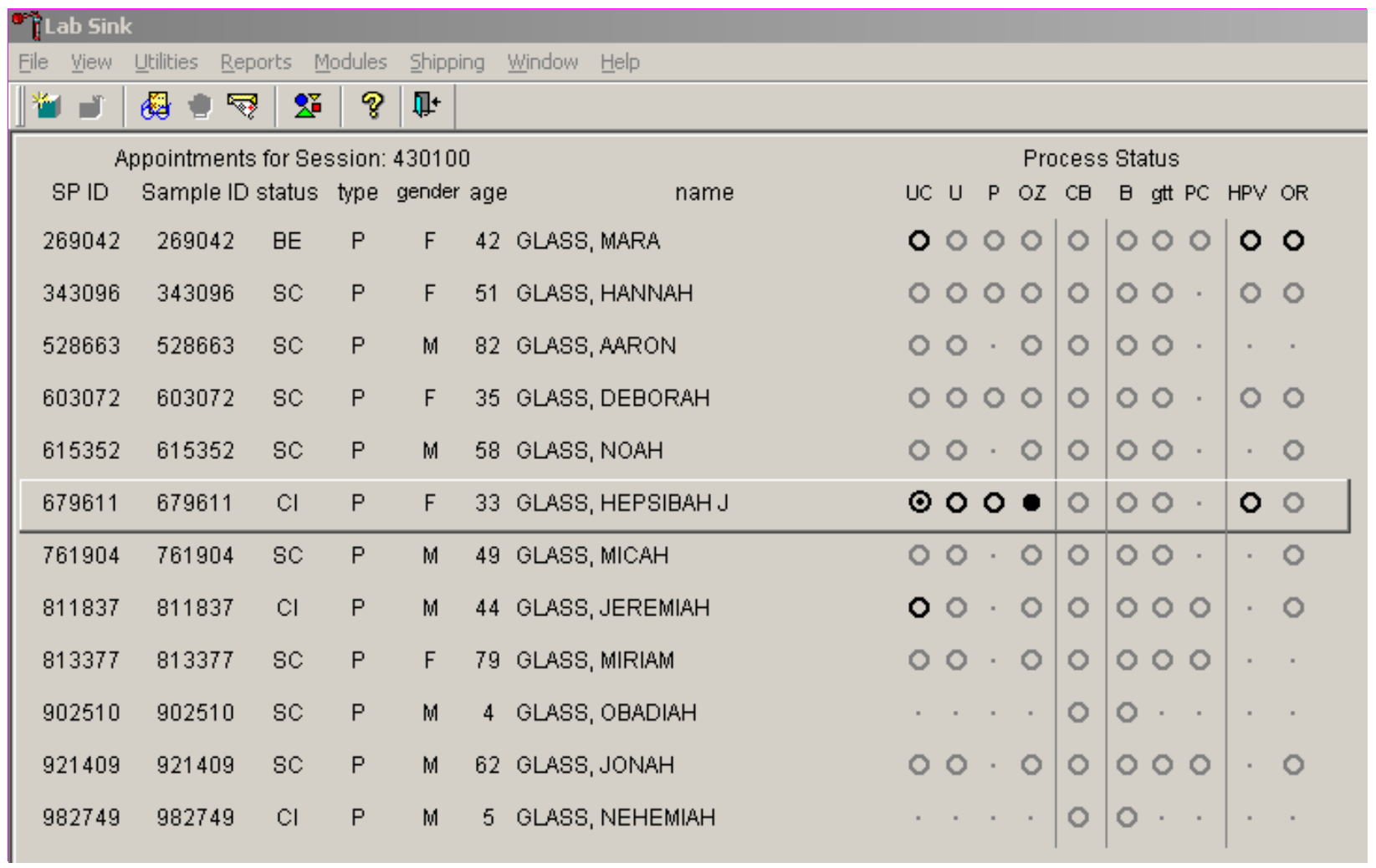

The bubble for osmolality will fill in as black on the heads-up screen if the module is marked as "not done" with a comment code.

\section{View Osmolality Module}

Open the module \{Process Osmolality Data\}. 


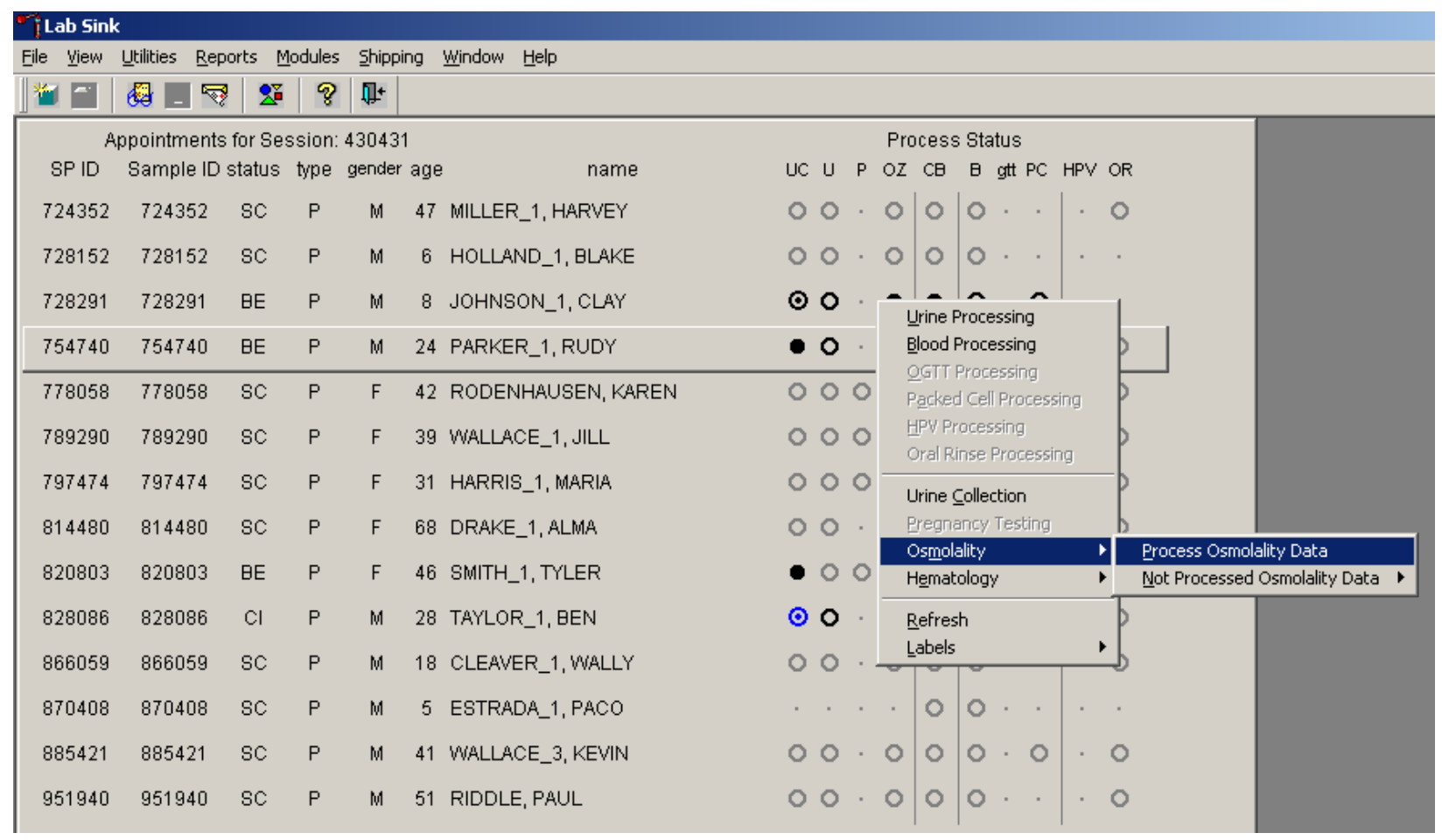

The Osmolality module displays. 


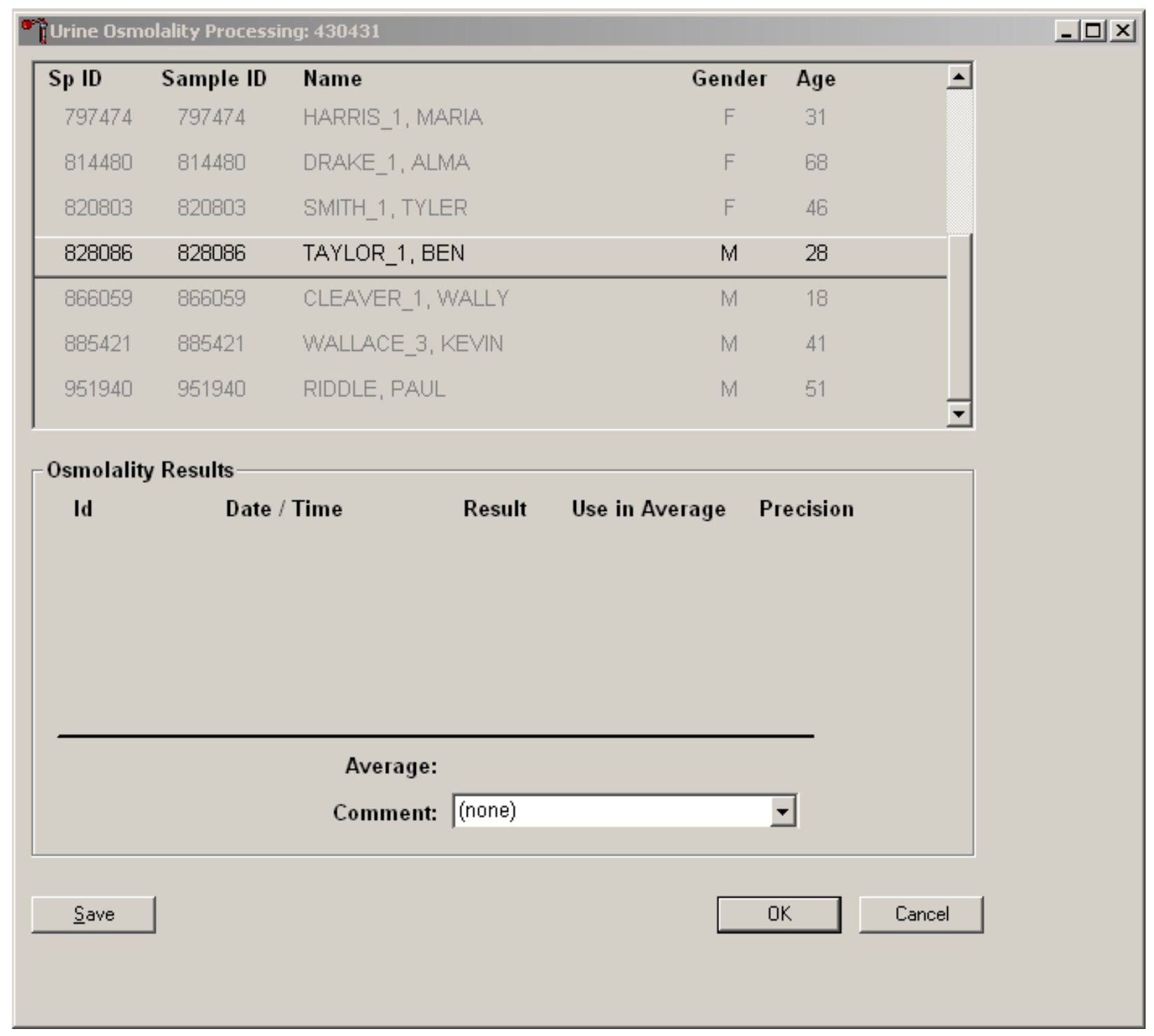

SPs eligible for osmolality testing appear in black on the upper part of the window. SPs are eligible for osmolality testing if their urine collection status is complete or QNS. If no osmolality runs have been transmitted from the Osmette II for a selected SP, no runs will be displayed in the lower portion of the screen. Press "OK" or "Cancel” to exit the screen.

3. Record an osmolality result when there is only enough sample for one run.

If a run has been transmitted from the Osmette II for a selected SP, the run(s) will display in the lower portion of the screen. 


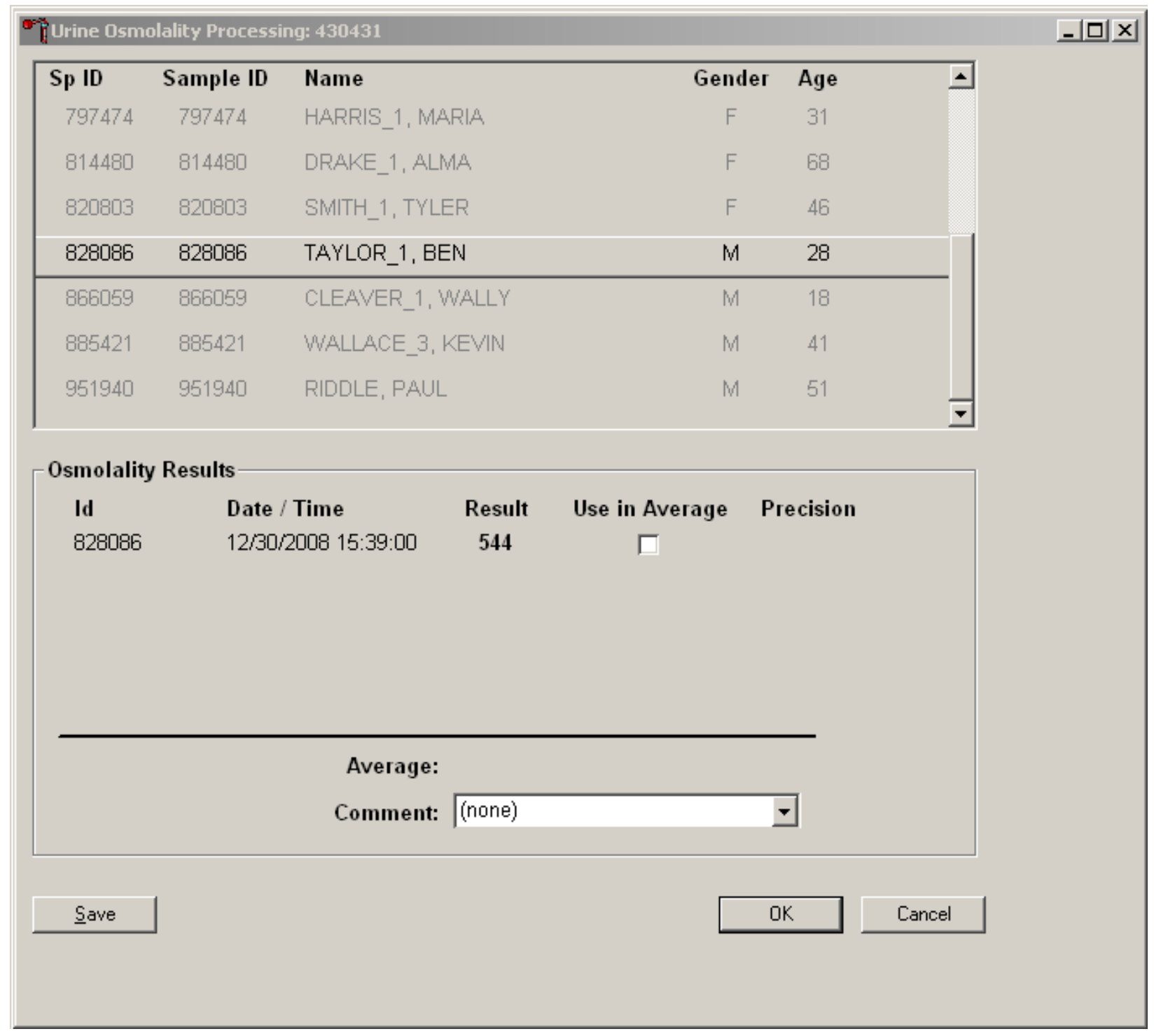

One run displays in the Osmolality Results portion of the screen. If there is only enough sample for one run, select the "Use in Average" box by directing the mouse arrow over the box and left click. The average will appear under the line. 


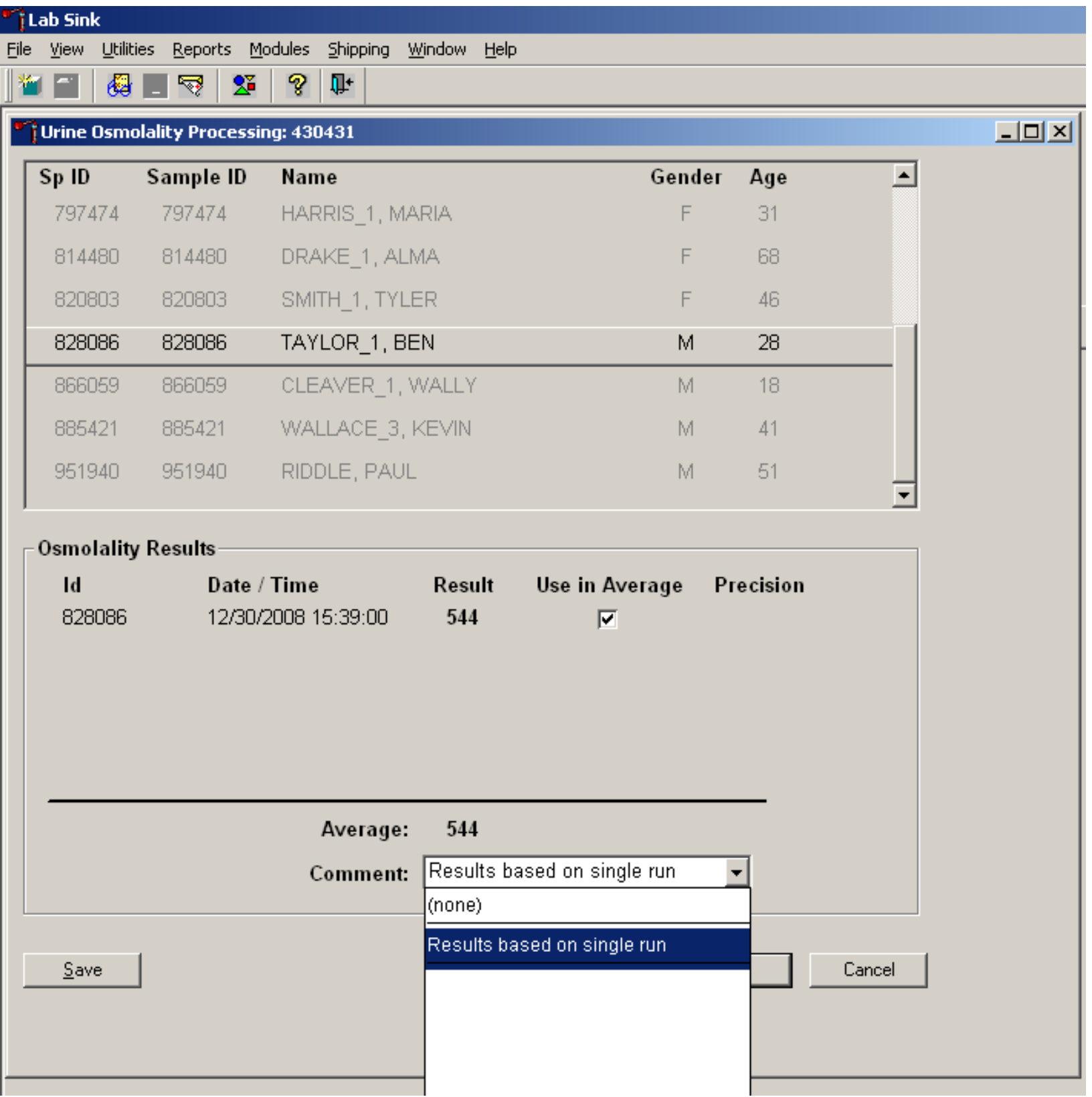

Select the comment "Results based on single run" by directing the mouse arrow to the down arrow and left click. Drag the mouse to the comment and left click. The comment will appear in the “Comment” field.

Save the result and comment code by directing the mouse arrow to the "Save" button and left click. Alternatively, direct the mouse arrow to the "OK" button and left click to save the result and exit the screen. 
The osmolality module will display as a solid black circle on the heads-up screen to indicate that the osmolality test has been completed for that SP.

4. Procedure for evaluating and handling precision limits

Multiple runs appear in the Osmolality Results section if more than one run has been transmitted from the Osmette II.

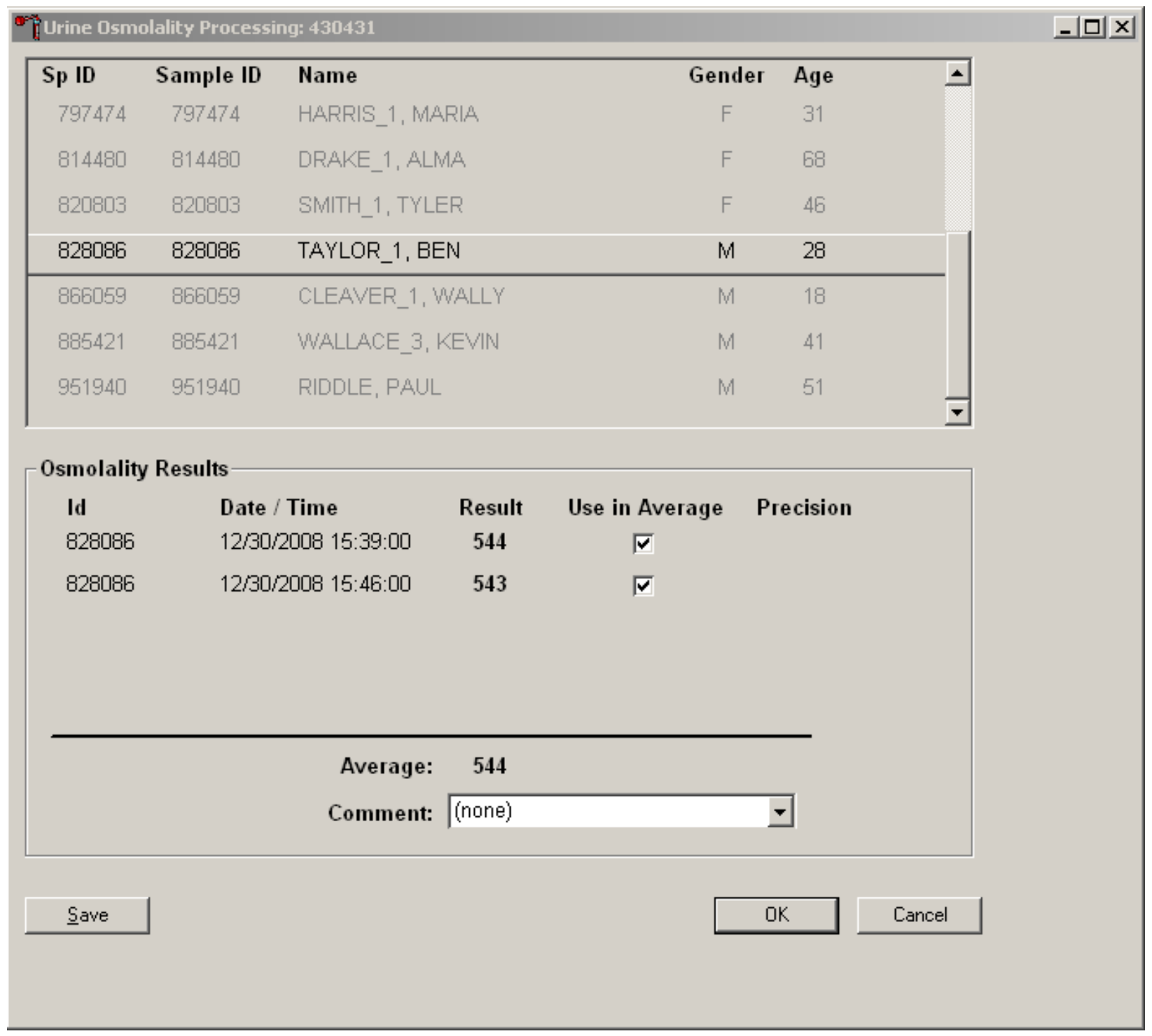

If both runs are within a range of 40 from each other, the application will automatically select the two runs and display the average under the line. Both results are within precision limits. No 
further runs are necessary to save the averaged result from a system perspective; however, each run should be evaluated by the medical technologist. It is the technologist's responsibility to determine if additional runs are necessary. To save the results without exiting the screen, direct the mouse arrow to the "Save" button and left click. To save the data and exit the screen, direct the mouse arrow to the "OK" button and left click. To cancel and exit without saving any changes made in the window, direct the mouse arrow to the "Cancel" button and left click.

Results are run that have a difference greater than 40 .

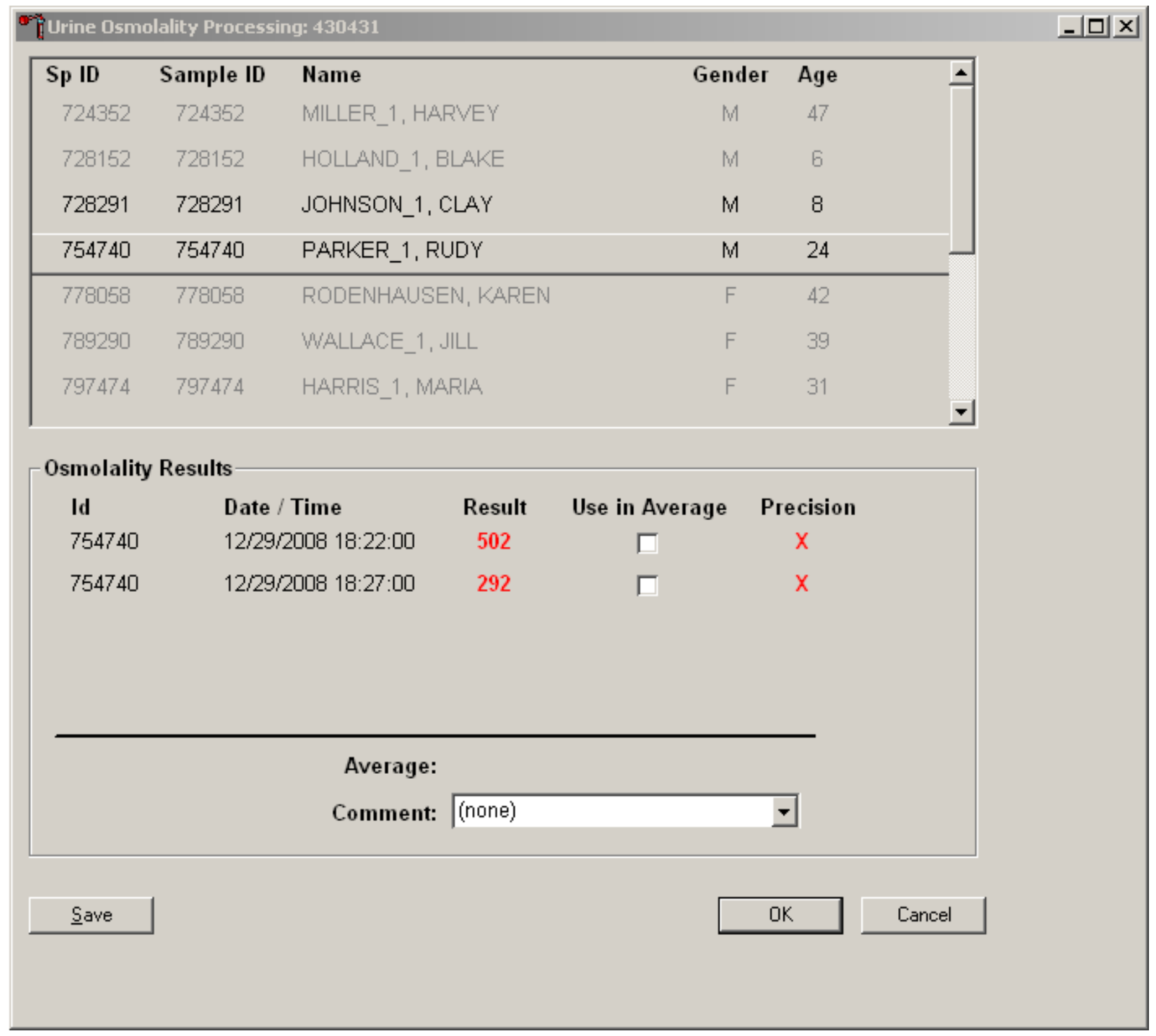


If two runs have a difference greater than 40 , the runs will both appear in red with an " $X$ " under the Precision column. Neither run will be selected to use in the average. The medical technologist should run the sample a third time.

\begin{tabular}{|c|c|c|c|c|c|}
\hline Sp ID & Sample ID & Name & Gender & Age & $\Delta$ \\
\hline 724352 & 724352 & MILLER_1, HARVEY & M & 47 & \\
\hline 728152 & 728152 & HOLLAND_1, BLAKE & M & 6 & \\
\hline 728291 & 728291 & JOHNSON_1, CLAY & $\mathrm{M}$ & 8 & \\
\hline 754740 & 754740 & PARKER_1, RUDY & $\mathrm{M}$ & 24 & - \\
\hline 778058 & 778058 & RODENHAUSEN, KAREN & $\mathrm{F}$ & 42 & \\
\hline 789290 & 789290 & WALLACE_1, JILL & $\mathrm{F}$ & 39 & \\
\hline 797474 & 797474 & HARRIS_1, MARIA & $\mathrm{F}$ & 31 & $=$ \\
\hline
\end{tabular}

Osmolality Results

$\begin{array}{llccc}\text { Id } & \text { Date } / \text { Time } & \text { Result } & \text { Use in Average } & \text { Precision } \\ 754740 & 12 / 29 / 200818: 22: 00 & \mathbf{5 0 2} & \text { 『 } \\ 754740 & 12 / 29 / 200818: 27: 00 & 292 & \square \\ 754740 & 12 / 29 / 200818: 29: 00 & \mathbf{5 0 7} & \text { 『 }\end{array}$

Average: $\quad 505$

Comment: (none)

Save

OK

Cancel

If the sample is run a third time and two of the results have a difference less than 40, those two runs will be selected to be averaged. No precision flags will appear. No further runs are necessary to save the averaged result from a system perspective; however, each run should be evaluated by the medical technologist. It is the technologist's responsibility to determine if additional runs are necessary. 
The runs can be unchecked manually if desired by directing the mouse over the check mark and left clicking. The run is deselected when no check mark appears in the "Use in Average" column. To select the run again, direct the mouse over the box and left click to display the check mark.

Osmolality has been repeated three times and all runs are out of precision.

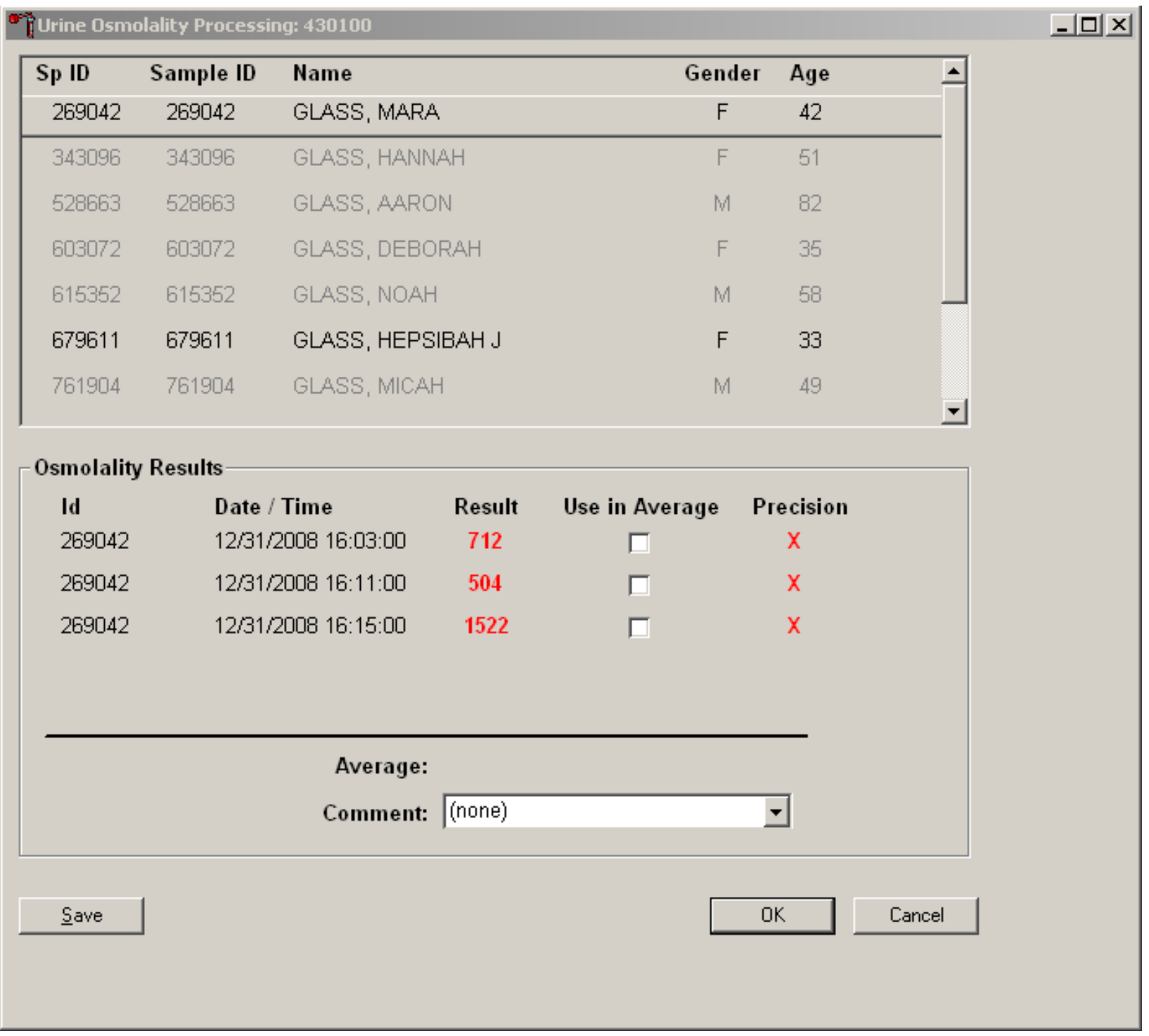

Three runs display in the Osmolality Processing screen and all are red with "X" flags in the Precision column. Three runs that are all out of precision should indicate to the medical technologist that the instrument is malfunctioning. Begin troubleshooting steps by checking the pipette functioning and calibration, and clean the Osmette II probe as described in Chapter 15, Operational Issues. 
5. Urine Osmolality Module Comments

\begin{tabular}{|l|l|}
\hline Comment & Use when: \\
\hline Laboratory error & There was a technologist error. \\
\hline Result based on single run & $\begin{array}{l}\text { The quantity of specimen is insufficient to run it } \\
\text { more than once. }\end{array}$ \\
\hline Blood present & $\begin{array}{l}\text { The urine is contaminated with blood. Do not run } \\
\text { an osmolality test. }\end{array}$ \\
\hline Not enough urine & $\begin{array}{l}\text { There is insufficient blood to perform at least one } \\
\text { complete osmolality test. }\end{array}$ \\
\hline
\end{tabular}

B. Osmette II Instrument Messages

1. Review the message and remedial action

The Osmette II displays several error messages. Note the error message and take remedial action.

\begin{tabular}{l|l}
\multicolumn{1}{c|}{ Display } & \multicolumn{1}{|c}{ Cause and Action } \\
\hline PREFREEZE & $\begin{array}{l}\text { Air bubbles or other particles caused the sample } \\
\text { to prefreeze. Check sample for particulate } \\
\text { matter that should be centrifuged. Rerun } \\
\text { sample, checking for proper pipetting technique. }\end{array}$ \\
\hline NO FREEZE & $\begin{array}{l}\text { The sample did not freeze. Rerun the sample. If } \\
\text { the instrument gives an extraordinary amount of } \\
\text { NO FREEZE messages, contact Precision } \\
\text { Systems for support. }\end{array}$ \\
\hline NO SAMPLE & $\begin{array}{l}\text { No sample was inserted into the cooling well, or } \\
\text { the cooling block is malfunctioning. Check to } \\
\text { make sure a sample was inserted into the } \\
\text { instrument. Rerun the sample. If the message } \\
\text { continues to appear, contact Precision Systems } \\
\text { for support. }\end{array}$ \\
\hline BUTTON ON & $\begin{array}{l}\text { One or more of the buttons were pressed during } \\
\text { the power-on sequence. Turn off the machine } \\
\text { and turn it back on, ensuring that nothing is } \\
\text { touching and accidentally depressing the SPEC, } \\
\text { CALIBRATION, or RUN keys. }\end{array}$ \\
\hline
\end{tabular}


The following messages require service from Precision Systems.

\begin{tabular}{l|l}
\hline Timer & \\
RAM & \\
\hline A/D Status & Contact the chief medical technologist and \\
MUX & Precision Systems for support.
\end{tabular}

XII. Limitations of Method: Specimen Rejection, Interfering Substances and Conditions

This method limits samples to human urine.

A. Specimen Rejection

1. Reject blood-contaminated specimens.

B. Interfering Substances and Conditions

1. Particulate matter will cause the sample to pre-seed when supercooled, interrupting the measurement cycle. Undissolved particles or precipitate in the sample may be eliminated by centrifuging the sample.

2. Samples that have bubbles in the pipette sample may give incorrect or not reproducible results. Double checking the pipetted sample for bubbles and discarding improperly pipetted samples will reduce the chance of inaccurate results due to air bubbles.

XIII. Reference Ranges

Not applicable.

XIV. $\quad$ Action Limits

Not applicable. 
XV. Specimen Storage and Handling during Testing

A. Specimen Storage

1. Store specimens refrigerated until processed.

2. Run within 4 hours after collection.

XVI. Alternative Method for Performing Test or Storing Specimens if Test System Fails

There is no alternative method for this test. Store urine in the refrigerator for no more than 4 hours.

XVII. Test Results Reporting System: Protocol for Reporting Action Limits

All records, including QA/QC data, will be maintained for 6 years. Use only numerical identifiers for SP results.

XVIII. Quality Control Summary Statistics and Graphs

Chapter 14 includes a separate detailed description of the comprehensive quality control plan. Compare all three instruments using the CAP proficiency results.

XIX. References

1. Precision Systems Osmette II Operating Manual Model 5005, Precision Systems, Inc. 2006 


\section{OGTT APPLICATION}

\subsection{OGTT Application - Section Two (Blood Draw)}

If an SP consumed the entire calibrated dose of the Trutol solution in 10 minutes and 1 hour and 110 minutes has elapsed, then he or she is eligible to return to phlebotomy to have (1) one additional 2-mL gray top drawn and (2) any tubes that were not drawn during the initial venipuncture examination drawn.

A pop-up message window will alert the coordinator and the phlebotomist when an SP is eligible to return to phlebotomy for the second blood draw. To close or remove the Message Center box, use the mouse to direct the mouse arrow to the Close button and left click, or use the mouse to direct the mouse's arrow to the upper right corner to the $\mathrm{X}$ and left click.

Logon to the OGTT application and log the SP into the component. 
The blood draw screen is divided into two sections.

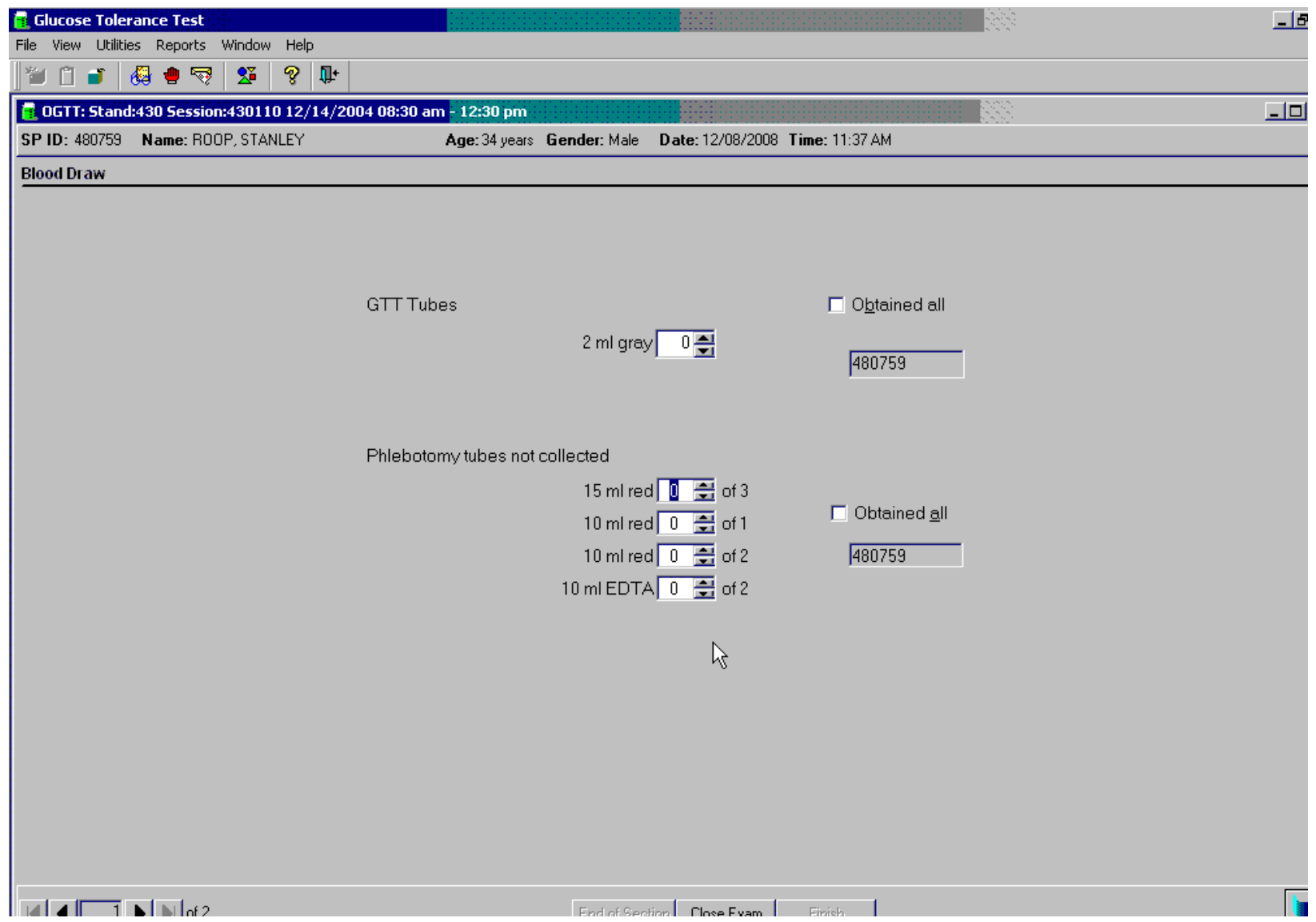

The upper section is identical to the venipuncture screen in the phlebotomy examination except that it contains only one 2-mL gray top tube. The bottom half of the screen lists any tubes that were not drawn during the initial venipuncture examination. The sections function exactly like the venipuncture screen in the phlebotomy examination. 
If all of the blood tubes were drawn during the phlebotomy examination, then only the first half of the blood draw screen will contain the 2-mL gray top tube. The lower section of the blood draw screen will indicate that there are "No phlebotomy tubes to collect."

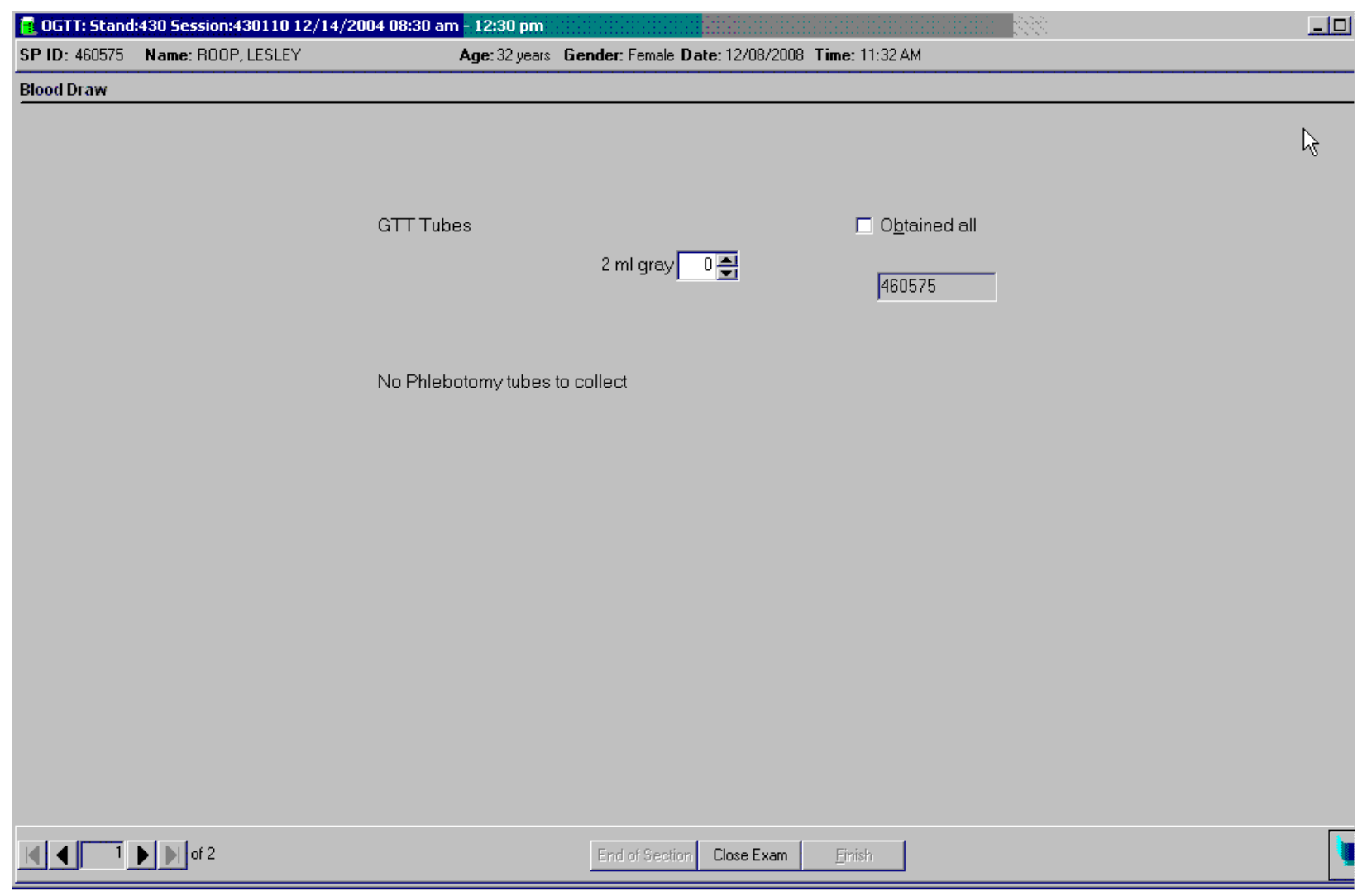

Draw the additional 2-mL gray top tube and then any tubes that were missed during the phlebotomy examination first. Draw all tubes according to the protocol described in Chapter 4.

Labels will print for all the tubes marked as collected, including the second 2-mL gray top tube. The label for the second 2-mL gray top tube contains a bar code with the SP ID concatenated with the vessel ID (\# 98) and includes the test name "OGTT.” 
Immediately after completing the venipuncture, enter the results of the blood draw and any comments about the venipuncture.

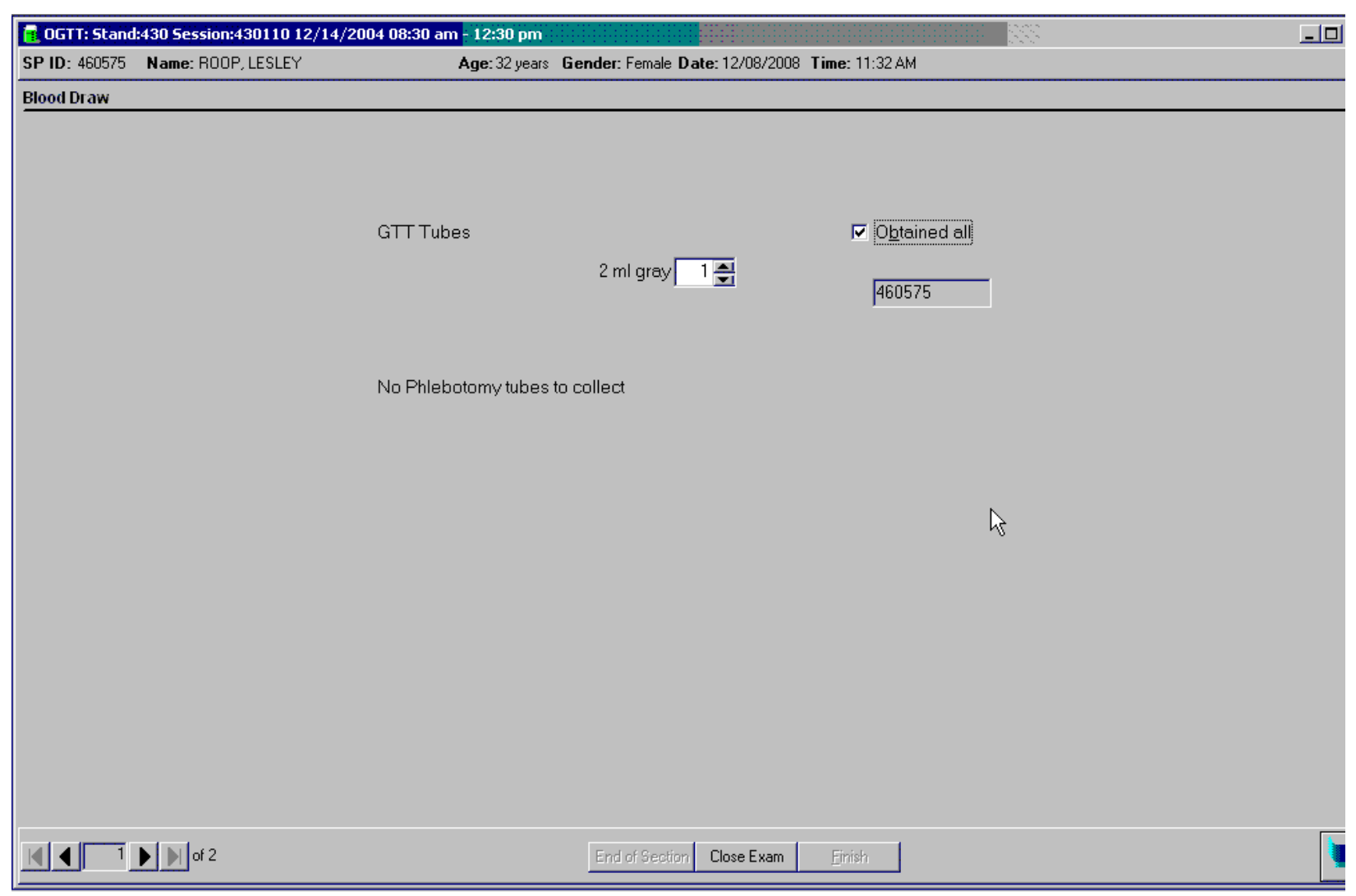

To record all tubes as filled or obtained, use the mouse to direct the mouse arrow to the “Obtained all” check box and left click, or type [Alt] [B/b]. This records a check mark in the box and marks all tubes as obtained. To mark individual tubes as filled or obtained, use the mouse to direct the mouse arrow to the up-down controls on the spin box and toggle the number of each tube up or down or type the correct number using the numeric keys. 
Verify the Blood Draw Section Status.

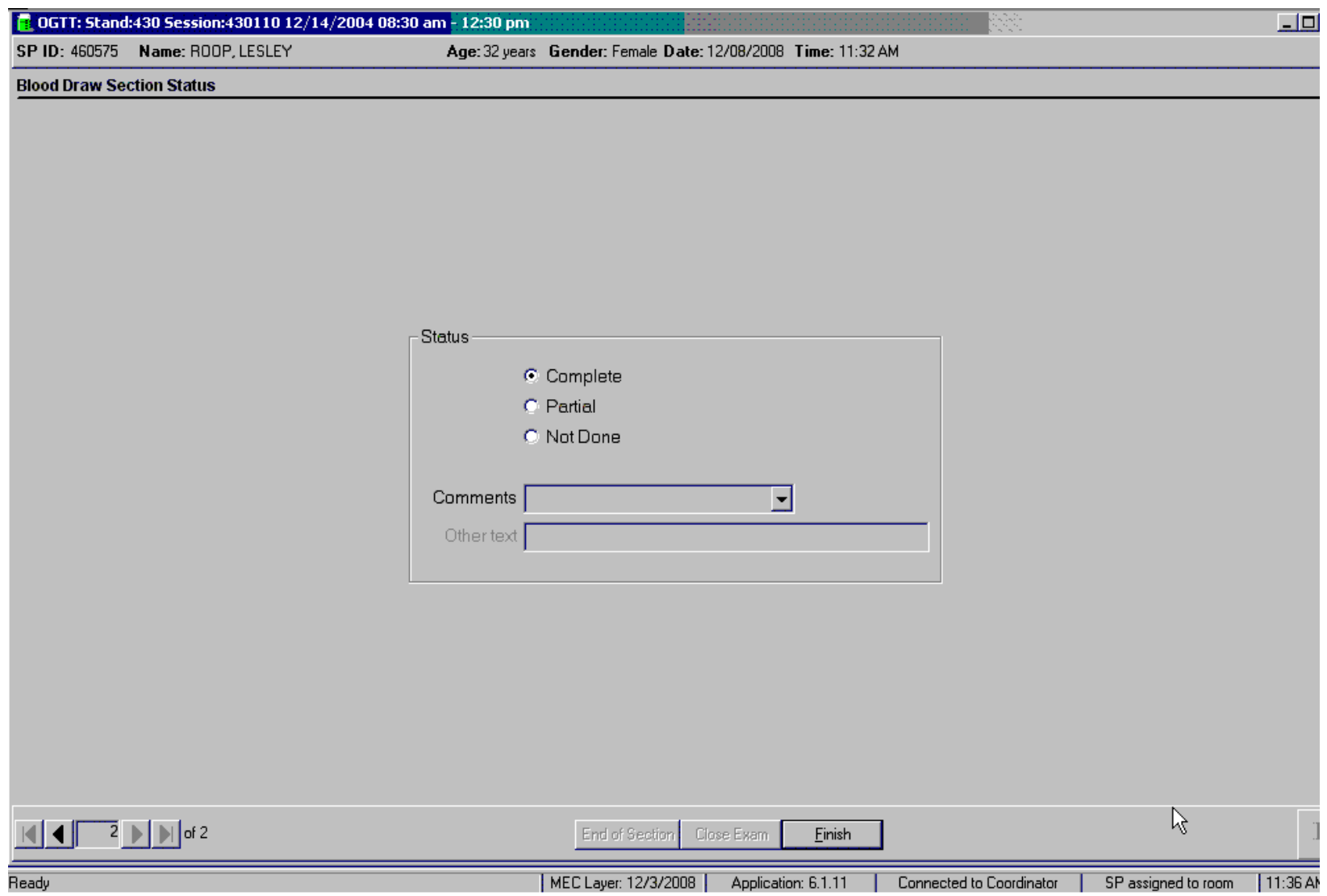

The Blood Draw Section Status is complete if the second 2-mL gray top tube is collected.

If any blood tubes were not drawn during the initial phlebotomy examination and they were drawn during the blood draw section of the OGTT examination, then the phlebotomy section status will automatically update to reflect the additional tubes drawn during the OGTT examination.

Remove the Dymo label from the SP's gown and ask the SP if he or she is allergic to peanuts. If not, then offer the SP peanut butter crackers and juice. If the SP is allergic to peanuts or does not know, then offer the standard crackers and juice. 
Use comment codes to explain Not Done status codes.

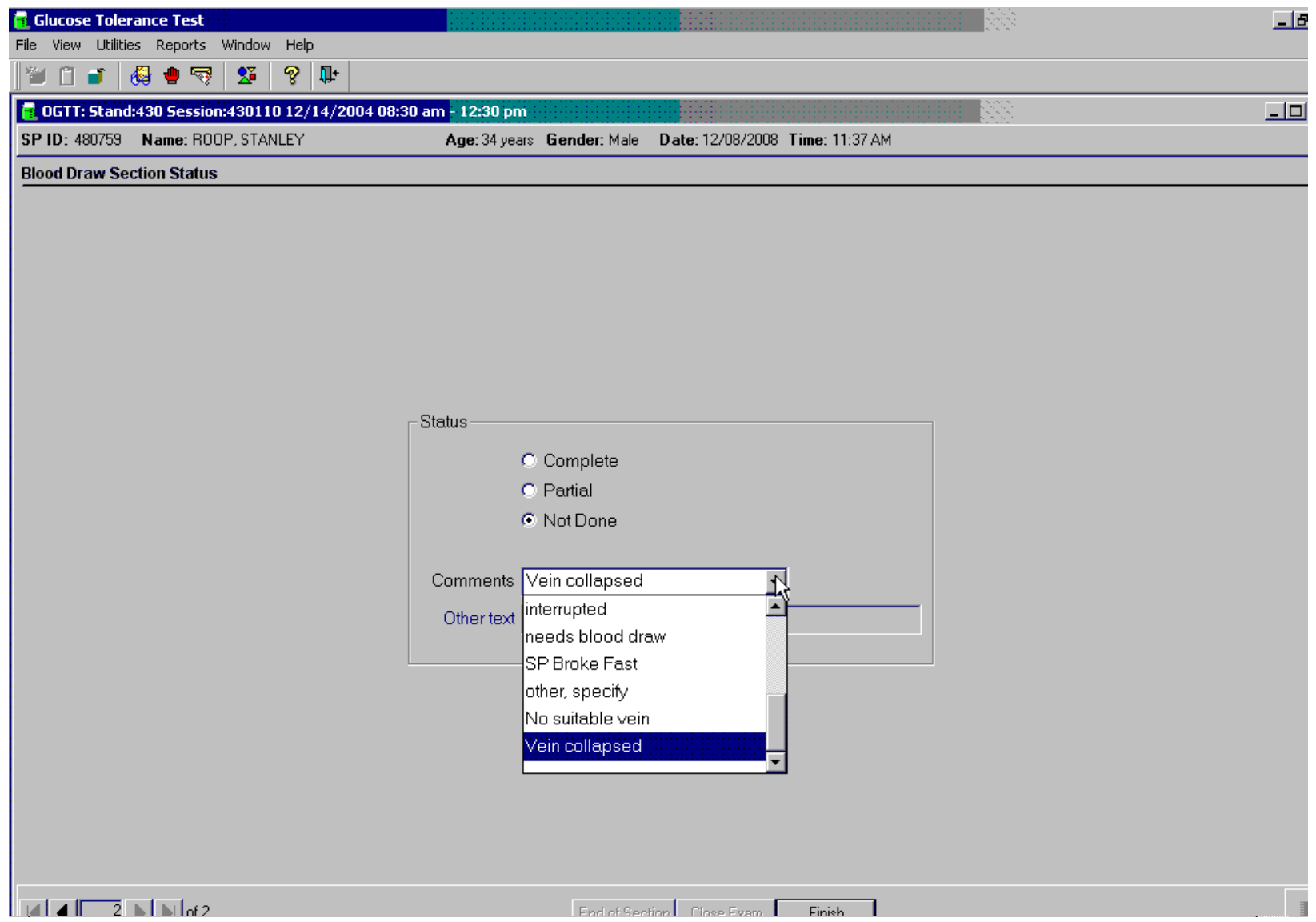

The blood draw section status is Not Done if the second 2-mL gray top tube is not collected.

Once the tube is marked as not obtained, the comment will default to "needs blood draw." Correct the comment by selecting the most appropriate comment code. To record a comment in the Comment text box, use the mouse to direct the mouse arrow to the scroll arrow on the drop-down list, left click, drag the mouse arrow to the desired choice, and left click. Use the scroll bar to view all choices. Alternatively, use the up and down keyboard arrows to scroll through the choices or type the first letter of the desired comment code. 


\begin{tabular}{|c|c|}
\hline Comment Code & Use when: \\
\hline Safety exclusion & $\begin{array}{l}\text { Not applicable } \\
\text { This is reserved for positive responses to the hemophilia and chemotherapy } \\
\text { exclusion questions and it is automatically coded by the application. The } \\
\text { coordinator may code exams using this comment. }\end{array}$ \\
\hline SP refusal & $\begin{array}{l}\text { The SP refuses to have his or her blood collected. This is an SP-initiated } \\
\text { nonresponse due to refusal. The SP refuses the component for any reason other } \\
\text { than an illness or emergency. If the SP refuses in the reception area, the } \\
\text { coordinator codes the exam. If the SP refuses after starting the exam, the } \\
\text { examiner codes the exam. Use this comment to code partial exams when the } \\
\text { SP refuses after one tube has been drawn, or the blood flow stops after one } \\
\text { tube but before all tubes have been successfully drawn. }\end{array}$ \\
\hline No time & Not applicable \\
\hline Physical limitation & Not applicable \\
\hline $\begin{array}{l}\text { Communication } \\
\text { problem }\end{array}$ & Not applicable \\
\hline Equipment failure & Not applicable \\
\hline SP ill/emergency & $\begin{array}{l}\text { Use this comment to code not done or partial when the SP faints or is about to } \\
\text { faint or the SP becomes ill or an emergency occurs and the test cannot be } \\
\text { performed on the SP. }\end{array}$ \\
\hline Interrupted & Not applicable \\
\hline Needs blood draw & $\begin{array}{l}\text { The system uses this code as a default after the SP consumed the Trutol. } \\
\text { Correct this code depending on the situation. }\end{array}$ \\
\hline SP broke fast & $\begin{array}{l}\text { Use this code if the SP ate or drank anything other than plain water after they } \\
\text { drank the Trutol and before the blood draw. }\end{array}$ \\
\hline $\begin{array}{l}\text { Error (technician/ } \\
\text { software/supply) }\end{array}$ & $\begin{array}{l}\text { Use this comment to code partial and not done exams when there are } \\
\text { phlebotomist errors, or software or supply issues. }\end{array}$ \\
\hline Other, specify & $\begin{array}{l}\text { If the above reason for a status Code of Not Done is not explained by one of } \\
\text { the Comment Codes, the examiner must choose Other, specify and record a } \\
\text { comment in the text field. }\end{array}$ \\
\hline No suitable vein & $\begin{array}{l}\text { Use this comment to code exams as not done (no tubes drawn) when the SP } \\
\text { does not have an accessible vein, any arms available, casts on both arms, there } \\
\text { is a rash over the entire area, or the blood draw was started but there was no } \\
\text { blood in the tubing. }\end{array}$ \\
\hline Vein collapsed & $\begin{array}{l}\text { Use this comment to code exams as partial when one or more tubes are } \\
\text { successfully drawn but blood flow stops before all tubes are drawn or when the } \\
\text { veins collapse. }\end{array}$ \\
\hline
\end{tabular}

When finished, use the mouse to direct the mouse arrow to the bright blue arrow in the bottom right hand corner and left click to exit or select [Enter] when this blue arrow is highlighted. 


\section{Laboratory Processing}

\subsubsection{Laboratory Overview}

The vessel for OGTT is \#98 (Exhibit 13-1). Use the gtt processing module to record the blood processing results. Labels for the OGTT vessel print for all SPs after the last blood processing set of labels. Keep the OGTT labels together and place them in the lab with the blood processing racks. Do not label the vessels in advance; label each vessel as it is processed.

Exhibit 13-1. Primary SP’s OGTT processing protocol

\begin{tabular}{|l|c|c|c|c|c|c|c|}
\hline ID & Test name & Ages & $\begin{array}{c}\text { Sample } \\
(\mathrm{mL})\end{array}$ & Sample type & $\begin{array}{c}\text { Collection } \\
\text { type }\end{array}$ & $\begin{array}{c}\text { Vessel } \\
\text { type }\end{array}$ & Remarks \\
\hline 98 & OGTT & $12+$ & 0.5 & Plasma & Gray & 2-mL & Morning SPs only \\
\hline
\end{tabular}

\subsubsection{Process the 2-mL Gray Top Tube for OGTT}

Centrifuge and separate the plasma from the 2-mL gray top tube as soon as possible. Process the specimen even if the contents of the gray tube clot.

- Place the tube in the centrifuge carrier of either tabletop centrifuge. Balance the tube in the centrifuge by using water-filled tubes if necessary. Centrifuge the 2-mL gray tube at $17-25^{\circ} \mathrm{C}$ or $11-8^{\circ} \mathrm{C}$ and $2,900 \mathrm{rpm}$ for 10 minutes to yield plasma for vessel 98 (OGTT.)

\section{Vessel 98}

- $\quad$ Use a calibrated plastic transfer pipette to transfer all plasma from this tube but at least 0.5 -mL plasma for vessel 98 (OGTT) on primary into a 2-mL vessel.

- Determine if the plasma is hemolyzed, turbid, lipemic, or icteric. If so, enter a comment to describe the plasma when entering the processing results.

- Close all vessels secured to prevent leakage and evaporation.

- Record results using the gtt module as described in Section 8.7.3 of the Laboratory Procedures Manual.

- Store all specimens immediately as outlined in Section 8.10 of the Laboratory Procedures Manual. 


\subsubsection{Record the Results of Specimen Processing}

Open and log onto the Laboratory application. Use the heads-up display to view the SPs, the modules for which they are eligible, and their current process status. Record blood processing results for vessel 98 using the "gtt" module.

\begin{tabular}{|c|c|c|c|c|c|c|c|c|c|c|c|c|c|}
\hline \multicolumn{14}{|l|}{ Lab Sink } \\
\hline \multirow{2}{*}{ 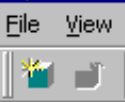 } & \multirow{2}{*}{ 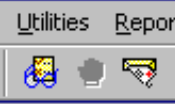 } & \multicolumn{2}{|c|}{ Modules } & \multicolumn{3}{|c|}{ Shipping window $\underline{\text { Help }}$} & & & & & & & \\
\hline & & 8 & 8 & 1]+ & & & & & & & & & \\
\hline & ppointments 1 & for Ses: & ssion: 4 & 430110 & & & & & & ces & s Status & & \\
\hline SPID & Sample ID s & status & type & gender & age & name & UC & $U P$ & $O Z$ & $\mathrm{CB}$ & $\mathrm{B}$ gtt $\mathrm{PC}$ & $\mathrm{CHPV}$ & OR \\
\hline 346104 & 346104 & $\mathrm{BE}$ & $\mathrm{P}$ & $\mathrm{F}$ & 28 & ROOP, NATALIE & 0 & 00 & 0 & 0 & 00 & 0 & 0 \\
\hline 460575 & 460575 & $\mathrm{BE}$ & $\mathrm{P}$ & $\mathrm{F}$ & & ROOP, LESLEY & 0 & 00 & 0 & 0 & 00. & $\circ$ & 0 \\
\hline 480759 & 480759 & $\mathrm{BE}$ & $\mathrm{P}$ & $M$ & 34 & ROOP, STANLEY & 0 & 0. & 0 & 0 & 00. & $\cdot$ & 0 \\
\hline 482080 & 482080 & $\mathrm{SC}$ & $P$ & $M$ & & ROOP, STUART & 0 & 0. & 0 & 0 & 00. & $\cdot$ & 0 \\
\hline 549961 & 549961 & $\mathrm{sc}$ & $P$ & $M$ & 26 & ROOP, WESLEY & 0 & 0. & 0 & 0 & 000 & & 0 \\
\hline 851056 & 851056 & $\mathrm{sc}$ & $P$ & $\mathrm{~F}$ & & ROOP, KELLEY & 0 & ho & 0 & 0 & 00. & 0 & 0 \\
\hline 860681 & 860681 & $\mathrm{SC}$ & $P$ & $\mathrm{~F}$ & 38 & ROOP, DONNA & 0 & 00 & 0 & 0 & 000 & 0 & 0 \\
\hline
\end{tabular}

The "gtt" module is grayed out until at least one SP has the second 2-mL gray top tube drawn. Once one SP has the second 2-mL gray top tube drawn, the processing circle for that SP turns black. 
Select the correct SP and access the OGTT Processing module.

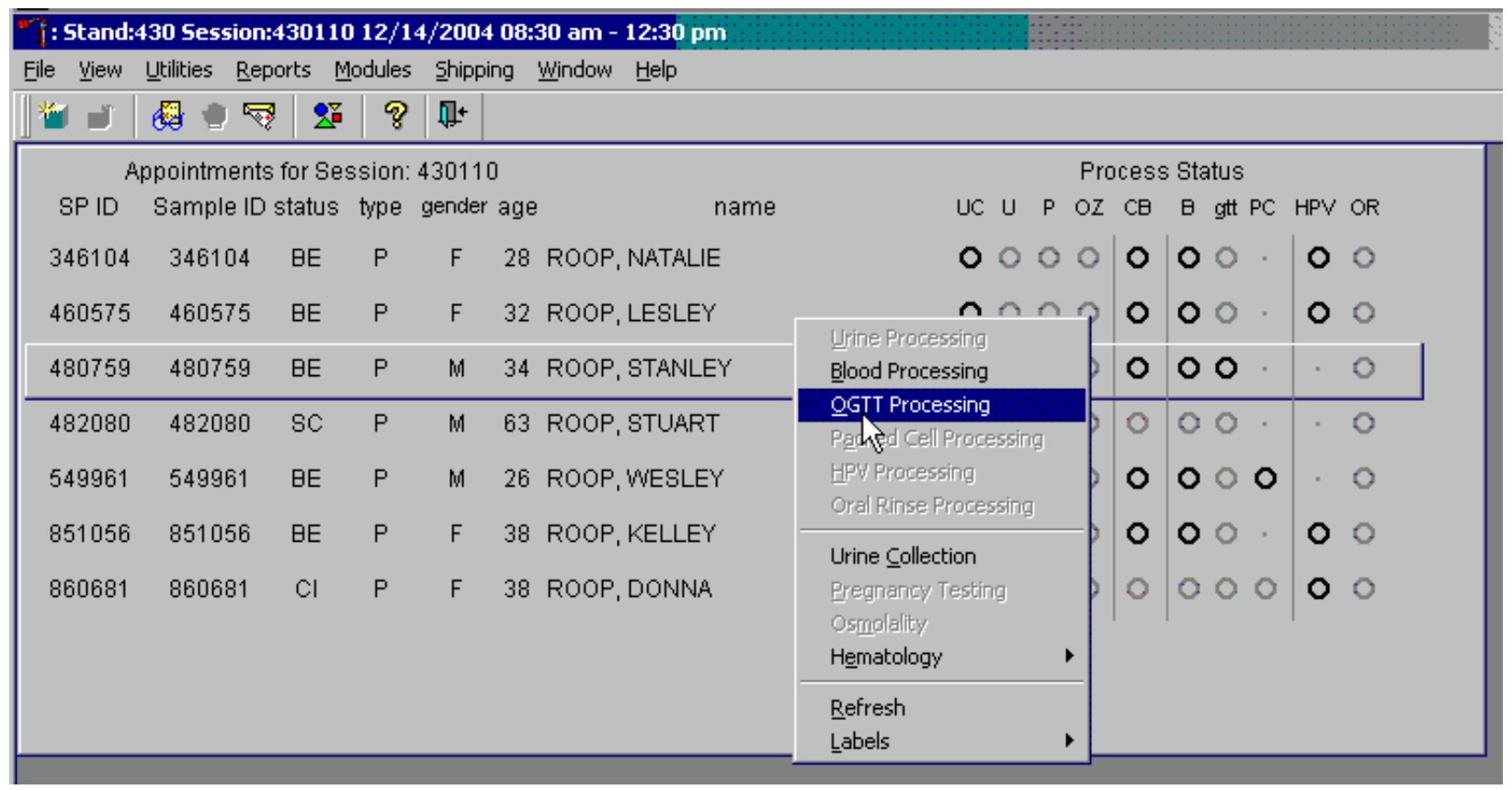

To access the OGTT Processing module, use the mouse to direct the mouse arrow to the SP on the heads-up display, left click, drag the mouse arrow to OGTT Processing, and right click. 
Record the vessel 98 processing results in the OGTT processing window. All SPs are included in the same window but are listed separately by rows.

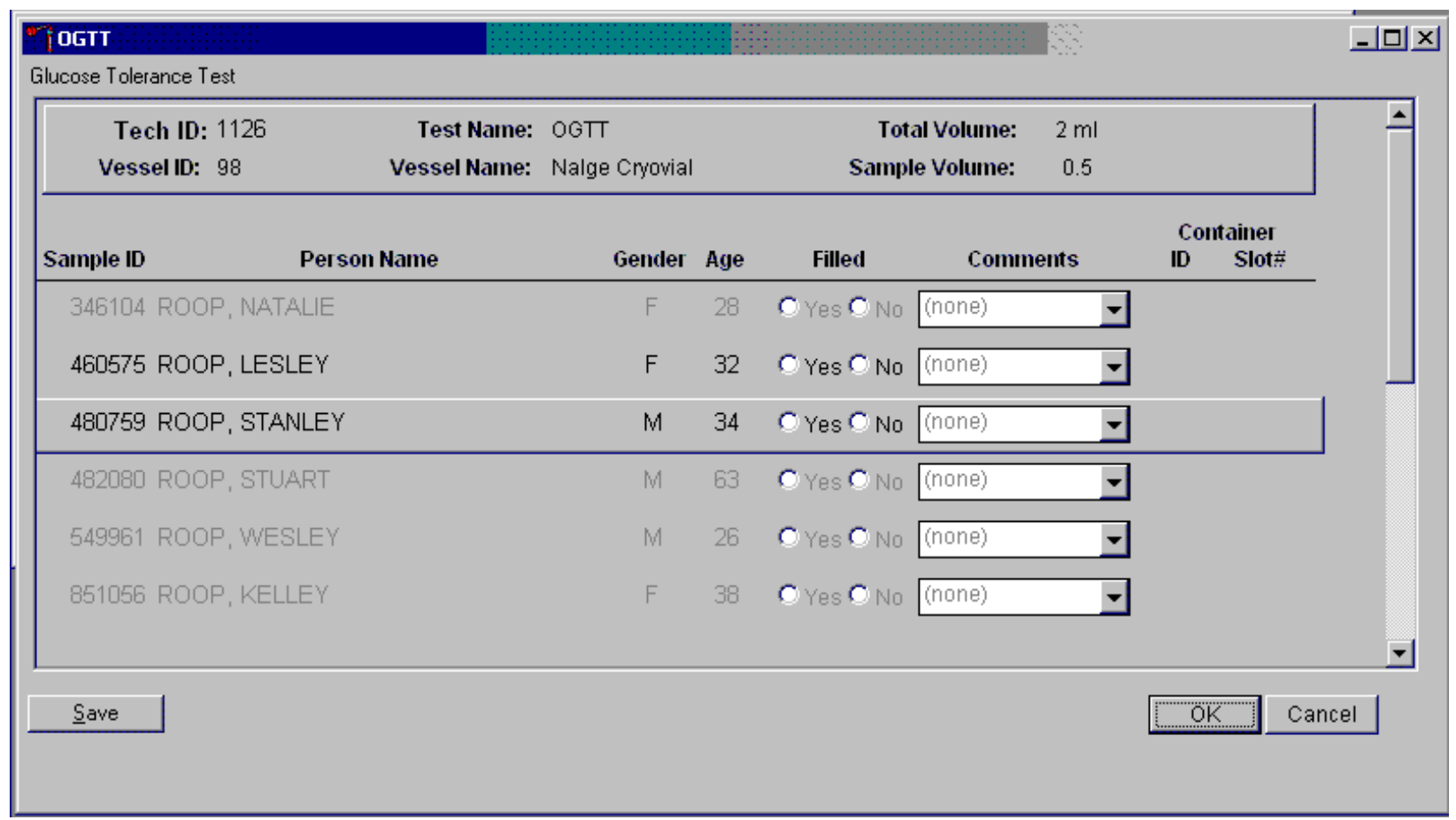

The OGTT processing window lists the vessel requirements across the top. SPs are listed separately in rows. The upper section of the Glucose Tolerance Test window contains the following information: Tech ID, Vessel ID (98), Test Name (OGTT), Total Volume (2 ml), Vessel Name (Nalge cryovial), and Sample Volume (0.5.) The lower section of the window contains columns for Sample ID, Person Name (Last, First), Gender, Age, Filled Yes and No radio buttons, Comments text box, and a Container ID/Slot \# column N. Use the Save button to save the results to the database. Use the OK button to save the results to the database and close the module. Use the Cancel button to close the window without saving any data to the database. 
Record blood processing results for each SP. Individually mark each vessel as Filled-“Yes.”

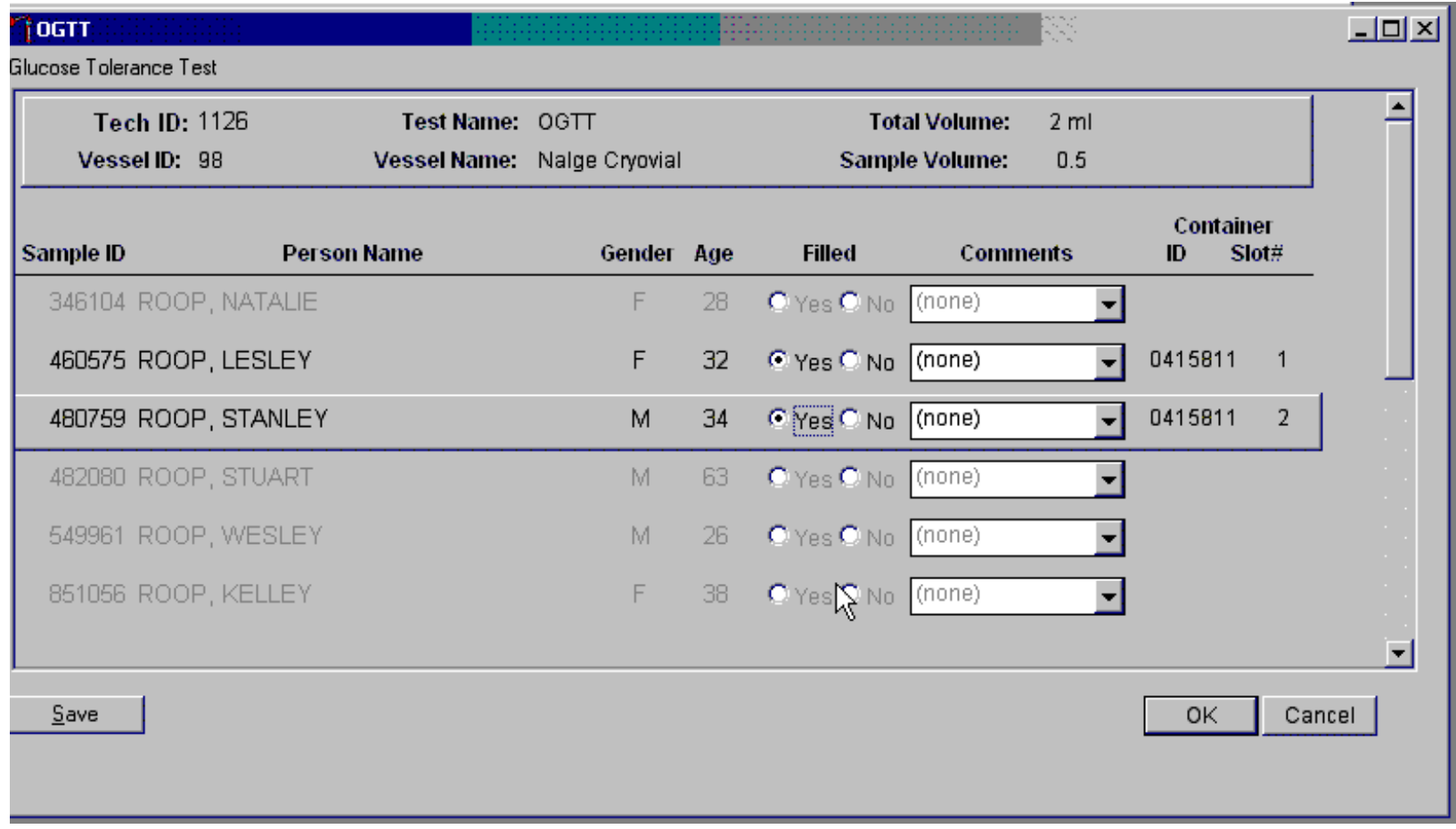

Only SPs who have had the second 2-mL gray top tubes marked as collected in the OGTT application are eligible to be processed. Once the tube is collected, the row changes from gray to black, indicating that the SP is ready to be marked as processed. To mark an individual vessel as collected or Filled-"Yes" use the mouse to direct the mouse arrow to the center of the "Yes" radio button and left click. As each vessel is marked as Filled-“Yes,” it is automatically assigned to a slot in an existing (open) container. 
Quality comment codes are available to describe the quality of the specimen.

\begin{tabular}{|c|c|}
\hline Comment for filled vessels & Use when: \\
\hline clotted & $\begin{array}{l}\text { The whole blood sample contains a fibrin formation. This is due to } \\
\text { inadequate mixing or inadequate clotting. }\end{array}$ \\
\hline equipment failure & Pipettes are malfunctioning. \\
\hline hemolyzed & $\begin{array}{l}\text { There is red appearance to the serum or plasma. Hemolysis refers to the } \\
\text { abnormal lysis of erythrocytes. Hemolysis can occur before venipuncture } \\
\text { (in vivo hemolysis) or during the analytic procedure (in vitro hemolysis). If } \\
\text { in vivo, hemolysis will give plasma a red color. Use when describing visible } \\
\text { hemolysis, which occurs when the concentration of hemoglobin exceeds } \\
200 \mathrm{mg} / \mathrm{L} \text {. }\end{array}$ \\
\hline icteric & $\begin{array}{l}\text { There is visible orange color to the serum or plasma. Icteric refers to orange } \\
\text { color imparted to a sample because of the presence of bilirubin. Bilirubin } \\
\text { present in serum results in visible "jaundice" color when the concentration } \\
\text { of bilirubin is above } 1130 \text { umol/L. }\end{array}$ \\
\hline lipemic & $\begin{array}{l}\text { There is turbid appearance to the serum or plasma. Lipemia refers to the } \\
\text { presence of lipid particles (usually very low-density lipoprotein) in a } \\
\text { sample, which gives the sample a turbid appearance. Plasma appears visibly } \\
\text { lactescent or lipemic when the concentration of triglycerides exceeds } 11.6 \\
\text { mmol/L. }\end{array}$ \\
\hline
\end{tabular}


Attach a comment to an individual filled vessel.

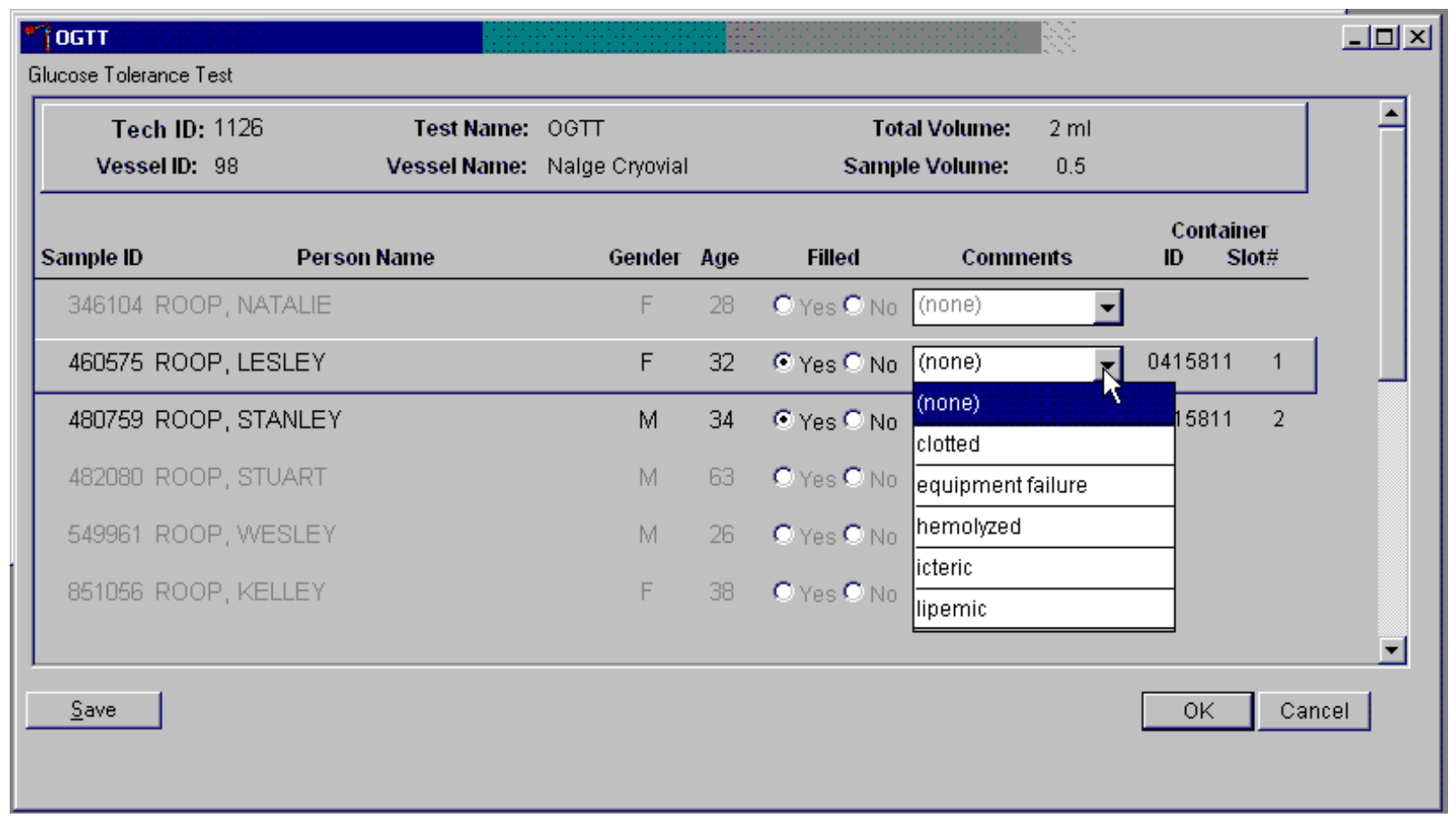

To attach a quality control comment to a filled vessel, use the mouse to direct the mouse arrow to the drop-down list under the Comments button to display the choices, drag the arrow to the correct choice, and left click or highlight the Comment text box and type the first letter of the desired choice (c, e, h, i, l). 
Record blood processing results for each SP. Individually mark each vessel as Filled-“No.”

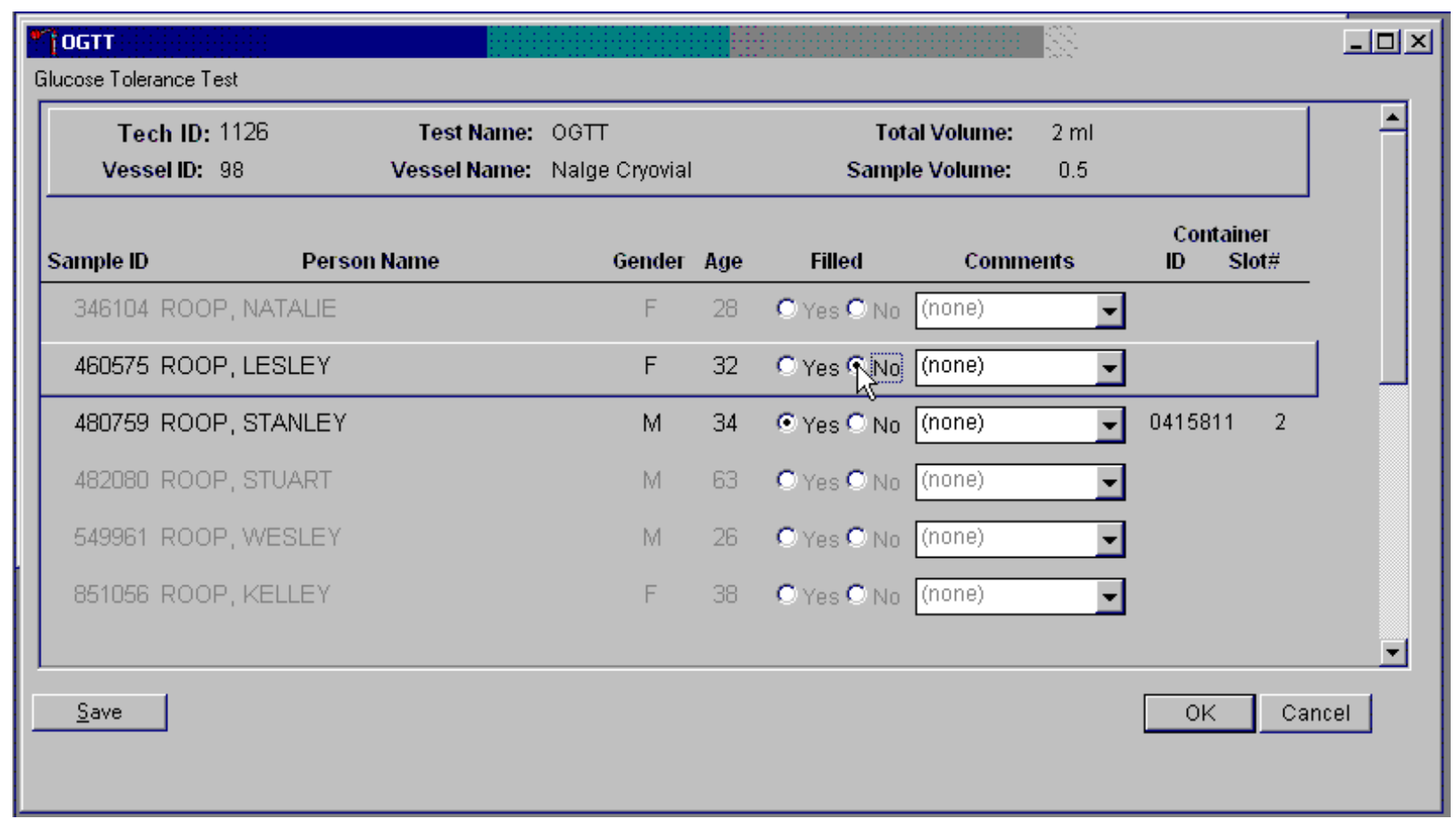

To mark vessel 98 as not collected or Filled-“No," use the mouse to direct the mouse arrow to the center of the "No" radio button and left click. Vessels marked as "No" require a comment.

Enter comment codes to describe the reasons why a vessel is marked as Filled-“No,” or why the protocol is incomplete.

\begin{tabular}{|l|l|}
\hline Comment for not filled vessels & \multicolumn{1}{c|}{ Use when: } \\
\hline laboratory error & $\begin{array}{l}\text { A tube breaks in the centrifuge, contents spill, or other technologist } \\
\text { error. }\end{array}$ \\
\hline quantity not sufficient & Quantity of blood is not sufficient to complete the protocol. \\
\hline SP refusal & The SP refused the blood draw. \\
\hline
\end{tabular}


Attach a comment to an individual Filled-“No” vessel.

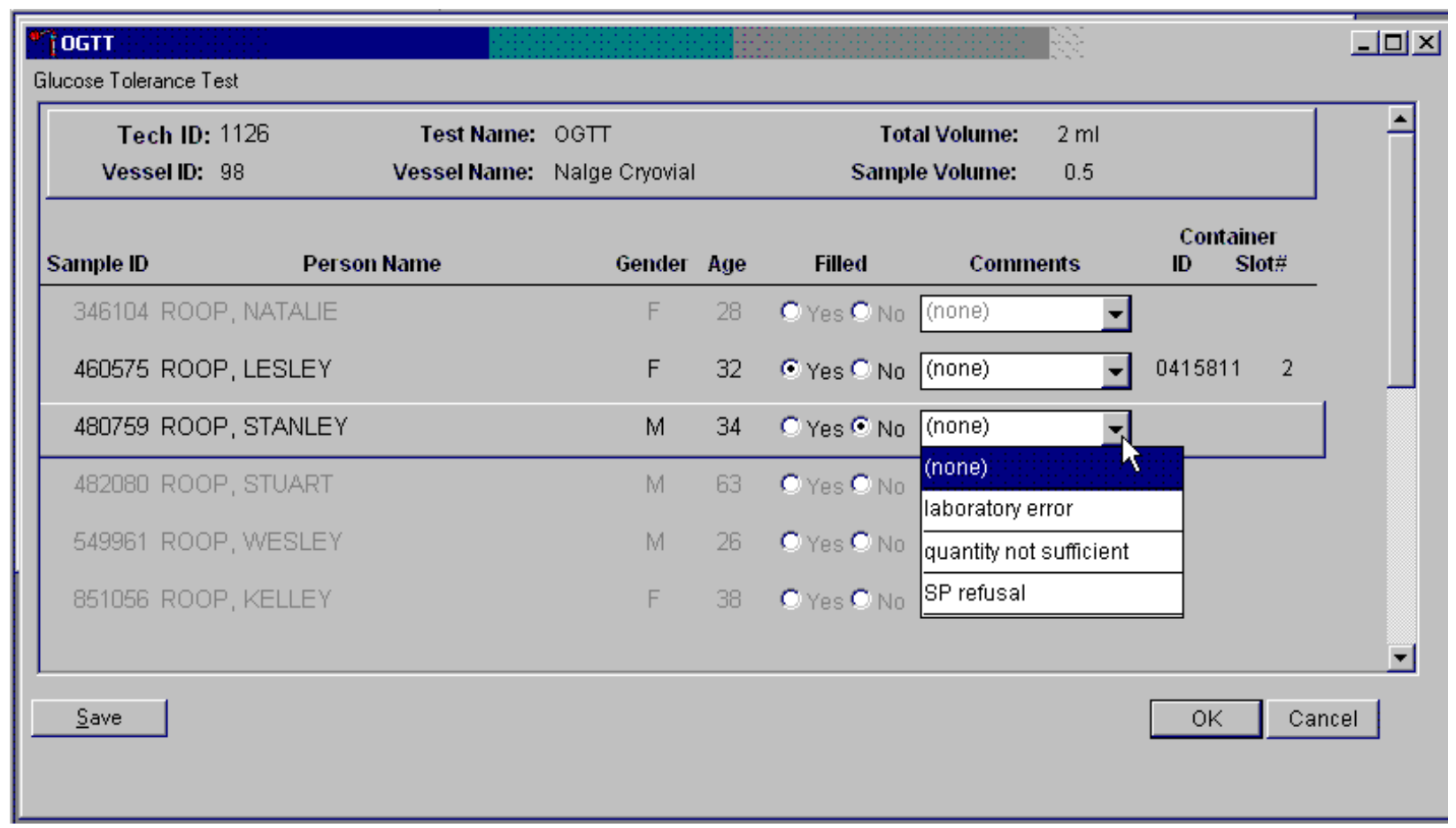

To attach a Filled-“No" comment to a vessel, use the mouse to direct the mouse arrow to the drop-down list under the Comments button to display the choices, drag the arrow to the correct choice, and left click. Alternatively, highlight the Comment text box and type the first letter of the desired choice. 
Continue to enter results as vessels are processed. Review the information in the OGTT processing window and save the data to the database.

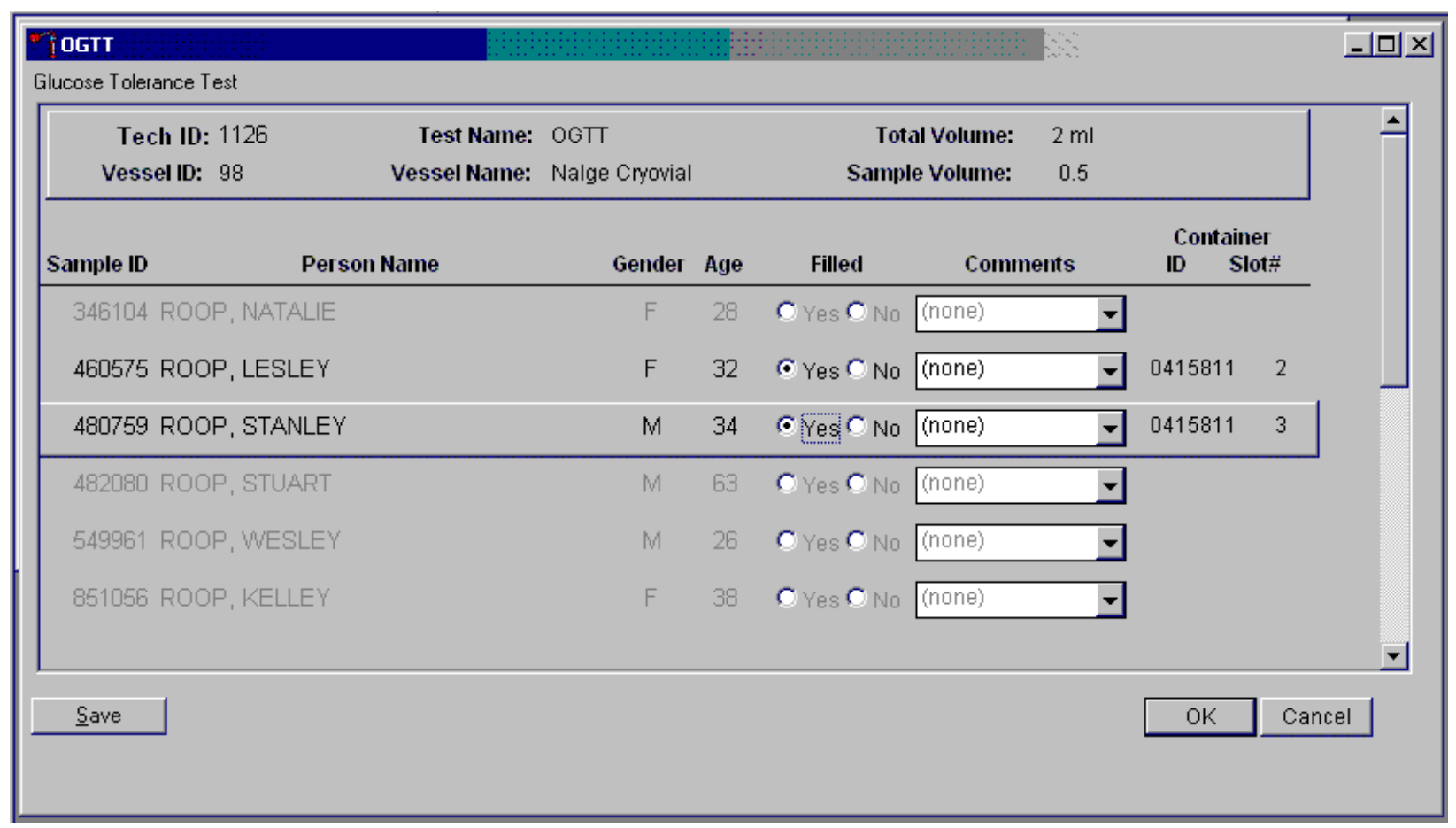

Store each filled vessel in the assigned slot in the assigned container. To record this action or to save this data to the database, use the mouse to direct the mouse arrow to the Save button and left click. To record this action or to save this data to the database and to exit the module, use the mouse to direct the mouse arrow to the OK button, and left click. To close the window without saving any data in the database, use the mouse to direct the mouse arrow to the Cancel button, and left click. 
It is not possible to mark vessels 9 (glucose) and 23 (insulin) in the blood processing module after the second 2-mL gray top tube has been marked as collected in phlebotomy. They are grayed out on the blood processing window.

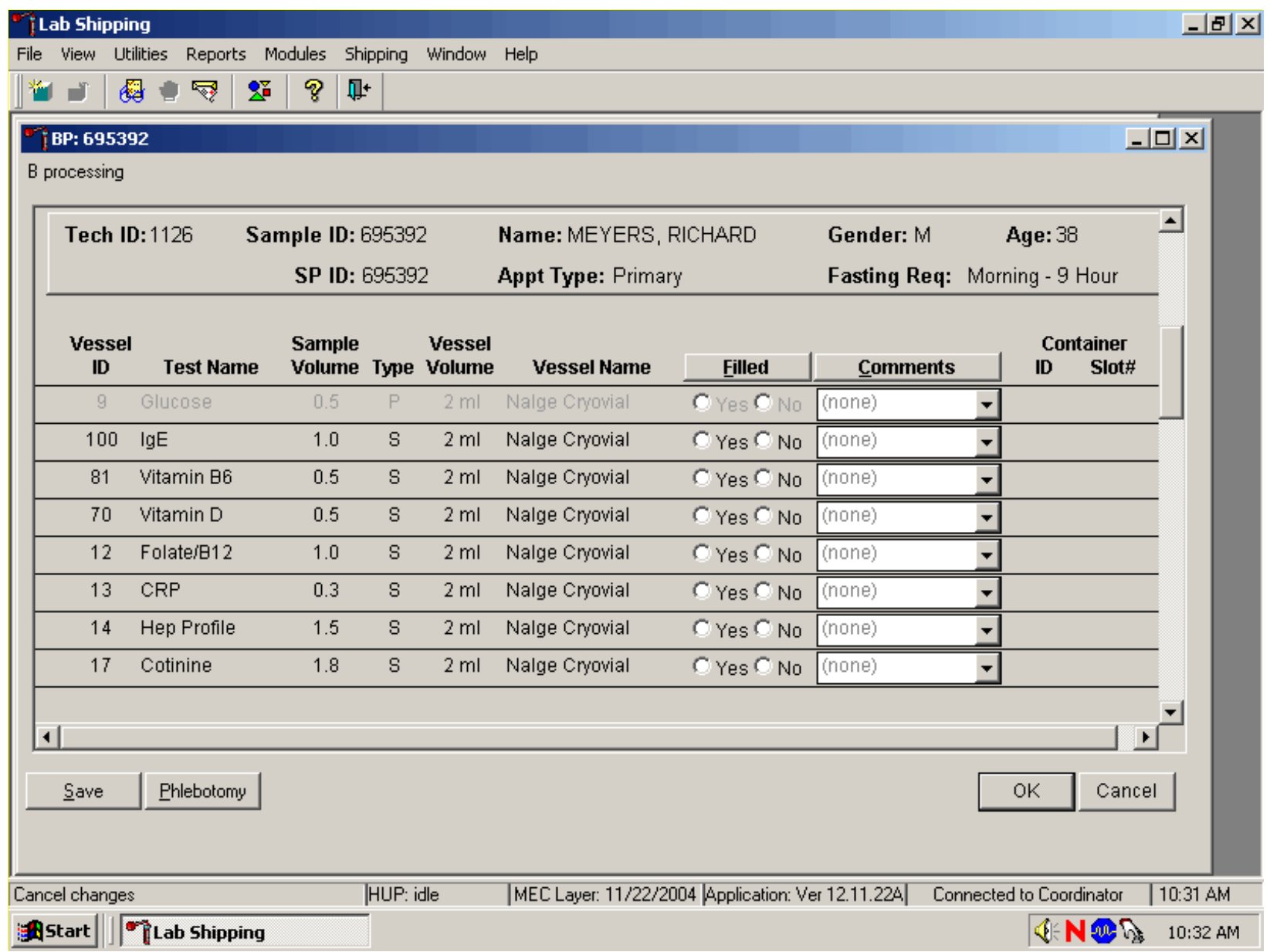

Process the blood from the first venipuncture as soon as possible. Contact the system programmer if the blood tubes for vessels 9 and 23 were drawn before the second 2-mL gray top tube was marked as collected in phlebotomy. 
During the OGTT blood draw (2 hours after the initial venipuncture and after the SP has consumed the Trutol), the phlebotomist will draw any tubes that were missed during the first venipuncture. Add the "Post OGTT draw" comment to any blood vessel processed or filled from blood collected during the OGTT blood draw.

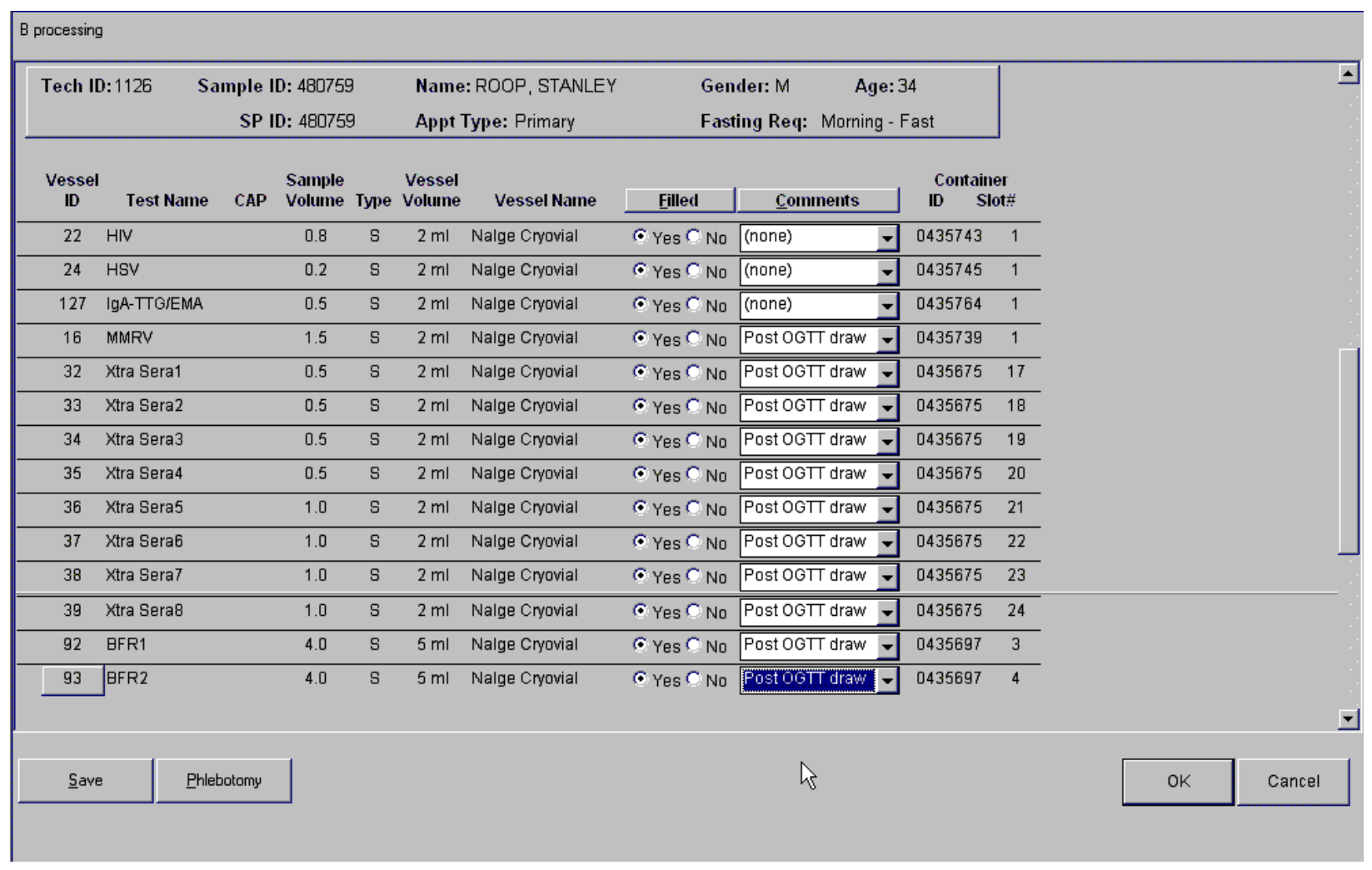


To attach a quality control comment to an individual filled vessel, use the mouse to direct the mouse arrow to the drop-down list under the Comments button to display the choices, drag the arrow to the correct choice, and left click or highlight the Comment text box and type the first letter of the desired choice (c, e, h, I, l, p).

To collectively assign the same comment to all vessels, use the mouse to direct the mouse arrow to the Comments button on the top of the radio buttons, left click, drag the arrow to \{Filled Comments\}, drag the mouse arrow to the correct choice, and left click. Alternatively, to assign the same comment to all filled vessels type [Shift] [C/c], and the first letter of the correct choice (i, l, e, c, h, p).

\subsubsection{Vessel 98 Storage Protocol}

Fill the vessels, enter the OGTT processing results, and prepare to store the vessels. Store vessels in numbered storage boxes according to test as indicated in Exhibit 13-2.

Exhibit 13-2. Storage protocol for GTT

\begin{tabular}{c|c|c|c}
\hline Shipping Location & Vessels & Conditions & Vessel Storage \\
\hline NHANES Diabetes Laboratory & 98 OGTT & Frozen & $9 \times 9$ two inch box \\
\hline
\end{tabular}

\subsubsection{Vessel 98 Shipping Protocol}

Ship the OGTT container weekly on dry ice to the NHANES Diabetes Laboratory. Exhibit 13-3 describes the shipping protocol.

Exhibit 13-3. GTT shipping protocol

\begin{tabular}{|l|l|l|l|l|}
\hline \multicolumn{1}{|c|}{ Location } & \multicolumn{1}{c|}{ Vessels } & \multicolumn{1}{c|}{ Conditions } & Vessel storage & \multicolumn{1}{c|}{$\begin{array}{c}\text { Shipping } \\
\text { frequency }\end{array}$} \\
\hline Blood & OGTT & Frozen & 9 x 9 two inch box & Weekly \\
$\begin{array}{l}\text { NHANES Diabetes } \\
\text { Vessoratory } 98\end{array}$ & & & \\
\hline
\end{tabular}


Pack the container in the same shipper as vessels 9, 23 and 21. Close the container using procedures described in Section 9.9 of the Laboratory Procedures Manual. Assign the closed container(s) to or associate closed containers with the same airbill that was created for the frozen shipper being sent to the NHANES Diabetes Lab. Exhibit 13-4 includes the contract laboratory address. Use procedures described in Section 9.10 of the Laboratory Procedures Manual. Follow the steps listed in Section 9.11 to create the shipping manifest and to send the electronic file. Pack and label the shipper using procedures described in Section 9.13 of the Laboratory Procedures Manual.

Exhibit 13-4. Contract laboratory addresses

\begin{tabular}{llll}
\hline $\begin{array}{l}\text { Laboratory/destination specimen } \\
\text { shipment address }\end{array}$ & Vessels/assays & Conditions & $\begin{array}{r}\text { Contact name } \\
\text { Telephone number }\end{array}$ \\
\hline NHANES Diabetes Laboratory & 9/Glucose & Frozen & Jean Bucksa \\
1120 Delaware St SE Mayo L275 & 23/Insulin & & (612) 273-36115 \\
Minneapolis, MN 551155 & 98/OGTT & & \\
& 21/Lipids & & \\
\hline
\end{tabular}




\subsubsection{Performing the OGTT on SPs Who Do Not Speak English}

When the MEC staff must conduct the OGTT procedure with an SP who does not speak English and the staff member does not speak the language of the SP, a translator who does speak the language of the SP assists the phlebotomist.

The translator stays with the phlebotomist and the SP for the entire procedure. It is very important that the phlebotomist be able to communicate with the SP if the SP becomes ill during the examination.

\subsubsection{Convert Screen Text to English or Spanish}

Convert exam screen text from English to Spanish or from Spanish to English at any time before, during, or after an exam.

To view the exam screen text in Spanish, use the mouse to direct the mouse arrow to \{Utilities\} in the menu bar, left click, drag the arrow to \{Spanish\}, and left click, or type [Ctrl] [S/s]. To return exam screen text to English, use the mouse to direct the mouse arrow to \{Utilities\} in the menu bar, left click, drag the arrow to \{English\}, and left click, or type [Ctrl] [E/e].

\begin{tabular}{ll}
\hline English & Toggle option to set exam language to English. \\
$C t r l+E$ & \\
\hline$\underline{\text { Spanish }}$ Ctrl $+S$ & Toggle option to set exam language to Spanish. \\
\hline
\end{tabular}




\title{
14. QUALITY CONTROL AND QUALITY ASSURANCE - POLICIES AND PROCEDURES
}

\author{
National Health and Nutrition Examination Survey \\ National Center for Health Statistics \\ Centers for Disease Control and Prevention \\ Public Health Service \\ Department of Health and Human Services
}

Current revision: January 2009

Approval: __ Date:

Dr. David Lacher, M.D.

Medical Officer, NCHS

Approval:

Date:

Brenda Lewis, MPH, MT (ASCP)

Laboratory Director, NCHS

Approval:

Date:

Renee Storandt

Technical Supervisor, Westat 


\author{
Abbreviations \\ NCHS - National Center for Health Statistics \\ CDC - Centers for Disease Control and Prevention \\ NHANES - National Health and Nutrition Examination Survey \\ MEC - mobile examination center \\ LPM - NHANES Laboratory Procedures Manual \\ U.S. - United States \\ CBC - complete blood count \\ ERB - ethics review board \\ QC - quality control \\ QA - quality assurance \\ IQAP- Interlaboratory Quality Assurance Program \\ PT - proficiency testing \\ CLIA - Clinical Laboratory Improvement Amendments of 1988 \\ HCFA - Health Care Financing Administration \\ ISIS - Integrated Survey and Information System \\ PC - personal computer \\ LAN - local area network \\ SP - survey participant
}


This policies and procedure quality control and quality assurance chapter is an adjunct to the Westat Policies Manual.

\subsection{Safety}

\subsubsection{Introduction}

NCHS and Westat maintain an ongoing Health and Safety Committee that acts in cooperation with the CDC Office of Health and Safety to assure safe working conditions for technologists in the NHANES mobile examination center (MEC). Safety precautions for all methods are documented in the Biosafety appendix in the NHANES Laboratory Procedures Manual, 2009. This manual is readily available to all laboratory staff. In addition to annual safety training, the NCHS Biomedical Engineer performs safety inspections at the beginning of each location. The technical supervisor assures that the primary laboratory analyst reads and is familiar with safety precautions.

\subsection{Specimen Submission and Handling}

\subsubsection{Introduction}

The goal of specimen submission and handling is to optimize the accurate and reliable measurement of analytes of interest. Each analytical procedure used in NHANES is documented in the NHANES Laboratory Procedures Manual (LPM). Each method in the LPM specifies the procedure to be used for preparation of the survey participant, specimen collection, labeling, processing, and preservation, and conditions for specimen transport that are appropriate for that method. The methods may refer to the NHANES blood collection protocol, or urine collection protocol, noting any special requirements specific to the particular method. These protocols are a starting point due to the special contamination requirements needed for collection of specimens analyzed for heavy metals. 


\subsubsection{Identification of Specimens}

Personal identifiers (e.g., names) are not included on test specimens. NHANES uses a random internal specimen ID number to identify and track individual samples. The Integrated Survey and Information System (ISIS) system at Westat and the National Center for Health Statistics (NCHS) maintains information that links the specimen ID number to the survey participant's name.

\subsubsection{Questions on Specimen Submission and Handling}

The chief medical technologist addresses questions concerning specimen collection including handling and testing.

\subsection{Test Requisition}

\subsubsection{Introduction}

Under a congressional mandate (Section 306 of the Public Health Service Act 42 U.S.C.242k) since 1960, NCHS has collected data on the health of the people of the United States through interviews and extensive physical examinations. Seven surveys using health examination procedures have been completed since 1960. As in previous NHANES programs, the survey's primary purpose is to produce descriptive statistics that can be used to measure and monitor the health and nutritional status of the civilian, noninstitutional U.S. population. Data collection for this survey involves about 7,000 survey participants per year, of whom about 5,000 per year are expected to be examined. Data collection began in January 2005 and continues for about 8 years at 15 different locations (stands) per year.

NHANES performs a complete blood count on survey participants 1 year and older, urine osmolality on SPs age 6+ and urine and/or serum pregnancy tests on female SPs aged 12-59 years, and girls 8-13 who report that they are menstruating when asked during the home interview. If a urine pregnancy test is positive on any female SP aged 8-17 years, the result is confirmed using a serum test. If no blood is drawn, repeat the urine test. Notify the physician of the second positive or negative confirmatory test or the inability to perform a confirmation test using the observation function. 
A record of the test result report is maintained for a period of 7 years by Westat, the primary NHANES contractor, and by NCHS for an indefinite period.

\subsubsection{Laboratory Tests}

"Panic" or abnormally high CBC results are reported to the survey participant. The report includes laboratory tests that will be performed. The NCHS medical officer or MEC physician involved in the measurement may also confer with the survey participant to obtain any other pertinent information, which might facilitate the laboratory measurement process.

Osmolality results are not reported to the survey participant. Pregnancy test results are reported immediately since other MEC components are not assigned until the results of the pregnancy test are documented.

\subsubsection{Human Subjects Review}

The NCHS ERB as defined by the CDC institutional review board (IRB) criteria shall perform all laboratory analyses conducted on human specimens, which are part of a "survey,” only after approval of the survey.

\subsection{Test Records}

\subsubsection{Introduction}

The purpose of test records is to ensure correct and reliable identification of survey participant specimens and to report accurate test results. The laboratory test record system is distinguished from the test report that is provided to the survey participant. 


\subsubsection{Components of Test Records}

Test records include:

- $\quad$ Person performing the test (may be identified by initials);

- Printed or electronically stored output from instrumentation that the technical supervisor judges as necessary to document the analytical result;

- Date of specimen analysis;

- Date and time that specimen was collected by the laboratory; and

- Condition of specimens that did not meet criteria for acceptable specimens (e.g., hemolyzed or insufficient quantity for test).

Test records are maintained for at minimum of 7 years. Records may be bound in laboratory notebooks, in loose-leaf form, or stored electronically.

\subsection{Test Report}

\subsubsection{Introduction}

The laboratory report is provided promptly to the survey participant. This occurs as soon as the quality control has been reviewed and approved. The laboratory maintains confidentiality of the survey participant results since it identifies samples only by a specimen ID number. Westat and NCHS maintain the link between the specimen ID number and the identity of the survey participant.

\subsubsection{Review, Approval, and Release of Test Results}

Upon completion of laboratory testing, results are reviewed by laboratory staff responsible for review of the quality control results to assure the measurement process was in-control for each of the survey participant's analyses.

For NHANES surveys, the initial evaluation of quality control (QC) results is accomplished electronically by comparing the QC results to QC limits stored in a relational database. The laboratory 
staff performing the CBC reviews these QC performance results and ensures that only acceptable survey participants' (SPs') data are reported.

Review and approval of the CBC and pregnancy test results by the MEC physician are documented. Review by the MEC physician includes an evaluation of the consistency of survey participant results with information that may be available on the survey participant (e.g., age, sex). All SPs' CBC samples are run in duplicate whenever possible. If inconsistent results are noted, and an adequate sample is available, the test is repeated a third time. If inconsistent results are again noted, the sample is run a fourth time. If the amount of sample is inadequate for repeat, then the survey participant is informed verbally and in writing of the inconsistent result.

The paper hard copy of the results with the NCHS medical officer's signature is filed by Westat and kept with the test record for 7 years. The NCHS medical officer signs all laboratory reports before release.

Laboratory reports are released only to the authorized survey participant. Each method includes a description of actions taken such as when results are markedly elevated or are "panic values." The survey participant is contacted immediately by the MEC physician with such results.

In addition to the written laboratory report, the data may be transmitted electronically in a

suitable format; for example, a SAS data set or an ASCII file by email to the Westat home office and to NCHS.

\subsubsection{List of Methods and Method Details}

NHANES maintains a Laboratory Procedures Manual (LPM) which includes detailed descriptions of each analytical procedure including analytical sensitivity, specificity, and accuracy, interfering substances, and other information. 


\subsubsection{Format of the Test Report}

The test report includes:

- $\quad$ Name and address of NCHS;

- Test result;

- Units of measurement;

- Reference range for each analyte, if available;

- Condition of specimens not meeting the laboratory’s criteria for acceptability; and

- Signature of the NHANES medical officer.

The laboratory report consists of:

- $\quad$ 1st page: a cover letter; and

- 2nd page: an analysis summary table.

A corrected laboratory report has the same format, except the words "corrected laboratory results" replace the words "laboratory results" in the letter and title of the pages. In addition, in the text of the cover letter, the date of the previous laboratory report is noted.

\subsubsection{Format for Laboratory Report Cover Letter}

An example of the Laboratory Report cover letter (report of findings) is available upon request.

\subsubsection{Format for Listing of Laboratory Results}

An example of the laboratory results is available upon request. 


\subsection{Quality Control}

\subsubsection{Introduction}

Most quantitative analytical procedures involve several operations, or steps, and each operation is subject to some inaccuracy or imprecision or to the possibility of a mistake. The immediate aim of quality control is to ensure that the analytical values produced by a clinical laboratory are sufficiently reliable for their intended purpose. A broader objective is to ensure that all laboratories produce analytical values that meet acceptable standards of precision and accuracy.

Quality control in laboratory medicine is the study of those errors that are the responsibility of the laboratory, and the procedures used to recognize and minimize them. An alternate term, "quality assurance," represents the techniques available to ensure with a specified degree of confidence that the result reported by the laboratory is correct. Both "precision control" (the agreement between replicate measurements) and "accuracy control” (the agreement between the mean estimate of a quantity and its true value) are evaluated to establish this degree of confidence.

A quality control program also monitors laboratory performance to detect excessive random or systematic error. Factors contributing to random error are those that affect the reproducibility of the measurement. These include instability of the instrumentation; variations in the temperature; variations in reagents and calibrators; handling techniques such as pipetting, mixing, and timing; and operators. Terms used to describe random error include precision, imprecision, reproducibility, and repeatability. Systematic error describes the error that is consistently low or high. Factors that contribute to systematic error are independent of the analyte concentration. Examples of these factors include interfering substances or incorrect assignment of the amount of substance in the calibrator.

A good quality control program includes both internal (intralaboratory) and external (interlaboratory) surveillance and monitors the following five parameters:

1. Clerical Error: This includes properly documented acknowledgment of transmittal and receipt of specimens (for example, "logging in"), proper labeling of all specimens, correct assignment of laboratory values to the proper SP ID number, and maintenance of proper records for all specimens for future reference.

2. Techniques: This includes continued assurance that all personnel performing an assay understand the principles underlying a particular assay and are cognizant of the proper 
technique for that assay; that all personnel use the same technique for a particular assay; that there is ready access to a current technique manual; and that periodic review is undertaken to ensure use of the most current and reliable techniques.

3. Reagents and Materials: This includes confirmation of commercial standards and controls before they reach the bench; proper labeling of reagents, particularly those prepared in the laboratory; ensuring all reagents in use are not outdated; having an adequate supply of current reliable reagents; proper calibration of equipment, such as pipettes; and proper washing of glassware.

4. Bench Performance: This includes the use of controls and standards for each assay performed, a technique based on sound statistical principles which allows the technologist performing the assay to detect error outside of previously determined limits before reporting data; documentation of daily bench performance for detection of less obvious error (particularly those which tend to accumulate over time, so-called “drift"); and established procedures to be followed wherever error is found to exceed previously determined limits.

5. Instrumentation: This includes periodic preventive maintenance of all instruments in use in the laboratory and documentation that each instrument is maintaining a previously determined level of each performance at each check.

In this context, QC procedures monitor and evaluate the quality of the analytical testing process of each method to ensure the accuracy and reliability of test results. Thus, QC procedures referenced here refer to the analytical phase of testing and not to the preanalytic or postanalytic phases. Quality assurance (QA) addresses all three phases of measurement: preanalytic, analytic, and postanalytic. QC is therefore viewed here as one part of the overall QA process.

\subsubsection{Method Specific QC Procedures}

Specific QC procedures exist for each analytical laboratory procedure in the Laboratory Procedures Manual. The following are some general aspects of QC procedures.

\subsubsection{QC Definitions}

\section{Internal (“Bench”) Quality Control}

For purposes of this document, internal quality control is the evaluation of analytical performance that includes QC bench samples for which the analyst knows the expected measurement 
result. Internal commercial quality control materials contain predetermined amounts of analyte in the matrix, qualify under Clinical Laboratory Improvement Amendments (CLIA) as "calibration materials," and may be used as a "calibration verification” as defined by CLIA.

- External ("Blind”) Quality Control - For purposes of this document, external quality control is the evaluation of analytical performance that includes QC samples for which the analyst does not know the expected measurement result. The analyst is "blind" to the expected measurement result. Examples include proficiency testing samples.

- Interlaboratory Quality Assurance Program (IQAP) - For purposes of this document, NHANES participates in Beckman Coulter's ${ }^{\circledR}$ IQAP program. Beckman Coulter ${ }^{\circledR}$ issues a report that contains a statistical analysis to evaluate performance.

- Proficiency Testing - For purposes of this document, proficiency testing is one method of external quality control in which the analytical performance of a method is evaluated using specimens provided on a periodic basis (usually every 3 months).

- Analytical Run (sometimes referred to just as a run) - An analytical run is a set of samples analyzed in a time within which the measurement system is considered to have stable accuracy and precision. The time for a run may not exceed 24 hours. An analytical run usually consists of both quality control specimens and survey participant specimens.

- Calibration - Defined by CLIA as "the process of testing and adjusting an instrument, kit, or test system to provide a known relationship between the measurement response and the value of the substance that is being measured by the test procedure."

- Calibration Material - Defined by CLIA as "a solution which has a known amount of analyte weighed in or has a value determined by repetitive testing using a reference or definitive test method.” Many standards are therefore calibration materials.

- Calibration Verification - Defined by CLIA as "the assaying of calibration materials in the same manner as survey participant samples to confirm that the calibration of the instrument, kit, or test system has remained stable throughout the laboratory's reportable range for survey participant test results.”

- QC Requirements of Each Analytical Run - Analysis of survey participant samples is organized into analytical runs. The minimum QC requirement for an analytical run, which is analyzing survey participant specimens, is that the run includes four internal (“bench”) QC specimens. 


\subsubsection{Overview of the Relationship between Internal QC, Proficiency Testing, and External QC}

A Beckman Coulter ${ }^{\circledR}$ technical representative performs HMX instrument setup, verification, and performs and verifies QC checks according to their procedure at the beginning of each stand. Medical technologists perform instrument calibration.

Most laboratory quality control programs include running internal (“bench”) QC with each analytical run to ensure each analytical run is in-control. The laboratory participates in proficiency testing programs administered by an outside scientific group to be sure on a periodic basis that accuracy and precision are within acceptable limits. The IQAP compares NHANES 5C cell control results to other laboratories. These QC systems are used within NHANES. The linearity of the instrument is evaluated at least once a year.

In addition to these systems, NHANES uses calibration materials at the beginning of each stand to standardize the three Beckman Coulter ${ }^{\circledR}$ HMX instruments and to provide additional objective evaluation of the quality of specimen analysis. S-CAL is a calibration material as defined by CLIA (i.e., "a solution, which has a known amount of analyte or has a value determined by repetitive testing using a reference or definitive test method") that is useful for monitoring method performance.

The Osmette II is used to perform osmolality on urine samples. The osmolality test is a determination of the number of osmotically active solutes present in the urine. It is performed to help evaluate the body's water balance, its ability to produce and concentrate urine. Urine osmolality is dynamic and will fluctuate as the body responds to and corrects temporary water imbalances.

The Osmette II is calibrated at the beginning of each stand using the manufacturer's procedure for total calibration. A calibration standards check is performed weekly. This procedure verifies that the two bottled Standards that are in use are accurate when compared to the same concentration of previously unopened reference standards (CON-TROL ampule Reference Standards). A calibration check procedure is performed daily. This involves running two Standards and two levels of QC material in duplicate. The pipettor is calibrated at the start of each stand and the Teflon tip is changed. 


\subsubsection{Internal (“Bench”) QC}

\section{Complete Blood Count}

The goal of internal QC is to provide a rapid feedback to the analyst on the performance of the measurement process to be sure analytical results and factors affecting analytical results are within acceptable limits. At least three internal QC specimens (5C® Cell Control) are included in each analytical run. The QC material used as internal QC specimen has levels of the analyte, which are useful for monitoring method performance.

The 5C® Cell Control is a hematology reference control used to monitor the performance of instruments with complete CBC and VCS differential technology. The 5C® Cell Control consists of human blood so repeated measurements to monitor daily performance of the instrument system are possible. The 5C® Cell Control Tri-Pack contains three levels-normal, abnormal I, and abnormal II. When used with Beckman Coulter's ${ }^{\circledR}$ blood diluent, Isoton III, they serve as a check on the accuracy of dilution, WBC counts, RBC counts, platelet counts, hemoglobin determinations, and differential counts.

The technologists run all three levels at the beginning of each session to monitor the precision of the instrument following procedures described in Chapter 7, Section X.B.

Standard Levy-Jennings QC charts are maintained for this internal QC specimen. A separate QC chart is to be maintained for each QC material used for this internal QC specimen. Standard criteria for run rejection based on statistical probabilities are used to declare a run either in-control or out-ofcontrol. No results for a given analyte are reported from an analytical run that has been declared out-ofcontrol for that analyte as assessed by internal ("bench”) QC. Rejection criteria are described in each method procedure in the LPM. Quality control results are captured by and stored in the ISIS. Any remedial actions for out-of-control conditions are documented in a QC logbook.

In addition, a latex control (Latron $\left.{ }^{\circledR}\right)$ is used to monitor the volume, conductivity, and light scatter (VCS) of the diff mode only. This control is run at the beginning of each day.

The Latron ${ }^{\circledR}$ control is a ready-to-use suspension of latex particles used to monitor differential parameters on the Beckman Coulter® HMX. These particles pass through the flow cell and 
produce characteristic electrical signals. It measures these signals as volume, conductivity, and light scatter. The technologist runs the Latron ${ }^{\circledR}$ control immediately following the Latron ${ }^{\circledR}$ primer.

\section{Osmolality}

Urine Osmolality Determination is performed on primary survey participants (SPs) who are 6 years and older and dry run odd and even volunteers age 12+. The test is performed using the random urine specimens collected at the MEC. There are no SP safety exclusions for this test. The test is performed in the MEC laboratory using the Osmette II instrument. SP samples are run in duplicate and the two results are averaged. If the difference between the two values exceeds a preset precision limit, then the sample is run a third time. The closest two results are reaveraged. It is possible to delete one or more results. If the final result is based on a single run, then a comment, "results based on single run” will automatically be appended to the result. Results are saved in whole numbers. Osmolality test results will not be reported back to the participant. If the measured value is $>2000 \mathrm{mOsm} / \mathrm{kg}$ then the result will be reported as “2000” with “out of range comment.”

Quality control materials include a set of standard bottled solutions with exact concentration of Sodium Chloride (NaCl - Inorganic Salt) and deionized water. In addition, two levels of a human urine controls [Quantimetrics Level 1 (484) and the Level 2 (828)] QC material are used as quality control material for the Osmette II. At the start of each stand the instrument is calibrated and the pipette is calibrated and the Teflon tip is changed.

Two levels of standards, 100 and 1500 milliosmoles, and the two levels of human urine controls (Quantimetrics Level 1 [484] and the Level 2 [828]) QC material are run daily during the first session or run just before the first sample. However, no sample can be imported or transmitted until the QC results are acceptable. The QC module captures the lot number, expiration date and any relevant statistics like mean and standard deviation of the Quantimetrics material. The Quantimetrics QC samples are run in duplicate and the results are averaged. If the difference between the two values exceeds a preset precision limit, then the sample is run a third time and the closest two results are then averaged. It is possible to delete one or more results. If the final result is based on a single run, then a comment, "results based on single run" will automatically be appended to the result. Results are saved in whole numbers. The limits for the 100 standard is \pm 1 SD with a spread $<6$. The limits for the 1500 standard is \pm 0.5 SD. 
Weekly quality control is a standard verification procedure. This includes running two 100 and 500 standards and comparing them to 100 and 500 CON-TROL reference standards. All results should be within 2SD of the target value. If results are > 2SD from the Standard target value, the instrument is recalibrated with CON-TROL Reference Standards, and Controls (I and II levels) are run.

\subsubsection{Coulter Interlaboratory Quality Assurance Program (IQAP)}

All HMX instruments participate in Beckman Coulter’s ${ }^{\circledR}$ Interlaboratory Quality Assurance Program (IQAP.) The Beckman Coulter ${ }^{\circledR}$ Data Management System saves results of $5 C \circledR$ cell controls in files. Technologists transfer the files to a disk and then send the disk to Beckman Coulter ${ }^{\circledR}$, who compares the results to other laboratories. Beckman Coulter ${ }^{\circledR}$ issues a report that contains a statistical analysis to evaluate performance. The technologists perform this procedure on the last day of each month, at the end of each stand, or when a control lot expires, using procedures described in Chapter 7 , Section X.D.

\subsubsection{Proficiency Testing}

Participation in the College of American Pathologist (CAP) proficiency-testing (PT) program is part of the comprehensive quality control program. Each MEC submits results for CAP specimens for evaluation. CAP compares the results to established values and issues a report.

The laboratory will satisfactorily participate in the HCFA-approved PT program for each method used to analyze human samples. Performance in the PT program, along with documentation of remedial action taken for unacceptable performance, is documented in a QC logbook that is available for review.

CAP samples are sent three times a year for hematology. Each shipment includes five 2.5$\mathrm{mL}$ whole blood specimens. Duplicate Beckman Coulter ${ }^{\circledR}$ CBC determinations are performed on each whole blood sample using procedures described in Chapter 7, Section X. PT samples are handled and analyzed in a manner identical to SP samples. Results are recorded on the CAP forms and sent to the address specified by CAP Surveys Program Support with a copy to NCHS. 
CAP samples are sent three times a year for qualitative serum and urine hCG. Each serum shipment includes five lyophilized sera specimens and each urine shipment contains five liquid specimens. PT samples are handled and analyzed in a manner identical to SP samples.

CAP samples for osmolality are shipped three times per year. Each Urine Chemistry (General) "U” shipment includes six 15.0-mL urine specimens.

\section{CAP Proficiency Testing}

For purposes of this document, proficiency testing is one method of external quality control in which the analytical performance of a method is evaluated using specimens provided on a periodic basis (usually every 3 months).

Participation in the College of American Pathologist (CAP) EXCEL proficiency-testing program is part of the comprehensive quality control program. Each MEC submits results for CAP specimens for evaluation. CAP compares the results to established values and issues a report.

These samples should be handled and analyzed in a manner identical to SP samples. Results are recorded on the CAP forms and are sent to the address specified by the CAP Surveys Program Support. A copy of the CAP form is sent to the home office at the end of the stand.

Results are recorded on the CAP forms and sent to the address specified by CAP Surveys Program Support with a copy to NCHS at the following address:

Brenda Lewis, MPH

National Center for Health Statistics

3313 Toledo Road

Hyattsville, MD 20782 


\subsubsection{Calibration Verification}

Calibration verification is defined by CLIA as "the assaying of calibration materials in the same manner as survey participant specimens to confirm that the calibration of the instrument, kit, or test system has remained stable throughout the laboratory's reportable range for survey participant test results." Thus, calibration verification assures that the accuracy of the measurement process across the reportable range is maintained over time. For methods approved by FDA, calibration verification is performed according to the manufacturer's specification and at the frequency recommended by the manufacturer.

Note that the calibration materials must bracket the reportable range; that is, results should not be reported as a numerical value unless they are within the reportable range. If a result is higher than the upper end of the reportable range and the method permits sample dilution, the sample may be diluted to bring it within the reportable range. If dilution has been necessary, the reported value will exceed the upper end of the reportable range. Each analytical procedure specifies acceptable limits for results of analyses of the calibration materials based on the method precision of these materials.

The reliability of analytical values obtained when using a procedure often depends on the quality of the standards and the calibration procedure. The College of American Pathologists (CAP) suggests that automated instruments be calibrated using multiple analytical whole blood specimens or a certified, stabilized whole-blood type preparation. The International Committee for Standardization of Hematology (ICSH) by its definition requires that a calibrator "be based on or traceable to a reference preparation or material.”

Beckman Coulter ${ }^{\circledR}$ provides the S-CAL ${ }^{\circledR}$ calibrator to meet these requirements. The HMX uses the S-CAL ${ }^{\circledR}$ kit that requires a calibrator to convert electronic measurements of each sample into accurate results expressed in clinical terms. S-CAL ${ }^{\circledR}$ calibrates the WBC, RBC, Hgb, MCV, Plt, and MPV parameters. It is a stabilized human-blood preparation. S-CAL ${ }^{\circledR}$ is an acceptable alternative to whole blood calibration. Hct, MCH, MCHC, RDW, and the DIFF parameters do not require calibration.

Calibration is a procedure to standardize the instrument by determining its deviation from calibration references and to apply any necessary correction factors. The calibration procedure uses replicate measurements of S-CAL ${ }^{\circledR}$ calibrator. The S-CAL ${ }^{\circledR}$ divides the average result into the calibrator 
Assigned Value to give an Adjustment Factor. Then, it obtains and adjusts an instrument reading according to the Adjustment Factor.

S-CAL ${ }^{\circledR}$ is used to calibrate the Beckman Coulter ${ }^{\circledR}$ HMX at the beginning of each stand (once every 6-8 weeks), after replacing any component dealing with dilution preparation, if the Beckman Coulter ${ }^{\circledR}$ representative suggests it, controls demonstrate unusual trends or are outside limits, or when the temperature varies by more than $10^{\circ} \mathrm{F}\left(5.5^{\circ} \mathrm{C}\right)$ from the room temperature during the last calibration.

Instructions for the performance of calibration procedures using S-CAL ${ }^{\circledR}$ calibration are in Section VII of Chapter 7.

The Osmette II is calibrated the start of each stand and after any major maintenance. Follow the calibration procedure described in Section VII of Chapter 12.

\subsubsection{Linearity}

The linearity controls verify the reportable range of Beckman Coulter ${ }^{\circledR}$ hematology systems. Linearity is performed at installation, at least yearly, and whenever experiencing an altitude change of 1 mile or more between locations using either Beckman Coulter ${ }^{\circledR}$ Lin-C ${ }^{\text {TM }}$ or CAP LN9 survey material.

Beckman Coulter® Lin-C ${ }^{\text {TM }}$ linearity controls are human blood components from which repeated measurements verify the reportable range of Beckman Coulter ${ }^{\circledR}$ hematology systems that use both Isoton ${ }^{\circledR}$ III diluent and Lyse S ${ }^{\circledR}$ III diff lytic reagent. Controls contain one each of Ultra Low Range, Low Range, Mid Range, and High Range, and Ultra High Range. Lin-C ${ }^{\mathrm{TM}}$ verifies ranges for the following parameters: WBC, RBC, Hgb, and Plt. Beckman Coulter ${ }^{\circledR}$ Lin-C ${ }^{\mathrm{TM}}$.

CAP Hematology Calibration Verification/Linearity Survey (LN9) materials are shipped twice per year and consist of 18 3.0-mL liquid specimens. The medical technologist follows all CAP instructions in preparing and running the materials before performing the test. They fill out the CAP result form, make a copy for the logbook, send results to CAP, and send the copy back to the home office at the end of the stand. 
CAP LN6 verifies the reportable range of the Precision Osmette II instrument. Survey LN6 corresponds to CAP Urine Chemistry (General) Survey U. Each shipment of Survey LN6 includes 15 4.0$\mathrm{mL}$ liquid specimens. Linearity was performed at installation and will be repeated in November of each year. The medical technologist follows all CAP instructions in preparing and running the materials before performing the test. They fill out the CAP result form, make a copy for the logbook, send results to CAP, and send the copy back to the home office at the end of the stand.

\subsubsection{Quality Control Records}

Records of all quality control results are maintained for at least 7 years. Quality control results are captured and stored in the ISIS and are available via the Intraweb. A QC logbook is maintained which documents out-of-control conditions and remedial actions taken to correct out-of-control conditions. The QC logbook also includes copies of results for Proficiency Testing and calibration.

\section{Test Methods, Equipment, Reagents, Supplies, and Facilities}

\subsubsection{Introduction}

Analytical procedures use equipment, reagents, materials, and supplies that are appropriate for achieving acceptable accuracy, precision, analytical sensitivity, and analytical specificity from the method. The documentation of individual analytical procedures included in the Laboratory Procedures Manual (LPM) specifies acceptable equipment, reagents, materials, and supplies. If special requirements concerning water quality, temperature, humidity, electrical power, or other conditions are required for acceptable method performance, then these are described for each procedure in the LPM. Special procedures to monitor these requirements are also included in the documentation of the individual procedure. A lack of requirements in the documentation of the analytical procedure means that the expected day-to-day variation in any of these parameters is acceptable for proper method performance.

Documentation of problems with conditions required (e.g., water quality) for acceptable method performance is maintained in the logbook of the primary instrument used in the analysis. This documentation includes remedial action. 


\subsubsection{Labeling of Reagents, Solutions, Supplies}

Reagents, solutions, and other supplies are labeled to indicate the identity of contents, the concentration (if significant), the recommended storage requirements, the preparation and expiration date, and any other pertinent information required for proper use. Reagent, solutions, and other supplies are not used when they have exceeded their expiration date.

If a method uses a manufacturer's kit, components of reagent kits of different lot numbers are not interchanged unless otherwise specified by the manufacturer.

Beckman Coulter ${ }^{\circledR}$ reagents are monitored and documented in the Beckman Coulter ${ }^{\circledR}$ Data Management System and are additionally located on the daily instrument startup result sheet. A copy of all startup results is filed in the quality control notebook and sent back to the home office at the end of each stand. The technologist documents any unusual occurrence in the automated Unusual Occurrence Log or repair of the Beckman Coulter ${ }^{\circledR}$ HMX in the automated Equipment Tracking System.

Logs are maintained for reagents other than the Beckman Coulter ${ }^{\circledR}$ reagents. The technologist documents reagent use by entering the reagent's name, date made, date put into use, expiration date, and tech ID in the automated Supply Use Control Log. The technical supervisor reviews the Supply Use Control Log at the end of each stand.

\subsubsection{Facilities}

Laboratories are arranged to ensure that adequate space, ventilation, and utilities are available for all phases of testing: preanalytic, analytic, and postanalytic. 


\subsection{Analytical Procedure Manual}

\subsubsection{Introduction}

All procedures performed in the laboratory on human specimens are documented in the LPM. A copy of the LPM is maintained at the Westat home office and NCHS. The individual procedure is also available at or nearby the bench site where the procedure is performed.

\subsubsection{Contents of the Analytical Procedure Documentation}

Each analytical procedure includes, when applicable:

- Requirements for specimen collection and processing including criteria for specimen rejection;

- Step-by-step performance of the procedure including test calculations and interpretation of results;

- Preparation of reagents, calibrators, controls, solutions, and other materials used in testing;

- Calibration and calibration verification procedures;

- The reportable range for survey participant test results;

- Quality control procedures;

- Remedial action to be taken when calibration or control results are outside acceptable limits;

- Limitation in methods, including interfering substances;

- $\quad$ Reference range (normal values);

- Life-threatening or “panic values”;

- Pertinent literature references;

- Specimen storage criteria;

- Protocol for reporting panic values;

- Course of action if test system becomes inoperable; and

- Safety considerations for performing the method. 


\subsubsection{Approval and Record Maintenance}

Each procedure is reviewed, approved, signed, and dated by the clinical consultant and technical supervisor and laboratory director of NHANES. Procedures are reapproved, signed, and dated if the director changes. Each change in a procedure is approved, signed, and dated by the current director. The procedure includes the dates of initial use and discontinuance, if discontinued. The procedure documentation is maintained for 7 years after discontinuance of the method.

\subsubsection{Method Performance Specifications}

Method performance specifications for each analytical method are established or verified:

- Accuracy - accuracy of each analytical method will be determined by analysis of calibrators and yearly linearity checks as described in the section of the LPM on quality control.

- Precision - precision of each analytical method is determined by analysis of QC reference materials as described in the section of this manual on quality control.

- Analytical specificity - analytical specificity is determined for each method including effects of potential interfering substances. This may be verified by testing the effect of potential interfering substances in method development, by analyzing reference materials, by comparing results on split samples with a method considered more definitive, and/or by analyzing a sample of persons $(n>20)$ and examining the measurement output searching for interference. The appropriate procedures for verifying analytical specificity will vary by analytical method. Substances that interfere with the analysis in the reportable range are listed in the method procedure.

- Reportable range of test results - the reportable range of test results is described in the documentation of the analytical procedure.

- Reference range (normal range) - if available, the reference range of test results is described in the documentation of the analytical procedure and on the test report.

- Other pertinent performance specifications - other performance specifications that are required for adequate method performance are specified in the documentation of the analytical procedure. 


\subsection{Equipment Maintenance and Function Checks}

\subsubsection{Introduction}

Laboratory equipment is checked regularly to ensure acceptable performance. Maintenance (including preventive maintenance) and function checks are documented in the Laboratory and Phlebotomy Quality Control modules in the Integrated Survey and Information System (ISIS). The frequency of maintenance and function checks follows manufacturers' recommendations, when available. Manufacturers' recommendations are included in the manuals that are located in notebooks in the laboratory.

Each analytical procedure outlines the equipment maintenance and function checks for proper method performance and acceptable results from the checks. These checks must be made at the interval specified in the documentation of the procedure. Failure of a function check is documented in the automated Unusual Occurrence Log.

\subsubsection{Eppendorf Pipette Calibration}

The Eppendorf Research pro electronic pipettes are calibrated once per year. Pipettes are sent to the manufacturer and calibration certificates are kept on file at the home office. 


\subsubsection{Temperature Monitoring}

Temperature recordings are required for storage and preservation of reagents and sample material (analytical specimens), as well as quality control and reference materials.

Beckman Coulter ${ }^{\circledR}$, Vacutainer, Precision Instruments, and Quantimetix have recommended the following storage conditions for reagents and tubes. Quality control temperature ranges have been established based on these recommendations.

\begin{tabular}{lll}
\hline \multicolumn{1}{c}{ Reagent } & \multicolumn{1}{c}{$\begin{array}{c}\text { Manufacturer's recommended } \\
\text { storage conditions }\end{array}$} & \multicolumn{1}{c}{ Established range } \\
\hline $\begin{array}{l}\text { Coulter }{ }^{\circledR} \text { reagents } \\
\text { Isoton } \\
\text { Coulter Clenz } \\
\text { HMX Pack }\end{array}$ & $2-25^{\circ} \mathrm{C}\left(36-77^{\circ} \mathrm{F}\right)$ & $17-25^{\circ} \mathrm{C}\left(63-77^{\circ} \mathrm{F}\right)$ \\
$\quad$ Lyse-S III & & \\
\hline $\begin{array}{l}\text { S-Cal at room temperature } \\
\text { S-Cal at refrigerated } \\
\text { temperature }\end{array}$ & $16-32^{\circ} \mathrm{C}\left(60-90^{\circ} \mathrm{F}\right)$ & $17-25^{\circ} \mathrm{C}\left(63\right.$ to $\left.77^{\circ} \mathrm{F}\right)$ \\
\hline Latron & $2-8^{\circ} \mathrm{C}\left(35-46^{\circ} \mathrm{F}\right)$ & $2-8^{\circ} \mathrm{C}\left(36\right.$ to $\left.46^{\circ} \mathrm{F}\right)$ \\
\hline $\begin{array}{l}5 \mathrm{C} \text { Cell Control } \\
\text { Vacutainers }\end{array}$ & $2-30^{\circ} \mathrm{C}\left(36-86^{\circ} \mathrm{F}\right)$ & $17-25^{\circ} \mathrm{C}\left(63\right.$ to $\left.77^{\circ} \mathrm{F}\right)$ \\
\hline $\begin{array}{l}\text { Precision standard } \\
\text { solutions }\end{array}$ & $2-8^{\circ} \mathrm{C}\left(36-46^{\circ} \mathrm{F}\right)$ & $2-8^{\circ} \mathrm{C}\left(36\right.$ to $\left.46^{\circ} \mathrm{F}\right)$ \\
\hline $\begin{array}{l}\text { Precision CON-TROL } \\
\text { reference standards }\end{array}$ & Not greater than $77^{\circ} \mathrm{F}$ & $17-25^{\circ} \mathrm{C}\left(63\right.$ to $\left.77^{\circ} \mathrm{F}\right)$ \\
\hline $\begin{array}{l}\text { Quantimetrics Level } 1 \text { and } \\
2 \text { material }\end{array}$ & Not greater than $77^{\circ} \mathrm{F}$ & $17-25^{\circ} \mathrm{C}\left(63\right.$ to $\left.77^{\circ} \mathrm{F}\right)$ \\
\hline
\end{tabular}


The following ranges have been established for monitoring instrument quality control.

\begin{tabular}{|c|c|c|}
\hline Instrument & $\begin{array}{l}\text { Manufacturer's instrument } \\
\text { range or limit }\end{array}$ & Established range \\
\hline \multicolumn{3}{|l|}{ Beckman centrifuge } \\
\hline (refrigerated) & -5 to $+25^{\circ} \mathrm{C}\left(23-77^{\circ} \mathrm{F}\right)$ & $2-8^{\circ} \mathrm{C}\left(35\right.$ to $\left.46^{\circ} \mathrm{F}\right)$ \\
\hline \multicolumn{3}{|l|}{ Beckman centrifuge } \\
\hline (room temperature) & -5 to $+25^{\circ} \mathrm{C}\left(23-77^{\circ} \mathrm{F}\right)$ & $17-25^{\circ} \mathrm{C}\left(62\right.$ to $\left.77^{\circ} \mathrm{F}\right)$ \\
\hline Revco freezer & $-30^{\circ} \mathrm{C}\left(-22^{\circ} \mathrm{F}\right)$ Limit & -26 to $-34^{\circ} \mathrm{C}\left(-15\right.$ to $\left.-29^{\circ} \mathrm{F}\right)$ \\
\hline Jewett freezer & $-20^{\circ} \mathrm{C}\left(-4^{\circ} \mathrm{F}\right)$ Limit & -18 to $-23^{\circ} \mathrm{C}\left(0\right.$ to $\left.-10^{\circ} \mathrm{F}\right)$ \\
\hline Jewett refrigerator & $2-5^{\circ} \mathrm{C}\left(35\right.$ to $\left.41^{\circ} \mathrm{F}\right)$ & $2-8^{\circ} \mathrm{C}\left(35\right.$ to $\left.46^{\circ} \mathrm{F}\right)$ \\
\hline LFBSC & 0.450 & 0.350 to 0.550 \\
\hline Phlebotomy refrigerator & None & $2-8^{\circ} \mathrm{C}\left(35\right.$ to $\left.46^{\circ} \mathrm{F}\right)$ \\
\hline Mettler Toledo Scale & $\begin{array}{l}\text { One } 100 \mathrm{~g} \text { weight (start of } \\
\text { stand QC) }\end{array}$ & +/- 1.0 grams \\
\hline Mettler Toledo Scale & $\begin{array}{l}\text { Two 300g weights (start of } \\
\text { stand QC) }\end{array}$ & +/- 6.0 grams \\
\hline Mettler Toledo Scale & One 500g weight (daily QC) & +/- 5.0 grams \\
\hline Mettler Toledo Scale & One 20g weight (daily QC) & +/- 0.2 grams \\
\hline
\end{tabular}

Two thermometers monitor ambient temperature in the laboratory and one thermometer monitors ambient temperature in the phlebotomy room. The allowable temperature range for these rooms is $17-25^{\circ} \mathrm{C}\left(63\right.$ to $\left.77^{\circ} \mathrm{F}\right)$.

The acceptable temperature range for refrigerators used for storage of CLIA-regulated specimens and reagents is $2-8^{\circ} \mathrm{C}$ and is checked and recorded daily. If temperatures are recorded automatically on a continuous monitoring system graph, the individual's initials are captured.

Freezer temperatures are monitored daily by visual inspection of either a thermometer or graphs and recorded. All freezers are monitored automatically by built-in visual systems (with thermographic or digital reading). The range of allowable temperature for the $-20^{\circ} \mathrm{C}$ Jewett model is -18 to $-23^{\circ} \mathrm{C}$. The range of allowable temperature for the $-30^{\circ} \mathrm{C}$ Revco model is -26 to $-34^{\circ} \mathrm{C}$. If the range is exceeded, the technologist contacts the MEC manager. 
A separate automated continuous Sensaphone system monitors the room temperature and the internal temperature of the laboratory refrigerators and freezers. When the system detects a significant increase in the temperature, it begins calling designated staff. The MEC managers and data managers have been identified as those individuals responsible for responding to the call by typing in a response code. The temperature increase could be confined to one freezer or refrigerator, to multiple units, or all units. The temperature increase could be due to any number of circumstances such as a loss of power because a circuit breaker tripped, a compressor malfunctioned, or a system failed. The MEC manager or data manager implements corrective actions that are warranted to prevent loss of biological specimens.

Freezers and refrigerators are regularly monitored for excessive ice deposit, inoperative cooling fans, and frayed or worn electrical power connections. Problems are reported to the MEC manager.

SPs are given a urine collection cup and instructed to void completely into the urine container when they check into the MEC and change into their exam clothing. The container is brought back to the MEC laboratory and placed on the counter near the Mettler Toledo scale at workstation 2.

No later than 15 minutes after the specimen arrives in the laboratory the medical technologist places the cup on the scale and scans in the specimen. The lab application calculates the volume of urine and compares the volume collected to the amount needed for the protocol. The volume and date and time of urine collection are also captured.

\subsection{Calibration and Calibration Verification}

\subsubsection{Introduction}

Calibration and calibration verification are specified in the documentation of the CBC analytical procedure. 


\subsubsection{Comparison of Methods Performed on Multiple Instruments or at Multiple Sites}

At least once every 6 to 8 weeks, each of the three HMX instruments is calibrated with $\mathrm{S}-\mathrm{Cal}$. CAP data are analyzed by regression analysis and at least two of the three instruments are compared to each other three times per year.

At least once every 6 to 8 weeks, each of the three Osmette II instruments is calibrated. CAP data are analyzed by regression analysis and at least two of the three instruments are compared to each other three times per year.

\subsection{Remedial Actions}

\subsubsection{Introduction}

Remedial actions are taken and documented when:

- Test systems perform outside acceptable performance specifications. Remedial action is documented in the appropriate QC manual.

- Results of control materials and calibration materials fail to meet quality control criteria. Remedial action is noted in the appropriate QC logbook.

- The laboratory cannot report survey participant test results within its established period. If the reporting of data will exceed the agreed upon time frame, the MEC and final Report of Findings indicate that test results are not available.

- Errors in the reported SP test results are detected. When errors are detected in the reporting of survey participant results, the MEC physician notifies the survey participant by phone, followed by the issuance of a corrected report within a time period suitable to the survey participant, but not to exceed 1 week. The corrected report clearly shows the corrected results in the title. Exact duplicates of the original as well as the corrected report are maintained for 7 years. 


\subsection{Integrated Survey Information System (ISIS)}

\subsubsection{Introduction}

One of the purposes of the ISIS is to store survey participant results for retrieval in a period acceptable for the intended use of the data. Survey participant test results are stored in a database. The ISIS consists of the PC databases used for storing survey participant results. This form for the ISIS facilitates the interfacing of varied and complex instrumentation used in many of the analytical procedures. The MEC LAN hardware serves as the hardware for the ISIS. The identification of the survey participant result will be by specimen ID number, and the result is accessible by this number.

The minimum amount of data stored for each analytical result is the specimen ID number, the analyte name, and the measured result. If the specimen ID does not provide sufficient information to relate the measured result to the QC data for the run, then the run number and run date must also be stored. The specimen ID, run date, and run number should be sufficient information to relate the measured result to the quality control information for that run. Access to the ISIS database is password protected so that only the persons authorized by the Westat and NCHS may access the data.

\subsubsection{Integrated Survey and Information System Data Down Times}

Each laboratory method is able to run and temporarily store results even if the MEC LAN is inoperable.

\subsubsection{Maintenance of LAN Hardware}

After failure of a LAN component, ISIS should be able to be repaired or replaced within a day by a functioning component. LAN hardware is readily available. PC components and the infrequent problems with this hardware are readily correctable in this period. Westat participates in the standard service contract arranged for NHANES for service of failing or failed computer equipment. 


\subsubsection{Backup Schedule of Integrated Survey and Information System Data}

ISIS data stored on LAN hard disk drives are backed up to tape once each weekday (Monday through Friday). If ISIS data are stored on local PC or minicomputer hard drives, they are archived to a second hard disk, tape, optical diskette, or CD-ROM at least once a week.

\subsection{Communications and Complaints}

\subsubsection{Introduction}

Good communication between the laboratory and persons reviewing laboratory analyses is essential for proper use of laboratory results. NHANES encourages collaborative relationships between technologists and physicians utilizing laboratory data. This collaborative interaction is the primary mechanism by which NHANES evaluates the effectiveness of laboratory protocols and other laboratory communications. If the laboratory has a persistent problem with meeting laboratory criteria (e.g., inadequate amount of specimen), the MEC manager, technical supervisor, or laboratory director will discuss the problem with the person in a collaborative spirit.

Similarly, complaints of laboratory performance are carefully considered. Usually, these complaints are fixed with minor adjustment or explanation. Each member of laboratory staff is responsive to complaints that have merit and relate to her or his responsibilities. Nontrivial complaints are reported to supervisors who will decide appropriate actions, including investigation and remedial action. The technical supervisor maintains documentation of nontrivial complaints concerning laboratory performance that are reported to her or him.

\section{Quality Promotion}

\subsubsection{Introduction}

High quality laboratory results are generated when all phases of the measurement process (i.e., preanalytic, analytic, and postanalytic) are conducted properly. Factors that promote high quality results include:

- Competent, well-trained, and motivated laboratory staff;

- Quality laboratory facilities; 
- Well-maintained, high quality laboratory equipment;

- High quality laboratory analytical methods; and

- Clear commitment of management to quality laboratory results.

NHANES is committed to supporting each one of these factors in order to enhance quality.

\subsection{Quality Assurance Program}

\subsubsection{Introduction}

The goal of the NHANES Quality Assurance Program (QAP) is to monitor and evaluate the overall quality of the testing process including preanalytic, analytic, and postanalytic phases.

The initial database and reporting system design included the following requirements:

- All data are released in a timely manner relative to its intended use. All CBC results are provided to the SP in a preliminary Report of Findings when the SP checks out of the MEC. If the results are not available by the time the SP checks out of the MEC, they are reviewed by the MEC physician as soon as they are available, and the SP is notified by telephone of any abnormal results. A hard copy of the CBC results is included in the final Report of Findings sent to the SP 6-12 weeks after the MEC examination. In the case where no blood was drawn, the preliminary or final Report of Findings states, "Not done.” In the case where blood was drawn but no results were transmitted, the preliminary or final Report of Findings states, "Data Not available at this time.”

- The preliminary and final Report of Findings are ISIS-generated and are based on results in the database; they are not predefined reports stored in the system. The format for the English and Spanish preliminary and final Report of Findings is tested annually to determine if the generic format is legible.

- The preliminary and final Report of Findings format includes reference ranges, units of measure, or notation for inadequate specimen. Sample preliminary and final English and Spanish Report of Findings are printed and the format is tested annually.

- NCHS is responsible for sending the final Report of Findings to each survey participant. Mailing labels are printed, the mailing label is matched to the final Report of Findings, and the final Report of Findings is inserted into the envelope. NCHS monitors communications from SPs who report that they have received the incorrect Report of Findings. 
- All results are generated based on results stored in the database. The ISIS system prohibits the MEC technologist from changing data in the database after the SP has checked out of the MEC or if the MEC Report of Findings has been printed. All subsequent copies of results should match the original results. If any result is ever changed in the database, a back-end editing request system form is submitted, tracked, and maintained by ISIS.

- A comprehensive security system guards against unauthorized access. A complete description of this system is maintained by and is available through NCHS.

The basic mechanism for the QAP is the quality audit. The quality audit is conducted by the NHANES technical supervisor or laboratory director, who maintains documentation of the results of the quality audits, including remedial actions taken to address conditions that merited corrective action.

\subsubsection{Quality Audit: Survey participant Test Management}

The quality audit for survey participant test management is conducted at least yearly and consists of:

- A survey of the laboratory staff, and of the analytical procedures used in NHANES. The survey includes requests for information on:

- $\quad$ Any changes which need to be made in survey participant preparation, specimen collection, labeling, preservation, and transportation;

- $\quad$ Any changes which need to be made in completeness and relevance of survey participant information obtained at the time the specimen is acquired, and the use and appropriateness of specimen rejection criteria based on their experience and interactions with persons requesting the tests;

- $\quad$ Laboratory-reporting errors encountered since the last survey;

- Whether the performance of the Integrated Survey and Information System (ISIS) is adequate for their measurement systems; and

- $\quad$ Nontrivial complaints from outside the laboratory about performance of the lab.

The technical supervisor and laboratory director assess the results of the audit and, in consultation with Beckman Coulter ${ }^{\circledR}$, implement corrective actions that are warranted. 


\subsubsection{Quality Audit: Quality Control}

Quality control addresses the analytic phase of measurement. The purpose of the internal ("bench") quality audit is to ensure that the QC analyses were performed and appropriate actions were taken when out-of-control conditions occurred. The quality audit of quality control shall be conducted at least yearly by the technical supervisor and laboratory director and shall consist of:

- A review of a random 10 percent sample of 5C Cell and Latron control results. The review evaluates whether appropriate corrective action was performed and documented for out-of-control conditions.

- An analysis of the proficiency testing results. The analysis and review will assess PT performance and, if unsatisfactory, whether the deficiencies have been adequately addressed. The technical supervisor and laboratory director compare results to determine the correlation coefficient. This correlation must be greater than 0.95 or the laboratory director may give written approval that the methods are sufficiently similar for the intended use of the data.

- The review of documents that demonstrate that at least once every 6 to 8 weeks the S-Cal calibrator was run on three instruments.

The technical supervisor and laboratory director assess the results of the audit and, in consultation with Beckman Coulter®, implement corrective actions that are warranted.

\subsubsection{Quality Audit: Personnel}

Well-trained and competent personnel are essential components of good laboratory performance. The quality audit of personnel is conducted at least yearly by the technical supervisor and laboratory director and shall consist of:

- A review of the 5C Cell and Latron control ("bench”) results or IQAP QC results for 10 percent of the total control runs covering a period of not less than 1 year. The internal ("bench") QC reflects the performance of persons performing the method. If out-of-control conditions occur with a frequency more than twice the expected statistical probability (e.g., 95\% control limits would normally be exceeded by $5 \%$ of the analytical runs), the technical supervisor and laboratory director will assess whether the unacceptable performance is due to the performance of the person in question or other factors (e.g., failing equipment). If the personnel performance is the cause, the technical supervisor and laboratory director determine and implement appropriate remedial action (e.g., training). 
- A review of all remedial action since the last audit to assess the effectiveness of the action. Acceptable performance of the method by the person involved for 1 month after remedial action is acceptable evidence of the effectiveness of remedial action.

- The technical supervisor and laboratory director audit the documentation of personnel training and evaluation to check whether the analyst has been appropriately evaluated in the last year (or 6 months if in first year performing a new method). The evaluation criteria are described in the Personnel Training and Evaluation section of the LPM (Chapter 10, Section 10.16).

The technical supervisor maintains documentation of the results of the audit.

\subsubsection{Quality Assurance Review with Staff}

The technical supervisor and laboratory director prepare a report of the results of quality audits within 3 weeks of the completion of the audit. The technical supervisor and laboratory director review pertinent information with appropriate staff to ensure that effective remedial actions are taken. The technical supervisor and laboratory director also notify staff members who have been involved in the audit if performance was acceptable and that no remedial action is needed. At least once every 6 months, the technical supervisor and laboratory director review the results of quality audits.

\subsection{Personnel Training and Evaluation}

The technical supervisor assures that persons performing laboratory tests receive appropriate training. The performance of persons performing laboratory tests is evaluated every 6 months for the first year testing is performed and annually thereafter. The evaluation shall consist of:

- Direct observation of test performance including survey participant preparation (if applicable), specimen handling, specimen processing, and testing;

- Direct observation of the recording and reporting of test results;

- Review of QC results, IQAP, PT, and linearity results and preventive maintenance records;

- Direct observation of performance of instrument maintenance and function checks; 
- Assessment of testing performance using blind QC materials; and

- Assessment of problem-solving skills.

The technical supervisor maintains documentation of the evaluations for at least 7 years. If remedial action is warranted, the technical supervisor shall discuss the results of a personnel evaluation with the person evaluated and implement appropriate action (e.g., training). 


\section{OPERATIONAL ISSUES}

\subsection{Start of Stand Activities}

The Westat warehouse manager sends supplies for all stands, schedules shipments so that they arrive on or before setup day, and ships supplies requiring refrigeration so that they arrive the day before set up. Store laboratory supplies in the laboratory cabinets or in the MEC trailer compartments.

The advance arrangement staff may assist in completing some setup activities. On setup day, the phlebotomists and medical technologists work together to complete all activities. These tasks include assisting in unpacking the warehouse pallets and boxes, sorting the laboratory supplies by storage location, completing an inventory of all existing and new supplies, organizing and storing all inventoried supplies in MEC cabinets or trailer compartments, and setting up each work area by stocking the area with appropriate supplies. Medical technologists and phlebotomists perform maintenance on and test the functionality of all instruments and equipment. Notify the MEC manager if additional supplies are required and document all instrument and equipment malfunctions in the automated Unusual Field Occurrence utility. Record all lot numbers and expiration dates for consumables (phlebotomy and lab) in the Supply Use Control Log, which is located in the respective QC module.

Mark and store miscellaneous hardware and original packing boxed in a safe location in the laboratory until the end of stand. Hardware includes wood blocks with Velcro, refrigerator and freezer bottle caps, temperature-recording pen protectors, restraining straps, wood boards, and carpeted counter supports and straps for the Beckman Coulter ${ }^{\circledR}$ HMX instrument and printer.

\section{Phlebotomy Room}

- The Phlebotomists' responsibilities include the following tasks:

- $\quad$ Inventory consumables and nonconsumables.

- $\quad$ Check the sink for hot and cold running water and proper drainage.

- $\quad$ Remove the wood block from the refrigerator door.

- $\quad$ Unlock all cabinets. 
- $\quad$ Release the phlebotomy chair restraint.

- Unlock all four casters.

- Pull up on the black lever on the lock and loosen just enough to separate the $\mathrm{S}$ bolts.

- $\quad$ Release the phlebotomy cart restraint.

- $\quad$ Turn on the refrigerator in phlebotomy, OGTT, and spare room.

- $\quad$ Verify the refrigerator’s operation.

- $\quad$ Check the function of the telephone intercom.

- $\quad$ Clean the refrigerators.

- $\quad$ Install the wall thermometer.

- $\quad$ Clean all work surfaces and storage areas.

- $\quad$ Stock and organize supplies.

- $\quad$ Record all lot numbers and expiration dates in the automated Supply Use Control Log.

- $\quad$ Check the computer operations.

- $\quad$ Store the chair and cart restraint and the wood block in the bottom right drawer.

\section{Laboratory}

- Medical technologists' responsibilities include the following tasks:

- $\quad$ Inventory consumables and nonconsumables.

- $\quad$ Check the sink and eyewash for cold running water and proper drainage.

- $\quad$ Check telephone operation.

- $\quad$ Check the wall thermometers for damage.

- $\quad$ Remove the wood door blocks from the refrigerators and freezers and store.

- $\quad$ Replace the refrigerator temperature probe solid bottle caps with the caps with holes and save solid caps for reuse at the end of the stand. 
- $\quad$ Place the refrigerator temperature probes back into the bottles.

- $\quad$ Plug in the refrigerators and freezers.

- $\quad$ Verify freezer and refrigerator operation.

- $\quad$ Check light, blowers, and magnehelic pressure gauge reading in the Laminar Flow Biological Safety Cabinet (LFBSC).

- $\quad$ Release the Beckman Coulter ${ }^{\circledR}$ restraining straps, remove the wood board, detach the bolted carpeted counter supports and store the straps, wood board, and counter supports until the end of the stand.

- $\quad$ Remove the restraining strap on the Beckman Coulter ${ }^{\circledR}$ printer and store the straps until the end of the stand.

- $\quad$ Contact Beckman Coulter ${ }^{\circledR}$ at 1-800-526-7694 to schedule instrument verification by a service technician. Provide the current address and instrument system ID.

- $\quad$ Perform all Beckman Coulter® beginning of stand operations as described in Chapter 7, Section VIII.F. This includes installing and priming all new reagents, performing a start-up procedure, and reviewing and verifying the system setup.

- $\quad$ Calibrate the Beckman Coulter ${ }^{\circledR}$ HMX. Complete the calibration before performing routine CBCs on SPs.

- $\quad$ Unpack the Mettler Toledo scale and store carrying case in an easily accessible area. Perform the QC on the Toledo Scale and complete the QC before performing routine urine flow rates on SPs

- $\quad$ Unpack the Osmette II osmometer. Change the Teflon tip of the Osmette II pipette. Run a total calibration on the Osmette II. Complete the calibration before performing routine osmolality tests.

- $\quad$ Refrigerate the serum and urine pregnancy controls, and Beckman Coulter ${ }^{\circledR}$ Latron and 5C Cell controls as soon as they arrive.

- $\quad$ Clean all work surfaces and storage areas.

- $\quad$ Stock and organize supplies.

- $\quad$ Clean the interior and exterior of refrigerators, freezers, centrifuges, and LFBSC.

- $\quad$ Perform centrifuge maintenance.

- $\quad$ Open and label all new containers using the laboratory application. 
- $\quad$ Mentally step through labeling, blood processing, urine processing, pregnancy testing, complete blood count, and specimen storage to make sure all the necessary equipment is functioning and all supplies are available in the phlebotomy/laboratory area.

- $\quad$ Document all readings and activities in the Laboratory QC module.

- $\quad$ Call FedEx to check availability (X-XXX-XXX-XXXX).

- NCHS biomedical engineer (annual) responsibilities include the following tasks:

- $\quad$ Determine the rpm of the centrifuges using a strobe or tachometer.

- $\quad$ Perform a timer check.

- $\quad$ Verify that both centrifuges are operating at $4-8^{\circ} \mathrm{C}$.

- $\quad$ Enter the results in the Laboratory QC module.

\subsection{End of Stand Activities}

For security reasons, lock all needles in the drawer each day after examinations are complete. Turn off the centrifuges. Reboot all computer terminals.

At the end of a stand, shut down all equipment and inventory all supplies. On teardown day, the phlebotomist and medical technologists work together to complete all activities. Carefully wrap all breakable supplies in preparation for the move. Lock all cabinets and drawers. Safely discard all biohazardous waste. Repack the Osmette II in its original box with protective padding and the Metler Toledo scale in the blue carrying case.

\section{Packing Phlebotomy Room}

- Phlebotomists' responsibilities include the following tasks:

- $\quad$ Inventory consumables and nonconsumables.

- $\quad$ Turn off and clean the refrigerators in the phlebotomy, OGTT, and spare rooms. 
- $\quad$ Place wood blocks with Velcro strips on the refrigerator doors to prevent the doors from closing or moving. Be careful not to damage the rubber gasket on the door.

- $\quad$ Pack all juice and crackers in labeled plastic boxes and store them inside the MEC.

- $\quad$ Remove all loose supplies (clock, telephone handset, etc.) and equipment from the countertops; pack loose supplies in boxes and label "Phlebotomy Room.” If possible, place the boxes in the cupboards above the counter, under the bench, or in the corners on the floor in phlebotomy. If these areas are full, place the boxes in the designated compartments under the trailers. Do not store Vacutainer ${ }^{\circledR}$ tubes in these trailer compartments.

- $\quad$ Bolt the room doors in the open position.

- $\quad$ Close and lock all cabinets.

- $\quad$ Lock all needles in the cabinets.

- $\quad$ Restrain the phlebotomy chair.

- Attach the S bolt on the end of the orange belt to each of the two wall brackets (under the drop-down chair).

- Attach the center S bolts to each other in the center of the orange belt.

- Tighten the belt by pulling on the free end of the belt.

- $\quad$ Restrain the phlebotomy cart in the staff lounge on the wall between the ISIS rack and the outside entry door to the staff lounge.

\section{Packing the Laboratory}

- Medical technologists' responsibilities include the following tasks:

- $\quad$ Inventory consumables and nonconsumables.

- $\quad$ Perform the Beckman Coulter ${ }^{\circledR}$ end of stand procedures as described in Chapter 7.

- Review, print, and clear the error file.

- Download the IQAP files.

- $\quad$ Print Latron and 5C Cell control cum statistics (files) and graphs. 
- $\quad$ Perform daily shut down.

- Bleach the apertures and flow cell.

- Bleach, rinse, and drain reagent lines.

- Turn off the power.

- Rinse exterior of the BSV.

- Wash exterior of the instrument.

- Dispose of all opened reagents.

- $\quad$ Check the expiration dates on the Beckman Coulter ${ }^{\circledR}$ Latron, Beckman Coulter ${ }^{\circledR}$ 5C Cell control, pregnancy test kit, pregnancy controls, and Quantimetrix urine controls. If unopened and they will not expire before the beginning of the next stand, pack them in a shipper with refrigerant packs, print a label using FedEx Ship, and ship back to the warehouse.

- $\quad$ Unplug the refrigerators and freezers.

- $\quad$ Clean refrigerators and freezers.

- $\quad$ Disinfect and clean the pipettes.

- $\quad$ Replace the refrigerator temperature probe bottle caps (with holes), with the bottle caps without holes, and save the caps with holes for reuse at the start of the next stand.

- $\quad$ Place wood blocks with Velcro strips on each refrigerator and freezer door to prevent the door from closing or moving. Be careful not to damage the rubber gasket on the door.

- $\quad$ Turn off the Mettler Toledo scale. Disconnect the scale from the tan serial cable and black AC adapter power cord. Tape the serial cable and power cord to the countertop. Repack the Mettler Toledo scale in the blue carrying case making sure to remove and store the weigh pan in the lid of the carrying case and cover the scale cone with the plastic cone protector.

- $\quad$ Turn off the Osmette II. Pack the Osmette II in the shipping box with foam padding. Make sure the foam padding surrounds the entire instrument so it is secure for transport.

- $\quad$ Unplug the LFBSC.

- $\quad$ Close and lock centrifuge lids and turn off centrifuges.

- $\quad$ Pack all tube rockers securely in boxes or in drawers. 
- $\quad$ Remove all small supplies from the counters and LFBSC and pack in boxes or cabinets.

- $\quad$ Close all cupboards and lock.

- $\quad$ Remove the fire extinguisher from the wall and lay inside the laboratory sink.

- $\quad$ Clean all counters, the LFBSC, and equipment with 1:100 bleach solution.

- $\quad$ Place chairs upside down or lay them down on the floor.

- $\quad$ Attach Beckman Coulter's bolted carpeted supports to the counter. Secure the Beckman Coulter ${ }^{\circledR}$ by laying the wood board across the top of the instrument and tightening the straps. Place foam blocks behind the instrument to cushion it during transit. Place a strap around the bottom front of the instrument. (Do not apply too much pressure.)

- $\quad$ Secure the Beckman Coulter® printer by strapping it to the printer shelf.

\section{Sending Laboratory Files and Supplies}

At the end of each stand, use FedEx to create a label to send the following hard-copy documentation to the technical supervisor at the home office:

- Beckman Coulter ${ }^{\circledR}$ Latron and C Cell Control printouts

- Beckman Coulter ${ }^{\circledR}$ Start Up printouts

- Beckman Coulter ${ }^{\circledR}$ Calibration printouts

- Beckman Coulter ${ }^{\circledR}$ Latex CBC/Diff cumulative statistic and graph printouts

- Beckman Coulter ${ }^{\circledR}$ Error file printout

- Beckman Coulter ${ }^{\circledR}$ Linearity printouts

- Proficiency testing copies (hematology, urine and serum hCG, and osmolality)

At the end of each stand use FedEx to create a label to send the following supplies to the warehouse supervisor:

- Unopened pregnancy test kits

- Unopened pregnancy urine controls - refrigerated 
- Pregnancy serum controls - refrigerated

- Unopened Coulter ${ }^{\circledR}$ 5C Cell and Latron controls - refrigerated

- Unopened Quantimetrix Human Urine controls - refrigerated

Pack supplies using procedures described in Chapter 9.

\subsection{Inventory Procedures and Supplies}

At the start and end of each stand, take a complete inventory. The warehouse uses this inventory to stock the MEC for the next stand.

The equipment and supplies associated with the MEC laboratory are listed in Exhibit 15-1, Equipment and supplies.

Exhibit 15-1. Equipment and supplies

-30 Revco freezer ULT 430A18

Two Beckman Coulter Allegra centrifuges

Two refrigerators (OGTT and right side of trailer 3)

Precision Instruments Osmette II Osmometer

Two Thermolyne Vari-Mix M48700 (lab)

Two Maxi-Mixer (lab)

Two solar wall thermometers (lab)

Two Jewett refrigerators UC5B
Five Jewett temperature monitoring units 8ER-1B

Laminar flow biological safety cabinet

One Jewett refrigerator (phlebotomy)

Mettler Toledo scale

One Thermolyne Speci-Mix (phlebotomy)

One Thermolyne Speci-Mix (lab)

One solar wall thermometer (phlebotomy)

Thermometer (centrifuge calibration)

Store supplies in the trailer compartments.

\subsubsection{General Inventory Procedures}

- Since item units can change, verify the "unit of measure" each time you record a count. Examples of units are pack, each, bag, box, case, roll, tray, bottle, pair, and pouch.

- Do not redefine or re-iterate the Unit of Measure. 
- "PAR Level” represents the number of units the component is to begin a stand with. Note that PAR Levels are set and adjusted by home component staff only. Any/all recommendations to adjust an item's PAR level should be directed to Home Component Staff.

- Do not write any notes, comments, etc. on the Start-of-Stand Inventory Count Sheets or the End-of-Stand Inventory Count Sheets. Comments or concerns should be written on a separate sheet of paper and attached to the inventory sheet.

- Rotate items by first using items you have in the rooms, then items in the belly compartment. Place items you just received in the back of the stock, unless you need them immediately. Only restock your rooms with items that were just shipped to the stand when you are out of that item in your room. Many items such as gloves, alcohol prep pads, sani-cloth wipes, blood tubes, etc. deteriorate over time. Always use inventory with the closest expiration date first.

- Multiple inventory items onboard the MEC contain lot numbers and expiration dates. When performing physical inventory counts, pay close attention to these items and mark inventory sheets accordingly for each lot number/expiration date per item.

- Do not purchase items listed on the inventory sheets. Every item listed on the inventory sheet is supplied by the NHANES Warehouse. Purchasing items locally creates chaos with the Inventory Management System.

- Do not borrow items from other components. Any additional inventory needed by a component should be requested from the NHANES Warehouse. Inventory usage is tracked by component. The "Counted By" tech id is entered into the database to track inconsistencies and trends. Everyone is accountable for his or her component counts.

\subsection{2 $\quad$ End of Stand Inventory Procedures}

The End-of Stand Inventory Count Sheet (EOSCS) includes the stand number, Site ID (MEC number), a “Counted By” line, and Exam Supply columns for item number, general item description, Unit designation, PAR level, and a count box. The MEC manager prints these EOSCSs for every component. The MEC staff is responsible for accurately counting (inventorying) exam supplies and entering this number into the count box. The MEC staff gives the completed sheets back to the MEC manager who sends them to the warehouse manager. The warehouse manager enters the item counts into the Inventory Management System.

- $\quad$ Print only one name in the "Counted By" field. This is the person responsible for taking and verifying the inventory count.

- $\quad$ Please write legibly for all counts. 
- Place a number in each "count” box. Do not leave any count box empty. If there is no stock remaining for a specific item, place a "zero" in the count box.

- Do not count partial units; record only whole numbers in the count box. Do not write entries such as: $1 / 2$, partial, some, many, a few, couple, .5, or multiple.

- If the PAR for an item is more than one (1) and the "unit of measure" is open, do not count that container. Only count the remaining number of items that have not been opened.

- If the PAR for an item is only one (1) unit and it is more than $1 / 2$ empty, place a zero (0) in the count box. A full unit will be supplied to the component at the opening of the next site.

- All active lot numbers and expiration dates are listed on the inventory count sheets if they are applicable for that item. Record the number of supplies remaining for each lot number/expiration date in the count box. If there is no inventory remaining for a listed lot number/expiration date, then place a "zero" in the count box.

- Any item that has expired during the MEC site or will expire before the MEC reopens at its next location should be removed from the component. If an item is removed, a "zero" should be entered in the count box on the End-of-Stand Inventory Count Sheet for that item. A Delete/Expired Inventory Count Sheet must be filled out, per component, showing the quantity for each item removed from the component. This additional inventory sheet must be attached to the End-of-Stand Inventory Count Sheet for that component when turned into the MEC manager.

\subsubsection{Mid-Stand Inventory Procedures}

Mid-Stand Inventory is performed by each component on the MEC. The Mid-Stand Inventory is performed approximately $2-2 \frac{1}{2}$ weeks into an operating site. This inventory provides the opportunity for MEC staff to request additional supplies from the warehouse that will be needed to complete the current MEC site. The Mid-Stand Inventory is conducted via UFO entry (per component).

NOTE: The mid-stand inventory is only to provide additional supplies needed to complete the current site, it is not designed to bring all supplies up to full par level.

- Each component is to review all remaining inventory to determine if additional supplies are needed to complete the current MEC site.

- A “UFO” will be entered (sighted) by one MEC staff member per component.

- MEC staff will enter a "UFO” by component name and then use "Inventory/Supplies" for the type of UFO entry. 
- In the description field, the MEC staff member will request the supply(s) needed for the component.

- Entries are to be made as: item description then quantity requested (per item).

- If no additional supplies are needed for the component, then the entry "Nothing Needed" is to be written in the description field.

- Do not request items in the UFO that are not currently on the component inventory list.

- $\quad$ Do not request changes to set par levels in mid-stand UFOs.

- $\quad$ Do not enter equipment issues in a UFO listed as "Inventory/Supplies."

- All completed UFO entries will be reviewed by the MEC manager for that MEC before the warehouse will pull and ship supplies requested.

- Once the supplies have been pulled by the warehouse and packed for shipping to the MEC, the warehouse manager will finalize the UFO.

- Mid-Stand deliveries are arranged between the MEC manager and the warehouse manager.

\subsubsection{Start of Stand Inventory Procedures}

The Start-of-Stand Pull Sheets (SOSPS) include the stand number, Site ID (MEC number), and Exam Supply columns for part number, general item description, Previous EOS Count number, PAR level, Unit designation, and a Ship to Stand box. The warehouse manager uses this report to bring each item back to par level. For example, if the Previous EOS Count (captured by the IMS) is four and the Par level is five, the Ship to Stand count box will contain the number one. The warehouse manager prints and packs one SOSPS for each component. The MEC staff is responsible for accurately reconciling the supplies and numbers in the SOSPS. The MEC staff gives the completed sheets back to the MEC manager who returns them to the warehouse manager. The warehouse manager reviews each sheet and sends additional supplies to the field if necessary.

- Reconcile the inventory for each item.

- Verify the Previous EOS Count for each item.

- Verify that the start of stand shipment contains the exact number of each item listed in the Ship to Stand box. 
- Previous EOS Count added to the Ship to Stand Count Box should equal PAR Level.

- If the total inventory equals the set PAR level, place a check mark on the right side of the Ship to Stand Count Box (per item).

- If there is a discrepancy, write the physical count on hand to the right of the Ship to Stand Count box (per item).

- If the quantity for an item is higher then the set PAR level, the excess should be removed from the component (leaving PAR) and returned to the warehouse. If an item(s) is being returned to the warehouse, have the MEC manager print a "Transfer Inventory to Warehouse Count Sheet” for the component. Print your name on the inventory sheet. Mark only the quantities for item(s) that are being returned. Give the excess inventory along with the "Transfer Inventory Sheet" to the MEC manager to ship supplies back to the warehouse.

\subsubsection{Consumables vs. Nonconsumables}

Inventory items are divided into two categories — consumable and nonconsumable. Inventory both types of items during each inventory. The definition for a consumable item is anything that is typically consumed during an examination. A nonconsumable item is one that can be used more then once (example: stopwatches, test tube racks, safety glasses, storage boxes, etc). There are times when nonconsumable items will need to be replaced. These instances would include items that are used in an emergency, when the item expires, or when the item breaks (example: physician medical kit supplies, stopwatches, thermometers, CPR masks, etc.).

\subsubsection{Shipping Excess Inventory Back to the Warehouse}

When shipping excess inventory back to the warehouse, please use the "Transfer Inventory to Warehouse Manifest," which is found on the Intraweb and can be printed by the MEC manager. This form looks similar to the "End of Stand Count Sheets." Print your name in the "Count By" field and mark only the items (with quantity being returned) in the count boxes that are being shipped back to the warehouse. Give the items to be returned along with the "Transfer Inventory to Warehouse Inventory Sheet” to the MEC manager to ship back to the warehouse. This information is entered into the Inventory Management System by the warehouse manager and is used to adjust the stand inventory and usage information as well as increase the warehouse inventory counts. 


\subsubsection{Tracking of Expired and Broken Inventory}

Complete the "Delete Expired/Broken Inventory Report” whenever inventory has expired and must be destroyed or has broken and is no longer usable. Print your name in the "Count By" field and mark only the item(s) in the count box that have expired or broken, and which are being removed from the MEC. This report is found on the Intraweb and should be completed and forwarded to the warehouse manager so that the expired or broken inventory can be removed from the stand inventory. Unless the item is a tool (piece of equipment), under most circumstances the item can be disposed of there in the field and there is no need to return the item to the warehouse. Check with the MEC manager or call the warehouse manager to verify the need to ship the expired/broken item back to the warehouse.

The chief medical technologist and the phlebotomist are responsible for notifying the MEC manager if additional supplies are needed during the stand. However, each technologist and phlebotomist is responsible for identifying any potential shortage of supplies at each workstation. Contact the warehouse manager directly at XXX-XXX-XXXX.

\subsection{Dry Run}

After setting up the phlebotomy and laboratory rooms at each new stand, the staff conducts a “dry run” session using volunteers to verify data and specimen collection and processing procedures. One purpose of the dry run session is to help identify any problem areas, particularly with equipment, and to resolve the problems before the stand begins. Another purpose is to provide the contract laboratories with blind split samples for quality control determinations. Two distinct IDs are assigned to each volunteerthe original SP ID and a second unique Sample ID. Document the results of the dry run session in a manner identical to an actual session except enter results twice, first using the SP ID, and second using the Sample ID.

Procedures

- Collect the amount of blood specified in the Dry Run Venipuncture Protocol (see Exhibit 15-1). This amount is less than the blood required from a routine examinee.

- $\quad$ Prepare split samples for each volunteer. 
- $\quad$ Collect 4-mL of urine and process as specified in Dry Run Urine Processing Protocol (see Exhibit 15-2).

- $\quad$ Process the blood as directed in the Dry Run Blood Processing Protocols (see Exhibits 15-3, 15-4, and 15-5).

- $\quad$ Store these split samples and ship them to contract laboratories for analysis with the first routine shipment.

\subsubsection{Phlebotomy Protocol and Procedures}

The dry run venipuncture protocol is dependent on the last digit in the SP ID. If the last digit is an odd number, the volunteer's protocol includes two 4-mL EDTA, two 2-mL gray, and four 15-mL red top tubes. If the last digit is an even number, then the volunteer's protocol includes four 3-mL EDTA, two 2-mL gray, and four 15-mL red top tubes. Since both even and odd dry run volunteers are eligible for the oral glucose tolerance test, their profiles include two additional 2-mL gray tubes.

On dry run day the phlebotomist refers to the following instructions to conduct the phlebotomy procedures:

- Access the phlebotomy application, open the exam, and wand the dry run volunteer into the component.

Exhibit 15-2. Dry run venipuncture protocol

\begin{tabular}{|c|c|c|}
\hline Tube type (in priority order) & $\begin{array}{c}\text { Last digit in SP ID } \\
=\text { Odd }\end{array}$ & $\begin{array}{c}\text { Last digit in SP ID } \\
=\text { Even }\end{array}$ \\
\hline 3-mL EDTA & 2 & 4 \\
\hline 2-mL Gray & 2 & 2 \\
\hline 15-mL Red & 4 & 4 \\
\hline 2-mL Gray (OGTT) & 2 & 2 \\
\hline
\end{tabular}

- Conduct the phlebotomy interview and administer the fasting questionnaire as described in Chapter 4.

- $\quad$ Perform the venipuncture using procedures described in Chapter 4. 
The last digit in the SP ID determines the dry run volunteer's venipuncture protocol. The correct venipuncture automatically displays. Note that there are two IDs - an SP ID (110355), and a Sample ID (616625). Both the SP ID and the Sample ID display under the “Obtained all” check box.

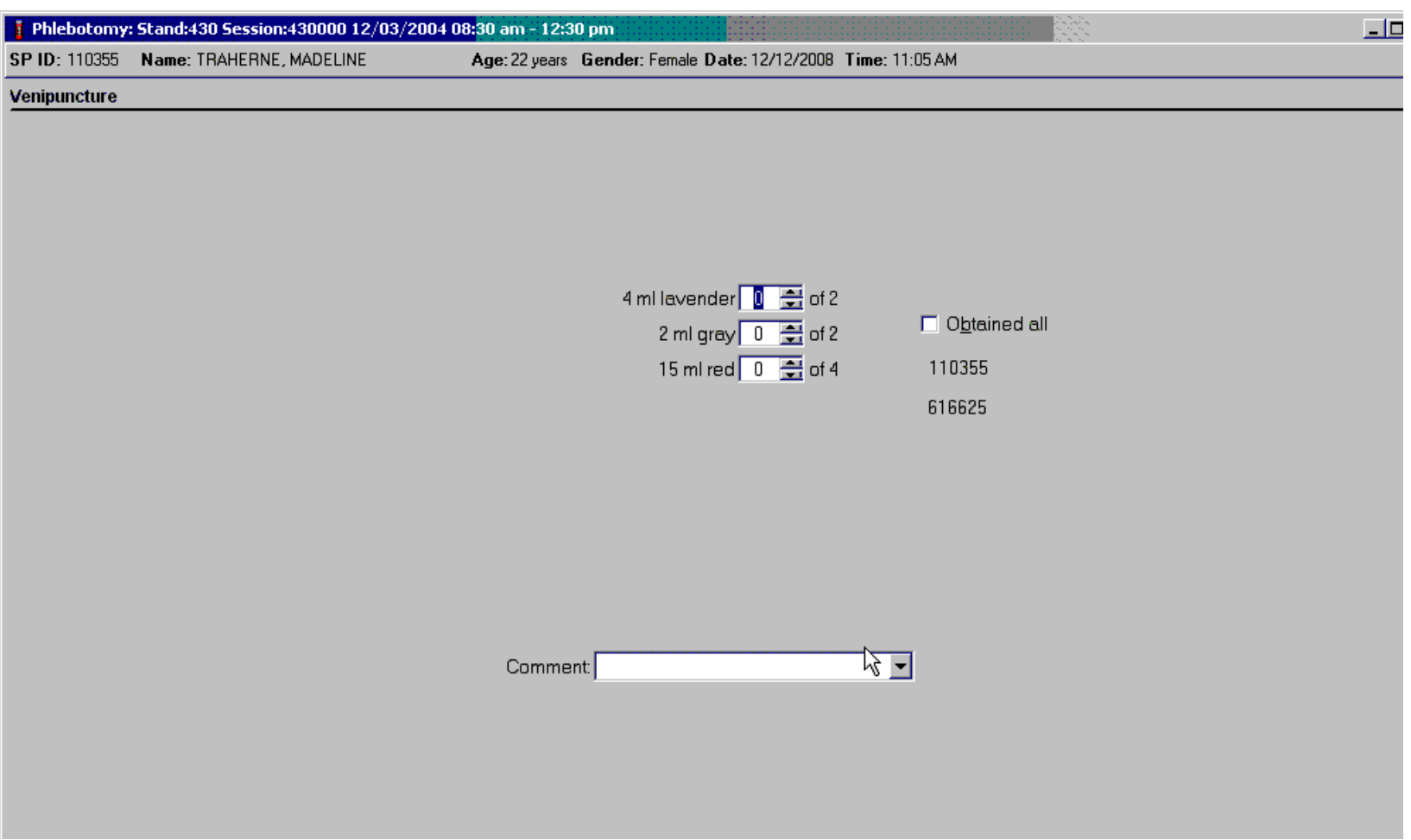

Enter the venipuncture results using procedures described in Chapter 4. Enter any reasons for a tube not being drawn according to protocol, and any comments about the venipuncture. Refer to Chapter 4 for specific instructions.

Label the Vacutainer ${ }^{\circledR}$ tubes for each volunteer; label half of the tubes with the SP ID and the other half with the Sample ID. If only one tube is drawn, discard the tube. If less than four of the 15$\mathrm{mL}$ red tubes are successfully drawn, label the first tube with the original SP ID and the second one with the Sample ID. If an odd number of tubes are drawn, discard the odd tube. 
For dry run volunteers with the SP ID and Sample ID ending in an odd number:

- $\quad$ Place one label with the original SP ID on one of the two 3-mL EDTA tubes and place the other label with the Sample ID on the other 4-mL EDTA tube.

- $\quad$ Place the label with the original SP ID on one tube of the pair of 2-mL gray tubes and place the label with the Sample ID on the other 2-mL gray tube.

- Place the labels with the original SP ID on two of the four 15-mL red tubes and place the labels with the Sample ID on the other two 15-mL red tubes.

For dry run volunteers with the SP ID and Sample ID ending in an even number:

- $\quad$ Place two of the labels with the original SP ID on one pair of 3-mL EDTA tubes and place the other two labels with the Sample ID on the other pair of 3-mL EDTA tubes.

- $\quad$ Place the label with the original SP ID on one tube of the pair of 2-mL gray tubes and place the label with the Sample ID on the other 2-mL gray tube.

- Place the labels with the original SP ID on two of the four 15-mL red tubes and place the labels with the Sample ID on the other two 15-mL red tubes. 


\subsubsection{Printing Labels, Urine Processing, and Pregnancy Testing}

Access the laboratory application and logon using the MEC Logon window.

\begin{tabular}{|c|c|c|c|c|c|c|c|c|c|c|c|c|c|c|c|c|}
\hline \multicolumn{17}{|c|}{ i: Stand:430 Session:430010 12/04/2004 08:30 am - 12:30 pm } \\
\hline 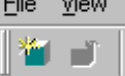 & Q Dilities Repo & \multicolumn{2}{|c|}{ of } & \multicolumn{2}{|c|}{\begin{tabular}{l}
\multicolumn{2}{c}{ Shipping } \\
$\mid$ DI $^{+} \mid$
\end{tabular}} & Window Help & & \multirow{2}{*}{\multicolumn{3}{|c|}{ ocess Status }} & \multirow{3}{*}{\multicolumn{2}{|c|}{$\mathrm{HPV}$ OF }} & \multirow[b]{2}{*}{$\Delta$} \\
\hline & ppointments 1 & for Ses & ssion: & 430010 & & & & & & & & & & & & \\
\hline SPID & Sample ID s & status & type & gender & age & name & UC & $\mathrm{u}$ & $P$ & $O Z$ & $\mathrm{CB}$ & $\mathrm{B} \mathrm{gtt}$ & $\mathrm{PC}$ & & & \\
\hline 119114 & 119114 & $\mathrm{SC}$ & $\mathrm{D}$ & $M$ & 72 & RAMOS, STEPHEN & 0 & 0 & . & 0 & 0 & 00 & & . & . & \\
\hline 119114 & 491426 & $\mathrm{SC}$ & $\mathrm{D}$ & $M$ & 72 & RAMOS, STEPHEN & $\cdot$ & 0 & . & 0 & 0 & 00 & & . & . & \\
\hline 281786 & 281786 & $\mathrm{sc}$ & $\mathrm{D}$ & M & 42 & RAMOS, MARTIN & 0 & 0 & . & 0 & 0 & 00 & & . & . & \\
\hline 281786 & 493106 & $\mathrm{sc}$ & $\mathrm{D}$ & M & 42 & RAMOS, MARTIN & & 0 & . & 0 & 0 & 00 & & . & . & \\
\hline 350948 & 350948 & $\mathrm{sc}$ & $\mathrm{D}$ & M & 74 & RAMOS, JACKSON & & 0 & . & 0 & 0 & 00 & & . & . & \\
\hline 350948 & 498908 & $\mathrm{sc}$ & D & M & 74 & RAMOS, JACKSON & $\cdot$ & 0 & . & 0 & 0 & 00 & · & · & . & \\
\hline 412754 & 412754 & $\mathrm{sc}$ & $\mathrm{D}$ & $\mathrm{F}$ & 25 & DEMARCO, JILL & 0 & 0 & 0 & 0 & 0 & 00 & & . & . & \\
\hline 412754 & 499102 & $\mathrm{sc}$ & $\mathrm{D}$ & $\mathrm{F}$ & & DEMARCO, JILL & & 0 & $\cdot$ & 0 & 0 & 00 & & . & . & \\
\hline 439242 & 439242 & $\mathrm{sc}$ & $\mathrm{D}$ & $\mathrm{F}$ & 49 & DEMARCO, LEANNE & 0 & 0 & 0 & 0 & 0 & 00 & & . & . & \\
\hline 439242 & 499492 & $\mathrm{sc}$ & $\mathrm{D}$ & $\mathrm{F}$ & & DEMARCO, LEANNE & & 0 & . & 0 & 0 & 00 & & . & . & \\
\hline
\end{tabular}

Two distinct IDs are assigned to each volunteer - the original SP ID and a second unique Sample ID. 
The Print All Labels pick list demonstrates that for each dry run volunteer, there are two entries, the original SP ID and the Sample ID.

\begin{tabular}{l} 
Print ALL Labels \\
\cline { 2 - 4 } \\
filter selection to:
\end{tabular}

There are two sets of urine and blood processing vessel labels and one pregnancy label (if eligible) for each dry run volunteer. The correct vessel labels automatically print based on the protocol, except for the labels for osmolality. Print these separately. Print labels using procedures described in Chapter 9. 
Print labels for the osmolality tests.

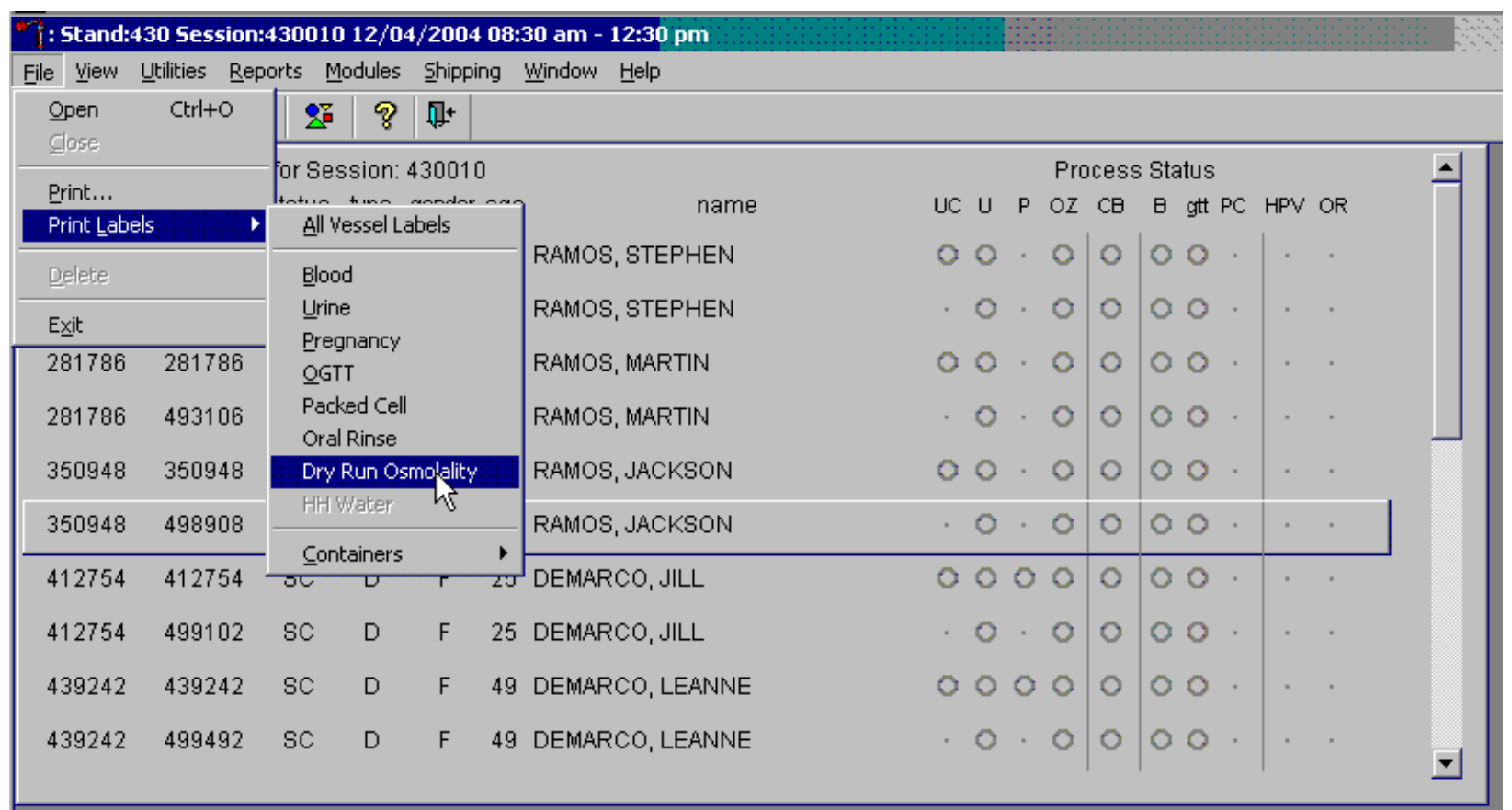

To print labels for osmolality, use the mouse to direct the mouse arrow to Reports, drag the arrow to Osmolality Dry Run Labels, and right click.

Place urine and pregnancy labels at workstation 2. Each volunteer has two unique blood processing racks - one with labels containing the SP ID and the second with labels containing the Sample ID. Assemble and label the blood processing racks for each dry run volunteer using procedures described in Chapter 9. 
Review the laboratory heads-up display. Use the heads-up display to view the modules for which dry run volunteer is eligible and the current process status.

\begin{tabular}{|c|c|c|c|c|c|c|c|c|c|c|c|c|c|c|c|c|}
\hline File View & Uيtilities Repo & orts $\underline{M}$ & odules & Shippi & ing & Window & & & & & & & & & & \\
\hline 仯 & 阙 - 穴 & 8 & $\vartheta$ & D? & & & & & & & & & & & & \\
\hline & ppointments & for Ses & sion: & $43001 c$ & & & & & & Pro & cess & Status & & & & 스 \\
\hline SPID & Sample ID s & status & type & gender & age & name & UC & $u$ & $\mathrm{P}$ & $\mathrm{OZ}$ & $\mathrm{CB}$ & $\mathrm{B}$ gtt $P$ & $\mathrm{PC}$ & HPV O & OR & \\
\hline 119114 & 119114 & $\mathrm{SC}$ & $\mathrm{D}$ & M & 72 & RAMOS, STEPHEN & 0 & 0 & $\cdot$ & 0 & 0 & 00 & . & $\cdot$ & . & \\
\hline 119114 & 491426 & $\mathrm{SC}$ & D & M & 72 & RAMOS, STEPHEN & $\cdot$ & 0 & $\cdot$ & 0 & 0 & 00 & . & $\cdot$ & . & \\
\hline 281786 & 281786 & $\mathrm{SC}$ & D & M & 42 & RAMOS, MARTIN & 0 & 0 & $\cdot$ & 0 & 0 & 00 & . & & . & \\
\hline 281786 & 493106 & SC & D & M & 42 & RAMOS, MARTIN & · & 0 & $\cdot$ & 0 & 0 & 00 & - & & . & \\
\hline 350948 & 350948 & $\mathrm{SC}$ & D & M & 74 & RAMOS, JACKSON & 0 & 0 & $\cdot$ & 0 & 0 & 00 & - & $\cdot$ & . & \\
\hline 350948 & 498908 & $\mathrm{SC}$ & D & M & 74 & RAMOS, JACKSON & . & 0 & $\cdot$ & 0 & 0 & 00 & - & . & · & \\
\hline 412754 & 412754 & $\mathrm{SC}$ & D & $\mathrm{F}$ & 25 & DEMARCO, JILL & 0 & 0 & 0 & 0 & 0 & 00 & - & $\cdot$ & · & \\
\hline 412754 & 499102 & $\mathrm{SC}$ & D & $\mathrm{F}$ & 25 & DEMARCO, JILL & $\cdot$ & 0 & $\cdot$ & 0 & 0 & 00 & & & & \\
\hline 439242 & 439242 & $\mathrm{SC}$ & D & $\mathrm{F}$ & 49 & DEMARCO, LEANNE & 0 & 0 & 0 & 0 & 0 & 00 & . & . & . & \\
\hline 439242 & 499492 & $\mathrm{SC}$ & D & $\mathrm{F}$ & 49 & DEMARCO, LEANNE & · & 0 & $\cdot$ & 0 & 0 & 00 & - & . & - & $\checkmark$ \\
\hline
\end{tabular}

The heads-up display lists each dry run volunteer twice. Note the difference between the SP ID and the Sample ID and the types of tests for which the dry run volunteer is eligible. Dry run volunteers with an SP ID and Sample ID ending in either an odd or even digit are eligible for urine collection and processing, osmolality, CBC, GTT, blood processing, and the pregnancy test (if female.) The urine and blood protocols constitute a limited subset of the primary SP's test protocol. Each test has the same age and gender restrictions as a primary SP. Notice that one urine collection result and one pregnancy test are required for each dry run volunteer when the SP ID and Sample ID end in an odd or even digit.

Collect approximately 4-5-mL urine from all dry run volunteers. The MEC coordinator instructs each volunteer to void as soon as they feel they are able to provide a urine specimen. The coordinator or local assistant transports the initial specimen to the laboratory. All dry run volunteers are eligible for at least one urine test.

Place the urine collection cup on the scale and scan in the urine collection specimen bar code as described in Section 5.4. 
Document the urine collection in the laboratory application.

\begin{tabular}{|c|c|c|c|c|c|c|c|c|c|c|c|c|c|}
\hline \multicolumn{7}{|c|}{ Appointments for Session: 430010} & \multicolumn{6}{|c|}{ Process Status } & \multirow[t]{3}{*}{$\Delta$} \\
\hline SP ID & Sample ID & status & type & gender & age & name & \multirow{4}{*}{$\begin{array}{l}\text { Wrine Processing } \\
\text { Blood Processing } \\
\text { ogst Processing } \\
\text { Packed Cell Processing } \\
\text { HPy Processing } \\
\text { Oral Ruinse Processing }\end{array}$} & $\mathrm{CB}$ & 日 & gtt $P$ & $\mathrm{HPV}$ & OR & \\
\hline 119114 & 119114 & $\mathrm{Cl}$ & $\mathrm{D}$ & M & 72 & RAMOS, STEPHEN & & 0 & 0 & 0 & . & . & \\
\hline 119114 & 491426 & $\mathrm{Cl}$ & $\mathrm{D}$ & M & 72 & RAMOS, STEPHEN & & 0 & 0 & 0 & . & · & \\
\hline 281786 & 281786 & $\mathrm{SC}$ & $\mathrm{D}$ & M & 42 & RAMOS, MARTIN & & 0 & & 0 & . & . & \\
\hline 281786 & 493106 & $\mathrm{SC}$ & D & $M$ & 42 & RAMOS, MARTIN & Urine Gollection & 0 & 0 & 0 & - & . & \\
\hline 350948 & 350948 & $\mathrm{sc}$ & D & M & 74 & RAMOS, JACKSON & $\begin{array}{l}\text { Bregnans Testing } \\
\text { osmolality }\end{array}$ & 0 & & 0 & . & . & \\
\hline 350948 & 498908 & $\mathrm{SC}$ & $\mathrm{D}$ & $M$ & 74 & RAMOS, JACKSON & Hernatology & 0 & 0 & 0 & . & . & \\
\hline 412754 & 412754 & $\mathrm{sc}$ & $\mathrm{D}$ & $\mathrm{F}$ & 25 & DEMARCO, JILL & Refresh & 0 & 0 & 0 & . & . & \\
\hline 412754 & 499102 & $\mathrm{SC}$ & $\mathrm{D}$ & $\mathrm{F}$ & 25 & DEMARCO, JILL & Labels & 0 & & 0 & . & . & \\
\hline 439242 & 439242 & $\mathrm{sc}$ & $\mathrm{D}$ & $\mathrm{F}$ & 49 & DEMARCO, LEANNE & 0000 & 0 & 0 & 0 & . & . & \\
\hline 439242 & 499492 & $\mathrm{SC}$ & $\mathrm{D}$ & $\mathrm{F}$ & 49 & DEMARCO, LEANNE & $\cdot 0 \cdot 0$ & 0 & 0 & 0 & . & . & -1 \\
\hline
\end{tabular}

To select the correct dry run volunteer's record, locate the record in the heads-up display that contains the urine collection circle. To access the urine collection module, use the up and down keys to move up and down the list until the correct record is highlighted or drag the mouse arrow to the correct volunteer and right click. Continue by dragging the mouse arrow to \{Urine Collection\}, then left click or type $[\mathrm{C} / \mathrm{c}]$. 
The urine collection window displays.

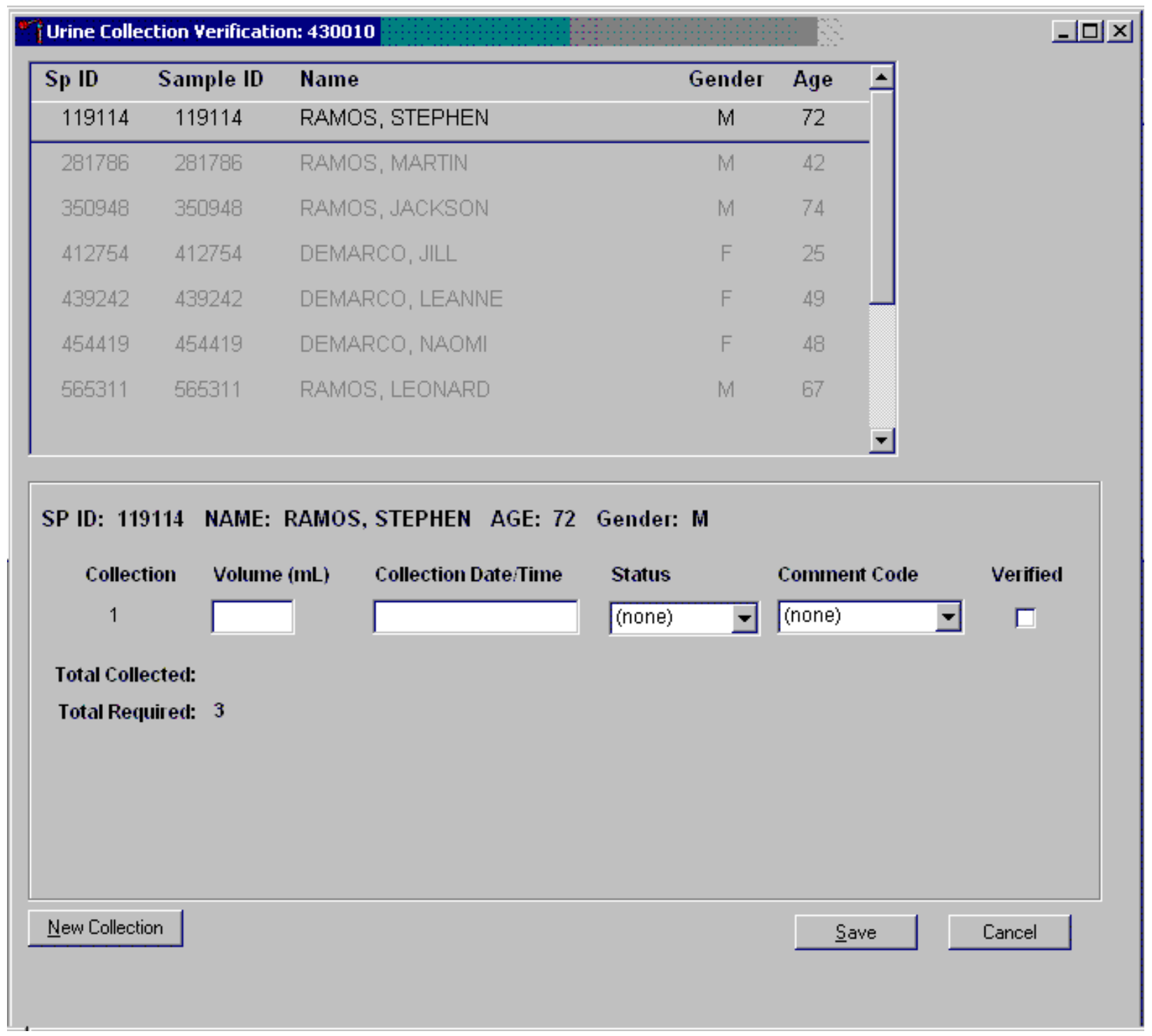

The top of the urine collection window contains columns for the SP ID, Sample ID, Person Name (last, first), Gender, and Age. The row for checked-in SPs is indicated in black, while SPs who have not checked into the MEC session are gray. Only dry run volunteers who have checked into the MEC session are available for processing. SPs who have a urine collection ready for verification display in blue. Use the scroll bar to view all SPs scheduled into the session. 
Verify the sufficiency of the quantity of urine received in the laboratory. Use the measuring tool to verify the amount of urine reported from the scale.

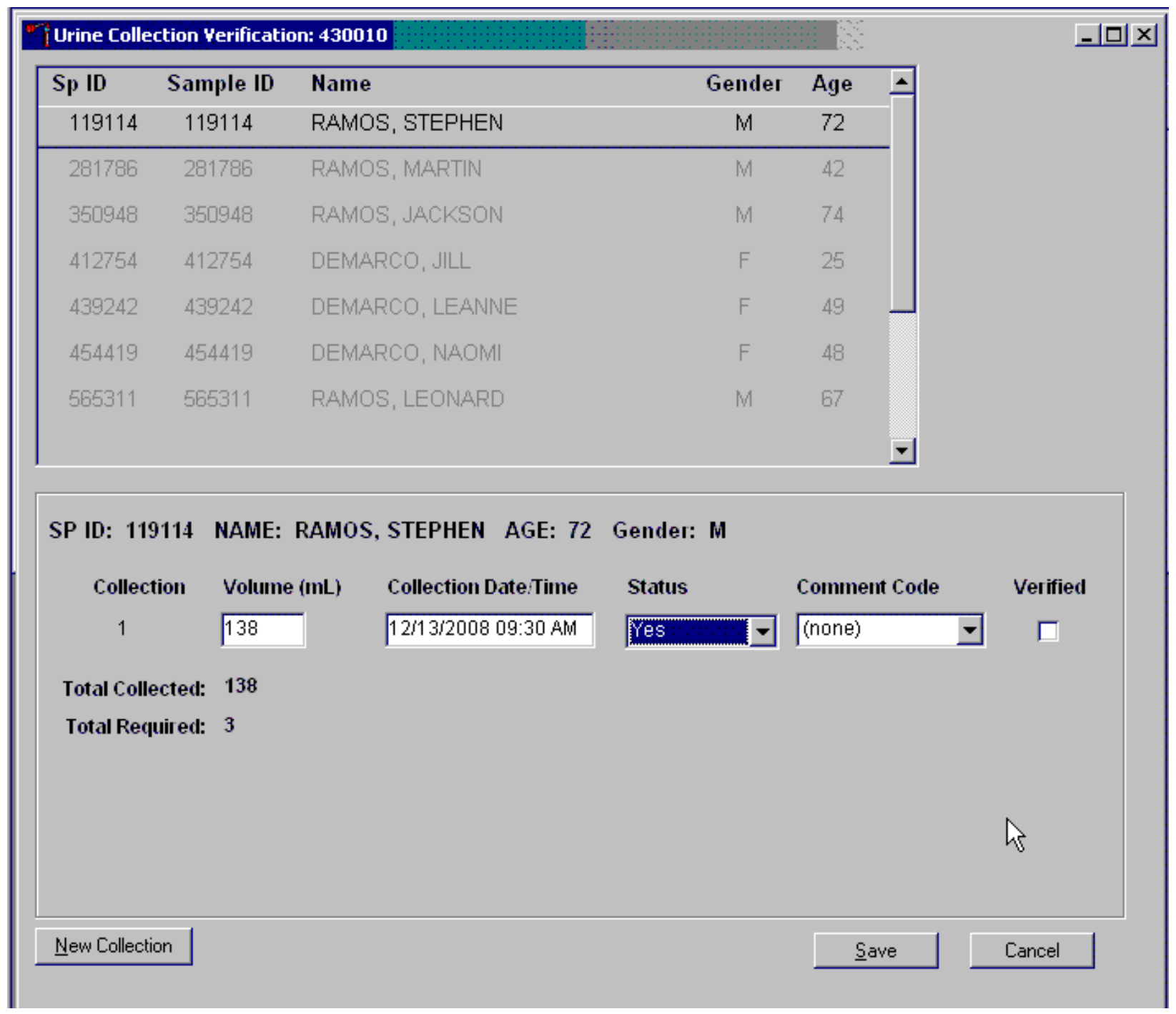

If the SP has submitted a urine collection that has been weighed and scanned into the database the volume, collection status, and collection date and time will automatically display in the lower portion of the Urine Collection screen. 
If the collection did not meet the minimum protocol requirement, the application will automatically insert "QNS" in the Status result text field and "quantity not sufficient" in the Comment Code text box.

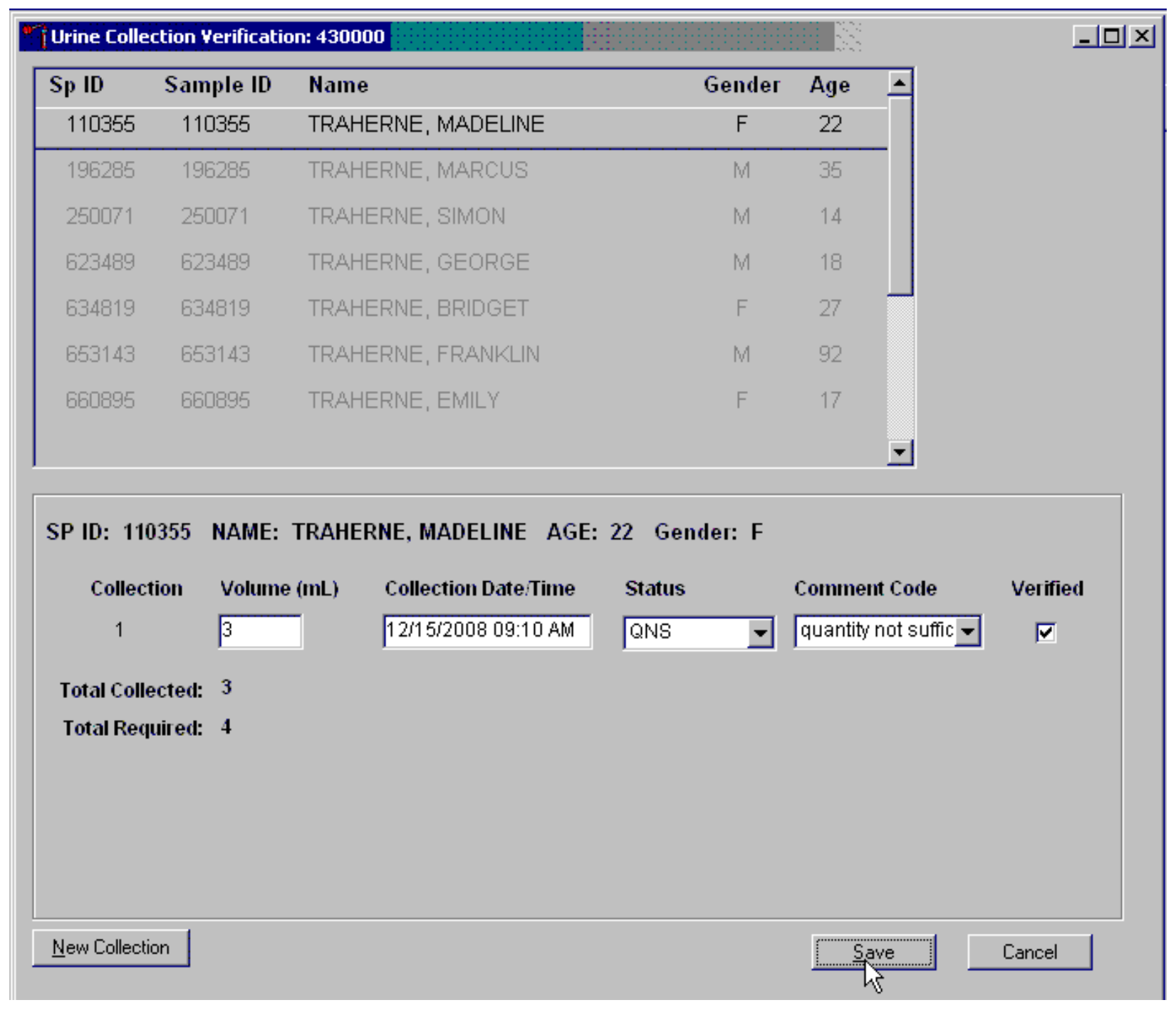

A QNS status immediately prompts and notifies the coordinator to ask the SP for an additional urine sample. 
A second instance of urine collection for the first QNS sample or third instance of urine collection for the second QNS sample will appear below the current sample.

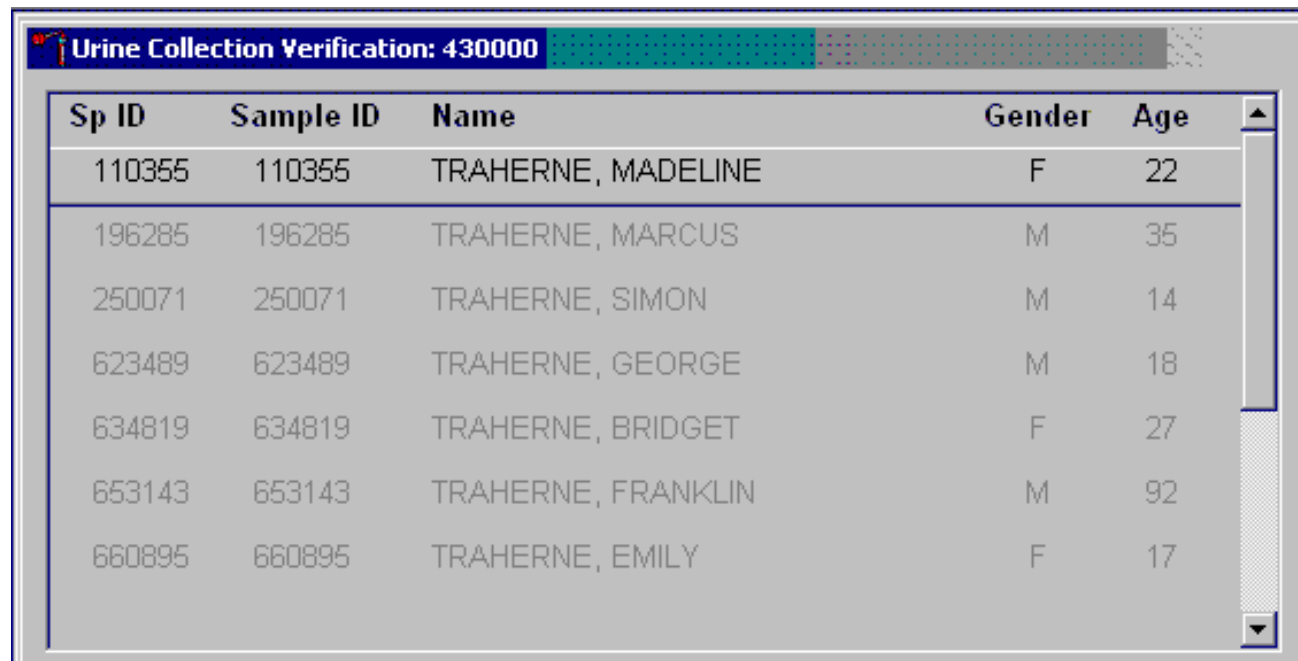

SP ID: 110355 NAME: TRAHERNE, MADELINE AGE: 22 Gender: F

\begin{tabular}{|c|c|c|c|c|c|c|}
\hline Collection & Volume $(\mathrm{mL})$ & Collection Date:Time & Statu: & & Comment Code & Verified \\
\hline 1 & 3 & $12 / 15 / 200809: 10 \mathrm{AM}$ & QNS & $\nabla$ & quantity not suffic - & $\nabla$ \\
\hline 2 & 25 & $12 / 15 / 200810: 30 \mathrm{AM}$ & Yes & $\nabla$ & (none) & Г \\
\hline
\end{tabular}

Total Collected: 28

Total Required: 4

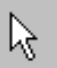

New Collection

Save

Cancel

If the quantity of urine collection does meet the requirement of the urine protocol, the application will automatically select a status of "Yes" and no additional collections will appear. 
Evaluate the quantity of urine received in the laboratory. Compare the number displayed in the Total Collected column and the actual volume of urine in the cup.

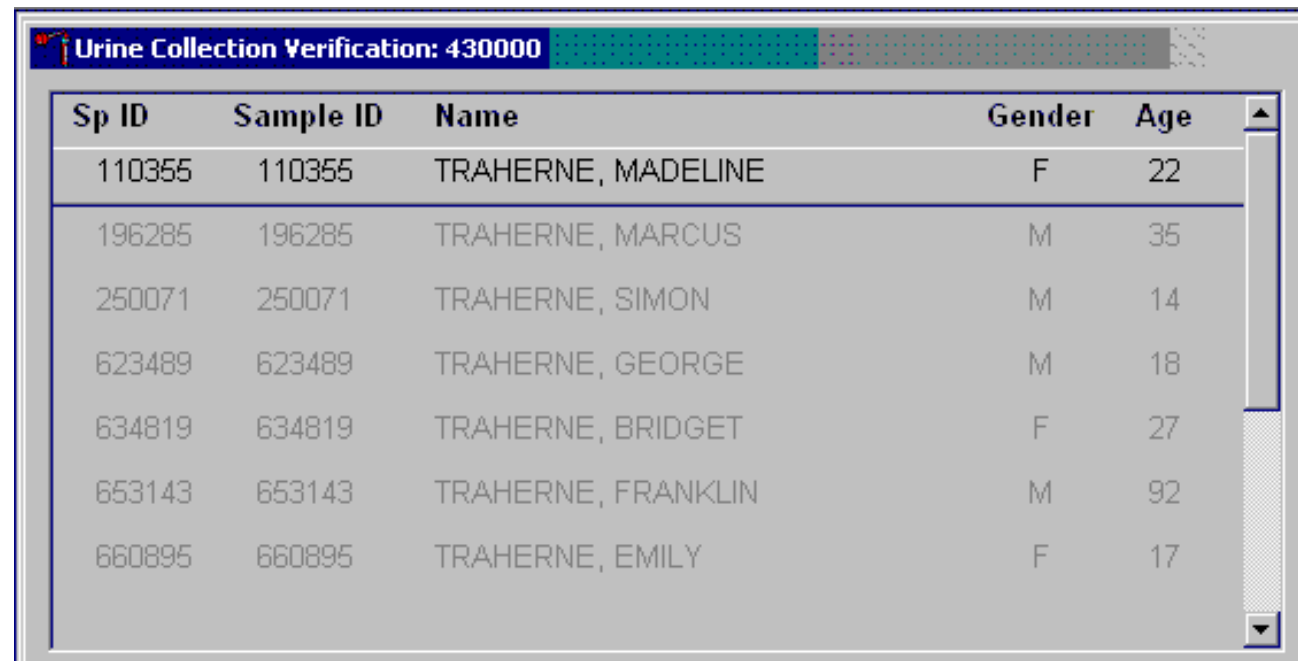

SP ID: 110355 NAME: TRAHERNE, MADELINE AGE: 22 Gender: F

\begin{tabular}{|c|c|c|c|c|c|c|}
\hline Collection & Volume $(\mathrm{mL})$ & Collection Date:Time & Statu: & & Comment Code & Verified \\
\hline 1 & 3 & $12 / 15 / 200809: 10 \mathrm{AM}$ & QNS & $\nabla$ & quantity not suffic - & $\nabla$ \\
\hline 2 & 25 & $12 / 15 / 200810: 30 \mathrm{AM}$ & Yes & $\nabla$ & (none) & Г \\
\hline
\end{tabular}

Total Collected: 28

Total Required: 4

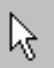

New Collection

Save

Cancel

Adjust the Volume (mL) if necessary by using the mouse to direct the mouse arrow to the text box under the Volume (mL) column. Right click on the text box to enable the cursor in the box. Make changes using the number pad on the keyboard. Once any necessary adjustments have been made, use the mouse to direct the mouse arrow to the box under the Collection Date/Time column or press the Tab key. 
A prompt will pop up asking if you would like to subtract the weight of the cup. If the volume of the sample was transmitted from the scale and you have made a manual adjustment to that reading, select "No."

Do not enter a result until urine arrives in the laboratory. At the end of each session, review the results for each dry run volunteer. Enter "No" in the result field for all volunteers who have not produced a urine sample. Record a result other than "(none)" for all records. Record a comment for all insufficient urine collections.

The laboratory application will automatically assign the comment code "quantity not sufficient” for all urine collections that are calculated as QNS by the scale application. The laboratory technician may also assign this comment code to all samples that are changed to QNS during the verification process. 


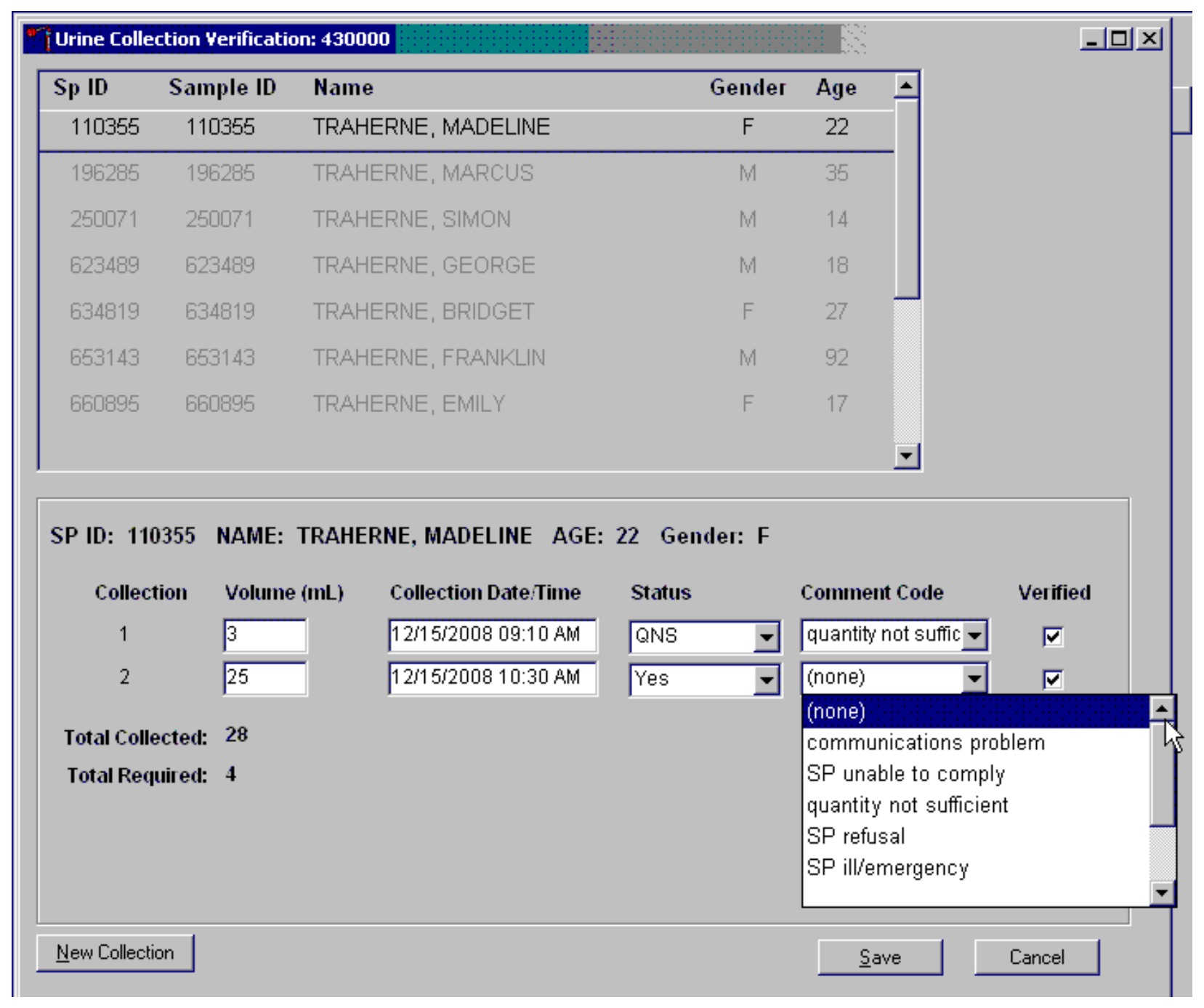

To record a comment, use the mouse to direct the mouse arrow to the drop-down list, click to display the comment codes, and select the most appropriate choice. Alternatively, use the up and down keyboard arrows to scroll through the choices or type the first letter of the desired comment code and when the correct choice is highlighted, left click. 
Verify the results of the urine collection.

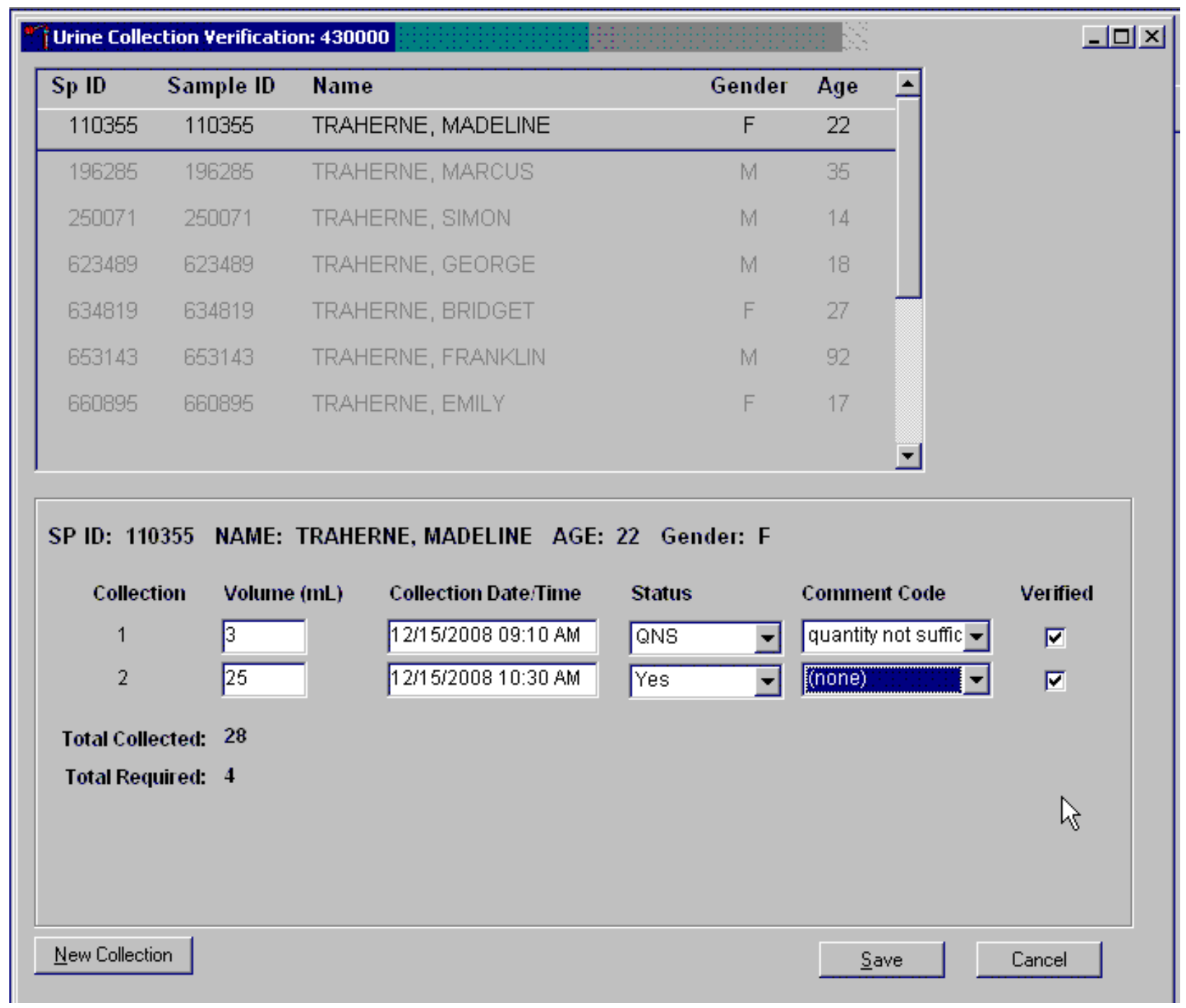

To verify, use the mouse to direct the mouse arrow to the "Verify" check box. Left click on the check box and a check mark will appear in the box. Verifying the results will save the results to the database.

To save a result to the database without exiting the module or removing the window, use the mouse to direct the mouse arrow to the "Verify" check box. Left click on the check box. Alternately, use the mouse to direct the arrow to the "Save" button and left click or press [Enter] when it is highlighted. To save a result to the database and exit the module and to remove the window, use the mouse to direct the mouse arrow to the "OK" button and left click. To exit the module and remove the window without 
saving any information to the database, use the mouse to direct the mouse arrow to the "Cancel" button and left click. To exit the module or close the window after saving results to the database and without recording any further actions, use the mouse to direct the mouse arrow to the $\mathrm{X}$ Close Window button and left click.

\subsubsection{Urine Processing}

Use the urine processing labels printed in advance to label the SP's urine vessels. The urine processing protocol is displayed in Exhibit 15-3. Dry run volunteers are eligible for at least one vessel 45. There are two sets of labels for each dry run volunteer. Label one set of vessels with the first set of labels (SP ID) and the second set of vessels with the second set of labels (Sample ID).

Exhibit 15-3. Dry run urine processing protocol

\begin{tabular}{|c|c|c|c|}
\hline ID & Assay & Sample size $\mathrm{mL}$ & Vessel \\
\hline 45 & Alb/Creat & 3 & 5-mL Cryovial \\
\hline
\end{tabular}

\section{Procedures for Dry Run Volunteers}

- Label one 5-mL cryovial for the first test - \#45. Label one tube with the volunteer's original SP ID. Label the other tube with the volunteer's Sample ID.

- Aliquot 3-mL urine into the vessels using a large plastic transfer pipette.

- Enter the results of urine processing for the original SP ID and again for the Sample ID. 
After filling the urine specimen vessels for each dry run volunteer, use the Urine Processing module to enter the urine processing results.

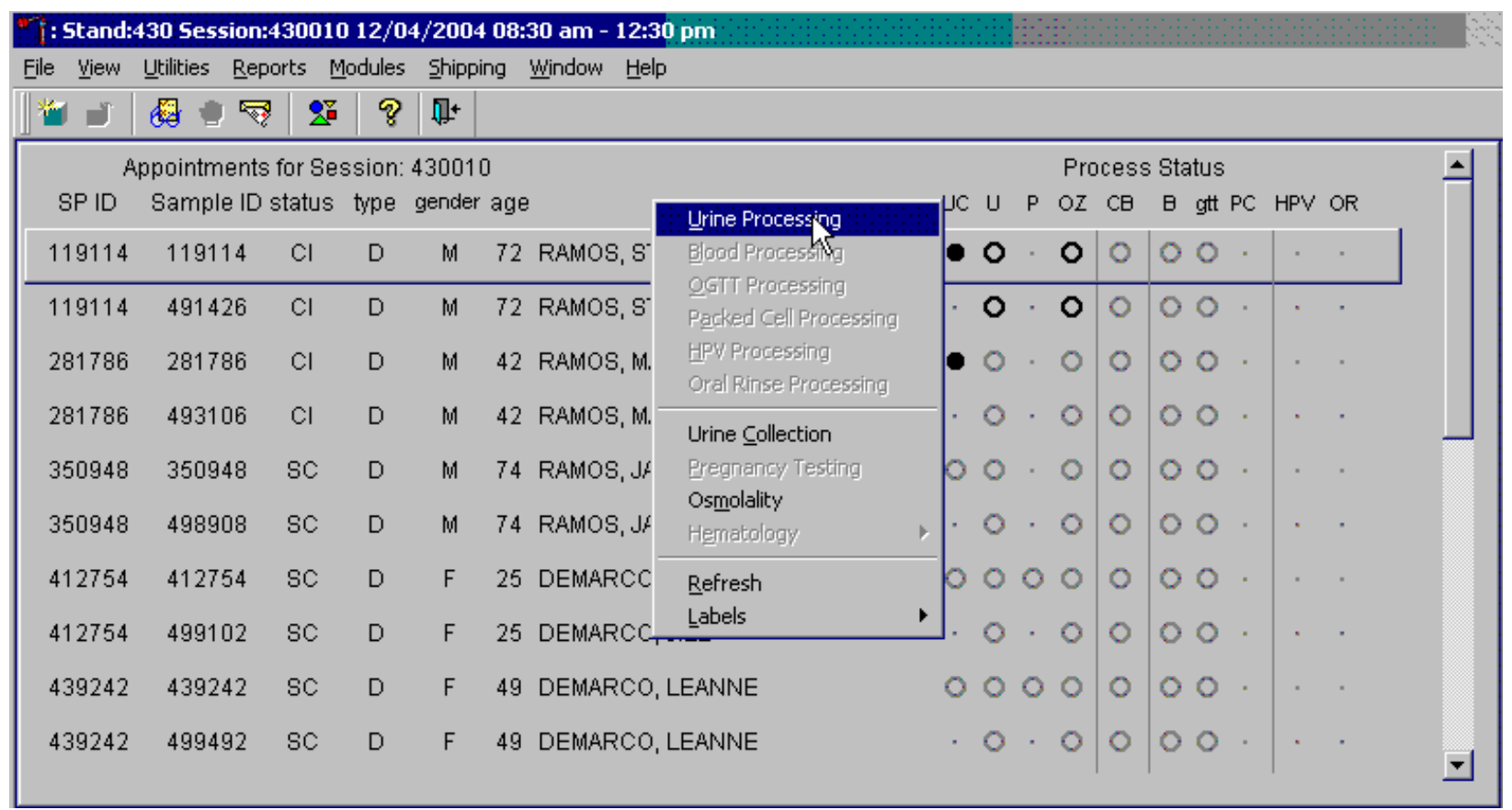

To access the Urine Processing module, use the mouse to direct the mouse arrow to the correct volunteer, right click, drag the mouse arrow to \{Urine Processing\}, and right click or right click and type [U/u]. Alternatively to access the Urine Processing module, use the up and down keys to move up and down the list until the correct volunteer is highlighted, right click, drag the mouse arrow to \{Urine Processing $\}$, and right click or right click and type [U/u]. 
The volunteer's urine processing screen displays.

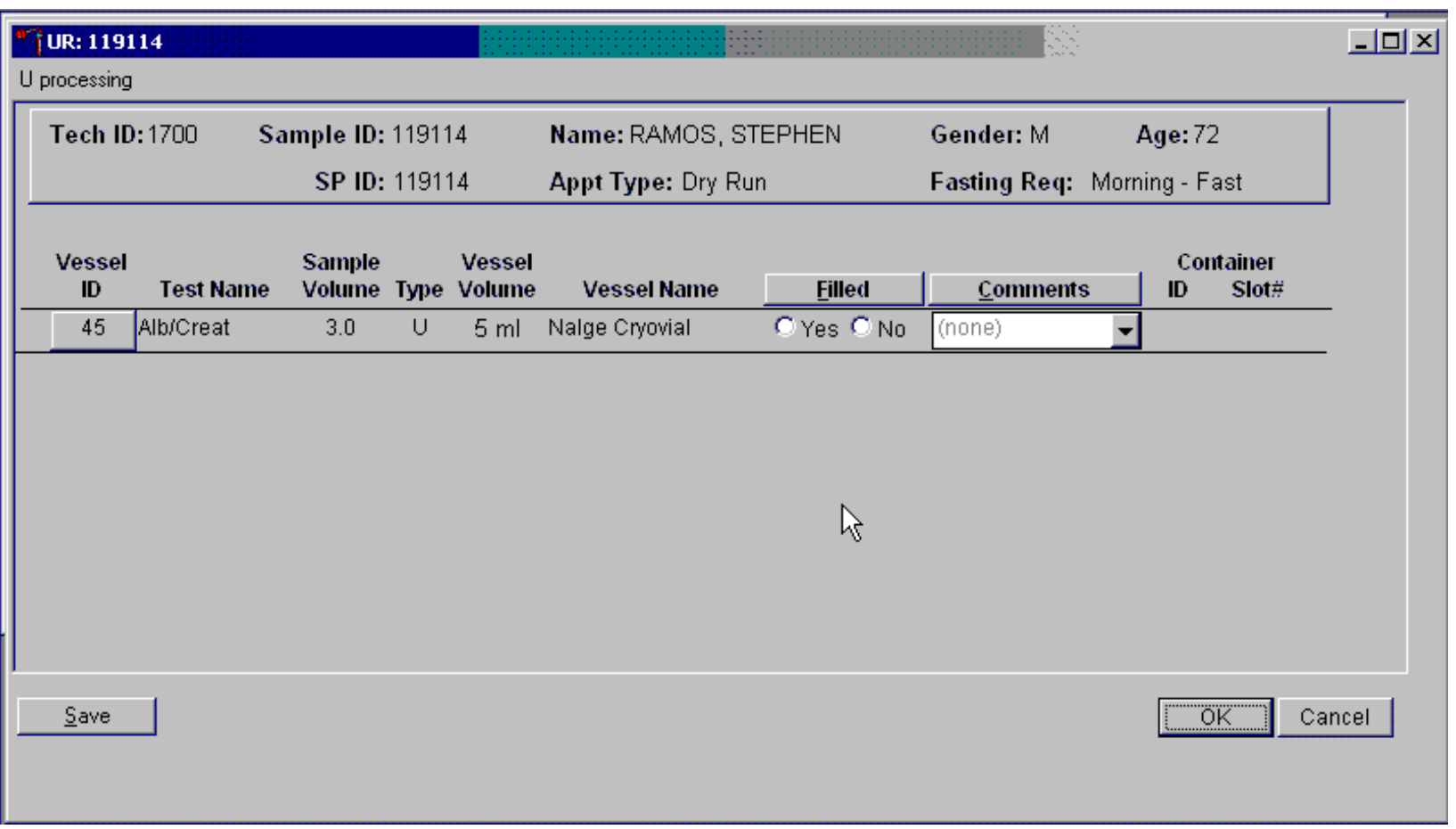

To mark an individual urine vessel as collected or Filled - "Yes," use the mouse to direct the mouse arrow to the "Yes" radio button and left click. As each vessel is marked as Filled - "Yes," it is automatically assigned to a slot in an existing (open) container. To mark an individual urine vessel as not collected or Filled - "No," use the mouse to direct the mouse arrow to the "No" radio button and left click. As each vessel is marked as Filled - "No," the comment, "quantity not sufficient” is automatically entered in the Comments column. To collectively mark all vessels as Filled - "Yes," use the mouse to direct the mouse arrow to the "Filled" button above the radio buttons, left click, and drag the arrow to \{Fill All\} and left click or type [Shift] [F/f]. To mark all vessels as Filled - "No," use the mouse to direct the mouse arrow to the "Filled" button above the radio buttons, left click, drag the mouse arrow to fNot Filled All\}, and left click or type [Shift] [F/f], [N/n]. Use the quality comment code to indicate if blood is present. Store each filled vessel in the assigned slot in the assigned container. To record this action or to save this data to the database, use the mouse to direct the mouse arrow to the "Save" button, and left click. To record this action or to save the data to the database and to exit the module, use the mouse to direct the mouse arrow to the "OK" button and left click. To close the window without saving any data in the database, use the mouse to direct the mouse arrow to the "Cancel” button, and left click. 
Access the Sample ID record on the heads-up display and record the processing results.

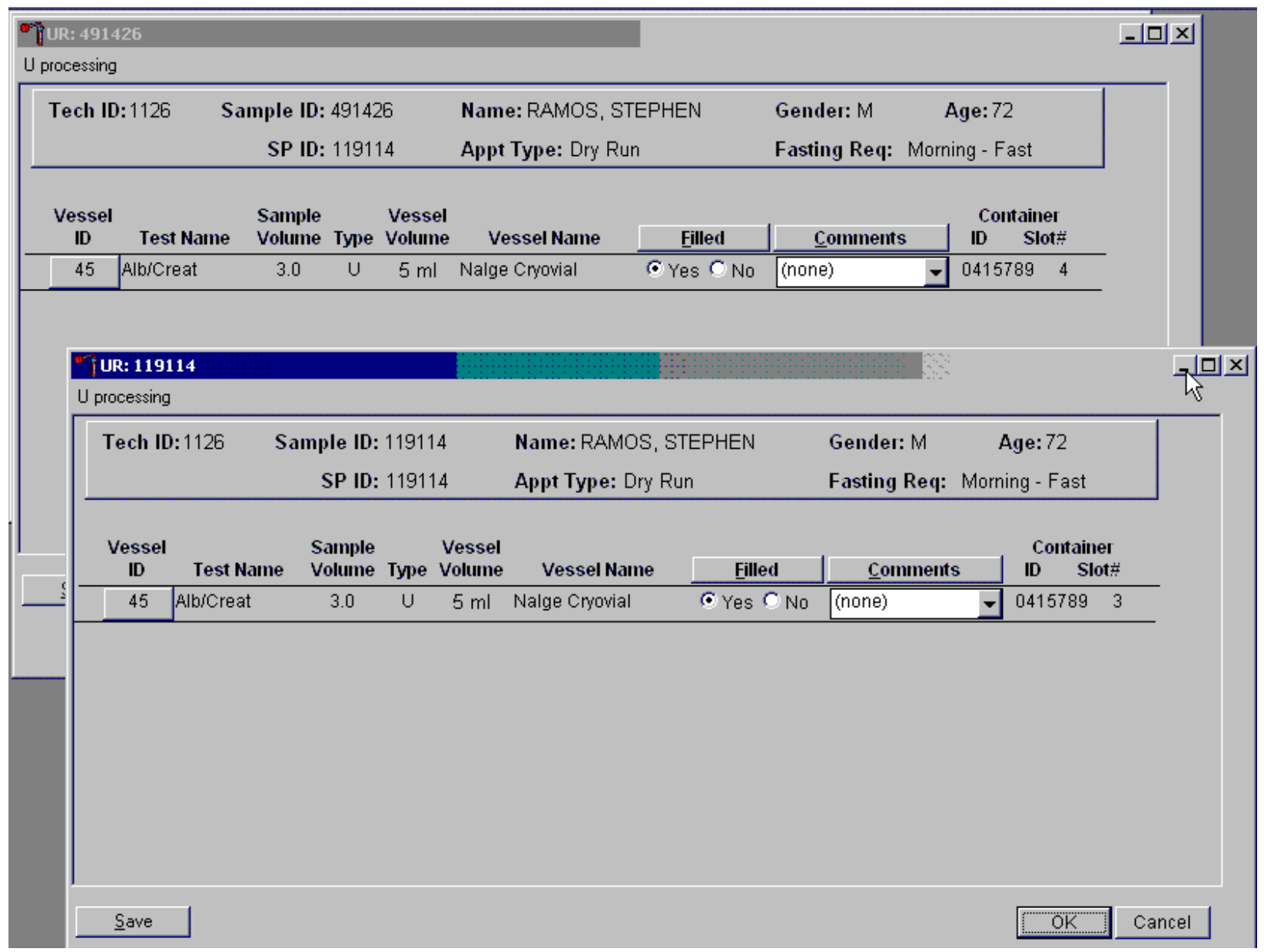

Remember to process vessels in pairs and, if the quantity of urine is insufficient to fill both vessels, do not record either vessel as filled.

Discard any remaining urine specimen at the end of each session. Pour urine down the drain in the laboratory sink. Flush the sink with water. Discard urine cup in the biohazard trash.

The urine-processing technologist performs the pregnancy test on female dry run volunteers aged 8-59 years and documents the results following the procedures in Chapter 6 . 


\subsubsection{Osmolality}

The urine processing technologist performs the osmolality test on dry run volunteers in duplicate-one test for each Sample ID_and documents the results following the procedures in Chapter 12. The osmolality test run on the sample where the SP ID and Sample ID are the same can be scanned in using the bar code initially labeled on the urine collection container. The duplicate osmolality test, run where the SP ID is not equal to the Sample ID, needs to have an additional label printed, placed on the container, and scanned.

Access the Dry Run Osmolality labels.

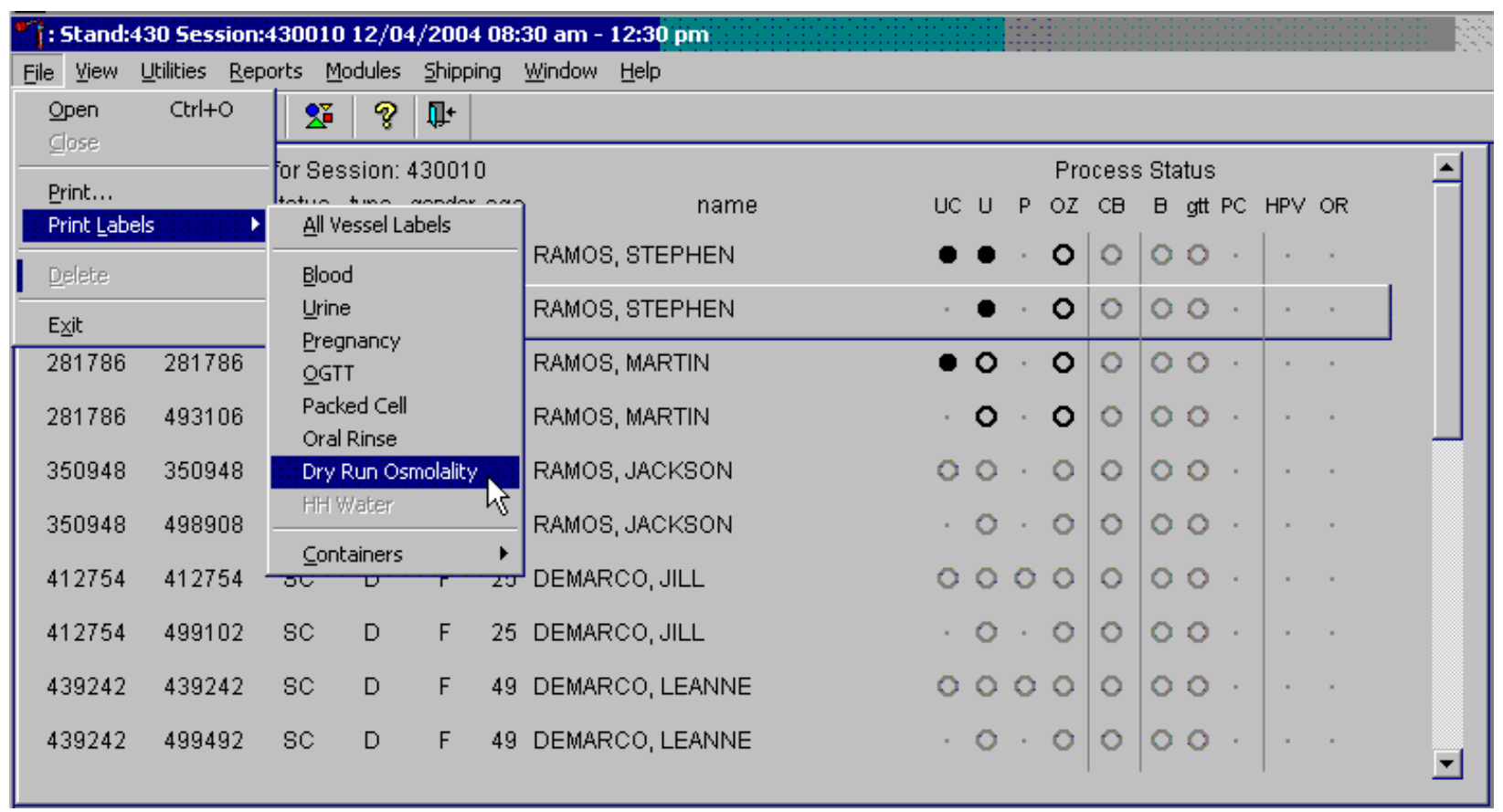

The labels print on the Dymo printer at workstation 2. Compare the printed label with the label on the urine cup. If the SP name and ID match, place the osmolality urine collection label on the lid of the urine collection cup. Scan this bar code in when running the duplicate osmolality test. When running the initial osmolality test, scan in the original urine collection label. 


\subsubsection{Hematology}

The technologist at workstation 1 runs two CBCs, in duplicate, for each dry run volunteer after the blood processing technologist processes whole blood from the 3-mL EDTA tubes according to the whole blood processing protocol (see Exhibit 15-4). Run the first CBC in duplicate on the first 3-mL EDTA tube with the original SP ID on the tube. Evaluate results, and save the data using procedures described in Chapter 7.

Run the second CBC in duplicate on the second 3-mL EDTA tube using the Sample ID. Evaluate results, and save the data using procedures described in Chapter 7.

\subsubsection{Blood Processing and Storage}

Print blood-processing labels using procedures described in Chapter 9. There are two sets of labels for each dry run volunteer; one set of labels contains the SP ID and the second set of labels contains the Sample ID. Prepare two blood processing racks (one with the SP ID labels and one with the Sample ID labels) using the blood-processing template for ages $12+$.

Exhibit 15-4. Dry run processing protocol - whole blood

\begin{tabular}{|l|l|l|c|l|l|c|}
\hline ID & \multicolumn{1}{|c|}{ Test name } & Groups & $\begin{array}{c}\text { Sample } \\
(\mathrm{mL})\end{array}$ & Sample type & Collection type & Vessel type \\
\hline 1 & Lead/Cad/HG & Both & 0.4 & Whole Blood & $\begin{array}{l}\text { 3 or 4-mL } \\
\text { EDTA }\end{array}$ & 2-mL \\
\hline 3 & RBC Folate & Even & 0.1 & Whole Blood & 3-mL EDTA & 2-mL \\
\hline 106 & RBC Fol1 & Odd & 0.1 & Whole Blood & 4-mL EDTA & 2-mL \\
\hline 4 & Glycohem & Odd & 0.4 & Whole Blood & 4-mL EDTA & 2-mL \\
\hline 5 & Mercury & Even & 0.5 & Whole Blood & 3-mL EDTA & 2-mL \\
\hline 125 & HLA-B27 & Even & 1.0 & Whole Blood & 4-mL EDTA & 2-mL \\
\hline
\end{tabular}


Treat each set of venipuncture tubes and prelabeled blood processing racks as two independent and separate dry run volunteers. Process the complete first set of venipuncture tubes and blood processing vessels on the dry run volunteer using the original SP ID, then process the second set of venipuncture tubes and blood processing vessels using the Sample ID.

For volunteers whose SP ID and Sample ID ends in an odd digit, process one of the 4-mL EDTA tubes for Lead/Cad/HG, RBC Fol1, Glycohem, and HLA-B27. For volunteers whose SP ID and Sample ID ends in an even digit, process one of the 3-mL EDTA tubes for Lead/Cad/HG, RBC Folate, and Mercury. Use processing procedures described in Chapter 8. Match the EDTA tube with the SP ID to the corresponding blood processing rack. Match the second EDTA tube with the Sample ID to the corresponding blood processing rack.

When the SP ID ends in an even digit, process the plasma in each of the other two 3-mL EDTA tubes for PUFA/TFA as described in Chapter 8. When the SP ID ends in an even or an odd digit, process each of the 2-mL gray top tubes for Glucose using the same procedures described in Chapter 8. When the SP ID ends in an even or an odd digit, process each of the 2-mL gray top tubes for OGTT using the same procedures described in the Oral Glucose Tolerance test in Chapter 13. 
Process pooled serum for the dry run volunteers using the Dry run blood processing protocol - serum (Exhibit 15-5).

Exhibit 15-5. Dry run blood processing protocol - serum

\begin{tabular}{|l|l|l|l|l|l|l|}
\hline \multicolumn{1}{|c|}{ ID } & Test name & \multicolumn{1}{|c|}{ Groups } & $\begin{array}{c}\text { Sample } \\
(\mathrm{mL})\end{array}$ & $\begin{array}{c}\text { Sample } \\
\text { type }\end{array}$ & \multicolumn{1}{|c|}{ Collection type } & \multicolumn{1}{|c|}{ Vessel type } \\
\hline 81 & Vit B6/Fol & Odd & 0.5 & Serum & 15-mL Red & 2-mL \\
\hline 70 & Vitamin D & Odd & 0.7 & 0.3 & Serum & 15-mL Red \\
\hline 13 & CRP & Odd & 0.3 & Serum & 15-mL Red & 2-mL \\
\hline 14 & Hep profile & Even & 1.5 & Serum & 15-mL Red & 2-mL \\
\hline 122 & HAV & Even & 1.5 & 0.3 & Serum & 15-mL Red \\
\hline 99 & Fer/TfR & Odd & 0.5 & Serum & 15-mL Red & 2-mL \\
\hline 17 & Cotinine & Even & 1.8 & Serum & 15-mL Red & 2-mL \\
\hline 21 & Lipids & Both & 1.5 & Serum & 15-mL Red & 2-mL \\
\hline 18 & Biochem & Both & 0.8 & Serum & 15-mL Red & 2-mL \\
\hline 23 & Insulin & Odd & 1.0 & Serum & 15-mL Red & 2-mL \\
\hline 75 & PSA & Even & 1.0 & Serum & 15-mL Red & 2-mL \\
\hline 118 & Thyroid & Both & 1.0 & 0.3 & Serum & 15-mL Red \\
\hline 127 & IgA-TTG/EMA & Both & 0.5 & 0.3 & Serum & 15-mL Red \\
\hline 16 & MMRV & Both & 1.5 & 0.3 & Serum & 15-mL Red \\
\hline 110 & Folate1 & Odd & 0.7 & Serum & 15-mL Red & 2-mL \\
\hline
\end{tabular}

- Fill each vessel as described in the blood processing protocol.

- Process the complete first set of venipuncture tubes and blood processing vessels on the dry run volunteer using the original SP ID. Continue to use the labeled vessels in the prepared rack.

- Process the second set of venipuncture tubes and blood processing vessels using the Sample ID. Continue to use the labeled vessels in the prepared rack.

- Use the same techniques for spinning and separating the blood as described in Chapter 8.

- Check the vessel labels before filling the vessel making sure that each vessel has the correct SP ID or Sample ID. 
Match the two processing racks to each other. Record the blood processing results.

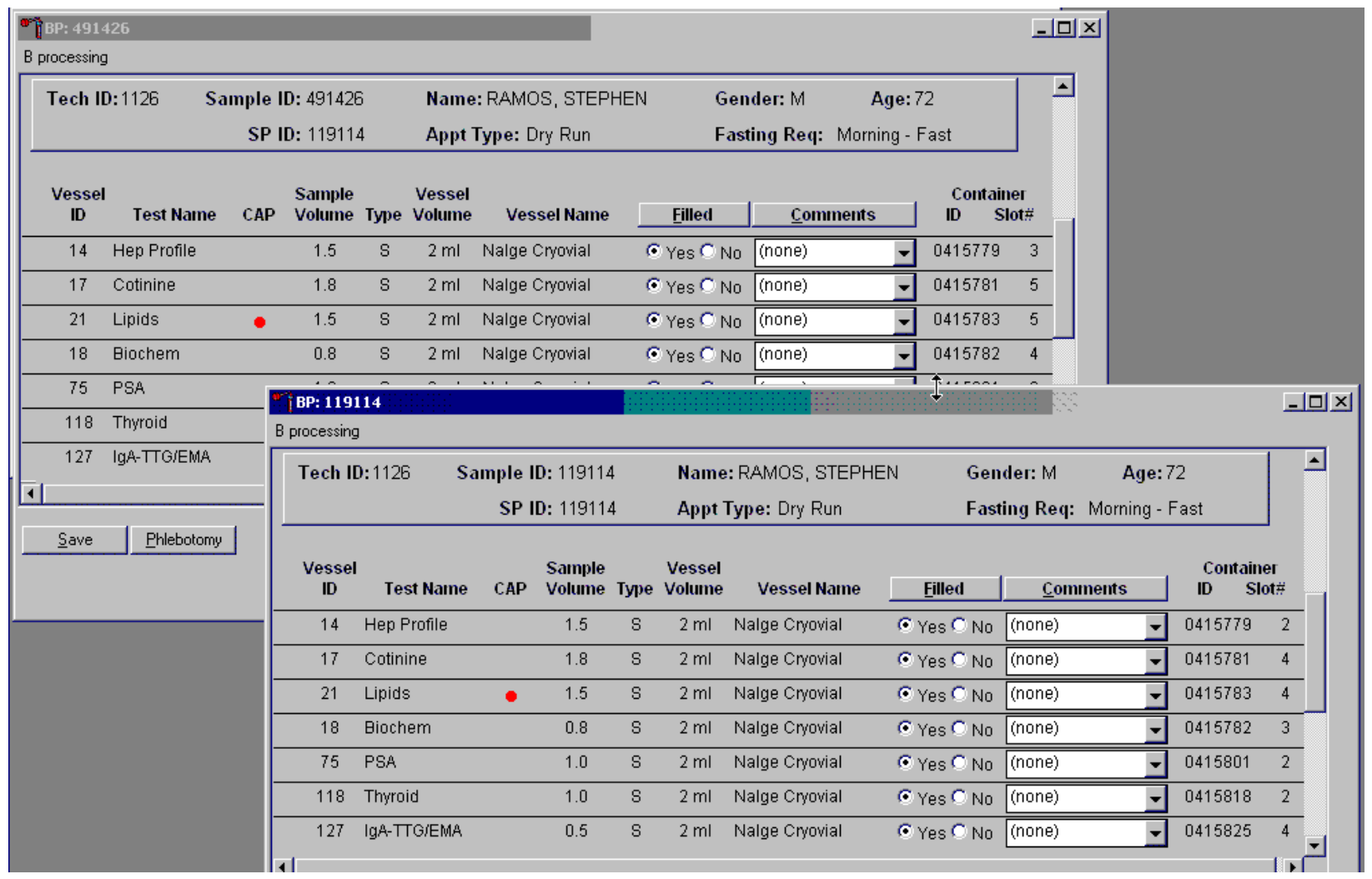

Enter results for those vessels where both vessels are filled for the whole blood, plasma, and serum protocol. Enter the results of blood processing using the correct Sample ID and SP ID numbers and procedures described in Chapter 8, Section 8.8. Store the blood vessels in a manner identical to blood vessels processed during a regular session.

\subsubsection{Shipping Dry Run Specimens}

Ship the split sample blood and urine vessels with samples from the regular sessions following procedures described in Chapter 9. 


\section{5}

\section{Centrifuges - Equipment Maintenance}

Technologists are responsible for inspecting the centrifuges at the beginning of each stand, and inspecting and cleaning the centrifuges at the end of each stand and whenever necessary. Clean the interior of the chamber with a mild soap, as time permits. Rinse thoroughly and dry completely.

1. Inspect the rotor yolk and buckets.

- Inspect for rough spots or pitting, white powder deposits or heavy discoloration. If any of these signs are evident, do not use the rotor. Use the second centrifuge and notify the NCHS engineer. Check the condition of the Oring in the tie-down nut; replace it if it is worn or damaged.

2. Clean the rotor.

- $\quad$ Use mild detergent, diluted 10 to 1 with water and a soft brush to clean the rotor yolk and tie-down nut. Thoroughly rinse the cleaned rotor and components with water and air-dry upside down. When the rotor is dry, lubricate the bucket sockets and pins by saturating a lintless tissue with TriFlow oil and wipe it on each bucket socket and pin. Also, put a drop of TriFlow oil between the pin and rotor yolk. Before reinstalling the rotor, lightly lubricate the drive hole of the rotor with Anti-Seize to prevent the rotor from sticking, as follows:

1. Apply the lubricant onto a swab.

2. Draw the coated swab through a paper towel to remove excess lubricant.

3 Lightly coat the inside of the drive hole with the lubricant remaining on the swab.

3. Clean the buckets.

- $\quad$ Remove the O-ring.

- $\quad$ Clean the bucket and O-ring with a mild detergent diluted 10 to 1 with water, then rinse thoroughly, and dry completely.

- $\quad$ Coat the O-ring with a light film of silicone vacuum grease. Place the O-ring on the ledge inside the bucket.

- $\quad$ Once a week and after every cleaning, lubricate contact areas between the buckets and the pins on the rotor. Saturate a lintless tissue with Tri-Flow oil and wipe it on each bucket socket and pin. Also, put a drop of Tri-Flow oil between the pin and rotor yolk. 
4. Inspect and clean Aerosolve canisters.

- $\quad$ Inspect the O-ring for nicks, abrasions, or other damage. Coat the O-ring with a light film of silicone vacuum grease before installing. Place the O-ring in the groove.

- $\quad$ Inspect the canister and lid threads and bottom of canister for heavy crazing or cracks.

- $\quad$ Inspect the tube racks for heavy scratches or cracks and inspect the rubber cushions for damage or for embedded glass from broken tubes.

- $\quad$ Clean the canisters, lids, and racks with a mild detergent, diluted 10 to 1 with water, and a soft brush. Thoroughly rinse the cleaned components with water and air-dry upside down. Coat the O-ring with a light film of silicone vacuum grease before reinstalling it into the groove.

The NCHS biomedical engineer certifies both centrifuges annually. The NCHS engineer determines the rpm of the instruments using a strobe light or a tachometer. The engineer records multiple readings for each centrifuge. The Beckman GS-6R centrifuge should exceed 3,750 rpm to ensure adequate centrifugal force development. The engineer checks each centrifuge timer against a stopwatch and records the values in the Laboratory QC module - NCHS QC.

Document beginning and end of stand cleaning procedures in the Laboratory QC module.

\subsection{Refrigerators and Freezers - Equipment Maintenance}

The freezers require proper ventilation in order to operate efficiently, and the air around the freezers must be free to circulate. Vents in the counters allow the heat from the freezers to rise and dissipate. Keep the vents open and do not obstruct the airflow. Do not store anything between the freezers or between the counter supports and the freezer. Do not store anything on the top grill.

Quality control procedures for the refrigerators and freezers include cleaning them at the beginning and end of the stand and whenever necessary. Continuously monitor the temperatures of refrigerators and freezers on a daily basis and record the results in the Laboratory QC module. Temperatures are automatically captured, graphed, and available in real time. 
The TempGuard system is a multiple device monitor and alarm system that is equipped with an integrated autodialer and data logging capabilities. The TempGuard system automatically monitors the interior temperature of the refrigerators, freezers, and room temperature.

The system can monitor up to 8 temperature sensors and each sensor must have a probe submerged into a bottle of resin. The configuration in the MEC, is as follows:

\begin{tabular}{|c|c|}
\hline TempGuard Port & Refrigerator/Freezer Name \\
\hline 1 & Refrigerator \# 1 \\
\hline 2 & Refrigerator \# 2 \\
\hline 3 & Freezer \# 3 \\
\hline 4 & Freezer \# 4 \\
\hline 5 & Freezer \# 5 \\
\hline 6 & Freezer \# 6 \\
\hline 7 & Room Temp \\
\hline
\end{tabular}

The TempGuard system has been programmed with the following: upper and lower limits for all units including the room temperature and a 60-second delay for the allowable time the freezer door can be left open before the system will alarm. The system has the capability to send out calls and emails to alert staff to temperature issues.

\section{Checking TempGuard Sensors}

All temperature sensors connected will have their temperature readings displayed next to the sensor number on the display

\section{Disabling Alarm}

1. Push the black button on the left side of the TempGuard to see any alarm conditions.

2. Hold the button for 5 seconds to confirm the alarm condition.

3. Alarm Conditions must be confirmed individually. 
4. The alarm relay will de-energize, and the TempGuard will stop making callouts for the current alarm condition.

\section{Start of Stand Set-Up (performed by the FES)}

Programming the Autodialer Functions (need to update MEC Manager's phone \# at each stand)

1. Enter the "Full Access" PIN (Enter 0000).

2. From the Program Menu, Select 1 to set telephone numbers.

3. $\quad$ You will hear "Select contact."

4. Select $\mathbf{1}$ for the first contact number (FES phone \#).

- $\quad$ Enter your phone number, using this format " $\mathbf{1}+$ area code $+\mathbf{7}$ digit \#”

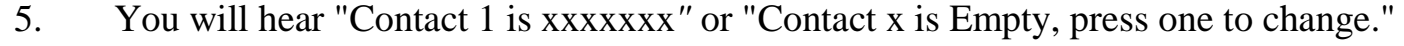

6. Press 1 to make a change or enter a telephone number.

7. You will hear "Enter number then press pound."

8. Enter the number, followed by the \# key.

- $\quad$ Entering the \# key will erase the currently programmed contact telephone number.

9. You will hear the telephone number you just entered.

10. You will be prompted to select another contact to program.

11. Select $\mathbf{2}$ for the second contact number (MEC Manager’s \#).

- $\quad$ For Dan enter “1-XXX-XXX-XXXX”

- $\quad$ For Lenea enter “1-XXX-XXX-XXXX”

12. Press 0 to return to the Program Menu.

The acceptable temperature range for refrigerators used for storage of CLIA-regulated specimens and reagents is $2-8^{\circ} \mathrm{C}$ and is checked and recorded daily. 
Freezer temperatures are monitored daily by visual inspection of either a thermometer or graphs and recorded. All freezers are monitored automatically by built-in visual systems (with thermographic or digital reading), which is determined by the probe in the antifreeze solution. The range of allowable temperature for the -30 Revco model is -26 to $-34^{\circ} \mathrm{C}$. If the range is exceeded, the technologist contacts the MEC manager.

\subsection{Use of the Class II Type A Biological Safety Cabinet}

The Labgard Model NU-425 Class II, Type A2, Laminar Flow Biological Safety Cabinet (LFBSC), with proper technique, provides an effective partial containment system for safe manipulation of moderate- and high-risk microorganisms. The vertical laminar airflow barrier prevents contaminated air from entering or escaping from the open-front work chamber, protecting the cabinet contents and the technologist. A high-efficiency filter system decontaminates both the incoming air and the exhaust.

\section{Installation, Certification, and Maintenance}

The NCHS biomedical engineer arranges for a certified engineer to perform the required annual certification that includes replacement of High Efficiency Particulate Air (HEPA) filters as necessary.

The basic concept of the LFBSC is to take air through a blower system, pressurize a plenum, and force air through a filter. Built-in supply and exhaust blowers come with separate HEPA filters. The recirculating airflow pattern directs a portion of the HEPA filtered air straight down over the central work area driving the airborne contaminants into the surrounding grills, allowing a pollution-free environment for particular specifications. If a filter is defective, the filtration system no longer filters and becomes a vacuum cleaner, taking in gross contamination and exploding it through the defective filter onto and around the critical work area. The filter of the LFBSC is a permanent part of the disposable plenum. A magnehelic gauge is calibrated in "inches of water gauge” pressure (w.g.). The gauge indicates when the supply HEPA filter is clogged. As the filter loads with particulate matter, the amount of pressure registered on the gauge will increase. The initial pressure reading is approximately 0.4 w.g. \pm 0.05 w.g. depending on the altitude from sea level. 
Clean the LFBSC at the beginning and end of each stand. Lift the front glass and wash both the interior and exterior glass. Remove all contents from the interior of the cabinet. Remove and wash the front grill. Lift the cover to the table and wash the base underneath. Put table cover and grill back into cabinet. Lock front glass into place. Document these activities in the Laboratory QC module. Take and record the pressure reading in the Laboratory QC module at the beginning of stand and at the start of each session.

Document all unscheduled maintenance for the LFBSC in the automated Unusual Field Occurrence utility. 


\section{Operating the NU-425, Laminar Flow Biological Safety Cabinet ${ }^{*}$}

\section{Operator Controls \& Indicators}

The following is a description of the controls and indicators found on both the front panel (see Drawing BCD-05322) and cabinet.

\subsubsection{Circuit Breaker-Blower (Top of Control Center)}

The motor/blower is protected with a circuit breaker. The circuit breaker in conjunction with the motor's thermal protector is designed to open under locked rotor or half-wave power conditions. Should the circuit breaker open (pop-out button will appear) merely depress to reset. If the circuit breaker continually opens, a failure has occurred in the motor or solid-state speed controller. Consult a qualified repair technician or NuAire, Inc. for replacement.

\subsubsection{Circuit Breaker-Outlets (Top of Control Center)}

The duplex outlet located in the sidewall of the work area is protected with a $3 \mathrm{amp}$ circuit breaker. The circuit breaker may trip at 110 percent of load rating but will trip at 145 percent of load rating in less than 2 seconds. Should the circuit breaker open, (pop-out button will appear), unplug the appliance plugged into the outlet and merely depress the pop-out button to reset.

\subsubsection{Fluorescent/U.V. Light Switch}

This switch provides on/off control for the fluorescent light and/or the ultraviolet (U.V.) light if present (optional). With the U.V. light option, the switch provides for on-center off-on operation so that both the fluorescent light and the U.V. light cannot be energized at the same time. The UV light is also interlocked, so it will only operate if the sliding window is closed. Proper care should be exercised when the U.V. light is on.

* Adapted from Operation and Maintenance Manual by NuAire, Inc., 2005. 


\subsubsection{Outlet Switch}

This switch provides on/off control for the 115 VAC power available in the outlet(s) within the cabinet workzone.

\subsubsection{Blower Switch}

The blower switch applies power to the internal motor/blower when in the ON position. The blower switch also has a second set of poles, which are available for use as a contact closure for an exhaust system. In addition, power can be applied to the accessory outlet located on the upper left corner of the cabinet, behind the front window assembly.

The purpose of the outlet is to provide "switched" power for the following (see also 8.1.11):

- Exhaust transition with integral blower

- To power a low power appliance, such as the low airflow alarm.

The rating of the outlet is 115 VAC at 2 amp maximum.

\subsubsection{Indicator Light}

An indicator light is located above the Blower on/off switch and indicates when power is applied to the blower.

\subsubsection{Audible Alarm Enable}

As the sliding window is raised above its normal working height, a micro switch located in the glide channel activates both an audible and visual alarm. This switch disables the audible alarm but will not disable the red indicator visual alarm. 


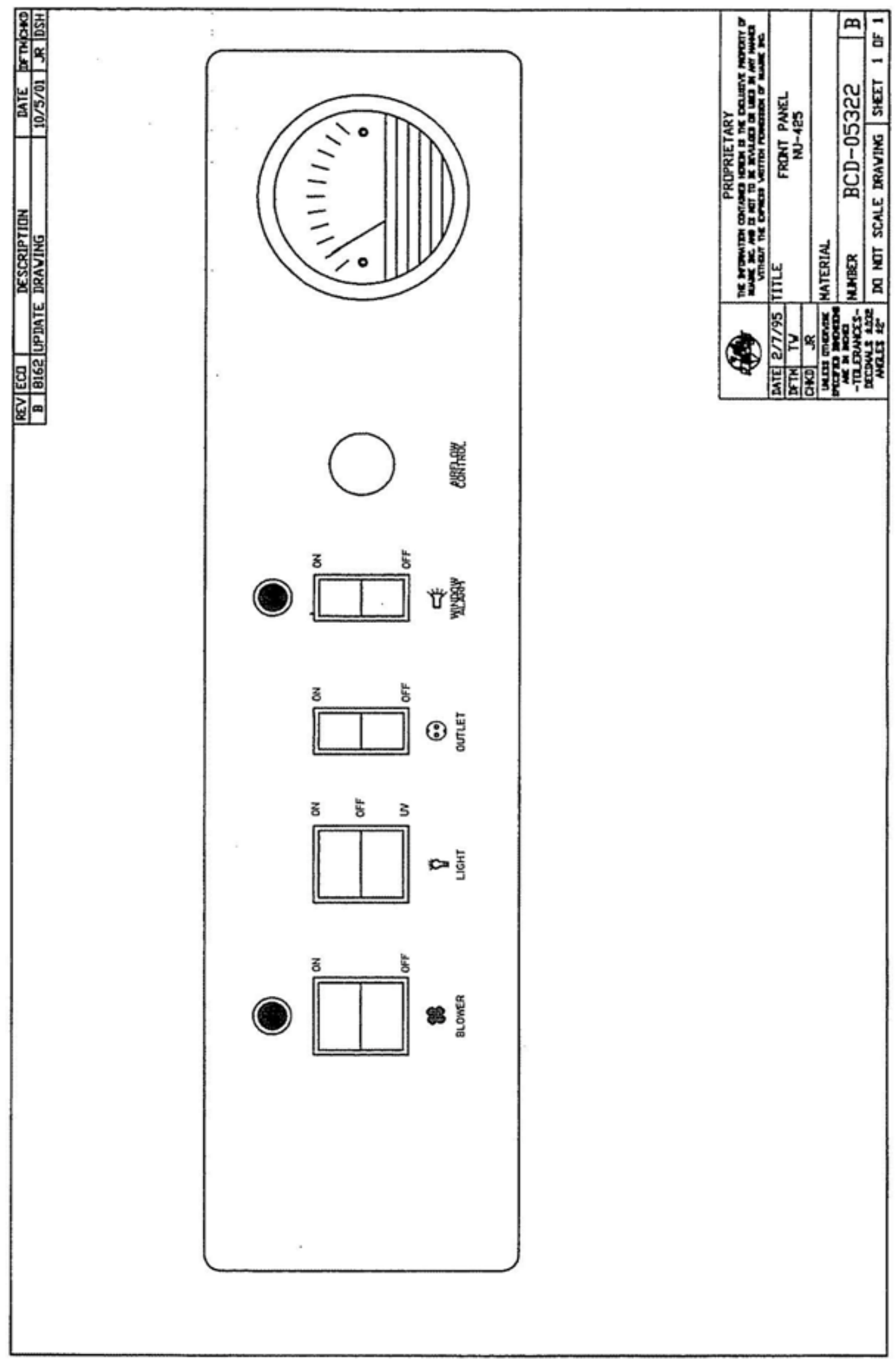




\subsubsection{Airflow Control}

The operating airflows within the cabinet (i.e. 70 LFPM (.35 m/s) downflow and 105 LFPM $(.53 \mathrm{~m} / \mathrm{s})$ air inflow barrier) are controlled by a potentiometer and an exhaust damper. The potentiometer controls the operating voltage applied to the motor/blower. The potentiometer is adjustable over 270 degrees with a slotted screwdriver, which varies the applied voltage from 70 to 115 VAC. THIS ADJUSTMENT SHOULD ONLY BE MADE BY A QUALIFIED TECHNICIAN EMPLOYING THE PROPER INSTRUMENTS IN ORDER TO INSURE AIRFLOWS PER NSF STD. NO. 49.

\subsubsection{Minihelic Gauge}

The unit is equipped with a minihelic gauge. The minihelic gauge displays the static pressure within the pressure plenum supplying the downflow and exhaust filters. The gauge is calibrated in "inches of water gauge" pressure. As the HEPA filters load with particulate matter, the amount of static pressure will increase, giving an indication of the "health" of the cabinet. The initial pressure reading will be approximately 0.5 ” w.g. + 0.1” w.g. depending on altitude from sea level. At each 0.1” w.g. increment increase, the cabinet airflow should be checked by a qualified technician, unless certified on a yearly (or sooner) basis.

\subsubsection{Sliding Window Operation}

The cabinet has a full counter balanced and removable sliding tempered glass window with two operational features. As the window is raised above its specified operating height, an audible alarm alerts the operator of possible compromised personnel protection. At the same time, an audible and visual alarm is activated. The audible alarm may be switched off while the visual alarm remains until lowered to the safe operating position. When the window is lowered below 2 inches $(50 \mathrm{~mm})$, the motor/blower automatically shuts down, to prevent stress on the motor/blower in the form of overheating. The maximum window height is $191 / 2$ inches (495mm). 


\subsubsection{Convenience/Accessory Outlet}

A convenience/accessory outlet is located in the upper left hand corner in back of the window assembly. The outlet is normally wired "hot" through the motor/blower circuit breaker. If an accessory is purchased, the outlet is defined as an accessory outlet supplying 115 VAC at 2.0 Amp maximum, and is wired to the blower switch (see electrical schematic).

\section{$1.2 \quad$ Operating Guidelines}

The intent herein is to present general operational guidelines that will aid in the use of the Laminar Flow Biological Safety Cabinet (LFBSC) to control airborne contaminants of low to moderate risk as stated in Technical Report No. FPS 56500000001 prepared by Dow Chemical U.S.A. for the National Cancer Institute, May 1, 1972.

Procedure protocols defined in terms of the barrier or control concepts unique to LFBSC must be developed in order to obtain a maximum potential for safety and protection. The pre-planning necessary to develop these protocols is based on several fundamental considerations, each of which will contribute to optimum benefits from the equipment:

- Know your "safe working area"

- Minimize disruption of "air curtain”

- Minimize room activity

- Utilize unidirectional airflow

- Employ aseptic techniques

\subsubsection{Know Your "Safe Working Area”}

The LFBSC safe working area is basically the worktray or depressed area. All work should be performed on or above the worktray. The area on or above the front grill is a non-safe working area. 


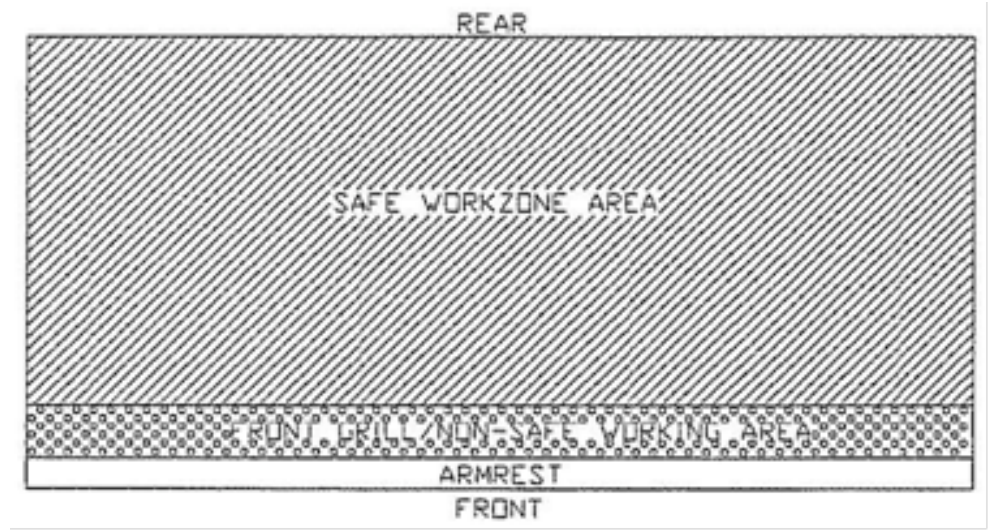

\subsubsection{Minimize Penetration of “Air Curtain”}

The minimum number of items necessary should be placed into the cabinet to prevent overloading, but the work should also be planned to minimize the number of times an operator's hands and arms must enter and leave the air curtain at the open face. The ideal situation is to have everything needed for the complete procedure placed in the hood before starting, so that nothing need pass in or out through the air barrier at the face until the procedure is completed. This is especially important in working with moderate risk agents.

Unnecessary raising of the hands inside the cabinet above the level of the work opening should be avoided. This presents an inclined plane from hands to elbows along which the downflow of air may run to, and possibly out, the open face.

NOTE: When working with agents of lower risk, it is not as important for all materials to be placed in the cabinet before starting, or for the procedure to be completely finished before materials are removed. Also, the time period for a unit may be continued over a more extended period during which entries and withdrawals from the cabinet may be made.

\subsubsection{Minimize Room Activity}

Activity in the room itself should be held to a minimum. Unnecessary activity may create disruptive air currents as well as interfere with the work of the operator. A person walking past the front 
of a cabinet can cause draft velocities up to $175 \mathrm{fpm}(.89 \mathrm{~m} / \mathrm{s})$, which are sufficient to disrupt the air balance of the laminar flow unit.

\subsubsection{Utilize Unidirectional Air Flow}

The operator must keep two important facts in mind: (I) The air, as supplied to the work area through filters from the top, is contaminant free and (2) Airborne contamination generated in the work area is controlled by the unidirectional flow of parallel air streams in a top-to-bottom direction.

A solid object placed in a laminar air stream will disrupt the parallel flow and consequently, the capability of controlling lateral movement of airborne particulates. A cone of turbulence extends below the object and laminarity of the air stream is not regained until a point is reached downstream, approximately equal to three to six times the diameter of the object. Within the parameters of this cone, particles may be carried laterally by multidirectional eddy currents.

Transfer of viable materials and manipulations which may generate aerosols should not be performed above sterile or uninoculated materials. Items should be localized on the work surface in “clean” and “dirty” groups.

\subsubsection{Employ Aseptic Technique}

The operator must not assume an attitude of "let the cabinet do it" when performing procedures within a LFBSC. Properly balanced and properly used cabinets will do an excellent job of controlling airborne contamination and containing viable agents, but the cabinet will not eliminate contact transmission of contamination. Normal laboratory contamination control procedures and basic aseptic techniques are necessary to obtain maximum benefit from the cabinet. For example, open bottle, tube or flask mounts should be kept as parallel as possible to the downflow to minimize capture of chance particulates. This precaution is merely an extension of good aseptic technique as practiced on open bench tops. The good laboratory practices designed to minimize creation and/or release of aerosols to the environment should not be discontinued. 
Items of equipment in direct contact with the etiologic agent must remain in the cabinet until enclosed or until surface-decontaminated. Trays of discard pipettes must be covered before removal from the cabinet (aluminum foil may substitute for fabricated covers).

If an accident occurs which spills or splatters suspensions of etiologic agent around the work area, all surfaces and items in the cabinet must be surface-decontaminated before being removed.

\section{$1.3 \quad$ Operating Sequence}

\subsubsection{Start Up}

Turn on cabinet blower and lights, check air intake and exhaust portals of the cabinet to make sure they are unobstructed.

Good procedure includes the decontamination or wipedown of cabinet surfaces with chemical disinfectant before work commences. This practice eliminates the need for UV lights, whose primary utility in this application is inactivation of surface contamination since the filters effectively remove all airborne contaminants. UV lights, therefore, are not recommended in the LFBSC.

Allow blowers to operate for a minimum of 15 minutes before aseptic manipulations are begun in the cabinet, If the filtered air exhausted from the unit is discharged into the room, as in some installations, an additional advantage is obtained from purification (filtration) of the room air circulated through the equipment. Because of this characteristic contributing to the quality of the laboratory environment, some owners of LFBSC leave them in operation beyond the time of actual use.

\subsubsection{Wipedown}

The interior surfaces of the workspace should next be disinfected (see cleaning procedures) by wiping them thoroughly with $70 \%$ alcohol or similar non-corrosive anti microbial agents. USE OF CHLORINATED OR HALOGEN MATERIALS IN THE CABINET MAY DAMAGE STAINLESS STEEL. 


\subsubsection{Materials \& Equipment}

The apparatus and materials should next be placed into the cabinet. Care must be exercised that no items be placed over the front intake grills. Materials should be arranged so that clean, dirty (used), and virus materials are well separated. Passage of contaminated materials over uninoculated cultures or clean glassware should be avoided and transfer of viable materials should be performed as deeply into the cabinet (away from open face) as possible.

\subsubsection{Air Purge}

Additional purging of the workspace without user activity should be allowed for 2-3 minutes after materials and apparatus have been placed in it. This will rid the area of all "loose" contamination that may have been introduced with the items.

\subsubsection{Perform Work}

The work can now be performed. The technician performing the work is encouraged to wear a long-sleeved gown with knit cuffs and rubber gloves. This will minimize the shedding of skin flora into the work area and concurrently protect the hands and arms from viable agent contamination. At a minimum, the hands and arms should be washed well with germicidal soap before and after work in the cabinet. For the preparation of antineoplastic drugs, the following procedures summarize those contained in OSHA Instruction PUB 8-1.1, "Work Practice Guidelines for Personnel Dealing with Cytotoxic (Antineoplastic) Drugs.” The above document should be thoroughly studied/reviewed prior to drug preparation in the cabinet.

- A sterile plastic-backed absorbent drape should be placed on the work surface during mixing procedures. The drape should be exchanged whenever significant spillage occurs, or at the end of each production sequence.

- Vials should be vented with a filter needle to eliminate internal pressure or vacuum.

- Before opening ampoules, care should be taken to insure that no liquid remains in the tip of the ampoule. A sterile gauze sponge should be wrapped around the neck of the ampoule while opening. 
- Final drug measurement should be performed prior to removing the needle from the stopper of the vial.

- A non-splash collection vessel should be available in the biological safety cabinet to discard excess drug solutions.

\subsubsection{Terminal Purging \& Wipedown}

Following completion of work, allow the cabinet to run for 2-3 minute period without personnel activity to purge the unit. The decontamination of the interior surfaces should be repeated after removal of all materials, cultures, apparatus, etc. A careful check of grills and diffuser grids should be made for spilled or splashed nutrients which may support fungus growth and resulting spore liberation that contaminates the protected work environment.

\subsubsection{Paper Catch/Prefilter}

A permanent paper catch is installed behind the rear divider panel of the work zone. This area forms the return air path to the motor/blower; and if the airflow is blocked, it could seriously affect the performance of the cabinet. Therefore, THE PAPER CATCH SHOULD BE CHECKED AND CLEANED NO LESS THAN A WEEKLY BASIS; DAILY basis if procedures dictate the use of paper

products. Any paper removed must be properly disposed of as Contaminated Hazardous Waste. The above procedures also apply to all units configured with a prefilter.

\subsubsection{Shut Down}

Turn off blowers and lights. Do not use cabinet as a depository for excess lab equipment during periods of non-operation. 


\section{$1.4 \quad$ Ergonomics}

Ergonomics, the study or accommodation of work practices is extremely important for proper cabinet usage and user health and safety. An evaluation of normal work practices should be performed with each user when working in a cabinet. Evaluation criteria should be at a minimum:

- $\quad$ Proper user posture

- Effective workzone layout for work practice

- Vision or sightlines

For each of the above evaluation criterion, several aids may be supplied to accommodate the user.

- Ergonomic chair - Be sure feet are resting on the floor, chair foot support or foot rest. Also be sure back is fully supported with proper chair adjustments.

- Forearm/armrest support - The cabinet is provided with a forearm support on the work access opening. Periodic mini-breaks during work practice should be taken resting forearm to avoid stress and fatigue.

- Effective workzone layout - Always prepare your work procedure to minimize reach to avoid neck and shoulder stress and fatigue. Rotating tables are optional to maximum workzone and minimize reach.

- Vision and sightline - Always prepare your work procedure to eliminate glare and bright reflections on the window. Keep your window clean and sightlines clear to your effect workzone.

\section{Cleaning Procedures}

Cleaning the cabinet is an important function in terms of both containment and sterility. Use the following procedure to effectively clean or surface disinfect the cabinet workzone surfaces.

- $\quad$ Raise the sliding window to a full-open position, if desired.

- Press the audible alarm silence or cleaning key on the front control panel to silence the audible alarm during the cleaning process.

- Apply appropriate disinfecting solution to cabinet surfaces. Most surface disinfectants require a specific contact time, depending upon the microbiological agents used 
within the cabinet. Consult appropriate disinfectant documentation for proper application and safety precautions.

- NOTE: Disinfectants that use chlorides and halogens will cause damage to the stainless steel surfaces if left on for long periods of time.

- After the specified contact time, wipe up excess disinfectant. 
Two thermometers monitor ambient temperature in the laboratory. Quality control procedures for ambient air temperatures involve checking these thermometers for damage, monitoring the temperature each session, and documenting any unusual occurrences. Check the two wall thermometers for damage at the beginning of each stand. Notify the MEC manager of any damage.

The allowable ambient temperature range for the laboratory is $17^{\circ}$ to $25^{\circ} \mathrm{C}\left(63^{\circ}\right.$ to $\left.77^{\circ} \mathrm{F}\right)$.

NOTE: If the room temperature is out of allowable range since the last reading, document this in the automated Unusual Field Occurrence utility and inform the MEC manager immediately.

\subsection{Precision Instruments Osmette II}

The Osmette II is a scientific instrument used to measure osmolality. Care must be used in packing and unpacking the instrument. When the instrument is unpacked, keep the foam cushions in the instrument box until the stand closes. Repack the instrument for transportation in the original box using the thick foam pads to cushion the instrument on all sides.

\section{Changing the Osmette II Pipette Teflon Tip}

Change the Teflon tip if any of the below are encountered:

- The beginning of each stand.

- The resistance of the tip when depressing the pipette appears to be too loose.

- The air bubble separation above the sample is gone after a reading.

- The readings are erratic.

Replace the Teflon pipette tip. Remove the pipette tip from the disposable pipette tip or the protective cover. The Teflon tip is the tiny white plastic on the end of the pipette wire. The tip can be removed several ways. Any method is acceptable as long as care is taken to not bend the pipette wire or 
damage the pipette in any other way. A razor blade can be used to carefully cut along the side of the tip to cause it to release from the end of the wire. The tip will also come off by using a fingernail and some patience to work a space between the tip and wire and then push the tip off.

Locate the small clear red box labeled "Osmette Teflon Tips and Calibrator." Use one of the new Teflon tips inside the box as the replacement. The Teflon tip has one end that is solid and one end with a hole. The end with the hole should be placed on the end of the pipette wire. Ensure that the wire is fully inserted into the new tip. There should be no gap between the white tip and the pipette wire.

\section{Verifying the Pipette Calibration}

The pipette requires two small instruments to verify that it is calibrated correctly and will draw the proper amount of solution into the disposable pipette tip. One calibrator resides in the clear red box with the replacement Teflon tips. This calibrator is made of a hard black plastic and is about an inch long. It has a nib on one end. This calibrator measures the size of the bubble that is held between the Teflon tip and the sample when in the disposable pipette tip. To ensure the bubble is the proper size, place a clean disposable pipette tip on the pipette. Depress the plunger. Insert the nib into the end of the disposable pipette tip. The end of the nib should just touch the end of the Teflon tip; the tip should not push the tool out, and oppositely there should be no space gap between the tool and the Teflon tip.

If the bubble is not properly calibrated, remove the disposable pipette tip and unscrew the lock nut at the base of the pipette wire. Hold the plunger firmly with one hand while unscrewing the lock nut with the other. Use a pair of pliers if necessary. Unscrew the lock nut until it is loose. The pipette wire will also feel loose. Be careful not to turn the pipette wire accidentally when unscrewing the lock nut. Controlled, small turns will adjust the pipette wire to change the calibration setting. Turn the pipette wire in a clockwise direction (if looking at the end of the pipette tip head on) to pull the pipette tip up into the pipette and therefore make the bubble size larger. Turn the wire clockwise to adjust the pipette so the calibration tool will insert further up into the end of the clean pipette tip. Turning the wire counterclockwise adjusts the pipette so that the calibration tool will close the gap between the calibration tool and the Teflon tip. The tool and the tip should just touch when properly calibrated. When the desired adjustment has been made, tighten the lock nut down with your fingers. 
Place a clean disposable pipette tip on the pipette. Use the black calibration tool again to check the bubble size. Readjust if needed by following the above procedure. Once the calibration is successful, tighten the lock nut so that it will not come loose during regular use. Be careful not to damage the pipette.

The second instrument required to calibrate the pipette resides on the pipette itself. The end of the plunger opposite the tip has a tool that reads "Calib Gage \& Key." This tool can be unscrewed from the end. The length of the tool measures the correct draw amount for the pipette. To measure the draw amount place a clean disposable pipette tip on the pipette. Without depressing the plunger, insert the calibration gauge into the end of the disposable pipette tip. The end of the calibration gauge should just touch the end of the Teflon tip.

If the draw amount is not properly calibrated, twist the pipette plunger around until the nut on the inside of the plunger aligns with the hole in the side of the pipette body. Use the tiny hex wrench on the end of the calibration gauge to carefully turn —usually not more than a quarter turn — the nut inside the hole. Redepress the piston and use the calibration gauge to see if the draw amount is now correct. Readjust if needed by following the above procedure. Be sure to replace the calibration gauge in the end of the pipette when calibration is complete.

\section{Cleaning, Opening, and Oiling the Osmette II Probe}

The Osmette II may need to have the probe cleaned for several reasons:

- The instrument displays a large amount of “NO FREEZE” errors.

- The instrument displays a large amount of “PRE-FREEZE” errors.

- The readings are erratic.

Clean the Osmette II probe. Wet the end of a Cleanette with an alcohol prep pad. Use as many pads as needed to ensure the end of the Cleanette is wet. Insert the wet end of the Cleanette into the probe well and wipe by rotating the Cleanette left and right. Remove the Cleanette. Wet the end of a Cleanette with distilled water. Insert the wet end of the Cleanette into the probe well and wipe by rotating the Cleanette left and right. Remove the Cleanette. Insert a dry Cleanette into the probe well and wipe by 
rotating the Cleanette left and right. Remove the Cleanette. This process should clean any residual proteins left in the well.

Over time some sample may leak out into the probe chamber because of leakage due to loss of surface tension (as in the case of proteins in the sample or improperly drawn samples). The only way to clean this residue is to open the probe chamber and wipe off the entire probe, base, and chamber. Open the Osmette II for cleaning the probe chamber. Ensure that the Power button on the back of the Osmette is switched to "Off." Unplug the instrument from both the electrical outlet and the computer. Very gently turn the instrument over and lay it on its top so that the underside is tilted toward you. The bottom of the instrument is exposed. There is a black plastic piece on the underside of the instrument known as the "coffin” for its shape. The coffin acts as a cover for the probe. Open the coffin by using a Phillips head screwdriver to loosen the single screw on the left, broad end of the coffin. Carefully remove the loose screw and place it somewhere secure so it will not get lost. The loose end of the coffin can now swing up to reveal an opening underneath.

Inside the opening is a large black piece with a white circle in the middle. The white circle is made out of a rubbery, softer material. In the white circle is a white, hard, plastic donut with a white wire coming out. Using gloved hands, use your fingertips to gently unscrew the white donut by turning it to the left. Once the donut is loose, gently pull it straight out of the probe chamber, being careful not to touch the probe tip to the sides of the chamber. The probe is on the end of the white plastic donut. It is made of a fine metal wire, much like a pin. At its base is a flat surface with a black circle surrounded by a metal circle.

To clean the Osmette II, use only a Cleanette to wipe around the base of the probe (black circle and metal circle) to remove any residual sample not picked up by normal cleaning. Look down into the probe chamber on the instrument. On each side of the chamber are threads to fasten the donut back in place. Use a cleanette to wipe around the threads and the ring at the bottom of the chamber to absorb any residual sample.

On a rare occasion the probe base may need to be oiled. Only oil the probe if recommended by the Precision Systems technician. To oil the probe, use a tiny dab of Dow 311 industrial lubricant on the end of a Cleanette. Wipe the lubricant around the base of the probe (black and metal circles). The lubricant should be spread very thinly. The purpose of the lubricant is to ensure that no sample seeps down into the break between the black circle and the metal circle at the base of the probe. The lubricant 
also helps to create surface tension to hold the sample in the pipette tip. Use a clean Cleanette to wipe off any globs of lubricant on the base of the probe.

Once cleaning and/or oiling is complete, put the Osmette II back together. Carefully direct the probe back down into the probe chamber. Use your fingers to gently tighten the white plastic donut into the white circle. The donut should only be finger tight; do not overtighten. Swing the coffin back down into place so that the hole in the coffin for the screw lines up with the hole in the osmette, and the probe opening is completely covered. Use the screw and Phillips head screwdriver to fasten in place. Do not over fasten.

\subsection{Mettler Toledo Scale}

The Mettler Toledo scale is a very sensitive precision weighing instrument with a weighing limit of 600 grams. Handle the scale with care at all times. Do not weigh anything on the scale other than urine cups and specimens. The weigh pan will get dirty during use. Clean the weigh pan as needed by removing the metal plate from the scale and cleaning with Windex. Replace the weigh pan when clean.

Always use the clear acrylic scale cover to protect the scale from damage while not in use. Use the scale cover to protect from drafts when weighing samples.

\section{Scale Set Up}

Open the blue carrying case. Remove scale from case and place on the counter next to the bar code scanner. Verify that the scale is free of dust and debris. If needed, use the brush in Care Pac M to brush away dust and debris. 
Locate the tan serial cable taped to counter. The serial cable has a broad end with a trapezoid input with a screw on either side. It looks like this:

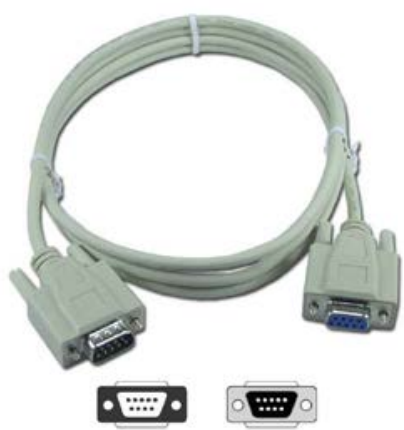

Insert the male end of the cable into the back of the scale. Use the screws to firmly fasten the cable to the back of the scale.

Locate the black AC adapter power cord taped to the counter. Place the round end in the round hole labeled "Power" on the back side of the scale. The LCD display on the front of the scale should say "OFF.” Remove the small, round, plastic cone protector from the top of the scale. Be sure to place the cone protector back into the blue carrying case so that it does not get lost.

Remove the weigh pan from the case lid. Gently place the pan on top of the scale, being sure to align the notch on the bottom of the pan with the bump on the right side of the cone. The pan should fit snugly and not wobble around when properly seated.

\section{Never move or turn over the scale without first placing the cone protector on the scale's} cone.

Balance the scale using the liquid level in the back right corner of the scale. Adjust the feet of the scale by screwing them in or out until the bubble in the liquid level stays within the center circle. 


\section{Scale Teardown}

Place the carrying case on the counter near the scale. Turn off the scale by pressing and holding the On/off button. Hold until "OFF" appears on the LCD display. Gently remove weigh pan from scale. Slide weigh pan back into slot in lid of carrying case. Remove the protective cone cover from the carrying case and place over the cone.

Turn the scale around on the counter so the back of the scale is visible. Unplug the AC adapter power cord from the back of the scale. Tape the end of the power cord to the counter so it is easily found at the next setup.

Unplug the serial cable from the back of the scale by unscrewing the two screws and gently pulling the end of the serial cable away from the back of the scale. Tape the cord to the counter so it is easily found at the next setup.

Place the scale in the protective carrying case. Close the lid and snap black locks tightly closed so that the lid and bottom of the case do not come apart. Place the carrying case back into the box for transport to the next stand.

Place the Care Pac M back into the box for transport. Make sure one Styrofoam bumper is on the bottom of the box and set the Care Pac M down into the bumper. Fit the second bumper on top of the Care Pac M and close the box. If Care Pac M has been packed properly, the box should close easily and completely. 


\subsection{Laboratory Quality Control Module}

Document the following items in the Laboratory QC module based on the frequency described in the following table.

\begin{tabular}{|c|c|c|}
\hline Frequency & Item & Acceptable result \\
\hline \multicolumn{3}{|c|}{ Start of Stand } \\
\hline & Freezer \#1 cleaned & Yes \\
\hline & Freezer \#2 cleaned & Yes \\
\hline & Freezer \#3 cleaned & Yes \\
\hline & Freezer \#4 cleaned & Yes \\
\hline & Refrigerator \#1 cleaned & Yes \\
\hline & Refrigerator \#2 cleaned & Yes \\
\hline & Centrifuge \#1 cleaned & Yes \\
\hline & Centrifuge \#2 cleaned & Yes \\
\hline & ULBSC cleaned & Yes \\
\hline & Magnahelic gauge reading & $0.350-0.550$ \\
\hline & Hood certification expiration date & After current date \\
\hline & Class 6 one hundred gram weight $+/-1.0 \mathrm{~g}$ & $99-101$ \\
\hline & Two Class 6 three hundred gram weight $+/-6.0 \mathrm{~g}$ & $594-606$ \\
\hline & Change Osmometer Teflon Pipette Tip & Yes \\
\hline & Osmette Total Calibration Distilled Water 1 & $\leq 1$ \\
\hline & Osmette Total Calibration Distilled Water 2 & $\leq 1$ \\
\hline & Osmette Total Calibration 100 Standard 1 & $99-101$ \\
\hline & Osmette Total Calibration 100 Standard 2 & $99-101$ \\
\hline & Osmette Total Calibration 500 Standard 1 & $499-501$ \\
\hline & Osmette Total Calibration 500 Standard 2 & $499-501$ \\
\hline & Osmette Total Calibration 500 Standard 3 & $499-501$ \\
\hline & Osmette Total Calibration 1500 Standard 1 & $1496-1504$ \\
\hline & Osmette Total Calibration 1500 Standard 2 & $1496-1504$ \\
\hline & Osmette Total Calibration 1500 Standard 3 & $1496-1504$ \\
\hline & Osmette Total Calibration 2000 Standard 1 & 1994-2006 \\
\hline
\end{tabular}




\begin{tabular}{|c|c|c|}
\hline Frequency & Item & Acceptable result \\
\hline & Osmette Total Calibration 2000 Standard 2 & 1994-2006 \\
\hline & Osmette Total Calibration 2000 Standard 3 & 1994-2006 \\
\hline \multicolumn{3}{|c|}{ Daily } \\
\hline & Freezer \#1 temperature reading & -18 to $-23^{\circ} \mathrm{C}$ or -28 to $-33^{\circ} \mathrm{C}$ \\
\hline & Freezer \#2 temperature reading & -18 to $-23^{\circ} \mathrm{C}$ or -28 to $-33^{\circ} \mathrm{C}$ \\
\hline & Freezer \#3 temperature reading & -18 to $-23^{\circ} \mathrm{C}$ or -28 to $-33^{\circ} \mathrm{C}$ \\
\hline & Freezer \#4 temperature reading & -18 to $-23^{\circ} \mathrm{C}$ or -28 to $-33^{\circ} \mathrm{C}$ \\
\hline & Centrifuge \#1 refrigerated reading & 4 to $8^{\circ} \mathrm{C}$ \\
\hline & Centrifuge \#2 room temperature reading & 17 to $25^{\circ} \mathrm{C}$ \\
\hline & Magnahelic gauge readings & $0.350-0.550$ \\
\hline & Refrigerator \#1 temperature reading & -18 to $-23^{\circ} \mathrm{C}$ or -28 to $-33^{\circ} \mathrm{C}$ \\
\hline & Refrigerator \#2 temperature reading & -18 to $-23^{\circ} \mathrm{C}$ or -28 to $-33^{\circ} \mathrm{C}$ \\
\hline & Workstation 1 room temperature reading & 17 to $25^{\circ} \mathrm{C}$ \\
\hline & Workstation 2 room temperature reading & 17 to $25^{\circ} \mathrm{C}$ \\
\hline & Flush eyewash for 5 minutes & Yes \\
\hline & Class F2 twenty gram weight $+/-0.2 \mathrm{~g}$ & $19.8-20.2$ \\
\hline & Class F2 five hundred gram weight $+/-5.0 \mathrm{~g}$ & $495-505$ \\
\hline & Verify Osmometer Pipette Calibration & Yes \\
\hline & Osmette Daily QC Check 100 Standard 1 & $96-104$ \\
\hline & Osmette Daily QC Check 100 Standard 2 & $96-104$ \\
\hline & $\begin{array}{l}\text { Osmette Daily QC Check } 1500 \text { Standard } 1 \text { (result } \\
1485 \text { - 1515) }\end{array}$ & $1485-1515$ \\
\hline & Osmette Daily QC Check 1500 Standard 2 & $1485-1515$ \\
\hline & Osmette Quantimetrix Level 1 Control & Yes \\
\hline & Osmette Quantimetrix Level 2 Control & Yes \\
\hline \multicolumn{3}{|c|}{ Weekly } \\
\hline & Freezer \#1 tempscribe chart paper changed & Yes \\
\hline & Freezer \#2 tempscribe chart paper changed & Yes \\
\hline & Freezer \#3 tempscribe chart paper changed & Yes \\
\hline & Freezer \#4 tempscribe chart paper changed & Yes \\
\hline & Refrigerator \#1 tempscribe chart paper changed & Yes \\
\hline
\end{tabular}




\begin{tabular}{|c|c|c|}
\hline Frequency & Item & Acceptable result \\
\hline & Refrigerator \#2 tempscribe chart paper changed & Yes \\
\hline & $\begin{array}{l}\text { HMX Latron and 5C Cell Control QC data } \\
\text { transmitted? }\end{array}$ & Yes \\
\hline & Osmette Standards Check 100 Standard 1 & $96-104$ \\
\hline & Osmette Standards Check 100 Standard 2 & $96-104$ \\
\hline & Osmette Standards Check 100 CON-TROL 1 (result) & $96-104$ \\
\hline & Osmette Standards Check 100 CON-TROL 2 & $96-104$ \\
\hline & Osmette Standards Check 500 Standard 1 & $496-504$ \\
\hline & Osmette Standards Check 500 Standard 2 & $496-504$ \\
\hline & Osmette Standards Check 500 CON-TROL 1 & $496-504$ \\
\hline & Osmette Standards Check 500 CON-TROL 2 & $496-504$ \\
\hline \multicolumn{3}{|c|}{ End of Stand } \\
\hline & Freezer \#1 cleaned & Yes \\
\hline & Freezer \#2 cleaned & Yes \\
\hline & Freezer \#3 cleaned & Yes \\
\hline & Freezer \#4 cleaned & Yes \\
\hline & Refrigerator \#1 cleaned & Yes \\
\hline & Refrigerator \#2 cleaned & Yes \\
\hline & Centrifuge \#1 cleaned? & Yes \\
\hline & Centrifuge \#2 cleaned? & Yes \\
\hline & LFBSC cleaned? & Yes \\
\hline & Temp charts sent to home office & Yes \\
\hline & Coulter printouts sent to home office & Yes \\
\hline & $\begin{array}{l}\text { HMX Latron and 5C Cell Control QC data } \\
\text { transmitted? }\end{array}$ & Yes \\
\hline
\end{tabular}


Document all instrument quality control activities in the Laboratory QC module.

Access the Laboratory QC module.

\begin{tabular}{|c|c|c|c|c|c|c|c|c|c|c|}
\hline \multicolumn{10}{|c|}{ i: Stand:430 Session:430110 12/14/2004 08:30 am - 12:30 pm } & \\
\hline$\| x$ & \multicolumn{10}{|c|}{ Quality Control Ctrl+Q } \\
\hline & \multirow{4}{*}{$\begin{array}{l}\text { Exam Pause } \\
\text { observations } \\
\text { IC Exclusions } \\
\text { Send Message } \\
\text { UFO Sighting } \\
\text { Set Session }\end{array}$} & \multirow{4}{*}{$\mathrm{Ctrl}+\mathrm{M}$} & \multirow{3}{*}{ gie name } & \multirow{2}{*}{\multicolumn{6}{|c|}{$\begin{array}{l}\text { Process Status } \\
\text { UC U } P \text { OZ } \quad \text { CB } \quad \text { a gtt } P C \quad H P V\end{array}$}} & \\
\hline SPID & & & & & & & & & & OR \\
\hline 346104 & & & & 00 & P & \multicolumn{2}{|c|}{\begin{tabular}{c|c}
$O Z$ & $C B$ \\
$O$ & 0
\end{tabular}} & $\begin{array}{l}\mathrm{B} \text { gtt PC } \\
00\end{array}$ & $\begin{array}{l}\text { HPV } \\
0\end{array}$ & 0 \\
\hline 460575 & & & 32 ROOP, LESLEY & 00 & 0 & 0 & 0 & 00 & 0 & 0 \\
\hline 480759 & $\checkmark$ English & $C t$ trl $+E$ & 34 ROOP, STANLEY & 00 & $\cdot$ & 0 & 0 & $0 \bullet$ & . & 0 \\
\hline 482080 & Spanish & $c t$ trit+5 & 63 ROOP, STUART & 00 & . & 0 & 0 & 00 & . & 0 \\
\hline 549961 & $\begin{array}{l}\text { Ioolbars... } \\
\text { System Configuration }\end{array}$ & & 26 ROOP, WESLEY & 00 & . & 0 & 0 & 000 & . & 0 \\
\hline 851056 & Pregnancy QC & & 38 ROOP, KELLEY & 00 & 0 & 0 & 0 & 00 & 0 & \\
\hline 860681 & $\begin{array}{l}\text { Coulter QC Lot Inio } \\
\text { Regen SP Data }\end{array}$ & & 38 ROOP, DONNA & 00 & 0 & 0 & 0 & 000 & & 0 \\
\hline
\end{tabular}

To access the QC module, use the mouse to direct the mouse arrow to \{Utilities\}, drag the arrow to \{Quality Control\}, and left click or type [Alt] [U/u], [Q/q] or [Ctrl] [Q/q]. 
Enter the required user ID.

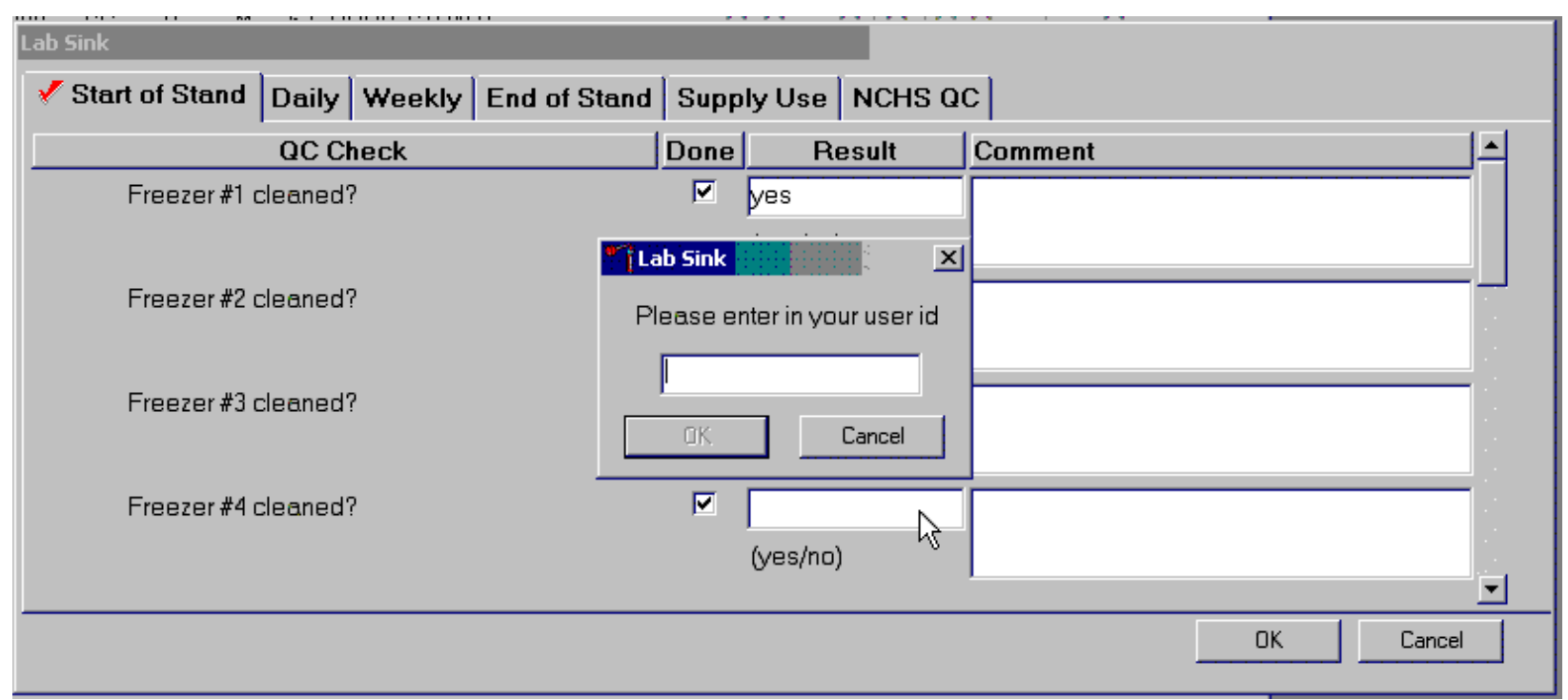

Enter user ID as last name_first initial. Type in the user ID and use the mouse to direct the mouse arrow to the "OK” button, and left click or select [Enter]. To exit without entering a user ID, use the mouse to direct the mouse arrow to the "Cancel" button, and left click. 
Enter quality control activities and readings.

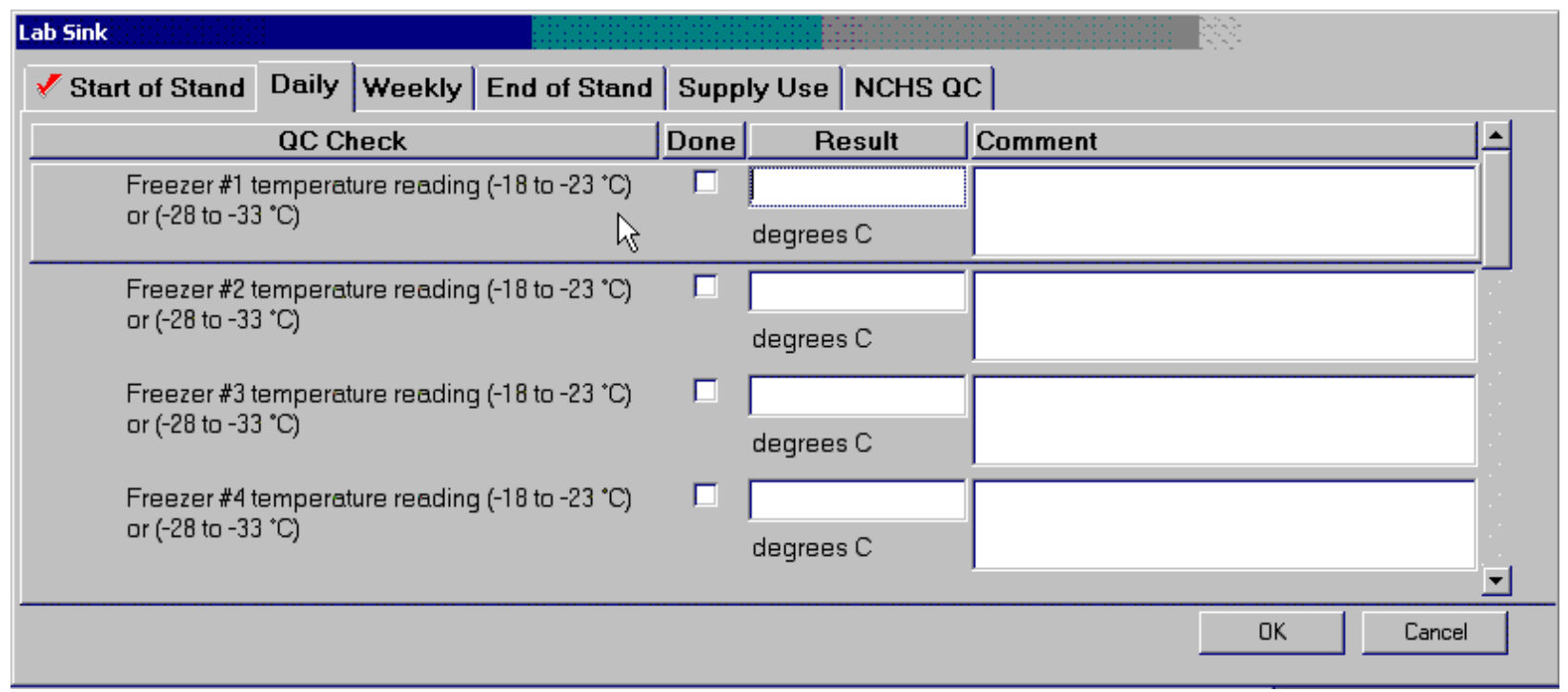

Select the correct activity (Start of Stand, Daily, Weekly, End of Stand, Supply Use, or NCHS QC) by using the mouse to direct the mouse arrow to the correct heading on the top of the file, and left click. At each stand, perform and record weekly activities on the same weekday as set up. 
Mark activities as done and enter the result.

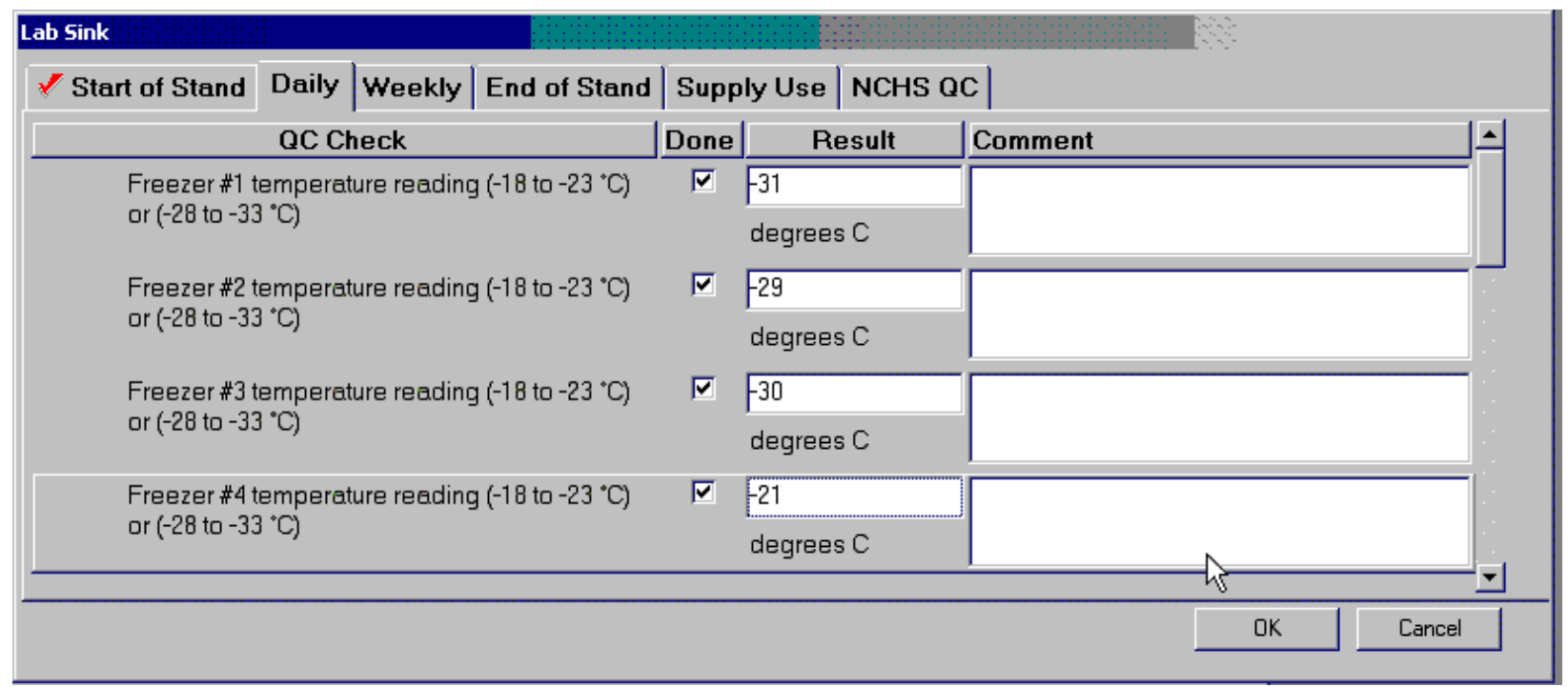

To record an activity as complete or done, use the mouse to direct the mouse arrow to the check box in the Done column, and left click to insert a check mark. Select [Tab] or [Enter] to move to the Result text box. 
Enter the response or reading in the Result text box.

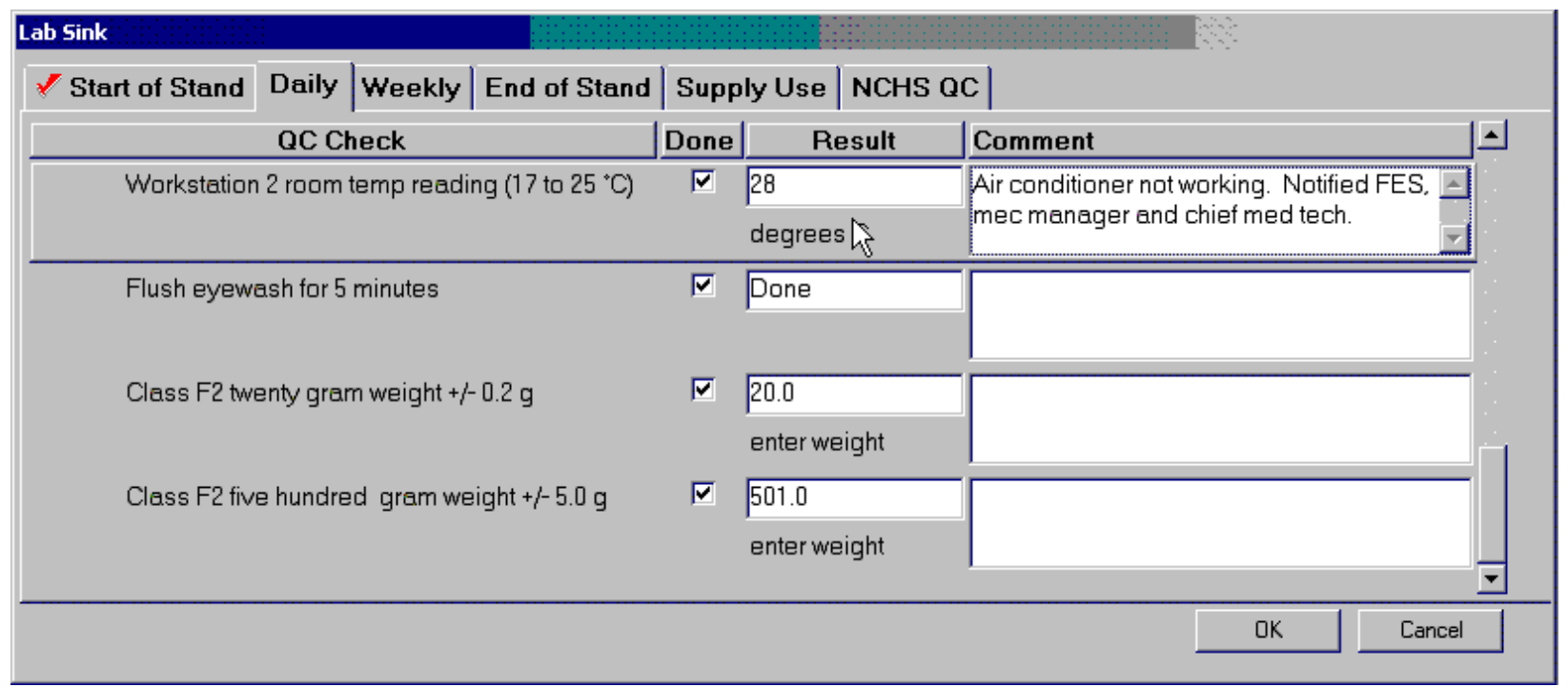

Enter "Yes” or "No" for text responses, whole numbers for rpm measurements, minutes, and seconds for timer readings, three decimals for w.g., and whole negative and positive numbers for thermometer readings in the Result text box. Select [Tab] or [Enter] to move to the Comment text box. Type any actions associated with an unexpected result in the Comment text box. Select [Tab] to move to the next line. 
Enter the lot numbers and expiration dates in the Supply Use activity. This activity lists every inventory item associated with the component. Record the pregnancy test kit and the serum and urine pregnancy control lot numbers and expiration dates even though these are captured in the Pregnancy QC module.

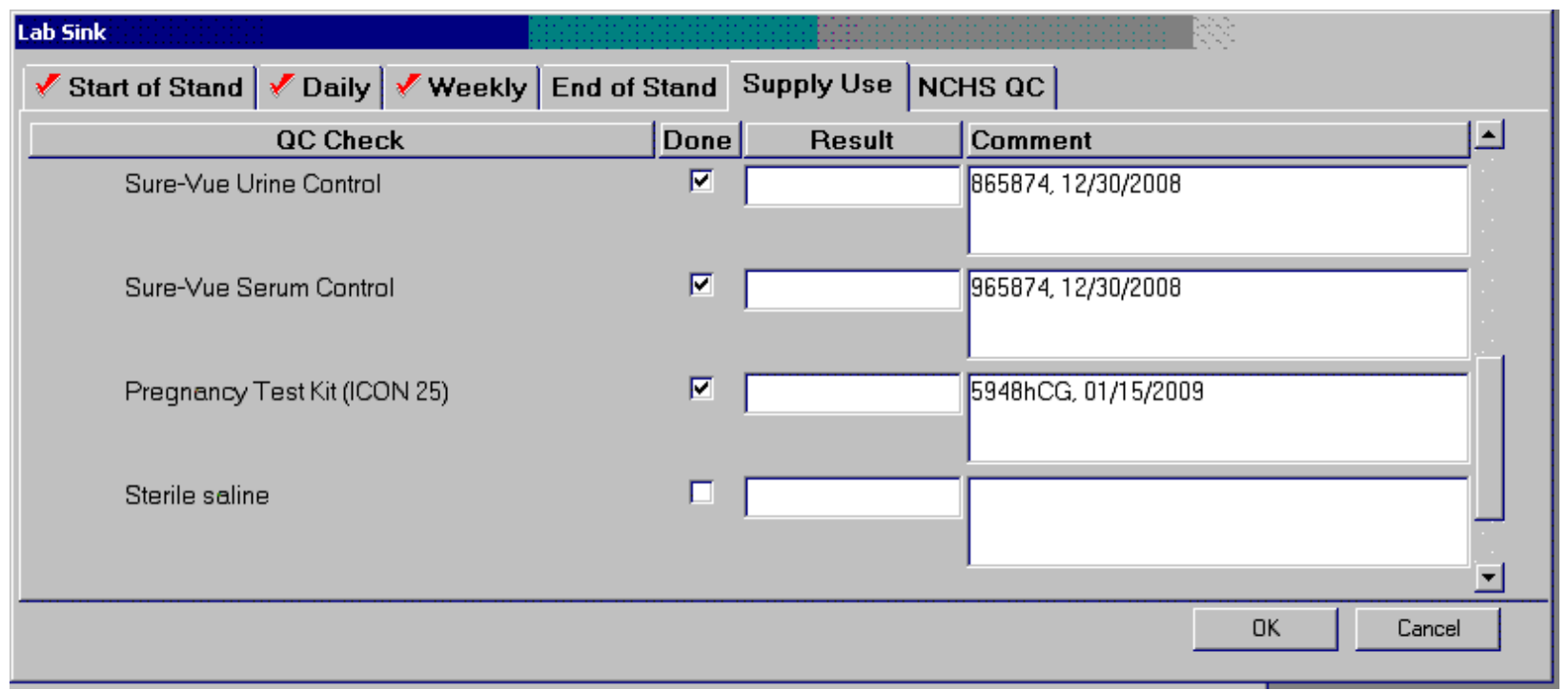

Mark each check box and record the lot and expiration date of each item in the Comments text box at the start of the stand and whenever a new lot is put into use. To record an activity as complete or done, use the mouse to direct the mouse arrow to the check box in the Done column, and left click to insert a check mark. Select [Tab] or [Enter] to move to the Result text box. Do not enter the response or reading in the Result text box. Select [Tab] or [Enter] to move to the Comment text box.

- For the ascorbic acid record the stand week (Week 1, Week 2, etc), add a comma, and record the date the reagent was reconstituted using the MM/DD/YYYY format.

- For the timers and thermometers, record the calibration date (14/06), add a comma, and record the expiration date using the MM/DD/YYYY format.

- For items with lot numbers, record the lot number, add a comma, and record the expiration date using the MM/DD/YYYY format.

There is no frequency requirement for this activity within the QC application. 
Update the information each time a new lot is put into use.

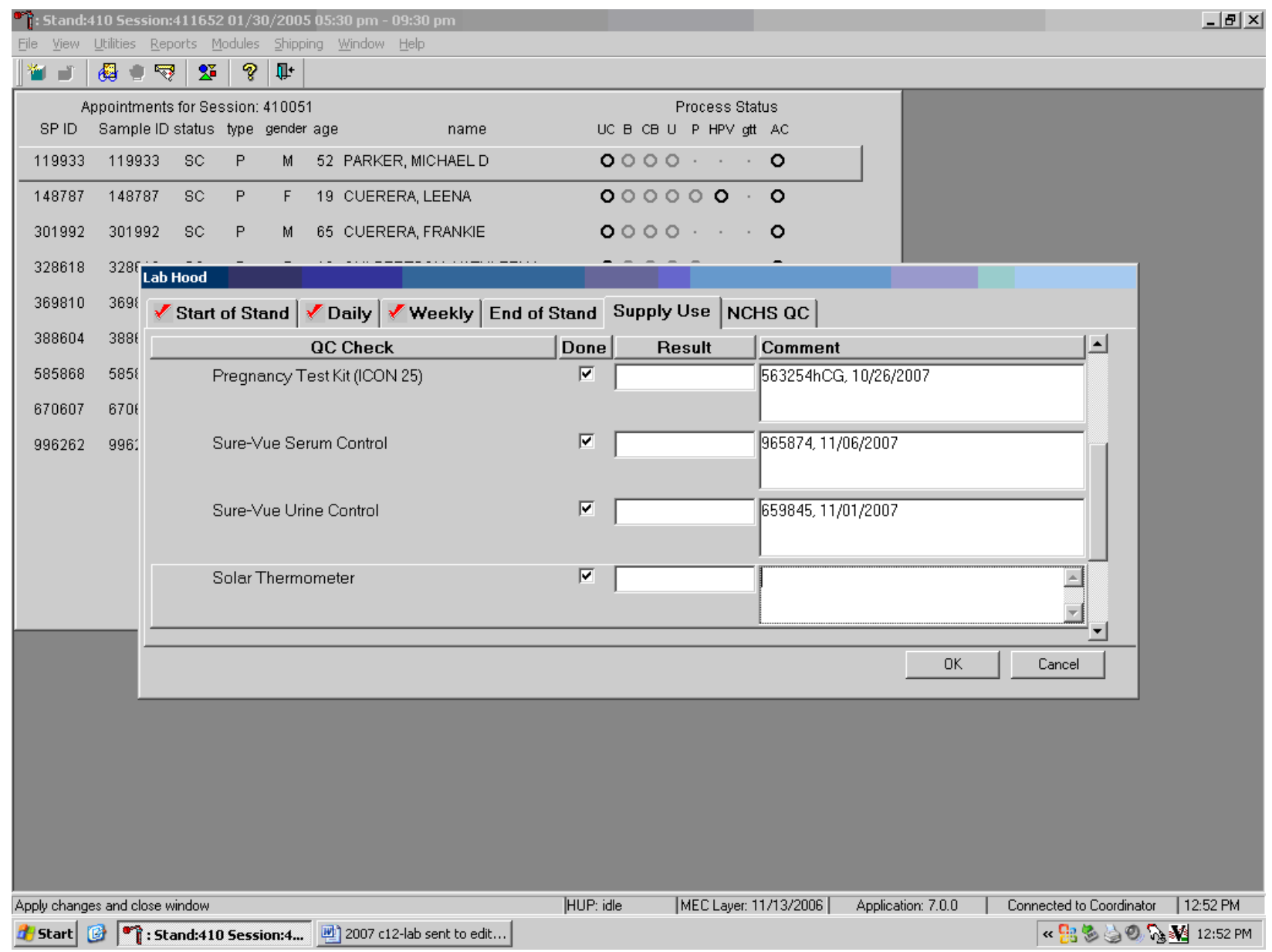

To save the results to the database, use the mouse to direct the mouse arrow to the "OK" button, and left click; to exit the QC module without saving the results, use the mouse to direct the mouse arrow to the "Cancel" button, and left click. Once results are saved to the database, they cannot be changed, except for the information contained in the Supply Use Comments text box. 
A Warning Message text box displays if required QC is incomplete.

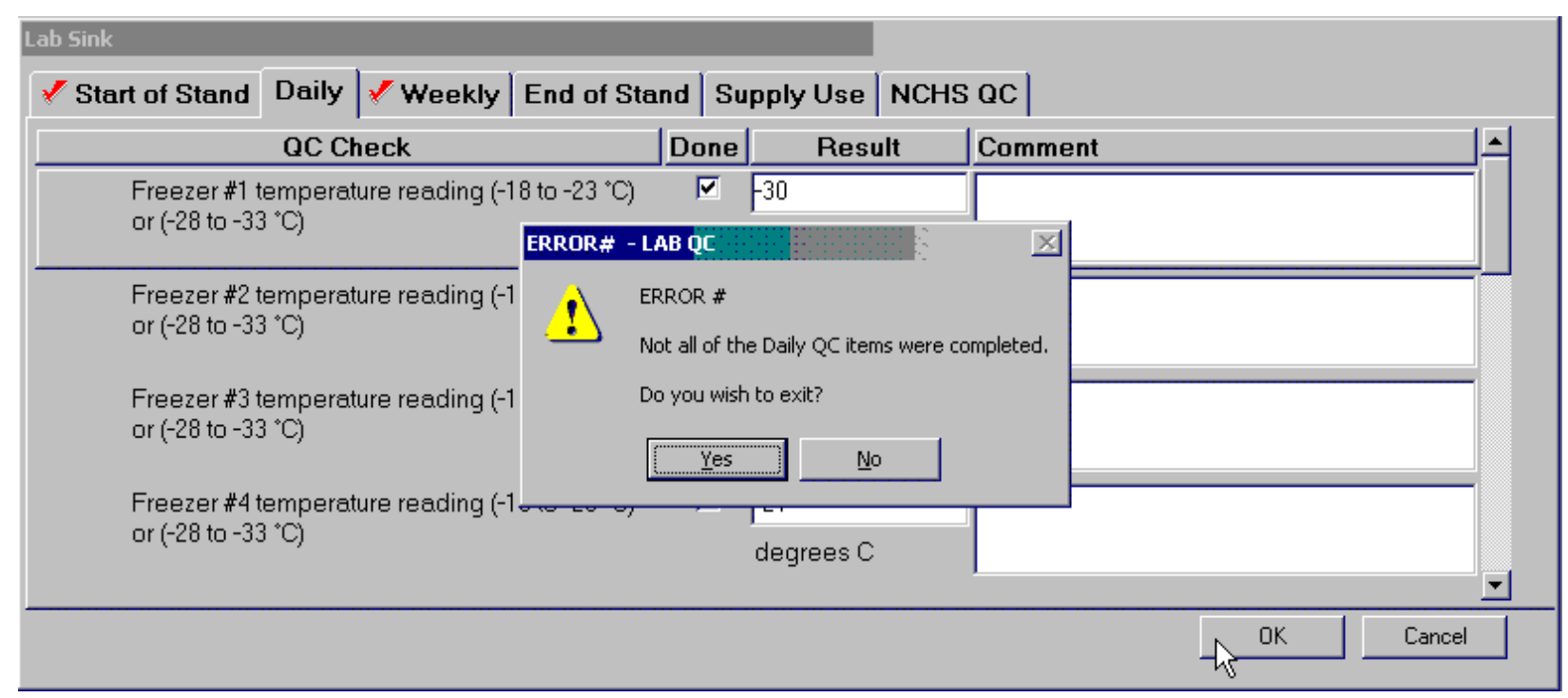

A Warning Message text box displays when an attempt is made to access a laboratory module if the required QC activities have not been performed or if some activities are incomplete. Respond to the Message text box. To exit the QC module, use the mouse to direct the mouse arrow to the "Yes" button, and left click or type [Y/y] or select [Enter]. To remain in the QC module, use the mouse to direct the mouse arrow to the "No" button, and left click or type [N/n]. Continue to perform the required QC activities and record the results. 
A red check mark on the top of the file header indicates that all QC activities associated with that requirement were successfully recorded and saved to the database.

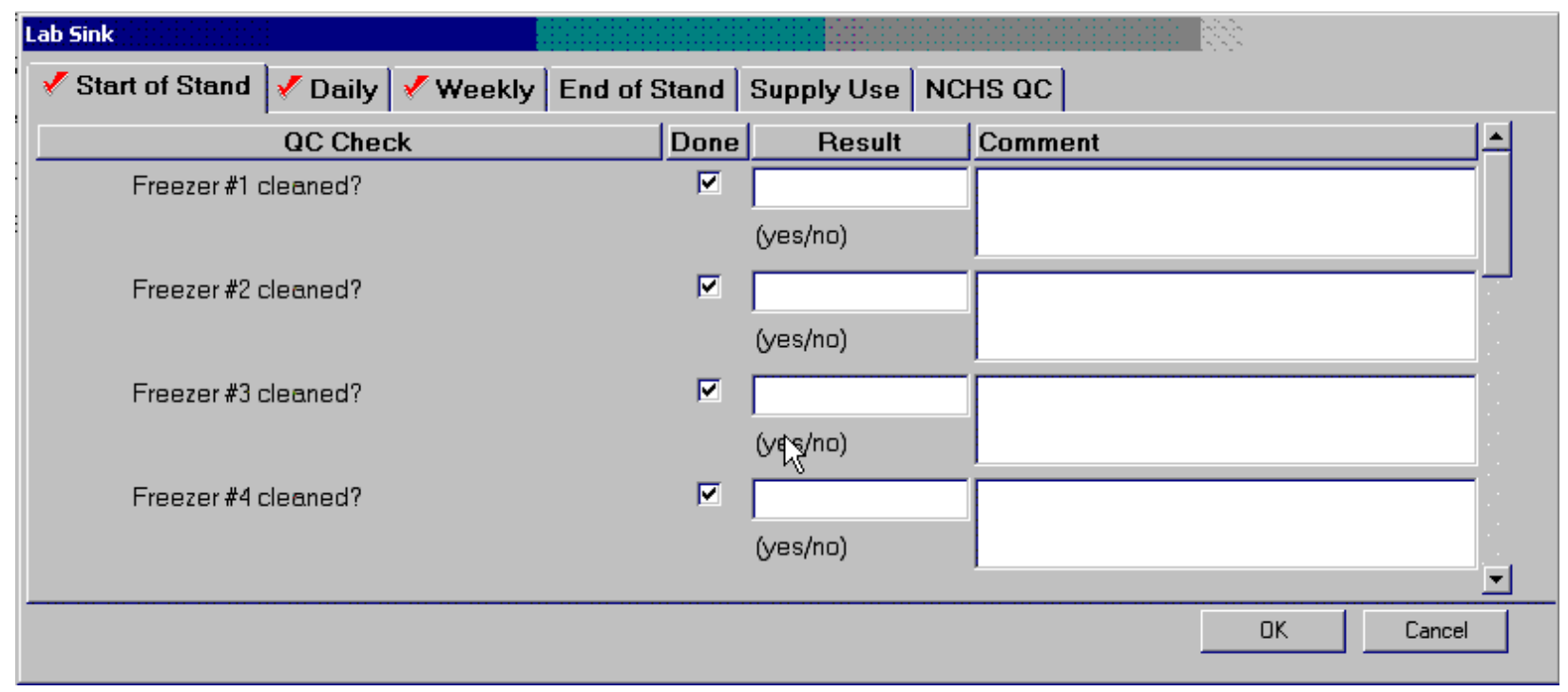

\subsection{Unusual Field Occurrence (UFO)}

Document any unusual occurrence using the Unusual Field Occurrence utility. The hard-copy Unusual Occurrence Log (see Exhibit 15-6) is available as a backup. Record the date, Tech ID number, an explanation of the incident, and the steps to resolve the incident. Be sure to record the model and the serial number of the instrument. Refer to the UFO User Guide for complete instructions. 
Exhibit 15-6. Hard-copy Unusual Occurrence Log

\section{NHANES \\ Unusual Occurrence Log}

Stand Number

Beginning Stand Date

Technologist Name and ID

Date

Time

Comments or Issue

Action Taken

Resolution

\section{Reviewed by}

Date reviewed 
Enter information into the appropriate line(s):

- Stand Number,

- Beginning Stand Date,

- Technologist Name and ID,

- Date,

- Time,

- Comments or Issue,

- Action Taken, and

- Resolution.

NOTE: Do not enter any text in the Reviewed by or Date reviewed lines.

Print these results and file in the QC notebook. Send to the home office at the end of each stand. The data manager can answer any questions about accessing, saving, and printing this document.

\subsection{Hard Copy Supply Use Control Log}

Document the lot number and expiration dates for all Vacutainer ${ }^{\circledR}$ tubes, needles, and reagents in the Supply Use Control activity of the Quality Control module. This hard-copy Supply Use Control Log (Exhibit 15-7) is available as a backup.

Enter information into the appropriate column(s):

- Reagent or Supply Name,

- Beginning Stand Date,

- Lot Number, 
Exhibit 15-7. Hard-copy Supply Use Control Log

\section{NHANES \\ Supply Use Control Log}

Stand Number

Beginning Stand Date

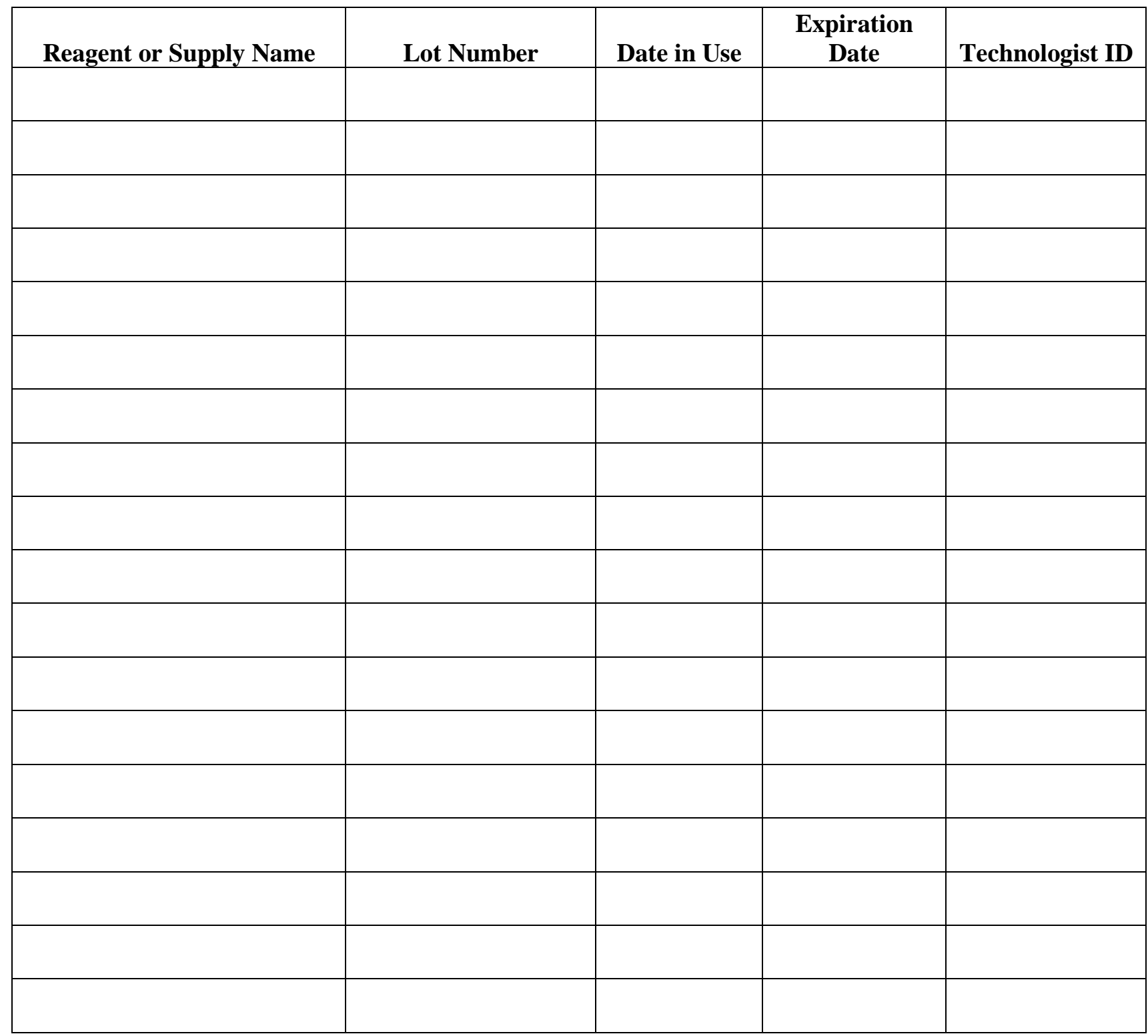


- Date in Use,

- Expiration Date, and

- Technologist ID.

Print these results and file in the QC notebook. Send to the home office at the end of each stand. The data manager can answer any questions about accessing, saving, and printing this document.

\subsection{Eppendorf Pipette Care, Sterilization, Maintenance, and Calibration}

The MEC laboratory has four Eppendorf Research pro electronic pipettes: one 5-100 $\mu \mathrm{L}$, two 50-1000 $\mu \mathrm{L}$, and one $100-5000 \mu \mathrm{L}$. The lab also uses $100 \mu \mathrm{L}$ and $1000 \mu \mathrm{L}$ Biopur pipette tips and 5-mL bulk pipette tips. Charge the pipettes in the 4-stand charger.

- Do not clean the display or any of the labeling using acetone or aggressive solutions.

- Do not allow any liquid to enter the device.

- Only authorized service personnel may carry out repairs.

- Use original spare parts and accessories (battery, pipette tips) only.

\section{Care}

Wipe the outside of the pipette with a moist cloth. The use of water with wetting agent is also permitted.

WARNING: DO NOT ALLOW ANY LIQUID TO ENTER THE PIPETTE. 
Rinse (with distilled water) and dry only the lower part of the pipette. The pipette may be wiped clean with 60 percent isopropanol.

Disassemble the lower half of the Research pro if the pipette is very contaminated or if very aggressive chemicals are dispensed. Rinse the individual parts in distilled water and then dry the parts. Lightly lubricate the piston using silicone grease.

After opening the pipette, check the position of the piston and execute a motor reset. Trigger the motor reset by holding down the Reset key until $\mathbf{R}$ appears in the display.

\section{Sterilization}

Disassemble only the lower half of the pipette. The individual parts of the lower part must be disassembled further into separate pieces. Please review the diagrams on page 27 of the Research Pro Instruction Manual before attempting to disassemble any pipette.

To loosen the lower part of the pipette (for all pipettes except the 5,000 $\mu \mathrm{L}$ ):

- Hold down the ejector and pull off the ejector sleeve. Force may be required.

- Unscrew the lower part using the key. During disassembly, do not damage the piston.

- Unscrew the piston. If necessary, first loosen the piston at the upper end using flatnose pliers. The piston is moved to the lowest position.

To loosen the lower part of the $5,000 \mu \mathrm{L}$ pipette:

- Hold down the ejector and pull off the ejector sleeve. Force may be required.

- Unscrew the cylinder. The piston is held in place magnetically. During reassemble, do not damage the piston.

- Pull the piston out of the upper part.

Assemble in reverse order. Engage the magnetic coupling of the 5,000 $\mu \mathrm{L}$ piston as shown in the diagram on page 27 of the Instruction Manual. Check that the dispensing function of the pipette is working correctly. 


\section{Maintenance and Calibration}

Apart from general care and occasional discharging (when the battery symbol starts flashing) and recharging of the pipette, no special maintenance is necessary if the pipette is used correctly. The lower part of the pipette may be replaced. Part B: “Ordering information/service parts," describes the process for replacing the seals in the lower part.

The pipettes are calibrated at least once per year, when the staff is on their break in June/July or December. When instructed, send the pipettes to the warehouse. The warehouse manager will contact the service department at Brinkmann Instruments at 800-645-3050, ext. 404 to schedule the calibration; send the pipettes to Brinkmann, and then back to the field.

A complete list of troubleshooting information is included on pages 28 and 29 of the Instruction Manual.

\subsection{Power Loss and Laboratory Operations}

Three scenarios may occur if power is lost to the MEC. Scenario 1 occurs when power is lost during an exam session and there is no backup generator. Scenario 2 occurs when power is lost when the MEC is not conducting a session. Scenario 3 occurs when power is lost but there is a backup generator available. Procedures to address each scenario are described in detail.

\subsubsection{Scenario 1 - Power Loss during Exam Session with No Backup Generator}

If a power loss occurs during a MEC session, action must be taken to either continue processing the blood or preserve the integrity of the processed specimens. The phlebotomist should not draw blood during a power loss because of safety concerns due to insufficient light. 


\section{Centrifuges}

- Overview - There are two centrifuges in each MEC: one centrifuge is operated at ambient temperature and the second is operated at refrigerated $\left(2-6^{\circ} \mathrm{C}\right)$ temperature. All blood tubes can be spun in the refrigerated centrifuge.

While power is generally required to open or unlock the centrifuge door, an emergency access procedure will allow the technologists to open the door to retrieve the blood tubes if a power failure occurs.

- Consequences - All blood tubes can remain in the locked centrifuge for up to 4 hours.

- Actions

1. It is not possible to routinely spin all tubes in one centrifuge; if a power loss is anticipated, spin all tubes in the refrigerated centrifuge.

2. Use the emergency access procedure to retrieve the blood tubes from the centrifuge. Aliquot the serum and plasma into the vessels if the serum or plasma has sufficiently separated from the cells.

- $\quad$ Move the secondary latch lever to UNLOCK position.

- $\quad$ Insert a 5-mm (3/16 in.) or smaller screwdriver into the override key slot in the front panel (see Figure 4-1 on page 4-4 of the Operations Manual).

- $\quad$ Turn the screwdriver clockwise until you hear a "click" or until the door pops open.

\section{Laminar Flow Hood}

- Overview - The technologists require the laminar flow hood to process the blood for two reasons: (1) the hood provides an uncontaminated or clean environment for processing environmental blood specimens; (2) the hood is required to provide a fullface shield in case of an accidental blood splash. In addition, the outlet in the hood provides power to the electronic pipettes. The electronic pipette is required to perform an accurate dilution for vessel 3 (RBC Folate).

- Consequences - The environmental specimens may become contaminated. The pipette will lose battery power after $\sim 60$ minutes. 


\section{- Actions}

1. Complete an Unusual Occurrence Log to document the SP IDs of the samples processed without a fully functioning hood. This information is subsequently recorded in an annual summary for NCHS.

2. Attach the comment "equipment failure" to vessels 1, 5, and 29.

3. Use the backup full-face shields when processing blood.

4. Process vessel 3 immediately so specimens are not lost due to battery failure.

\section{Freezers and Refrigerators}

- Overview - There are four freezers and two refrigerators in each MEC lab. Each unit is marked with a label. Refrigerant gel packs are distributed and stored in the freezers. Most specimens are stored in various plastic vials and the vials are placed in cardboard boxes with grids; the cardboard boxes are then stored in the freezers and refrigerators. In addition, some blood tubes (vessels 54 and 55), swabs (vessels 73 and 76), the VOC water (vessel 56), and the Coulter and pregnancy controls are stored in the refrigerator.

- Consequences - Specimen integrity is affected if frozen specimens thaw or if refrigerated specimens reach ambient temperature. Some analytes are more sensitive than other analytes. The temperature-monitoring chart provides a constant readout of the actual temperature. A full freezer takes approximately 3 hours to rise from $-20^{\circ} \mathrm{C}$ to $-10^{\circ} \mathrm{C}$; a $1 / 2$ filled freezer takes 2 hours and 25 minutes to rise to $-10^{\circ} \mathrm{C}$; and a $1 / 4$ filled freezer takes 1 hour and 45 minutes to rise to $-10^{\circ} \mathrm{C}$. Once the temperature reaches $-10^{\circ} \mathrm{C}$ the staff has approximately 30 minutes before the specimens thaw.

\section{- Actions}

1. Do not open the freezer or refrigerator doors, if possible.

2. Refrigerators - After 30 minutes or when the temperature recording chart reading reaches $7^{\circ} \mathrm{C}$, add 14 frozen gel packs (stored in the bottom of each freezer) to the center of each refrigerator. Alternatively, add 25 pounds of ice cubes or blocks to each refrigerator. Do not add dry ice to the refrigerators; this will cause the refrigerator to explode.

3. Freezers - Contact the local dry ice company and order 200 pounds of dry ice. Obtain four large ice chests or coolers; each cooler should hold the boxes from one freezer and 50 pounds of dry ice. Within 2 hours or when the temperature recording chart reading reaches $-10^{\circ} \mathrm{C}$, move the containers (boxes) to the ice chests and add 50 pounds of dry ice to each ice chest. Alternatively, move the 
containers to Styrofoam shippers and distribute the dry ice across the shippers. Do not add dry ice to the freezers; this will cause the freezer to explode.

\section{Coulter HMX}

- Overview - The Coulter consists of the HMX, the monitor, hard drive, and printer. The HMX will not function without the hard drive and monitor. The blood components for the CBC (complete blood count) are stable for a maximum of 24 hours when stored at room temperature.

- Consequences - None.

- Actions - None.

NOTE: The data manager should disable the Sensaphone system before a scheduled power loss.

\subsubsection{Scenario 2 - Power Loss when the MEC is Not Conducting Exams}

If the power loss occurs when the MEC is not conducting a session, the freezers and refrigerators require action to protect the integrity of the specimens. The lab staff can continue to ship if all the ISIS processes are complete. If the ISIS processes are not complete, the lab staff can still pack the shippers and complete the ISIS process once power is restored.

\section{Freezers and Refrigerators}

- Overview - All three MECs now have the Temperature Guard system installed. This system monitors the room temperature and the internal temperature of the refrigerators and freezers in the MEC laboratory. When the system detects a significant increase in the temperatures, it begins calling designated staff. The MEC managers and data managers have been identified as those individuals responsible for responding to the call by typing in the response code. The temperature increase could be confined to one freezer or refrigerator, to multiple units, or all units. The temperature increase could be due to any number of circumstances such as a loss of power because a circuit breaker tripped, or due to a compressor malfunction or failure. Whoever enters the code must act to prevent loss of biological specimens. The Temperature Guard temperature trigger was established to allow 30 minutes of travel time. After 30 minutes, frozen specimens may begin to thaw.

- Consequences - Same as scenario \#1. 


\section{- Actions}

1. Examine each recording unit chart to verify which unit(s) is/are affected. The red pen line on the affected freezer or refrigerator chart should show a distinct movement toward the center of the graph.

2. Refrigerators - If one refrigerator has been affected, move all the specimens to the other refrigerator. If both refrigerators have been affected, keep the specimens cold by adding 14 frozen gel packs (stored in the bottom of each freezer) to the center of each refrigerator. Alternatively, add 25 pounds of ice cubes or blocks to each refrigerator. Do not add dry ice to the refrigerators; this will cause the refrigerator to explode.

3. Freezers - If only one freezer has been affected, move all the specimens from the affected unit to an unaffected unit. If more than one freezer has been affected and there is sufficient space in the unaffected freezers to accommodate the specimens, move the specimens to the other unaffected freezers. If there is insufficient space to accommodate the frozen specimens in the unaffected units, or if all four freezers are affected, try to reach the chief medical technologist or any of the staff medical technologists to assist. Contact the local dry ice company and order 200 pounds of dry ice. Obtain four large ice chests or coolers; each cooler should hold the boxes from one freezer and 50 pounds of dry ice. Within 2 hours or when the temperature recording chart reading reaches $-10^{\circ} \mathrm{C}$, move the containers (boxes) to the ice chests and add 50 pounds of dry ice to each ice chest. Alternatively, move the containers to Styrofoam shippers and distribute the dry ice across the shippers. Do not add dry ice to the freezers; this will cause the freezer to explode.

4. Leave a note on the unit indicating the new location of the specimens.

5. Unplug the affected unit. Each plug is bound to an extension cord or is labeled and assigned to a corresponding wall outlet.

\subsubsection{Scenario 3 - Power Loss with a Backup Generator}

If the power loss occurs when the MEC is conducting a session and there is a backup generator available, continue to monitor the temperature of the freezers and refrigerators and assess the situation with the MEC manager. If the temperature approached the established thresholds:

- Retrieve the generator from the trailer belly compartment,

- Retrieve the power strip,

- Plug the freezers into the power strip, 
- Start the generator, and

- If both refrigerators have been affected, keep the specimens cold by adding 14 frozen gel packs (stored in the bottom of each freezer) to the center of each refrigerator. Alternatively, add 25 pounds of ice cubes or blocks to each refrigerator.

If a power loss occurs and the MEC is not conducting a session, the freezers and refrigerators require action to protect the integrity of the specimens. The Sensaphone system alerts the MEC and data managers that the temperature of the freezers and/or refrigerators has exceeded established thresholds. The MEC or data managers must respond to the call, travel to the MEC immediately, and do the following:

- Retrieve the generator from the trailer belly compartment,

- Retrieve the power strip,

- Plug the freezers into the power strip,

- Start the generator, and

- If both refrigerators have been affected, keep the specimens cold by adding 14 frozen gel packs (stored in the bottom of each freezer) to the center of each refrigerator. Alternatively, add 25 pounds of ice cubes or blocks to each refrigerator. 
Appendix A

\section{International Air Transport Association (IATA) Dangerous Goods Regulations \\ January 2009}




\section{APPENDIX A \\ INTERNATIONAL AIR TRANSPORT ASSOCIATION (IATA) DANGEROUS GOODS REGULATIONS}

\section{A.1 General Provisions}

Dangerous goods can be transported safely by air transport provided certain principles are strictly followed. The International Air Transport Association (IATA) publishes dangerous goods regulations. NHANES shipments fall under these regulations because diagnostic specimens (UN 3373) are packed in dry ice, which is classified as dangerous goods, and shipped via Federal Express, which uses air transport.

Dangerous goods are articles or substances that are capable of posing a significant risk to health, safety, or to property, when transported by air and which are classified as to the type of hazard. The IATA Dangerous Goods Regulations are applicable to all shippers and agents that offer consignments of dangerous goods to airlines. A shipper must comply fully with these regulations when offering a consignment of dangerous goods to IATA member airlines, and to airlines participating in IATA interline agreements for cargo. Before offering any package for air transport, the shipper must comply with the following specific responsibilities (1.3.2).*

- A shipper must provide such information to his employees as will enable them to carry out their responsibilities with regard to the transport of dangerous goods by air.

- The shipper must ensure that the articles or substances are not prohibited for transport by air.

- The articles or substances must be properly identified, classified, packed, marked, labeled, and documented in accordance with IATA regulations.

- Before a consignment of dangerous goods is offered for air transport, all relevant persons involved in its preparation must have received training to enable them to carry out their responsibilities.

The successful application of regulations concerning the transport of dangerous goods and the achievement of their objectives are greatly dependent on the appreciation of all individuals concerning

\footnotetext{
* Notations in parentheses throughout this appendix represent the appropriate section in the IATA Dangerous Goods Regulations.
} 
the tasks involved and on a detailed understanding of the regulations. Initial and recurrent training programs achieve this for all persons concerned in the transport of dangerous goods. Training must be provided or verified upon the employment of a person in a position involving the transport of dangerous goods by air (1.5.0.2). Recurrent training must take place within 24 months of previous training to ensure knowledge is current. There is a provision for a 3-month window that allows for recurrent training conducted within the final 3 months of the 24-month period to be considered to have been completed on the expiry date of the 24-month period (1.5.0.3). A test must be undertaken following dangerous goods training to verify understanding of the regulation. A certificate must be issued confirming successful completion of the test (1.5.0.4).

Training curricula must include (1.5.2.2):

- General familiarization aimed at providing familiarity with IATA general provisions;

- Function specific training in the requirements applicable to the function for which that person is responsible; and

- Safety training to cover the hazards presented by dangerous goods, safe handling, and emergency response procedures.

A record of training must be maintained, which must include the following (1.5.5):

- The individual's name;

- The most recent training completion date;

- A description, copy, or reference to training materials used to meet the training requirement;

- The name and address of the organization providing the training; and

- Evidence which shows that a test has been completed satisfactorily.

\section{Training Curricula (table 1.5A)}

Aspects of transport of dangerous goods by air which packers should be familiar, as a minimum include:

- General philosophy;

- Limitations; 
- General requirements for shippers;

- Classifications;

- List of dangerous goods;

- General packing requirements;

- Packing instructions;

- Labeling and marking;

- Shipper's Declaration and other relevant documentation;

- Recognition of Undeclared Dangerous Goods;

- Provisions for passengers and crew; and

- Emergency procedures.

\section{A.2 Limitations}

Some dangerous goods are too dangerous to be carried by aircraft, others may be carried on cargo aircraft only, and some are acceptable on both cargo and passenger aircraft. A number of limitations are placed on dangerous goods, which are permitted to be transported by air. These limitations are established by these Regulations (2.0).

The following goods are permitted on aircraft as checked baggage only and with the approval of the operator(s) (2.3.2).

- $\quad$ Dry Ice - Carbon Dioxide, solid (dry ice) in quantities not exceeding $2.5 \mathrm{~kg}$ (5 lbs.) per person when used to pack perishables not subject to these Regulations, provided the checked baggage (package) permits release of carbon dioxide gas (2.3.4.6).

\section{A.3 Classifications}

Dangerous goods are defined as those goods which meet the criteria of one or more of the nine UN hazard classes and, where applicable, to one of the three UN packing groups according to 
provisions of IATA regulations. The nine classes relate to the type of hazard, whereas the packing groups relate to the degree of danger within the class (3.0.1). The nine classes are: Class 1 Explosives; Class 2 Gases; Class 3 Flammable Liquids; Class 4 Flammable Solids, Substances Liable to Spontaneous Combustion, Substances Which, in Contact with Water, Emit Flammable Gases; Class 5 Oxidizing Substances and Organic Peroxide; Class 6 Toxic and Infectious Substances; Class 7 Radioactive Material; Class 8 Corrosives; and Class 9 Miscellaneous Dangerous Goods.

Dangerous goods are assigned to the relevant packing group according to the degree of hazard that they present (3.0.3). The three packing groups are: Packing Group 1 - high danger; Packing Group 2 - medium danger; and Packing Group 3 - low danger.

\section{A.3.1 Division 6.2 - Infectious Substances (3.6.2)}

Division 6.2 defines infectious substances as substances which are known or are reasonably expected to contain pathogens. Pathogens are defined as microorganisms (including bacteria, viruses, rickettsiae, parasites, fungi) and other agents such as prions, which can cause disease in humans or animals. (3.6.2.1).

Infectious substances must be classified in Division 6.2 and assigned to UN 2814, UN 2900, or UN 3373, as appropriate (3.6.2.2.1). Infectious substances are divided into two categories, Category A and Category B. Category A is an infectious substance which is transported in a form that, when exposure to it occurs, is capable of causing permanent disability, or life-threatening or fatal disease in otherwise healthy humans or animals. The proper shipping name for UN 2814 is Infectious substance, affecting humans. The proper shipping name for UN 2900 is Infectious substance, affecting animals. Category B is an infectious substance which does not meet the criteria for inclusion in Category A (3.6.2.2.2.1). Infectious substances in Category B must be assigned to UN3373. The proper name of UN 3373 is Biological substance Category B (3.6.2.2.2.2).

Patient specimens for which there is minimal likelihood that pathogens are present are not subject to these Regulations if the specimen is packed in packaging that will prevent any leakage and that is marked with the words "Exempt human specimen" or "Exempt animal specimen” (3.6.2.2.3.6). The packaging must consist of three components: a leakproof primary receptacle(s), a leakproof secondary receptacle, and an outer packaging of adequate strength for its mass and intended use, and with at least 
one surface having minimum dimensions of $100 \mathrm{~mm}$ x $100 \mathrm{~mm}$. For liquids, absorbent material in sufficient quantity to absorb the entire contents must be placed between the primary receptacle(s) and the secondary packaging so that, during transport, any release or leak of a liquid substance will not reach the outer packaging and will not compromise the integrity of the cushioning material. When multiple fragile

primary receptacles are placed in a single secondary packaging, they must be either individually wrapped or separated to prevent contact between them (3.6.2.2.3.6).

\section{A.3.2 Class 9 - Miscellaneous Dangerous Goods}

Class 9 defines miscellaneous dangerous goods as substances and articles, which, during air transport, present a danger not covered by other classes (3.9.1.1). Included in this class is solid carbon dioxide, or dry ice. Dry ice is regulated by packing instruction 904 .

\section{A.3.3 List of Dangerous Goods}

UN 3373 Biological substance Category B specimens must comply with packing instruction 650 (Pg 463).

Dry ice is classified as UN number 1845 and is classified as Class 9 dangerous goods. Shipping packaging must be labeled with a Miscellaneous Class 9 label (pg. 167). Dry ice is included within Packing Group III, which is classified as a minor danger. A maximum net quantity per package is $200 \mathrm{~kg}$ or $441 \mathrm{lb}$. The relevant packing instruction is 904 (Pg 499). A special provision directs that packing tests are not considered necessary (4.4 A48 Pg 317) and when dry ice is used as a refrigerant for other dangerous goods loaded in a unit load device or other type of pallet, the quantity limits per package of $200 \mathrm{~kg}$ do not apply. (4.4 A151 Pg 323).

\section{A.4 General Packing Provisions}

The shipper is responsible for all aspects of the packing of dangerous goods in compliance with these Regulations (5.0.1.1). For packing purposes, Packing Group numbers I, II, or III are assigned 
to dangerous goods in Classes 3, 4, 5, 6, 8, and 9 according to the relative degree of danger presented by the article or substance (5.0.2.1).

- Packing Group I - Substances presenting high danger

- $\quad$ Packing Group II - Substances presenting medium danger

- Packing Group III - Substances presenting low danger

Dry ice is reassigned to packing group III. Dry ice (solid carbon dioxide) is produced by expanding liquid carbon dioxide to vapor and "snow" in presses that compact the product into blocks. Dry ice is used primarily for cooling and can cause severe burns (its temperature is about $-79^{\circ} \mathrm{C}$ ) to skin in case of direct contact. When dry ice (solid carbon dioxide) converts (sublimates) directly to gaseous carbon dioxide, it takes heat from its surroundings. The resulting gas is heavier than air and can cause suffocation in confined areas as it displaces air. Packages containing dry ice must be designed and constructed to prevent buildup of pressure due to release of carbon dioxide gas.

Dangerous goods must be packed in good quality packaging, which must be strong enough to withstand the shocks and loadings normally encountered in transport. Packages must be constructed and closed as to prevent any loss of contents when prepared for transport, which might be caused under normal conditions of transport, by vibration, or by changes in temperature, humidity, or pressure (resulting from altitude, for example) (5.0.2.4.1).

New, remanufactured, reused, or reconditioned packaging must be manufactured and tested under a program of quality assurance to ensure that such packaging meets requirements (5.0.2.5).

Packaging for which retention of liquid is a basic function, must be capable of withstanding, without leakage, an internal pressure that produces a pressure differential of not less than $95 \mathrm{kPa}$ for liquids in packing group III, Class 3, or Division 6.1 (5.0.2.9).

Packaging used for solids, which may become liquid at temperatures likely to be encountered during air transport, must also be capable of containing that substance in the liquid state (5.0.2.10).

Inner packaging - cushioning material: Inner packaging must be packed, secured, or cushioned in an outer packaging in such a way that, under normal conditions of transport, they cannot 
break, be punctured, or leak their contents into outer packaging and so as to control their movement (5.0.2.12.1).

\section{A.4.1 Packing Instruction 650 - UN 3373 (Pg 461-463)}

Packaging must be of good quality materials, strong enough to withstand the shocks and loading normally encountered during transport, including trans-shipment between transport units and warehouses as well as removal from a pallet or overpack for subsequent manual or mechanical handling. Packaging must be constructed and closed to prevent any loss of contents when prepared for transport, which might be caused under normal conditions of transport, by vibration, or by changes in temperature, humidity, or pressure.

The packaging must consist of three components:

1. A primary receptacle(s);

2. A secondary packaging; and

3. A rigid outer packaging.

Primary receptacles must be packed in secondary packaging in such a way that, under normal conditions of transport, they cannot break, be punctured, or leak their contents into secondary packaging. Secondary packaging must be secured in outer packaging with suitable cushioning material. Any leakage of the contents must not substantially impair the protection properties of the cushioning material or of the outer packaging.

Packages must be prepared as follows:

\section{0(a) For Liquids}

- The primary receptacle(s) must be leakproof and must not contain more than $1 \mathrm{~L}$.

- The secondary packaging must be leakproof.

- If multiple fragile primary receptacles are placed in a single secondary packaging, they must be individually wrapped or separated to prevent contact between them. 
- Absorbent material must be placed between the primary receptacle and the secondary packaging. The absorbent material, such as cotton wool, must be in sufficient quantity to absorb the entire contents of the primary receptacle (so that any release of the liquid substance will not compromise the integrity of the cushioning material or of the outer packaging.

- The primary receptacle or the secondary packaging must be capable of withstanding, without leakage, an internal pressure producing a pressure differential of not less than $95 \mathrm{kPa}$ in the range of $-40^{\circ} \mathrm{C}$ to $+55^{\circ} \mathrm{C}\left(-40^{\circ} \mathrm{F}\right.$ to $\left.130^{\circ} \mathrm{F}\right)$.

- The outer packaging must not contain more than $4 \mathrm{~L}$.

\section{0(b) for Solids}

- The primary receptacles must be siftproof and must not exceed the outer packaging weight limit.

- The secondary packaging must be siftproof.

- If multiple fragile primary receptacles are placed in a single secondary packaging, they must be individually wrapped or separated to prevent contact between them.

- The outer packaging must not contain more than $4 \mathrm{~kg}$. This includes ice, dry ice, or liquid nitrogen when used to keep specimens cold.

- If there is any doubt as to whether or not residual liquid may be present in the primary receptacle during transport then a packaging suitable for liquids, including absorbent material, must be used.

\section{Package Testing}

- Each completed package must be capable of successfully passing the following drop tests described in 6.5.1.1 except that the height of the drop must not be less than $1.2 \mathrm{~m}$. Following the appropriate drop sequence, there must be no leakage from the primary receptacle(s) which must remain protected by absorbent material, when required, in the secondary packaging.

\section{For Liquids and Solids}

- An itemized list of contents must be enclosed between the secondary packaging and the outer packaging.

- Packages must have one side with dimensions of not less than $100 \mathrm{~mm}$ x $100 \mathrm{~mm}$ (4 in. $x 4$ in.) 
- For transport, the mark illustrated below must be displayed on the external surface of the outer packaging on a background of contrasting color and must be clearly visible and legible. The mark must be in the form of a square set at an angle of $45^{\circ}$ (diamondshaped) with each side having a length of at least $50 \mathrm{~mm}$ (2 in.), the width of the line must be at least $2 \mathrm{~mm}$ and the letters and numbers must be at least $6 \mathrm{~mm}$ high.

- The proper shipping name "Biological Substance, Category B" letters at least $6 \mathrm{~mm}$ high must be marked on the outer package adjacent to the diamond-shaped mark.

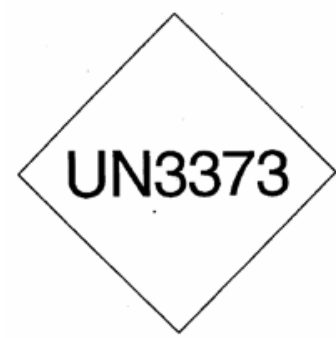

Unless all package markings are clearly visible, the following conditions apply when packages are placed in an overpack:

- The overpack must be marked with the work "Overpack;” and

- The package markings must be reproduced on the outside of the overpack.

Specific Requirements - refrigerated or frozen specimens: ice, dry ice, and liquid nitrogen

- When dry ice or liquid nitrogen is used to keep specimens cold, all applicable requirements of these Regulations must be met. When used, ice or dry ice must be placed outside the secondary packaging or in the outer packaging or an overpack. Interior supports must be provided to secure the secondary packagings in the original position after the ice or dry ice (solid carbon dioxide) has been dissipated. If ice is used, the packaging or overpack must be leakproof. If dry ice (solid carbon dioxide) is used, the outside packaging or overpack must be designed and constructed to permit the release of carbon dioxide gas to prevent a build-up of pressure that could rupture the packaging.

- The primary receptacle and the secondary packaging must maintain their integrity at the temperature of the refrigerant used as well as the temperatures and the pressures, which could result if refrigeration were to be lost. 
- Infectious substances assigned to UN 3373 which are packed and marked in accordance with this packing instruction are not subject to any other requirement of these regulations except for the following:

(a) The name, address, and telephone number of the shipper and consignee must be provided on each package;

(b) The name, and telephone number of a person responsible must be provided on the air waybill or on the package;

(c) The classification must be in accordance to 3.6.2 (see Section A.3.1);

(d) The incident reporting requirements in 9.6.1 must be met; and

(e) The inspection for damage or leakage requirements in 9.4.1 and 9.4.2.

9.6.1 - An operator must report dangerous goods accidents or incidents to the appropriate authorities of the State of the operator and the State in which the accident or incident occurred, in accordance with the reporting requirements of those appropriate authorities.

9.4.1 - Packages and overpacks containing dangerous goods must be inspected for signs of damage or leakage upon unloading from the aircraft or unit load device. If evidence of damage or leakage is found, the position where the dangerous goods or unit load was stowed on the aircraft must be inspected for damage or contamination and any hazardous contamination removed. The special responsibilities of operators regarding infectious substances are detailed in 9.4.2. (9.4.1)

9.4.2 - If any person responsible for the carriage of packages containing infectious substances becomes aware of damage to or leakage from such a package, that person must:

- $\quad$ Avoid handling the package or keep handling to a minimum;

- $\quad$ Inspect adjacent packages for contamination and put aside any that may be contaminated;

- $\quad$ Inform the appropriate public health authority, and provide information on any other countries of transit where persons may have been exposed to danger; and

- $\quad$ Notify the shipper and/or consignee.

- If an air waybill is used, the "Nature and Quantity of Goods" box should show the text "BIOLOGICAL SUBSTANCE, CATEGORY B.”

- A Shipper's Declaration for Dangerous Goods is not required. 
- Clear instructions on filing and closing such packages must be provided by packaging manufacturers and subsequent distributors to the shipper or to the person who prepares the package to enable the package to be correctly prepared for transport.

\section{A.4.2 Packing Instruction 904 - Dry Ice (Pg 499)}

This instruction applies to UN 1845 on passenger and cargo aircraft. The General Packaging Requirements of 5.0.2 must be met.

Dry ice (solid carbon dioxide), when offered for transport by air, must be in packaging designed and constructed to permit the release of carbon dioxide gas and to prevent a build-up of pressure that could rupture the packaging.

The net weight of dry ice must be marked on the outside of the package.

Arrangements between the shipper and operator(s) must be made for each shipment to ensure that ventilation and safety procedures are followed.

When a Shipper's Declaration is not required, the following information as required by 8.2.3 for dry ice (solid carbon dioxide) must be contained in the "Nature and Quantity of Dangerous Goods" box on the air waybill:

- UN 1845;

- $\quad$ Proper shipping name (Dry Ice or Carbon Dioxide, solid);

- 9 (the word “Class” may be included prior to the number " 9 ”);

- The number of packages; and

- The net weight of dry ice in each package.

The net weight of the dry ice must be marked on the outside of the package. 


\section{A.5 Packing Specifications and Performance Tests}

Performance tests are designed to ensure that there will be no loss of contents under normal transport conditions. The severity of the tests on a packaging is dependent on the intended contents, taking account of the degree of danger; that is, packing group, relative density (specific gravity), and vapor pressure (for liquids) (6.3.0.2).

The design of each packaging type must be tested as provided in accordance with procedures established by IATA regulation (6.3.1). Tests must be successfully performed on each packaging type before such packaging is used (6.3.1.1.1).

\section{A.6 Marking and Labeling}

All markings must be so placed on packages or overpacks that they are not covered or obscured by any part of or attachment to the packaging or any other label or marking (7.1.3.1).

All markings must be:

- Durable and printed or otherwise marked on, or affixed to, the external surface of the package or overpack;

- Readily visible and legible;

- Able to withstand open weather exposure without a substantial reduction in effectiveness; and

- Displayed on a background of contrasting color (7.1.3.2).

Unless all markings representative of all dangerous goods in the overpack are clearly visible, the overpack must be marked with the word "Overpack” (7.1.4).

Each package must be marked durably and legible on the outside with each of the following:

- The proper shipping name of the contents and the corresponding UN numbers (7.1.5.1a) (BIOLOGICAL SUBSTANCE, CATEGORY B is UN 3373 and dry ice is UN 1845;

- The full name and address of the shipper and the consignee (7.1.5.1b); 
- The NET Quantity of dry ice contained in each package or overpack must be shown. The quantity must be marked on each package or overpack adjacent to the UN number and Proper Shipping Name (7.1.5.1e

- The name and telephone number of a responsible person (7.1.5.1f); and

- For packages containing UN 3373: - "BIOLOGICAL SUBSTANCE, CATEGORY B.” (7.1.5.1h).

\section{Empty Packaging}

- Other than Class 7, a packaging which previously contained dangerous goods must be marked as required for those dangerous goods unless steps such as cleaning, purging of vapors, or refilling with a nondangerous substance are taken to nullify any hazard (7.1.5.6).

There are two types of labels: hazard labels (in the shape of a square set at $45^{\circ}$ ) which are required for most dangerous goods in all classes and handling labels (in various rectangular shapes), which are required, wither alone or in addition to hazard labels for some dangerous goods (7.2.2.2).

A primary hazard label is specified for each listed article and substance. A secondary hazard label or labels must also be applied for each article and substance having subsidiary risk (7.2.3.1). For Class 9 materials, the package must bear the class 9, "Miscellaneous Dangerous Goods” label as required for all dry ice shipments (7.2.3.10).

All labels must be securely affixed or printed on the packaging so that they are readily visible and legible and not obscured by any part of the packaging or by any other label (7.2.6.1a). Each label must be affixed on a background of contrasting color or must have a dotted or solid line outer border (7.2.6.1b). The label must not be folded or affixed in such a manner that different parts of the label appear on different faces of the package (7.2.6.1c). The package must be of such a size that there is adequate space to affix all required labels (7.2.6.1e).

Before an empty packaging which had previously contained infectious substances is referred to the shipper, or sent elsewhere, it must be thoroughly disinfected or sanitized and any label or marking indicating that it had contained an infectious substance must be removed or obliterated. (7.2.3.12.2)

Labels must be located on the same surface of the package near the proper shipping name marking if the package dimensions are adequate (7.2.6.2.1). 


\section{A.7 Documentation}

Except as otherwise specified, a "Shipper's Declaration for Dangerous Goods form and an “Air Waybill” must be completed for each consignment of dangerous goods (8.0.1). A "Shipper's Declaration for Dangerous Goods" is not required for shipments containing diagnostic specimens (Pg 463) or dry ice (Pg 499).

If a Shipper's Declaration is not required for dangerous goods, the Nature and Quantity of Goods” box of the Air Waybill must show in sequence (8.2.3):

- UN or ID number;

- $\quad$ Proper Shipping Name;

- Class or Division number;

- Number of packages; and

- $\quad$ Net quantity per package.

For UN 3373, it is only necessary to show the text "BIOLOGICAL SUBSTANCE; CATEGORY B" and "UN 3373.” For dry ice, it is not necessary to show the packing group and packing instruction number on the air waybill.

\section{A.8 References}

1. IATA Dangerous Goods Regulations, 50th Edition, effective January 1, 2009, ISBN 978-92-9233-006-4

2. FedEx Service Guide Vol. 1, effective June 1, 2000. 


\section{A.9 IATA Regulations Examination}

Name

Date

1. IATA is the acronym for:

2. Dangerous goods are articles or substances that are capable of posing a significant risk to health, safety or to property, when transported by air and which are classified as to the type of hazard. (T or F)

3. What are the four responsibilities the shipper must comply with before offering any package for air transport?

4. List the three training requirements:

5. How many UN hazard classes are there?

6. What are the two categories of infectious substances?

7. What is the proper shipping name of UN 3373 ?

8. To what UN hazard class does dry ice belong? 
9. What is the dry ice UN number?

10. To what packing group does dry ice belong?

11. Solid carbon dioxide or dry ice is produced by expanding liquid carbon dioxide to vapor and "snow" in presses that compact the product into blocks. Dry ice is used primarily for cooling and can cause severe burns (its temperature is about $-79^{\circ} \mathrm{C}$ ) to skin in case of direct contact. When dry ice (solid carbon dioxide) converts (sublimates) directly to gaseous carbon dioxide, it takes heat from its surroundings. The resulting gas is heavier than air and can cause suffocation in confined areas as it displaces air. Packages containing dry ice must be designed and constructed to prevent buildup of pressure due to release of carbon dioxide gas.

( $\mathrm{T}$ or F)

12. Define "Exempt human specimen.”

13. What packing instruction regulates UN3373?

14. List the three packaging components in packing instruction 650:

15. Properly prepared shipments containing liquids must meet the following 6 conditions: 
16. An itemized list of contents must be placed between the secondary packaging and the outer packaging. ( $\mathrm{T}$ or $\mathrm{F}$ )

17. The proper shipping name "Biological Substance, Category B" letters at least $6 \mathrm{~mm}$ high must be marked on the outer package adjacent to the diamond-shaped mark. (T or F)

18. When used, ice or dry ice must be placed outside the secondary packaging or in the outer packaging or overpack. Interior supports must be provided to secure secondary packagings in the original position after all the ice or dry ice has dissipated. (T or F)

19. The weight of dry ice is required on the outside of the packaging. ( $\mathrm{T}$ or $\mathrm{F}$ )

20. The performance tests are designed to ensure that there will be under normal transport conditions.

21. All markings must be:

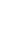

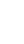

22. For Class 9 goods (dry ice), the package is required to bear the label. (T or F)

23. All labels must be securely affixed or printed on the packaging so that they are readily visible and legible and not obscured by any part of the packaging or by any other label. (T or F)

24. An "air waybill” must be completed for each consignment of dangerous goods (8.0.1). (T or F) 


\section{A.10 IATA Regulations Examination (Answer Key)}

Name

Date

1. IATA is the acronym for the International Air Transport Association

2. Dangerous goods are articles or substances that are capable of posing a significant risk to health, safety or to property, when transported by air and which are classified as to the type of hazard. (T or F) True

3. What are the four responsibilities the shipper must comply with before offering any package for air transport?

- Information to employees as will enable them to carry out their responsibilities.

- $\quad$ Ensure that articles or substances are not prohibited for transport by air.

- $\quad$ Articles must be properly identified, classified, packed, marked, labeled, and documented.

- $\quad$ All persons involved in preparing consignment must have received training.

4. List the three training requirements:

- General familiarization aimed at providing familiarity with IATA general provisions;

- $\quad$ Function specific training in the requirements applicable to the function for which that person is responsible; and

- Safety training to cover the hazards presented by dangerous goods, safe handling, and emergency response procedures.

5. How many UN hazard classes are there? 9 (nine)

6. What are the two categories of infectious substances?

- $\quad$ Category A

- $\quad$ Category B

7. What is the proper shipping name of UN 3373? Biological Substance Category B

8. To what UN hazard class does dry ice belong? Class 9 
9. What is the dry ice UN number? UN 1845

10. To what packing group does dry ice belong? Packing group 3

11. Solid carbon dioxide or dry ice is produced by expanding liquid carbon dioxide to vapor and "snow" in presses that compact the product into blocks. Dry ice is used primarily for cooling and can cause severe burns (its temperature is about $-79^{\circ} \mathrm{C}$ ) to skin in case of direct contact. When dry ice (solid carbon dioxide) converts (sublimates) directly to gaseous carbon dioxide, it takes heat from its surroundings. The resulting gas is heavier than air and can cause suffocation in confined areas as it displaces air. Packages containing dry ice must be designed and constructed to prevent buildup of pressure due to release of carbon dioxide gas.

(T or F) True

12. Define "Exempt human specimen."

Patient specimens for which there is minimal likelihood that pathogens are present are not subject to these Regulations if the specimen is packed in packaging that will prevent any leakage and that is marked with the words "Exempt human specimen."

13. What packing instruction regulates UN3373? Packing instruction 650

14. List the three packaging components in packing instruction 650 .

- $\quad$ Primary receptacle

- A secondary packaging

- $\quad$ A rigid outer packaging

15. Properly prepared shipments containing liquids must meet the following 6 conditions:

- The primary receptacle must be leakproof.

- The secondary packaging must be leakproof.

- $\quad$ Fragile receptacles must be individually wrapped or separated to prevent contact.

- $\quad$ Absorbent material must be placed between primary receptacle and secondary packaging.

- $\quad$ Primary receptacle or secondary packaging must be capable of withstanding an internal pressure producing a pressure differential of not less than $95 \mathrm{kPa}$ in the range of $-40^{\circ} \mathrm{C}$ to $+55^{\circ} \mathrm{C}$.

- $\quad$ The outer package must not contain more than $4 \mathrm{~L}$.

16. An itemized list of contents must be placed between the secondary packaging and the outer packaging. (T or F) True 
17. The proper shipping name "Biological Substance, Category B" letters at least $6 \mathrm{~mm}$ high must be marked on the outer package adjacent to the diamond-shaped mark. ( $\mathrm{T}$ or F) True

18. When used, ice or dry ice must be placed outside the secondary packaging or in the outer packaging or overpack. Interior supports must be provided to secure secondary packagings in the original position after all the ice or dry ice has dissipated. (T or F) True

19. The weight of dry ice is required on the outside of the packaging.

(T or F) True

20. The performance tests are designed to ensure that there will be no loss of contents__ under normal transport conditions.

21. All markings must be:

- Durable and printed or otherwise marked on, or affixed to, the external surface of the package or overpack;

- Readily visible and legible;

- $\quad$ Able to withstand open weather exposure without a substantial reduction in effectiveness; and

- Displayed on a background of contrasting color.

22. For Class 9 goods (dry ice), the package is required to bear the Class 9 "Miscellaneous" dangerous goods label. ( $\mathrm{T}$ or F) True

23. All labels must be securely affixed or printed on the packaging so that they are readily visible and legible and not obscured by any part of the packaging or by any other label. ( $T$ or F) True

24. An "air waybill” must be completed for each consignment of dangerous goods (8.0.1). (T or F) True

$50^{\text {th }}$ Edition International Air Transport Association. 


\section{WESTAT \\ Certificate of Test Completion}

International Air Transport Association (IATA) Dangerous Goods Regulations

Name

Date of Successful Exam

Examiner 


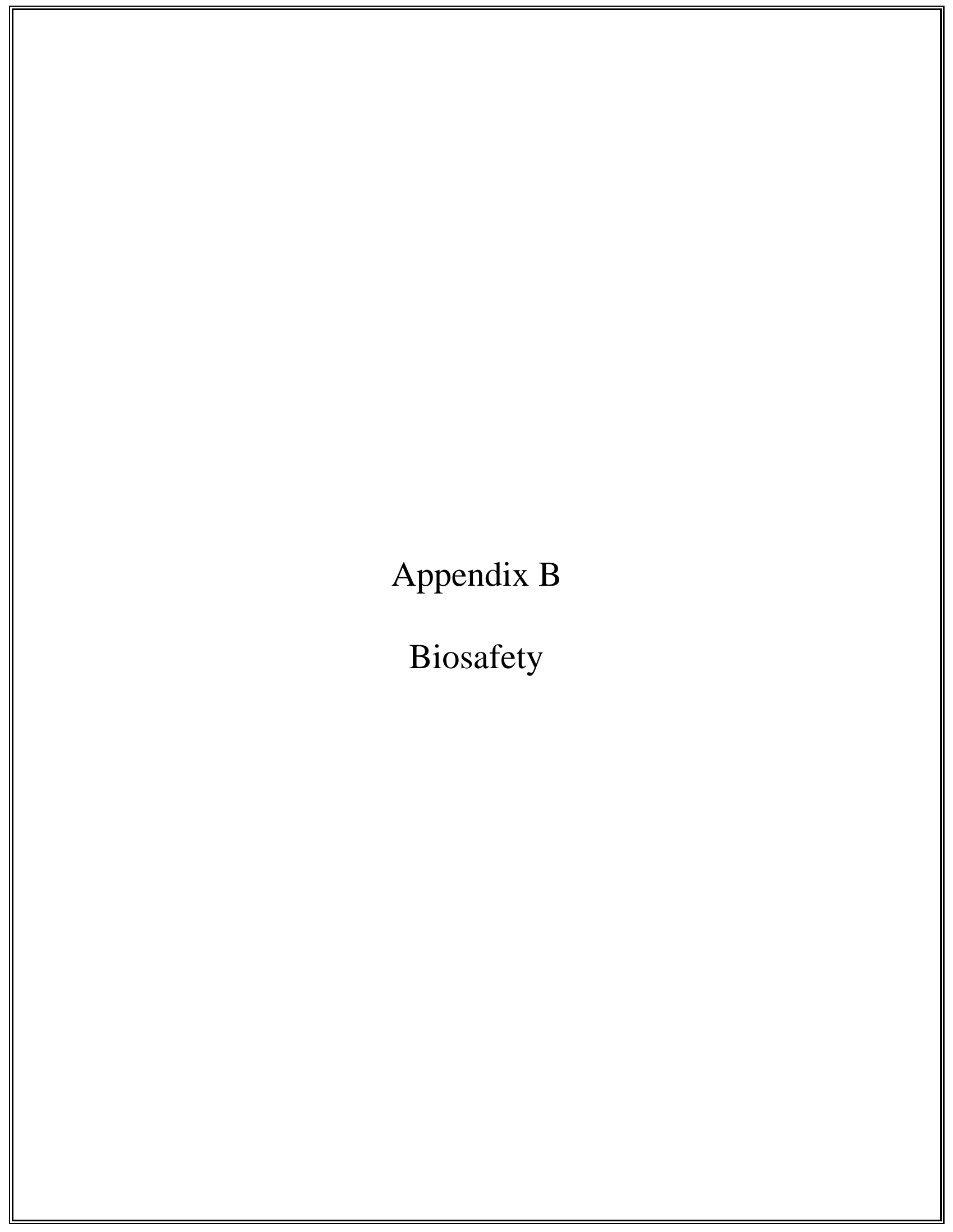




\section{APPENDIX B. BIOSAFETY}

\section{B. POLICY FOR MANAGEMENT OF EXPOSURE TO BLOODBORNE PATHOGENS}

\section{Exposure Control Plan for NHANES Training and Examinations}

\section{B.1 Introduction}

The policy presented in this section is intended to serve as the written policy for the NHANES mobile examination center staff training and main study period. It is based on the OSHA Regulations for exposure to bloodborne pathogens as published in the Federal Register (29CFR 1910.1030), the Updated U.S. Public Health Service Guidelines for the Management of Occupational Exposure to HBV, HCV, and HIV and Recommendations for Postexposure Prophylaxis (MMWR, June 29, 2001), the CDC recommendations, Immunization of Health-Care Workers: Recommendations of the Advisory Committee on Immunization Practices (ACIP) and the Hospital Infection Control Practices Advisory Committee (HICPAC) (December 1997) and the revised Occupational Exposure to Bloodborne Pathogens: Needlestick and Other Sharps injuries; Final Rule.

This manual describes the procedures required to treat and document blood exposure injuries to MEC staff. Universal precautions are the foundation of standard operating procedures and emergency procedures throughout the exam center. MEC staff, the home office staff, and NCHS monitor compliance with these precautions and the pertinent OSHA regulations.

OSHA's rule applies to all persons occupationally exposed to blood or other potentially infectious materials. Blood means human blood, blood products, or blood components. On NHANES, the only body fluids that are collected are blood, vaginal swabs, and urine. Urine is not considered infectious unless the specimens are visibly contaminated with blood or are known to contain an infectious agent.

The Occupational Safety and Health Administration revised the Bloodborne Pathogens standard in conformance with the requirements of the Needlestick Safety and Prevention Act. This Act directs OSHA to: (1) revise the Bloodborne Pathogens standard to include new examples in the definition of engineering controls along with two new definitions; (2) require that Exposure Control Plans reflect how employers implement new developments in control technology; (3) require employers to solicit 
input from employees responsible for direct patient care in the identification, evaluation, and selection of engineering and work practice controls; and (4) require certain employers to establish and maintain a log of percutaneous injuries from contaminated sharps.

\section{B.2 Exposure Determination}

\section{B.2.1 MEC Employees by Group}

Exposure determination is based on the definition of occupational exposure without regard to personal protective clothing and equipment. Job classifications within the work environment are reviewed for exposure and two groups of exposures are identified: Category I job classifications in which all the employees have occupational exposure, and Category II classifications in which some of the employees have occupational exposure. Where all employees have occupational exposure, it is not necessary to list specific work tasks. Where only some employees have occupational exposure, procedures causing occupational exposure must be listed.

Occupational exposure for NHANES staff is categorized as follows:

\section{Category I Exposures}

- Phlebotomist

- Medical technologist

- Dental hygienist

\section{Category II Exposures}

- Physician

- $\quad$ MEC manager

The physician may risk exposure in the event of an emergency in the MEC. The physician is expected to respond to any emergency and perform appropriate procedures, but this does not include the initiation of IV fluids. The MEC manager is responsible for insuring that biohazardous waste is removed and thus may occasionally handle bagged biohazardous waste. The MEC manager is also expected to respond to emergencies and thus may risk exposure if the emergency involves a laceration. 


\section{B.3 Methods of Compliance}

\section{B.3.1 Control for Category I and Category II Exposures}

Risks posed by bloodborne pathogens may be reduced through vaccination, engineering controls, employee work practices, personal protective equipment, and housekeeping procedures. Bloodborne pathogens means pathogenic microorganisms that are present in human blood and can cause disease in humans. These pathogens include, but are not limited to, hepatitis B virus (HBV), hepatitis C virus (HCV), and human immunodeficiency virus (HIV).

Because some infectious diseases can be communicable through contact with SPs and blood, all Category I and Category II staff members are advised to:

- Obtain the hepatitis B vaccine if recommended by Westat for their category of exposure; and

- Use universal precautions (see Section B.3.2) when there is a potential for exposure to blood or body fluids.

\section{B.3.2 General - Universal Precautions}

Universal Precautions is an approach to infection control. According to the concept of Universal Precautions, all human blood and certain human body fluids are treated as if known to be infectious for HIV, HBV, and other bloodborne pathogens. Universal precautions are observed to prevent contact with blood or other potentially infectious materials. Other Potentially Infectious Materials means: (1) the following human body fluids: semen, vaginal secretions, cerebrospinal fluid, synovial fluid, pleural fluid, pericardial fluid, peritoneal fluid, amniotic fluid, saliva in dental procedures, any body fluid that is visibly contaminated with blood, and all body fluids in situations where it is difficult or impossible to differentiate between body fluids; (2) any unfixed tissue or organ (other than intact skin) from a human (living or dead); and (3) HIV-containing cell or tissue cultures, organ cultures, and HIV- or HBVcontaining culture medium or other solutions; and blood, organs, or other tissues from experimental animals infected with HIV or HBV. Universal precautions are applied to procedures involving all sample persons. 


\section{B.3.3 Engineering and Work Practice Controls}

Engineering and work practice controls are the primary methods used to prevent occupational transmission of HBV, HIV, and other bloodborne pathogens. Sharps disposal containers, self-sheathing needles, and biological safety cabinets are examples of engineering controls used in the NHANES.

Biological safety cabinets are similar in appearance to laboratory fume hoods and protect employees from exposure to airborne biohazards by providing an enclosed, mechanically ventilated space for them to work. A laminar flow biological safety cabinet is located in the laboratory in every MEC.

Sharps containers are liquid-tight, puncture-resistant vessels used to minimize the hazards posed by discarded needles, broken glass, scalpels, and other contaminated sharp objects. Light plastic bottles such as those that hold consumer products are unacceptable because the walls are easily penetrated by sharps. Metal, heavy-gauge plastic or laminated multi-ply cardboard containers are a much better choice.

Engineering controls isolate or remove the bloodborne pathogen hazard from the workplace. Work Practice Controls are controls that reduce the likelihood of exposure by altering the manner in which a task is performed (e.g., prohibiting recapping of needles by a two-handed technique). These include procedures for processing and handling blood and blood products, waste disposal, and personal hygiene.

\section{Category I}

Specific procedures for preventing exposures in Category I staff are as follows:

- All materials intended for reuse are inspected and decontaminated on a regular basis and are cleaned and decontaminated immediately upon visible contamination.

- Sinks that can be used for hand washing are provided in the bathrooms in trailers 1 and 4 and in the hallways in trailers 1 and 2, in the laboratory, phlebotomy room, and staff lounge in trailer 3 , and in the dental exam room in trailer 4 . The laboratory and dental sinks include an electronic eye on/off mechanism. 
- All MEC exam staff are instructed to wash hands with soap and water after removing gloves or upon accidental skin contact with blood or body fluids, and between each SP exam.

- Employees are instructed to wash hands and any other skin with soap and water, or flush mucous membranes with water immediately or as soon as feasible following contact of such body areas with blood or other potentially infectious materials.

- Sharps should be handled with extreme care and discarded into the needle disposal containers provided in the MEC. Staff members are instructed that contaminated needles and other contaminated sharps shall not be bent, recapped, or removed. Shearing or breaking of contaminated needles is prohibited. They are required to use the shielding mechanism. Contaminated sharps means any contaminated object that can penetrate the skin including, but not limited to, needles, scalpels, broken glass, broken capillary tubes, and exposed ends of dental wires. Use of the safety shielding mechanism is mandatory.

- Discarded needles and other waste sharps must be placed in an "approved" sharps container. Care must also be taken when mounting a sharps container on the walls and cabinets to make sure the container is accessible to everyone. Immediately or as soon as possible after use, contaminated reusable sharps are placed in puncture resistant and leakproof containers until properly reprocessed. These containers are identified as containing potentially infectious materials and are not allowed to overfill.

- Employees are instructed not to eat, drink, smoke, handle contact lenses, or apply cosmetics or lip balm in the exam rooms.

- No food or drinks are kept in refrigerators, freezers, shelves, cabinets, or work surfaces in the examination rooms. An employee lounge with a refrigerator is provided for personal food storage.

- Procedures involving blood or other potentially infectious materials are performed in such a manner as to minimize splashing, spraying, spattering, or aerosolizing. For example, the splattering of blood or serum that occurs when rubber stoppers are pulled out of specimen tubes is minimized by covering the stopper with gauze before removing it and performing this procedure under the biosafety hood.

- Mouth pipetting/suctioning of blood or other potentially infectious materials is prohibited.

- Equipment should be checked and decontaminated before servicing and shipping. Decontamination means the use of physical or chemical means to remove, inactivate, or destroy bloodborne pathogens on a surface or item to the point where it is no longer capable of transmitting infectious particles and the surface or item is rendered safe for handling, use, or disposal. 
- Work surfaces and or equipment contaminated with blood or body fluids are washed with detergent and disinfected with a 1:10 or 1:100 dilution of bleach at the end of every examination session, as described in the examiner manuals.

- Protective coverings used to cover equipment and environmental surfaces are removed and replaced as soon as feasible when overtly contaminated with blood or body fluids during the exam session.

- Broken glassware is never picked up by hand, but swept up with a brush and dustpan. To minimize aerosol formation, broken glass is not dropped into a disposal container but gently placed in the container.

\section{Category II}

Although the MEC is not set up to treat or manage medical problems, medical emergencies may occur. If the staff must administer CPR, it is recommended that all staff observe the following procedures to prevent exposures:

- Wear gloves and a lab coat when performing emergency procedures. Disposable impervious lab jackets are available in the lab if needed.

- Use the disposable resuscitation masks found in designated areas.

- Follow the instructions of the MEC physician, who will direct the emergency response.

- Decontaminate and/or dispose of all emergency medical supplies.

- Change any protective equipment contaminated with blood or body fluids.

- Wash hands after removing gloves and before working with the next SP.

\section{B.3.4 Personal Protective Equipment}

Personal Protective Equipment is used when occupational exposure remains after instituting engineering and work practice controls. Personal protective equipment is specialized clothing or equipment worn by an employee for protection against a hazard. General work clothes (e.g., uniforms, pants, shirts, or blouses) not intended to function as protection against a hazard are not considered personal protective equipment. 
The selection of protective equipment depends on the nature of the potential exposure but generally includes gloves, lab coats, and gowns. If there is a chance for potentially infectious material to splash into the eyes or mouth, staff members are instructed to wear face protection in the form of a mask and/or safety glasses with side shields. The employer provides all protective equipment in appropriate sizes and sufficient quantities for staff use. Hypoallergenic gloves or other alternatives are available to employees with allergic sensitivity to gloves.

Staff members other than those in Categories I or II are not expected to perform work procedures that carry risk for exposure to blood or body fluids but the following precautions are communicated to the staff in training and monitored:

- Staff should not perform any procedure or task for which they have not been trained.

- Gloves, disposable impervious lab jackets, and/or safety glasses are worn when performing venipuncture, and laboratory procedures. Personal protective equipment is provided to the staff. Disposable impervious lab jackets are provided to the medical technologists, phlebotomists, and health technologists performing the oral health examination. Reusable cloth lab coats are provided to the physician and MEC manager and are laundered by the employer if contaminated with blood or body fluids. Safety glasses are optional for the phlebotomist and are available onsite. Disposable, powder-free hypoallergenic nonlatex powder-free gloves are provided to the medical technologists, phlebotomist, and dental examiners. Wear appropriate gloves if contact with blood, or other potentially infective materials is anticipated. Never wash or decontaminate disposable gloves for reuse.

- Gloves are changed after each sample person exam or when visibly soiled with blood and/or body fluids. Disposable impervious lab jackets worn by the laboratory and phlebotomy staff are discarded when soiled and at the end of each workweek. Disposable impervious lab jackets worn by the health technologists during the oral health examination are changed when visibly soiled with blood and body fluids. All disposable impervious lab jackets are removed before leaving the work area and after a garment becomes contaminated. Place used protective equipment in appropriately designated areas or containers when being stored or discarded.

\section{B.3.5 Housekeeping}

Under the OSHA standard, all areas must be kept clean and sanitary. A cleaning schedule that includes appropriate methods of decontamination procedures has been developed for every component and is included in the component-specific procedure manuals. These procedures are included in component training programs and are part of daily component procedures. Cleaning procedures are 
based on the type of surfaces, the type of contamination present, the tasks or procedures, and their location in the facility. Housekeeping staff also receive a written set of instructions for cleaning designated areas of the MEC.

The following housekeeping procedures must be observed and will be monitored by project staff:

- Clean and decontaminate all equipment and environmental and work surfaces contaminated with blood or other potentially infectious materials.

- Decontaminate work surfaces with an appropriate disinfectant after completion of procedures, immediately when overtly contaminated, after any spill of blood or other potentially infectious material, and at the end of the work shift when surfaces have become contaminated since the last cleaning.

- Remove and replace protective coverings such as plastic wrap.

- Inspect and decontaminate, on a regular basis, reusable receptacles such as bins, pails, and cans that have the likelihood for becoming contaminated. When contamination is visible, clean and decontaminate receptacles immediately, or as soon as possible.

- Place other regulated waste in closable and labeled or color-coded containers. When storing, handling, transporting, or shipping, place other regulated waste in containers that prevent leakage.

- When discarding contaminated sharps, place them in containers that are closable, puncture-resistant, appropriately labeled or color-coded, and leakproof on the sides and bottom.

- Ensure that sharps containers are easily accessible to personnel and located as close as feasible to the use area. Keep sharps containers upright throughout use, replace routinely, close when moved, and do not allow to overfill.

- Never manually open, empty, or clean contaminated sharps disposable containers.

- Discard regulated waste into appropriate containers, labeled as biohazardous waste, and seal to prevent leakage during handling, storage, and transport. Regulated Waste means liquid or semiliquid blood or other potentially infectious materials; contaminated items that would release blood or other potentially infectious materials in a liquid or semiliquid state if compressed; items that are caked with dried blood or other potentially infectious materials and are capable of releasing these materials during handling; contaminated sharps; and pathological and microbiological wastes containing blood or other potentially infectious materials. A hazardous waste company is responsible for the disposal of biohazardous waste from the MEC. 
Specific methods and regular schedules for cleaning bench tops, equipment, and other environmental surfaces contaminated with potentially infectious materials are documented in the Infection Control Manual and in the respective component procedure manuals. The time required for cleanup may be reduced if surfaces are covered before work with an impermeable, disposable covering such as plastic sheeting or absorbent bench pads.

Good housekeeping procedures are not limited to areas in which infectious materials are handled. Prudent safety practice dictates that halls and passageways are kept clear of obstructions such as carts, obsolete equipment, and shipping containers. Fire doors, other than those interconnected to smokedetection systems, are kept closed. Stairs are not obstructed and exits are clearly marked.

Contaminated laundry is handled as little as possible and placed in labeled bags at locations where it is used. Leakproof bags are used to transport wet laundry. In addition, all employees who work with contaminated laundry wear gloves.

\section{B.4 Hepatitis B Vaccine}

The hepatitis B vaccine is made available to Category I and II MEC staff at no cost and at a reasonable time and place within 10 working days of initial assignment, and to any MEC staff following an exposure incident. Employees who refuse the hepatitis vaccine must sign the Declination Form, which is kept with the employee's medical records. Employees who have previously received the vaccine should provide evidence of antibody testing or completion of the vaccine series.

If an exposure incident occurs, the MEC manager and MEC physician must be notified immediately. The MEC physician must complete a Medical Incident Form, and the employee must receive an immediate medical evaluation to assess their level of vaccine protection, their level of exposure, and to recommend an appropriate treatment regimen per the Updated U.S. Public Health Service Guidelines for the Management of Occupational Exposure to HBV, HCV, and HIV and Recommendations for Postexposure Prophylaxis (MMWR, June 29, 2001). 


\section{B.4.1 Procedures for Evaluating Exposure Incidents}

The OSHA standard requires that following a report of an exposure incident, the employer shall make immediately available to the exposed employee a confidential medical evaluation and followup. Exposure Incident means a specific eye, mouth, other mucous membrane, nonintact skin, or parenteral contact with blood or other potentially infectious materials that results from the performance of an employee's duties. The exposure report must include at least the following elements:

1. Date and time of exposure;

2. Documentation of the route(s) of exposure, and the circumstances under which the exposure incident occurred;

3. Identification and documentation of the source individual, unless the employer can establish that identification is infeasible or prohibited by state or local law;

a. The source individual's blood shall be tested as soon as feasible and after consent is obtained in order to determine HBV, HCV, and HIV infectivity. If consent is not obtained, the employer shall establish that legally required consent cannot be obtained. When the source individual's consent is not required by law, the source individual's blood, if available, shall be tested and the results documented.

b. Results of the source individual's testing shall be made available to the exposed employee's physician, and the employee shall be informed of applicable laws and regulations concerning disclosure of the identity and infectious status of the source individual.

4. Collection and testing of blood for HBV, HCV, and HIV serological status;

a. The exposed employee's blood shall be collected as soon as feasible and tested after consent is obtained.

b. If the employee consents to baseline blood collection, but does not give consent at that time for HIV serologic testing, the sample shall be preserved for at least 90 days. If, within 90 days of the exposure incident, the employee elects to have the baseline sample tested, such testing shall be done as soon as feasible.

5. Post-exposure prophylaxis, when medically indicated, as recommended by the U.S. Public Health Service;

6. Counseling; and

7. Evaluation of reported illnesses. 
It is important that the employee have access to treatment for possible HBV, HCV, or HIV exposure as soon as possible after the incident because HBIG (Hepatitis B immunoglobulin), hepatitis B vaccine, and HIV PEP (postexposure prophylaxis) are most likely to be effective if administered as soon after the exposure as possible. During training, the training supervisor, the Director of MEC Operations, Catherine Novak, and/or the Director of Examinations, Carla Maffeo, Ph.D., will oversee the response to a needlestick or other exposure. During the examination period, the MEC physician is responsible for initiating the response, referring the employee to the nearest emergency department, notifying home office staff, and documenting the incident.

At a minimum, the evaluation and followup must include the following elements:

- Treatment of Exposure Site

- $\quad$ Wounds and skin sites that have been in contact with blood or body fluids should be washed with soap and water; mucous membranes should be flushed with water. No evidence exists that using antiseptics for wound care or expressing fluid by squeezing the wound further reduces the risk of bloodborne pathogen transmission; however, the use of antiseptics is not contraindicated. The application of caustic agents (e.g., bleach) or the injection of antiseptics or disinfectants into the wound is not recommended.

- Exposure Report

- $\quad$ Document the routes of exposure and how the exposure occurred on a Medical Incident Form and OSHA's Forms 300 and 301 (See Section 5.6 in Chapter 5 of the Infection Control Procedures Manual). The Medical Incident Form can be accessed from any exam room by clicking on the ambulance icon on the toolbar.

- Evaluation of the Employee and the Exposure

- The employee should proceed immediately to the closest emergency department where they can be evaluated and counseled. Hospitals are available during all working hours, including nights and weekends. HBIG, hepatitis B vaccine, and antiretroviral agents for HIV PEP should be available for timely administration (i.e., either by providing access on site or by creating linkages with other facilities or providers to make them available off site). The evaluating emergency physician is responsible for providing postexposure management and counseling. They are familiar with evaluation and treatment protocols and whether the hospital has access to HBIG, hepatitis B vaccine, and antiretroviral drugs for HIV PEP.

- $\quad$ The exposure will be evaluated by the hospital physician for the potential to transmit HBV, HCV, and HIV based on the type of body substance involved 
and the route and severity of the exposure. Blood, fluid containing visible blood or other potentially infectious fluid, can be infectious for bloodborne viruses. Exposures to these fluids through a percutaneous injury (i.e., needlestick or other penetrating sharps-related event) or through contact with a mucous membrane are situations that pose a risk for bloodborne virus transmission and require further evaluation. For HCV and HIV, exposure to a blood-filled hollow needle or visibly bloody device suggests a higher risk exposure than exposure to a needle that was most likely used for giving an injection.

The physician evaluating the employee must be provided with a copy of the OSHA bloodborne pathogen regulations, a description of the employee's duties relevant to the exposure incident, and documentation of the route and circumstances of exposure. Within 15 days after the evaluation of the exposed employee, the employer must obtain a copy of the report from the healthcare professional responsible for the postexposure evaluation and insure that the employee receives a copy of the written opinion. The evaluating physician must provide a written opinion regarding vaccination status and postexposure evaluation and followup. The written opinion to the employer is limited to whether the vaccine is necessary, documentation of administration, and whether the employee was informed of the results of the evaluation, including any medical conditions resulting from exposure which require further evaluation or treatment. However, all diagnoses not related to the exposure incident are protected by patient/physician confidentiality and should not be included in the written report to the employer.

- $\quad$ For skin exposure, followup is indicated only if it involves exposure to a body fluid previously listed and evidence exists of compromised skin integrity (e.g., dermatitis, abrasion, or open wound).

- Evaluation of the Exposure Source

- The person whose blood or body fluid is the source of an occupational exposure should be evaluated for HBV, HCV, and HIV infection.

- $\quad$ The HBV, HCV, and/or HIV infection status of the source is unknown. The source person should be informed of the incident and asked to undergo testing at the emergency department or to consent to have their blood tested by an independent reference laboratory. It will be explained to the SP that a blood test is needed to determine treatment options for the exposed person. Offer to use serum that has already been collected for testing so that a second venipuncture will not be necessary. The SP may also choose to go to their physician to obtain the test at NHANES project expense. The SP will be asked to consent to release the results of the tests to the exposed staff person by sending the results to the staff person's physician. The SP should sign the consent form (See Exhibit 5-3 in Chapter 5 of the Infection Control Procedures Manual.) and a copy of the consent should be provided to the SP. If the SP 
refuses to be tested, the MEC physician or other supervisor named above should document the refusal in writing.

- $\quad$ Procedures should be followed for testing source persons, including obtaining informed consent, in accordance with applicable state and local laws. Any persons determined to be infected with HBV, HCV, or HIV should be referred for appropriate counseling and treatment. Confidentiality of the source person should be maintained at all times.

- $\quad$ Testing to determine the HBV, HCV, and HIV infection status of an exposure source should be performed as soon as possible. An FDA-approved rapid HIVantibody test kit should be considered for use in this situation, particularly if testing by EIA cannot be completed within $24-48$ hours. Repeatedly reactive results by EIA or rapid HIV-antibody tests are considered highly suggestive of infection, whereas a negative result is an excellent indicator of the absence of HIV antibody. Confirmation of a reactive result by Western blot or immunofluorescent antibody is not necessary to make initial decisions about postexposure management but should be done to complete the testing process and before informing the source person. Repeatedly reactive results by EIA for anti-HCV should be confirmed by a supplemental test (i.e., recombinant immunoblot assay [RIBA ${ }^{\mathrm{TM}}$ ] or HCV PCR). Direct virus assays (e.g., HIV p24 antigen EIA or tests for HIV RNA or HCV RNA) for routine HIV or HCV screening of source persons are not recommended.

- If the exposure source cannot be tested or refuses to provide consent, information about where and under what circumstances the exposure occurred should be assessed epidemiologically for the likelihood of transmission of $\mathrm{HBV}, \mathrm{HCV}$, or HIV. Certain situations as well as the type of exposure might suggest an increased or decreased risk; an important consideration is the prevalence of HBV, HCV, or HIV in the population group (i.e., institution or community) from which the contaminated source material is derived.

- $\quad$ Testing of needles or other sharp instruments implicated in an exposure, regardless of whether the source is known or unknown, is not recommended. The reliability and interpretation of findings in such circumstances are unknown, and testing might be hazardous to persons handling the sharp instrument.

- The exposed employee's physician is provided with the source individual's test result and information about the applicable disclosure laws and regulations concerning the source identity and infectious status. (This will be accomplished through the evaluating physician and not through the employer.)

A copy of all documentation related to the incident, including the Medical Incident Form and OSHA's Forms 300 and 301, should be sent to the Director of Mobile Examination Center Operations, Catherine Novak, as soon as possible. 
A more detailed description of these recommendations are in the Updated U.S. Public Health Service Guidelines for the Management of Occupational Exposure to HBV, HCV, and HIV and Recommendations for Postexposure Prophylaxis (MMWR, June 29, 2001). Copies of these guidelines are available in Appendix C of the Infection Control Manual.

\section{B.5 Labeling}

The OSHA standard requires that fluorescent orange and orange-red warning labels be attached to containers of regulated waste, to refrigerators and freezers containing blood and other potentially infectious materials, and to other containers used to store, transport, or ship blood or other potentially infectious materials. These labels are not necessary when (1) using red bags or red containers; (2) labels designate the contents of containers of blood, blood components, or blood products; and (3) individual containers of blood or other potentially infectious materials are placed in a labeled container during storage, transport, or disposal. The warning label must be fluorescent orange or orange-red, contain the biohazard symbol and the word BIOHAZARD, in a contrasting color, and be attached to each object by string, wire, adhesive, or other method to prevent loss or unintentional removal of the label.

In the MEC, labels are affixed to containers of infectious waste, centrifuges, refrigerators and freezers containing blood or body fluids and containers used to store, transport, or ship blood or body fluids.

\section{B.6 Communication of Hazards to Employees - Training}

All MEC employees, including those designated, as Category I and II will receive information and training during their working hours regarding the OSHA regulation and their exposure category at the time of initial assignment and at no cost to the employee. The training program will be provided at least once a year thereafter. Additional training will be provided as necessary when existing tasks are modified or tasks are added that affect the employee's exposure risk. Persons conducting the training will be knowledgeable about the subject matter, and the information will be appropriate in context and vocabulary to the education level, literacy, and language of the audience. All training programs for MEC staff will include instruction in the OSHA regulations, general infection control 
practices, and specific infection control procedures relevant to each examination component. The training must include the following elements:

- How to obtain a copy of the regulatory text and an explanation of its contents;

- Information on the epidemiology and symptoms of bloodborne diseases;

- Ways in which bloodborne pathogens are transmitted;

- Explanation of the exposure control plan and how to obtain a copy;

- Information on how to recognize tasks that might result in occupational exposure (categories of exposure);

- Explanation of the use and limitations of work practice and engineering controls, and personal protective equipment;

- Information on the types, selection, proper use, location, removal, handling, decontamination, and disposal of personal protective equipment;

- Information on hepatitis B vaccination such as safety, benefits, efficacy, methods of administration, and availability;

- Information on who to contact and what to do in an emergency;

- Information on how to report an exposure incident and on postexposure evaluation and followup;

- Information on warning labels, and signs, where applicable, and color-coding;

- Information on other infectious disease concerns in the MEC; and

- Question and answer session on any aspect of the training.

\section{B.7 Recordkeeping}

The exposure control plan is reviewed and updated at least annually or whenever new tasks and procedures affect occupational exposure. A copy of the Exposure Plan is kept in every copy of the Infection Control Manual. Copies of this manual are kept in the staff lounge and in the physician exam room, and in each field office. The plan must be made available to the Assistant Secretary for OSHA and to the Director of the National Institute for Occupational Safety and Health for examination and copying if required. 
Employers also must preserve and maintain for each employee an accurate record of occupational exposure. Under the OSHA bloodborne pathogens standard, medical records must include the following information:

- Employee's name and Social Security number;

- Employee's hepatitis B vaccination status including vaccination dates and any medical records relating to the employee's ability to receive vaccinations;

- Results of examinations, medical testing, and postexposure evaluation and followup procedures;

- Health care professional's written opinion; and

- A copy of the information provided to the health care professional.

Medical records must be kept confidential and maintained for at least the duration of employment plus 30 years.

Training records must also be maintained and kept accurate by the employer for 3 years and must include the following:

- Training dates;

- Content or a summary of the training;

- Names and qualifications of trainer(s); and

- Names and job titles of trainees.

Upon request, both medical and training records must be available to the Director of the National Institute for Occupational Safety and Health and to the Assistant Secretary of Labor for Occupational Safety and Health. Training records must be available to employees or employee representatives upon request. The employee or anyone having the employee's written consent can obtain an employee's medical records. In addition, if the employer ceases to do business, medical and training records must be transferred to the successor employer. If there is no successor employer, the employer must notify the Director, NIOSH, U.S. Department of Health and Human Services, for specific directions regarding disposition of the records at least 3 months before intended disposal. 


\section{B.8 Schedule and Methods for Implementing Sections of the Standard Covering the Methods of Compliance}

Procedures for compliance with OSHA bloodborne pathogen recommendations are included in initial NHANES training programs and incorporated into all specific component procedures and operations procedures in the MEC. All procedures should be operational at the time of training and during the main study.

\section{B.9 Sharps Injury Log}

29 CFR Part 1904 - Recording and Reporting Occupational Injuries and Illnesses requires establishment and maintenance of a sharps injury log. All work-related needlestick injuries and cuts from sharp objects that are contaminated with another person's blood or other potentially infectious material must be entered on the OSHA's Forms 300 and 301 as an injury. The OSHA's Forms 300 and 301 serve as the sharps injury log. Do not enter the employee's name to maintain confidentiality. The sharps injury log must contain, at a minimum:

1. The type and brand of device involved in the incident;

2. The department or work area where the exposure incident occurred; and

3. An explanation of how the incident occurred.

The sharps injury log is maintained for the period required by 29 CFR 1904.03; the OSHA's Forms 300 and 301 are maintained for five (5) years following the end of the calendar year that these records cover.

\section{B.10 Needlestick and other Sharps Injuries}

In January 2001, the Occupational Safety and Health Administration revised the Bloodborne Pathogens (BBP) standard in conformance with the requirements of the Needlestick Safety and Prevention Act. The revisions to OSHA's BBP standard required under the Needlestick Safety and Prevention Act can be broadly categorized into four areas: (1) modification of definitions relating to 
engineering controls; (2) revision and updating of the Exposure Control Plan; (3) solicitation of employee input; and (4) recordkeeping.

The standard has been revised to add new requirements to the annual review and update of the Exposure Control Plan. Consideration and implementation of safer medical devices must be documented in the Exposure Control Plan by describing the safer devices identified as candidates for adoption, the method or methods used to evaluate devices and the results of evaluations, and justification for selection decisions. The revised standard requires that solicitation of input from employees be documented in the Exposure Control Plan. This information must be updated at least annually. The recordkeeping requirements of the standard have been amended by adding a paragraph to require that employers maintain a sharps injury log to serve as a tool for identifying high risk areas and evaluating devices.

In November 1999, NIOSH published ALERT, Preventing Needlestick Injuries in Health Care Settings. A comprehensive plan and program to prevent transmission of bloodborne pathogens and to meet (and exceed, whenever possible) regulations was implemented to identify and evaluate various new safer needle technologies and devices. The intent was to replace the existing nonshielded blood collection needles.

Annual formal evaluations have been conducted since 2000. A notebook detailing each evaluation is kept at the Westat home office and is available for review and inspection.

The latest memo describing the process that was conducted in November 2008 follows. 
TO: $\quad$ Catherine Novak, Sharon Lash

November 22, 2008

FROM: $\quad$ Barbara Lindstrom

SUBJECT: Annual 2008 Shielded Needle Evaluation

\section{Background}

In January 2001, the Occupational and Safety and Health Administration (OSHA) revised the Bloodborne Pathogens (BBP) standard in conformance with the requirements of the Needlestick Safety and Prevention Act. The revisions to OSHA's BBP standard required under the Needlestick and Safety Act can be broadly categorized into four areas: modification of definitions relating to engineering controls, revision and updating the Exposure Control Plan (ECP), solicitation of employee input, and recordkeeping.

The standard was revised to add new requirements to the annual review and update of the ECP. Consideration and implementation of safer medical devices must be documented in the ECP by describing the safer devices identified as candidates for adoption, the methods used to evaluate the devices and the results of the evaluation, and justification for selection decisions. This information must be updated annually.

\section{Previous Evaluations}

Products selected for evaluation are based on three sources of research: information provided by existing vendors, information provided by supply representative, and an Internet search. A variety of blood collection needles and sharps containers have been included in all previous evaluations. Preference is given to those products that use newer and safer technologies.

A variety of blood collection needles and sharps containers were evaluated during the first annual evaluation process that began in February 2000. Specific products were approved and put into use in August 2000. The evaluation process was repeated in July 2001, July 2002, September 2002, February 2004, August 2005, and August 2006. The needles and sharps containers that are currently being used were approved during the August 2006 evaluation cycle. Documentation supporting each evaluation cycle is contained in a notebook at the home office.

The NHANES survey currently maintains one approved sharps container and four shielded blood collection needles:

$>$ Becton Dickinson ${ }^{\mathrm{TM}}$ (BD) Vacutainer Eclipse Multi-sample needle (21G x 1 1/4")

$>$ Becton Dickinson ${ }^{\mathrm{TM}}$ Butterfly Needle 19G Saf-T EZ Set and Luer Adapter

$>$ Becton Dickinson ${ }^{\mathrm{TM}}$ Becton Dickinson ${ }^{\mathrm{TM}}$ Butterfly Needle 21G Safety-Lok Blood Collection Set

$>$ Becton Dickinson ${ }^{\mathrm{TM}}$ Becton Dickinson ${ }^{\mathrm{TM}}$ Butterfly Needle 23G Safety-Lok Blood Collection Set

$>$ Bio-Hazard Container (8 Gallon) 


\section{Candidates for Adoption - 2008}

This year's research yielded several new shielded needles for consideration. In addition the phlebotomists had requested optional 3 and 7-mL red top tubes to evaluate as an alternative to the existing BD tubes. There were no new sharps containers that offered additional safety features so the medical technologists and phlebotomists reevaluated the current sharps container.

\section{Methods for Evaluation Devices}

On November 3, 2008 the warehouse staff shipped the following supplies to the chief medical technologists at stands 245 and 246.

- $\quad$ VENOJECT® II (Standard) Tube Holder. Use with the multi-sample luer adapter.
o P-1316R 50 pieces

- $\quad$ Winged Infusion Sets, 19G x 3/4" Thin Wall Needle, 12" Tubing

$$
\text { o } \mathrm{SV} * \text { S19BLN } 12 \text { pieces }
$$

- $\quad$ Winged Infusion Sets, 25G x 1/2" Regular Wall Needle, 8" Tubing
o $\mathrm{SV} * \mathrm{~S} 23 \mathrm{BL}$
12 pieces

- $\quad$ VENOJECT® Multi-Sample Luer Adapter (male). For use with Standard Tube Holder and SampLok ${ }^{\circledR}$ Tube Holder.

o XX*MN2000T 50 pieces

- Surshield ${ }^{\text {TM }}$ Safety Winged Blood Collection Set with 21G x 3/4" Needle and 12" Tubing o $\quad$ MN*SVS21B30 12 pieces

- $\quad$ Surshield ${ }^{\mathrm{TM}}$ Safety Winged Blood Collection Set with 23G x 3/4" Needle and 12" Tubing o $\quad \mathrm{MN} *$ SVS23B30 12 pieces

- $\quad$ Kendall / Tyco 3mL Red Top Blood Collection Tube $8881301215 \quad 50$ pieces

- Kendall / Tyco 7mL Red Top Blood Collection Tube $8881301512 \quad 50$ pieces

The MEC phlebotomists and all backup phlebotomists printed and reviewed the package insert instructions before using these products. They followed the instructions and completed the evaluation by testing the various products and filling out a separate evaluation form (attachment A) for each item. They used the Venoject tube holder and not the BD tube holder with these products. The staff evaluated every product at least once and the backup phlebotomists evaluated one each of the 19G winged infusion set and the 21 or 23G safety winged blood collection sets. The staff conducted this evaluation at stands 245 and 246 from November 3 through November 21. The completed evaluation forms for each product were sent to the technical supervisor on November 21. 


\section{Sharps Container Evaluation}

The phlebotomists and medical technologists used the Sharps Disposal Evaluation Form (see Attachment B) to reevaluate the 8-gallon biohazard sharps container currently used in the lab and phlebotomy. Each medical technologist and phlebotomist completed at least one evaluation form for the 8-gallon container. The evaluation forms were sent to the technical supervisor on November 21.

\section{Results of the Evaluation and Justification for Selection Decisions}

The evaluation forms demonstrated serious problems with all winged infusion sets and winged blood collection sets. In all cases they required more than one hand to engage the safety feature which rendered the needle less safe than the current sets which require a one-handed technique. This evaluation cycle reaffirmed the staff's confidence in the consistently reliable safety feature of the existing blood collection sets and their comfort when using these safety features. The decision is to continue to use the existing BD infusion and blood collection sets.

The staff maintains a small supply of the Becton Dickinson ${ }^{\mathrm{TM}}$ Vacutainer Eclipse Multi-sample needle (21G x 1 1/4"). These are intended for use when a longer needle is indicated, such as in obese participants. The staff uses less than one or two per year. Repeated evaluations have never revealed any issues with these needles so we will continue to stock them.

The evaluation revealed that the Kendall 3 and 7-mL red top tubes were cumbersome to handle and did not fit well into the tube holder so the decision is to continue to use the existing BD tubes.

All phlebotomists and medical technologists reevaluated the 8-gallon biohazard sharps container used in the lab and phlebotomy. The evaluation forms revealed no problem with this item. The decision is to continue to use the existing sharps containers.

All evaluation forms will be kept in a notebook at the home office and are available for inspection. 


\section{ATTACHMENT A \\ Safety Blood Collection Set \\ Evaluation Form - Stands 245 and 246 \\ Page 1}

\section{Your Name \\ Today's Date \\ Product Name \\ Product Needle Size}
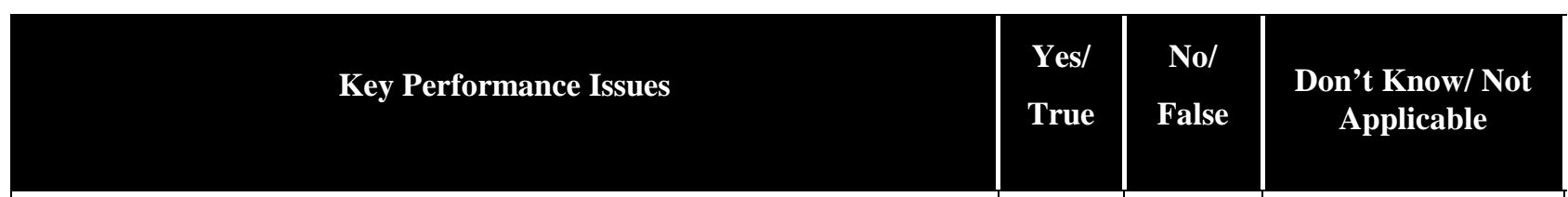
1. The product is satisfactory for standard phlebotomy purposes
2. The product is equally satisfactory for different or diverse patient populations (adults, children, heavy, thin, etc.)

Comment:

3. What percentage of typical clinical procedures does this product address?

4. The product is satisfactory for SPs with fragile veins

5. The product is simple to operate

6. The use of this product requires no training

7. The safety feature activated with a one-handed technique

8. The safety feature worked reliably

9. Both hands remain behind the needle during engagement of safety feature

10. The safety feature did not interfere with the blood draw

11. This product allowed me to see what I needed to see during the blood draw

12. The safety feature could not be bypassed

13. The use of this product did not create any extra risk of sprays, blood leakage, and/or drips

14. The product could be disposed of into the sharps container that is available for my use

\section{Feedback}

15. About how many times did you use the blood collection set before you were comfortable using it? (check one)

1 time

5 times

10 times

15 times

20 times

I never felt comfortable

16. Did you have any problems with this device? If yes, please briefly explain below.

$\square$ Yes $\quad \square$ No 


\section{Key Performance Issues}

Yes/

True

Don't Know/ Not

Applicable

17. Which device would you rather use: (Please check one)
$\square$ 1. The product we normally use
$\square$ 2. This test one
$\square$ 3. Other, please indicate

Comments: 


\section{ATTACHMENT B \\ Sharps Disposal \\ Evaluation Form}

Date:

Position:

Manufacturer:
Name:

Stand:

Capacity:

Indicate the degree to which you agree or disagree with each statement by circling the most appropriate number for each statement.

Not applicable (N/A) may be used if the question does not apply to this product.

\section{Please Rate:}

1. The container is puncture resistant

2. The container does not leak

3. The container is stable

4. The container is accessible from any direction

5. The container is located where the needles are used

6. The container allows single handed use

7. It is possible to drop sharps or tubes into the container vertically

8. No force is required to put sharps or tubes into the container

9. Needles/tubing do not get caught on the opening or interior shape

10. The container opens and closes easily

11. The container has a solid tight-fitting cover

12. The box accommodates a range of sharp sizes or tubes

13. The size of the container is appropriate for its use

14. No one (including a child) can access the contents

15. There is a permanent lock for final disposal that is not reversible

16. The user can determine the fill level visually

17. The container is appropriately labeled

18. The box is not threatening to participants

19. Use of this container does not compromises infection control practices agree disagree

$\begin{array}{llllll}1 & 2 & 3 & 4 & 5 & \mathrm{~N} / \mathrm{A}\end{array}$

$\begin{array}{llllll}1 & 2 & 3 & 4 & 5 & \mathrm{~N} / \mathrm{A}\end{array}$

$\begin{array}{lllll}1 & 2 & 3 & 4 & 5\end{array} \mathrm{~N} / \mathrm{A}$

$\begin{array}{lllllll}1 & 2 & 3 & 4 & 5 & \mathrm{~N} / \mathrm{A}\end{array}$

$\begin{array}{llllll}1 & 2 & 3 & 4 & 5 & \mathrm{~N} / \mathrm{A}\end{array}$

$\begin{array}{llllll}1 & 2 & 3 & 4 & 5 & \mathrm{~N} / \mathrm{A}\end{array}$

$\begin{array}{llllll}1 & 2 & 3 & 4 & 5 & \mathrm{~N} / \mathrm{A}\end{array}$

$\begin{array}{llllll}1 & 2 & 3 & 4 & 5 & \mathrm{~N} / \mathrm{A}\end{array}$

$\begin{array}{llllll}1 & 2 & 3 & 4 & 5 & \mathrm{~N} / \mathrm{A}\end{array}$

$\begin{array}{llllll}1 & 2 & 3 & 4 & 5 & \mathrm{~N} / \mathrm{A}\end{array}$

$\begin{array}{llllll}1 & 2 & 3 & 4 & 5 & \mathrm{~N} / \mathrm{A}\end{array}$

$\begin{array}{llllll}1 & 2 & 3 & 4 & 5 & \mathrm{~N} / \mathrm{A}\end{array}$

$\begin{array}{lllllll}1 & 2 & 3 & 4 & 5 & \mathrm{~N} / \mathrm{A}\end{array}$

$\begin{array}{llllll}1 & 2 & 3 & 4 & 5 & \mathrm{~N} / \mathrm{A}\end{array}$

$\begin{array}{llllll}1 & 2 & 3 & 4 & 5 & \mathrm{~N} / \mathrm{A}\end{array}$

$\begin{array}{llllll}1 & 2 & 3 & 4 & 5 & \mathrm{~N} / \mathrm{A}\end{array}$

$\begin{array}{llllll}1 & 2 & 3 & 4 & 5 & \mathrm{~N} / \mathrm{A}\end{array}$

$\begin{array}{llllll}1 & 2 & 3 & 4 & 5 & \mathrm{~N} / \mathrm{A}\end{array}$

$\begin{array}{llllll}1 & 2 & 3 & 4 & 5 & \mathrm{~N} / \mathrm{A}\end{array}$

Did this sharps container interfere with your ability to complete the venipuncture or blood processing protocol?

Yes or No

Are there other questions that you feel should be asked regarding the safety/utility of this product? 


\section{B.11 Laboratory and Phlebotomy Infection Control}

This section contains a summary of all procedures used for infection control in the MEC laboratory and phlebotomy components. For detailed information regarding these procedures, refer to the component's manual of operation.

\section{B.11.1 Glossary}

Decontamination: The use of physical or chemical means to remove, inactivate, or destroy bloodborne pathogens on a surface or item to the point where they are no longer capable of transmitting infectious particles and the surface or item is rendered safe for handling, use, or disposal.

Detergent: $\quad$ A cleansing agent used to facilitate the removal of gross debris from an object.

Disinfect: Inactivation of virtually all recognized pathogenic microorganisms but not necessarily all microbial forms (e.g., bacterial endospores) on inanimate objects.

Engineering Controls (e.g., sharps disposal containers, self-sheathing needles, safer Controls: medical devices, such as sharps with engineered sharps injury protections and needleless systems) that isolate or remove the bloodborne pathogens hazard from the workplace.

Mucous membrane Exposure that occurs from droplet contamination (splashes, spatters) exposure: of the eyes, nose, or mouth.

Parenteral Exposure that occurs from piercing mucous membranes or the skin exposure: $\quad$ barrier through such events as needlesticks, human bites, cuts, and abrasions.

Personal Protective Specialized clothing or equipment worn by an employee for protection Equipment: $\quad$ against a hazard. General work clothes (e.g., uniforms, pants, shirts or blouses) not intended to function as protection against a hazard are not considered personal protective equipment.

Regulated Waste: $\quad$ Liquid or semiliquid blood or other potentially infectious materials; contaminated items that would release blood or other potentially infectious materials in a liquid or semiliquid state if compressed; items that are caked with dried blood or other potentially infectious materials and are capable of releasing these materials during handling; contaminated sharps; and pathological and microbiological wastes containing blood or other potentially infectious materials. 
Sharps with Non-needle sharps or a needle device used for withdrawing body engineered sharps fluids, accessing a vein or artery, or administering medications or injury protections: other fluids, with a built-in safety feature or mechanism that effectively reduces the risk of an exposure incident.

Sterilize: $\quad$ The use of a physical or chemical procedure to destroy all microbial life including highly resistant bacterial endospores.

Universal An approach to infection control. According to the concept of precautions: $\quad$ Universal Precautions, all human blood and certain human body fluids are treated as if known to be infectious for HIV, HBV, and other bloodborne pathogens.

Work Practice Controls that reduce the likelihood of exposure by altering the manner Controls: $\quad$ in which a task is performed (e.g., prohibiting recapping of needles by a two-handed technique).

In order to comply with Federal regulations, minimize the spread of infectious diseases, and provide a safe working environment, follow defined safety procedures at all times.

\section{B.11.2 Laboratory Access}

- Unauthorized people should not enter the MEC laboratory.

- All authorized persons must wear gloves when working with equipment, and when handling biological specimens such as blood, blood products, or urine in the laboratory. Examples are lab staff, biomedical engineers, Coulter technical representatives, NCHS or observers, coordinator, assistant coordinator, health techs, and MEC manager. People who visit the lab to observe should not touch countertops, equipment, or supplies if they are not wearing gloves.

\section{B.11.3 Laboratory/Phlebotomy - Universal Precautions}

A complete description of Universal Precautions is described in Section B.3.2. Laboratory staff, including the phlebotomist, observe Universal Precautions at all times. 


\section{B.11.4 Laboratory/Phlebotomy - Specific Engineering and Work Practice Controls}

Engineering and work practice controls are described in Section B.3.3. Engineering controls eliminate hazards at their source. The biological safety cabinet and waste containers for sharps are examples of engineering controls used in the NHANES. A laminar flow biological safety cabinet is located in the laboratory in every MEC.

All health care workers take precautions to prevent injuries caused by needles and other sharp instruments or devices during procedures, when cleaning used instruments, during disposal of used needles, and when handling sharp instruments after procedures. It is mandatory and essential to use the shielded mechanism of the needle.

Specific measures of protection for the laboratory staff (including the phlebotomist) are described below.

Hang outside street clothing in a designated separate clean area.

\section{Work Practice Controls}

- Do not eat, drink, smoke, handle contact lenses, or apply cosmetics or lip balm in the MEC laboratory or in the examination rooms.

- Do not keep food or drinks in refrigerators, freezers, shelves, cabinets, or work surfaces in the examination rooms. An employee lounge with a refrigerator is provided for personal food storage.

- Do not mouth pipette.

- Procedures involving blood or other potentially infectious materials are performed in such a manner as to minimize splashing, spraying, or aerosolizing. For example, the splattering of blood or serum that occurs when rubber stoppers are pulled out of specimen tubes is minimized by covering the stopper with gauze before removing it and performing this procedure under the biosafety hood.

- Perform all blood processing tasks in the LSBSC.

- Use Aerosolve containers when centrifuging blood tubes.

- Used needles must never be sheared, bent, clipped, broken, or recapped by hand. Discarded needles and other waste sharps must be placed in an "approved" sharps container. Either use a one-handed "scoop" technique or a mechanical device designed for holding the needle sheath. 
- Use of the safety shielding mechanism on the blood collection needle is mandatory.

- Never point (direct) or move the unsheathed tip of a needle toward anyone.

- Always be alert and extremely cautious when handling unsheathed needles.

- Immediately after use, dispose of sharps in sharps containers.

- When discarding contaminated sharps, place them in containers that are closable, puncture-resistant, appropriately labeled or color-coded, and leakproof on the sides and bottom.

- Ensure that sharps containers are easily accessible to personnel and located as close as feasible to the use area.

- Keep sharps containers upright throughout use, replace routinely, close when moved, and do not overfill. Do not overstuff the biohazard waste containers by poking the trash down with another object. Shaking the container back and forth is acceptable.

- Never manually open, empty, or clean contaminated sharps disposable containers.

- Never discard needles directly into a container used for ordinary ("noninfectious”) waste.

\section{B.11.5 Laboratory/Phlebotomy - Personal Protective Equipment}

A complete description of personal protective equipment is contained in Section B.3.4.

- Wear impervious lab jackets when performing venipuncture and laboratory procedures. Disposable impervious lab jackets are provided to the medical technologists and phlebotomists.

- Remove protective equipment before leaving the work area and after a garment becomes contaminated.

- Place used protective equipment in appropriately designated areas inside the laboratory and place in designated biohazardous containers when being discarded.

- Discarded lab jackets when soiled and at the end of each workweek.

- If the LFBSC is nonfunctional, then wear safety glasses when processing blood. Safety glasses are optional for the phlebotomist but safety glasses are available onsite.

- Wear appropriate face and eye protection such as a mask with glasses with solid side shields or a chin-length face shield when splashes, sprays, spatters, or droplets of 
blood or other potentially infectious materials pose a hazard to the eye, nose, or mouth.

- It is mandatory to wear gloves when performing venipuncture, processing blood specimens, processing urine, processing vaginal swabs, and performing hematology testing. Disposable, powder-free hypoallergenic nonlatex powder-free gloves are provided to the medical technologists and phlebotomist.

- Change gloves after each sample person's exam or when visibly soiled with blood and/or body fluids.

- $\quad$ Never wash or decontaminate disposable gloves for reuse.

- Discard utility gloves at the first sign of deterioration.

- Remove gloves before leaving the laboratory or phlebotomy room.

Personal Hygiene - Infection Control

- Wash hands with soap and water after removing gloves or upon accidental skin contact with blood or body fluids, and before working with the next SP.

- Hand washing sinks are located in the bathrooms in trailers 1 and 4, in the hallways in trailers 1 and 2, in the laboratory, phlebotomy room, and staff lounge in trailer 3, and in the dental exam room in trailer 4 . The laboratory and dental sinks include an electronic eye on/off mechanism.

- If using liquid soap for hand washing, replace with fresh product when empty. Do not add liquids to a partially full dispenser.

\section{B.11.6 Laboratory/Phlebotomy - Waste Management}

A complete description of the waste management procedures is described in Section B.3.5.

- Discard hazardous waste into appropriate containers. Biohazardous waste containers are labeled and sealed to prevent leakage during handling storage and transport. A hazardous waste company is responsible for the disposal of biohazardous waste from the MEC. Do not overstuff the biohazard waste containers by poking the trash down with another object. Shaking the container back and forth is acceptable.

- Dispose of all potentially infectious materials in a biohazard waste container.

- When discarding glass blood tubes, place them in sharps containers that are closable, puncture-resistant, appropriately labeled or color-coded, and leakproof on the sides and bottom. 
- Place solid waste from the laboratory in biohazard bags and transport to an area designated to hold these bags. A single bag is adequate if the bag is sturdy (not easily penetrated) and if the waste can be put in the bag without contaminating the outside of the bag; otherwise, double bagging is indicated.

- To minimize the potential risk for accidental transmission of disease or injury, store infective waste waiting terminal processing in a pre-incineration storage area in the MEC accessible only to personnel involved in the disposal process.

- Place regulated waste in closable and labeled or color-coded containers. When storing, handling, transporting or shipping, place other regulated waste in containers that prevent leakage.

- Transport closed sharps containers and biohazard bags destined for incineration or steam sterilization to the designated pre-incineration storage.

- Discard all regulated waste according to Federal, state, and local regulations.

- Refer to "infectious waste" in the glossary for any questions about identifying wastes for which special precautions appear prudent.

- Liquid Wastes

- $\quad$ Pour urine down the sink connected to the sewer before discarding the cup into biohazard container.

- $\quad$ Pour Coulter waste down the sink connected to a sewer. Add 1 3/4 cups (3.75$\mathrm{mL}$ ) undiluted bleach to the 10-gallon waste container and reattach to the instrument.

\section{B.11.7 Laboratory/Phlebotomy - Labeling}

In the MEC, labels are affixed to containers of infectious waste, centrifuges, refrigerators, and freezers containing blood or body fluids and containers used to store, transport, or ship blood or body fluids.

A complete description of the OSHA label requirements is described in Section B.5. 


\section{B.11.8 Laboratory/Phlebotomy - Housekeeping}

A complete description of OSHA's housekeeping requirements is described in Section B.3.5.

All housekeeping procedures must be observed and will be monitored by project staff:

- Wash work surfaces and or equipment contaminated with blood or body fluids with detergent and disinfected with a 1:10 dilution of bleach.

- At the end of every examination session, disinfect work surfaces with a 1:10 dilution of bleach.

- Check and decontaminate equipment before servicing and shipping.

- Remove protective coverings used to cover equipment and environmental surface and replace as soon as feasible when overtly contaminated with blood or body fluids during the exam session.

- Inspect and decontaminate all materials intended for reuse on a regular basis and clean and decontaminate immediately upon visible contamination.

- Inspect and decontaminate, on a regular basis, reusable receptacles such as bins, pails, and cans that have the likelihood for becoming contaminated. When contamination is visible, clean and decontaminate receptacles immediately, or as soon as possible.

- Place other regulated waste in closable and labeled or color-coded containers. When storing, handling, transporting or shipping, place other regulated waste in containers that prevent leakage.

- Clean and decontaminate all equipment and environmental and work surfaces contaminated with blood or other potentially infectious materials.

- Decontaminate work surfaces with an appropriate disinfectant after completion of procedures, immediately when overtly contaminated, after any spill of blood or other potentially infectious material, and at the end of the work shift when surfaces have become contaminated since the last cleaning.

- Remove and replace protective coverings such as plastic wrap and aluminum foil when contaminated.

- Clean and disinfect equipment and working surfaces, including keyboard covers, with a 1:10 bleach solution (5-mL bleach to 50 -mL tap water) or 1:100 bleach solution (1$\mathrm{mL}$ bleach to $100-\mathrm{mL}$ water) after completion of procedures, when surfaces are 
overtly contaminated, immediately after any spill of blood or other potentially infectious materials, and at the end of the work shift.

- Remove and replace nonabsorbent paper used to cover equipment and environmental surfaces at the end of the work shift or when they become overtly contaminated.

- Check equipment routinely and decontaminate as necessary before servicing or shipping.

- Decontaminate reusable items contaminated with blood or other potentially infectious materials before washing and/or reprocessing. Routinely check equipment and decontaminate it before servicing and shipping.

- Clean and decontaminate all equipment and work surfaces contaminated with blood or other potentially infectious materials.

- On a regular basis inspect and decontaminate reusable receptacles such as bins, pails, and cans that have likelihood for becoming contaminated. When contamination is visible, clean and decontaminate receptacles immediately, or as soon as possible.

\section{B.11.9 Laboratory/Phlebotomy - Cleaning a Blood Spill}

If a blood spill occurs, wear gloves, and if needed, a gown and facial protection during the cleanup procedure.

If spill contains broken glass use dustpan and broom to collect glass pieces. Dispose of broken glass and contaminated paper towels in sharps containers that are closable, puncture-resistant, appropriately labeled or color-coded, and leakproof on the sides and bottom. Disinfect dustpan and

broom with bleach solution. Always use mechanical means such as forceps or a brush and dust pan to pick up contaminated broken glass; never pick up broken glass with hands even if wearing gloves. To minimize aerosol formation, broken glass is not dropped into a disposal container but gently placed in the container.

To clean a blood spill perform the following procedure:

- Wear gloves;

- Cover spill with disposable paper towels;

- Soak paper towels with 10 percent bleach solution; 
- Let soak for at least 30 seconds;

- Dispose of blood/bleach soaked paper towels in biohazard container;

- Allow area to dry and then remove gloves; and

- Wash hands.

\section{B.12 References}

The following references were used to prepare the Exposure Plan:

1. Occupational Exposure to Bloodborne Pathogens, U.S. Department of Labor, Occupational Safety and Health Administration. OSHA 3127, 1996 (Revised). Title 29 Code of Federal Regulations, Part 1910.1030. [56 FR 64004, Dec. 06, 1991, as amended at 57 FR 12717, April 13, 1992; 57 FR 29206, July 1, 1992; 61 FR 5507, Feb. 13, 1996; 66 FR 5325 Jan., 18, 2001]

2. Immunization of Health-Care Workers, USPHS, CDC,, MMWR 1997;46(No. RR-18).

3. Updated U.S. Public Health Service Guidelines for the Management of Occupational Exposure to HBV, HCV, and HIV and Recommendations for Postexposure Prophylaxis (MMWR, June 29, 2001)

4. Occupational Exposure to Bloodborne Pathogens, Needlestick and other Sharps injuries: Final Rule, U.S. Department of Labor, Occupational Safety and Health Administration. OSHA, 2000 (Revised).

5. Title 29 Code of Federal Regulations, Part 1904.

6. CDC. Guideline for Hand Hygiene in Health-Care Settings Recommendations of the Healthcare Infection Control Practices Advisory Committee and the HICPAC/SHEA/APIC/IDSA Hand Hygiene Task Force. MMWR 2002:51(RR-16).

Copies of these materials are included in the Appendixes of the Infection Control Manual. 
Appendix C

Laboratory Protocol 
Table C-1. Phlebotomy protocol

\begin{tabular}{|c|c|c|c|c|c|c|c|c|c|c|c|c|c|}
\hline Tube Type & $\begin{array}{c}\text { Primary } \\
1-2\end{array}$ & $\begin{array}{c}\text { Primary } \\
\text { 3-5 }\end{array}$ & $\begin{array}{c}\text { Primary } \\
6-11\end{array}$ & $\begin{array}{c}\text { Primary } \\
12+\end{array}$ & $\begin{array}{c}\text { Primary } \\
20+\end{array}$ & $\begin{array}{l}\text { Dry Run } \\
\text { Odd } 12+\end{array}$ & $\begin{array}{c}\text { Dry Run } \\
\text { Even 12+ }\end{array}$ & $\begin{array}{c}\text { VIP } \\
\text { Guest }\end{array}$ & $\begin{array}{c}\text { VIP } \\
\text { Guest }\end{array}$ & $\begin{array}{c}\text { VIP } \\
\text { Guest }\end{array}$ & $\begin{array}{c}\text { VIP } \\
\text { Guest } \\
12+\end{array}$ & $\begin{array}{c}\text { Guests } \\
1-11\end{array}$ & $\begin{array}{c}\text { Guests } \\
12+\end{array}$ \\
\hline 3-mL EDTA & 1 & 2 & 1 & 2 & 1 & & 4 & 1 & 1 & 1 & 1 & 1 & 1 \\
\hline 4-mL EDTA & & & 1 & 1 & 2 & 2 & & & & & & & \\
\hline 2-mL Gray & & & & 1 & 1 & 2 & 2 & & & & 1 & & \\
\hline 3-mL Red & 2 & & 1 & & & & & 1 & 1 & & & & 1 \\
\hline 7-mL Red & & 2 & & & & & & & & 1 & & & \\
\hline 15-mL Red & & & & 3 & 3 & 4 & 4 & & & & & & \\
\hline 10-mL Red & & & 2 & 3 & 3 & & & & & & 1 & & \\
\hline 7-mL EDTA & & & & 1 & 1 & & & & & & & & \\
\hline 10-mL EDTA & & & & & 2 & & & & & & & & \\
\hline 10-mL Gray & & & & 1 & 1 & & & & & & & & \\
\hline 2-mL Gray & & & & 1 & 1 & 2 & 2 & & & & & & \\
\hline Total ml & 9 & 20 & 30 & 106 & 127 & 76 & 78 & 6 & 6 & 10 & 15 & 3 & 6 \\
\hline Total ounces & 0.3 & 0.7 & 1.0 & 3.6 & 4.3 & 2.6 & 2.6 & 0.2 & 0.2 & 0.3 & 0.5 & 0.1 & 0.2 \\
\hline Total tablespoons & 0.6 & 1.4 & 2.0 & 7.2 & 8.6 & 5.2 & 5.4 & 0.4 & 0.4 & 0.6 & 1.0 & 0.2 & 0.4 \\
\hline
\end{tabular}




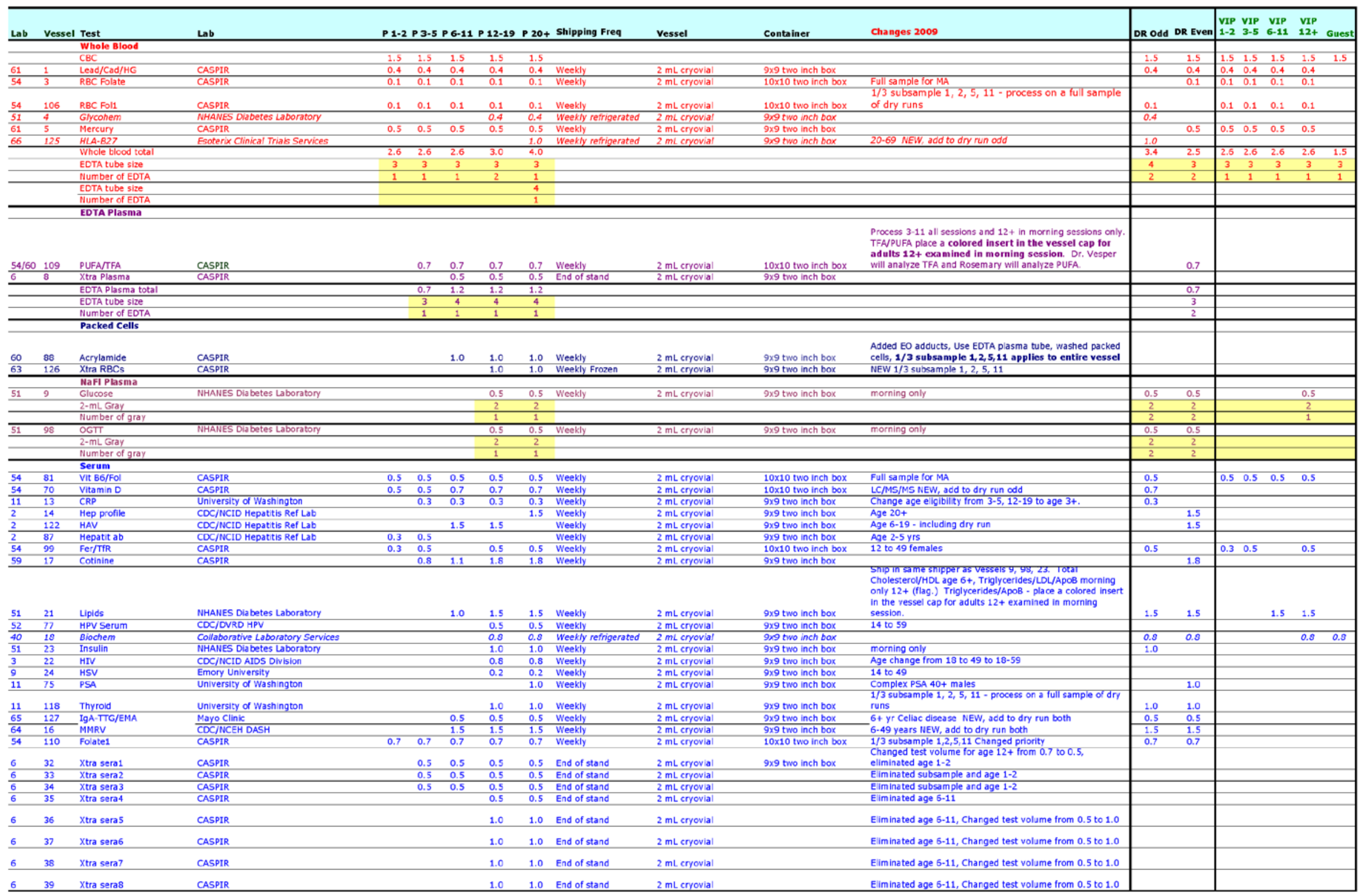




\section{Table C-2. Volumes (continued)}

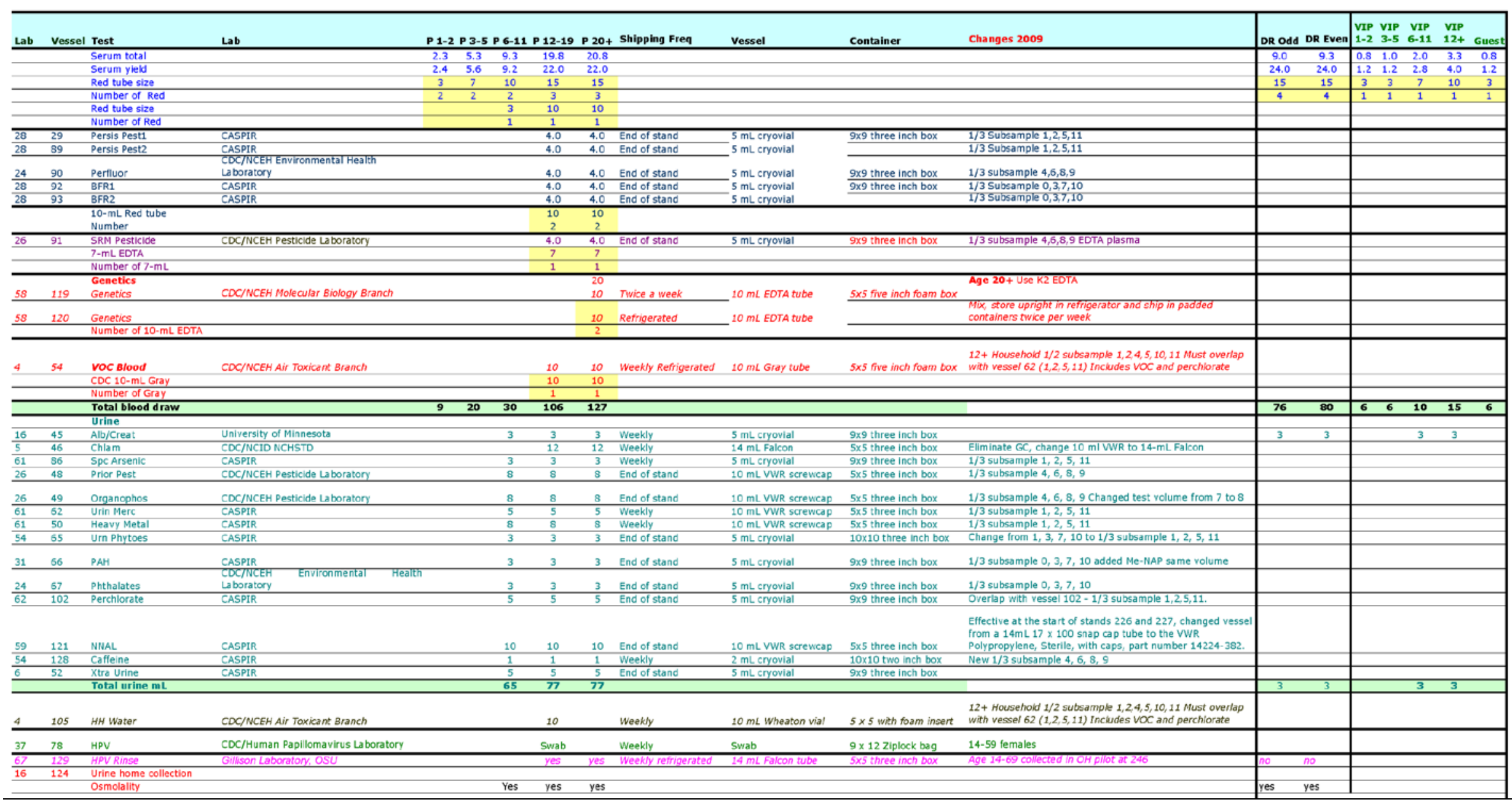




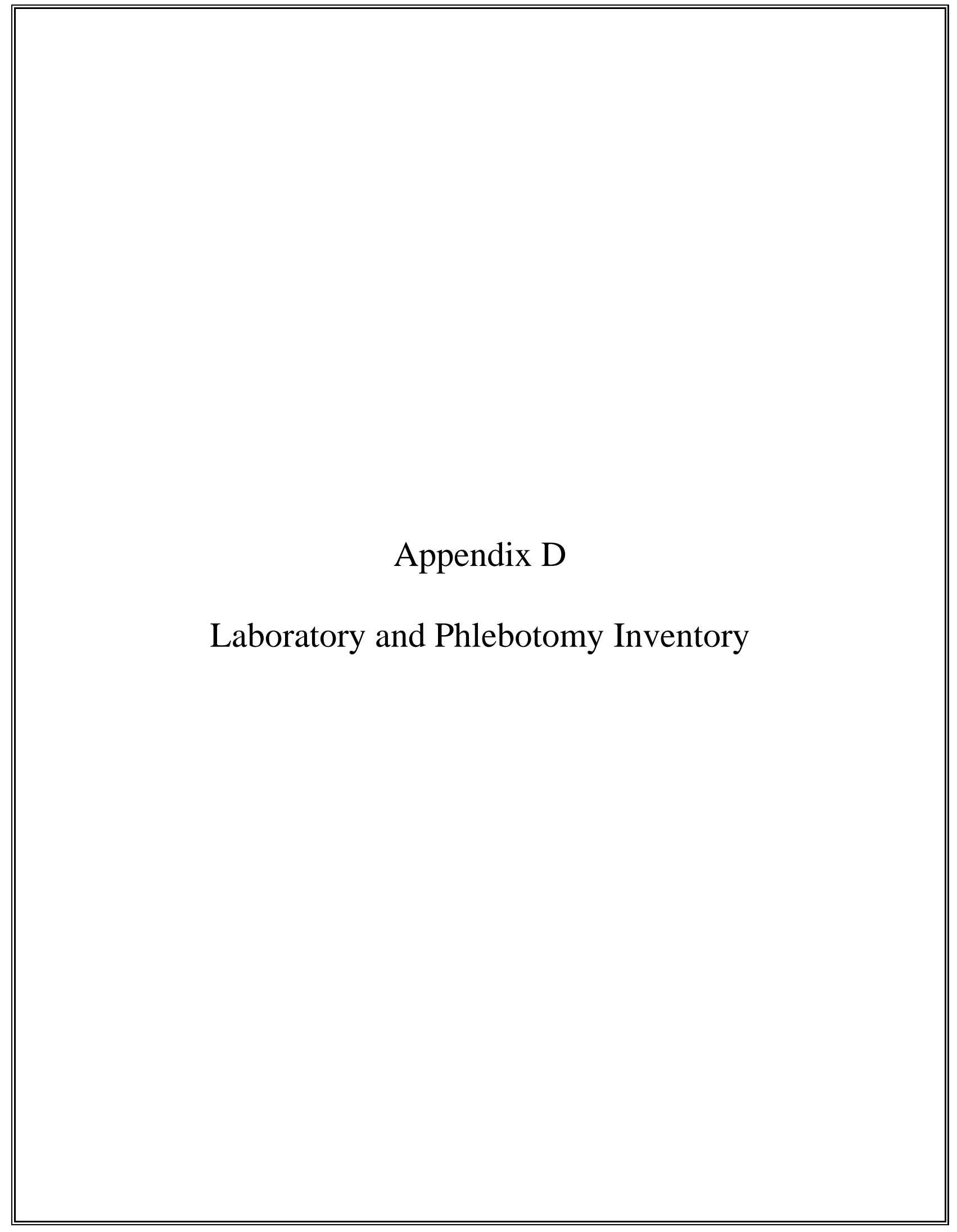




\section{APPENDIX D. LABORATORY AND PHLEBOTOMY INVENTORY}

\section{LABORATORY INVENTORY}

CONSUMABLE ITEMS:

Part number Description

EXAM SUPPLIES

56616-032

FA201-10

SO - AA

$\mathrm{RD} 06173$

24-54

$19-086333$

$12-007-187$

9A-7793

$108 \mathrm{M}$

14-830-132

SO-Bleach

1441266

23-751610

WAL78742

9876239

9872495

365062

365061

365060

G31-500

34155

47745-078

47745-074

$47745-180$

721032

RDR024

306239H01

414004-012

86.1171

15-235-61
Absorbent Bench Pad - blue chuck

Absorbent Sheets (2" x 2")

Absorbic Acid

Alluminum Foil - 50 Sq. Feet Roll

Applicator Sticks, cotton tipped

Applicator Sticks, wooden

Bench Kote Paper - 46" x 57" Paper for Lab Counter

Bio-Hazard Bag (19" x 23")

Bio-Hazard Bags (25" x 35")

Bio-Hazard Container (8 Gallon)

Bleach - Regular - 5 - 5.25 Hydrochloride, Fragra

Bleach Right Disinfecting Spray - 32 Ounce Spray Bottle

Deionized Water - 1 Gallon Plastic Bottle

Distilled Water - Plastic Gallon Jugs

Gloves, Maxxim Latex Free (Lg.)

Gloves, Maxxim Latex Free (Sm.)

Gloves, Nitrile Large - Purple Glove

Gloves, Nitrile Medium - Purple Glove

Gloves, Nitrile Small - Purple Glove

Glycerin - 500mL Brown Glass Bottle

Kimwipes (4 1/2 x 8 1/2)

Pipette Tips 1000uL - 5 Racks of 96 tips each / per case

Pipette Tips 200uL

Pipette Tips 5mL (Bulk Pack) - 500 Pcs. per case

Saline Solution 0.9\% - 1 Liter Plastic Bottle

Temperature Chart Pens (Mark-A-Matic II)

Temperature Charts (Refrigerator/Freezer)

Transfer Pipette (15mL ,8.0 Bulb Clear) - 250 per box

Transfer Pipette (3.5 clear, plastic)

Wipes, Terri (23" x 11") $\underline{\text { Par level Unit distribution }}$

15

5

12

1

1

1

2

1

1

20

4

2

1

5

10

10

10

8

10

1

3

2

2

1

3

1

2

2

6

1 each

pack

each

roll

bag

box

case

box

box

each

gallon

bottle

gallon

gallon

box

box

box

box

box

bottle

box

box

box

case

bottle

pack

pack

box

box

box 
Part number

OFFICE SUPPLIES

2251

DUR PC1604

MAXMF2-HD-FRMTD

107708

AVE 5165

RFS 1X2625

AVE 5160

SAN 30001

AVE 08888

95.954

MMM 670-5AU

FGA10230

SPR-33-1LB

HEWQ2610A

PK-122-5Z2F005 $\underline{\text { Description }}$

$\underline{\text { Par level Unit distribution }}$

\section{HEMATOLOGY SUPPLIES}

7547116

8546931

8547166

8546733

7546914

7546915

8546983

624519
3-Hole Copier Paper - 500 sheets per pack

Batteries, 9-Volt - do not count what is in equip.

Diskettes, 3.5

Dymo Costar Label (1"x2 1/8") - 500 labels per roll

Label - Full Sheet 8.5" x 11" - Lab Label

Labels, Datamax (1.125", clear) - Black Bar Added

Labels, Mailing Label 1" x 2 5/8"

Magic Markers - Sharpie - Fine Point

Magic Markers, Black

Magic Markers, Felt Tip Fine Line (Waterproof)

Post-It Page Markers - 100 pcs per pad / 5 colors

Printer Ribbon, Datamax (Thermal Transfer)

Rubber Bands, \#33

Toner Cartridge - HP2300dn - Spare Only

Toner Cartridge - Kyocera - For Lab Coulter Printer pack

each

box

box

box

roll

box

each

each

each

pack

roll

box

each

each

Coulter 5C Cell Control - REFRIGERATED - New

Machine

Coulter Clenz - 10 Liter

Coulter HMX Pak

Coulter Isoton III

Coulter Latron Control - REFRIGERATED

Coulter Latron Primer - REFRIGERATED

Coulter Lyse-S

Coulter S-Cal - REFRIGERATED

pack

box

box

box

pack

pack

bottle

pack

SPECIALIZED STICKERS \& LABELS

FEDEX Class 9 Label - Dry Ice Label

8CD-130239R

Sticker, "Keep Frozen " - 500 / roll

8CD-130242R

Sticker, "Refrigerate Do Not Freeze" - 500 / roll

roll

roll

roll

\section{PREGNANCY TEST SUPPLIES}

22247114

SA087712

SA087413
Pregnancy Test Kit (ICON 25) - Non-Refrigerated

Sure-Vue hCG Serum Control Set - Refrigerated

Sure-Vue hCG Urine Control Set - Refrigerated box

pack

pack 
Part number Description

$\underline{\text { Par level Unit distribution }}$

JACKETS, GOWNS \& SLIPPERS

$\begin{array}{llcc}\text { 1A-32187 } & \text { Disposable Lab Jackets - 48 Inches Long, Lite Blue } & 1 & \text { box } \\ \text { 18-022838 } & \text { Lab Jackets, Disp. (Blue, 2XL) } & 7 & \text { each } \\ \text { 1721L } & \text { Lab Jackets, Disp. (Blue, Lg) } & 15 & \text { each } \\ 1721 \mathrm{P} & \text { Lab Jackets, Disp. (Blue, Petite) } & 20 & \text { each } \\ 1721 \mathrm{XL} & \text { Lab Jackets, Disp. (Blue, X-Lg) } & 10 & \text { each }\end{array}$

TUBES, VIALS, FILTERS

\begin{tabular}{|c|c|c|c|}
\hline 62.559 .001 & Conical Tube (50mL, red top) - 25 Pieces Per Bag/300 per case & 16 & bag \\
\hline $15-350-49$ & Cryogenic Vial Color Coders - Red Only & 4 & pack \\
\hline SO-10mL & CryoPro 10mL Vial - WITH ADDITIVE (urine/mercury) & 300 & each \\
\hline 14224-382 & CryoPro Cryogenic 10mL Vial - Screw On Cap & 4 & case \\
\hline 14-959-11B & Falcon Culture Test Tube $(14 \mathrm{~mL})$ & 12 & bag \\
\hline $679800-5840$ & Nalgene Cryovial (2.0 mL, Non-Sterile) & 12 & case \\
\hline 03-337-7D & Nalgene Cryovial (2.0 mL, Sterile) & 2 & case \\
\hline 03-337-7H & Nalgene Cryovial (5.0 mL, Sterile) & 10 & se \\
\hline $02-681-50$ & Serum Filter Tube (13mm x 4") & 2 & ack \\
\hline $02-681-52$ & Serum Filter Tube (16mm x 6") & 20 & pack \\
\hline
\end{tabular}

\section{SHIPPING SUPPLIES}

$\begin{array}{llcc}\text { 1A-55968 } & \text { Bag, Ziplock (9" x 12" Clear) - Bitran Replacement Bag } & 1 & \text { case } \\ \text { RB-2-100 } & \text { Box, Cardboard 2" with 10 x 10 grid } & 45 & \text { each } \\ \text { BX-2-D-81 } & \text { Box, Cardboard 2" with 9 x 9 grid } & 200 & \text { each } \\ \text { RB-3-100 } & \text { Box, Cardboard 3" with 10 x 10 grid } & 10 & \text { each } \\ \text { NC335SBC81 } & \text { Box, Cardboard 3" with 9 x 9 grid } & 40 & \text { each } \\ \text { RB-3 } & \text { Box, Cardboard 3" with foam insert - VOC Water Tube Box } & 20 & \text { each } \\ \text { NC335SB3C } & \text { Box, Cardboard 3" without grid } & 70 & \text { each } \\ \text { 95.064.925 } & \text { Box, Cardboard 5.25" x 5.25" with foam insert } & 10 & \text { each } \\ \text { CU-D-25 } & \text { Cell Dividers 3" (5 x 5) } & 70 & \text { each } \\ \text { STP 710 } & \text { Combination Bag and Envelope System - 50 Units per case } & 2 & \text { case } \\ \text { STP 730 } & \text { Combination Bag and Envelope System - 50 Units per case } & 4 & \text { case } \\ \text { STP 740 } & \text { Combination Bag and Envelope System - 50 Units per case } & 1 & \text { case } \\ \text { SEL 10190 } & \text { Envelopes, Large Padded - 10.5" x 16" } & 10 & \text { each } \\ & \text { FEDEX Airbill Pouches } & 2 & \text { pack } \\ & \text { FEDEX Blank Airbills } & 1 & \text { pack } \\ & \text { FEDEX Large Box } & 1 & \text { each } \\ \text { 240-20 } & \text { FEDEX Preprinted Airbill - Warehouse - Stand } & 200 & \text { each } \\ \text { 03-528-18 } & \text { Specific/Throw Away At } & 1 & \text { roll } \\ \text { 03-530-71 } & \text { News-Pak (newsprint on roll) } & 48 & \text { each } \\ \text { MMM 3710-2-TT } & \text { Shipper (Insulated, Styrofoam, Medium) - 16 x 16 x 16 } & 40 & \text { each } \\ \text { 03-528C } & \text { Shipper (Insulated, Styrofoam, Small) - 14 x 14 x 10 } & 10 & \text { roll } \\ & \text { U-Tek Large Refrigerant Packs (24 oz.) } & 10 & \text { case }\end{array}$




\section{COMPONENT TOOLS}

415S0023BY NOTRAX, Floor Mat - Sheilded Sof-Tred, 2x3

6 Y762 Valve Grease - Dow Corning Lubricant \#111

1

1 tube

\section{OSMOLALITY SUPPLIES}

2101

2115

2120

2105

2023

2201

2205

$1432-31$

$1431-31$

2048
Osmometer 100 milliosmoles - Std Solution - 125 mL Bottle Osmometer 1500 milliosmoles - Std Solution - $125 \mathrm{~mL}$ Bottle Osmometer 2000 milliosmoles - Std Solution - $125 \mathrm{~mL}$ Bottle Osmometer 500 milliosmoles - Std Solution - 125 mL Bottle Osmometer 50uL Disposable Sample Tubes - 500/pack Osmometer CON-TROL 100 mOsm - Ref Solution - 5mlL ampules, 12/pk Osmometer CON-TROL 500 mOsm - Ref Solution - 5mlL ampules, 12/pk Osmometer Human Urine Control - Level 2 - 3 bottles per box (refrigerate) Osmometer Human Urine Control - Level 1 - 3 bottles per box (refrigerate) Osmometer Pipette Tips and Cleanettes - 1000 per pack bottle bottle bottle bottle pack

pack

1

pack

1

box

box

pack

\section{SPARE PARTS \& TOOLS}

11458

22-46-139-7

22-46-138-9

22-46-136-2

99833

NC9818625

22461559

40822

66-039-6

Cordless Screwdriver w/ Socket - NEW - Craftsman 4.8 Volt

Unit

Eppendorf Pipettor 100-5000uL

Eppendorf Pipettor 50-1000uL

Eppendorf Pipettor 5-100uL

Hammer (16 oz. Claw) - Used for breaking down dry ice

Pipettor Battery - Spare / Backup Battery

Pipettor Charging Stand

Screwdriver (4 in 1 multi-tip screwdriver)

Screwdriver Set - 6 piece precision - Mfg. Name: Stanley each

each

each

each

each

each

each

each

each

\section{SHIPPING SUPPLIES}

14-241B

8A-18116

11-394-200

11-394-305

1E-24039

SPR 01750
Aluminum Ice Scoop

Full Face Shield

Gloves, Cryo Insulated, Large

Gloves, Cryo Insulated, Medium

Leather Utility Gloves

Tape Gun, 2" each

each

pair

pair

pair

each 


\section{COMPONENT TOOLS}

\begin{tabular}{|c|c|c|}
\hline BPT - Custom & Blood Processing Template - All Ages 1-2, 3-5, 6-11, 12+ & 100 \\
\hline $21-102-52$ & Cube Rack (Clear) for Absorbic Acid - 4 Way Flipper Rack & 2 \\
\hline 03-409-20E & Distilled Water Bottle - Vented & 1 \\
\hline 15-059-147 & $\begin{array}{l}\text { Exact -Temp Refrigerator Thermometer - Red Liquid Therm. } \\
\text { with Bottle } \\
\text { Exact-Temp Freezer Thermometer (Ultra-Low, -90C) - Red }\end{array}$ & 1 \\
\hline 15-059-145 & Liquid Therm. with Bottle & 2 \\
\hline OA-9188 & Flashlight - Black & 1 \\
\hline 08-572-7C & Graduated Cylinder 50mL & 1 \\
\hline $60985-444$ & Multicolor 4-Way Flip Racks - Set of 4 Racks & 1 \\
\hline 14-809-101 & Nalge 13mm Half Rack - Unwire Plastic & 3 \\
\hline 14-809-134 & Nalge 16mm Full Rack - Unwire Plastic & 4 \\
\hline 14-809-117 & Nalge 16mm Half Rack - Unwire Plastic & 5 \\
\hline 03-337-7E & Nalgene Cryovial Rack - Plastic, 50 Well Tray & 100 \\
\hline 9A-33810 & Protective Eyewear & 3 \\
\hline WX-167 & Public Alert Radio - Weather Radio & 1 \\
\hline $15-078 J$ & Thermometer, Calibration - For Centerfuge & 1 \\
\hline 15-077-19 & Thermometer, Solar & 2 \\
\hline 06-662-51 & Timer & 1 \\
\hline 06-662-3 & Timer, Triple Display & 2 \\
\hline 7BC-12097-2 & Wash Bottle - Deionized Water - Clear Squeeze Bottle & 1 \\
\hline 7BC-12097-2 & Wash Bottle - Saline - Clear Squeeze Bottle & 1 \\
\hline
\end{tabular}

\section{OSMOLALITY SUPPLIES}




\section{PHLEBOTOMY INVENTORY}

\section{CONSUMABLE ITEMS:}

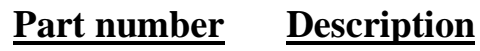

$\underline{\text { Par level Unit distribution }}$

\section{EXAM SUPPLIES}

\begin{tabular}{|c|c|c|c|}
\hline $7000 \mathrm{HP}$ & Air Sickness Bags & 2 & each \\
\hline $58-104$ & Alcohol Wipes & 3 & box \\
\hline 17-98797E & Ammonia Ampules & 1 & box \\
\hline $\mathrm{SO}-\mathrm{BO}$ & Baby Oil & 1 & bottle \\
\hline 496278 & Bandaid, Liquid Bandage - 10 applications per box & 5 & box \\
\hline AB95 & Bandaids - Cartoon Characters & 2 & box \\
\hline 7FQ-47126 & $\begin{array}{l}\text { Bandaids (3/4" x 3" Flex Adhesive) - Coverlet } \\
\text { Butterfly Needle 19G Saf-T EZ Set - } 50 \text { pcs. box / } 4 \text { boxes per }\end{array}$ & 5 & box \\
\hline 387359 & case & 5 & box \\
\hline 367281 & $\begin{array}{l}\text { Butterfly Needle } 21 \text { g Safety - Lok - } 50 \text { pcs. box / } 4 \text { boxes per } \\
\text { case } \\
\text { Butterfly Needle } 23 \text { g Safety - Lok - } 50 \text { pcs. box / } 4 \text { boxes per }\end{array}$ & 6 & box \\
\hline 367283 & case & 1 & box \\
\hline 19-003-428D & Co-Flex NL Cohesive Bandage 1Inch - Multi - Colored Rolls & 1 & case \\
\hline Wet Wipes & Disposable Baby Wipes - Plastic Container & 1 & box \\
\hline 82004-826 & Gauze Pads $(2 \times 2)$ & 6 & pack \\
\hline GOJ-9639 12CT & Handsoap-Waterless (Purrell) - & 1 & bottle \\
\hline 209269 & Heel Warmers (Infant) & 5 & each \\
\hline 531968 & Luer Adapter - Vacutainer - 100 pcs. box / 10 boxes per case & 3 & box \\
\hline $18-917$ & Pillow Cover - for 17" x 23" pillows & 20 & each \\
\hline 934-0852 & Pillow, (disposable, 17 x 23) & 1 & each \\
\hline 530212 & Squeeze Ball (for drawing blood) - Foam 3" Ball & 2 & each \\
\hline Sticker & Sticker, Cartoon (Kids) & 4 & roll \\
\hline TB-1-18 & Tourniquets (latex, lg. Str, 1") & 10 & each \\
\hline Feb-76 & Tourniquets, Stretch (Blue, Vcutnr.) & 20 & box \\
\hline $1530-0$ & Transpore Paper (1/2 " x 10 yds) & 1 & oll \\
\hline $1527-0$ & Transpore Tape (1/2 " x $10 \mathrm{yds})$ & 1 & \\
\hline
\end{tabular}

OFFICE SUPPLIES

107708 Dymo Costar Label (1"x2 1/8") - 500 labels per roll

$7 \quad$ box

FORMS

List of Reported Tests (Handout) - Counted Individually

10

each

VOC SUPPLIES

BD Vacutainer 10mL Gray - VOC Blood Draw Tube

each 
Part number $\quad \underline{\text { Description }}$

FASTING SNACK SUPPLIES

132-745
PC9904
188670
TGP401526P
401025FB

401223FB
Animal Crackers

Juice, Apple - Motts

Juice, Orange - Minute Maid

Plastic Drinking Straw - Individually Wrapped

Plastic Medicine Cup - 1 Ounce

Trutol Glucose Bevarage - Fruit Punch - 24 per case

Trutol Glucose Bevarage - Lemon / Lime - 24 per case

Trutol Glucose Bevarage - Orange - 24 per case

$\underline{\text { Par level Unit distribution }}$

$\begin{array}{cc}288 & \text { each } \\ 200 & \text { each } \\ 150 & \text { each } \\ 2 & \text { box } \\ 2 & \text { sleeve } \\ 36 & \text { each } \\ 48 & \text { each } \\ 72 & \text { each }\end{array}$

\section{TUBES, VIALS, FILTERS}

366643

367921

367856

366668

367861

366450

366431

366430

02-683-101

22-289-953

$14219-240$
BD Hemogard 10 mL EDTA Lavender Top

BD Hemogard 2 mL Gray Top

BD Hemogard 3mL EDTA Lavender Top

BD Hemogard 3mL Red Top

BD Hemogard 4 mL EDTA Purple Top

BD Hemogard 7mL EDTA Lavender Top

BD Hemogard 7mL Red Top

BD Vacutainer $10 \mathrm{~mL}$ Red Top

BD Vacutainer Eclipse (21g x 1 1/4") - 48 needles per box

BD Vacutainer Tube Holder - 250 pcs. bag / 4 bags per case

KS Vacutainer 15mL Red Top

$\begin{array}{cc}5 & \text { tray } \\ 3 & \text { tray } \\ 6 & \text { tray } \\ 1 & \text { tray } \\ 6 & \text { tray } \\ 1 & \text { tray } \\ 2 & \text { tray } \\ 7 & \text { tray } \\ 1 & \text { box } \\ 500 & \text { each } \\ 8 & \text { tray }\end{array}$

\section{COMPONENT TOOLS}

415S0023BY NOTRAX, Floor Mat - Sheilded Sof-Tred, 2x3

each

\section{EMERGENCY SUPPLIES}

CPR Mask - Adult

each

\section{COMPONENT TOOLS}

ML4042

14-809-130

14-809-101

$14-809-117$

30-1310-05

15-077-19
Footstool - Metal

Nalge 13mm Full Rack - Unwire Plastic

Nalge 13mm Half Rack - Unwire Plastic

Nalge 16mm Half Rack - Unwire Plastic

Scissors - Lister bandage (5 1/2 " Stainless Steel)

Thermometer, Solar each

each

each

each

each

each 